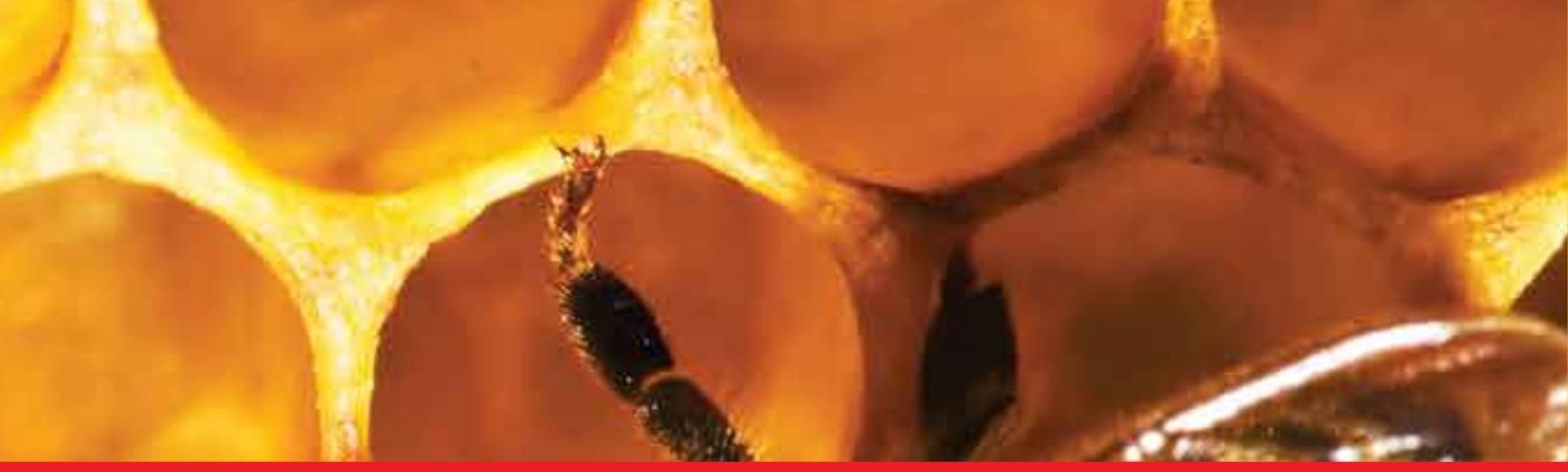

\title{
IntechOpen
}

\section{Biomimetic Based Applications}

Edited by Anne George

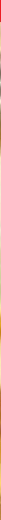





\section{BIOMIMETIC BASED APPLICATIONS}

Edited by Anne George 


\section{Contributors}

Katia ciuffi, Emerson de Faria, Gustavo Ricci, Frederico Matias Lemos, Marcio LuÃs Andrade Silva, Ademar Alves, Paulo Calefi, Eduardo Nassar, Wantai Yang, Tofig Nagiev, Rumiana Dimova, Peng Yang, Reinaldo de Bernardi, José Jaime Jaime Da Cruz, Arturo Forner-Cordero, Can-Cheng Guo, Guo-Fang Jiang, Ming-Hsi Chiang, Rong Xiu Li, Renate Naumann, Denise Schach, Marc GRosserueschkamp, Christoph Nowak, Carola Hunte, Wolfgang Knoll, Yu Takano, Kizashi Yamaguchi, Haruki Nakamura, Mariusz Trytek, Marek Majdan, Jan Fiedurek, Hongbing Ji, Xiantai Zhou, Fernando P. Lima, Nicholas Burnett, Brian Helmuth, David Wethey, Nicole Kish, Kyle Aveni-Deforge, daqing wei, yu zhou, Ana Maria Carmona-Ribeiro, Lilian Barbassa, Letícia Melo, Ryo Yoshida, Chao-hai Wei, Yuan Ren, Xiao-xuan Zhang, Xu-biao Yu, Benjamin Evans, Rich Superfine, Maura Pellei, Carlo Santini, Andreas Katsiamis, Emmanuel Drakakis

\section{(c) The Editor(s) and the Author(s) 2011}

The moral rights of the and the author(s) have been asserted.

All rights to the book as a whole are reserved by INTECH. The book as a whole (compilation) cannot be reproduced, distributed or used for commercial or non-commercial purposes without INTECH's written permission.

Enquiries concerning the use of the book should be directed to INTECH rights and permissions department (permissions@intechopen.com).

Violations are liable to prosecution under the governing Copyright Law.

\section{(cc) BY}

Individual chapters of this publication are distributed under the terms of the Creative Commons Attribution 3.0 Unported License which permits commercial use, distribution and reproduction of the individual chapters, provided the original author(s) and source publication are appropriately acknowledged. If so indicated, certain images may not be included under the Creative Commons license. In such cases users will need to obtain permission from the license holder to reproduce the material. More details and guidelines concerning content reuse and adaptation can be foundat http://www.intechopen.com/copyright-policy.html.

\section{Notice}

Statements and opinions expressed in the chapters are these of the individual contributors and not necessarily those of the editors or publisher. No responsibility is accepted for the accuracy of information contained in the published chapters. The publisher assumes no responsibility for any damage or injury to persons or property arising out of the use of any materials, instructions, methods or ideas contained in the book.

First published in Croatia, 2011 by INTECH d.o.o.

eBook (PDF) Published by IN TECH d.o.o.

Place and year of publication of eBook (PDF): Rijeka, 2019.

IntechOpen is the global imprint of IN TECH d.o.o.

Printed in Croatia

Legal deposit, Croatia: National and University Library in Zagreb

Additional hard and PDF copies can be obtained from orders@intechopen.com

Biomimetic Based Applications

Edited by Anne George

p. cm.

ISBN 978-953-307-195-4

eBook (PDF) ISBN 978-953-51-6007-6 


\section{We are IntechOpen, \\ the world's leading publisher of Open Access books}

\section{Built by scientists, for scientists}

\section{$4,400+$}

Open access books available

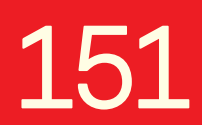

Countries delivered to

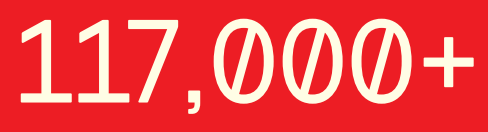

International authors and editors
$130 \mathrm{M}+$

Downloads

Our authors are among the

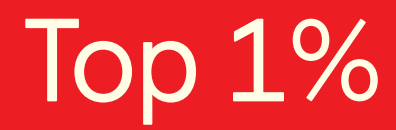

most cited scientists

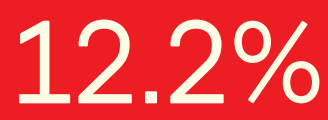

Contributors from top 500 universities

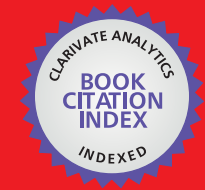

WEB OF SCIENCE ${ }^{\text {IM }}$

Selection of our books indexed in the Book Citation Index in Web of Science ${ }^{\mathrm{TM}}$ Core Collection (BKCI)

\section{Interested in publishing with us? \\ Contact book.department@intechopen.com}

Numbers displayed above are based on latest data collected.

For more information visit www.intechopen.com 



\section{Meet the editor}

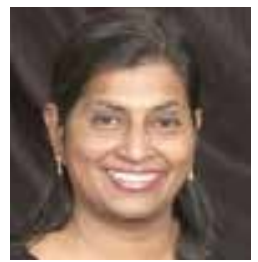

Anne George received her PhD in Physical Chemistry from Madras University, India in 1983. She then did her Postdoctoral work with Dr. Arthur Veis at Northwestern University on determining the structure of type I collagen in solution using Fourier Transform Infrared Spectroscopy. She joined as an Assistant Professor in 1993 at Northwestern University where she started work on the cloning of the dentin matrix proteins. She was instrumental in identifying the family of dentin matrix proteins from the rat odontoblasts. She was awarded a "Teaching Excellence Award" from Northwestern University. She then moved to the University of Illinois at Chicago in 1998 as an Associate Professor, became a Full Professor in 2003 , and continued her work on noncollagenous proteins and their role in biomineralization. She is now an Allan G. Brodie Endowed Professor at the University of Illinois at Chicago. Her work was reported in Chicago Tribune "Calcium Link-Genes may solve mystery of how teeth harden" in 1994 and Scientific Year Book of Encyclopedia Britannica "Tooth Gene Studied" in 1998. A documentary on her work was produced by Dallas TV. She is the recipient of the IADR Basic Research Award in "Pulp Biology and Regeneration" in 2008. In 2011 she was conferred with the honor of doctor honoris causa from the University Paris Descartes. Dr. George is the author of over 90 papers in peer-reviewed journals. Her research focuses on biomineralization related proteins and their application as templates in biomimetic mineralization studies and as protein-based templates for bone and dentin regeneration. 



\section{Contents}

Preface XIII

Chapter 1 Biomimetic Epoxidation of Olefins Catalyzed by Metalloporphyrins with Molecular Oxygen 1 Hong-Bing Ji and Xian-Tai Zhou

Chapter 2 Biomimetic Oxidation of Hydrocarbons with Air over Metalloporphyrins 31 Guofang Jiang, Qiang Liu and Cancheng Guo

Chapter 3 Homogeneous and Heterogeneous Free-Based Porphyrins Incorporated to Silica Gel as Fluorescent Materials and Visible Light Catalysts Mimic Monooxygenases 59 Mariusz Trytek, Marek Majdan and Jan Fiedurek

Chapter 4 Physicochemical Peculiarities of Iron Porphyrin - Containing Electrodes in Catalase - and Peroxidase

- Type Biomimetic Sensors 105

T.M.Nagiev

Chapter 5 Design of Biomimetic Models Related to the Active Sites of Fe-Only Hydrogenase 123

Yu-Chiao Liu, Ling-Kuang Tu, Tao-Hung Yen and Ming-Hsi Chiang

Chapter 6 The Improvement of LC-MS/MS Proteomic Detection with Biomimetic Affinity Fractionation 141 Rong-Xiu Li, Qing-Qiao Tan and De-Xian Dong

Chapter 7 Green Oxidation Reactions of Drugs Catalyzed by Bio-inspired Complexes as an Efficient Methodology to Obtain New Active Molecules Emerson Henrique de Faria, Gustavo Pimenta Ricci, Frederico Matias Lemos, Marcio Luis Andrade e Silva, Ademar Alves da Silva Filho, Paulo Sérgio Calefi, Eduardo José Nassar and Katia Jorge Ciuffi 
Chapter 8 Chemical Indices of the Biomimetic Models

of Oxyhemocyanin and Oxytyrosinase 183

Yu Takano, Kizashi Yamaguchi and Haruki Nakamura

Chapter 9 Bioactive Microarc Oxidized TiO2-based

Coatings for Biomedical Implication 201

Daqing Wei and Yu Zhou

Chapter 10 Antimicrobial Biomimetics 227

Ana Maria Carmona-Ribeiro,

Lilian Barbassa and Letícia Dias de Melo

Chapter 11 Biomimetic Adsorbents: Enrichment of Trace Amounts of Organic Contaminants (TAOCs) in Aqueous Solution

Chao-Hai Wei, Xiao-Xuan Zhang, Yuan Ren and Xu-Biao Yu

Chapter 12 Spectro-Electrochemical Investigation of the $\mathbf{b c}_{1}$ Complex from the Yeast Saccharomyces cerevisiae using Surface Enhanced B-Band Resonance Raman Spectroscopy 311 Denise Schach, Marc Großerüschkamp, Christoph Nowak, Carola Hunte, Wolfgang Knoll and Renate L. C. Naumann

Chapter 13 Self-Oscillating Gel as Novel Biomimetic Materials $\mathbf{3 3 3}$ Ryo Yoshida

Chapter 14 Non-Calcium Inorganic Materials Fabrication by Surface-Immobilized Organic Molecular Template $\mathbf{3 4 9}$ Peng Yang, Wantai Yang, Xu Zhang and Jinchun Chen

Chapter 15 Biomimetic Applications of Metal Systems Supported by Scorpionates 385 Maura Pellei and Carlo Santini

Chapter 16 Analogue CMOS Cochlea Systems:

A Historic Retrospective $\mathbf{4 2 9}$

Andreas Katsiamis and Emmanuel Drakakis

Chapter 17 Design Considerations for Magnetically Actuated Biomimetic Cilia 473 Benjamin Evans and Rich Superfine

Chapter 18 Monitoring the Intertidal Environment with Biomimetic Devices 499

Fernando P. Lima, Nicholas P. Burnett, Brian Helmuth, Nicole Kish, Kyle Aveni-Deforge and David S. Wethey

Chapter 19 Nanoparticle Synthesis in Vesicle Microreactors $\mathbf{5 2 3}$ Peng Yang and Rumiana Dimova 
Chapter 20 Biologically Inspired Locomotion Control of a Climbing Robot 553

Reinaldo de Bernardi, Arturo Forner-Cordero and José Jaime Da Cruz 



\section{Preface}

Biomimetics is the science of emulating nature's design. In nature, living organisms synthesize mineralized tissues and this process of biomineralization is under strict biological control. It involves the interactions of several biological macromolecules among themselves and with the mineral components. Generally, natures design principles are based on a "Bottom-Up" strategy. Such processes lead to the formation of hierarchically structured organic-inorganic composites with mechanical properties optimized for a given function. A common theme in mineralized tissues is the intimate interaction between the organic and inorganic phases and this leads to the unique properties seen in biological materials. Therefore, understanding natures design principles and ultimately mimicking the process may provide new approaches to synthesize biomaterials with unique properties for various applications. Biomimetics as a scientific discipline has experienced an exceptional development. Its potential in several applications such as medical, veterinary, dental science, material science and nanotechnology bears witness to the importance of understanding the processes by which living organisms exert an exquisite control on the fabrication of various materials. Despite several breakthroughs, there exist only a limited number of methods for the preparation of advanced materials. Consequently, precisely controlling the architecture and composition of inorganic materials still remain enigmatic. Biological organisms have the extraordinary ability to fabricate a wide variety of inorganic materials into complex morphologies that are hierarchically structured on the nano, micro and macroscales with high fidelity. The next generation of biologically inspired materials fabrication methods must draw inspiration from complex biological systems.

The interaction between cells, tissues and biomaterial surfaces are the highlights of the book "Advances in Biomimetics". In this regard the effect of nanostructures and nanotopographies and their effect on the development of a new generation of biomaterials including advanced multifunctional scaffolds for tissue engineering are discussed. The 2 volumes contain articles that cover a wide spectrum of subject matter such as different aspects of the development of scaffolds and coatings with enhanced performance and bioactivity, including investigations of material surface-cell interactions.

Anne George

University of Illinois at Chicago, Department of Oral Biology,

Chicago,

USA 



\title{
Biomimetic Epoxidation of Olefins Catalyzed by Metalloporphyrins with Molecular Oxygen
}

\author{
Hong-Bing Ji and Xian-Tai Zhou \\ School of Chemistry and Chemical Engineering, Sun Yat-sen University, 510275, \\ Guangzhou, China
}

\section{Introduction}

The direct oxidation of hydrocarbon is a field of both academic and industrial importance and challenge.[1-3] Catalytic oxidation is a key technology for converting petroleum-based feedstock to useful chemicals of a high oxidation state such as alcohols, carbonyl compounds, and epoxides. Millions of tons of these compounds are annually produced worldwide and find applications in all areas of chemical industries.[4-6]

Epoxidation of olefins is an important reaction in organic synthesis because the formed epoxides are intermediates that can be converted to a variety of products.[7-10] Access to a variety of epoxides has largely been successful due to the remarkable catalytic activity of transition metal complexes, which have a unique ability to bring the alkene substrate and the oxygen source within the coordination sphere of the metal leaving to a facial transfer of oxygen atom to the carbon-carbon double bond.[11-15]

Cytochrome P-450 enzymes are heme-containing monooxygenases and play a key role in the oxidative transformation of endogeneous and exogeneous molecules.[16-20] They are virtually ubiquitous in nature and are present in all forms of life like plants and mammals, as well as in some prokaryotic organisms such as bacteria.[21-23] The active site of P-450s contains a highly conserved prosthetic heme IX complex coordinated by a thiolate ligand from a cysteine residue (Figure 1).

The primary function of cytochrome P-450 enzymes is the oxygenation of a wide variety of organic substrates by inserting one oxygen atom from $\mathrm{O}_{2}$ to the substrate and reducing the other oxygen atom with reducing equivalents to a water molecule, utilizing two electrons that are provided by $\mathrm{NAD}(\mathrm{P}) \mathrm{H}$ via a reductase protein (Scheme 1).

$$
\mathrm{R}-\mathrm{H}+\mathrm{O}_{2}+\mathrm{NAD}(\mathrm{P}) \mathrm{H}+\mathrm{H}^{+} \stackrel{\text { cytochrome P-450 }}{\longrightarrow} \mathrm{R}-\mathrm{OH}+\mathrm{H}_{2} \mathrm{O}+\mathrm{NAD}(\mathrm{P})^{+}
$$

Scheme 1. Overall oxygenation reaction catalyzed by cytochrome P-450

Being a triplet (two unpaired electrons in ground state), molecular oxygen is unreactive toward organic molecules at low temperatures. The reaction of dioxygen with the single state of organic substrates is spin-forbidden.[24] Consequently, the oxygenation of organic molecules at physiological temperatures must involve the modification of the electronic structure of one of the partners. Living systems mainly use enzymes like cytochromes P-450 to modify the electronic structure of dioxygen to form which is adapted for the desired 


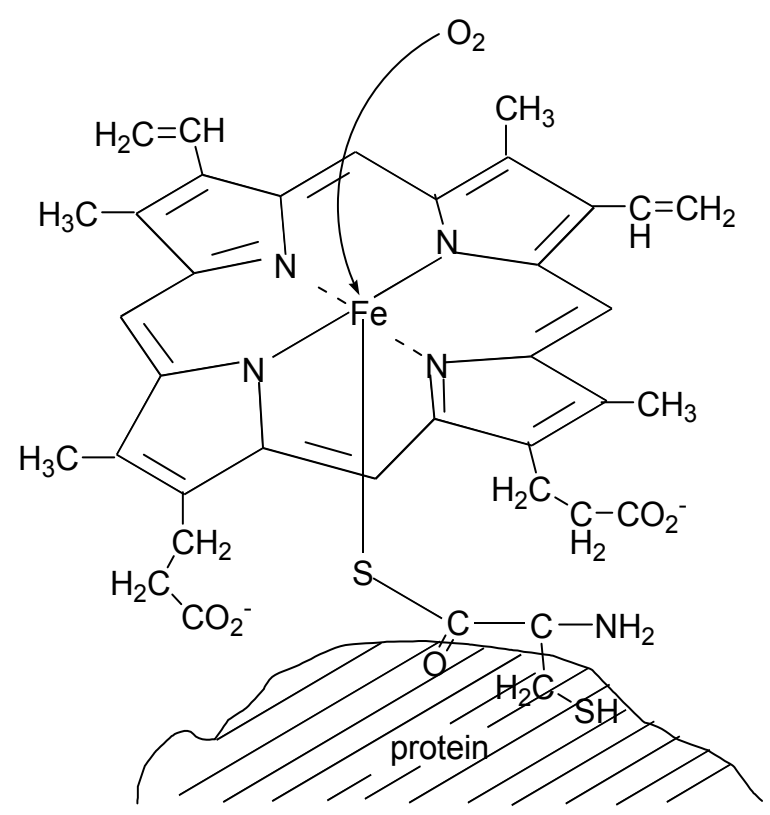

Fig. 1. Prosthetic of cysteinato-heme enzymes: an iron(III) protoporphyrin-IX covalently linked to the protein by the sulfur atom of a proximal cysteine ligand.

oxidation reaction. The mechanism of its catalytic activity and structural functions has been the subject of extensive investigation in the field of biomimetic chemistry. The high-valent iron(IV)-oxo intermediate, formed by the reductive activation of molecular oxygen via peroxoiron(III) and hydroperoxy-iron(III) intermediates by cytochrome P-450, is responsible for the in vivo oxidation of drugs and xenobiotics. This high valent iron(IV)-oxo intermediate and probably other intermediates of the $\mathrm{P} 450$ catalytic cycle can be formed by the reaction of iron(III) porphyrins with different monooxygen donors.[25-27] Therefore, cytochrome P-450 enzymes are potent oxidants that are able to catalyze the hydroxylation of saturated carbonhydrogen bonds, the epoxidation of double bonds, the oxidative dealkylation reactions of aminies, oxidations of aromatics, and the oxidation of heteroatoms. [28-30]

As the isolation of P-450 enzymes from plants is extremely difficult, the first reactions employing this hemoprotein's enzymes were carried out with bacterial and mammalian P-450. Only in recent years have genes of P-450 enzymes been isolated from plants, and the first reactions confirmed that these enzymes take an active part in herbicide detoxification. [31] The use of chemical model systems mimicking P-450 might therefore be a very useful tool for overcoming the difficulty in working with enzymes in vivo and vitro.[32] The synthesis of cytochrome P-450 models is a formidable challenge for chemist to establish a system that is structurally equivalent to the enzymes. The synthetic mimic not only is a structural analogue exhibiting spectroscopic features close to the enzyme's cofactor but also displays a similar reactivity and catalysis.[33] In recent years, the development of efficient catalytic systems for oxidation reactions that mimic the action of cytochrome P-450 dependent momooxygenases has attracted much attention.[34-42] Synthetic metalloporphyrins have been used as cytochrome P-450 models and have been found to be highly efficient homogeneous or heterogeneous catalysts for oxidation reactions, especially for the alkane hydroxylation and alkene epoxidation.[43-45] 
During the past two decades, the use of metalloporphyrins as catalysts for the epoxidation of olefins has received increasing attention since the leading works of Groves and co-workers by using iodosylbenzene (PhIO) as oxygen atom donor.[46] A variety of oxidants, such as hydrogen peroxides,[47-49] iodosylbenzene,[50-52] magnesium monoperoxyphthalate, [53-54] tetrabutylammonium monosulfate and eriodate,[55-56] in combination with a large variety of metalloporphyrin catalysts have been employed as oxygen atom donors.

For economic and environmental viewpoints, the aerobic epoxidation of olefins catalyzed by metalloporphyrins is attracting more interests. The chapter will try to cover the biomimetic homogeneous and heterogeneous aerobic epoxidation of olefins catalyzed by metalloporphyrins in the recent years. It will focus on the modeling of the monooxygenase catalytic circle with synthetic metalloporphyrins. Since the stioichiometry of a monooxygenase-mediated oxygenation requires two electrons and two protons to reduce the second oxygen atom of dioxygen to water, most of works reported involve an electron source: borohydride, hydrogen and colloidal platinum, zinc powder, electrons from an electrode or aldehyde as reductant.[57-61] According to the sacrificial use of electrons, the recent advances in this section will focus on presenting the metalloporphyrin- mediated epoxidation in the presence of zinc powder, aldehyde as reductant or in absence of reductant. Both practical and mechanistic point of view for the epoxidation of olefins catalyzed by metalloporphyrins will be presented.

\section{Zinc powder as reductant}

A viologen-linked $\mathrm{Mn}(\mathrm{III})$ porphyrin complex $\left(\mathrm{MnPC}_{\mathrm{x}} \mathrm{MV}\right.$, Scheme 2) with a short methylene-chain, in which a viologen is covalently linked by the methylene-chain into one phenyl group of 5,10,15,20- tetraphenylporphyrinatomanganese(III) chloride (MnTPPCl), was used as catalyst for a monooxygenase of cyclohexene in an air-equilibrated acetonitrile solution containing insoluble zinc powder as reductant, more cyclohexene oxide was obtained as a single product than when $\mathrm{MnTPPCl}$ was used as catalyst.[62] According to the reaction of an air-equilibrated acetonitrile suspension containing $1 \times 10^{-4} \mathrm{M} \mathrm{MnTPPCl}, 1 \times 10^{-4}$ M 1-MeIm (1-methylimidazole), $7.3 \times 10^{-2} \mathrm{M}$ zinc powder, $2 \times 10^{-2} \mathrm{M}$ benzoic acid (the cleaving reagent of dioxygen double-bond) and $0.47 \mathrm{M}$ cyclohexene for $3 \mathrm{~h}$ at $30^{\circ} \mathrm{C}$, about $1 \times 10^{-3} \mathrm{M}$ epoxide was obtained as the single oxidation product of cyclohexene. Since the turnover number of $\mathrm{MnTPPCl}$ was about 10, it was found that $\mathrm{MnTPPCl}$ acted as catalyst.

Further, when $1 \times 10^{-4} \mathrm{M} \mathrm{MnPC}_{2} \mathrm{MV}$ or the mixture of $1 \times 10^{-4} \mathrm{M} \mathrm{MnTPPCl}$ and $1 \times 10^{-4} \mathrm{M} \mathrm{MV}^{2+}$ was used as the catalyst, the amount of the product epoxide remarkably increased and the turnover number reached about 40 for $3 \mathrm{~h}$. The time-dependence of the amount of the product epoxide is shown in Figure 2. This suggests that viologen and the viologen moiety in $\mathrm{MnPC}_{2} \mathrm{MV}$ acted as the mediator for the electron transfer from zinc powder to $\mathrm{Mn}$ porphyin.

Enhancement of cyclohexene oxide was produced by adding an axial ligand for MnTPPCl such as $\mathrm{Cl}^{-}, \mathrm{Br}^{-}$in the catalytic system.[63] The $\mathrm{Cl}^{-}$and $\mathrm{Br}^{-}$promoted this epoxidation probably by assisting the oxygen transfer from $\mathrm{Mn}(\mathrm{V})$-oxo complex, that is an intermediate in this reaction cycle, to cyclohexene, and $\mathrm{HV}^{2+}$ functioned as the mediator of electron transfer from zinc to Mn(II)TPP-dioxygen adduct, enhancing the production of epoxide. However, when a small amount of $1-\operatorname{MeIm}\left(<10^{-2} \mathrm{M}\right)$ was added in this system containing $\mathrm{HV}^{2+}$, the epoxide was not produced and zinc was hardly consumed. Moreover, a larger amount of epoxide was obtained by adding 1-MeIm further $\left(>10^{-2} \mathrm{M}\right)$. A plausible mechanism was proposed as shown in Figure 3. 


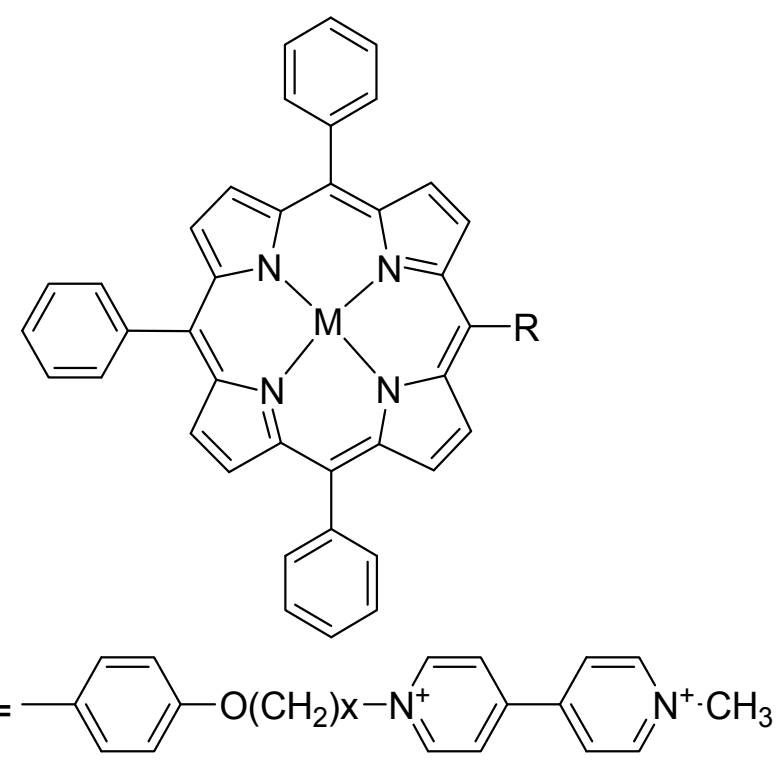

Scheme 2. Structure and abbreviation of covalently linked manganese(III) porphyrinsviologen

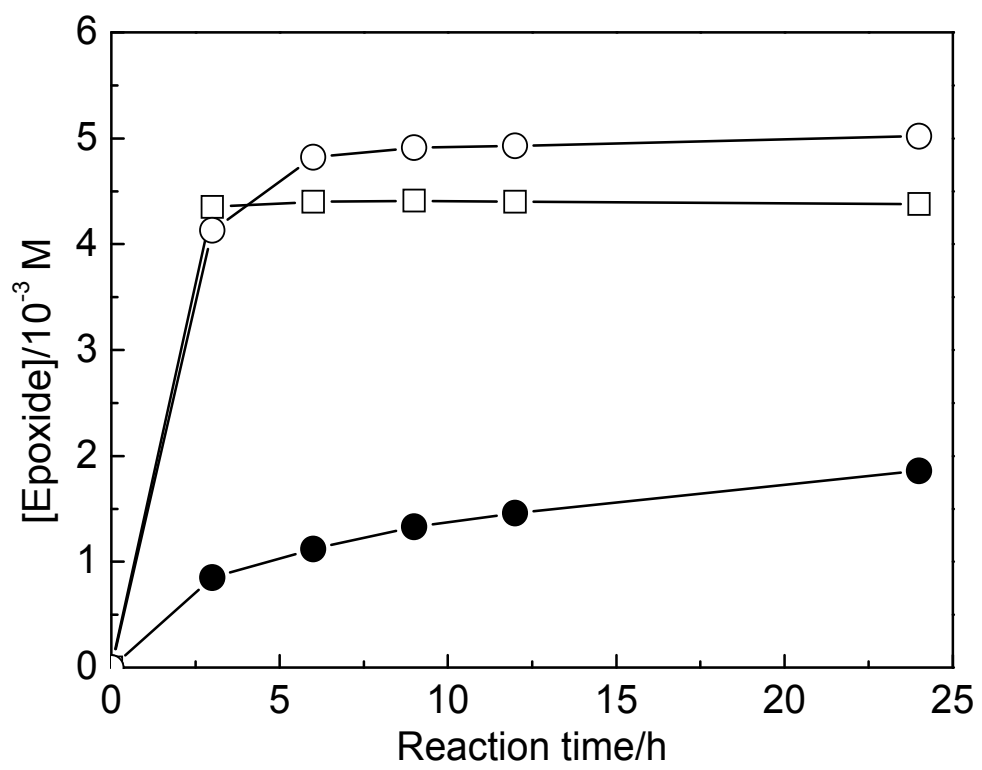

Fig. 2. Time-dependence of the amount of produced epoxide in air-equilibrated acetonitrile suspension containing $1 \times 10^{-4} \mathrm{M}$ Mn porphyrin, $5 \times 10^{-3} \mathrm{M} 1-\mathrm{MeIm}, 7.3 \times 10^{-2} \mathrm{M}$ zinc powder, $2 \times 10^{-2} \mathrm{M}$ benzoic acid and $0.47 \mathrm{M}$ cyclohexene at $30^{\circ} \mathrm{C}$. Catalyst: $\operatorname{MnTPPCl}(\mathbf{O})$, $\mathrm{MnPC}_{2} \mathrm{MV}(\mathrm{O})$ and $\mathrm{MnTPPCl}+1 \times 10^{-4} \mathrm{M} \mathrm{MV}^{2+}(\square)$ 


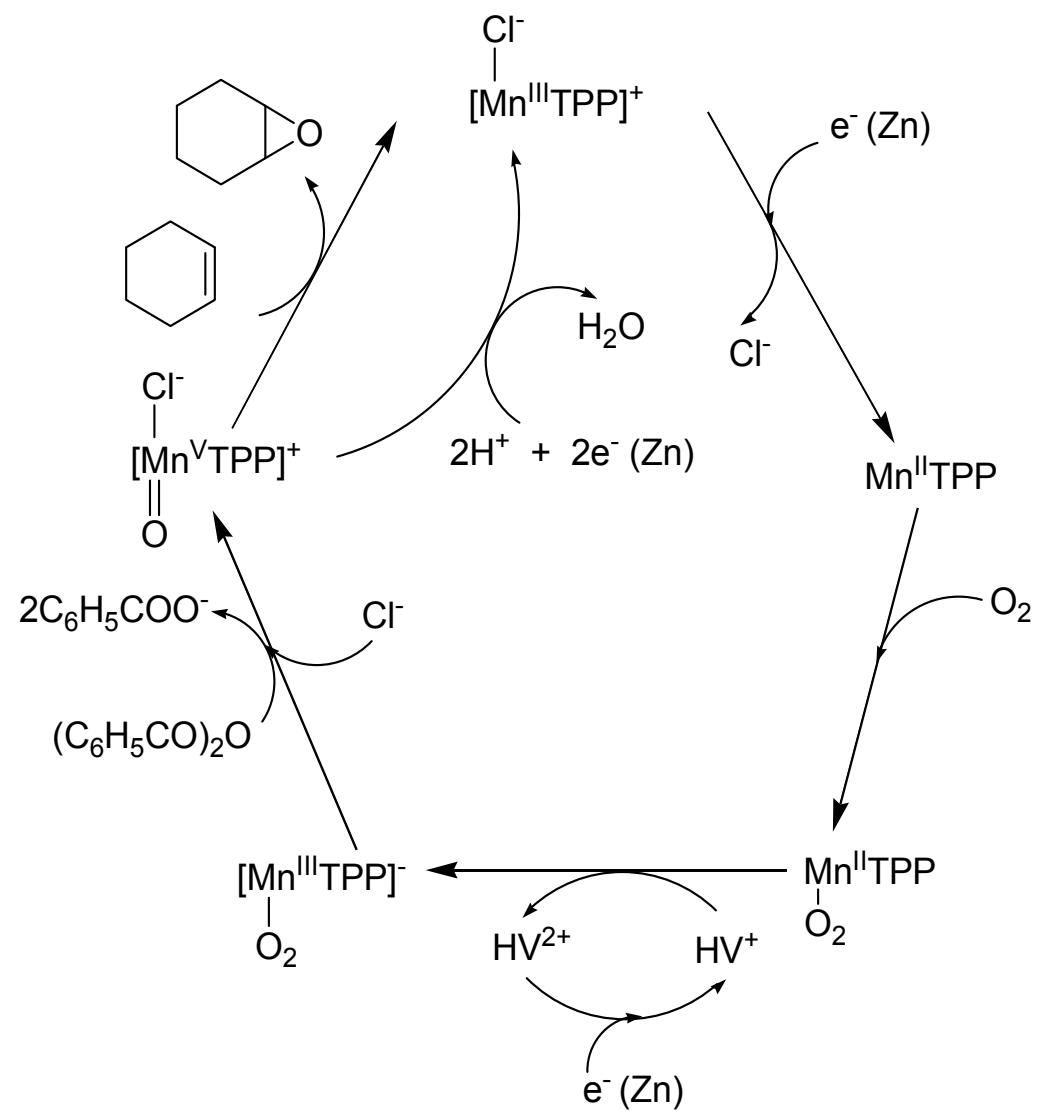

Fig. 3. Catalytic epoxidation cycle of cyclohexene using $\mathrm{MnTPPCl}$ as catalyst in the absence of 1-MeIm

The hexylviologen acted effectively as the mediator of electron transfer from zinc powder to $\mathrm{Mn}(\mathrm{II}) \mathrm{TPP}-\mathrm{O}_{2}$ adduct. Since the halogen ion can coordinate easily to the manganese ion in $[\mathrm{Mn}(\mathrm{V}) \mathrm{TPP}=\mathrm{O}]^{+}$cation due to an additive electrostatic interaction, the oxygen transfer proceeded by the assistance of its coordinate bond. Thus, when $\mathrm{HV}^{2+}$ was added in the catalytic system using the Mn porphyrin catalyst with the $\mathrm{Cl}^{-}$counter ion, the dioxygenactivated reductive epoxidation of cyclohexene occurred even in the absence of 1-MeIm.

According to the mechanism, the decrease of the epoxide production with increasing 1MeIm $\left(<10^{-2} \mathrm{M}\right)$ may be explained from the factor that the coordination of 1-MeIm to $\mathrm{Mn}(\mathrm{II}) \mathrm{TPP}$ depressed the formation of the $\mathrm{Mn}(\mathrm{II}) \mathrm{TPP}-\mathrm{O}_{2}$ adduct. Further, the epoxide was obtained by adding a large amount of 1-MeIm $\left(>10^{-2} \mathrm{M}\right)$ because the reducing power of $\mathrm{Zn}$ may become strong by a complex formation between $\mathrm{Zn}^{2+}$ and 1-MeIm.

\section{Aldehydes as reductant}

Aldehyde is another effective reducing agent for the epoxidation of olefins with dioxygen as oxidant. Mukaiyama reported an efficient approach for epoxidation of olefins using dioxygen as oxidant under ambient conditions. The process involved use of $\beta$-diketonate complexes of $\mathrm{Ni}^{2+}, \mathrm{Co}^{2+}$, and $\mathrm{Fe}^{3+}$ as catalysts and an aldehyde as oxygen acceptor.[64-66] 
Subsequently, many metal catalysts e.g. manganese complex, cobalt-containing molecular sieves and metalloporphyrins demonstrated highly catalytic performance for the aerobic oxidation in the presence of aldehyde.[67-70]

Mandal and co-workers reported the epoxidation of various olefins using cobalt porphyrins (Scheme 3) in ambient molecular oxygen and 2-methylpropanal.[71]

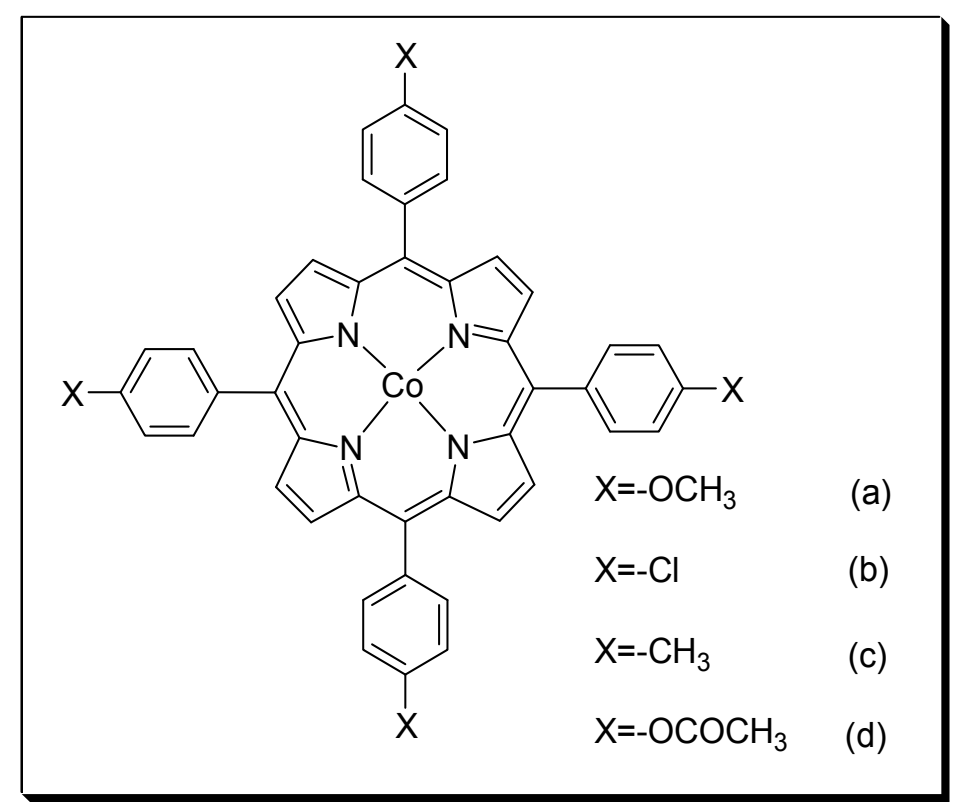

Scheme 3. Structures of cobalt prophyrins used in the epoxidation of olefins

Methyl styrene, stilbene and farnesyl acetate were transformed to the corresponding epoxides in nearly quantitative yield (Table 1). It is noteworthy that trans-stilbene afforded the corresponding trans-epoxide (entry 2). While highly regioselective monoepoxidation of farnesyl acetate to give epoxide was observed under these conditions (entry 3). Similarly, limonene was readily transformed to a mixture of mono and diepoxide in 1:2.3 ratio in quantitative yield (entry 4). Interestingly, the $\alpha, \beta$ unsaturated carbonyl compounds i.e. chalcone (entry 5) and ethyl cinnamate (entry 6) were epoxidized in good yields to give stereochemically pure trans epoxides respectively.

In order to probe the substitute effect in the para position of aromatic ring of porphyrin, the catalytic activities of (a)-(c) were studied in the epoxidation of cyclohexene. Thus the oxidation of cyclohexene using (a) as catalyst afforded a mixture of corresponding epoxide, cyclohexenol, cyclohexenone in 1:3:1.2 ratio. On the other hand oxidation of cyclohexene in the presence of (b) yielded only a mixture of cyclohexenol and cyclohexenone whereas catalysis under (c) gave rise to a mixture of epoxide, cyclohexenol and cyclohexenone respectively. These results could be conceivable that the oxidation are proceeding via an analogous cobalt oxo species.

The simple structural metalloporphyrins has proven to be an excellent catalyst for the epoxidation of olefins in the presence of molecular oxygen and isobutylaldehyde. As a part of metalloporphyrins-catalyzed oxidations of our group works, the epoxidation of olefins catalyzed by very small amount of MnTPP (manganese meso-tetraphenyl porphyrin) was 


\begin{tabular}{|c|c|c|c|}
\hline Entry & Substrate & Productb & Yields (\%)a \\
\hline 1 & $\mathrm{Ph}$ & & 94 \\
\hline 2 & $P$ & $P$ & 100 \\
\hline 3 & & & 80 \\
\hline 4 & & & $100 c$ \\
\hline 5 & O & 0 & 83 \\
\hline 6 & 0 & 0 & 51 \\
\hline
\end{tabular}

${ }^{a}$ Isolated yield. ${ }^{b}$ Yield determined from ${ }^{1} \mathrm{H}-\mathrm{NMR}$ of the crude reaction mixture.

Cbtained as a mixture of syn-anti diastereomers

Table 1. Co(II) porphyrin catalyzed oxidation of olefins using 2-methylpropanal and dioxygen reported (Scheme 4), in which extremely high turnover number that could be comparable to most enzyme catalysis was obtained.[72] When the amount of manganese mesotetraphenylporphyrin catalyst was $2.5 \times 10^{-8} \mathrm{mmol}$, the cyclohexene oxide could be obtained with the isolated yield of $90 \%$.

It should be mentioned that the turnover number of the present catalyst could reach $731,470,480$. Since commonly, TOF is used to express the catalytic efficiency of enzyme with the definition as converted substrate $(\mathrm{mol})$ per enzyme $(\mathrm{mol})$ per minute. The TOF of most enzymes is about $1000 \mathrm{~min}^{-1}$ or more. For example, the TOF of catalase is $6 \times 10^{6} \mathrm{~min}^{-1}$, and the TOF of $\beta$-galactosidase is $1.25 \times 10^{4} \mathrm{~min}^{-1}$. In the present manganese meso-tetraphenylporphyrin catalyzed system, the TOF reaches up to $1.2 \times 10^{6} \mathrm{~min}^{-1}$, which is the range for enzyme activity. Also, various olefins could be smoothly converted to corresponding epoxides in the catalytic system under ambient conditions. As shown in Table 2, it seems that the efficiency of epoxidation in this catalytic system is very dependent on the steric structure of substrates. The influence of steric effects could further be found when styrene and its derivatives were oxidized, the conversion rates of styrene, trans- $\beta$-methylstyrene and trans-stilbene were $95 \%$, $89 \%$ and $86 \%$ after reacting for $4.5,7.0$ and $8.0 \mathrm{~h}$, again demonstrating a steric effect (entries 4-6). 


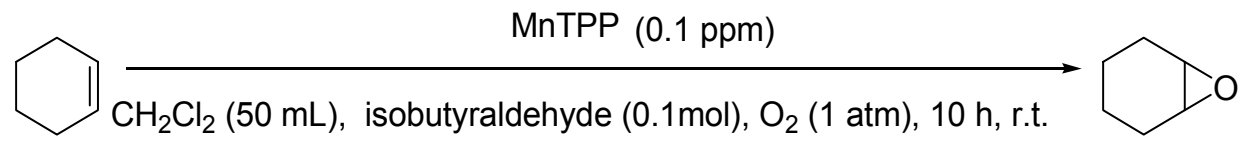

$20 \mathrm{mmol}$

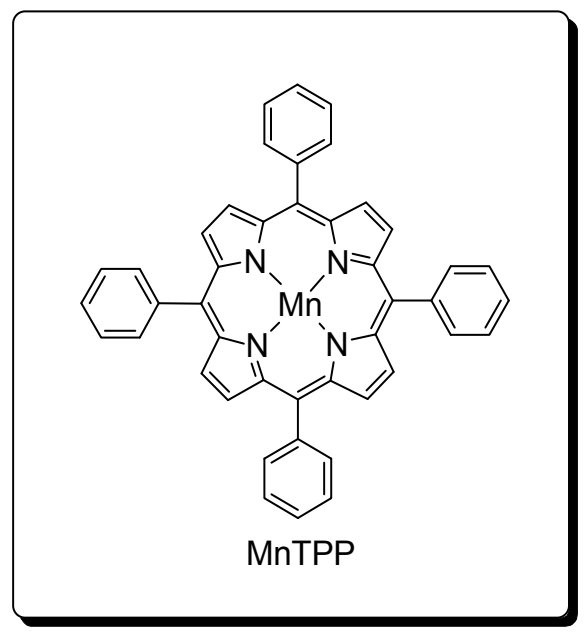

isolated yield: $90 \%$

TON: $731,470,480$

TOF: $1.2 \times 10^{6} \mathrm{~min}^{-1}$

Scheme 4. Manganese meso-tetraphenylporphyrin catalyzed epoxidation of olefins

Similarly, in the epoxidation of other cycloolefin e.g. cyclooctene, the reaction system exhibits high catalytic performance with 93\% yield of cyclooctene epoxide (entry 7). Epoxidation of linear chains e.g. 1-octene and trans-2-octene smoothly proceeded with high conversion and yield, and similar catalytic activities for the two substrates shows the located position of $\mathrm{C}=\mathrm{C}$ bond on linear chain alkenes could hardly influence their catalytic performance (entries 8-9).

Despite of the high efficiency of the catalyst system, another salient feature of the present epoxidation is its high regioselectivity (entry 10). In addition, the catalyst system exhibits specific selective oxidation performance towards $\mathrm{C}=\mathrm{C}$ bond and hydroxyl group activation. $\mathrm{C}=\mathrm{C}$ bond was preferentially activated and the corresponding epoxide as the only product with $90 \%$ yield could be obtained for the cinnamyl alcohol oxidation, and no products from hydroxyl group oxidation could be detected (entry 11).

The extremely high catalytic performance (comparable to enzymes) exhibited by manganese meso-tetraphenylporphyrin is most interesting. In order to gain insight into the likely reasons, the epoxidation processes of cyclohexene in the presence of isobutyraldehyde and molecular oxygen with different amounts of catalyst of 10000, 100, 10, 1, $0.1 \mathrm{ppm}$ (based on substrate) were tracked. The reaction profiles are shown in Figure 4.

Although the amount of the catalyst decreased exponentially, cyclohexene could be nearly stoichiometrically oxidized within the reaction times ranged from 2.0 to $5.0 \mathrm{~h}$, as shown in Figure 1. When the amount of catalyst was 1 or $0.1 \mathrm{ppm}$, the reaction displays an induction period, then followed by a sharp acceleration until completion. When a radical scavenger such as tetrachloromethane was added to the reaction system, the conversion was stopped and no product could be detected after $5 \mathrm{~h}$, even with $10000 \mathrm{ppm}$ catalyst. Therefore, the epoxidation catalyzed by metalloporphyrin should involve radical species. The result is consistent with those of Qi, Nam, and Ravikumar using ruthenium complex, cyclam or manganese acetate dihydrate as catalysts.[73-76] 


\begin{tabular}{|c|c|c|c|c|c|}
\hline Entry & Substrate & Product & $\begin{array}{l}\text { Reaction } \\
\text { time }(\mathrm{h})\end{array}$ & $\begin{array}{c}\text { Conv. } \\
(\%)\end{array}$ & $\begin{array}{c}\text { Yield } \\
(\%)\end{array}$ \\
\hline 1 & & & 4.5 & 97 & 95 \\
\hline 2 & & & 5.0 & 93 & 90 \\
\hline 3 & & & 6.0 & 83 & 72 \\
\hline 4 & & & 4.5 & 95 & 93 \\
\hline 5 & & & 7.0 & 89 & 87 \\
\hline 6 & & & 8.0 & 86 & 85 \\
\hline 7 & & & 5.0 & 95 & 93 \\
\hline 8 & & & 5.0 & 94 & 93 \\
\hline 9 & & & 5.0 & 93 & 89 \\
\hline 10 & & & 5.0 & 92 & 91 \\
\hline 11 & & & 6.0 & 92 & 90 \\
\hline
\end{tabular}

a substrate $(2 \mathrm{mmol})$, isobutyraldehyde $(0.01 \mathrm{~mol}), \mathrm{CH}_{2} \mathrm{Cl}_{2}(5 \mathrm{~mL}), \mathrm{O}_{2}$ bubbling, r.t.

Table 2. Epoxidation of alkenes catalyzed by manganese meso-tetraphenylporphyrin in the presence of molecular oxygen and isobutyraldehydea 


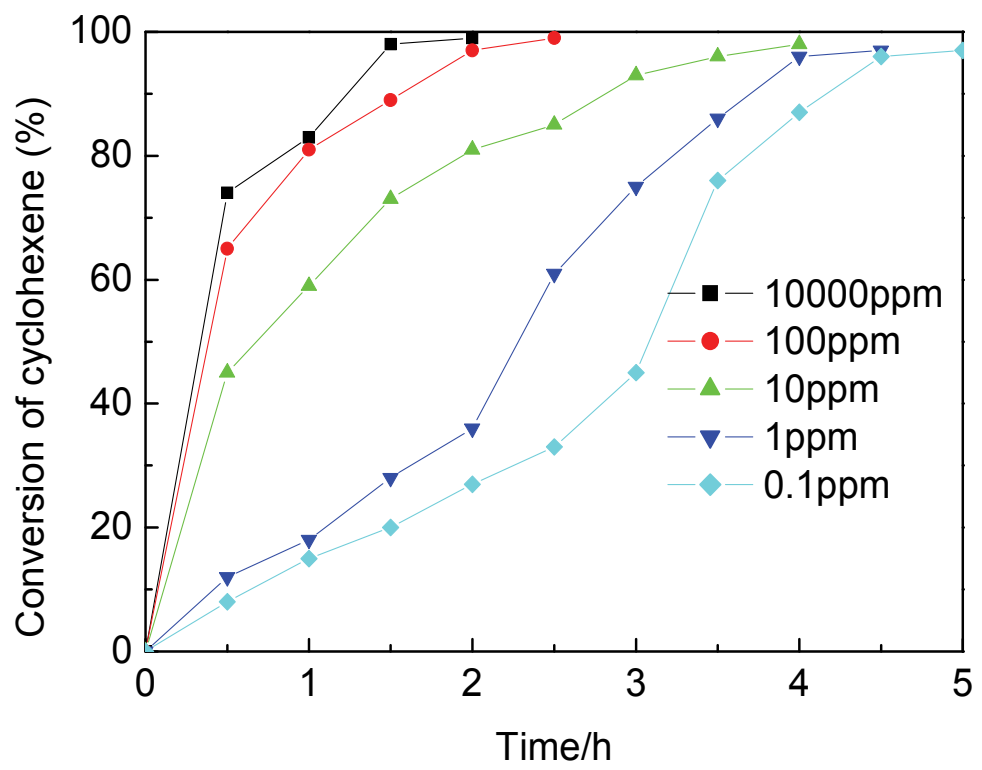

Fig. 4. Conversion rates profile of cyclohexene oxide with different amounts of Mn(TPP) a a substrate ( $2 \mathrm{mmol})$, isobutyraldehyde $(0.01 \mathrm{~mol}), \mathrm{CH}_{2} \mathrm{Cl}_{2}(5 \mathrm{~mL}), \mathrm{O}_{2}$ bubbling (1atm), r.t.

Iron, ruthenium and cobalt also showed excellent activity for cyclohexene epoxidation by molecular oxygen (Table 3). [77] Comparing the catalytic activities of different porphyrin catalysts, it was found that manganese porphyrin was the most effective since cyclohexene could be completely converted within 4.0 hours. The catalytic activity of different metalloporphyrins is probably influenced by their electric potential and the stability of different valences of metal atoms.

\begin{tabular}{cccc}
\hline Entry & Catalyst & Conv. $(\%)$ & Yield (\%) \\
\hline 1 & $\mathrm{Mn}(\mathrm{TPP}) \mathrm{Cl}$ & $>99$ & $>99$ \\
2 & $\mathrm{Fe}(\mathrm{TPP}) \mathrm{Cl}$ & 93 & 92 \\
3 & $\mathrm{Co}(\mathrm{TPP}) \mathrm{Cl}$ & 90 & 90 \\
4 & $\mathrm{Ru}(\mathrm{TPP}) \mathrm{Cl}$ & 86 & 85 \\
5 & - & 15 & 13 \\
\hline
\end{tabular}

a Olefins $(2 \mathrm{mmol})$, aldehyde $(0.01 \mathrm{~mol})$, catalyst $\left(2 \times 10^{-6} \mathrm{mmol}\right), \mathrm{CH}_{2} \mathrm{Cl}_{2}(5 \mathrm{~mL}), \mathrm{O}_{2}$ bubbling, r.t., $4 \mathrm{~h}$

Table 3. Epoxidation of cyclohexene by molecular oxygen in the presence of various metalloporphyrins and isobutyraldehyde ${ }^{a}$

The mechanisms of alkene epoxidation by molecular oxygen in the presence of metal complexes and aldehyde have been already investigated. For such oxidation system, all evidence indicates that the reaction proceeded via free radical process. Oxygenation of substrates is assumed to occur via reactive high-valent metal oxo intermediates that are produced by the reaction of peroxyacid with the metal catalysts from this mechanism. 
In the system of olefin epoxidation by dioxygen, the process of epoxidation can follow different pathways depending on catalyst and reaction conditions. Nam and coauthors concluded that acylperoxy radicals from the autoxidation reaction or complexes of metal with the acylperoxy radicals were the active epoxidants, [74] and the metal complexes could accelerate the free radical autoxidation of aldehyde during the initiation step. Ravikumar reported that the epoxidation of olefin in perfluoro-2-butyltetrahydrofuran occurred either by the direct reaction of metal-peroxy species with olefins or through metal oxo species.[75] However, Kaneda et al. proposed that the key epoxidizing agent was not the metal oxo species, the peroxyacid was generated during the autoxidation of aldehyde, and the role of transition metal catalyst could be omitted.[78]

For the manganese porphyrin catalyzed epoxidaiton system, the typical product compositions of aerobic oxidation of cyclohexene are shown in Figure 5. Except the epoxide, isobutyric acid and perisobutyric acid generated from isobutyraldehyde could be detected in the reaction mixture. The formation of perisobutyric acid could be hardly detected within the first two-hour period. After reacting for 2 hours, the concentration of perisobutyric acid increased slightly while that of epoxidized cyclohexene increased sharply. It seems that the epoxidation occurred first with induction period, and then followed by sharp acceleration till completion, which agree with the feature of radical-involved reaction. In order to verify the free radical mechanism for the aerobic epoxidation of cyclohexene catalyzed by manganese porphyrins, a free radical inhibitor, 2,6-di-tert-butylphenol $(2 \mathrm{mmol})$ was added to the oxidation system. It has been found that the running oxidation of cyclohexene could be subsequently quenched. In addition, epoxide, isobutyric acid or perisobutyric acid could not be found when the reaction was carried out under nitrogen atmosphere.

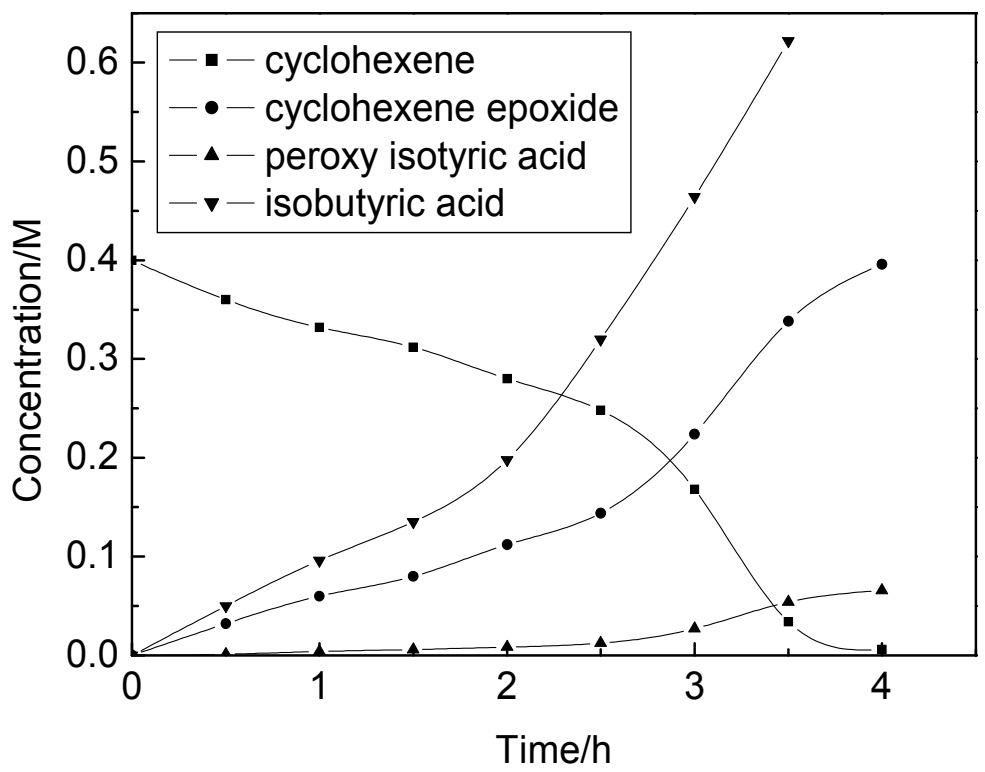

Fig. 5. Oxidation of cyclohexene by molecular oxygen in the presence of $\mathrm{Mn}(\mathrm{TPP}) \mathrm{Cl}$ and isobutyraldehyde: concentration of cyclohexene, $0.4 \mathrm{M}$; concentration of isobutyraldehyde, $2.0 \mathrm{M}$; concentration of catalyst, $4 \times 10^{-5} \mathrm{M}$; $\mathrm{O}_{2}$ bubbling; room temperature. 
In contrary to Kaneda's results, the metalloporphyrins catalyst is absolutely necessary for the epoxidation of cyclohexene from the fact that only $13 \%$ yield of epoxide can be obtained in the blank experiment. Therefore, the mechanism of epoxides production via metallporphyrins-peroxy from the reaction of acylperoxy radical with metallporphyrins is impossible. The epoxide (yields up to $13 \%$ ) in the absence of catalyst should be attributed to the reaction of acylperoxy radical with olefins directly. In the none-catalysis process, the acylperoxy radical was generated from acyl radical and dioxygen, and acyl radical was initiated from the auto-oxidation of aldehyde. A mechanism for the epoxidation of olefins by dioxygen in the presence of metalloporphyrins and isobutyraldehyde has been proposed from the experimental results, as shown in Figure 6.

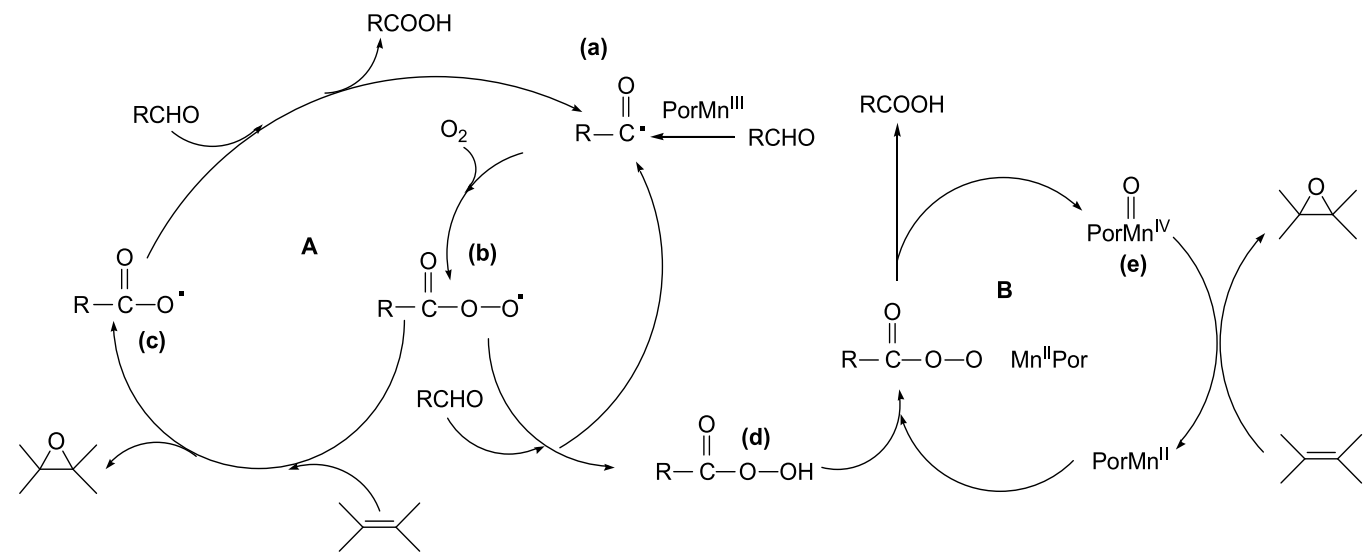

Fig. 6. Plausible mechanism of olefin epoxidation catalyzed by $\mathrm{Mn}(\mathrm{III})$ porphyrins in the presence of molecular oxygen and isobutyraldehyde

Based on the proposed mechanism, the manganese porphyrin reacts with the aldehyde to generate an acyl radical (a) at first. The acyl radical then reacts with dioxygen to give an acylperoxy radical (b). The acylperoxy radical is assumed to play two roles. Firstly, it reacts with olefins to yield epoxides directly accompanying the generation of carboxyl radical (c), which reacts with aldehyde to generate another acyl radical and carboxylic acid (pathway A). Secondly, in pathway B, the acylperoxy radical acts as a carrier by reacting with another aldehyde molecule to give peroxyacid (d), thereby generating another acyl radical. Formation of epoxide is assumed by active high-valent Mn porphyrin intermediates (e), which are formed by the reaction of the peroxyacid with the manganese porphyrin. The evidence described above suggests that pathway B is usually superior over pathway A.

In order to obtain further information on the role of the high-valent Mn porphyrin species for the present oxidation, in stiu EPR measurement was used for the aerobic oxidation of cyclohexene. The catalytic epoxidation in $\mathrm{CH}_{3} \mathrm{CN}$ was carried out in a sealed Wilmad WG810-A quartz-flat cell filled with dioxygen. EPR spectra were recorded with 20 min intervals. Figure 7 exemplifies the EPR results observed for $\mathrm{Mn}(\mathrm{III})$ porphyrin. Figure 7a shows the initial EPR spectrum of the $\mathrm{Mn}(\mathrm{TPP}) \mathrm{Cl}$ and the silent spectrum is characteristic of $\mathrm{Mn}(\mathrm{III})$ species from integer-spin (non-Kramers) $\mathrm{Mn}$ (III) $S=2$ porphyrin compound.[79-81] When isobutyraldehyde was added into $\mathrm{Mn}(\mathrm{TPP}) \mathrm{Cl}$ solution, a broad EPR signal of $\mathrm{Mn}$ (II) was observed (Figure 7b).[82] The change of EPR signal was attributed to the reaction of Mn (III) porphyrin with aldehyde, from which an acyl radical was generated and the Mn (III) 
porphyrin was converted to $\mathrm{Mn}(\mathrm{II})$ species. This is in agreement with the first step of the proposed mechanism. The six lines shown in Figure 7c are a typical signal of $\mathrm{Mn}(\mathrm{IV})$ species, which indicates the catalytic process should involve high-valent oxo intermediate. [83-84]

As the reaction went on, it was interesting to find that the intensity of signal $(g=2.02)$ increased, the peak became stronger and sharper, coupling with the decreasing intensity of adjacent signal $(g=1.98)$. The EPR spectra are shown in Figure $7 \mathrm{~d}-\mathrm{f}$. The fact demonstrated that $\mathrm{Mn}$ (II) porphyrins species might be there in the mixture, which are formed by the reduction of $\mathrm{Mn}(\mathrm{IV})$ intermediate.
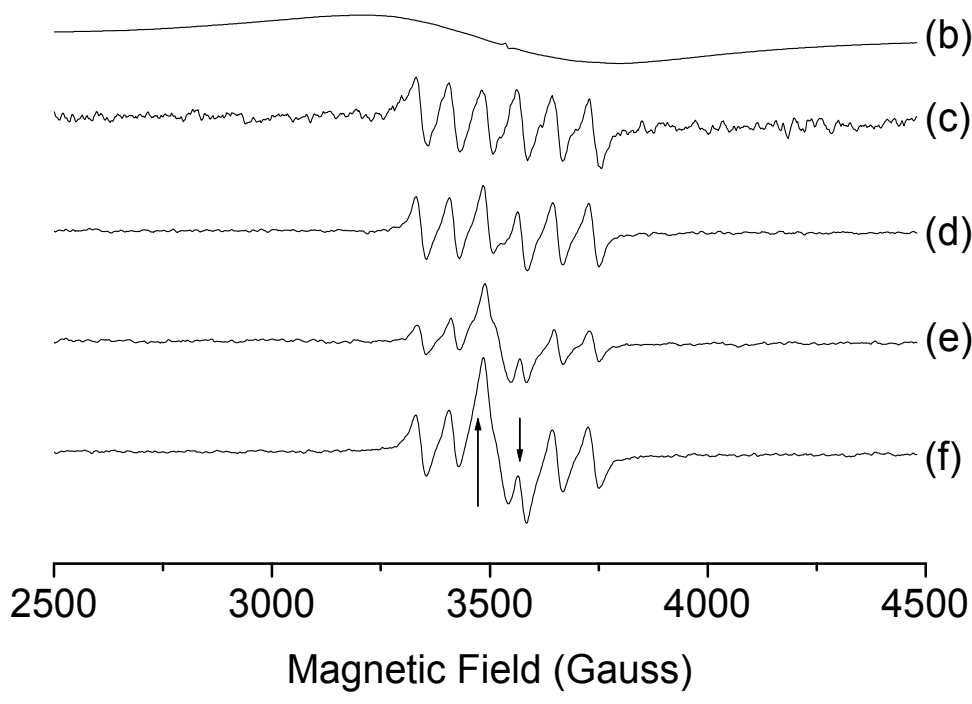

Fig. 7. In-situ EPR spectra of cyclohexene epoxidation catalyzed by $\mathrm{Mn}(\mathrm{TPP}) \mathrm{Cl}$ in the presence of dioxygen and isobutyraldehyde. (a) spectrum of $\mathrm{Mn}(\mathrm{TPP}) \mathrm{Cl}$; (b) spectrum of $\mathrm{Mn}(\mathrm{TPP}) \mathrm{Cl}$ plus isobutyraldehyde; (c) initial spectrum of cyclohexene epoxidation; (d) epoxidation spectrum after $20 \mathrm{~min}$; (e) epoxidation spectrum after $40 \mathrm{~min}$; (f) epoxidation spectrum after $60 \mathrm{~min}$.

The curves of UV-vis spectra for metalloporphyrins during the reaction process are shown in Figure 8. The spectrophotometer was programmed to acquire UV-vis spectrum every 10 minutes. As shown in Figure 8, a decrease in the Soret band at $445 \mathrm{~nm}$ was observed, with a concomitant slight increase in the typical Soret band at $\sim 417 \mathrm{~nm}$. The disappearance of the peak $(445 \mathrm{~nm})$ suggested that oxidant active species $\left(\mathrm{Mn}^{\mathrm{IV}=} \mathrm{O}\right)$ were consumed by substrate. [85-87] In addition, during the reaction, the color changes in the reaction mixture also indicate the presence of such high-valence species. GC analysis revealed the formation of the epoxide, which is indicative of the presence of an active oxidation species.

The epoxidation of cyclohexene in the presence of isobutyraldehyde and dioxygen to produce epoxidized cyclohexene with $\mathrm{Mn}(\mathrm{TPP}) \mathrm{Cl}$ as catalyst is a combination of oxidation of aldehyde and epoxidation of cyclohexene. Following the reaction mechanism given in Figure 6, the reaction equations can be listed as below: 


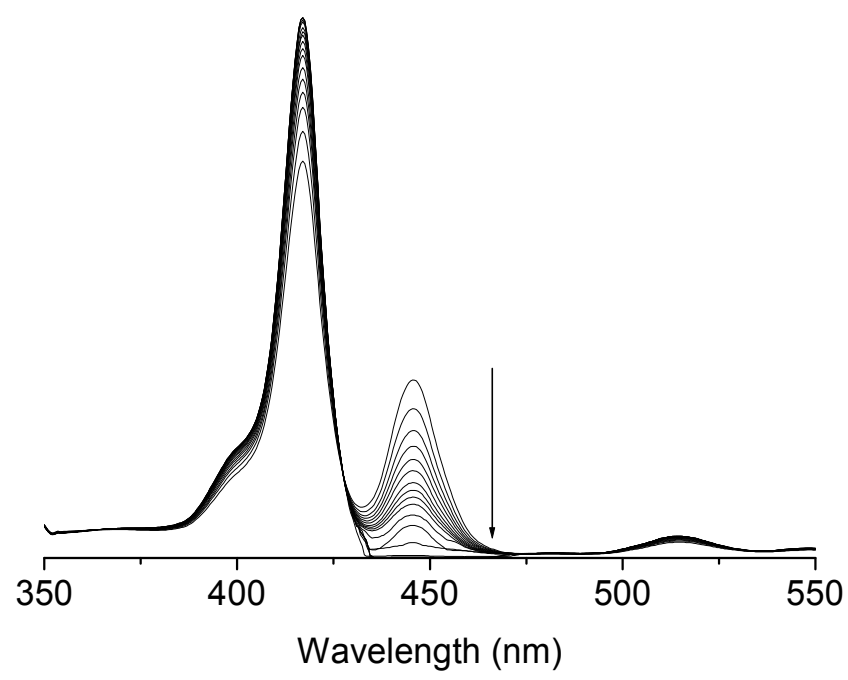

Fig. 8. In situ UV-vis spectra of the aerobic epoxidation of cyclohexene catalyzed by $\mathrm{Mn}(\mathrm{TPP}) \mathrm{Cl}(0.04 \mathrm{mM})$ in dichloromethane solution (time scan: $120 \mathrm{~min}$, interval $10 \mathrm{~min})$. Reaction conditions: cyclohexene $\left(0.4 \mathrm{~mol} \mathrm{~L}^{-1}\right)$, catalyst $(0.04 \mathrm{mM})$ and isobutyraldehyde $(4$ mol L-1)

Initiation: Equation 1 is the initiation step.

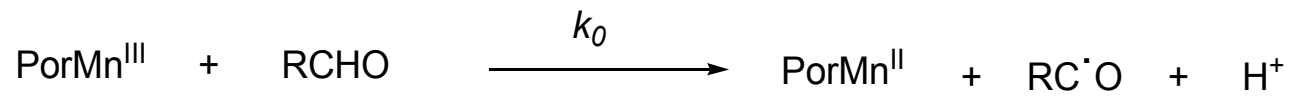

Propagation:

$$
\begin{aligned}
& \mathrm{RCO}^{\circ}+\mathrm{O}_{2} \stackrel{k_{1}}{\longrightarrow} \mathrm{RCO}_{3}{ }^{\circ} \\
& \mathrm{RCO}_{3}{ }^{\circ}+\mathrm{RCHO} \stackrel{k_{2}}{\longrightarrow} \mathrm{RCO}_{3} \mathrm{H}+\mathrm{RC}^{\circ} \mathrm{O}
\end{aligned}
$$

Termination: The main termination step is:

$$
2 \mathrm{RCO}_{3} \cdot \stackrel{k_{3}}{\longrightarrow} \text { inactive }
$$

The other termination steps are:

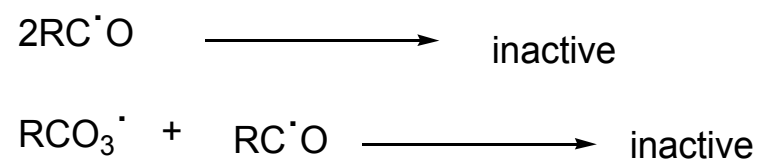

Epoxidation: The epoxidation steps include pathway A and B. 
Pathway B:

$$
\begin{aligned}
& \text { PorMn" }+\mathrm{RCO}_{3} \mathrm{H} \longrightarrow \mathrm{k}_{4} \text { PorMn }^{\mathrm{IV}}=\mathrm{O}+\mathrm{RCOOH} \\
& \text { PorMn }{ }^{\mathrm{IV}}=\mathrm{O}+\longrightarrow \mathrm{k}+\mathrm{C}_{5}+\mathrm{PorMn}^{\mathrm{II}}
\end{aligned}
$$

Pathway A:

$$
\begin{aligned}
& \mathrm{RCO}_{3}{ }^{\circ} \longrightarrow \mathrm{CO}_{6}+\mathrm{RCO}_{2}{ }^{\circ} \\
& \mathrm{RCO}_{2}{ }^{\cdot}+\mathrm{RCHO} \longrightarrow k_{7} \mathrm{RCOOH}+\mathrm{RC}^{\circ} \mathrm{O}
\end{aligned}
$$

The molar balance equations for each component of the reactions are presented as follows: First, the changes of the free radicals concentrations are:

$$
\begin{aligned}
& \mathrm{d}\left[\mathrm{RC}^{\circ} \mathrm{O}\right] / \mathrm{d} t=k_{0}[\mathrm{RCHO}]\left[\mathrm{PorMn}^{\prime \prime \prime}\right]-k_{1}\left[\mathrm{RC}^{\circ} \mathrm{O}\right]\left[\mathrm{O}_{2}\right]+k_{2}\left[\mathrm{RCO}_{3}{ }^{\circ}\right][\mathrm{RCHO}]+k_{7}\left[\mathrm{RCO}_{2}{ }^{\circ}\right][\mathrm{RCHO}] \\
& \mathrm{d}\left[\mathrm{RCO}_{2}{ }^{\circ}\right] / \mathrm{d} t=k_{6}\left[\mathrm{RCO}_{3}{ }^{*}\right][\text { cyclohexene }]-k_{7}\left[\mathrm{RCO}_{2}{ }^{\circ}\right][\mathrm{RCHO}] \\
& \mathrm{d}\left[\mathrm{RCO}_{3}{ }^{\circ}\right] / \mathrm{d} t=k_{1}\left[\mathrm{RC}^{\circ} \mathrm{O}\right]\left[\mathrm{O}_{2}\right]-k_{2}\left[\mathrm{RCO}_{3}{ }^{\circ}\right][\mathrm{RCHO}]-2 k_{3}\left[\mathrm{RCO}_{3}{ }^{\circ}\right]^{2}-k_{6}\left[\mathrm{RCO}_{3}{ }^{\circ}\right][\text { cyclohexene }]
\end{aligned}
$$

Assuming that the system is in pseudo steady state, eqs 11-13 become

$$
\mathrm{d}\left[\mathrm{RC}^{*} \mathrm{O}\right] / \mathrm{d} t=\mathrm{d}\left[\mathrm{RCO}_{2}{ }^{*}\right] / \mathrm{d} t=\mathrm{d}\left[\mathrm{RCO}_{3}{ }^{\circ}\right] / \mathrm{d} t=0
$$

Summing eqs. 11,12 and 13 ,

$$
k_{0}[\mathrm{RCHO}]\left[\mathrm{PorMn}^{\mathrm{II}}\right]=2 k_{3}\left[\mathrm{RCO}_{3}{ }^{\circ}\right]^{2}
$$

In addition, eqs. 16 and 17 can be deduced from eqs. 7 and 8:

$$
\begin{aligned}
& \mathrm{d}\left[\mathrm{Mn}^{\mathrm{IV}}=\mathrm{O}\right] / \mathrm{d} t=k_{4}\left[\text { PorMn }^{\mathrm{I}}\right]\left[\mathrm{RCO}_{3} \mathrm{H}\right]-k_{5}\left[\mathrm{Mn}^{\mathrm{IV}}=\mathrm{O}\right][\text { cyclohexene }] \\
& \mathrm{d}\left[\mathrm{RCO}_{3} \mathrm{H}\right] / \mathrm{d} t=k_{2}\left[\mathrm{RCO}_{3}{ }^{\circ}\right][\mathrm{RCHO}]-k_{4}\left[\mathrm{PorMn}^{\mathrm{I}}\right]\left[\mathrm{RCO}_{3} \mathrm{H}\right]
\end{aligned}
$$

Based on pathways $A$ and $B$, the total rate equation of epoxide $(\operatorname{Re})$ can be expressed as:

$$
\mathrm{Re}=\mathrm{d}[\text { epoxide }] / \mathrm{d} t=k_{5}\left[\mathrm{Mn}^{\mathrm{IV}}=\mathrm{O}\right][\text { cyclohexene }]+k_{6}\left[\mathrm{RCO}_{3}{ }^{\circ}\right][\text { cyclohexene }]
$$


Through the pseudo steady state hypothesis for the Mn(IV) high-valent intermediate and $\mathrm{RCO}_{3} \mathrm{H}$, eqs.16, 17 and 18 can be summarized to get the generating rate of epoxide as:

$\mathrm{Re}=k_{2}\left(k_{6} / 2 k_{3}\right)^{0.5}[\mathrm{RCHO}]^{1.5}\left[\mathrm{PorMn}{ }^{11 \prime}\right]^{0.5}+k_{6}\left(k_{6} / 2 k_{3}\right)^{0.5}[\mathrm{RCHO}]^{0.5}\left[\mathrm{PorMn}^{\mathrm{III}}\right]^{0.5}[$ cyclohexene $]$

Then, eqs. 19 can be simplified as:

$$
\mathrm{Re}=K_{1}[\mathrm{RCHO}]^{1.5}\left[\mathrm{PorMn}^{\prime \prime \prime}\right]^{0.5}+K_{2}[\mathrm{RCHO}]^{0.5}\left[\mathrm{PorMn}^{\prime \prime \prime}\right]^{0.5}[\text { cyclohexene }]
$$

From equation 20, it can be seen that the rate of epoxidation is related with the concentration of cyclohexene, manganese porphyrins catalyst and isobutyraldehyde with 1, 0.5 and 1.5 power respectively. In order to verify the equation of the epoxidation rate further, the factors that influence epoxidation rate were investigated using cyclohexene as model substrate. The rate of epoxidation was obtained based on the following assumption: (1) There is very large excess of cyclohexene compared to catalyst (the [catalyst]/ [cyclohexene] ratio is $1 / 10^{6}$ ) in the catalytic system; (2) The conversion rate of cyclohexene should be controlled within $5 \%$.

The catalytic epoxidation of cyclohexene in the presence of isobutyraldehyde and molecular oxygen was carried out with the range of cyclohexene concentration from 0.1 to $0.5 \mathrm{M}$. The results of epoxidation rate with different concentrations of cyclohexene are listed in Table 4. It indicated that increasing concentration of cyclohexene accelerated the epoxidation rate from $2.30 \times 10^{-3}$ to $2.83 \times 10^{-3} \mathrm{~mol} \mathrm{~L}^{-1} \mathrm{~min}^{-1}$.

\begin{tabular}{ccc}
\hline Entry & Concentration of cyclohexene $(\mathrm{M})$ & $\mathrm{R} \times 10^{3}\left(\mathrm{~mol} \mathrm{~L}^{-1} \mathrm{~min}^{-1}\right)$ \\
\hline 1 & 0.1 & 2.30 \\
2 & 0.2 & 2.42 \\
3 & 0.3 & 2.60 \\
4 & 0.4 & 2.76 \\
5 & 0.5 & 2.83 \\
\hline
\end{tabular}

${ }^{a}$ Temperature: $308 \mathrm{~K}$; Concentration of isobutyraldehyde: $2.0 \mathrm{M}$; Concentration of $\mathrm{Mn}(\mathrm{TPP}) \mathrm{Cl}, 0.4 \times 10^{-6}$ $\mathrm{M} ; \mathrm{O}_{2}$ bubbling.

Table 4. Effect of cyclohexene concentration on the aerobic epoxidation rate in the presence of isobutyraldehyde $a$

A plot of epoxidation rate against the concentration of cyclohexene yields a straight line, as shown in Figure 9.

As seen in Figure 9, linear correlation between epoxidation rate and [cyclohexene] was evident using $\mathrm{Mn}(\mathrm{TPP}) \mathrm{Cl}$ as catalyst, in which correlation coefficient was 0.992 . Consequently, the catalytic reaction also exhibited first-order dependence in relation to the concentration of cyclohexene. Thus, the experimental results are in good agreement with the theoretical analysis as shown in equation 20.

The results indicated that increasing the concentration of catalyst results in an increase of the initial reaction rate. Increasing the concentration of catalyst from $0.8 \times 10^{-4}$ to $4.0 \times 10^{-4} \mathrm{M}$ increases the epoxidation rate from $1.98 \times 10^{-3}$ to $4.47 \times 10^{-3} \mathrm{~mol} \mathrm{~L}^{-1} \mathrm{~min}^{-1}$ as shown in Table 5 . 


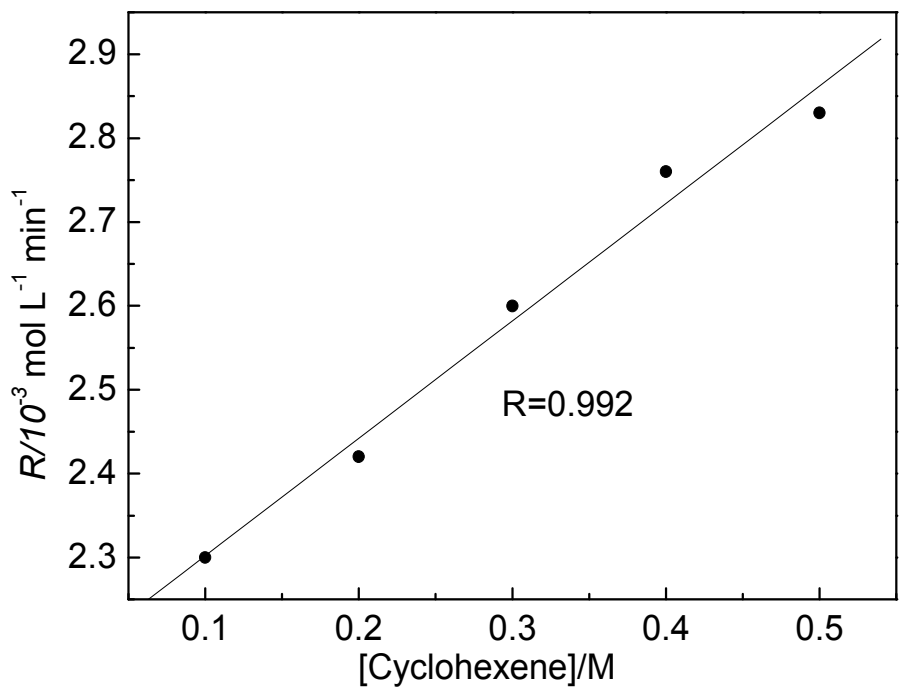

Fig. 9. Effect of cyclohexene concentration on the epoxidation rate in the presence of isobutyraldehyde and molecular oxygen: concentration of isobutyraldehyde, $2.0 \mathrm{M}$; concentration of $\mathrm{Mn}(\mathrm{TPP}) \mathrm{Cl}, 0.4 \times 10^{-6} \mathrm{M} ; \mathrm{O}_{2}$ bubbling; room temperature.

\begin{tabular}{cccc}
\hline Entry & {$[\mathrm{Mn}(\mathrm{TPP}) \mathrm{Cl}], \mathrm{M}$} & {$[\mathrm{Mn}(\mathrm{TPP}) \mathrm{Cl}]^{0.5} \times 10^{2}, \mathrm{M}^{0.5}$} & $\mathrm{R} \times 10^{3}, \mathrm{~mol} \mathrm{~L}^{-1} \mathrm{~min}^{-1}$ \\
\hline 1 & $0.8 \times 10^{-4}$ & 0.89 & 1.98 \\
2 & $1.6 \times 10^{-4}$ & 1.26 & 2.94 \\
3 & $2.4 \times 10^{-4}$ & 1.55 & 3.61 \\
4 & $3.2 \times 10^{-4}$ & 1.79 & 4.01 \\
5 & $4.0 \times 10^{-4}$ & 2.00 & 4.47 \\
\hline
\end{tabular}

a Temperature: $308 \mathrm{~K}$; Concentration of isobutyraldehyde: $2.0 \mathrm{M}$; Concentration of cyclohexene: $0.4 \mathrm{M}$; $\mathrm{O}_{2}$ bubbling.

Table 5. Effect of concentration of $\mathrm{Mn}(\mathrm{TPP}) \mathrm{Cl}$ catalyst on the aerobic epoxidation rate in the presence of isobutyraldehyde ${ }^{a}$

Plot of the initial rate of epoxidized cyclohexene versus the concentration of $[\mathrm{Mn}(\mathrm{TPP}) \mathrm{Cl}]^{0.5}$ yields a straight line as shown in Figure 10.

As can be observed in Figure 10, linear correlation between epoxidation rate and $[\mathrm{Mn}(\mathrm{TPP}) \mathrm{Cl}]^{0.5}$ was evident, in which correlation coefficient was 0.997 . The line can be expressed by:

$$
R e=0.081 \times 10^{-3}+2.217 \times[\mathrm{Mn}(\mathrm{TPP}) \mathrm{Cl}]^{0.5}
$$

The results indicated that increasing of isobutyraldehyde concentration from 0.8 to $2.4 \mathrm{M}$ resulted in an increase of the initial rate of epoxidized cyclohexene from $1.11 \times 10^{-3}$ to $3.41 \times 10^{-}$ ${ }^{3} \mathrm{~mol} \mathrm{~L}^{-1} \mathrm{~min}^{-1}$ as shown in Table 6 . 


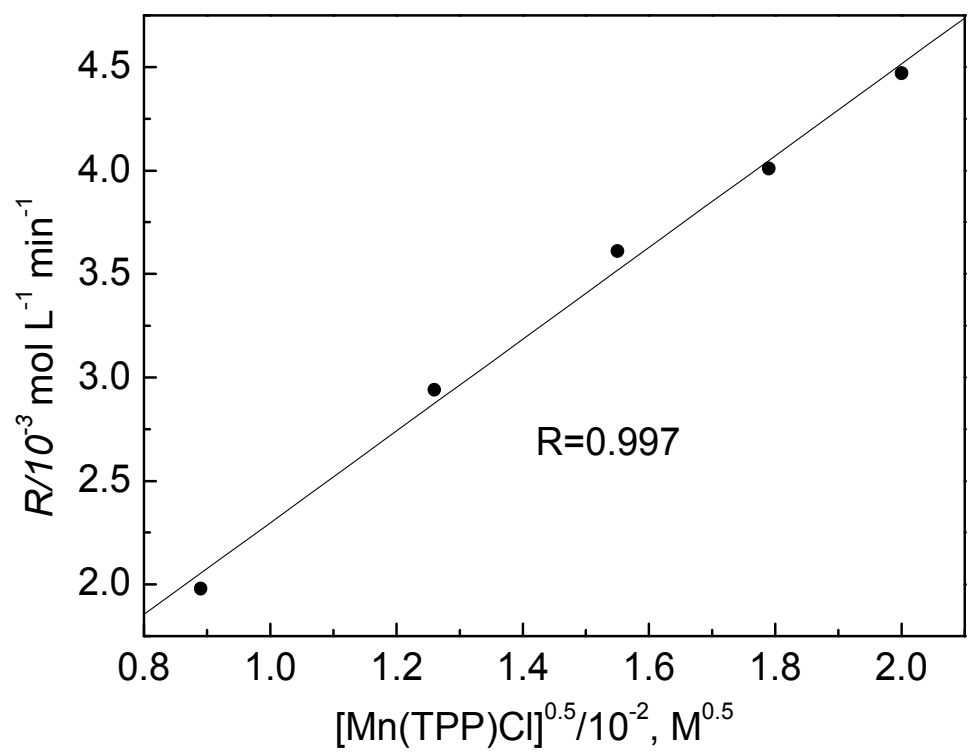

Fig. 10. Effect of the concentration of $\mathrm{Mn}(\mathrm{TPP}) \mathrm{Cl}$ catalyst on the epoxidation rate in the presence of isobutyraldehyde and molecular oxygen: concentration of isobutyraldehyde, $2.0 \mathrm{M}$; concentration of cyclohexene, $0.4 \mathrm{M} ; \mathrm{O}_{2}$ bubbling; room temperature.

\begin{tabular}{ccccc}
\hline Entry & [aldehyde], $\mathrm{M}$ & [aldehyde] ${ }^{1.5, \mathrm{M}^{1.5}}$ & [aldehyde] ${ }^{0.5}, \mathrm{M}^{0.5}$ & $\begin{array}{c}\mathrm{R} \times 10^{3}, \mathrm{~mol} \mathrm{~L}^{-1} \\
\mathrm{~min}^{-1}\end{array}$ \\
\hline 1 & 0.80 & 0.72 & 0.89 & 1.11 \\
2 & 1.20 & 1.31 & 1.10 & 1.64 \\
3 & 1.60 & 2.02 & 1.26 & 2.16 \\
4 & 2.00 & 2.83 & 1.41 & 2.75 \\
5 & 2.40 & 3.72 & 1.55 & 3.41 \\
\hline
\end{tabular}

${ }^{a}$ Temperature: $308 \mathrm{~K}$; Concentration of cyclohexene: $0.4 \mathrm{M}$; Concentration of $\mathrm{Mn}(\mathrm{TPP}) \mathrm{Cl}, 0.4 \times 10^{-6} \mathrm{M}$; $\mathrm{O}_{2}$ bubbling.

Table 6. Effect of isobutyraldehyde concentration on the aerobic epoxidation rate in the presence of molecular oxygen ${ }^{a}$

The plot of the epoxidation reaction rate versus [isobutyraldehyde] ${ }^{0.5}$ is shown in Figure 11.

The graph shown in Figure 11 illustrated clearly that the reaction rate versus [isobutyraldehyde] ${ }^{0.5}$ yielded a cube curve, which was consistent with the analysis as shown in equation 20.

As shown above, the experimental results correlate the theoretical analysis well. The good agreement for kinetics could further explain that the metalloporphyrin-catalyzed epoxidaiton of cyclohexene is via a high-valent metal intermediate.

Bismetalloporphyrin dimers are composed of two covalently bound monometalloporphyrins molecules. Investigations over the past decades indicated that $\mu$-oxobisiron porphyrins (Scheme 5) are inactive or have low activity for hydroxylation of alkanes. Recently, high catalytic activities of $\mu$-oxo dimeric metalloporphyrins for the oxidation of cyclohexane, ethylbenzene and toluene have been reported.[88-90] 


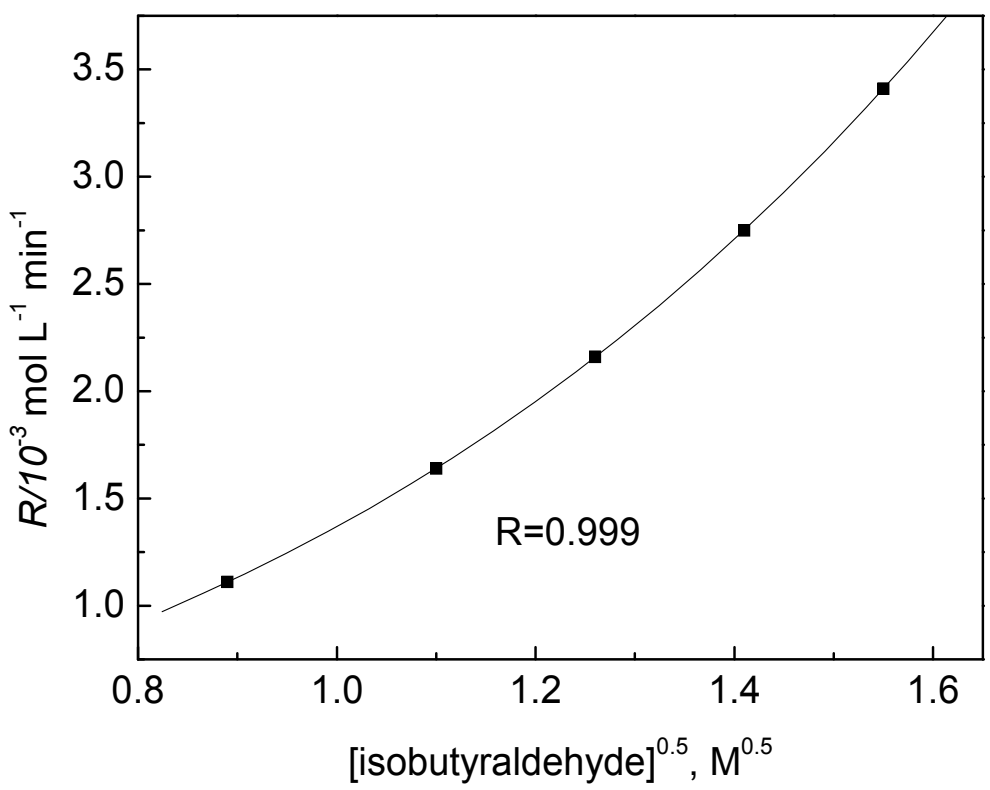

Fig. 11. Effect of isobutyraldehyde concentration on the epoxidation rate in the presence of molecular oxygen: concentration of cyclohexene, $0.4 \mathrm{M}$; concentration of $\mathrm{Mn}(\mathrm{TPP}) \mathrm{Cl}$, $0.4 \times 10^{-6} \mathrm{M} ; \mathrm{O}_{2}$ bubbling; room temperature.

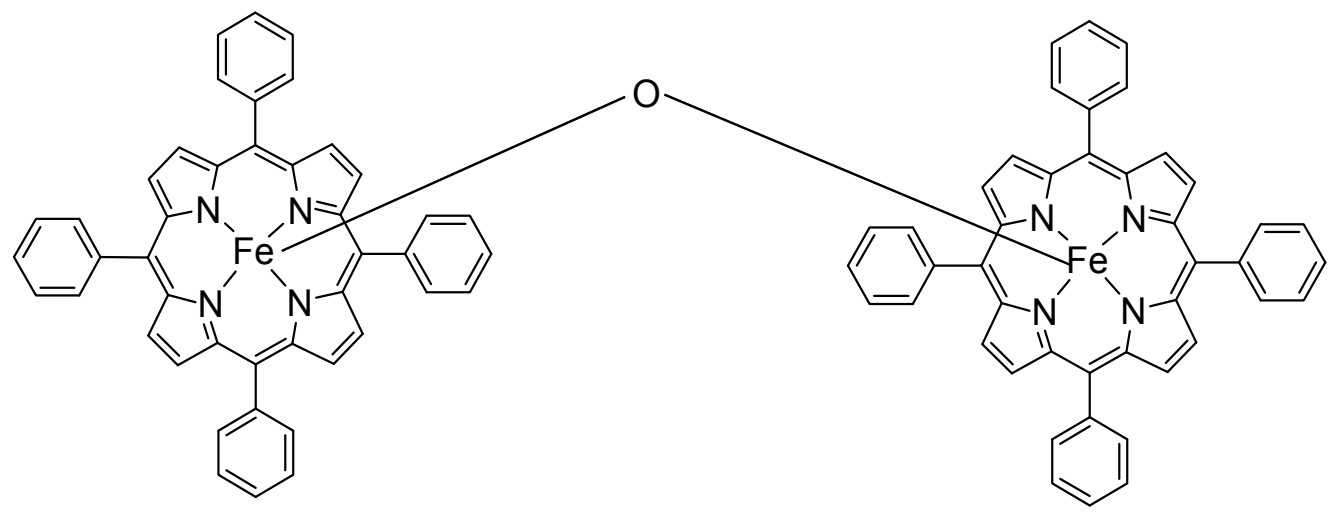

Scheme 5. Structure of $\mu$-oxo-bisiron(III) porphyrin

With cyclohexene as model compound, the effects of reaction conditions on the epoxidation in the presence of $(\mathrm{Fe} \text { IIITPP })_{2} \mathrm{O}$ with molecular oxygen as oxidant have been investigated. Table 7 summarizes the main results obtained under different reaction conditions. It could be found from entry 1 of Table 7 that only $21 \%$ cyclohexene could be converted in the absence of catalyst, indicating that the (FeIIITPP) $)_{2} \mathrm{O}$ catalyst is crucial for the epoxidation. As depicted in Table 7, it was observed that the yield is considerably enhanced when the catalyst was used. The conversion of cyclohexene could reach up to $96 \%$ even the amount of catalyst was only $0.01 \mathrm{ppm}$ (entry 2). The optimal amount of catalyst for the epoxidation was $0.05 \mathrm{ppm}$ (entry 3). On the contrary, attempts for enhancing the yield by increasing the 
amount of catalyst were unsuccessful. The yield decreased with the increase of catalyst amount (entries 4-6). This is the typical characteristics of metalloporphyrins-catalyzed oxidation, which caused by the shielding effect due to the more catalyst intermediates by increasing amount of metalloporphyrins.

\begin{tabular}{cccc}
\hline Entry & Amount of catalyst/ppm & Conv. $\%$ & Yield/\% \\
\hline 1 & 0 & 21 & 21 \\
2 & 0.01 & 96 & 93 \\
3 & 0.05 & $>99$ & 96 \\
4 & 0.1 & 90 & 88 \\
5 & 1.0 & 81 & 80 \\
6 & 10.0 & 84 & 81 \\
$7 b$ & 0.05 & 0 & 0 \\
$8^{c}$ & 0.05 & 92 & 90 \\
$9 d$ & 0.05 & $>99$ & 96 \\
$10^{e}$ & 0.1 & 85 & 82 \\
\hline
\end{tabular}

a Substrate $(2 \mathrm{mmol})$, isobutylaldehyde $(0.011 \mathrm{~mol})$, acetonitrile $(6 \mathrm{~mL}), \mathrm{O}_{2}$ bubbling, $4 \mathrm{~h}, \mathrm{rt}$

${ }^{b}$ No isobutylaldehyde

${ }^{c}$ Substrate /isobutylaldehyde $=1: 5$

${ }^{d}$ Substrate /isobutylaldehyde $=1: 6$

${ }^{e} \mathrm{Fe}{ }^{\text {IIITPPCl }}$ as catalyst

Table 7. Aerobic epoxidation of cyclohexene catalyzed by $(\mathrm{Fe} \text { IIITPP })_{2} \mathrm{O}$ in the presence of isobutyraldehyde ${ }^{a}$

The effects of isobutyraldehyde on the reaction were also investigated. As shown in Table 7, the reaction could not take place in the absence of isobutyraldehyde (entry 7), strongly implying that (FeIIITPP) ${ }_{2} \mathrm{O}$ could not activate molecular oxygen without aldehyde as an oxygen acceptor under ambient conditions. The epoxidation with various molar ratio of substrate to isobutyraldehyde were examined. When the substrate/isobutyraldehyde molar ratio reached 1:5, 92\% cyclohexene could be converted (entry 8). Increasing molar ratio gave higher conversion (entry 9). However, no significant difference was observed when the substrate/isobutyraldehyde molar ratio exceeds 1:5.5.

Compared with the aerobic epoxidation catalyzed by mono-metalloporphyrin, (FeIIITPPCl, entry 10), it is clear that $\mu$-oxo dimeric iron(III) porphyrin exhibited higher catalytic performance for the aerobic epoxidation of cyclohexene, probably because (Fe ${ }^{\text {IIITPP }}{ }_{2} \mathrm{O}$ has superior stability for epoxidation than Fe ${ }^{\mathrm{IIITPPCl}}$.

Solvent played an important role for the epoxidation by using $\mu$-oxo-bisiron(III) porphyrin as the catalyst (Table 8).

It has been observed that solvent played an important role for the oxidation reaction. Polar solvents e.g. acetonitrile and benzotrifluoride seemed to be more favorable for the epoxidation of cyclohexene. Dichloromethane was not suitable for this catalytic epoxidation, although it was commonly used in metalloporphyrins-catalyzed epoxidation systems. [9193] It may be due to the instability of (FeIIITPP $)_{2} \mathrm{O}$ in dichloromethane, which could be partly converted to monometalloporphyrins.

Most olefins could be smoothly converted to corresponding epoxides with high conversion rate and excellent selectivity in the $\mu$-oxo-bisiron(III) porphyrin catalyzed system (Table 9). It seems that the efficiency of the epoxidation in this catalytic system is not closely related 


\begin{tabular}{ccccc}
\hline Entry & Solvent & Conv. $\%$ & Yield/\% & Selectivity/\% \\
\hline 1 & acetonitrile & $>99$ & 96 & 96 \\
2 & benzotrifluoride & 73 & 71 & 97 \\
3 & methanol & 66 & 64 & 97 \\
4 & dichloromethane & 60 & 57 & 95 \\
5 & toluene & 61 & 59 & 96 \\
\hline
\end{tabular}

${ }^{a}$ Substrate $(2 \mathrm{mmol})$, substrate / isobutylaldehyde =1:5.5 (molar ratio), ( $\left.\mathrm{Fe}^{\mathrm{IIITPP}}\right)_{2} \mathrm{O}(0.05 \mathrm{ppm})$, acetonitrile $(6 \mathrm{~mL}), \mathrm{O}_{2}$ bubbling, $4 \mathrm{~h}$, $\mathrm{rt}$

Table 8 . Effect of solvent on the aerobic epoxidation of cyclohexene catalyzed by $\left(\mathrm{Fe} \mathrm{IIITP}_{2} \mathrm{O} a\right.$

Entry

a Substrate $(2 \mathrm{mmol})$, isobutylaldehyde $(0.011 \mathrm{~mol})$, acetonitrile $(6 \mathrm{~mL}), \mathrm{O}_{2}$ bubbling, $\mathrm{rt}$ ${ }^{b}\left(\mathrm{Fe}^{\mathrm{IIT} T P P}\right)_{2} \mathrm{O}(1.0 \mathrm{ppm})$

Table 9. Aerobic epoxidation of various alkenes catalyzed by $\left(\mathrm{Fe} \mathrm{IIITP}_{2} \mathrm{O} a\right.$ 
with the steric conformation of substrates. For instance, slightly longer reaction time was required for 1-methylcyclohexene than cyclohexene (entries 1-2). However, slow oxidation occurred and an unsatisfactory conversion could be obtained for the simple $\alpha$-olefins or terminal alkenes such as 1-octene, 1-hexene and styrene (entries 3, 5 and 7). When the amount of catalyst increased to $1.0 \mathrm{ppm}$, remarkable enhancement for conversion and yield was observed (entries 4, 6 and 8). While for the non-terminal linear olefin e.g. trans-2-octene, the catalytic system showed excellent catalytic performance, in which trans-2-octene was converted to the corresponding epoxide in 4 hours (entry 9). The catalytic system also seems favorable for the conversion of other cycloolefin e.g. cyclooctene, in which the reaction system exhibited highly catalytic performance with $92 \%$ yield of cyclooctene epoxide (entry 11).

Another salient feature of the present epoxidation is its high regioselectivity. When (+)limonene was subjected to the epoxidation, the monoepoxide with the epoxide group on the ring was the main product and its selectivity was $72 \%$ (entry 12 ).

To further investigate the efficiency of the catalytic system, a large scale experiment for the aerobic epoxidation of cyclohexene was carried out. When the amount of (FeIIITPP $)_{2} \mathrm{O}$ catalyst used was $6.0 \times 10^{-9} \mathrm{mmol}$, cyclohexene oxide could be obtained with the isolated yield of $87 \%$. It should be mentioned that the turnover number of the $\mu$-oxo dimeric porphyrin could reach $1,432,993,256$, and its TOF was $2.4 \times 10^{6} \mathrm{~min}^{-1}$. Comparing with monometallporphyrins as catalyst, remarkable enhancement of reactivity was obtained for the present olefin epoxidation system, in which the turnover number (TON) of the catalyst has doubled from about 700 million to 1,400 million.

As reported previously, the oxygenation of organic substrates catalyzed by metalloporphyrin complexes plus aldehyde is usually considered to involve a radical and high-valent metal intermediate mechanism.[94-95] 2,6-Di-tert-butyl-4-methylphenol as a radical trap has been used in the epoxidation of cyclohexene, and it was found that the reaction was completely inhibited. Therefore, the aerobic epoxidation should involve radical species.

In order to obtain further information about the role of the high-valent iron porphyrin on the epoxidation, in situ UV-vis spectra was used. The catalytic epoxidation in $\mathrm{CH}_{3} \mathrm{CN}$ was carried out in a quartz colorimetric cell with dioxygen bubbling. UV-vis spectra were recorded with 5 min intervals. Figure 12 exemplifies the UV-vis results observed for $\mu$-oxo dimeric iron(III) porphyrins during the epoxidation process. The initial spectrum of (FeIITPP $)_{2} \mathrm{O}$ catalyst with a characteristic of Soret band at $408 \mathrm{~nm}$ was shown in Figure 12a. When isobutyraldehyde was added into the mixture, a shift from 408 to $417 \mathrm{~nm}$ with loss in intensity was observed, as shown in Figure 12b. Besides, the disappearance of the two Qband peaks at 570 and $610 \mathrm{~nm}$ was found with the addition of isobutyraldehyde. As the reaction continued, a decreasing in the Soret band at $417 \mathrm{~nm}$ was recorded. The changes of $\mathrm{UV}$-vis spectra are indicative of the existence of an active species expected as the high-valent iron porphyrin.[96-98]

It is usually considered that $\mu$-oxo dimeric metalloporphyrins could easily form monometalloporphyrins during the catalytic process. In fact, whether the dinuclear intermediate or the mononuclear intermediate is the active species remain controversial for the oxygenation of hydrocarbons catalyzed by $\mu$-oxo dimeric metalloporphyrins. The possible mononuclear metalloporphyrins existed in the catalytic system are FeTPPCl or FeTPPOH. Attempts to obtain some useful information from UV-vis spectrum had been tried. 


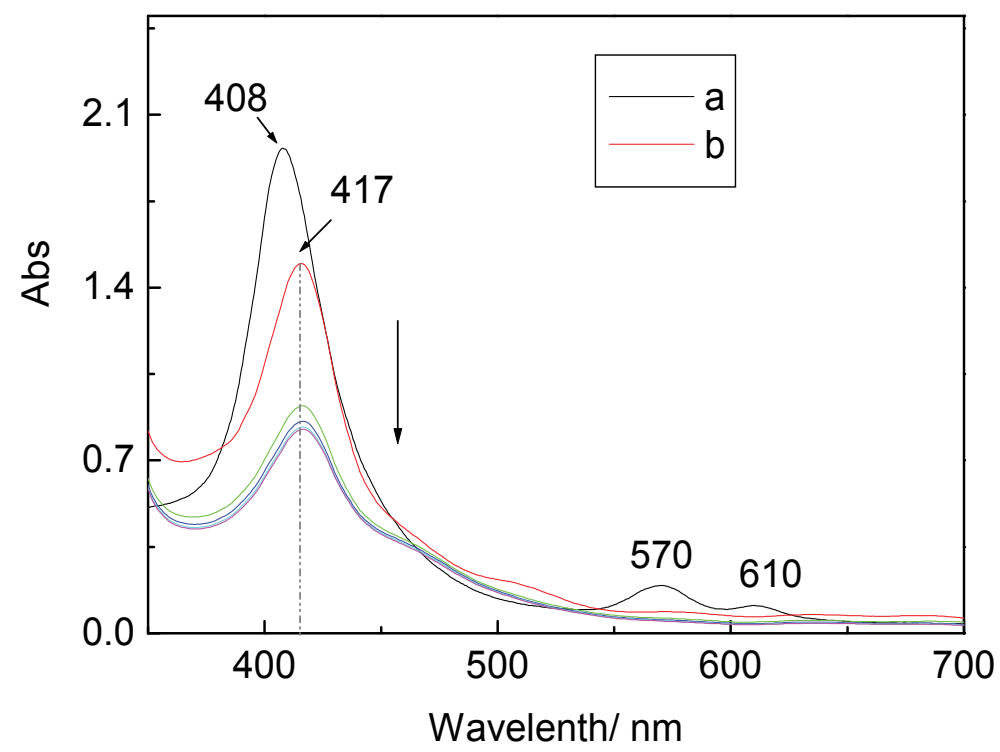

Fig. 12. In situ UV-vis spectra of cyclohexene epoxidation of catalyzed by $\left(\mathrm{Fe}^{\mathrm{IIITPP}}\right)_{2} \mathrm{O}$ in the presence of molecular oxygen and isobutyraldehyde (time scan with 5 min intervals), cyclohexene $(1 \mathrm{mmol})$, acetonitrile $(6 \mathrm{~mL})$, catalyst $\left(1 \times 10^{-8} \mathrm{~mol} / \mathrm{L}\right)$, isobutyraldehyde $(10 \mathrm{mmol}), \mathrm{rt},(\mathrm{a})(\mathrm{Fe} \text { IIITPP })_{2} \mathrm{O},(\mathrm{b})$ initial point of reaction in the presence of isobutyraldehyde From the UV-vis spectra, FeTPPOH as the mononuclear intermediate in the process is impossible. The active intermediate of FeTPPOH would form a new peak at $461 \mathrm{~nm}$, while no such peak was found from the in situ UV-vis spectra as shown in Figure 12. Figure 13a shows the UV-vis spectra of $\left(\mathrm{Fe} \mathrm{IIITP}_{2} \mathrm{O}\right.$ with isobutyraldehyde and cyclohexene after reacting for $20 \mathrm{~min}$. Similar UV-vis curves could be obtained for FeTPPCl catalyst as shown in Figure 13b. If the high-valent intermediate was mononuclear iron porphyrin (FeTPPCl), the Soret peak at $416 \mathrm{~nm}$ would disappear as the reported previously. However, a certain degree loss of intensity for the Soret peak at $416 \mathrm{~nm}$ could be observed for the present system. As shown in Figure 13, the intensity of Soret peak at 416 remains unchanged after 20 min. The above results indicate that both the dinuclear and the mononuclear high-valent intermediate exist in the catalytic system. Probably two kinds of oxo-iron intermediates, that is $\mathrm{O}=\mathrm{V} F e-\mathrm{O}-\mathrm{Fe}=\mathrm{O}$ and $\mathrm{Fe}=\mathrm{O}$ were formed by series of free radical processes. Formation of epoxide is assumed by the reaction of oxo-iron intermediates with olefins.

In the biomimetic catalytic oxidation of olefins using metalloporphyrins, catalysts have been fixed on various supports in order to suppress the dimerization of oxo intermediates or inactivation due to the oxidation of the porphyrin ring to inhibit this reaction. However, in the oxidation using immobilized catalysts, the activity decreases due to suppression of the degree of freedom of the complexes or diffusion of reactants onto the catalytic sites. Aiming to develop a highly selective catalyst, composite materials were synthesized, in which porphyrin complexes with various substituents were intercalated between clay layers to maintain a space on the axis of the central metal of porphyrin as a reaction field.[99] Specifically, cationic porphyrinato cobalt complexes with the substituents of the quaternary ammonium salt of heterocyclic amine (Scheme 6) were synthesized, and these complexes were fixed between the montmorillonite layers. By using these pillared clay catalysts, the 
epoxidation of cyclohexene with molecular oxygen in the presence of a reducing agent were examined for high activity and selectivity.

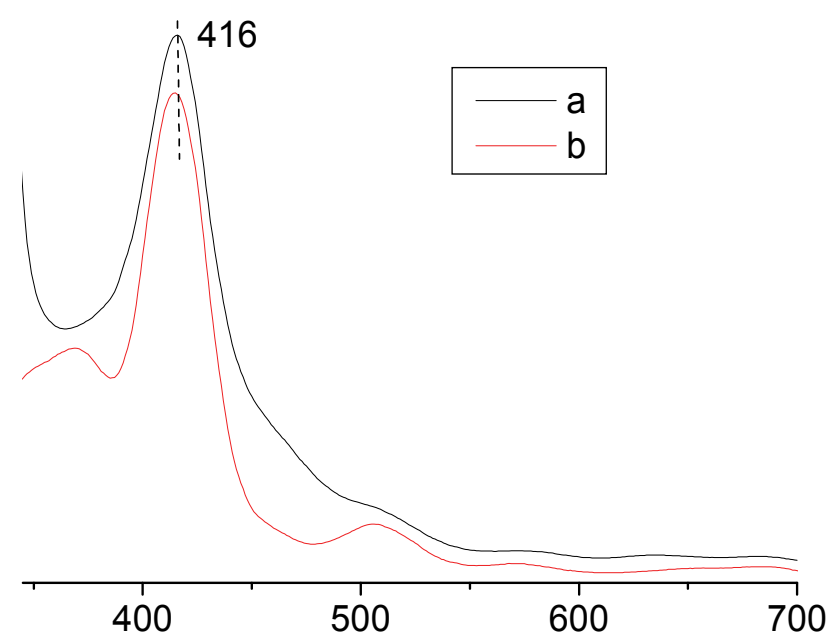

Fig. 13. The UV-vis spectra of (FeIIITPP $)_{2} \mathrm{O}$ with isobutyraldehyde and cyclohexene after 20 min of reaction time (a), FeTPPCl (b)

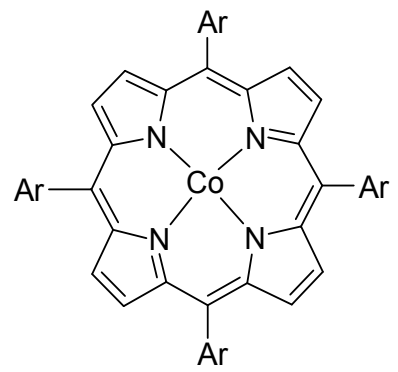

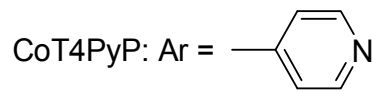

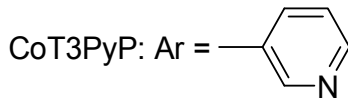

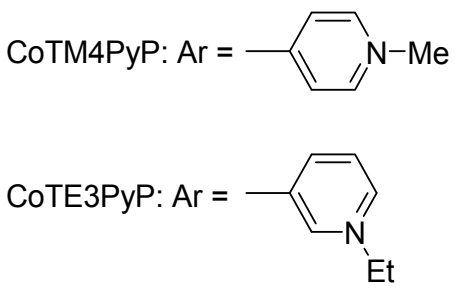

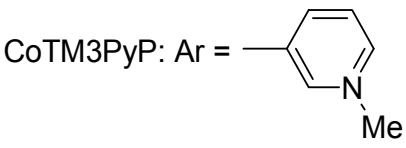

Scheme 6. Structure of cobalt porphyrin complexes

Figure 14 shows the relationship between the yield of epoxide and reaction time in the oxygen oxidation of cyclohexene in the presence of isobutyraldehyde using the CoTE3PyP/MT catalyst, in comparison with the cases of the reaction in a homogeneous system using the CoTE3PyP complex and the reaction of pure montmorillonite.

Although the homogeneous complex catalyst contained about 20 times as much CoTE3PyP as the interlayer-fixed catalyst, its activity for the oxidation was very low. Although a slight quantity of epoxide was generated in the presence of pure MT or T4PyP/MT free of a metal, the reaction stopped in about $5 \mathrm{~h}$; the yield of 1,2-epoxycyclohexane was quite similar to that in the oxidation without catalyst. Therefore, it is suggested that peroxides contained in the substrate or reducing agent were involved in the reaction, and that neither MT nor T4PyP/MT was active for the oxygen oxidation. In the reaction using CoTE3PyP/MT as a catalyst, there 


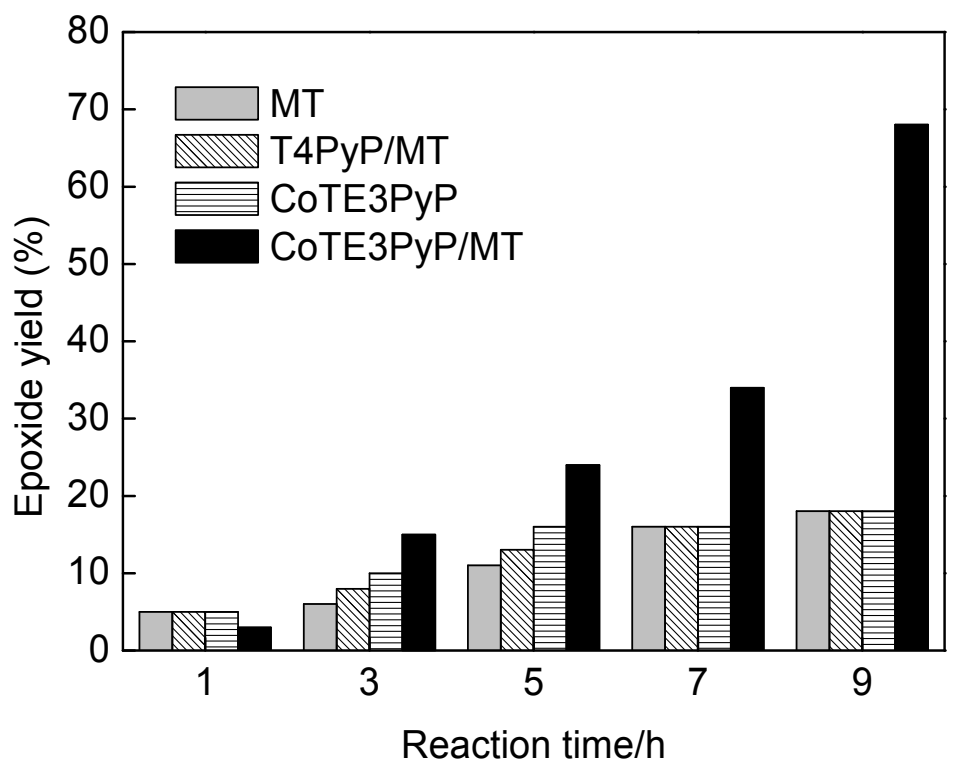

Fig. 14. Relationship between the yield of 1,2-epoxycyclohexane and reaction time. Conditions: cat., $20 \mathrm{mg}$ (in case of T4PyP/MT and CoTE3PyP/MT, [porphyrin]: $6.4 \times 10^{-5}$ $\mathrm{mol} / \mathrm{g}$ ); cyclohexene, $0.04 \mathrm{~mol}$; isobutyraldehyde, $0.06 \mathrm{~mol}$; acetonitrile, $10 \mathrm{~mL}$.

was an induction period in the initial stage of the reaction and the yield of epoxide remarkably increased after $5 \mathrm{~h}$. This result is attributed to the interlayer expansion by the swelling of montmorillonite to enhance the degree of freedom of catalytic sites and diffusion of reactants.

The results of the oxidation of cyclohexene by using various interlayer-fixed porphyrinato cobalt complexes, that is, the catalysts having the different clay basal spacing are shown in Table 10.

CoTE3PyP/MT, which has the largest interlayer spacing, gave the highest epoxide yield. Moreover, the oxygen oxidation of cyclohexene was carried out using CoTE3PyP/MT catalysts containing intercalated CoTE3PyP in the range of $(1.6-6.4) \times 10^{-5} \mathrm{~mol} / \mathrm{g}$. The epoxide yield improved with the quantity of CoTE3PyP and by using CoTE3PyP/MT catalyst with a content of $6.4 \times 10^{-5} \mathrm{~mol} / \mathrm{g}$ the epoxide yield reached $65.9 \%$ in a reaction time of $9 \mathrm{~h}$. On the other hand, the use of CoTM4PyP/MT and CoTM3PyP/MT, of which the interlayer spacing are 0.42 and $0.54 \mathrm{~nm}$, respectively, as catalyst gave the low yield of epoxide. Their space on the central metal for the oxidation to occur must be restricted because of the thickness of the porphyrin molecule. Thus, it is difficult for cylcohexene of which the size is about $0.45 \mathrm{~nm}$ to pull in this space. By contrast, CoTE3PyP/MT having the interlayer spacing of $0.68 \mathrm{~nm}$ seems to provide sufficient space so that cyclohexene approaches the central metal of porphyrin. These results indicate that the formation of a reaction field can be controlled by a kind of meso-substitutent of the porphyrinato cobalt complex intercalated between montmorillonite layers and the oxidation can proceed selectively in the molecule size of the substrate by means of this reaction field. 


\begin{tabular}{|c|c|c|c|c|}
\hline \multirow{2}{*}{ Catalyst } & \multirow{2}{*}{$\begin{array}{c}\text { Time } \\
\text { (h) }\end{array}$} & \multicolumn{2}{|c|}{ Yield (\%) } & \multirow{2}{*}{$\begin{array}{c}\text { Turnover } \\
\text { number }\end{array}$} \\
\hline & & 1,2-Epoxycyclohexane & cyclohexanone & \\
\hline \multirow[t]{2}{*}{ CoTM4PyP/MT } & 9 & 17.6 & 2.6 & 25250 \\
\hline & 24 & 36.6 & 7.7 & 55375 \\
\hline \multirow[t]{2}{*}{ СоТМЗРуP/MT } & 9 & 23.8 & 2.8 & 33250 \\
\hline & 24 & 31.1 & 5.1 & 45250 \\
\hline \multirow[t]{2}{*}{ СоТЕЗРуP/МТ } & 9 & 41.5 & 4.9 & 58000 \\
\hline & 24 & 56.1 & 9.8 & 82375 \\
\hline \multirow[t]{2}{*}{ CoTE3РyP/MT $b$} & 9 & 48.8 & 7.3 & 23375 \\
\hline & 24 & 56.1 & 12.2 & 28458 \\
\hline \multirow[t]{2}{*}{ СоТЕЗРуP/МТc } & 9 & 65.9 & 9.8 & 23656 \\
\hline & 24 & 61.0 & 12.2 & 22875 \\
\hline \multirow[t]{2}{*}{ CoTEЗРyP } & 9 & 31.7 & 4.9 & 580 \\
\hline & 24 & 34.1 & 4.9 & 618 \\
\hline \multirow[t]{2}{*}{ MT } & 9 & 17.1 & 2.4 & - \\
\hline & 24 & 22.0 & 4.9 & - \\
\hline
\end{tabular}

${ }^{a}$ Cat. $20 \mathrm{mg}$ ([porphyrin]: $1.6 \times 10^{-5} \mathrm{~mol} / \mathrm{g}$ ); cyclohexene $0.04 \mathrm{~mol}$; isobutyraldehyde $0.06 \mathrm{~mol}$; acetonitrile $10 \mathrm{~mL}$.

${ }^{b}$ Cat. $20 \mathrm{mg}$ ([porphyrin]: $\left.4.8 \times 10^{-5} \mathrm{~mol} / \mathrm{g}\right)$

${ }^{c}$ Cat. $20 \mathrm{mg}$ ([porphyrin]: $\left.6.4 \times 10^{-5} \mathrm{~mol} / \mathrm{g}\right)$

Table 10. Oxidation of cyclohexene by using various interlay-fixed porphyirnato cobalt complexes as catalyst ${ }^{a}$

\section{Epoxidation without reductant}

The main goal in catalytic olefin epoxidations is to use both oxygen atoms of molecular oxygen to produce selectively epoxides without using reducing agents as co-factors. Such a reaction is particularly important for the large scale production of various epoxides. In the field of metalloporphyrin-catalyzed olefins epoxidation with molecular oxygen, the breakthrough was reported by Groves group. [100] $\mathrm{Ru}$ VI (TMP $)(\mathrm{O})_{2}$ catalyzes the aerobic epoxidation of olefins at room temperature and normal pressure. Measurement of oxygen uptake during the oxidation of cyclooctene indicated that $2 \mathrm{~mol}$ of epoxide were produced per mole of molecular oxygen consumed. The proposed mechanism is shown in Scheme 7. The active oxidant $\mathrm{Ru}{ }^{\mathrm{VI}}(\mathrm{TMP})(\mathrm{O})_{2}$ reacts with an olefin to afford the epoxide and $\mathrm{Ru}^{\mathrm{IV}}(\mathrm{TMP})(\mathrm{O})$, and the disproportionation of the latter generates $\mathrm{Ru}^{\mathrm{II}}(\mathrm{TMP})$ and the active oxidant $\mathrm{Ru}^{\mathrm{VI}}(\mathrm{TMP})(\mathrm{O})_{2}$. The $\mathrm{Ru}^{\mathrm{II}}(\mathrm{TMP})$ re-enters the catalytic cycle after being oxidized by dioxygen to $\mathrm{Ru}^{\mathrm{IV}}(\mathrm{TMP})(\mathrm{O})$.

Since then, few examples on the metalloporphyrins-catalyzed epoxidation by dioxygen in the absence of a co-reductant could be found. Polymeric sheet porphyrin: polymeric mesotetra-(1,5- naphthalene bisulfonyloxo) phenyl cobalt porphyrins (PTNBSOPPCo) showed good activity in the oxidation of styrene with dioxygen.[101]

Simple metalloporphyirns were employed as the catalyst for the aerobic oxidation of $\alpha$ pinene in the absence of co-catalyst and solvent. Among various metalloporphyrins, $\mathrm{MnTPPCl}$ presented the best selectivity regarding the epoxide. When the reaction was carried out under $80^{\circ} \mathrm{C}$ for $5 \mathrm{~h}$, the conversion of $\alpha$-pinene was $23 \%$ and the selectivity for 
the product of $\mathrm{C}=\mathrm{C}$ bond was $60 \%$. With increasing the electron- donating abilities of the peripheral substituents, the selectivity regarding the oxidation of $\mathrm{C}=\mathrm{C}$ bond decreased. The mechanism involves a high-valence metal-oxygen positive radical, which plays an important role in the epoxidation of alkenes.[102]
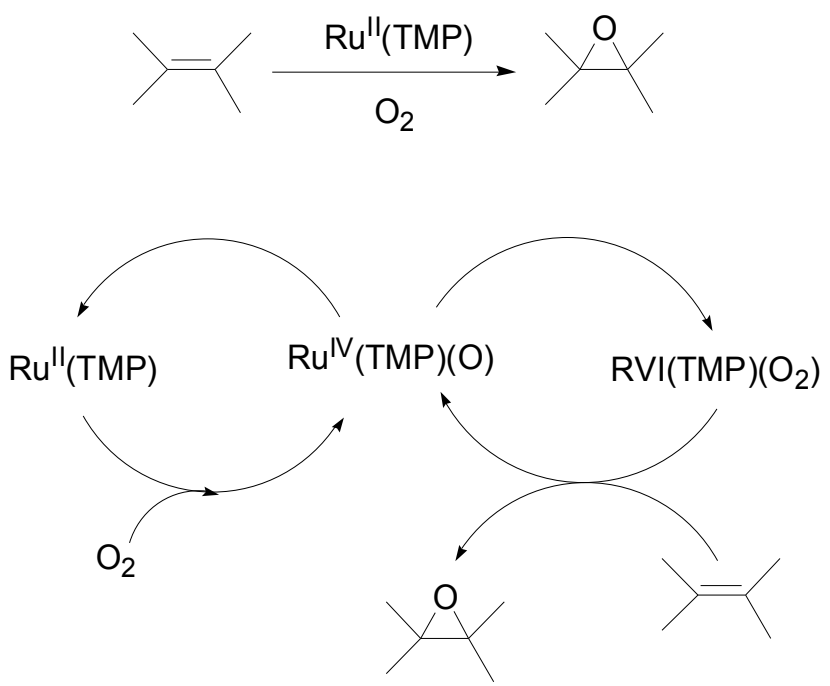

Scheme 7. $\mathrm{Ru}(\mathrm{TMP})$-catalyzed olefins epoxidation with dioxygen

\section{Conclusion}

From this chapter, it is convincing that metalloporphyrins are very versatile oxidation catalysts which can be used in epoxidation of olefins. It clearly demonstrated the impressive progress made in the area of metalloporphyrins-catalyzed aerobic epoxidation of olefins with different reductant, especially using aldehydes as reductant. Attempts have been made to avoid the sacrificial use electrons or reductant in the epoxidation of olefins with molecular oxygen. These methodologies described based on various epoxidation reactions have clearly opened new avenues for major growth in the area of epoxidation using molecular oxygen. The use of molecular oxygen as a source of oxygen atom and the formation of few neutral byproducts clearly make aerobic epoxidation a highly desirable, economically viable, and environmentally acceptable reaction.

Biomimetic catalysis of metalloporphyrins is now a meeting point for chemistry, chemical, catalysis and biology. Metalloporphyrins catalyzed aerobic epoxidation of olefins becoming an important and highly rewarding protocol for important feedstock for petrochemical and fine chemical industries. The aerobic epoxidation process of biomimetic catalysis will be performed at the industrial scale in the future due to the high efficiency and selectivity.

\section{Acknowledgement}

The authors thank the National Natural Science Foundation of China (21036009 and 20976203), Higher-level talent project for Guangdong provincial universities and the Fundamental Research Funds for the Central Universities for providing financial support to this project. 


\section{References}

[1] Backvall, J. E. Modern Oxidation Methods; Wiley-VCH: Weinheim, 2004.

[2] Labinger, J. A.; Bercaw, J. E. Nature 2002, 417, 507-514.

[3] Olah, G. A.; Molnar, A. Hydrocarbon Chemistry; John Wiley \& Sons Inc.: Hoboken, 2003.

[4] Parshall, G. H. Homogeneous Catalysis: The Application of Catalysis by Soluble Transition Metal Complexes; Wiley: New York, 1980.

[5] Punniyamurthy, T.; Velusamy, S.; Iqbal, J. Chem. Rev. 2005, 105, 2329-2363.

[6] Sheldon, R. A.; Kochi, J. K. Metal-Catalyzed Oxidation of Organic Compounds; Academic Press: New York, 1981.

[7] Burke, A. J. Coordin. Chem. Rev. 2008, 252, 170-175.

[8] Xia, Q. H.; Ge, H. Q.; Ye, C. P.; Liu, Z. M.; Su, K. X. Chem. Rev. 2005, 105, 1603-1662.

[9] Chatterjee, D. Coordin .Chem. Rev. 2008, 252, 176-198.

[10] Wong, O. A.; Shi, Y. Chem. Rev. 2008, 108, 3958-3987.

[11] Lane, B. S.; Burgess, K. Chem. Rev. 2003, 103, 2457-2473.

[12] Luts, T.; Frank, R.; Suprun, W.; Fritzsche, S.; Hey-Hawkins, E.; Papp, H. J. Mol. Catal. A 2007, 273, 250-258.

[13] Rubajlo, V. L.; Maslov, S. A.; Zaikov, G. E. Liquid-Phase Oxidation of Unsaturated Compounds; Nova Science Publishers, Inc.: Hauppauge, 1993.

[14] Castaman, S. T.; Nakagaki, S.; Ribeiro, R. R.; Ciuffi, K. J.; Drechsel, S. M. J. Mol. Catal. A 2009, 300, 89-97.

[15] Serafimidou, A.; Stamatis, A.; Louloudi, M. Catal. Commun. 2008, 9, 35-39.

[16] Loew, G. H.; Harris, D. L. Chem. Rev. 2000, 100, 407-420.

[17] Sono, M.; Roach, M. P.; Coulter, E. D.; Dawson, J. H. Chem. Rev. 1996, 96, 2841-2888.

[18] Gunsalus, I. C.; Pederson, T. C.; Sligar, S. G. Annu. Rev. Biochem. 1975, 44, 377.

[19] Ortiz de Montellano, P. R.; De Voss, J. J. Nat. Prod. Rep. 2002, 19, 477-493.

[20] Ortiz de Montellano, P. R. Cytochrome P450: structure, mechanism and biochemistry; Plenum: New York, 1996.

[21] Hayaishi, O. Molecular mechanism of oxygen activation; Academic Press: New York, 1974.

[22] Meunier, B.; Bernadou, J. Top .Catal. 2002, 21, 47-54.

[23] Omura, T.; Ishimura, Y.; Fujii-Kuriyama, Y. Cytochrome P450; Kodansha: Tokyo, 1993.

[24] Filatov, M.; Reckien, W.; Peyerimhoff, S. D.; Shaik, S. J. Phys. Chem. A 2000, 104, 1201412020.

[25] Tshuva, E. Y.; Lippard, S. J. Chem. Rev. 2004, 104, 987-1012.

[26] Meunier, B.; de Visser, S. P.; Shaik, S. Chem. Rev. 2004, 104, 3947-3980.

[27] Costas, M.; Mehn, M. P.; Jensen, M. P.; Que, L., Jr. Chem. Rev. 2004, 104, 939-986.

[28] Meunier, B. Biomimetic Oxidations Mediated by Metal Complexes; Imperial College Press: London, 2000.

[29] Meunier, B. Chem. Rev. 1992, 92, 1411-1456.

[30] Meunier, B. Metalloporphyrin Catalyzed Oxidation; Kluwer Academic Publisher: 1994.

[31] Reichhart, D. W.; Hehn, A.; Didieriean, L. Trends Plant Sci. 2000, 5, 116-123.

[32] Bernadou, J.; Meunier, B. Adv. Synth. Catal. 2004, 346, 171-184.

[33] Woggon, W. D. Acc.Chem. Res. 2005, 38, 127-136.

[34] Chen, H. Y.; Ji, H. B.; Zhou, X. T.; Xu, J. C.; Wang, L. F. Catal. Commun. 2009, 10, 828-832.

[35] Zhou, X. T.; Ji, H. B.; Yuan, Q. L. J. Porphyr. Phthalocya. 2008, 12, 94-100.

[36] Zhou, X. T.; Ji, H. B.; Yuan, Q. L.; Xu, J. C.; Pei, L. X.; Wang, L. F. Chin. J. Chem. 2008, 26, 1114-1118.

[37] Ji, H. B.; Yuan, Q. L.; Zhou, X. T.; Pei, L. X.; Wang, L. F. Bioorg. Med. Chem. Lett. 2007, 17, 6364-6368. 
[38] Zhou, X. T.; Ji, H. B.; Cheng, Z.; Xu, J. C.; Pei, L. X.; Wang, L. F. Bioorg. Med. Chem. Lett. $2007,17,4650-4653$.

[39] Zhou, X. T.; Yuan, Q. L.; Ji, H. B. Tetrahedron Lett. 2010, 51, 613-617.

[40] Turner, M.; Vaughan, O. P. H.; Kyriakou, G.; Watson, D. J.; Scherer, L. J.; Davidson, G. J. E.; Sanders, J. K. M.; Lambert, R. M. J. Am. Chem. Soc. 2009, 131, 1910-1914.

[41] Alkordi, M. H.; Liu, Y. L.; Larsen, R. W.; Eubank, J. F.; Eddaoudi, M. J. Am. Chem. Soc. 2008, 130, 12639-12641.

[42] Mirkhani, V.; Moghadam, M.; Tangestaninejad, S.; Kargar, H. Appl. Catal. A 2006, 303, 221-229.

[43] Mansuy, D. Coord. Chem. Rev. 1993, 125, 129-141.

[44] Ji H.B.; She Y.B. Green Oxidation and Reduction; China Petrochemical Press : Beijing, 2005.

[45] Zhou, X. T.; Ji, H. B.; Pei, L. X.; She, Y. B.; Xu, J. C.; Wang, L. F. Chin. J. Org. Chem. 2007, $27,1039-1049$.

[46] Groves, J. T.; Nemo, T. E. J. Am. Chem. Soc. 1983, 105, 5786-5791.

[47] Campestrini, S.; Tonellato, U. J. Mol. Catal. A 2001, 171, 37-42.

[48] Nam, W.; Oh, S.-Y.; Sun, Y. J.; Kim, J.; Kim, W.-K.; Woo, S. K.; Shin, W. J. Org. Chem. 2003, 68, 7903-7906.

[49] Stephenson, N. A.; Bell, A. T. J. Mol. Catal. A 2007, 275, 54-62.

[50] Rahiman, A. K.; Bharathi, K. S.; Sreedaran, S.; Narayanan, V. Catal. Lett. 2009, 127, 175182.

[51] Castaman, S. T.; Nakagaki, S.; Ribeiro, R. R.; Ciuffi, K. J.; Drechsel, S. M. J. Mol. Catal. A 2009, 300, 89-97.

[52] Li, Z.; Xia, C. G.; Ji, M. Appl. Catal. A 2003, 252, 17-21.

[53] Haber, J.; Iwanejko, R.; Poltowicz, J.; Battioni, P.; Mansuy, D. J. Mol. Catal. A 2000, 152, 111-115.

[54] Haber, J.; Iwanejko, R.; Poltowicz, J.; Battioni, P.; Mansuy, D. J. Mol. Catal. A 2000, 152, $117-120$.

[55] Mohajer, D.; Rezaeifard, A. Tetrahedron Lett. 2002, 43, 1881-1884.

[56] Mohajer, D.; Karimipour, G.; Bagherzadeh, M. New J. Chem. 2004, 28, 740-747.

[57] Tabushi, I.; Morimitsu, K. Tetrahedron Lett. 1986, 27, 51-54.

[58] Tabushi, I.; Yazaki, A. J. Am. Chem. Soc. 1981, 103, 7371-7373.

[59] Tabushi, I.; Kodera, M.; Yokoyama, M. J. Am. Chem. Soc. 1985, 107, 4466-4473.

[60] Bedioui, F.; Devynck, J.; Bied-Charreton, C. J. Mol. Catal. A 1996, 113, 3-11.

[61] Leduc, P.; Battioni, P.; Bartoli, J. F.; Mansuy, D. Tetrahedron Lett. 1988, 29, 205-208.

[62] Tsuda, Y.; Takahashi, K.; Yamaguchi, T.; Matsui, S.; Komura, T. J. Mol. Catal. A 1998, 130, 285-295.

[63] Tsuda, Y.; Takahashi, K.; Yamaguchi, T.; Matsui, S.; Komura, T.; Nishiguchi, I. J. Mol. Catal. A 1999, 138, 145-153.

[64] Mukaiyama, T.; Yamada, T.; Nagata, T.; Imagawa, K. Chem. Lett. 1993, 327-330.

[65] Yamada, T.; Takai, T.; Rhode, O.; Mukaiyama, T. Bull. Chem. Soc. Jpn. 1991, 64, 21092117.

[66] Nagata, T.; Imagawa, K.; Yamada, T.; Mukaiyama, T. Chem. Lett. 1994, 1259-1262.

[67] Qi, J. Y.; Li, Y. M.; Zhou, Z. Y.; Che, C. M.; Yeung, C. H.; Chan, A. S. C. Adv. Synth. Catal. 2005, 347, 45-49.

[68] Ravikumar, K. S.; Barbier, F.; Begue, J. P.; Delpon, D. B. Tetrahedron 1998, 54, 7457-7464.

[69] Raja, R.; Sankar, G.; Thomas, J. M. Chem.Commun. 2009, 9, 829-830.

[70] Ellis, S.; Kozhevnikov, I. V. J. Mol. Catal. A 2002, 187, 227-235.

[71] Mandal, A. K.; Iqbal, J. Tetrahedron 1997, 53, 7641-7648. 
[72] Zhou, X. T.; Ji, H. B.; Xu, H. C.; Pei, L. X.; Wang, L. F.; Yao, X. D. Tetrahedron Lett. 2007, 48, 2691-2695.

[73] Qi, J. Y.; Qiu, L. Q.; Lam, K. H.; Yip, C. W.; Zhou, Z. Y.; Chan, A. S. C. Chem.Commun. 2003, 1058-1059.

[74] Nam, W.; Baek, S. J.; Lee, K. A.; Ahn, B. T.; Muller, J. G.; Burrows, C. J.; Valentine, J. S. Inorg. Chem. 1996, 35, 6632-6633.

[75] Ravikumar, K. S.; Barbier, F.; Begue, J. P.; Delpon, D. B. Tetrahedron 1998, 54, 7457-7464.

[76] Nam, W.; Kim, H. J.; Kim, S. H.; Ho, R. Y. N.; Valentine, J. S. Inorg. Chem. 1996, 35, 10451049.

[77] Zhou, X. T.; Ji, H. B. Chem. Eng. J. 2010, 156, 411-417.

[78] Kaneda, K.; Haruna, S.; Imanka, T.; Hamamoto, M.; Nishiyama, Y.; Ishii, Y. Tetrahedron Lett. 1992, 33, 6827-6830.

[79] Harvey, J. D.; Ziegler, C. J.; Telser, J.; Ozarowski, A.; Krzystek, J. Inorg. Chem. 2005, 44, 4451-4453.

[80] Goldberg, D. P.; Telser, J.; Krzystek, J.; Montalban, A. G.; Brunel, L. C.; Barrett, A. G. M.; Hoffman, B. M. J. Am. Chem. Soc. 1997, 119, 8722-8723.

[81] Lansky, D. E.; Mandimutsira, B.; Ramdhanie, B.; Clausen, M.; Penner-Hahn, J.; Zvyagin, S. A.; Telser, J.; Krzystek, J.; Zhan, R.; Ou, Z.; Kadish, K. M.; Zakharov, L.; Rheingold, A. L.; Goldberg, D. P. Inorg. Chem. 2005, 44, 4485-4498.

[82] Caudle, M. T.; Mobley, C. K.; Bafaro, L. M.; Lobrutto, R.; Yee, G. T.; Groy, T. L. Inorg. Chem. 2004, 43, 506-514.

[83] Friedermann, G. R.; Halma, M.; Dias de Freitas Castro, K.; Benedito, F. L.; Doro, F. G.; Drechsel, S. M.; Mangrich, A. S.; Assis, M. D.; Nakagaki, S. Appl. Catal. A 2006, 308, 172-181.

[84] Konishi, S.; Hoshino, M.; Imamura, M. J. Phys. Chem. 1982, 86, 4537-4539.

[85] Nunes, G. S.; Mayer, I.; Toma, H. E.; Araki, K. J. Catal. 2005, 236, 55-61.

[86] Rebelo, S. L. H.; Pereira, M. M.; Simoes, M. M. Q.; Neves, M. G. P. M.; Cavaleiro, J. A. S. J. Catal. 2005, 234, 76-87.

[87] Haber, J.; Mlodnicka, T.; Poltowicz, J. J. Mol. Catal. 1989, 54, 451-461.

[88] Guo, C. C.; Liu, Q.; Wang, X. T.; Hu, H. Y. Appl. Catal. A 2005, 282, 55-59.

[89] Guo, C. C.; Liu, X. Q.; Liu, Y.; Liu, Q.; Chu, M. F.; Zhang, X. B. J. Mol. Catal. A 2003, 192, 289-294.

[90] Guo, C. C. J. Catal. 1998, 178, 182-187.

[91] Reginato, G.; Di Bari, L.; Salvadori, P.; Guilard, R. Eur. J. Org. Chem. 2000, 1165-1171.

[92] Zhao, Y. C.; Xiang, Y. Z.; Pu, L.; Yang, M.; Yu, X. Q. Appl. Catal. A 2006, 301, 176-181.

[93] Elemans, J. A. A. W.; Bijsterveld, E. J. A.; Rowan, A. E.; Nolte, R. J. M. Eur. J. Org. Chem. 2007, 751-757.

[94] Wentzel, B. B.; Alsters, P. L.; Feiters, M. C.; Nolte, R. J. M. J. Org. Chem. 2004, 69, 3453-3464.

[95] Wentzel, B. B.; Gosling, P. A.; Feiters, M. C.; Nolte, R. J. M. J. Chem. Soc., Dalton Trans 1998, 2241-2246.

[96] Song, W. J.; Seo, M. S.; George, S. D.; Ohta, T.; Song, R.; Kang, M. J.; Kitagawa, T.; Solomon, E. I.; Nam, W. J. Am. Chem. Soc. 2007, 129, 1268-1277.

[97] Nam, W.; Lim, M. H.; Moon, S. K.; Kim, C. J. Am. Chem. Soc. 2000, 122, 10805-10809.

[98] Shimazaki, Y.; Nagano, T.; Takesue, H.; Ye, B. H.; Tani, F.; Naruta, Y. Angew. Chem. Int. Ed. 2004, 129, 98-100.

[99] Kameyama, H.; Narumi, F.; Hattori, T.; Kameyama, H. J. Mol. Catal. A 2006, 258, 172-177.

[100] Groves, J. T.; Quinn, R. Inorg. Chem. 1984, 23, 3844-3846.

[101] He, Y. F.; Wang, R. M.; Wang, Y. P.; Xia, C. G. Eur. Polym. J. 2002, 38, 2129-2132.

[102] Guo, C. C.; Yang, W. J.; Mao, Y. L. J. Mol. Catal. A 2005, 226, 279-284. 


\title{
Biomimetic Oxidation of Hydrocarbons with Air over Metalloporphyrins
}

\author{
Guofang Jiang, Qiang Liu and Cancheng Guo* \\ School of Chemistry and Chemical Engineering, Hunan University, \\ Changsha 410082, Hunan, \\ P. R. China
}

\section{Introduction}

The oxidation of $\mathrm{C}-\mathrm{H}, \mathrm{C}-\mathrm{C}$ and $\mathrm{C}=\mathrm{C}$ of hydrocarbons, from the syntheses of many fine chemicals to the manufacture of various commodities in large scale, plays great important role in transformation of basic materials to useful synthetic building in organic chemistry and in conversion of hydrocarbon to oxygen-containing industrial production especially the hydroxylation, epoxidation, degradation of hydrocarbon derivatives[1-6]. Conventional oxidation methodologies often suffer low chemo- and/or regio-selectivity with low conversion and low turnover number (TON) in an unfriendly way from both economical and environmental aspects which the catalyst is very expensive and generates amounts of hazardous waste. Oxidation reactions catalyzed by both non-metal[7-8] and metal[9-14] catalysts have been receiving increasing attention, particularly for the highly selective aerobic oxidations catalyzed by metal complexes[15-17]. In nature, aerobic oxidation processes are carried out in a highly selective manner by mono- or dioxygenases under mild conditions[18-19]. A well-known type of monooxygenase is cytochrome P-450(CP-450)[2023], which features an iron porphyrin core, and can catalyze a wide variety of oxidation reactions including epoxidation, hydroxylation, dealkylation, degradation, dehydrogenation, and oxidation of amines, sulfides, alcohols and aldehydes, even for unreactive substrates such as unactivated hydrocarbons. This stimulates numerous efforts in developing biomimetic oxidation systems[24-26].

Metalloporphyrins, with a core structure closely resembling that of the iron porphyrin core of CP-450, have been extensively studied as catalysts to oxidate a series kind of reactions mimic the natural style[24,27]. In 1979, Groves and co-workers[28] reported the first oxidation system with synthetic metalloporphyrin as a catalyst. They developed the oxidation system of terminal oxidant iodosylbenzene(PhIO) and ironporphyrin catalyst [FeIII(por)Cl], which can effect both epoxidation of styrene and cyclohexene, and hydroxylation of cyclohexane and adamantane. Subsequently, many reports focusing on metalloporphyrin-catalyzed oxidation systems have appeared in the literature, as described in previous reviews[29-34]. As documented in the literature, the hydroxylation of alkanes and epoxidation of alkenes catalyzed by iron, manganese and ruthenium porphyrins with the traditional terminal oxidants $\mathrm{PhIO}, \mathrm{NaOCl}$ and 2,6-dichloropyridine- $\mathrm{N}$-oxide, including enantioselective oxidations are the most extensively studied systems. 
It has been well documented that the active species in the oxidation reactions catalyzed by CP-450 are high-valent iron-oxo species. High-valent oxometalloporphyrins have been observed in the reactions of synthetic metalloporphyrins with PhIO, $m$-CPBA and $\mathrm{O}_{2}$, and have been reported to be able to oxidize alkanes, alkenes, phosphines, amines and sulfides by oxygen atom transfer and/or insertion reactions[31,35].

In the past several decades, oxyfunctionalization of hydrocarbons in biomimetic systems with molecular oxygen in the presence of metalloporpyrins as catalysts is one of the most attractive transformations in synthetic organic chemistry and manufacture of hydrocarbon derivatives. The behavior of metalloporphyrin-catalyzed hydrocarbon oxidation under mild conditions has been systematically studied as a model of CP-450 monooxygenase by many researchers[21,24,36,37] with the ultimate goal of searching for the most effective metalloporphyrin catalysts for hydrocarbon oxidation. Chemoselective oxidation process of hydrocarbons is of great importance in fine chemicals industry and oil product industry. Aerobic oxidation of alkanes, alkenes and aromatic hydrocarbons, an extremely important reaction in chemical industries, has attracted more and more attention during recent decade due to the oxidation products are widely used in pharmaceutical, dyestuff, perfume, resin and textile fiber industries, such as the very important Nylon-6 and Nylon-66. Investigation of the mechanism, industrial procedure and the pertinent fields for biomimetic oxidation of hydrocarbons with air over metalloporphyrins attracts more and more attentions.

This chapter mainly summarized the systematic researches of our group focusing on biomimetic oxidation of hydrocarbon with air over simple metalloporphyrins, $\mu$-oxometalloporphyrins including oxidation of alkanes, alkenes, aromatic hydrocarbons, mechanism study and industrial-scale biomimetic oxidation of hydrocarbons.

\section{Biomimetic oxidation of $\mathrm{C}-\mathrm{H}$ of alkanes}

Recent work on catalytic hydroxylation using metalloporphyrin-based oxidation systems is mainly focused on $\mathrm{C}-\mathrm{H}$ bonds of alkane. A few research groups reported a catalytic system consisting of monometalloporphyrin and molecular oxygen[38-39]. Unfortunately, this system needs to use the reducers. To improve this system, Lyons et al. synthesize the perhalogenated metalloporphyrin complexes and use the complexes as catalysts for isobutane and propane hydroxylation with molecular oxygen[40]. These results open a door for the biomimetic oxidation of hydrocarbons with molecular oxygen over metalloporphyrins in which coreductants are not required, but more and more researching work must to be investigated because perhalogenated metalloporphyrin complexes is very expensive and in high catalyst loading with low turn over number(TON), which makes it difficult for industrialization.

\subsection{Biomimetic oxidation of C-H of cyclohexane}

The liquid-phase aerobic oxidation of cyclohexane to cyclohexanol and cyclohexanone (the so-called KA oil) has always been an area of intensive research[41-42] because it is one of the key steps in the industrial production of $\varepsilon$-caprolactam and Nylon[43-44]. For this particular transformation, current industrial methods either employ cobalt salts as catalysts or use no catalyst at all (autoxidation at higher temperature). However, there are several major shortcomings associated with the current technology, such as low conversion $(\sim 4 \%)$ and low selectivity for the desired product KA oil $(<80 \%)$. Based on above unsolved problems, many scientists regarded that the industrial production of KA oil from cyclohexane has been 
considered as the least efficient procedure of all major industrial processes. The development of a more efficient and chemo-selective process is highly desirable. Lately, particularly in the last 10 years, scientists have developed many new heterogeneous or homogeneous catalytic processes for the aerobic oxidation of cyclohexane, such as $\mathrm{N}$ hydroxyphthalimide(NHPI)[45-47], metalloaluminophosphates [58-50], Zeolite Y[51-52], etc., to overcome these shortcomings, and much progress has been made.

For the purpose of solving the bottle-neck problem in oxidation of hydrocarbons, our group has been engaging development novel biomimetic and more efficient method using porphyrin complexes as catalysts during the last two decades. After systematical investigation, till now, our research and development results have not only improved the reaction conversion to $>7 \%$, but also increased the selectivity of KA oil to $>85 \%$ in industrial scale. The aerobic oxidation of cyclohexane over tetraphenylporphinatocobalt(TPPCo) is currently employed by the Sina-Petro Chemical-Baling Petro chemical Company to produce more than 124,000 tons of KA oil annually.

In 2003, inspired by the success of the work of Lyons et al., we tried to use the simple $\mu$-oxobismetalloporphyrin (TPPFe $\left.{ }^{\mathrm{III}}\right)_{2} \mathrm{O}$ to catalyze hydroxylation of hydrocarbons using air as the terminal oxidant to gain a better understanding of the possibility of using simple $\mu$-oxo dimeric metalloporphyrin to catalyze the hydrocarbon oxidation directly by air. We developed a new reaction system for the aerobic oxidation of the inactive $\mathrm{C}-\mathrm{H}$ bond of cyclohexane catalyzed by a very small amount of simple $\mu$-oxo-ironporphyrin $\left(\mathrm{TPPFe}^{\mathrm{III}}\right)_{2} \mathrm{O}[53]($ Scheme 1$)$.
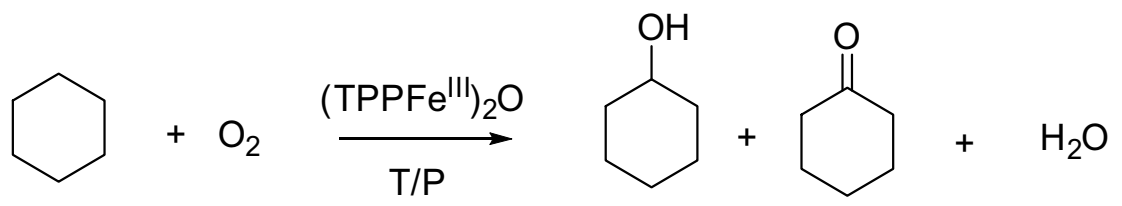

Scheme 1. Aerobic oxidation of cyclohexane by $\mu$-oxo-ironporphyrin

Cyclohexane was oxidized into cyclohexanol and cyclohexanone with air catalyzed by a very small amount of $\left(\mathrm{TPPFe}^{\mathrm{III}}\right)_{2} \mathrm{O}$ in the absence of solvent and cocatalyst. The results showed that the selectivity to cyclohexanol and cyclohexanone is $90 \%$ with a conversion of $12.55 \%$ and catalyst turnover numbers of 90,000 (based on the metal) under the optimum conditions. The reaction of cyclohexane oxidation catalyzed by $\mu$-oxo-bisironporphyrin was carried out in a $500 \mathrm{ml}$ of autoclave with $3 \mathrm{mg}$ of catalyst, $350 \mathrm{ml}$ of cyclohexane under the temperature of $398 \mathrm{~K}$ and pressure of $0.6 \mathrm{MPa}$.

The $\mu$-oxo-bisironporphyrin is synthesized from the corresponding simple metalloporphyrin, we wanted to know that whether the simple tetraphenylporphyrins could catalyzed the cyclohexane hydroxylation or not in the absence of any cocatalysts or reductants. After further investigation, we developed a catalysis system for the cyclohexane oxidation into KA oil with air catalyzed by some simple tetraphenylporphyrins in the absence of any cocatalysts or reductants[37]. After systematical researches on this catalytic oxidation at different temperatures and pressures in the presence of different catalysts, we obtained the optimum reaction conditions and found that cyclohexane conversion was up to $16.2 \%$, general yields of cyclohexanol and cyclohexanone was $82 \%$, catalyst turnover number reached 400,000.

Also the catalysis of chitosan-supported iron(III) tetraphenylporphyrin for cyclohexane oxidation with air in the absences of solvent, reductant and co-catalyst became true[54]. The 
research result showed $10.48 \%$ conversion of cyclohexane, $79.20 \%$ cyclohexanol and cyclohexanone selectivity and $1.40 \times 10^{5}$ catalyst turnover number, respectively under the relatively moderate conditions of $418 \mathrm{~K}$ and $0.8 \mathrm{MPa}$. We found that the coordination between partly deacetylated chitosan and ironporphyrin increased the yields and selectivity of the aerobic oxidation of cyclohexane catalyzed by ironporphyrin.

In order to investigate the reason why the nitrogenous biopolymers can enhance the catalytic ability of metalloporphyrins, both chitin-supported ironporphyrin and completely deacetylated chitosan-supported ironporphyrin were used to study the Fe-N coordinate behavior between ironporphyrin and the nitrogenous group of polysaccharides[55]. The corresponding coordinate constants of the ironporphyrin with different nitrogenous polysaccharides by means of Langmuir's adsorption isotherm equation were determined, and then the effects of the coordination on the cyclohexane oxidation catalyzed by polysaccharide-supported ironporphyrin were investigated. It is shown that the coordination of the nitrogenous polysaccharide to ironporphyrin had an important influence on both the conversion and selectivity of the aerobic oxidation of cyclohexane catalyzed by ironporphyrin. The selectivity to the cyclohexanone and cyclohexanol production, the catalyst turnover, and the rate constants of cyclohexane oxidation increased with increasing coordination constant as compared in table 1.

\begin{tabular}{ccc}
\hline & Conversion, $\%$ & TON $^{* *}$ \\
\hline Chitosan-ironporphyrin & 8.76 & 39328 \\
Chitin-ironporphyrin & 5.01 & 21760 \\
Ironporphyrin & 1.81 & 8076 \\
Chitin & 0 & - \\
Chitosan & 0 & - \\
\hline
\end{tabular}

Notes: * Reaction conditions: $\mathrm{TPPFeCl}, 0.0071 \mathrm{mmol}$; cyclohexane, $350 \mathrm{ml}$; temperature, $418 \mathrm{~K}$; pressure, $0.8 \mathrm{MPa}$; reaction time, $1.5 \mathrm{~h}$.

** Referred to one mole of iron atom.

Table 1. The results of cyclohexane oxidations catalyzed by chitosan- and chitin-supported ironporphyrin and ironporphyrin with air*

Also the chitosan-supported cobalt tetraphenylporphyrin can catalyzed the aerobic oxidation of cyclohexane under the relatively moderate conditions of $418 \mathrm{~K}$ and $0.8 \mathrm{Mpa}$ with $11 \%$ conversion of cyclohexane, $87 \%$ selectivity for the main product cyclohexanol and cyclohexanone and $2.9 \times 10^{5}$ catalyst turnover number[56]. Chitosan-supported iron(III)/or cobalt tetraphenylporphyrin can be prepared easily. Chitosan is cheap and plentiful, and possesses particular microstructure and excellent function; the preparation technology of catalyst is simple also. Chitosan-supported iron(III)/or cobalt tetraphenylporphyrin will have a bright future in industrial applications.

Manganeseporphyrin supported on imidazole-modified silica gel(Scheme 2) can highly catalyze the oxidation of cyclohexane efficiently with yield of $7.1 \%$ and selectivity above $90 \%$ to cyclohexanol and cyclohexanone, and the catalyst can be reused several times[57].

A systematic study on the effect of a catalyst on the aerobic oxidation of cyclohexane was carried out using cobalt isooctanoate, metalloporphyrin $p$-ClTPPCo and $[p-\mathrm{ClTPPFe}]_{2} \mathrm{O}$ as catalysts[58]. The results showed that the metalloporphyrin-catalyzed system performed better than the traditional cobalt salt (Co isooctanoate) in terms of both the reaction 

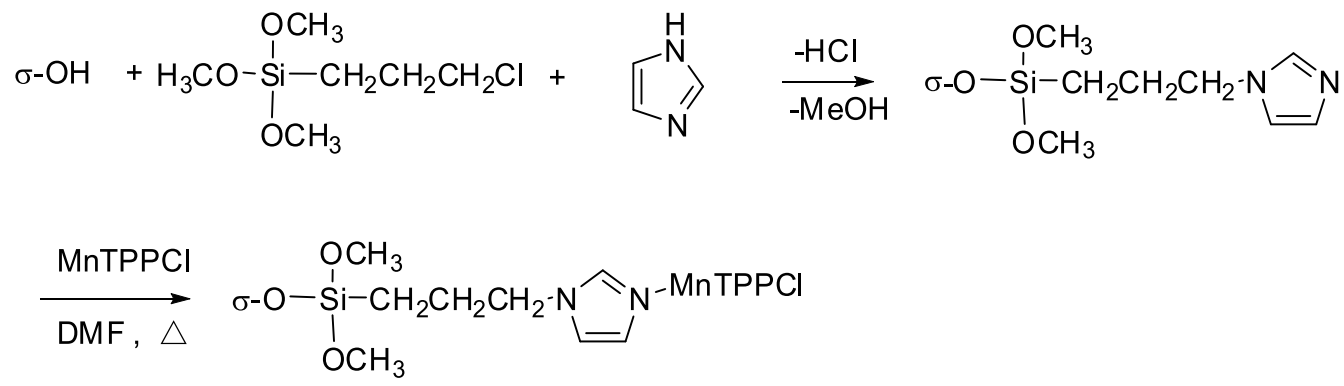

Scheme 2. Synthetic route to manganeseporphyrin supported on imidazole-modified silica gel

conversion and the selectivity of cyclohexanol and cyclohexanone. More importantly, bisironporphyrin complex, which was traditionally considered to be either non-active or not-so-active for hydrocarbon oxidation, excelled at $155^{\circ} \mathrm{C}$, achieving high selectivity for $\mathrm{KA}$ oil $(80 \%)$ and good reaction conversion $(13.8 \%)$. Meanwhile, the production of adipic acid was also increased and good selectivity of adipic acid among the by-products was also obtained. The study suggested that $[p \text {-ClTPPFe }]_{2} \mathrm{O}$ catalyzed aerobic oxidation could be used to produce adipic acid in addition to KA oil. Some mechanistic rationales were proposed to explain the superior performance of the $[p \text {-ClTPPFe }]_{2} \mathrm{O}$ catalyst based on its unique structural and chemical properties.

Biomimetic oxidation of cyclohexane via air catalyzed by metalloporphyrins has already been the subject of a Chinese patent application[59] and had successfully achieved industrial scale production.

\subsection{Biomimetic oxidation of $\mathrm{C}-\mathrm{H}$ of methylcyclohexane}

Methylcyclohexanol and methylcyclohexanone also play an important role in fine chemicals and in relative fields. Our group had been engaged in preparation of methylcyclohexanone and methylcyclohexanol via selective aerobic oxidation of methylcyclohexane catalyzed by metalloporphyrin encouraged by the success of biomimetic oxidation of cyclohexane. We reported first[60] that catalyzed by $\mathrm{Mn}(\mathrm{III})$ porphyrin, methylcyclohexane could be oxidized by air(Scheme 3).

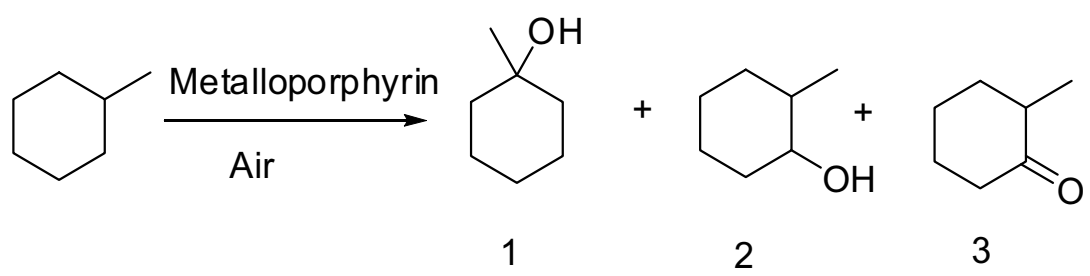

Scheme 3. Aerobic oxidation of methylcyclohexane by metalloporphyrin

The main products were 1-methylcyclohexanol, 2-methylcyclohexanol and 2methylcyclohexanone. The effect on the conversion and selectivity was investigated when reaction conditions or porphyrin structure were changed. With the alkoxylsubstituting metalloporphyrins as catalysts, the selectivity of methylcyclohexanone and methylcyclohexanol was better than using TPPMnOAc. Moreover, the larger the substitute's 
volume, the higher the conversion, and selectivity still remained satisfactory. Using 5,10,15,20tetra( $p$-2-heptanoxylphenyl)porphyrinatomanganese as catalyst, the conversion was up to 20 $\%$ and the overall selectivity of methylcyclohexanone and methylcyclohexanol was $76 \%$. Further researching for the selective catalysis of metalloporphyrins for the aerobic oxidation of different $\mathrm{C}-\mathrm{H}$ bonds of hydrocarbons in the system of manganeseporphyrinmethylcyclohexane-air was investigated in detail in our group(Scheme 4)[61].

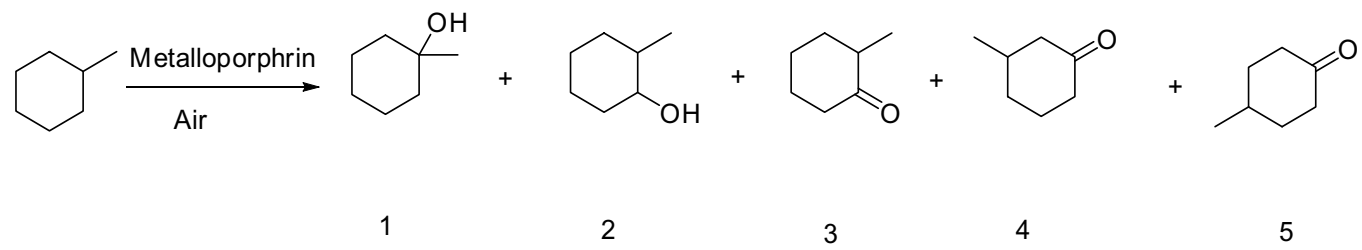

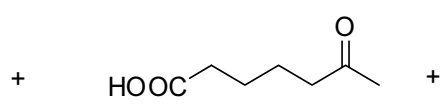

6

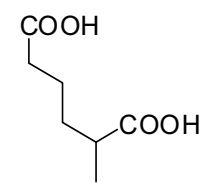

7<smiles>CC(CCC(=O)O)CC(=O)O</smiles>

8

Scheme 4. Biomimetic aerobic oxidation of methylcyclohexane

After systematic investigation, it showed that the oxidation reaction of alkanes catalyzed by metalloporphyrin is kept to the classic radical chain mechanism [62-64]. While at the initial stage of methylcyclohexane aerobic oxidation, metalloporphyrin turns into high-valent oxometal radical cation $[\mathrm{PMIV}=\mathrm{O}]^{\bullet+}$, which is responsible for the velocity of the reaction. The selective oxidation of different $\mathrm{C}-\mathrm{H}$ bond is mainly affected by two factors: activation energy and regioselectivity of different $\mathrm{C}-\mathrm{H}$ bond. Obviously, the activation energy of secondary $\mathrm{C}$ $\mathrm{H}$ bond is greater than that of tertiary $\mathrm{C}-\mathrm{H}$ bond, so the factors such as temperature, catalyst activity can affect the activation energy. For the selective oxidation of secondary $\mathrm{C}-\mathrm{H}$ bond, higher temperature and stronger catalyst activity is preferred. Though the pressure in the reaction system does not affect the two factors, it can shift the equilibrium of the reaction. Therefore, it can change the oxidation selectivity of different $\mathrm{C}-\mathrm{H}$ bonds.

\section{Biomimetic oxidation of alkenes}

In the field of olefin oxidations with molecular oxygen catalyzed by metalloporphyrins, the catalytic system metalloporphyrins- $\mathrm{O}_{2}-\mathrm{BH}_{4}^{-}$were developed[65]. In this catalytic system, sodium borohydride $\left(\mathrm{NaBH}_{4}\right)$ used as reductant was essential for oxygenation of olefins[66]. Lately, some papers reported the aerobic oxidation of olefins catalyzed by perhalogenated metalloporphyrins or multisubstituent dioxoruthenium porphyrins catalysts [67-69]. Recently, the catalysis of simple porphyrins for oxidation of cyclohexene and the carbon-carbon double bond oxidation with air in the absence of cocatalysts or reductants using the styrenemanganeseporphyrin-air system were reported by our group. The selectivities among epoxidation of $\mathrm{C}=\mathrm{C}$, oxidation of allyl $\mathrm{C}-\mathrm{H}, \mathrm{C}=\mathrm{C}$ breaking were investigated in detail. 


\subsection{Biomimetic oxidation of $\mathrm{C}-\mathrm{H}$ of cyclohexene}

The selective oxidation of $\mathrm{C}-\mathrm{H}$ of olefins is one of the paramount reactions in organic chemistry. Many hydroxyl, carbonyl, unsaturated ketone and other compounds could be synthesized by this methodologies[70-73]. We reported a selective oxidation of allylic C-H of cyclohexene catalyzed by simple porphyrin over air (Scheme 5)[74].<smiles></smiles>

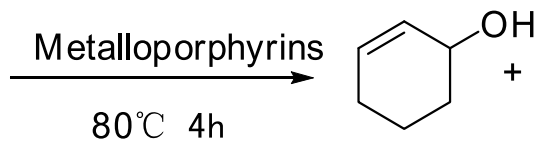<smiles>O=C1C=CCCC1</smiles><smiles>C1CCC2OC2C1</smiles>

Scheme 5. Aerobic oxidation of cyclohexene

The simple cobalt tetraphenylporphyrins can catalyze cyclohexene oxidation into cyclohexenone with air in the absence of additives and solvents. The temperature, air velocity, catalyst concentration and pressure influenced the yield and conversion of cyclohexene oxidation with air. The result of effect of temperature, air velocity, pressure and catalyst concentration were summarized in the table 2-5 respectively.

\begin{tabular}{ccccc}
\hline Temperature $/{ }^{\circ} \mathrm{C}$ & $\begin{array}{c}\text { Cyclohexene } \\
\text { Conv./\% }(\mathrm{mol})\end{array}$ & $\begin{array}{c}\text { Select.of } \\
\text { Cyclohexenone/\% }\end{array}$ & $\begin{array}{c}\text { Time at } \\
\text { maximum } \\
\text { yield/h }\end{array}$ & TON \\
\hline 60 & 5.2 & 80 & 8.0 & $2.1 \times 10^{4}$ \\
70 & 12 & 78 & 6.0 & $5.0 \times 10^{4}$ \\
80 & 21 & 75 & 4.0 & $8.7 \times 10^{4}$ \\
90 & 23 & 63 & 3.5 & $9.5 \times 10^{4}$ \\
\hline
\end{tabular}

Table 2. Effect of temperature on cyclohexeneoxidation reaction

\begin{tabular}{ccccc}
\hline $\begin{array}{c}\text { Air velocity } \\
/ \mathrm{m}^{3} \mathrm{~h}^{-1}\end{array}$ & $\begin{array}{c}\text { Cyclohexene } \\
\text { Conv. } \\
/ \%(\mathrm{~mol})\end{array}$ & $\begin{array}{c}\text { Select.of } \\
\text { Cyclohexenone } \\
/ \%(\mathrm{~mol})\end{array}$ & $\begin{array}{c}\text { Time at } \\
\text { maximum } \\
\text { yield } / \mathrm{h}\end{array}$ & TON \\
\hline 0.04 & 12 & 58 & 5.0 & $5.0 \times 10^{4}$ \\
0.06 & 18 & 69 & 4.5 & $7.4 \times 10^{4}$ \\
0.08 & 21 & 75 & 4.0 & $8.7 \times 10^{4}$ \\
0.10 & 21 & 74 & 4.0 & $8.7 \times 10^{4}$ \\
\hline
\end{tabular}

Table 3. Effect of air velocity on cyclohexene oxidation reaction

\begin{tabular}{ccccc}
\hline $\begin{array}{c}\text { Pressure } \\
/ \mathrm{MPa}\end{array}$ & $\begin{array}{c}\text { Cyclohexene } \\
\text { Conv. } \\
/ \%(\mathrm{~mol})\end{array}$ & $\begin{array}{c}\text { Select.of } \\
\text { Cyclohexenone } \\
/ \%(\mathrm{~mol})\end{array}$ & $\begin{array}{c}\text { Time at } \\
\text { maximum } \\
\text { yield } / \mathrm{h}\end{array}$ & TON \\
\hline 0.2 & 20 & 71 & 4.0 & $8.25 \times 10^{4}$ \\
0.4 & 21 & 75 & 4.0 & $8.7 \times 10^{4}$ \\
0.6 & 20 & 76 & 4.0 & $8.25 \times 10^{4}$ \\
\hline
\end{tabular}

Table 4. Effect of pressure on cyclohexene oxidation reaction 


\begin{tabular}{ccccc}
\hline $\begin{array}{c}\text { Catalyst } \\
\text { Concentration } \\
\times 10^{5} /(\mathrm{mol} / \mathrm{L})\end{array}$ & $\begin{array}{c}\text { Cyclohexene } \\
\text { Conv. } \\
/ \%(\mathrm{~mol})\end{array}$ & $\begin{array}{c}\text { Select.of } \\
\text { Cyclohexenone } \\
/ \%(\mathrm{~mol})\end{array}$ & $\begin{array}{c}\text { Time at } \\
\text { maximum } \\
\text { yield } / \mathrm{h}\end{array}$ & TON \\
\hline 57 & 14 & 56 & 2.0 & $2.3 \times 10^{3}$ \\
23 & 17 & 63 & 2.5 & $7.0 \times 10^{3}$ \\
4.5 & 19 & 69 & 3.5 & $3.9 \times 10^{4}$ \\
2.3 & 21 & 75 & 4.0 & $8.7 \times 10^{4}$ \\
\hline
\end{tabular}

Table 5. Effect of catalyst concentration on cyclohexene oxidation reaction

The conversion of cyclohexene was up to $21 \%$, the selectivity of cyclohexenone reached $75 \%$ under the optimum conditions of $0.4 \mathrm{MPa}, 80{ }^{\circ} \mathrm{C}$ and $2.3 \times 10^{-5} \mathrm{~mol} / \mathrm{L}$ cobalt tetraphenylporphyrin.

\subsection{Biomimetic oxidation of $\mathbf{C}=\mathrm{C}$ of styrene}

In 2006, We[75] reported a novel aerobic oxidative cleavage of $\mathrm{C}=\mathrm{C}$ double bonds of styrene catalyzed by simple manganese porphyrin(Scheme 6).

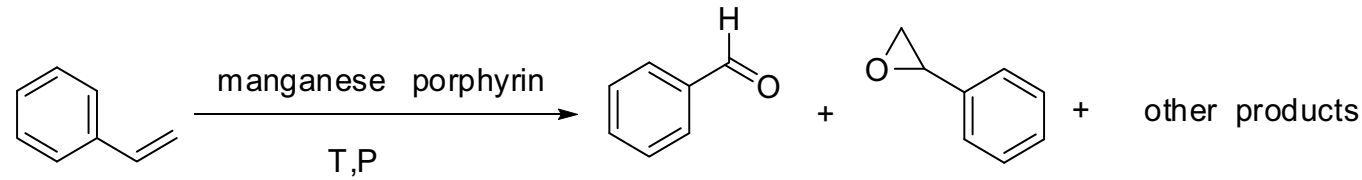

Scheme 6. Aerobic oxidation of styrene by metalloporphyrin

Under the catalysis of chloro(tetraphenylporphinato)manganese, the oxidative cleavage of the carbon-carbon double bond of the styrene with air yields the main product benzaldehyde. The results show that the oxidative cleavage and the epoxidation of the styrene double bond are the competition reactions in the styrene-manganese porphyrin-air system. The reaction temperature decided the product distribution. Under the conditions of $0.4 \mathrm{MPa}$ air and $30 \mathrm{ppm}$ of chloro(tetraphenylporphinato)- manganese, the styrene conversion was $20.0 \%$ and the selectivity of benzaldehyde and styrene oxide was $81.7 \%$ and $12.7 \%$ respectively when the reaction temperature was $110{ }^{\circ} \mathrm{C}$. Styrene conversion was $92.5 \%$ and the selectivity of benzaldehyde and styrene oxide was $48.1 \%$ and $41.2 \%$ respectively when the reaction temperature was $120^{\circ} \mathrm{C}$.

\subsection{Biomimetic oxidation of $\mathrm{C}=\mathrm{C}$ and allylic $\mathrm{C}-\mathrm{H}$ bonds in $\alpha$-pinene}

a-pinene is the single largest renewable terpene in nature and an important industrial raw material in the terpene industry. With oxidation, isomerization, and hydrogenation, apinene can produce a variety of important products. The a-pinene epoxide has great industrial value. We[76] reported simple metalloporphyrins(TPPMnCl, TPPCoCl, TPPFeCl, TPPNi, TPPCu or TPPZn) catalyzed the aerobic oxidation of a-pinene in the absence of cocatalyst and solvents, and the catalytic selectivity of metalloporphyrins for aerobic oxidation of $\mathrm{C}=\mathrm{C}$ double bond and allylic $\mathrm{C}-\mathrm{H}$ of $\mathrm{a}$-pinene was investigated(Scheme 7).

When TPPMnCl loaded as the catalyst with a concentration of $6 \times 10^{-5} \mathrm{~mol} / \mathrm{L}, 16 \mathrm{~L} / \mathrm{h}$ of the air flow rate at ambient pressure and $5 \mathrm{~h}$ of reaction time, the results are summarized in table 6. 


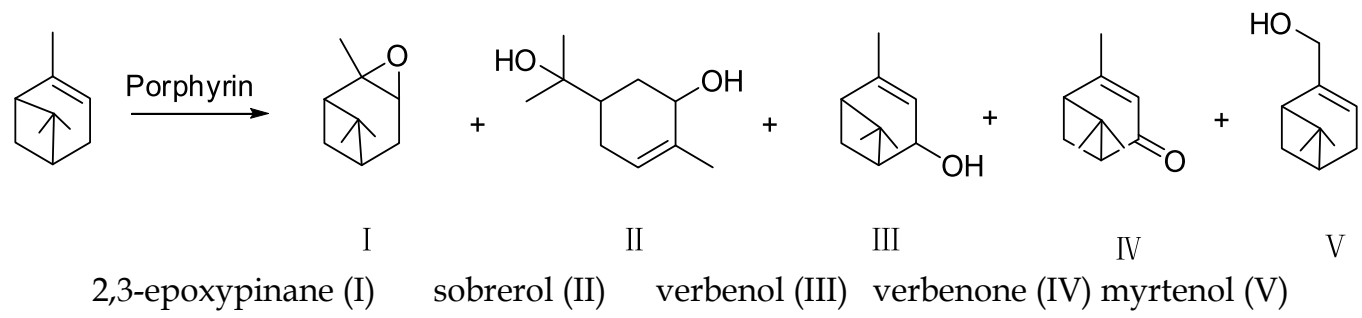

Scheme 7. Aerobic oxidation of a-pinene by metalloporphyrin

\begin{tabular}{ccccc}
\hline \multirow{2}{*}{ Temperature $\left({ }^{\circ} \mathrm{C}\right)$} & \multirow{2}{*}{ Conversion $(\%)$} & \multicolumn{3}{c}{ Selectivity $(\%)^{\mathrm{a}}$} \\
\cline { 3 - 5 } & & Products I and II & Products III and IV & Product V \\
\hline 80 & 23.02 & 60.19 & 36.58 & 3.23 \\
70 & 19.87 & 60.45 & 35.86 & 3.69 \\
60 & 16.22 & 60.99 & 35.67 & 3.33 \\
50 & 14.78 & 62.00 & 34.55 & 3.45 \\
40 & 8.77 & 66.86 & 30.12 & 3.23 \\
\hline
\end{tabular}

Table 6. Effect of temperature on aerobic oxidation of a-pinene

Different metal core in TPPMCl got the different conversion and selectivity(Table 7).

\begin{tabular}{ccccc}
\hline \multirow{2}{*}{ Catalysts } & \multirow{2}{*}{ Conversion(\%) } & \multicolumn{3}{c}{ Selectivity(\%) } \\
\cline { 3 - 5 } & & Products I and II & Products III and IV & Product V \\
\hline TPPMnCl & 16.22 & 60.99 & 35.67 & 3.33 \\
TPPCoCl & 13.84 & 59.11 & 37.05 & 3.84 \\
TPPFeCl & 12.40 & 51.30 & 43.83 & 4.87 \\
\hline
\end{tabular}

Conditions: the concentration of the catalyst, $6 \times 10^{-5} \mathrm{~mol} / \mathrm{L}$; the temperature, $60 \circ \mathrm{C}$; ambient pressure; the flow rate of air, $16 \mathrm{~L} / \mathrm{h}$; reaction time, $5 \mathrm{~h}$.

Table 7. Effect of metal core of porphyrin on aerobic oxidation of a-pinene

Over TPPMnCl catalyst, the selectivity regarding the oxidation of $\mathrm{C}=\mathrm{C}$ bond increased with decreasing temperature. Among various metalloporphyrins, $\mathrm{TPPMnCl}$ presented the largest selectivity regarding the oxidation of $\mathrm{C}=\mathrm{C}$ bond and $\mathrm{TPPFeCl}$ the largest selectivity regarding the oxidation of allylic $\mathrm{C}-\mathrm{H}$ bonds. With increasing the electron-donating abilities of the peripheral substituents in $(p-\mathrm{X}) \mathrm{PPMnCl}\left(\mathrm{X}=-\mathrm{Cl},-\mathrm{CH}_{3},-\mathrm{OCH}_{3},-\mathrm{OH}\right)$, the selectivities regarding the oxidation of $\mathrm{C}=\mathrm{C}$ bond decreased. Manganese tetraphenylporphyrin supported on nano- $\mathrm{TiO}_{2}$ has been used as catalyst for the aerobic oxidation of a-pinene[77]. Experimental results showed that this much enhanced activity could arise from possible cocatalysis between metalloporphyrin and the nano- $\mathrm{TiO}_{2}$ support. The catalyst can be reused several times with minor loss to its catalytic activity. Table 8 summarized the results of different catalysts catalyzed aerobic oxidation of a-pinene.

\section{Biomimetic oxidation of $\mathrm{C}-\mathrm{H}$ of aromatic hydrocarbons}

The aerobic liquid-phase oxidation of toluene, $p$-xylene (PX), $p$-toluic acid over metalloporphyrin, metalloporphyrin/ $\mathrm{Co}(\mathrm{OAc})_{2}$, metalloporphyrin/NHPI and other systems were studied in our group. 


\begin{tabular}{|c|c|c|c|c|c|c|c|c|}
\hline Catalyst & $\begin{array}{c}\text { pinene } \\
\text { epoxide, } \%\end{array}$ & $\begin{array}{c}\text { Sobrerol, } \\
\%\end{array}$ & $\begin{array}{c}\text { Verbenol, } \\
\%\end{array}$ & $\begin{array}{c}\text { Verbenone } \\
, \%\end{array}$ & $\begin{array}{l}\text { Myrtenol, } \\
\%\end{array}$ & $\begin{array}{c}\text { Others, } \\
\%\end{array}$ & $\begin{array}{c}\text { Conversion, } \\
\%\end{array}$ & $\begin{array}{c}\text { Epoxide } \\
\text { selectivity } \\
\% \\
\end{array}$ \\
\hline No catalyst & 1.63 & 2.12 & 1.04 & 1.34 & 0.29 & 0.45 & 6.87 & 23.72 \\
\hline $\mathrm{TiO}_{2}$ & 7.13 & 3.59 & 1.71 & 0.51 & 0.48 & 0.24 & 13.66 & 52.20 \\
\hline Ti-Si & 4.38 & 1.83 & 0.53 & 1.34 & 0.58 & 0.34 & 9.00 & 48.67 \\
\hline $\mathrm{T}(p-\mathrm{Cl}) \mathrm{PPMnCl} \mathrm{l}^{\mathrm{a}}$ & 3.85 & 3.26 & 2.71 & 1.56 & 0.41 & 0.81 & 12.60 & 30.56 \\
\hline $\mathrm{TiO}_{2}+(p-\mathrm{Cl}) \mathrm{PPMnCl}^{\mathrm{b}}$ & 5.84 & 3.39 & 1.61 & 2.39 & 0.93 & 2.01 & 16.17 & 36.11 \\
\hline $\begin{array}{c}\mathrm{TiO}_{2}+(p- \\
\mathrm{Cl}) \mathrm{PPMnCl}+\text { imidazole }^{\mathrm{c}}\end{array}$ & 6.52 & 3.96 & 1.84 & 2.50 & 0.85 & 1.93 & 17.50 & 37.25 \\
\hline Ti-Si-PMn & 12.88 & 8.20 & 11.45 & 4.48 & 1.01 & 1.24 & 39.26 & 32.81 \\
\hline
\end{tabular}

a Concentration, $3.56 \times 10^{-5} \mathrm{M}$; the amount of $\mathrm{T}(p-\mathrm{Cl}) \mathrm{PPMnCl}$ is the same as Ti-Si-PMn.

b Mixture of nano- $\mathrm{TiO}_{2}$ and $\mathrm{T}(p-\mathrm{Cl}) \mathrm{PPMnCl}((1.55 \mathrm{mg} \mathrm{T}(p-\mathrm{Cl}) \mathrm{PPMnCl}) /(100 \mathrm{mg} \mathrm{TiO} 2))$.

c Mixture of " $b$ " and imidazole, mol ratio of imidazole $/ \mathrm{T}(p-\mathrm{Cl}) \mathrm{PPMnCl}=5 / 1$

Table 8. Results of a-pinene oxidation in the presence of different catalysts

\subsection{Biomimetic oxidation of $\mathrm{C}-\mathrm{H}$ of toluene}

Toluene is the most important aromatic hydrocarbon. Scientists have been interested in the selective oxidation of toluene with air to synthesize benzaldehyde and benzyl alcohol, which are versatile intermediates in the chemical industry[78]. But the present oxidation of toluene with air could not produce benzaldehyde and benzyl alcohol because it was difficult to effectively prevent the over-oxidation of benzaldehyde into benzoic acid during the oxidation of toluene. So far, the oxidation of toluene with air is mainly used to synthesize benzyl acid, and benzaldehyde and benzyl alcohol are synthesized by the chlorination of toluene followed by hydrolysis, a serious polluting process[79]. Some researchers tried the selective aerobic oxidation of toluene catalyzed by the transition metal salts. But these technologies are companied by drawbacks such as low selectivity and use of large amounts of solvents and bromides [80].

We[81] firstly reported catalysis of simple cobalt tetraphenylporphyrin for selective liquid phase oxidation of toluene with air in the absence of any solvents or promoters to produce benzaldehyde and benzyl alcohol(Scheme 8).

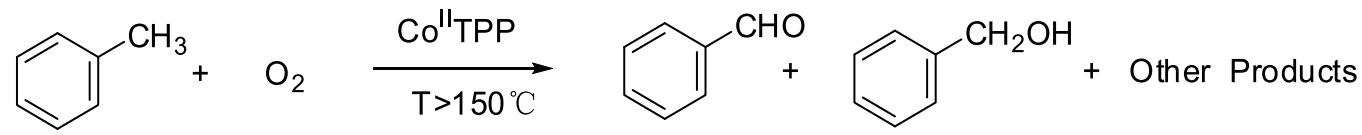

Scheme 8. Aerobic oxidation of toluene by metalloporphyrin

Compared with the present synthetic method of benzaldehyde and benzyl alcohol by the chlorination of toluene followed by hydrolysis, which has been used in the world industry, our new method has the advantage of friendly environmental effects. We found that the reaction time, temperature, air pressure, the amount of catalyst and the flowing rates of air influenced the toluene conversion and the selectivity of benzaldehyde and benzyl alcohol. By the use of $3.2 \times 10^{-5} \mathrm{M}$ cobalt tetraphenylporphyrin as catalyst, toluene oxidation with air under the optimum conditions of $160^{\circ} \mathrm{C}$ and $0.8 \mathrm{MPa}$ and $0.04 \mathrm{~m}^{3} / \mathrm{h}$ air flow produced benzaldehyde and benzyl alcohol at $60 \%$ selectivity and $8.9 \%$ conversion of toluene. The mole turnover numbers of the catalyst was about 25,000. Compared with the $\mathrm{Co}(\mathrm{OAc})_{2}$ catalyst, Cobalt tetraphenylporphyrin is effective both in conversion and selectivity (Table 9). 


\begin{tabular}{|c|c|c|c|c|c|}
\hline \multirow[b]{2}{*}{ Catalyst } & \multicolumn{3}{|c|}{ Selectivity(mol \%) } & \multirow[b]{2}{*}{ Conversion $(\mathrm{mol} \%)$} & \multirow{2}{*}{$\begin{array}{c}\text { Turnover } \\
\text { number }(\mathrm{mol} / \mathrm{mol})\end{array}$} \\
\hline & Benzaldehyde & $\begin{array}{l}\text { Benzyl } \\
\text { alcohol }\end{array}$ & $\begin{array}{c}\text { Benzoic } \\
\text { acid }\end{array}$ & & \\
\hline Co(II)TPP & 33 & 27 & 39 & 8.9 & 24275 \\
\hline $\mathrm{Co}(\mathrm{OAc})_{2}$ & 29 & 20 & 50 & 2.9 & 3125 \\
\hline
\end{tabular}

Table 9. Comparison of aerobic oxidation of toluene catalyzed by $\mathrm{Co}(\mathrm{II}) \mathrm{TPP}$ and $\mathrm{Co}(\mathrm{OAc})_{2}$

The further investigationresulted that $\mu$-oxo-bis[porphyriniron(III)] is another effective catalyst for aerobic oxidation of toluene(Scheme 9)[82].<smiles>Cc1ccccc1</smiles>

Scheme 9. Aerobic oxidation of toluene by $\mu$-oxo-bis metalloporphyrin

Catalyzed by $\mu$-oxo-bis[porphyriniron(III)] under the reaction conditions of $438 \mathrm{~K}$ and 0.8 $\mathrm{MPa}$, the molar total yields for the products benzaldehyde and benzyl alcohol and the turnover number of the catalyst are $4.35 \%$ and 21,830 (based on the metal ion), respectively. Compared with the reaction catalyzed by the corresponding monometalloporphyrin $\mathrm{TPPFe}^{\mathrm{III}} \mathrm{Cl}$, the total yields of the oxidation products and the catalyst turnover number by the dimeric ironporphyrin were almost twice those by the former(Table 10).

\begin{tabular}{ccccc}
\hline Catalyst & $\begin{array}{c}\text { Turnover } \\
\text { number }\end{array}$ & $\begin{array}{c}\text { Toluene } \\
\text { convension, } \%\end{array}$ & $\begin{array}{c}\text { Selectivity } \\
\text { (-aldehyde +-ol), \% }\end{array}$ & -aldehyde /-ol \\
\hline$[\mathrm{TPPFe}]_{2} \mathrm{O}$ & 21830 & 7.36 & 59.06 & 1.15 \\
$\mathrm{TPPFe}^{\mathrm{III}} \mathrm{Cl}$ & 12593 & 4.08 & 60.94 & 0.84 \\
\hline
\end{tabular}

Oxidation carried out by the reaction of $7.5 \mathrm{mg}$ catalyst with toluene $(350 \mathrm{~mL})$ at $438 \mathrm{~K}, 0.8 \mathrm{MPa}$ for 225 min.

Table 10. Comparison of catalytic property of $\left[\mathrm{TPPFe}^{\mathrm{III}}\right]_{2} \mathrm{O}$ and $\mathrm{TPPFe} \mathrm{IIICl}^{\mathrm{C}} \mathrm{in}$ toluene oxidation

Possible mechanism of toluene oxidation catalyzed by $\mu$-oxo-bis[porphyriniron(III)] is proposed as bellowing(Scheme 10).

After systematical investigation, it showed that toluene oxidation catalyzed by $\mu$-oxobis[porphyriniron(III)] may proceed via a mechanism similar to the hydrocarbon oxidation with molecular oxygen over monometalloporphyrins.

Meanwhile, we[83] got the optimized reaction condition for toluene oxyfunctionalization with air over metalloporphyrins. The effects of different central metal ions in metalloporphyrins $[\mathrm{T}(p-\mathrm{C} 1) \mathrm{PPMCl}(\mathrm{M}=\mathrm{Fe}, \mathrm{Co}, \mathrm{Mn}, \mathrm{Cu})]$ on the reaction course had been examined and it was found that $\mathrm{T}(p-\mathrm{C} 1) \mathrm{PPC}$ presented the highest catalytic activity in the reaction. The reaction conditions of toluene oxidation were optimized by using orthogonal experiment design. Five relevant factors were investigated: temperature, air pressure, catalyst loading, air flow rate and reaction time. The effects of the five factors on both toluene conversion and total yield of benzaldehyde and benzyl alcohol were discussed. The research results showed that the reaction temperature was the most significant factor influencing toluene oxidation. On the basis of the margin analysis, the optimum conditions for the toluene conversion and the total yield of benzaldehyde and benzyl alcohol respectively were achieved, under which the toluene conversion was up to $14.67 \%$ and the total yield of benzaldehyde and benzyl alcohol reached $5.89 \%$.The aerobic oxidation of 
toluene over metalloporphyrin is currently employed by Balaji Amines LTD in India to produce more than 10,000 tons of benzaldehyde annually.
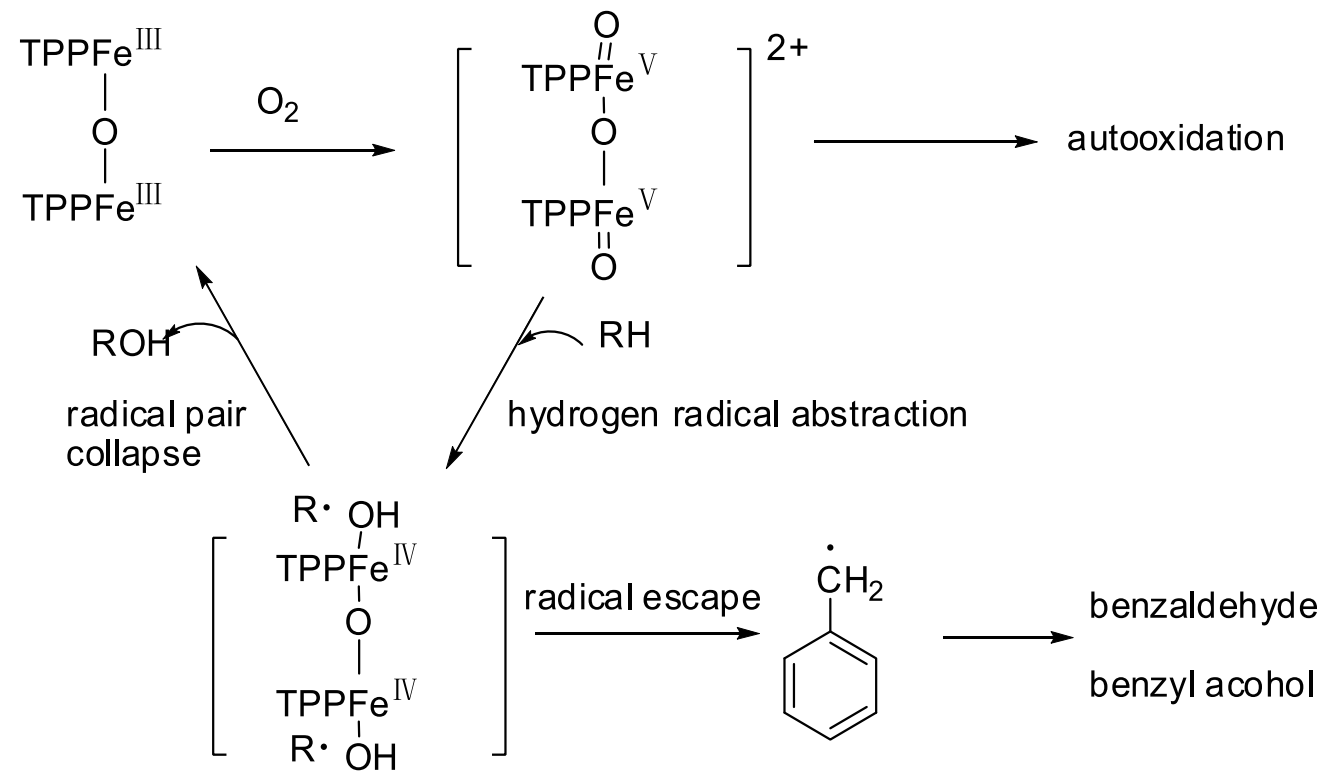

Scheme 10. Possible mechanism for aerobic oxidation of toluene by $\mu$-oxo-bis metalloporphyrin

\subsection{Biomimetic oxidation of $\mathrm{C}-\mathrm{H}$ of $p$-xylene}

Liquid-phase aerobic oxidation of $p$-xylene is an important process for the production of terephthalic acid, which is widely used in the commercial production of polyesters. So far, $p$ xylene oxidation with air has been carried out in acetic acid, using $\mathrm{Co}(\mathrm{OAc})_{2} / \mathrm{Mn}(\mathrm{OAc})_{2} / \mathrm{HBr}$ as catalysts in industry. However, the high environmental impact of the catalysts, corrosion of reactors due to the halides, low space-time yield and the oxidative combustion of acetic acid present in the process of $p$-xylene oxidation, are the major shortcomings. We reported the aerobic liquid-phase oxidation of $p$-xylene over metalloporphyrins(Scheme 11)[84].<smiles>Cc1ccc(C(=O)O)cc1</smiles>

Scheme 11. Aerobic oxidation of $p$-xylene by metalloporphyrin in acetic acid

The oxidation was carried out in $20 \%$ acetic acid with 10 ppm metalloporphyrins as catalyst, and corrosive bromide was omitted. Effect of different metalloporphyrins on the oxidation reaction was investigated and got the result below.

$\mathrm{T}(p-\mathrm{Cl}) \mathrm{PPMnCl}$ was the most efficient catalyst for the oxidation of $p$-xylene with air under the chosen conditions. It gave the highest $p$-xylene conversion and yield of $p$-toluic acid and terephthalic acid. A possible reason was related to its stability and the higher oxidation- 
reduction potential of $\mathrm{T}(p-\mathrm{Cl}) \mathrm{PPMnCl}$. Under the conditions of $180{ }^{\circ} \mathrm{C}$ and $2.0 \mathrm{MPa}, 44 \%$ conversion of $p$-xylene and $85 \%$ selectivity of $p$-toluic acid were obtained. The oxidation of $p$-xylene with air to terephthalic acid was a series of reactions, and some intermediate products such as the $p$-toluic alcohol, $p$-toluic aldehyde and $p$-toluic acid were formed during the oxidation. The formation, accumulation and conversion of these products all affected the overall $p$-xylene oxidation. The catalytic oxidation of $p$-xylene with air over metalloporphyrins was a "green" reaction with potential industrial applications.

Recently, We reported an aerobic liquid-phase oxidation of p-xylene(PX) over metalloporphyrin and $\mathrm{Co}(\mathrm{OAc})_{2}[85]$.

\begin{tabular}{|c|c|c|c|c|c|c|c|}
\hline \multirow[t]{2}{*}{ Catalyst } & \multicolumn{2}{|c|}{$\begin{array}{c}p \text {-toluic aldehyde, } \\
\%\end{array}$} & \multicolumn{2}{|c|}{$p$-toluic acid, \% } & \multicolumn{2}{|c|}{$\begin{array}{c}\text { Terephthalic acid, } \\
\%\end{array}$} & \multirow[t]{2}{*}{ Conversion, $\%$} \\
\hline & Yield & Selectivity & Yield & Selectivity & Yield & Selectivity & \\
\hline $\begin{array}{c}\mathrm{T}(p- \\
\mathrm{Cl}) \mathrm{PPMnCl}\end{array}$ & 1.76 & 5.7 & 27.09 & 86.9 & 2.31 & 7.4 & 32.05 \\
\hline $\mathrm{T}(p-\mathrm{Cl}) \mathrm{PPCo}$ & 2.07 & 7.4 & 25.08 & 89.3 & 0.92 & 3.4 & 28.54 \\
\hline $\begin{array}{c}\mathrm{T}(p- \\
\mathrm{Cl}) \mathrm{PPFeCl}\end{array}$ & 2.31 & 8.8 & 22.77 & 86.9 & 1.12 & 4.3 & 26.76 \\
\hline $\mathrm{T}(p-\mathrm{Cl}) \mathrm{PPCu}$ & 1.94 & 36.6 & 3.08 & 58.1 & 0.28 & 5.3 & 5.36 \\
\hline $\mathrm{T}(p-\mathrm{Cl}) \mathrm{PPZn}$ & 2.44 & 59.2 & 1.51 & 36.7 & 0.17 & 4.1 & 4.19 \\
\hline
\end{tabular}

Table 11. Comparison of catalytic property of different porphyrin in $p$-xylene oxidation<smiles>[R]OC(=O)COc1ccc(C)cc1</smiles>

Scheme 12. Aerobic oxidation of $p$-xylene by metalloporphyrin-Co(OAc $)_{2}$ in acetic acid

When $\mathrm{T}(p-\mathrm{Cl}) \mathrm{PPMnCl}$ was used as the catalyst, less then $1 \%$ TPA was produced while $12 \%$ of the PX reacted. When the catalyst was switched to $\mathrm{Co}(\mathrm{OAc})_{2}$, the yield of TPA increased to $8 \%$ while the reaction conversion reached $47 \%$. Much to our delight, when $\mathrm{T}(p$ $\mathrm{Cl}) \mathrm{PPMnCl}$ and $\mathrm{Co}(\mathrm{OAc})_{2}$ were used together, the PX conversion increased to $67 \%$ and the TPA yield almost doubled (17\%). It is understandable that the PX conversion and TPA yield by $\mathrm{T}(p-\mathrm{Cl}) \mathrm{PPMnCl}-\mathrm{Co}(\mathrm{OAc})_{2}$ are higher than those by either $\mathrm{T}(p-\mathrm{Cl}) \mathrm{PPMnCl}$ or $\mathrm{Co}(\mathrm{OAc})_{2}$ alone. The results showed that both the PX conversion and TPA yield could be increased significantly even though only a minute amount of metalloporphyrin is added to the reaction mixture besides the cobalt catalyst. The results suggested that the PX oxidation was improved because of the acceleration of the chain initiation of PX oxidation by metalloporphyrin catalyst, and the acceleration of the chain initiation itself was due to the ease of peroxide formation over metalloporphyrin.

\subsection{Biomimetic oxidation of $p$-toluic acid}

The preparation of terephthalic acid (TPA) via the aerobic oxidation of $p$-xylene (PX) is a very important industrial process from both economical and environmental aspects. But the difficulty of the aerobic oxidation of PX to TPA lies in the oxidation of $p$-toluic acid (PTA) with 
air to TPA. Our previous work has solved the step of the oxidation of PX to PTA, however, the oxidation of PTA to TPA still remains a challenge to the oxidation of PX to TPA. For the purpose of exploring how to oxidize PTA to TPA effectively under moderate conditions, we reported for the first time the aerobic oxidation of $p$-toluic acid to terephthalic acid over tetra $(p$ chlorophenylporphinato)-manganese $(\mathrm{T}(p-\mathrm{Cl}) \mathrm{PPMnCl}) /$ cobalt acetate(Scheme 13)[86].<smiles>Cc1ccc(C(=O)O[Na])cc1</smiles>

PTA
TPA<smiles>O=Cc1ccc(C(=O)O)cc1</smiles>

4-CBA

Scheme 13. Aerobic oxidation of $p$-toluic acid by metalloporphyrin-Co(OAc $)_{2}$ in acetic acid

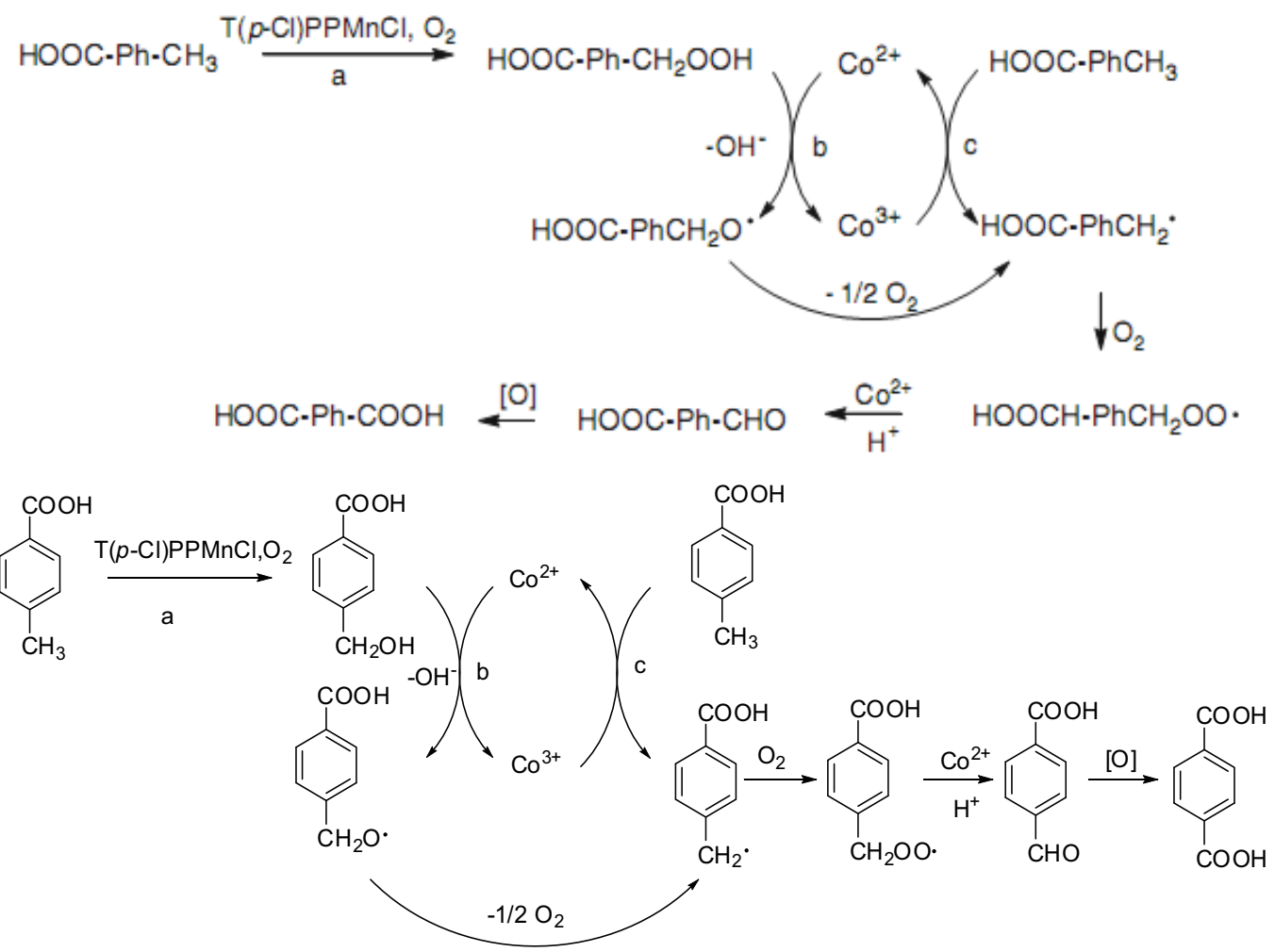

Scheme 14. Preliminary mechanism of co-catalysis between $\mathrm{T}(p-\mathrm{Cl}) \mathrm{PPMnCl}$ and $\mathrm{Co}(\mathrm{OAc}) 2$ in the aerobic oxidation of PTA to TPA

The studies indicated that the PTA oxidation was influenced by the catalyst composition, catalyst concentrations and reaction conditions. The co-catalysis of $\mathrm{T}(p-\mathrm{Cl}) \mathrm{PPMnCl} / \mathrm{Co}(\mathrm{OAc})_{2}$ was also studied. Both the PTA conversion and TPA yield increased significantly when the a small amount of $\mathrm{T}(\mathrm{p}-\mathrm{Cl}) \mathrm{PPMnCl}$ was added to the PTA- $\mathrm{O}_{2}-\mathrm{Co}(\mathrm{OAc})_{2}$ system. By the use of 548 ppm $\mathrm{Co}(\mathrm{OAc})_{2}$ and $16 \mathrm{ppm} \mathrm{T}(p-\mathrm{Cl}) \mathrm{PPMnCl}$ as the catalysts, PTA oxidation under the optimum conditions of $190^{\circ} \mathrm{C}, 1.2 \mathrm{MPa}$ and $160 \mathrm{~L} / \mathrm{h}$ airflow produced TPA at $96.8 \%$ selectivity 
and $62.9 \%$ conversion of PTA in $5 \mathrm{~h}$. For the co-catalysis between metalloporphyrin and cobalt acetate, the function of $\mathrm{T}(p-\mathrm{Cl}) \mathrm{PPMnCl}$ might be to produce more peroxides from the PTA, and then more $\mathrm{Co}^{3+}$ was formed from the oxidation of the $\mathrm{Co}^{2+}$ with peroxides. Moreover, $\mathrm{Co}^{3+}$ promoted the PTA oxidation due to the ease of the free radicals' formation.

\subsection{Biomimetic oxidation of $\mathbf{C}-\mathrm{H}$ of ethylbenzene}

We reported selective oxidation of ethylbenzene with air catalyzed by simple $\mu$-oxometalloporphyrins under mild conditions in the absence of additives(Scheme 15)[87].

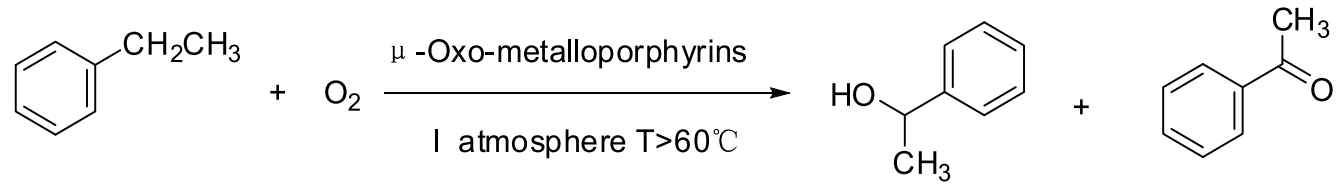

Scheme 15. Aerobic oxidation of ethylbenzene by $\mu$-oxo-metalloporphyrins

Two simple $\mu$-oxo dimeric metalloporphyrins, $\mu$-oxo-bis(tetraphenylporphinato)iron $[\mathrm{TPPFe}]_{2} \mathrm{O}$ and $\mu$-oxo-bis(tetraphenylporphinato)manganese [TPPMn $]_{2} \mathrm{O}$ were used as catalyst in the oxidation of ethylbenzene with air under mild condition. The results showed that the products of ethylbenzene oxidation catalyzed by [TPPFe $]_{2} \mathrm{O}$ or [TPPMn $]_{2} \mathrm{O}$ with air are acetophenone and a-phenylethanol exclusively under $\mathrm{T}>60{ }^{\circ} \mathrm{C}$ and atmospheric pressure in absence of any additives. [TPPMn $]_{2} \mathrm{O}$ is an active catalyst with high selectivity and turnover number of 2134 for oxidation of ethylbenzene by air when temperature reacts at $70{ }^{\circ} \mathrm{C}$. The kinetic study shows that the ethylbenzene oxidation is zero-order for oxygen and first-order for ethylbenzene. The activation energy of the reaction is $72.7 \mathrm{~kJ} / \mathrm{mol}$. Comparing with the corresponding monometalloporphyrins $\mathrm{TPPFeCl}$ and $\mathrm{TPPMnCl}$, $[\mathrm{TPPFe}]_{2} \mathrm{O}$ and [TPPMn $]_{2} \mathrm{O}$ have the better catalytic activity for ethylbenzene oxidation with air without any additive under mild conditions.

\begin{tabular}{lcccc}
\hline Metalloporphyrin & $(\mathrm{TPPMn})_{2} \mathrm{O}$ & $\mathrm{TPPMnCl}$ & $(\mathrm{TPPFe})_{2} \mathrm{O}$ & $\mathrm{TPPFeCl}$ \\
\hline Maximum yield(h) & 20 & 23 & 5 & 6 \\
Ethylbenzene conversion & 8.08 & 6.48 & 3.73 & 5.41 \\
Maximum yield of acetophenone(\%) & 4.89 & 3.62 & 1.97 & 2.91 \\
Maximum yield of & 3.19 & 2.86 & 1.76 & 2.50 \\
Phenylethanol $(\%)$ & 3.76 & 3.10 & 2.74 & 2.85 \\
Ketone/alcohol $(\mathrm{mol} / \mathrm{mol})$ & 2024 & 783 & 878 & 642 \\
Total turnover number $(\mathrm{mol} / \mathrm{mol})$ & 3.33 & 2.30 & 7.68 & 5.61 \\
Total yields at $2 \mathrm{~h}(\mathrm{mmol})$ &
\end{tabular}

Reaction temperature is $85^{\circ} \mathrm{C}$. The flow rate of air is $0.06 \mathrm{~m}^{3} / \mathrm{h}$. Ethylbenzene is $30 \mathrm{ml}$. Catalysts are $10 \mathrm{mg}$.

Table 12. Effects of metalloporphyrins on oxidation products of ethylbenzene

\section{Mechanism of biomimetic oxidation of hydrocarbons over metalloporphyrins}

The oxygenation mechanism operating in CP-450 and in synthetic metalloporphyrin models has received much attention in recent decades [21,88-90]. However, the putative character of 
the active oxidants recently remains a controversial matter in the catalytic cycles of reductive oxygen activation and reaction by CP-450[35,91] (Scheme 16), three different species have been considered as possible active oxidants[2]: one presenting a nucleophilic character, a peroxy species (d), one with electrophilic character, a iron(IV) oxo porphyrin pcation radical intermediate (7) similar to the Compound I (Cpd I) of horseradish peroxidase, and the last with both nucleophilic and electrophilic character, a hydroperoxy species ( $\mathrm{Fe}^{\mathrm{III}}$ $\mathrm{OOH}(\mathrm{R})$, 6) known as Compound 0 (Cpd 0). Recently, iron (IV) oxo-porphyrin complexs $\left(\mathrm{Fe}^{\mathrm{IV}}=\mathrm{O},(8)\right)$, a model of a peroxidase Compound $\mathrm{II}(\mathrm{CpdII})$, is believed to participate in hydroxylation of hydrocarbon[35].

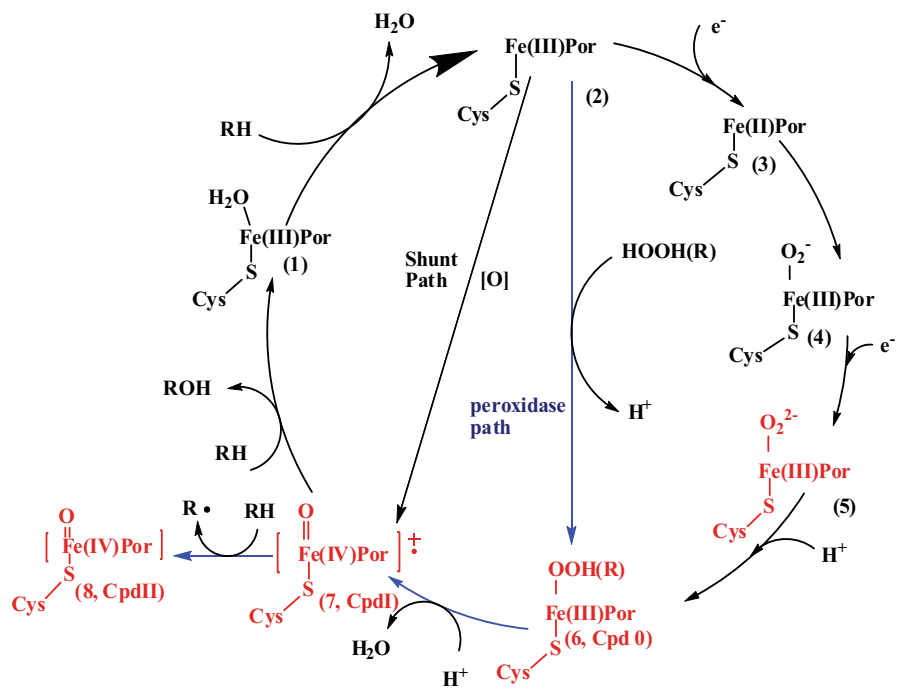

Scheme 16. Proposed catalytic cycle of CP-450(Black) and Peroxidase(Blue).

To synthesize and distinguish the reactivity of these active oxidants, many oxygen-atomtransfer reactions catalyzed by synthetic metalloporphyrin complexes as CP-450 models have been extensively studied[92-93].

\subsection{Mechanism of oxidation reactions with oxygen donors catalyzed by metalloporphyrins}

Since the pioneering work of Groves et.al. on the synthesis and characterization of metal-oxo porphyrin $\Pi$-cation radicals[31,94], a number of metal-oxo porphyrin complexs, especially iron(IV)-oxo porphyrin complexs have been synthesized and characterized with various spectroscopic techniques and isotopically labeled experiments, and their reactivities have been extensively investigated in oxygenation reactions.[35,92,95] Similarly to CP-450 catalytic cycle mechanism, the metal-oxo porphyrin n-cation radicals formed via shunt path (Scheme 16, (7)) have been proposed as ultimate reactive species under different oxidation reactions with single oxygen atom donors such as $\mathrm{PhIO}, \mathrm{NaClO}, \mathrm{KHSO}_{5}, m-\mathrm{CPBA}, t_{-}$ $\mathrm{BuOOH}, \mathrm{H}_{2} \mathrm{O}_{2}$ etc. However, iron (III)-hydroperoxo species (analogous to (6) in Scheme 16) was postulated recently as the second electrophilic oxidant in biomimetic oxidation reaction with $\mathrm{H}_{2} \mathrm{O}_{2}$ and alkyl peroxide as oxygen donor[35]. The action of these metal-oxo porphyrin species seems to be dependent on the nature of the porphyrin ligand, the axial ligand, the solvent and the oxygen donor $[35,92]$. 
Our research group has also been involved in elucidating the formation mechanisms of metal-oxo porphyrin $\Pi$-cation radicals. We attempted to apply the linear free-energy relationships to metalloporphyrin-catalyzed biomimetic oxidation of hydrocarbon. Forty metalloporphyrins with different substituents (R) on the porphyrin ring, $\mathrm{RTPPM}^{\mathrm{II}} \mathrm{Cl}$ and (RTPPM $\left.{ }^{\mathrm{III}}\right)_{2} \mathrm{O}(\mathrm{M}=\mathrm{Fe}, \mathrm{Mn})$ were synthesized and were used to catalyze cyclohexane hydroxylation with PhIO under mild conditions[96-98]. A Hammett relationship, $\log k=\rho \sigma+c$, was shown to exist between the rate constants $k$ of the cyclohexane hydroxylation and Hammett constants $\sigma$ of substituents on the porphyrin rings. The value of the reaction constant $\rho$ changed from 0.3 to 0.5 for the cyclohexane oxidation catalyzed by metalloporphyrins, and electron-withdrawing substituents increased the reaction rates. This means that the active oxidant was a radical cation species analogue with the high-valent oxo-metal $\Pi$-cation radicals intermediate (Scheme 17,(3)). Further quantitative structureactivity relationship (QSAR) studies[99] on ironporphyrin-catalyzed cyclohexane oxidation with $\mathrm{PhIO}$ indicate that the electron withdrawing substituents could lower $\mathrm{E}_{\text {LUMO-b }}$ and disperse the electron density of around the centro-metal core of porphyrin better, they can facilitate ironporphyrin binding with the oxidant and increase the catalytic activity of ironporphyrin consequently.

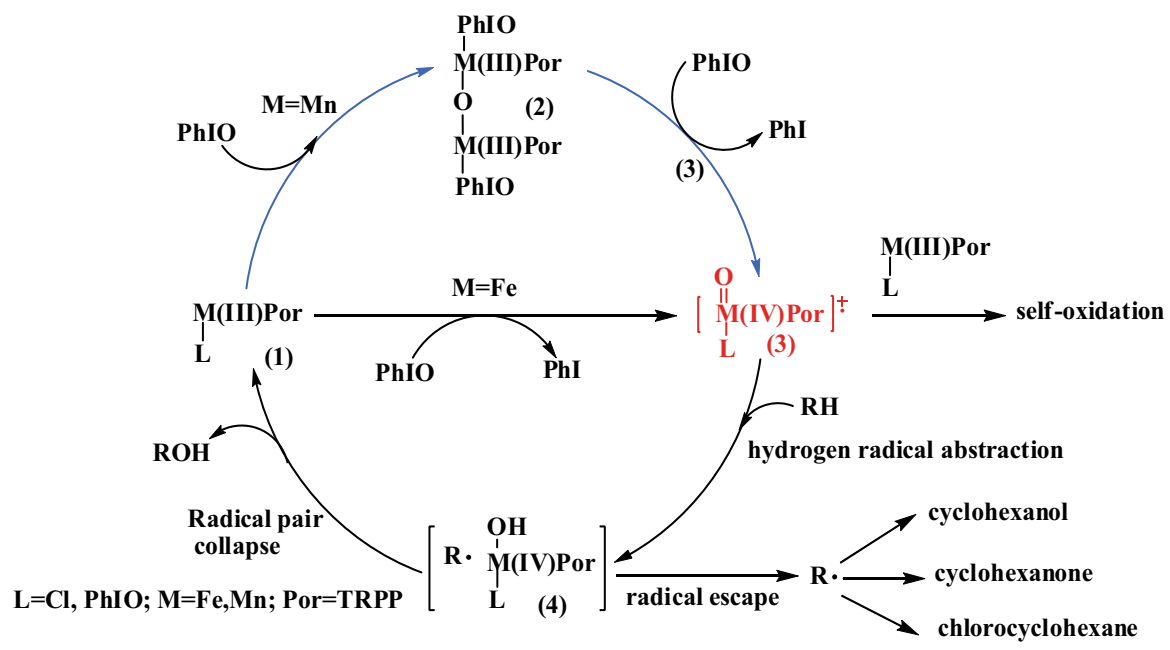

Scheme 17. Proposed reaction mechanism of metalloporphyrin biomimetic catalytic of alkane hydroxylation with oxygen donors

Although high value metal-oxo porphyrin complexs exhibit a versatile reactivity to catalyze the epoxidation of alkenes and the hydroxylation of alkane under different circumstances, many metalloporphyrin-based catalysts suffer from limited lifetime activity due to the oxidative self-destruction of porphyrin ligands[100]. However, the self-oxidation of metalloporphyrins is less studied. Our group's further study[35] on the linear free-energy relationships of self-oxidation of metalloporphyrins show that high value metal-oxo porphyrin complexs as active oxidants are ascribed to the oxidative destruction of porphyrin ring, and the self-oxidation of metalloporphyrins and hydrogen radical abstraction in the system of the cyclohexane hydroxylation are two competition reactions via the same active oxidant. The reaction rates depend on the porphyrin ligands, axial ligands and oxidants. The oxidation of monometalloporphyrins is highly sensitive to 
substituents of porphyrin rings, both electron-withdrawing substituents and electrondonating substituents of porphyrin ligands could accelerate the self-oxidation reaction rate. On the contrary, the oxidation of $\mu$-oxo bismetalloporphyrin is not sensitive to substituents of porphyrin rings. Electron-withdrawing substituents slightly favor the oxidation reaction. In addition of the porphyrin ligand effect, the catalytic hydroxlation of cyclohexane was also markedly affected by the axial ligands of metalloporphyrins, different oxygen donors and solvents[101], indicating that the neutral organic axial ligands, strong polarity of solvent and the oxidation ability are important factors in the oxidation ability of high value metal-oxo porphyrin complexs.

\subsection{Mechanism of oxidation reactions using molecular oxygen without a reductant catalyzed by metalloporphyrins}

Although metalloporphyrin-catalyzed biomimetic oxidation of hydrocarbon with $\mathrm{O}_{2}$ without reductants is analogue with the catalytic cycle of $\mathrm{CP}-450$ systems, this catalytic system named as Lyons system do not require reductants or photochemical and electrochemical technical assistance. In order to search after more evidence of the mechanism in Lyons catalyst system, Lyons [44,102-103], Labinger [68,104-105], Therien [106-107], and Harber [108-109] have made many ground-breaking work. There are three main reaction mechanisms in Lyons catalytic system. One is a high-value metal-oxo species mechanism, one is metal-bound alkylperoxide mechanism, and the last is a radical-chain autoxidation mechanism.

Lyons[44,102] and Therien[107] have found that an iron (IV)-oxo porphyrin complex $\left(\mathrm{PFe}{ }^{\mathrm{IV}}=\mathrm{O}, \mathrm{Cpd}\right.$ II) generated by $\mu$-oxo iron porphyrin thermal disproportionation (Scheme 18, marked by blue line), is involved in polyhalogenated ironporphyrins oxidation of isobutane through the UV-vis and high pressure NMR measurements. Haber et al[109] proposed that homolytic cleavage of alkyl peroxide manganese(III)porphyrin complexes PMn ${ }^{\mathrm{III} O O R}$ result in ketone as the main reaction product in the study of manganeseporphyrin-catalyzed oxidation of cyclooctane. Takeuchi [110] and Mansuy [111] have also found a similar alkylperoxide-bound porphyrin complexs in metalloporphyrin$\mathrm{O}_{2}-\mathrm{NaBH}_{4}$ system and a metalloporphyrin- $\mathrm{O}_{2}$-photocatalytic system. However, by means of alkyl hydroperoxide characterization[107], products comparition[73], and a simple kinetic model calculation[105], a radical-chain autoxidation mechanism proposed by Labinger et.al.[104] is likely operative in isobutane oxidation where the role of metalloporphyrin is to generate radicals by catalyzing the decomposition of alkyl hydroperoxides via Haber-Weiss cycle[103]. (Shceme 18, chemical cycle)This cycle is also present in cycloalkane[109] and alkylaromatics oxidation[112].

Reductive conversion of ferric complexs to ferrous complex in CP-450 catalytic cycle needs for co-reductants, which plays a key role in activation of molecular oxygen. In general, polyhalogenation[113], photochemical reduction, and electrochemical reduction of metalloporphyrin[114] can facilitate the ferric complexs reduction. Through the temperature effect research of different metalloporphyrin catalytic oxidation of hydrocarbons[41], our group found that trivalent metal porphyrins (such as PorFe(III) and PorMn(III)) at low temperature cannot be converted to divalent metalloporphyrin, but increase of reaction temperature facilitate the thermal decomposition of PorM(III) to divalent PorM(II).

Our group always found that a small amount of cyclohexanone was obtained in the hydroxidation of cyclohexane with metalloporphyrins and PhIO. However, the 
hydroxylation of hydrocarbon in CP-450 only obtains alcohol via high value metal-oxo complexs(Scheme 16). Cyclohexanol is the product formed by the cage collapse of radical pairs( Scheme 17,(4)), and where the cyclohexanone comes from? By means of studying the effects of the structure of metalloporphyrins[98] and the reaction conditions on the kinetics of ketones formation[115], our group founded that cyclohexanone is the product formed by the reaction of the escaped radical $R \cdot($ Scheme 17) and oxidants. The experiments showed that cyclohexanone yield increased when the above reaction was carried out in air [104] at elevated temperature [115]. Moreover, alky radicals could escape more easily from the solvent cage at the higher temperature to participate in the radical-chain autoxidation[112].

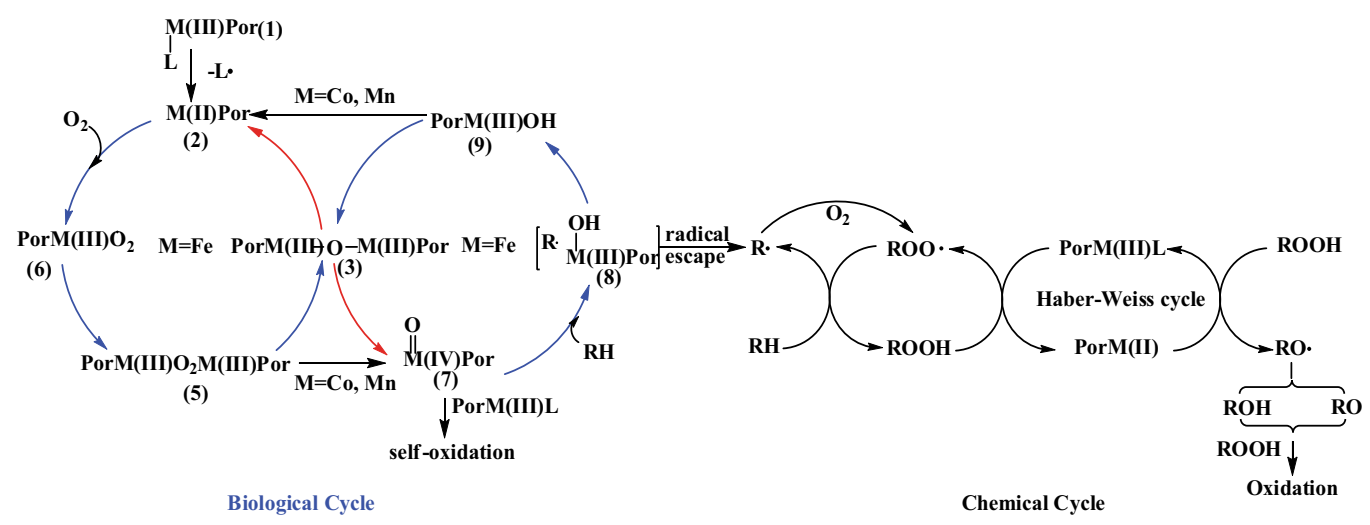

Scheme 18. Biological-chemical-cycle coupling mechanism of metalloporphyrin biomimetic catalytic of hydrocarbon oxygenation with $\mathrm{O}_{2}$ without a reductant

We thus propose a novel mechanism referred to as the biological-chemical-cycle coupling mechanism (Scheme 18). The main function of the biological cycle is to initiate the radical species and further amplify them through a cycle in analogous with CP-450 catalytic cycle. After escaping from the $\mathrm{CP}-450$ catalytic cycle, the alkyl radical may then enter the chemical cycle to be further amplified and oxidized through a Haber-Weiss cycle to afford the products. Therefore, one molecule of metalloporphyrin catalyst could catalyze several hundred thousands of molecules of cyclohexane through coupling of the biological and chemical cycles. The total turnover number should be geometric growth in the biologicalchemical-cycle coupling cycle.

\section{Industrial-scale biomimetic oxidation of hydrocarbons}

The ultimate goal for basic researching of aerobic oxidation of hydrocarbons is to find their application for the green environment. As the selectivity and activity for oxidation of C-H bond of alkane, alkene and aromatic hydrocarbon is poor, biomimetic oxidation using porphyrin as catalyst now surmounted above shortcomings and realized the industrial application.

Current industrial hydrocarbon oxidation methods generally suffer from extremely low efficiency and environment-unfriendly problems. For example, aerobic oxidation of cyclohexane to cyclohexanol and cyclohexanone mixture (KA oil), a key step in polyamide nylon production, is a very significant industrial process not only because KA oil is an extremely important commercial raw material of nylon- 6 and nylon-66, but also because the 
reaction is among the least efficient of major industrial chemical processes. So far, general industrial cyclohexane oxidation has a $4 \%$ conversion rate with no higher than $80 \%$ yield of cyclohexanone, using a two-step process without catalyst, or with a soluble cobalt salt catalyst[47].

In the past 40 years, the discovery of novel methods aiming to solve the reciprocal inhibition relationship between the reaction conversion and the cyclohexanol and cyclohexanone yield in aerobic oxidation of cyclohexane remain a daunting task for the cyclohexane oxidation industry[46,116], and significant efforts have been made to develop the technology for selective oxidation of alkane with air [16].

The oxidation of hydrocarbon catalyzed by metalloporphyrins has been known for several decades[117] , and aerobic oxidation of hydrocarbon by polyhalogenated metalloporphyrins has also been reported[44]. However, the catalytic activity of the metalloporphyrins was generally poor, the catalyst life was short, and catalysts were extremely expensive. Due to these seemingly insurmountable shortcomings, it was widely believed that profitable industrial-scale oxidation of hydrocarbon catalyzed by metalloporphyrins was impossible [118].

Our group reported firstly all over the world [119] a novel industrial approach of hydrocarbon oxidation with air based on a metalloporphyrins as CP-450 monooxygenase model and its industrial application to cyclohexane oxidation. Upon addition of extremely low concentrations (1-5 ppm) of simple cobalt porphyrin (tetraphenylporphinatocobalt, TPPCo) to the commercial cyclohexane oxidation system, and decrease of the reaction temperature and pressure about $20{ }^{\circ} \mathrm{C}$ and $0.4 \mathrm{MPa}$ respectively, the conversion of the cyclohexane increased from $4.8 \%$ to $7.1 \%$, the yield of cyclohexanol and cyclohexanone increased from $77 \%$ to $87 \%$. The aerobic oxidation of cyclohexane was carried out using a 70,000-ton cyclohexanone equipment set comprised of five series-wound stirring reactors using extremely low concentrations (2 ppm) of simple cobaltporphyrin TPPCo as catalyst, under $145{ }^{\circ} \mathrm{C}$ and 0.8 $\mathrm{MPa}$ air pressure. The industrial-scale oxidation results are shown in table 13.

\begin{tabular}{lcc}
\hline Catalyst & Cobalt porphyrin & No Catalyst \\
\hline Production output, kt/a & 125 & 70 \\
Conversion,mol, \% & $\geq 7.1$ & 4.8 \\
Turnover number, mol/mol & 308800 & - \\
Cyclohexanone yield, \% & $\geq 87$ & 77 \\
Cyclohexanol; Cyclohexanone & $1.2: 1$ & $0.9: 1$ \\
Temperature, ${ }^{\circ} \mathrm{C}$ & 145 & 165 \\
Pressure, $\mathrm{MPa}$ & 0.8 & 1.2 \\
\hline
\end{tabular}

Table 13. Comparison of cyclohexane aerobic oxidation over 70,000-ton cyclohexanone equipment set

The advantages of this industrial procedure includes: (1) no need to modify the cyclohexanone equipment currently used in industry, (2) lower reaction temperature and pressure, (3) extremely low (1-5 ppm) concentrations of simple metalloporphyrin catalysts used, (4) cyclohexane conversion increasing from $4.8 \%$ to $7.1 \%$, (5) cyclohexanone yield elevating from $77 \%$ to $87 \%$, and (6) no catalyst reclaimed process and setups.. Therefore, this procedure significantly improves the low conversion rate and low yield the cyclohexane oxidation industry has been facing for the past 40 years. 


\section{Conclusion}

Metalloporphyrin-based oxidation systems not only hold great importance in biomimetic studies but also, as bioinspired oxidation systems, provide a useful tool for organic oxidation reactions. A highly important reaction is the aerobic oxidation catalyzed by metalloporphyrins. Such reactions will have an impact if further increases in product turnovers are realized.

Progress is still required to further improve these metalloporphyrin-based oxidation systems, mainly in two directions. Improvement of the selectivity of substrate recognition by the catalyst or the support of the catalyst, which is performed by the protein in CP-450, should lead to more regio-, stereo- and enantioselective oxygenations. Major improvements are also necessary for the construction of efficient systems for substrate hydroxylation by $\mathrm{O}_{2}$ itself, because of the difficulty to mimic the catalytic cycle of $\mathrm{O}_{2}$ activation by CP-450 that involves a tight coupling between the electron-and proton-transfer steps.

\section{Reference}

[1] J. E. Backvall, Modern Oxidation Methods, Wiley-VCH, Weinheim, 2004

[2] M. Hudlicky, Oxidations in Organic Chemistry, American Chemical Society, Washington, DC, 1990

[3] E. T. Denisov and I. B. Afanas'ev, Oxidation and Antioxidants in Organic Chemistry and Biology, CRC Taylor\&Francis, Boca Raton, 2005

[4] S. Bhaduri and D. Mukesh, Homogeneous Catalysis: Mechanisms and Industrial Applications, John Wiley\&Sons, New York, USA, 2000

[5] S. M. Roberts and G. Poignant, Catalysts for Fine Chemical Synthesis, Hydrolysis Oxidation and Reduction, John Wiley\&Sons Ltd, Chichester, UK, 2002, 1

[6] S. Caron, R. W. Dugger, S. G. Ruggeri, J. A. Raganand, D. H. B. Ripin, Large-Scale Oxidations in the Pharmaceutical Industry,Chem. Rev, 2006, 106, 2943

[7] W. Adam, C. R. Saha-Moller and P. A. Ganeshpure, Synthetic Applications of Nonmetal Catalysts for Homogeneous Oxidations, Chem. Rev, 2001, 101, 3499

[8] A. Wong and Y. Shi, Organocatalytic Oxidation. Asymmetric Epoxidation of Olefins Catalyzed by Chiral Ketones and Iminium Salts,Chem. Rev, 2008, 108, 3958

[9] R. A. Sheldon and J. K. Kochi, Metal Catalyzed Oxidations of Organic Compounds, Academic Press, New York, 1981

[10] K. A. Jørgensen, Transition-Metal-Catalyzed Epoxidations, Chem. Rev, 1989, 89, 431

[11] S. S. Stahl, J. A. Labinger and J. E. Bercaw, Homogeneous Oxidation of Alkanes by Electrophilic Late Transition Metals, Angew Chem. Int. Ed, 1998, 37, 2180

[12] A. Corma and H. Garcia, Lewis Acids as Catalysts in Oxidation Reactions: From Homogeneous to Heterogeneous Systems, Chem Rev, 2002, 102, 3837

[13] B. Limberg, The Role of Radicals in Metal-Assisted Oxygenation Reactions, Angew. Chem. Int. Ed, 2003, 42, 5932

[14] Q. H. Xia, H. Q. Ge, C. Ye, Z. M. Liu and K. X. Su, Advances in Homogeneous and Heterogeneous Catalytic Asymmetric Epoxidation, Chem. Rev, 2005, 105, 1603 
[15] E. Marko, P. R. Giles, M. Tsukazaki, S. M. Brown and C. J. Urch, Copper-Catalyzed Oxidation of Alcohols to Aldehydes and Ketones: An Efficient, Aerobic Alternative, Science, 1996, 274, 2044

[16] I. M. Thomas, R. Raja, G. Sankar and R. G. Bell, Molecular-sieve Catalysts for the Selective Oxidation of Linear Alkanes by Molecular Oxygen, Nature, 1999, 398, 227

[17] D. I. Enache, J. K. Edwards, P. Landon, B. Solsona-Espriu, A. F. Carley, A. A. Herzing, M. Watanabe, C. J. Kiely, D. W. Knight and G. J. Hutchings, Solvent-Free Oxidation of Primary Alcohols to Aldehydes Using Au-Pd/ $\mathrm{TiO}_{2}$ Catalysts, Science, 2006, 311, 362

[18] E. I. Solomon, U. M. Sundaram and T. E. Machonkin, Multicopper Oxidases and Oxygenases, Chem. Rev, 1996, 96, 2563

[19] T. D. H. Bugg, Dioxygenase Enzymes: Catalytic Mechanisms and Chemical Models, Tetrahedron, 2003, 59, 7075

[20] M. Sono, M. P. Roach, E. D. Coulter and J. H. Dawson, Heme-Containing Oxygenases, Chem. Rev, 1996, 96, 2841

[21] B. Meunier, S. P. de Visser and S. Shaik, Mechanism of Oxidation Reactions Catalyzed by Cytochrome P450 Enzymes, Chem. Rev, 2004, 104, 3947

[22] V. B. Urlacher and S. Eiben, Cytochrome P450 Monooxygenases: Perspectives for Synthetic Application, Trends. Biotechnol, 2006, 24, 324

[23] D. Mansuy, Biocatalysis and Substrate Chemodiversity: Adaptation of Aerobic Living Organisms to Their Chemical Environment, Catal. Today, 2008, 138, 2

[24] B. Meunier, Biomimetic Oxidations Catalyzed by Transition Metal Complexes, Imperial College Press, London, 2000

[25] R. V. Eldik and J. Reedijk, Homogeneous Biomimetic Oxidation Catalysis, Adv. Inorg. Chem, Elsevier, Amsterdam, 2006, 58

[26] P. Gamez, P. G. Aubel, W. L. Driessen and J. Reedijk, Homogeneous Bio-inspired Copper-Catalyzed Oxidation Reactions, Chem. Soc. Rev, 2001, 30, 376

[27] R. A. Sheldon, D. Marcel, Metalloporphyrins in Catalytic Oxidations, New York, 1994

[28] I. T. Groves, T. E. Nemo and R. S. Myers, Hydroxylation and Epoxidation Catalyzed by Iron-Porphine Complexes. Oxygen Transfer from Iodosylbenzene, J. Am. Chem. Soc, $1979,101,1032$

[29] T. J. McMurry and J. T. Groves, Cytochrome P-450: Structure, Mechanism, and Biochemistry, Plenum Publishers, New York, 1986,

[30] J. T. Groves, K. Shalyaev and J. Lee, The Porphyrin Handbook, Academic Press, 2000, 4, 17

[31] J. T. Groves, Cytochrome P-450: Structure, Mechanism, and Biochemistry, Kluwer Academic/Plenum Publishers, New York, 2005, 3, 1

[32] D. Ostovic and T. C. Bruice, Mechanism of Alkene Epoxidation by Iron, Chromium, and Manganese Higher Valent Oxo-Metalloporphyrins, Acc. Chem. Res, 1992, 25, 314

[33] I. M. Smith and R. Guilard, The Porphyrin Handbook, Academic Press, 2000, 4, 41

[34] W. Nam, High-Valent Iron(IV)-Oxo Complexes of Heme and Non-Heme Ligands in Oxygenation Reactions, Acc. Chem. Res, 2007, 40, 522 
[35] D. Ostovic and T. C. Bruice, Mechanism of Alkene Epoxidation by Iron, Chromium, and Manganese Higher Valent Oxo-Metalloporphyrins,Acc. Chem. Res, 1992, 25, 314

[36] H. B. Ji, Y. B. She, Green Oxidation and Reduction, China Petrochemical Press, Beijing, 2005

[37] C. C. Guo, M. F. Chu, Q. Liu, Y. Liu, D. C. Guo, X. Q. Liu, Effective Catalysis of Simple Metalloporphyrins for Cyclohexane Oxidation with Air in the Absence of Additives and Solvents, App1. Cata1. A, 2003, 246, 303

[38] D. Mansuy, M. Fontecave, J.F. Bartoli, Mono-Oxigenase-Like Dioxygen Activation Leading to Alkane Hydroxylation and Olefin Epoxidation by an $\mathrm{Mn}{ }^{\mathrm{III}}$ (porphyrin)Ascorbate Biphasic System, J. Chem. Soc. Chem. Commun, 1983,1, 253

[39] I. Fontecave, D. Mansuy, Monooxygenase-Like Oxidations of Olefins and Alkanes Catalyzed by Manganese Porphyrins : Comparison of Systems Involving either $\mathrm{O}_{2}$ and Ascorbate or Iodosylbenzene, Tetrahedron, 1984, 40, 4297

[40] J. E. Lyons, P. E. Ellis, H. K. Mayers, Halogenated Metalloporphyrin Complexes as Catalysts for Selective Reactions of Acyclic Alkanes with Molecular Oxygen, J. Catal, 1995, 155, 59

[41] U. Schuchardt, W. A. Carvalho, and E. V. Spinacé, Why Is It Interesting to Study Cyclohexane Oxidation?, Synlett, 1993, 10, 713

[42] U. Schuchardt, D. Cardoso, R Sercheli, R. D. Pereira, R. S. Cruz, M. C. Guerreiro, D. Mandelli, E. V. Spinacé and E. L. Pires, Cyclohexane Oxidation Continues to Be a Challenge, App1. Cata1. A, 2001, 211, 1.

[43] K. Suresh, M. M. Sharma and T. Sridhar, Engineering Aspects of Industrial LiquidPhase Air Oxidation of Hydrocarbons, Ind. Eng.Chem. Res, 2000, 39, 3958.

[44] A. Castellan, J. C. J. Bart and S. Cavallaro, Industrial Production and Use of Adipic Acid, Catal. Today, 1991, 9, 237

[45] Y. Ishii, A Novel Catalysis of N-Hydroxyphthalimide (NHPI) Combined with Co(acac) $(n=2$ or 3$)$ in the Oxidation of Organic Substrates with Molecular Oxygen, J. Mol. Catal. A: Chem. 1997, 117, 123

[46] T. Iwahama, K. Syojyo, S. Sakaguchi and Y. Ishii, Direct Conversion of Cyclohexane into Adipic Acid with Molecular Oxygen Catalyzed by N-Hydroxyphthalimide Combined with Mn(acac) $)_{2}$ and Co(OAc) 2, Org. Process Res. Dev, 1998, 2, 255

[47] I. Sawatari, T. Yokota, S. Sakaguchi and Y. Ishii, Alkane Oxidation with Air Catalyzed by Lipophilic N-Hydroxyphthalimides without Any Solvent, J. Org. Chem, 2001, 66,7889

[48] J. M. Thomas, R Raja, G. Sankar and R. G. Bell, Redox Molecular Sieve Catalysts for the Aerobic Selective Oxidation of Hydrocarbons, Stud. Surf. Sci. Catal,2000, 130, 887

[49] M. Dugal, G. Sankar, R. Raja and J. M. Thomas, Designing a Heterogeneous Catalysts for the Production of Adipic Acid by Aerial Oxidation of Cyclohexane, Angew. Chem. Int. Ed. Engl, 2000, 39, 2310

[50] R. Raja, G. Sankar and J. M. Thomas, Powerful Redox Molecular Sieve Catalysts for the Selective Oxidation of Cyclohexane in Air, J. Am. Chem.Soc, 1999, 121, 11926

[51] E. L. Pires, M. Wallau and U. Schuchardt, Selective Oxidation of Cyclohexane over Rare Earth Exchanged Zeolite Y, Stud. Surf. Sci. Catal, 1997, 110, 1025 
[52] E. L. Pires, M. Wallau and U. Schuchardt, Cyclohexane Oxidation over Rare Earth Exchanged Zeolite Y, J. Mol. Chem. A: Chem, 1998, 136, 69

[53] C. C. Guo, X. Q. Liu, Y. Liu, Q. Liu, M. F. Chu, X. B. Zhang, Studies of Simple $\mu-O x o-$ Bisiron(III)porphyrin as Catalyst of Cyclohexane Oxidation with Air in Absence of Cocatalysts or Coreductants, J. Mol. Chem. A: Chem,2003, 192, 289

[54] C. C. Guo, G. Huang, X. B. Zhang, D. C. Guo, Catalysis of Chitosan-Supported Iron Tetraphenylporphyrin for Aerobic Oxidation of Cyclohexane in Absence of Reductants and Solvents, App1. Cata1. A, Gene, 2003, 247, 261

[55] C. C. Guo, G. Huang, D. C. Guo, Preparation of Nitrogenous Polysaccharide-Supported Ironporphyrins and Their Catalysis for the Aerobic Oxidation of Cyclohexane, Kinet. Catal, 2006, 47(1), 93

[56] G. Huang, X. J. Li, C. C. Guo, Aerobic Oxidation of Cyclohexane Catalyzed by Chitosansupported Cobalt Tetraphenylporphyrin, Chin J. Catal, 2005, 26(9), 765

[57] X. T. Wang, M. F. Chu, C. C. Guo, Catalysis of Manganeseporphyrin Supported on Imidazole-modified Silica Gel for Cyclohexane Oxidation with Air, Chem. J. Chin. Univ., 2005, 26(1), 64

[58] B. Y. Hu, Y. J. Yuan, J. Xiao, C. C. Guo, Q. Liu, Z. Tan and Q. H. Li, Rational Oxidation of Cyclohexane to Cyclohexanol, Cyclohexanone and Adipic Acid with Air over Metalloporphyrin and Cobalt Salt, J. Porphyrins Phthalocyanines, 2008, 12, 27

[59] C. C. Guo, Q. Liu, X. B. Zhang, Method for Oxidation of Alkanes and Cycloalkane Useing Air, CN 1269343A, 2000

[60] J. M. Ma, C. C. Guo, Preparation of Methylcyclohexanol and Methylcyclohexanone from Aerobic Oxidation of Methylcyclohexane Catalyzed by Metalloporphyrins, J. Chem. Indu. Eng, (China), 2005, 56(5), 835

[61] K. W. Xu, J. Y. Ma, Q. Jiang, H. Y. Hu, C. C. Guo, Selective Catalysis of Manganeseporphyrins on Aerobic Oxidation of Different Carbon-Hydrogen Bonds of Methylcyclohexane, J. Mol. Catal. A: Chem., 2006, 243, 194

[62] K.T. Moore, I. T. Horvath, M. J. Therien, Mechanistic Studies of (Porphinato)IronCatalyzed Isobutane Oxidation. Comparative Studies of Three Classes of ElectronDeficient Porphyrin Catalysts, Inorg. Chem, 2000, 39, 3125

[63] R. B. Brown Jr, C. L. Hill, Catalytic Homogeneous Functionalization of Adamantane. Influence of Electronic and Structural Features of the Metalloporphyrin Catalyst on Atom Transfer Selectivity (Oxygenation versus Acidification/Halogenation), J. Org. Chem, 1988, 53, 5762

[64] C. C. Guo, Q. Liu, X. T. Wang, H. Y. Hu, Selective Liquid Phase Oxidation of Toluene with Air, App1. Cata1. A, 2005, 282, 55

[65] I, Tabushi and N. Koga, P-450 Type Oxygen Activation by Porphyrin-Manganese Complex, J. Am. Chem.Soc,1979, 101, 6456

[66] K. Kano, H. Takagi, M. Takeuchi, S. Hashimoto and Z. Yoshida, PorphinatoironCatalyzed Oxygenation of Styrene in Aqueous Solution, Chem. Lett, 1991,519

[67] J. T. Groves, and R. Quinn, Aerobic Epoxidation of Olefins with Ruthenium Porphyrin Catalysts, J. Am. Chem.Soc,1985, 107, 5790

[68] E. R. Birnbaum, M. W. Grinstaff, J. A. Labinger, J. E. Bercaw and H. B. Gray, On the Mechanism of Catalytic Alkene Oxidation by Molecular Oxygen and Halogenated Iron Porphyrins, J. Mol. Catal. A: Chem., 1995, 104, 119 
[69] E. R. Birnbaum, J. A. Labinger, J. E. Bercaw and H. B. Gray, Catalysis of Aerobic Olefin Oxidation by a Ruthenium Perhaloporphyrin Complex, Inorg. Chim. Acta, 1998, 270,433

[70] E. N. Jacobsen, L. Deng, Y. Furukawa and L. E. Martinez, Enantioselective Catalytic Epoxidation of Cinnamate Esters, Tetrahedron 1994, 50, 4323

[71] T. Katasuki, Catalytic Asymmetric Oxidations Using Optically Active (Salen)manganese(III) Complexes as Catalysts, Coord. Chem. Rev, 1995, 140, 189

[72] T. Takemori, A. Inagaki and H. Suzuki, A Novel Type of Carbon-Carbon Double Bond Cleavage of 1,1-Disubstituted Alkenes on a Triruthenium Polyhydrido Cluster, J. Am. Chem. Soc, 2001, 123, 1762

[73] D. Yang and C. Zhang, Ruthenium-Catalyzed Oxidative Cleavage of Olefins to Aldehydes, J. Org. Chem, 2001, 66, 4814

[74] W. Liu, Q. Liu and C. C. Guo, Catalysis of Simple Cobaltporphyrin for Aerial Oxidation of Cyclohexene to Cyclohexenone, J. Chem. Indus. Eng. (China), 2005, 59(5), 1537

[75] Y. F. Li, C. C. Guo, X. H. Yan and Q. Liu, Aerobic Oxidative Cleavage of C=C Double Bond of Styrene Catalyzed by Simple Manganese Porphyrin, J. Porphyrins Phthalocyanines, 2006, 10, 942

[76] C. C. Guo, W. J. Yang, Y. L. Ming, Selectively Aerobic Oxidation of C=C and Allylic C-H Bonds in a-Pinene over Simple Metalloporphyrins, J. Mol. Catal. A: Chemical, 2005, 226, 279

[77] W. J. Yang, C. C. Guo, Z. Y. Li and N. Y. Tao, Aerobic Oxidation of a-Pinene Catalyzed by Nano-Titania-Supported Manganese Tetraphenylporphyrin, J. Porphyrins Phthalocyanines 2009; 13, 973

[78] R. A. Sheldon, Fine Chemicals by Catalytic-oxidation, Chemtech, 1991, 556

[79] W. Partenheimer, Methodology and Scope of Metal/Bromide Autoxidation of Hydrocarbons, Catal. Today, 1995, 23, 69

[80] R. A. Sheldon, J. K. Kochi, Metal Catalysed Oxidation of Organic Compounds, Academic Press, New York, 1981

[81] C. C. Guo, Q. Liu, X. T. Wang, H. Y. Hu, Selective Liquid Phase Oxidation of Toluene with Air, App1. Cata1. A, 2005, 282, 55

[82] H. Y. Hu, Q. Jiang, Q. Liu, J. X. Song, W. Y. Lin and C. C. Guo, Catalysis of $\mu-O x o-$ Bis[porphyriniron(III)] for Toluene Oxidation with Molecular Oxygen, J. Porphyrins Phthalocyanines 2006, 10, 948

[83] L. Y. Li, X. H. Yang, G. F. Jiang, Q. Liu, J. X. Song and C. C. Guo, Toluene Oxyfunctionalization with Air over Metalloporphyrins and Reaction Condition Optimization, Chin. J. Chem. Eng., 2007, 15(3), 453

[84] Q. Jiang, H. Y. Hu, C. C. Guo, Q. Liu, J. X. Song and Q. H. Li, Aerobic Liquid-Phase Oxidation of $p$-Xylene over Metalloporphyrins, J. Porphyrins Phthalocyanines 2007, 11,524

[85] Q Jiang, Y. Xiao, Z. Tan, Q. H. Li, C. C. Guo, Aerobic Oxidation of $p$-Xylene over Metalloporphyrin and Cobalt Acetate: Their Synergy and Mechanism, J. Mol. Catal. A: Chem, 2008, 285, 162

[86] Y. Xiao, W. P. Luo, X. Y. Zhang, C. C. Guo, Q. Liu, G. F. Jiang, Q. H. Li, Aerobic Oxidation of $p$-Toluic Acid to Terephthalic Acid over $\mathrm{T}(p-\mathrm{Cl}) \mathrm{PPMnCl} / \mathrm{Co}(\mathrm{OAc})_{2}$ Under Moderate Conditions, Catal. Lett, 2010, 134, 155 
[87] C. C. Guo, Q. J. Peng, Q Liu, G. F. Jiang, Selective Oxidation of Ethylbenzene with Air Catalyzed by Simple $\mu$-Oxo Dimeric Metalloporphyrins Under Mild Conditions in the Absence of Additives, J. Mol. Catal. A: Chem, 2003, 192, 295

[88] P.R. Ortiz de Montellano (Ed.), Cytochrome P450: Structure, Mechanism and Biochemistry, third ed., Kluwer Academic/ Plenum Publisher, New York, 2005.

[89] K.M. Kadish, K.M. Smith, R. Guilard (Eds.), The Porphyrin Handbook, vol. 3, Academic Press, 2000.

[90] S.shaik, W. Z. Lai, H. Chen, Y. Wang, The Valence Bond Way: Reactivity Patterns of Cytochrome P450 Enzymes and Synthetic Analogs, Acc. Chem. Res. 2010, 43(8), 1154.

[91] T.M. Makris, I. Denisov, I. Schlichting, S.G. Sligar, in: P.R. Ortiz de Montellano (Ed.), Cytochrome P450: Structure, Mechanism and Biochemistry, third ed., Kluwer Academic/ Plenum Publisher, New York, 2005, p. 149.

[92] B. Meunier, A. Robert, G. Pratviel, J. Bernadou, in: K.M. Kadish, K.M. Smith, R. Guilard (Eds.), The Porphyrin Handbook, vol. 4, 2000, p. 119.

[93] D. Mansuy, A Brief History of the Contribution of Metalloporphyrin Models to Cytochrome P450 Chemistry and Oxidation Catalysis, C. R. Chimie. 2007, 10, 392.

[94] J.T. Groves, in: P.R. Ortiz de Montellano (Ed.), Cytochrome P450: Structure, Mechanism and Biochemistry, third ed., Kluwer Academic/Plenum Publisher, New York, 2005, p. 1 .

[95] Y.Watanabe, in: K.M. Kadish, K.M. Smith, R. Guilard (Eds.), The Porphyrin Handbook, vol. 4, 2000, p. 97.

[96] C. C. Guo, Synthesis of $\mu$-Oxo-bisiron(III)Porphyrin Compounds and Their Catalysis for Cyclohexane Hydroxylation, J.Catal.1998, 178(1): 182.

[97] C. C. Guo, H. P. Li, J. B. Xu, Study of Synthesis of $\mu$-Oxo-bismanganese(III)Porphyrin Compounds and Their Catalysis of Cyclohexane Oxidation by PhIO, J. Catal. 1999, 185(2): 345 .

[98] C.C. Guo, J. X.Song, X. B Chen, G. F Jiang, A New Evidence of the High-Valent OxoMetal Radical Cation Intermediate and Hydrogen Radical Abstract Mechanism in Hydrocarbon Hydroxylation Catalyzed by Metalloporphyrins, J. Mol. Catal. A: Chem. 2000, 157, 31.

[99] N. Liu, G. F. Jiang, C. C. Guo, Z. Tan, Quantitative Structure-Activity Relationship Studies on Ironporphyrin-Catalyzed Cyclohexane Oxidation with PhIO, J. Mol. Catal. A: Chem. 2009, 304, 40.

[100] M. H. Alkordi, Y. L. Liu, R. W. Larsen, J. F. Eubank, M. Eddaoudi, Zeolite-like Metal-Organic Frameworks as Platforms for Applications: On MetalloporphyrinBased Catalysts, J. Am. Chem.Soc. 2008, 130, 12639.

[101] C.C. Guo, X. Y. Liu, M. S. Yang, S. J. Zhu, M. D. Gui, Study on the Mechanism of Cyclohexanone Formation in Cyclohexane Hydroxylation Catalyzed by Metalloporphyrins, Chem. J. Chin. Univ. 1991, 12(12), 1617.

[102] P. E. Ellis Jr., J. E.Lyons, Selective Air Oxidation of Light Alkanes Catalyzed by Activated Metalloporphyrins, The search for a Suprabiotic System, Coord. Chem. Rev. 1990, 105(2): 181. 
[103] J. E. Lyons, P. E. Ellis Jr., H. K. Mayers, R. W. Wagner, Halogen Substituent Effects on the Catalytic Activity of Iron Porphyrin Complexes for the Decomposition of tertButyl Hydroperoxide, J. Catal. 1993, 141, 311.

[104] M. W. Grinstaff, M. G. Hill, J. A. Labinger, H. B. Gray, Mechanism of Catalytic Oxygenation of Alkanes by Halogenated Iron Porphyrins, Science, 1994, 264, 1311.

[105] J. A. Labinger, A Simplified Model for Catalyzed Isobutane Autoxidation: Implications for the Mechanism of Catalysis by Halogenated Porphyrin Complexes, Catal. Lett. 1994, 26(1-2): 95.

[106] K. T. Moore, I. T. Horvath, M. J. Therien, High-Pressure NMR Studies of (Porphinato)iron-Catalyzed Isobutane Oxidation Utilizing Dioxygen as the Stoichiometric Oxidant, J. Am. Chem. Soc. 1997, 119(7), 1791.

[107] K. T. Moore, I. T. Horvath, M. J. Therien, Mechanistic Studies of (Porphinato)IronCatalyzed Isobutane Oxidation. Comparative Studies of Three Classes of ElectronDeficient Porphyrin Catalysts, Inorg. Chem. 2000, 39(15): 3125.

[108] J. Haber, L. Matachowski, K. Pamin, J. Połtowicz, Manganese Porphyrins as Catalysts for Oxidation of Cyclooctane in Lyons System, J. Mol. Catal. A: Chem. 2000, 162, 105.

[109] J. Haber, L. Matachowski, K. Pamin, J. Połtowicz, The Effect of Peripheral Substituents in Metalloporphyrins on Their Catalytic Activity in Lyons System, J. Mol. Catal. A: Chem. 2003, 198, 215.

[110] M. Takeuchi, M. Kodera, K. Kano, Z. I. Yoshida, Mechanisms for (Porphyrinato)iron(III)-Catalyzed Oxygenation of Styrenes by O2 in Presence of BH-4, J Mol. Catal. A: Chem. 1996, 113, 51.

[111] A. Maldotti, B. G. Varani, A. Molinari, P. Battioni, D. Mansuy, Oxidation of Cyclohexane by Molecular Oxygen Photoassisted by meso-Tetraarylporphyrin Iron(III)-Hydroxo Complexes , Inorg. Chem. 1996, 35, 1126.

[112] S. Evans, J. R. Lindsay Smith, The Oxidation of Ethylbenzene and Other Alkylaromatics by Dioxygen Catalysed by Iron(III) tetrakis(pentafluorophenyl) porphyrin and Related Iron Porphyrins, J. Chem. Soc., Perkin Trans. 2, 2000, 11, 1541.

[113] D. Dolphin, T. G. Traylor, L. Y. Xie, Polyhaloporphyrins: Unusual Ligands for Metals and Metal-Catalyzed Oxidations, Acc. Chem. Res. 1997, 30, 251

[114] D. Hendrickson; M. G. Kinnaird, K. S. Suslick, Photochemistry of (5,10,15,20tetraphenylporphyrinato)iron(III) halide complexes, Fe(TPP)(X), J. Am. Chem. Soc. $1987,109,1243$

[115] C.C. Guo, X. B. Zhang, L. B. Hou, J. B. Xu, X. D. Hao, G. M. Guo, B. X. Liang, X. B. Chen, Study on the Mechanism of Cyclohexanone Formation in Cyclohexane Hydroxylation Catalyzed by Metalloporphyrins, Chem. J. Chin. Univ. 1991, 12(12), 1617

[116] J.M.,Thomas, R, Raja, G. Sankar and R. G. Bell, Molecular Sieve Catalysts for the Regioselective and Shape- Selective Oxyfunctionalization of Alkanes in Air, Acc. Chem. Res, 2001, 34, 191

[117] B. Meunier, Metalloporphyrins as Versatile Catalysts for Oxidation Reactions and Oxidative DNA Cleavage, Chem. Rev, 1992, 92, 1411 
[118] J. A. Labinger and J. E. Bercaw. Understanding and Exploiting C-H Bond Activation, Nature, 2002, 417, 507

[119] C. C. Guo, X. Q. Liu, Q. Liu, Y. Liu, M. F. Chu and W. Y. Lin, First Industrial-Scale Biomimetic Oxidation of Hydrocarbon with Air over Metalloporphyrins as Cytochrome P-450 Monooxygenase Model and Its Mechanistic Studies, J. Porphyrins Phthalocyanines, 2009, 13, 1250 


\title{
Homogeneous and Heterogeneous Free-Based Porphyrins Incorporated to Silica Gel as Fluorescent Materials and Visible Light Catalysts Mimic Monooxygenases
}

\author{
Mariusz Trytek $^{1^{*}}$, Marek Majdan ${ }^{2}$ and Jan Fiedurek ${ }^{1}$ \\ ${ }^{1}$ Department of Industrial Microbiology, \\ Institute of Microbiology and Biotechnology, \\ Maria Curie-Skłodowska University, \\ Akademicka St. 19, 20-033 Lublin \\ ${ }^{2}$ Faculty of Chemistry, Maria Curie-Skłodowska University, \\ M.Curie-Skłodowskiej Sq. 2, 20-031 Lublin \\ Poland
}

\section{Introduction}

The use of enzymes and whole cells as biocatalysts in biotechnology and organic chemistry is recognized as being of great economic potential in the increasing production of unique and valuable compounds present in nature in an insufficient amount. Bioconversion with living microbial cells, which have the ability to regenerate their own respective cofactors and exhibit a spectrum of enzymatic activity, is a very useful tool in bioorganic synthesis. There is no doubt that the advantage of microbial biotransformations is the possibility to induce enzymes of defined, desired activity, simply by the suitable preincubation methods (Griffin et al., 2001). However, this kind of biocatalysts has several limitations in application to oxidative biotransformations, including stability and activities, especially to those substrates and products that are toxic and require being performed in reaction media other than water. A disadvantage might be the membrane barrier and diffusion problems. Recent advances in addressing these problems include molecular and reaction engineering approaches (Burton, 2003).

A broad field of activity of biological heme-containing oxygenases like cytochromes P450 has provoked the development of the invention of artificial catalytic systems based on porphyrins mimicking the controlled and selective oxidation reactions of these hemoproteins. The concept of artificial enzymes and biomimetic chemistry was introduced by Breslow in 1972 (Breslow, 1972), that started the field of bioinspired or biomimetic catalysis, wherein researchers try to copy Nature in designing the catalyst with the structure and properties similar to those of the active sites of enzymes. Such biomimetic systems are expected to work in organic media and have high stability toward toxic organic compounds in comparison with traditional catalysis employing enzymes or microorganisms.

The porphyrins, due to their synthetic versatility and reactivity, are especially attractive in the construction of biomimetic analogues of monooxygenases through elaboration of the 
superstructure of the macrocycle or chelate complexes. Firstly, the brief description of the physicochemical properties of porphyrins is given in this chapter together with the main contributions of metalloporphyrin model studies to the cytochromes P450 chemistry and oxidation catalysis. The reactions of terpenes oxidation are singled out. Secondly, we elaborated a matter of porphyrins working as photoactive catalysts in the oxidative transformation of limonene and a-pinene into the products attracting great interest by the fragrance and flavour industry. The effects of substrate concentration, wavelength of visible light during the photochemical excitation, kind of solvent and oxidant on the efficiency of the porphyrin-based homogeneous system was discussed. Finally, two types of porphyrins (water-soluble and water-insoluble) incorporated into the silica gel are described pointing out to the advantages and limitations of the silica matrices prepared by the sol-gel method. Here, the immobilization of porphyrin catalyst in an insoluble organic or inorganic support is also briefly outlined. Among the topics included in this part of the chapter are: the effect of porphyrins immobilization on the changes of their photophysical properties, photodegradation or aggregation, as well as correlation between photoactivity of free-base porphyrins in various organic solutions and those entrapped in silica matrices. The correlation between the biocatalytic efficiency of porphyrins entrapped in the sol-gel matrix and their intensity of fluorescent emission and absorbance was also examined. The reasons for much better photooxidation of a-pinene by $\mathrm{OEP} / \mathrm{SiO}_{2}$ than by $\mathrm{HmP} / \mathrm{SiO}_{2}$ are discussed, and the mechanism of pinene photooxidation based on proton transfer and then on two possible routes namely, electron abstraction by the light-excited $\mathrm{OEP} / \mathrm{SiO}_{2}$ matrix and the photosensitized singlet oxygen generation is proposed. It is worth emphasizing that the systems investigated are new and have not been reported before.

\section{The biological monooxygenation. New opportunities and innovations for the improvement biocatalysis efficiency}

The use of enzymes, either derived from natural sources or generated through directed evolution methods, has increased significantly over the past decades (Tao \& Cornisch, 2002; Wackett, 2004). There are essentially two approaches to choosing a biocatalyst: the screening for novel biocatalysts, and the screening for new activities among the existing biocatalysts. The latter approach also includes the improvement of known biocatalysts through protein engineering, either through molecular biology or direct modification of the protein, and the modification of biocatalyst properties by »media engineering (Adamczak \& Krishna, 2004; Turner, 2009).

Replacing the aqueous milieu with organic solvents, ionic liquids, supercritical fluids, fluoro-hydrocarbons etc. enables biocatalysis to increase the chemo-, regio- and enantiospecificity as well as catalytic efficiency of many enzymes (Castro \& Knubovets, 2003). It should be kept in mind that supremely good selectivity of enzymes is weighted against their lack of stability (Thomas \& Raja, 2005). As the application of enzymes continues to grow within both academia and industry, it is evident that, in order to satisfy the stringent requirement of industrial processes with respect to catalyst turnover and productivity, protein engineering techniques will be essential to the growing incorporation of this technology into synthetic chemical processes. This requires a profound understanding of protein structure and molecular biology techniques for manipulation of proteins, as well as the computational methods. The rational mutagenesis of amino acids based on a knowledge of structure or mechanism is targeted at specific residues and is hence popularly known as 
side-directed mutagenesis. This technique is a good tool for investigating enzyme mechanism or altering catalytic attributes. It may be possible to change the electrostatic environment of an active side, perhaps by changing a positively charged lysine to negatively charged glutamic acid. Of course, some amino acid side chains are more susceptible to damage by oxidation than others, notably those containing sulfur, such as cysteine and methionine. It was postulated that mutating the methionine residue in the active side of subtilisin at position 222 to other residues like alanine, serine and cysteine may decrease susceptibility of the enzyme to inactivation through oxidation (Estell et al., 1985).

As the number of structures of enzymes increases and more is learned about the molecular and structural determination of substrate recognition and transformation by enzymes, there is increasing move towards the computational design for enzyme activities that as yet have no equivalent in natural enzyme chemistry (Arnold, 2001; Rothlisberger et al., 2008). The powerful combination of in silico design and directed evolution suggests that protein evolution strategies in future may be credibly targeted towards entirely new enzymes for which no natural enzymes currently exist. Directed evolution involves repeated rounds of (i) random gene library generation, (ii) expression of genes in a suitable host and (iii) screening of libraries of variant enzymes for the property of interest. Both in vitro screeningbased methods and in vivo selection-based methods have been applied to the evolution of enzyme function and properties (Turner, 2009).

Another aspect of diversity that attracts an increasing level of attention within the biocatalysis community is that of catalytic promiscuity. This can loosely be defined as the ability of an enzyme to catalyze a new chemical reaction additional to that to which it is usually attributed. Such reactions of new substrates have been recognized as a valuable research and synthesis tool (Hult \& Berglund, 2007). Many examples of catalytic promiscuity exist within the literature (Bornshcheuer \& Kaslauskas, 2004). One theory suggests that a low level of promiscuous activity in an enzyme can assist the evolution of that enzyme into one that has optimum levels of activity for new reaction. For example, a phosphotriesterase (PTE) enzyme that catalyses the degradation of the herbicide paraoxon is thought only to have acquired this ability relatively recently, as previously, the pesticide substrate would not have occurred in the environment.

Among enzymes, heme monooxygenases (such as cytochrome P450 and peroxidases) are probably the most versatile biocatalysts because of their capability of catalyzing the regioand stereoselective transfer of an oxygen atom from $\mathrm{O}_{2}$ into a vast range of substrates, including monoterpenes. It is one of the most widespread enzymatic activities occurring in all forms of life including both prokaryotes and eukaryotes (Li et al., 2002). Cytochrome P450 was found in a wide range of organisms from bacteria to mammals where it exists in both soluble and membrane-bound states playing at least two key roles. Firstly, it catalyzes many oxidation steps involved in the biosynthesis or biodegradation of endogenous substrates such as steroid hormones, fatty acids or prostaglandins. Secondly, they participate in the crucial step in the oxidative metabolism of exogenous compounds such as drugs and other xenobiotic products, allowing their elimination out from living organism (Mansuy, 1990). The family of these enzymes is involved in the reactions as diverse as e.g. hydroxylation, N-, O- and S-dealkylation, sulphoxidation, epoxidation, deamination, desulphuration, dehalogenation, peroxidation, and N-oxide reduction.

Most monooxygenases are metal-enzymes where metallic complex active center is located in the prosthetic site of the molecule. The active centre of $\mathrm{P} 450$ contains the heme prosthetic group (protoporphyrin IX), which is bound to the apoprotein by the coordination to the iron 
of the tiolate group of cysteine at the proximal side of porphyrin ring, (and also through salt bridges and hydrogen bonds) (Poulos at al., 1987) (Fig. 1). Unlike P450, a heme in peroxidases (except for chloroperoxidase) is ligated with a proximal histidine residue and uses hydrogen peroxide as an oxidant instead of $\mathrm{O}_{2}$.

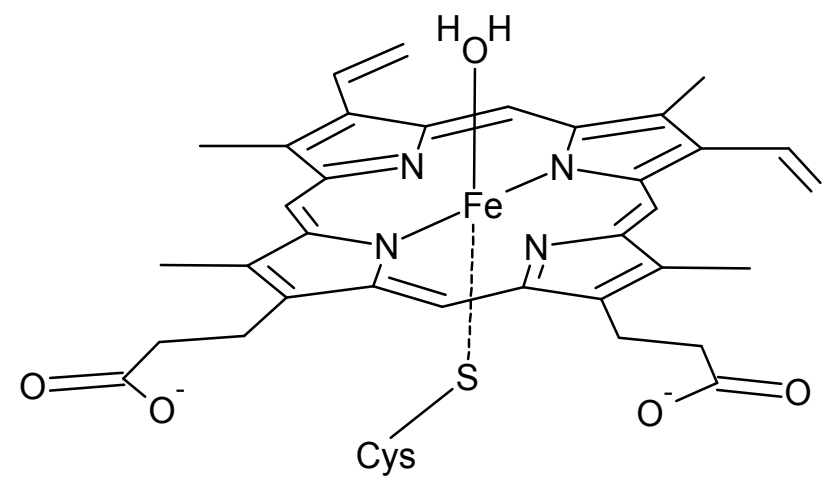

Fig. 1. Protoporphyrin IX. The prosthetic group of cytochrome P450.

One should bear in mind that despite impressive biotechnological impact, the potential of monooxygenases has not been fully exploited. Isolation and purification of the relevant enzymes is difficult and expensive. Moreover, instability and other difficulties associated with using monooxygenases under industrial conditions limit their implementation in applied biocatalysis.

Notwithstanding progress of enzymology and biochemistry which permits to work directly with the proteins themselves, it is still usually difficult to purify the cytochrome P450 dependent enzymes and isolate the corresponding gene using amino acid sequence information for the purified protein. Application of isolated P450-monooxygenases as oxidation biocatalysts is also limited due to the requirement for the costly cofactor $\mathrm{NAD}(\mathrm{P}) \mathrm{H}$.

Site-directed mutagenesis, directed evolution and chimeragenesis, design of substrate binding cavities, chemical modification of prosthetic groups and covalent attachment of metal cofactors were applied in improving the activities and changing of the substrate specificity of various P450s or in inducing a new reactivity in a non-metal protein (Bell, 2001; Kato et al., 2002; van de Velde et al., 2001; Branco et al., 2008). Despite the fact that some efforts meet with spectacular success, it seems to be still too early for these improved P450 systems to be applied in the industry (Bühler \& Schmid, 2004).

\section{Porphyrins as monooxygenase mimics in hydrocarbon oxidative biocatalysis under homogeneous conditions}

A wide variety of activity of biological heme-containing monoxygenases has inspired the preparation, characterization, and application of artificial catalytic systems resembling the structure as well as the activity of these enzymes. There are two main, strictly connected, objectives in the investigation of biomimetic catalysis: i) insight into the nature of the enzymatic processes and ii) the necessity in both biotechnology and industrial chemistry to obtain more efficient biocatalysts working with high regio- and stereo- selectivity, under mild temperature and pressure (Bartocci et al., 1996). There has been observed growing 
interest in the use of metalloporphyrins as biomimetic catalysts in recent years (Que \& Tolman, 2008).

Porphyrins belong to the naturally occurring compounds participating in many important biological processes such as: oxygen transport and different catalytical conversions. Porphyrins can be treated as derivatives of porphine, which consists of four pyrrole subunits joined via $-\mathrm{CH}=$ bridges. This aromatic macrocycle has 22 n electrons, although according to Húckel rule, only 18 of them undergo delocalization $(4 n+2, n=4)$. The size of the cavity in this macrocycle is suitable for the adaptation of different metal ions. The exchange of two protons in $\mathrm{H}_{2}$-porphyrin by a metal ion leads to the origination of metalloporphyrin, a complex compound with a binegative macrocyclic anion. The compounds of $\mathrm{Fe}, \mathrm{Co}, \mathrm{Zn}$ and $\mathrm{Ni}$ are the best known metaloporphyrins. Hemes (iron complexes) and chlorophylles (magnesium complexes) are the examples of porphyrins having special importance in biological systems, the latter in photosynthesis. In turn, vitamin $B_{12}$ has a cobalt complex in its structure with coryne ligand structurally similar to porphyrin. The well known is the role of $B_{12}$ in catalysis of isomerization reactions of methyl group transfer in biological systems. Hemoproteins (complexes of Fe) serve as oxygen carriers (hemoglobin) or oxygen stores (mioglobin) and participate in: electron transfer processes (cytochrome $\mathrm{b}$ and $\mathrm{c}$ ), activation of $\mathrm{O}_{2}$ (cytochrome $\mathrm{P} 450$ ). The porphyrins have become an indispensable component in the evolution of living organisms, due to many types of chemical reactions, characteristic of this group of compounds, such as coordination, polymerization, aggregation, oxidation and reduction, catalysis, sorption and photochemical changes.

Several synthetic models based on porphyrins for heme oxidases have been developed in order to understand principles of the complex mechanism of dioxygen activation and oxygen atom transfer processes, and to obtain valuable information on the nature and reactivity of intermediates produced in the catalytic cycles of metalloenzymes in living organisms (Hessenauer-Ilicheva at al., 2007; Dallacosta et al, 2007; Nam, 2007; Suzuki et al., 1999). The extensive investigation of metalloporphyrins catalyzed oxidation reaction has concentrated on using iron (III) and manganese (III) and the role of pyridine and imidazole ligands. The proposed mechanism for the reaction proceeds through the formation of a high valent metal-oxo species, the first model of which was reported by Groves et al. in 1981 using the system based on the Fe(III) complex of the meso-tetramethylphenylporphyrin $\mathrm{Fe}(\mathrm{TMP}) \mathrm{Cl}$ with peroxyacids (Groves et al., 1981) and later confirmed by iron tetraphenylporphyrinate, $\mathrm{Fe}(\mathrm{TPP}) \mathrm{Cl}$ in the presence of iodosobenzene $(\mathrm{PhI}=\mathrm{O})$ as an oxidant or acetic anhydride as a donor of acyl cations (Mandon et al., 1989; Oligario et al., 2002). The mechanistic developments in biomimetic research have been supported by cross-linked investigation (directed mutagenesis, computational methods, physical biochemistry) using a broad array of the spectroscopic techniques. The detailed mechanistic aspects and structural characterization of intermediates are very well described elsewhere (Shteinman, 2001; Watanabe, 2001; Sligar et al., 2005).

Monooxygenases require a metal center (transition metal ions, predominantly iron and copper) and two electrons to reductively cleave atmospheric dioxygen, producing only a single water molecule in the process while saving the second atom for substrate functionalization and formal oxidation:

$$
\mathrm{RH}+\mathrm{O}_{2}+2 \bar{e}+\mathrm{H}^{+} \rightarrow \mathrm{ROH}+\mathrm{H}_{2} \mathrm{O}
$$


In cytochrome P450, the electrons necessary for the reduction of diooxygen are transferred from a cellular reductant NADH. They are delivered directly one by one by the cytochrome P450-reductase, a huge protein which contains two flavin cofactors, FAD and FMN. The role of metal is reduced to the activation of molecular oxygen and decrease in the kinetic barrier to its reaction with hydrocarbons due to the formation of reactive metal-oxygen intermediates.

The commonly accepted catalytic mechanism operating in the P450 monooxygenases involves six steps (Shteinman, 2001; Sligar et al., 2005; Bernhardt, 2006). Catalytic turnover starts with the binding of substrate to the active site with displacement of water coordinated to the open axial ligand position thereby moving the iron (FeIII) to a high-spin ferric manifold, which increases the redox potential of the complex and facilitates the transfer of the first electron forming the ferrous heme ( $\mathrm{Fe}^{\mathrm{II}}$ ) that could bind oxygen (Sligar et al., 2005). In the next steps, further reduction and protonation of the resulted oxy-ferrous intermediate leads to the generation of the so called "active oxygen species", ferric peroxoanion, ferric hydroperoxo, high-valent iron-oxo complex (compound I-ferryl porphyrin cation radical), responsible for the hydroxylation of the substrates. The last species was also found in peroxidases and well characterized (Penner-Hahn et al., 1986). It was postulated (by the heme model systems according to the similar assumption for P450) that two different electrophilic oxidants can hydroxylate alkanes in the catalytic cycle of P450. One of them, hydroperoxide, is a predominantly epoxidizing agent, and another oxidant, ferryl, is a predominantly hydroxylating agent (Vaz et al., 1998; Newcomb \& Toy, 2000; Watanabe, 2001; Jin et al., 2003), although the density functional theory (DFT) calculations excluded the former intermediate as a reactive, electrophilic oxidant (Groves, 2003).

Despite the increasing knowledge of structure-function relationship and physico-chemistry of monooxygenase function, the question how the formation and ultimate chemical reactivity of the intermediate states of metal, oxygen and substrate is controlled to have an effect on efficient catalytic processing still remains open.

The second reason for the considerable interest in monooxygenases mimetic is connected with the design of the perfect chemical model system for a growing number of synthetic transformations (e.g. for oxidation of aliphatic and olefinic hydrocarbons). Such catalyst could be applied in the conversion of petroleum products to valuable commodity chemicals (Groves, 2000), preparation of hydroxylated metabolites of drugs, oxidation of agrochemicals, pollutants, and other xenobiotics (Meunier et al., 2004), as well in the catalytic production of unique and valuable compounds present in nature in an insufficient amount. Thus, one of the most extensively studied groups of compounds in both the enzymatic and biomimetic transformations are terpenes, hydrocarbons derived from the isoprene units, which are the largest class of plant secondary metabolites. Together with terpenoids (oxidized terpenes), terpenes are the main flavour and fragrance impact molecules in the essential oils of higher plants and flowers, however, their natural resources are limited to a few plant growing regions. In the perfumery, as well as in the flavour industry at present, a lot of effort is devoted to the replacement of expensive naturally occurring raw materials or the reproduction of original organoleptic effects by the use of new chemicals. Terpenes have also drawn increasing commercial attention because of their beneficial healthcare effects. Numerous terpenoids have been found to be effective in chemoprevention and chemotherapy of several diseases and to exhibit antimicrobial, antiviral, antihyperglycemic, anti-inflammatory and antiparasitic activities (Paduch et al., 2007). Therefore, a special attention should be paid to introduction of new terpenoid 
products in modern therapies. Monoterpenes are known to play an important role in chemical ecology, where they act as pollinator attractants, repellents, sex pheromones, alerting pheromones, antifungal defences, or as part of defense secretion systems against predators (Banthorpe, 1994).

Most of the microbial hydroxylation of terpenes and other exogenous substrates described so far are initiated by cytochrome P450 dependent monooxygenases (Duetz et al., 2003; Bicas at al., 2009). A number of microorganisms have been reported to convert limonene and pinene to notable monoterpenoid compounds (Chatterjee and Bhattacharyya, 2001; Yoo \& Day, 2002; Duetz et al., 2003; Adams et al., 2003; Trytek \& Fiedurek, 2005; Bicas at al., 2009), yet the amounts obtained (a few milligrams per litre) were insufficient for industrial applications. The drawbacks of this type of biotransformation systems are excess volatility of terpenes, insolubility in aqueous solutions, and their toxicity towards microorganisms (Krings \& Berger, 1998).

The development of synthetic metallic complexes that reproduce the activity of P450 enzymes and enable to carry out the reaction (in the case of water insoluble precursors) in organic solvents has been the subject of intense research (Meunier, 1992; Collman et al., 1993; Suslick, 1999; Bernadou \& Meunier, 2004; Simões et al., 2006). Metalloporphyrins in homogeneous systems, with the structures based on the well known mesotetraphenylporphyrin (called the first-generation catalyst), are amongst the catalysts commonly studied (Maraval et al., 2002; Guo et al., 2005; Milaeva et al., 2007). The largest bulk of examples has been published for the manganese (III) and iron (III) porphyrins (Traylor \& Tsuchiya, 1987; Gonsalves et al. 1991; Mansuy\& Fontecave, 1984; Martins et al., 2001; Maraval et al., 2002; Rebelo et al., 2005), but the complexes of porphyrins with $\mathrm{Cr}$ (Groves \& Krupper, 1979; Schmidt et al., 2005), and Ru(II) (Groves et al., 1997; Murahashi \& Komiya, 1998; Simonneaux \& Maux, 2002) have also been demonstrated as useful catalysts for the oxygen atom transfer reactions to olefin or alkane-type substrates. A wide variety of oxidative reactions (mainly epoxidation and hydroxylation of the olefins) catalyzed by metalloporphyrins has been studied (Montanari \& Casella, 1994; Kadish at al., 2000, Meunier, 2000; Simões et al., 2006), and numerous classes of substrates have been given in transformation including pre- and post-emergence herbicides (Chauhan \& Kumari, 2007), amino acids (Mukherjee \& Ray, 2007), monoterpenes such as: geraniol, nerol, limonene, apinene, a-terpinene, 1,8-cineole, carvacrol, thymol, and cymene (Martins et al., 2001; Maraval et al., 2002, Guo et al., 2005; Simões et al., 2006). In the specific case of terpenes, Skrobot et al. (2003) showed the production of epoxides from these compounds. In the similar work, the oxidation of monoterpenes by hydrogen peroxide catalyzed by porphyrins was also described (Martins et al., 2001).

Almost in all investigations, strong oxidants were used as oxygen atom transfer reagents to metalloporphyrins, such as $\mathrm{PhIO}$, alkylhydroperoxides, peroxyacids, hypochlorites, $\mathrm{C}_{6} \mathrm{~F}_{5} \mathrm{IO}$, potassium monopersulfate. Lately, the use of more environmentally benign, $\mathrm{H}_{2} \mathrm{O}_{2}$ has been preferred. This concept is favoured by the fact that cytochromes P450 (in the absence of $\mathrm{NAD}(\mathrm{P}) \mathrm{H})$ and peroxidases ${ }^{1}$ in vitro can also use hydrogen peroxide and other exogenous peroxides to catalyze the hydroxylation of substrates under mild conditions (Yuchun et al., 2001; Trytek \& Fiedurek, 2002). The monooxygenation by metalloporphyrins with these oxidants requires the presence of pyridine or 1-methylimidazol, 4-butylpyridine, imidazole,

1 Though peroxidase forms the ferryl intermediate, it cannot hydroxylate alkanes because of steric hindrance for the attack to the $\mathrm{C}-\mathrm{H}$ bond by $\mathrm{O}$ atom in this enzyme. 
or ammonium acetate, which were shown to act both as axial ligands and cocatalyst used to promote the desired heterolytic cleavage of peroxide and also to stabilize the active species $\mathrm{Mn}(\mathrm{V})=\mathrm{O}$ formed in the oxidation cycle (Meunier, 1992; Gonsalves et al., 1996; Maraval et al., 2002; Susana et al., 2003; Cantonetti et al., 2004). These agents substantially increased the rate of the regio- and stereoselectivity reaction, and their role resembles somewhat the action of the strongly nucleophilic thiolate axial ligand in cyt-P450, which is crucial for heterolytic cleavage of the $\mathrm{O}-\mathrm{O}$ bond of hydroperoxo intermediate by donating the electron density to this bond (Suzuki at al., 1999; Watanabe, 2000). Cytochromes P450 mimics using $\mathrm{O}_{2}$ itself as an oxygen atom donor has also been developed. In this case a reducing agent and/or proton source must be used (especially $\mathrm{Zn}$ powder, $\mathrm{CH}_{3} \mathrm{COOH}$, acetaldehyde or borohydrides) (Mansuy, 1990, Groves, 2000) and higher degree of organization of the catalyst is required due to competition between the substrate and reducing agent for the active oxidant (Shteinman, 2001).

Models with a tight coordination of the axial ligand are in general not good catalysts as thiolate or porphyrin is rapidly oxidized in the presence of oxidants (Meyer et al., 2005). However, for some porphyrins-based systems, the oxidation reactions usually provide very high conversions of the substrates (often exceeding $90 \%$ after a few hours), and even some systems are able to convert olefin to epoxides in a stereospecific manner, and alkanes to the corresponding alcohols and ketones in a good yield. Nevertheless, application of conventional homogeneous systems in the porphyrin catalysis is frequently complicated by the oxidative degradation instability of the metalloporphyrin rings, difficulty of recovering this rather expensive catalyst, and poor degree of regioselectivity. The systems also lead to generation of chemically contaminated effluents that have a great environmental impact.

The stability of porphyrin catalysts was substantially improved by the introduction of electron-withdrawing substituents into the phenyl group of the porphyrins and then by the complete halogenation of the porphyrin ring (second and third generation of porphyrin) (Fig 2). The decreased oxidative degradation of the porphyrin ligand and the increased reactivity of the intermediate were also achieved to some extent when the bulky or chiral groups were attached to the porphyrin periphery (ortho-position of the phenyl ring of $\mathrm{H}_{2}$ TPP or meso-position of the porphyrin), which provides some steric protection for the active oxygen species preventing from a too fast destruction of the porphyrin ring (Suslick, 1999). In practice, both electronic and steric properties of the substituents are manipulated to improve oxidative robustness of the porphyrins and must also be considered along with the regio- and stereochemical factors (Suslick, 1999; Maraval et al., 2002; Guo et al., 2005).

The two main strategies towards a new generation of more regioselective and efficient artificial hemoproteins have been envisioned. The first exploits the systems with simple sterically hindered porphyrins and shaped-selective catalysts that have a superstructure designed to recognize and bind incoming substrates. As an extension of the basic structure of the porphyrin macrocycle, there has been a multitude of approaches to synthesize porphyrins with a variety of peripheral substituents or even with more than four pyrrole rings, leading to the modification of the macrocyclic ring size, planarity, number of $\Pi$ electrons and aromaticity, and ultimately, the biocatalytic activity. Diverse structures have been created in this fashion (Fig. 3.) and more elegant systems including stereoselective substituents are described and shown in greater details in the literature (Kadish et al., 2000). 


\section{$\mathrm{Fe}(\mathrm{III})\left(\mathrm{TDCPCl}_{8} \mathrm{P}\right) \mathrm{L}: \quad \mathbf{X}=\mathrm{Cl}$ \\ Fe(III)(TDCPP)L: $\quad \mathbf{X}=\mathrm{H}$}

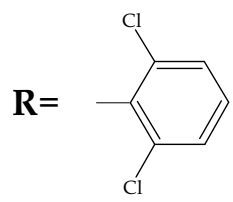

$\mathrm{Fe}(\mathrm{III})\left(\mathrm{TF}_{5} \mathrm{PPBr}_{8}\right) \mathrm{L}: \quad \mathbf{X}=\mathrm{Br}$<smiles></smiles>

Fe(III)(TMP)L: $\quad \boldsymbol{X}=\mathrm{H}$

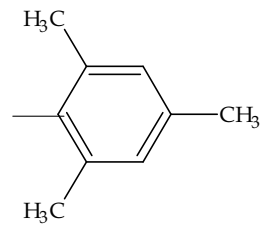

Fig. 2. The second and third generations of porphyrin. Halogenated porphyrins exhibit the increased stability.

The examples of extraordinary compounds which can be used as efficient catalysts in the future are: amino acid functionalized porphyrins (Robertson et al., 1994; Geier. \& Sasaki, 1997), porphyrins with the substituents at a pyrrolenic nitrogen atom, the so-called " $N$ substituted porphyrins" (Ito et al., 1996), the porphyrin complexes composed of more than one porphyrin ring, for example "sandwich" or "shish kebab" porphyrins, where several macrocyclic rings are joined by metal ions. The porphyrins are also suitable elements of more complicated systems, which can find application in biomimetic catalysis like that of the larger macrocyles prepared by covalent bonding of porphyrins with hydrophobic cavities (cyclodextrins, cyclophanes) (Hartmman et al., 1999; Feiters et al., 2000; Woggon, 2010), cobaltacarborane-porphyrin and carbohydrate-porphyrin conjugates, carboranylporphyrins, multiporphyrin arrays (e.g. cofacial bisporphyrins) (Chang et al., 2004; Hao, 2007). The porphyrin complexes with other compounds, such as dendrimer metalloporphyrins (Suslick, 1999) as fullerenes (Nierengarten et al., 1998), as well as "expanded" porphyrins, for example texaphyrins (Sessler et al., 1994; Lisowski et al., 1995), "porphyrin wheels" (Schenning et al., 1996), saphyrins, platyrins and others (Jasat \& Dolphin, 1997; Lim et al., 2009) seem to be also very promising.

Secondly, supramolecular mimetic approaches with modulation of heterogeneous environment to restrict substrate orientation or access have been used. Many interesting examples of activity of porphyrins and metalloporphyrins in such systems have been reported, for example: amphiphilized metalloporphyrins in the micellar media for 
epoxidation of limonene (Mancini et al., 2001; Cantonetti et al., 2004), complex imbedded in a self-assembled lipid or surfactant bilayer (Groves \& Neumann, 1989; Feiters et al., 2000), porphyrins appended with $\beta$-cyclodextrines (Breslow, 1997; Woggon, 2010), polyethylene glycol-modified hemin (Takahashi et al., 1986), surfactant-heme complex (Kamiya et al., 1997), association of polyoxometalates with porphyrins (Santos et al., 2005), association of monoclonal anti-porphyrin antibodies (so-cold hemoabzymes) with metalloporphyrin cofactor (Nimri \& Keinan, 1999; Ricoux et al., 2007). The most promising solution to improve the stability of porphyrin catalysts is to immobilize or encapsulate in a solid, usually inorganic, matrix.

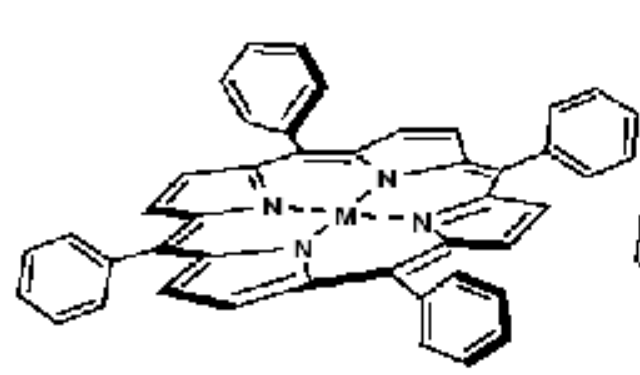

tetraaryl porphyrin

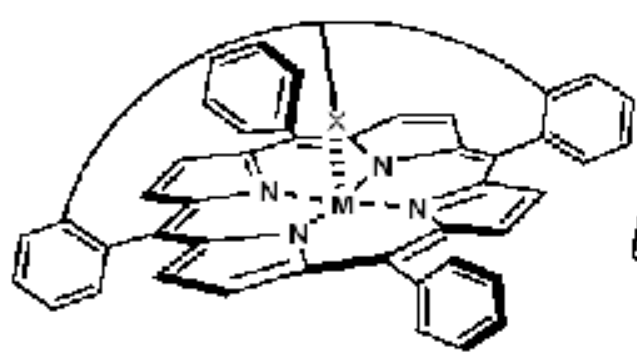

strapped porphyrin
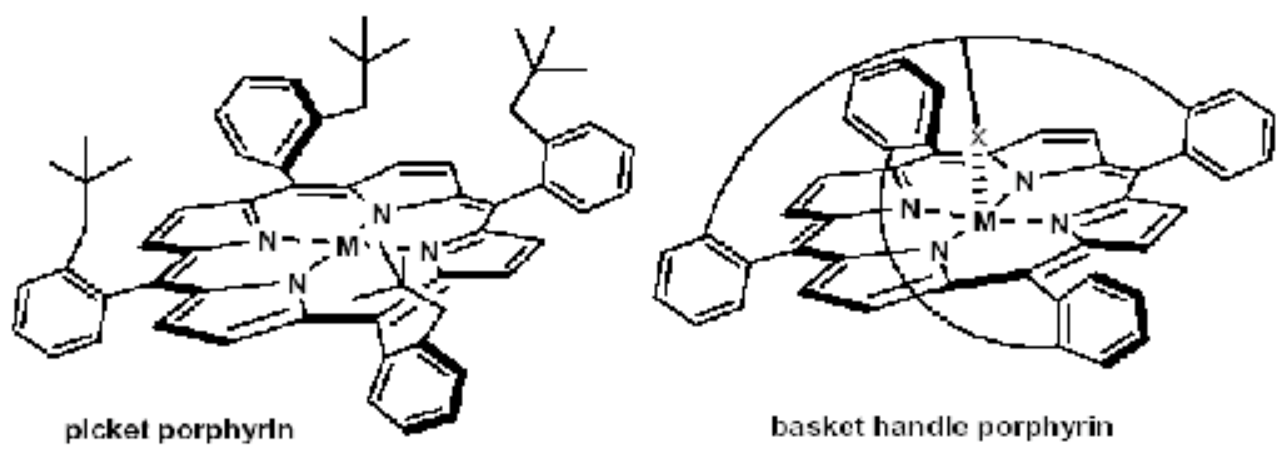

Fig. 3. Examples of the most frequently used porphyrin classes as monooxygenase mimics. 


\subsection{Porphyrins as photocatalysts for oxidation of organic compounds}

The subsequent synthetic method searching for biomimetic monooxygenase analogues is the use of photoactive porphyrins as the sensitizer catalysts for the aerobic oxidation. The conceptually attractive entry for the employment of porphyrins as photocatalysts and photons as the energy source in biomimetic catalysis consists in the fact that in plants and living organisms four crucial prerequisites are found, which favour the photoprocess on bioactive molecules. These criteria are: (I) presence of natural sunlight providing visible spectrum irradiation; (II) good light-absorbing properties and proliferation of photosensitizers (e.g., tannins, porphyrins, and chlorophyll) in the environment; (III) pervasive molecular dioxygen; and, finally, (IV) abundance of oxidizable substrates, such as terpenes, in the immediate vicinity (Margaros et al., 2006).

The role of porphyrins in photocatalysis and photosynthesis processes or in the function of solar cells is related to the intensive absorption of UV-visible light by these compounds. It is known that the colour of many simple complex compounds is the result of electronic transitions with the participation of metal d-electrons. The transitions between d-orbitals are forbidden in respect to symmetry and therefore their intensity is low. However, the porphyrins case is somewhat different, i.e. the absorption spectra of porphyrins are dominated by the strong bands corresponding with the $\Pi-\Pi^{*}$ transitions in the porphyrin ligand. The two kinds of bands are recognized in the absorption spectra of porphyrins: $B$ (Soret band) and Q. According to Gouterman (1961), the essential features of these spectra can be explained based on the model of four molecular orbitals.

The absorption bands in the porphyrin systems result from the electronic transitions between two HOMOs and two LUMOs energy levels. The metal center and the substituents on the ring affect the relative energies of these transitions. Mixing splits these two states of energy, forming a higher energy 1 eu state with larger oscillator strength (Soret band) and a lower energy 1 eu state with smaller oscillator strength (Q-bands). The shape and position of $\mathrm{B}$ and $\mathrm{Q}$ are closely related to the aggregation of porphyrins, their protonation with the formation of dications or axial ligation, as well as their metallation. The Soret band in the blue and the Q-band in the red are major porphyrin bands, which represent an important component of sunlight.

Peculiar spectral properties and ability of the porphyrins to undergo photoinduced multielectron transfer without changing their structure has opened new fields of their application not only for light-driven biocatalysis but also for application in solar energy conversion, photodegradation of a wide range of organic and inorganic chemicals in air and water, elimination of bacteria, viruses, cancer cells and splitting of water as well as construction of sensing materials to mimic the human nose (electronic nose) and photosynthetic systems (Paolesse et al., 2002).

There are a lot of contributions of porphyrin model studies to the photooxidation catalysis in homogeneous systems (Weber et al., 1994; Quici et al., 1993; Maldotti et al., 1996; Funyu et al., 2003). Simple photoexcited FeIII porphyrins were used to induce hydrocarbon oxygenation to the corresponding ketones under aerobic conditions. For example, in pure cyclohexane, high yields of cyclohexanone with small amounts of cyclohexanol as a by-product were obtained (Bartocci at al., 1996). The photo-oxidation of phenol and monochlorophenols by irradiation with visible light in aqueous alkaline solution in the presence of 5,10,15,20-tetrakis(4-carboxyphenyl)porphyrin and oxygen has been described by Wöchrle and co-workers (Gerdes, et al., 1997). For the visible light-photocatalytic oxidation of hydrocarbons, a bis-iron(III)-1-oxo pacman porphyrin was used (Rosenthal et al., 2006). 
Trytek et al. (2007) described a comparative study of photoexcited system involving metal free and metal-containing porphyrin catalysts in the photooxidation of monoterpene, $R-(+)-$ limonene using dioxygen from the air dissolved in organic solvents. The largest photocatalytic activity was registered by using metal free porphyrins such as 5,10,15,20tetraphenylporphyrin $\left(\mathrm{H}_{2} \mathrm{TPP}\right)$ and hematoporphyrin IX (HmP-IX). Later investigations of light-promoted biooxidation of a-pinene have revealed that octaethylporphine $\left(\mathrm{H}_{2} \mathrm{OEP}\right)$ and porphyrins chelating a metal ion with a closed-shell electron configuration, especially zinc 5,10,15,20-Tetra (4-pyridyl)-21H,23H-porphine are also very efficient photocatalysts. Pyridyl porphyrin (II) and aryl porphyrin (III) with relatively longer substituents had twice as low catalytic efficiency. Also Zn(II) and Co(II) tetraphenyl porphyrins had lower catalytical properties. A mixture of carvone and another unknown product (1) (with a mass spectrum similar to that of verbenone) in the concentrations of up to $3.4 \mathrm{~g} / \mathrm{L}$ and $6.0 \mathrm{~g} / \mathrm{L}$, respectively was obtained from the chloroform solution consisting of catalytic amount of $\mathrm{H}_{2} \mathrm{TPP}$ and $90 \%$ $(\mathrm{v} / \mathrm{v})$ limonene, within the period of 70-hour exposition to the sunlight. The authors have checked the effects of substrate concentration and kind of oxidant on the efficiency of the porphyrin-based homogeneous system, as well as determined some photoexcitation conditions and mechanism for monoterpene transformation. At lower substrate concentration of about $1-5 \%$, and high photoirradiation intensity $\left(>1600 \mu \mathrm{mol} / \mathrm{m}^{2} \mathrm{~s}\right.$, where $\mathrm{mol}$ is the number of photons), the yield of by-products, especially $(1 \mathrm{~S}, 4 \mathrm{R})-p$-mentha-2,8diene 1-hydroperoxide and 1,2-limonene oxide, increases significantly. Substantial amounts of trans- and cis-carveol, perilla aldehyde, perilla alcohol, and other hydroperoxides were also identified. The productivity of terpenoinds has been improved (about 1.6-fold), when $0.5 \%(\mathrm{v} / \mathrm{v})$ of $\mathrm{H}_{2} \mathrm{O}_{2}$ or tert- $\mathrm{BuOOH}$ was added to the solution. An increase in oxidant concentration caused oxidative degradation of the porphyrin ring under the strong oxidizing conditions.

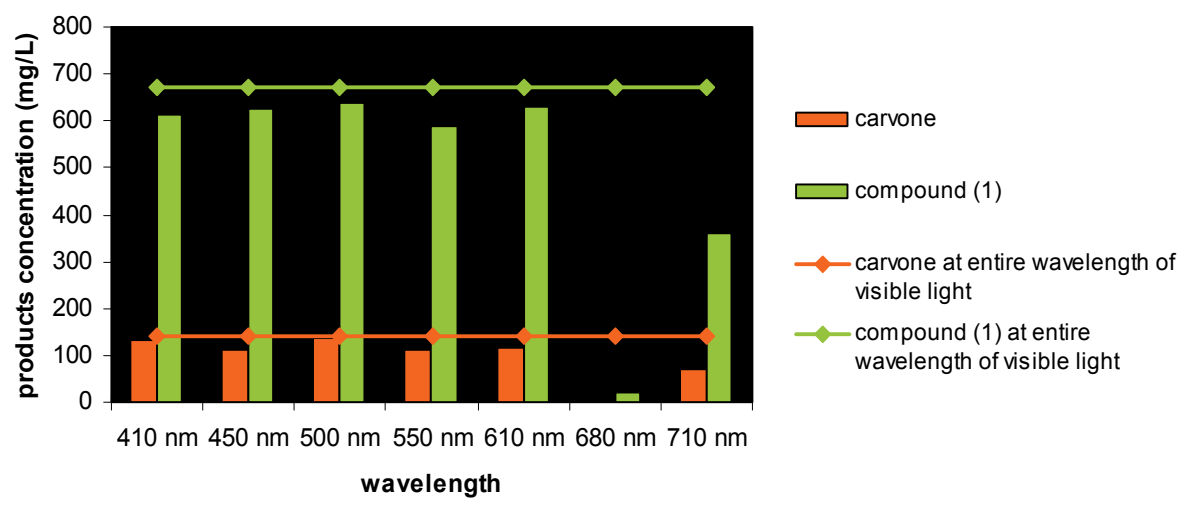

Fig. 4. Effect of wavelengths of visible light on limonene photooxidation with $\mathrm{H}_{2} \mathrm{TPP}$.

It has been shown that the photooxidation of limonene does not depend significantly on the particular wavelengths of visible light. The best yields of products were achieved within the entire wavelength range of the visible light which is profitable from an economic point of view, since the costs of the kind of light in photocatalytic reactions are taken into consideration. However, at $670 \mathrm{~nm}$ remarkable decrease in terpenoids accumulation was detected in the course of the photooxidation process (Fig 4). This phenomenon was explained by low absorption coefficient of $\mathrm{H}_{2} \mathrm{TPP}$ in this spectral region and the formation 
of the protonated porphyrin in the system, which is known to have the enhanced deactivation of the excited state (Chirvony et al., 2000). In fact, it was established based on the absorption spectra of porphyrins recorded before and after photocatalysis (Fig 5) that porphyrin in the system with a low concentration of limonene exists in a protonated form of
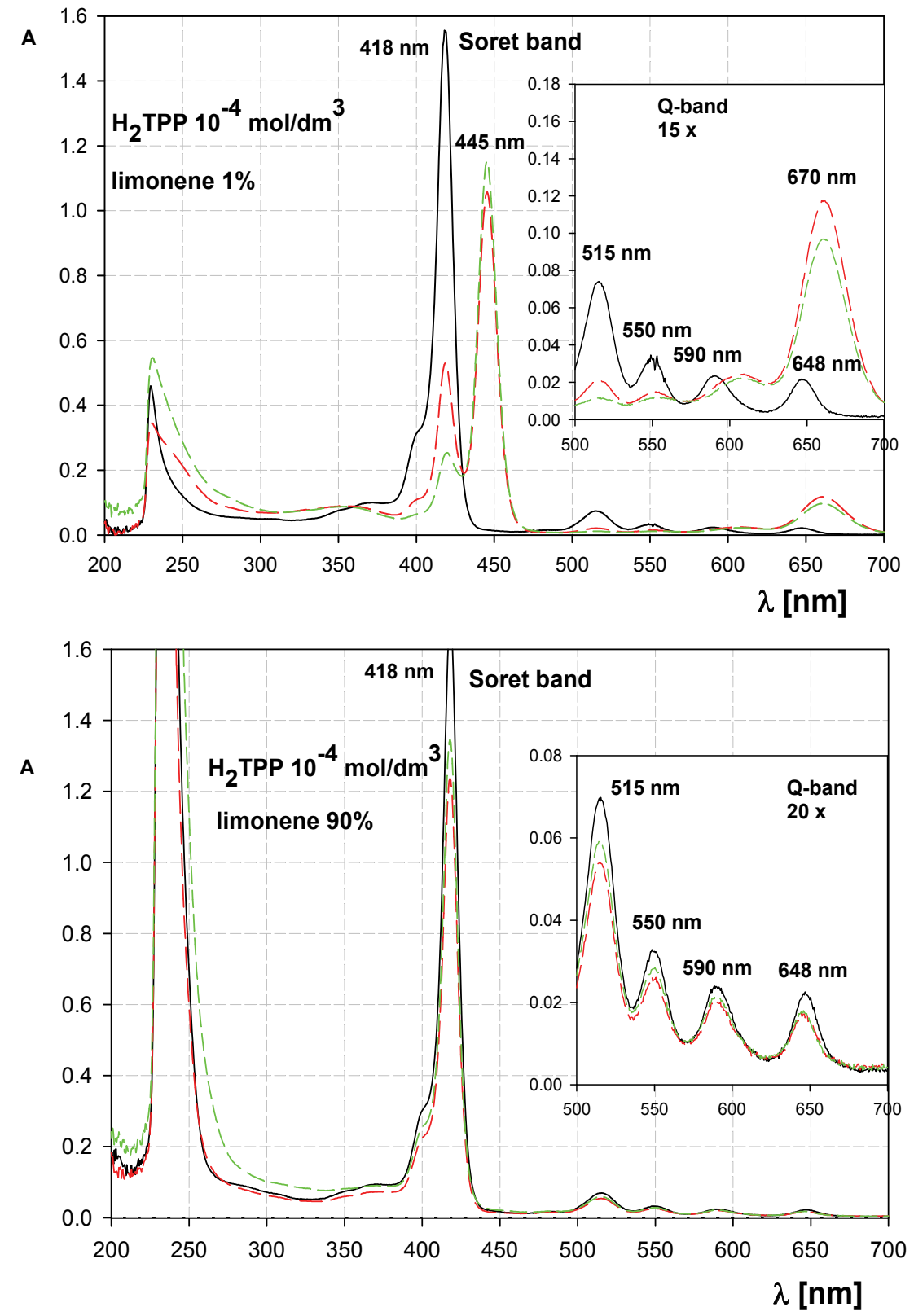

Fig. 5. Absorption spectra of the $\mathrm{H}_{2} \mathrm{TPP}$-limonene system (at two different substrate concentrations) in chloroform; before photoreaction (black solid line), after $36 \mathrm{~h}$ (red dash line) and $126 \mathrm{~h}$ (green dash line) exposition to the sunlight (Trytek at al., 2007). 
dication $\mathrm{H}_{4} \mathrm{TPP}^{2+}$. As a characteristic Q-band was only observed for free-base $\mathrm{H}_{2} \mathrm{TPP}$ (500$650 \mathrm{~nm})$ and dication $\mathrm{H}_{4} \mathrm{TPP}^{2+}(670 \mathrm{~nm})$, this means that the catalytic system is based just on photoexcited porphyrine and the metal contamination is excluded. The porphyrin spectra with a high $90 \%$ concentration of limonene during photoexcitation showed that, neither Soret nor $\mathrm{Q}$ bands were shifted, and only their intensity was slightly lower after irradiation than before it, which does not indicate that the catalyst is readily decomposed under the applied oxidizing conditions. In this context, it is worth taking into account that the presence of a large excess of readily oxidized substrates will provide kinetic protection against oxidative destruction of the biomimetic catalysts (Suslick, 1999).

The authors concluded that such spectral changes are the evidence for the existence of two different mechanisms of monoterpenes photooxidation by $\mathrm{H}_{2} \mathrm{TPP}$, to the extent extremely dependent on the substrate concentration. For the systems with a lower substrate concentration, a proton accepting mechanism is more important, whereas in the systems with a larger amount of monoterpene, $\mathrm{H}_{2} \mathrm{TPP}$ rather plays a role of a photosensitizer and oxidative transformation is most probably based on a free radical chain pathway initiated by photoexcited porphyrin producing singlet oxygen. Singlet oxygen is a powerful electrophile, so the reaction may proceed just as well by electron transfer from the electron rich monoterpenes to it. The common mechanism of action of the sensitizer is rather complex. The absorbed light promotes the excited state of the porphyrin catalyst. The singlet-excited state $\left(S_{1}\right)$ undergoes intersystem crossing to the triplet state $\left(T_{1}\right)$, which, in turn, reacts with oxygen (or first with the substrate) producing singlet oxygen $\left({ }^{1} \mathrm{O}_{2}\right)$, superoxide radical anions $\left(\mathrm{O}^{2 \cdot-}\right)$, and hydroxyl radicals (Fig. 6). In most of the cases, the concurrent involvement of these reactive oxygen species determines the mechanism of the photooxidation (Chacon et al., 1988; Krishna et al., 1991; Ahmad et al., 2004).

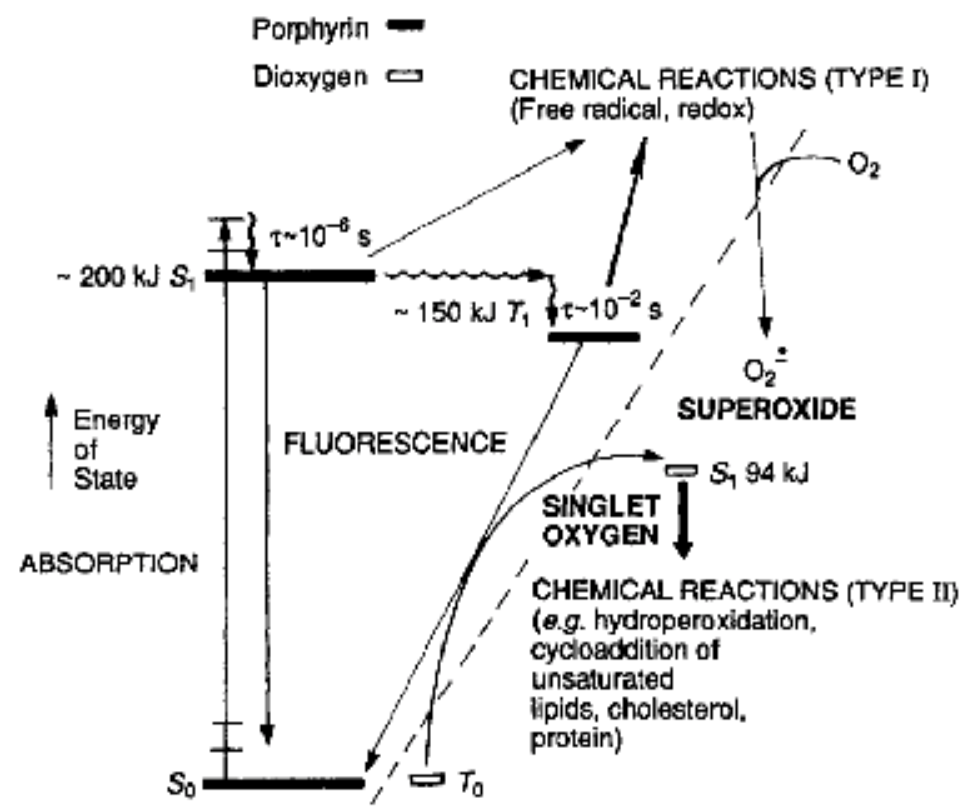

Fig. 6. Generation of excited porphyrin states and reactive diooxygen species (Bonnett, 1995) - Reproduced by permission of The Royal Society of Chemistry, copyright 1995. 
We applied the photoexcited porphyrin using diooxygen under atmospheric pressure to oxidize $\alpha-$ and $\beta$-pinene. The system enables the production of pinocarvone, transpinocarveol and myrtenol from a-pinene, whereas from $\beta$-pinene, myrtenol as the most abundant and pinocarvone being by-product, were obtained. Some porphyrins have been found to be characterized by unusually high reactivity toward pinene hydroxylation. For instance, in the a-pinene oxidation studies, up to $17 \mathrm{mg} / \mathrm{L}$ of pinocarvone was achieved after 24-hour photoirradiation of $50 \%(\mathrm{v} / \mathrm{v})$ of the substrate solution in the presence of $\mathrm{H}_{2} \mathrm{OEP}$ (Trytek et al., in preparation).

Homogeneous catalysts in general are inclined towards an easy deactivation and are difficult to be recovered from reaction mixtures, leading to many restrictions in large scaleprocesses. Furthermore, the porphyrins typically used as photosensitizers are poorly soluble and together with their decomposition derivatives, can contaminate the reaction products and make the purification step really troublesome. For example, the sensitizer hematoporphyrin is only moderately soluble in most organic solvents (e.g., ether, chloroform), and nearly insoluble in water. In this case, one possible solution might be the use of heterogeneous catalysts through the immobilization of the porphyrins on organic and inorganic solid supports.

\section{Heterogeneous catalysis using biomimetic porphyrins}

Much work has been devoted to investigation of solid supports for the immobilization of porphyrins with respect to easier recovery, recycling and increased stability of this expensive catalyst. Numerous proposals have appeared: the use of cross-linked polymers (Griesbeck \& El-Idreesy, 2005; Ribeiro et al., 2008), clays and layered materials (Bedioui, 1995; Evans \& Lindsay Smith, 2001), biopolymers (Huanga et al., 2005), cationic ionexchange resins (Campestrini, S. \& Meunier, 1992); silanized kaolinite (Nakagaki et al., 2004); different types of zeolites (Skrobot et al., 2003; Haber et al., 2004), modified silica (Milaeva et al., 2007), and also microporous porphyrin framework solids (Suslick et al., 2005). Noteworthy results for the immobilized porphyrins have been obtained with ionic porphyrins immobilized on cationically functionalized polystyrene (Inbaraj et al., 2003), porphyrins copolymerized with polystyrene (Griesbeck et al., 2004), a soluble polyethylene glycol-supported tetrahydroxyphenyl porphyrin (Benaglia et al., 2002), photosensitizers ionically bound at polymeric ion-exchanging resins (Gerdes et al., 2001), and a cross-linked polyacrylamide hydrogel serve as a scaffold for the photosensitizer hematoporphyrin (Rogers et al., 2005) and as well with chitosan-supported metallotetraphenylporphyrin complexes (Huanga et al., 2005).

Manganese(III) 5-(pentafluorophenyl)-10,15,20-tri(2,6 dichlorophenyl)porphyrin, and manganese(II) 2,3,7,8,12,13,17,18-octachloro-5-(pentafluorophenyl)-10,15,20-tri(2,6dichlorophenyl)porphyrin, have been covalently attached to aminopropylated silica and used in hydrocarbon oxidation by iodosylbenzene and hydrogen peroxide (Doro et al., 2000).

A few supported porphyrins have been tested in the oxidation of several monoterpenes. A tetracationic porphyrin, meso-tetrakis(4-Nbenzylpyridyl) porphyrinato manganese(III) being supported in a zeolite, $\mathrm{NaY}$, have proved to be an active catalyst for the epoxidation of $(R)$ $(+)$-limonene and a-pinene, as well as in the hydroxylation of carvacrol and thymol, using $\mathrm{H}_{2} \mathrm{O}_{2}$ and ammonium acetate in $\mathrm{CH}_{3} \mathrm{CN}$ at room temperature and under atmospheric pressure. This catalyst has enabled the $40-50 \%$ conversion of a-pinene after 6 hours of reaction, not exceeding $25 \%$ of conversion for the other substrates, even after 24 hours of 
reaction. The 24-hour oxidation of limonene ( $>21 \%$ of conversion) gave rise to the mixture of 1,2- and 8,9-epoxide products. The oxidation of a-pinene afforded mainly the formation of corresponding epoxide and campholenic aldehyde, whereas carvacrol ( $>25 \%$ of conversion) and thymol ( $>18 \%$ of conversion) produced thymoquinone with $100 \%$ selectivity. However, in the presence of $\mathrm{H}_{2} \mathrm{O}_{2}$, the irreversible deactivation of catalyst took place, by leaching of the porphyrin complex from zeolite, followed by a loss of activity when the catalyst was reused (Skrobot et al., 2003).

Tangestaninejad et al. have described the tetra(4-pyridyl)porphyrinato manganese(III) anchored in chloromethylated styrene-divinylbenzene copolymer as an efficient and reusable catalyst for the $(R)-(+)$-limonene epoxidation by sodium periodate, among several other alkenes (Moghadam et al., 2004).

The Chinese team has utilized manganese(III) 5,10,15-tris(tolyl)-20-(4-hydroxyphenyl) porphyrin covalently attached to the Merrifield's peptide resin for the highly diastereoselective epoxidation of cholest-5-ene derivatives ( $\mathrm{Du}$, et al., 2004). The similar sort of material has been used by Gonsalves et al. (2008) in photooxidation of monoterpenes. They have covalently linked the chlorosulfonation activated porphyrins to the Merrifieldmodified polymers. These immobilised porphyrins were used then as effective photosensitizers in monoterpenes oxidation using chloroform as the solvent. Photooxygenation of a-terpinene originated ascaridole as the main product, citronellol gave isomeric hydroperoxides. The latter oxidative transformation represents an important industrial application of a singlet-oxygen ene reaction as the first step of the route to the fragrant chemical specialty rose oxide (Pickenhagen \& Schatkowski, 1998). From a-pinene and $\beta$-pinene, followed by reduction of the corresponding hydroperoxide products with triphenylphosphine, trans-pinocarveol and myrtenol have been obtained. These products are expected as a result of the singlet oxygen ene addition to terpenoic olefins, although in the case of pinenes, non-ene products were also formed probably through a pathway involving a mechanism of electron transfer between the excited photosensitizers and the substrates (Ribeiro et al., 2008).

Presently, the sol-gel processes offer new and promising possibilities of physical trapping or covalent bonding of porphyrin catalysts inside the silica network. The process is based on the evolution of inorganic networks through the formation of a sol and gelation with formation of a network in a continuous liquid phase. The precursors of the synthesis of these sols consist of a metal or a metalloid element bonded with various reactive ligands. The most popular are metal alkoxides since they react readily with water. The most known alkoxides are the alkoxysilanes, such as teramethoxysilane (TMOS) and tetraethoxysilane (TEOS). Aluminates, titanates or borates are also used in the sol-gel process.

Besides simplicity of preparation, low-temperature encapsulation, silica sol-gels are also characterized by optical transparency, chemical and photochemical inertness, as well as photochemical, thermal, and mechanical stability. Moreover, those materials have a negligible swelling in organic solvents and aqueous solution compared to most organic polymers. The matrices appeared to have additional two roles: in protection of porphyrin from oxidative degradations and in induction, by tunable porosity, of the selective positioning of the substrate with respect to the porphyrin cofactor (Kandimalla et al., 2006). The sol-gel process allowing the preparation of optically transparent porous silica gel seems to be excellent technology for development of the photoactive porphyrin catalyst.

The classical work by Avnir et al. (1984) concerning the spectra of rhodamine 6G entrapped in silica prepared by the sol-gel method, started successful research on direct 
bioencapsulation in silica, organosiloxanes and hybrid sol-gel polymers, also active biological substances with heat-sensitive and fragile molecules, such as enzymes, proteins, DNA, RNA, catalytic antibodies and even the whole cells of microbes (Kandimalla et al., 2006). Such materials have many potential applications due to their catalytic, biosensing, optical, photo-electronic, photonic, and nanobiophotonic properties (Lan et al., 1999; Gill \& Ballesteros, 2000; Ariga et al., 2007; Escribano et al., 2008).

Porphyrins have been encapsulated in sol-gel materials (either in monolithic blocks or as thin layers on various supports) because of their ability of photoconduction and photoemission, in the context of their application as fluorescent materials (Papkovsky et al., 2000; García-Sánchez et al., 2006; De la Luz et al., 2007) optical sensors (Delmarre \& BiedCharreton, 2000; Im et al., 2005), dye sensitive solar cells (Grätzel, 2001; Ray et al., 2001), and non-linear absorption (Sun et al., 1997), mesoporous materials (Ariga et al., 2007), as well as sensitizers in photodynamic therapy (PDT) (Reisfeld, 2001; Podbielska et al., 2006).

\section{Spectral and biocatalytical properties of porphyrins immobilized in the silica gels}

The physicochemical characterization of the active element is considered of primary importance at the initial stages of the design of a new optical and photocatalytic device. The most commonly employed techniques in the investigation of porphyrin systems are: nuclear magnetic resonance (NMR), absorption, excitation and emission spectroscopy (Oulmi et al., 1989) or circular magnetic dichroism (Purrello et al., 2000). The influence of different factors on the absorption, emission and luminescent spectral properties of the porphyrins can be shown in the best way when the cases of porphyrins intercalated in different matrices are considered. The most spectacular examples of spectroscopic properties of porphyrin intercalates are reported below.

Dargiewicz et al. (2002) studied the UV-Vis absorption spectra of water soluble porphyrins: tetrakis[4-(trimethylammonio)phenyl] $\quad\left(\mathrm{H}_{2} \mathrm{TTMePP}\right)$ and tetrakis(1-methyl-4-pirydyl)( $\mathrm{H}_{2}$ TmePyP) in the monolith silica aerogel and solid thin films obtained by the sol-gel method (Figs. 7-8).The authors found the bands intensity decrease, their broadening and shift in both porphyrins toward longer wavelengths when the sol solution was compared with the aerogel and monolithic sol-gel material. The changes in $\mathrm{Q}$ bands were more complex, i.e. the bands $Q_{y(1,0)}$ and $Q_{y(0,1)}$ were blue shifted while the bands $Q_{x(1,0)}$ and $Q_{x(0,1)}$ were red shifted for aerogel, which was interpreted as a result of silica matrix polarity decrease in comparison with sol. In turn, the decrease of components in $Q$ bands from four to two when the spectra of porphyrins in sol were compared with these for the monolithic sol-gel material was explained as a consequence of free base porphyrins protonation in acidic soda-lime glass with formation of dications $\mathrm{H}_{4} \mathrm{P}^{2+}$. The dimerization constants $\mathrm{K}_{\mathrm{d}}$ of both porphyrins in the monolithic sol-gel glass, calculated based on the Soret bands intensity changes, were found to be in the range: $1-6.8 \times 10^{5} \mathrm{M} \cdot{ }^{-1}$. Their decrease with time of gel drying was the result of steric obstacle in dimers formation when the $\mathrm{SiO}_{2}$ network was formed and monomer porphyrins were preferable.

Pereira et al. (2005) studied the spectra of mesoporphyrin IX in the $\mathrm{TiO}_{2}$ and $\mathrm{SiO}_{2}$ matrices (Fig. 9). The authors found that in the initially formed titania matrices the porphyrin exists in a similar environment and a significant proportion relates to its neutral monomer, especially within the matrices with DMF. Without DMF more of the ionised porphyrine is present, both cation and dication. The quantity of the neutral form decreases with time and 
without DMF the spectrum of porphyrine within the titania monolith broadens and shifts to red accompanied by a decrease of fluorescence time. In the presence of DMF this effect is not observed, although the amount of monomer decreases and the presence of aggregates, photoproducts as well as ionised porphyrine is possible. The final forms of the silica matrix also show the effect of DMF addition. In the absence of DMF the main form of porphyrine is the cation.

Sanchez et al. (2004; 2006; 2009) reported about the free bases of the substituted tetraphenylporphyrins $\mathrm{H}_{2} \mathrm{~T}\left(\mathrm{o}-\mathrm{NH}_{2}\right) \mathrm{PP}, \mathrm{H}_{2} \mathrm{~T}(\mathrm{p}-\mathrm{COOH}) \mathrm{PP}$ and $\mathrm{H}_{2} \mathrm{~T}(\mathrm{p}-\mathrm{OH}) \mathrm{PP}$, which were bound to the silica matrix by the use of the functionalized alkoxides 3isocyanatopropyltriethoxysilane or 3-amminopropyltriethoxysilane, by the sol-gel method. It was shown, based on the UV-Vis spectra of all xerogels, that porphyrins are in stable forms inside the xerogel pores. The red fluorescence observed in the solutions of the substituted porphyrins was preserved even after these species were covalently bound to the
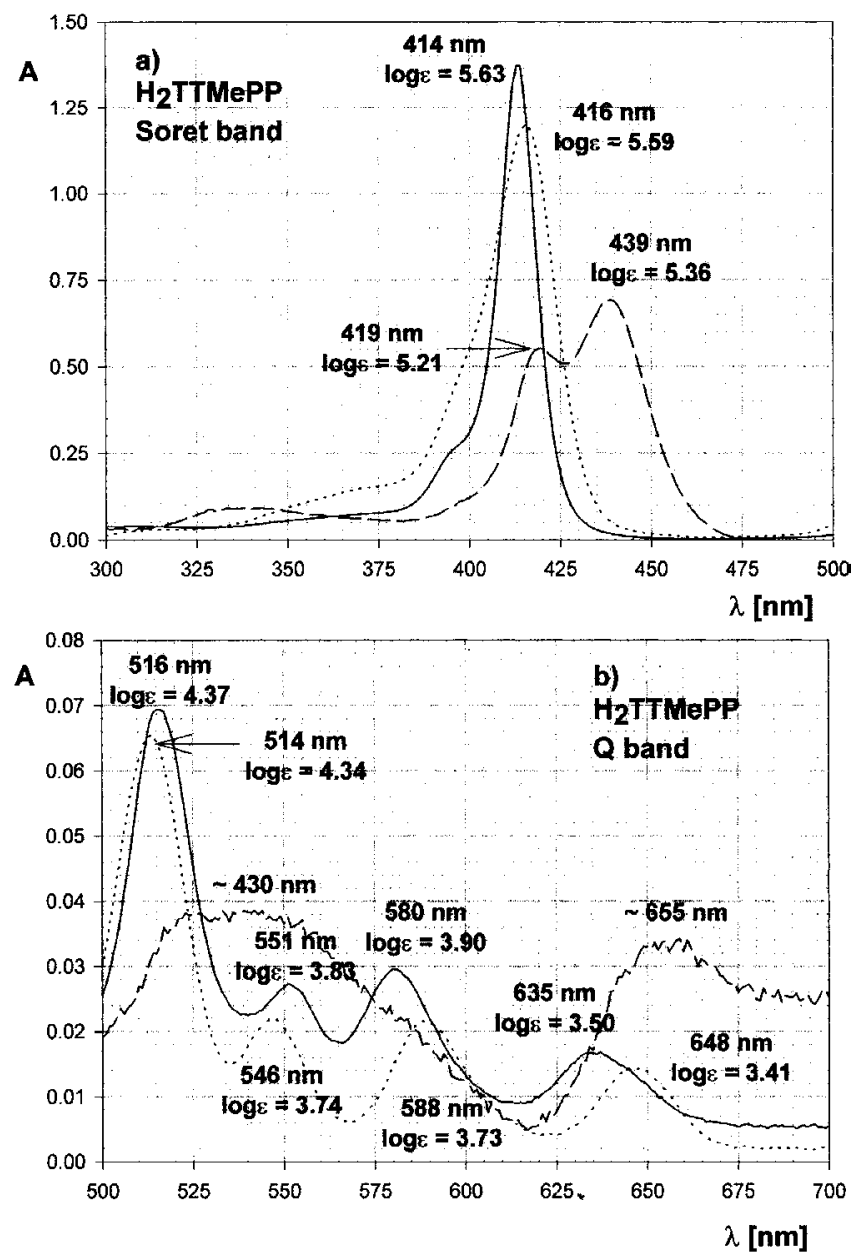

Fig. 7. Absorption spectra of the $\mathrm{H}_{2}$ TTMePP in: aqueous solution (solid line), monolithic solgel material (dotted line) and thin film (dash line); $\varepsilon=$ molecular extinction coefficient; reproduction with permission from Colloids and Surfaces A, copyright 2002. 

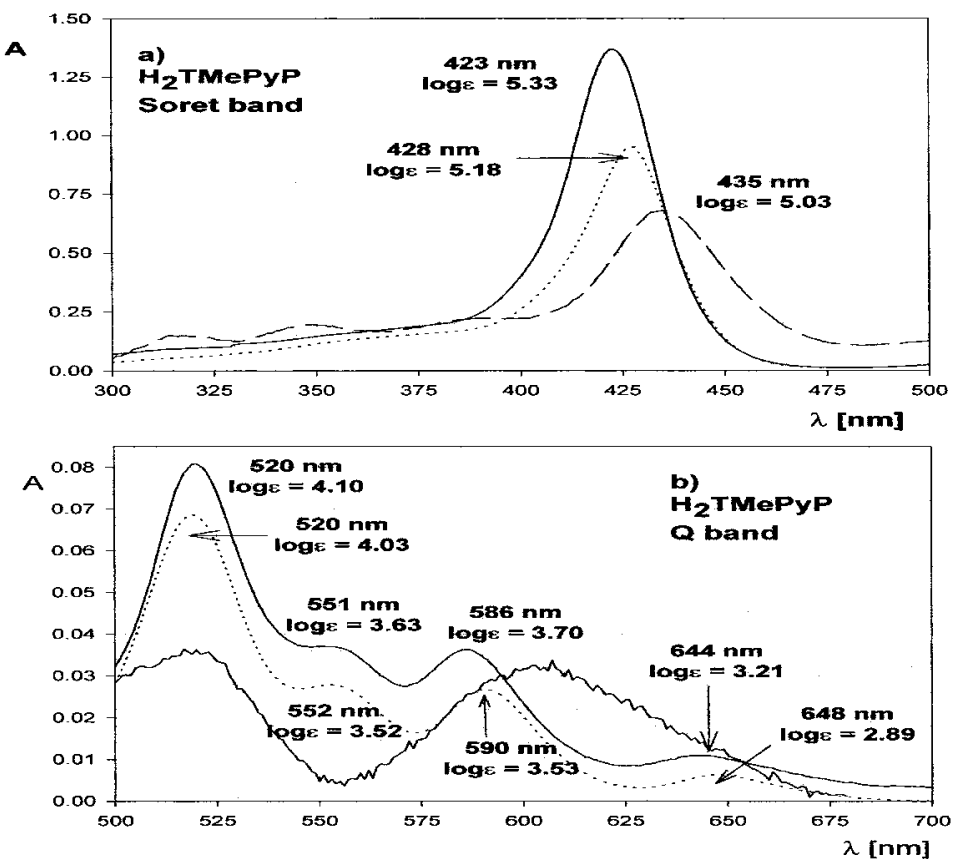

Fig. 8. Absorption spectra of the $\mathrm{H}_{2} \mathrm{TMePyP}$ in: aqueous solution (solid line), monolithic solgel material (dotted line) and thin film (dash line); $\varepsilon=$ molecular extinction coefficient; reproduction with permission from Colloids and Surfaces A, copyright 2002.

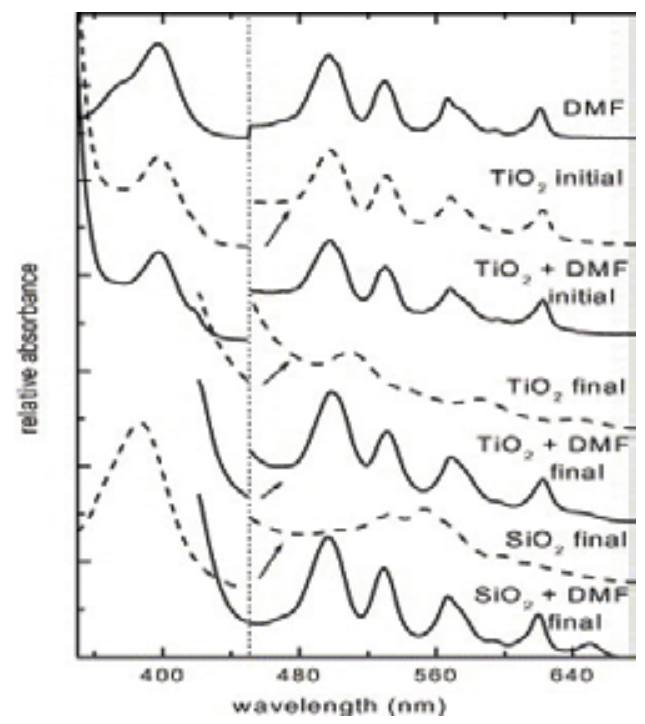

Fig. 9. Absorption spectra for MP in sol-gel-derived matrices, both with and without addition DMF. The absorption spectrum in DMF is given for comparison and the $Q$ band region has been amplified for clarity; reproduction with permission from Journal of Photochemistry and Photobiology A: Chemistry, copyright 2005. 
silica matrix. The best results, in terms of fluorescence preservation, were obtained by using a TPP substituted with $-\mathrm{NH}_{2}$ groups in the ortho position of the phenyl rings, i.e $\mathrm{H}_{2} \mathrm{~T}(\mathrm{o}-$ $\mathrm{NH}_{2}$ )PP. The UV-Vis spectrum of these species showed no signs of protonation. The authors found that cobalt tetraphenylporphyrins substituted with hydroxyl groups: (a) in ortho and para positions, and (b) with amino groups in para positions can be trapped in $\mathrm{SiO}_{2}$ by the solgel method. The addition of DMF, methanol or pyridine is necessary for successful immobilization of macrocycle in the silica network. The addition of pyridine to the gelling mixture results in the inhibition of demetallation and protonation of the macrocycles.

Dargiewicz et al. (2004) reported the strong luminescence of Eu complex with methylpyridylporphyrin $\left(\mathrm{H}_{2} \mathrm{TmePyP}\right)$ intercalated in the silica gel. The authors observed strong bands in the emision spectrum of Eu complex located at 604, 560 and $554 \mathrm{~nm}$ when the excitations: 423, 443 and $530 \mathrm{~nm}$ were applied. Pure $\mathrm{EuCl}_{3}$ intercalated in gel did not show the luminescence, whereas the free base porphyrin spectra had the pairs of peaks placed at 653,$716 ; 658,719$ and $655,715 \mathrm{~nm}$ respectively (at the excitation wavelengths: 423, 443, 530 $\mathrm{nm})$, red shifted when compared with the Eu complex. The authors suggested that the red luminescence of Eu complex results partially from the $\pi-\pi$ electron interaction of porphyrin rings and from the interaction of methyl pyridyl porphyrin peripherals with $\mathrm{Si}-\mathrm{O}-\mathrm{Si}$ or $\mathrm{Si}-\mathrm{O}$ groups located at the inner surface of gel pores.

Delmaire et al. (1999) studied the UV-Vis spectra of two porphyrins: 5,10,15,20-tetra(4-Nmethylpyridinium)porphyrin $\left(\mathrm{H}_{2} \mathrm{TMPyP}^{4+}\right)$ and 5,10,15,20-tetra(4-sulfonato)porphyrin $\left(\mathrm{H}_{2}\right.$ TPPS4-) in the silica sol-gel matrix. The Soret band underwent a remarkable shift upon the $\mathrm{pH}$ change of buffer solutions, which were equilibrated with gel samples. In the case of $\mathrm{H}_{2} \mathrm{TMPyP}^{4+}$, a shift of $12 \mathrm{~nm}$ was observed from $422 \mathrm{~nm}$ in the solution to $434 \mathrm{~nm}$ in the solgel materials. This was probably the result of the presence of positive charges on the porphyrin ring favouring strong interactions with the deprotonated silanols of the surface. In turn when a solution of $\mathrm{H}_{2}$ TPPS4- at $\mathrm{pH} 6$ was incorporated in the sol-gel material a green colour was observed probably due to the protonation of the central nitrogen. It was possible to determine the dissociation constant $\mathrm{pK}$ of protonated $\mathrm{H}_{4}$ TPPS2- species inside a sol-gel matrix and from this determination to find the local $\mathrm{pH}$ in the sol-gel. It was concluded that $\mathrm{pH}$ sensor is a possible application.

Polska and Radzki (2008) found very strong fluorescence enhancement of tetrakis[4(trimethylammonio)phenyl]porphyrin $\left(\mathrm{H}_{2} \mathrm{TTMePP}\right)$ by the addition of concavaline A both in the solution and in the silica sol-gel matrix. Based on the absorption spectra in the solution authors concluded about the 1:1 associate formation between the porphyrine and concavaline A with the binding constant $\mathrm{K}=4.35 \times 10^{5} \mathrm{~mol}^{-1}$. During inspection of the emission spectra of transparent monolithic silica gels doped with concavaline $A$ and porphyrin associate (Fig. 10) authors found that emission of concavalin is excited at the absorption near $260 \mathrm{~nm}$, while excitation at $430 \mathrm{~nm}$ is not observed. The excitation at the Soret band results in the remarkable enhancement of porphyrin fluorescence in the associate, and this phenomenon probably results from energy transfer from protein to porphyrin. Fluorescence of the associate was 10-fold more intense than luminescence of the gel doped by porphyrin alone. The intensification of fluorescence was observed only for the low concentration of the associate $\left(1.25 \times 10^{-5} \mathrm{~mol} / \mathrm{dm}^{3}\right)$. For the higher concentrations of porphyrin and porphyrin-protein associate the luminescence quenching was observed. The authors suggested practical application of the studied system in luminescent materials. 

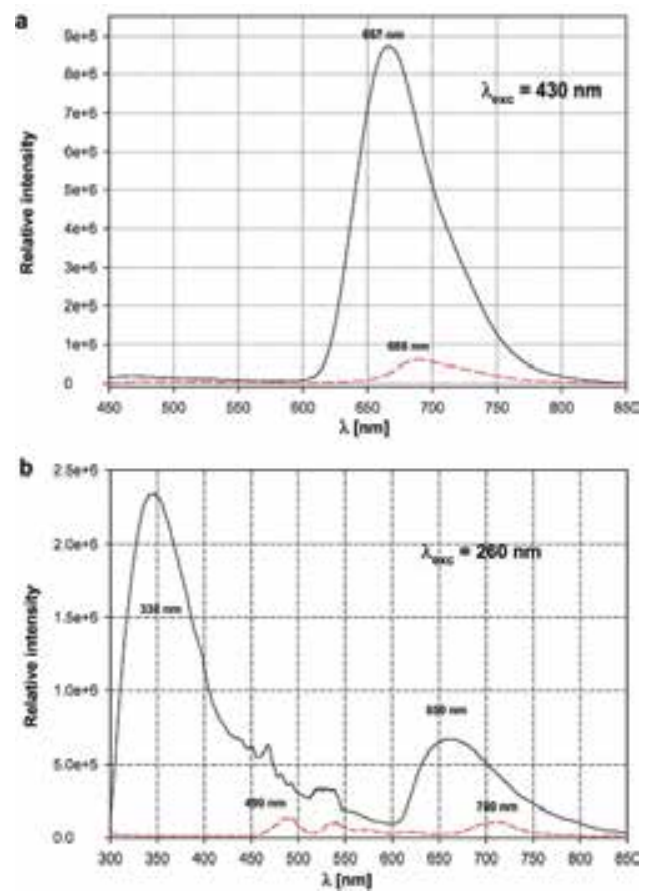

Fig. 10. Emission spectra of the monolithic silica gels: doped with $1.25 \times 10^{-5} \mathrm{~mol} \mathrm{dm}^{-1} \mathrm{H}_{2}$ TTMePP (dashed line) and 1.25 $\times 10^{-5} \mathrm{~mol} \mathrm{dm}^{-1} \mathrm{H}_{2}$ TTMePP/Con A 1:1 associate (solid line) excited at $430 \mathrm{~nm}$ (a) and doped with $5 \times 10^{-5} \mathrm{~mol} \mathrm{dm}^{-1}$ Con A (solid line) and $5 \times 10^{-5} \mathrm{~mol}$ $\mathrm{dm}^{-1} \mathrm{H}_{2}$ TTMePP/Con A 1:1 associate (dashed line) excited at $260 \mathrm{~nm}$ (b); reproduction with permission from Optical Materials, copyright 2008.<smiles>CC1=C(CCC(=O)O)C(/C=c2\[nH]/c(=C\C3=N/C(=C\c4[nH]c(/C=C5\N=C(CCC(=O)O)C(C)=C5C)c(C)c4C(C)O)C(C(C)O)=C3C)c(C)c2C)=N1</smiles>

HmP-IX<smiles></smiles>

$\mathrm{H}_{2} \mathrm{OEP}$

Fig. 11. Chemical structures of porphyrins encapsulated in silica gel showing biomimetic activity in photooxygenation of monoterpenes. 
Trytek et al. (2009) studied the fluorescent and biocatalytical properties of water soluble and insoluble prophyrins intercalated in the silica sol-gel matrix. The inspection of UV-Vis absorption spectra of tetraphenylporphyrin $\mathrm{H}_{2} \mathrm{TPP}$ showed that the intensity of the Soret band is lower in sol than in a solution. After gelation and 33 days of drying further lowering and broadening of the Soret band was observed, which was explained on the one hand by the changing polarity of the gel environment (the transition from hydro to alcogel) and by the dimerization and agglomeration of the porphyrin. The spectrum of a dried sample differed from sol as well as from the samples before drying. The Soret band was shifted from 414 to $437 \mathrm{~nm}$, whereas in the Q band region the new, very intensive band at $655 \mathrm{~nm}$ was observed and its existence was probably the result of porphyrin protonation with consequent dication formation. In the emission spectrum of $\mathrm{H}_{2} \mathrm{TPP}$ the fluorescence of sol and the dried gel was evidently less intensive than luminescence in the DMSO solution as a result of porphyrin agglomeration. The shift of the luminescence maximum from 650 to $667 \mathrm{~nm}$, when sol was compared with the dried sample, confirmed the dication existence in the dried gel.

$\mathrm{HmP} \mathrm{IX/SiO}{ }_{2}$, one week after gelation
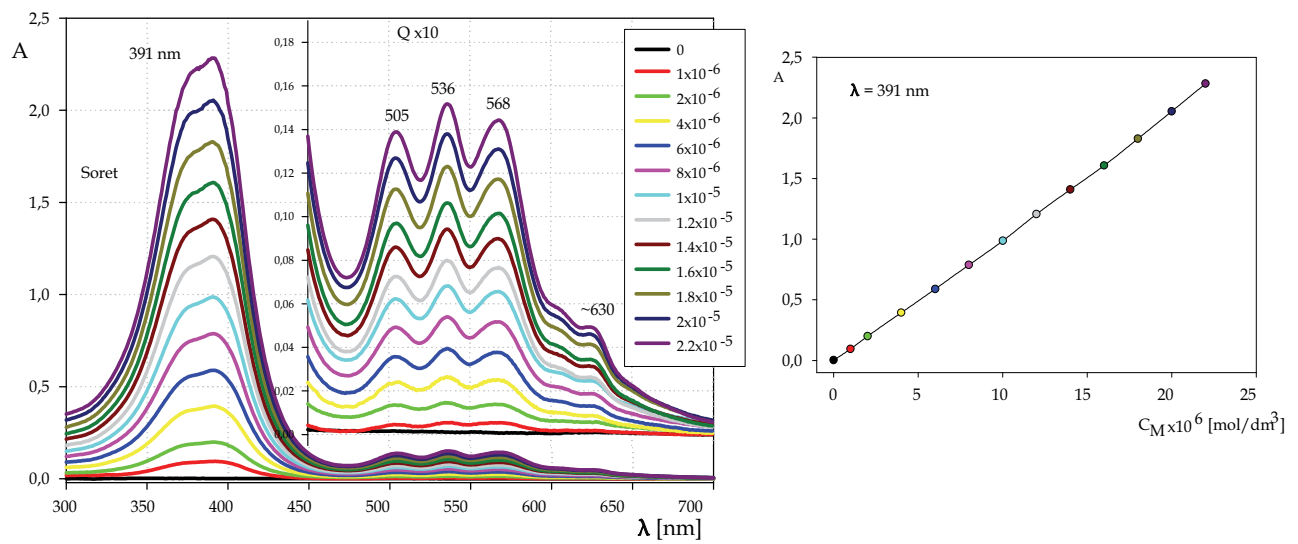

$\mathrm{H}_{2} \mathrm{OEP} / \mathrm{SiO}_{2}$, one week after gelation

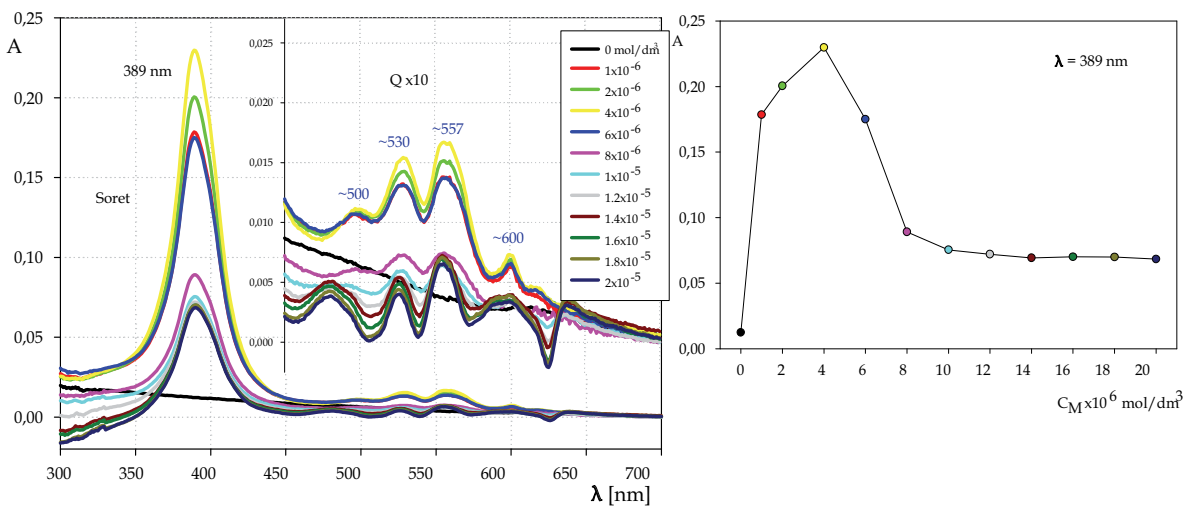

Fig. 12. UV-Vis absorption spectra of $\mathrm{HmP} I X$ and OEP intercalated in $\mathrm{SiO}_{2}$ alcogel (the numbers denote the initial concentrations of porphyrins in silica sol). 
The authors compared the behaviour of Hemato-IX porphyrin (HmP-IX) and octaethylporphyrin ( $\left.\mathrm{H}_{2} \mathrm{OEP}\right)$ (Fig. 11) in silica alco-gel (Fig. 12) (Trytek et al., manuscript in preparation). There was an evident straightline increase of absorbance at $391 \mathrm{~nm}$ with the concentration of Hemato-IX porphyrine, which probably was the result of its solvation by ethanol molecules and therefore its lack of aggregation in the alcogel matrix. It has been reported that the porphyrin molecules tend to aggregate by hydrophobic and $\pi-\pi$ interactions, leading to loss of linearity in the Beer's plot (Takagi et al., 2002).

The essential difference in the spectral behaviour of hematoporphyrine in comparison with $\mathrm{H}_{2} \mathrm{OEP}$ resulted probably from the presence of polar groups in its structure, i.e. peripheral hydroxyl and carboxyl groups, attracting strongly solvent molecules in alcogel. In turn, for octaethyl porphyrin the drastic decrease of the Soret band intensity with the porphyrin concentration was the basis for the evaluation of the molar fractions of monomers, dimers and trimers of porphyrin (Fig. 13). It was found that trimers are a predominant form of free base porphyrin in sol, whereas dimers molar fraction in alcogel was much higher than monomers and trimers, and this fact is probably a consequence of steric hindrance in trimers formation in the silica pores.

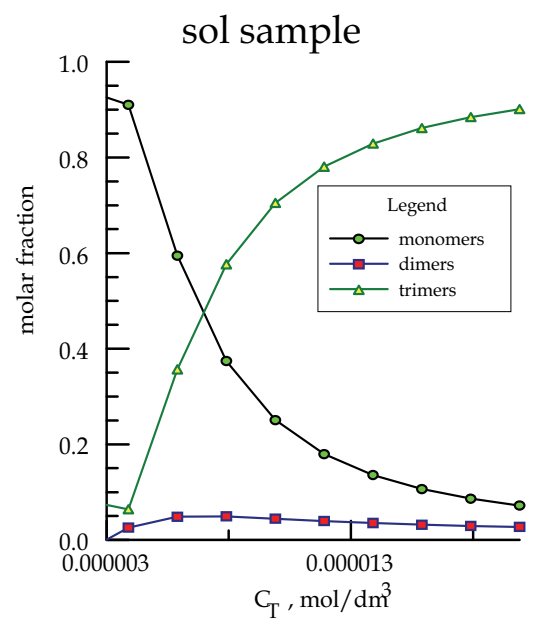

alcogel, 12 weeks after gelation

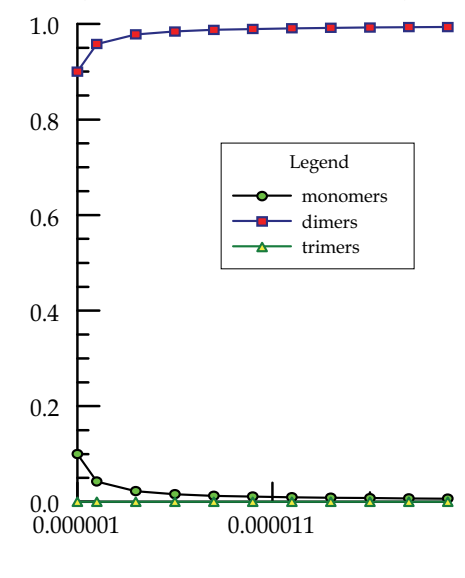

Fig. 13. The molar fractions of monomers, dimers and trimers of octaethylporphyrin in sol and alcogel.

In xerogel both porphyrines: $\mathrm{H}_{2} \mathrm{OEP}$ and $\mathrm{HmP}$-IX behave similarly relating to the character of their UV-Vis absorption spectra. There is observable red shift of the Soret bands with their characteristic broadening at the higher concentration of porphyrins (Fig. 14), when the xerogel is compared with alcogel (Fig. 12), i.e. from 391 to $400 \mathrm{~nm}$ for HmP-IX and from 389 to $400 \mathrm{~nm}$ for $\mathrm{H}_{2} \mathrm{OEP}$. This fact is explained by many investigators by the agglomeration of tetrapyrrole macrocycles. On the other hand it can not be precluded that the existence of the above mentioned Soret bands red shift may be also referred to the interaction of porphyrin molecules with the silica walls. Based on the FT-IR and Raman spectra formation of hydrogen bonds between the porphyrine and silica walls has been found. The band $1083 \mathrm{~cm}^{-1}$ in FT-IR spectrum of pure $\mathrm{SiO}_{2}$ was shifted toward $1078 \mathrm{~cm}^{-1}$ in silica gel doped with porphyrine as a result of interaction of porphyrin with siloxane oxygens via hydrogen bond. In Raman spectrum the band responsible for the O-Si-O bending mode was shifted from 810 
$\mathrm{cm}^{-1}$ in $\mathrm{SiO}_{2}$ to $816 \mathrm{~cm}^{-1}$ in O-SiO.... $\mathrm{H}_{2} \mathrm{OEP}$ species. What is more interesting the band $599 \mathrm{~cm}^{-}$ 1 , characteristic of the $(\mathrm{SiO})_{3}$-ring breathing mode, was observed merely in the case of $\mathrm{SiO}_{2} / \mathrm{H}_{2} \mathrm{OEP}$ case as a result of partial rearrangement of tetrahedral silica structure after incorporation of porphyrin and formation of three-membered rings.

$\mathrm{HmP} \mathrm{IX/SiO}{ }_{2}$ xerogel, 7 months after gelation

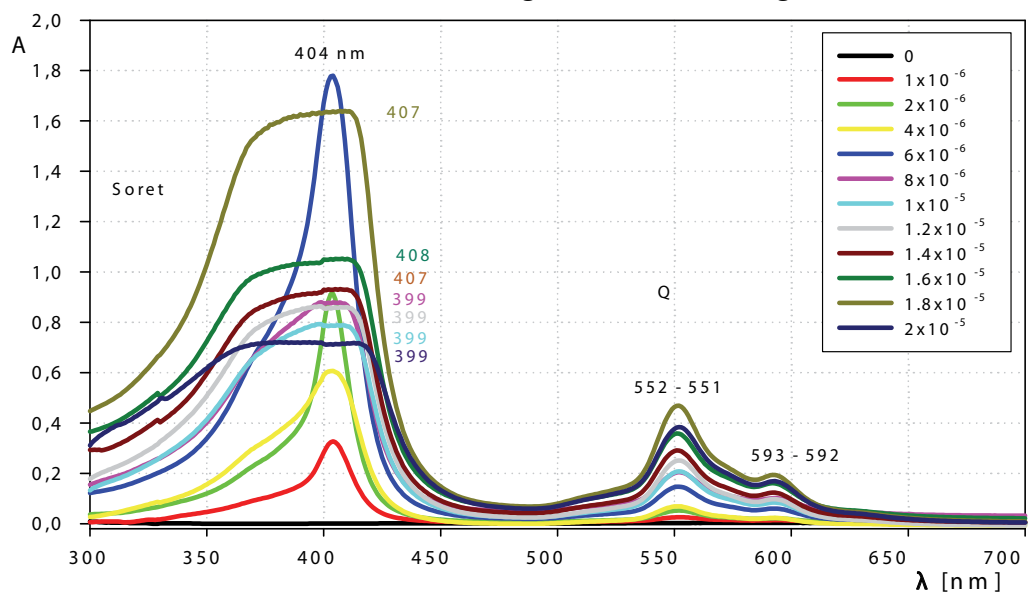

$\mathrm{H}_{2} \mathrm{OEP} / \mathrm{SiO}_{2}$ xerogel, 7 months after gelation

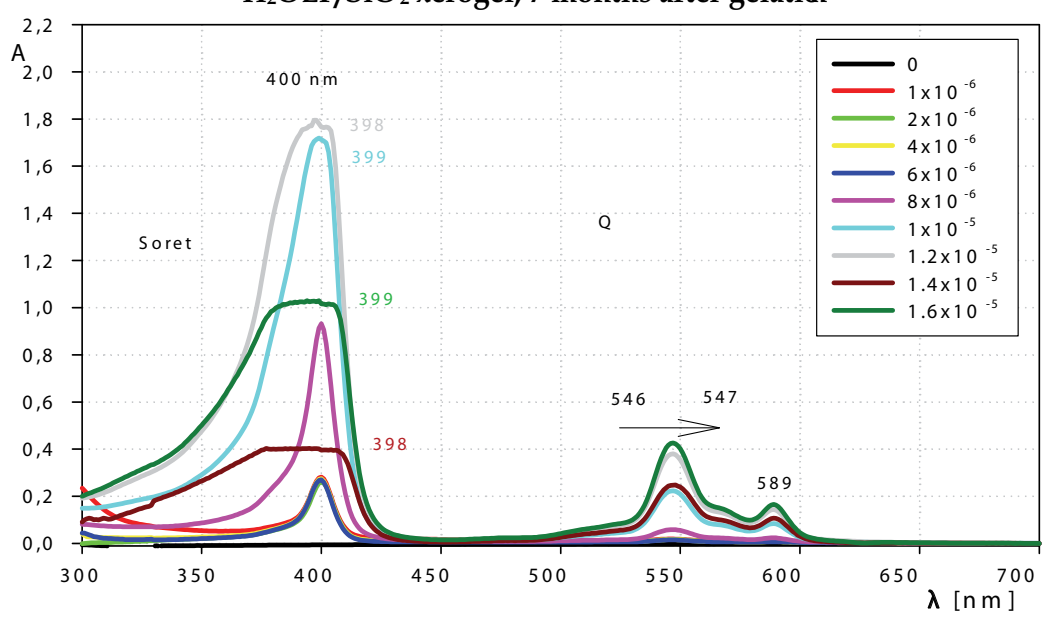

Fig. 14. UV-Vis absorption spectra of $\mathrm{HmP}$ IX and $\mathrm{H}_{2} \mathrm{OEP}$ intercalated in $\mathrm{SiO}_{2}$ xerogel (the numbers denote the initial concentrations of porphyrins in silica sol).

Formation of H- (face to face) and J-type (face to tail) agglomerates of free base porphyrin and its dications is very probable, since splitting of the Soret band in the excitation spectrum into two peaks (Fig. 15), both for octaethyl- and hematoporphyrin IX was observed. This is in agreement with the exciton theory, in which the excited-state energy level of a monomeric dye splits into two during aggregation. Separation of the Soret band into two components is not the same for both porphyrins. For HmP-IX porphyrine the intensity of the bands 345 and $425 \mathrm{~nm}$ is similar, which means that the concentrations of $\mathrm{H}$ - and J-aggregates are 
approximately equal. In the case of $\mathrm{H}_{2} \mathrm{OEP}$ porphyrin the 355 band intensity is significantly higher than that for $412 \mathrm{~nm}$. Formation of H-aggregate is therefore more advanced in comparison with the J-aggregate.

$\mathrm{HmP}$ IX/SiO 2 xerogel, 16 months after gelation

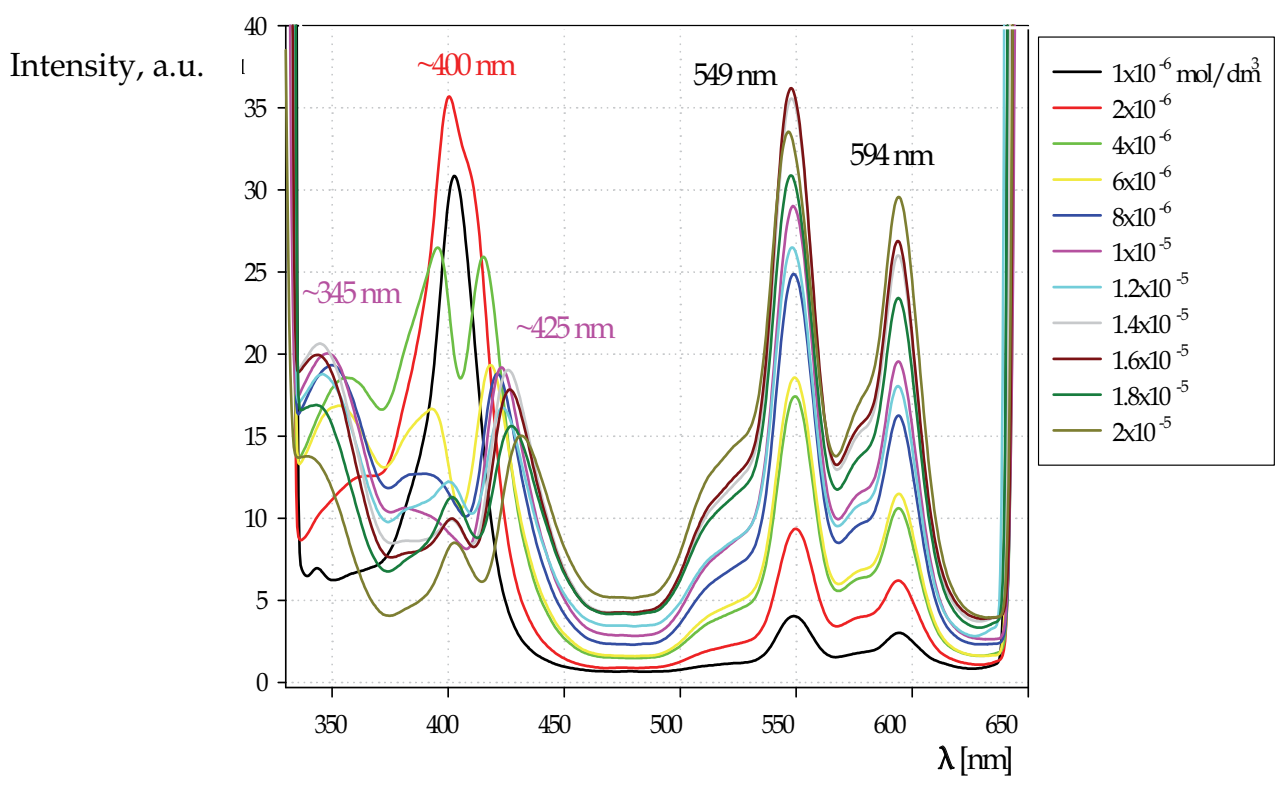

$\mathrm{H}_{2} \mathrm{OEP} / \mathrm{SiO}_{2}$

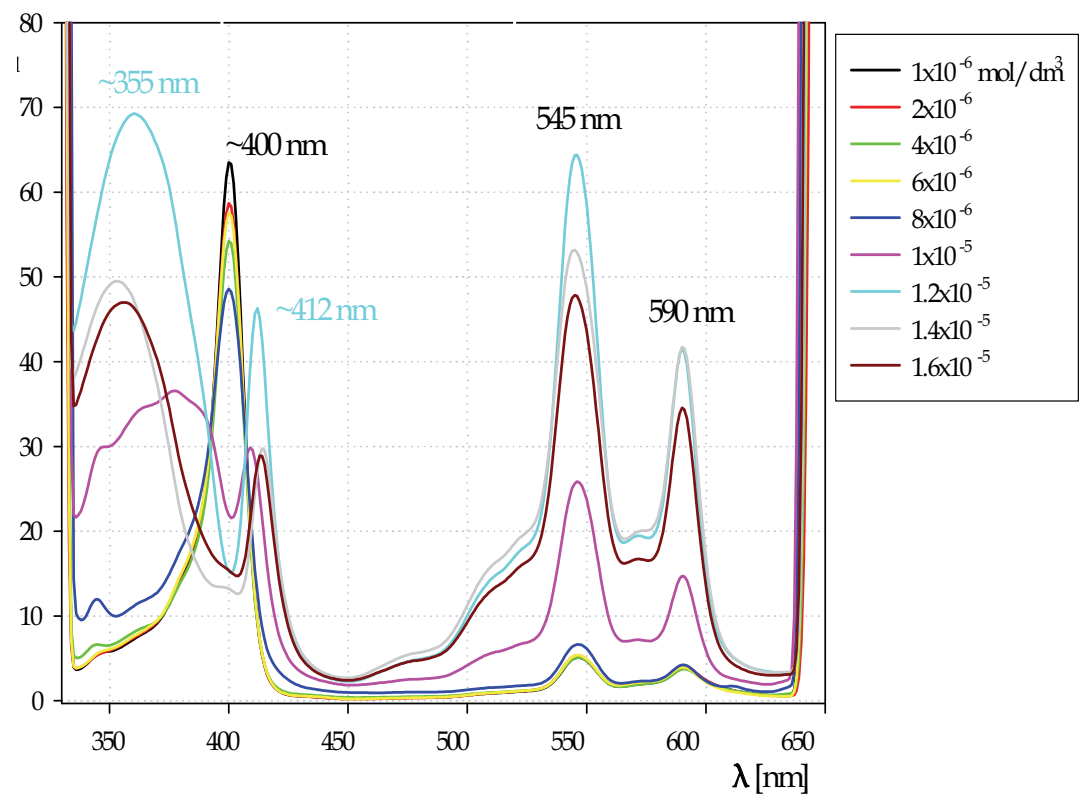

Fig. 15. Excitation spectra of porphyrins intercalated in silica xerogel (16 months after gelation, emission at $650 \mathrm{~nm}$ ). 

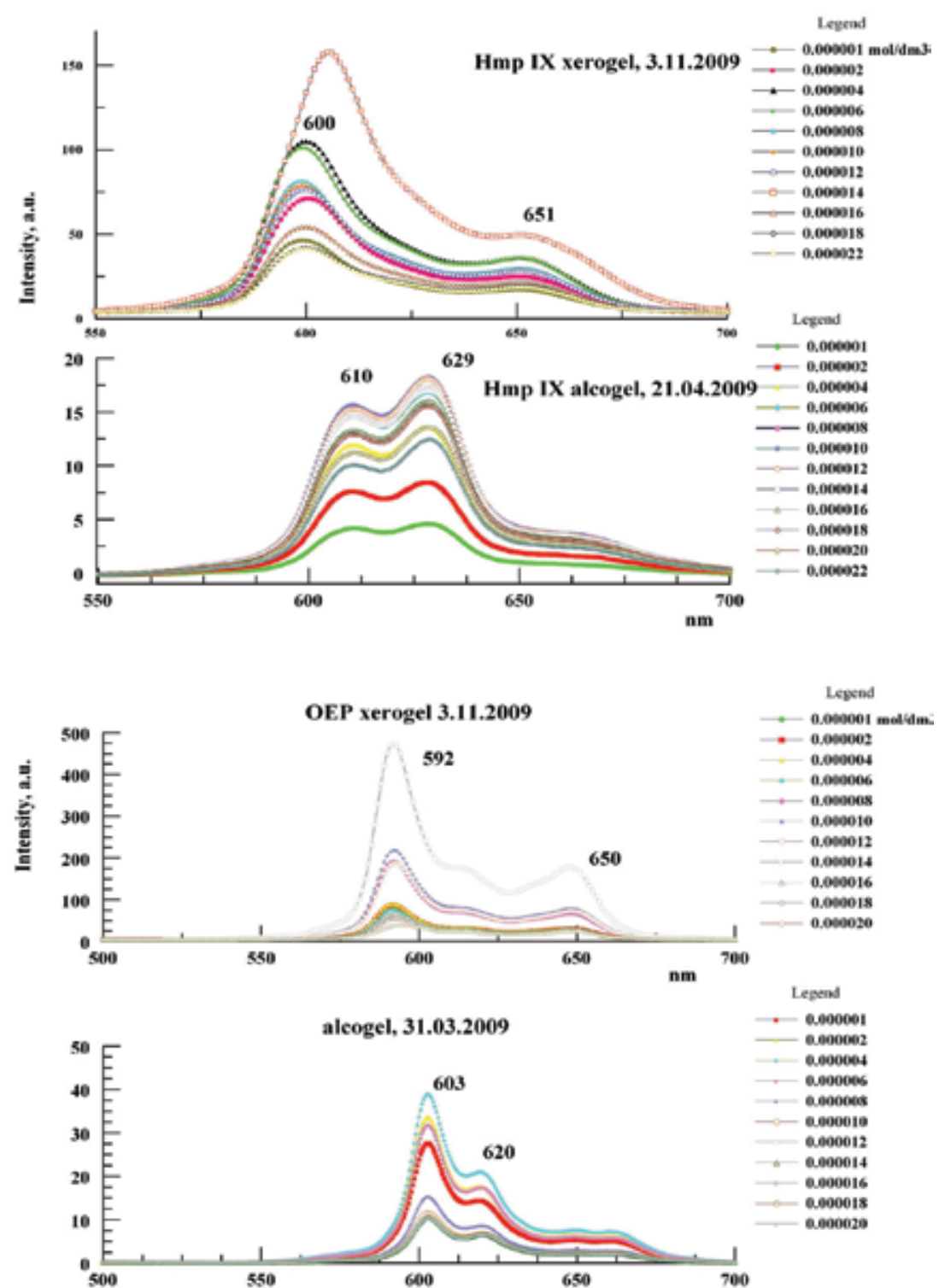

Fig. 16. The emission spectra comparison of $\mathrm{HmP} I X, \mathrm{H}_{2} \mathrm{OEP}$ in alco- and xerogel (excitation wavelength $390 \mathrm{~nm}$ ).

Textural characteristics of silica xerogel based on $\mathrm{N}_{2}$ adsorption revealed the microporous character of adsorbent, however, apart from the micro-, the meso- and macropores were present in the silica structure with the pore size diameter: 17-3000 $\AA$; therefore the accommodation of $\mathrm{J}$ - and $\mathrm{H}$-dimers of porphyrines inside the gel structure is possible without steric obstacles. It must be emphasized that besides optical applications, the silica materials should have very small pore sizes and if the functionalized porphyrin systems are used as biocatalysts or chemical sensors, the passage of reagents and products in and out of 
the pores becomes really important (Garcia-Sanchez et al., 2009). Control of many operational factors involved in the hydrolysis and condensation during the sol-gel process is essential for achieving a proper balance between non-leaching of the entrapped bioactive porphyrins and their accessibility to the reagents.

Emission spectra (Fig. 16) contain two bands at 610, $629 \mathrm{~nm}$ for Hemato IX porphyrin and $603,620 \mathrm{~nm}$ for $\mathrm{H}_{2} \mathrm{OEP}$. These bands correspond to the presence of dications and free base porphyrins. When passing from alco- to xerogel there can be seen the blue shift of bands to 600 and $592 \mathrm{~nm}$ and the emergence of a new band at $650 \mathrm{~nm}$, which probably is related to the agglomeration of free base porphyrins and their protonated forms. On the other hand, it is well known that the agglomerated and protonated forms of porphyrins have less intensive fluorescence compared to free-based porphyrin monomers. In our case if they really exist in the dried gel (xerogel), protonated porphyrin dimers possess characteristic of very strong red fluorescence with greater intensity in compared to that porphyrins dissolved in sol and to those with the aryl substituents immobilized in gel such as $\mathrm{H}_{2} \mathrm{TPP} / \mathrm{SiO}_{2}$.

Lee and Okura (1997) reported the sensor oxygen prepared from immobilized platinum octaethylporphyrin. The luminescence spectra of porphyrin in the silica matrix were very sensitive to the oxygen presence. The strong luminescence quenching was found with the oxygen concentration rise, i.e. intensity of the $645 \mathrm{~nm}$ band decreased up to $97.5 \%$ changing from the totally deoxyganated to oxygenated conditions. This result seems to be dependent on the porous structure of the inorganic matrix prepared by the sol-gel process, having channels for oxygen. According to us, it is most probable that in the presence of oxygen there occur non-radiative processes concurrent to fluorescence, for example quenching of the porphyrin excited state by ${ }^{3} \mathrm{O}_{2}$ leading to energy transfer and ${ }^{1} \mathrm{O}_{2}$ formation. The UV-Vis absorpion spectra of PtOEP-doped silica glass and initial PtOEP/dimethylformamide solutions differed markedly, i.e. the bands 400, 550, $586 \mathrm{~nm}$ in the sol-gel glass were red shifted in comparison with those of the initial PtOEP/DMF solution, which appeared at 375, $500,534 \mathrm{~nm}$. This fact was explained as arising from the less polar silica matrix.

The investigations of tetrakis-(N-methyl-4-pyridinium)porphyrin encapsulated in mesoporus silica by Yoshida and co-workers (2003) showed the evident changes in the UVVis absorbance and fluorescence spectrum when free base porphyrin in solution is compared with that in gel. Shift of the Soret band in direction of shorter wavelengths was observed and explained by the dimerization of porphyrin and interaction of porhyrine with the solid surface. Shift of the flurescence band from 650 to $665 \mathrm{~nm}$ was also found with the increase in the adsorbed TMPyP amounts. The luminescence intensity varied depending on the adsorbed amounts and the variation was not simple. Occurence of luminescence quenching including self-quenching was suggested.

$\mathrm{Xu}$ et al. (2001) reported about the formation of monomeric and aggregated terakis(psulphonatophenyl)porphyrin encapsulated within an aluminosilicate mesostructure, i.e in MCM-41, under different $\mathrm{pH}$ conditions. Stabilization of MCM-41 was necessary through the use of a silylation reagent, (aminopropyl)triethoxysilane, which cross-linked oxygen on the surface, rigidified the mesoporous material walls and functionalized the interface for proper guest-host interaction. The authors found that the encapsulated free-base monomer exhibited the blue shift for its Soret band (396 nm) when compared with the Soret band (413 $\mathrm{nm}$ ) of the solution monomeric species. The blue shift for the absorption was also established for the encapsulated aggregate when compared with the solution aggregate, i.e. from 490 to $487 \mathrm{~nm}$ respectively. Such shifts were explained in terms of charge transfer 
caused by the host-guest interaction, connected with the steric effects associated with the MCM pore structure. The broad structure for the Soret band for TSPP-M/(MCM-41) was interpreted as site-specific absorptions for encapsulated TSPP, suggesting that the monomeric species is distributed in various positions within the cage and experiences a range of perturbations. Based on the analysis of absorbance and fluorescence spectra the authors concluded about the formation of J-aggregates by porphyrin in gel, which had the zigzag or spread deck of cards structure (Fig. 17).

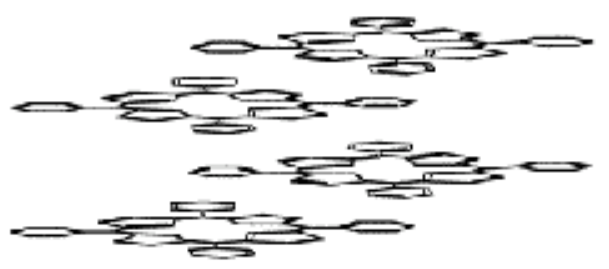

$\boldsymbol{A}$

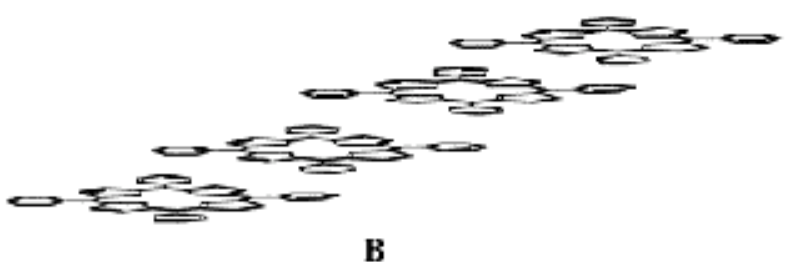

Fig. 17. Two possible linear J-aggregates (A and B, relating to the zigzag and spread deck of cards, respectively) resulting upon protonation of the macrocycle; reproduction with permission from Journal of Physical Chemistry B, copyright 2001.

Holland et al. (1998) investigated the encapsulation of meso-Tetrakis(5trimethylammoniopentyl)porphyrin (TMAP) in the molecular sieve MCM-41 via the hydrothermal synthesis or by the surfactant/porphyrin ion-exchange reaction with MCM41. Both methods allowed for the incorporation of porphyrin into the mesoporous channel system. The inspection of the UV-Vis spectra showed that porphyrin dimers or aggregates were formed during ion-exchange, whereas TMAP molecules remained isolated during hydrothermal synthesis. In the latter the UV-Vis spectra were similar to those of TMAP in dilute solution and no significant broadening of Soret and $Q$ bands was noticed.

Murata et al. (2000) modified the surface silanol groups of FSM-type mesoporous silica (folded sheet mesoporous material) with 1,4-butanediol and used this material as the adsorbent to accommodate chlorophyll a molecules. Pheophytinization (removal of magnesium atom from the porphyrin ligand) was suppressed by the presence of the surface organic groups intercalated into the mesoporous silica. Pheophytinization occurred in the unmodified silica. The UV-Vis spectra showed that the $Q_{y}$ transition occurred at $665 \mathrm{~nm}$, suggesting that the encapsulated $\mathrm{Chl} a$ molecules are well dispersed in the mesopores of $1,4 \mathrm{BD}-\mathrm{C}_{18} \mathrm{FSMs}$. The UV-vis spectrum of $1,4 \mathrm{BD}-\mathrm{C}_{18} \mathrm{FSM}$ containing $\mathrm{Chl} a$ stored under dark and cold $\left(0^{0}\right)$ conditions for 6 months, was registered in order to estimate the stability of incorporated Chl $a$ and the spectrum showed no change. The authors concluded that the obtained results may lead to the construction of in vitro biomimetic solar energy conversion and storage systems. 
Itoh et al. (2002) investigated the adsorption of chlorophyll $a$ to the mesoporous silica FSM. The chlorophyll-FSM conjugate was formed with a nanometer-scale interaction between chlorophyll molecules. There was found the enhancement of photostability for the chlorophyll $a$ adsorbed to the pores FSM, along with decrease in the pore volume and specific surface area, as well as a shift in the adsorption maximum to a longer wavelength, i.e from 671 to $675 \mathrm{~nm}$. Such a shift was explained as a consequence of an interaction between two chlorophyll molecules. The evolution of hydrogen gas was observed when the aqueous solution containing chlorophyll-FSM, methyl viologen, 2-mercaptoethanol, and platinum was illuminated with visible light.

As follows from the presented results the spetroscopic behaviour of the porphyrins in the solutions and solid matrices is evidently different but the interpretation of changes in the position and intensity of Soret and $Q$ bands of porphyrins spectra after their intercalation in various matrices is very complexed. The same applies to the formation of UV-Vis absorption bands referred to the porphyrins agglomeration. There are too many effects, such as formation of dications, agglomeration and interactions of porphyrins with the matrix, which prevent the identification of the electronic transitions. Despite the lack of theoretical explanation for complexed porphyrins spectroscopic behaviour, the fact of their high affinity for the solid matrices and the preservation of their luminescent properties during insertion into the solid matrices structure has been used in biocatalytic applications and this is the issue of the next topic.

As most of the silica-gels are transparent to visible light, the mechanisms governing the photoprocesses are strongly dependent on the characteristics of the photosensitizer. Studies on the change of structure conformation and physicochemical properties of porphyrin macrocycles entrapped in silica matrices can be especially helpful in elucidation of mechanism of their photocatalytic oxidation in heterogeneous systems, and in attempts at achievement of more powerful biocatalysts as well as in rationalizing the reaction manifolds accessible to the porphyrin-silica catalysts.

\section{Photocatalytic oxygenation using porphyrins intercalated in the sol-gel matrix}

Only a few works have been reported on porphyrins immobilized in the sol-gel matrix as the new functional catalytic devices for the production of commercially important compounds.

Iron porphyrin catalysts were anchored on the spherical silica gel beads obtained by the solgel route (Biazzotto et al., 2002; Moreira et al., 2005; Papacídero et al., 2006) for the oxidation of $(\mathrm{Z})$-cyclooctene and cyclohexane with $\mathrm{PhIO}$ or $\mathrm{H}_{2} \mathrm{O}_{2}$. Better results were obtained with the electronegatively substituted porphyrin on the $\mathrm{SiO}_{2}$ supports rendering $86 \%$ epoxide yields (Moreira et al., 2005). A similar carrier covalently modified with the metal-free monopyridyltriphenylporphyrin, introduced into a polymer microchannel and the microchip were applied for photodecomposition of phenol under solution-flow conditions (Kitamura et al., 2006). Very recently a visible light photocatalytical system based on waterinsoluble tin porphyrin $\mathrm{Sn}(\mathrm{OH})_{2}(\mathrm{TPP})$ immobilized on $\mathrm{SiO}_{2}$ has been successfully used for the degradation of 4-chlorophenol and acid orange 7 (Kim et al., 2008). Silica microspheres functionalized with 5-(4-allyloxy)phenyl-10,15,20-tri(2,6-dichlorophenyl)porphyrin have been prepared by Huang et al. for the photooxidation of 1,5-dihydroxynaphthalene under visible light irradiation in the aerated aqueous solution (Cai et al., 2009). 
Trytek et al. (2009) have presented the results concerning two types of porphyrins (watersoluble and water-insoluble) incorporated into the silica gel which act on the one hand as fluorophores, and on the other hand as efficient catalysts in biomimetic photocatalysis of monoterpene, a-pinene, in organic solvent under molecular oxygen. In the presented experiments the authors confirmed that the photochemical excitation of sol-gel immobilized porphyrins is crucial for catalyzed oxidation of monoterpenes, and it can proceed with sunlight or by using an artificial light source. The catalysts based on the porphyrin copper (II) complexes proved to be ineffective in the photochemical system. The largest level of light promoted biotransformation was achieved with water insoluble $\mathrm{H}_{2} \mathrm{TPP}$ and water soluble, cationic tetrakis(1-methyl-4-pyridinio)porphyrin $\left(\mathrm{H}_{2} \mathrm{TMePyP}\right)$.

We decided to develop the biomimetic system using $\mathrm{H}_{2} \mathrm{TMePyP} / \mathrm{SiO}_{2}$ because $\mathrm{H}_{2} \mathrm{TPP}$ in silica gel was rapidly protonated in the initial course of the photooxygenation, and without regeneration could not be reused. Among four solvents tested for this reaction, chloroform and dichloromethane proved to be the best with respect to the enhancement of the system productivity. Although the molar yields of products were rather low (about 15\%), $\mathrm{H}_{2} \mathrm{TMePyP} / \mathrm{SiO}_{2}$ in the powdered form could be repeatedly used for 11 successive biotransformations of each 24-hour cycle without a significant loss of activity, rendering up to $10.5 \mathrm{~g} / \mathrm{L}$ yield of pinocarvone, $2 \mathrm{~g} / \mathrm{L}$ of trans-pinocarveol and about $1 \mathrm{~g} / \mathrm{L}$ of myrtenol. It was proved by the invariance of the UV-Vis spectra of the filtered reaction mixture and also on the basis of luminescent intensity for the powder both before and after the reaction that the porphyrin catalyst remains inside the gel pores for thirteen cycles.

The effect of porphyrins immobilization in the silica matrices on the changes and correlation between their photophysical properties and biomimetic activities can be shown from the comparative study on water insoluble $\mathrm{SiO}_{2}$-encapsulated $\mathrm{HmP}-\mathrm{IX}$ and $\mathrm{H}_{2} \mathrm{OEP}$ (Fig. 11), bearing substituents of different polarity (Trytek et al., in preparation). The photoxidation of a-pinene in the organic solvent by molecular oxygen was demonstrated successfully for only octaethylporphine $/ \mathrm{SiO}_{2}$. Significant differences in photobiocatalytic performance of these both intensive luminescent materials may result from strong interaction of the electron-accepting carboxyl groups of HmP-IX with silica, and much stronger electron donating ability of $\mathrm{H}_{2} \mathrm{OEP}$ making the central cavity of the porphyrin ring less electrophilic in contrast to $\mathrm{HmP}$ (Kane et al., 1998). Such results may be also interpreted in terms of the earlier described spectral investigations, which revealed the contrasting capabilities of $\mathrm{HmP}$ IX and $\mathrm{H}_{2} \mathrm{OEP}$ aggregation in both the sol solution and sol-gel matrix, as well as differences in the formation of $\mathrm{H}$ - and J-type agglomerates in xerogel.

Since the HmP in silica preserves the fluorescence, we can not preclude the formation of CO-O-Si ester bridges with the siloxane network by $\mathrm{HmP}$, because according to the other authors the covalent bond between the porphyrin molecules and the silica walls in the interior pores of the gel diminish the interaction of porphyrin molecules with silanol groups $(\mathrm{Si}-\mathrm{OH})$ and then the possibility of non-radiating decay of the fluorescent process of porphyrins (Garcia-Sanchez et al., 2009). Thus, the significant drop of the photocatalytic activity of the HmP-IX in silica may be attributed to its chemical binding with $\mathrm{SiO}_{2}$, probably hindering the undergoing intersystem passing from the singlet excited state of porphyrin to its triplet state, which is an important criterion for a photosensitizer and photochemical activation of dioxygen. As a result, concurrent radiative transition from the excited $\mathrm{HmP} / \mathrm{SiO}_{2}$ state to its singlet ground state may occur in the photochemical system. 
Maximal yields of all photooxidation products occurred in more non-polar solvents e.g. methyl chloride and chloroform. Hydrophilic solvents such as, for example, $\mathrm{MeOH}$ are substantially less efficient in terms of their ability to maintain active porphyrins in the solgel. A possible explanation is based on the generally accepted rule that singlet oxygen $\left({ }^{1} \mathrm{O}_{2}\right)$ is the primary oxygen agent responsible for photooxidation by light-sensitive catalysts (DeRosa and Crutchley, 2002). In chloroform, singlet oxygen has a relatively long lifetime and thus photooxygenation resulting faster in comparison with other solvents (Hurst et al., 1982).

However, the GC-MS analysis revealed that the products (pinocarvone, trans-pinocarveol and myrtenol were rather not directly obtained in the ene type reaction characteristic of light promoted singlet oxygen reactions where ${ }^{1} \mathrm{O}_{2}$ reacts with olefins causing a double bond shift and hydroperoxides or alcohols formation (Kenney \& Fisher, 1973; Ribeiro et al., 2008). In this context, the behaviour of the photocatalysts encapsulated in silica gel toward a-pinene is unusual and can not be compared to that of several groups of porphyrin photocatalysts and hem iron biocatalyst, which afford the mixtures of hydroperoxides with pinene oxide and verbenol or the pinocarveol and myrtenol as main products after the reduction of the corresponding hydroperoxides (Ribeiro et al., 2008).

In the photooxidation reactions a free radical chain mechanism is also suggested and in this case, competition between the abstraction of the allylic hydrogen to give allylic oxidation products and the addition of the alkylperoxy radical to the double bond resulting in epoxide products is expected. A broad product mixture usually arises from typical radical-initiated autooxidation (Henning et al., 1990; Nakagaki et al., 2004). Since we obtained merely the ketone and alcohol with no hydroperoxides and neither epoxide nor corresponding glycol derivatives being detected in the reaction solutions, we suggest that the cooperative mechanism of a-pinene photooxidation based on two possible routes participates in phototransformation of pinene by porphyrins in silica gel. The first pathway involving electron abstraction by the light-excited $\mathrm{OEP} / \mathrm{SiO}_{2}$ matrix can lead to the formation of minor components during a radical autoxidation mechanism, while the most abundant compounds - pinocarveol and pinocarvon are formed through a photosensitized reaction with singlet oxygen (either by electron transfer from pinene to ${ }^{1} \mathrm{O}_{2}$ or via a radical mechanism).

Photophysical properties, photodegradation and aggregation changes may have a possible effect on the profile and yields of photooxidation products of substrate (Karapire et al., 2005). The biocatalytic efficiency of porphyrins entrapped in the sol-gel matrix was found to be positively correlated with their intensity of fluorescent emission and absorbance over the range of $\mathrm{H}_{2} \mathrm{OEP}$ concentration studied in sol. The maximum turnover number (defined as a mole of product to a mole of porphyrin per hour) was observed to be reached at $10^{-6}$ for OEP and $10^{-5}$ for the HmP-IX (Tab. 1). Close to these $\mathrm{H}_{2} \mathrm{OEP}$ concentrations, the largest luminescence intensity and sharpness of the relative Soret band was noticed, which ruled out the formation of porphyrin aggregated species, in contrary to the larger amount of catalysts in the silica medium. This is in an agreement with the more general rule that the formation of a dication and dimers or higher order aggregates decreases photoactivity of the photosensitizers (Gerdes et al., 1997; Iliev et al., 2000; Tanielian et al., 2001) as the presence of porphyrin stacking does not allow an optimal interaction of the pinene with macrocycles (Paolesse et al., 2002). 


\begin{tabular}{|c|c|c|c|c|c|c|}
\hline \multirow{2}{*}{$\begin{array}{c}\text { Concentrat } \\
\text { ion of } \\
\mathrm{H}_{2} \text { OEP in } \\
\text { sol (M) }\end{array}$} & \multicolumn{2}{|c|}{ Concentration of products $(\mathrm{g} / \mathrm{L})$} & \multicolumn{3}{|c|}{ Turnover number } \\
\cline { 2 - 7 } & pinocarvone & $\begin{array}{c}\text { trans- } \\
\text { pinocarveol }\end{array}$ & myrtenol & pinocarvone & $\begin{array}{c}\text { trans- } \\
\text { pinocarveol }\end{array}$ & myrtenol \\
\hline $1 \times 10^{-6}$ & 0.087 & 0.024 & - & 2285.7 & 615.3 & - \\
\hline $2 \times 10^{-6}$ & 0.206 & 0.045 & - & 2717.1 & 579.2 & - \\
\hline $4 \times 10^{-6}$ & 0.546 & 0.093 & 0.06 & 3537.4 & 588.0 & 383.9 \\
\hline $6 \times 10^{-6}$ & 0.96 & 0.156 & 0.111 & 4225.7 & 670.1 & 478.0 \\
\hline $8 \times 10^{-6}$ & 0.769 & 0.128 & 0.086 & 2514.2 & 408.4 & 277.7 \\
\hline $1 \times 10^{-5}$ & 0.856 & 0.137 & 0.102 & 2212.8 & 345.6 & 260.5 \\
\hline $1.2 \times 10^{-5}$ & 0.618 & 0.107 & 0.058 & 1343.0 & 226.9 & 124.5 \\
\hline $1.4 \times 10^{-5}$ & 0.686 & 0.116 & 0.068 & 1279.8 & 211.2 & 125.3 \\
\hline $1.6 \times 10^{-5}$ & 0.974 & 0.180 & 0.125 & 1575.6 & 284.2 & 199.7 \\
\hline $1.8 \times 10^{-5}$ & 0.797 & 0.123 & 0.068 & 1132.1 & 170.5 & 110.8 \\
\hline $2 \times 10^{-5}$ & 1.3 & 0.219 & 0.198 & 1666.2 & 273.9 & 250.6 \\
\hline
\end{tabular}

Table 1. Effect of initial concentration of octaethylporphyrin in silica on its biocatalytic efficiency in the a-pinene photooxidation.

However, the results show that the yield of all products increased with an increase in $\mathrm{H}_{2} \mathrm{OEP}$ porphyrin concentration from 0.087 up to $1.3 \mathrm{mg} / \mathrm{L}$ (Tab. 1). We suggested that the dimeric complex of $\mathrm{H}_{2} \mathrm{OEP}$ in $\mathrm{SiO}_{2}$ participates in photooxidation of monoterpenes; if not, the catalytic performance should result in much lower yield of products compared with the homogeneous system in which the same moles of the porphyrin were taken into the reaction (Fig. 18). Intercalation of silica with $\mathrm{H}_{2} \mathrm{OEP}$ may be expected to originate a system in which the catalytic abilities of the two species (dimers and dications) reinforce each other. As a conclusion, we propose a daring hypothetical primary mechanism step of a-pinene photooxidation by $\mathrm{H}_{2} \mathrm{OEP} / \mathrm{SiO}_{2}$. The essential, photoinduced process, would involve transfer of protons from the dimeric form of porphyrin dication to the lone electron pairs of siloxane oxygen (in $\equiv \mathrm{Si}-\mathrm{O}-\mathrm{Si} \equiv$ ) or passing protons to the not-bound silanol groups of silica network. The resulting excited porphyrin in the triplet state can then react in one of

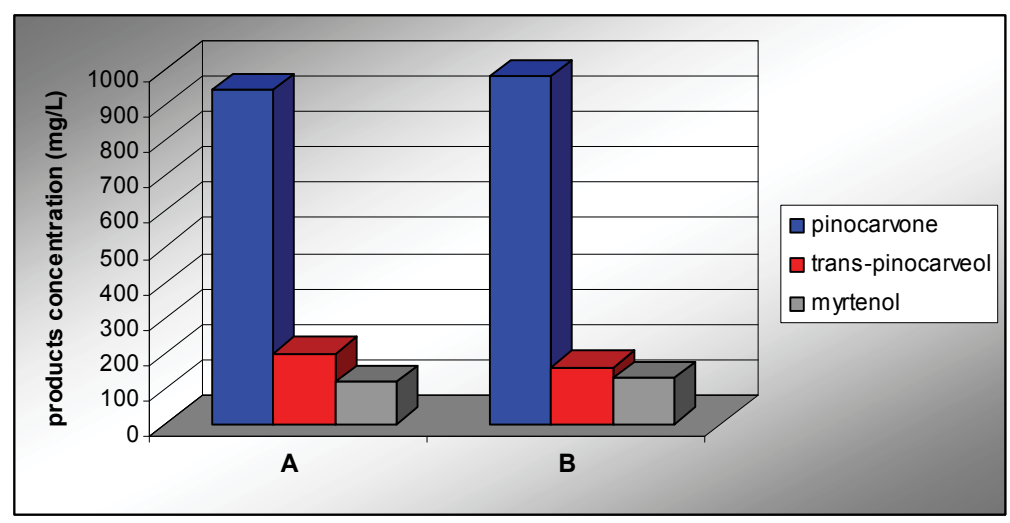

Fig. 18. Comparison of $\mathrm{H}_{2} \mathrm{OEP}$ activity in the homogeneous system (A) to the heterogeneous based on the silica immobilized catalyst (B) in the process of a-pinene photooxidation. 
two ways, via type I photooxygenation (electron transfer between the excited photosensitizer and the substrate) or II mechanism (energy transfer process during the collision of the excited photosensitizer molecule with triplet oxygen) leading to singlet oxygen generation. These two alternative pathways are well documented in literature (DeRosa and Crutchley, 2002; Griesbeck et al., 2005).

Undoubtedly, the real mechanism of the photooxidation by porphyrin in silica xerogel is more complex, and for example, in the charge transfer inside the silica, the contribution of the holes transport should also be taken into account (Mele et al., 2003).

The kinetics of this process is of the first order in respect to pinene concentration. The straight line dependence of $\ln (\mathrm{c} / \mathrm{co}$ ) on time $\mathrm{t}$ (where co is the initial concentrations of pinene) proves the first order character of biotransformation reaction. The rate constant $\mathrm{K}=0.013 / \mathrm{h}$. An independent reactivity experiment corroborated this suggestion, since no oxidative products were detected under typical reaction conditions using trans-pinocarveol or myrtenol as the substrates. Therefore one can conclude that all three products: transpinocarveol, myrtenol, pinocarvone are the result of independent biotransformation reactions of pinene at different oxidation rates. Based on the concentrations of the products of biotransformation the following interesting straight line dependences were found: $\log [$ trans-pinocarveol] vs log [myrtenol], log[pinocarvon] vs $\log [$ myrtenol], log[transpinocarveol] vs $\log [$ pinocarvon]. Hence, it is possible to evaluate the concentration of one product based on the other one. Of course, this will not be an accurate evaluation, since it is difficult to maintain identical reaction conditions, i.e. the same gel, its porosity, and the mixing rate of phases.

The correlation between the photoactivity of free-base porphyrins in organic solutions and those entrapped in the silica matrices indicates that the strategy of incorporation of different porphyrins into the transparent matrix was successful, as the photoactivity of water insoluble $\mathrm{H}_{2} \mathrm{OEP}$ in the heterogeneous system was exactly the same as in the homogeneous one (Fig. 18). The supplementary characterization of silica pores dimension by the $\mathrm{N}_{2}$ adsorption method enabled the appreciation of porphyrins encapsulation inside the sol-gel matrix structure and access of the reagents to the active site of catalyst.

Similarly, the high biocatalytic efficiency was achieved for water-soluble $\mathrm{H}_{2} \mathrm{TMePyP} / \mathrm{SiO}_{2}$ with no leaching of the porphyrin complex, followed by retaining catalytic activity in the sol-gel-matrix, when the catalyst was reused. This occurred despite the fact that silica matrix polarity and structure with part of the closed pores might hinder the access of oxygen and pinene to the catalytic site, and diffusion of the oxidation products into the reaction medium. This stability was not only due to physical entrapment but also to additional multiple interactions with $\mathrm{SiO}_{2}$ through hydrogen, ionic or hydrophobic interactions. The question remains whether the improved photostability of these catalysts is a factor of their immobilization, or simply a result of lower singlet oxygen yields.

Porphyrins in the silica gel work as photoactive catalysts in the oxidative transformation of monoterpenes into the products attracting great interest by the fragrance and flavour industry. Pinocarvone, pinocarveol and myrtenol, the compounds of large commercial value, were the products obtained in the biomimetic oxidation of a-pinene by all porphyrins photoactive in the silica gel, as confirmed by the GC-MS analysis. These structures were previously formed in the a-pinene biotransformation with the use of genera of Basidiomycetes and with the intermediates of enzyme degradation pathways of a-pinene occurring in some bacteria strains such as Pseudomonas putida, P. fluorescens or in the case of Bacillus pallidus (Busman \& Berger, 1994; Savithiry et al., 1998). Therefore, the catalytic 
activity of the examined porphyrins in the sol-gel matrix bears resemblance to enzymatic catalysis involving a-pinene monooxygenase and pinocarveol dehydrogenase.

\section{Conclusions}

The presence of the porphyrins as the active site of many natural enzymes makes them enticing targets for biocatalysis. Many attempts have been made to design the active site analogues of heme monooxygenases. The huge number of studies performed on iron porphyrin models and on hemoproteins themselves had a strong impact on the elucidation of the enzymatic reaction mechanisms in the catalytic pathway of monooxygenases. Besides the important role they have played in understanding these complex enzymes, porphyrins have allowed to develop many efficient bioinspired catalysts for a variety of oxidation reactions that are of importance for many different industrial processes. The biomimetic oxidation of hydrocarbons by porphyrins under condition of homogeneous catalysis compares very favourably in the efficiency with that catalyzed with enzymes. However, the attempts at process implementation and scale-up to pilot or industrial scales have not been successful yet.

A challenging problem in this area is the construction of the well organized biomimetics showing high regio- and stereoselectivity as well as stability. The development of heterogeneous catalysts, with two types of porphyrins (water-soluble and water-insoluble) as the catalytic units incorporated into the silica gel would allow overcoming some of these limitations with the challenge of improving the catalytic activity and stability. As shown the sol-gel process has proved to be a valid methodology to produce a transparent and stable, designed luminescent porphyrin-based hybrid material, which can be shaped not only for the application in the fields of biomimetic catalysis for the enzyme like cytochrome P450 but also for the electroluminescence, light emitting diodes and lasers, as mentioned in the literature. Although the product analysis has only been conducted for pinene, the catalytic activity extends to a large variety of olefins including terpenes.

From the Raman, FTIR and UV-Vis data it was concluded that the porphyrins form a complex with silica, probably by the hydrogen bond formation of protonated porphyrin molecules, e.g. monomers or dimers of $\mathrm{H}_{2} \mathrm{OEP}$ with the $\mathrm{Si}-\mathrm{O}-\mathrm{Si}$ or $\mathrm{Si}-\mathrm{O}$ groups in the pore structure. The dimerization process in $\mathrm{SiO}_{2}$ alcogel is advanced for the $\mathrm{H}_{2} \mathrm{OEP}$ case whereas for HmP IX the lack of dimerization was observed.

As follows from the comparison of biocatalytic activity of $\mathrm{H}_{2} \mathrm{OEP}$ and $\mathrm{HmP}$ IX the main goal for the preparation of porphyrin-introduced silica gel, which may be efficiently used in biocatalysis, should be based on the selection of porphyrin with the weak molecular interaction with silanol or siloxane groups; therefore the resulting excited state conformation would facilitate the intersystem crossing from a singlet excited state of porphyrin to its triplet state which, in turn, would affect photocatalyst functionality and therefore oxygen activation, responsible for the oxidation reaction.

The aggregation of the porphyrins is unfavourable for the biocatalytic process. Therefore based on the excitation spectrum of the porphyrins in xero gel it may be possible to select the range of porphyrins concentration where the single Soret band is observed instead of two, that are characteristic of $\mathrm{H}$ - and J-aggregates and therefore to obtain the acceptable yield of biocatalytic conversion with these concentrations of catalyst. This statement is valid for biotransformation of a-pinene in the $\mathrm{H}_{2} \mathrm{OEP} / \mathrm{SiO}_{2}$ system where the appearance of Soret band splitting at $10^{-5} \mathrm{~mol} / \mathrm{dm}^{3}$ of $\mathrm{H}_{2} \mathrm{OEP}$ accompanied the decrease of its biocatalytic 
activity. The confirmation of correlation between the character of the excitation spectra of other porphyrins and their catalytic activity is necessary.

While spectral characterizations have provided considerable insight into the porphyrin-host matrix interaction and the relationship between the structures and the spectroscopic behaviour of porphyrins entrapped in silica matrices, more sophisticated experimental and theoretical methods are needed if the effect of free-base porphyrins immobilization on the changes of their photophysical properties, photodegradation or aggregation, as well as a photocatalytic activity in various organic solutions are to be more fully understood. These studies surely will allow take one step closer to the directed oxidation of organic compounds with dioxygen in the kinetically controlled way.

\section{References}

Adamczak M. \& Krishna, S. (2004). Enzyme for efficient biocatalysis. Food Technology and Biotechnology, Vol. 42, 251-264.

Adams, A. Demyttenaere, J.C.R. \& de Kimpe, N. (2003). Biotransformation of $(R)-(+)-$ and (S)-(-)-limonene to a-terpineol by Penicillium digitatum - Investigation of the culture conditions. Food Chemistry, Vol. 80, 525-534.

Ahmad, I., Fasihullah, Q.; Noor, A.; Ansari, I. \& Ali Q. (2004). Photolysis of riboflavin in aqueous solution: A kinetic study. International Journal of Pharmaceutics, Vol. 280, 199-208.

Ariga, K.; Vinu, A.; Hill JP. \& Mori, T. (2007). Coordination chemistry and supramolecular chemistry in mesoporous nanospace. Coordination Chemistry Reviews, Vol. 251, 25622591.

Arnold, F.H. (2001). Combinatorial and computational challenges for biocatalyst design. Nature, Vol. 409, 253-257.

Avnir, D.; Levy, D. \& Reisfeld, R. (1984). The nature of silica glass cage as reflected by spectral changes and enhanced photostability of trapped rhodamine 6G. Journal of Physical Chemistry, Vol. 88, 5956-5959.

Banthorpe, D.V. (1994). Terpenoids. In: Natural Products: Their Chemistry and Biological Significance, J. Mann, R.S. Davidson, J.B. Hobbs, D.V. Banthorpe, J.B. Harborne (Eds.), Longman Scientific \& Technical, Longman Group, Harlow, UK, pp. 289-359.

Bartocci, C., Maldotti, A. \& Varani, G. (1996). Photoexcited iron porphyrin as biomimetic catalysts. G. La Chimica e I'Industria, Vol. 78, 1097-1104.

Bedioui, F. (1995). Zeolite-encapsulated and clay-intercalated metal porphyrin, phthalocyanine and Schiff-base complexes as models for biomimetic oxidation catalysts: an overview. Coordination Chemistry Reviews, Vol. 144, 39-68.

Bell, S., Sowden, R., Wong L. (2001). Engineering the haem monooxygenase cytochrome P450 $0_{\text {cam }}$ for monoterpene oxidation. Chemical Communications, Issue 7, 635-636.

Benaglia, M.; Danelli, T.; Fabris, F.; Sperandio, D. \& Pozzi, G. (2002). Poly(ethylene glycol)supported tetrahydroxyphenyl porphyrin: A convenient, recyclable catalyst for photooxidation reactions. Organic Letter, Vol. 4, 4229-4232.

Bernadou, J. \& Meunier, B. (2004). Biomimetic Chemical Catalysts in the Oxidative Activation of Drugs. Advanced Synthesis \& Catalysis, Vol. 346 (2-3), 171-184.

Bernhardt, R. (2006). Cytochromes P450 as versatile biocatalysts. Journal of Biotechnology, Vol. $124,128-145$. 
Biazzotto, J. C.; Vidoto, E.A.; Nascimento, O.R.; Iamamoto, Y. \& Serra, O.A. (2002). Iron(III)-tetra-o-ureaphenylporphyrinosilica obtained by a sol-gel process: A study of EPR, surface area and catalytic activity. Journal of Non-Crystalline Solids, Vol. 304(1-3), 101-108.

Bicas, J.L.; Dionìsio, A.P. \& Pastore, G.M. (2009). Bio-oxidation of terpenes: An approach for the flavor industry. Chemical Reviews, Vol. 109(9), 4518-4531.

Bonnett, R. (1995). Photosensitizers of the Porphyrin and Phthalocyanine Series for Photodynamic Therapy. Chemical Society Reviews, Vol. 24, 19-33.

Bornshcheuer, U.T. \& Kaslauskas, R.J. (2004). Catalytic promiscuity in biocatalysis: using old enzymes to form new bonds and follow new pathway. Angewandte Chemie International Edition, Vol. 43(45), 6032-6040.

Branco, R.; Seifert A.; Budde, M.; Urlacher, V.; Ramos, M.; Pleiss, J. (2008). Anchoring effects in a wide binding pocket: The molecular basis of regioselectivity in engineered cytochrome P450 monooxygenase from B. megaterium. Proteins: Structure, Function, and Bioinformatics, Vol. 73(3), 597-607.

Breslow, R, (1972). Biomimetic Chemistry. Chemical Society Reviews, Vol. 1, 533-580.

Breslow, R.; Zhang, X. \& Huang, Y. (1997). Selective Catalytic Hydroxylation of a Steroid by an Artificial Cytochrome P-450 Enzyme. Journal of the American Chemical Society, Vol. 119, 4535-4536.

Bühler, B. \& Schmid, A. (2004). Process implementation aspects for biocatalytic hydrocarbon oxyfunctionalization. Journal of Biotechnology, Vol. 113(1-3), 183-210.

Burton, S.G. (2003). Oxidizing enzymes as biocatalysts. Trends in Biotechnology, Vol. 21(12), 543-549.

Busman, D. \& Berger, R. (1994) In: Maarse H, van der Heij DG (eds) Trends in Flavour Research, Elsevier Science.

Cai, J-H.; Huang, J-W.; Zhao, P.; Ye, Y-J.; Yu, H-C. \& Ji, L-N. (2009). Silica microspheres functionalized with porphyrin as a reusable and efficient catalyst for the photooxidation of 1,5-dihydroxynaphthalene in aerated aqueous solution. Journal of Photochemistry and Photobiology A: Chemistry, Vol. (2-3), 236-243.

Campestrini, S. \& Meunier, B. (1992). Olefin epoxidation and alkane hydroxylation catalyzed by robust sulfonated manganese and iron porphyrins supported on cationic ion-exchange resins. Inorganic Chemistry, Vol. 31, 1999-2006.

Cantonetti, V.; Monti, D.; Venanzi, M.; Bombelli, C.; Ceccacci, F. \& Mancini, G. (2004). Interaction of a chirally functionalised porphyrin derivative with chiral micellar aggregates. Construction of a system with stereoselective cytochrome-P450 biomimetic activity. Tetrahedron: Asymmetry, Vol. 15(13), 1969-1977.

Castro, G.R. \& Knubovets T. (2003). Homogeneous biocatalysis in organic solvents and water-organic mixtures. Critical Reviews in Biotechnology, Vol. 23(3), 195 -231.

Chacon, J.; McLearie, J. \& Sinclair, R. (1988). Singlet oxygen yields and radical contributions in the dye-sensitised photo-oxidation in methanol of esters of polyunsatured fatty acids (oleic, linoleic, linolenic and arachidonic). Photochemistry and Photobiology, Vol. 47, 647-656.

Chang, C.J.; Loh, Z-H.; Shi, C.; Anson, F.C. \& Nocera, D.G. (2004). Targeted proton delivery in the catalyzed reduction of oxygen to water by bimetallic pacman porphyrins. Journal of the American Chemical Society, Vol. 126, 10013-10020.

Chatterjee, T. \& Bhattacharyya, D.K. (2001). Biotransformation of limonene by Pseudomonas putida. Applied Microbiology and Biotechnology, Vol. 55, 541-546. 
Chauhan, S. \& Kumari, P. (2007). Biomimetic oxidation of metribuzin with hydrogen peroxide catalyzed by 5,10,15,20-tetraarylporphyrinatoiron(III) chlorides. Tetrahedron Letters, Vol. 48(29), 5035-5038.

Chirvony, V.; van Hoek, A.; Galievsky,V.; Sazanovich, I.; Schaafsma, T.; \& Holten, D. (2000). Comparative Study of the Photophysical Properties of Nonplanar Tetraphenylporphyrin and Octaethylporphyrin Diacids. Journal of Physical Chemistry B Vol. 104(42), 9909-9917.

Collman, J.P.; Zhang, X.; Lee, V.J.; Uffelman, E. \& Brauman, J.I. (1993). Regioselective and Enantioselective Epoxidation Catalyzed by Metalloporphyrins. Science, Vol. 261, 1404-1411.

Dallacosta, C.; Alves W.; Ferreira, A.; Monzania, E. \& Casella, L. (2007). A new dinuclear heme-copper complex derived from functionalized protoporphyrin IX. Dalton Transactions, Vol. 21, 2197-2206.

Dargiewicz, J., Makarska, M., Radzki, St. (2002). Spectroscopic characterization of watersoluble cationic porphyrins in sol-gel silica matrices and coatings. Colloids and Surfaces A: Physicochemical and Engineering Aspects Vol. 208(1-3), 159-165.

Dargiewicz-Nowicka, J., Makarska, M., Villegas, M.A., Legendziewicz, J., Radzki, St. (2004). Photophysics of the porphyrins; unusual fluorescence of europium porphyrin complex entrapped in sol-gel silica matrix. Journal of Alloys and Compounds, Vol. 380(1-2), 380-388.

De la Luz, V.; García-Sánchez, M. \& Campero, A. (2007). Luminescent porphyrinosilica obtained by the sol-gel metod. Journal of Non-Crystalline Solids, Vol. 353(22-23), 2143-2149.

Delmaire, D., Meallet-Renault, R., Bied-Charreton, C., Pasternack, R.F. (1999). Incorporation of water-soluble porphyrins in sol-gel matrices and application to $\mathrm{pH}$ sensing. Analytica Chimica Acta, Vol. 401(1-2), 125-128.

Delmarre, D. \& Bied-Charreton, C. (2000). Grafting of cobalt porphyrins in sol-gel matrices: application to the detection of amines. Sensors and Actuators B, Vol. 62, 136-142.

DeRosa, M. \& Crutchley, R. (2002). Photosensitized singlet oxygen and its applications. Coordination Chemistry Reviews, Vol. 233-234, 351-371.

Doro, F.G.; Smith, J.R..; Ferreira, A.G. \& Assis, M..D. (2000). Oxidation of alkanes and alkenes by iodosylbenzene and hydrogen peroxide catalysed by halogenated manganese porphyrins in homogeneous solution and covalently bound to silica. Journal of Molecular Catalysis A-Chemical, Vol.164(1-2), 97-108.

Du, C-P.; Li, Z-K.; Wen, X-M.; Wu, J.; Yu, X-Q.; Yang, M. \& Xi, RG. (2004). Highly diastereoselective epoxidation of cholest-5-ene derivatives catalyzed by polymersupported manganese(III) porphyrins. Journal of Molecular Catalysis A: Chemical, Vol. 216, 7-12.

Duetz, W.; Bouwmeester, H.; van Beilen, J.B. \& Witholt, B. (2003). Biotransformation of limonene by bacteria, fungi, yeast, and plants. Applied Microbiology and Biotechnology, Vol. 61, 265-277.

Escribano, P.; Julián-López, B.; Planelles-Aragó, J.; Cordoncillo, E.; Viana, B. \& Sanchez, C. (2008). Photonic and Nanobiophotonic Properties of Luminescent LanthanideDoped Hybrid Organic-Inorganic Materials. Journal of Materials Chemistry, Vol. 18, 23-40. 
Estell, D.A.; Graycar, T.P. \& Wells, J.A. (1985). Engineering an enzyme by side-directed mutagenesis to be resistant to chemical oxidation. Journal of Biological Chemistry, Vol. 260(11), 6518-6521.

Evans, S. \& Lindsay Smith, J.R. (2001). The oxidation of ethylbenzene by dioxygen catalysed by supported iron porphyrins derived from iron(III) tetrakis(pentafluorophenyl) porphyrin. Journal of the Chemical Society, Perkin Transactions 2, Issue 2, 174-180.

Feiters, M.; Rowan, A. \& Nolte, R. (2000). From simple to supramolecular cytochrome P450 mimics. Chemical Society Reviews, Vol. 29(6), 375-384.

Funyu, S.; Isobe, T.; Takagi, S.; Tryk, D. \& Inoue, H. (2003). Highly effcient and selective epoxidation of alkenes by photochemical oxygenation sensitized by a ruthenium(II) porphyrin with water as both electron and oxygen donor. Journal of the American Chemical Society, Vol. 125, 5734-5740.

Garcia-Sanchez, M.A. \& Campero, A. (2004). Cobalt ortho- and para-subsituted tetraphenylporphyrins inserted in $\mathrm{SiO}_{2}$ gels. Journal of Non-Crystalline Solids, Vol. 333(2), 226-230.

Garcia-Sanchez, M.A.; De la Luz, V.; Estrada-Rico, M.L.; Murillo-Martinez, M.M.; CoahuilaHernandez, M.I.; Sosa-Fonseca, R.R.; Tello-Solis, S.R.; Rojas, F. \& Campero, A. (2009). Fluorescent porphyrins covalently bound to silica xerogel matrices. Journal of Non-Crystalline Solids, Vol. 355(2), 120-125.

Garcia-Sanchez, M.A.; Tello-Solis, S.R.; Sosa, R. \& Campero, A. (2006). Fluorescent porphyrins trapped in monolitic $\mathrm{SiO}_{2}$ gels. Journal of Sol-Gel Science and Technology, Vol. 37(2), 93-97.

Geier, G. \& Sasaki, T. (1997). The design, synthesis and characterization of a porphyrinpeptide conjugate. Tetrahedron Letters, Vol. 38(22), 3821-3824.

Gerdes, R.; Bartels, O.; Schneider, G.; Wöhrle, D. \& Schulz-Ekloff, G. (2001). Photooxidations of phenol, cyclopentadiene and citronellol with photosensitizers ionically bound at a polymeric ion exchanger. Polymers Advanced Technologies, Vol. 12, 152-160.

Gerdes, R.; Wöhrle, D.; Spiller, W.; Schneider, G.; Schnurpfei, G. \& Schulz-Ekloff, G. (1997). Photo-oxidation of phenol and monochlorophenols in oxygen-saturated aqueous solutions by different photosensitizers. Journal of Photochemistry and Photobiology A: Chemistry, Vol. 111(1), 65-74.

Gill, I. \& Ballesteros, A. (2000). Bioencapsulation within synthetic polymers (Part 1): Sol-gel encapsulated biologicals. Trends in Biotechnology, Vol. 18, 282-296.

Gonsalves, A.M; Johnstone, R.; Pereira, M.M.; Shaw, J. \& Sobral, A. (1991). Metal-assisted reactions. Part 22. Synthesis of perhalogenated prophyrins and their use as oxidation catalysts. Tetrahedron Letters, Vol. 32(10), 1355-1358.

Gonsalves, A.M. \&. Pereira, M.M. (1996). State of the art in the development of biomimetic oxidation catalysts. Journal of Molecular Catalysis A: Chemical, Vol. 113, 209-221.

Gouterman, M. (1961). Spectra of porphyrins. Journal of Molecular Spectroscopy, Vol. 6, 138163.

Grätzel, M. (2001). Sol-Gel processed $\mathrm{TiO}_{2}$ films for photovoltaic applications. Journal of SolGel Science and Technology, Vol. 22, 7-13.

Griesbeck, A. \& El-Idreesy, T. (2005). Solvent-free photooxygenation of 5-methoxyoxazoles in polystyrene nanocontainers doped with tetrastyrylporphyrine and protoporphyrine-IX. Photochemical and Photobiological Sciences, Vol. 4, 205-209. 
Griesbeck, A.; El-Idreesy, T. \& Bartoschek, A. (2004). Photooxygenation in Polystyrene Beads with Covalently and Non-Covalently Bound Tetraarylporphyrin Sensitizers. Advanced Synthesis \& Catalysis, 346(2-3), 245-251.

Griesbeck, A.G.; El-Idreesy, T.T. E Bartoschek, A. (2005). Photooxygenation in polymer matrices: En route to highly active antimalarial peroxides. Pure and Applied Chemistry, Vol. 77(6), 1059-1074.

Griffin, D.R.; Gainer J.L. \& Carta, G. (2001). Asymmetric ketone reduction with immobilized yeast in hexan: biocatalyst deactivation and regeneration. Biotechnology Progress, Vol. 17(2), 304-310.

Groves, J. \& Neumann, R. (1989). Regioselective oxidation catalysis in synthetic phospholipid vesicles. Membrane-spanning steroidal metalloporphyrins. Journal of the American Chemical Society, Vol. 111(8), 2900-2909.

Groves, J. (2000). Reactivity and mechanisms of metalloporphyrin- catalyzed oxidations. Journal of Porphyrins and Phthalocyanines, Vol. 4(4), 350-352.

Groves, J. (2003). The bioinorganic chemistry of iron in oxygenases and supramolecular assemblies. Proceedings of the National Academy of Science, Vol. 100(7), 3569-3574.

Groves, J.T. \& Kruper, W.J. (1979). Preparation and characterization of an oxoporphinatochromium(V) complex. Journal of American Chemical Society, Vol. 101(25), 7613-.7615.

Groves, J.; Haushalter, R.; Nakamura, M.; Nemo, T. \& Evans, B. (1981). High-valent ironporphyrin complexes related to peroxidase and cytochrome P-450. Journal of the American Chemical Society, Vol. 103(10), 2884-2886.

Groves, J.T.; Shalyaev, KV.; Bonchio M. \& Carofiglio T. (1997). Rapid catalytic oxygenation of hydrocarbons with perhalogenated ruthenium porphyrin complexes. Studies in Surface Science and Catalysis, Vol. 110, 865-872.

Guo, C-C.; Yang W-J. \& Mao, Y-L. (2005). Selectively aerobic oxidation of C=C and allylic $\mathrm{C}-\mathrm{H}$ bonds in a-pinene over simple metalloporphyrins. Journal of Molecular Catalysis A: Chemical, Vol. 226(2), 279-284.

Haber, J.; Pamin, K. \& Połtowicz, J. (2004). Cationic metalloporphyrins and other macrocyclic compounds in zeolite matrix as catalysts for oxidation with dioxygen. Journal of Molecular Catalysis A: Chemical, Vol. 224(1-2), 153-159.

Hao, E. (2007). Syntheses and evaluation of porphyrin derivatives for applications in medicine and in material science. Ph.D. Dissertation: Louisiana State University, USA.

Hartmmann, P.; Collet, A. \& Viguier, M. (1999). Synthesis and characterization of model fluoroacylated poly(ethylene oxide). Journal of Fluorine Chemistry, Vol. 95, 145-151.

Henning, H.; Rehorek, D.; Stich, R. \& Weber, L. (1990). Photocatalysis induced by lightsensitive coordination compounds. Pure and Applied Chemistry, Vol. 62(8), 14891494.

Hessenauer-Ilicheva, N.; Franke, A.; Meyer, D.; Woggon, W. \& van Eldik, R. (2007). Lowtemperature rapid-scan detection of reactive intermediates in epoxidation reaction catalyzed by a new enzyme mimic of cytochrome p450. Journal of the American Chemical Society, Vol. 129(41), 12473-12479.

Holland, B.T.; Walkup, C. \& Stein, A. (1998). Encapsulation, Stabilization and Catalytic Properties pf Flexible Metal Porphyrin Complexes in MCM-41 with Minimal Electronic Perturbation by the Environment. Journal of Physical Chemistry B, Vol. 102(22), 4301-4309. 
Huanga, G.; Guo, C-C. \& Tang, Si-Si. (2007). Catalysis of cyclohexane oxidation with air using various chitosan-supported metallotetraphenylporphyrin complexes. Journal of Molecular Catalysis A: Chemical, Vol. 261, 125-130.

Hult K. \& Berglund P. (2007). Enzyme promiscuity: mechanism and applications. Trends Biotechnology, Vol. 25, 231-238.

Hurst, J.; McDonald, J. \& Schuster, G. (1982). Lifetime of singlet oxygen in solution directly determined by laser spectroscopy. Journal of the American Chemical Society, Vol. 104(7), 2065-2067.

Iliev, V.; Prahov, L.; Bilyarska, L.; Fischer, H.; Schulz-Ekloff, G.; Wöhrle, D. \& Petrov, L. (2000). Oxidation and photooxidation of sulfide and thiosulfate ions catalyzed by transition metal chalcogenides and phthalocyanine complexes. Journal of Molecular Catalysis A: Chemical, Vol. 151(1-2), 161-169.

Im, S.; Khalil, G.; Callis, J.; Ahn, B.; Gouterman. M. \& Xia, Y. (2005). Synthesis of polystyrene beads loaded with dual luminophors for self-referenced oxygen sensing. Talanta, Vol. 67(3), 492-497.

Inbaraj, J.J.; Vinodu, M.V.; Gandhidsan, R.; Murugesan, R. \&. Padmanabhan, M. (2003). Photosensitizing properties of ionic porphyrins immobilized on functionalized solid polystyrene support. Journal of Applied Polymer Science, Vol. 89(14), 3925-3930.

Ito, A.; Konishi, K. $\mathcal{E}$ Aida, T. (1996). Free bases of chiral $\mathrm{N}$-substituted porphyrins as catalysts for asymmetric reaction. Tetrahedron Letters, Vol. 37(15), 2585-2588.

Itoh, T.; Yano, K.; Inada, Y. \& Fukushima, Y. (2002). Photostabilized Chlorophyll $a$ in a Mesoporous Silica:Adsorption Properties and Photoreduction Activity of Chlorophyll a. Journal of the American Chemical Society, Vol. 124(45), 13437-13441.

Jasat, A. \& Dolphin, D. (1997). Expanded porphyrins and their heterologs. Chemical Reviews, Vol. 97(6), 2267-2340.

Jin, S.; Makris, T.; Bryson, T.; Sligar, S. \& Dawson, J. (2003). Epoxidation of olefins by hydroperoxo-ferric cytochrome P450. Journal of the American Chemical Society, Vol. 125(12), 3406- 3407.

Kadish, K.M.; Smith, K. M.; Guilard, R. (eds.), "The Porphyrin Handbook", Academic Press, San Diego, 2000, Vol. 4.

Kamiya, N.; Furusaki, S. \& Goto, M. (1997). Peroxidase activity and stability of surfactantheme complex in nonaqueous media. Biotechnology Letters, Vol. 19(10), 1015-1018.

Kandimalla, V.; Tripathi, V. \& Ju, H. (2006). Immobilization of Biomolecules in Sol-Gels: Biological and Analytical Applications. Critical Reviews in Analytical Chemistry, Vol. 36, 73-106.

Kane, K.M.; Lorenz, C.R.; Heilman, D.M. \& Lemke, F.R. (1998). Substituent effects on the spectroscopic properties and reactivity of hexacoordinate silicon(IV) porphyrin complexes. Inorganic Chemistry, Vol. 37(4), 669-673.

Karapire, C.; Kus, M.; Turkmen, G.; Trevithick-Sutton, C.; Foote, C. \& Icli, S. (2005). Photooxidation studies with perylenediimides in solution, PVC and sol-gel thin films under concentrated sun light. Solar Energy, Vol. 78, 5-17.

Kato, S.; Yang, H.; Ueno, T.; Ozaki, S.; George, J.; Phillips, N.; Fukuzumi, S.; Watanabe, Y. (2002). Asymmetric sulfoxidation and amine binding by H64D/V68A and H64D/V68S Mb: Mechanistic insight into the chiral discrimination step. Journal of the American Chemical Society, Vol. 124(29), 8506-8507.

Kenney, R. \& Fisher, G. (1973). Preparation of trans-Pinocarveol and Myrtenol. Industrial \& Engineering Chemistry Produsts Research Development, Vol. 12(4), 317-319. 
Kholdeeva, O.A.; Zalomaeva, O.V.; Sorokin, A.B.; Ivanchikova, I.D.; Della Pina, C. \& Rossi, C.M. (2007). New routes to Vitamin $K_{3}$. Catalysis Today, Vol. 121, 58-64.

Kim, W.; Park, J.; Jo, HJ.; Kim, H-J. \& Choi, W. (2008). Visible Light Photocatalysts Based on Homogeneous and Heterogenized Tin Porphyrins. Journal of Physical Chemistry C, Vol. 112, 491-499.

Kitamura, N.; Yamada, K.; Ueno, K. \& Iwata, S. (2006). Photodecomposition of phenol by silica-supported porphyrin derivative in polymer microchannel chips. Journal of Photochemistry and Photobiology A: Chemistry, Vol. 184, 170-176.

Krings, U. \& Berger R.G. (1998). Biotechnological production of flavors and fragrances. Applied Microbiology and Biotechnology, Vol. 49, 1-8.

Krishna, C.; Uppuluri, S.; Riesz, P.; Zigler, J. \& Balasubramanian, D. (1991) A study of the photodynamic efficiencies of some eye lens constituents. Photochemistry and Photobiology, Vol. 54, 51-58.

Lan, E.; Dave, B.; Fukuto, J.; Dunn, B.; Zink J. \& Valentine, J. (1999). Synthesis of sol-gel encapsulated heme proteins with chemical sensing properties. Journal of Materials Chemistry, Vol. 9, 45-53.

Lee, S.K. \& Okura, I. (1997). Porphyrin-doped sol-gel glass as a probe for oxygen sensing. Analytica Chimica Acta, Vol. 342(2-3), 181-188.

Li, Z.; van Beilen, J.B.; Duetz, W.A.; Schmid, A.; de Raadt, A.; Griengl, H. \& Witholt, B. (2002). Oxidative biotransformations using oxygenases. Current Opinion in Chemical Biology, Vol. 6(2), 136-144.

Lim, J.; Yoon, Z.; Shin, J.; Kim, K.; Yoon, M. \& Kim, D. (2009). The photophysical properties of expanded porphyrins: relationships between aromaticity, molecular geometry and non-linear optical properties. Chemical Communications, Vol. 3, 261-273.

Lisowski, J.; Sessler, J.L. \& Mody, T.D. (1995). ${ }^{13} \mathrm{C}$ and ${ }^{31 P}$ NMR study of paramagnetic lanthanide (III) texaphyrins. Inorganic Chemistry, Vol. 34(17), 4336-4342.

Maldotti, A.; Bartocci, C.; Varani, G.; Molinari, A.; Battioni, P. \& Mansuy, D. (1996). Oxidation of cyclohexane by molecular oxygen photoassisted by mesotetrarylporphyrin iron(III)-hydroxo complexes. Inorganic Chemistry, Vol. 35, 11261131.

Mancini, G.; Monti, D. \& Pastorini, A. (2001). Selectivity in the Oxidation of Limonene by Amphiphilized Metalloporphyrins in Micellar Media. Langmuir, Vol. 17(23), 71987203.

Mandon, D.; Weiss, R.; Franke, M.; Bill, E. \& Trautwein A. (1989). Oxoiron porphyrin species with high-valent iron: formation by solvent-dependent protonation of a peroxoiron(III) porphyrinate derivative. Angewandte Chemie International Edition, Vol. 28(12), 1709-1711.

Mansuy, D. (1990). Biomimetic catalysts for selective oxidation in organic chemistry. Pure and Applied Chemistry, Vol. 62(4), 741-746.

Mansuy, D. \& Fontecave, M. (1984). Monooxygenase-like oxidations of olefins and alkanes catalyzed by manganese porphyrins: comparison of systems involving either $\mathrm{O}_{2}$ and ascorbate or iodosylbenzene. Tetrahedron, Vol. 40(1-2), 253-254.

Maraval, V.; Ancel, J. \& Meunier, B. (2002). Manganese(III) porphyrin catalysts for the oxidation of terpene derivatives: a comparative study. Journal of Catalysis, Vol. 206(2), 349-357. 
Margaros, I.; Montagnon, T.; Tofi, M.; Pavlakos, E. \& Vassilikogiannakis G. (2006). The power of singlet oxygen chemistry in biomimetic syntheses. Tetrahedron, Vol. 62, 5308-5317.

Martins, R.; Neves, M.; Silvestre, A.; Simões, M.; Silva, A.; Tomé, A.; Cavaleiro, J.; Tagliatesa, P. \& Crestini, C. (2001). Oxidation of unsaturated monoterpenes with hydrogen peroxide catalysed by manganese(III) porphyrin complexes. Journal of Molecular Catalysis A: Chemical, Vol. 172(1-2), 33-42.

Mele, G.; Del Sole, R.; Vasapollo, G.; García-López, E.; Palmisano, L. \& Schiavello, M. (2003). Photocatalytic degradation of 4-nitrophenol in aqueous suspension by using polycrystalline $\mathrm{TiO}_{2}$ impregnated with functionalized $\mathrm{Cu}(\mathrm{II})$-porphyrin or $\mathrm{Cu}(\mathrm{II})$ phthalocyanine. Journal of Catalysis, Vol. 217, 334-342.

Meunier, B. (1992). Metalloporphyrins as versatile catalysts for hydrocarbon oxygenations and oxidative DNA cleavage. Chemical Reviews, Vol. 92, 1411-1456.

Meunier, B. (ed.), "Biomimetic Oxidations Catalysed by Transition Metal Complexes", Imperial College Press, London, 2000.

Meunier, B.; de Visser, S.P. \& Shaik, S. (2004). Mechanism of oxidation reactions catalyzed by cytochrome P450 enzymes. Chemical Reviews, Vol. 104(9), 3947-3980.

Meyer, D.; Leifels, T.; Sbaragli, L. \& Woggon, W. (2005). Reactivity of a new class of P450 enzyme models. Biochemical and Biophysical Research Communications, Vol. 338(1), 372-377.

Milaeva, E.R.; Gerasimova, O.A.; Maximov, A.L.; Ivanova, E.A.; Karachanov, E.A.; Hadjiliadis, N. \& Louloudi, M. (2007). The catalytic activity of immobilized on modified silica metalloporphyrins bearing antioxidative 2,6-di-tert-butylphenol pendants. Catalysis Communications, Vol. 8(12), 2069-2073.

Moghadam, M.; Tangestaninejad, S.; Habibi, M.H. \& Mirkhani, V. (2004) A convenient preparation of polymer-supported manganese porphyrin and its use as hydrocarbon monooxygenation catalyst. Journal of Molecular Catalysis A: Chemical, Vol. 217(1-2), 9-12.

Montanari, F. \& Casella L. (Eds.), Metalloporphyrins Catalysed Oxidations, Kluwer Academic Publishers, Dordrecht, 1994.

Moreira, MSM.; Martins, P.R.; Curi, R.B.; Nascimento, O.R. \& Iamamoto, Y. (2005). Iron porphyrins immobilised on silica surface and encapsulated in silica matrix: a comparison of their catalytic activity in hydrocarbon oxidation. Journal of Molecular Catalysis A: Chemical, Vol. 233, 73-81.

Mukherjee, M. \& Ray, A. (2007). Biomimetic oxidation of l-arginine with hydrogen peroxide catalyzed by the resin-supported iron (III) porphyrin. Journal of Molecular Catalysis A: Chemical, Vol. 266(1-2), 207-214.

Murahashi, S-I. \& Komiya, N. (1998). New types of catalytic oxidations in organic synthesis. Catalysis Today, Vol. 41(4), 339-349.

Murata, S.; Hata, H.; Kimura, T.; Sugahara, Y.; Kuroda, K. (2000). Effective adsorption of chlorophyll $a$ by FSM-type mesoporous silica modified with 1,4-Butanediol. Langmuir, Vol. 16(18), 7106-7108.

Nakagaki, S.; Benedito, F. \& Wypych, F. (2004). Anionic iron(III) porphyrin immobilized on silanized kaolinite as catalyst for oxidation reactions. Journal of Molecular Catalysis A: Chemical, Vol. 217, 121-131.

Nam, W. (2007). High-valent iron(IV)-oxo complexes of heme and non-heme ligands in oxygenation reactions. Accounts of Chemical Research, Vol. 40(7), 522-531. 
Newcomb, M. \& Toy, H. (2000). Hypersensitive Radical Probes and the Mechanisms of Cytochrome P450-Catalyzed Hydroxylation Reactions. Accunts of Chemical Research, Vol. 33(7), 449-455.

Nierengarten, J.F.; Schall, C. \& Nicoud, J.F. (1998). A tetraphenylporphyrin with four fullerene substituents. Angewandte Chemie International Edition, Vol. 37(13/14), 19341936.

Nimri, S. \& Keinan, E. (1999). Antibody-Metalloporphyrin Catalytic Assembly Mimics Natural Oxidation Enzymes. Journal of the American Chemical Society. Vol. 121(91), 8978-8982.

Oligario, F.; de Visser, S.; Cohen, S.; Sharma, P. \& Shaik, S. (2002). Searching for the second oxidant in the catalytic cycle of cytochrome P450: A theoretical investigation of the iron(III)-hydroperoxo species and its epoxidation pathways. Journal of the American Chemical Society, Vol. 124, 2806-2817.

Oulmi, D.; Maillard, P.; Vever-Bizet, C. \& Brault, D. (1989). Glycosylated porphyrins: characterisation of association in aqueous solutions by absorption and fluorescence spectroscopies and determination of singlet oxygen yield in organic media. Photochemistry and Photobiology, Vol. 67(5), 511-518.

Paduch, R.; Kandefer-Szerszeń, M.; Trytek, M. \& Fiedurek, J. (2007). Terpenes - substances useful in human health care. Archivum Immunologiae et Therapiae Experimentalis, Vol. $55,315-327$.

Paolesse, R.; Monti, D.; Monica, L.; Venanzi, M.; Froiio, A.; Nardis, S.; DiNatale, C.; Martinelli, E. \& D'Amico, A. (2002). Preparation and self-assembly of chiral porphyrin diads on the gold electrodes of quartz crystal microbalances: A novel potential approach to the development of enantioselective chemical sensors. Chemistry - A European Journal, Vol. 8(11), 2476-2483.

Papacídero, AT.; Rocha, LA.; Caetano, BL.; Molina, E.; Sacco, HC.; Nassar, EJ.; Martinelli, Y.; Mello, C.; Nakagaki, S. \& Ciuffi, KJ. (2006). Preparation and characterization of spherical silica-porphyrin catalysts obtained by the sol-gel methodology. Colloids and Surfaces A: Physicochemical and Engineering Aspects, Vol. 275, 27-35.

Papkovsky, D.; O'Riordan, T. \& Soini, A. (2000). Phosphorescent porphyrin probes in biosensors and sensitive bioassays. Biochemical Society Transactions, Vol. 28, 74-77.

Penner-Hahn, J.E.; Eble, K.S.; McMurry, T.J.; Renner, M.; Balch, A.L.; Groves, J.T.; Dawson, J.H. \&. Hodgson, K.O. (1986). Structural characterization of horseradish peroxidase using EXAFS spectroscopy. Evidence for $\mathrm{Fe}=\mathrm{O}$ ligation in compounds I and II. Journal of American Chemical Society. Vol. 108(24), 7819-7825.

Pereira, M.R.; Ferreira, J.A.; Hungerford, G. (2005). Photophysics of mesoporphyrin IX in solution and confined in sol-gel-derived matrices. Journal of Photochemistry and Photobiology A: Chemistry, Vol. 172(1), 7-17.

Pickenhagen, W. E Schatkowski, D. (1998). (Dragoco Gerberding \& Co AG) Ger. Offen. DE 19645922 A1.

Podbielska, H.; Bindig, U.; Ulatowska-Jarza, A.; Hołowacz, I.; Müller, G. \& Scheller, E. (2006). Optical properties of sol-gel fiber optic applicators for laser interstitial therapy. Laser Physics, Vol. 16(5), 816-826.

Polska, K., Radzki, St. (2008). Spectral and AFM characterization of trimethylammoniophenylporphyrin and concanavalin A associate in solution and monolithic $\mathrm{SiO}_{2}$ gels obtained by the sol-gel method. Optical Materials, Vol. 30(10), 1644-1654. 
Poulos, N.; Finzel, B. \& Howard, P. (1987). High-resolution crystal structure of cytochrome P450cam. Journal of Molecular Biology, Vol. 195(3), 687-700.

Purrello, R.; Raudino, A.; Scolaro, L.M.; Loisi, A.; Bellacchio, E. \& Lauceri, R. (2000). Ternary porphyrin aggregates and their chiral memory. Journal of Physical Chemistry B, Vol. 104, 10900-10908.

Que, L. \& Tolman, W. (2008). Biologically inspired oxidation catalysis. Nature, Vol. 455, 333340.

Quici, S.; Banfi, S.; Pozzi, G. (1993). Simple synthetic models of cytochrome P-450: efficient catalyst in hydrocarbon oxidation. Gazzetta Chimica Italiana, Vol. 123, 597-612.

Ray, AK.; Tracey, SM.; Hodgson, SN. (2001). Photoelectric measurements on chloroaluminium phthalocyanine/titanium oxide heterojunctions. Journal of Sol Gel Science and Technology, Vol. 22, 15-22.

Rebelo, S.L.; Simões, M.M.; Neves, G.M. \& Cavaleiro, J.A. (2003). Oxidation of alkylaromatics with hydrogen peroxide catalysed bymanganese(III) porphyrins in the presence of ammonium acetate. Journal of Molecular Catalysis A: Chemical, Vol. 201, 9-22.

Rebelo, S.L.; Simões, M.M; Neves, G.M.; Silva, A.; Tagliatesta, P. \& Cavaleiro, J.A. (2005). Oxidation of bicyclic arenes with hydrogen peroxide catalysed by manganese(III) porphyrins. Journal of Molecular Catalysis A: Chemical, Vol. 232, 135-142.

Reisfeld, R. (2001). Prospects of sol-gel technology towards luminescent materials. Optical Materials, Vol. 16, 1-7.

Ribeiro, S.; Serra, A. \& Gonsalves, A. (2008). Immobilised porphyrins in monoterpene photooxidations. Journal of Catalysis, Vol. 256, 331-337.

Ricoux R.; Raffy, Q. \& Mahy JP. (2007). New biocatalysts mimicking oxidative hemoproteins: Hemoabzymes. Comptes Rendus Chimie, Vol. 10, 684-702.

Robertson, D.; Farid, R.; Moser, C.; Urbauer, J.; Mulholland, S.; Pidikiti, R.; Lear, J.; Wand, A.; DeGrado W. \& Dutton P. (1994). Design and synthesis of multi-haem proteins. Nature, Vol. 368, 425 - 432.

Rogers, J.C.; Dickerson, T.J; Wentworth Jr., P.; \& Janda, K.D. (2005). A high-swelling reagent scaffold suitable for use in aqueous and organic solvents. Tetrahedron, Vol. 61, 12140-12144.

Rosenthal, J.; Luckett, T.; Hodgkiss, J. \& Nocera, D. (2006). Photocatalytic oxidation of hydrocarbons by a bis-iron(III)-l-oxo pacman porphyrin using $\mathrm{O}_{2}$ and visible light. Journal of the American Chemical Society, Vol. 128, 6546-6547.

Rothlisberger, D.; Khersonsky, O.; Wollacott, A.M.; Jiang, L.; DeChancie, J.; Betker, J.; Gallaher, J.L.; Althoff, E.A.; Zangehellini, A.; Dym, O.; Albeck, S.; Houk, K.N.; Tawfik, D.S. \& Baker, D. (2008). Kemp elimination catalysts by computational enzyme design. Nature, Vol. 453, 190-195.

Santos, I.; Rebelo, S.; Balula, M.; Martins, R.; Pereira, M.; Simões, M.; Neves, M.; Cavaleiro, J. \& Cavaleiro, A. (2005). Association of Keggin-type anions with cationic mesosubstituted porphyrins: synthesis, characterization and oxidative catalytic studies. Journal of Molecular Catalysis A: Chemical, Vol. 231, 35-45.

Savithiry, N.; Gage, D.; Fu, W. \& Oriel, P. (1998). Degradation of pinene by Bacillus pallidus BR425. Biodegradation, Vol. 9(5), 337-341.

Schenning, A.; Benneker, F.B.; Geurts, H.P.; Liu, X.Y. \& Nolte, R.J. (1996). Porphyrin wheels. Journal of the American Chemical Society, Vol. 118, 8549-8552. 
Schmidt, J.; Lobkovsky, E. \& Coates, G. (2005). Chromium(III) octaethylporphyrinato tetracarbonylcobaltate: a highly active, selective, and versatile catalyst for epoxide carbonylation. Journal of the American Chemical Society, Vol. 127(32), 11426-11435.

Sessler, JL.; Hemmi, G.; Mody, TD., Murai, T.; Burrell, A. \& Young, SW. (1994). Texaphyrins: synthesis and applications. Accounts of Chemical Research, 27(2), 43-50.

Shteinman, A. (2001). The role of metal-oxygen intermediates in biological and chemical monooxygenation. Russian Chemical Bulletin, International Edition, Vol. 50(10), 17951810.

Simões, M.; Neves, M. \& Cavaleiro, J. (2006). Metalloporphyrins as catalysts in the oxidation of terpenes. Jordan Journal of Chemistry, 1(1), 1-12.

Simonneaux, G. \& Maux, P. (2002). Optically active ruthenium porphyrins: chiral recognition and asymmetric catalysis. Coordination Chemistry Reviews, 228(1), 43-60.

Skrobot, F.C.; Valente, A.A.; Neves, G.; Rosa, I.; Rocha, J. E Cavaleiro, J.A.S. (2003). Oxidation of aromatic monoterpenes with monoterpenes oxidation in the presence of Y zeolite-entrapped manganese(III) tetra(4- $N$-benzylpyridyl)porphyrin. Journal of Molecular Catalysis A: Chemical, Vol. 201, 211-222.

Sligar, S.; Makris, T. \& Denisov, I. (2005). Thirty years of microbial P450 monooxygenase research: Peroxo-heme intermediates - The central bus station in heme oxygenase catalysis. Biochemical and Biophysical Research Communications, Vol. 338, 346-354.

Sorokin, A.B.; Mangematin, S. \& Pergrale, C., J. (2002). Selective oxidation of aromatic compounds with dioxygen and peroxides catalyzed by phthalocyanine supported catalysts. Journal of Molecular Catalysis A: Chemical, Vol. 182-183, 267-281.

Sun, X.D; Wang, X-J.; Shan, W.; Song, JJ.; Fan, MG. \& Knobbe, ET. (1997). Nonlinear effects in chromophore doped sol-gel photonic materials. Journal of Sol-Gel Science and Technology, Vol 9(2), 169-181.

Suslick, K.S. (1999). Shape-Selective oxidation by metalloporphyrins, In: The Porphyrin Handbook; Kadish, K.; Smith, K.; Guillard, R., eds., Academic Press: New York, pp. 41-60.

Suzuki, N.; Higuchi T.; Urano, Y.; Kikuchi, K.; Uekusa, H.; Ohashi, Y.; Uchida, T.; Kitagawa, T. \& Nagano, T. (1999). Novel iron porphyrin-alkanethiolate complex with intramolecular $\mathrm{NH} \cdots \mathrm{S}$ hydrogen bond:synthesis, spectroscopy, and reactivity. Journal of the American Chemical Society, Vol. 121(49), 11571-11572.

Takagi, S.; Tryk, D. \& Inoue, H. (2002). Photochemical Energy Transfer of Cationic Porphyrin Complexes on Clay Surface. Journal of Physical Chemistry B, Vol. 106(21), 5455-5460.

Takahashi, K; Matsushima, A.; Saito, Y. \& Inada, Y. (1986). Polyethylene glycol-modified hemin having peroxidase activity in organic solvents. Biochemical and Biophysical Research Communications, Vol. 138(1), 283-288.

Tanielian, C.; Schweitzer, C.; Mechin, R. \& Wolff, C. (2001). Quantum yield of singlet oxygen production by monomeric and aggregated forms of hematoporphyrin derivative. Free Radical Biology \& Medicine, 30(2), 208-212.

Tao, H. \& Cornisch. V.W. (2002). The milestones in directed enzyme evolution. Current Opinion in Chemical Biology, Vol. 6(6), 858-864.

Thomas, J.M. \& Raja, R. (2005). Designing catalysts for clean technology, green chemistry, and sustainable development. Annual Review of Materials Research, Vol. 35, 315-350.

Traylor, T. \& Tsuchiya, S. (1987). Perhalogenated tetraphenylhemins: stable catalysts of high turnover catalytic hydroxylations. Inorganic Chemistry, 26(8), 1338-1339. 
Trytek, M., \& Fiedurek, J. (2002). Biotransformation of d-limonene to carvone by means of glucose oxidase and peroxidase. Acta Microbiologica Polonica, Vol. 51, 57-62.

Trytek, M. \& Fiedurek, J. (2005). A novel psychrotrophic fungus, Mortierella minutissima, for D-limonene biotransformation. Biotechnology Letters, Vol. 27, 149-153.

Trytek, M., Fiedurek, J. \& Radzki, St. (2007). A Novel Porphyrin-Based Photocatalytic System for Terpenoids Production from $(R)-(+)-L i m o n e n e . ~ B i o t e c h n o l o g y ~ P r o g r e s s$, Vol. 23, 131-137.

Trytek, M.; Majdan, M.; Lipke, A. \& Fiedurek, J. Manuscript in preparation.

Trytek, M., Fiedurek, J., Lipke, A., Radzki, St. (2009). Porphyrins incorporated to $\mathrm{SiO}_{2}$ gels as fluorescent materials and efficient catalysts in biomimetic photocatalytic system. Journal of Sol-Gel Science and Technology, Vol. 51(3), 272-286.

Turner, N.J. (2009). Directed evolution drives the next generation of biocatalysts. Nature Chemical Biology, Vol. 5, 567 - 573.

van de Velde, F., van Rantwijk, F., Sheldon, R. (2001). Improving the catalytic performance of peroxidases in organic synthesis. Trends of Biotechnology, Vol. 19(2), 73-80.

Vaz, A.; McGinnity G. \& Coon, M. (1998). Epoxidation of olefins by cytochrome P450: Evidence from site-specific mutagenesis for hydroperoxo-iron as an electrophilic oxidant. Proceedings of the National Academy of Science, Vol. 95, 3555-3560.

Wackett, L.P. (2004). Novel biocatalysis by database mining. Current Opinion in Biotechnology, Vol. 15(4), 280-284.

Watanabe Y. (2001). Alternatives to the oxoferryl porphyrin cation radical as the proposed reactive intermediate of cytochrome P450: two-electron oxidized Fe(III) porphyrin derivatives. Journal of Biological Inorganic Chemistry, Vol. 6(8), 846-856.

Watanabe, Y. In: The Porphyrin Handbook, eds. Kadish, K.; Smith, K. \& Guilard Vol. 4, Academic Press, New York, London, 2000, pp. 97.

Weber, L.; Hommel, R.; Behling, J.; Haufe, G. \& Henning, H. (1994). Photocatalytic oxygenation of hydrocarbons with (tetraarylporphyrinato)iron(III) complexes and molecular oxygen. Comparison with microsomal cytochrome P-450 mediated oxygenation reactions. Journal of the American Chemical Society, Vol. 116(6), 24002408.

Woggon, W-D. (2010). Metal complexes covalently linked to $\beta$-cyclodextrin. Current Organic Chemistry, Vol. 14(13), 1362-1379.

$\mathrm{Xu}$, W., Guo, H., Akins, D. (2001). Aggregation of tetrakis(p-sulfonatophenyl)porphyrin within modified mesoporous MCM-41. Journal of Physical Chemistry B, Vol. 105(8), 1543-1546.

Yoo, S. \& Day, D. (2002). Bacterial metabolism of a- and $\beta$-pinene and related monoterpenes by Pseudomonas sp. strain PIN. Process Biochemistry, Vol. 37(7), 739-745.

Yoshida, A., Kakegawa, N., Ogawa, M. (2003). Adsorption of a cationic porphyrin onto mesoporous silicas. Research on Chemical Intermediates, Vol. 29(7-9), 720-731.

Yuchun, X.; Das, P. \& Klibanov, A. (2001). Excipients activate peroxidases in specific but not in non-specific reactions in organic solvents. Biotechnology Letters, Vol. 23(18), 14511454. 


\title{
Physicochemical Peculiarities of Iron Porphyrin - Containing Electrodes in Catalase - and Peroxidase - Type Biomimetic Sensors
}

\author{
T.M.Nagiev \\ Institute of Chemical Problems, National Academy of Sciences of Azerbaijan, Baku, \\ Azerbaijan
}

\section{Introduction}

High sensitivity and substrate specificity of sensors based on biological systems make them irreplaceable as applied measuring devices. Unfortunately, their high sensitivity to environmental impacts, short operation time and high price are the main disadvantages of the biological systems. If the active component of biosensors (biopolymers) is replaced by their chemical analogs, i.e. biomimics which are now being developed in the framework of mimetic catalysis [1], the majority of these and other disadvantages can be eliminated.

Biomimetic analogs of existing enzymatic biosensors have been designed with broader functional abilities using inorganic biomimics, which is one of the strategic directions in the branch of super-sensitive analytical systems. The development of model systems is the only means of succeeding in this direction. Following achievements in mimetic catalysis, biomimetic electrodes of the catalase, peroxidase and monooxygenase type have been effectively designed [2].

At present, enzymatic and enzyme-based biosensor methods $[3,4]$ are widely employed for qualitative and quantitative determinations of lower aliphatic alcohols, in particular, methanol-ethanol mixtures. Biosensors have some obvious advantages: preliminary separation of the sample components is not required; they are highly selective and sensitive; the determinations with their use are fast and do not require complicated apparatus; and they are economical. Sensors based on biological materials (enzymes, cells, tissues, antibodies, receptors, nucleic acids, etc.) have, however, some substantial disadvantages, which, as a rule, restrict their practical applications. These are high sensitivity to the action of the environment, short service time, high cost, the occasional application of complex enzymatic systems, multistep determinations, and some others.

Advances in the field of mimetic catalysis allow synthesis of the biomimetic analogues of corresponding enzymes. Their use in sensors will avoid many of the drawbacks mentioned above.

In this work, we used, as an example, a model peroxidase reaction of oxidation of ethanol into acetaldehyde in the electrochemical regime to elaborate the basic principles of functioning of peroxidase-mimetic sensor devices.

The phenomenon of $\mathrm{H}_{2} \mathrm{O}_{2}$ synthesis as an intermediate product of substrate biooxidation with the participation of many oxidases has formed the foundation for the creation and development of many biosensors possessing immobilized peroxidase as the active substance [5]. 
In general, electrochemically realized biological processes, in which the central place is devoted to $\mathrm{H}_{2} \mathrm{O}_{2}$ synthesis and dissociation reactions, can be presented as follows:

$$
\begin{aligned}
& \mathrm{S} \text { (glucose, amino acids) }+\mathrm{O}_{2} \stackrel{\substack{\text { oxidase } \\
\downarrow}}{\longrightarrow} \mathrm{S}^{\prime}+\mathrm{H}_{2} \mathrm{O}_{3} \\
& \mathrm{H}_{2} \mathrm{O}_{2} \underset{\substack{\uparrow \\
\text { peroxidase }}}{\longrightarrow} \mathrm{H}_{2} \mathrm{O}+2 \bar{e}
\end{aligned}
$$

As a rule, the combined immobilization of oxidase and peroxidase on the electrode allows the determination of metabolite $(S)$ concentration, even if negligibly small.

Additional sufficient capabilities also occur as a result of the chemical conjugation mechanism, on which the overwhelming majority of enzymatic reactions are based. There is a firm belief that, in principle, a sequence of conjugated chemical processes can be selected for any reagent, starting from the substance to be detected and ending with an active product of enzymatic reaction. To put it another way, the corresponding enzymatic electrode may always be prepared.

The need to develop concise methods for determining the concentration of $\mathrm{C}_{2} \mathrm{H}_{5} \mathrm{OH}$ in aqueous solutions arises from the requirements imposed by the quality control of alcohol drinks.

Another reason for developing these sensors is for detecting microquantities of $\mathrm{C}_{2} \mathrm{H}_{5} \mathrm{OH}$ in aqueous solutions of various origins.

The authors devoted their investigations to the development of a peroxidase-biomimetic sensor for determining trace quantities of ethyl alcohol in various solutions. In all tests the reaction system represented a mixture of microamounts of hydrogen peroxide and ethyl alcohol in an aqueous medium. The task was to determine the effect of the $\mathrm{H}_{2} \mathrm{O}_{2}: \mathrm{C}_{2} \mathrm{H}_{5} \mathrm{OH}$ ratio on the detection ability of the biomimetic electrode.

Figure 1. shows an electrochemical system - a model of a catalase-biomimetic sensor, consisting of the reference electrode $\left(\mathrm{Ag} / \mathrm{AlCl} / \mathrm{Cl}^{-}\right)$and biomimetic electrode. In this system, the electrochemical potential changed as a result of mimetic electrode interaction with $\mathrm{H}_{2} \mathrm{O}_{2}$ injected into the system. Initially, the system potential is measured in twice distilled water (the background solution) and then, as a definite amount of $\mathrm{H}_{2} \mathrm{O}_{2}$ is added, a change of aqueous $\mathrm{H}_{2} \mathrm{O}_{2}$ potential is observed.

\section{Experimental, results and discussion}

Biomimetic electrode was prepared according to two techniques:

1. inorganic biomimic-catalase adhesion to aluminum foil;

2. based on the active fragment of catalase (the most complicated way to obtain the working element of biomimetic sensor) [6].

A biomimetic sensor was created with electrodes from aluminum wire ( $2 \mathrm{~mm}$ thick) and aluminum foil (size $20 \times 10 \times 1 \mathrm{~mm}$ ), to which the working element was applied by two methods:

1. on an adhesive covering the electrode with a thin layer;

2. mixed with adhesive.

In order to obtain a fragment of catalase, it was immobilized by adsorption on various organic and inorganic carriers (such as aluminum oxide, diasorb DEAE, agarose), with farther trypsine treatment. Aluminum wire or foil was used as the electrode. Such electrodes 


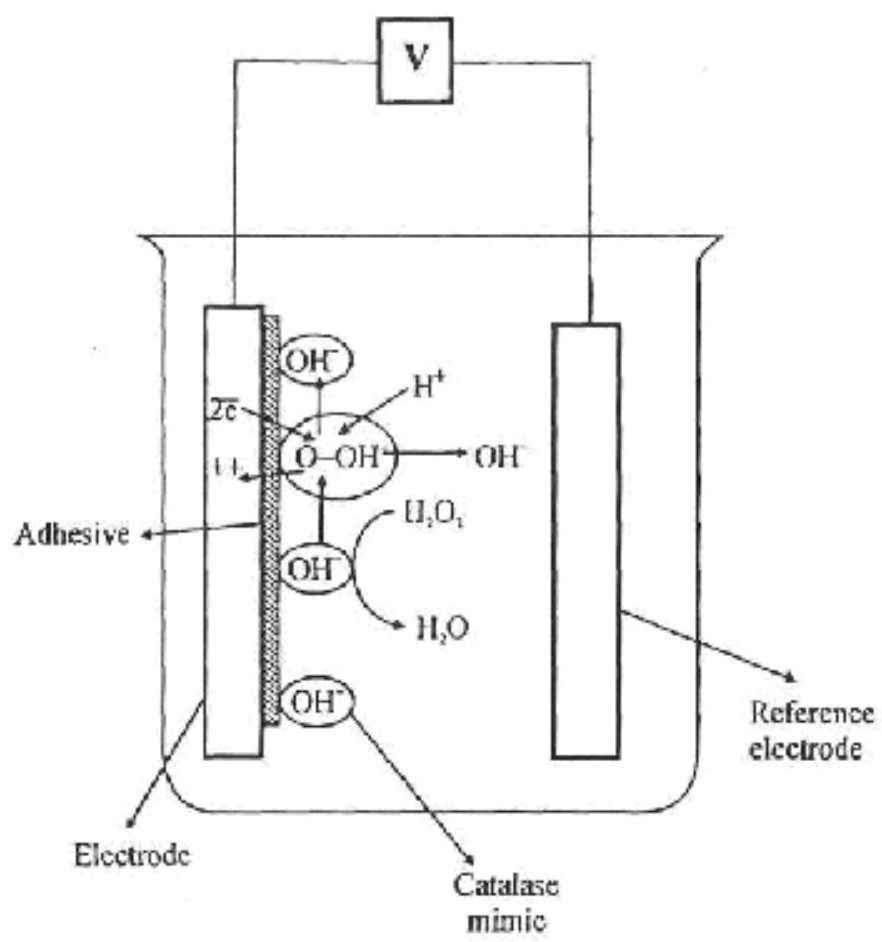

Fig. 1. Catalase-mimetic electrochemical cell.

were selected because of their low price and inertness in relation to hydrogen peroxide. The binding agent between the electrode and biomimic was Pattex adhesive and 7.5\% polyacrylamide gel.

The electrochemical system consisting of a biomimetic selector of catalase or peroxidase type, which was part of the electrode, allowed us to determine the low concentrations of $\mathrm{H}_{2} \mathrm{O}_{2}$ and $\mathrm{C}_{2} \mathrm{H}_{5} \mathrm{OH}$ in aqueous solutions at $22^{\circ} \mathrm{C}$. This formed the basis for inexpensive and easily handled potentiometric biomimetic sensors.

Inorganic mimic of catalase was prepared according to the well-known technique $[1,2]$. Partly purified catalase of human blood erythrocytes and trypsine (Sigma Company) were used for the catalase sources.

The change of electrode potential $(\mathrm{E})$ of the catalase reaction with time was measured by a voltmeter. $\mathrm{pH}$ and $\mathrm{E}$ values for aqueous hydrogen peroxide were determined simultaneously for possible correlations between $\mathrm{pH}$ metric and potentiometric results of enzymatic activity of catalase-biomimetic sensors. The electrochemical unit was also equipped with a magnetic mixer.

For the purpose of determining low hydrogen peroxide concentrations, the authors have designed the most cost-effective and simple to use potentiometric-biomimetic sensors based on immobilized catalase mimics. These sensors possess high hydrodynamic properties and the fastest speed of response. Figure 8.3 shows experimental data on catalase activity of biomimetic electrode in $0.03 \%$ aqueous $\mathrm{H}_{2} \mathrm{O}_{2}$. For the sake of comparison, catalase activities of aluminum electrode and aluminum electrode with applied adhesive are also shown. 
Peroxidase mimetic, like its catalase analogue, consists of two parts: the active part and the support. The support is a neutral activated aluminum oxide onto which an active site (hematin with $8.6 \mathrm{wt} \%$ iron, Sigma) is deposited. The biomimetic is synthesized by the known procedure.

The biomimetic sensors $[7,8]$ on the basis of catalase and peroxidase bioimitators for determining ultralow concentrations of $\mathrm{H}_{2} \mathrm{O}_{2}$ and $\mathrm{C}_{2} \mathrm{H}_{5} \mathrm{OH}$ are still at the development stage because of disadvantages that hinder their practical applications. For example, the presence of glue material [7] on the surface of an electrode led to quick deactivation of the biomimetic selector because of protoporphyrin's insufficient stability to the oxidant $\left(\mathrm{H}_{2} \mathrm{O}_{2}\right)$ and its intermediates and decomposition of the biomimetic electrode.

It is important to develop more active and stable biomimetic iron porphyrin electrodes free of the above drawbacks: to create and study the physicochemical features of new variants of catalase - and peroxidase-type iron porphyrin electrodes to determine ultralow concentrations of $\mathrm{H}_{2} \mathrm{O}_{2}$ and $\mathrm{C}_{2} \mathrm{H}_{5} \mathrm{OH}$ in aqueous solutions.

The oxide coating $\left(\mathrm{A}_{2} \mathrm{O}_{3}\right)$ of the aluminum electrode was prepared by electrochemical and chemical methods [9]. Iron porhyrin derivatives were deposited by adsorption on the coatings. In the biomimetic selector, the matrix was the oxidized surface of the aluminum electrode $\left(\mathrm{A}_{2} \mathrm{O}_{3}\right)$. This was a new design, which allowed us to avoid using glue materials and increased the activity and stability of the biomimetic selector.

\section{Catalase biomimetic sensors}

In all three cases (Al, $\mathrm{Al}$ with applied adhesive and biomimetic electrode), as observed from Figure 2., the presence of hydrogen peroxide in the system causes an initial sharp increase of the system potential. This is most likely associated with the formation of a new surface layer at the electrode-solution interface. Some time later an equilibrium surface layer is formed and the potential at Al-solution and Al-adhesive-solution barely changes. Refracto-metric analysis shows that $\mathrm{Al}$ does not cause dissociation of $\mathrm{H}_{2} \mathrm{O}_{2}$ at low concentration of the latter, whereas electrochemical potential in biomimetic electrode- $\mathrm{H}_{2} \mathrm{O}_{2}-\mathrm{Cl} \sim-\mathrm{AgCl}-\mathrm{Ag}$ system continues changing to almost full $\mathrm{H}_{2} \mathrm{O}_{2}$ dissociation (Figure 1). Dissociation of $\mathrm{H}_{2} \mathrm{O}_{2}$ was tested by solution titration with potassium permanganate.

According to the author's ideas and experiments, the following reactions are implemented in electrochemical system:

Catalase reaction

$$
2 \mathrm{H}_{2} \mathrm{O}_{2} \stackrel{\text { biomimic }}{\longrightarrow} 2 \mathrm{H}_{2} \mathrm{O}+\mathrm{O}_{2}
$$

Electrochemical reaction

$$
\mathrm{O}_{2}+4 \bar{e}+2 \mathrm{H}^{+} \stackrel{\text { electrode }}{\longrightarrow} 2 \mathrm{OH}^{-}
$$

It is common knowledge that hydrogen peroxide is a soft dibasic acid. Therefore, catalase activity of biomimetic electrodes may change the $\mathrm{pH}$ of the $\mathrm{H}_{2} \mathrm{O}_{2}$ solution by both reactions (1) and (2). If these reactions take place on the biomimetic electrode, at the end of the process the $\mathrm{pH}$ of the solution will equal the $\mathrm{pH}$ of the distilled water. 


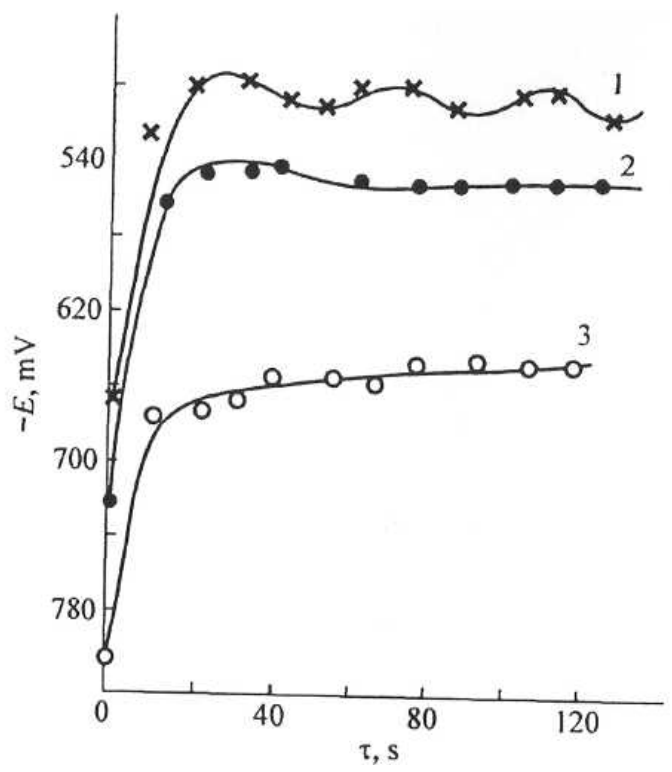

Fig. 2. Time dependence of electrochemical potential in the system. $\mathrm{T}=20^{\circ} \mathrm{C} 003 \mathrm{wt} \% \mathrm{H}, 0$ (1: biomimetic electrode; 2: aluminum electrode; 3 : aluminum electrode with applied adhesive)

For the purpose of determining $\mathrm{H}_{2} \mathrm{O}_{2}$ dissociation mechanism on the electrode the authors followed up the $\mathrm{pH}$ variation dynamics in the system until complete hydrogen peroxide dissociation. Some time after the start of the process the course of the curve in Figure 3 indicates a $\mathrm{pH}$ higher than for redistilled water (background solution) approaching 7. Titration by $\mathrm{KMnO}_{4}$ showed that a trace concentration of hydrogen peroxide below the sensitivity of biomimetic electrode still remained in the solution.

The fact that the $\mathrm{pH}$ of the reaction mixture exceeds the value for redistilled water testifies to the implementation of both reactions (1) and (2) in the system. Note that in the absence of reaction (2) and full $\mathrm{H}_{2} \mathrm{O}_{2}$ dissociation, the $\mathrm{pH}$ observed for the solution must correspond to the $\mathrm{pH}$ of the redistilled water (6.2).

These data present an experimental proof of proceses (1) and (2) run on the biomimetic electrode and the consequences:

1. A definite quantity of oxygen molecules accumulated on the surface of the biomimetic electrode (catalase reaction) must diffuse to the volume of the adhesive layer, toward the electrode surface. Hence, the specific requirements to the adhesive follow: on the one hand, it must provide strong enough adhesion of a mimic to electrode; on the other hand, it must possess low oxygen adsorption ability.

2. The adhesive must be highly inert in the catalase reaction.

3. $\mathrm{OH} \sim$ anions, formed in electrode reaction (2), must display higher adsorption ability to the adhesive.

4. The exclusive role of surface oxygen in electrochemical reaction (2). 


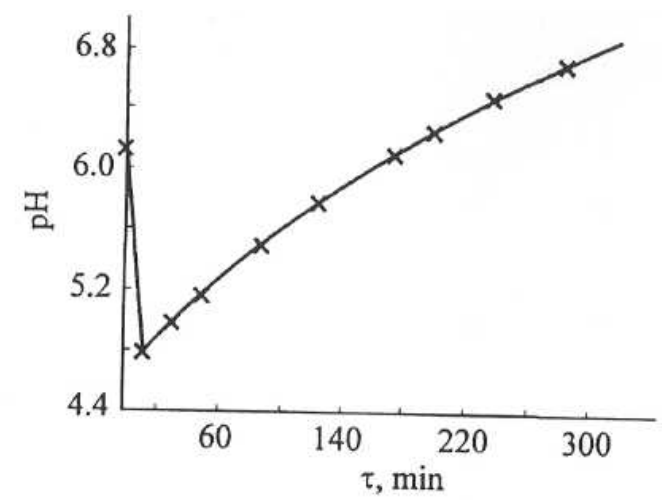

Fig. 3. Time dependence of $\mathrm{pH}$ for aqueous $\mathrm{H}_{2} \mathrm{O}_{2}$ dissociation. $T=20^{\circ} \mathrm{C}, 0.01$ wt. $\% \mathrm{H}_{2} \mathrm{O}_{2}$.

The additional experimental proof of the last statement is given by data in Figure 4. Curve (a) shows the change of $\mathrm{pH}$ and potential in the 'mimetic electrode- $\mathrm{H}_{2} \mathrm{O}_{2}-\mathrm{CI}-\mathrm{AgCl}-\mathrm{Ag}$ ' system. Curve (b) shows the results of tests carried out for the mimic applied on the electrode surface. According to these data, the potential abruptly decreases in the system, whereas curve (a) indicates its increase.

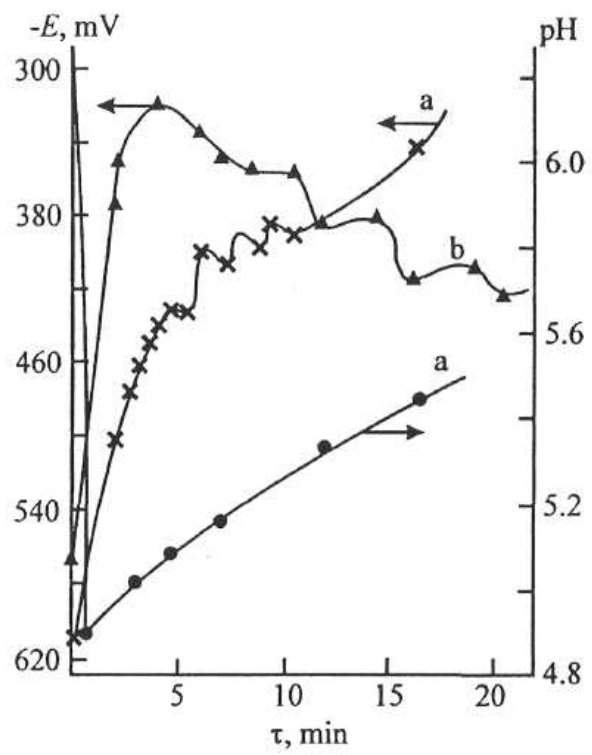

Fig. 4. Time dependence of electrochemical potential in the system and aqueous $\mathrm{H}_{2} \mathrm{O}_{2} \mathrm{pH}$, $\mathrm{T}=20^{\circ} \mathrm{C}, 0.01 \mathrm{wt}$. $\% \mathrm{H}_{2} \mathrm{O}_{2}$ (a: biomimic is adhered to aluminum foil; b: biomimic is present in aqueous $\mathrm{H}_{2} \mathrm{O}_{2}$ ). 
With regard to the above, of special interest are test results for unmixed system (in all other experiments the system was mixed by a magnet mixer). It turns out that part of the molecular oxygen synthesized in the catalase reaction (1) releases from the solid, and forms a gas 'cover' around the mimetic electrode in the interface layer between the solid and the reaction mixture. This circumstance will decelerate $\mathrm{H}_{2} \mathrm{O}_{2}$ diffusion to the biomimetic electrode and decrease the rate of the catalase reaction (8.1), hence promoting electric potential drop. Most of these hindrances can be eliminated by mixing the reaction system. The efficiency of the system mixing is illustrated by data in Fig. 5 .

Although mixing of the solution reduces the diffusion effect on the course of the catalase reaction, a special experiment must be set up to determine the predominance of one of two possible limitations of diffusion and kinetic origin. For this purpose, knowing that the diffusion rate is almost independent of temperature in the system and the chemical reaction rate increases with temperature by $2-A$ times for every $10 \mathrm{~K}$, the temperature influence on the change of potential and $\mathrm{pH}$ in the system was studied. The results of the experiments are presented in Figure 6. The $\mathrm{pH}$ curves are practically unchanged, although the temperature of the mixture was twice increased. Such regularity is typical of reactions proceeding under diffusion complication conditions, where the catalase reaction rate is limited by diffusion (i.e. depends on $\mathrm{H}_{2} \mathrm{O}_{2}$ delivery to and elimination from the mimetic electrode), but not by kinetics of the catalase reaction. Apparently, electrochemical indices deteriorate as a consequence of an increase in the molecular oxygen desorption rate in the volume which reduces the rate of electrochemical reaction (2). It is also probable that temperature causes any other effect on the physical conditions of the electrode.

In all Figures electrochemical potentials possess clear maxima and minima. Such curve shapes conform to the shape for catalase and electrochemical reactions in the diffusion zone of the system. As mentioned above, molecular oxygen accumulated on the surface of the mimetic electrode during catalase reaction (1) diffuses through the adhesive and mimic layers to the electrode surface, where it is activated and interacts with $\mathrm{H}^{+}$. Anions $\mathrm{OH}^{-}$ formed in this process may set the electrode surface free for the next portion of oxygen by diffusion only. Thus, the rate of electrochemical reaction (2) will be defined by the ratio of the rates of molecular oxygen diffusion to the electrode surface and reverse diffusion of $\mathrm{OH}^{-}$ anions from the surface.

The maxima observed on $E$ curves correspond to the highest rates of electrochemical reaction (2), which are functions of $\mathrm{O}_{2}$ concentration on the electrode, testifying to its highest values under current conditions. During reaction (2) the $\mathrm{O}_{2}$ concentration decreases and the rate of electrochemical reaction (2) is reduced simultaneously. It begins increasing as soon as a new portion of molecular oxygen reaches the electrode surface, set free from $\mathrm{OH}^{-}$anions. Finally, this catalytic process connected to diffusion events will have a sinusoidal shape until $\mathrm{H}_{2} \mathrm{O}_{2}$ is fully dissociated, and the reaction is terminated.

Based on the analysis of the obtained experimental regularities of the electrochemical process, it may be fundamentally concluded that the process at electrode displays a selloscillation mechanism. Obviously, self-oscillations happen due to internal diffusion of the surface components of the mimetic electrode, which is not affected by solution mixing intensity. Mixing causes a strong influence on the external diffusion and $\mathrm{OH} \sim$ anion drainage from the interface layer.

Biomimetic sensors, prepared from catalase adsorbed on diasorb and $\mathrm{A}_{2} \mathrm{O}_{3}$, treated with trypsine and adhered to an aluminum electrode surface using $7.5 \%$ polyacrylamide gel of Pattex adhesive, were found to be the most effective sensors among appliances of this type 


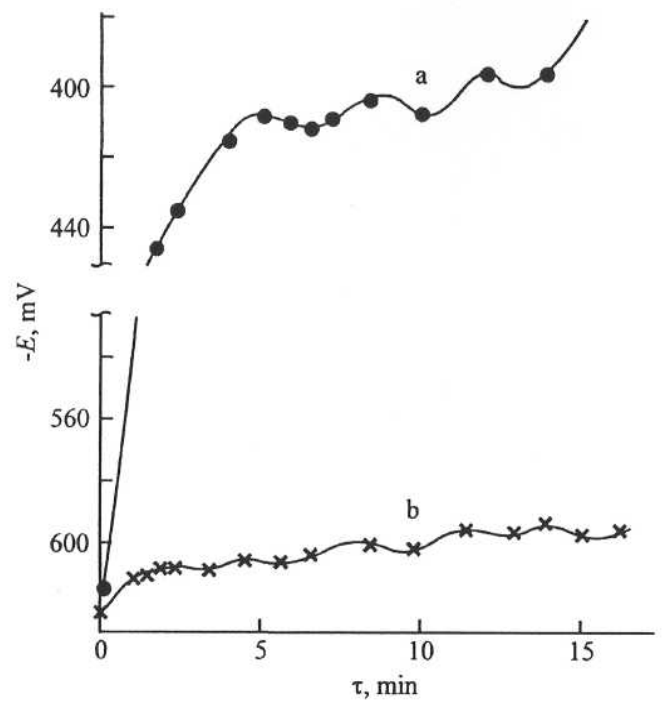

Fig. 5. The mixing effect on the change of electric potential in the system. $T-20^{\circ} \mathrm{C}, 0.01$ wt. $\% \mathrm{H}_{2} \mathrm{O}_{2}$ (a: mixed system; b: without mixing).

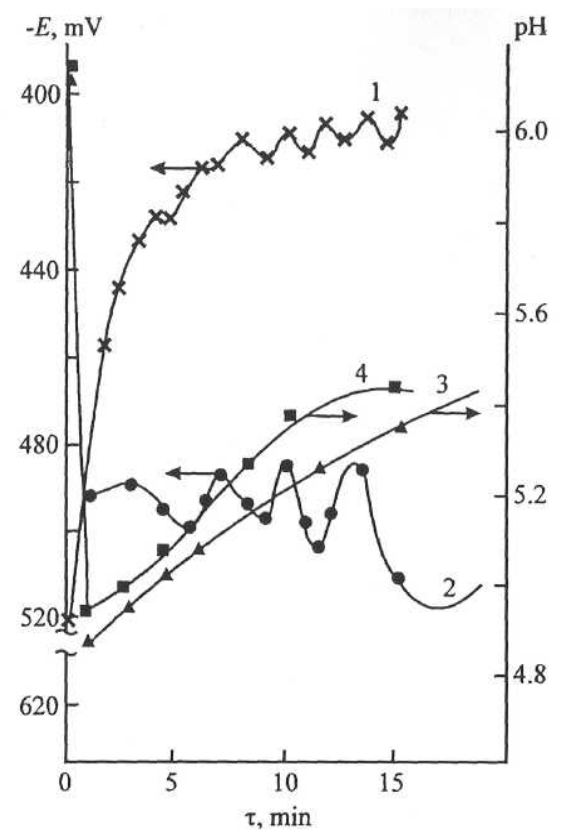

Fig. 6. Temperature dependencies of electrochemical potential $(1,2)$ and $\mathrm{pH}(3,4)$ at $20 \mathrm{~T}$ $(1,3)$ and $40^{\circ} \mathrm{C}(2,4) ; 0.01$ wt. \% $\mathrm{H}_{2} \mathrm{O}_{2}$.

(Figure 7.). These biomimetic sensors are distinguished by high hydrodynamic properties, operation stability and rapid speed of response. A tendency to an increase of the electrode potential is observed for biomimetic sensors, derived from catalase adsorbed on diasorb (without trypsine treatment), agarose (treated with trypsine and without it), and adhered to 
an aluminum electrode surface by $7.5 \%$ polyacrylamide gel. It is also observed for biomimetic sensors, derived from catalase adsorbed on diasorb (without trypsine treatment), $\mathrm{Si}_{2} \mathrm{O}_{3}$ (treated with trypsine and without it), and adhered to aluminum electrode surface by Pattex adhesive.

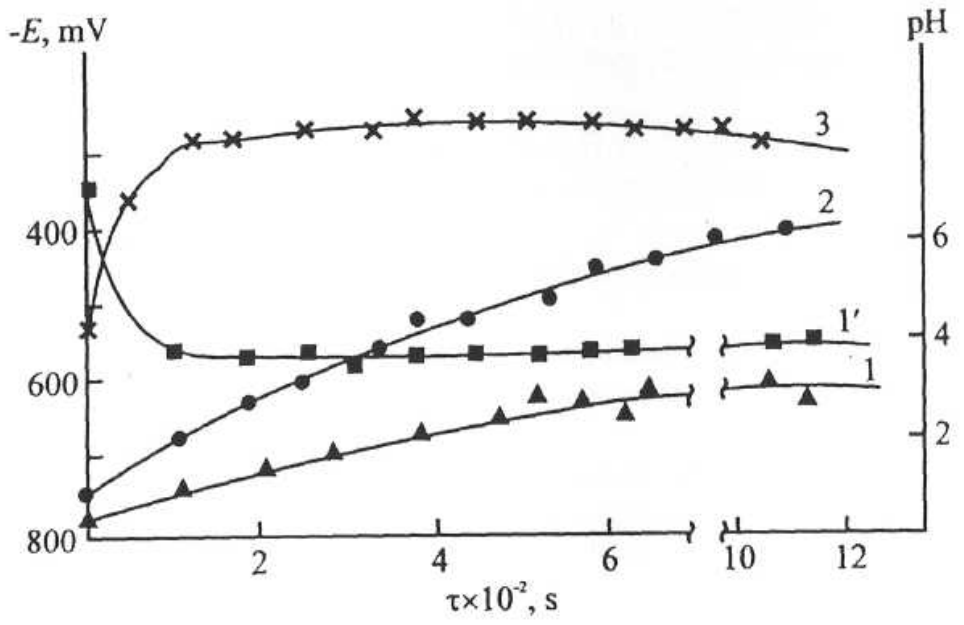

Fig. 7. Time dependencies of electrochemical potential (1-3) and $\mathrm{pH}$ of aqueous $\mathrm{H}_{2} \mathrm{O}_{2}\left(1^{\prime}\right)$ in the system. $T=20^{\circ} \mathrm{C}, 0.06 \mathrm{wt} . \% \mathrm{H}_{2} \mathrm{O}_{2}$.. Catalase is adsorbed on diasorb $\left(1,1^{\prime}\right)$ or $\mathrm{A}_{2} \mathrm{O}_{3}(2,3)$, treated by trypsine and adhered to aluminum foil with $7.5 \%$ polyacrylamide gel $\left(1,1^{\prime}, 2\right)$ or Pattex (3).

Biomimetic sensors, prepared by catalase adsorption on diasorb and agarose (treated with trypsine) and adhered to an aluminum electrode surface by Pattex adhesive, displayed an abrupt decrease of the electrode potential. Sensors prepared by catalase adsorption on $\mathrm{A}_{2} \mathrm{O}_{3}$ (without trypsine treatment) and adhesion to the aluminum electrode with Pattex adhesive displayed a high oscillation of the electrode potential, which induces extreme instability of the operation. Hence, it should be noted that sensor operation was always better in the case of enzyme treatment with trypsine.

When the carrier is selected, aluminum oxide should be preferred, because biomimetic sensors on it display higher characteristics (Figure 7). Moreover, they are low in price, long lived and stable, and possess high hydrodynamic properties.

Pattex adhesive combines well with such carriers as $\mathrm{AI}_{2} \mathrm{O}_{3}$ and $\mathrm{Si}_{2} \mathrm{O}_{3}$, but is absolutely useless for agarose and diasorb.

Thus, two reactions (catalase and electrochemical) are implemented in the biomimetic electrode $\mathrm{H}_{2} \mathrm{O}_{2} / \mathrm{Cl}^{-} / \mathrm{AgCl} / \mathrm{Ag}$ system. In the case of inorganic support, it is not the kinetic but the diffusion (external and internal) factor that is predominant, owing to which the electrochemical reaction is of a self-oscillation type $[7,8]$.

As can be seen from the data of Fig. 8, due to the presence of hydrogen peroxide in a water solution, the potential of the electrochemical system increased drastically at first in all cases because of the formation of a new surface layer on the electrode-solution boundary and then because of electrochemical reactions (1) and (2). After a certain period of time, an equilibrium surface layer formed, as indicated by the constant values of potentials on the electrode-solution and biomimetic electrode-solution boundaries. 


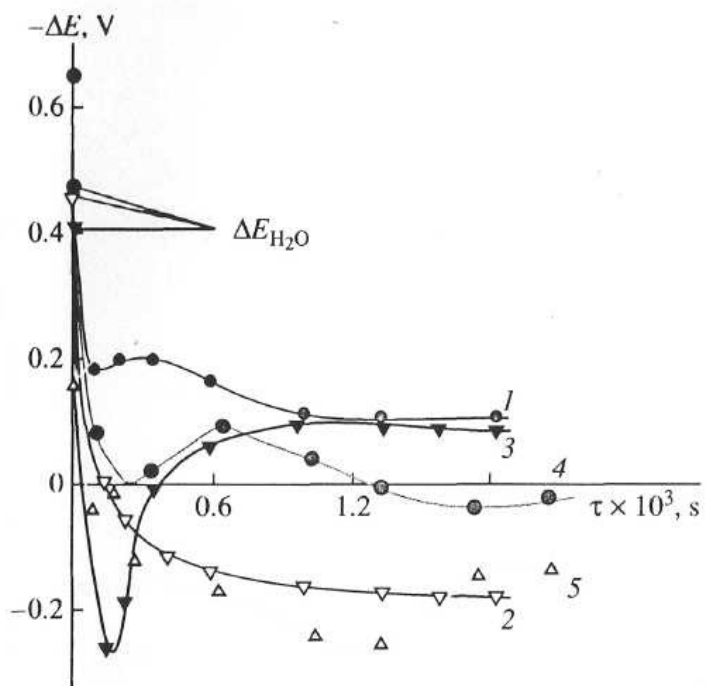

Fig. 8. Time dependences of the electrochemical potential $(\Delta E)$ in a reaction medium of $1 \%$ aqueous $\mathrm{H}_{2} \mathrm{O}_{2}$ at $22^{\circ} \mathrm{C}$ for electrodes prepared by the electrochemical procedure: (1) $\mathrm{Al}_{2} \mathrm{O}_{3} / \mathrm{Al}$; (2) hemin $\left(33.6 \mathrm{mg}\right.$ ) deposited by glueing on the surface of the $\mathrm{Al}_{2} \mathrm{O}_{3} / \mathrm{Al}$ electrode; (3) hemin (0.88 mg) and (4) and (5) TPhPFe(III) (0.084 and $0.44 \mathrm{mg}$, respectively) deposited by adsorption on the surface of the $\mathrm{A}_{2} \mathrm{O}_{3} / \mathrm{AI}$ electrode.

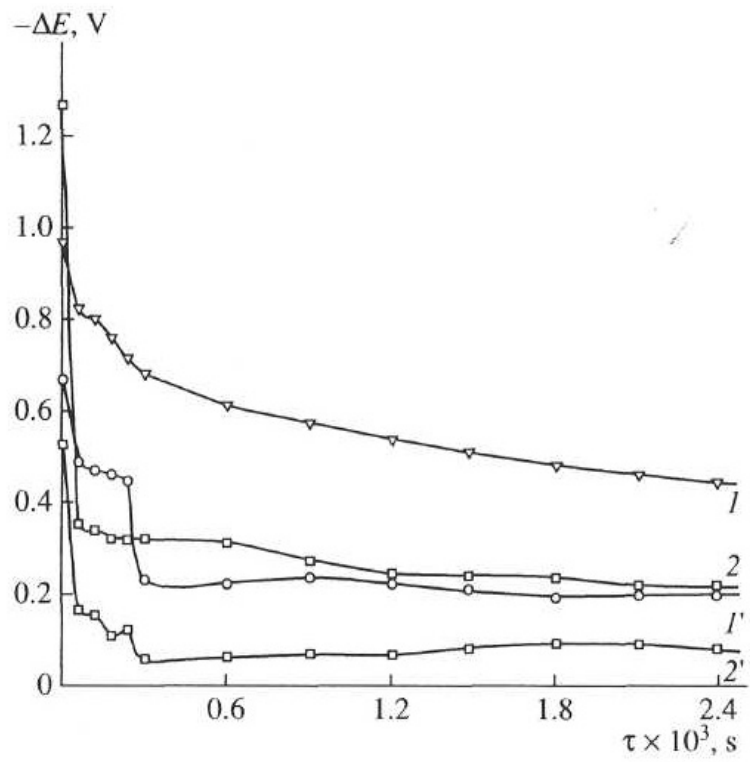

Fig. 9. Time dependences of the electrochemical potential $(\Delta E)$ in a reaction medium of $1 \%$ aqueous $\mathrm{H}_{2} \mathrm{O}_{2}$ at $22^{\circ} \mathrm{C}$ for electrodes prepared by the chemical procedure: $(1,2) \mathrm{Al}_{2} \mathrm{O}_{3} \mathrm{Al}$ electrodes prepared by the hydrothermal and aluminate methods, respectively; (1', 2') $\mathrm{TPhPFe}(\mathrm{III})$ ( 0.6 and $0.23 \mathrm{mg}$, respectively) deposited by adsorption on the surface of the $\mathrm{A}_{2} \mathrm{O}_{3} / \mathrm{A} 1$ electrode. 
As follows from curves 1 and 2, the electrochemical potential increased with time. After two sequential runs, the hemin selector prepared by the glue method was completely deactivated.

The tendency of curves 1 and 3 (Fig. 8) toward a constant value of $\Delta E$, identical for both, obviously showed that a catalase reaction was almost completely absent for curve 1 and vanished after $600 \mathrm{~s}$ for 3 . Moreover, the reproducibility of results and the activity of the electrode prepared by adsorption did not change after all experiments.

Irrespective of the amount of the deposited working element, biomimetic electrodes with TPhPFe(III) (curves 4 and 5, Fig. 8) also had a long lifetime, and their activity was almost independent of the number of experiments. As follows from the values of $\Delta E$ on curves 4 and 5 , they depend considerably on the amount of TPhPFe(III) deposited on $\mathrm{A}_{2}{ }_{2} \mathrm{O}_{3} / \mathrm{A} 1$. The hemin biomimetic electrode was more active than TPhPFe(III), but the latter was much more stable after prolonged service [9].

Figure 9 shows the catalase activities of biomimetic electrodes (the $\mathrm{A}_{2} \mathrm{O}_{3} / \mathrm{A} 1$ electrode was prepared by a chemical procedure under the same conditions as in the previous experiment). As can be seen from Fig. 9, the presence of $\mathrm{H}_{2} \mathrm{O}_{2}$ in the system always leads to an increase in the electrochemical potential of the system.

The amount of TPhPFe(III) deposited by hydrothermal processing of the surface in the presence of hydrogen peroxide was higher than the amount of the compound deposited by the aluminate method, but the catalytic activity of the latter was appreciably higher. This indicates that the deposition procedure plays an important role.

The electrode prepared by the aluminate method was most stable and sensitive, although the active surface layer was slightly destroyed during both experiments.

A comparison of the data in Figs. 8 and 9 shows that electrodes prepared by the chemical method were inferior, in catalase activity and stability, to biomimetic electrodes prepared by the electrochemical procedure.

The electrodes prepared by the electrochemical method have a limiting sensitivity to the concentration of $\mathrm{H}_{2} \mathrm{O}_{2}, 10^{-2}$ wt \%, while the electrodes obtained by the chemical procedure showed activity in the vibrational mode probably because of diffusion (their sensitivity was $10^{-6}$ wt \%, $\mathrm{H}_{2} \mathrm{O}_{2}$ ).

Note that the electrodes prepared by the chemical technique proved rather sensitive, but they were much less stable than the electrodes obtained by the electrochemical method.

The supposed electrochemical mechanism of the catalase reaction was stepwise (Fig. 10), as noted in $[6,7]$. At the first stage, highly active hydroperoxide particles formed, which were responsible for the transfer of electrons from the cathode to the iron ion and their subsequent distribution. As a result, at the last stage, hydroxide anions were generated to the volume (reaction medium) with concurrent regeneration of the biomimetic. This mechanism shows how $\mathrm{pH}$ in the electrochemical system becomes higher than in bidistilled water ( $\mathrm{pH}$ 6.2).

As a result, catalase and electrochemical reactions proceed consecutively in the system. These reactions can be represented as

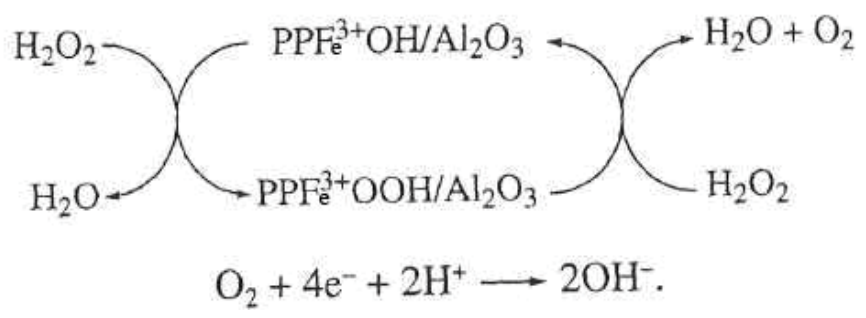




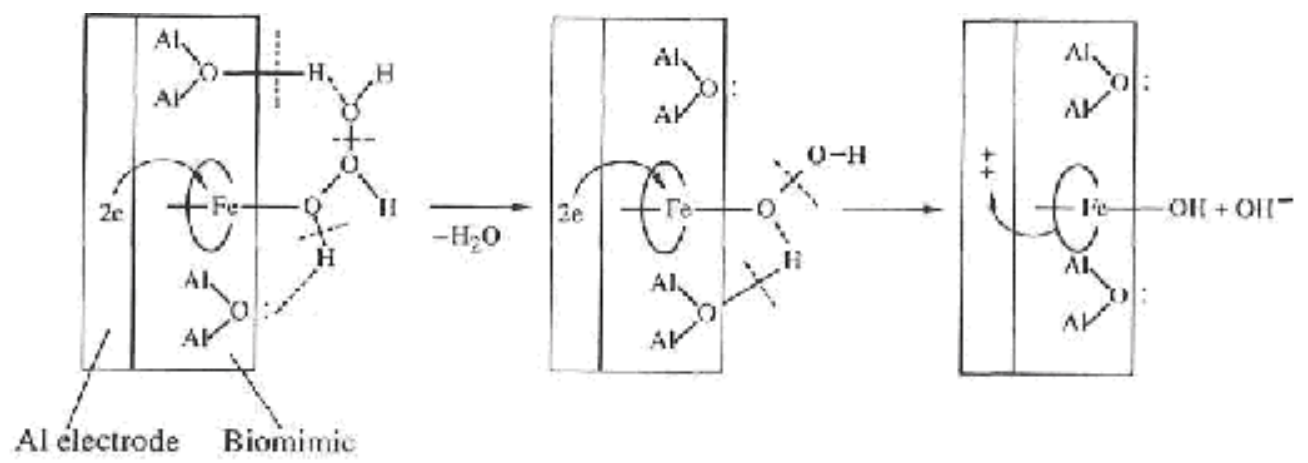

Fig. 10. Mechanism of the decomposition of $\mathrm{H}_{2} \mathrm{O}_{2}$ on the catalase biomimic electrode.

\section{Peroxidase biomimetic sensors}

The peroxidase-mimetic electrode was studied in the following way. First, the potential of twice distilled water (base solution) was measured. Then, the required amount of $\mathrm{C}_{2} \mathrm{H}_{5} \mathrm{OH}$ was added and the variation of the electrode potential was recorded. Next a certain amount of $\mathrm{H}_{2} \mathrm{O}_{2}$ (oxidizer) was introduced into the solution and the potential shift was measured. The potential of the electrochemical system changed as a result of synchronous reactions (catalase and peroxidase) that occurred in the system.

As in $[3,4]$, the choice of the electrode was dictated by the availability and low cost of the material and by the fact that it is inert with respect to $\mathrm{H}_{2} \mathrm{O}_{2}$. A magnetic stirrer was employed in all experiments.

The ethanol concentration was varied from $10^{-8}$ to 5 wt \% while the hydrogen peroxide concentration remained unchanged (1 wt \%). The results are displayed in Fig. 11, where the potential curve of twice distilled water is also given for comparison. According to $[7,8]$ the electrochemical reaction environment possesses catalase activity. For this reason, Fig 11 also shows the curve of the electrochemical potential shift of $1 \%$ aqueous solution of $\mathrm{H}_{2} \mathrm{O}_{2}$ in the absence of $\mathrm{C}_{2} \mathrm{H}_{5} \mathrm{OH}$.

To examine the curves of the peroxidase reaction, it was necessary to measure the electrochemical potential of a $1 \%$ aqueous solution of ethanol. Its value fully coincides with the potentials of twice distilled water. It follows that the water-alcohol solution virtually remains inert with respect to the electrode. The peroxidase curves (Fig. 11) describing the potential shift of the reactive mixture $\mathrm{C}_{2} \mathrm{H}_{5} \mathrm{OH}+\mathrm{H}_{2} \mathrm{O}_{2}$ under conditions when the value of $\left[\mathrm{H}_{2} \mathrm{O}_{2}\right]$ is maintained unchanged $(1 \mathrm{wt} \%)$ while $\left[\mathrm{C}_{2} \mathrm{H}_{5} \mathrm{OH}\right]$ is varied from $10^{-6}$ to $5 \mathrm{wt} \%$, show a more substantial potential shift to the positive side than the catalase curves of $\mathrm{H}_{2} \mathrm{O}_{2}$. The highest difference between the potentials of $\mathrm{H}_{2} \mathrm{O}_{2}$ and the $\mathrm{C}_{2} \mathrm{H}_{5} \mathrm{OH}+\mathrm{H}_{2} \mathrm{O}_{2}$ mixture is observed at $\tau=10 \mathrm{~s}$. For $1 \%$ solutions of $\mathrm{H}_{2} \mathrm{O}_{2}$ and $\mathrm{C}_{2} \mathrm{H}_{5} \mathrm{OH}, \Delta \mathrm{E}=0.2 \mathrm{~V}$. In virtually all the experiments, the potentials measured in the initial period of the peroxidase reaction are also higher than those at later periods of the reaction. Note that the potential of the $\mathrm{C}_{2} \mathrm{H}_{5} \mathrm{OH}+$ $\mathrm{H}_{2} \mathrm{O}_{2}$ mixture never coincided with that of $\mathrm{H}_{2} \mathrm{O}_{2}$ and was never below it. Thus, a very important conclusion can be derived: a reaction between $\mathrm{C}_{2} \mathrm{H}_{5} \mathrm{OH}$ and $\mathrm{H}_{2} \mathrm{O}_{2}$ undoubtedly occurs in the system; this reaction is responsible for an increase in the potential of the peroxidase reaction, the increase being higher than that for the catalase reaction. In the absence of the peroxidase reaction, the system potential would coincide with the potential of the catalase reaction. The curves of peroxidase potentials are, however, arranged relative to 
each other in such a manner that a certain periodicity in the dependence of peroxidase reaction potentials on the low concentrations of $\mathrm{C}_{2} \mathrm{H}_{5} \mathrm{OH}$ can be detected. This arrangement may be attributed to the inadequate procedure of the fixation of the peroxidase mimetic on the surface of the aluminum electrode. This problem can presumably be solved by eliminating possible systematic errors involved in the preparation of the biomimetic electrode.

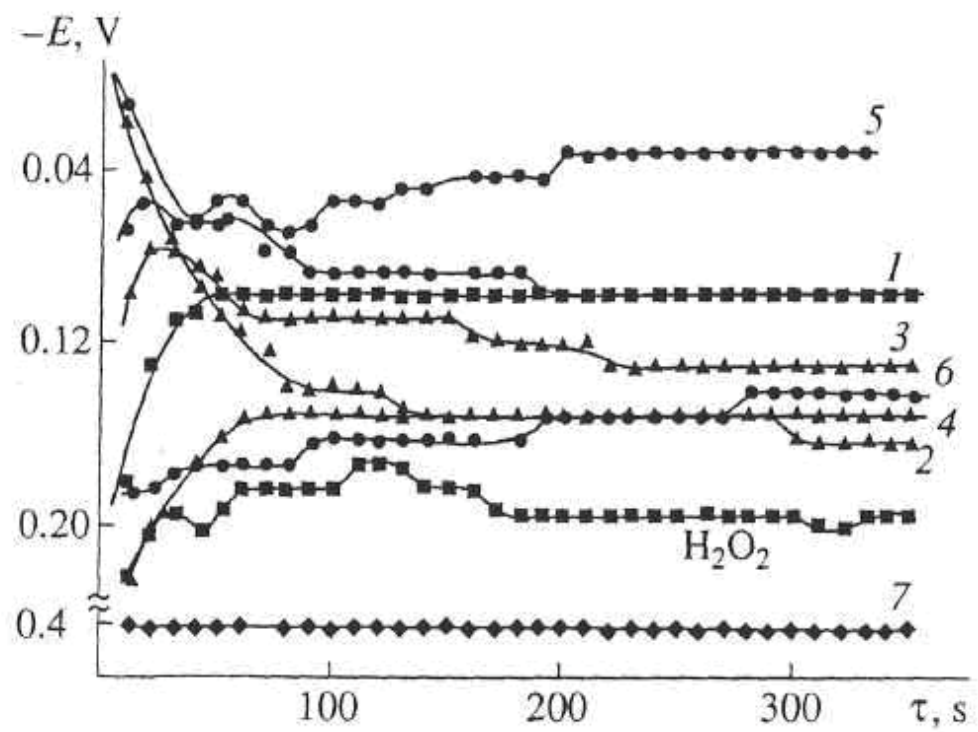

Fig. 11. Time dependences of the electrochemical potential at $1 \mathrm{wt} \% \mathrm{H}_{2} \mathrm{O}_{2}$ and various concentrations of $\mathrm{C}_{2} \mathrm{H}_{5} \mathrm{OH}$ : (1) $5 \times 10^{-6}$, (2) $5 \times 10^{-5}$, (3) $5 \times 10^{-4}$, (4) $5 \times 10^{-2}$, (5) $5 \times 10^{-1}$, and (6) $5 \mathrm{wt} \%$; (7) twice distilled water.

It is well known that acetaldehyde in the $\mathrm{C}_{2} \mathrm{H}_{5} \mathrm{OH}+\mathrm{H}_{2} \mathrm{O}_{2}$ system can appear not only as a result of a peroxidase reaction in the presence of a catalyst, but also by the monooxygenase mechanism. To solve this problem, we used the standard method of identification of the peroxidase reaction [10]. For this purpose, we added the indicator thymolphthalein to a $1 \%$ aqueous solution of $\mathrm{H}_{2} \mathrm{O}_{2}$ containing a biosimulator sample and identified the peroxidase mechanism of acetaldehyde formation by monitoring how the color of the solution changes from colorless to dark blue.

Another series of experiments was undertaken to study the effect of the product of peroxidase reaction (acetaldehyde) on the electrochemical potential of the system.

Figure 12 (curve 3) shows that the electrochemical potential of acetaldehyde is belower than the potential of water and ethyl alcohol (acetaldehyde is a nonpolar compound). The curve 1 of the potential shift for the $\mathrm{CH}_{3} \mathrm{CHO}+\mathrm{H}_{2} \mathrm{O}_{2}$ reactive mixture is higher than that for hydrogen peroxide. This suggests that a reaction (presumably .involving monooxygenase) occurs between $\mathrm{H}_{2} \mathrm{O}_{2}$ and $\mathrm{CH}_{3} \mathrm{CHO}$ to yield a more polar compound $\left(\mathrm{CH}_{3} \mathrm{COOH}\right)$.

The experimental data presented in Fig. 12, curve 2 were obtained in the following manner. An aqueous solution of $\mathrm{H}_{2} \mathrm{O}_{2}$ was introduced into the $\mathrm{C}_{2} \mathrm{H}_{5} \mathrm{OH}+\mathrm{CH}_{3} \mathrm{CHO}$ reactive mixture at $\tau=130 \mathrm{~s}$. In the segment of curve 2, the potential of aqueous $\mathrm{C}_{2} \mathrm{H}_{5} \mathrm{OH}$ and $\mathrm{CH}_{3} \mathrm{CHO}$ (up to $\mathrm{T}=130 \mathrm{~s}$ ) is noticeably higher than that for each of these compounds, which are usually at 
the level of the water potential or lower. This phenomenon may be attributed to the formation of a new surface layer on the electrode. If $\mathrm{C}_{2} \mathrm{H}_{5} \mathrm{OH}$ and $\mathrm{CH}_{3} \mathrm{CHO}$ separately formed the electrode surface, curve 2 (Fig. 12) with a characteristic segment up to $\tau=130 \mathrm{~s}$ would hardly be obtained. Ethanol evidently reacts in water with acetaldehyde to yield associates whose activity is higher than that of each of them. Introduction of $\mathrm{H}_{2} \mathrm{O}_{2}$ into the $\mathrm{C}_{2} \mathrm{H}_{5} \mathrm{OH}+\mathrm{CH}_{3} \mathrm{CHO}$ mixture at $\tau=130$ s produces a sufficient jump in the potential with the formation of maxima and minima, which is typical of complex electrochemical reactions. We may presume the presence of acetaldehyde molecules in two states in the reaction system. One of the states is produced in the peroxidase reaction and exists at the electrode surface or in the surface layer, in which $\mathrm{CH}_{3} \mathrm{CHO}$ concentration is higher than in the bulk. The other state is produced when $\mathrm{CH}_{3} \mathrm{CHO}$ is in the reaction volume. We believe that a combination of these two states is responsible for the observed maxima and minima in curve 2, Fig. 12.

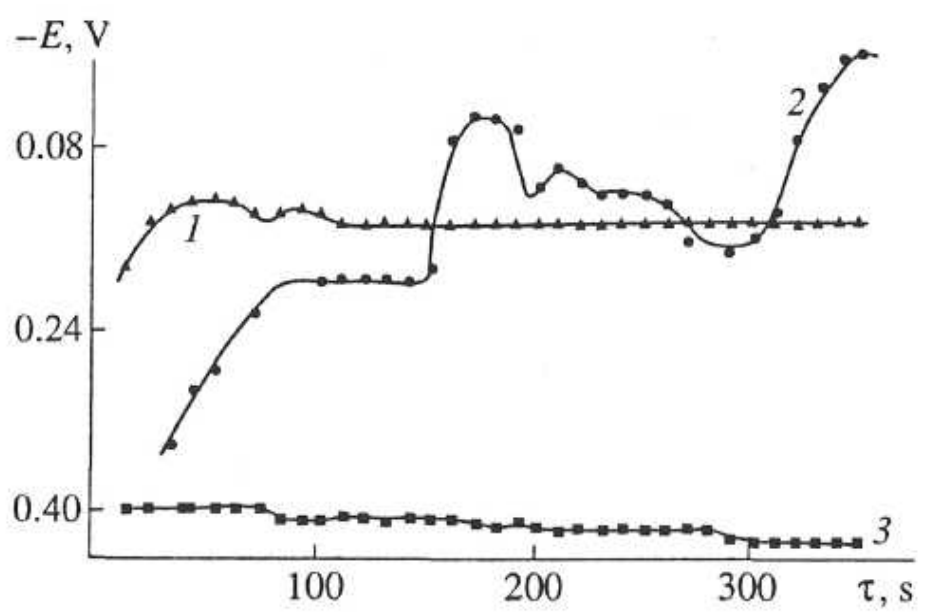

Fig. 12. Time dependences of the potential of the peroxidase electrode at $1 \mathrm{wt} \% \mathrm{H}_{2} \mathrm{O}_{2}$ and various concentrations of the product of the electrochemical reaction $\left(\mathrm{CH}_{3} \mathrm{CHO}\right)$ : (1) $0.25,(2)$ $0.25 \cdot 10^{-2} \mathrm{wt} \% \mathrm{C}_{2} \mathrm{H}_{5} \mathrm{OH}$, and (3) $0.025 \%$.

The peroxidase reaction, which is synchronous with the catalase reaction, also occurs in the studied electrochemical system. Both reactions interact (are conjugate) with each other via the common intermediate $\mathrm{PPFe}^{3+} \mathrm{OOH} / \mathrm{Al}_{2} \mathrm{O}_{3}$.

It consists of several elementary stages displayed in Fig. 10. The first stage yields the highly active hydroperoxide species, which is responsible for the electron transfer from the cathode to the iron ion followed by their further redistribution. As a result, at the last stage in the bulk (reaction environment), hydroxide anions are generated with simultaneous regeneration of a biomimetic. This mechanism convincingly shows how the $\mathrm{pH}$ value in the system becomes higher than that for twice distilled water ( $\mathrm{pH}$ 6.2) $[7,8]$.

The proposed mechanism of peroxidase reaction was considered by analogy with the mechanism of the catalase reaction (Fig. 13). Note that a proton transferred to the active site of the biomimetic electrode can be replaced by $\mathrm{H}^{+}$from the bulk of the reaction environment. The mechanisms of catalase and peroxidase reactions give us ideas about the pathways of their implementation in the electrochemical regime. The ratio of the products of 
both reactions $\left(\mathrm{O}_{2}\right.$ and $\left.\mathrm{CH}_{3} \mathrm{CHO}\right)$ depends on the ratio of the rates of the interaction of $\mathrm{H}_{2} \mathrm{O}_{2}$ and $\mathrm{CH}_{3} \mathrm{CHO}$ with the surface intermediate.

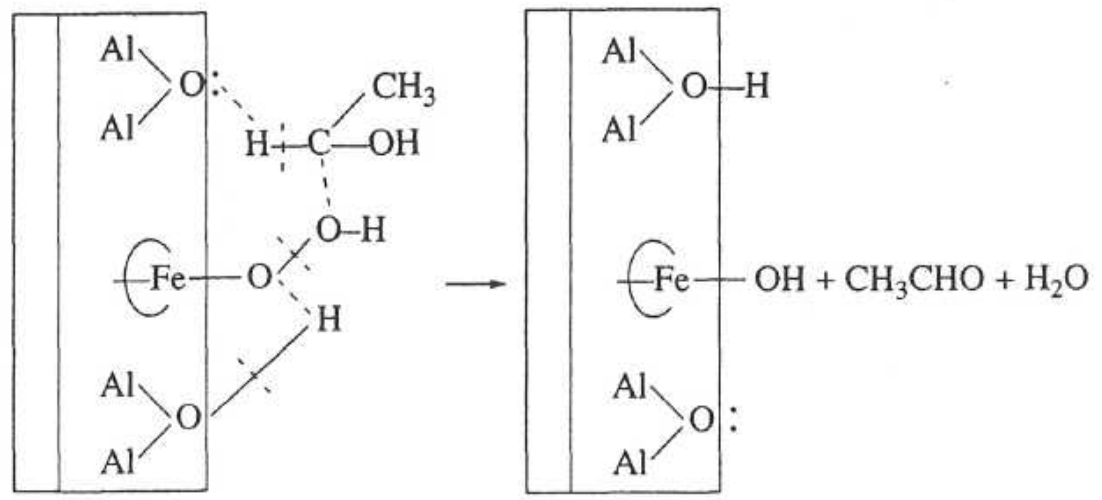

Fig. 13. Suggested mechanism of operation of the peroxidase-mimetic electrode under electrocatalytic conditions.

The schemes shown in Fig 10. explain the coherent and simultaneous mechanism of catalase and peroxidase reactions, which can be represented as [1]

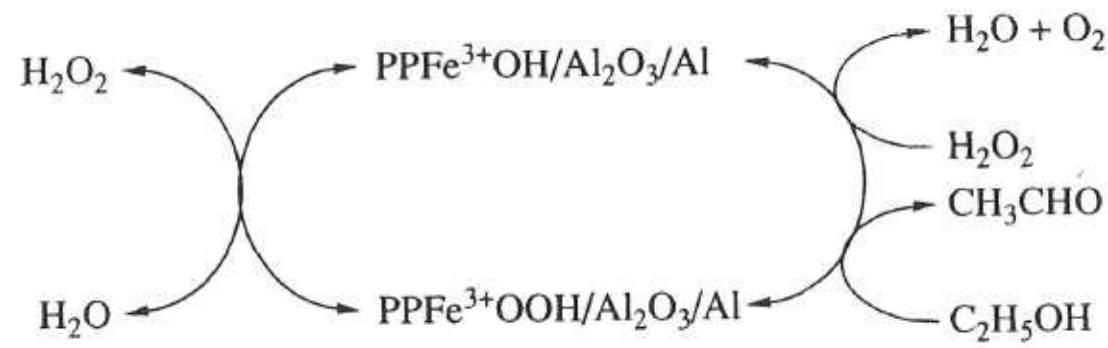

For comparison, we give the results of experiments with biomimetic electrodes prepared by the chemical method under the identical conditions.

Note that the electrodes prepared in different ways proved stable against the action of the oxidant.

In another series of experiments, we determined the lowest (threshold) sensitivity of the system to ethanol. Biomimetic electrodes with TPhPFe(III) made it possible to detect ethanol in an amount of $10^{-6} \mathrm{wt} \%$ in an aqueous solution, while the stability of the electrode remained high.

Figure 14 presents the results of experiments in which $\mathrm{H}_{2} \mathrm{O}_{2}$ concentration was varied from 0.01 to $1 \mathrm{wt} \%$ for constant $\mathrm{C}_{2} \mathrm{H}_{5} \mathrm{OH}$ concentration ( $1 \mathrm{wt} \%$ ). It can readily be seen that the electrode potential of the $\mathrm{C}_{2} \mathrm{H}_{5} \mathrm{OH}+\mathrm{H}_{2} \mathrm{O}_{2}$ reactive mixture grows as $\mathrm{H}_{2} \mathrm{O}_{2}$ concentration increases. This is associated with the fact that the growth of the $\mathrm{H}_{2} \mathrm{O}_{2}$ concentration leads to an increase in the rates of all three reactions considered above. The observed complex pattern of the curves of the potential shift and the scatter of the experimental data can presumably be attributed to the fact that, when the biomimetic electrode is made using a gluing material, the active mass is deposited nonuniformly in thickness. 
The experimental data involved in the determination of the sensitivity threshold of the elaborated peroxidase-mimetic sensor for the analysis of $\mathrm{C}_{2} \mathrm{H}_{5} \mathrm{OH}$ traces are given in Fig. 15 . The electrode potentials in Fig. 15 correspond to various $\mathrm{C}_{2} \mathrm{H}_{5} \mathrm{OH}$ traces and are the best values of $\Delta E$ selected from the experimental curves in Fig. 11. Note that the entire experimental material is obtained on one model of an aluminum electrode. Each time a new peroxidase-mimetic electrode was prepared, we mechanically scraped off the depleted peroxidase mimetic from its surface and then deposited a fresh portion of it with glue. This procedure for the preparation of a biomimetic electrode may be a source of systematic errors. For example, the aluminum electrode itself may be worn out. Moreover, we have no assurance that the spent material is completely removed from the electrode surface. For this reason, the alternation of maxima and minima in Fig. 15 may be due to both the systematic errors involved in the preparation of biomimetic electrodes and the complex nature of the physicochemical processes that occur on the electrode surface.

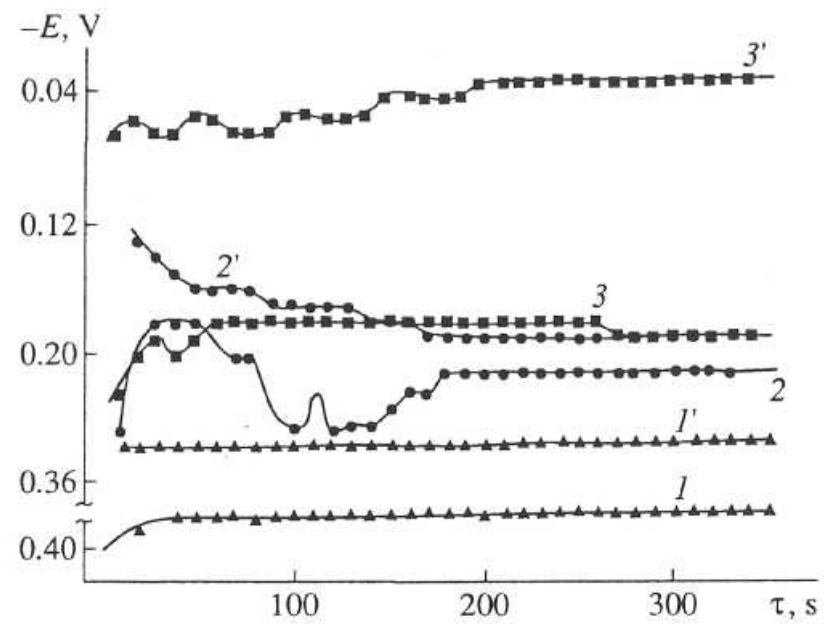

Fig. 14. Dependences of the potentials of (1-3) catalase and $\left(1^{\prime}-3^{\prime}\right)$ peroxidase reactions on the concentration of $\mathrm{H}_{2} \mathrm{O}_{2}$ in a mixture at 1 wt \% $\mathrm{C}_{2} \mathrm{H}_{5} \mathrm{OH} ;\left(1,1^{\prime}\right) 0.01,\left(2,2^{\prime}\right) 0.1,\left(3,3^{\prime}\right) 1$ wt \% $\mathrm{H}_{2} \mathrm{O}_{2}$. 
We verified some of these suppositions by an experiment with a peroxidase-mimetic sensor made on a new aluminum electrode under the conditions corresponding to one of the minima in the curve displayed in Fig. 15. The result of this experiment is shown in Fig. 15 as a separate point $A$. It follows that the minimum observed at a $\mathrm{C}_{2} \mathrm{H}_{5} \mathrm{OH}$ concentration of $10^{-3}$ wt $\%$ depends on the state of aluminum foil: the fresh electrode shows a sharp jump of the potential to the positive side. This effect suggests how the physicochemical properties of the proposed biomimetic sensor and the technique of preparation of biomimetic electrodes can be improved. The data in Fig. 15 show that the sensitivity threshold of the elaborated sensor with respect to ethanol in aqueous solutions is $10^{-8} \mathrm{wt} \%$.

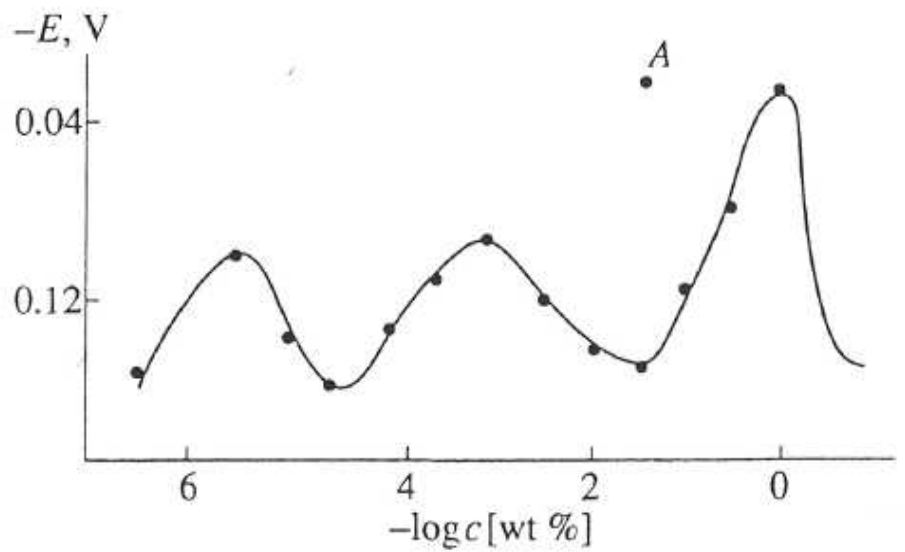

Fig. 15. Potential of the peroxidase sensor as a function of ethanol concentration (c) in an aqueous solution at $0.01 \mathrm{wt} \% \mathrm{H}_{2} \mathrm{O}_{2} ; A$ is the result obtained on a new aluminum electrode.

\section{Conclusion}

As a result of our studies, we developed biomimetic electrodes that did not lose their activity for prolonged periods of time under the action of the oxidant and its intermediates and final products of the decomposition of $\mathrm{H}_{2} \mathrm{O}_{2}$ and oxidation of $\mathrm{C}_{2} \mathrm{H}_{5} \mathrm{OH}$.

The designed biomimetic sensors proved stable, due to which they could be reused many times.

\section{Reference}

[1] Nagiev T.M., Coherent Synchronized Oxidation Reactions by Hydrogen Peroxide. Elsevier. Amsterdam. 2007. 325 p.

[2] T.M. Nagiev, Russian Journal of Physical Chemistry, Vol. 70, No. 6, 1996, pp. 895 - 903.

[3] Sorochinsky, V.V. and Kurganov, B.I., Prikl. Biokhim. MikrobioL, 1997, Vol. 33, No. 6, p. 579 (in Russian).

[4] Glazkov, V.V., Mizgunov, U.M., Zolotova, G.A. and Dolmanova, I.F., Zh. Analit. Khim., 1997, Vol. 52, No. 1, p. 83 (in Russian).

[5] Varfolomeev, S.D., Kurichkin, I.N. and Aropolov A.I., 1996, Vol. 11, No. 9, pp. 863-871. 
[6] Nagiev, T.M., Abbasova, M.T., Baba-zade, S.N., Kuliev, S.A., Stepanova, E.V. and Agamamedova, L.M., J. Appl. Biochem. Biotechnol, 2000, Vol. 88, p. 275.

[7] T.M. Nagiev, M.T. Abbasova, S.N. Baba-zade, E.V. Stepanova and L.M. Agamamedova, Russian Journal of Physical Chemistry, Vol. 73, No. 12, 1999, pp. 2032-2036.

[8] L.M. Agamamedova, M.T. Abbasova and T.M. Nagiev, Russian Journal of Physical Chemistry, Vol. 76, No. 12, 2002, pp. 1993-1997.

[9] T.M. Nagiev, N.A. Sardarly, Russian Journal of Physical Chemistry, 2008, vol. 83, No 8, pp. 1400-1404.

[10] Waker, J.F., Formaldehyde, Goskomkhimizdat, Moscow, 1957, 423 p. (in Russian). 


\title{
Design of Biomimetic Models Related to the Active Sites of Fe-Only Hydrogenase
}

\author{
Yu-Chiao Liu, Ling-Kuang Tu, Tao-Hung Yen and Ming-Hsi Chiang \\ Institute of Chemistry, Academia Sinica, \\ Taiwan
}

\section{Introduction}

Fe-only hydrogenase is a metalloenzyme that is found in a variety of organisms such as acetogenic, photosynthetic, nitrogen-fixing, methanogenic and sulfate reducing bacteria (Adams, 1990; Vignais \& Billoud, 2007). It plays an important role on energy cycling in the biological systems. Fe-only hydrogenase can either metabolize hydrogen molecules to produce reducing equivalents or store reducing power in the format of molecular hydrogen (Fontecilla-Camps et al., 2007). The most intriguing part of Fe-only hydrogenase is its high efficiency $\left(6 \times 10^{3} \mathrm{~s}^{-1}\right)$ in $\mathrm{H}_{2}$ production at a mild potential $(-0.1$ to $-0.5 \mathrm{~V}$ vs NHC) (Holm et al., 1996). Understanding mechanism of enzymatic hydrogen production will facilitate design of better biomimetic models of Fe-only hydrogenase for substitutes to expensive platinum working electrodes used in industrial hydrogen production (Cammack et al., 2001; Vincent et al., 2007).

$$
2 \mathrm{H}^{+}+2 \mathrm{e}^{-} \leftrightarrow \mathrm{H}_{2}
$$

Recent structural characterization of Fe-only hydrogenase isolated from Clostridium pasteurianum (CpI) (Peters et al., 1998) and Defulfovibrio desulfuricans (DdH) (Nicolet et al., 1999) shows the active site that is a [6Fe6S] unit called the H-cluster consists of a [2Fe2S] subunit including two square-pyramidal Fe centers bridged by a dithiolate ligand, and a [4Fe4S] cluster (Fig. 1). These [2Fe2S] and [4Fe4S] clusters are linked to each other via a cysteinyl thiolate on the peptide chain. In addition to the Scys bridge, several cyanide and
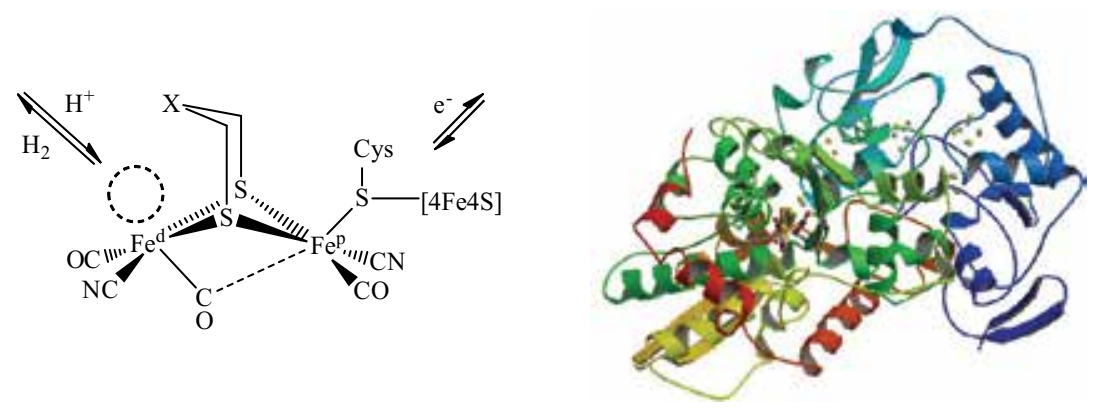

Fig. 1. Protein structure of Fe-only hydrogenase from Clostridium pasteurianum (1FEH) (Peters et al., 1998) on the right and the Chemdraw structure of the active site on the left. 
carbonyl ligands occupy the primary coordination sphere of the diiron unit. The dithiolate bridge is an abiological ligand that is presumed to dithiomethyl amine $\left(\mathrm{HN}\left(\mathrm{CH}_{2} \mathrm{~S}\right)_{2}\right)(\mathrm{Nicolet}$ et al., 2001; Silakov et al., 2009). Two CN- groups are arranged in the trans configuration and form hydrogen bonding to nearby amino residues of the peptide backbone. One metal-metal bond is present between two low-spin Fe $\mathrm{I}^{\mathrm{I}}$ centers that are separated by 2.5-2.6 $\mathrm{A}$. One vacant site is open to coordination of substrates at the $\mathrm{Fe}^{\mathrm{d}}$ center that is the metal site distal to the [4Fe4S] ferredoxin cluster. To the trans position of the catalytic site one $\mathrm{CO}$ ligand within the $\mathrm{Fe}_{2}$ subunit coordinates underneath the Fe-Fe vector for a possible function: distributing electron density between two Fe centers.

When protons are transported through a proton channel within the protein to the active site of Fe-only hydrogenase, the aza nitrogen site being a Lewis base relays protons to the catalytic Fe center as the N-protonated (NH) (Ezzaher et al., 2009; Wang et al., 2005; Wang et al., 2008) and the N-protonated, Fe-hydride (NHFeH) intermediates are generated (Barton et al., 2008; Chiang et al., 2009; Siegbahn et al., 2007) (Fig. 2). Migration of the NH protons is probably initiated by agostic interaction. Another advantage to have the aza nitrogen within the active site of Fe-only hydrogenase is decrease of the reduction potential upon protonation of the $\mathrm{N}$ sites (Capon et al., 2008; Liu et al., 2010). In the recent studies of model complexes, the protonated Fe adt (adt $=$ azadithiolate) derivative is reduced at $-1.82 \mathrm{~V}$ compared with $-2.43 \mathrm{~V}$ of the un-protonated species (Chiang et al., 2009). Such energy difference as well as ability of proton relays is the reason for protein crystallographers and chemists to choose nitrogen as the putative bridgehead over oxygen and carbon atoms since they are not distinguishable in crystallography (Nicolet et al., 1999; Pandey et al., 2008). In the reversible reaction, the lone pair of the aza nitrogen site assists heterolytic cleavage of molecular hydrogen as the catalytic Fe acts as a Lewis acidic site (Olsen et al., 2009).
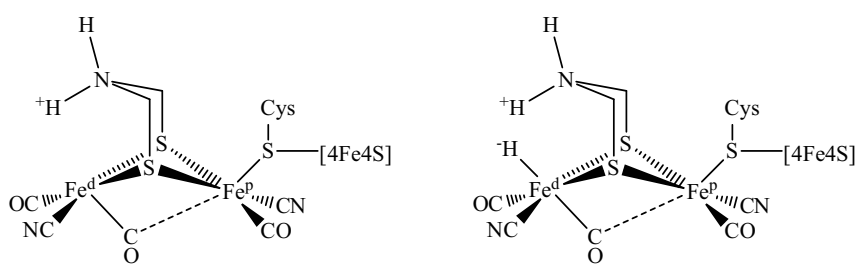

Fig. 2. Possible intermediates in mechanism of enzymatic $\mathrm{H}_{2}$ production by Fe-only hydrogenase.

If the aza nitrogen site is to assist the enzymatic $\mathrm{H}_{2}$ production/uptake, availability of the vacant site on the nearby Fe center is a compliment necessity for the high catalytic turnover frequency of the entatic state. Isolation of the model complexes possessing the azadithiolate bridges has been successfully accomplished (Lawrence et al., 2001; Li \& Rauchfuss, 2002; Ott et al., 2004). On the contrary, difficulties have been encountered when the model compounds with the apical empty site are prepared albeit few unstable examples have been reported.

The organometallic complexes composed of $\{2 \mathrm{Fe} 2 \mathrm{~S}\}$ moiety have been extensively studied in the recent years (Darensbourg et al., 2003; Tard \& Pickett, 2009). Numerous synthetic routes have been developed to better approach the active site of Fe-only hydrogenase (Tard et al., 2005). CO substitution by cyanides, phosphines and other $\sigma-/ \Pi$-donors are performed in order to enrich electron density about the Fe centers (Lawrence et al., 2001; Liu et al., 2005; Lyon et al., 1999). $\mathrm{H}_{2}$ production in the presence of these synthetic models is studied by 
voltammetric techniques such as cyclic voltammetry and controlled-potential electrolysis (Capon et al., 2009; Capon et al., 2005; Ezzaher et al., 2009; Felton et al., 2009). Unfortunately, the hydride within the identifiable Fe-hydride species is in the bridging fashion for most of the examples (Gloaguen \& Rauchfuss, 2009; Greco et al., 2007). The most possible mechanism for formation of the $\mu$-hydride products is addition of protons to the electron enriched Fe-Fe orbitals (Gloaguen et al., 2001). Theoretical calculations have shown that greater activation energy is required to generate the hydride in the terminal manner and the $t$-hydride species is less stable (Zampella et al., 2009). In order to overcome the kinetic hindrance, the apical empty site is a key component.

When the $\left\{\mathrm{Fe}_{2} \mathrm{~S}_{2}\right\}$ unit of $\mathrm{Fe}_{2}(\mathrm{SS})(\mathrm{CO})_{6-2 x} \mathrm{~L}_{2 x}$ and its derivatives (SS = dithiolate linker) is viewed along the Fe-Fe axis, two $\mathrm{Fe}(\mathrm{CO})_{3-x} \mathrm{~L}_{x}$ moieties are in eclipse configuration. If one of the $\mathrm{Fe}(\mathrm{CO})_{3-\mathrm{x}} \mathrm{L}_{\mathrm{x}}$ moieties is twisted and one of its $\mathrm{CO}$ ligands is located underneath the $\mathrm{Fe}-\mathrm{Fe}$ vector, the apical site on the Fe center is opened up. In this chapter, we would like to deal with the "rotated" structure of the diiron dithiolato carbonyl complexes. Factors that are in control of the rotated geometry will be discussed.
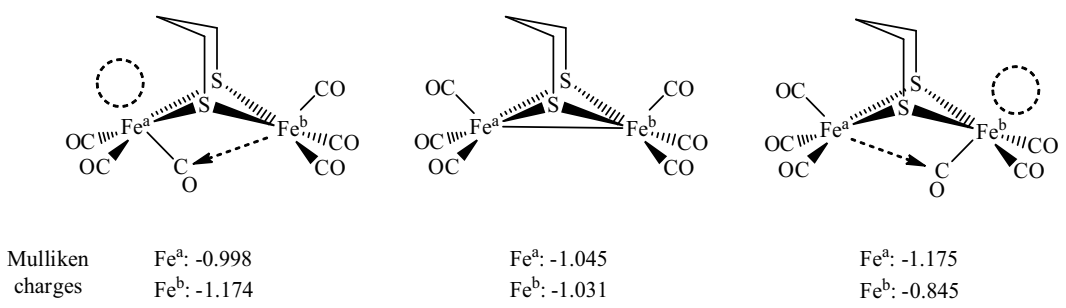

Fig. 3. Changes of Mulliken charges of the Fe centers upon rotation of the Fea and Feb sites (Georgakaki et al., 2003).

\section{The rotated structure}

The computed results for the optimized structure of $\left[\mathrm{Fe}_{2}(\mu-\mathrm{pdt})(\mathrm{CO})_{6}\right]$ (pdt $=1,3-$ propanedithiolate) that agree with the experimentally determined data show electronic equivalence between two metal centers (Georgakaki et al., 2003). The Mulliken charges for both Fe sites are -1.045 and -1.031 . HOMO of the ground state structure is predominated by $\mathrm{Fe}-\mathrm{Fe}$ bond character with symmetric metal contribution, shown in Fig. 3. Once one of the $\mathrm{Fe}(\mathrm{CO})_{3}$ moieties is rotated by $60^{\circ}$, the Fe-Fe bond is disrupted and its distance increases by $0.07 \AA$. Asymmetric Mulliken charges are obtained as the Fe site of the rotated subunit becomes more positive. In the unrotated structure, all $\angle \mathrm{Fe}-\mathrm{C}-\mathrm{O}$ are nearly $180^{\circ}$ whereas $\angle \mathrm{Fe}-\mathrm{C}-\mathrm{O}$ (semi-bridged) is decreased to as small as $168^{\circ}$ as the $\mathrm{Fe}(\mathrm{CO})_{3}$ unit is rotated and a semi-bridging $\mathrm{CO}$ group is formed. The $\mathrm{Fe} \bullet \bullet \mathrm{CO}$ (semi) distance is determined to $2.67 \AA$. Such short distance indicates some interaction is present between the Fe center and this carbonyl group. It is expected the more negative unrotated Fe center distributes some degree of electron density to the more positive rotated Fe center via the $\mathrm{CO}$ semi-bridge, which probably provides stabilizing incentive to the rotated structure.

In order to determine degree of the distortion between two Fe subunits, two parameters, $\psi$ and $\Phi$, are employed. Crabtree has introduced the $\psi$ angle to evaluate the coordination mode of CO (Crabtree \& Lavin, 1986), displayed in Fig. 4. It is defined to the angle between the Fe-Fe vector and the $\mathrm{CO}$ group underneath it. A typical $\psi$ value around $100^{\circ}$ is observed 
for the terminal carbonyl, whereas it decreases as the carbonyl spins about the Fe-Fe axis to form a $\mathrm{CO}$ semi-bridge as the $\mathrm{CO}$ continues approaching the second Fe center. A symmetrically coordinated and bent $\mathrm{CO}$ bridge $\left(\angle \mathrm{Fe}-\mathrm{C}-\mathrm{O}(\right.$ bri $\left.) \approx 140^{\circ}\right)$ is formed eventually. The second parameter introduced by Darensbourg is the $\Phi$ angle which is defined as the torsion angle of $\mathrm{L}_{\mathrm{ap}}-\mathrm{Fe}-\mathrm{Fe}-\mathrm{L}_{\mathrm{ap}}$ ( $\mathrm{L}_{\mathrm{ap}}=$ the apical ligand) (Singleton et al., 2008).
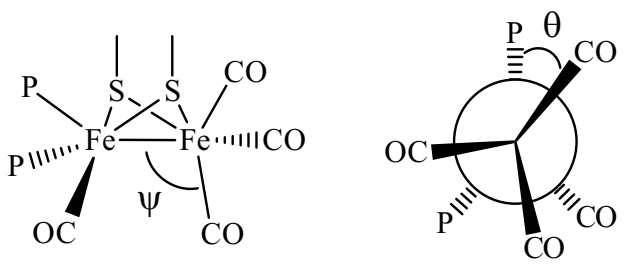

Fig. 4. Schematic presentations of the $\psi$ and $\Phi$ angles.

\section{Electronic asymmetry}

\subsection{Electronic asymmetry created by inequivalent substitution}

As indicated by DFT calculations (Georgakaki et al., 2003), the structure in the lower energy consists of apical substitution when one $\mathrm{CO}$ group is replaced by a $\sigma$-donating ligand. Owing to the steric repulsion of the bridgehead the conformer with an apical $\sigma$ donor at the less hindered side has the lowest energy. This species also has the smallest activation energy for rotation of the $\mathrm{Fe}(\mathrm{CO})_{3}$ subunit among all isomers of $\left[\mathrm{Fe}_{2}(\mu-\mathrm{pdt})(\mathrm{CO})_{5}(\mathrm{CN})\right]$. It suggests the $\mathrm{Fe} \cdot \bullet \mathrm{CO}$ (semi) interaction is enhanced and the semi-bridging $\mathrm{CO}$ is stabilized in the presence of the apical $\sigma$ donor. Addition of a substrate is then favored if so. In fact, monocyanation of $\left[\mathrm{Fe}_{2}(\mathrm{CO})_{5}\left\{\mathrm{MeSCH}_{2} \mathrm{C}(\mathrm{Me})\left(\mathrm{CH}_{2} \mathrm{~S}\right)_{2}\right\}\right]$ occurs at a rate $10^{4}$ times faster than the same reaction for $\left[\mathrm{Fe}_{2}(\mathrm{CO})_{6}\left\{\mathrm{CH}_{2}\left(\mathrm{CH}_{2} \mathrm{~S}\right)_{2}\right\}\right]$ (Zampella et al., 2005). The former has a thioether coordinated to the trans position of the Fe-Fe bond in contrast to the all-CO environment of the latter. This apical coordination facilitates facile turnstile rotation of the adjacent $\mathrm{Fe}(\mathrm{CO})_{3}$ subunit to accept $\mathrm{CN}$ - coordination. In this associative mechanism, one bridging/semibridging $\mathrm{CO}$ intermediate is generated, which has been spectroscopically characterized (Razavet et al., 2001). Besides, it would be interesting to compare the reaction rate for the complex with mono-substitution at the basal position. Only small amount of the di-cyanide product is formed when $\left[\mathrm{Fe}_{2}(\mathrm{CO})_{5}(\mathrm{CN})\left\{\mathrm{MeSCH}_{2} \mathrm{C}(\mathrm{Me})\left(\mathrm{CH}_{2} \mathrm{~S}\right)_{2}\right\}\right]$ reacts with cyanide (George et al., 2002). In this molecule, the thioether group is merely a dangling end. Less electron density supplied by the basal cyanide to LUMO of the $\mathrm{Fe}_{2}$ core makes rupture of the Fe-Fe bond more difficult so the $\mathrm{Fe}(\mathrm{CO})_{3}$ rotation is hindered. In addition, formation of the semi-bridging CO is destabilized. It is found that 300 -fold excess of cyanide is required to force clean formation of the di-cyanide species. These results emphasize importance of the apical substitution within $\left[\mathrm{Fe}_{2}(\mu-\mathrm{SS})(\mathrm{CO})_{5} \mathrm{~L}\right]$.

As appropriate bidentate ligands are employed, larger asymmetric electronic environment is created since the $\mathrm{Fe}(\mathrm{CO}) \mathrm{L}_{2}$ moiety is much electron-enriched. Greater tendency to constitute the inverted geometry is observed for a few known examples. Compared to $\left[\mathrm{Fe}_{2}(\mu-\mathrm{pdt})(\mathrm{CO})_{6}\right]$, $\mathrm{PMe}_{3}$ substitution occurs at the faster rate for $\left[\mathrm{Fe}_{2}(\mu-\mathrm{pdt})(\mathrm{CO})_{4}\left(\mathrm{~K}^{2}-\mathrm{dppv}\right)\right](\mathrm{dppv}=\mathrm{cis}-1,2-$ bis(diphenylphosphino)ethylene) (Justice et al., 2007). It is estimated the rotated transition state is stabilized by $5 \mathrm{kcal} / \mathrm{mol}$ in the presence of dppv. On the contrary, the complexes with symmetrically di-substitution are generally inert toward further substitution. 

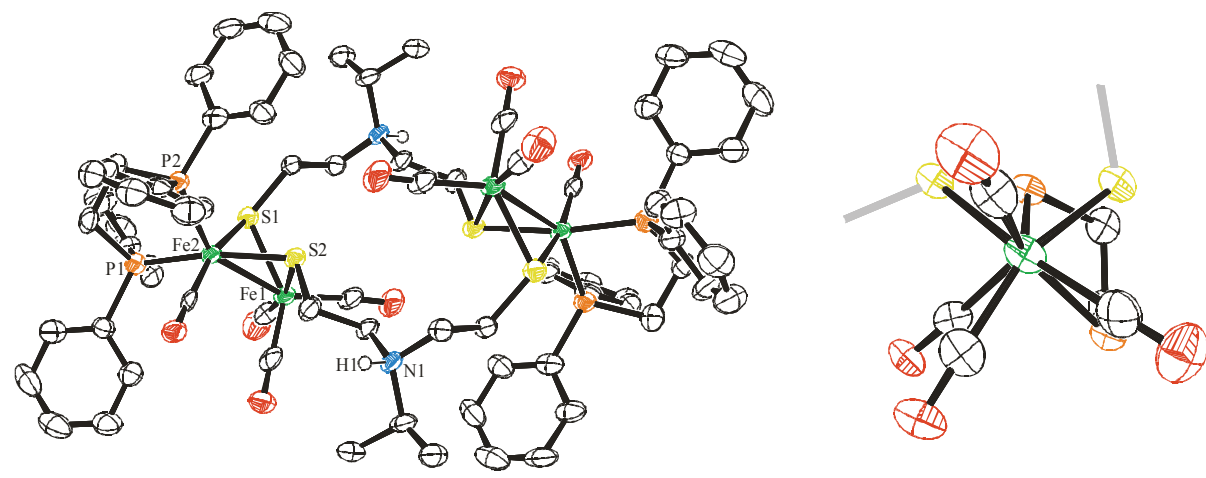

Fig. 5. Molecular structures of $\left[\mathrm{Fe}_{2}\left(\mu-\mathrm{S}\left(\mathrm{CH}_{2}\right)_{2} \mathrm{Ni} \operatorname{Pr}(\mathrm{H})\left(\mathrm{CH}_{2}\right)_{2} \mathrm{~S}\right)(\mathrm{CO})_{4}\left(\mathrm{~K}^{2}-\mathrm{dppe}\right)\right]_{2}{ }^{2+}$, thermal ellipsoids drawn at $30 \%$ probability level. All hydrogen atoms are omitted for clarity. The side view of partial molecular structure is shown to highlight the distortion.

Computational analysis has shown the highest-energy and lowest-energy IR bands of carbonyls are associated with the $\mathrm{Fe}(\mathrm{CO})_{3}$ and $\mathrm{Fe}(\mathrm{CO}) \mathrm{L}_{2}$ moieties, respectively, in $\left[\mathrm{Fe}_{2}(\mu-\right.$ $\left.\mathrm{SS})(\mathrm{CO})_{4}\left(\mathrm{~K}^{2}-\mathrm{P}_{2}\right)\right]\left(\mathrm{P}_{2}=\right.$ diphosphine chelates) (Justice et al., 2007). The energy difference, $\Delta \mathrm{v}_{\mathrm{CO}}$, therefore can be treated as an approximate gauge of electronic asymmetry between two Fe subunits and act a compliment to the $\psi$ and $\Phi$ angles. For the complexes with inequivalent phosphine substitution, the $\Delta \mathrm{v}_{\mathrm{CO}}$ value is commonly greater than $100 \mathrm{~cm}^{-1}$ (Justice et al., 2007; Liu et al., 2010; Song et al., 2005; Wang et al., 2008). On the other hand, smaller energy difference of 70-90 $\mathrm{cm}^{-1}$ is observed for the symmetrical diphosphine species (Gao et al., 2007; $\mathrm{Li}$ et al., 2007). Among the known phosphine-substituted complexes, $\left[\mathrm{Fe}_{2}(\mu-\right.$ $\left.\mathrm{S}\left(\mathrm{CH}_{2}\right)_{2} \mathrm{NiPr}(\mathrm{Me})\left(\mathrm{CH}_{2}\right)_{2} \mathrm{~S}\right)(\mathrm{CO})_{4}\left(\mathrm{~K}^{2}\right.$-dppe $\left.)\right]_{2}{ }^{2+}$ (dppe = 1,2-bis(diphenylphosphino)ethane) is the only $\mathrm{K}^{2}$-diphosphine example with the most distorted structure, characterized by crystallography, where the $\psi$ and $\Phi$ angles are $85.9^{\circ}$ and $36.3^{\circ}$, respectively. Other than its related derivative, $\left[\mathrm{Fe}_{2}\left(\mu-\mathrm{S}\left(\mathrm{CH}_{2}\right)_{2} \mathrm{Ni} \operatorname{Pr}(\mathrm{H})\left(\mathrm{CH}_{2}\right)_{2} \mathrm{~S}\right)(\mathrm{CO})_{4}\left(\mathrm{~K}^{2} \text {-dppe }\right)\right]_{2}{ }^{2+}$ with $\psi=91.4^{\circ}$, no example has an angle smaller or close to $90^{\circ}$. The $\Phi$ angle of the same molecule is measured to $34.1^{\circ}$ (Fig. 5). The $\Delta v_{C O}$ value for both complexes is about $120 \mathrm{~cm}^{-1}$. These results rank these two complexes to the top two examples for the most twisted $\mathrm{Fe}(\mathrm{CO})_{3-\mathrm{x}} \mathrm{P}_{\mathrm{x}}$ configuration. Two other complexes are also reported to possess the large $\Phi$ angle: $30.2^{\circ}$ and $27.7^{\circ}$ for $\left[\mathrm{Fe}_{2}(\mu-\right.$ edt) $\left.(\mathrm{CO})_{4}\left(\mathrm{~K}^{2}-\mathrm{dppv}\right)\right]$ (edt $=1$,2-ethanedithiolate) (Justice et al., 2007) and $\left[\mathrm{Fe}_{2}(\mu-\mathrm{pdt})(\mathrm{CO})_{4}\left(\mathrm{~K}^{2}-\right.\right.$ $\mathrm{dppm})](\mathrm{dppm}=$ bis(diphenylphosphino)methane) (Adam et al., 2007), respectively. The two phosphine ends in these complexes occupy the apical and basal positions within one Fe center, which would probably assist rotation of the different Fe subunit.

Free energy profile reveals how the electronic factor dictates the reaction mechanism and products upon protonation of $\left[\mathrm{Fe}_{2}(\mu-\mathrm{SS})(\mathrm{CO})_{6-\mathrm{x}} \mathrm{P}_{\mathrm{x}}\right]$ (Zampella et al., 2009), as shown in Table 1. For both of the all-CO and di-substituted species, a high activation is required to form a $\mu-$ hydride product. This species in addition is thermodynamically disfavored for the former but is stable for the latter. Even so, no computed energy-minimum $t$-hydride structure can be reached for both cases. In other words, the more electron enriched metal centers are required to achieve the goal. In solution, no protonation proceeds unless the all-CO species is reduced first. Formation of the $t$-hydride intermediates are characterized by spectroscopy in the reaction of $\left[\mathrm{Fe}_{2}(\mu-\mathrm{pdt})(\mathrm{CO})_{4}\left(\mathrm{k}^{2}\right.\right.$-dppe)] with $\mathrm{HBF}_{4}$ (Ezzaher et al., 2007). Two species with hydrides located at either the all-CO or the P-substituted Fe portion are observed at the temperature below $220 \mathrm{~K}$. They quickly convert to the $\mu$-isomer as the temperature is 
increased to above $243 \mathrm{~K}$. The experimental results are well consistent with the computed data. Additional phosphine substitution can significantly reduce the reaction energy barrier of protonation. In the presence of four phosphine ligands, $\Delta G^{\ddagger}$ is lowered by about 10 $\mathrm{kcal} / \mathrm{mol}$. The $t$-hydride products become stable thermodynamically. The most striking point is formation of the $t$ - and $\mu$-hydrides is now kinetically equally favored. $\left[\mathrm{Fe}_{2}(\mu-\right.$ $\mathrm{pdt})(\mathrm{CO})_{2}\left(\mathrm{~K}^{2}-\mathrm{dppv}\right)_{2}$ ] reacts with $\mathrm{HBF}_{4}$ to generate the $t$-hydride product, which is slowly converted to its most stable species of the $\mu$-form (Barton \& Rauchfuss, 2008). Similarly, a small rate constant of $2 \times 10^{-4} \mathrm{~s}^{-1}(294 \mathrm{~K})$ is measured for the intramolecular isomerization of $\left[\mathrm{Fe}_{2}(\mu-\mathrm{edt})(\mathrm{CO})(\mu-\mathrm{CO})(\mathrm{H})\left(\mathrm{PMe}_{3}\right)_{4}\right]^{+}$to the $\mu$-species (van der Vlugt et al., 2005). Inequivalent $(3+1)$ substitution favors the terminal protonation route albeit the bridging form remains the thermodynamically more stable product.

\begin{tabular}{ccccc}
\hline \hline & \multicolumn{2}{c}{$\mu$-hydride } & \multicolumn{2}{c}{ t-hydride } \\
& $\Delta G$ & $\Delta G^{\ddagger}$ & $\Delta G$ & $\Delta G^{\ddagger}$ \\
\hline$\left[(\mathrm{CO})_{3} \mathrm{Fe}(\mu\right.$-edt $\left.) \mathrm{Fe}(\mathrm{CO})_{3}\right]$ & 11.3 & 17.1 & - & - \\
\hline$\left[(\mathrm{CO})_{3} \mathrm{Fe}\left(\mu\right.\right.$-edt) $\left.\mathrm{Fe}(\mathrm{CO})\left(\mathrm{PH}_{3}\right)_{2}\right]$ & -2.3 & 18.9 & - & - \\
\hline$\left[\left(\mathrm{PH}_{3}\right)_{2}(\mathrm{CO}) \mathrm{Fe}(\mu\right.$-edt $\left.) \mathrm{Fe}(\mathrm{CO})\left(\mathrm{PH}_{3}\right)_{2}\right]$ & -13.3 & 8.1 & -3.4 & 10.9 \\
\hline$\left[\left(\mathrm{PH}_{3}\right)(\mathrm{CO})_{2} \mathrm{Fe}(\mu\right.$-edt $\left.) \mathrm{Fe}\left(\mathrm{PH}_{3}\right)_{3}\right]$ & -19.4 & 6.0 & -8.1 & 0.0 \\
\hline \hline
\end{tabular}

Table 1. Kinetic and thermodynamic data for formation of the $\mu$ - and $t$-hydride species, as energies in $\mathrm{kcal} / \mathrm{mol}$ (Zampella et al., 2009).

A different class of the complexes with asymmetric electronic structure consists of nitrosyl substitution. When $\mathrm{PMe}_{3}$ replaces one $\mathrm{CO}$ group of the $\mathrm{Fe}(\mathrm{CO})_{3}$ moiety within $\left[\mathrm{Fe}_{2}(\mu-\right.$ $\mathrm{pdt})(\mathrm{CO})_{4}\left(\mathrm{~K}^{2}\right.$-dppv)], the torsion angle of $30.2^{\circ}$ decreases to $9.0^{\circ}$ owing to counterbalance of electronic asymmetry by $\mathrm{PMe}_{3}$ addition (Justice et al., 2007). In the presence of $\mathrm{NO}^{+}$, a significant twist of $36.1^{\circ}$ is observed in $\left[\mathrm{Fe}_{2}(\mu-\mathrm{pdt})(\mathrm{CO})_{3}(\mathrm{NO})\left(\mathrm{K}^{2} \text {-dppv) }\right]^{+}\right.$instead (Olsen et al., 2008). The most distorted configuration is observed in a series of diiron dithiolato trimethylphosphine complexes. For $\left[\mathrm{Fe}_{2}(\mu-\mathrm{xdt})(\mathrm{CO})_{3}(\mathrm{NO})\left(\mathrm{PMe}_{3}\right)_{2}\right]^{+}(\mathrm{xdt}=\mathrm{edt}$, pdt) where two $\mathrm{PMe}_{3}$ ligands occupy the apical and basal positions of two different Fe centers, the $\psi$ angles of $76 \sim 81^{\circ}$ are obtained (Olsen et al., 2008). When the bis-trans-basal $\mathrm{PMe}_{3}$ conformer is crystallographically characterized, the smallest $\psi$ angle of $62^{\circ}$ is measured (Fig. 6). This is the $\mathrm{FeIFe}^{\mathrm{I}}$ structure most resemble to the inverted geometry of the active site of Fe-only hydrogenase.
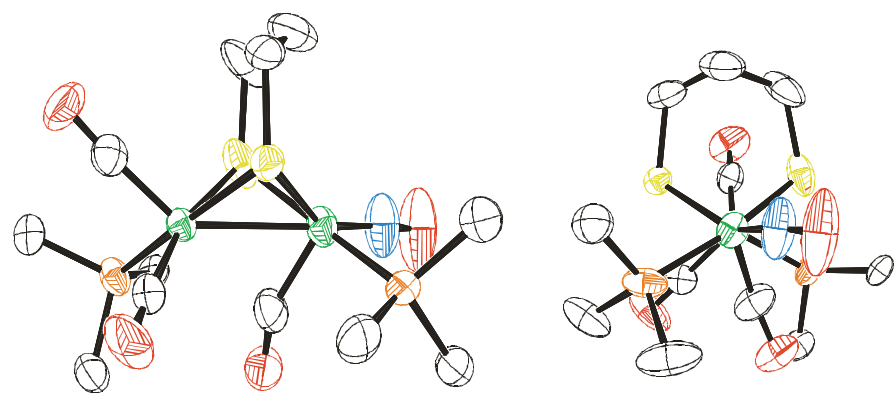

Fig. 6. Molecular structures of $\left[\mathrm{Fe}_{2}(\mu-\mathrm{pdt})(\mathrm{CO})_{3}(\mathrm{NO})\left(\mathrm{PMe}_{3}\right)_{2}\right]^{+}$, thermal ellipsoids drawn at $35 \%$ probability level (Olsen et al., 2008). The side view of molecular structure is shown to highlight the distortion. All hydrogen atoms are omitted for clarity. 


\subsection{The mixed-valence structures}

In addition to tuning by ligand management, the most straightforward route to create an electronic asymmetry is formation of the mixed-valence structures. Single-electron reduction of the $\mathrm{Fe}^{\mathrm{IFe}} \mathrm{Fe}^{\mathrm{I}}$ complexes causes $\mathrm{CO}$ liberation (Darchen et al., 1988) in accompany to Fe-S bond cleavage (Borg et al., 2008). The products such as $\left[\mathrm{Fe}_{2}\left(\mu, \mathrm{k}^{2}-\mathrm{xdt}\right)(\mu-\mathrm{CO})(\mathrm{CO})_{5}\right]^{-}(\mathrm{xdt}=$ pdt, edt, bdt; bdt $=1$,2-benzenedithiolate) identified by theoretical calculations (Capon et al., 2007; Felton et al., 2009; Felton et al., 2007), [Fe $\left.(\mu-\mathrm{pdt})(\mathrm{CO})_{6}\right]^{-}$spectroscopically characterized (Borg et al., 2004; Borg et al., 2007), and $\left[\left(\mu, \mathrm{K}^{1}-\mathrm{pdt}\right)\left\{\mathrm{Fe}_{2}(\mu-\mathrm{CO})(\mathrm{CO})_{6}\right\}\left\{\mathrm{Fe}_{2}(\mu-\right.\right.$ $\left.\left.\mathrm{pdt})(\mathrm{CO})_{5}\right\}\right]^{2-}$ and $\left[(\mu-\mathrm{CO})_{2}\left\{\mathrm{Fe}_{2}(\mu-\mathrm{pdt})(\mathrm{CO})_{4}\right\}_{2}\right]^{2-}$ isolated from experiments (Aguirre de Carcer et al., 2006; Best et al., 2007; Borg et al., 2004) have been obtained or proposed (Fig. 7).
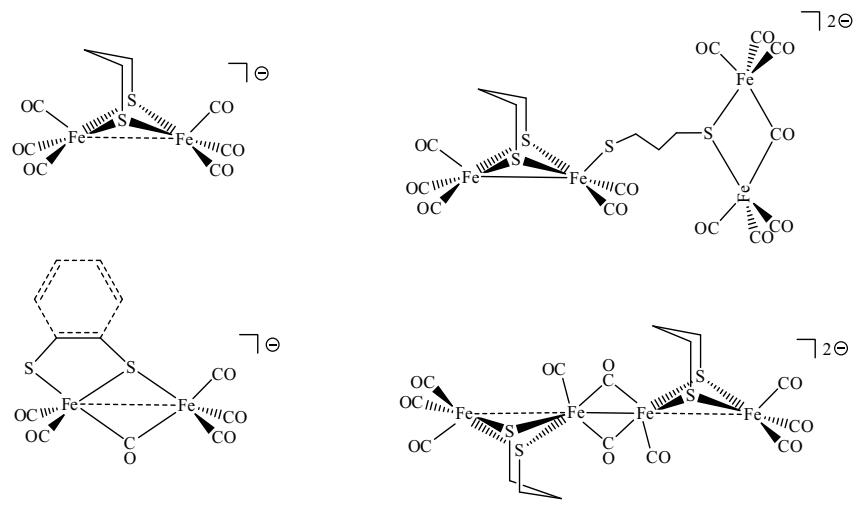

Fig. 7. Single-electron reduction products of the $\mathrm{Fe}^{\mathrm{IF}} \mathrm{Fe}^{\mathrm{I}}$ species. See the contents for references.
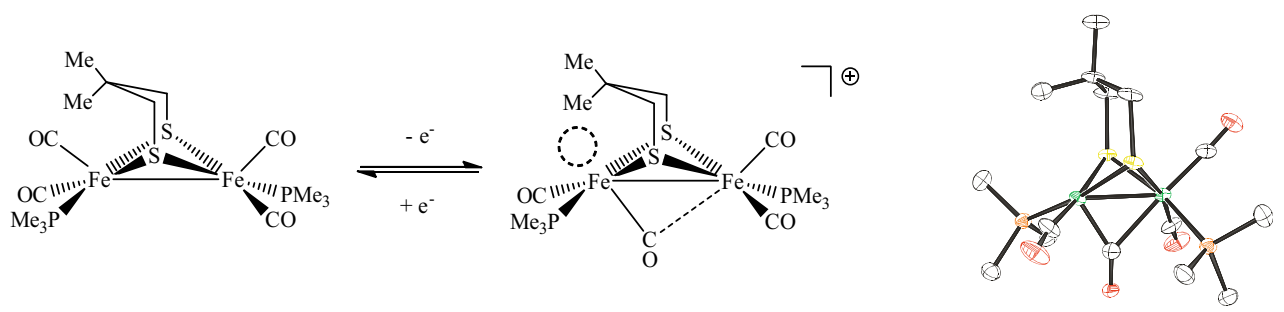

Fig. 8. Synthetic scheme of $\left[\mathrm{Fe}_{2}(\mu-\mathrm{dmpdt})(\mu-\mathrm{CO})(\mathrm{CO})_{3}\left(\mathrm{PMe}_{3}\right)_{2}\right]^{+}$and its molecular structure. All hydrogen atoms are omitted for clarity (Singleton et al., 2008).

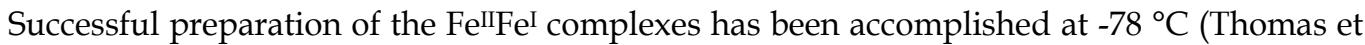
al., 2008). These species are only stable at very low temperature. It is assumed the steric influence from either the bulky bridgehead in $\left[\mathrm{Fe}_{2}(\mu-\mathrm{dmpdt})(\mathrm{CO})_{4}\left(\mathrm{PMe}_{3}\right)_{2}\right](\mathrm{dmpdt}=2,2-$ dimethyl-1,3-propanedithiolate) (Singleton et al., 2008) or the large structural hindrance ligand in $\left[\mathrm{Fe}_{2}(\mu-\mathrm{pdt})(\mathrm{CO})_{4}\left(\mathrm{PMe}_{3}\right)\right.$ (IMes)] (IMes = 1,3-bis(2,4,6-trimethylphenyl)imidazol-2ylidene) (Liu \& Darensbourg, 2007; Thomas et al., 2008) in addition to the mixed valence assists isolation of the empty apical site (Fig. 8). The ease of oxidation is decreased when additional phosphine ligands replace $\mathrm{CO}$ groups of $\left[\mathrm{Fe}_{2}(\mu-\mathrm{xdt})(\mathrm{CO})_{4}\left(\mathrm{~K}^{2}-\mathrm{dppv}\right)\right]$ (Justice et al., 2008). The oxidation potential for $\left[\mathrm{Fe}_{2}(\mu-\mathrm{edt})(\mathrm{CO})_{2}\left(\mathrm{~K}^{2}-\mathrm{dppv}\right)_{2}\right]$ is $-195 \mathrm{mV}$ milder than that of $\left[\mathrm{Fe}_{2}(\mu\right.$-edt $)(\mathrm{CO})_{3}\left(\mathrm{PMe}_{3}\right)\left(\mathrm{K}^{2}\right.$-dppv)] (Justice et al., 2008). The semi-bridging $\mathrm{CO}$ group is characterized in these complexes. The empty apical site is also confirmed by addition of 


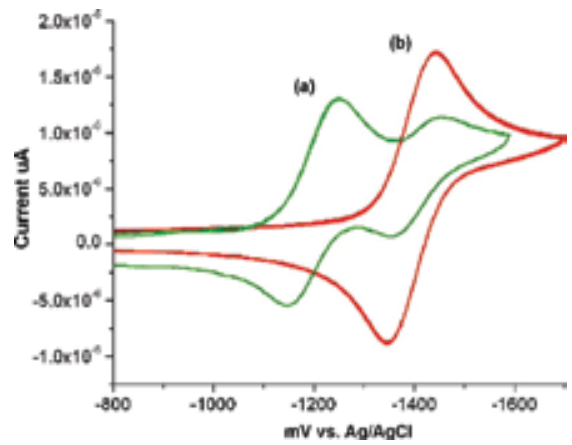

Fig. 9. Cyclic voltammograms of $\left[\mathrm{Fe}_{2}(\mu-\mathrm{pdt})(\mathrm{H})(\mathrm{CO})_{2}\left(\mathrm{k}^{2}-\mathrm{dppv}\right)_{2}\right]^{+}$. (a) a mixture of the $t$ - and $\mu$-isomers and (b) the $\mu$-isomer after isomerization (Barton \& Rauchfuss, 2008). (copyright 2008 American Chemical Society)

exogenous $\mathrm{CO}$ and $\mathrm{NO}$ molecules to the first coordination sphere within the $\mathrm{Fe}^{\mathrm{II}} \mathrm{Fe}^{\mathrm{I}}$ complex (Justice et al., 2007). The $\mathrm{CO}$ adduct product resembles the $\mathrm{H}_{\mathrm{ox}} \mathrm{CO}$ state of the Fe-only hydrogenase. When $\left[\mathrm{Fe}_{2}\left\{\mathrm{MeSCH}_{2} \mathrm{C}(\mathrm{Me})\left(\mathrm{CH}_{2} \mathrm{~S}\right)_{2}\right\}(\mathrm{CN})_{2}(\mathrm{CO})_{4}\right]^{2-}$ is oxidized by one electron, one $\mathrm{CO}$ group turns to the bridging mode, which allows the thioether pendant end to coordinate to the apical position (Razavet et al., 2002). The reduction potential can benefit

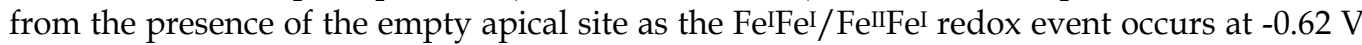
for $\left[\mathrm{Fe}_{2}(\mu-\mathrm{pdt})(\mathrm{CO})_{4}\left(\mathrm{PMe}_{3}\right)(\mathrm{IMes})\right]$ vs $-0.10 \mathrm{~V}$ for $\left[\mathrm{Fe}_{2}(\mu-\mathrm{pdt})(\mathrm{CO})_{4}\left(\mathrm{PMe}_{3}\right)_{2}\right]$ (Singleton et al., 2008). For the sake of comparison, the $t$-hydride species of $\left[\mathrm{Fe}_{2}(\mu-\mathrm{pdt})(\mu-\mathrm{CO})(\mathrm{H})(\mathrm{CO})\left(\mathrm{k}^{2}-\right.\right.$ dppv $\left.)_{2}\right]^{+}$is reduced at a potential $200 \mathrm{mV}$ milder than its $\mu$-isomer of $\left[\mathrm{Fe}_{2}(\mu-\mathrm{pdt})(\mu-\right.$ $\left.\mathrm{H})(\mathrm{CO})_{2}\left(\mathrm{~K}^{2}-\mathrm{dppv}\right)_{2}\right]^{+}$(Barton \& Rauchfuss, 2008), shown in Fig. 9.

\section{Bridgehead effects of dithiolate linkers}

\subsection{Steric influences of the bridgeheads}

Significance of the bridgehead's sterics on the activation energy to rotate one $\mathrm{Fe}(\mathrm{CO})_{3}$ moiety in $\left[\mathrm{Fe}_{2}(\mu-\mathrm{SS})(\mathrm{CO})_{6}\right]$ is shown by DFT calculations, where $\Delta G^{\ddagger}$ of $14.10 \mathrm{kcal} / \mathrm{mol}$ for $\left[\mathrm{Fe}_{2}(\mu-\mathrm{edt})(\mathrm{CO})_{6}\right]$ is decreased to 13.71 and $12.25 \mathrm{kcal} / \mathrm{mol}$ for $\left[\mathrm{Fe}_{2}(\mu-\mathrm{pdt})(\mathrm{CO})_{6}\right]$ and $\left[\mathrm{Fe}_{2}(\mu-\right.$ $o$-xyldt)(CO) $)_{6}$ (o-xyldt $\left.=o-\mathrm{C}_{6} \mathrm{H}_{4}\left(\mathrm{CH}_{2} \mathrm{~S}\right)_{2}\right)$, respectively (Georgakaki et al., 2003). These theoretical results indicate the more hindered bridgehead lowers the barrier to rotation. In the experimental aspects, this factor has been concurred with crystallographical results of a series of $\left[\mathrm{Fe}_{2}(\mu-\mathrm{SS})(\mathrm{CO})_{6-\mathrm{x}} \mathrm{L}_{x}\right]$ complexes. The $\Phi$ angle practically increases from zero in $\left[\mathrm{Fe}_{2}(\mu-\mathrm{pdt})(\mathrm{CO})_{6}\right]$ to $5.7^{\circ}$ in $\left[\mathrm{Fe}_{2}(\mu-\mathrm{dmpdt})(\mathrm{CO})_{6}\right]$ (Singleton et al., 2008). A larger $\Phi$ value of $15.8^{\circ}$ is observed in the bulkier bridgehead derivative, $\left[\mathrm{Fe}_{2}(\mu-\mathrm{depdt})(\mathrm{CO})_{6}\right]$ (depdt $=2,2-$ diethyl-1,3-propanedithiolate). In addition, a dramatic twist occurs from $4.26^{\circ}$ to $40.7^{\circ}$ when the pdt bridge in $\left[\mathrm{Fe}_{2}(\mu-\mathrm{pdt})(\mathrm{CO})_{5}(\mathrm{IMes})\right]$ is replaced by the dmpdt bridge. The axial configuration of the butyl substituent in $\left[\mathrm{Fe}_{2}(\mu-\mathrm{adt})(\mathrm{CO})_{4}(\mu-\mathrm{dppm})\right]\left(\mathrm{adt}=\mathrm{BuN}\left(\mathrm{CH}_{2} \mathrm{~S}\right)_{2}\right)$ causes a large torsion angle of $31^{\circ}$ compared with $5.2^{\circ}$ of $\left[\mathrm{Fe}_{2}(\mu-\mathrm{pdt})(\mathrm{CO})_{4}(\mu-\mathrm{dppm})\right](\mathrm{Gao}$ et al., 2007). Increased steric bulk of the bridgehead leads to larger repulsion between the substituents and the apical carbonyl in the unrotated structure. To lower it, the $\mathrm{Fe}(\mathrm{CO})_{3}$ subunit is forced to spin and eventually a rotated structure in relatively lower energy is obtained (Fig. 10). The calculated ground state energy of the unrotated and rotated optimized structures can be compared. For the smallest substituent, the rotated structure of $\left[\mathrm{Fe}_{2}(\mu-\mathrm{pdt})(\mathrm{CO})_{6}\right]$ is of $12.2 \mathrm{kcal} / \mathrm{mol}$ higher than its unrotated parent molecule. This energy 
decreases to $9.4 \mathrm{kcal} / \mathrm{mol}$ when one proton is replaced by a methyl group (Tye et al., 2006). Large stabilization energy of $4.8 \mathrm{kcal} / \mathrm{mol}$ is gained with for a $t$-Bu substituent.
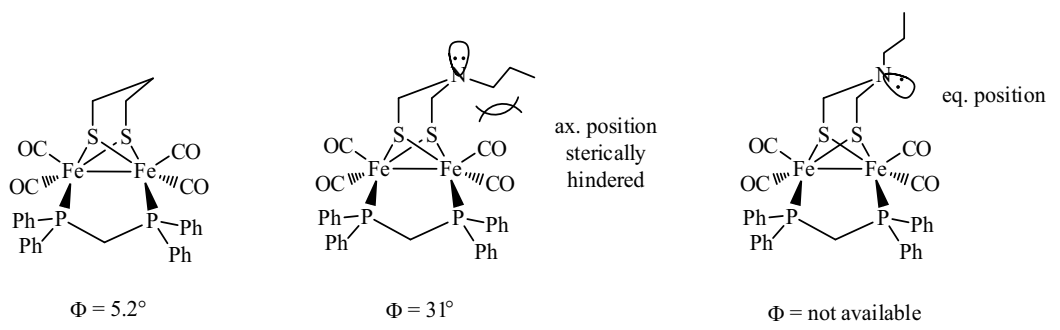

Fig. 10. The Chemdraw structures of $\left[\mathrm{Fe}_{2}(\mu-\mathrm{xdt})(\mathrm{CO})_{4}(\mu-\mathrm{dppm})\right]$ and their corresponding $\Phi$ angles (Gao et al., 2007).

Stabilization of the higher energy conformer by the steric hindrance is also observable in the oxidized complexes. In contrast to unavailability of $\left[\mathrm{Fe}_{2}(\mu-\mathrm{pdt})(\mathrm{CO})_{4}\left(\mathrm{PMe}_{3}\right)_{2}\right]^{+}$, the rotated geometry of $\left[\mathrm{Fe}_{2}(\mu-\mathrm{dmpdt})(\mathrm{CO})_{4}\left(\mathrm{PMe}_{3}\right)_{2}\right]^{+}$is stable for structural characterization (Singleton et al., 2008). However, the edt derivative of $\left[\mathrm{Fe}_{2}(\mu-x \mathrm{dt})(\mu-\mathrm{CO})(\mathrm{CO})_{2}\left(\mathrm{PMe}_{3}\right)(\mathrm{MeCN})\left(\mathrm{\kappa}^{2}-\right.\right.$ $\mathrm{dppv})]^{2+}$ is robust at room temperature whereas its pdt analogue is merely detectable at low temperature (Justice et al., 2007).

Length of the dithiolate linker has the similar influence to that exerted by the sterically demanding bridgehead. The calculated ground state energy of the rotated structure of $\left[\mathrm{Fe}_{2}\left(\mu-\mathrm{S}\left(\mathrm{CH}_{2}\right)_{n} \mathrm{~S}\right)(\mathrm{CO})_{6}\right]$ is stabilized as $n$ is increased (Tye et al., 2006). The rotated conformer is destabilized by $14.7 \mathrm{kcal} / \mathrm{mol}$ for $n=2$. The energy is decreased to 12.2 and 11.6 for $n=3$ and 4 , respectively. For the case of 1,5-pentanedithiolate, the energy is down to 7.4 $\mathrm{kcal} / \mathrm{mol}$.

\subsection{Lewis acidity and basicity of the bridgehead}

When a heteroatom replaces carbon as the central atom of the bridgehead, not only the steric hindrance but also Lewis acidity/basicity affects kinetics and stability of the rotated structure. For the three-atom dithiolate linkers, no variation in energy is observable if the $\mathrm{CH}_{2}$ group is replaced by the NH group (Tye et al., 2006). The relative short linker length limits any large destabilization caused by the central bridgehead. For the oxa analogue, lacking of the hydrogen atom is probably the reason for its increased ground state energy of the rotamer.

Longer backbones facilitate interaction between the bridgehead and the apical carbonyl of the unrotated structure as well as the metal site of the rotated structure. Among the $\mathrm{CH}_{2}$, $\mathrm{NH}$ and $\mathrm{O}$ derivatives of $\left[\mathrm{Fe}_{2}\left(\mu-\mathrm{X}\left(\mathrm{CH}_{2} \mathrm{CH}_{2} \mathrm{~S}\right)_{2}\right)(\mathrm{CO})_{6}\right]$, similar energies are obtained. In contrast, the $\mathrm{BH}$ analogue is destabilized by $7.5 \mathrm{kcal} / \mathrm{mol}$. A higher energy of $10.9 \mathrm{kcal} / \mathrm{mol}$ is required for its rotated species compared to the lowest-energy $\mathrm{NH}$ analogue. It is to say that the electron deficient $\left\{\mathrm{Fe}_{2}(\mathrm{CO})_{6}\right\}$ unit does not accommodate too well with the Lewis acid, i.e. the $\mathrm{BH}$ part. On the other hand, to the Lewis acidic units, $\mathrm{CO}$ and Fe, the aza nitrogen site forms a good Lewis acid-base pair. Both of its unrotated and rotated structures are of the same energy. A dangling aza nitrogen unit attached to the bridgehead will exert the similar effects on the ground state energy.

In the molecular structures of $\left[\mathrm{Fe}_{2}\left(\mu-\mathrm{S}\left(\mathrm{CH}_{2}\right)_{2} \mathrm{NR}\left(\mathrm{CH}_{2}\right)_{2} \mathrm{~S}\right)(\mathrm{CO})_{6}\right]_{2}\left(\mathrm{R}={ }_{n} \mathrm{Pr}, i \mathrm{Pr}\right)$, pointing lone pairs of the aza nitrogens toward the apical carbonyl groups reveals the $\mathrm{Fe}_{2}$ units are 

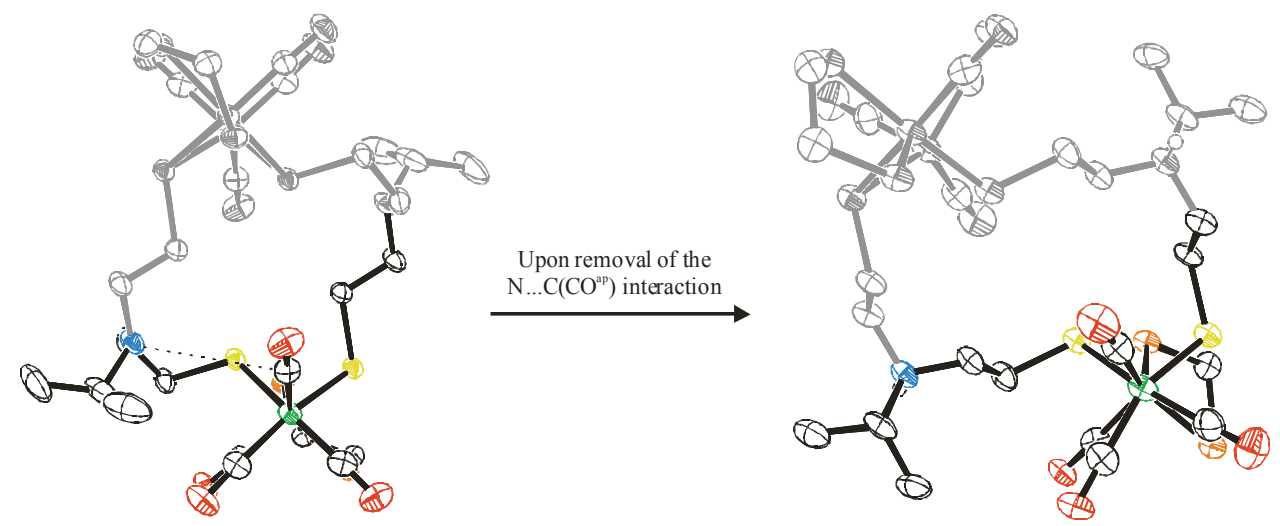

Fig. 11. Presentation of the distortion along the Fe-Fe axis upon removal of the $\mathrm{N} \bullet \bullet \mathrm{CO}(\mathrm{CO} a \mathrm{p})$ interaction within $\left[\mathrm{Fe}_{2}\left(\mu-\mathrm{S}\left(\mathrm{CH}_{2}\right)_{2} \mathrm{Ni} \operatorname{Pr}\left(\mathrm{CH}_{2}\right)_{2} \mathrm{~S}\right)(\mathrm{CO})_{4}\left(\mathrm{k}^{2}-\mathrm{dppe}\right)\right]_{2}$. Only the partial molecules are highlighted for the clearer view.

relatively electron poor (Liu et al., 2010). The presence of the $\mathrm{N} \bullet \bullet \mathrm{CO}(\mathrm{CO}$ ap) interaction would assist stabilization of the molecules and function as an indicator of electron richness about the Fe center. The $\mathrm{N} \bullet \bullet \mathrm{CO}(\mathrm{CO} a \mathrm{p})$ distance is increased when $\mathrm{CO}$ is substituted by phosphines. The stronger the $\sigma$-donating ability, the longer the distance. Besides, this $\mathrm{N} \bullet \bullet \mathrm{CO}(\mathrm{CO} a \mathrm{p})$ interaction could counterbalance electronic asymmetry within the molecule of $\left[\mathrm{Fe}_{2}\left(\mu-\mathrm{S}\left(\mathrm{CH}_{2}\right)_{2} \mathrm{Ni} \operatorname{Pr}\left(\mathrm{CH}_{2}\right)_{2} \mathrm{~S}\right)(\mathrm{CO})_{4}\left(\mathrm{~K}^{2} \text {-dppe }\right)\right]_{2}$. The distance of 3.721(7) $\AA$ is shorter than $4.011(3) \AA$ of the all-CO parent molecule, suggesting a stronger interaction is present. It is probably originated from asymmetric electronic distribution due to the presence of $\mathrm{K}^{2}$-dppe so the $\mathrm{Fe}(\mathrm{CO})_{3}$ subunit is more electron deficient than the same part in the all-CO molecule. Unequal distributed electron density is revealed by the distortion angle between two $\mathrm{Fe}$ subunits that changes from $10.8^{\circ}$ to $\sim 35^{\circ}$ upon removal of the $\mathrm{N} \bullet \bullet \mathrm{CO}(\mathrm{CO}$ ap) interaction (Fig. 11). These results stress importance of the aza nitrogen site on the structures.

\section{Coordination effect of ligands}

\subsection{Ligand field caused by the coordinated groups}

The active site of Fe-only hydrogenase is encapsulated inside the protein pocket wherein numerous hydrogen bonds between the bound $\mathrm{CN}^{-}$ligands and the peptide chain are present. Two amino acid residues, Lys ${ }^{358}$ and Ser 232 , form hydrogen bonds with the CNgroups of the H-cluster in CpI (Peters et al., 1998). As for DdH, Lys ${ }^{237}$ and the peptide-chain nitrogen atoms of Ala ${ }^{109}$ and Ile $^{204}$ are in close contacts with the cyanides (Nicolet et al., 1999). In addition to the functional role being to modulate electronic structure of the Fe centers, these weak intramolecular interactions serve the structural purpose to support the $\mathrm{H}$-cluster. The peptide backbone that is in control of the rotated geometry of the active site probably via hydrogen bonding would slightly adjust the twist as morphology of the protein changes to adopt the optimal stereo configuration during catalysis. In the synthetic aspects, the di-phosphine bridges with an appropriate length in $\left[\mathrm{Fe}_{2}(\mu-\mathrm{SS})(\mathrm{CO})_{4} \mathrm{P}_{2}\right]$ can well resemble a combination of cyanides and the peptide chain as the donor ends and the chain backbone are already present. Their donor ability can be tuned by different substituents on the phosphines (Fig. 12). 

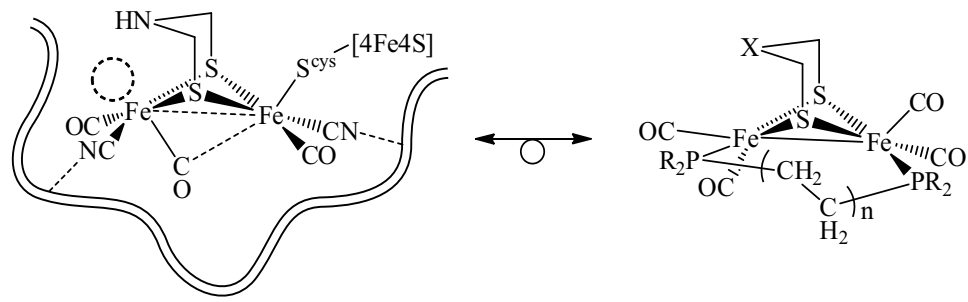

Fig. 12. Schematic presentation of the structural resemblance between the active site and the model complexes.

The distortion angles in $\left[\mathrm{Fe}_{2}\left(\mu-\mathrm{S}\left(\mathrm{CH}_{2}\right)_{2} \mathrm{NiPr}\left(\mathrm{CH}_{2}\right)_{2} \mathrm{~S}\right)(\mathrm{CO})_{4}(\mu-\mathrm{PP})\right]_{2}(\mathrm{PP}=\mathrm{dppe}$, dppm) can be compared. For the shorter phosphine, the $\mathrm{Fe}_{2}(\mathrm{CO})_{4} \mathrm{P}_{2}$ unit with a dppm bridge has the more compact structure: 2.522 and $2.549 \AA$ for the $\mathrm{Fe}-\mathrm{Fe}$ bond distances in $\left[\mathrm{Fe}_{2}(\mu-\right.$ $\left.\left.\mathrm{S}\left(\mathrm{CH}_{2}\right)_{2} \mathrm{~N} i \operatorname{Pr}\left(\mathrm{CH}_{2}\right)_{2} \mathrm{~S}\right)(\mathrm{CO})_{4}(\mu-\mathrm{dppm})\right]_{2}$ and its dppe analogue, respectively (Liu et al., 2010). Since dppm is a relatively rigid bridging ligand, only the eclipse configuration between two Fe moieties is allowed to be achieved. The $\Phi$ angle and $\mathrm{N} \bullet \bullet \mathrm{CO}(\mathrm{CO}$ ap) distance of the dppm derivative therefore is comparable to those of the all-CO parent molecule: $3.9 \mathrm{vs} 6.0^{\circ}$ and 3.987(7) vs 4.011(3) $\AA$ for $\left[\mathrm{Fe}_{2}\left(\mu-\mathrm{S}\left(\mathrm{CH}_{2}\right)_{2} \mathrm{Ni} \operatorname{Pr}\left(\mathrm{CH}_{2}\right)_{2} \mathrm{~S}\right)(\mathrm{CO})_{4}(\mu-\mathrm{dppm})\right]_{2}$ and $\left[\mathrm{Fe}_{2}(\mu-\right.$ $\left.\left.\mathrm{S}\left(\mathrm{CH}_{2}\right)_{2} \mathrm{Ni} \operatorname{Pr}\left(\mathrm{CH}_{2}\right)_{2} \mathrm{~S}\right)(\mathrm{CO})_{6}\right]_{2}$, respectively. The other dppm example, $\Phi=5.2^{\circ}$ for $\left[\mathrm{Fe}_{2}(\mu-\right.$ pdt) $(\mu$-dppm $\left.)(C O)_{4}\right]$, is reported (Gao et al., 2007).

Dppe, on the other hand, consists of a two-carbon backbone, giving it higher degree of freedom to adopt the least strained structure in $\left[\mathrm{Fe}_{2}\left(\mu-\mathrm{S}\left(\mathrm{CH}_{2}\right)_{2} \mathrm{NiPr}\left(\mathrm{CH}_{2}\right)_{2} \mathrm{~S}\right)(\mathrm{CO})_{4}(\mu \text {-dppe })\right]_{2}$. The distances between two phosphorus atoms of $3.787 \AA$ within the $\mathrm{Fe}_{2}$ subunits is longer than those of the two basal carbonyl carbons and Fe-Fe bond of 3.137 and $2.549 \AA$, respectively. Combined with assistance of the $\mathrm{N} \bullet \bullet \cdot \mathrm{CO}(\mathrm{CO}$ ap $)$ interaction $(\mathrm{d}=3.721(7) \AA)$, two Fe moieties are twisted by $26.5^{\circ}$. The most distorted structure from employment of phosphine ligands is observed in $\left[\mathrm{Fe}_{2}(\mu-\mathrm{pdt})(\mathrm{CO})_{3}\left(\mu, \mathrm{K}^{2}\right.\right.$-triphos $\left.)\right]$ (triphos = bis $(2-$ diphenylphosphinoethyl)phenylphosphine) (Adam et al., 2007). In this molecule, two phosphorus ends occupy the apical and basal positions of one Fe site while the third phosphorus coordinates to the basal site of the other Fe center in a cis fashion. The $\Phi$ and $\psi$ angles of the triphos complex are measured to 49.4 and $88^{\circ}$, respectively. A combined influence from the bridging and chelating manner is expected to result in the large distortion.

\subsection{Influences of the secondary coordination sphere interactions}

As described in the previous section in which the $\mathrm{N} \bullet \bullet \mathrm{CO}(\mathrm{CO} a \mathrm{p})$ interaction exerts observable impacts on the structure, influences of the second coordination sphere interaction should not be neglected. In the presence of Lewis acids, $\mathrm{AlBr}_{3}$ adduct of $\left[\mathrm{Fe}_{2}(\mu-\right.$ $\left.x d t)(\mu-C O)(d p p v)_{2}(C O)\right]$ is formed (Justice et al., 2007). Binding of $\mathrm{AlBr}_{3}$ leads to a $80 \mathrm{~cm}^{-1}$ shift on the IR band energy, which is as significant as the change upon protonation of the $\mathrm{Fe}^{\mathrm{I} F e} \mathrm{I}$ core. In calculations of the $\left[\mathrm{Fe}_{2}(\mu\right.$-adt $\left.)(\mathrm{CO})_{5}\left(\mathrm{CH}_{3} \mathrm{SH}\right)\right]-\mathrm{AlCl}_{3}$ pair, the rotated structure with an asymmetrical $\mathrm{CO}$ bridge bound to $\mathrm{AlCl}_{3}$ is more stable than the unrotated model possessing an adduct of an apical terminal CO group by $1.2 \mathrm{kcal} / \mathrm{mol}$ (Lee \& Jo, 2009). Assisted by the bridging $\mathrm{CO}-\mathrm{AlCl}_{3}$ group, activation energy to transfer the $\mathrm{NH}$ proton to the Fe center is as low as $2.6 \mathrm{kcal} / \mathrm{mol}$ in the vacuum. In addition, energies of LUMOs in the $\mathrm{NH}$ and $\mathrm{NHFeH}$ species are decreased, which in turn makes reductions easier. Effects of interactions in the secondary coordination sphere on reduction potentials have been studied 
in several classes of metalloproteins such as iron-sulfur proteins (ferredoxins and highpotential iron proteins) (Low \& Hill, 2000) and cupredoxins (Berry et al., 2003). A shift of the working potential greater than $1 \mathrm{~V}$ is observed.

\section{Conclusion}

DFT calculations have indicated that high efficiency of $\mathrm{H}_{2}$ production is associated with formation of the $t$-hydride species. Since protonation can occur either directly onto the Fe-Fe bond or via the relay route by the aza nitrogen bridgehead, suppression of the $\mu$-hydride form could be achieved in the presence of the rotated structure. From theoretical and experimental studies, several factors in the stereochemical and electronic aspects have been examined. It is apprehended that steric influences of bulky dithiolate linkers, availability and strength of back-donation from the unrotated Fe center to the semi-bridging carbonyl, and structural distortion caused by ligand coordination sphere are critical to the transient inverted-geometry species. A challenge we face now is how to make these factors play in harmony within the biomimetic model complexes.

\section{Acknowledgments}

The work is supported by National Science Council of Taiwan and Academia Sinica.

\section{References}

Adam, F. I.; Hogarth, G. \& Richards, I. (2007). Models of the iron-only hydrogenase: Reactions of $\left[\mathrm{Fe}_{2}(\mathrm{CO})_{6}(\mu-\mathrm{pdt})\right]$ with small bite-angle diphosphines yielding bridge and chelate diphosphine complexes $\left[\mathrm{Fe}_{2}(\mathrm{CO})_{4}(\right.$ diphosphine $\left.)(\mu-\mathrm{pdt})\right]$. Journal of Organometallic Chemistry, 692, 3957-3968.

Adam, F. I.; Hogarth, G.; Richards, I. \& Sanchez, B. E. (2007). Models of the iron-only hydrogenase: Structural studies of chelating diphosphine complexes $\left[\mathrm{Fe}_{2}(\mathrm{CO})_{4}(\mu-\right.$ pdt)( $\mathrm{\kappa}^{2} P, P^{\prime}$-diphosphine)]. Dalton Transactions, 2495-2498.

Adams, M. W. W. (1990). The structure and mechanism of iron-hydrogenases. Biochimica et Biophysica Acta, 1020, 115-145.

Aguirre de Carcer, I.; DiPasquale, A.; Rheingold, A., L. \& Heinekey, D. M. (2006). Active-site models for iron hydrogenases: Reduction chemistry of dinuclear iron complexes. Inorganic Chemistry, 45, 8000-8002.

Barton, B. E.; Olsen, M. T. \& Rauchfuss, T. B. (2008). Aza- and oxadithiolates are probable proton relays in functional models for the [FeFe]-hydrogenases. Journal of the American Chemical Society, 130, 16834-16835.

Barton, B. E. \& Rauchfuss, T. B. (2008). Terminal hydride in [FeFe]-hydrogenase model has lower potential for $\mathrm{H}_{2}$ production than the isomeric bridging hydride. Inorganic Chemistry, 47, 2261-2263.

Berry, S. M.; Ralle, M.; Low, D. W.; Blackburn, N. J. \& Lu, Y. (2003). Probing the role of axial methionine in the blue copper center of azurin with unnatural amino acids. Journal of the American Chemical Society, 125, 8760-8768.

Best, S. P.; Borg, S. J.; White, J. M.; Razavet, M. \& Pickett, C. J. (2007). On the structure of a proposed mixed-valent analogue of the diiron subsite of [FeFe]-hydrogenase. Chemical Communications, 4348-4350. 
Borg, S. J.; Behrsing, T.; Best, S. P.; Razavet, M.; Liu, X. \& Pickett, C. J. (2004). Electron transfer at a dithiolate-bridged diiron assembly: Electrocatalytic hydrogen evolution. Journal of the American Chemical Society, 126, 16988-16999.

Borg, S. J.; Ibrahim, S. K.; Pickett, C. J. \& Best, S. P. (2008). Electrocatalysis of hydrogen evolution by synthetic diiron units using weak acids as the proton source: Pathways of doubtful relevance to enzymic catalysis by the diiron subsite of [FeFe] hydrogenase. Comptes Rendus Chimie, 11, 852-860.

Borg, S. J.; Tye, J. W.; Hall, M. B. \& Best, S. P. (2007). Assignment of molecular structures to the electrochemical reduction products of diiron compounds related to [Fe-Fe] hydrogenase: A combined experimental and density functional theory study. Inorganic Chemistry, 46, 384-394.

Cammack, R.; Frey, M. \& Robson, R. (2001). Hydrogen as a fuel: Learning from nature, Taylor \& Francis, 0-415-24242-8, London and New York.

Capon, J.-F.; Ezzaher, S.; Gloaguen, F.; Pétillon, F. Y.; Schollhammer, P. \& Talarmin, J. (2008). Electrochemical insights into the mechanisms of proton reduction by $\left[\mathrm{Fe}_{2}(\mathrm{CO})_{6}\{\mu-\right.$ $\left.\left.\mathrm{SCH}_{2} \mathrm{~N}(\mathrm{R}) \mathrm{CH}_{2} \mathrm{~S}\right\}\right]$ complexes related to the $[2 \mathrm{Fe}]_{\mathrm{H}}$ subsite of [FeFe]hydrogenase. Chemistry-A European Journal, 14, 1954-1964.

Capon, J.-F.; Ezzaher, S.; Gloaguen, F.; Pétillon, F. Y.; Schollhammer, P.; Talarmin, J.; Davin, T. J.; McGrady, J. E. \& Muir, K. W. (2007). Electrochemical and theoretical investigations of the reduction of $\left[\mathrm{Fe}_{2}(\mathrm{CO})_{5} \mathrm{~L}\left\{\mu-\mathrm{SCH}_{2} \mathrm{XCH}_{2} \mathrm{~S}\right\}\right]$ complexes related to [FeFe] hydrogenase. New Journal of Chemistry, 31, 2052-2064.

Capon, J.-F.; Gloaguen, F.; Pétillon, F. Y.; Schollhammer, P. \& Talarmin, J. (2009). Electron and proton transfers at diiron dithiolate sites relevant to the catalysis of proton reduction by the [FeFe]-hydrogenases. Coordination Chemistry Reviews, 253, 14761494.

Capon, J.-F.; Gloaguen, F.; Schollhammer, P. \& Talarmin, J. (2005). Catalysis of the electrochemical $\mathrm{H}_{2}$ evolution by di-iron sub-site models. Coordination Chemistry Reviews, 249, 1664-1676.

Chiang, M.-H.; Liu, Y.-C.; Yang, S.-T. \& Lee, G.-H. (2009). Biomimetic model featuring the $\mathrm{NH}$ proton and bridging hydride related to a proposed intermediate in enzymatic $\mathrm{H}_{2}$ production by Fe-only hydrogenase. Inorganic Chemistry, 48, 7604-7612.

Crabtree, R. H. \& Lavin, M. (1986). Structural analysis of the semibridging carbonyl. Inorganic Chemistry, 25, 805-812.

Darchen, A.; Mousser, H. \& Patin, H. (1988). Two-electron transfer catalysis of carbon monoxide exchange with a ligand in hexacarbonyldiiron compounds $[(\mu-$ $\left.\mathrm{RS})_{2} \mathrm{Fe}_{2}(\mathrm{CO})_{6}\right]$. Journal of the Chemical Society, Chemical Communications, 968-970.

Darensbourg, M. Y.; Lyon, E. J.; Zhao, X. \& Georgakaki, I. P. (2003). The organometallic active site of [Fe]hydrogenase: Models and entatic states. Proceedings of the National Academy of Sciences of the United States of America, 100, 3683-3688.

Ezzaher, S.; Capon, J.-F.; Dumontet, N.; Gloaguen, F.; Pétillon, F. Y.; Schollhammer, P. \& Talarmin, J. (2009). Electrochemical study of the role of a H-bridged, unsymmetrically disubstituted diiron complex in proton reduction catalysis. Journal of Electroanalytical Chemistry, 626, 161-170.

Ezzaher, S.; Capon, J.-F.; Gloaguen, F.; Pétillon, F. Y.; Schollhammer, P.; Talarmin, J. \& Kervarec, N. (2009). Influence of a pendant amine in the second coordination 
sphere on proton transfer at a dissymmetrically disubstituted diiron system related to the $[2 \mathrm{Fe}]_{\mathrm{H}}$ Subsite of $[\mathrm{FeFe}] \mathrm{H}_{2}$ ase. Inorganic Chemistry, 48, 2-4.

Ezzaher, S.; Capon, J.-F.; Gloaguen, F.; Pétillon, F. Y.; Schollhammer, P.; Talarmin, J.; Pichon, R. \& Kervarec, N. (2007). Evidence for the formation of terminal hydrides by protonation of an asymmetric iron hydrogenase active site mimic. Inorganic Chemistry, 46, 3426-3428.

Felton, G. A. N.; Mebi, C. A.; Petro, B. J.; Vannucci, A. K.; Evans, D. H.; Glass, R. S. \& Lichtenberger, D. L. (2009). Review of electrochemical studies of complexes containing the $\mathrm{Fe}_{2} \mathrm{~S}_{2}$ core characteristic of [FeFe]-hydrogenases including catalysis by these complexes of the reduction of acids to form dihydrogen. Journal of Organometallic Chemistry, 694, 2681-2699.

Felton, G. A. N.; Petro, B. J.; Glass, R. S.; Lichtenberger, D. L. \& Evans, D. H. (2009). One- to two-electron reduction of an [FeFe]-hydrogenase active site mimic: The critical role of fluxionality of the [2Fe2S] core. Journal of the American Chemical Society, 131, 11290-11291.

Felton, G. A. N.; Vannucci, A. K.; Chen, J.; Lockett, L. T.; Okumura, N.; Petro, B. J.; Zakai, U. I.; Evans, D. H.; Glass, R. S. \& Lichtenberger, D. L. (2007). Hydrogen generation from weak acids: Electrochemical and computational studies of a diiron hydrogenase mimic. Journal of the American Chemical Society, 129, 12521-12530.

Fontecilla-Camps, J. C.; Volbeda, A.; Cavazza, C. \& Nicolet, Y. (2007). Structure/function relationships of [NiFe]- and [FeFe]-hydrogenases. Chemical Reviews, 107, 4273-4303.

Gao, W.; Ekströem, J.; Liu, J.; Chen, C.; Eriksson, L.; Weng, L.; Åkermark, B. \& Sun, L. (2007). Binuclear iron-sulfur complexes with bidentate phosphine ligands as active site models of Fe-hydrogenase and their catalytic proton reduction. Inorganic Chemistry, 46, 1981-1991.

Georgakaki, I. P.; Thomson, L. M.; Lyon, E. J.; Hall, M. B. \& Darensbourg, M. Y. (2003). Fundamental properties of small molecule models of Fe-only hydrogenase: Computations relative to the definition of an entatic state in the active site. Coordination Chemistry Reviews, 238-239, 255-266.

George, S. J.; Cui, Z.; Razavet, M. \& Pickett, C. J. (2002). The di-iron subsite of all-iron hydrogenase: Mechanism of cyanation of a synthetic $\{2 \mathrm{Fe} 3 \mathrm{~S}\}$ - carbonyl assembly. Chemistry-A European Journal, 8, 4037-4046.

Gloaguen, F.; Lawrence, J. D. \& Rauchfuss, T. B. (2001). Biomimetic hydrogen evolution catalyzed by an iron carbonyl thiolate. Journal of the American Chemical Society, 123, 9476-9477.

Gloaguen, F. \& Rauchfuss, T. B. (2009). Small molecule mimics of hydrogenases: Hydrides and redox. Chemical Society Reviews, 38, 100-108.

Greco, C.; Zampella, G.; Bertini, L.; Bruschi, M.; Fantucci, P. \& De Gioia, L. (2007). Insights into the mechanism of electrocatalytic hydrogen evolution mediated by $\mathrm{Fe}_{2}\left(\mathrm{~S}_{2} \mathrm{C}_{3} \mathrm{H}_{6}\right)(\mathrm{CO})_{6}$ : The simplest functional model of the Fe-hydrogenase active site. Inorganic Chemistry, 46, 108-116.

Holm, R. H.; Kennepohl, P. \& Solomon, E. I. (1996). Structural and functional aspects of metal sites in biology. Chemical Reviews, 96, 2239-2314.

Justice, A. K.; De Gioia, L.; Nilges, M. J.; Rauchfuss, T. B.; Wilson, S. R. \& Zampella, G. (2008). Redox and structural properties of mixed-valence models for the active site 
of the [FeFe]-hydrogenase: Progress and challenges. Inorganic Chemistry, 47, 74057414.

Justice, A. K.; Nilges, M. J.; Rauchfuss, T. B.; Wilson, S. R.; De Gioia, L. \& Zampella, G. (2008). Diiron dithiolato carbonyls related to the $\mathrm{H}_{\mathrm{ox}}{ }^{\mathrm{CO}}$ state of [FeFe]-hydrogenase. Journal of the American Chemical Society, 130, 5293-5301.

Justice, A. K.; Rauchfuss, T. B. \& Wilson, S. R. (2007). Unsaturated, mixed-valence diiron dithiolate model for the $\mathrm{H}_{\mathrm{ox}}$ state of the [FeFe] hydrogenase. Angewandte Chemie International Edition, 46, 6152-6154.

Justice, A. K.; Zampella, G.; De Gioia, L. \& Rauchfuss, T. B. (2007). Lewis vs. Brønstedbasicities of diiron dithiolates: spectroscopic detection of the "rotated structure" and remarkable effects of ethane-vs. propanedithiolate. Chemical Communications, 20192021.

Justice, A. K.; Zampella, G.; De Gioia, L.; Rauchfuss, T. B.; van der Vlugt, J. I. \& Wilson, S. R. (2007). Chelate control of diiron(I) dithiolates relevant to the [Fe-Fe]-hydrogenase active site. Inorganic Chemistry, 46, 1655-1664.

Lawrence, J. D.; Li, H. \& Rauchfuss, T. B. (2001). Beyond Fe-only hydrogenases: Nfunctionalized 2-aza-1,3-dithiolates $\mathrm{Fe}_{2}\left[\left(\mathrm{SCH}_{2}\right)_{2} \mathrm{NR}\right](\mathrm{CO})_{x}(x=5,6)$. Chemical Communications, 1482-1483.

Lawrence, J. D.; Li, H.; Rauchfuss, T. B.; Bénard, M. \& Rohmer, M.-M. (2001). Diiron azadithiolates as models for the iron-only hydrogenase active site: Synthesis, structure, and stereoelectronics. Angewandte Chemie International Edition, 40, 17681771.

Lee, J. W. \& Jo, W. H. (2009). Effect of Lewis acid on the structure of a diiron dithiolate complex based on the active site of [FeFe]-hydrogenase assessed by density functional theory. Dalton Transactions, 8532-8537.

Li, H. \& Rauchfuss, T. B. (2002). Iron carbonyl sulfides, formaldehyde, and amines condense to give the proposed azadithiolate cofactor of the Fe-only hydrogenases. Journal of the American Chemical Society, 124, 726-727.

Li, P.; Wang, M.; He, C.; Liu, X.; Jin, K. \& Sun, L. (2007). Phosphane and phosphite unsymmetrically disubstituted diiron complexes related to the Fe-only hydrogenase active site. European Journal of Inorganic Chemistry, 3718-3727.

Liu, T. \& Darensbourg, M. Y. (2007). A mixed-valent, Fe(II)Fe(I), diiron complex reproduces the unique rotated state of the [FeFe]hydrogenase active site. Journal of the American Chemical Society, 129, 7008-7009.

Liu, X.; Ibrahim, S. K.; Tard, C. \& Pickett, C. J. (2005). Iron-only hydrogenase: Synthetic, structural and reactivity studies of model compounds. Coordination Chemistry Reviews, 249, 1641-1652.

Liu, Y.-C.; Tu, L.-K.; Yen, T.-H.; Lee, G.-H. \& Chiang, M.-H. (2010). Submitted.

Liu, Y.-C.; Tu, L.-K.; Yen, T.-H.; Lee, G.-H.; Yang, S.-T. \& Chiang, M.-H. (2010). Secondary coordination sphere interactions within the biomimetic iron azadithiolate complexes related to Fe-only hydrogenase: Dynamic measure of electron density about the Fe sites. Inorganic Chemistry, 49, 6409-6420.

Low, D. W. \& Hill, M. G. (2000). Backbone-Engineered High-Potential Iron Proteins: Effects of Active-Site Hydrogen Bonding on Reduction Potential. Journal of the American Chemical Society, 122, 11039-11040. 
Lyon, E. J.; Georgakaki, I. P.; Reibenspies, J. H. \& Darensbourg, M. Y. (1999). Carbon monoxide and cyanide ligands in a classical organometallic complex model for Feonly hydrogenase. Angewandte Chemie International Edition, 38, 3178-3180.

Nicolet, Y.; De Lacey, A. L.; Vernède, X.; Fernandez, V. M.; Hatchikian, E. C. \& FontecillaCamps, J. C. (2001). Crystallographic and FTIR spectroscopic evidence of changes in Fe coordination upon reduction of the active site of the Fe-only hydrogenase from Desulfovibrio desulfuricans. Journal of the American Chemical Society, 123, 15961601.

Nicolet, Y.; Piras, C.; Legrand, P.; Hatchikian, C. E. \& Fontecilla-Camps, J. C. (1999). Desulfovibrio desulfuricans iron hydrogenase: The structure shows unusual coordination to an active site Fe binuclear center. Structure, 7, 13-23.

Olsen, M. T.; Barton, B. E. \& Rauchfuss, T. B. (2009). Hydrogen activation by biomimetic diiron dithiolates. Inorganic Chemistry, 48, 7507-7509.

Olsen, M. T.; Bruschi, M.; De Gioia, L.; Rauchfuss, T. B. \& Wilson, S. R. (2008). Nitrosyl derivatives of diiron(I) dithiolates mimic the structure and Lewis acidity of the [FeFe]-hydrogenase active site. Journal of the American Chemical Society, 130, 1202112030.

Olsen, M. T.; Justice, A. K.; Gloaguen, F.; Rauchfuss, T. B. \& Wilson, S. R. (2008). New nitrosyl derivatives of diiron dithiolates related to the active site of the [FeFe]hydrogenases. Inorganic Chemistry, 47, 11816-11824.

Ott, S.; Kritikos, M.; Åkermark, B.; Sun, L. \& Lomoth, R. (2004). A biomimetic pathway for hydrogen evolution from a model of the iron hydrogenase active site. Angewandte Chemie International Edition, 43, 1006-1009.

Pandey, A. S.; Harris, T. V.; Giles, L. J.; Peters, J. W. \& Szilagyi, R. K. (2008). Dithiomethylether as a ligand in the hydrogenase H-cluster. Journal of the American Chemical Society, 130, 4533-4540.

Peters, J. W.; Lanzilotta, W. N.; Lemon, B. J. \& Seefeldt, L. C. (1998). X-ray crystal structure of the Fe-only hydrogenase ( $\mathrm{CpI}$ ) from Clostridium pasteurianum to 1.8 angstrom resolution. Science, 282, 1853-1858.

Razavet, M.; Borg, S. J.; George, S. J.; Best, S. P.; Fairhurst, S. A. \& Pickett, C. J. (2002). Transient FTIR spectroelectrochemical and stopped-flow detection of a mixed valence $\{\mathrm{Fe}(\mathrm{I})-\mathrm{Fe}(\mathrm{II})\}$ bridging carbonyl intermediate with structural elements and spectroscopic characteristics of the di-iron sub-site of all-iron hydrogenase. Chemical Communications, 700-701.

Razavet, M.; Davies, S. C.; Hughes, D. L. \& Pickett, C. J. (2001). $\{2 \mathrm{Fe} 3 S\}$ clusters related to the di-iron sub-site of the $\mathrm{H}$-centre of all-iron hydrogenases. Chemical Communications, 847-848.

Siegbahn, P. E. M.; Tye, J. W. \& Hall, M. B. (2007). Computational studies of [NiFe] and [FeFe] hydrogenases. Chemical Reviews, 107, 4414-4435.

Silakov, A.; Wenk, B.; Reijerse, E. \& Lubitz, W. (2009). ${ }^{14}$ N HYSCORE investigation of the Hcluster of [FeFe] hydrogenase: Evidence for a nitrogen in the dithiol bridge. Physical Chemistry Chemical Physics, 11, 6592-6599.

Singleton, M. L.; Bhuvanesh, N.; Reibenspies, J. H. \& Darensbourg, M. Y. (2008). Synthetic support of de novo design: Sterically bulky [FeFe]-hydrogenase models. Angewandte Chemie International Edition, 47, 9492-9495. 
Singleton, M. L.; Jenkins, R. M.; Klemashevich, C. L. \& Darensbourg, M. Y. (2008). The effect of bridgehead steric bulk on the ground state and intramolecular exchange processes of $\left(\mu-\mathrm{SCH}_{2} \mathrm{CR}_{2} \mathrm{CH}_{2} \mathrm{~S}\right)\left[\mathrm{Fe}(\mathrm{CO})_{3}\right]\left[\mathrm{Fe}(\mathrm{CO})_{2} \mathrm{~L}\right]$ complexes. Comptes Rendus Chimie, 11, 861-874.

Song, L.-C.; Yang, Z.-Y.; Bian, H.-Z.; Liu, Y.; Wang, H.-T.; Liu, X.-F. \& Hu, Q.-M. (2005). Diiron oxadithiolate type models for the active site of iron-only hydrogenases and biomimetic hydrogen evolution catalyzed by $\mathrm{Fe}_{2}\left(\mu-\mathrm{SCH}_{2} \mathrm{OCH}_{2} \mathrm{~S}-\mu\right)(\mathrm{CO})_{6}$. Organometallics, 24, 6126-6135.

Tard, C.; Liu, X.; Ibrahim, S. K.; Bruschi, M.; De Gioia, L.; Davies, S. C.; Yang, X.; Wang, L.-S.; Sawers, G. \& Pickett, C. J. (2005). Synthesis of the H-cluster framework of iron-only hydrogenase. Nature, 433, 610-613.

Tard, C. \& Pickett, C. J. (2009). Structural and functional analogues of the active sites of the [Fe]-, [NiFe]-, and [FeFe]-hydrogenases. Chemical Reviews, 109, 2245-2274.

Thomas, C. M.; Liu, T.; Hall, M. B. \& Darensbourg, M. Y. (2008). Regioselective ${ }^{12} \mathrm{CO} /{ }^{13} \mathrm{CO}$ exchange activity of a mixed-valent $\mathrm{Fe}(\mathrm{II}) \mathrm{Fe}(\mathrm{I})$ model of the $\mathrm{H}_{\mathrm{ox}}$ state of [FeFe]hydrogenase. Chemical Communications, 1563-1565.

Thomas, C. M.; Liu, T.; Hall, M. B. \& Darensbourg, M. Y. (2008). Series of mixed valent $\mathrm{Fe}(\mathrm{II}) \mathrm{Fe}(\mathrm{I})$ complexes that model the $\mathrm{H}_{\mathrm{ox}}$ state of [FeFe]hydrogenase: Redox properties, density-functional theory investigation, and reactivities with extrinsic CO. Inorganic Chemistry, 47, 7009-7024.

Tye, J. W.; Darensbourg, M. Y. \& Hall, M. B. (2006). De novo design of synthetic di-iron(I) complexes as structural models of the reduced form of iron-iron hydrogenase. Inorganic Chemistry, 45, 1552-1559.

van der Vlugt, J. I.; Rauchfuss, T. B.; Whaley, C. M. \& Wilson, S. R. (2005). Characterization of a diferrous terminal hydride mechanistically relevant to the Fe-only hydrogenases. Journal of the American Chemical Society, 127, 16012-16013.

Vignais, P. M. \& Billoud, B. (2007). Occurrence, classification, and biological function of hydrogenases: An overview. Chemical Reviews, 107, 4206-4272.

Vincent, K. A.; Parkin, A. \& Armstrong, F. A. (2007). Investigating and exploiting the electrocatalytic properties of hydrogenases. Chemical Reviews, 107, 4366-4413.

Wang, F.; Wang, M.; Liu, X.; Jin, K.; Dong, W.; Li, G.; Åkermark, B. \& Sun, L. (2005). Spectroscopic and crystallographic evidence for the N-protonated FeIFel azadithiolate complex related to the active site of Fe-only hydrogenases. Chemical Communications, 3221-3223.

Wang, N.; Wang, M.; Liu, T.; Li, P.; Zhang, T.; Darensbourg, M. Y. \& Sun, L. (2008). COmigration in the ligand substitution process of the chelating diphosphite diiron complex $(\mu-\mathrm{pdt})\left[\mathrm{Fe}(\mathrm{CO})_{3}\right]\left[\mathrm{Fe}(\mathrm{CO})\left\{(\mathrm{EtO})_{2} \mathrm{PN}(\mathrm{Me}) \mathrm{P}(\mathrm{OEt})_{2}\right\}\right]$. Inorganic Chemistry, 47, 6948-6955.

Wang, N.; Wang, M.; Zhang, T.; Li, P.; Liu, J. \& Sun, L. (2008). A proton-hydride diiron complex with a base-containing diphosphine ligand relevant to the [FeFe]hydrogenase active site. Chemical Communications, 5800-5802.

Zampella, G.; Bruschi, M.; Fantucci, P.; Razavet, M.; Pickett, C. J. \& De Gioia, L. (2005). Dissecting the intimate mechanism of cyanation of $\{2 \mathrm{Fe} 3 \mathrm{~S}\}$ complexes related to the active site of all-iron hydrogenases by DFT analysis of energetics, transition states, intermediates and products in the carbonyl substitution pathway. Chemistry- $A$ European Journal, 11, 509-520. 
Zampella, G.; Fantucci, P. \& De Gioia, L. (2009). Unveiling how stereoelectronic factors affect kinetics and thermodynamics of protonation regiochemistry in [FeFe] hydrogenase synthetic models: A DFT investigation. Journal of the American Chemical Society, 131, 10909-10917. 


\title{
The Improvement of LC-MS/MS Proteomic Detection with Biomimetic Affinity Fractionation
}

\author{
Rong-Xiu Li, Qing-Qiao Tan and De-Xian Dong \\ Key Laboratory of MOE for Microbial metabolism and \\ College of Life Science and Biotechnology, Shanghai Jiao Tong University, \\ 800 Dong-chuan Road, Shanghai 200241
}

China

\section{Introduction}

The systematic study of all proteins, referred to as proteomics, is performed to identify the components of a particular proteome and analyze global changes in protein expression in response to different stimuli. Over the past two decades, mass spectrometry has become an important tool for the analysis of proteins (Aebersold and Mann, 2003; Yates, 2004). It is estimated that a cell contains at least 10 000-30 000 different proteins that span a wide range of sizes, relative abundance, acidity/basicity, and hydrophobicity (Badock et al., 2001). As is known, the low-abundance and high-abundance proteins differ in concentration over nine orders of magnitude (Omenn, 2004), which results in the high-abundance proteins impeding the investigation of middle- or low-abundance proteins. The separation of the protein mixture into organelles or other multi-protein complex fractions prior to a proteomics analysis is usually the first step to increase the probability of detecting low-copy-number proteins (Rabilloud et al., 1998; Taylor et al., 2000; Cronshaw et al., 2002; Jiang et al., 2004; Gao et al., 2005). Efficient and highly resolving separation of the protein mixture into organelles or other multi-protein complex fractions prior to a mass spectrometry analysis is usually used as reducing the complexity of samples, which highly increases the probability of detecting low-copy-number proteins (Rabilloud et al., 1998; Taylor et al., 2000; Cronshaw et al., 2002; Jiang et al., 2004; Gao et al., 2005). Before the widespread use of shotgun methods, proteins were fractionated by 2D gel electrophoresis (2D-gel), a method that separates them according to pI and molecular weight (Harry et al., 2000; Gygi, et al., 2000; Motoyama \& Yates, 2008). Although 2D-gel has excellent resolving power for intact proteins, the process is difficult to automate, laborious, and suffers from low throughput (Klose \& Kobalz, 1995). The more fundamental drawbacks are the limited dynamic range and the exclusion of certain classes, such as low-abundance proteins (Gygi et al., 2000), integral membrane proteins (Braun et al., 2007), and proteins with extremes in isoelectric point (pI) and molecular weight (MW) (Corthals et al., 2000; Oh-Ishi et al., 2000).

Another explored proteins pre-fractionation methods is multidimensional liquid chromatography technique combined ion-exchange chromatography and/or hydrophobic chromatography and/or reverse-phase chromatography, which has been a powerful 
technique to simplify the proteins in sample to 2D-gel in the proteomics research(Shen et al., 2004; Bergh et al., 2003; Jacobs et al., 2004).

One current method for the analysis of protein mixtures is proteolytic digestion followed by LC/MS/MS, often referred to as "shotgun" strategy. It has been proved powerful (Washburn et al., 2001; Wu et al., 2003; Shen et al., 2004). The heart of the method is the use of MS/MS to identify proteolytic peptides. However, it results in a complexity of millions of trypsin digest peptides, in which the signal of peptides of high-abundance proteins would mask the signal of middle- or low-abundance proteins. To simplify the sample and increase the detection probability of middle- and low-abundance proteins, suitable high abundance protein depletion and prefractionation methods were often adopted prior to trypsin digestion (Bergh et al., 2003; Jacobs et al., 2004; Gao et al., 2006; Liu and Zhang, 2007; Gao et al., 2008).

Affinity chromatography (AC) can be also used to reduce the complexity of the protein/peptide by selecting the specific target protein/peptide (Gygi et al., 1999; Pieper et al., 2003; Greenough et al., 2004, Tan et al.,2009a,2009b \& 2010). Traditional affinity adsorbents comprise natural biological ligands, which are expensive to produce, show low binding capacities, have limited life cycles, and low scale-up potential. As a new affinity fraction, biomimetic chromatography uses synthetic affinity ligands for matrix, which could mimic the properties of natural ligands and circumvent these drawbacks of natural ligands by imparting resistance to chemical and biochemical degradation, displaying ease and low cost of production, and withstanding harsh sterilization without loss of performance (Lowe, 2001). It has been verified that many specific synthetic affinity ligands could be designed to purify different single proteins in our lab and other labs (Li et al., 1998; Teng et al., 1999; Gupta and Lowe, 2004; Melissis et al., 2007; Xin et al., 2007; Dong et al., 2008a).

Our lab has constructed affinity ligand library (thousands of ligands), and different ligands showed different absorbance effect to proteins such as purification, depletion of high abundance proteins, and enrichment of low abundance proteins (Dong et al, 2008b), which gives a imaginable application prospect in proteomics. In this Chapter, from biomimetic affinity ligands library in our lab, we first screened out some affinity ligands showing large absorption differences in band distribution, then, selected the affinity ligands having medium absorption ability; and finally a cascade and a tandem composition of these affinity ligands were contructed and applied in prefractionation of complex tissue proteins. Fractionated samples were analyzed by LTQ-MS/MS. After reducing the complicity of tissue protein sample, more protein information was able to be obtained from LTQ-MS /MS analysis. The cascade and tandem affinity biomimetic affinity chromatography exhibits wide application prospect in proteomics because of its low price, simplicity, high throughput, specificity of ligand absorbance and repeatability.

\section{Materials and methods}

\subsubsection{Materials}

Adult male Sprague-Dawley rats were purchased from Shanghai Laboratory Animal Center (Jiu-Ting, Shanghai, China). Sequencing grade modified trypsin was from Promega Corporation. The water used was Milli-Q grade (Millipore, Bedford, MA). Phenylmethylsulfonyl fluoride (PMSF), dithiothreitol (DTT), guanidine hydrochloride, formic acid, acetonitrile, trifluoroacetic acid, and iodoacetamide were obtained from Sigma (St. Louis, MO). SDS, acrylamide and N,N'methylen-bis-acrylamide were purchased from 
Amersham Pharmacia company. Unless stated otherwise, all reagents and chemicals were of the highest purity available.

\subsubsection{Sample preparation}

The rat liver tissue was cut into small pieces and cleaned with cold physiological saline solution $(0.9 \% \mathrm{NaCl})$ to remove blood and some possible contaminants. Then, $5 \mathrm{~g}$ of tissue debris was rapidly mixed with $50 \mathrm{ml} \mathrm{pH}$ 7.6 Tris- $\mathrm{HCl}$ lysis buffer, containing $1 \mathrm{mM}$ PMSF, $5 \mathrm{mM} \mathrm{MgCl}, 250 \mathrm{mM}$ Sucrose, $1 \mathrm{mM}$ DTT. Tissue sample was homogenized in an ice bath, then sonicated for $800 \mathrm{~s}$ with the JY92-II Ultrasonic Cell Disrupter (Ningbo Haishu Kesheng Ultrasonic Equipments Co.,Ltd, China) at 50\% of duty cycle and $450 \mathrm{~W}$ output. After being

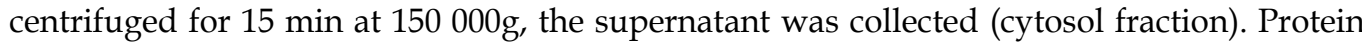
concentration of the sample was determined by the modified Bradford method described by Qu et al(1997), using bovine serum albumin (BSA) as standard.

\subsubsection{Synthesis of affinity ligands}

The affinity ligands library was constructed according to the methods of our lab(Dong et al 2008a). Briefly, Sepharose 4B was first activated with epichlorohydrin, then cyanuric chloride was coupled as a large spacer, and substitution of chloride in spacer with different amino compounds provides lots of ligands which have different sizes, shapes, and functionalities.

\subsubsection{Evaluating of synthetic affinity ligands}

Different gel-immobilized synthetic ligands $(1 \mathrm{ml})$ were respectively packed into polystyrene columns with porous discs at both the bottom and the top of the gel. The column was equilibrated with a 10 -fold column volume of $10 \mathrm{mM}$ phosphate buffered saline (pH7.0). 4mg Cytosol fraction of rat liver protein was loaded onto the column, and left bound for 30 minutes. $10 \mathrm{mM}$ phosphate buffered saline ( $\mathrm{pH7.0)}$ was applied to flush the column until the baseline of UV monitor (A280) went down to the bottom, and the flowthrough was collected. Then, 20mM Glycine-NaOH buffer (pH12.0) was used to elute out the bound proteins. Finally, $0.1 \mathrm{M} \mathrm{NaOH}$ plus $30 \%$ ethanol was applied to wash and clean the column until the baseline of UV monitor went down to the bottom. All the elution fractions collected were used for subsequent SDS-PAGE electrophoresis. After comparison with absorbance protein profiles, selecting the ligands having large difference in absorbance characterization.

\subsubsection{Trypsin digestion of each protein mixture fraction}

Appropriate volumes of protein sample for each fraction were desalted and concentrated as above, lyophilized to dryness. $30 \mu \mathrm{g}$ of lyophilized protein sample was redissolved in reducing solution $(6 \mathrm{M}$ guanidine hydrochloride, $50 \mathrm{mM}$ Tris- $\mathrm{HCl}, 3 \mathrm{mM}$ DTT, $\mathrm{pH} 8,30 \mu \mathrm{l}$ ). The mixture was incubated at $60^{\circ} \mathrm{C}$ for $1 \mathrm{~h}$ and then $1.5 \mu \mathrm{l}$ of $1 \mathrm{M}$ iodoacetamide was added and incubated for an additional $30 \mathrm{~min}$ at room temperature in darkness. After denaturation, allow the reaction to cool and add $270 \mu 1$ of $50 \mathrm{mM}$ ammonium bicarbonate buffer( $\mathrm{pH}$ 8.5). Then, modified trypsin was added to a final proteinase: protein ratio of 1:30(W/W), and the mixture was incubated at $37^{\circ} \mathrm{C}$ overnight. The peptide mixture was lyophilized to dryness. 


\subsubsection{LTQ-MS/MS analysis}

Redissolved peptides mixture was injected onto a Zorbax 300 SB-C18 peptide traps (Agilent Technologies, Wilmington, DE) to desalt, and separation was performed on a Zorbax 300SBC18 reverse phase capillary column $(300 \mu \mathrm{m}$ inner diameter $\times 15 \mathrm{~cm}$, Agilent Technologies) at $(250 \mathrm{nl} / \mathrm{min})$ with a linear gradient of $4-50 \%$ B over $50 \mathrm{~min}$ (A: $0.1 \%$ formic acid; B: $84 \%$ $\mathrm{CH} 3 \mathrm{CN}$ and $0.1 \%$ formic acid), a step up to $100 \% \mathrm{~B}$ in $4 \mathrm{~min}$, and then holding at $100 \% \mathrm{~B}$ for $10 \mathrm{~min}$. The peak was online injected into a Finnigan LTQ (single linear quadrupole ion trap) mass spectrometer for peptide identification.

Mass spectrometry was on a Finnigan LTQ linear ion trap. The MS method consisted of a cycle combining one full MS scan with three MS/MS events (25\% collision energy) followed by an MS3 event (35\% collision energy). Dynamic exclusion duration was set to $30 \mathrm{~s}$. The MS/MS and MS3 spectra from all the runs were searched using BIOWORKS protein identification software against database of ipi Rat. The SEQUEST filter was set to Charge +1 , Xcorr $\geq 1.9$; Charge +2 , Xcorr $\geq 2.2$; Charge +3, Xcorr $\geq 3.75$; and DelCN $\geq 0.1$ 。

\subsubsection{Bioinformatics annotation tools}

The theoretical $\mathrm{pI}$ and molecular mass values of proteins were from ExPASy Proteomics Server (http://cn.expasy.org/tools/pi_tool.html). The GRAVY values (http://www.bioinformatics.org/sms2/protein_gravy.html)were determined according to Kyte \& Doolittle (1982). The TMHMM (http://www.cbs.dtu.dk/services/TMHMM/) (Krogh et al., 2001) was used to predict protein TM domains.

\section{Prefractionation of rat liver cytosol proteins using tandem biomimetic affinity chromatography}

\subsection{Tandem mimetic affinity prefractionation}

According to absorbance protein profiles (SDS-PAGE), we selected 5 affinity ligand columns having large band difference, and tandem combination of 5 columns could be applied as prefractionation of tissue lysis prior to LTQ shot -gun LC-MS proteome analysis. A schematic illustration of the workflow is shown in Fig. 1. 4mg Cytosol fraction of rat liver protein was loaded into the tandem columns. After rat liver proteins being coupled for 30 minutes, $10 \mathrm{mM}$ phosphate buffered saline ( $\mathrm{pH} 7.0)$ was applied to flush the tandem columns until the baseline of UV monitor (A280) went down to the bottom. Then, 5 tandem columns were divided into single column, and bound protein of each column was respectively eluted by 2-fold column volume of 20mM Glycine-NaOH buffer (pH12.0). 5 elution fractions were instantly adjusted into $\mathrm{pH}$ 7-8. After being desalted and concentrated using Microcon ultrafiltration membranes (3,000 Nominal Molecular Weight Limit, Millipore), all the elution fractions collected were used for subsequent SDS-PAGE electrophoresis and LTQ analysis.

\subsection{Evaluating for differential protein absorbance profiles of mimetic affinity ligands}

After analyzing the screening datas of our lab in serum and leech proteins, we selected the ligands showing medium absorbance ability. 60 ligands were selected from affinity ligands library of our lab, and the absorbance characterization of these ligands was reevaluated using rat liver cytosol proteins as materials. From the SDS-PAGE profiles of each ligand, we found that some ligands showed large absorbance differences in band distribution to rat liver cytosol proteins (FIG. 2). Partial examples of proteins absorbance profiles were shown in FIG. 2, and each ligand showed its own specific binding ability to some proteins, but low 


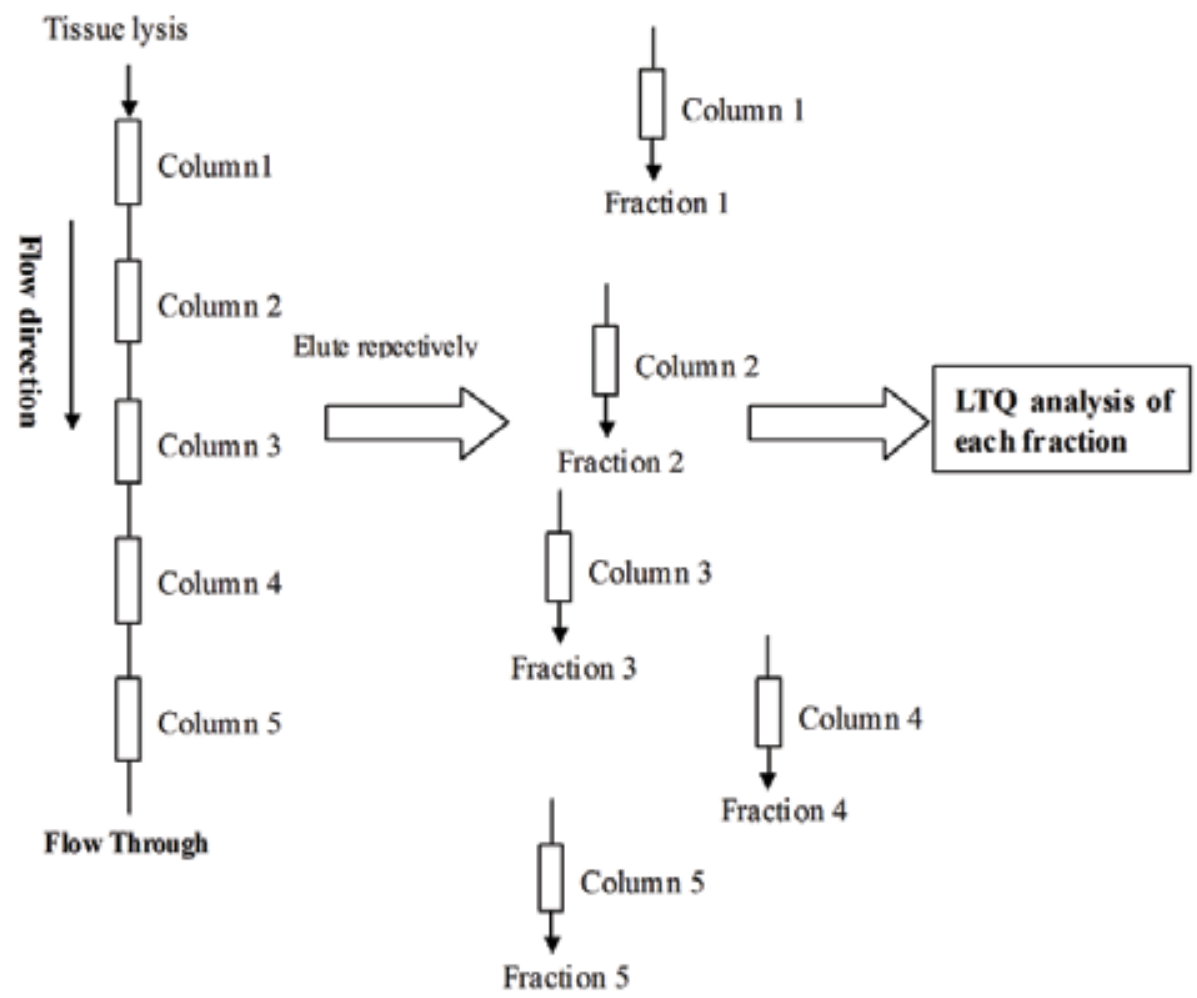

Fig. 1. Schematic illustration of tandem biomimetic affinity fraction used in this work

absorbance characterization to other proteins. At the same time, these ligands showed different absorbance ability. Some ligands exhibited relatively low absorbance ability such as A1-4 and A7-56, but other ligands showed relatively high absorbance ability such as A6, A17-56 and A29-32. According to the affinnity specificity of these ligands, composition of different ligands could be applied in well-distribution of complex tissue proteins. After reducing the complicity of tissue protein samples, more protein information was able to be obtained from proteome analysis. In present research, tandem combination of a few ligands was used as prefractionation of complex protein samples prior to LTQ-MS /MS proteome analysis. A7-56, A84, A11-70, A6, and A29-32 were selected for tandem composition in the follow experiments.

\subsection{Tandem biomimetic affinity fractionation and LTQ-MS/MS analysis}

The flowchart in FIG. 1 gives a more detailed overview of the tandem procedure, and the ligand column showed relatively low absorbance ability was put front. A7-56, A84, A11-70, A6, and A29-32 were orderly connected. 4mg Cytosol fraction of rat liver proteins was loaded into the tandem columns, after binding and flushing fully 5 tandem columns were divided into single column, and bound protein of each column was respectively eluted by 2 fold column volume of $20 \mathrm{mM}$ Glycine- $\mathrm{NaOH}$ buffer (pH12.0). Flowthrough (fraction 6) and all the elution fractions (fraction1 to 5) collected were used for subsequent SDS-PAGE electrophoresis (FIG. 2). From proteins band distribution, complex rat liver cytosol proteins were well-distributed into six fractions. 


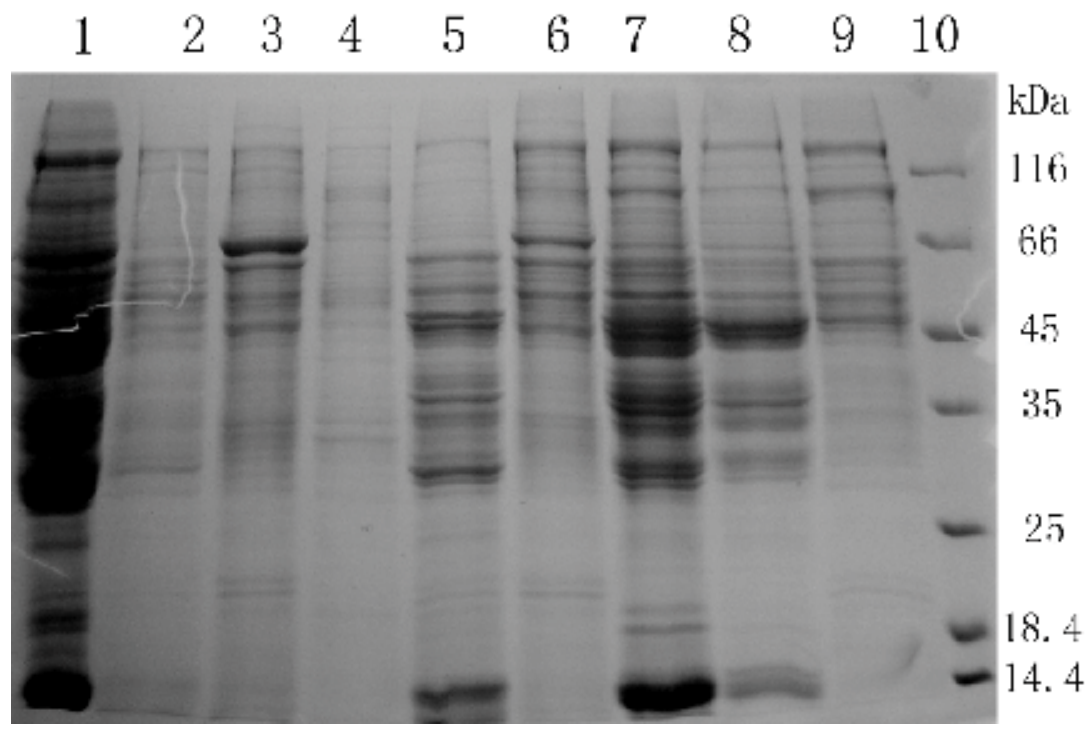

Fig. 2. Partial examples of rat liver proteins absorbance profiles having large band distribution difference. Lane 1 is crude rat liver cytosol. Lane 10 is the protein marker (Fermentas, USA). Lanes2-9show the $20 \mathrm{mM}$ Glycine-NaOH buffer (pH12.0) eluates of columns A1-4, A6, A7-56, A84, A11-70, A17-56, A29-32, and A25-35 respectively.

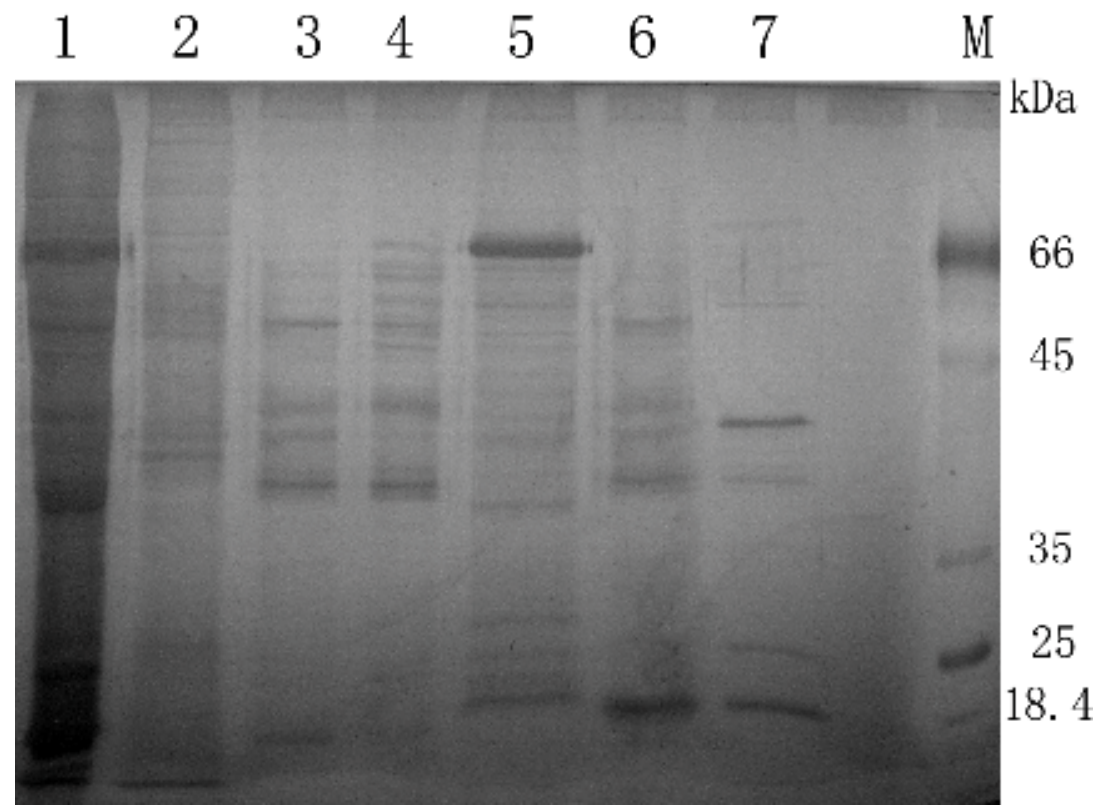

Fig. 3. Protein profiles of tandem affinity fraction. Lane 1 is crude rat liver cytosol. $\mathrm{M}$ is the protein marker (Fermentas, USA). Lanes2-6 show the $20 \mathrm{mM}$ Glycine-NaOH buffer ( $\mathrm{pH} 12.0$ ) eluates of 5 tandem columns A7-56, A84, A11-70, A6, and A29-32 respectively (fraction 1 to 5). Lane 7 was flowthrough of tandem affinity chromatography (fraction 6). 
After being desalted and concentrated using Microcon ultrafiltration membranes $(3,000$ Nominal Molecular Weight Limit, Millipore), all the fractions collected were used for subsequent LTQ-MS/MS analysis. The technical route was evaluated by comparing the protein numbers detected from the unfractionated cytosol sample with those from six welldistributed fractions. The MS/MS spectra acquired from equivalent normalized aliquots of the six respective fractions were searched against the rat database extracted from SwissProt, GenPept, and PIR entries and a six-way translation of dbEST using the program SEQUEST running on a DECA workstation. In order to avoid false-positive hits, we referred to the parameters reported in previous studies and applied more strict criteria for peptide identification (Charge +1,Xcorr $\geq 1.9$; Charge +2 , Xcorr $\geq 2.2$; Charge +3 , Xcorr $\geq 3.75$; and DelCN $\geq 0.1$ ). This resulted in highly confident identification of a total of 665 unique rat proteins in the six fractions (Table 1), over eight times as protein numbers detected in unfractionated cytosol sample (only 74 unique proteins). Of these, 290 proteins (827 unique peptides) were characterized in fraction 1(A7-56), 123 proteins (476 unique peptides) in fraction 2(A84), 152 proteins (605 unique peptides) in fraction 3(A11-70), 279 proteins (818 unique peptides) in fraction 4 (A6), 155 proteins (439 unique peptides) in fraction 5 (A29-32), and 60 proteins ( 344 unique peptides) in fraction 6 (Flow through). At the same time, only 5 proteins were characterized in all the six fractions and 177 proteins were only characterized in fraction1, 24 only in fraction 2, 30 only in fraction 3, 140 only in fraction 4, 42 only in fraction 5, and 17 only in the Flow through fraction (FIG. 4A). Each ligand showed different absorbance characterization to tissue proteins. About 430 unique proteins $(64.7 \%)$ only characterized in specific fractions, which exhibited well-distribution effect of tandem biomimetic affinity prefractionation. Table 2 showed the number of proteins identified according to $1,2,3$, and $>3$ unique peptides. About $40 \%$ proteins in each fraction were identified from a unique peptide. It is noted that most of the proteins identified by a single peptide was also only found in a certain fraction, which indicates those proteins are lowabundant components in the cell and enriched after fractionation. FIG. 4B presented sensitivity of this tandem affinity method to a wide variety of protein classes. Of these 665proteins, 61 proteins detected were with extreme size (MW<10kD, or $>100 \mathrm{kD}), 14$ with extreme $\mathrm{pI}$ value $(\mathrm{pI}<4.3$, or $>10)$, 55 with hydrophobic characterization (GRAVY $>0$ ), and 41 with TM domain (Predicted TM Helices $\geq 1$ ). Compared with the traditional 2D-PAGE method, the shotgun strategy presents a number of data with very rapid speed and limited sample consumption. In present research, the tandem affinity prefractionation could obviously enhance the detection ability of shotgun strategy. Most importantly, the tandem affinity prefractionation could be finished in 4 hours, and one-dimension LTQ-MS/MS analysis employed would consumed only 5 hours, which was far lower than 2D-LCMS/MS. Including other processes (such as concentration, trypsin digestion), only data from single run of $20 \mathrm{~h}$ were used in this work.

\begin{tabular}{|c|c|c|c|c|c|c|c|}
\hline Fraction & F1(A7-56) & F2(A84) & F3(A11-70) & F4(A6) & F5(A29-32) & F6(FT) & Total \\
\hline $\begin{array}{c}\text { Unique } \\
\text { peptides }\end{array}$ & 827 & 476 & 605 & 818 & 439 & 344 & 2413 \\
\hline $\begin{array}{c}\text { Unique } \\
\text { Proteins }\end{array}$ & 290 & 123 & 152 & 279 & 155 & 60 & 665 \\
\hline
\end{tabular}

Table 1. Peptides and proteins identified in six rat liver tandem fractions using LTQ -MS/MS 


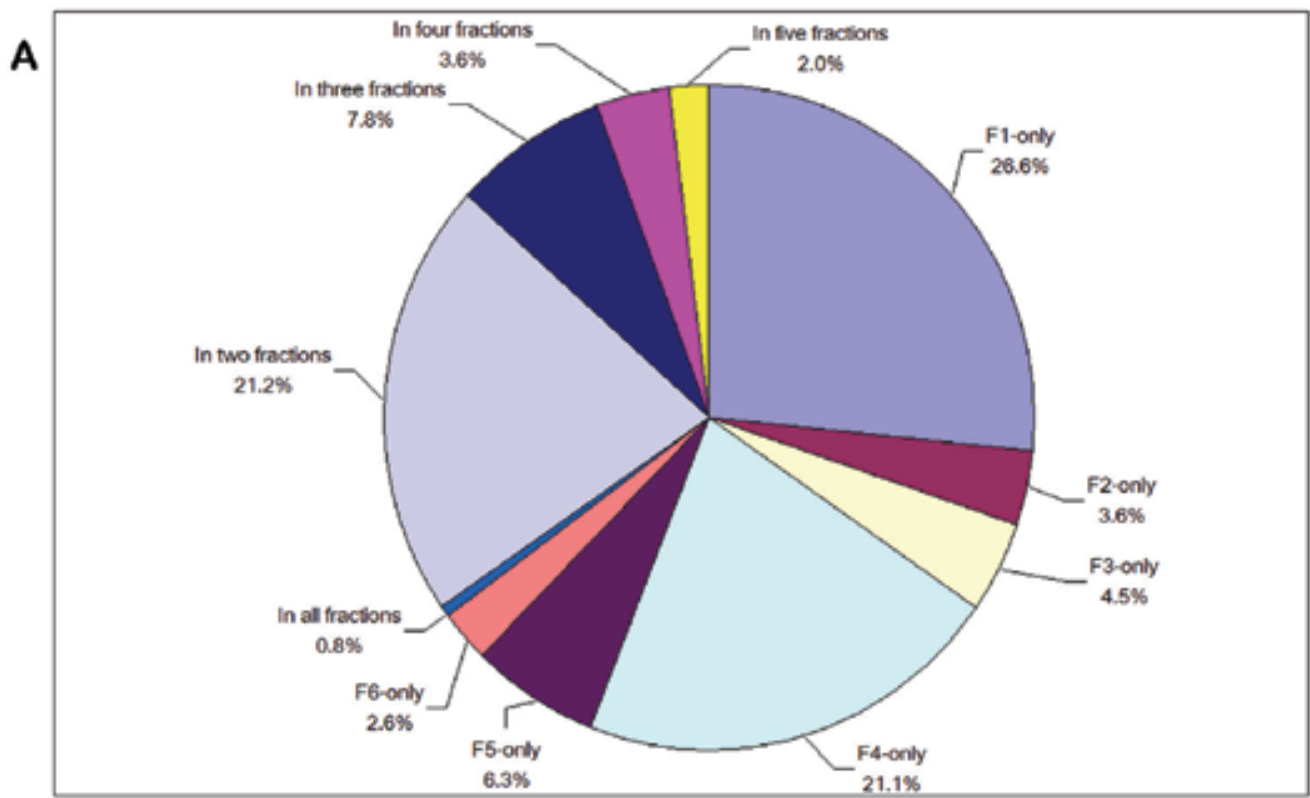

B

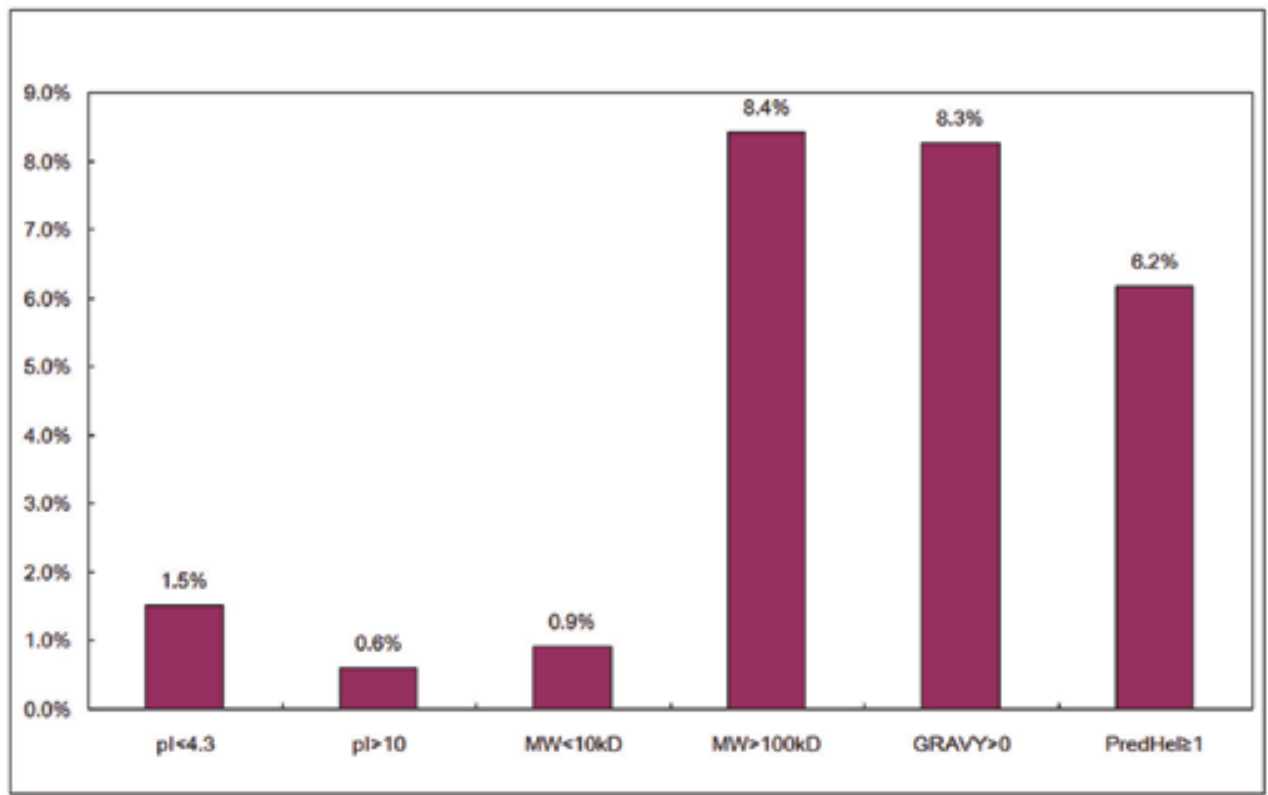

Fig. 4. A total of 665 rat proteins identified from the six tandem fractions of rat liver cytosol. A shows the percentage of the proteins identified from the each fraction and overlapping of different fractions. For example, F1-only represents the proteins identified appeared only in the F1 fraction, not in other fractions; In two fractions means the identified proteins appeared in two different fractions; In three fractions means the identified proteins appeared in three fractions; In all fractions means the identified proteins appeared in all the six fractions. B presents sensitivity to a wide variety of protein classes. The bars indicate the percentage of proteins identified in this study from a variety of protein classes. 


\begin{tabular}{|c|c|c|c|c|c|c|c|c|c|c|c|c|c|}
\hline \multirow{2}{*}{$\begin{array}{l}\text { Unique } \\
\text { Peptides }\end{array}$} & \multirow[b]{2}{*}{ Total $^{b}$} & \multicolumn{12}{|c|}{ Fraction $^{a}$} \\
\hline & & $\mathrm{F} 1^{\mathrm{c}}$ & $\mathrm{F} 2 \mathrm{c}$ & F3c & F4c & F5c & F6 & $\begin{array}{c}\text { F1- } \\
\text { onlyd }\end{array}$ & $\begin{array}{c}\text { F2- } \\
\text { only }^{\mathrm{d}}\end{array}$ & $\begin{array}{c}\text { F3- } \\
\text { only }^{\text {d }}\end{array}$ & $\begin{array}{c}\text { F4- } \\
\text { only }^{\mathrm{d}}\end{array}$ & $\begin{array}{c}\text { F5- } \\
\text { onlyd }\end{array}$ & $\begin{array}{l}\text { F6- } \\
\text { only }\end{array}$ \\
\hline 1 & 255 & 124 & 39 & 49 & 123 & 62 & 13 & 86 & 13 & 17 & 77 & 22 & 5 \\
\hline 2 & 122 & 66 & 27 & 23 & 57 & 39 & 9 & 37 & 7 & 7 & 26 & 12 & 5 \\
\hline 3 & 74 & 31 & 10 & 26 & 24 & 23 & 9 & 16 & 2 & 3 & 9 & 4 & 1 \\
\hline$>3$ & 214 & 69 & 47 & 54 & 75 & 31 & 29 & 38 & 2 & 3 & 28 & 5 & 6 \\
\hline Total & 665 & 290 & 123 & 152 & 279 & 155 & 60 & 177 & 24 & 30 & 140 & 43 & 17 \\
\hline
\end{tabular}

a Six fractions-------F1(A7-56), F2(A84), F3(A11-70), F4(A6), F5(A29-32), F6(Flow Through).

$\mathrm{b}$ A total of 665 proteins identified in the six fractions. The peptides attributed to the identification of certain protein in different fractions were combined to calculate the unique peptides.

c Proteins identified in certain fraction.

d Proteins identified only in each fraction.

Table 2. Proteins identified in six tandem rat liver fractions according to different number of unique peptides

\subsection{Physicochemical characteristics of the identified proteins}

The 665 identified proteins were classified according to different physicochemical characteristics such as molecular mass, pI, hydrophobicity (GRAVY value), and TM domain predicted by TMHMM (FIG. 5).

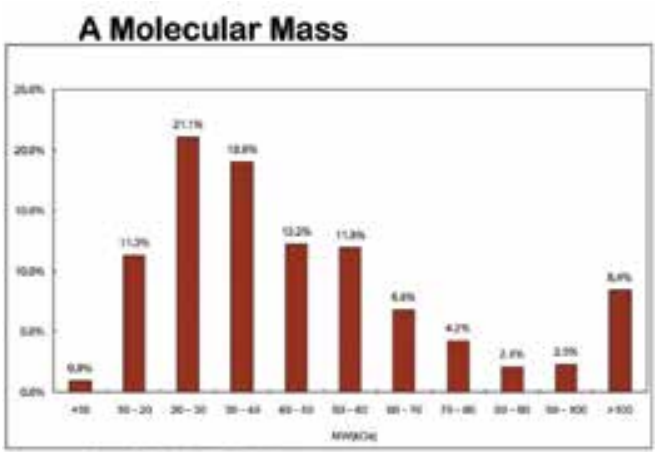

C GRAVY Value

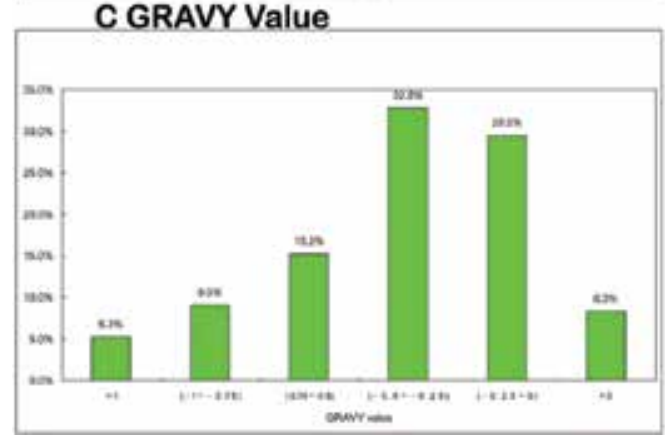

B pl Value

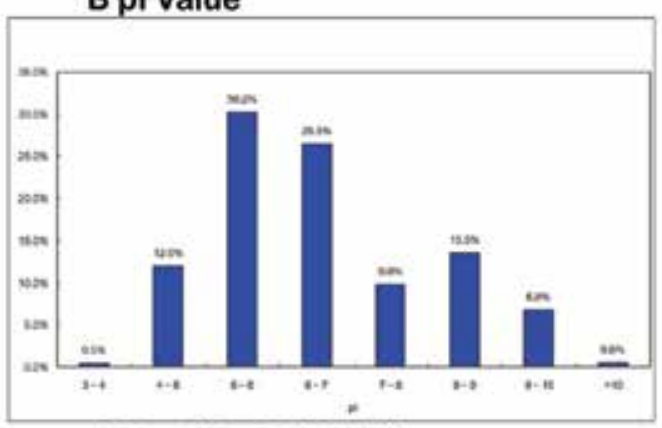

D Predicted TM Helices

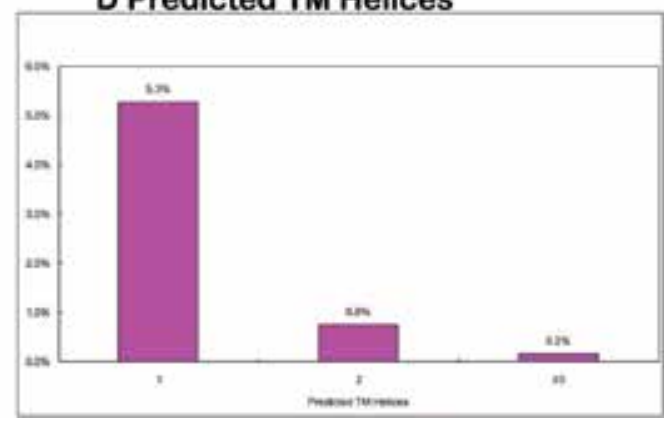

Fig. 5. Distribution of the total proteins identified in relation to their theoretical molecular mass (A), pI (B), GRAVY values (C), and the number of predicted TM helices (D). The bars indicate the percentage of proteins in total proteins identified. 
The smallest and the largest molecular mass obtained are $6.6 \mathrm{kDa}$ and $550.8 \mathrm{kDa}$, respectively. For the 665 proteins, 501 proteins $(75.3 \%)$ distribute among10-60kDa molecular mass intervals, which are compatible with general 2D-PAGE, while there are six $(0.9 \%)$ proteins with mass $<10 \mathrm{kDa}$ and $56(8.4 \%)$ proteins with mass $>100 \mathrm{kDa}$, beyond the general 2D-PAGE separation limits (FIG. 5A). It was more interesting that all proteins with mass $<10$ $\mathrm{kDa}$ only found in a certain fraction, and forty-five $(80.4 \%)$ proteins with mass $>100 \mathrm{kDa}$ only found in a certain fraction, which indicated those proteins also were low-abundant components and enriched after tandem affinity prefractionation.

Regarding the $\mathrm{pI}$ distribution, the total 665 proteins distribute across a wide pI range (3.7511.86) (FIG. 5B). A total of 377 proteins (58\%) distribute among pI 5-7 intervals, but only ten $(1.5 \%)$ proteins have $\mathrm{pI}<4.3$ and four $(0.6 \%)$ proteins have pI> 10. Interestingly, Most of proteins with extreme $\mathrm{pI}$ value were only found in a certain fraction, especially in Fraction 1. The GRAVY values (http://www.bioinformatics.org/sms2/protein_gravy.html) were determined according to Kyte and Doolittle (1982). The proteins detected in 2D-PAGE gels are generally hydrophilic, thus with negative GRAVY values $(37,38)$. For the total 665 proteins identified, their GRAVY values vary in the range of $-2.04 \sim 0.36$ (FIG. 5C). Interestingly, Fifty-five $(8.3 \%)$ hydrophobic proteins were identified with positive GRAVY values.

The TMHMM (http://www.cbs.dtu.dk/services/TMHMM/) (Krogh et al.,2001) was used to predict protein TM domains. Without specific methods for enrichment or treatment of membrane proteins, we still identified $41(6.2 \%)$ proteins of the total 665 proteins have one or more predicted TM domain (FIG. 5B), of which 35 proteins have one TM domain, 5 have two TM domains, and one protein has seven TM domains (FIG. 5D). The protein with seven TM domains was detected as Glucose-dependent insulinotropic receptor (IPI00382339.3, $\mathrm{MW}=51.7 \mathrm{kDa}$, $\mathrm{pI}=6.43$, GRAVY value=0.36).

In our study, we first combined the tandem biomimetic affinity prefractionation and LTQMS/MS (one dimension). From biomimetic affinity ligands library, we screened out some ligands showing large absorbance differences in band distribution to rat liver cytosol proteins. According to the affinnity specificity of these ligands, tandem composition of different ligands could be applied in well-distribution of complex tissue proteins. After reducing the complicity of tissue protein samples from tandem affinity prefractionation, more protein information was able to be obtained from LTQ-MS/MS analysis. With the strict and widely accepted SEQUEST criterion and only the rat protein database, a total of 665 unique rat proteins were identified in the six fractions, which was far higher than proteins indentified in unfractionated cytosol (74 proteins). Only 170 proteins were identified in rat liver or its cytosol sample through 2D-PAGE ( Fountoulakis \& Suter, 2002). Although the number of proteins identified from all or each rat liver fraction in our study are far from the theoretical protein number of rat liver cytosol, which may have suffered from a limited protein database for rat compared with those of human and mouse.

In present research, the tandem affinity prefractionation could obviously enhance the detection ability of shotgun strategy. Most importantly, the tandem affinity prefractionation could be finished in 4 hours, and one-dimension LTQ-MS/MS analysis employed would consumed only 5 hours, which was far lower than 2D-LC-MS/MS. Including other processes (such as concentration, trypsin digestion), only data from single run of $20 \mathrm{~h}$ were used in this work. 


\section{Prefractionation of of rat liver cytosol proteins utilizing cascade composition of affinity chromatography}

\subsection{Cascade affinity prefractionation of tissue samples}

From biomimetic affinity ligands library in our lab, we first screened out some affinity ligands showing large absorbance differences in band distribution to rat liver cytosol proteins. Then, the affinity ligands having medium absorbance ability were selected, cascade composition of these affinity ligands could be adopted for the prefractionation of tissue proteins. In this study, cascade composition of three affinity ligands was adopted, and the schematic illustration of the workflow was shown in FIG. 6. Briefly, after complex tissue sample was loaded to the first affinity ligand column, flowthrough and elution were collected for the two new fractions. Then, after above two new fractions were parallel loaded to the second affinity liand column, flowthrough and elution were collected for the four new fractions. At last, above four new fractions were loaded to the third affinity ligands, and 8 new fractions could be obtained. The binding and elution conditions were same as above. After being desalted and concentrated using Microcon ultrafiltration membranes (3,000 Nominal Molecular Weight Limit, Millipore), all the fractions collected were used for subsequent SDS-PAGE electrophoresis and LTQ-MS/MS analysis.

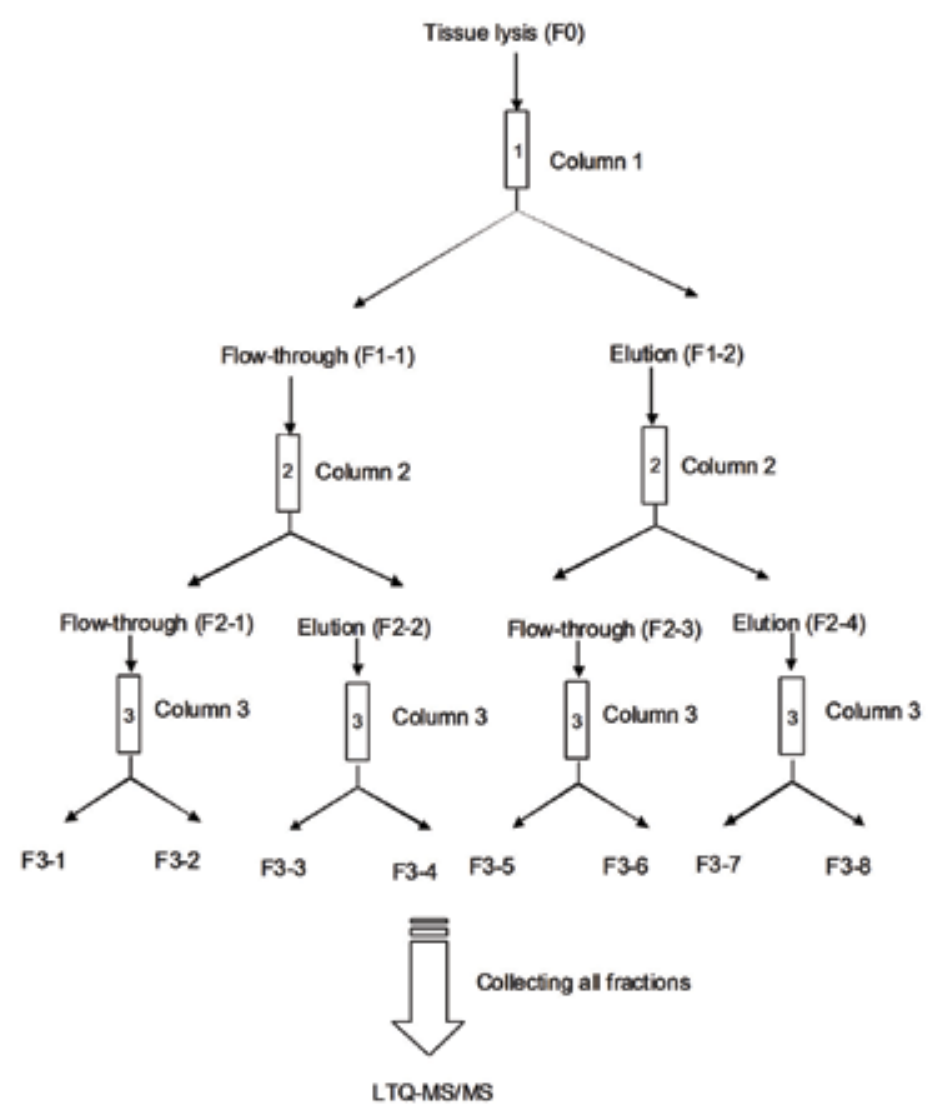

Fig. 6. Schematic illustration of Cascade affinity fraction used in this work 


\subsection{Evaluating for differential protein absorbance profiles of mimetic affinity ligands}

After analyzing the screening data of our lab in serum and leech proteins, we selected the affinity ligands having medium absorbance ability. Sixty affinity ligands were selected from the affinity ligands library of our lab, and the protein absorbance characterization of these ligands was reevaluated using rat liver cytosol sample as materials. From the SDS-PAGE profiles of each ligand, we found that some ligands showed large absorbance differences in band distribution to rat liver cytosol proteins (FIG.7). Partial examples of proteins absorbance profiles were shown in FIG.7, and each ligand showed its own specific binding ability to some group of proteins, but low absorbance characterization to others. At the same time, some affinity ligands exhibited relatively low absorbance ability such as A11-70 and A7-56, but other ligands showed relatively high absorbance ability such as A6, A15 and A29-32. According to the affinnity specificity of these ligands, composition of different ligands could be used to fractionate complex tissue proteins into different group of proteins. After reducing the complicity of tissue protein samples, more protein information was able to be obtained from proteome analysis. In present research, cascade combination of several affinity ligands was used as prefractionation of complex protein samples prior to LTQ-MS /MS proteome analysis. Three affinity ligands (A15, A8-54, A11-70) were selected for threecascade composition in the follow experiments.

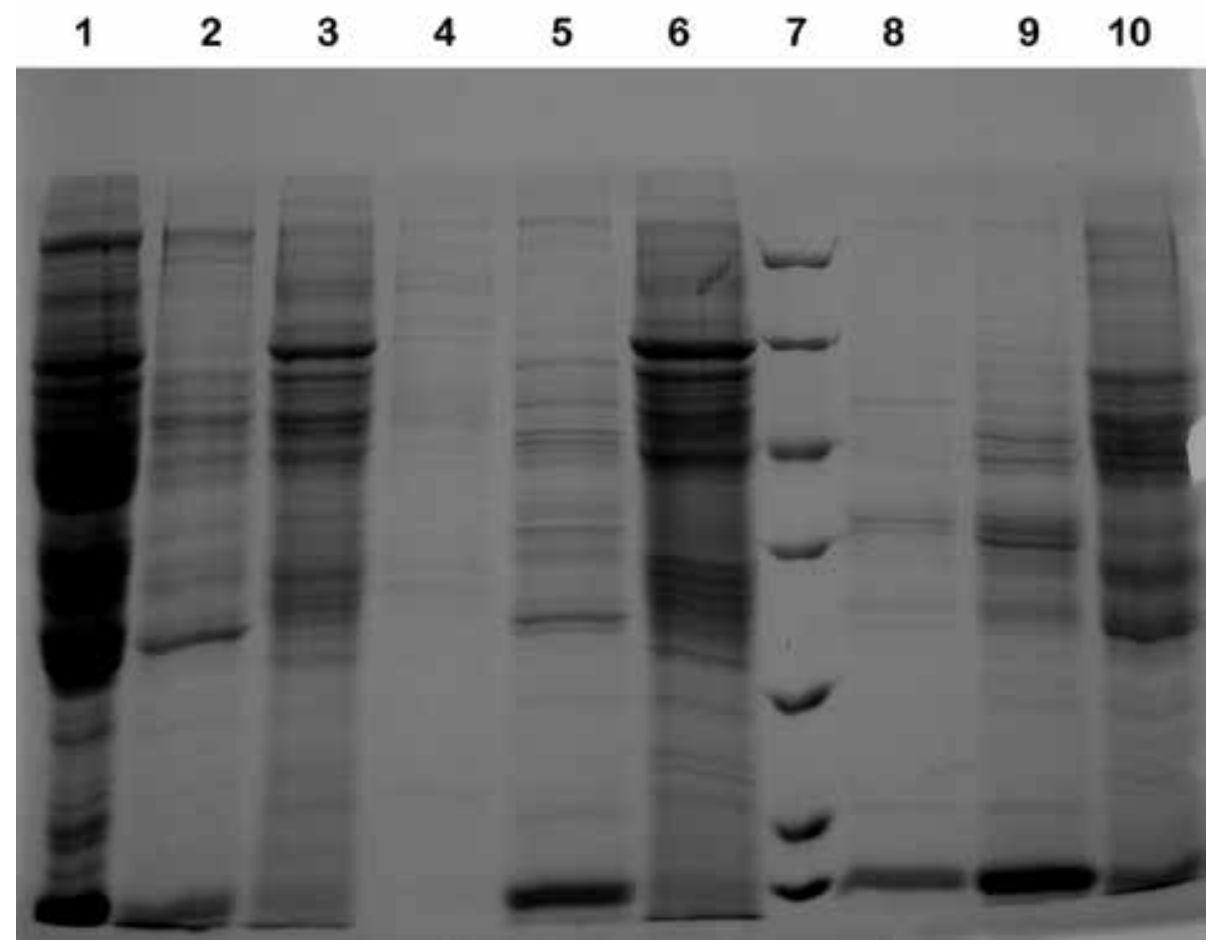

Fig. 7. Partial examples of rat liver proteins absorbance profiles having large band distribution difference. Lane 1 is crude rat liver cytosol. Lane 7 is the protein marker: 116, $66.2,45,31,25,18.4,14.4 \mathrm{kDa}$ (Fermentas, USA). Lanes2-6, 8-10show the $20 \mathrm{mM}$ Glycine$\mathrm{NaOH}$ buffer (pH12.0) eluates of columns A1-4, A6, A7-56, A8-54, A15, A11-70, A22-83, and A25-35, respectively. 


\subsection{Cascade affinity fractionation and LTQ-MS/MS analysis}

The flowchart in FIG. 6 gave a more detailed overview of the cascade fractionation procedure. Affinity ligand column A15, having relatively high absorbance ability, was put in the first affinity fractionation. After $4 \mathrm{mg}$ rat liver cytosol was loaded to the column A15, the flow-through and elution were collected (adjusted to $\mathrm{pH} 7.0$ instantly), named as F1-1 and F1-2. Then, F1-1 and F1-2 were parallel loaded to the column A8-54, four fractions (F2$1 \sim \mathrm{F} 2-4)$ were obtained from collecting the flow-through and elution. At last, the four fractions in the second affinity fractionation were loaded to A11-70, eight new fractions (F31 F3-8) were obtained from collecting the flow-through and elution. All fractions were collected for subsequent SDS-PAGE electrophoresis (FIG. 8). From proteins band distribution profiles in FIG. 8, the three-cascade-fractionation reduced the complexity of tissue sample gradually. In the end, complex rat liver cytosol proteins were well-distributed into eight simple fractions (F3-1 F3-8), and each fraction exhibited its own specific proteins distribution characterization.
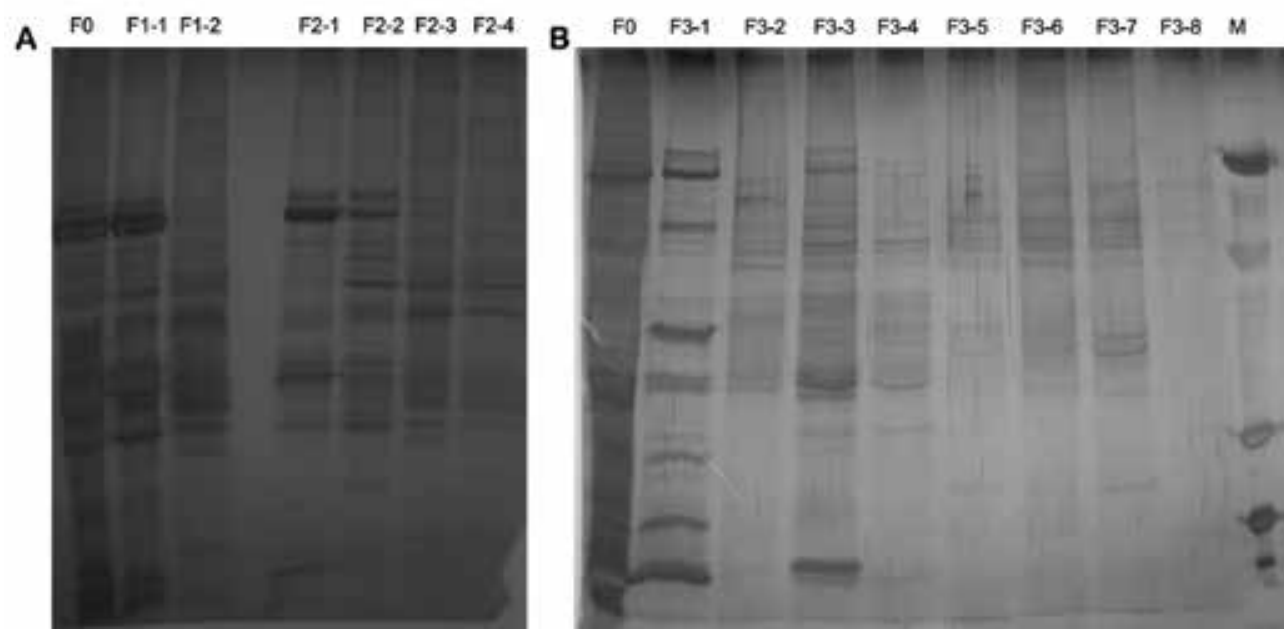

Fig. 8. Protein profiles of cascade affinity fractionation (A15 A8-54 A11-70). A shows the proteins distribution of all the fractions in the first and second fractionation, F0 is crude rat liver cytosol, F1-1 and F1-2 are flowthrough and elution of A15, F2-1 F2-4 are four new fractions of the second fractionation(A8-54). B shows the proteins distribution of all the fractions in the third fractionation, F0 is crude rat liver cytosol, F3-1 F3-8 are eight new fractions of the third fractionation (A11-70), $\mathrm{M}$ is the protein marker: 116, 66.2, 45, 31, 25, 18.4, 14.4kDa (Fermentas, USA).

After being desalted and concentrated using Microcon ultrafiltration membranes $(3,000$ Nominal Molecular Weight Limit, Millipore), all the fractions collected were used for subsequent LTQ-MS/MS analysis. The technical route was evaluated by comparing the protein numbers detected from the unfractionated rat liver cytosol sample with those from the fractions of each grade in the cascade affinity fractionation. The MS/MS spectra acquired from equivalent normalized aliquots of the 15 respective fractions were searched against the IPI rat database using the program SEQUEST running on ISB/SPC Proteomics workstation. As most sequence search engines return results even for 'unmatchable' spectra, proteome researchers must devise ways to distinguish correct from incorrect peptide 
identifications. The target-decoy search strategy represents a straightforward and effective way to manage this effort. In this work, The ISB/SPC Proteomics Tools-TPP V4.2 was applied, which combined the decoy database searching approach with automated filter criteria optimization. Peptides with a $\mathrm{p}$ value of equal or bigger than 0.95 , protein probability with a $\mathrm{p}$ value of equal or bigger than 0.99 , were used for filtering conditions. Three hundred ninety-one unique proteins were identified in unfractionated rat liver cytosol, 499 unique proteins were identified (27.6\% increased) in two fractions (F1-1and F12 ) of the first affinity fractionation, 616 unique proteins were identified $(23.4 \%$ increased) in four fractions (F2-1 F2-4) of the second affinity fractionation, and 732 unique proteins $(18.8 \%$ increased) were identified in eight fractions (F3-1 F3-8) of the third affinity fractionation (Table 1). In the end, the cascade affinity fractionation resulted in highly confident identification of a total of 859 unique rat protein groups in cascade affinity fractions (Table 1), over two times as protein numbers detected in unfractionated rat liver cytosol sample. It was noted that most of the proteins identified in unfractionated sample (364 proteins, 93.1\%) also emerged in the cascade affinity fractions, and 495 new proteins were identified in the cascade affinity fractions. Table 2 showed the increasing percentage of protein numbers identified in all affinity fractionation processes, and the effect of each fractionation was obvious. The minimal increasing percentage was $14.4 \%$, and the maximum of increasing percentage was $113.3 \%$. Therefore, the cascade affinity fractionation resulted in the highly increasing of the protein numbers identified, and much more new proteins were detected.

\begin{tabular}{llllll}
\hline Fraction & $\mathrm{F}^{\mathrm{a}}$ & $\mathrm{F} 1-1 \sim \mathrm{F} 1-2^{\mathrm{b}}$ & $\mathrm{F} 2-1 \sim \mathrm{F} 2-4^{\mathrm{c}}$ & $\mathrm{F} 3-1 \sim \mathrm{F}^{2}-8^{\mathrm{d}}$ & Totale \\
\hline Unique peptides & 1511 & 1432 & 1637 & 1686 & 1951 \\
\hline Unique Proteins & 391 & 499 & 616 & 738 & 859 \\
\hline
\end{tabular}

a F0 was unfractionated rat liver cytosol.

b Two fractions(F1-1 F1-2) in the first affinity fractionation.

c Four fractions(F2-1 F2-4) in the second affinity fractionation.

d Eight fractions(F3-1 F3-8) in the third affinity fractionation.

e Total proteins identified in all fractions(no including F0).

Table 3. Peptides and proteins identified in all rat liver fractions of the cascade affinity fractionation using LTQ-MS/MS

\begin{tabular}{llllllll}
\hline \multirow{2}{*}{ Firsta } & F0 & F1-1 & F1-2 & F2-1 & F2-2 & F2-3 & F2-4 \\
\cline { 2 - 7 } & 391 & 298 & 374 & 360 & 165 & 302 & 359 \\
\hline \multirow{2}{*}{ Second $^{b}$} & F1- & F2- & F2- & F3- & F3- & F3- & F3- \\
& $1+F 1-2$ & $1+F 2-2$ & $3+F 2-4$ & $1+F 3-2$ & $3+F 3-4$ & $5+F 3-6$ & $7+F 3-8$ \\
\cline { 2 - 7 } & 499 & 387 & 443 & 412 & 352 & 353 & 430 \\
\hline $\begin{array}{l}\text { Increasing } \\
(\%)\end{array}$ & 27.6 & 29.9 & 18.4 & 14.4 & 113.3 & 16.9 & 19.8 \\
\hline
\end{tabular}

a First showed the protein numbers identified in the fractions before affinity fractionation .

$\mathrm{b}$ Second showed the protein numbers identified in the collected fractions (including flow-through and elution) after affinity fractionation.

Table 4 . Increasing percentage of protein numbers identified in all affinity fractionation processes 
Table 5 showed the protein numbers identified according to 1, 2, 3, and $>3$ unique peptides. About $40 \%$ proteins in each fraction were identified from a unique peptide, and a total of 497 unique protein groups $(56.09 \%)$ in all fractions were identified from a unique peptide. About $20 \%$ proteins in each fraction were identified from two unique peptides, $10 \%$ from three unique peptides, and 15 30\% from $>3$ unique peptides. It was noted that most of the proteins identified from two or more unique peptides were often repeated in different fractions, which indicated those proteins are high-abundant components in the cell. On the contrary, the proteins identified by a single peptide showed relatively low repeated proportion, which indicated that most of those proteins were low-abundant components in the cell and enriched after fractionation. Comparing with the traditional 2D-PAGE method, the shotgun strategy presents a number of data with very rapid speed and limited sample consumption. In present research, the cascade affinity prefractionation could obviously enhance the detection ability of shotgun strategy. Most importantly, the tandem affinity prefractionation could be finished in 8 hours, and one-dimension LTQ-MS/MS analysis employed would consumed only 5 hours, which was far lower than 2D-LC-MS/MS. Including other processes (such as concentration, trypsin digestion), only data from single run of $24 \mathrm{~h}$ were used in this work.

\begin{tabular}{|c|c|c|c|c|c|c|c|c|c|c|c|c|c|c|c|c|}
\hline \multirow{2}{*}{$\begin{array}{c}\text { Unique } \\
\text { Peptides }\end{array}$} & \multirow{2}{*}{ Totalb } & & \multicolumn{14}{|c|}{ Fraction a } \\
\hline & & F0 & F1-1 & F1-2 & F2-1 & $F 2-2$ & F2-3 & F2-4 & F3-1 & F3-2 & F3-3 & F3-4 & F3-5 & F3-6 & F3-7 & F3-8 \\
\hline 1 & 479 & 122 & 120 & 141 & 159 & 78 & 136 & 128 & 126 & 119 & 105 & 87 & 152 & 93 & 101 & 147 \\
\hline 2 & 154 & 79 & 59 & 82 & 58 & 37 & 53 & 57 & 55 & 56 & 55 & 49 & 53 & 48 & 57 & 76 \\
\hline 3 & 85 & 43 & 32 & 48 & 42 & 16 & 41 & 53 & 20 & 35 & 28 & 26 & 31 & 31 & 32 & 45 \\
\hline$>3$ & 141 & 147 & 87 & 103 & 101 & 34 & 72 & 121 & 78 & 68 & 81 & 78 & 52 & 56 & 62 & 114 \\
\hline Total & 859 & 391 & 298 & 374 & 360 & 165 & 302 & 359 & 279 & 278 & 269 & 240 & 288 & 228 & 252 & 382 \\
\hline
\end{tabular}

a fifteen fractions - F0 was unfractionated rat liver cytosol. F1-1 and F1-2 were two fractions in the first affinity fractionation. F2-1 F2-4 were four fractions in the second affinity fractionation. F3-1 F3-8 were eight fractions in the third affinity fractionation.

b A total of 859 proteins identified in the fourteen fractions(no including F0). The peptides attributed to the identification of certain protein in different fractions were combined to calculate the unique peptides.

Table 5. Proteins identified in all fractions of cascade affinity fractionation of rat liver cytosol according to different number of unique peptides

\subsection{Physicochemical characteristics of the identified proteins}

The 859 identified proteins were classified according to different physicochemical characteristics such as molecular mass, pI, hydrophobicity (GRAVY value), and TM domain predicted by TMHMM (FIG. 9).

The smallest and largest molecular mass obtained are $6.1 \mathrm{kDa}$ and $419.6 \mathrm{kDa}$, respectively. For the 859 proteins, 617 proteins $(71.83 \%)$ distribute among 20-70kDa molecular mass intervals, which are compatible with general 2D-PAGE, while there are 75 proteins $(8.73 \%)$ with mass $<20 \mathrm{kDa}$ and 73 proteins $(8.5 \%)$ with mass $>100 \mathrm{kDa}$, beyond the general 2D-PAGE separation limits (FIG. 9A). Regarding the pI distribution, the total 859 proteins distribute across a wide pI range (3.75-11.56) (FIG. 9B). A total of 594 proteins (69.16\%) distribute among pI 5-8 intervals, but only 85 proteins $(9.9 \%)$ have pI $<5$ and 180 proteins $(20.95 \%)$ have $\mathrm{pI}>8$. 
The GRAVY values (http://www.bioinformatics.org/sms2/protein_gravy.html) were determined according to Kyte and Doolittle (Kyte and Doolittle, 1982). The proteins detected in 2D-PAGE gels are generally hydrophilic, thus with negative GRAVY values (Fountoulakis, and Takacs, 2001; Fountoulakis and Suter, 2002). For the total 859 proteins identified, their GRAVY values vary in the range of $-2.047 \sim 0.322$ (FIG. 9C). It is noted that most of the proteins are hydrophilic (787 proteins with negative GRAVY values), and 72 $(8.38 \%)$ hydrophobic proteins were identified with positive GRAVY values.
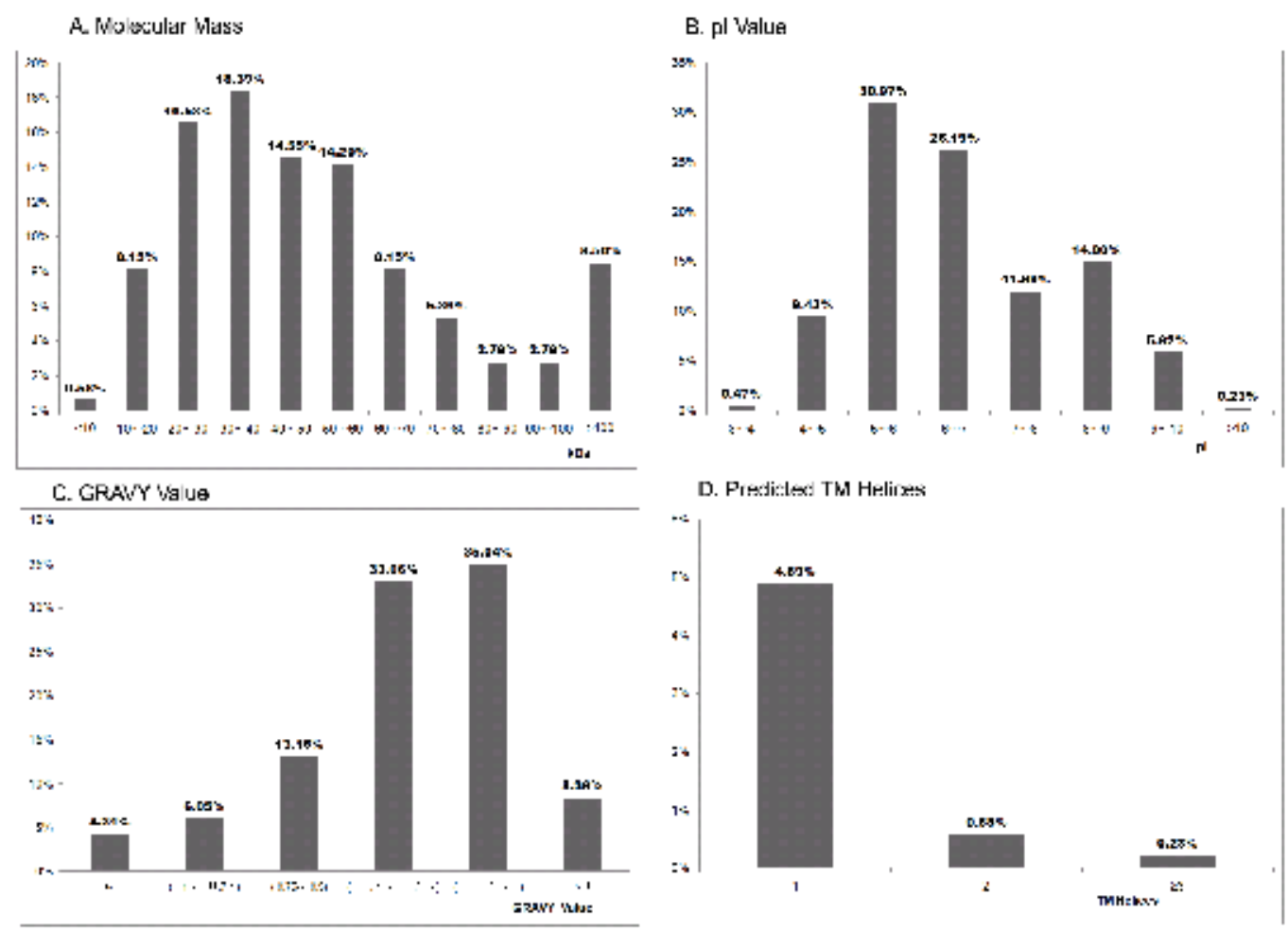

D. PIe:di:lex: TM Helirsts
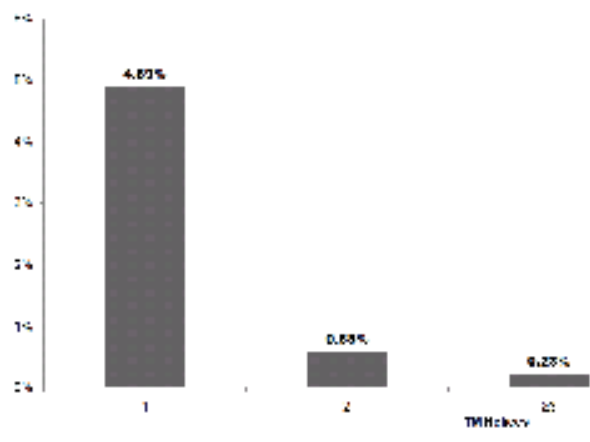

Fig. 9. Distribution of the total proteins identified in relation to their theoretical molecular mass (A), pI (B), GRAVY values (C), and the number of predicted TM helices (D). The bars indicate the percentage of proteins in total proteins identified.

Analysis of membrane proteins is an important field in proteomics because membrane proteins are represented by $30 \%$ of the genome and constitute approximately $70 \%$ of all human protein based drug targets (Wallin and von Heijne, 1998; Hopkins and Groom, 2002). Analysis of membrane proteins has been notoriously difficult, which has been demonstrated by their under-representation in 2D gels (Molloy et al., 1998; Santoni et al., 2000). The TMHMM software (http://www.cbs.dtu.dk/services/TMHMM/) was used to predict protein TM domains of membrane proteins (Krogh et al., 2001). Without specific methods for enrichment or treatment of membrane proteins, we still identified $49(5.7 \%)$ proteins of the total 859 proteins have one or more predicted TM domain (FIG. 9D). Of these, 42 proteins have one TM domain, 5 have two TM domains, and two proteins have three TM domains (FIG. 9D). 
A<smiles>[R]NCC(O)COCCOC</smiles>

An<smiles>[R]Nc1nc(N[R])nc(NCC(O)COC(C)(C)C)n1</smiles>

Am-n

B

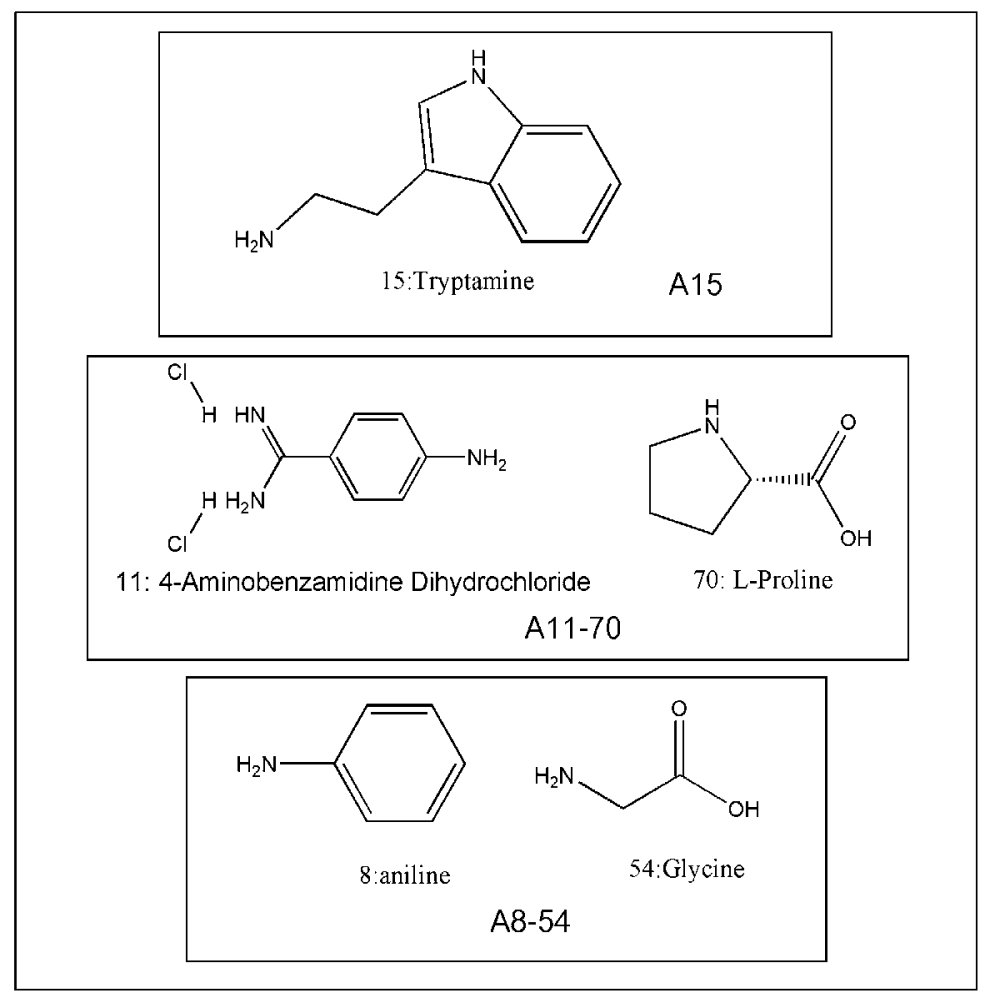

Fig. 10. The structure of affinity ligands used in cascade affinity prefractionation. Sepharose 4B was matrix, which was activated with epichlorohydrin. A showed two kinds of synthetic ligands: first, single amino compound was directely linked to activated sepharose, named as An; second, cyanuric chloride was coupled to activated sepharose as a large spacer, and two chloride in spacer could be substituted by two amino compounds, named as Am-n. B gave the chemical structures of amino compounds used in three selected affinity ligands (A15, A11-70, A8-54). 


\section{Discussion}

In our study, we first combined the cascade biomimetic affinity prefractionation with LTQMS/MS analysis. From biomimetic affinity ligands library, we screened out three ligands showing large absorbance differences in band distribution to rat liver cytosol proteins. The structures of the these elected ligands were shown in Fig. 10, and the structure difference was obvious. According to the difference of these ligands in size, shape, structure, and biochemical characterization, each ligand could exhibit the affinity specificity to some protein groups. Therefore, the cascade composition of different ligands could be applied in well-distribution of complex tissue proteins. After reducing the complicity of tissue protein samples, more protein information was able to be obtained from LTQ-MS/MS analysis. In this report, only three ligands were used in the cascade composition of affinity chromatography. However, after simple affinity fractionation for three times, the crude rat liver cytosol was fractionated into eight relatively simple fractions, and a total of 859 unique rat protein groups were identified in the cascade affinity fractions, which was far higher than proteins indentified in unfractionated cytosol (391 proteins). According to previous research reports, in rat liver cytosol sample Only 170 proteins were identified through 2DPAGE (Fountoulakis and Suter, 2002), and 222 proteins were identified through 2D-LCMS/MS (Jiang et al., 2004). We applied much more strict filtering condition compared with previous references, but our results gave more non-redundant rat liver cytosol protein groups (859).

In present research, the cascade affinity fractionation could obviously enhance the detection ability of shotgun strategy. For more complex tissue samples, much more affinity ligands could be selected fot cascade composition. Combined usage of the cascade affinity fractionation and LTQ-MS/MS was simple, low-cost, and effective, which gave a broad application prospect in proteomics.

\section{Acknowledgments}

This work was supported by the National S \& T Major Projects of China (Key Innovative Drug Development, No. 2009ZX09306-008), National Basic Research Program of China (973 Program, No. 2007CB936004 \& 2009CB118906), National High Technology Research and Development Program of China (863 Program, No. 2007AA100506), Natural Science Foundation of China (No. 30630012), Shanghai Leading Academic Discipline Project (No. B203) and Shanghai Science and Technology Innovation Action Program (No. 072312048 \& 08DZ1204400).

\section{References}

Aebersold, R \& Mann, M. (2003). Mass spectrometry-based proteomics. Nature 422,198-207

Badock, V, Steinhusen, U, Bommert, K \& Otto, A. (2001) Prefractionation of protein samples for proteome analysis using reversed-phase high-performance liquid chromatography. Electrophoresis 22, 2856-2864.

Bergh, GV, Clerens, S, Vandesande, F \& Arckens, L. (2003). Reversed-phase highperformance liquid chromatography prefractionation prior to two-dimensional 
difference gel electrophoresis and mass spectrometry identifies new differentially expressed proteins between striate cortex of kitten and adult cat. Electrophoresis 24: 1471-1481.

Braun, RJ, Kinkl, N, Beer, M \& Ueffing, M. (2007). Two-dimensional electrophoresis of membrane proteins. Anal. Bioanal. Chem. 389: 1033-1045.

Corthals, GL, Wasinger, VC, Hochstrasser, DF \& Sanchez, JC. (2000). The dynamic range of protein expression: a challenge for proteomic research. Electrophoresis 21:1104-1115.

Cronshaw, JM, Krutchinsky, AN, Zhang, W, Chait, BT \& Matunis, MJ. (2002). Proteomics analysis of the mammalian nuclear pore complex. J. Cell Biol. 158:915-927.

Dong, D, Liu, H, Xiao, Q, Wang, T, Liu, H \& Li, R. (2008a). Biomimetic affinity purification of cardiotoxin and its pharmacological effects on the nervous system. J. Mol. Recognit. 21:148 - 153

Dong, D, Gui, Y, Chen, D \& Li, R. (2008b). Utilizing a library of synthetic affinity ligands for the enrichment, depletion and one-step purification of leech proteins. J. Mol. Recognit. 21:163-8.

Fountoulakis, M, Juranville, JF, Jiang, L, Avila, D, RÖder, D, Jakob, P, Berndt, P, Evers, S \& Langen, H. (2004). Depletion of the high-abundance plasma proteins. Amino Acids 27: $249-259$.

Fountoulakis, M \& Suter, L.( 2002). Proteomic analysis of the rat liver. J. Chromatogr. B Analyt. Technol. Biomed. Life Sci. 782: 197-218.

Fountoulakis, M \& Takács, B. (2001). Effect of strong detergents and chaotropes on the detection of proteins in two-dimensional gels. Electrophoresis 22:1593-1602.

Gao, M, Deng, C, Yu, W, Zhang, Y, Yang, P \& Zhang, X. (2008). Large scale depletion of the high-abundance proteins and analysis of middle- and low-abundance proteins in human liver proteome by multidimensional liquid chromatography. Proteomics 8: 939-947

Gao, M, Hong, J, Yang, P \& Zhang, X. (2005). Chromatographic prefractionation prior to two-dimensional electrophoresis and mass spectrometry identifies: Application to the complex proteome analysis in rat liver. Anal. Chim. Acta 553:83-92.

Gao, M, Zhang, J, Deng, C, Yang, P \& Zhang, X. (2006). Novel Strategy of High-Abundance Protein Depletion Using Multidimensional Liquid Chromatography. J. Proteome Res. 5: 2853-2860.

Greenough, C, Jenkins, RE, Kitteringham, NR, Pirmohamed, M, Park, BK \& Pennington, SR. (2004). A method for the rapid depletion of albumin and immunoglobulin from human plasma. Proteomics 4: 3107-3111.

Gupta, G \& Lowe, CR. (2004). An artificial receptor for glycoproteins. J. Mol. Recognit. 17:218-235.

Gygi, SP, Corthalas, GL, Zhang, Y, Rochon, Y \& Aebersold, R. (2000). Evaluation of twodimensional gel electrophoresis-based proteome analysis technology. Proc. Natl. Acad. Sci. U. S. A. 97: 9390-9395.

Gygi, SP, Rist, B, Gerber, SA, Turecek, F, Gelb, MH \& Aebersold, R. (1999). Quantitative analysis of complex protein mixtures using isotope-coded affinity tags. Nat. Biotechnol. 17:994-999. 
Harry, J, Wilkins, MR, Herbert, BR, Packer, NH, Gooly, AA \& Williams, KL. (2000). Proteomics: Capacity versus utility. Electrophoresis 21: 1071-1081.

Hinerfeld, D, Innamorati, D, Pirro, J \& Tam, SW. (2004). Serum/Plasma Depletion with Chicken Immunoglobulin YAntibodies for Proteomic Analysis from Multiple Mammalian Species. J. Biomol.Tech. 15:184-190.

Hopkins, AL \& Groom, CR. (2002).The druggable genome. Nat Rev. Drug Discovery. 1:72730.

Jacobs, JM, Mottaz, HM, Yu, LR, Anderson, DJ, Moore, RJ, Chen, WN, Auberry, KJ, Strittmatter, EF, Monroe, ME, Thrall, BD, Camp, DG 2nd \& Smith, RD. (2004). Multidimensional proteome analysis of human mammary epithelial cells. J. Proteome Res. 3: 68-75.

Jiang, XS, Zhou, H, Zhang, L, Sheng, QH, Li, SJ, Li, L, Hao, P, Li, YX, Xia, QC, Wu, JR \& Zeng, R. (2004). A High-throughput approach for subcellular proteome: Identification of rat liver proteins using subcellular fractionation coupled with twodimensional liquid chromatography tandem mass spectrometry and bioinformatic analysis. Mol. Cell Proteomics. 3: 441-455.

Klose, J \& Kobalz, U. (1995). Two-dimensional electrophoresis of proteins: an updated protocol and implications for a functional analysis of the genome. Electrophoresis 16:1034-1059.

Krogh, A, Larsson, B, von Heijne, G \& Sonnhammer, EL. (2001). Predicting transmembrane protein topology with a hidden Markov model: Application to complete genomes. J. Mol. Biol. 305: 567-580.

Kyte, J \& Doolittle, RF. (1982). A simple method for displaying the hydropathic character of a protein. J. Mol. Biol. 157: 105-132.

Li, R, Dowd, V, Stewart, DJ, Burton, SJ \& Lowe, CR. (1998). Design, synthesis, and application of a protein A mimetic. Nat. Biotechnol. 16:190-195.

Liu, C \& Zhang, X. (2007). Multidimensional capillary array liquid chromatography and matrix-assisted laser desorption/ionization tandem mass spectrometry for highthroughput proteomic analysis. J.Chromatogr. A 1139: 191-198.

Lowe, CR. (2001). Combinatorial approaches to affinity chromatography. Curr. Opin. Chem. Biol. 5:248-256.

Melissis, S, Labrou, NE \& Clonis, YD. (2007). One-step purification of Taq DNA polymerase using nucleotide-mimetic affinity chromatography. Biotechnol. J. 2: 121-132.

Gao, MX, Hong, J, Yang, PY \& Zhang, XM. (2005). Chromatographic prefractionation prior to two-dimensional electrophoresis and mass spectrometry identifies: Application to the complex proteome analysis in rat liver Analytica Chimica Acta. $553,83-92$

Molloy, MP, Herbert, BR, Walsh, BJ, Tyler, MI, Traini, M, Sanchez, JC, Hochstrasser, DF, Williams, KL \& Gooley, AA. (1998). Extraction of membrane proteins by differential solubilization for separation using two-dimensional gel electrophoresis. Electrophoresis 19: 837-44.

Motoyama, A \& Yates, JR 3rd. (2008). Multidimensional LC separations in shotgun proteomics. Anal. Chem. 80: 7187-7193. 
Oh-Ishi, M, Satoh, M \& Maeda T. (2000). Preparative two-dimensional gel electrophoresis with agarose gels in the first dimension for high molecular mass proteins. Electrophoresis 21:1653-1669.

Pieper, R, Su, Q, Gatlin, CL, Huang, ST, Anderson, NL \& Steiner, S. (2003). Multi-component immunoaffinity subtraction chromatography: an innovative step towards a comprehensive survey of the human plasma proteome. Proteomics 3: 422-432.

Omenn, G. S., (2004).The Human Proteome Organization Plasma Proteome Project pilot phase: Reference specimens, technology platform comparisons, and standardized data submissions and analyses. Proteomics, $4: 1235-1240$.

Qu, Y, Moons, L \& Vandesande, F. (1997). Determination of serotonin, catecholamines and their metabolites by direct injection of supernatants from chicken brain tissue homogenate using liquid chromatography with electrochemical detection. J. Chromatogr. B Biomed. Sci. Appl. 704: 351-358.

Rabilloud, T, Kieffer, S, Procaccio, V, Louwagie, M, Courchesne, PL, Patterson, SD, Martinez, P, Garin, J \& Lunardi, J. (1998). Two-dimensional electrophoresis of human placental mitochondria and protein identification by mass spectrometry: Toward a human mitochondrial proteome. Electrophoresis 19:10061014.

Santoni, V, Molloy, M \& Rabilloud, T. 2000. Membrane proteins and proteomics: un amour impossible. Electrophoresis 21:1054-70.

Shen, Y, Jacobs, JM, Camp, DG 2nd, Fang, RH, Moore, RJ \& Smith RD. (2004). Ultra-highefficiency strong cation exchange LC/RPLC/MS/MS for high dynamic range characterization of the human plasma proteome. Anal. Chem. 76:1134 1144.

Tan, QQ, Dong, DX, Ye, L, Li, RX , (2009a), Combined usage of cascade affinity fractionation and LC-MS/MS for the proteomics of adult mouse testis. J. Sep. Sci. 32, 1-9.

Tan, QQ, Dong, DX \& \& Li, RX, (2009b),A novel fractionation method prior to MS-based proteomics analysis using cascade biomimetic affinity chromatography, $J$ Chromatogr B, 877, 3799-3805

Tan, QQ, Dong, DX, Ye, L, Huo, CX, Huang FY \& Li, RX, (2010),Pre-fractionation of rat liver cytosol proteins prior to mass spectrometry-based proteomic analysis using tandem biomimetic affinity chromatography, J. Mol. Recognit., 23, 93 - 100

Taylor, RS, Wu, CC, Hays, L, Eng, JK, Yate, JR \& Howell, KE. (2000). Proteomics of rat liver Golgi complex: Minor proteins are identified through sequential fractionation. Electrophoresis 21:3441-3459.

Teng, SF, Sproule, K, Hussain, A \& Lowe, CR. (1999). A strategy for the generation of biomimetic ligands for affinity chromatography combinatorial synthesis and biological evaluation of an IgG binding ligand. J. Mol. Recognit. 12: 67-75.

Wallin, E \& von Heijne, G. (1998). Genome-wide analysis of integral membrane proteins from eubacterial, archaean, and eukaryotic organisms. Protein Sci. 7:1029-38.

Washburn, MP, Wolters, D \& Yates, JR 3rd. (2001). Large-scale analysis of the yeast proteome by multidimensional protein identification technology. Nat. Biotechnol. 19: 242-247.

Wu, CC, MacCoss, MJ, Howell, KE \& Yates, JR 3rd. (2003). A method for the comprehensive proteomic analysis of membrane proteins. Nat. Biotechnol. 21: 532-538. 
Xin, Y, Dong, D, Wang, T \& Li, R. (2007). Affinity purification of serine proteinase from Deinagkistrodon acutus venom. J. Chromatogr. B Analyt. Technol. Biomed. Life Sci. 859: 111-118.

Yates, JR 3rd. (2004). Mass spectral analysis in proteomics. Annu. Rev. Biophys. Biomol. Struct. 33:297-316. 


\title{
Green Oxidation Reactions of Drugs Catalyzed by Bio-inspired Complexes as an Efficient Methodology to Obtain New Active Molecules
}

\author{
Emerson Henrique de Faria ${ }^{1}$, Gustavo Pimenta Ricci ${ }^{1}$, Frederico Matias \\ Lemos ${ }^{1}$, Marcio Luis Andrade e Silva ${ }^{1}$, Ademar Alves da Silva Filho ${ }^{2}$, \\ Paulo Sérgio Calefi ${ }^{1}$, Eduardo José Nassar ${ }^{1}$ and Katia Jorge Ciuffi ${ }^{1}$ \\ 1 Universidade de Franca - Av. Dr. Armando Salles Oliveira.201. Parque Universitário, \\ Franca- SP Brasil - CEP - 14.404-600 \\ ${ }^{2}$ Universidade Federal de Juiz de Fora - Faculdade de Farmácia e Bioquímica, \\ Departamento Farmacêutico, Campus Universitário, Martelos, Juiz de Fora-MG.
} Brasil

\section{Introduction}

The cytochromes P450 (P450s) are an important class of heme-containing enzymes that act as monooxigenases. P450s have a variety of critical roles in biology and are considered the most versatile enzymes in nature because of their key part in the metabolism of biomolecules and xenobiotics.

The $\mathrm{P} 450$ reacts with one oxygen atom from distinct oxidants such as dioxygen $\left(\mathrm{O}_{2}\right)$, forming the active oxidant species, the so-called metal-oxo complex (high-valent iron(IV)oxo intermediate), which is able to transfer the oxygen atom to several substrates like alkanes, alkenes, aromatic compounds and amines, among others. This active species is also responsible for the cleavage of $\mathrm{C}-\mathrm{C}$ bonds in organic substrates. The key feature of P450 enzymes is their ability to perform this reaction selectively, under mild conditions.

Synthetic metalloporphyrins have been extensively used as biomimetic catalysts due to their ability to act as P450 models. Ironporphyrins, in particular, have been employed as models because they are capable of catalyzing organic oxidations. Besides their biological and catalytic properties, ironporphyrins can also be immobilized onto organic polymers as well as amorphous and crystalline inorganic materials, such as silica, alumina, and clays.

The immobilization of metalloporphyrins onto inorganic supports has been found to yield efficient, selective catalysts for the oxidation of oxidizable groups, promoting a special environment for the approach of the substrate to the catalytically active species, thus mimicking the "site-isolation principle" of biological enzymes. The sol-gel process is considered an ideal methodology to prepare the ironporphyrin heterogeneous catalysts.

Synthetic metalloporphyrins also have potential application in the design of green processes for the accomplishment of many kinds of oxidations mimicking the P450 reactions.

Over the last decade the search for efficient and environmentally friendly oxidation procedures that could be used to develop green processes for many kinds of oxidation 
reactions has been intensified. Also, since the oxidation of organic compounds with high selectivity is of extreme importance in synthetic chemistry, many attempts have been made in order to oxidize compounds and produce target components that cannot be easily obtained by conventional routes. A very interesting and promising field for the application of metalloporphyrin catalysts lies in the area of natural product oxidation, with potential use as drugs for clinical practice. This can be achieved by employing clean oxidants like hydrogen peroxide or molecular oxygen.

Lignans have attracted much interest over the years on account of their broad range of biological activities, including antitumoral, trypanocidal, antimicrobial, and antiinflammatory activities, so alternatives that promote and improve their production are necessary.

(-)-Hinokinin, a dibenzylbutyrolactone lignan, possesses antileishmanial, anti-inflammatory, antigenotoxicity [5-9], and significant in vitro and in vivo trypanocidal activities against Trypanosoma cruzi, the etiologic agent of Chagas`disease.

Licarin-A, a neolignan obtained from the oxidative coupling of isoeugenol, displays significant antiparasitic activity against the adult forms of Schistosoma mansoni.

Taking into account the antiparasitic potential and the possible production of derivatives from Licarin-A and (-)-Hinokinin, there is great interest in the search for new approaches that will enable the oxidation of these lignans to be carried out, since the oxidized products are probably biologically active.

Enzymatic biological models have been widely employed throughout all the phases of drug design and development. In vivo oxidations carried out by enzymes like P450s can be easily mimicked under mild conditions by using synthetic metalloporphyrin systems. More specifically, ironporphyrins have been utilized as P450 models due to their ability to catalyze countless organic reactions.

In this chapter, the catalytic activity of ironporphyrins supported on an alumina or silica matrix, prepared by the sol-gel methodology, in the oxidation of lignans such as licarin-A and (-)-cubebin is investigated, in the search for novel systems for the accomplishment of green oxidation reactions by bio-inspired catalysts. Special attention is given to the reaction products and their potential biological activities.

\section{Cytochromes P450 (P450s)}

The cytochromes P450 (P450s) are an important class of heme-containing enzymes that contain a protoporphyrin IX as the active center and act as monooxigenases. As observed in Figure 1, the protoporphyrin presents one $S$ of a cysteine as the fifth ligand and a free sixth coordination site available for the binding of molecular oxygen (McMurry \& Groves,1986; Lewis, 2001; Lohmann \&Karst, 2008;). The P450s have a relatively hydrophobic active site cavity.

Cytochrome P450 enzymes are present in all five biological kingdoms. In mammalian species, P450s are present in most tissues and are largely founded in the liver (McMurry \& Groves, 1986; Lewis, 2001; Siroká \&Drastichová, 2004;Mansuy, 2007; Munro et al.,2007; Lohmann \&Karst, 2008).

P450s have a variety of critical roles in biology and are considered the most versatile enzymes in nature because of their key part in the metabolism and degradation of biomolecules and xenobiotics. Their role in the endogenous metabolism of steroids is considered the primary function of the organism (McMurry \& Groves,1986; Lewis, 2001; 


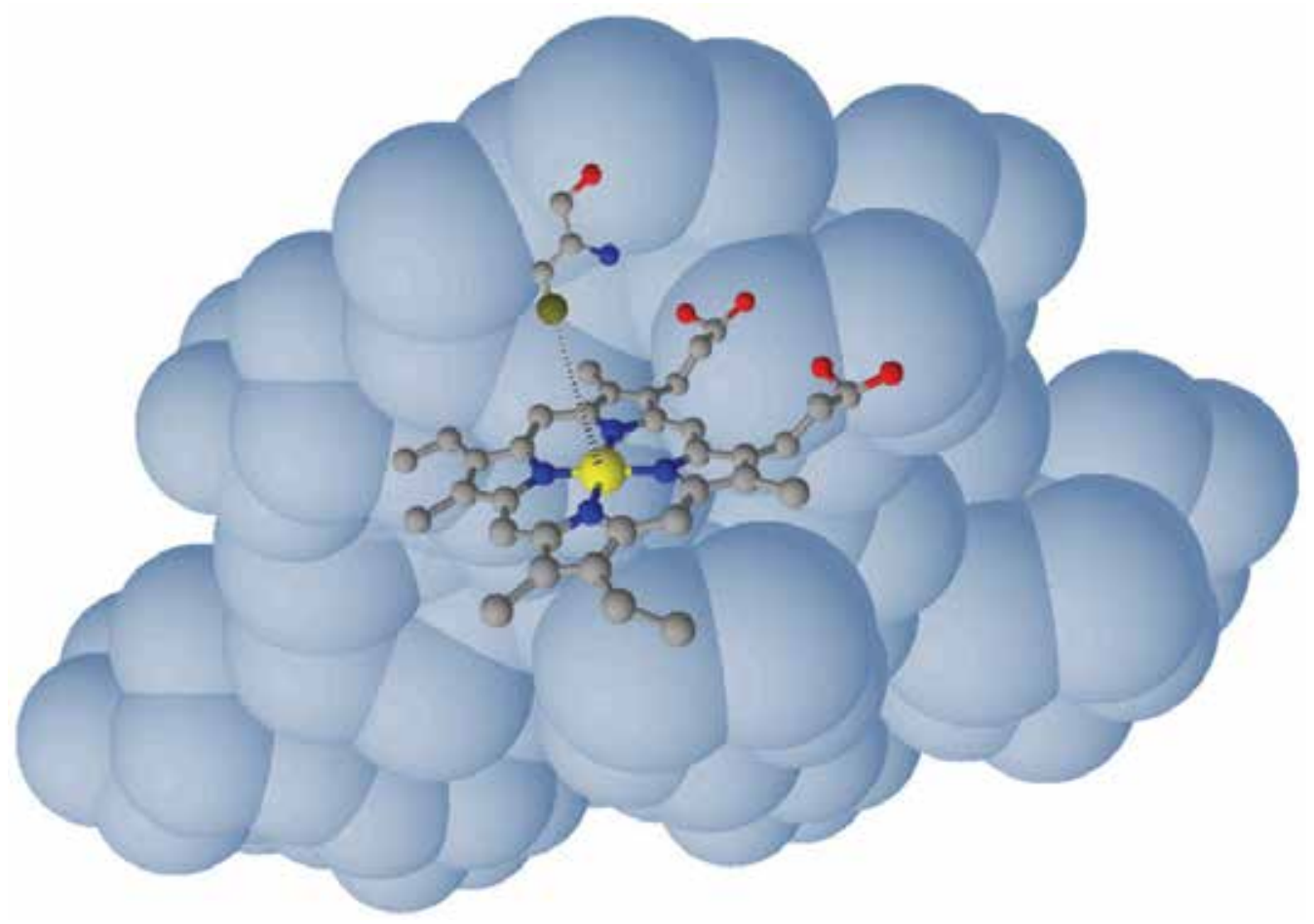

Fig. 1. Representative structure of cytochrome P450.

Siroká \&Drastichová, 2004; Mansuy, 2007; Munro et al., 2007; Lohmann \&Karst, 2008), a crucial step in the adaptation of living organisms to their always changing chemical environment (Mansuy, 2007). It is accepted that in the prehistoric organisms one function of the P450 enzymes was the hydroxylation of organic substrates, so that the oxidized products could be employed as energy source (McMurry \& Groves,1986; Lewis, 2001; Siroká \&Drastichová, 2004; Mansuy, 2007; Munro et al.,2007; Lohmann \&Karst, 2008).

Usually, the exposure of an organism to xenobiotics implies biological responses, generally in the form of biotransformation of the pharmacological or toxic substance, which generally depends on the conversion of the absorbed compound into an active metabolite or not, with a view to its elimination. During the biotransformation, a lipid-soluble xenobiotic or endobiotic compound is enzymatically transformed into polar, water-soluble, and excretable metabolites. The metabolic products are often less active than the parent drug or even inactive. However, some biotransformation products (metabolites) may have enhanced activity or toxic effects compared with the initial compound. A key enzymatic system that determines the body's ability to deal with drugs and chemicals is represented by P450 (Meyer, 1996).

Briefly, drugs and other xenobiotics are transformed via multiple reactions in two distinct stages, namely phase I and phase II reactions. Phase I reactions are regarded as being responsible for preparing the drug for phase II reactions. Phase II reactions are usually the true "detoxification" pathways, leading to compounds that account for most of the inactive, excreted drug products (Tanaka, 2001).

In plant organisms, the production of some significant secondary metabolites, such as lignin, terpenoids, steroids, essential oils, and opioid precursors (Lohmann \&Karst, 2008), which 
are essential for the production of some drugs for use in humans, is also based on cytochrome functions (Lewis, 2001).

The P450s react with one oxygen atom from distinct oxidants such as dioxygen $\left(\mathrm{O}_{2}\right)$, forming the active oxidant species, the so-called metal-oxo complex (high-valent iron(IV)oxo intermediate, $\left.\mathrm{Fe}^{\mathrm{IV}}(\mathrm{O}) \mathrm{P}^{+}\right)$, which is able to transfer the oxygen atom to several substrates like alkanes, alkenes, aromatic compounds and amines, among others. This active species is also responsible for the cleavage of C-C bonds in organic substrates. In the mechanism called "rebound mechanism", the hydrogen abstraction from substrates such as alkanes (R$\mathrm{H})$ by the $\mathrm{Fe}^{\mathrm{IV}}(\mathrm{O}) \mathrm{P}^{+}$species takes place, producing an alkyl radical $\mathrm{R}$. and an iron(III)hydroxo complex as intermediates. This is followed by the caged alkyl radical rebound to the hydroxyl group, generating the alcohol (Lewis, 2001; Mansuy, 2007). (Figure 2).

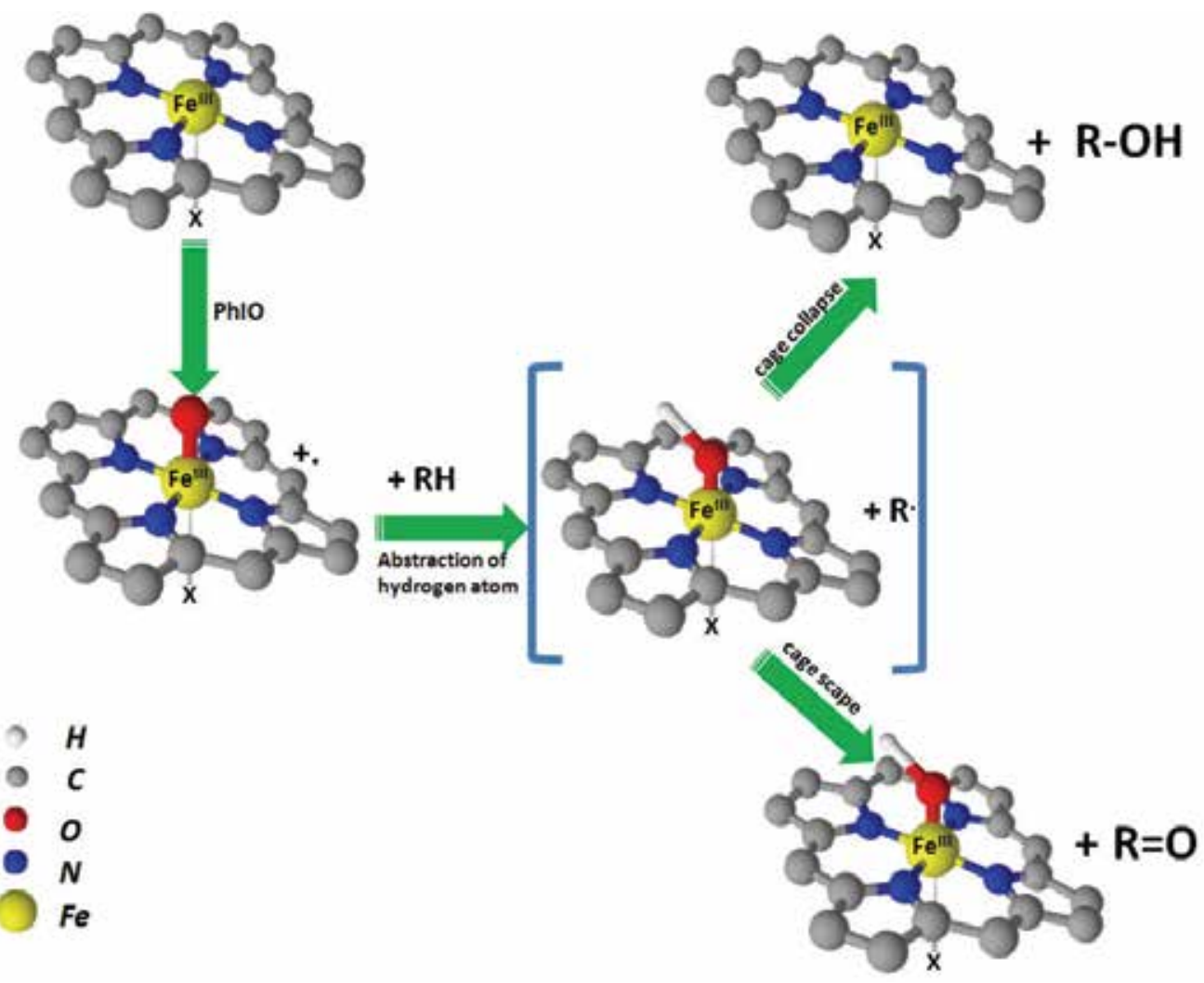

Fig. 2. Rebound mechanism of metalloporphyrin-catalyzed hydroxilations

The key feature of P450 enzymes is their ability to perform this reaction selectively, under mild conditions, by monooxygenation of the substrate (McMurry \& Groves,1986; Lewis, 2001; Siroká \& Drastichová, 2004; Mansuy, 2007; Munro et al.,2007; Lohmann \&Karst, 2008)

\section{Cytochromes P450 models}

The development of research on P450s has largely paralleled that on drug metabolism, and there are strong connections between these two areas (Gibson \& Skett, 1994). 
Indeed, it is very important to know and follow the mechanisms organisms undergo after exposure to a xenobiotic. Deeper understanding of the catalytic reactions carried out by enzymes, especially with respect to the nature of the active oxidizing species, has recently been achieved thank to studies on enzyme models (Bernardou \&Meunier, 2004; Nam, 2007; Lohmann \&Karst, 2008), particularly synthetic enzymes.

By means of synthetic models, it is possible to quickly predict information about the biotransformation of a drug during the search for and development of new therapeutic agents, which in turn enables prediction of their toxic effect (Lewis, 2001; Mansuy, 2007). Furthermore, the use of enzyme models diminishes animal testing, not to mention the fact that problems related to the isolation of natural enzymes and cells are also eliminated (Bernardou \&Meunier, 2004).

Enzymatic biological models have been widely employed throughout all the phases of drug design and development. In vivo oxidations carried out by enzymes like P450s can be easily mimicked under mild conditions by using synthetic metalloporphyrin systems. More specifically, ironporphyrins and manganeseporphyrins have been utilized as P450 models due to their ability to catalyze countless organic reactions (Bernardou et al., 1991).

Several porphyrin systems have been reported in the literature, and over the years three main generations of these catalysts have been prepared (Mansuy, 2007). Figure 3 shows some structures, representing the three generations of porphyrins. Ironporphyrins, in particular, have been more employed as models because they are capable of catalyzing organic oxidations pretty similarly to the biological enzyme.

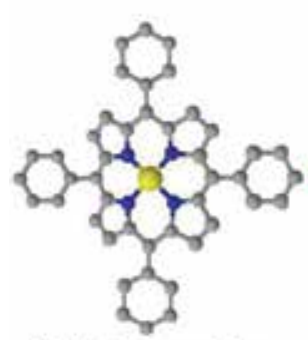

(a) 1st generation

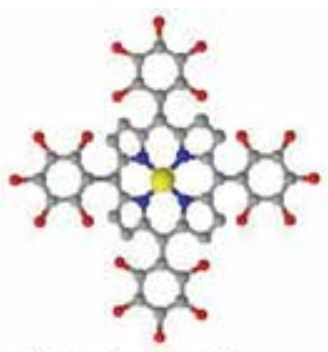

(b) 2nd generation

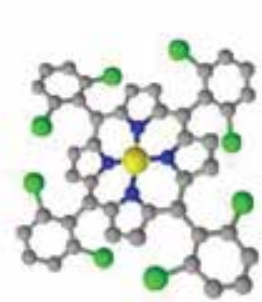

(c) 2nd generation

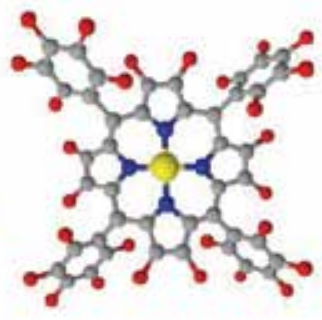

(d) 3th generation

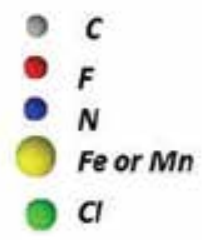

Fig. 3. Strucutral formula of several metalloporphyrins: (a) meso-tetrakisphenylporphinato iron III (FeTPP), meso tetrakisphenylporphinato manganese III (MnTPP), (b) meso tetrakis(pentafluorophenyl)porphinato iron III (FeTFPP), tetrakis(pentafluorophenyl)porphinato manganse III (MnTFPP), (c) meso-Tetrakis( 2,6dichlorophenyl)porphinato iron III, (d) $\beta$-octa- fluoro-mesotetrakis(pentafluorophenyl)porphinato iron III.

Besides their biological and catalytic properties, synthetic metalloporphyrins can also be immobilized onto organic polymers as well as amorphous and crystalline inorganic materials, such as silica, alumina, and clays (Figure 4). 


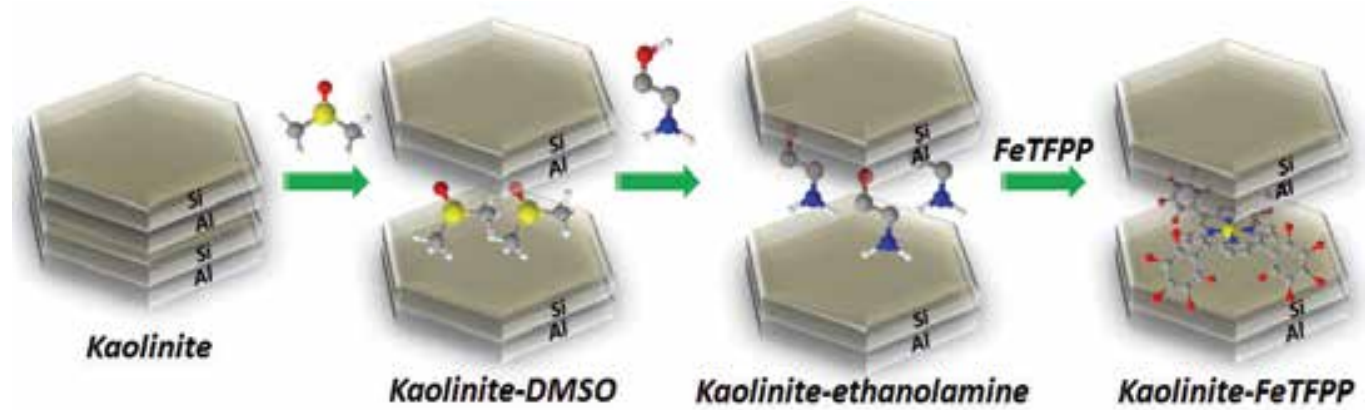

Fig. 4. Kaolinite functionalized with a second-generation of metalloporphyrin, Fe(TPFPP) [adapted from (Bizaia et al.,2009) ]

The immobilization of metalloporphyrins onto inorganic supports has been found to yield efficient, selective catalysts for the oxidation of oxidizable groups, promoting a special environment for the approach of the substrate to the catalytically active species, thus mimicking the "site-isolation principle" of biological enzymes (Lewis, 2001; Mansuy, 2007). The sol-gel process is considered a practical methodology for the preparation of heterogeneous ironporphyrin catalysts. We ( de Lima et al., 2001; de Oliveira, et al., 2001; Sacco et al.; 2001; Bizaia et al., 2009; de Faria et al., 2004 ; Mac Leod et al., 2006 ; Machado et al., 2009) have presented interesting results regarding the efficiency of ironporphyrin catalysts supported on silica or alumina (Figure 5).

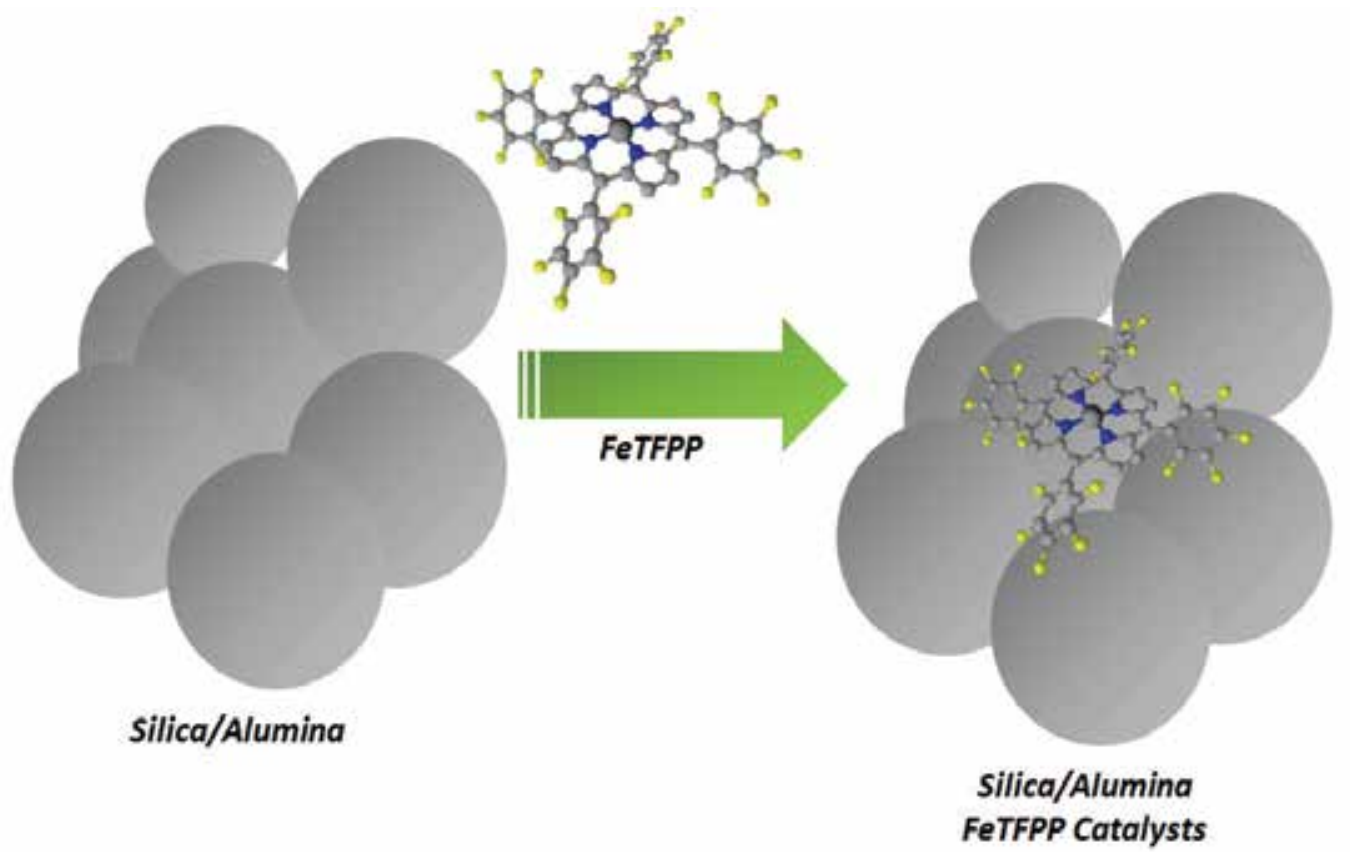

Fig. 5. Porphyrins entrapped in an alumina matrix by non-hydrolitic sol-gel process [adapted from (Lima et al., 2001). 
Synthetic metalloporphyrins also have potential application in the design of green processes for the accomplishment of many kinds of oxidations that mimic the P450 reactions.

Since the pioneering work of Groves (Groves et al., 1979), synthetic metalloporphyrins have been used as biomimetic catalysts of a multitude of reactions, mainly of oxidations of satured and unsaturated hydrocarbons (Mansuy, 2007). Over the last years, the great versatility of these biomimetic metalloporphyrins has been extended to the oxidation of countless substrates, thus generating important molecules for application in fine chemistry and in the pharmaceutical industry. Important examples of such reactions are selective DNA cleavage, oxidation of pesticides and lignins, and oxidation of chlorinated aromatic compounds (Mansuy, 2007).

During the last decade, the development of synthetic systems mimicking the activity of cytochrome P450 by using metalloporphyrin catalysts and various oxygen atom donors has been reported. Some catalytic oxidation reactions described in the literature are summarized in Table 1, but this is not an overview from the literature data. A major review can be found in references (Lewis, 2001; Bernardou \&Meunier, 2004; Mansuy, 2007; Lohmann \&Karst, 2008).

Table 1 gives some examples of the use of these model systems in the the metabolism of xenobiotics. It is noteworthy that first- and second-generation ironporphyrins are more often employed as catalysts, using Iodosylbenzene (PhIO), $\mathrm{NaOCl}$, metachloroperoxybenzoic acid (m-CPBA), or $\mathrm{O}_{2} / \mathrm{Pt}$-colloid as oxygen donor.

It is also worthy of note that synthetic metalloporphyrin catalysts are also very interesting for application in sophisticated organic synthesis and high-value products, such as fine chemicals. Apart from studies on xenobiotics, these catalysts can also be employed to promote chemical transformations that can potentially replace expensive industrial processes and which normally would not lead to the same product selectivity achieved by means of synthetic metallorporphyrins or biological systems.

It is always preferable to use oxidants containing only one oxygen atom when the study of the reaction model is concerned. Iodosylbenzene, for instance, is a widely employed oxidant in the academic field. This polymeric solid does not contain a weak $\mathrm{O}-\mathrm{H}$ bond, thus eliminating the occurrence of free radical chain reactions normally initiated by oxidants like alkyl hydroperoxides R-O-O-H (Groves, 2006). However, this oxidant is expensive and hazardous. In fact, while iodosylbenzene is the preferred oxidant in studies related to oxidative metabolism, because it is very good for predicting drug metabolism, it is no longer indicated for research on bio or chemotransformation.

Over the last decade the search for efficient and environmentally friendly oxidation procedures that could be used to develop green processes for many kinds of oxidation reactions has been intensified. Also, because the highly selective oxidation of organic compounds is of extreme importance in synthetic chemistry, many attempts have been made in order to oxidize compounds and produce target components that cannot be easily obtained by conventional routes.

A very interesting and promising field for the application of metalloporphyrin catalysts lies in the area of natural product oxidation, with potential use in the discovery of new drugs for utilization in clinical practice. This could be achieved by employing clean oxidants like hydrogen peroxide or molecular oxygen. Nevertheless, although dioxygen is considered an ideal oxidant, it is difficult to use this very reactive compound because it is hard to control reaction selectivity, not to mention the security issues related to this gas. 


\begin{tabular}{|c|c|c|c|c|c|}
\hline Porphyrin & Matrix & Substrate & Reaction & Oxidant & References \\
\hline $\begin{array}{l}{[\mathrm{Fe}(\mathrm{TPFPP})]} \\
{[\mathrm{Fe}(\mathrm{TDFSPP})]} \\
{[\mathrm{Fe}(\mathrm{TCFSPP})]}\end{array}$ & $\begin{array}{l}\text { Homogeneous } \\
\text { Kaolinite } \\
\text { Si-APTS } \\
\text { Chrys } \\
\end{array}$ & $\begin{array}{l}\text { Cyclohexane } \\
\text { Heptane }\end{array}$ & \multirow{5}{*}{ Oxidation } & PhIO & $\begin{array}{l}\text { Nakagaki } \\
\& \\
\text { Wypych, } \\
2007\end{array}$ \\
\hline $\mathrm{Mn}(\mathrm{TDCPP}) \mathrm{Cl}$ & \multirow{8}{*}{ Homogeneous } & $\begin{array}{l}\text { Cyclooctene } \\
\text { Non-1-ene } \\
\text { cis-stilbene } \\
\text { Cyclohexane }\end{array}$ & & $\mathrm{H}_{2} \mathrm{O}_{2}$ & $\begin{array}{l}\text { Thellend } \\
\text { et al., } 1994\end{array}$ \\
\hline $\begin{array}{l}{[\mathrm{Fe}(\mathrm{TPP})] \mathrm{Cl}} \\
{[\mathrm{Mn}(\mathrm{TPP})] \mathrm{Cl}} \\
{[\mathrm{Fe}(\mathrm{TFPP})] \mathrm{Cl}} \\
{[\mathrm{Mn}(\mathrm{TFPP})] \mathrm{Cl}}\end{array}$ & & $\begin{array}{l}\text { Piperine } \\
\text { Piplartine }\end{array}$ & & PhIO & $\begin{array}{l}\text { Schaab et } \\
\text { al., } 2010\end{array}$ \\
\hline $\begin{array}{l}\text { Mn-TMPyP } \\
\text { Fe-TMPyP }\end{array}$ & & DNA & & $\begin{array}{l}\mathrm{O}_{2} \\
\mathrm{~K}_{2} \mathrm{O}\end{array}$ & $\begin{array}{l}\text { Pitié et al., } \\
2006\end{array}$ \\
\hline $\begin{array}{l}\mathrm{Fe}(\mathrm{TCPP}) \mathrm{Cl} \\
\mathrm{Mn}(\mathrm{TCPP}) \mathrm{Cl} \\
\mathrm{Mn}(\mathrm{TFPP}) \mathrm{Cl}\end{array}$ & & Primidone & & $\begin{array}{l}t- \\
\mathrm{BOOH}\end{array}$ & $\begin{array}{l}\text { Mac Leod } \\
\text { et al., } 2008\end{array}$ \\
\hline $\begin{array}{l}\text { Horseradish- } \\
\text { peroxidase }\end{array}$ & & Chlorophyll & \multirow{3}{*}{ Degradation } & & $\begin{array}{l}\text { Hynninen } \\
\text { et al., } 2010 \\
\end{array}$ \\
\hline $\begin{array}{l}{\left[\mathrm{Fe}\left(\mathrm{TPPF}_{20}\right)\right]} \\
{\left[\mathrm{Fe}\left(\mathrm{TPPCl}_{8}\right)\right]} \\
{\left[\mathrm{Fe}\left(\mathrm{TPPCl}_{8} \beta-\mathrm{Br}_{8}\right)\right]}\end{array}$ & & $\begin{array}{l}\text { Fenitrothion } \\
\text { Cyanophos } \\
\text { Tolclofos- } \\
\text { methyl } \\
\text { butamifos } \\
\text { Fenthion } \\
\end{array}$ & & $\mathrm{H}_{2} \mathrm{O}_{2}$ & $\begin{array}{l}\text { Fujisawa } \\
\& \text { Katagi, } \\
2005\end{array}$ \\
\hline $\begin{array}{ll}\beta-\quad \text { and } & \text { meso- } \\
\text { substituted } & \text { iron- } \\
\text { porphyrins } & \\
\end{array}$ & & $\begin{array}{l}\text { Permethrin } \\
\text { Fenvalerate }\end{array}$ & & $\begin{array}{l}\mathrm{H}_{2} \mathrm{O}_{2} \\
m \mathrm{CPBA}\end{array}$ & \begin{tabular}{|l} 
Fujisawa \\
$\& \quad$ Katagi, \\
2009 \\
\end{tabular} \\
\hline Gold(III)porphyrin & & Carcinomas & $\begin{array}{l}\text { Anticancer } \\
\text { activity }\end{array}$ & --- & $\begin{array}{l}\text { van Rijt \& } \\
\text { Sadler, } \\
2009\end{array}$ \\
\hline
\end{tabular}

Table 1. Examples of model systems based on P450 in the metabolism of xenobiotics.

It is widely accepted that hydrogen peroxide is another ideal oxidant because of its high active oxygen content, availability, and non-toxicity. Moreover, it is considered to be nonpolluting, since it produces only water as product (Goti \& Cardona, 2008). Oxidations with hydrogen peroxide are highly atom-economic. Furthermore, hydrogen peroxide is a safe, readily available, cheap reagent.

Although the oxidation of natural products by synthetic ironorphyrins is a new area, it is a very promising field because it is well established that the chemical transformation of abundant and cheap natural products can make other more valuable compounds with interesting biological activities available. 


\section{Synthetic metalloporphyrins in the oxidation of plants}

Natural products, especially medicinal plants, have great importance for the development of new drugs used in human and animal medicines. According to Rates (Rates, 2001) about $25 \%$ of the drugs prescribed worldwide are sourced from natural plants, and of the 252 drugs considered as basic and essential by the World Health Organization (WHO), $11 \%$ are exclusively originated from plants. In addition, many synthetic drugs have been and are being obtained from natural precursors, since they represent a valuable alternative for the development of new medicines (Elisabetsky et al.,1995; Balunas \& Kinghorn, 20005). Examples of important drugs obtained from plants are digoxin (obtained from Digitalis sp. and used in heart failure) and taxol (obtained from Taxus breviola and used as antitumor). Thus, medicinal plants are an important source of new chemical substances with potential therapeutic applicability.

Concerning the production of active compounds, the diversity of the plant kingdom represents an extraordinary reserve of new molecules, but only a small percentage of plant species in the world have been investigated from a chemical viewpoint, and the biological and pharmacological screenings of their constituents are even scarcer (Cordell, 2000).

Pharmacological research on the active principles of medicinal plants has provided important advances in the therapeutics of many diseases, and various substances found in plants have been used as useful tools in pharmacological, physiological, and biochemical studies (Souza Brito, 1996; Rates, 2001).

The transformation of abundant natural products can transform them into economically valuable compounds, boosting their application in several areas of human heath. One interesting review can be found in the reference (Simões et al., 2009). In this subject area some interesting research has been carried out on essential oils from plants. Terpenoids, which are widely distributed in nature, play an important role as fragrance, flavoring, and medicaments for the treatment of various diseases. Their chemical transformation by means of oxidation with several oxidants using synthetic metalloporpyrin as biomimetic catalysts of P450s has been reported in the literature (Toshiro et al., 1999). Konoike et al. (Konoibe et al., 1999) have described the use of the ironporpyrin (FeTFPP, Figure 3)/metachloroperbenzoic acid system in the allylic hydroxylation of position 11 of triterpenes bearing a sterically hindered olefin. This oxidation position was obtained with the oleanolic acid, ursolic acid, and dihydrolanosterol, and their derivatives were efficiently $(67 \%)$ converted to the corresponding allylic alcohols. Despite the high conversion rate achieved with the homogeneous catalytic system, the reaction presented some drawbacks, such as the employed temperature $-78^{\circ} \mathrm{C}$, and no possibility of catalyst reuse, since in fact no reuse of the homogeneous catalyst was mentioned.

The paper by Rosália et al. (Rosália et al., 1999) has described oxidation of the monoterpenes: carvacrol, thymol, and p-cymene, Figure 6, using manganseporphyrins (MnTDCPP and MnTFPP), Figure 3, /hydrogen peroxide as catalyst/oxidant. The oxidation of carvacrol and thymol selectively produced the thymoquinone, a high-value product compared with the starting reagents, because it has anticancer, anti-inflammatory, and hepatoprotective effects (Ravindran et al., 2010). The homogeneous manganese catalyst /hydrogen peroxide systems were very efficient for the production of thymoquinone, but no catalyst reuse was mentioned.

Millos (Milos, 2001) has reported that good thymoquinone production, Figure 6, $(\sim 60.0 \%)$ was achieved by oxidizing the essential oil of oregano with the oxidant potassium 


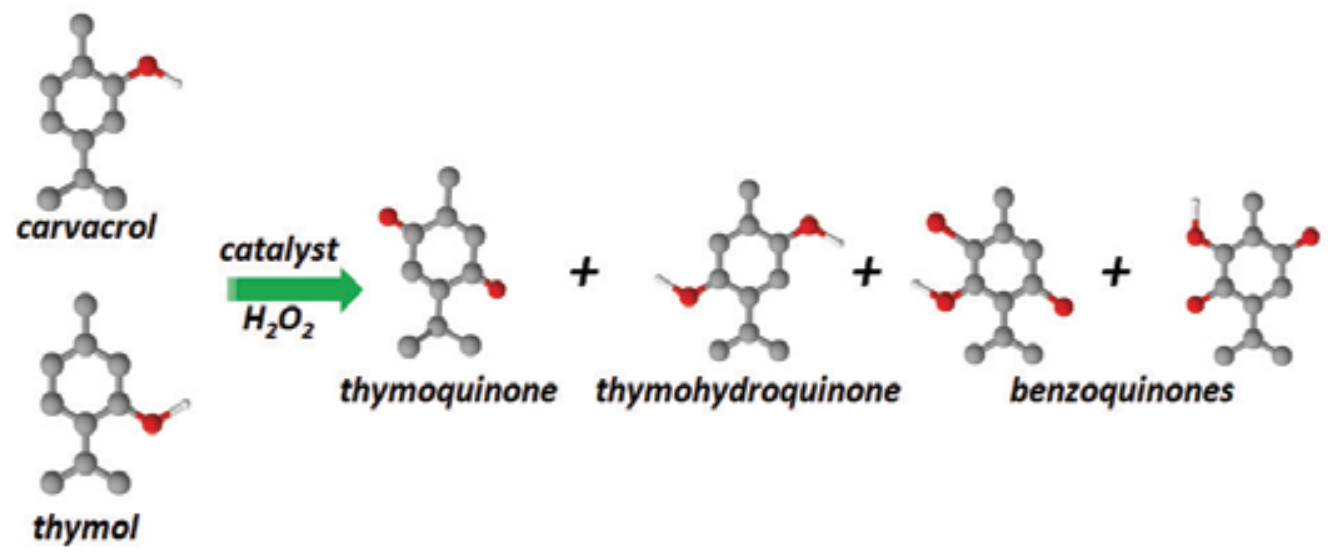

Fig. 6. Schematic representation of carvacrol and thymol oxidation to production of thymoquinone and possible byproducts.

monopersulfate, using an ironporphyrin (FeTPP, Figure 2) as biomimetic catalyst. The great differential in this work (Milos, 2001) is that, contrary to Rosália et al. (Rosália et al., 1999), Millo et al. worked directly with the oregano essential oil isolated from dried plant material, whereas Rosália worked with commercially available reagents.

Another major accomplishment of Milos's work (Milos, 2001) is the fact that a firstgeneration ironporphyrin, which is usually cheaper and easier to synthesize, was employed. However, this ironporphyrin can be easily destroyed under drastic reaction conditions, which would impair catalyst reuse.

Cavaleiro et al .(Cavaleiro, et al.,1996) have been one of the first to report the processing of 1 , 8-cyneole, Figure 7, one of the major components of the essential oils of Eucalyptus globules, using the clean oxidant, hydrogen peroxide. Although this oil is abundant, it is inert and had little economic significance. The oxidation of cyneole by a manganseporphyrin (MnTDCPP or MnTFPP, Figure 3)/hydrogen peroxide system generated oxygenated products with high added value. The homogeneous catalysts were very efficient, and the use of a cocatalyst improved hydrogen peroxide efficiency, consequently allowing for higher substrate conversion using shorter reaction times and smaller amounts of $\mathrm{H}_{2} \mathrm{O}_{2}$, without loss of selectivity. No catalyst reuse was reported.

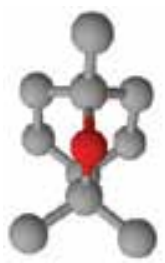

Fig. 7. Structure of 1,8-cyneole.

Another greatly important and largely available terpenoid is limonene, Figure 8, which is a source of a great variety of oxyfunctionalized derivatives. Some literature works have reported the chemical transformation of this substrate by manganesoporphyrins $/ \mathrm{H}_{2} \mathrm{O}_{2}$ systems (Skrobot et al., 2003). Metalloporphyrin systems have also been employed as efficient catalysts in the epoxidation of pinene to pinene oxide, Figure 8, which is a useful 
intermediate in the synthesis of several sandalwood fragrances (Maraval et al., 2002; Skrobot et al., 2003) have reported on excellent studies about this substrate using these systems.
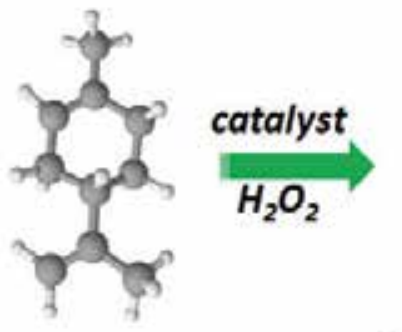

limoneneoxide

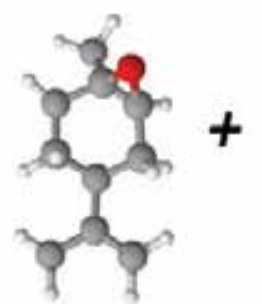

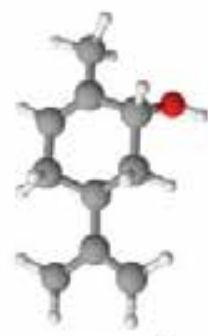

carveol

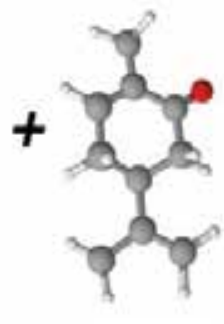

carvone

Fig. 8. Schematic representation of limonene oxyfunctionalization and possible derivatives: limoneneoxide, carveol and carvone

Skrobot et al. (Skrobot et al., 2003) have described the use of manganeseporphyrins (manganese(III) tetra(4-N-benzylpyridyl)porphyrin, in particular) as catalysts for the epoxidation of pinene and for the hydroxylation of carvacrol, Figure 6, using hydrogen peroxide as oxidant. The catalysts were used in either homogeneous solution or supported on $\mathrm{Y}$ zeolite. The results reported by the authors were very interesting, since the heterogeneous system gave similar results to those achieved with the homogeneous system. This was considered promising because it is normally expected that heterogenous catalysts will lead to reduced conversion rates, due to the difficult access of the substrate to the active site and diffusion of the products back into solution. However, despite the use of a clean oxidant, re-use of the heterogeneous catalyst prepared by Skrobot et al. (Skrobot et al., 2003) revealed loss of catalytic activity, attributed to leaching of the manganeseporphyrin from the zeolite.

Even though literature works have described several tests using metalloporphyrin models, we have verified that only a few works have tested the substrate directly extracted from plants. Recently Schaab et al. (Schaab et al., 2010) employed the system ironporphyrin/iodosylbenzene in the oxidation of the substrate piplartine, a potential anticancer plant (isolated from Piper tuberculatum). They showed that ironporphyrin is a good catalyst for prediction of drug metabolism by P450. Nevertheless, most studies were accomplished using standardized, commercially available compounds. Moreover, the oxidant of choice in the majority of the studies is iodosylbenzene, which is very good for predicting drug metabolism, but is no longer indicated for studies on bio or chemical transformation of substances for medicinal uses. Also, many works have employed homogeneous catalysts and there is no mention of catalyst reuse, probably because of the relative difficulty in isolating it from the reaction medium.

Still, among the works reported above involving the chemical transformation of plants, it can be noted that most papers focus on studies of terpenoids and their derivatives. Thus, increasing the research on the oxidation of active ingredients from other medicinal plants and establishing larger association between chemistry and pharmacology are still necessary, in view of the possibility of discovering new pharmacologically active chemicals. Lignans are among the chemicals produced by plants that deserve special interest and therefore must be chemically and pharmacologically exploited. 


\section{Oxidation of lignans by ironporphyrins}

Lignoid term is a generic name, which features a group of secondary metabolites whose skeleton is formed exclusively by the group phenylpropanoid (C3-C6) $n$, where $n$ is restricted to a few units (Ward,1999). Because of the wide diversity of occurrence, the broad spectrum of biological activities, and the important role they play in plant development, the lignoids have been the target of several studies (MacRae \& Towers, 1984; Saleem et al., 2005). Among them, there are lignans and neolignans. Lignans are dimers derived from condensation by oxidative coupling of cinnamyl alcohols with each other or with cinnamic acids, whose carbon $\gamma$ (C3) of side chain is oxidized (Ward, 1999; Gottlieb \& Yoshida, 1989). Thus, lignans are biosynthesized by dimerization, via oxidative coupling of phenylpropanoids units (C6C3), forming a variety of structurally distinct subclasses. An example of these substances is cubebin (Figure 9a), a dibenzylbutyrolactone lignan obtained from the seeds of Piper cubeba (Piperaceae), which has anti-inflammatory (Souza et al., 2004; Silva et al.,2005); and trypanocidal (Bastos et al., 2001) activities.

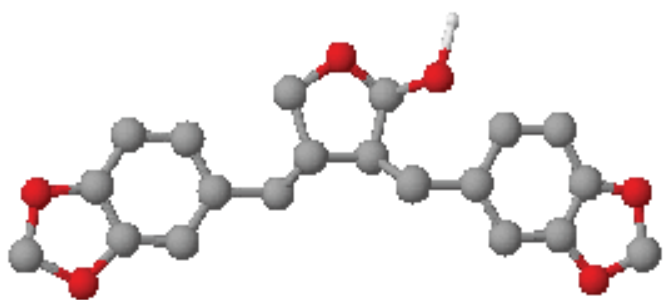

(a)

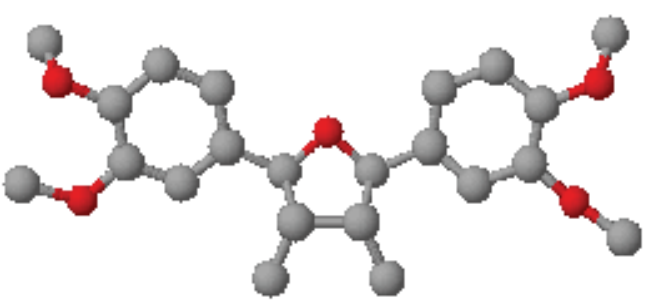

(b)

Fig. 9. Structure of cubebin (a) and veraguensin (b).

Neolignans are dimers derived from condensation by oxidative coupling of allyl and /or propenyl phenols and, unlike lignans, are free of oxidation in the $\gamma$ carbon atom. An example of this class is veraguensin (Figure 9b), a tetrahydrofuran neolignan found in Nectandra megapotamica (Lauraceae), which has anti-inflammatory (Da Silva Filho et al., 2004 a) trypanocidal (Lopes, et al. 1998; Da Silva Filho et al., 2004), properties.

In general, the biological and commercial importance of these compounds can be represented by derivatives of podophyllotoxin (Figure 10), a lignan obtained from Podophyllum peltatum (Berberidaceae) and used in the treatment of cancer (Bastos et al.,1996).

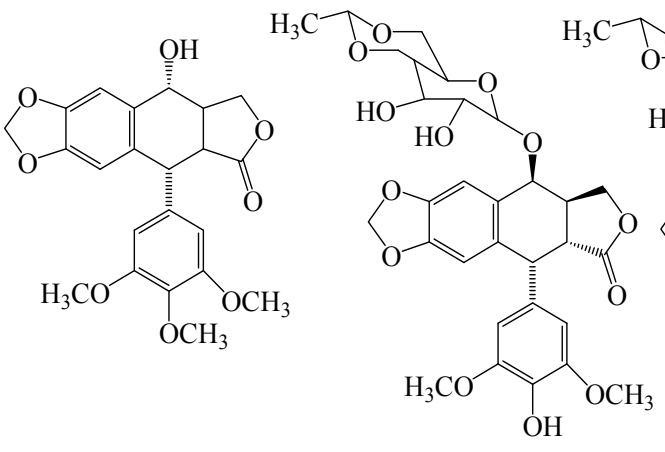

(a) (b)

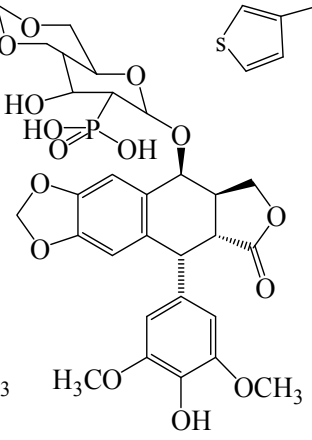

(c)

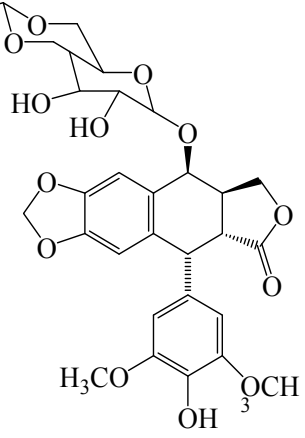

(d)

Fig. 10. Chemical strucutures of podophyllotoxin (a), etoposide (b), etopophos (c) e teniposide (d). 
Actually, lignans have interesting biological properties, and useful drugs have been developed from the well-known lignans podophyllotoxin and steganacin (of the phenyltetraline and dibenzocyclooctane classes, respectively) for the treatment of cancer as well as other ailments. Crude plant materials containing lignans have long been used in folk medicine (Rehnberg \& Magnusson, 1990).

Another major focus of the search for active natural products has been the antiparasitic activities of compounds, especially the schistosomicidal , trypanocidal (De Souza et al., 2005), and leishmanicidal (Barata et al., 2000) activities of these substances. Such activities are respectively represented by methylcubebin (Figure 11a), veraguensin (Figure 9b), and surinamensin (Figure 11b), showing that lignans and neolignans prototypes are promising for the development of new antiparasitic drugs.

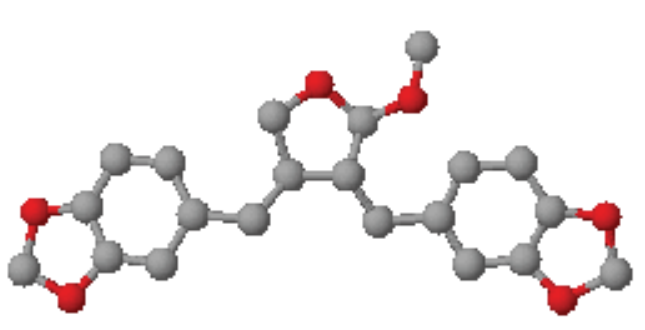

(a)

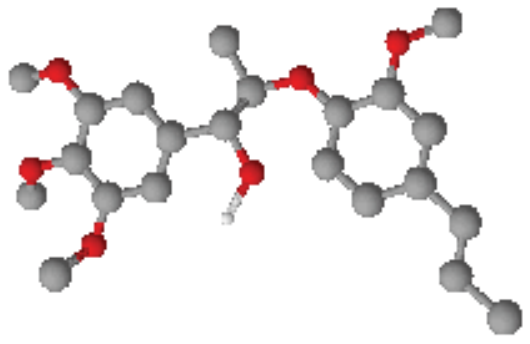

(b)

Fig. 11. Structure of methylcubebin (a) and surinamensin (b).

On account of the biological activities of lignans, including their anticancer, antitumoral, trypanocidal, antimicrobial, and anti-inflammatory activities, some alternatives that will promote and improve their production are necessary.

(-)-Hinokinin, Figure 12, a dibenzylbutyrolactone lignan, possesses antileishmanial, antiinflammatory, antigenotoxic (Souza et al.,2004 ; Da Silva et al.,2005 ; Medola et al., 2007), and significant in vitro and in vivo trypanocidal activities against Trypanosoma cruzi, the etiologic agent of Chagas` disease (Saraiva et al., 2007). Considering its promising biological activities, as well as its potential use as trypanocidal drug, large quantities of (-)-hinokinin are needed. (-)-Hinokinin and (-)-cubebin co-occur in $P$. cubeba, where they are among the major components of the biomass (Souza et al., 2004; Silva et al., 2007). In a traditional process, the oxidation of (-)-cubebin, catalyzed by pyridinium chlorochromate, PCC, ( De Souza et al., 2005) can produce additional quantities of (-)-hinokinin. However, PCC is toxic and contains chromium (VI), a potential carcinogen. Besides its direct toxicity, the use of heavy metals (chromium) is a potential environmental problem to both water and soil qualities, and consequently to plant, animal, and human life (Barros et al., 2007).

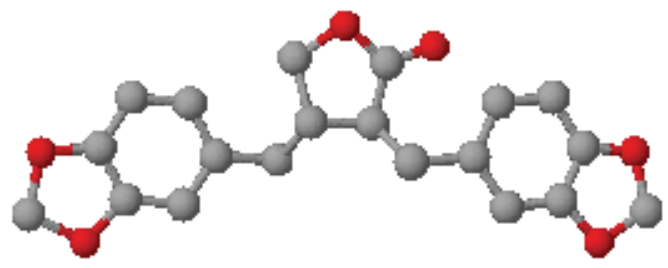

Fig. 12. Chemical strucuture of (-)-Hinokinin 
As part of our work on oxidative catalytic systems involving ironporphyrins (Papacídero et al., 2006), as well as of our studies on the synthesis and biological activities of lignans (Bastos, et al., 1996; Da Silva Filho et al., 2004; Souza et al., 2005) our group has developed a synthetic method for production of (-)-hinokinin consisting of the oxidation of (-)- cubebin catalyzed by biomimetic heterogeneous metalloporphyrin catalytic systems. Cubebin was isolated by us directly from Piper cubeba. (Powdered seeds of Piper cubeba L., bought from the market, were exhaustively extracted by maceration with $96 \%$ ethanol. The concentrated crude extract was partitioned between the hexane and methanol/water (9:1) phases and purified by liquid chromatography and crystallization).

The genus Piper has over 700 species distributed in both hemispheres. Piper cubeba, belonging to the Piperaceae family, is one of the folkloric plants used as a spice in many countries, including Indonesia, India, Europe in the middle ages, and Morocco. It has also been used for the treatment of dysentery, syphilis, abdominal, pain, diarrhea, enteritis, and asthma (Usia et al., 2005). P. cubeba has been investigated by our research group, too, aiming to evaluate the biological activities of extracts, isolated compounds, and dibenzylbutyrolactone semi-synthetic derivatives.

Similarly to what was reported previously by our group in the case of the epoxidation of alkenes ( De Lima et al., 2001), the immobilization of ironporphyrin on alumina prepared by the non-hydrolytic sol-gel route (NHG) is a very interesting and efficient method for the construction of selective catalysts for cubebin oxidation. The ironporphyrin was efficiently supported on the matrix and it did not leach from the support in the employed reaction conditions.

The oxidation of (-)-cubebin (a), catalyzed by the heterogeneous FeTFPP-NHG system, was performed with two oxidants, namely iodosylbenzene or $\mathrm{H}_{2} \mathrm{O}_{2}$, at atmospheric pressure and room temperature (Figure 13).

(a)

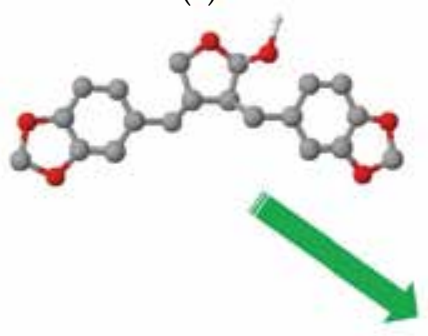

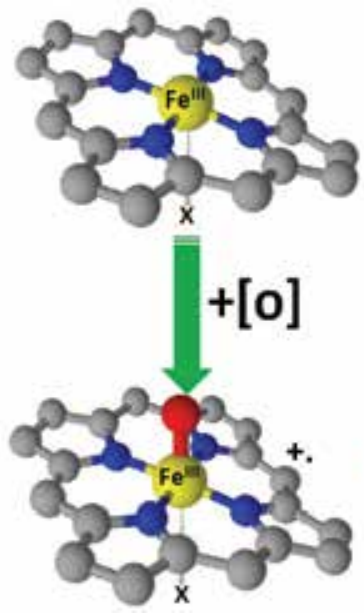

(b)

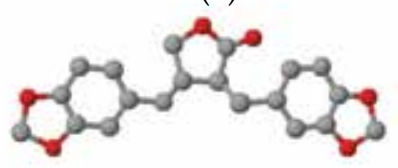

Fig. 13. Schematic representation of cubebin oxidation to (-)-hinokinin catalyzed by ironporphyrin.

The oxidation of (-)-cubebin was efficiently catalyzed in the case of both oxidants, leading to the sole production of (-)-hinokinin (b). Higher yields of this product and $100 \%$ selectivity were obtained when PhIO was used as the oxygen donor, compared with $\mathrm{H}_{2} \mathrm{O}_{2}(70 \%)$ (Figure 13). It is noteworthy that the catalytic activity of the FeTFPP- 
NHG/acetonitrile/PhIO system was similar to the oxidizing activity of PCC (98\% hinokinin yield). Considering that the formation of (-)-hinokinin catalyzed by ironporphyrins was performed under mild and environmentally-friendly conditions, the results obtained here are better than those achieved using PCC. These results are quite interesting and, to our knowledge, there are no similar works reported in the literature.

The great advantage of the heterogeneous catalyst is its reuse, and the high efficiency and stability of a catalytic system can be confirmed via catalyst reuse. To this end, the solid catalysts were separated from the reaction mixture after each experiment by simple filtration and dried before being used in a subsequent run. In the case of our system, the catalyst was reused in five consecutive runs, without loss in terms of selectivity. So it can be claimed that the non-hydrolytic sol-gel method allowed for contruction of an economically viable catalyst that can be successfully reused. SEM techniques revealed that catalyst structure was preserved after the five reuse experiments, a clear indication of the stability and robustness of the catalytic system prepared by the non-hydrolytic sol gel process.

The results obtained here give strong evidence that the active catalytic species responsible for the oxidation of cubebin is the metal-oxo complex (high-valent iron(IV)-oxo intermediate, $\left.\mathrm{Fe}^{\mathrm{IV}}(\mathrm{O}) \mathrm{P}^{++}\right)$, so it can be said that an efficient catalyst bioinspired on $\mathrm{P} 450$ s was built.

Licarin-A, a neolignan obtained from the oxidative coupling of isoeugenol, displays significant antiparasitic activity against the adult forms of Schistosoma mansoni.

Taking into account the antiparasitic potential and the possible production of derivatives from licarin-A, such as (-)-hinokinin, a dibenzylbutyrolactone lignan, there is great interest in the search for new approaches that will enable the oxidation of these lignans to be carried out, since the oxidized products are probably biologically active.

Our group has also examined the oxidation of licarina-A, Figure 14, by a system bioinspired on the P450s, more specifically the second-generation ironporphyrin FeTFPP+ immobilized on silica and the oxidants iodosylbenzene, or hydrogen peroxide.

The licarin-A was obtained by oxidative coupling, using (E)-isoeugenol (10.5 mmol), dissolved in methanol $(50 \mathrm{~mL})$, citrate-phosphate buffer $(450 \mathrm{~mL}, 20 \mathrm{mM}, \mathrm{pH} 3)$ and horseradish peroxidase (HRP, $20 \mathrm{~mL}, 2500 \mathrm{U}$ ) (Nascimento et al., 2001). The mixture was stirred while $\mathrm{H}_{2} \mathrm{O}_{2} 30 \%(0.57 \mathrm{~mL})$ was added dropwise over $10 \mathrm{~min}$. Licarin-A (a white solid) was purified by column chromatography (silica gel $600.040-0.063 \mathrm{~mm}$ ) with hexaneethyl acetate $(8: 2 \mathrm{v} / \mathrm{v})$.

Initially, FeTFPP+ was covalently bound to the silica support by means of the sol-gel method, which consisted in reacting the metalloporphyrin with 3aminopropyltriethoxysilane (APTES), as previously described by Ciuffi et al (Ciuffi, et al., 2000). The oxidation of licarin-A catalyzed by silica-FeTFPP occurred in mild conditions, and the products were isolated by high performance liquid chromatography.

The ironporphyrin was efficiently supported on the matrix and did not leach from the support under the employed reaction conditions.

As in the case of cubebin, the oxidation of licarin A catalyzed by the heterogeneous FeTFPPsilica was performed by using one of the following oxidants: PhIO or $\mathrm{H}_{2} \mathrm{O}_{2}$, at atmospheric pressure and room temperature (Figure 5). Although the studies using the oxidant $\mathrm{H}_{2} \mathrm{O}_{2}$ are only preliminary, the oxidation was efficiently catalyzed in the case of both oxidants, but high selectivity was obtained only with iodosylbenzene (100\%), compared with 30\% selectivity achieved with $\mathrm{H}_{2} \mathrm{O}_{2}$, since in the latter case other products (alcohols) were also detected.

The loss of selectivity in the reactions carried out with hydrogen peroxide as oxidant could be a strong indication of the involvement of free radical mechanisms, a consequence of the 
homolytic cleavage of the peroxide. Therefore, as already done in an earlier work by our group, tests in the presence of radical trap were accomplished, since these traps would eliminate the occurrence of any free radical mechanisms in this type of reaction.

As in the case of cubebin oxidation, the results obtained for licarin A oxidation strongly suggest that the active catalytic species responsible for the oxidation is the metal-oxo complex (high-valent iron(IV)-oxo intermediate, $\mathrm{Fe}^{\mathrm{IV}}(\mathrm{O}) \mathrm{P}^{++}$) .

Because our studied on licarin A oxidation are in the initial stage, recyclability of the catalyst has not yet been investigated.

It is noteworthy that metalloporphyrin catalysts are able to oxidize the products directly extracted from plants or previously purified.

It is also worth mentioning that the experiments were repeated at least three times in the case of both substrates, and total reproducibility was achieved.

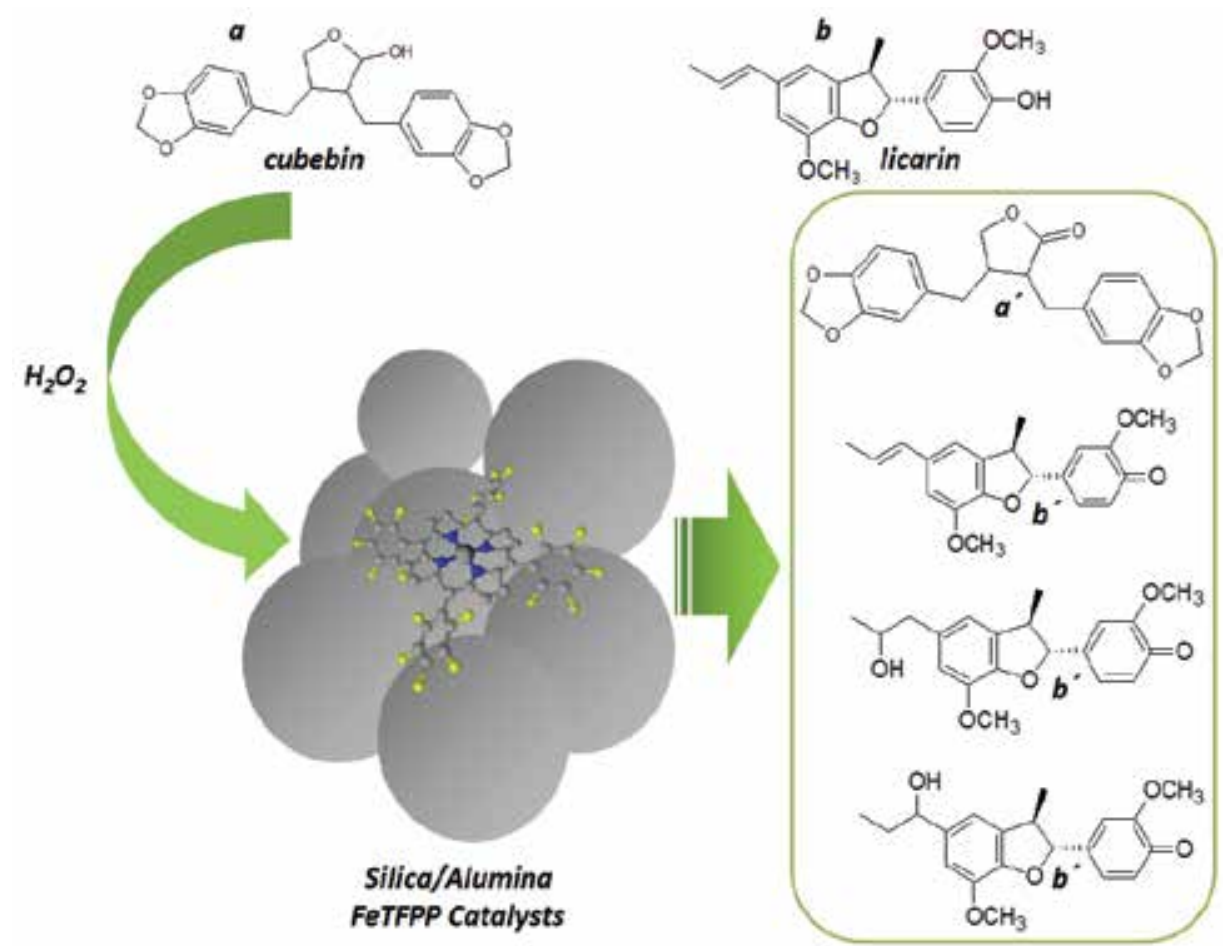

Fig. 14. Schematic representation of cubebin and licarin oxidation by iroporphyrin heterogeneous catalysts and possible products.

\section{Conclusions}

Natural products, especially those found in medicinal plants, have great importance for the development of new drugs for use in human and animal medicines.

On account of the biological activities of natural products, including anticancer, antitumoral, trypanocidal, antimicrobial, and anti-inflammatory activities, some alternatives that promote and improve their production are necessary and many attempts have been made in order to oxidize natural plant compounds and produce target components that are not easily obtained by conventional routes. 
The use of metalloporphyrin catalysts, biomimics of P450s, is very promising. However, a lot of work remains to be done regarding the use of the clean oxidant hydrogen peroxide and catalyst reuse. One approach to overcoming the latter difficulty is immobilization of the synthetic metalloporphyrins on solid supports.

\section{References}

Balunas, M. J. \& Kinghorn, A. D. (2005). Drug discovery from medicinal plants. Life Sciences, 78, 5, (2005) 431-441, 0024-3205.

Barata, L.E.S.; Santos, L.S.; Ferri, P.H.; Phillipson, J.D.; Paine, A. \& Croft, S.L. (2000). Antileishmanial activity of neolignans from Virola species and synthetic analogues. Phytochemistry 55,6,( 2000) 589-595, 0031-9422 .

Barros, A.J.M., Prasad, S., Leite, V.D. \& Souza, A.G. (2007). Biosorption of heavy metals in upflow sludge columns. Bioresource Technology, 98, 7, (2007)1418-1425, 0960-8524.

Bastos, J. K.; Burandt, C. L.; Nanayakkara, N.P.D.; Bryant, L. \& McChesney, J.D. (1996). Quantitation of aryltetralin lignans in plant parts and among different populations of Podophyllum peltatum by reversed-phase high-performance liquid chromatography. Journal of Natural Products, 59, 4, (1996) 406-408, 0163-3864.

Bastos, J.K., Gottlieb, O.R., Sarti, S.J. \& Filho, D.S. (1996). Isolation of lignans and sesquiterpenoids from leaves of Zanthoxylum naranjillo, Natural Product Letters 9, 1, (1996) 65-70, 1478-6419.

Bastos, J.K.; Carvalho, J.C.T.; De Souza, G.H.B.; Pedrazzi, A.H.P. \& Sarti, S.J (2001). Antiinflammatory activity of cubebin, a lignan from the leaves of Zanthoxyllum naranjillo Griseb. Journal of Ethnopharmacology, 75, 2-3, 279-282, 0378-8741.

Bernadou J.; Bonnafous M.; Labat, G.; Loiseau, P. \& Meunier, B. (1991). Model systems for metabolism studies. Biomimetic oxidation of acetaminophen and ellipticine derivatives with water-soluble metalloporphyrins associated to potassium monopersulfate. Drug Metabolism and Disposition. 19,2, (1991) 360-365, 0090-9556.

Bernadou, J. \& Meunier, J. (2004). Biomimetic Chemical Catalysts in the Oxidative Activation of Drugs Advanced.Synthesis \& Catalysis, 346, (2004) 171-184. 1615-4150.

Bizaia, N.; de Faria, E. H.; Ricci, G. P.; Calefi, P. S.; Nassar, E. J.; Castro K. A. D. F.; Nakagaki, S.; Ciuffi, K. J.; Trujillano, R.; Vicente, M. A.; Gil. A. \& Korili, S. A. (2009). Porphyrin-Kaolinite as Efficient Catalyst for Oxidation Reactions, ACS Applied Materials \& Interfaces, 1, 11, (2009) 2667-2678. 1944-8244.

Cavaleiro, J. A. S.; Nascimento, G. M. S. F. C.; Neves, M. G. P. M. S.; Pinto, M. T.; Silvestre, A. J. D. \& Vicente, M. G. H. (1996). Oxidation of natural compounds catalyzed by Mn(III) porphyrin complexes. Tetrahedron Letters, 37, 11, (1996) 1893-1896, 0040-4020.

Ciuffi, K.J., Sacco, H.C., Biazzotto, J.C., Vidoto, E.A., Nascimento, O.R., Leite, C.A.P., Serra, O.A. \& Iamamoto, Y. (2000) Synthesis of fluorinated metalloporphyrinosilica imprinted with templates through sol-gel process. Journal of Non-Crystalline Solids, 273, 1-3, (2000) 100-108, 0022-3093.

Cordell, G. A. (2000). Biodiversity and drug discovery. Asymbiotic relationship. Phytochemistry, 55, (2000) 463-480, 0031-9422.

Da Silva Filho, A.A., Albuquerque, S., Silva, M.L.A.E., Eberlin, M.N., Tomazela, D.M. \& Bastos, J.K. (2004). Tetrahydrofuran Lignans from Nectandra megapotamica with Trypanocidal Activity. Journal of Natural Products, 67, 1, 42-45, 0163-3864.

Da Silva, R.; De Souza; G.H.B.; Da Silva, A.A.; De Souza, V.A.; Pereira, A.C.; Royo, V.D.A.; Silva, M.L.A.; P.M. Donate, P.M.; A.L.M. Araújo, A.L.M. ; J.C.T. Carvalho, J.C.T. \& Bastos, J.K. (2005). Synthesis and biological activity evaluation of lignan lactones 
derived from (-)-cubebin. Bioorganic and Medicinal Chemistry Letters, 15, 4,(2005) 1033-1037, 0960-894X.

de Faria, A. L.; Airoldi, C.; Doro, F. G.; Fonseca, M. G. \& das Dores Assis, M. (2004). Anchored ironporphyrins - the role of talc-aminofunctionalyzed phyllosilicates in the catalysis of oxidation of alkanes and alkenes. Applied Catalysis A: General, 268, 12, (2004) 217-226, 0926-860X.

de Lima, O. J. ; De Aguirre, D. P. ; De Oliveira, D. C. ; Da Silva, M. A. ; Mello C. ; Leite, C. A. P. ; Sacco, H. C. \& Ciuffi, K. J. (2001). Porphyrins entrapped in an alumina matrix. Journal of Materials. Chemistry, 11, (2001) 2476-2481, 0959-9428.

de Oliveira, D. C.; Sacco, H. C.; Nascimento, O. R.; Iamamoto, Y. \& Ciuffi, K. J. (2001). Amino ironporphyrinosilica hybrid materials. Journal of Non-Crystalline Solids, 284, 1-3 (2001), 27-33, 0022-3093.

De Souza, V.A.; Da Silva, R.; Pereira, A.C.; Royo, V.D.A.; Saraiva, J.; Montanheiro, M.; De Souza, G.H.B.; Da Silva Filho, A.A.; Grando, M.D.; Donate, P.M.; Bastos, J.K.; Albuquerque, S.; Silva \& M.L.A. (2005). Trypanocidal activity of (-)-cubebin derivatives against free amastigote forms of Trypanosoma cruzi. Bioorganic and Medicinal Chemistry Letters, 15, 2, (2005) 303-307, 0960-894X.

De Souza, V.A.; Da Silva, R.; Pereira, A.C.; Royo, V.D.A.; Saraiva, J.; Montanheiro, M., De Souza, G.H.B., da Silva Filho, A.A.; Donate, P.M. \& Bastos, J.K.; Albuquerque S., Silva, M.L.A. ( 2005) Synthesis and biological activity evaluation of lignan lactones derived from (-)-cubebin. Bioorganic and Medicinal Chemistry Letters,15, 2, (2005) 303307, 0968-0896.

Elisabetsky, E.; Amador, T. A.; Albuquerque, R. R.; Nunes, D. S. \& Carvalho, A. C. T. (1995). Analgesic activity of Psychotri colorata (Wild ex R and S) Muell Arg. Alkaloids. J. Ethnopharmacology, 148, (1995)77-83, 0378-8741.

Gibson, G. G. \& Skett, P. (1994). Introduction to Drug Metabolism, Chapman and Hall, 0412399105, London.

Goti, A. \& Cardona, F. (2008). Chemistry and Materials Science, Green Chemical Reactions, NATO Science for Peace and Security Series, 9781-1-4020-8458-4, Leece.

Gottlieb, O.R. \& Yoshida, M. (1989). Natural products of woods plants. Chemicals extraneous to the lignocellulosic cell wall. Springer -Verlag, 0302-9743, Berlin.

Groves, J. T. (2006). High-valent iron in chemical and biological oxidations. Journal of . Inorganic Biochemistry, 100, 2006,434 - 447, 0162-0134.

Groves, J. T.; Nemo, T. E. \& Myers, R. S. (1979). Hydroxylation and epoxidation catalyzed by iron-porphine complexes. Oxygen transfer from iodosylbenzene. Journal of. American Chemical Society, 101, 4 (1979), 1032-1033 , 0002-7863.

Lewis, D. F. V. (2001). Guide to Cytochrome P450 Structure and Function, Taylor \& Francis, 0 74840897 5, London.

Lohmann, W. \& Karst, U. (2008). Biomimetic modeling of oxidative drug metabolism strategies, advantages and limitations. Anaytical and. Bioanalytical Chemistry, 391, (2008), 79-96, 1618-2642

Mac Leod, T. C. O.; Guedes, D. F.C.; Lelo, M. R.; Rocha, R. A.; Caetano, B. L.; Ciuffi, K. J. \& Assis, M. D. (2006). Catalytic activity of Jacobsen catalyst encapsulated in an alumina matrix by the sol-gel process. Journal of Molecular Catalysis A: Chemical, 259, 1-2, (2006), 319-327., 1381-1169.

Machado, G. S.; de Freitas Castro, K. A. D.; de Lima, O. J.; Nassar, E. J.; Ciuffi, K. J. \& Nakagaki, S. (2009). Aluminosilicate obtained by sol-gel process as support for an anionic iron porphyrin: Development of a selective and reusable catalyst for 
oxidation reactions. Colloids and Surfaces A: Physicochemical and Engineering Aspects, 349, 1-3, (2009) 162-169, 0927-7757.

Macrae, W.D. \& TOWERS, G.H.N. (1984). Biological activities of lignans. Phytochemistry, 23, 6,(1984) 1207-1220, 0031-9422.

Mansuy, D. (2007). A brief history of the contribution of metalloporphyrin models to cytochrome P450 chemistry and oxidation catalysis. Comptes Rendus Chimie, 10, (2007) 392-413. 1631-0748.

Maraval, V.; Ancel, J. E. \& Meunier, B. (2002). Manganese(III) Porphyrin Catalysts for the Oxidation of Terpene Derivatives: A Comparative Study. Journal of Catalysis, 206,(2002) 349-357, (2002), 0021-9517.

McMurry, T.J \& Groves, J.T. (1986). Cytochrome P-450, Structure, Mechanism and Biochemistry, Plenum Press, 0-306-48324-6, New York. pp. 1-28.

Medola, J.F.; Cintra, V.P.; Pesqueira e Silva, E.P.; de Andrade Royo, V.; da Silva, R.; Saraiva, J.; Albuquerque, S.; Bastos, J.K.; Silva, M.L.A. \& Tavares, D.C. (2007). (-)-Hinokinin causes antigenotoxicity but not genotoxicity in peripheral blood of Wistar rats. Food and Chemical Toxicology, 45, 4, (2007) 638-642, 0278-6915.

Meyer, Urs A. (1996). Overview of enzymes of drug metabolism, Journal of Pharmacokinetics and Pharmacodynamics, 24, 5, (1996) 449-459, 1567-567X.

Milllos, M. (2001). A comparative study of biomimetic oxidation of oregano essential oil by $\mathrm{H} 2 \mathrm{O} 2$ or KHSO5 catalyzed by Fe (III) meso-tetraphenylporphyrin or Fe (III) phthalocyianine. Applied Catalysis A: General , 216, (2001) 157-161, 0926-860X.

Munro, A. W.; Girvan, H. M. \& McLean, K. J. (2007). Variations on a (t)heme-novel mechanisms, redox partners and catalytic functions in the cytochrome P450 superfamily. Natural Product Reports, 24, (2007) 585-609, 0265-0568.

Nam, W. (2007). High-Valent Iron (IV)-Oxo Complexes of Heme and Non-Heme Ligands in Oxygenation Reactions, Accounts of Chemical Research, 40, (2007) 522-531, 0001-4842.

Nascimento, I.R.; Lopes, L. M.X. , Davin, L. B. \& Lewis, N.G. (2001). Stereoselective synthesis of 8,9-licarinediols. Tetrahedron, 2001, 56, (2001) 9181-9193, 0040-4020

Papacídero, A. T.; Rocha, L. A.; Caetano, B. L.; Molina, E.; Sacco, H. C.; Nassar, E. J.; Martinelli, Y.; Mello, C.; Nakagaki, S. \& Ciuffi, K. J. (2006). Preparation and characterization of spherical silica-porphyrin catalysts obtained by the sol-gel methodology. Colloids and Surfaces A: Physicochemical and Engineering Aspects, 275, 13, (2006), 27-35, 0927-7757.

Rates, S. M. K. (2001). Plants as source of drugs. Toxicon, 39, 5,(2001) 603-61, 0041-0101.

Ravindran, J.; Nair, H. B.; Prasad, B. S. S.; Tekmal, R. R. \& Aggarwal, B. B. (2010). Bisdemethylcurcumin and structurally related hispolon analogues of curcumin exhibit enhanced prooxidant, anti-proliferative and anti-inflammatory activities in vitro. Biochemical Pharmacology. 79, 11, (2010), 1658-1666, 0006-2952.

Rehnberg, N. \& Magnusson, G.( 1990). Total synthesis of the lignans (-)- and (+)-burseran, $(-)$-cubebin, and (-)-hinokinin by diastereoselective conjugate addition of benzyl anions to 2-(R) and (s)-benzyloxy-2,5-dihydro-4-(3,4-methylenedioxybenzoyzfuran. Tetrahedron Letters, 29, 29, (1988) 3599-3602, 0040-4020.

Rosália, R. L.; Martins, M. G. P. M. S.; Neves, A.; Silvestre, J. D.; Silva; A. M. S. \& Cavaleiro, J. A. S. (1999). Oxidation of aromatic monoterpenes with hydrogen peroxide catalysed by $\mathrm{Mn}(\mathrm{III})$ porphyrin complexes. Journal of Molecular Catalysis A: Chemical,137, 1-3, (1999) 41-47. 1381-1169.

Sacco, H. C.; Ciuffi, K. J.; Biazzotto, J. C.; Mello, C.; de Oliveira, D. C.; Vidoto, E. A.; Nascimento, O. R.; Serra, O. A. \& Iamamoto, Y. (2001). Ironporphyrins trapped sol- 
gel glasses: a chemometric approach. Journal of Non-Crystalline Solids, 284, (2001) 174-182, 0022-3093.

Saleem, M.; Kim, H.J.; Ali, M. S. \& Lee, Y. S. (2005). An update on bioactive plant lignans. Natural Product Reports, 22, 6, (2005) 696-716, 0265-0568.

Saraiva, J.; Vega, C.; Rolon, M.; Da Silva, R.; E Silva, M.L.A.; Donate, P.M.; Bastos, J.K.; Gomez-Barrio A. \& De Albuquerque, S. (2007). In vitro and in vivo activity of lignan lactones derivatives against Trypanosoma cruzi. Parasitology Research, 100, 4, (2007) 791-795, 0932-0113.

Schaab, E. H.; Crotti, A. E. M.; Iamamoto,Y.; Kato, M. J.; Lotufo, L. V. C. \& Lopes, N. P. (2010). Biomimetic oxidation of Piperine and Piplartine catalyzed by Iron(III) and Manganese(III) Porphyrins. Biological \& Pharmaceutical Bulletin, 33, 5, (2010) 912916, 0918-6158.

Silva, M.L.A.; Coímbra, H.S.; Pereira, A.C.; Almeida, V.A.; Lima, T.C.; Costa, E.S.; Vinhólis, A.H.C.; Royo, V. A.; Silva, R.; da Silva Filho, A.A.; Cunha, W.R.; Furtado, N.A.J.C.; Martins, C.H.G.; Carvalho, T.C. \& Bastos, J.K. (2007). Evaluation of Piper cubeba extract, (-)-cubebin and its semi-synthetic derivatives against oral pathogens. Phytotherapy Research, 21, 5, 2007, 420-422, 0951-418X.

Silva, R.; Souza, G.H.B.; Da Silva, A.A.; De Souza, V.A.; Pereira, A.C.; Royo, V.de A.; E Silva, M.L.A.; Donate, P.M.; De Matos Araújo, A.L.S.; Carvalho, J.C.T. \& Bastos, J.K. (2005). Synthesis and biological activity evaluation of lignan lactones derived from (-)-cubebin. Bioorganic \& Medicinal Chemistry Letters, 15, (2005) 1033-1037, 0960-894X.

Simões, M. M. Q.; De Paula, R.; Neves, M. G. P. M. S. \& Cavaleiro, J. A. S. (2009). Metalloporphyrins in the biomimetic oxidative valorization of natural and other organic substrates. Journal of Porphyrins and Phthalocyanines, 13, (2009) 589-596, 1088-4246.

Siroká, Z. \& Drastischová, J. (2004). Biochemical Markers of Aquatic Environment Contamination - Cytochrome P450 in Fish. A Review. Acta Veterinaria Brno, 73, (2004) 123-132, 0001-7213

Skrobot, F. C.; Valente, A. A.; Neves, G.; Rosa, I.; Rocha, J. \& Cavaleiro, J. A. S. (2003). Monoterpenes oxidation in the presence of $\mathrm{Y}$ zeolite-entrapped manganese(III) tetra(4-N-benzylpyridyl) porphyrin. Journal of Molecular Catalysis A: Chemical, 201, 1-2, (2003) 211-222, 1381-1169.

Souza Brito, A. R. M. (1996). How to study the pharmacology of medicinal plants in underdeveloped countries. The Journal of.Ethnopharmacoogyl, 54, 2-3, ( 1996) 131-138, 0378-8741.

Souza, G.H.B.; Da Silva Filho, A.A.; De Souza, V.A.; Pereira, A.C.; Royo, V.de A.; E Silva, M.L.A.; Da Silva, R.; Donate, P.M.; Carvalho, J.C.T. \& Bastos, J.K. (2004). Analgesic and anti-inflammatory activities evaluation of (-)-Oacetyl, O-methyl, (-)-Odimethylethylamine cubebin and their preparation from (-)-cubebin. II Farmaco, 59, (2004) 55-61, 0014-827X.

Tanaka, E. (2001). Polymorphism of Drug Metabolizing Enzymes in Humans. Sepsis, 4, (2001) 247-254. 1385-0229.

Toshiro, K.; Yoshitaka, A. \& Yasuhiko, K. (1999). A novel allylic hydroxylation of sterically hindered olefins by Fe-porphyrin-catalyzed mCPBA oxidation. Tetrahedron Letters, 40 (1999) 6971-6974. 0040-4020.

Usia T.; Watabe, T.; Kadota, S. \& Tezuka,Y. (2005). Potent CYP3A4 inhibitory constituents of Piper cubeba. Journal of Natural Products, 68 1, (2005) 64-68, 0163-3864.

Ward, R. S. (1999). Lignans, neolignans and related compounds. Natural Product Reports, 16, 1, (1999) 75-96, 0265-0568. 


\title{
Chemical Indices of the Biomimetic Models of Oxyhemocyanin and Oxytyrosinase
}

\author{
Yu Takano ${ }^{1}$, Kizashi Yamaguchi ${ }^{2}$ and Haruki Nakamura ${ }^{1}$ \\ 1 Institute for Protein Research, Osaka University \\ ${ }^{2}$ Graduate School of Science, Osaka University \\ Japan
}

\section{Introduction}

The $\mathrm{Cu}_{2}\left(\mu-\eta^{2}: \eta^{2}-\mathrm{O}_{2}\right)$ species has attracted attention in the model studies of type III copper proteins because this structure is suggested as an important motif in biological systems (Karlin and Tyeklár, 1993; Kitajima and Moro-oka, 1994). Although oxyhemocyanin (oxyHc) and oxytyrosinase (oxyTy) have this species in the active site, they show different functions: oxygen transport and oxygen activation, respectively (Cooksey et al., 1997; Cuff et al., 1998; Holm et al., 1996; Solomon et al., 1992).

Synthetic modeling approaches have greatly developed our understanding of the chemical characters of the $\mathrm{Cu}_{2}\left(\mu-\eta^{2}: \eta^{2}-\mathrm{O}_{2}\right)$ species (Cahoy et al., 1999; Funahashi et al., 2008; Hu et al., 2001; Kitajima et al., 1992; Kitajima et al., 1989; Kodera et al., 2004; Kodera et al., 1999; Lam et al., 2000). Kitajima et al. succeeded in synthesizing $\left[\left(\mathrm{HB}\left(3,5-\mathrm{iPr}_{2}-\mathrm{Pz}_{3}\right) \mathrm{Cu}\right]_{2}\left(\mathrm{O}_{2}\right)\left(\mathrm{HB}\left(3,5-\mathrm{iPr}_{2}-\right.\right.\right.$ $\mathrm{Pz})_{3}=$ hydrotris $\{3,5$-diisopropyl-pyrazolyl\}borate (Trofimenko, 1999)) (1), which showed remarkable physicochemical similarities to oxyHc and oxyTy, and determining the crystal structures of the complex, which first characterized a $\mathrm{Cu}_{2}\left(\mu-\eta^{2}: \eta^{2}-\mathrm{O}_{2}\right)$ structure for the active site of oxyHc, before the X-ray crystallographic studies of Hc were reported (Kitajima et al., 1989). Tolman and his collaborators synthesized $\left[\left(\mathrm{iPr}_{3} \mathrm{TACD}\right) \mathrm{Cu}\right]_{2}\left(\mu-\eta_{2}: \eta_{2}-\mathrm{O}_{2}\right)\left(\mathrm{iPr} \operatorname{Pr}_{3} \mathrm{TACD}=\right.$ 1,5,9-triisopropyl-1,5,9-triazacyclodecane (2) to understand how the nature of the tridendate macrocyclic supporting ligand influences the relative stability of the isomeric $\mu-\eta^{2}: \eta^{2}$ peroxo- and bis( $\mu$-oxo)dicopper complexes (Lam et al., 2000). They showed that lowtemperature oxygenation of $\left.\left[\left(\mathrm{iPr}_{3} \mathrm{TACD}\right) \mathrm{Cu}\right]_{2}\left(\mathrm{CH}_{3} \mathrm{CN}\right)\right] \mathrm{SbF}_{6}$ yielded a $\mu-\eta^{2}: \eta^{2}$ product with no trace of the bis( $\mu$-oxo) isomer and concluded that the size of the ligand substituents and the ligand macrocycle ring size are key factors in controlling the relative stabilities of the $\mu-\eta_{2}: \eta_{2}-\mathrm{O}_{2}$-peroxo and bis $(\mu$-oxo) forms. Kodera et al. reported the reversible dioxygen binding by the room-temperature-stable complex, $[(\mathrm{L} 1) \mathrm{Cu}]_{2}\left(\mu-\eta_{2}: \eta_{2}-\mathrm{O}_{2}\right)(\mathrm{L} 1=1,2-$ bis$[2-(1,1-$ bis(6-methyl-2-pyridyl)ethyl)-6-pyridyl]ethane) (3), and showed that the long $\mathrm{Cu}-\mathrm{O}$ bonds and the strong $\mathrm{O}-\mathrm{O}$ bond of 3 are favorable for easy release of $\mathrm{O}_{2}$ since the $\mathrm{O}-\mathrm{O}$ stretch of 3 is the strongest for $\mathrm{Cu}_{2}\left(\mu-\eta^{2}: \eta^{2}-\mathrm{O}_{2}\right)$ complexes including oxyHc from the resonance Raman spectrum (Kodera et al., 2004). Masuda and his coworkers synthesized a new $\mathrm{Cu}_{2}\left(\mu-\eta_{2}: \eta_{2}-\mathrm{O}_{2}\right)$ complex formed with $\alpha$-isosparteine and benzoate (4) and determined the novel coordination structure of a carboxylate-bridged butterfly type $\mu-\eta^{2}: \eta^{2}$-peroxide dicopper core (Funahashi et al., 2008). It was shown that the butterfly core is supported by 
benzoate in the axial position, losing its planarity despite the Jahn-Teller effect on the $\mathrm{Cu}(\mathrm{II})$ $d^{9}$-configuration. Fig. 1 illustrates the coordinating ligands to the $\mathrm{Cu}_{2}\left(\mu-\eta^{2}: \eta^{2}-\mathrm{O}_{2}\right)$ core of the biomimetic models 1-4, and Fig. 2 shows the X-ray crystallographic structures of 1-4.<smiles></smiles>

1<smiles>CC(C)N1CCCN(C(C)C)CCN(C(C)C)CC1</smiles>

2<smiles>Cc1cccc(C(C)(c2cccc(C)n2)c2cccc(CCc3cccc(C(C)(c4cccc(C)n4)c4cccc(C)n4)n3)n2)n1</smiles><smiles>OC(O)c1ccccc1</smiles>

Fig. 1. Coordinating ligands utilized for the biomimetic model of Hc 1-4.

Several theoretical investigations have also been performed on models of the dicopper active site (Aullón et al., 2006; Benson et al., 2002; Cramer et al., 2003; Cramer and Pak, 2001; Gresh et al., 2002; Siegbahn, 2006; Siegbahn and Wirstam, 2001). Siegbahn studied the catalytic mechanisms of tyrosinase and catechol oxidase using $\mathrm{Cu}_{2} \mathrm{O}_{2}$ complex coordinated by six imidazole ligands at the UB3LYP level of theory (Siegbahn, 2006; Siegbahn and Wirstam, 2001). Cramer et al. investigated the mechanism of the intramolecular $\mathrm{C}-\mathrm{H}$ bond cleavage in $[\mathrm{LCu}]_{2}(\mu-\mathrm{O})_{2}(\mathrm{~L}=1,4,7$-tribenzyl-1,4,7-triazacyclononane) (Cramer et al., 2003; Cramer and Pak, 2001) using the integrated molecular orbital molecular mechanics (Maseras and Morokuma, 1995) with DFT and universal force field (Rappe et al., 1992). Aullón et al. performed UB3LYP calculations on binuclear $[(\mathrm{Tp}) \mathrm{Cu}]_{2}(\mu-\mathrm{O})_{2}$ complexes $(\mathrm{Tp}=$ tris(pyrazolyl)borate) to examine the effect of the substituents of the Tp ligands on dioxygen activation and stabilization (Aullón et al., 2006).

Previously, we investigated the magnetic couplings and the potential energy surface for the reversible binding process of the model of Hc using unrestricted Hartree-Fock and spinpolarized density functional theory (DFT) (Becke's half and half LYP (UBHandHLYP), UB3LYP, and UBLYP) (Takano et al., 2009; Takano et al., 2001; Takano and Yamaguchi, 2007). In this model, each $\mathrm{Cu}$ ion is coordinated by three methylimidazole. We concluded that the superexchange interaction via the bridging dioxygen causes a strong antiferromagnetic couplings between the two copper ions of the model of oxyHc and that the structural conversion from oxy to deoxyHc controls the reversible binding of dioxygen for Hc.

To comprehend the chemical character of the $\mathrm{Cu}_{2}\left(\mu-\eta^{2}: \eta^{2}-\mathrm{O}_{2}\right)$ complexes, we have investigated the magnetic interaction and the nature of the chemical bond of the biomimetic 
models of oxyHc and oxyTy, 1-4, from the viewpoint of the shape and symmetry of the natural orbitals and chemical indices. We compared the geometries, magnetic couplings, and nature of chemical bonds of the optimized structures to those of the X-ray structures.

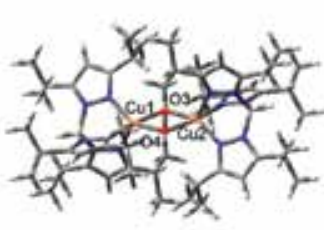

1

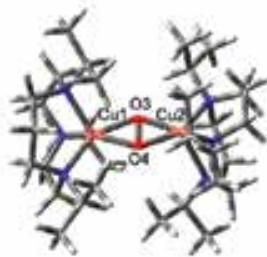

2

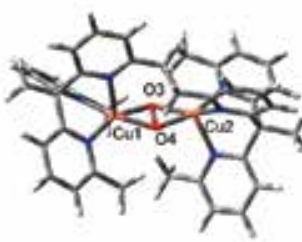

3

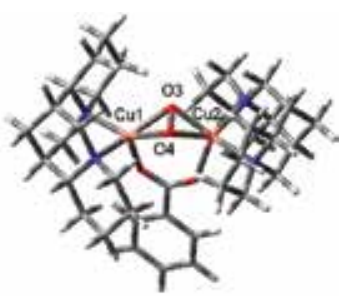

4

X-ray strucuture

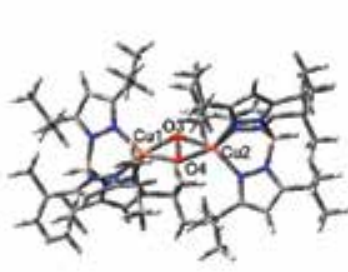

1

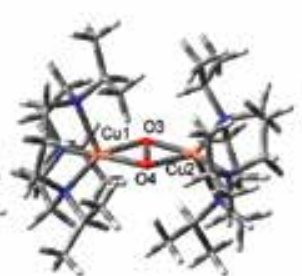

2

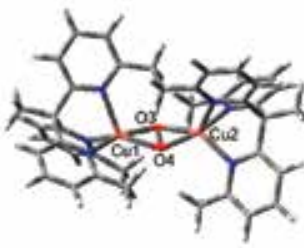

3

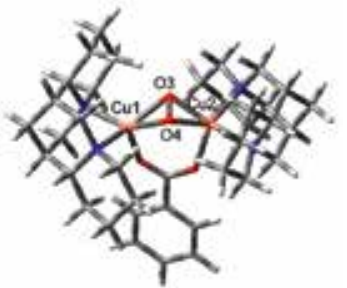

4

Optimized strucuture

Fig. 2. X-ray crystallographic structures and optimized structures of 1-4. Site numbers Cu1, $\mathrm{Cu} 2, \mathrm{O} 3$, and $\mathrm{O} 4$ are also illustrated.

\section{Theoretical background}

\subsection{Magnetic coupling constant $\left(J_{\mathrm{ab}}\right)$}

Since a large number of transition metal complexes have unpaired d electrons, magnetic properties of transition metal complexes have been investigated in order to understand the molecular structures and the electronic structures. Magnetic coupling constants $\left(J_{\mathrm{ab}}\right)$ can be experimentally determined by the measurement of magnetic susceptibility. Recent development of computational techniques of quantum chemistry has made theoretical calculations of $J_{\mathrm{ab}}$ values possible (Takano et al., 2001). Let us consider the superexchange interactions between $\mathrm{Cu}(\mathrm{II}) \mathrm{L}_{3}$ (L: ligand) fragments via peroxide. Cupric ion, $\mathrm{Cu}(\mathrm{II})$, has the $\mathrm{d}^{9}$ configuration with half spin $(S=1 / 2)$. If each spin is mainly localized on one site (a or $\mathrm{b}$ site), as illustrated in I of Fig. 3, we can estimate $J_{\mathrm{ab}}$ values between magnetic sites on the basis of the Heisenberg model (Onishi et al., 2001; Salem, 1982; Soda et al., 2000; Takano et al., 2008; Takano et al., 2002a; Takano et al., 2001; Takano et al., 2000; Takano and Yamaguchi, 2007) using the energy gap by spin-polarized DFT calculations between the highest spin (HS) and lowest spin (LS) states,

$$
H=-2 \sum J_{\mathrm{ab}} \mathbf{S}_{\mathrm{a}} \cdot \mathbf{S}_{\mathrm{b}},
$$

where $S_{a}$ and $S_{b}$ represent the spins at sites a and $b$, respectively. However, spin-polarized DFT solutions in the LS states usually exhibit the broken symmetry problem (Isobe et al., 
2002; Mitani et al., 2000a; Onishi et al., 2001; Salem, 1982; Takano et al., 2008; Takano et al., 2002a; Takano et al., 2001; Takano et al., 2000; Takano and Yamaguchi, 2007). Spin projection of the broken symmetry solutions should be carried out to eliminate the spin contaminations.

$$
J_{\mathrm{ab}}=\frac{{ }^{\mathrm{LS}} E_{\mathrm{X}}-{ }^{\mathrm{HS}} E_{\mathrm{X}}}{{ }^{\mathrm{HS}}\left\langle S^{2}\right\rangle_{\mathrm{x}}-{ }^{\mathrm{LS}}\left\langle S^{2}\right\rangle_{\mathrm{x}}},
$$

where ${ }^{Y} E_{X}$ and $Y<S^{2}>_{X}$ denote the total energy and total angular momentum of the spin state $\mathrm{Y}$ by spin-polarized DFT, respectively.

\subsection{Natural orbitals}

The molecular orbital picture is also feasible for elucidation of the origin of the magnetic interaction between the $\mathrm{Cu}(\mathrm{II})$ ions. In order to obtain molecular orbital-theoretical explanation of the magnetic interactions, the natural orbitals of the spin-polarized DFT solutions were determined by diagonalizing their first-order density matrices (Onishi et al., 2001; Salem, 1982; Takano et al., 2008; Takano et al., 2002a; Takano et al., 2001; Takano et al., 2000; Takano and Yamaguchi, 2007) as

$$
\rho\left(\mathbf{r}, \mathbf{r}^{\prime}\right)=\sum n_{i} \phi_{i}^{*}(\mathbf{r}) \phi_{i}\left(\mathbf{r}^{\prime}\right)
$$

where $n_{i}$ denotes the occupation number of the natural orbital $\phi_{i}$. The occupation numbers of the bonding and anti-bonding natural orbitals were almost 2.0 and 0.0 , respectively, except for the two singly occupied natural orbitals (SONOs), for which the occupation numbers were close to 1.0. The broken symmetry bonding orbitals are generally given by the in- and out-of phase combinations of the bonding $(\phi)$ and antibonding $\left(\phi^{*}\right)$ DFT natural orbital

$$
\psi_{i}^{ \pm} \cong(\cos \omega) \phi_{i} \pm(\sin \omega) \phi_{i}^{*}
$$

where $\omega$ is the orbital mixing coefficient (Onishi et al., 2001; Salem, 1982; Takano et al., 2008; Takano et al., 2002a; Takano et al., 2001; Takano et al., 2000; Takano and Yamaguchi, 2007).

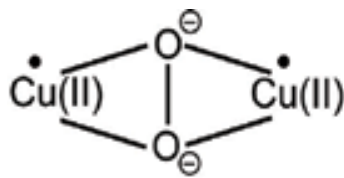

(I)<smiles>O1[Ge][Ge]2O[Ge]12</smiles>

$\mathrm{Cu}(\mathrm{I})$

(III)<smiles>O=O</smiles>

(II)<smiles>[GeH3]O[GeH3]</smiles>

(IV)

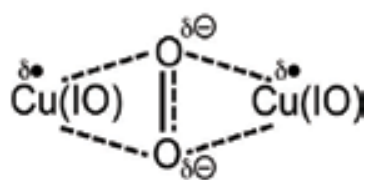

(V)

$$
(\mathrm{I}<\mathrm{IO}<\mathrm{II})
$$

Fig. 3. Valence bond description of the $\mathrm{Cu}_{2} \mathrm{O}_{2}$ bond. 
The orbital overlap $T_{i}=\left\langle\psi_{i}^{+} \mid \psi_{i}^{-}\right\rangle$between magnetic orbitals is a measure of the SE interaction, namely $T_{i}=0$ for pure metal diradical and $T_{i}=1$ for the closed-shell pair. The occupation numbers of the bonding and antibonding DNO are expressed by the $T_{i}$ value;

$$
n_{i}=1+T_{i}, \quad n_{i}^{*}=1-T_{i}
$$

The total spin angular momentum for the BS solution is given by

$$
\mathrm{LS}<\mathrm{S}^{2}>=S_{\min }\left(S_{\min }+1\right)+\Sigma\left(1-T_{i}^{2}\right)
$$

\subsection{Chemical indices}

In theoretical and coordination chemistries, an important issue is to understand the nature of the chemical bonds in transition metal complexes. Chemical indices are useful criteria for the nature of chemical bonds. Since the broken symmetry solutions are employed in this study, several chemical indices, which are equally defined by the symmetry-adapted CASCI and CASSCF, are expressed in terms of the occupation numbers of DFT natural orbital (Isobe et al., 2003; Mitani et al., 2000a; Yamaguchi, 1990). These indices are used as common criteria for the nature of the chemical bonds of the $\mathrm{Cu}_{2}\left(\mu-\eta^{2}: \eta^{2}-\mathrm{O}_{2}\right)$ core.

The effective bond order (Isobe et al., 2003; Yamaguchi, 1990) is defined by

$$
b_{i}=\frac{n_{i}-n_{i}^{*}}{2}
$$

The effective bond order is a measure of the superexchange interaction; that is, $b_{i}=0$ for pure metal diradical and $b_{i}=1$ for the closed-shell pair.

Classical and quantum information theories and generalized entropies are now well-defined in several different fields to extract information. In chemistry, information entropy $(I)$ is employed to express the characteristic of chemical bonds (Ramirez et al., 1997). The Jaynes information entropy is defined by the occupation number of SONOs to express electron correlation

$$
I=-n_{i} \ln n_{i}
$$

The Jaynes information entropy for the spin-restricted DFT solution of closed-shell systems is given by

$$
I_{\mathrm{C}}=-2 \ln 2
$$

Then, the normalized Jaynes information entropy is defined as a measure of correlation correction as

$$
I_{\mathrm{n}}=\frac{I_{\mathrm{C}}-I}{I_{\mathrm{C}}} \quad\left(0 \leq I_{\mathrm{n}} \leq 1\right)
$$

The $I_{\mathrm{n}}$ value increases with the decrease of $T_{i}$ in this definition, and it is parallel to the decrease of the effective bond order $\Delta b=1-b=1-T$ (Isobe et al., 2002). The $I_{\mathrm{n}}$ and $\Delta b$ values are, therefore, responsible for loss of bond information or covalent bonding. 
The unpaired electron density $U$ is defined as the deviation of the post HF and CI wavefunctions from the single Slater determinant (Takatsuka et al., 1978), and it is also expressed by the occupation number as

$$
U=n_{i}\left(2-n_{i}\right)
$$

Very recently, the $U$-value is utilized as a measure of electron correlation by Staroverov and Davidson (Staroverov and Davidson, 2000).

The CASCI and CASSCF methods (Roos et al., 1982) are well-accepted approaches to openshell species. Therefore, it is desirable to clarify mutual relations between the CI and BS methods. The diradical character $(Y)$ is defined by the weight of doubly excited configuration $\left(W_{D}\right)$ involved in the projected BS solution in comparison with CASCI and CASSCF (Isobe et al., 2002; Mitani et al., 2000b) as

$$
Y=2 W_{\mathrm{D}}=1-\frac{2 T_{i}}{1+T_{i}^{2}}=\frac{n_{i}^{2}-4 n_{i}+4}{n_{i}^{2}-2 n_{i}+2}
$$

Thus, all the chemical indices introduced here are related each other through the occupation numbers of natural orbitals, which can be calculated by both symmetry-adapted solutions such as CASSCF and broken-symmetry solutions. These indices are used as common criterion for chemical bonds of biomimetic complexes 1-4.

\section{Computational details}

\subsection{Computational procedure}

All quantum chemical calculations were carried out on the synthetic $\mathrm{Cu}_{2} \mathrm{O}_{2}$ complexes 1-4 with Gaussian03 program (Frisch et al., 2003).

In DFT calculations, exchange-correlation potentials are generally defined by

$$
E_{\mathrm{xC}}=C_{1} E_{\mathrm{X}}^{\mathrm{HF}}+C_{2} E_{\mathrm{x}}^{\mathrm{Slater}}+C_{3} \Delta E_{\mathrm{x}}^{\mathrm{Becke88}}+C_{4} E_{\mathrm{C}}^{\mathrm{VWN}}+C_{5} \Delta E_{\mathrm{C}}^{\mathrm{LYP}}
$$

where $E_{\mathrm{X}}^{\mathrm{HF}}$ is the Hartree-Fock exchange, $E_{\mathrm{X}}^{\text {Slater }}$ is the Slater exchange, $\Delta E_{\mathrm{X}}^{\text {Becke88 }}$ is the gradient part of the exchange functional of Becke (Becke, 1988), $E_{\mathrm{C}}^{\mathrm{VWN}}$ is the correlation functional of Vosko, Wilk, and Nusair (Vosko et al., 1980), and $\Delta E_{\mathrm{C}}^{\mathrm{LYP}}$ is the correlation functional of Lee, Yang, and Parr (Lee et al., 1988) which includes the gradient of the density. Parameters, $C_{i}(i=1-5)$, are the mixing coefficients. The parameter sets $\left(C_{1}, C_{2}, C_{3}\right.$, $C_{4}$, and $\left.C_{5}\right)$ are taken as $(0.00,1.00,1.00,1.00$, and 1.00) for BLYP, $(0.20,0.80,0.72,1.00$, and 0.81) for B3LYP (Becke, 1993), and (0.50, 0.50, 0.50, 1.00, and 1.00) for BHandHLYP (Becke, 1993). Since we showed that UBHandHLYP (referred to as UB2LYP in the previous papers (Takano et al., 2001; Takano and Yamaguchi, 2007)) can be regarded as a reliable method to examine the magnetic couplings and the dioxygen binding process of Hc and the nature of chemical bonds of the biomimetic complexes in the previous papers (Takano et al., 2009; Takano et al., 2001; Takano and Yamaguchi, 2007), we used the UBHandHLYP exchangecorrelation functionals to investigate the magnetic couplings and the chemical indices: it is noteworthy that UBHandHLYP is much more reliable than conventional UB3LYP for strongly correlated electron systems such as $\mathrm{CuO}$ plane in oxide superconductors (Onishi et al., 2001). 
Basis sets employed in all the calculations were Tatewaki-Huzinaga MIDI $(533(21) / 53(21) /(41))$ (Tatewaki and Huzinaga, 1979) plus Hay's d diffuse function $(\alpha=$ 0.1491) (Hay, 1977) for $\mathrm{Cu}(\mathrm{II})$ ions, Pople's 6-31G(d) (Hariharan and Pople, 1973) for C, O, N, and $\mathrm{B}$ atoms, and 6-31G (Hehre et al., 1972) for $\mathrm{H}$ atoms.

\subsection{Geometrical parameters for $1-4$}

The geometrical parameters of 1-4 were taken from the X-ray crystallographic data of Cambridge Structural Database (Allen, 2002) as shown in Fig. 2 (Refcode for 1: KECZEX, Refcode for 2: NAPGEQ, Refcode for 3: BEMNIR, and Refcode for 4: QOSRAS). Full geometry optimizations of 1-4 were also performed in highest- and lowest-spin states in the gas phase by using the UBHandHLYP functionals with the basis sets mentioned above. The optimized structures were characterized by using harmonic frequency calculations.

\section{Comparison between X-ray structures and optimized structures}

The geometrical parameters of the X-ray and optimized structures for 1-4 in the most stable state are listed in Table 1. In the geometry optimizations, models 1-3 preferred the lowest spin state to the highest spin state, while model 4 favored the highest spin state over the lowest spin state. The differences between the X-ray and optimized bond distances were within $0.1 \AA$ except for the $\mathrm{Cu} 1-\mathrm{Cu} 2, \mathrm{O} 3-\mathrm{O} 4$, and $\mathrm{Cu} 2-\mathrm{O} 3$ parts of $2(0.11,0.11$, and $0.15 \AA$, respectively) and the $\mathrm{Cu}-\mathrm{N}$ part of $3(0.12 \AA)$. In the optimization, the changes of the dihedral angles of the $\mathrm{Cu}_{2} \mathrm{O}_{2}$ core were less than $10^{\circ}$ except for the $\mathrm{d}(\mathrm{O} 3-\mathrm{O} 4-\mathrm{Cu} 1-\mathrm{Cu} 2)$ and $\mathrm{d}(\mathrm{Cu} 1-\mathrm{O} 3-\mathrm{O} 4-\mathrm{Cu} 2)$ of $\mathbf{1}\left(21.3^{\circ}\right.$ and $\left.-17.4^{\circ}\right)$ and $\mathrm{d}(\mathrm{Cu} 1-\mathrm{O} 3-\mathrm{O} 4-\mathrm{Cu} 2)$ of $4\left(-10.3^{\circ}\right)$. These differences between the optimized geometries and the X-ray structures would be due to the effects of crystal packing and the spin contamination errors during the optimization as shown below.

\begin{tabular}{|c|c|c|c|c|c|c|c|c|}
\hline & \multicolumn{2}{|c|}{1} & \multicolumn{2}{|c|}{2} & \multicolumn{2}{|c|}{3} & \multicolumn{2}{|c|}{4} \\
\hline & X-raya & $\mathrm{Opt}^{\mathrm{b}}$ & X-raya & $\mathrm{Opt}^{\mathrm{b}}$ & X-raya & $\mathrm{Opt}^{\mathrm{b}}$ & X-raya & $\mathrm{Optb,c}$ \\
\hline $\mathrm{Cu} 1-\mathrm{Cu} 2^{\mathrm{d}}$ & 3.56 & 3.58 & 3.52 & 3.63 & 3.52 & 3.61 & 3.27 & 3.24 \\
\hline O3-O4d & 1.41 & 1.48 & 1.37 & 1.48 & 1.49 & 1.47 & 1.46 & 1.46 \\
\hline $\mathrm{Cu} 1-\mathrm{O} 3 \mathrm{~d}$ & 1.90 & 1.95 & 1.96 & 1.96 & 1.92 & 1.93 & 1.98 & 2.08 \\
\hline $\mathrm{Cu} 1-\mathrm{O} 4 \mathrm{~d}$ & 1.93 & 1.96 & 1.92 & 1.97 & 1.91 & 2.00 & 1.87 & 1.92 \\
\hline $\mathrm{Cu} 2-\mathrm{O} 3 \mathrm{~d}$ & 1.93 & 1.94 & 1.81 & 1.96 & 1.96 & 1.98 & 1.91 & 1.92 \\
\hline $\mathrm{Cu} 2-\mathrm{O} 4^{\mathrm{d}}$ & 1.90 & 1.98 & 1.89 & 1.96 & 1.90 & 1.94 & 1.98 & 2.05 \\
\hline $\mathrm{Cu}-\mathrm{N}(\mathrm{O})^{\mathrm{d}, \mathrm{e}}$ & 2.08 & 2.11 & 2.11 & 2.17 & 2.05 & 2.17 & 2.03 & 2.09 \\
\hline $\begin{array}{c}\mathrm{d}(\mathrm{O} 3-\mathrm{O} 4-\mathrm{Cu} 1- \\
\mathrm{Cu} 2)^{\mathrm{f}}\end{array}$ & 0.0 & 21.3 & 10.37 & 5.30 & -15.8 & -19.6 & 48.2 & 53.7 \\
\hline $\begin{array}{c}\mathrm{d}(\mathrm{Cu} 1-\mathrm{O} 3-\mathrm{O} 4- \\
\mathrm{Cu} 2)^{\mathrm{f}}\end{array}$ & 180.0 & 162.6 & 171.9 & 176.0 & -168.2 & -164.7 & 132.3 & 122.0 \\
\hline
\end{tabular}

a The parameters are taken from the $X$-ray structures. ${ }^{b}$ The parameters were fully optimized at the UBHandHLYP level of theory. ${ }^{c}$ Geometry optimization was performed in the highest spin state. ${ }^{d}$ Distances are given in angstroms. e Average value for bond distances between $\mathrm{Cu}$ ion and $\mathrm{N}$ or $\mathrm{O}$ atom coordinating to $\mathrm{Cu}_{2}\left(\mu-\eta^{2}: \eta^{2}-\mathrm{O}_{2}\right)$ core. ${ }^{\mathrm{f}}$ Dihedral angles are given in degrees.

Table 1. Selected Geometrical Parameters for 1-4 in the most stable state at the UBHandHLYP level of theory. 
In the biomimetic model $\mathbf{1}$, the large changes of the dihedral angles mean that the structure of the $\mathrm{Cu}_{2} \mathrm{O}_{2}$ core converts from the planar structure to the butterfly one in the geometry optimization. The geometry optimization of 2 kept the core structure planar but expanded the $\mathrm{Cu}_{2} \mathrm{O}_{2}$ core, indicating that the coordinating ligand of 2 weakly suppresses the $\mathrm{Cu}_{2} \mathrm{O}_{2}$ core compared to the other ligands. As opposed to 1, though the dihedral angles of the $\mathrm{Cu}_{2} \mathrm{O}_{2}$ core of 3 hardly changed in the optimization, the $\mathrm{Cu} 1-\mathrm{Cu} 2$ distance elongated to 0.09 $\AA$, the O3-O4 length slightly shrank, the mean bond distance between the coordinating ligands and the $\mathrm{Cu}_{2} \mathrm{O}_{2}$ core increased to $0.12 \AA$. These results imply easy release of oxygen molecule from 3. The core structure of 4 is the most distorted of all the models 1-4. Optimization made the $\mathrm{Cu}_{2} \mathrm{O}_{2}$ core of 4 to be distorted more. It causes the strongly mixing of the character of triplet oxygen molecule, resulting in the stabilization of the highest spin state more than the lowest spin state because triplet oxygen molecule is much more stable than singlet oxygen molecule.

\section{Magnetic couplings of the biomimetic model complexes 1-4}

\subsection{Magnetic coupling constants $\left(J_{\mathrm{ab}}\right)$}

Table 2 summarizes the $J_{\mathrm{ab}}$ values for 1-4. All $J_{\mathrm{ab}}$ values calculated with the $X$-ray structures show strong antiferromagnetic couplings between the $\mathrm{Cu}(\mathrm{II})$ ions. The absolute values of the $J_{\mathrm{ab}}$ are in the order $\left|J_{\mathrm{ab}}(\mathbf{2})\right|>>\left|J_{\mathrm{ab}}(\mathbf{1})\right|>\left|J_{\mathrm{ab}}(\mathbf{3})\right|>>\left|J_{\mathrm{ab}}(\mathbf{4})\right|$. This result is mainly brought from the distortion of the $\mathrm{Cu}_{2} \mathrm{O}_{2}$ core structure because the discrepancies from the planar structure $\left(\mathrm{d}(\mathrm{O} 3-\mathrm{O} 4-\mathrm{Cu} 1-\mathrm{Cu} 2)=0^{\circ}\right.$ and $\left.\mathrm{d}(\mathrm{Cu} 1-\mathrm{O} 3-\mathrm{O} 4-\mathrm{Cu} 2)=180^{\circ}\right)$ for the $\mathrm{Cu}_{2} \mathrm{O}_{2}$ core of 1-4 are in the order: $1 \approx 2<3<<$. The $J_{\mathrm{ab}}$ values are sensitive to the change of the $\mathrm{Cu}_{2} \mathrm{O}_{2}$ structure because the $\mathrm{Cu}_{2} \mathrm{O}_{2}$ core lies in the labile bonding region (Takano and Yamaguchi, 2007), and the core structure strongly affects the strength of the symmetry-allowed orbital interactions between the symmetric $(S) d_{x y}-d_{x y}$ orbital of the dicopper site and the $\sigma^{*}$ and $\pi_{v}{ }^{*}$ orbitals of $\mathrm{O}_{2}$ and between the antisymmetric (A) $\mathrm{d}_{\mathrm{xy}}+\mathrm{d}_{\mathrm{xy}}$ orbital and the $\pi_{\mathrm{h}}{ }^{*}$ orbital, as shown in Fig. 4.

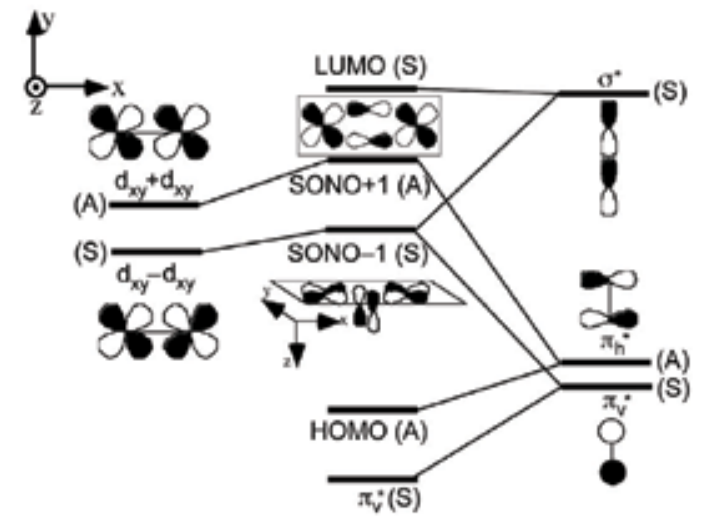

Fig. 4. Orbital diagram of the $\mathrm{Cu}_{2} \mathrm{O}_{2}$ bond.

The magnetic couplings weakened after full geometry optimization, and, in particular, the $J_{\mathrm{ab}}$ value for 4 became positive, indicating a ferromagnetic coupling. This is because of the elongation of the $\mathrm{Cu}-\mathrm{O}-\mathrm{Cu}$ lengths $(\mathrm{Cu} 1-\mathrm{O} 3+\mathrm{Cu} 2-\mathrm{O} 3$ and $\mathrm{Cu} 1-\mathrm{O} 4+\mathrm{Cu} 2-\mathrm{O} 4)$ and the change of the dihedral angles. Geometry optimization of 1 remarkably changed the $\mathrm{Cu}_{2} \mathrm{O}_{2}$ 
structure from the planar form to the butterfly form. The structural conversion affects the orbital interactions between the $\mathrm{Cu}$ (II) ions via peroxide, leading to drastic reduction of the $J_{\mathrm{ab}}$ values in magnitude. The larger elongation of the $\mathrm{Cu}-\mathrm{O}-\mathrm{Cu}$ lengths in $2(0.15 \AA$ for $\mathrm{Cu} 1-$ O3-Cu2 and $0.12 \AA$ for Cu1-O4-Cu2) and 3 (0.13 $\AA$ for Cu1-O4-Cu2) made the magnetic coupling weak, though the shape of the $\mathrm{Cu}_{2} \mathrm{O}_{2}$ core kept almost planar during geometry optimization. During geometry optimization of 4 , the $\mathrm{Cu}-\mathrm{O}$ bond lengths elongated by about $0.1 \AA$, showing that the dioxygen part tends to dissociate from the dicopper core and that the spin crossover from the lowest spin state to the highest spin state occurs (Takano and Yamaguchi, 2007). However, this spin crossover during geometry optimization might be attributed to the spin contamination error (Kitagawa et al., 2007; Saito et al., 2010; Saito et al., 2008). These indicate that the ligands that can hold the $\mathrm{Cu}_{2} \mathrm{O}_{2}$ core planar lead to the strong antiferromagnetic complexes.

\begin{tabular}{ccc}
\hline Model & \multicolumn{2}{c}{$J_{\mathrm{ab}^{a}}$} \\
\cline { 2 - 3 } & $\mathrm{X}^{a}$-ray $^{\mathrm{b}}$ & $\mathrm{Opt}^{\mathrm{c}}$ \\
\hline $\mathbf{1}$ & -1902 & -678.1 \\
$\mathbf{3}$ & -1967 & -1363 \\
$\mathbf{4}$ & -1508 & -1020 \\
\hline
\end{tabular}

${ }^{a} J_{\mathrm{ab}}$ is shown in $\mathrm{cm}^{-1} .{ }^{b}$ The parameters are taken from the X-ray structures. ${ }^{c}$ The parameters were fully optimized at the UBHandHLYP level of theory.

Table 2. Magnetic Coupling constants $\left(J_{\mathrm{ab}}\right)^{a}$ Calculated for 1-4 at the UBHandHLYP level of theory.

\subsection{Charge and spin density distributions}

We have investigated the charge and spin density distributions for 1-4 to understand the characteristics of the magnetic couplings. As listed in Table 3, the charge density on the O-O group varies from the formal charge $(-2.0)$ in I to 0.0 in the valence bond configuration II in Fig. 3, indicating the back charge transfer from peroxide to cupric ions. The back charge transfer implies that the superexchange interactions are responsible for the strong antiferromagnetic coupling of the biomimetic models.

In Table 3, the spin densities on $\mathrm{Cu}(\mathrm{II})$ ions were about 0.7 for $\mathbf{1 - 4}$. The spin density populations indicate the resonance state between the one-electron transfer valence bond configurations III and IV in Fig. 3. The spin densities on the O-O group are cancelled out in this resonance state $\mathbf{V}$; that is, intermediate valence state.

The broken symmetry orbitals in Eq. (4) are approximately expressed by the fragment orbitals responsible for these valence bond configurations in Fig. 3.

$$
\begin{aligned}
& \psi^{+} \cong(\cos \theta) \pi_{\mathrm{h}}^{*}+(\sin \theta) d_{\mathrm{xy}}^{\mathrm{Cu} 2} \\
& \psi^{-} \cong(\cos \theta) \pi_{\mathrm{h}}^{*}+(\sin \theta) d_{\mathrm{xy}}^{\mathrm{Cu} 1}
\end{aligned}
$$

where $\theta$ is the orbital mixing parameter and $d_{\mathrm{xy}}^{\mathrm{Cu} 1}$ or $d_{\mathrm{xy}}^{\mathrm{Cu} 2}$ denote the localized $\mathrm{d}_{\mathrm{xy}}$ orbital at $\mathrm{Cu} 1$ or $\mathrm{Cu} 2$, respectively. The broken symmetry solution is approximately given by $\left|\psi^{+} \bar{\psi}^{-} d_{\mathrm{xy}}^{\mathrm{Cu}} \bar{d}_{\mathrm{xy}}^{\mathrm{Cu} 2}\right|$. The spin density at Cu1 is calculated by 


\begin{tabular}{|c|c|c|c|c|c|c|}
\hline Model & & & $\mathrm{Cu}^{a}$ & $\mathrm{Cu} 2^{a}$ & $\mathrm{O}^{a} a$ & $\mathrm{O} 4^{a}$ \\
\hline \multirow[t]{4}{*}{1} & \multirow[t]{2}{*}{ X-ray } & Charge & 1.082 & 1.082 & -0.594 & -0.594 \\
\hline & & Spin & 0.709 & -0.708 & 0.006 & -0.007 \\
\hline & \multirow[t]{2}{*}{ Opt } & Charge & 1.023 & 1.075 & -0.591 & -0.572 \\
\hline & & Spin & 0.726 & -0.727 & 0.000 & -0.000 \\
\hline \multirow[t]{4}{*}{2} & \multirow[t]{2}{*}{ X-ray } & Charge & 0.985 & 1.044 & -0.641 & -0.612 \\
\hline & & Spin & 0.661 & -0.651 & -0.009 & -0.008 \\
\hline & \multirow[t]{2}{*}{ Opt } & Charge & 0.929 & 0.937 & -0.604 & -0.601 \\
\hline & & Spin & 0.689 & -0.676 & -0002 & -0.005 \\
\hline \multirow[t]{4}{*}{3} & \multirow[t]{2}{*}{ X-ray } & Charge & 1.127 & 1.107 & -0.594 & -0.611 \\
\hline & & Spin & 0.701 & -0.713 & 0.022 & -0.013 \\
\hline & \multirow[t]{2}{*}{ Opt } & Charge & 1.071 & 1.063 & -0.610 & -0.586 \\
\hline & & Spin & 0.715 & -0.712 & 0.025 & -0.029 \\
\hline \multirow[t]{4}{*}{4} & \multirow[t]{2}{*}{ X-ray } & Charge & 0.873 & 0.887 & -0.584 & -0.601 \\
\hline & & Spin & 0.688 & -0.687 & -0.034 & 0.030 \\
\hline & \multirow[t]{2}{*}{ Opt (HS) ${ }^{b}$} & Charge & 0.841 & 0.841 & -0.545 & -0.545 \\
\hline & & Spin & 0.666 & 0.667 & 0.215 & 0.215 \\
\hline
\end{tabular}

${ }^{a}$ The site numbers are shown in Fig. 2. ${ }^{b}$ Geometry optimization was performed in the highest spin state.

Table 3. Charge and Spin Densities of 1-4 at the UBHandHLYP level of theory.

$$
Q_{\mathrm{Cu} 1}=\left(1-\sin ^{2} \theta\right) \cong 0.7
$$

The $\theta$-parameter was determined to be $0.58 \mathrm{rad}$. The broken symmetry solution after spin projection, namely the resonance state $\mathbf{V}$, is obtained by

$$
\left.{ }^{1} \Phi \cong(\text { spin-projected })\right) \psi^{+} \bar{\psi}^{-} d_{\mathrm{xy}}^{\mathrm{Cu1}} \bar{d}_{\mathrm{xy}}^{\mathrm{Cu} 2} \mid=N\left(\sum C_{\mathrm{x}}{ }^{1} \Phi_{\mathrm{VB}}(\mathrm{X})\right)
$$

where ${ }^{1} \Phi_{\mathrm{VB}}(X)$ denotes the pure singlet valence bond wavefunction responsible for the valence bond structure $\mathrm{X}(=\mathbf{I}-\mathbf{I V})$, and $N$ is the normalizing factor. The configuration mixing parameters $C_{X}$ are defined by the orbital mixing parameter $\omega$.

$$
\begin{gathered}
C_{\mathrm{II}}=1+\cos 2 \theta \\
C_{\mathrm{II}}=1-\cos 2 \theta \\
C_{\mathrm{III}}=C_{\mathrm{IV}}=\sin 2 \theta
\end{gathered}
$$

These values are determined as

$$
C_{\mathrm{I}}=1.4, C_{\mathrm{II}}=0.6 C_{\mathrm{III}}=C_{\mathrm{IV}}=0.9
$$

The ratios of the valence bond configurations were 5 (I) $: 1$ (II) $: 2$ (III = IV) under the drastic approximation. The antiferromagnetically coupled singlet state (V) in Fig. 3 is expressed by the superposition of the four valence bond structures I-IV. However, the broken symmetry molecular orbitals partly involve the contribution from $\pi_{\mathrm{v}}{ }^{*}$ orbital as illustrated in Fig. 4 . This implies that much more valence bond configurations are required to express the spin projected broken symmetry state, quantitatively. Judging from the charge and spin densities 
on the $\mathrm{Cu}_{2} \mathrm{O}_{2}$ core, the copper ion lies in the intermediate oxidation $(\mathrm{IO})$ state $(\mathrm{I}<\mathrm{IO}<\mathrm{II})$, while dioxygen in turn exists in the intermediate reduction (IR) state $\left(\mathrm{O}_{2}{ }^{1-}<\mathrm{O}_{2}{ }^{\mathrm{m}-}<\mathrm{O}_{2}{ }^{2-} ; 1<\right.$ $\mathrm{m}<2$ ) as shown in $\mathbf{V}$ of Fig. 3. The intermediate valence structure $\mathbf{V}$ expresses the coupling between $\mathrm{Cu}(\mathrm{IO})$ and $\mathrm{O}_{2}(\mathrm{IR})$ anion.

In the models 1-4, the total charge densities on the $\mathrm{Cu}$ sites ( $\mathrm{Cu} 1$ and $\mathrm{Cu} 2)$ estimated with the X-ray structures were 2.16, 2.03, 2.23, and 1.76, respectively, showing that the back charge transfer from the peroxide to the $\mathrm{Cu}$ (II) sites in 4 is much stronger than those in 1-3. This tendency is attributable to the conversion from peroxide to oxygen molecule due to the distorted $\mathrm{Cu}_{2} \mathrm{O}_{2}$ core, which causes the strong mixing of the character of triplet oxygen molecule to the electronic structure of the $\mathrm{Cu}_{2} \mathrm{O}_{2}$ core. After geometry optimizations of 1-4, the charge densities on the $\mathrm{Cu}$ sites reduced, namely the oxidation number of the $\mathrm{Cu}$ ions become close to monovalent cation. It indicates the decrease of the magnetic couplings between the $\mathrm{Cu}$ ions as shown above.

\section{Natural orbitals and chemical indices}

\subsection{Natural orbitals}

The orbital correlation diagram is exhibited in Fig. 4. Fig. 5 illustrates the SONOs of 1-4 in the LS state. The bonding symmetric $(S) d_{x y}-d_{x y}$ orbital of the dicopper site interacts with S-type orbitals $\left(\sigma^{*}, \pi_{\mathrm{v}}{ }^{*}\right.$, etc) of peroxide in SONO-1, while SONO+1 consists of the antisymmetric (A) $\mathrm{d}_{\mathrm{xy}}+\mathrm{d}_{\mathrm{xy}}$ orbital and the $\pi_{\mathrm{h}}{ }^{*}$ one. The $\pi_{\mathrm{h}}{ }^{*}$ orbital is stabilized by the symmetry-allowed orbital interactions to afford HOMO as shown in Fig. 4. The SONOs delocalize on the whole $\mathrm{Cu}_{2} \mathrm{O}_{2}$ core. The delocalized orbital on the $x y$-plane can be attributable to the antiferromagnetic superexchange interactions between the $\mathrm{Cu}(\mathrm{II})$ ions via dioxygen. The SONO-1 of $\mathbf{1}$ was composed of the $d_{x y}-d_{x y}$ and $\sigma^{*}$ orbitals because of the planar $\mathrm{Cu}_{2} \mathrm{O}_{2}$ core structures. On the other hand, that of 4 consisted of the $d_{x y}-d_{x y}$ and $\pi^{*}$ orbitals because the butterfly core structure allows the d orbitals to interact with $\pi^{*}$ orbitals. In 2 and 3 , both the $\sigma^{*}$ and $\pi^{*}$ orbitals of peroxide was involved in the formation of the SONO-1. These results indicate that the architecture of the $\mathrm{Cu}_{2} \mathrm{O}_{2}$ core strongly influences the $\mathrm{d}-\mathrm{p}$ orbital interaction, attributing to the magnetic couplings and the chemical bond characters.

\subsection{Occupation numbers and chemical indices}

The natural orbital analysis clearly demonstrates that the $\mathrm{Cu}_{2} \mathrm{O}_{2}$ bonds exhibit intermediate bonding. Chemical indices such as effective bond order should be a useful index for the investigation of bond character (Isobe et al., 2003; Takano et al., 2008; Takano and Yamaguchi, 2007; Yamaguchi, 1990). Using the occupation numbers of SONOs (Table 4), these indices for 1-4 can be estimated with Eqs. (7), (10), (11), and (12).

The $b$ values for 1-4 are summarized in Table 5 . The $b_{\text {somo }}$ values indicate that the $\mathrm{Cu}$ sites intermediately interact with each other through the binding dioxygen. The $\mathrm{Cu}_{2} \mathrm{O}_{2}$ bond is not a closed-shell type molecular orbital configuration $\left(b_{\mathrm{SOMO}}=1.0\right)$, but an open shell configuration, where electrons partially occupy the antibonding SONO+1. This open-shell molecular orbital configuration ensures the strong antiferromagnetic superexchange

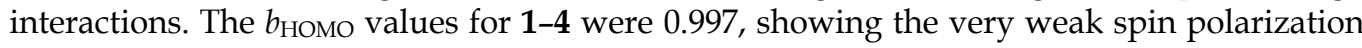
effect. The $b_{\mathrm{SOMO}}$ values increases as $4<<3<\mathbf{1}<2$, indicating the same tendency of the $\left|J_{\mathrm{ab}}\right|$ values. Judging from the charge densities and the $b_{\text {SOMO }}$ values, 4 estimates much smaller superexchange interaction between $\mathrm{Cu}(\mathrm{II})$ ions via peroxide than $\mathbf{1 - 3}$, showing the smaller absolute value of $J_{\mathrm{ab}}$. On the other hand, the $b_{\mathrm{HOMO}}$ values in models 1-4 are close to each 
other, indicating that the spin polarization effects are insensitive to the ligand coordination. The natural orbital analysis clearly demonstrates that the antiferromagnetic couplings of these $\mathrm{Cu}_{2} \mathrm{O}_{2}$ system are dominated by the superexchange interaction and that the $\mathrm{Cu}$ (II) ions and the peroxide ion show an intermediate orbital interaction $\left(0<b_{\mathrm{SOMO}}<1.0\right)$.
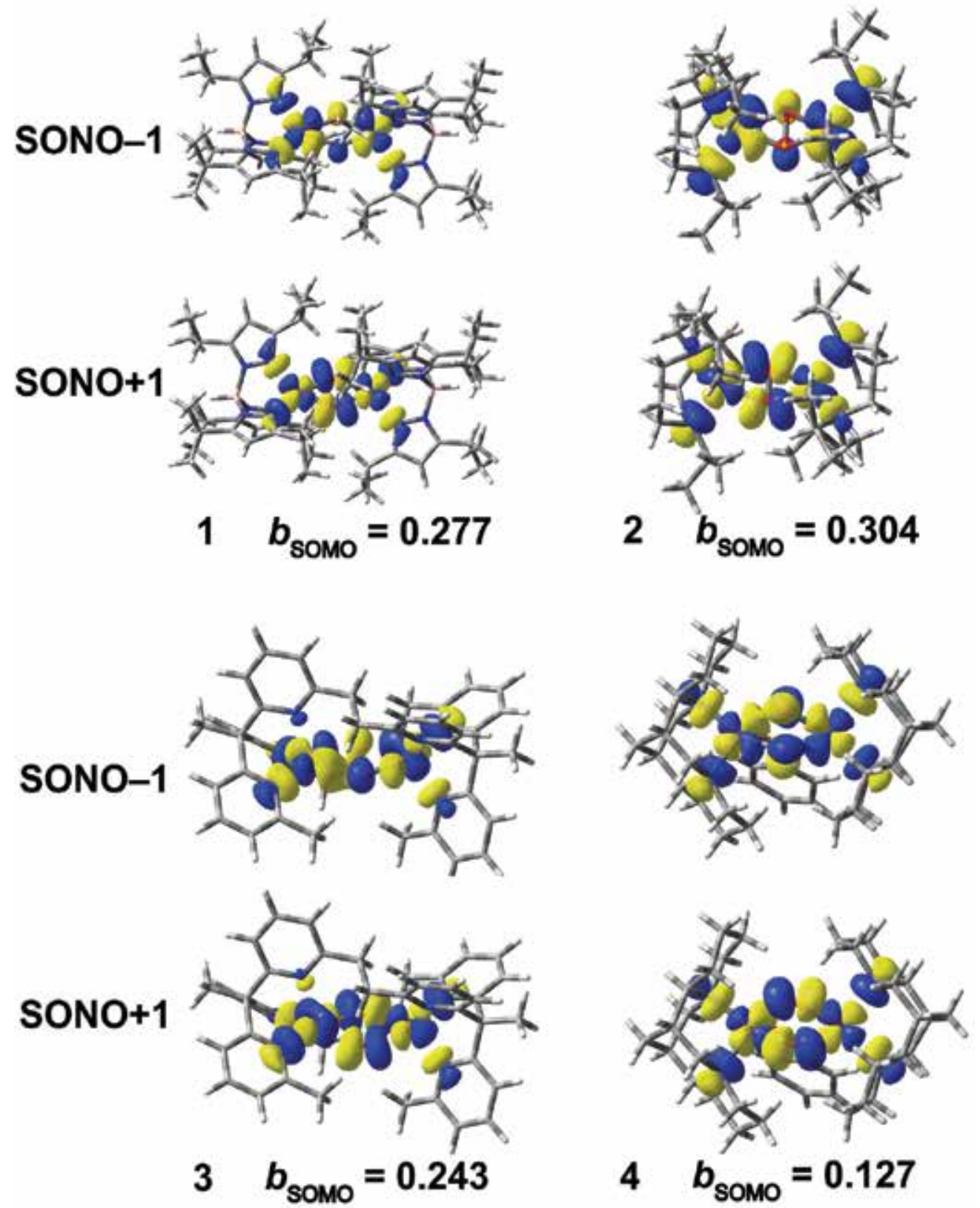

Fig. 5. Singly occupied bonding (SONO-1) and antibonding (SONO+1) natural orbitals (SONOs) obtained from UBHandHLYP calculations in the LS state with the X-ray crystallographic structures of 1-4. 


\begin{tabular}{cccccc}
\hline Model & & HOMO & SOMO-1 & SOMO+1 & LUMO \\
\hline $\mathbf{1}$ & X-ray & 1.997 & 1.277 & 0.723 & 0.003 \\
& Opta $^{\mathrm{a}}$ & 1.997 & 1.236 & 0.764 & 0.003 \\
$\mathbf{2}$ & X-ray & 1.997 & 1.304 & 0.696 & 0.003 \\
& Opta $^{\mathrm{a}}$ & 1.997 & 1.253 & 0.747 & 0.003 \\
$\mathbf{3}$ & X-ray & 1.997 & 1.243 & 0.757 & 0.003 \\
& Opta $^{\mathrm{a}}$ & 1.997 & 1.222 & 0.778 & 0.003 \\
$\mathbf{4}$ & X-ray & 1.997 & 1.127 & 0.873 & 0.003 \\
& Opta $^{\mathrm{a}}$ & 1.998 & 1.091 & 0.909 & 0.002 \\
\hline
\end{tabular}

${ }^{a}$ Geometrical parameters were fully optimized.

Table 4. Occupation Numbers of HOMO and SOMOs (SONOs) of 1-4 by UBHandHLYP in the LS State.

\begin{tabular}{crcc}
\hline Model & & $b_{\text {HOMO }}$ & $b_{\text {SOMO }}$ \\
\hline $\mathbf{1}$ & X-ray & 0.997 & 0.277 \\
& Opta $^{\mathrm{a}}$ & 0.997 & 0.236 \\
$\mathbf{2}$ & X-ray & 0.997 & 0.304 \\
& Opta $^{\mathrm{a}}$ & 0.997 & 0.253 \\
\multirow{3}{*}{$\mathbf{4}$} & X-ray & 0.997 & 0.243 \\
& Opta $^{\mathrm{a}}$ & 0.997 & 0.222 \\
& X-ray & 0.997 & 0.127 \\
& Opta $^{\mathrm{a}}$ & 0.998 & 0.091 \\
\hline
\end{tabular}

${ }^{a}$ Geometrical parameters were fully optimized.

Table 5. Effective bond orders of HOMOs and SOMOs (SONOs) of 1-4 by UBHandHLYP in the LS State.

The information entropy $(I)$, unpaired electron density $(U)$, and diradical character $(Y)$ are also useful indices for the investigation of the character of the chemical bond (Soda et al., 2000). Using the occupation numbers of the SONOs, the indices were estimated with Eqs. (10)-(12). In Table 6, the I values express the loss of the covalency of the chemical bonds. The $U$ values are equivalent to the deviation from exact singlet value $\mathrm{LS}<\mathrm{S}^{2}>=0$ for the brokensymmetry solution. The $Y$ values, which characterize the double excitation of electrons occupying the bonding NOs, indicate how much the unoccupied electrons can localize on the spin sites. They can be regarded as useful indices to diagnose the bond nature and measures of the strength of orbital interactions. Previously, we investigated the nature of the chemical bonds of organic systems, showing that intermolecular radical character of organic radicals, phenalenyl radical dimeric pair, were represented as 0.65 of the $I$ value, 0.838 of the $U$ value, and 0.30 of the $Y$ value (Takano et al., 2002b, c). Comparing to the organic system, we found that the biomimetic complexes, 1-4, show strong radical character, that is, rather weak orbital interactions; however, the weak bond character is sufficient for the cooperative intramolecular (through-bond) charge transport for the oxygen trapping, namely the dioxygen binding in the biomimetic complexes, 1-4. The estimated effective bond orders and information entropies exhibit similar behavior responsible for the bond formation via electron delocalization. On the other hand, the evaluted unpaired electron densities are responsible for the electron correlation effects, and therefore they are about 1.0 for all the complexes, indicating an important role of the electron correlation effect. The electron 
delocalization and electron correlations are competitive in the copper-oxygen systems. This is the reason why we must use UBHandHLYP instead of UB3LYP and UBLYP as discussed in detail in our previous paper (Takano et al., 2001; Takano and Yamaguchi, 2007). The optimized structure provided the larger chemical indices for 1-4 than the X-ray structure due to the relaxation of the $\mathrm{Cu}_{2} \mathrm{O}_{2}$ core. It indicates that crystal environment strengthens the orbital interactions of the dicopper core of the biomimetic complexes, 1-4. The evaluated information entropies, unpaired electron densities, and diradical characters show the following tendency: $4>>3>1>2$, qualitatively corresponds to the order of the magnetic coupling: $J_{\mathrm{ab}}(\mathbf{4})>>J_{\mathrm{ab}}(\mathbf{3})>J_{\mathrm{ab}}(\mathbf{1})>J_{\mathrm{ab}}(2)$.

\begin{tabular}{ccccc}
\hline Model & & $I_{\mathrm{n}}$ & $U$ & $Y$ \\
\hline $\mathbf{1}$ & X-ray & 0.775 & 0.923 & 0.485 \\
& Opta $^{\mathrm{a}}$ & 0.811 & 0.944 & 0.553 \\
$\mathbf{2}$ & X-ray $^{\mathrm{O}}$ & 0.75 & 0.908 & 0.443 \\
& Opta $^{\mathrm{a}}$ & 0.796 & 0.936 & 0.524 \\
$\mathbf{3}$ & X-ray & 0.805 & 0.941 & 0.541 \\
& Opta $^{\mathrm{a}}$ & 0.823 & 0.951 & 0.577 \\
$\mathbf{4}$ & X-ray & 0.903 & 0.984 & 0.75 \\
& Opta $^{\mathrm{a}}$ & 0.931 & 0.992 & 0.819 \\
\hline
\end{tabular}

${ }^{a}$ Geometrical parameters were fully optimized.

Table 6. Information entropies $(I)$, unpaired electron densities $(U)$, and diradical characters $(Y)$ of SOMOs (SONOs) of 1-4 by UBHandHLYP in the LS State.

\section{Concluding remarks}

The magnetic couplings and the nature of the chemical bonds of the $\mathrm{Cu}_{2}\left(\mu-\eta^{2}: \eta^{2}-\mathrm{O}_{2}\right)$ core of the biomimetic models 1-4 were investigated by hybrid DFT calculations such as UBHandHLYP calculations.

We estimated the magnetic coupling constants and examined the electronic structures for 14 from the viewpoint of the shape and symmetry of the natural orbitals and chemical indices. Analysis of natural orbitals and effective bond orders provide us useful insights that the antiferromagnetic couplings of the $\mathrm{Cu}_{2} \mathrm{O}_{2}$ systems are dominated by the superexchange interaction, that $\mathrm{Cu}(\mathrm{II})$ ions and peroxide show an intermediate orbital interaction $(0<b<$ 1.0), and that the distortion of the $\mathrm{Cu}_{2} \mathrm{O}_{2}$ core from a planar structure to a butterfly structure and elongation of the $\mathrm{Cu}-\mathrm{O}$ bonds cause the reduction of orbital interactions between the symmetric $d_{x y}-d_{x y}$ orbital of the dicopper site and the $\pi_{v}{ }^{*}$ orbital of $\mathrm{O}_{2}$ and between the antisymmetric $d_{x y}+d_{x y}$ orbital and the $\pi_{h}{ }^{*}$ orbital, weakening the magnetic coupling between the $\mathrm{Cu}$ sites via $\mu-\eta^{2}: \eta^{2}$-peroxide. The information entropy, unpaired electron density, and diradical character exhibited the useful information about the chemical bonds in the dicopper core of biomimetic complexes, 1-4. Especially, the evaluted unpaired electron densities indicates the reason why we must use UBHandHLYP instead of UB3LYP and UBLYP. Thus, natural orbitals and chemical indices such as effective bond order, information entropy, unpaired electron density, and diradical character are useful for elucidation of the nature of chemical bonds based on the broken symmetry DFT calculation. 


\section{References}

Allen, F.H. (2002). The Cambridge Structural Database: a quarter of a million crystal structures and rising. Acta Crystallogr Sect B-Struct Sci 58, 380-388.

Aullón, G., Gorun, S.M., \& Alvarez, S. (2006). Effects of tris(pyrazolyl)borato ligand substituents on dioxygen activation and stabilization by copper compounds. Inorganic Chemistry 45, 3594-3601.

Becke, A.D. (1988). Density-functional exchange-energy approximation with correct asymptotic behavior. Phys Rev A 38, 3098.

Becke, A.D. (1993). Density-functional thermochemistry. III. The role of exact exchange. The Journal of Chemical Physics 98, 5648-5652.

Benson, D.E., Haddy, A.E., \& Hellinga, H.W. (2002). Converting a maltose receptor into a nascent binuclear copper oxygenase by computational design. Biochemistry 41, 3262-3269.

Cahoy, J., Holland, P.L., \& Tolman, W.B. (1999). Experimental studies of the interconversion of $\mu-\eta^{2}: \eta^{2}$-peroxo- and bis( $($-oxo $)$ dicopper complexes. Inorganic Chemistry 38, 2161-2168.

Cooksey, C.J., Garratt, P.J., Land, E.J., Pavel, S., Ramsden, C.A., Riley, P.A., \& Smit, N.P.M. (1997). Evidence of the indirect formation of the catecholic intermediate substrate responsible for the autoactivation kinetics of tyrosinase. Journal of Biological Chemistry 272, 26226-26235.

Cramer, C.J., Kinsinger, C.R., \& Pak, Y. (2003). Mechanism of intramolecular C-H bond activation in $\left[(\mathrm{LCu})_{2}\left((-\mathrm{O})_{2}\right]^{2+}(\mathrm{L}=1,4,7\right.$-trialkyl-1,4,7-triazacyclononane): Quantum mechanical/molecular mechanical modeling. Journal of Molecular Structure: THEOCHEM 632, 111-120.

Cramer, C.J., \& Pak, Y. (2001). Transition state for intramolecular C-H bond cleavage in $\left[(\mathrm{LCu})_{2}(\mu-\mathrm{O})_{2}\right]^{2+}(\mathrm{L}=1,4,7$-tribenzyl-1,4,7-triazacyclononane). Theoretical Chemistry Accounts 105, 477-480.

Cuff, M.E., Miller, K.I., Van Holde, K.E., \& Hendrickson, W.A. (1998). Crystal structure of a functional unit from Octopus hemocyanin. Journal of Molecular Biology 278, 855870.

Frisch, M.J., Trucks, G.W., Schlegel, H.B., Scuseria, G.E., Robb, M.A., Cheeseman, J.R., Montgomery, J.A., Vreven, T., Kudin, K.N., Burant, J.C., et al. (2003). Gaussian 03, Revision C.02.

Funahashi, Y., Nishikawa, T., Wasada-Tsutsui, Y., Kajita, Y., Yamaguchi, S., Arii, H., Ozawa, T., Jitsukawa, K., Tosha, T., Hirota, S., et al. (2008). Formation of a bridged butterflytype $\mu-\eta^{2}: \eta^{2}$-peroxo dicopper core structure with a carboxylate group. Journal of the American Chemical Society 130, 16444-16445.

Gresh, N., Policar, C., \& Giessner-Prettre, C. (2002). Modeling copper(I) complexes: SIBFA molecular mechanics versus ab initio energetics and geometrical arrangements. J Phys Chem A 106, 5660-5670.

Hariharan, P.C., \& Pople, J.A. (1973). The influence of polarization functions on molecular orbital hydrogenation energies. Theor Chim Acta 28, 213-222.

Hay, P.J. (1977). Gaussian basis sets for molecular calculations. The representation of $3 \mathrm{~d}$ orbitals in transition-metal atoms. The Journal of Chemical Physics 66, 4377-4384. 
Hehre, W.J., Ditchfield, R., \& Pople, J.A. (1972). Self-Consistent Molecular Orbital Methods. XII. Further Extensions of Gaussian-Type Basis Sets for Use in Molecular Orbital Studies of Organic Molecules. The Journal of Chemical Physics 56, 2257-2261.

Holm, R.H., Kennepohl, P., \& Solomon, E.I. (1996). Structural and Functional Aspects of Metal Sites in Biology. Chemical Reviews 96, 2239-2314.

$\mathrm{Hu}$, Z., George, G.N., \& Gorun, S.M. (2001). Flourine encapsulation and stabilization of biologically relevant low-valence copper-oxo cores. Inorganic Chemistry 40, 48124813.

Isobe, H., Takano, Y., Kitagawa, Y., Kawakami, T., Yamanaka, S., Yamaguchi, K., \& Houk, K.N. (2002). Extended Hartree-Fock (EHF) theory of chemical reactions VI: hybrid DFT and post-Hartree-Fock approaches for concerted and non-concerted transition structures of the Diels-Alder reaction. Molecular Physics 100, 717-727.

Isobe, H., Takano, Y., Kitagawa, Y., Kawakami, T., Yamanaka, S., Yamaguchi, K., \& Houk, K.N. (2003). Systematic comparisons between broken symmetry and symmetryadapted approaches to transition states by chemical indices: A case study of the Diels-Alder reactions. J Phys Chem A 107, 682-694.

Karlin, K.D., \& Tyeklár, Z. (1993). Bioinorganic Chemistry of Copper (New York, Chamman \& Hall).

Kitagawa, Y., Saito, T., Ito, M., Shoji, M., Koizumi, K., Yamanaka, S., Kawakami, T., Okumura, M., \& Yamaguchi, K. (2007). Approximately spin-projected geometry optimization method and its application to di-chromium systems. Chemical Physics Letters 442, 445-450.

Kitajima, N., Fujisawa, K., Fujimoto, C., Moro-oka, Y., Hashimoto, S., Kitagawa, T., Toriumi, K., Tatsumi, K., \& Nakamura, A. (1992). A new model for dioxygen binding in hemocyanin. Synthesis, characterization, and molecular structure of the $\mu-\eta^{2}: \eta^{2}$ peroxo dinuclear copper(II) complexes, $\left[\mathrm{Cu}\left(\mathrm{HB}\left(3,5-\mathrm{R}_{2} \mathrm{pz}\right)_{3}\right)\right]_{2}\left(\mathrm{O}_{2}\right)(\mathrm{R}=i-\mathrm{Pr}$ and $\mathrm{Ph})$. Journal of the American Chemical Society 114, 1277-1291.

Kitajima, N., Fujisawa, K., Moro-oka, Y., \& Toriumi, K. (1989). $\mu-\eta^{2}: \eta^{2}-$ Peroxo binuclear copper complex, $\left[\mathrm{Cu}\left(\mathrm{HB}\left(3,5-\mathrm{iPr}_{2} \mathrm{pz}\right)_{3}\right)\right]_{2}\left(\mathrm{O}_{2}\right)$. Journal of the American Chemical Society 111, 8975-8976.

Kitajima, N., \& Moro-oka, Y. (1994). Copper-dioxygen complexes. Inorganic and bioinorganic perspectives. Chemical Reviews 94, 737-757.

Kodera, M., Kajita, Y., Tachi, Y., Katayama, K., Kano, K., Hirota, S., Fujinami, S., \& Suzuki, M. (2004). Synthesis, Structure, and Greatly Improved Reversible $\mathrm{O}_{2}$ Binding in a Structurally Modulated $\mu-\eta^{2}: \eta^{2}-$ Peroxodicopper(II) Complex with RoomTemperature Stability. Angewandte Chemie - International Edition 43, 334-337.

Kodera, M., Katayama, K., Tachi, Y., Kano, K., Hirota, S., Fujinami, S., \& Suzuki, M. (1999). Crystal structure and reversible $\mathrm{O}_{2}$-binding of a room temperature stable $\mu-\eta^{2}: \eta^{2-}$ peroxodicopper(II) complex of a sterically hindered hexapyridine dinucleating ligand. Journal of the American Chemical Society 121, 11006-11007.

Lam, B.M.T., Halfen, J.A., Young Jr, V.G., Hagadorn, J.R., Holland, P.L., Lledós, A., Cucurull-Sánchez, L., Novoa, J.J., Alvarez, S., \& Tolman, W.B. (2000). Ligand macrocycle structural effects on copper-dioxygen reactivity. Inorganic Chemistry 39, 4059-4072.

Lee, C., Yang, W., \& Parr, R.G. (1988). Development of the Colle-Salvetti correlation-energy formula into a functional of the electron density. Physical Review B 37, 785. 
Maseras, F., \& Morokuma, K. (1995). IMOMM: A new integrated ab initio + molecular mechanics geometry optimization scheme of equilibrium structures and transition states. J Comput Chem 16, 1170-1179.

Mitani, M., Mori, H., Takano, Y., Yamaki, D., Yoshioka, Y., \& Yamaguchi, K. (2000a). Density functional study of intramolecular ferromagnetic interaction through $m$-phenylene coupling unit (I): UBLYP, UB3LYP, and UHF calculations. Journal of Chemical Physics 113, 4035-4051.

Mitani, M., Yamaki, D., Takano, Y., Kitagawa, Y., Yoshioka, Y., \& Yamaguchi, K. (2000b). Density-functional study of intramolecular ferromagnetic interaction through $\mathrm{m}$ phenylene coupling unit (II): Examination of functional dependence. Journal of Chemical Physics 113, 10486-10504.

Onishi, T., Takano, Y., Kitagawa, Y., Kawakami, T., Yoshioka, Y., \& Yamaguchi, K. (2001). Theoretical study of the magnetic interaction for M-O-M type metal oxides. Comparison of broken-symmetry approaches. Polyhedron 20, 1177-1184.

Ramirez, J.C., Soriano, C., Esquivel, R.O., Sagar, R.P., Ho, M.H., \& Smith, V.H. (1997). Jaynes information entropy of small molecules: Numerical evidence of the Collins conjecture. Phys Rev A 56, 4477-4482.

Rappe, A.K., Casewit, C.J., Colwell, K.S., Goddard, W.A., \& Skiff, W.M. (1992). UFF, a full periodic table force field for molecular mechanics and molecular dynamics simulations. Journal of the American Chemical Society 114, 10024-10035.

Roos, B.O., Linse, P., Siegbahn, P.E.M., \& Blomberg, M.R.A. (1982). A simple method for the evaluation of the 2nd-order perturbation energy from external double-excitations with a CASSCF reference wavefunction. Chem Phys 66, 197-207.

Saito, T., Kataoka, Y., Nakanishi, Y., Matsui, T., Kitagawa, Y., Kawakami, T., Okumura, M., \& Yamaguchi, K. (2010). Which hybrid GGA DFT is suitable for $\mathrm{Cu}_{2} \mathrm{O}_{2}$ systems if the spin contamination error is removed? Chem Phys 368, 1-6.

Saito, T., Kitagawa, Y., Shoji, M., Nakanishi, Y., Ito, M., Kawakami, T., Okumura, M., \& Yamaguchi, K. (2008). Theoretical studies on the structure and effective exchange integral $\left(\mathrm{J}_{\mathrm{ab}}\right)$ of an active site in oxyhemocyanin (oxyHc) by using approximately spin-projected geometry optimization (AP-opt) method. Chemical Physics Letters $456,76-79$.

Salem, L. (1982). Electrons in Chemical Reactions: First Principle (New York, Wiley).

Siegbahn, P.E.M. (2006). The performance of hybrid DFT for mechanisms involving transition metal complexes in enzymes. Journal of Biological Inorganic Chemistry 11, 695-701.

Siegbahn, P.E.M., \& Wirstam, M. (2001). Is the Bis-m-Oxo Cu2(III,III) state an intermediate in tyrosinase? Journal of the American Chemical Society 123, 11819-11820.

Soda, T., Kitagawa, Y., Onishi, T., Takano, Y., Shigeta, Y., Nagao, H., Yoshioka, Y., \& Yamaguchi, K. (2000). Ab initio computations of effective exchange integrals for $\mathrm{H}$ $\mathrm{H}, \mathrm{H}-\mathrm{He}-\mathrm{H}$ and $\mathrm{Mn}_{2} \mathrm{O}_{2}$ complex: comparison of broken-symmetry approaches. Chemical Physics Letters 319, 223-230.

Solomon, E.I., Baldwin, M.J., \& Lowery, M.D. (1992). Electronic structures of active sites in copper proteins: Contributions to reactivity. Chemical Reviews 92, 521-542.

Staroverov, V.N., \& Davidson, E.R. (2000). Transition regions in the cope rearrangement of 1,5-hexadiene and its cyano derivatives. Journal of the American Chemical Society $122,7377-7385$. 
Takano, Y., Isobe, H., \& Yamaguchi, K. (2008). Theoretical studies on electronic structures and chemical indices of the active site of oxygenated and deoxygenated hemerythrin. Bulletin of the Chemical Society of Japan 81, 91-102.

Takano, Y., Kitagawa, Y., Onishi, T., Yoshioka, Y., Yamaguchi, K., Koga, N., \& Iwamura, H. (2002a). Theoretical studies of magnetic interactions in $\mathrm{Mn}(\mathrm{II})(\mathrm{hfac})_{2}\{\mathrm{di}(4-$ pyridyl)phenylcarbene $\}$ and $\mathrm{Cu}(\mathrm{II})(\mathrm{hfac})_{2}\{\mathrm{di}(4$-pyridyl)phenylcarbene $\}$. Journal of the American Chemical Society 124, 450-461.

Takano, Y., Koizumi, K., \& Nakamura, H. (2009). Theoretical studies of the magnetic couplings and the chemical indices of the biomimetic models of oxyhemocyanin and oxytyrosinase. Inorg Chim Acta 362, 4578-4584.

Takano, Y., Kubo, S., Onishi, T., Isobe, H., Yoshioka, Y., \& Yamaguchi, K. (2001). Theoretical studies on the magnetic interaction and reversible dioxygen binding of the active site in hemocyanin. Chemical Physics Letters 335, 395-403.

Takano, Y., Soda, T., Kitagawa, Y., Onishi, T., Yoshioka, Y., \& Yamaguchi, K. (2000). Theoretical study on magnetic interactions of Mn-pi conjugated system. Molecular Crystals and Liquid Crystals 342, 291-296.

Takano, Y., Taniguchi, T., Isobe, H., Kubo, T., Morita, Y., Yamamoto, K., Nakasuji, K., Takui, T., \& Yamaguchi, K. (2002b). Effective exchange integrals and chemical indices for a phenalenyl radical dimeric pair. Chemical Physics Letters 358, 17-23.

Takano, Y., Taniguchi, T., Isobe, H., Kubo, T., Morita, Y., Yamamoto, K., Nakasuji, K., Takui, T., \& Yamaguchi, K. (2002c). Hybrid density functional theory studies on the magnetic interactions and the weak covalent bonding for the phenalenyl radical dimeric pair. Journal of the American Chemical Society 124, 11122-11130.

Takano, Y., \& Yamaguchi, K. (2007). Hybrid density functional study of ligand coordination effects on the magnetic couplings and the Dioxygen binding of the models of hemocyanin. International Journal of Quantum Chemistry 107, 3103-3119.

Takatsuka, K., Fueno, T., \& Yamaguchi, K. (1978). Distribution of odd electrons in groundstate molecules. Theor Chim Acta 48, 175-183.

Tatewaki, H., \& Huzinaga, S. (1979). A systematic preparation of new contracted Gaussian type orbital set. I. Transition metal atoms from Sc to Zn. The Journal of Chemical Physics 71, 4339-4348.

Trofimenko, S. (1999). Scorpionates: the coordination chemistry of polypyrazolborate ligands (London, Imperial College Press).

Vosko, S.H., Wilk, L., \& Nusair, M. (1980). Accurate spin-dependent electron liquid correlation energies for local spin-density calculations-a critical analysis. Can J Phys $58,1200-1211$.

Yamaguchi, K. (1990). In Self-Consistent Field Theory and Applications, R. Carbo, and M. Klobukowski, eds. 


\title{
Bioactive Microarc Oxidized $\mathrm{TiO}_{2}$-based Coatings for Biomedical Implication
}

\author{
Daqing Wei and Yu Zhou \\ Harbin Institute of Technology
}

China

\section{Introduction}

Hydroxyapatite $\left(\mathrm{Ca}_{10}\left(\mathrm{PO}_{4}\right)_{6}(\mathrm{OH})_{2}, \mathrm{HA}\right)$ and bioactive glass-ceramic $\left(\mathrm{SiO}_{2}-\mathrm{CaO}-\mathrm{P}_{2} \mathrm{O}_{5}-\mathrm{MO}\right.$ $(\mathrm{M}=\mathrm{Na}, \mathrm{Mg}$, etc.)) both exhibit good bioactivity. Unfortunately, these bioactive ceramic materials are not suitable for load-bearing conditions on account of their poor mechanical properties (Liu et al., 2004; Morais et al., 2007). As compared to these brittle ceramics, metal materials such as titanium and its alloys exhibit excellent mechanical toughness and strength (Liu et al., 2004). However, they have poor osteoinductive properties because of their bioinert feature. Thus, to prepare bioactive coatings on titanium and its alloys is an approach to resolving the disadvantages of ceramic and metal biomaterials. Many surface modifying techniques (e.g., plasma spraying (Zheng et al., 2000), sol-gel method (Wen et al., 2007; Balamurugan et al., 2007), biomimetic and electrochemical deposition (Zhang et al., 2005)) have been developed to deposit bioactive coating on titanium and its alloys.

Microarc oxidation (MAO) is a relatively convenient and effective technique to deposit bioceramic coatings on the surfaces of Ti and its alloys (Yerokhin et al., 1999). This technique can introduce various desired elements into titania-based coatings and produce various functional coatings with a porous structure (Yerokhin et al., 1999). Additionally, MAO coatings usually exhibit good interfacial bonding to substrates (Wang et al., 2009). Moreover, it is very suitable to modify various substrates with complex geometries. Most of MAO coatings mainly contained anatase, rutile and amorphous or crystalline calcium phosphate phase (at high applied voltage). It is difficult to form HA phase during MAO process because of a high temperature and a rapid cooling rate at anodic surfaces. And the apatite-forming ability of the MAO coatings is not very good. Thus, the subsequent modifications have been developed such as sol-gel, ultraviolet (UV) irradiation and hydrothermal treatment (Li et al., 2005; Han et al., 2008; Ishizawa et al., 1995). Authors have developed a simple method of chemical-treatment to modify the surfaces of the MAO coatings for improving the inducation ability for the formation of biomimetic apatite (Weia et al., 2007; Wei et al., 2008).

In addition, the formation process of biomimetic apatite on the bioactive surface has been noted for long time. However, some important problems were also not solved until now. Thus, systematic thermodynamic and kinetic calculations of Gibbs free energy $(\Delta G)$ and nucleation rate $(\log J)$ for the formation of various calcium phosphates (CaPs) in different simulated body fluids (SBFs) was conducted by authors. The structure and formation process of biomimetic apatite were reported. 


\section{Formation bioactive MAO coatings on titanium and its alloys for inducing biomimetic apatite}

Using MAO technique to deposit bioactive ceramic coatings on titanium and its alloys has received much attention in recent years (Fini et al., 1999; Zhu et al., 2001; Zhu et al., 2002; Zhu et al., 2002; Han et al., 2003; Frauchiger et al., 2004; Li et al., 2004; Song et al., 2004; Li et al., 2005; Zhang et al., 2004; Rodriguez et al., 2003). To prepare the bioactive coatings on titanium and its alloys, introductions of $\mathrm{Ca}$ and $\mathrm{P}$ elements into MAO were taken into account. In previous studies, various calcium salts such as calcium acetate, $\beta$ glycerophosphate disodium salt pentahydrate, calcium glycerophosphate and calcium dihydrogen phosphate were used in MAO process.

In the previous research, titania-based coatings on titanium were prepared by MAO at various applied voltages $(250-500 \mathrm{~V})$ in an electrolytic solution containing $\beta$ glycerophosphate disodium salt pentahydrate and calcium acetate monohydrate (Song et al. 2004). Ca- and P-containing titania-based coatings were formed on the titanium substrates. The phase, $\mathrm{Ca}$ and $\mathrm{P}$ content, morphology and thickness of the coatings were strongly dependent on the applied voltage. In particular, Ca- and P-containing compounds such as $\mathrm{CaTiO}_{3}, \mathrm{Ca}_{2} \mathrm{P}_{2} \mathrm{O}_{7}$ and $\mathrm{Ca}_{3}\left(\mathrm{PO}_{4}\right)_{2}$ were produced at higher voltages $(>450 \mathrm{~V})$. Besides the $\beta$ glycerophosphate disodium salt pentahydrate, calcium glycerophosphate was also used. In the electrolyte of calcium glycerophosphate and calcium acetate, the titanium anodic oxide coating is porous, highly crystalline, and rich in $\mathrm{Ca}$ and $\mathrm{P}$. The optimum condition is that the concentration of the electrolyte is $0.02 \mathrm{M}$ calcium glycerophosphate and $0.15 \mathrm{M}$ calcium acetate, and current density and final voltage are $70 \mathrm{~A} / \mathrm{m}^{2}$ and $350 \mathrm{~V}$, respectively (Zhu et al., 2001). To achieve high amounts of $\mathrm{Ca}$, researchers (Frauchiger et al., 2004) used a new electrolyte containing Ca-EDTA chelate complex to prepare MAO coating. Based on the previous researches, authors have developed the MAO coatings only containing P. The MAO coating formed at 300 and $350 \mathrm{~V}$ after oxidizing for $5 \mathrm{~min}$ show good surface properties.

In addition, as mentioned above, to achieve high amounts of Ca, EDTA was used to add into the electrolyte. Authors further investigated the effects of applied voltage and electrolyte composition and concentrations on the structures of the MAO coatings (e.g., phase composition, surface morphology, micropore number and size, element distribution on the surface and cross-sectional characters, etc) (Weib et al., 2007). In the MAO coating formed on Ti6Al4V, $\mathrm{TiO}_{2}$ nanocrystals were randomly distributed in $\mathrm{Ca}$ - and P-doped matrix, and their crystal size was $30-40 \mathrm{~nm}$. With increasing the applied voltage, the surface toughness, micropores size, $\mathrm{Ca}$ and $\mathrm{P}$ concentrations, coating thickness are increased. High applied voltage promoted the formation of rutile. However, besides the $\mathrm{TiO}_{2}$ and amorphous phase were observed, no other phase were detected at different applied voltages, not similar the previous researches. In this electrolyte, the EDTA impeded the crystallization of $\mathrm{TiO}_{2}$ crystals during the MAO process. Besides the above electrolyte, the similar electrolyte with addition of HA was also investigated. It was found that the addition of HA promoted the formation of anatase (Weic et al., 2007).

Recently, bioactive glass components (BGC) doped MAO coatings on titanium were noticed by authors. This electrolyte was composed of $\mathrm{Ca}\left(\mathrm{CH}_{3} \mathrm{COO}\right)_{2} \cdot \mathrm{H}_{2} \mathrm{O}, \mathrm{Na}_{2} \mathrm{SiO}_{3}$, EDTA-2Na and $\mathrm{NaOH}$. And the results indicated that the surface morphology, phase composition and elemental concentrations are highly depended on the applied voltages. And BGC doped $\mathrm{TiO}_{2}$-based coatings exhibit good interface bonding with titanium, showing a graded 
structure in elemental concentrations of $\mathrm{Si}, \mathrm{Ca}, \mathrm{Na}, \mathrm{O}$, Ti, etc.. Moreover, these elements were distributed uniformly on the surface of $\mathrm{BGC}$ doped $\mathrm{TiO}_{2}$-based coatings. During the MAO treatment process, Ti could not be oxidized sufficiently, thus the TiO phase was also formed according to XPS resutls.

Based on the above researches, generally speaking, the structures such as phase composition, surface morphology and elemental composition of the MAO coatings are highly dependent on the MAO process parameters such as the applied voltage, oxidizing time, frequency, duty cycle and electrolyte composition, etc., especially the applied voltage and electrolyte composition.

\section{The improvement of bioactivity of MAO coatings by surface modifications}

Most of MAO coatings mainly contain anatase, rutile and amorphous or crystalline calcium titanate and calcium phosphate phase, etc. (at high applied voltage). It is difficult to form HA phase during MAO process because of a high temperature and a rapid cooling rate at anodic surfaces. In fact, MAO coating may possess specific surface structures such as amorphous phase, nonequilibrium solid, complex mixed-compounds, etc, since complex plasma physical and chemical reactions such as ionization and plasma condensation, etc occur at electrode surfaces. Based on the previous researches (Han et al., 2008), the apatiteforming ability and bioactivity of most of MAO coatings are not very good. Thus, subsequent activation methods such as sol-gel, ultraviolet (UV) irradiation and hydrothermal treatments have been investigated to improve the surface bioactivity of MAO coatings, as mentioned above.

In the previous research (Song et al., 2004; Han et al., 2003), the MAO coating containing Ca and $\mathrm{P}$ formed at higher voltages $(>450 \mathrm{~V})$ was immersed in a simulated body fluid for 28 days, a carbonated hydroxyapatite was induced on the surfaces. This MAO coating contained $\mathrm{CaTiO}_{3}, \mathrm{Ca}_{2} \mathrm{P}_{2} \mathrm{O}_{7}$ and $\mathrm{Ca}_{3}\left(\mathrm{PO}_{4}\right)_{2}$, which play key roles for the formation of the biomimetic apatite (Han et al., 2003).

Researchers have sought the methods to improve the ability of the MAO coatings to induce the formation of biomimetic apatite. The hydrothermal treatment is the common method to change the surface structure of the MAO coatings to formation of hydroxyaptite/titania composite coatings. Regarding to the hydrothermal treatment of the MAO coatings, researches have been extensively reported in the past. As reported, a MAO coating containing $\mathrm{Ca}$ and $\mathrm{P}$ was formed on commercially pure titanium which was anodized in an electrolytic solution of dissolved $\beta$-glycerophosphate and calcium acetate. HA crystals were precipitated by hydrothermally treating the MAO coating at $300^{\circ} \mathrm{C}$. The morphology, composition, and amount of HA crystals were significantly dependent on the electrolytes composition (Ishizawa et al., 1995).

In the previous research (Huang et al. 2004), surface modification of titanium implant is processed by microarc oxidation and hydrothermal treatment. A porous surface with a biologically active bone-like apatite layer was formed. The apatite layer consists of very fine crystals and high crystallinity and is integrated with the titanium alloy substrate with a graded structure without a distinct interface. Such a bioactive layer is expected not only to enhance the bone ingrowth into the porous structure, but also to improve the interlocking between implant and bone.

The Ca-doped MAO coating without addition of P was also investigated (Song et al., 2005). This MAO coating showed no apatite-formation during the testing process. However, after a 
hydrothermal treatment at $250^{\circ} \mathrm{C}$, apatite was formed on the surfaces of the $\mathrm{CaTiO}_{3}$ embedded titania after 28 days, which was closely related to the formation of amorphous $\mathrm{Ca}(\mathrm{OH})_{2}$ and presumably surface Ti-OH groups.

The other treatment method is using UV irradiation to modify MAO titania coating. Based on the previous researches (Han et al., 2008), an enhanced bioactivity and cell response to titania after UV irradiation was achieved. Compared to the MAO coating, the UV-irradiated MAO coatings do not exhibit any obvious change in surface roughness, morphology, grain size and phase component; however, they have more abundant basic $\mathrm{Ti}-\mathrm{OH}$ groups and become more hydrophilic because the water contact angle decreases significantly. In SBF, bonelike apatite-forming ability is significantly stronger on the UV-irradiated coatings than the MAO coating.

Sol-gel was also used to change the bioactivity of the MAO coatings. Thin HA layers could be deposited on MAO coatings by sol-gel method ( $\mathrm{Li}$ et al., 2005). The bioactivity of the MAO coating was improved further by the sol-gel HA coating on the MAO treated Ti. The porous morphology and roughness of the MAO coatings was changed slightly after sol-gel treatment.

Based on the above researches, authors have developed a simple chemical-treatment to modify the surface of the MAO coatings (Weia et al., 2007; Wei et al., 2008). In this process, the MAO coatings were treated by $\mathrm{NaOH}$ aqueous solution with different concentrations. After alkali-treatment, the surfaces of the MAO coatings containing $\mathrm{Ca}$ and $\mathrm{P}$ become rough, and the $\mathrm{Ca}$ and $\mathrm{P}$ concentrations decrease with increasing the concentration of $\mathrm{NaOH}$ solution. When $5 \mathrm{~mol} / \mathrm{L} \mathrm{NaOH}$ solution was used, amorphous calcium titanate hydrogel was formed on the surface, showing a nanoflake-like morphology with an approximately oriented structure. During the alkali-treatment process, Ca and P on the surface of the MAO coating show a process of dissolution. At the same time, negatively charged $\mathrm{HTiO}_{3}$ - ions are formed on the MAO coating surface due to the attack of $\mathrm{OH}^{-}$on the $\mathrm{TiO}_{2}$ phase of the $\mathrm{MAO}$ coating. Then, the negatively charged ions could incorporate sodium from the alkali solution and calcium from the alkali solution and MAO coating to form the titanates hydrogels.

In the case of MAO coatings containing P after alkali-treatment (Wei et al., 2008), ribbon-like products with an interlaced morphology were found on the surface. During the chemical etching process, $\mathrm{P}$ of the surface of the MAO coating dissolved into the $\mathrm{NaOH}$ aqueous solution. Negatively charged $\mathrm{HTiO}_{3}$ - ions are formed on the $\mathrm{MAO}$ coating surface due to the attack of $\mathrm{OH}^{-}$ions on $\mathrm{TiO}_{2}$ phase of the $\mathrm{MAO}$ coating. The negatively charged $\mathrm{HTiO}_{3}$ - ions could incorporate sodium ions from the $\mathrm{NaOH}$ aqueous solution to form sodium titanate.

\section{Structure and formation of biomimetic apatite on the surface of chemical- treated MAO coatings}

\subsection{The composition of biomimetic apatite}

Apatites have been used in medicine and dentistry for long time. The interest in one group member, HA, arises from its similarity to bone apatite, the major component of the inorganic phase of bone, which plays a key role in the calcification and resorption processes of bone. Different phases of calcium phosphate ceramics such as $\mathrm{HA}$ and $\mathrm{Ca}_{3}\left(\mathrm{PO}_{4}\right)_{2}$ (TCP) can be used in medicine, depending on whether a bioactive or a resorbable material is desired. Apatite is the name given to a group of crystals of the general chemical formula $\mathrm{M}_{10}\left(\mathrm{RO}_{4}\right) \mathrm{X}_{2}$, where $\mathrm{R}$ is most commonly phosphorus, $\mathrm{M}$ could be one of several metals, although it 
usually calcium, and $\mathrm{X}$ is commonly hydroxide or a halogen such as fluorine or chlorine. Hydroxyapatite belongs to the apatite family, which is the most commonly used calcium phosphate in the medical field, as it possesses excellent biocompatibility and is osteoconductive.

The apatite structure is very hospitable in allowing the substitutions of many other ions, which makes people to bethink of biological apatites. The biological apatites constitute the mineral phase of calcified tissues such as bone, dentine and enamel in the body. They are similar to synthetic HA, but they differ from HA in composition, stoichiometry, and physical and mechanical properties. Biological apatites are usually calcium-deficient as a result of various substitutions at regular $\mathrm{HA}$ lattice points. The general chemical formula for biological apatites is $(\mathrm{Ca}, \mathrm{M})_{10}\left(\mathrm{PO}_{4}, \mathrm{CO}_{3}, \mathrm{Y}\right)_{6}(\mathrm{OH}, \mathrm{F}, \mathrm{Cl})_{2}$. Where $\mathrm{M}$ represents metallic elements such as $\mathrm{Na}, \mathrm{K}$, and $\mathrm{Mg}$, and $\mathrm{Y}$ represents functional groups such as acid phosphate, sulfates, etc..

As well known, the bones and teeth of all vertebrates are natural composite materials, where one of the components is an inorganic solid, carbonate hydroxyapatite. Thus, the formation of the carbonate hydroxyapatite in vitro by biomimetic method is worthy to be noticed. According to the previous researches [Müller et al., 2006; Weia et al., 2007; Wei et al., 2008], the biomimetic apatites of A-type slightly substituted carbonated-HA (SCHA-A) and B-type slightly substituted carbonated-HA with $\mathrm{HPO}_{4}{ }^{2-}$ group (HPO4-SCHA-B) have been reported extensively. At the same time, $\mathrm{Na}, \mathrm{K}, \mathrm{Mg}$ and $\mathrm{Cl}$ elements could be also introduced into these apatites, however, the concentrations of these elements are very small. In addition, it is interesting that the induced biomimetic apatite presented two-level porous structure on microand nano-scales, which has less been reported according to authors' studies [Wei et al., 2009].

\subsection{The formation of $\mathrm{Ti}-\mathrm{OH}$ group and its induction for biomimetic apatites}

During the SBF immersion process, a variety of reactions such as dissolution, ion exchange and precipitation occur on the surface of the chemical-treated MAO coatings. The Ca could release from the surface of the MAO coating. An ionic exchange between $\mathrm{Ca}^{2+}$ of the chemical-treated MAO coatings and $\mathrm{H}_{3} \mathrm{O}^{+}$of the SBF takes place during SBF immersion process. As a result, abundant $\mathrm{Ti}-\mathrm{OH}$ groups are formed on the surface of the chemicaltreated MAO coating. Also, the $\mathrm{Na}^{+}$ions on the chemical-treated $\mathrm{MAO}$ coating could participate in the ionic exchange process, promoting the formation of $\mathrm{Ti}-\mathrm{OH}$ groups.

Generally, substrates with functionalized surfaces such as $-\mathrm{OH}, \mathrm{PO}_{4} \mathrm{H}_{2}, \mathrm{COOH}, \mathrm{SO}_{3} \mathrm{H}$, and $\mathrm{CONH}_{2}$ groups facilitate the formation of bonelike apatite in SBF or solutions containing various ions with respect to apatite [Toworfe et al., 2006; Liu et al., 2002].

The nucleation and growth of biomimetic apatite generate from an interfacial molecular recognition between functionalized surface and ions with respect to apatite in solutions. The interfacial molecular recognition involves certain aspects such as electrostatic potential interaction [Toworfe et al., 2006]. According to the previous researches [Weia et al., 2007; Wei et al., 2008; Toworfe et al., 2006], the electrostatic potential interaction is that the Ti-OH groups incorporate calcium ions, and then absorb the phosphate and carbonic acid ions in the SBF. The absorbed negative ions such as phosphate and carbonic acid ions could further attract the calcium ions etc.. With increasing the immersion time, the supersaturation degree of solution with respect to apatite near the vicinity of the Ti-OH group occurs, which triggers the apatite nucleation. Once the apatite nuclei are formed, they grow spontaneously by assembling the remaining calcium, phosphate and carbonic acid ions around apatite nuclei in the SBF. 


\section{Theoretical calculation and understanding the mechanism of biomimetic apatite formation in SBF on the surfaces of bioactive coatings}

In order to understand the formation of various calcium phosphates (CaPs) thoroughly, theoretical calculations were conducted by many researchers. In the previous results, thermodynamic analyses of $\mathrm{CaPs}$ have been reported in the solutions including $\mathrm{CaCl}_{2}+\mathrm{NaCl}+\mathrm{NaH}_{2} \mathrm{PO}_{4}+\mathrm{NaOH}$ [Boistelle et al., 1990], $\mathrm{HA}$ powder $+\mathrm{HCl}+\mathrm{KOH}+\mathrm{NaCl}[\mathrm{Feng}$ et al., 2000], $\mathrm{NaCl}+\mathrm{CaCl}_{2}+\mathrm{KH}_{2} \mathrm{PO}_{4}$ [Wu et al., 1997] $\mathrm{CaCl}_{2}+\mathrm{KH}_{2} \mathrm{PO}_{4}+\mathrm{KOH}$ [Koutsoukos et al., 1980; Koutsoukos et al., 1981], $\mathrm{Ca}\left(\mathrm{NO}_{3}\right)_{2}+\mathrm{KH}_{2} \mathrm{PO}_{4}+\mathrm{NaOH}$ [aHeughebaert et al., 1984; bHeughebaert et al., 1984], $\mathrm{CaCl}_{2}+\mathrm{KH}_{2} \mathrm{PO}_{4}+\mathrm{NaCl}+\mathrm{KOH}$ [Koutsopoulos et al., 1994; Koutsopoulos et al., 2000;]. In fact, understanding the formation of CaPs in SBF is very important and valuable, since SBFs were widely used to obtain biomimetic apatite or evaluate the apatite-inducing ability of biomaterials. Thus, the thermodynamics and kinetics for the fomation of various CaPs in SBF are necessary. Based on this purpose, researchers [Boistelle, et al., 1990; Lu et al., 2005] have conducted the thermodynamic and kinetics of $\mathrm{Ca}_{4}\left(\mathrm{HPO}_{4}\right)\left(\mathrm{PO}_{4}\right)_{2}(\mathrm{OCP}), \mathrm{CaHPO}_{4}(\mathrm{DCPD})$ and HA, etc..

Besides OCP, DCPD and HA, researches indicated that biomimetic apatites such as carbonated HA containing $\mathrm{HPO}_{4}{ }^{2-}$ group were formed on the samples after SBF immersion [Müller et al., 2006; Weia et al., 2007; Wei et al., 2008;]. In addition, the relationships among the ions concentration, supersaturation, Gibbs free energy, critical nucleation are not very clear. We feel that there lacks an overall and systematic thermodynamic and kinetic guidelines for various CaPs such as DCPD, OCP, $\mathrm{Ca}_{3}\left(\mathrm{PO}_{4}\right)_{2}(\mathrm{TCP}), \mathrm{HA}, \mathrm{Ca} 9\left(\mathrm{HPO}_{4}\right)\left(\mathrm{PO}_{4}\right)_{5} \mathrm{OH}$ (DOHA), A-type slightly substituted carbonated-HA (SCHA-A) and B-type slightly substituted carbonated-HA with $\mathrm{HPO}_{4}{ }^{2-}$ group (HPO4-SCHA-B), etc. formation in SBF. In this work, authors calculated the thermodynamic and kinetic processes of common $\mathrm{CaPs}$ mentioned above. According to the calculated results, the apatites induced by bioactive coatings were explained.

\subsection{Analytical model}

In this work, various SBFs with different ion concentrations were considered as shown in Table 1, because the composition, concentration, $\mathrm{pH}$, etc. of the SBFs could affect the phase composition, crystallinity and crystal growth rate of biomimetic apatites. In the text, if no other explanation, the used SBF for calculation is referred to the c-SBF.

\begin{tabular}{|l|c|c|c|c|c|c|c|c|}
\hline \multirow{2}{*}{ Blood plasma and SBF } & \multicolumn{7}{|c|}{ Ion concentration $(\mathrm{mmol} / \mathrm{L})$} \\
\cline { 2 - 10 } & $\mathrm{Na}^{+}$ & $\mathrm{K}^{+}$ & $\mathrm{Mg}^{2+}$ & $\mathrm{Ca}^{2+}$ & $\mathrm{Cl}^{-}$ & $\mathrm{HCO}^{3-}$ & $\mathrm{HPO}_{4}^{2-}$ & $\mathrm{SO}_{4}{ }^{2-}$ \\
\hline Blood plasma & 142.0 & 5.0 & 1.5 & 2.5 & 103.0 & 27.0 & 1.0 & 0.5 \\
\hline c-SBF & 142.0 & 5.0 & 1.5 & 2.5 & 147.8 & 4.2 & 1.0 & 0.5 \\
\hline r-SBF & 142.0 & 5.0 & 1.5 & 2.5 & 103.0 & 27.0 & 1.0 & 0.5 \\
\hline i-SBF & 142.0 & 5.0 & 1.0 & 1.6 & 103.0 & 27.0 & 1.0 & 0.5 \\
\hline m-SBF & 142.0 & 5.0 & 1.5 & 2.5 & 103.0 & 10.0 & 1.0 & 0.5 \\
\hline HBSS & 142 & 5.81 & 126 & 0.898 & 146 & 4.17 & 0.779 & 0.406 \\
\hline
\end{tabular}

Table 1. Composition of different SBFs and blood plasma

If the supersaturation regarding to apatites of the SBFs is high enough, the formation of the apatites in the SBFs is possible on thermodynamically. The classical equation of Gibbs free energy change in supersaturated sultions was shown below [Mullin et al., 2001]: 


$$
\Delta G=-\frac{\mathrm{R} T}{\mathrm{n}} \ln (S)=-\frac{\mathrm{R} T}{\mathrm{n}} \ln \left(\frac{A_{\mathrm{P}}}{K_{\mathrm{sp}}}\right)
$$

where $\Delta G$ is the Gibbs free energy per mole of ionic units that compose CaPs in solution, $R$ is the gas constant $\left(8.314 \mathrm{JK}^{-1} \mathrm{~mol}^{-1}\right), \mathrm{n}$ is the number of ion units in a CaPs molecule, $T$ is the absolute temperature and $S$ is the supersaturation that is defined by the ratio of the activity product of ion units composing precipitates $(A p)$ to the corresponding solubility product (Ksp).

In this work, the $\Delta G$ for the formation of the CaPs including DCPD, OCP, DOHA, TCP, HA, SCHA-A-0.5, SCHA-A-1, SCHA-B-1 and HPO4-SCHA-B-0.5 were investigated, as shown in Table 2.

\begin{tabular}{|c|c|c|c|}
\hline $\begin{array}{l}\text { Calcium } \\
\text { phosphates }\end{array}$ & Reactions & Ksp & Reference \\
\hline DCPD & $\mathrm{Ca}^{2+}+\mathrm{HPO}_{4}^{2-=} \mathrm{CaHPO}_{4}$ & $10^{-6.622}$ & [Lu et al., 2005] \\
\hline $\mathrm{OCP}$ & $4 \mathrm{Ca}^{2+}+\mathrm{HPO}_{4}{ }^{2-+}+2 \mathrm{PO}_{4}^{3-}=\mathrm{Ca}_{4}\left(\mathrm{HPO}_{4}\right)\left(\mathrm{PO}_{4}\right)_{2}$ & $10^{-36.48}$ & [Lu et al., 2005] \\
\hline DOHA & $\begin{array}{l}9 \mathrm{Ca}^{2+}+5 \mathrm{PO}_{4}^{3-+}+\mathrm{HPO}_{4}^{2-+} \mathrm{OH}- \\
=\mathrm{Ca}_{9}\left(\mathrm{HPO}_{4}\right)\left(\mathrm{PO}_{4}\right)_{5} \mathrm{OH}\end{array}$ & $10^{-85.1}$ & [Lu et al., 2005] \\
\hline TCP & $3 \mathrm{Ca}^{2+}+2 \mathrm{PO}_{4}^{3-}=\mathrm{Ca}_{3}\left(\mathrm{PO}_{4}\right)_{2}$ & $10^{-117.2}$ & [Müller et al., 2006] \\
\hline HA & 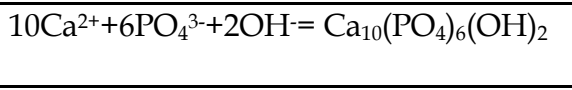 & $2.83 \times 10^{-30}$ & $\begin{array}{l}\text { [Koutsopoulos et } \\
\text { al., 2000] }\end{array}$ \\
\hline SCHA-A-0.5 & $\begin{array}{l}10 \mathrm{Ca}^{2+}+6 \mathrm{PO}_{4}^{3-+1} / 2 \mathrm{CO}_{3}{ }^{2-+} \mathrm{OH}^{-} \\
=\mathrm{Ca}_{10}\left(\mathrm{PO}_{4}\right)_{6}(\mathrm{OH})\left(\mathrm{CO}_{3}\right)_{0.5}\end{array}$ & $10-115.6$ & [Lu et al., 2005] \\
\hline SCHA-A-1 & $10 \mathrm{Ca}^{2+}+6 \mathrm{PO}_{4}^{3-}+\mathrm{CO}_{3}^{2-}=\mathrm{Ca}_{10}\left(\mathrm{PO}_{4}\right)_{6} \mathrm{CO}_{3}$ & $10^{-85}$ & [Ito et al., 1997] \\
\hline SCHA-B-1 & $\begin{array}{l}9 \mathrm{Ca}^{2+}+5 \mathrm{PO}_{4}{ }^{3-}+\mathrm{CO}_{3}{ }^{2-}+\mathrm{OH}^{-} \\
=\mathrm{Ca}_{9}\left(\mathrm{PO}_{4}\right)_{5}\left(\mathrm{CO}_{3}\right)(\mathrm{OH})\end{array}$ & $10^{-73.7}$ & [Ito et al., 1997] \\
\hline $\begin{array}{l}\text { HPO4- } \\
\text { SCHA-B-0.5 }\end{array}$ & 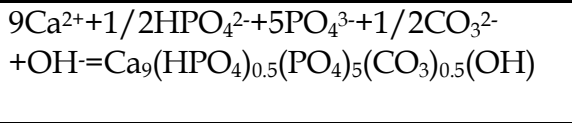 & 10-115.4 & $\begin{array}{l}\text { [Lu et al., 2005; } \\
\text { Koutsopoulos et } \\
\text { al., 2000] }\end{array}$ \\
\hline
\end{tabular}

Table 2. Kinds of calcium phosphates and their synthesized reactions

For examples, the supersaturation $S$ of OCP and SCHA-A- 0.5 were calculated by the following equations:

$$
\begin{gathered}
S(\mathrm{OCP})=\frac{\left(\gamma_{\mathrm{Ca}^{2+}}\right)^{4}\left(\gamma_{\mathrm{HPO}_{4}^{2-}}\right)\left(\gamma_{\mathrm{PO}_{4}^{3-}}\right)^{2}\left[\mathrm{Ca}^{2+}\right]^{4}\left[\mathrm{HPO}_{4}^{2-}\right]\left[\mathrm{PO}_{4}^{3-}\right]^{2}}{K_{\mathrm{SP}}(\mathrm{OCP})} \\
S(\mathrm{SCHA}-\mathrm{A}-0.5)=\frac{\left(\gamma_{\mathrm{Ca}^{2+}}\right)^{10}\left(\gamma_{\mathrm{PO}_{4}^{3-}}\right)^{6}\left(\gamma_{\mathrm{CO}_{3}^{2-}}\right)^{0.5}\left(\gamma_{\mathrm{OH}^{-}}\right)\left[\mathrm{Ca}^{2+}\right]^{10}\left[\mathrm{PO}_{4}^{3-}\right]^{6}\left[\mathrm{CO}_{3}^{2-}\right]^{0.5}\left[\mathrm{OH}^{-}\right]}{K_{\mathrm{SP}}(\mathrm{SCHA}-\mathrm{A}-0.5)}
\end{gathered}
$$

The $S$ of the other CaPs could be calculated by similar method.

In these equations, $\left[\mathrm{Ca}^{2+}\right],\left[\mathrm{PO}_{4}^{3-}\right],\left[\mathrm{CO}_{3}^{2-}\right],\left[\mathrm{OH}^{-}\right]$and $\left[\mathrm{HPO}_{4}^{2-}\right]$ are the equilibrium concentrations of the corresponding ions in the SBF. The $\gamma_{\mathrm{Ca} 2}+, \gamma_{\mathrm{PO}} 43-, \gamma_{\mathrm{CO}} \mathrm{C}_{-}, \gamma_{\mathrm{OH}} \mathrm{H}$ and $\gamma_{\mathrm{HPO}} \mathrm{H}_{-}$ are the activity coefficients of the corresponding ions in the SBF. In order to obtain the equilibrium concentrations, the possible chemical association/dissociation reactions 
regarding to CaPs were should be taken into account firstly as shown in Table 3 [Oyane, et al., 2003].

\begin{tabular}{|l|l|}
\hline Chemical reaction & $\mathrm{K}$ \\
\hline $\mathrm{H}_{2} \mathrm{CO}_{3}$ (aq.) $\leftrightarrow \mathrm{H}^{+}+\mathrm{HCO}_{3}^{-}$ & $10^{-6.31}$ \\
\hline $\mathrm{HCO}_{3}^{-} \leftrightarrow \mathrm{H}^{+}+\mathrm{CO}_{3}^{2-}$ & $10^{-10.25}$ \\
\hline $\mathrm{HPO}_{4}$ (aq.) $\leftrightarrow \mathrm{H}++\mathrm{H} 2 \mathrm{PO} 4-$ & $10^{-2.196}$ \\
\hline $\mathrm{H}_{2} \mathrm{PO}_{4}^{-} \leftrightarrow \mathrm{H}^{+}+\mathrm{HPO}_{4}^{2-}$ & $10^{-7.185}$ \\
\hline $\mathrm{HPO}_{4}^{-} \leftrightarrow \mathrm{H}^{+}+\mathrm{PO}_{4}^{3-}$ & $10^{-12.19}$ \\
\hline $\mathrm{Ca}^{2+}+\mathrm{HCO}_{3}{ }^{-} \leftrightarrow \mathrm{CaHCO}_{3}{ }^{+}$ & $10^{1.16}$ \\
\hline $\mathrm{Ca}^{2+}+\mathrm{CO}_{3}^{2-} \leftrightarrow \mathrm{CaCO}_{3}$ (aq.) & $10^{3.38}$ \\
\hline $\mathrm{Ca}^{2+}+\mathrm{OH}^{-} \leftrightarrow \mathrm{CaOH}^{+}$ & 25.12 \\
\hline $\mathrm{Ca}^{2+}+\mathrm{H}_{2} \mathrm{PO}_{4}^{-} \leftrightarrow \mathrm{CaH}_{2} \mathrm{PO}_{4}{ }^{+}$ & 31.9 \\
\hline $\mathrm{Ca}^{2+}+\mathrm{HPO}_{4}{ }^{2-} \leftrightarrow \mathrm{CaHPO}_{4}$ (aq.) & $6.81 \times 10^{2}$ \\
\hline $\mathrm{Ca}^{2+}+\mathrm{PO}_{4}^{3-} \leftrightarrow \mathrm{CaPO}_{4}^{-}$ & $3.46 \times 10^{6}$ \\
\hline
\end{tabular}

Table 3. The possible chemical association/dissociation reactions in SBF [Oyane, et al., 2003] Based on these association/dissociation reactions, three mass balance equations are used to calculate the ions equilibrium concentration in SBF:

$$
\begin{gathered}
\mathrm{C}_{\mathrm{Ca}_{2+}=}=\left[\mathrm{Ca}^{2+}\right]+\left[\mathrm{CaOH}^{+}\right]+\left[\mathrm{CaCO}_{3}\right]+\left[\mathrm{CaH}_{2} \mathrm{PO}_{4}{ }^{+}\right]+\left[\mathrm{CaHPO}_{4}\right]+\left[\mathrm{CaPO}_{4}^{-}\right]+\left[\mathrm{CaHCO}_{3}{ }^{+}\right] \\
\mathrm{CHCO}-_{-}=\left[\mathrm{HCO}_{3}^{-}\right]+\left[\mathrm{CO}_{3}{ }^{2-}\right]+\left[\mathrm{H}_{2} \mathrm{CO}_{3}\right]+\left[\mathrm{CaHCO}_{3}{ }^{+}\right]+\left[\mathrm{CaCO}_{3}\right] \\
\mathrm{C}_{\mathrm{HPO}_{4}{ }_{-}=}=\left[\mathrm{HPO}_{4}{ }^{2-}\right]+\left[\mathrm{HPO}_{4}\right]+\left[\mathrm{PO}_{4}{ }^{3-}\right]+\left[\mathrm{CaH}_{2} \mathrm{PO}_{4}^{+}\right]+\left[\mathrm{CaHPO}_{4}\right]+\left[\mathrm{H}_{2} \mathrm{PO}_{4}^{-}\right]+\left[\mathrm{CaPO}_{4}^{-}\right]
\end{gathered}
$$

Where the $\mathrm{C}_{\mathrm{Ca} 2+}, \mathrm{C}_{\mathrm{HCO}-}$ and $\mathrm{C}_{\mathrm{HPO} 42-}$ is the initial addition concentration shown in Table 1 and the [] is the equilibrium concentration.

According to the equation of Debye-Hückel, the $\gamma_{i}$ can be calculated by the following equations [Mullin et al., 2001]:

$$
\begin{gathered}
\lg \gamma_{i}=-\mathrm{A} z_{i}^{2}\left[\frac{I^{1 / 2}}{1+I^{1 / 2}}-0.3 I\right] \\
I=\frac{1}{2} \sum_{i} c_{i} z_{i}^{2}
\end{gathered}
$$

where $A$ is the Debye-Hückel constant dependent on temperature $A=0.5211\left(37{ }^{\circ} C\right), I$ is the total ionic strength of the SBF, $z_{i}$ is the charge number of ions and $c_{i}$ is the molar concentration of each ion unit.

According to the previous results [Weia et al., 2007; Wei et al., 2008], the apatite formation on the surface of the bioactive coatings is a heterogeneous nucleation process. And the heterogeneous nucleation energy $\left(\Delta G_{\text {het }}\right)$ is calculated by the following equation [Mullin et al., 2001]:

$$
\Delta G_{\text {het }}=\frac{16 \pi \sigma^{3} v^{2} f(\theta)}{3(\mathrm{k} T \ln S)^{2}}
$$


Where $\sigma$ is the surface energy of per unit area (surface tension), $v$ is the molecule volume of crystal, $\mathrm{k}$ is the Boltzmann constant and $T$ is the absolute temperature, the $f(\theta)$ is a function of contacting angle, $\theta$, between crystal body and substrate, and was calculated by the following equation [Mullin et al., 2001]:

$$
f(\theta)=\left(\frac{(2+\cos \theta)(1-\cos \theta)^{2}}{4}\right)
$$

In the Equ. 19, the $\cos \theta$ was calculated by the following equation [Mullin et al., 2001]:

$$
\cos \theta=\frac{\sigma_{\mathrm{L} \beta}-\sigma_{\mathrm{\alpha} \beta}}{\sigma_{\mathrm{\alpha L}}}
$$

where $\sigma_{\mathrm{L} \beta}$ is the interface tension between liquid phase and substrate, $\sigma_{\alpha \beta}$ is the interface tension between nuclear and substrate and $\sigma_{\mathrm{\alpha L}}$ is the interface tension between liquid phase and nuclear. In this work, the $\theta$ was assumed as $90^{\circ}$ based on the previous researches [Wei et al., 2007; Wei et al., 2008; Lu et al., 2005].

In this work, the critical nucleus, $r_{c}$, of various $\mathrm{CaPs}$ were calculated by the following equation [25]:

$$
r_{\mathrm{c}}=\frac{2 \sigma v}{\mathrm{k} T \ln S}
$$

Based on the above data, the nucleation rates of various CaPs were calculated by the following equation [Mullin et al., 2001]:

$$
J=K \cdot \exp \left(-\frac{\Delta G}{\mathrm{k} T}\right)=K \cdot \exp \left(-\frac{16 \pi v^{2} \sigma^{3} f(\theta)}{3 \mathrm{k}^{3} T^{3}(\ln S)^{2}}\right)
$$

In this equation, $K$ is a kinetic factor, which is independent of the substrates and not a constant. For different chemical reactions, the $K$ value is different. Boistelle et al. [Boistelle, et al., 1990] proposed a mathmatics mode to calculate the $K$, as shown in the following equation:

$$
K=K^{\prime} P
$$

where $\mathrm{K}^{\prime}$ is a constant $\left(13.64 \times 10^{-24} \mathrm{~cm}^{-3} \mathrm{~s}^{-1}\right)$ and $P$ is nucleation probability. When the molecular formula of $\mathrm{CaP}$ is $\mathrm{A} 1(\mathrm{n} 1) \mathrm{A} 2(\mathrm{n} 2) \ldots \mathrm{A} i(\mathrm{n} i)$ (where $\mathrm{A} i$ is atom species/molecule and $\mathrm{n} i$ is the amount of the $\mathrm{A} i$ atom/molecule), $P$ was calculated by the following equation [Boistelle, et al., 1990]:

$$
P=\frac{(\mathrm{n} 1+\mathrm{n} 2+\cdots+\mathrm{n} i) ! \times\left[\mathrm{A} 1^{z 1}\right]^{\mathrm{n} 1}\left[\mathrm{~A} 2^{z 2}\right]^{\mathrm{n} 2} \cdots\left[\mathrm{A} i^{z i}\right]^{\mathrm{n} i}}{\mathrm{n} 1 ! \mathrm{n} 2 ! \cdots \mathrm{n} i ! \times\left\{\left[\mathrm{A} 1^{z 1}\right]+\left[\mathrm{A} 2^{z 2}\right]+\cdots+\left[\mathrm{A} i^{z i}\right]\right\}^{(\mathrm{n} 1+\mathrm{n} 2+\cdots+\mathrm{n} i)}}
$$

Where, the $\left[\mathrm{A} i{ }^{i}\right]$ denoted the ion concentration corresponding to the $\mathrm{A} i$ atom/molecule. For example, $P$ of the HPO4-SCHA-B- 0.5 was calculated by the following Equ. 25 : 


$$
P=\frac{(9+0.5+5+0.5+1) ! \times\left[\mathrm{Ca}^{2+}\right]^{9}\left[\mathrm{HPO}_{4}{ }^{2-}\right]^{0.5}\left[\mathrm{PO}_{4}{ }^{3-}\right]^{5}\left[\mathrm{CO}_{3}{ }^{2-}\right]^{0.5}[\mathrm{OH}]^{1}}{9 ! 0.5 ! 5 ! 0.5 ! 1 ! \times\left\{\left[\mathrm{Ca}^{2+}\right]+\left[\mathrm{HPO}_{4}{ }^{2-}\right]+\left[\mathrm{PO}_{4}{ }^{3-}\right]+\left[\mathrm{CO}_{3}{ }^{2-}\right]+[\mathrm{OH}]\right\}^{(9+0.5+5+0.5+1)}}
$$

Boistelle et al. used this equation to calculate the P of DCPD, OCP and HA [Boistelle, et al., 1990]. Leng et al. [Lu et al., 2005] used this mode to further calculate the $P$ of DOHA and SCHA-A-0.5. In this work, the $P$ of TCP, SCHA-A-1, SCHA-B-1 and HPO4-SCHA-B-0.5 were calculated by this equation.

In the previous researches [Lu et al., 2005], the measurements could affect the value of $\sigma$ of the apatite. Even different researchers provided different $\sigma$ values using same measurement [Lu et al., 2005]. Thus, it brought out the difficulty to obtain the accurate value of $\sigma$. In addition, there has no available data for SCHA-A-0.5 and HPO4-SCHA-B-0.5 et al.. Thus it was considered to obtain the $\sigma$ value by theoretical calculation.

In 1990s, Mersmann et al. [Mullin et al., 2001] proposed an equation to calculate the $\sigma$ value:

$$
\sigma=0.414 \mathrm{k} T\left[\frac{\rho_{\mathrm{c}} \mathrm{N}}{M}\right]^{2 / 3} \ln \left[\frac{c_{\mathrm{S}}}{c_{\mathrm{L}}}\right]
$$

where $\mathrm{k}$ is boltzmann constant $\left(1.38 \times 10^{-23} \mathrm{JK}-1\right), \mathrm{N}$ is Avogadro constant $\left(6.02 \times 10^{26} \mathrm{kmol}^{-1}\right), M$ is molar mass $\left(\mathrm{kgkmol}^{-1}\right), \rho_{\mathrm{c}}$ is the crystal density $\left(\mathrm{kgm}^{-3}\right), c_{\mathrm{S}}$ and $c_{\mathrm{L}}$ are the solute concentrations $\left(\mathrm{kmolm}^{-3}\right)$ in the solid and liquid phases.

In order to calculate conveniently, the above equation was deduced further. The following two equations were introduced into the Equ.26.

$$
\begin{gathered}
\rho_{\mathrm{c}}=\frac{M}{\mathrm{~N} \cdot v} \\
c_{\mathrm{S}}=\frac{\rho_{\mathrm{c}}}{M}=\frac{1}{v \mathrm{~N}}
\end{gathered}
$$

And the Equ.26 was rewritten as shown below:

$$
\sigma=-0.414 \mathrm{kT} v^{-2 / 3} \ln \left(v \mathrm{~N} c_{\mathrm{L}}\right)
$$

where $\mathrm{k}$ is boltzmann constant $\left(1.38 \times 10^{-23} \mathrm{JK}^{-1}\right), T$ is absolute temperature, $v$ is molecular volume, $\mathrm{N}$ is Avogadro constant $\left(6.02 \times 10^{26} \mathrm{kmol}^{-1}\right)$ and $c_{\mathrm{L}}$ is the solute concentrations $\left(\mathrm{kmolm}^{-3}\right)$ in the liquid phase.

When the molecular formula of the products is written as $\mathrm{A} 1(\mathrm{n} 1) \mathrm{A} 2(\mathrm{n} 2) \ldots \mathrm{A} i(\mathrm{n} i)$ (where $\mathrm{A} i$ is atom/molecule and $\mathrm{n} i$ is the number of the $\mathrm{A} i$ atom/molecule), $c_{\mathrm{L}}$ was calculated by the following equation:

$$
c_{\mathrm{L}}=\left(\frac{K_{\mathrm{sp}}}{\mathrm{n} 1^{\mathrm{n} 1} \times \mathrm{n} 2^{\mathrm{n} 2} \times \cdots \times \mathrm{n} i^{\mathrm{n} i}}\right)^{\left(\frac{1}{\mathrm{n} 1+\mathrm{n} 2+\cdots+\mathrm{ni}}\right)}
$$

According to the previous researches, there has no available data of the $v$ value of the SCHA-A-0.5, SCHA-A-1, SCHA-B-1 and HPO4-SCHA-B-0.5. Thus it was an assumption that the $v$ values of the SCHA-A-0.5, SCHA-A-1, SCHA-B-1 and HPO4-SCHA-B-0.5 are same to that of HA. The values of $v$ and $c_{\mathrm{L}}$ of various apatites were shown in Table 4 . 


\begin{tabular}{|l|l|l|}
\hline Apatite & $v\left(\mathrm{~m}^{-3}\right)$ & $c_{\mathrm{L}}(\mathrm{mol} / \mathrm{L})$ \\
\hline DCPD & $3.09 \mathrm{E}-28$ & $4.88652 \mathrm{E}-04$ \\
\hline OCP & $3.106 \mathrm{E}-28$ & $2.28312 \mathrm{E}-06$ \\
\hline DOHA & $5.31 \mathrm{E}-28$ & $8.43452 \mathrm{E}-07$ \\
\hline TCP & $1.48 \mathrm{E}-28$ & $4.82695 \mathrm{E}-07$ \\
\hline HA & $5.3 \mathrm{E}-28$ & $4.09944 \mathrm{E}-08$ \\
\hline SCHA-A-0.5 & $5.3 \mathrm{E}-28$ & $3.66998 \mathrm{E}-08$ \\
\hline SCHA-A-1 & $5.3 \mathrm{E}-28$ & $1.37127 \mathrm{E}-06$ \\
\hline SCHA-B-1 & $5.3 \mathrm{E}-28$ & $4.35071 \mathrm{E}-06$ \\
\hline HPO4-SCHA-B-0.5 & $5.3 \mathrm{E}-28$ & $1.96054 \mathrm{E}-08$ \\
\hline
\end{tabular}

Table 4 . The values of $v$ and $c_{\mathrm{L}}$ of various apatites

\subsection{Calculated results}

Fig. 1 shows that the $\Delta G$ of various CaPs decrease with increasing the $\mathrm{pH}(4 \sim 10)$, except of DCPD, of which the $\Delta G$ decreased when $\mathrm{pH}<8$ and increased when $\mathrm{pH}>8$ always being higher than zero, agreeing with the previous study [Lu et al., 2005]. At $\mathrm{pH}=7.4$, the $\Delta G$ of various $\mathrm{CaPs}$ increased as the following sequence: HPO4-SCHA-B-0.5 < SCHA-A-0.5 < HA $<$ TCP $<$ OCP $<$ DOHA $<0<$ DCPD $<$ SCHA-A- $1<$ SCHA-B- 1 . And the $\Delta$ G of HPO4-SCHAB-0.5 and SCHA-A-0.5 are always lower than that of HA with increasing $\mathrm{pH}$.

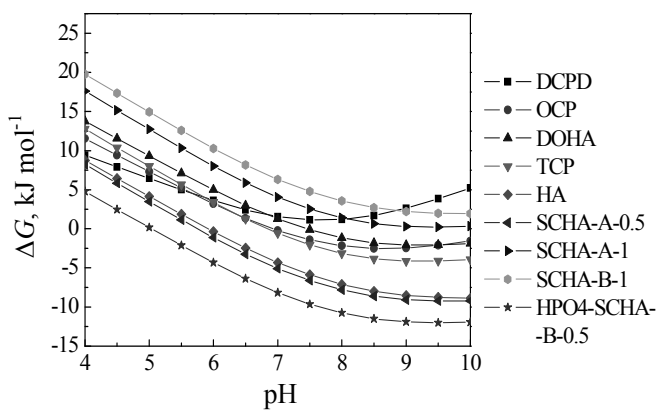

Fig. 1. $\Delta G$ for the formation of various CaPs provided by the SBF

This result suggested that the formation of HPO4-SCHA-B-0.5 and SCHA-A-0.5 is thermodynamically easier than the other $\mathrm{CaPs}$. The theoretical calculation results are agreed with the previous experimental result [Weia et al., 2007; Wei et al., 2008]. In Fig. 1, it was also noticed that the $\Delta G$ increased obviously when the content of the $\mathrm{CO}_{3}{ }^{2-}$ ions in the CaPs is high by comparison of the $\Delta G$ of the HA and SCHA-A-1. Similar result was also observed by comparison of HA and SCHA-B-1.

Fig. 2 shows the change in $\Delta G$ with raising the concentrations of $\mathrm{Ca}^{2+}, \mathrm{HPO}_{4}{ }^{2-}$ and $\mathrm{HCO}_{3}-$ ions and $\mathrm{SBF}$ at $\mathrm{pH}=7.4$. The $\Delta G$ of various $\mathrm{CaPs}$ increased as the following sequence: HPO4SCHA-B-0.5 < SCHA-A-0.5 < HA $<$ TCP $<$ OCP $<$ DOHA $<$ DCPD $<$ SCHA-A-1 < SCHA-B-1 at different ions concentration. Increasing the concentrations of $\mathrm{Ca}^{2+}$ and $\mathrm{HPO}_{4}^{2-}$ ions leaded to the drop of $\Delta G$ of various CaPs as shown Fig. $2 a$ and $b$, because the augment of $\mathrm{Ca}^{2+}$ and $\mathrm{HPO}_{4}{ }^{2-}$ ions concentration improve the $\ln S$ of various CaPs according to Equs.11,12. The increase of the $\mathrm{HCO}_{3}$ - ions seemed to increase the $\Delta G$ of various CaPs slightly, as shown in Fig. 2c. 


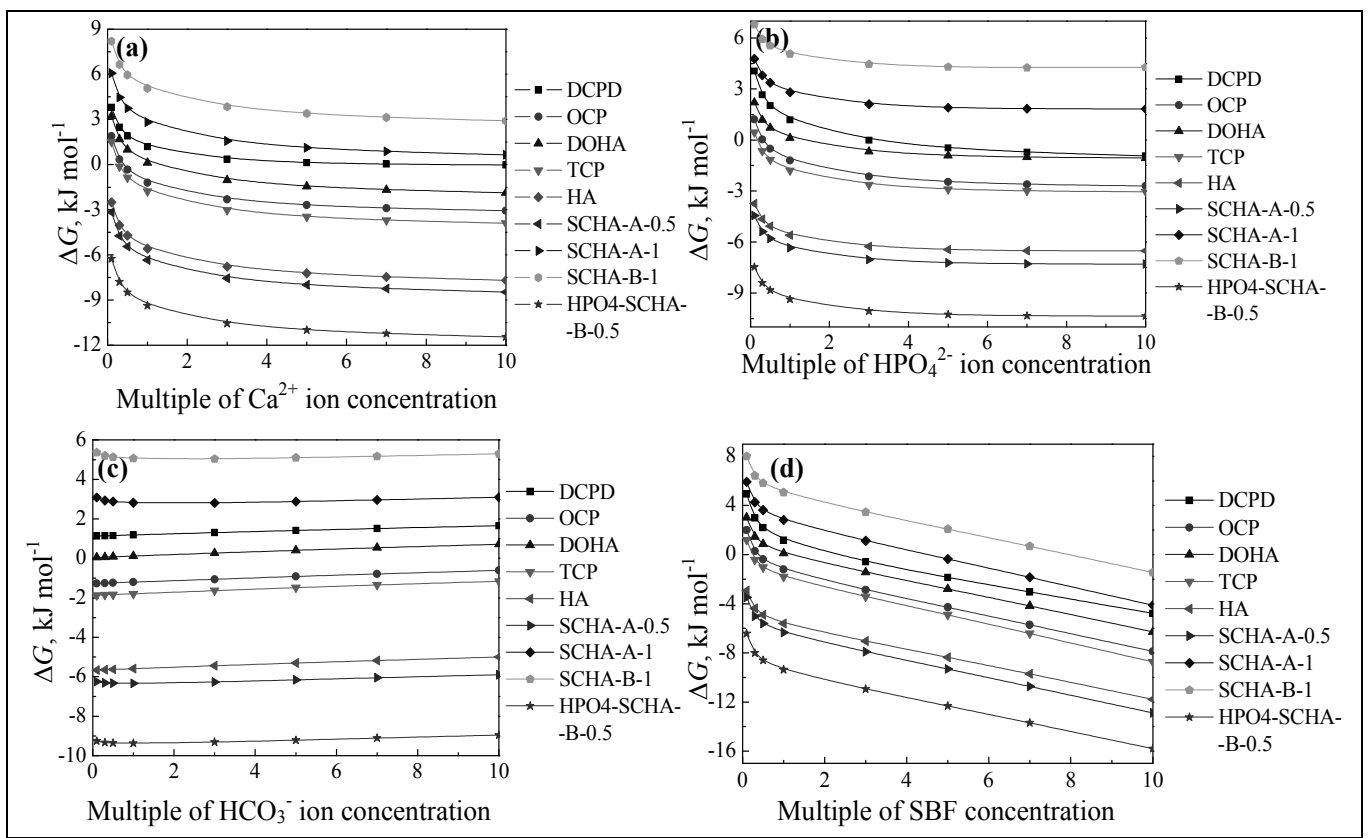

Fig. 2. Change in the $\Delta \mathrm{G}$ with increasing the multiple of $\mathrm{Ca}^{2+}, \mathrm{HPO}_{4}{ }^{2-}$ and $\mathrm{HCO}_{3}$ - ions concentrations and SBF at $\mathrm{pH}=7.4$ : (a) $\mathrm{Ca}^{2+}$, (b) $\mathrm{HPO}_{4}{ }^{2-}$, (c) $\mathrm{HCO}_{3}{ }^{-}$and (d) $\mathrm{SBF}$ concentrations.

Fig. $2 \mathrm{~d}$ shows the decrease in the $\Delta G$ with increasing the multiple of SBF concentrations at $\mathrm{pH}=7.4$. The decrease of $\Delta G$ is also owing to the increase of $\ln S$ as result of the increase in SBF concentration according to Equs.11,12. At $\mathrm{pH}=7.4$, the $\Delta G$ of the various CaPs always ascend as the following sequence: HPO4-SCHA-B-0.5 < SCHA-A-0.5 $<$ HA $<$ TCP $<$ OCP $<$ DOHA $<$ DCPD $<$ SCHA-A-1 < SCHA-B-1 when changing the SBF concentration.

Besides of $\mathrm{Ca}^{2+}, \mathrm{HPO}_{4}{ }^{2-}$ and $\mathrm{HCO}_{3}-$ ions, the effect of other ions in SBF on the $\Delta G$ was also taken into account (The figures not shown here). According to the calculated results, when $\mathrm{Na}^{+}$ions increased from 0 to 20 multiples, a maximum of the $\Delta G$ was observed at the $\mathrm{Na}^{+}$ ions concentration increasing to about 4 multiples. In the cases of the $\mathrm{K}^{+}$and $\mathrm{Mg}^{2+}$ ions, the $\Delta G$ of the various CaPs increased slightly with increasing concentration. These results suggested that the augment of the $\mathrm{K}^{+}$and $\mathrm{Mg}^{2+}$ ions is not suitable for the formation of the $\mathrm{CaPs}$, agreeing the previous experimental results [ $\mathrm{Lu}$ et al., 2005]. The effect of $\mathrm{Cl}^{-}$ions concentration on the $\Delta G$ of various CaPs is similar to that of $\mathrm{Na}^{+}$ions. And the effect of $\mathrm{SO}_{4}{ }^{2-}$ ions concentration on the $\Delta G$ of various CaPs is not obviously.

Based on the calculation of the $S$ (Equs. 11,12), the values of the $S$ is greatly dependent on the $\gamma_{i}$ corresponding ions in the CaPs, since these CaPs did not contain $\mathrm{Na}^{+}, \mathrm{K}^{+}, \mathrm{Mg}^{2+}, \mathrm{Cl}^{-}$ and $\mathrm{SO}_{4}{ }^{2-}$ ions. According to the calculated results, it was found that the change of the $\gamma_{i}$ is basically contrary with the change of $\Delta G$ with increasing the concentrations of $\mathrm{Na}^{+}, \mathrm{K}^{+}, \mathrm{Mg}^{2+}$ and $\mathrm{Cl}^{-}$. According to the Equs. 16 and 17, the $\gamma_{i}$ is related with the $I$. In other words, the change in the concentrations of $\mathrm{Na}^{+}, \mathrm{K}^{+}, \mathrm{Mg}^{2+}, \mathrm{Cl}-$ and $\mathrm{SO}_{4}{ }^{2-}$ ions could result in the alteration of $I$, thus eventually, affect the value of $\gamma_{i}$. Based on the Equs. 11,12, $\gamma_{i}$ is larger, and $S$ is higher. Thus the $\Delta G$ is lower according to the Equ. 1 . In the case of $\mathrm{SO}_{4}^{2-}$ ions, the effect of its concentration on the $\gamma_{i}$ is not obvious, thus the $\Delta G$ did not alter basically. 
Here, two events were also to be noticed. One is that $\gamma i$ has a minimum when changing the ions of the SBF. In Equ. 16, it was set that:

$$
\mathrm{Y}=\frac{I^{1 / 2}}{1+I^{1 / 2}}-0.3 I
$$

if $\gamma_{i}$ has a minimum, thus the $\mathrm{Y}$ should has a maximum with increasing the $I$. In fact, $Y$ exactly has a maximum based on the calculation of the Equ. 31 . When $I$ is at $0.3 \sim 0.4$, the $Y$ shows a maximum about 0.25 .

The other event is that the augment of $\mathrm{K}^{+}$and $\mathrm{Mg}^{2+}$ ions concentration leaded to a very slight increase of $\Delta G$ of the various $\mathrm{CaPs}$, unlike changing the $\mathrm{Na}^{+}$and $\mathrm{Cl}$ - ions. The reason for this is mainly that the $I$ corresponding to $\mathrm{K}^{+}$and $\mathrm{Mg}^{2+}$ ions did not change greatly, because the concentrations of $\mathrm{K}^{+}$and $\mathrm{Mg}^{2+}$ ions is greatly lower compared to the $\mathrm{Na}^{+}$and $\mathrm{Cl}$ ions in the SBFs. Thus the slight change in the $I$, as result of the change of $\mathrm{K}^{+}$and $\mathrm{Mg}^{2+}$ ions concentration, is insufficient for alter the $\mathrm{Y}$ value. In fact, if the concentrations of $\mathrm{K}^{+}$and $\mathrm{Mg}^{2+}$ ions increased to about $1.25 \mathrm{~mol} / \mathrm{L}$ and $0.3 \mathrm{~mol} / \mathrm{L}$, respectively, the $\Delta G$ of the various CaPs became to decrease apparently.

Fig. 3 shows that the $\triangle G$ of CaPs in the c-SBF, r-SBF, i-SBF and m-SBF are at similar level at $\mathrm{pH}=7.4$; while $\Delta \mathrm{G}$ of CaPs in HBSS are higher than in the other SBFs. In all SBFs, the CaPs always increased as the following sequence: HPO4-SCHA-B- $0.5<$ SCHA-A-0.5 < HA < OCP.

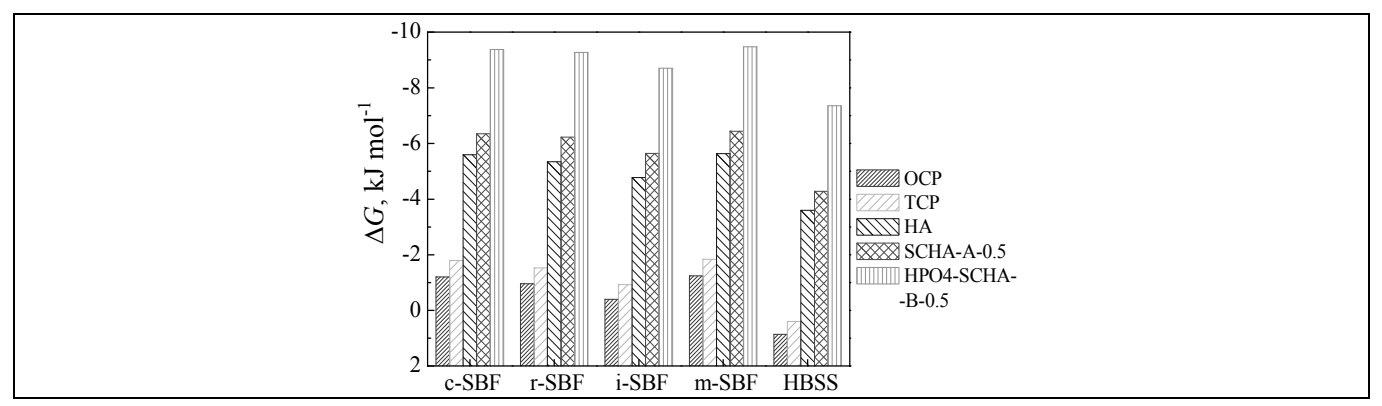

Fig. 3. $\triangle \mathrm{G}$ for the formation of OCP, HA, SCHA-A-0.5 and HPO4-SCHA-B-0.5 in the different $\mathrm{SBF}$ at $\mathrm{pH}=7.4$.

Fig. 4a shows effect of the $\mathrm{pH}$ on the $\log J$ of various CaPs. Because the $\triangle \mathrm{G}$ of DCPD, SCHA-A1 and SCHA-B-1 is higher than zero in SBF at $\mathrm{pH}=7.4$, the $\log J$ of these CaPs did not taken into account. The $\log$ J of various CaPs increased as the following sequence: $\mathrm{OCP}<\mathrm{HA}<\mathrm{HPO} 4-$ SCHA-B-0.5 < SCHA-A-0.5 at $\mathrm{pH}=7.4$. This result revealed that the formation of SCHA-A-0.5 and HPO4-SCHA-B-0.5 is kinetically favorable. In fact, the previous experimental results also found the trace of A-type substituted carbonated-HA and B-type substituted carbonated-HA containing $\mathrm{HPO}_{4}{ }^{2-}$ group [Müller et al., 2006; Weia et al., 2007; Wei et al., 2008].

Totally, the $\log J$ of various CaPs ascended firstly and then descended when increasing $\mathrm{pH}$. And the maximum was observed at $\mathrm{pH}=9 \sim 10$, except of $\mathrm{OCP}$, whose maximum occurred at about $\mathrm{pH}=8.5$. When $\mathrm{pH}<9.5, \log$ J of HA, HPO4-SCHA-B-0.5 and SCHA-A-0.5 increased as following sequence: HA < HPO4-SCHA-B-0.5 < SCHA-A-0.5. In general, the logJ of the three kinds of $\mathrm{CaPs}$ is higher than that of other CaPs.

To understand the effect of various factors on $\log J$, the following Equation was used according to Equ. 22: 


$$
M=\exp \left(-\frac{16 \pi v^{2} \sigma^{3} f(\theta)}{3 \mathrm{k}^{3} T^{3}(\ln S)^{2}}\right)
$$

Thus, the $\log J$ is determined by the $P$ and $M$ based on the Equs. 22 and 32, neglecting the exact value of $\mathrm{K}^{\prime}$. Fig. $4 \mathrm{~b}$ and c shows the effect of the $\mathrm{pH}$ on the $\log P$ and $\ln M$ of various CaPs. With increasing the $\mathrm{pH}$, the $\log P$ of TCP, HA, HPO4-SCHA-B-0.5 and SCHA-A-0.5 increased firstly when $\mathrm{pH}<9 \sim 10$ and then decreased when $\mathrm{pH}>9 \sim 10$; while that of OCP and DOHA increased slightly. According to Fig. 4c, the $\ln M$ of HA, HPO4-SCHA-B-0.5 and SCHA-A-0.5 did not change with increasing $\mathrm{pH}$; while that of other CaPs increased firstly when $\mathrm{pH}<9$ and then decreased when $\mathrm{pH}>9$, especially OCP. These results indicated that the $\log J$ of TCP, is mainly dependent on the $\log P$ when $\mathrm{pH}$ was changed; while that of OCP and DOHA is highly determined by the $\ln M$.

From Fig. $4 \mathrm{~d}$, the $\ln M$ value changed greatly when $\ln S$ ranged from 0 to 0.5 , and did not alter basically when $\ln S>0.5$. According to the calculated results, the $\ln S$ of HA, HPO4SCHA-B-0.5 and SCHA-A-0.5 is basically higher than 40 when $\mathrm{pH}>7$; while that of the OCP and DOHA is just near zero when $\mathrm{pH}>7$. Thus the $\ln M$ of OCP and DOHA is sensitive to the change of $\mathrm{pH}$ according to Fig. $4 \mathrm{~d}$.

Fig. 5 shows the change in the $\log J$ with increasing the ions concentrations at $\mathrm{pH}=7.4$. The $\log$ J of HA and HPO4-SCHA-B-0.5 and SCHA-A-0.5 has a tendency to decrease with increasing $\mathrm{Ca}^{2+}$ concentrations; while that of OCP, DOHA and TCP increased, especially $\mathrm{OCP}$ as shown in Fig. 5a. However, the $\log$ J of HPO4-SCHA-B-0.5 and SCHA-A-0.5 is always higher than that of other $\mathrm{CaPs}$ when $\mathrm{Ca}^{2+}$ ions concentration changed in the range of 0 10 multiples. The descent in the logJ of HA and HPO4-SCHA-B-0.5 and SCHA-A-0.5 mainly resulted from the decrease of $\log P$ according to calculated results (not shown here), because the $\ln M$ of these CaPs did not alter basically due to the high $\ln S$ (>20) compared to OCP, DOHA and TCP. The $\ln S$ of OCP, DOHA and TCP near zero caused a great variety of $\ln M$ value, which further brought out the augment of $\log J$ of these CaPs since the $\log P \operatorname{did}$ not alter basically according to calculated results (not shown here).

Fig. $5 \mathrm{~b}$ shows the change in the $\log J$ with increasing the concentrations of $\mathrm{HPO}_{4}{ }^{2-}$ ions in $\mathrm{SBF}$ at $\mathrm{pH}=7.4$. Except of HPO4-SCHA-B-0.5 showing a tendency to decrease, the logJ of other $\mathrm{CaPs}$ mounted up with increasing the concentrations of $\mathrm{HPO}_{4}^{2-}$ ions. And the logJ of SCHAA-0.5 and HPO4-SCHA-B-0.5 is higher than that of other CaPs when changing concentrations of $\mathrm{HPO}_{4}{ }^{2-}$ ions. According to calculated results (Figures not shown here), the change of $\log P$ of OCP, DOHA and HPO4-SCHA-B-0.5 are similar due to the presence of $\mathrm{HPO}_{4}{ }^{2-}$ ion in their structures and showed a slight decrease. Furthermore, the $\log P$ of other $\mathrm{CaPs}$ increased slightly. The effects of the $\mathrm{HPO}_{4}{ }^{2-}$ ions on $\ln M$ and $\ln S$ are similar to those of $\mathrm{Ca}^{2+}$ ions. The $\log$ J of OCP, DOHA and TCP is highly dependent on the $\ln M$; while those of HA and SCHA-A- 0.5 is mainly determined by the $\log P$.

Fig. $5 \mathrm{c}$ shows that the change in the $\log J$ with increasing the concentrations of $\mathrm{HCO}_{3}$ - ions in $\mathrm{SBF}$ at $\mathrm{pH}=7.4$ is not obviously. Also, the change in the $\log P, \ln M$ and $\ln S$ are not evident according to the calculated results (Figures not shown here).

Fig. $5 \mathrm{~d}$ shows the change in the $\log J$ with increasing the SBF concentrations at $\mathrm{pH}=7.4$. The $\log J$ of OCP, TCP and DOHA increased and those of other CaPs did not alter basically. Compared to $\log J$, a similar result of the effect of SBF concentrations on $\ln M$ was observed according to the calculated results (not shown here). When the multiple of SBF concentration is larger than four, the $\log J$ of OCP is higher than those of other CaPs. In 


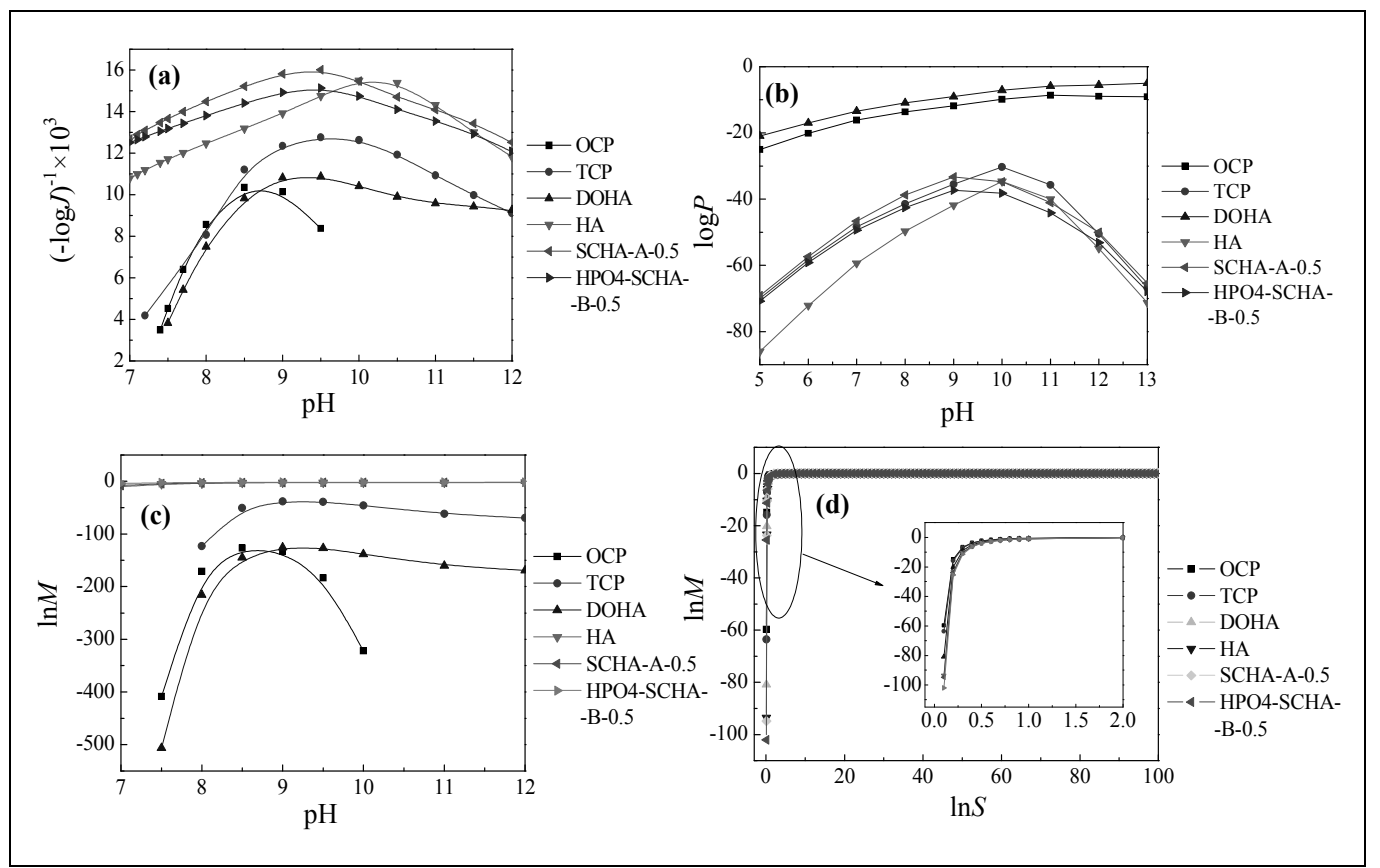

Fig. 4. Effect of $\mathrm{pH}$ on the $\log J, \log P$ and $\ln M$ of various CaPs with increasing $\mathrm{pH}$ : (a) $\log J$, (b) $\log P$, (c) $\ln M$ and (d) the relation between the $\ln S$ and $\ln M$.

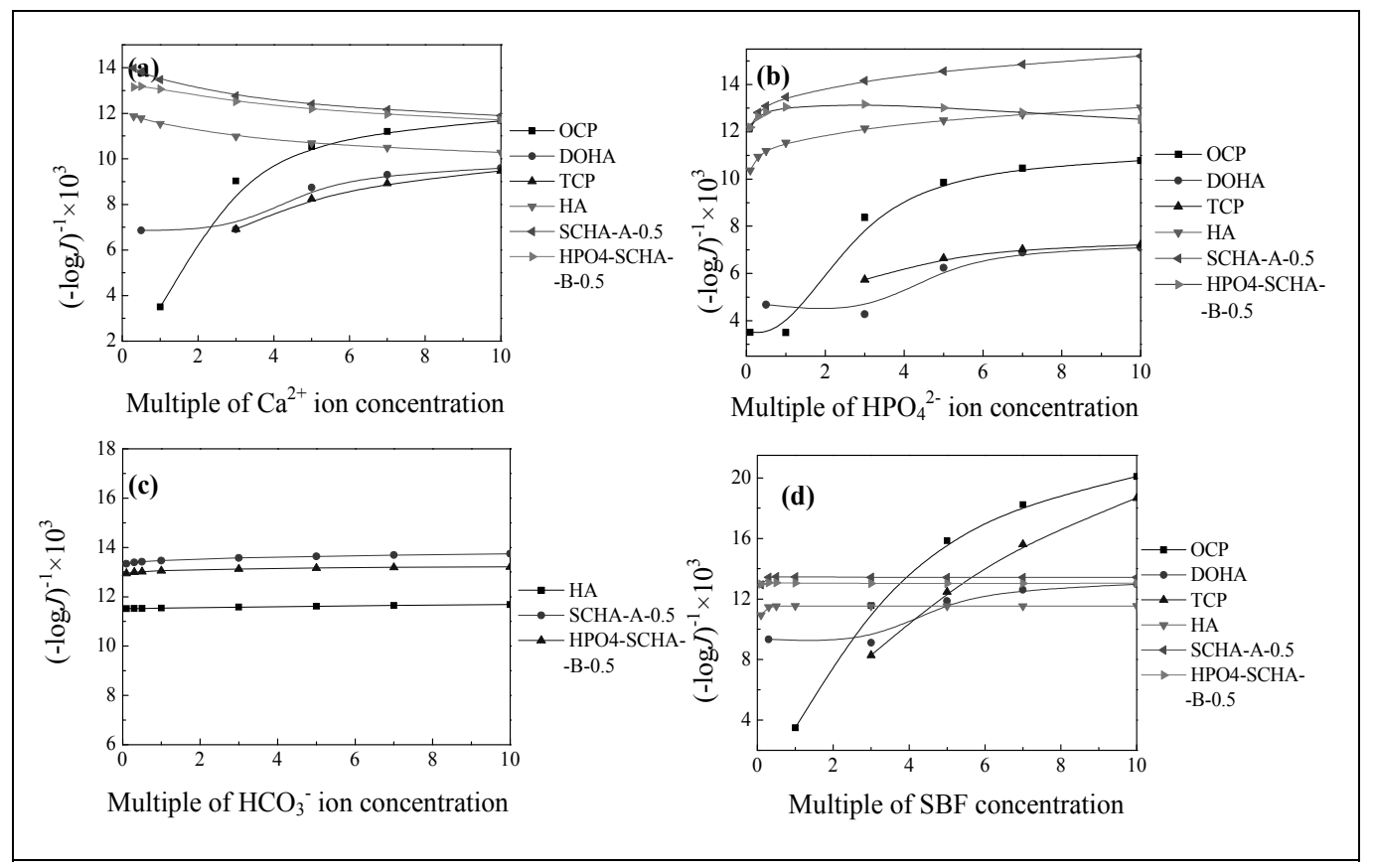

Fig. 5. Change in the $\log J$ with increasing the concentrations of various ions and SBF at $\mathrm{pH}=7.4$ : (a) $\mathrm{Ca}^{2+}$, (b) $\mathrm{HPO}_{4}{ }^{2-}$, (c) $\mathrm{HCO}_{3}{ }^{-}$and (d) SBF. 
addition, the effect of the SBF concentrations on the $\log P$ is not obvious (not shown here). The calculated results demonstrated that the $\ln S$ of HA, SCHA-A-0.5 and HPO4-SCHA-B0.5 is higher than 20; while that of OCP, DOHA and TCP varied near the zero, leading to a great variation of $\ln M$.

The effect of $\mathrm{Na}^{+}, \mathrm{K}^{+}, \mathrm{Mg}^{2+}, \mathrm{Cl}^{-}$and $\mathrm{SO}_{4}{ }^{2-}$ ions concentration on the logJ of HA, SCHA-A-0.5, SCHA-A-1, SCHA-B-1 and HPO4-SCHA-B-0.5 is not apparent. However, these ions have effect on the logJ of OCP, DOHA and TCP, where the logJ of OCP and TCP decreased firstly, and then increased. And the $\log J$ for DOHA increased with increasing $\mathrm{Cl}^{-}$ions concentration. With increasing $\mathrm{K}^{+}, \mathrm{Mg}^{2+}$ and $\mathrm{SO}_{4}{ }^{2-}$ ions concentration, the $\log$ J of OCP and TCP lowered slightly.

The logJ of DOHA, OCP and TCP is sensitive for the change of $\mathrm{Na}^{+}$and $\mathrm{Cl}^{-}$ions. The reason for this is that the change of $\mathrm{Na}^{+}$and $\mathrm{Cl}^{-}$ions also has a great effect on the $\ln M$ of the DOHA, OCP and TCP (not shown here). The change in $\ln M$ is associated with the $\ln S$ at here. According to the calculated results, the change of $\ln S$ of DOHA, OCP and TCP nearby the zero, resulting in the great variation in $\ln M$, and $\ln S$ of the other CaPs is greatly higher than zero, which leaded to an unobvious change in $\ln M$.

Fig. 6 shows the relationships between $\log J$ and $v$ of CaPs. In Fig. 6a, with increasing the $v$ from 200 to $1200 \AA^{3}$, the logJ of OCP and TCP increased slowly. The change of $v$ did not alter the $\log J$ of other $\mathrm{CaPs}$ significantly. At the same time, the $\log J$ increased as the following sequence: TCP < OCP < HA < HPO4-SCHA-B-0.5 < SCHA-A-0.5 as increasing $v$. This result suggested that the hypothesis of the $v$ of carbonated-HA being equal to that of HA was feasible. In fact, the difference of the $v$ between the carbonated-HA and HA is not significant [Fleet et al., 2004; Kumar et al., 2000]. Further calculating on $\log J$ of CaPs with different $v$ as increasing $\mathrm{pH}$ was considered as shown in Fig. $6 \mathrm{~b}$. With increasing $\mathrm{pH}$, the change of $v$ did not alter the logJ of HA, HPO4-SCHA-B-0.5 and SCHA-A-0.5. While in the case of OCP, the increase of $v$ improved the $\log$ J slightly.

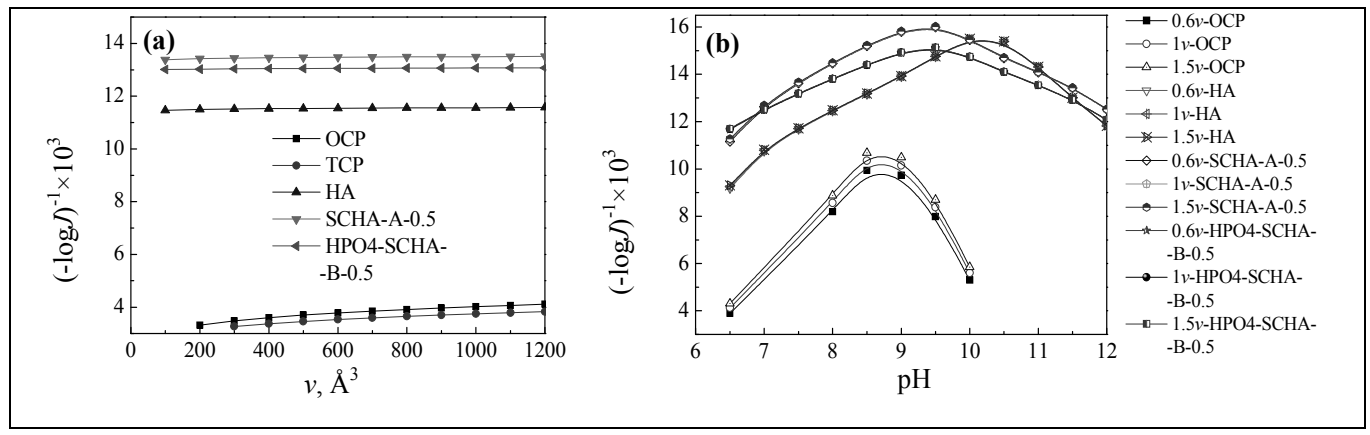

Fig. 6. The relationships between $\log J$ and $v$ of CaPs: (a) change in the logJ with increasing the $v$ of apatites at $\mathrm{pH}=7.4$ and (b) change in the $\log$ J with increasing $\mathrm{pH}$ at vaious $v$ of apatites.

The change in the $\log J$ with increasing the $\sigma$ at $\mathrm{pH}=7.4$ is shown in Fig. 7a. It was evident that the $\log J$ of the CaPs decreased with increasing the $\sigma$, especially of OCP and TCP. The reason for the great decrease in $\log$ J of OCP and TCP is that the change of $\sigma$ has a great effect on the $\ln M$ as shown in Fig. $7 \mathrm{~b}$, which can further influence the $\log J$ of these CaPs. In fact, the previous calculated results also indicated that the effect of $\sigma$ variation on the $\log J$ was greatly [Lu et al., 2005]. 
On the other hand, it was difficult to obtain the accurate $\sigma$ values of the CaPs based on the previous studies [Lu et al., 2005]. Wu and Nancollas summarized the $\sigma$ of HA, OCP and DCPD acquired by various measurement methods such as contact angle, crystal growth kinetics, dissolution kinetics, etc. [Wu et al., 1999]. Their results showed $\sigma$ of HA in the range of $9.3 \sim 87 \mathrm{mJm}^{-2}$, and those of OCP and DCPD in the ranges of $3 \sim 45$ and $0.4-70 \mathrm{mJm}^{-2}$, respectively. In this work, the calculated values of $\sigma$ are basically in these ranges.

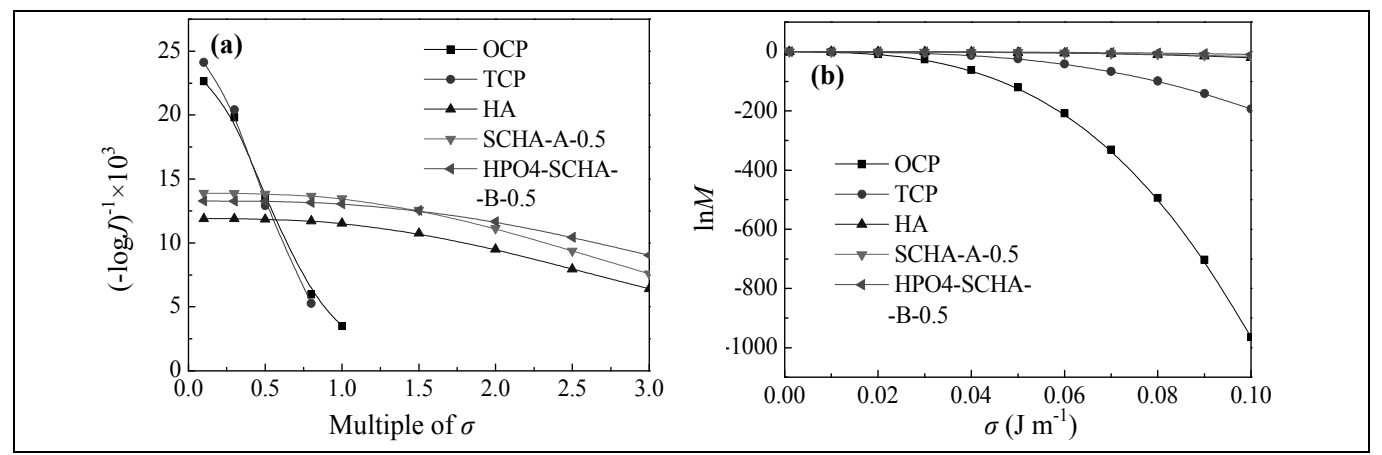

Fig. 7. The relationships between $\log J$ and $\ln M$ and $\sigma$ of CaPs: (a) change in the logJ with increasing the multiple of $\sigma$ at $\mathrm{pH}=7.4$ and (b) relation between $\ln \mathrm{M}$ and $\sigma$ at $\mathrm{pH}=7.4$.

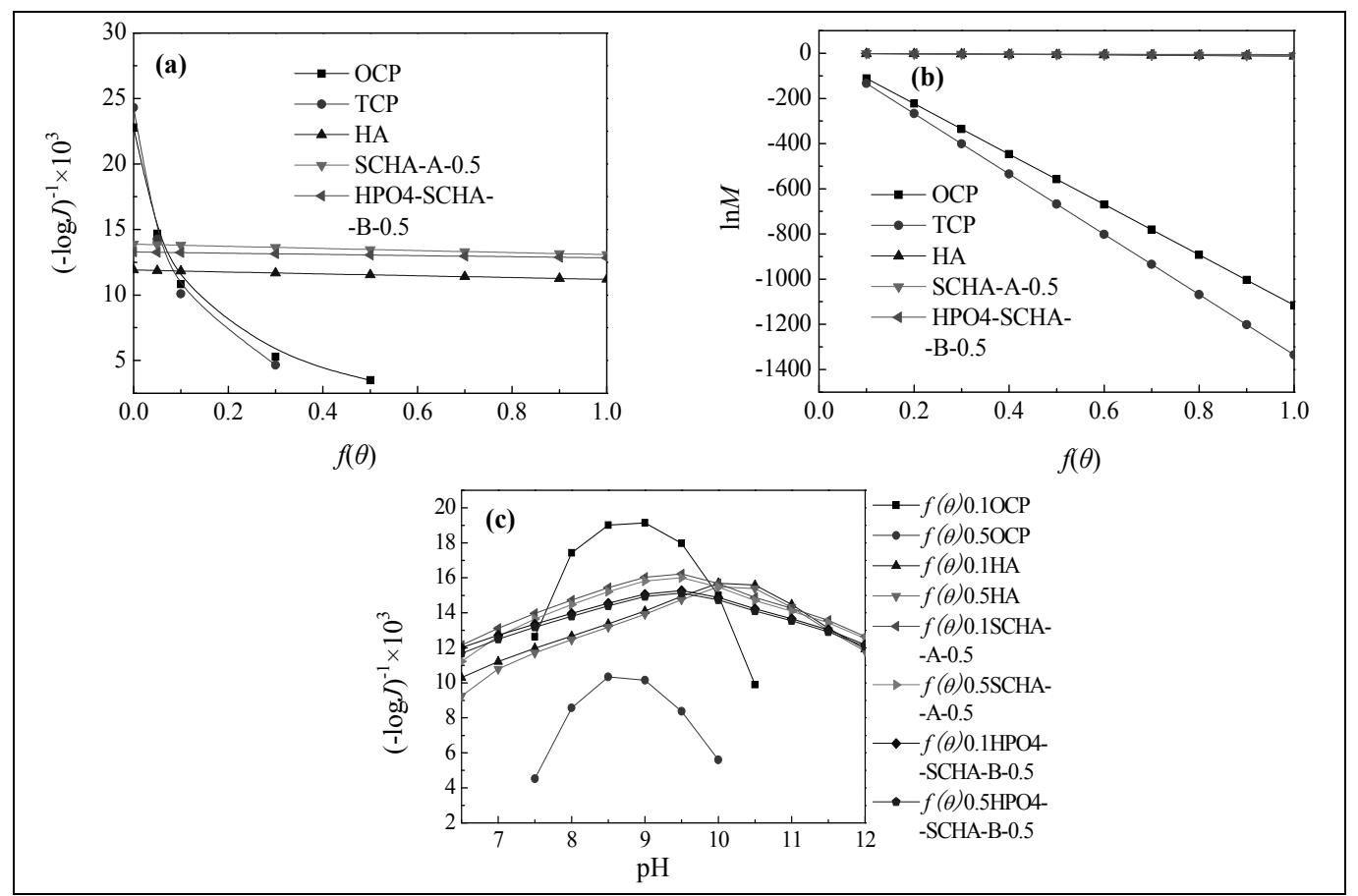

Fig. 8. The relationships among $\log J, f(\theta), \ln M$ and $\mathrm{pH}$ of CaPs: (a) change in the logJ with increasing the $f(\theta)$ at $\mathrm{pH}=7.4$, (b) relation between $\ln M$ and $f(\theta)$ at $\mathrm{pH}=7.4$ and (c) change in the $\log J$ with increasing $\mathrm{pH}$ as using different $f(\theta) . f(\theta) 0.1$ and $f(\theta) 0.5$ means the value of the $f(\theta)$ used at here is 0.1 and 0.5 , respectively. 
Fig. 8a shows the change in the $\log J$ with increasing $f(\theta)$ at $\mathrm{pH}=7.4$. The $\log J$ of OCP and TCP decreased obviously with increasing $f(\theta)$. However, the $\log J$ of other CaPs did not change basically. The reason for this is that the change of $f(\theta)$ has a great effect on the $\ln M$ of OCP and TCP as shown in Fig. 8b, which can further influence the logJ of these CaPs. The results shown in Fig. 8c indicated that the $\log J$ of OCP is highly dependent on the $f(\theta)$ with increasing $\mathrm{pH}$, and change of $f(\theta)$ has no obvious effects on the $\log J$ of the other CaPs.

Fig. 9 shows that the SBFs species did not markedly affect the logJ of HA, SCHA-A-0.5 and HPO4-SCHA-B-0.5 at $\mathrm{pH}=7.4$. Fig. 10a shows the $\log$ J of OCP is highly dependent on the $\mathrm{SBF}$ species with increasing $\mathrm{pH}$, and change of SBF species has no obvious effects on the logJ of HA (Fig. 10b).

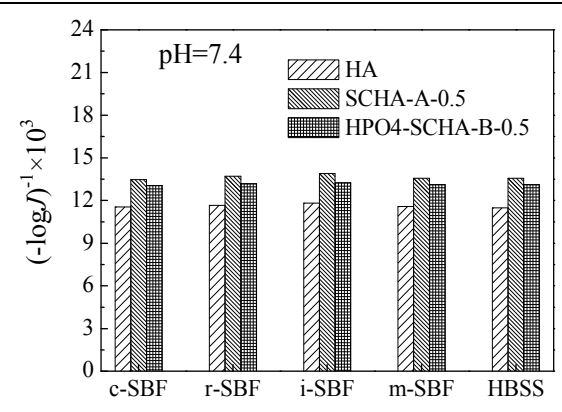

Fig. 9. $\log$ J of HA, SCHA-A-0.5 and HPO4-SCHA-B-0.5 in the different SBFs

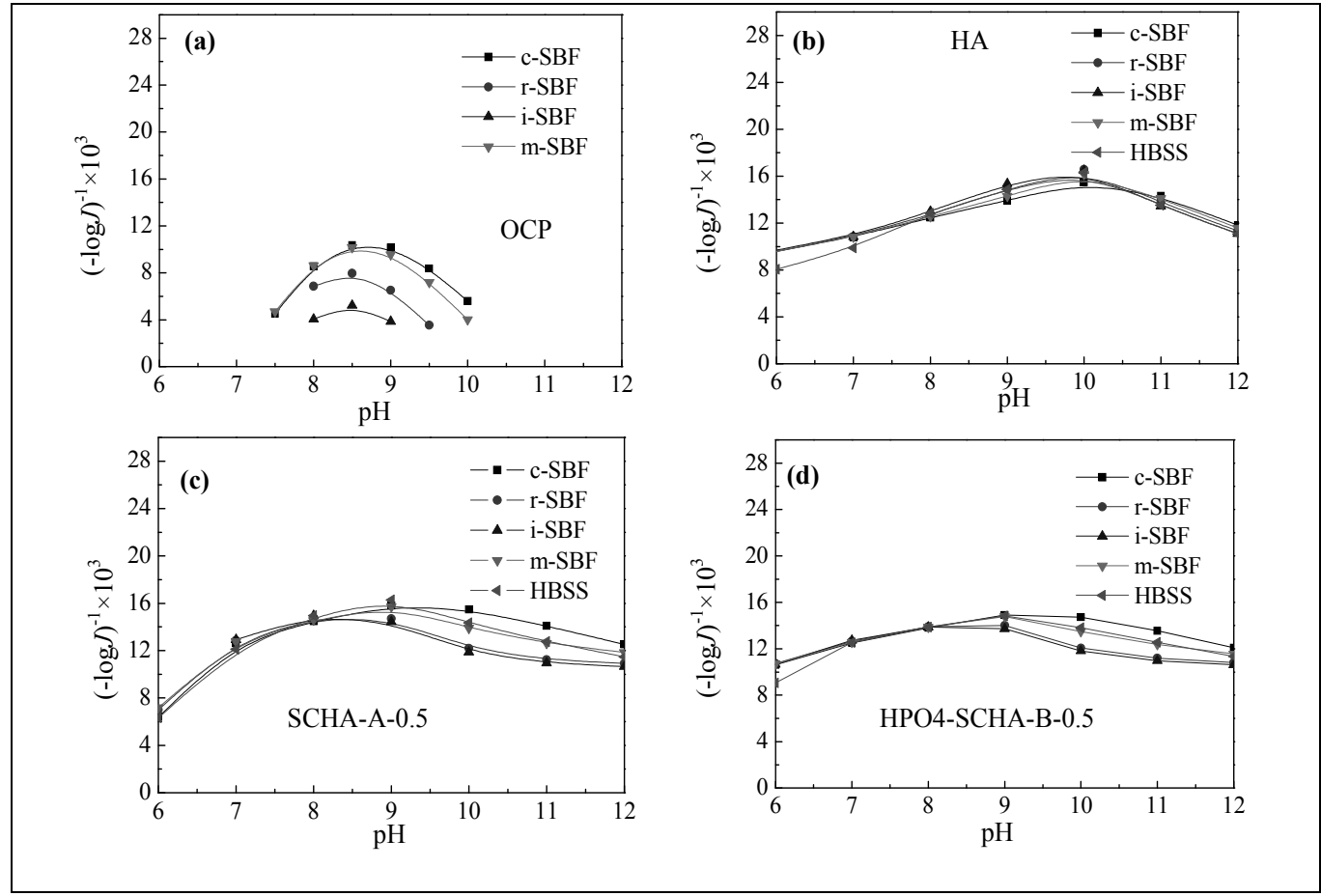

Fig. 10. $\log J$ of various apatites in different SBFs with increasing $\mathrm{pH}$ : (a) OCP, (b) HA, (c) SCHA-A-0.5 and (d) HPO4-SCHA-B-0.5. 
In the case of OCP, the logJ increased in the four SBFs as the following sequence: i-SBF < $r$ $\mathrm{SBF}<\mathrm{m}-\mathrm{SBF}<\mathrm{c}-\mathrm{SBF}$ (Fig. 10a). For SCHA-A-0.5 and HPO4-SCHA-B-0.5, the effect of SBF species on the $\log J$ is not obvious when $\mathrm{pH}<9$; while distinction was presented when $\mathrm{pH}>9$, where the $\log J$ increased as the following sequence: i-SBF $\approx \mathrm{r}-\mathrm{SBF}<\mathrm{m}-\mathrm{SBF} \approx \mathrm{HBSS}<\mathrm{c}-\mathrm{SBF}$ (Fig. 10c and d).

In order to understand the change of $\log J$ of $\mathrm{OCP}$, the effect of $\mathrm{pH}$ on the $\log P, \ln M$ and $\ln S$ were considered. Based on the calculated results, the $\log J$ is mainly determined by the $\ln S$, which determined the change of $\ln M$ here. And the curve of $\ln M$ is similar to the $\log J$ of OCP.

In the case of HA, the $\mathrm{pH}$ has no significant effect on $\log P$ and $\ln M$, thus the $\log J$ of HA did not alter obviously.

However the distinguish of $\log J$ of SCHA-A-0.5 and HPO4-SCHA-B-0.5 in various SBFs appeared when $\mathrm{pH}>9$. The reason for this is that the change of $\log P$ caused by the SBF species, where the SBF species has no apparent effects on the $\ln M$. The change of $\log P$ is mainly resulted from the different concentrations of $\mathrm{HCO}_{3}$ - ions of SBFs. As shown in the Table 1, the concentrations of $\mathrm{HCO}_{3}$ - ions in various SBFs decreased as following sequence: $\mathrm{i}-\mathrm{SBF}=\mathrm{r}-\mathrm{SBF}>\mathrm{m}-\mathrm{SBF}=\mathrm{HBSS}>\mathrm{c}-\mathrm{SBF}$, which is just contrary to the change of logJ.

\subsection{Structure of the bone apatite and biomimetic apatite}

As well known, the bones and teeth of all vertebrates are natural composite materials, where one of the components is an inorganic solid, carbonate hydroxyapatite. Thus the formation of the carbonate hydroxyapatite in vitro by biomimetic method is worthy to be noticed. These results indicated whether the induced apatite is A-type or B-type carbonated-HA, the high concentration of $\mathrm{CO}_{3}{ }^{2-}$ ions in carbonated-HA deter its formation. In other words, the dissolution of highly carbonated-HA could be serious relatively. In fact, the previous results indicated that $\mathrm{CO}_{3}{ }^{2-}$ ions concentration in carbonated-HA is higher, the dissolving of carbonated HA is faster [Okazaki, et al., 1999].

Besides $\mathrm{CO}_{3}^{2-}$ ions, $\mathrm{HPO}_{4}{ }^{2-}$ ions also is one component of the bone apatite. The carbonatedHA of bones ranges between $4 \%$ and $8 \%$ in carbonate content, which increases with age while $\mathrm{HPO}_{4}{ }^{2-}$ ions decreases. In this work, the theoretical calculation results indicated that the introduction of $\mathrm{HPO}_{4}{ }^{2-}$ ions lowered the $\Delta G$ of carbonated HA. In fact, biomimetic apatite containing $\mathrm{HPO}_{4}{ }^{2-}$ ions have been reported.

Also, $\mathrm{Na}^{+}, \mathrm{K}^{+}, \mathrm{Mg}^{2+}$ and $\mathrm{Cl}^{-}$ions can incorporate the structure of apatite. Thus, the chemical composition of apatite could be more complex. If $\mathrm{Na}^{+}, \mathrm{K}^{+}, \mathrm{Mg}^{2+}$ and $\mathrm{Cl}^{-}$ions were considered, more equations regarding to calculating their equilibrium concentrations were needed. Thus mathematic method and computer language are needed to solve the high order equation such as quintic equation. In addition, no details parameters of these CaPs in the thermodynamic and kinetic calculations such as $K_{\mathrm{sp}}, v, \sigma, f(\theta)$ etc. can be available. According to these, it was difficult to conduct the thermodynamic and kinetic calculations of apatite formation when the chemical compositions of apatite become more abundant.

\subsection{Nuclear and growth}

Due to SBF with high concentration, the apatite body was formed at the early stage of the $\mathrm{SBF}$ immersion process as shown Fig. 11a. With increasing the SBF immersion time, the apatite body grew, resulting in a decrease in SBF concentration (red line). When the size of apatite body reached $r_{\mathrm{c}}$, and the $\mathrm{SBF}$ concentration is higher than $c_{\mathrm{c}}$. Thus the apatite nuclear 
was formed, which could further grow due to the high concentration of SBF. And the SBF concentration continuously decreased due to the growth of the apatite nuclear. When the $\mathrm{SBF}$ concentration reached an equilibrium concentration, the sized of the apatite particle did not increase.

During total process of apatite formation, the apatite grew by consuming calcium ions and phosphate radicals, et al.. At the same time, the apatite could was dissolved, releasing calcium ions and phosphate radicals, et al.. When SBF concentration is higher than equilibrium concentration, the deposition rate of apatite is greater than dissolution rate of apatite. When SBF concentration is equal to equilibrium concentration, the deposition rate of apatite is equal to the dissolution rate of apatite.

If the initial concentration of SBF is lower than $c_{c}$, thus the apatite nuclear can not be formed. Even if the apatite body (its size less than $r_{\mathrm{c}}$ ) was formed, it usually disappeared due to no superfluous ions to supply the deposition of apatite body. Thus, the apatite formation is highly dependent on the ions concentration near the surface of bioactive coatings based on the above discussion. Just the attraction of functionalized surfaces for other ions caused the increase in ions concentration near the local surface. Based on the above equation, the $r_{\mathrm{c}}$ of $\mathrm{CaPs}$ was calculated. It was observed that the $r c$ decreased as the following sequence: $\mathrm{OCP}>$ TCP >> HA > SCHA-A-0.5 > HPO4-SCHA-B-0.5. These results suggested that the formation of SCHA-A-0.5 and HPO4-SCHA-B-0.5 is easier compared with other CaPs.

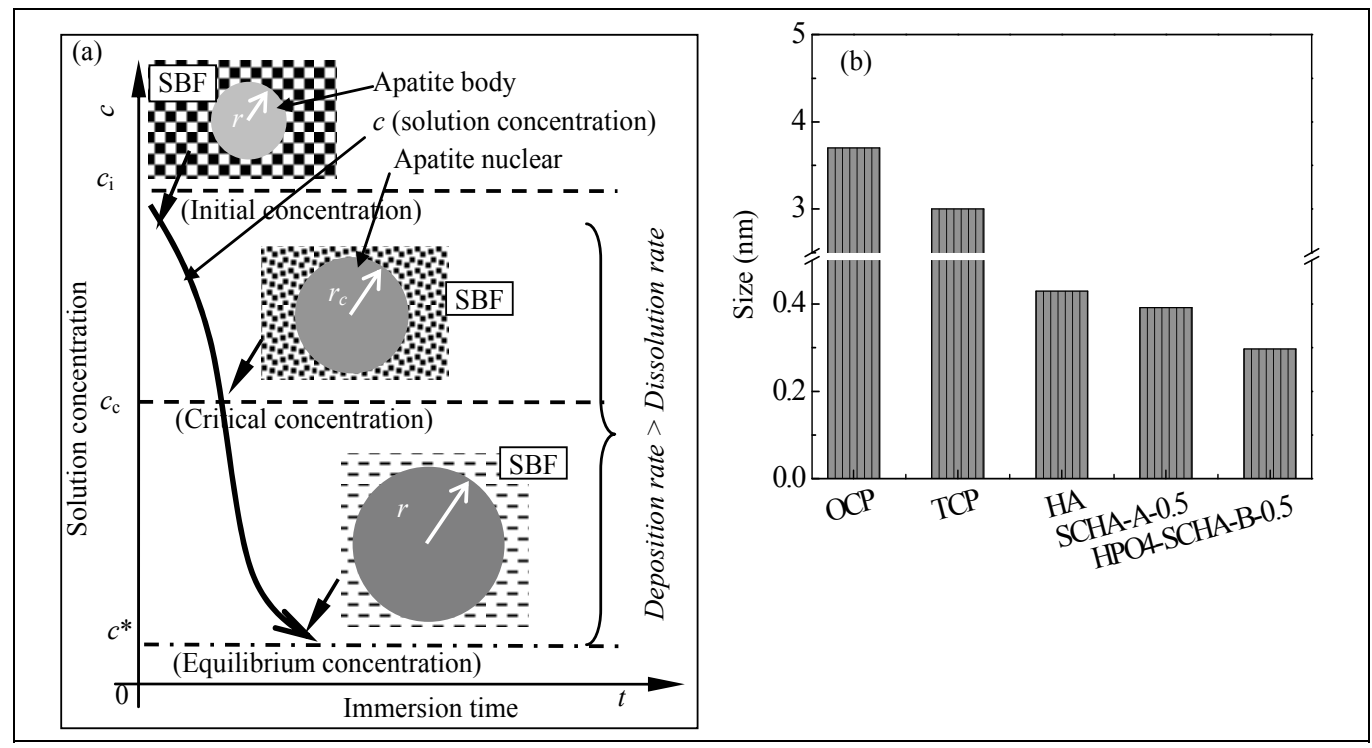

Fig. 11. Nuclear and growth of apatites in SBF: (a) schametical diagram for the formation and growth of apatite nuclear during the SBF immersion process and $(b) r_{c}$ of various CaPs nuclear in the SBF.

\subsection{Phase transformation of biomimetic apatite}

The previous results indicated that the gel-like matter was formed on the surface of the chemical-treated MAO coatings at the early stage of the SBF immersion process. The gel-like matter mainly contained $\mathrm{Ca}$ and $\mathrm{P}$ elements with an amorphous structure. After further immersion, the amorphous $\mathrm{Ca}$ and $\mathrm{P}$ could transform to crystal phase. 
In fact, the formation of apatite on the surface of substrates is a very complex process, since various factors have effect on the apatite nucleation and growth such as supersaturation or the solution compositions, interface energy and contacting angle of apatites and substrates, etc..

The precipitation of apatite from SBF or the supersaturated calcium phosphate solution (differing from SBF in composition containing higher $\mathrm{Ca}$ and $\mathrm{P}$ concentrations) has been widely investigated in the past [Feng et al., 1999; Li et al., 2002; Barrère et al., 1999; Lu et al., 2004; Lu et al., 2007; Horváthová et al., 2008; Xie et al., 2006]. Experimental observation indicated that some important characteristics of the initial behaviors of apatite formation. Feng et al. [Feng et al., 1999; Li et al., 2002] observed various apatites such as DCPD, OCP and HA phases on the surfaces of alkali-treated titanium when the samples were immersed in supersaturated calcification solution. Leng [Lu et al., 2004] used transmission electron microscopy (TEM) to investigate the formation of calcium phosphate on alkali- and heattreated titanium surfaces in a revised SBF. In their results, electron diffraction of the precipitates revealed that $\mathrm{OCP}$, instead of $\mathrm{HA}$, directly nucleates from amorphous $\mathrm{CaP}$. The OCP crystals continuously grew on the titanium surfaces rather than transforming into apatite. Lu et al. [Lu et al., 2007] found that amorphous CaP coatings were formed on the surfaces by immersing the nitric acid treated Ti specimens in SBF, while OCP coatings were formed in the supersaturated calcium phosphate solution after 3 days of immersion. And, OCP could further transfer into carbonated-HA in SBF [Horváthová et al., 2008].

Based on the this investigation, compared to HA and carbonated-HA, the formation of OCP is highly dependent on $f(\theta), \sigma$ and local ions concentration near substrate surfaces. This probably is the main reason for forming OCP and sometimes not during the immersion process in $\mathrm{Ca}$ - and $\mathrm{P}$-containing solutions. In other words, $\mathrm{OCP}$ is not the necessary intermediate phase for the formation of HA or carbonated-HA, and the formation of OCP is associated with the circumstance, especially the contacting angle between OCP and substrate and local ions concentration.

\section{Conclusion}

MAO is a relatively convenient and effective technique to deposit bioceramic coatings on the surfaces of $\mathrm{Ti}$ and its alloys. The structures such as phase composition, surface morphology and elemental composition of the MAO coatings are highly dependent on the applied voltage, oxidizing time, frequency, duty cycle and electrolyte composition, etc., especially the applied voltage and electrolyte composition.

The apatite-forming ability and bioactivity of most of MAO coatings are not very good. Thus, subsequent activation methods such as sol-gel, UV irradiation and hydrothermal treatments have been investigated to improve the surface bioactivity of MAO coatings. Authors have developed a simple chemical-treatment to modify the surface of the MAO coatings. A variety of reactions such as dissolution, ion exchange and precipitation took place on the surface of the chemical-treated MAO coating during the SBF immersion process to form $\mathrm{Ti}-\mathrm{OH}$ groups, which greatly improved the apatite-forming ability of the MAO coatings.

The induced biomimetic apatites were SCHA-A and HPO4-SCHA-B. At the same time, Na, $\mathrm{K}, \mathrm{Mg}$ and $\mathrm{Cl}$ elements could be also introduced into these apatites, however, the concentrations of these elements are very small. In addition, the induced biomimetic apatite presented two-level porous structure on micro- and nano-scales. 
The theoretical calculated result indicated that the formation of A-type slightly substituted carbonated-HA and B-type slightly substituted carbonated-HA containing $\mathrm{HPO}_{4}{ }^{2-}$ ions is thermodynamically and kinetically easier than other $\mathrm{CaPs}$, consistent with the experimental results. The $\mathrm{pH}$, ions concentrations and species of SBFs greatly affected the $\Delta G$ and $\log J$ of $\mathrm{CaPs}$. Totally, the $\Delta \mathrm{G}$ of $\mathrm{CaPs}$ descend with increasing $\mathrm{pH}$ and concentration multiple of SBF and $\mathrm{Ca}^{2+}$ and $\mathrm{HPO}_{4}{ }^{2-}$ ions concentrations. Moreover, other factors such as surface tension, contacting angle and molecular volume can affect the logJ of CaPs, especially $\mathrm{Ca}_{4}\left(\mathrm{HPO}_{4}\right)\left(\mathrm{PO}_{4}\right)_{2}, \mathrm{Ca}_{3}\left(\mathrm{PO}_{4}\right)_{2}$ and $\mathrm{Ca} 9\left(\mathrm{HPO}_{4}\right)\left(\mathrm{PO}_{4}\right)_{5} \mathrm{OH}$.

The surface topography, surface energy and wetting properties etc. are important factors to affect the osteoblast adhesion and proliferation. The current results indicated that MAO coatings and modified MAO coatings show good biocompatibility according to the cell proliferation tests in vitro.

\section{References}

Balamurugan, A.; Balossier, G.; Kannan, S.; Michel, J.; Rebelo, A.H.S. \& Ferreira, J.M.F. (2007). Development and in vitro characterization of sol-gel derived $\mathrm{CaO}-\mathrm{P}_{2} \mathrm{O}_{5}-$ $\mathrm{SiO}_{2}-\mathrm{ZnO}$ bioglass. Acta Biomater, Vol. 3, No. 2, March 2007, 255-262, ISSN: 17427061

Barrère, F.; Layrolle, P.; Blitterswijk, C.A.V. \& Groot, K.D. (1999). Biomimetic calcium phosphate coatings on Ti6Al4V: a crystal growth study of octacalcium phosphate and inhibition by $\mathrm{Mg}^{2+}$ and $\mathrm{HCO}_{3}{ }^{2-}$. Bone, Vol. 25, No. 2, Supplement 1, August 1999, 107S-111S, ISSN: 8756-3282

Boistelle, R. \& Lopez-valero, I. (1990). Growth units and nucleation: the case of calcium phosphates. J of Crystal Growth., Vol. 102, No. 3, May 1990, 609-617, ISSN: 0022-0248

Feng, Q.L.; Wang, H.; Cui, F.Z. \& Kim, T.N. (1999). Controlled crystal growth of calcium phosphate on titanium surface by $\mathrm{NaOH}$-treatment. Journal of Crystal Growth, Vol. 200, No. 3-4, April 1999, 550-557, ISSN: 0022-0248

Feng, Q.L.; Cui, F.Z.; Wang, H.; Kim, T.N. \& Kim, J.O. (2000). Influence of solution conditions on deposition of calcium phosphate on titanium by $\mathrm{NaOH}$-treat ment. Journal of Crystal Growth, Vol. 210, No. 4, March 2000, 735-740, ISSN: 0022-0248

Fini, M.; Cigada, A. \& Rondelli, G. (1999). In vitro and in vivo behaviour of Ca- and Penriched anodized titanium. Biomaterials, Vol. 20, No. 17, September 1999, 15871594, ISSN: 0142-9612

Fleet, M.E. \& Liu, X.Y. (2004). Location of type B carbonate ion in type A-B carbonate apatite synthesized at high pressure. Journal of Solid State Chemistry, Vol. 177, No. 9, September 2004, 3174-3182, ISSN: 0022-4596

Frauchiger, V.M.; Schlottig, F.; Gasser, B. \& Textor, M. (2004). Anodic plasma-chemical treatment of CP titanium surfaces for biomedical applications. Biomaterials, Vol. 25, No. 4, February 2004, 593-606, ISSN: 0142-9612

Han, Y.; Hong, S.H. \& Xu, K.W. (2003). Structure and in vitro bioactivity of titania-based films by micro-arc oxidation. Surf Coat Technol., Vol. 168, No. 2-3, 22 May 2003, 249258, ISSN: 0257-8972 
Han, Y.; Chen, D.H.; Sun, J.F.; Zhang, Y.M. \& Xu, K.W. (2008). UV-enhanced bioactivity and cell response of micro-arc oxidized titania coatings. Acta Biomaterialia, Vol. 4, No. 5, September 2008, 1518-1529, ISSN: 1742-7061

Heughebaert, J.Ca. \& Nancollas, G.H. (1984). Kinetics of crystallization of octacalcium phosphate. J Phys Chem, June 1984, 88 (12), 2478-2481.

Heughebaert, J.Cb. \& Nancollas, G.H. (1984). Mineralization kinetics-the role of octacalcium phosphate in the precipitation of calcium phosphates. Colloid Surf, Vol. 9, No. 1, 1 March 1984, 89-93.

Horváthová, Renáta.; Müller, Lenka.; Helebrant, A.; Greil, P. \& Müller, F.A. (2008). In vitro transformation of OCP into carbonated HA under physiological conditions. Materials Science and Engineering C, Vol. 28, No. 8, December 2008, 1414-1419, ISSN: 0928-4931

Huang, P.; Zhang, Y.; Xu, K.W. \& Han, Yong. (2004). Surface modification of titanium implant by microarc oxidation and hydrothermal treatment. J Biomed Mater Res Part B: Appl Biomater 70B: Vol. 70B, No. 2, 15 August 2004, 187-190, ISSN: 0021-9304

Ishizawa, H. \& Ogino, M. (1995). Characterization of thin hydroxyapatite layers formed on anodic titanium oxide coatings containing $\mathrm{Ca}$ and $\mathrm{P}$ by hydrothermal treatment. Journal of Biomedical Materials Research, Vol. 29, No. 9, September 1995, 1071-1079, ISSN: 0021-9304

Koutsopoulos, S.; Paschalakis, P.C. \& Dalas, E. (1994). The calcification of elastin in vitro. Langmuir, Vol. 10, No. 7, July 1994, 2423-2428, ISSN: 0743-746

Koutsopoulos, S. \& Dalas, E. (2000). The calcification of fibrin in vitro. J Cryst Growth, Vol. 216, No. 1-4, 15 June 2000, 450-458, ISSN: 0022-0248

Koutsoukos, P.G.; Amjad, Z.; Tomson, M.B. \& Nancollas, G.H. (1980). Crystallization of calcium phosphates - a constant composition study. J Am Chem Soc, Vol. 102, No. 5, February 1980, 1553-1557

Koutsoukos, P.G. \& Nancollas, G.H. (1981). Crystal growth of calcium phosphatesepitaxial considerations. J Cryst Growth Vol. 53, No. 1, May 1981, 10-19

Kumar, T.S.S.; Manjubala, I. \& Gunasekaran, J. (2000). Synthesis of carbonated calcium phosphate ceramics using microwave irradiation. Biomaterials, Vol. 21, No. 16, August 2000, 1623-1629, ISSN: 0142-9612

Li, F.; Feng, Q.L.; Cui, F.Z.; Li, H.D. \& Schubertb, H. (2002). A simple biomimetic method for calcium phosphate coating. Surface and Coatings Technology, Vol. 154, No. 1, May 2002, 88-93, ISSN: 0257-8972

Li, L.H.; Kong, Y.M. \& Kim, H.W. (2004). Improved biological performance of Ti implants due to surface modification by micro-arc oxidation. Biomaterials. Vol. 25, No. 14, June 2004, 2867-2875, ISSN: 0142-9612

Li, L.H.; Kim H.W.; Lee, S.H.; Kong, Y.M. \& Kim, H.E. (2005). Biocompatibility of titanium implants modified by microarc oxidation and hydroxyapatite coating. Wiley Periodicals, Inc. J Biomed Mater Res A, Vol. 73, No. 1, April 2005, 48-54, ISSN: 15493296

Liu, Q.; Ding, J.; Mante, F.K.; Wunde, S.L. \& Baran, G.R. (2002). The role of surface functional groups in calcium phosphate nucleation on titanium foil: a self- 
assembled monolayer technique. Biomaterials, Vol. 23, No. 15, August 2002, 31033111, ISSN: 0142-9612

Liu, X.Y.; Paul, K.C. \& Ding, C.X. (2004). Surface modification of titanium, titanium alloys, and related materials for biomedical applications. Mater Sci Eng R, Vol. 47, No. 3-4, December 2004, 49-121, ISSN: 0927-796X

Lu, X. \& Leng, Y. (2004). TEM study of calcium phosphate precipitation on bioactive titanium surfaces. Biomaterials, Vol. 25, No. 10, May 2004, 1779-1786, ISSN: 01429612

Lu, X.; Zhao, Z.F. \& Leng, Yang. (2007). Biomimetic calcium phosphate coatings on nitricacid-treated titanium surfaces. Materials Science and Engineering C, Vol. 27, No. 4, May 2007, 700-708, ISSN: 0928-4931

Morais, L.S.; Serra, G.G.; Muller, C.A.; Andrade, L.R.; Palermo, E.F.A.; Elias, C.N. \& Meyers, M. (2007). Titanium alloy mini-implants for orthodontic anchorage: Immediate loading and metal ion release. Acta Biomater, Vol. 3, No. 3, May 2007, 331-339, ISSN: 1742-7061

Müller, L. \& Müller, F.A. (2006). Preparation of SBF with different $\mathrm{HCO}_{3}$ - content and its influence on the composition of biomimetic apatites. Acta Biomater, Vol. 2, No. 2, March 2006, 181-189, ISSN: 1742-7061

Mullin, J.W. (2001). Crystallization. Butterworth-Heinemann. UK :86-214

Okazaki, M. \& Takahashi, J. (1999). Synthesis of functionally graded $\mathrm{CO}_{3}$ apatite as surface biodegradable crystals. Biomaterials., Vol. 20, No. 12, June 1999, 1073-1078 ISSN: 0142-9612

Oyane, A.; Kim, H.M.; Furuya, T.; Kokubo, T.; Miyazaki, T. \& Nakamura, T. (2003). Preparation and assessment of revised simulated body fluids. J Biomed Mater Res A, Vol. 65A, No. 2, 188-195, 1 May 2003 ISSN: 0021-9304

Rodriguez, R.; Kim, K.H. \& Ong, J.L. (2003). In vitro osteoblast response to anodized titanium and anodized titanium followed by hydrothermal treatment. J Biomed Mater Res A, Vol. 65, No. 3, June 2003, 352-358, ISSN: 0021-9304

Song, W.H.; Jun, Y.K.; Han, Y. \& Hong, S.H. (2004). Biomimetic apatite coatings on microarc oxidized titania. Biomaterials, Vol. 25, No. 17, August 2004, 3341-3349, ISSN: 01429612

Song, W.H.; Ryu, H.S. \& Hong, S.H. (2005). Apatite induction on Ca-containing titania formed by micro-arc oxidation. J Am Ceram Soc, Vol. 88, No. 9, September 2005, 2642-2644, ISSN: 0002-7820

Toworfe, G.K.; Composto, R.J.; Shapiro, I.M. \& Ducheyne, P. (2006). Nucleation and growth of calcium phosphate on amine-, carboxyl- and hydroxyl-silane self-assembled monolayers. Biomaterials, Vol. 27, No. 4, February 2006, 631-642, ISSN: 0142-9612

Wang, Y.M.; Guo, L.X.; Ouyang, J.H.; Zhou, Y. \& Jia, D.C. (2009). Interface adhesion properties of functional coatings on titanium alloy formed by microarc oxidation method. Applied Surface Science, Vol. 255, No. 15, May 2009, 6875-6880, ISSN: 01694332

Wei, D.Q.a.; Zhou, Y.; Jia, D.C. \& Wang, Y.M. (2007). Characteristic and in vitro bioactivity of microarc oxidized $\mathrm{TiO}_{2}$-based coating after chemical treatment. Acta Biomater., Vol. 3, No. 5, September 2007, 817-827, ISSN: 1742-7061 
Wei, D.Qb.; Zhou, Y.; Jia, D.C. \& Wang, Y.M. (2007). Effect of applied voltage on the structure of microarc oxidized $\mathrm{TiO}_{2}$-based bioceramic films. Mater. Chem. Phys., Vol. 104, No. 1, July 2007, 177-182, ISSN: 0254-0584

Wei, D.Qc.; Zhou Y.; Wang Y.M. \& Jia D.C. (2007). Characteristic of microarc oxidized coatings on titanium alloy formed in electrolytes containing chelate complex and nano-HA. Appl. Surf. Sci., Vol. 253, No. 11, March 2007, 5045-5050, ISSN: 0169-4332

Wei, D.Q.; Zhou, Y.; Wang, Y.M. \& Jia, D.C. (2008). Chemical etching of micro-plasma oxidized titania film on titanium alloy and apatite deposited on the surface of modified titania film in vitro. Thin Solid Films, Vol. 516, No. 8, February 2008, 18181825, ISSN: 0040-6090

Wei, D.Q.; Zhou, Y. \& Yang, C.H. (2009). Structure, cell response and biomimetic apatite induction of gradient $\mathrm{TiO}_{2}$-based/nano-scale hydrophilic amorphous titanium oxide containing Ca composite coatings before and after crystallization. Colloids and Surfaces B: Biointerfaces, Vol. 74, No. 1, November 2009, 230-237, ISSN: 0927-7765

Wen, C.E.; Xu, W.; Hu, W.Y. \& Hodgson, P.D. (2007). Hydroxyapatite/titania sol-gel coatings on titanium-zirconium alloy for biomedical applications. Acta Biomater, Vol. 3, No. 3, May 2007, 403-410, ISSN: 1742-7061

$\mathrm{Wu}, \mathrm{W}$. \& Nancollas, G.H. (1997). Nucleation and crystal growth of octacalcium phosphate on titanium oxide surfaces. Langmuir, Vol. 13, No. 4, February 1997, 861-865, ISSN: 0743-7463

Wu, W. \& Nancollas, G.H. (1999). Determination of interfacial tension from crystallization and dissolution data: a comparison with other methods. Adv Colloid Interface Sci, Vol. 79, No. 2-3, February 1999, 229-279, ISSN: 0001-8686

Xie, Y.T.; Liu, X.Y.; Chu, P.K. \& Ding, C.X. (2006). Nucleation and growth of calciumphosphate on Ca-implanted titanium surface. Surface Science, Vol. 600, No. 3, February 2006, 651-656, ISSN: 0039-6028

Xiong, Lu. \& Yang, Leng. (2005). Theoretical analysis of calcium phosphate precipitation in simulated body fluid. Biomaterials, Vol. 26, No. 10, April 2005, 1097-1108, ISSN: 0142-9612

Yerokhin, A.L.; Nie, X.; Leyland, A.; Matthews, A. \& Dowey, S.J. (1999). Plasma electrolysis for surface engineering. Surf Coat Technol, Vol. 122, No. 2-3, December 1999, 73-93, ISSN: 0257-8972

Zhang, Q.Y.; Leng, Y. \& Xin, R.L. (2005). A comparative study of electrochemical deposition and biomimetic deposition of calcium phosphate on porous titanium. Biomaterials, Vol. 26, No. 16, June 2005, 2857-2865, ISSN: 0142-9612

Zhang, Y.M.; Bataillon-Linez, P.; Huang, P.; Zhao, Y.M.; Han, Y.; Traisnel, M.; Xu, K.W. \& Hildebrand, H.F. (2004). Surface analyses of micro-arc oxidized and hydrothermally treated titanium and effect on osteoblast behavior. J Biomed Mater Res A, Vol. 68A, No. 2, February 2004, 383-391, ISSN: 0021-9304

Zheng, X.B.; Huang, M.H. \& Ding, C.X. (2000). Bond strength of plasma-sprayed hydroxyapatite/Ti composite coatings. Biomaterials, Vol. 21, No. 8, April 2000, 841849, ISSN: 0142-9612 
Zhu, X.L.; Kim, K.H. \& Jeong, Y.S. (2001). Anodic oxide films containing Ca and P of titanium biomaterial. Biomaterials, Vol. 22, No. 16, August 2001, 2199-2206, ISSN: 0142-9612

Zhu, X.L.; Ong, J.L.; Kim, S.Y. \& Kim, K.H. (2002). Surface characteristics and structure of anodic oxide films containing $\mathrm{Ca}$ and $\mathrm{P}$ on a titanium implant material. J Biomed Mater Res, Vol. 60, No. 2, May 2002, 333-338, ISSN: 0021-9304 


\title{
Antimicrobial Biomimetics
}

\author{
Ana Maria Carmona-Ribeiro, Lilian Barbassa and Letícia Dias de Melo \\ Biocolloids Lab, IQUSP, Universidade de São Paulo, \\ Caixa Postal 26077, CEP05513-970 São Paulo SP \\ Brazil
}

\section{Introduction}

A vast territory for research is open from mimicking the behaviour of microorganisms to defend themselves from competitors. Antibiotics secreted by bacteria or fungi can be copied to yield efficient molecules which are active against infectious diseases. On the other hand, nanotechnology provides novel techniques to probe and manipulate single atoms and molecules. Nanoparticles are finding a large variety of biomedical and pharmaceutical applications, since their size scale can be similar to that of biological molecules (e.g. proteins, DNA) and structures (e.g. viruses and bacteria). They are currently being used in imaging (El-Sayed et al., 2005), biosensing (Medintz et al.,2005), biomolecules immobilization (Carmona-Ribeiro, 2010a), gene and drug delivery (Carmona-Ribeiro, 2003; CarmonaRibeiro, 2010b) and vaccines (O’Hagan et al., 2000; Lincopan \& Carmona-Ribeiro, 2009; Lincopan et al., 2009). They can also incorporate antimicrobial agents (antibiotics, metals, peptides, surfactants and lipids), can be the antimicrobial agent or used to produce antimicrobial devices. Antimicrobial agents found in Nature can sucessfully be copied for synthesis of novel biomimetic but synthetic compounds. In this review, synthetic cationic surfactants and lipids, natural and synthetic peptides or particles, and hybrid antimicrobial films are overviewed unraveling novel antimicrobial approaches against infectious diseases.

\section{Biofilms, antimicrobial films and surfaces}

\subsection{Impregnation of materials and coatings with antimicrobials}

The minimal conditions required for life on a given material are the presence of water or wet air, with a little dissolved gas, mineral salts and organic molecules so that in natural environments, biofilms can form on surfaces of materials (Lejeune, 2003). A biofilm is a structured consortium of bacteria embedded in a self-produced polymer matrix consisting of polysaccharide, protein and DNA. For human societies, the most detrimental property of surface-associated contaminants as biofilms is probably the expression of specific characters, such as increased resistance to detergents, disinfectants, antibiotics and immunological defenses (Hoiby et al., 2010). Many nosocomial infections are considered consequences of surface contaminations, since biofilms can be formed in indwelling medical devices and biomaterials, or even in equipments such as air conditioning and water-distribution networks (Mah \& O’Toole, 2001; Hoiby et al., 2010). Besides, contaminated common hospital surfaces, such as door handles (Oie et al., 2002), stethoscopes (Cohen et al., 1997) and ward 
fabrics and plastics (Neely \& Maley, 2000) can act as reservoirs of potentially harmful microbes. Upon being touched, these contaminated surfaces could lead to the spread of infection and propagate the contamination to other surfaces and patients in the vicinity (Page et al., 2009). Moreover, the device-related infections are usually associated with increased morbidity, mortality and additional hospital cost to patient (Tamilvanan et al., 2008). Antimicrobial films are required for packaging of food products, since microbial contaminations are responsible for enormous losses in food safety, conservation and shelf life (Cha \& Chinnan, 2004; Dutta et al., 2009). Much effort has been devoted to the design of antimicrobial materials in form of particles, coatings or surfaces able to prevent surface contamination and/or erradicate the biofilm consortia (Tiller et al., 2001; Francolini et al., 2004; Furno et al., 2004; Zivanovic et al., 2007; Pereira et al., 2008; Ye et al., 2008; Caro et al., 2009; Avila-Sosa et al., 2010).

The first combinations of dental cements and resins with antibiotic drugs were described in the fifties (Colton \& Ehrlich, 1953) whereas resorbable or soluble polymeric carriers which could deliver active drugs directly at the site of infection started to be described in the late seventies (Kopecek, 1977; Arai et al., 2010; Campoccia et al., 2010; Feng et al., 2010; Noel et al., 2010). Control, eradication or inhibition of biofilms and surface-related contaminations included development of novel materials, ranging from synthetic (Park et al., 1998; Hirota et al., 2005; Jampala et al., 2008; Pereira et al., 2008; Bryaskova et al., 2010) to natural and biodegradable compounds (Zhang et al., 1994; Cha \& Chinnan, 2004; Pranoto et al., 2005; Maizura et al., 2007; Noel et al., 2010). Local antibiotic delivery systems have been proposed as alternative therapies to typical prophylactic antibiotic dosing (Frank et al., 2005). The most common drug carrier has been polymethylmethacrylate (PMMA) (McLaren, 2004; Nelson, 2004) but other materials could also be used as a local drug delivery system, especially in orthopaedic area, such as calcium sulfate (Heijink et al., 2006), collagen (Diefenbeck et al., 2006) and chitosan (Khor \& Lim, 2003; Noel et al., 2010).

Different strategies for producing active films or surfaces have been described as summarized in Figure 1. The layer-by-layer (LbL) deposition consists in a LbL assembly of multiple thin films based on intermolecular electrostatic, hydrogen bonding, and/or covalent interactions between film components (Decher, 1997; Cui et al., 2008; Picart, 2008; Dvoracek et al., 2009; Agarwal et al., 2010; Shukla et al., 2010; Kharlampieva et al., 2009). Among other coating techniques, LbL assembly has the advantage of being a gentle, aqueous assembly process with nanometer level control over the composition of the layers. For example, Shukla et al. (2010) assembled films on silicon substrates which were plasma etched and immediately immersed in a solution containing a cationic polyelectrolyte, then water rinsed and then submerged in the polyanion of choice for the particular architecture being constructed also followed by water rinse step. After this the substrate was immersed in water solution containing the cationic antimicrobial amine-terminated ponericin G1 peptide and water rinsed again. Four deposition steps produced a tetralayered film with sucessive layers: (polycation/ polyanion/ ponericin G1/ polyanion)n, where n represents the number of deposited tetralayer repeats (Shukla et al., 2010). Another approach to produce antimicrobial films and coatings is the spin-coating technique (Pereira et al., 2008; Jausovec et al., 2008), which is based on the solubilization of polymer and antimicrobial compound in a volatile solvent, e.g. chloroform, followed by spin-coating of the solution on the surface of a glass slide or silicon wafer. The hybrid film is obtained from spinning and solvent evaporation (Pereira et al., 2008) (Figure 1). Graftings or covalent attachments of certain antimicrobial chemical moieties, such as pyridinium groups (Tiller et al., 2001) or 
lysozyme (Caro et al., 2009) to surfaces can also be used to prepare antimicrobial materials. In an example of the plasma-enhanced method (Jampala et al., 2008), stainless steel substrates were pretreated with oxygen plasma and received a hexamethyldisiloxane layer. Residual gases were then pumped out and ethylene diamine plasma films deposited before substrate immersion in a hexyl bromide solution for deposition of the antimicrobial layer (Figure 1).

\section{LbL deposition}
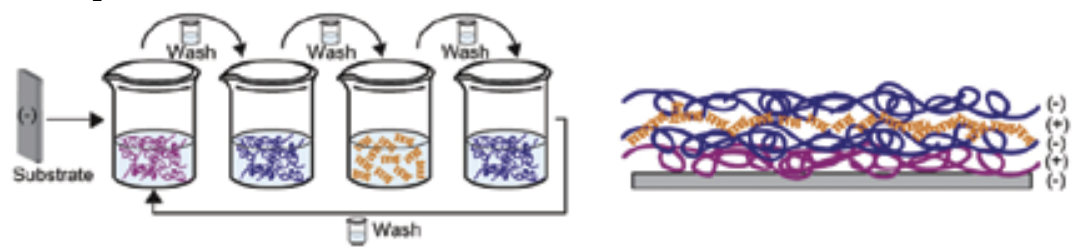

Shukla

et al.,

2010

Spin-coating

Grafting
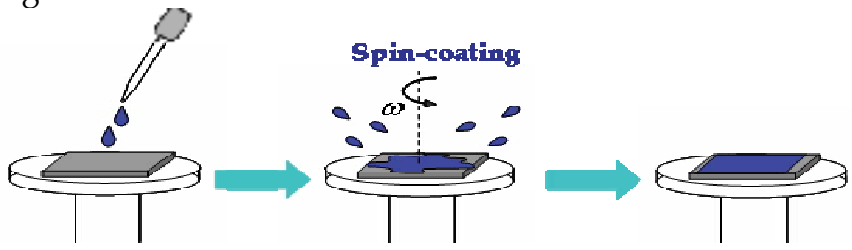

Pereira

et al.,

2008

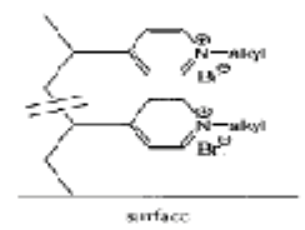

Tiller et al., 2001

Plasma-enhanced deposition

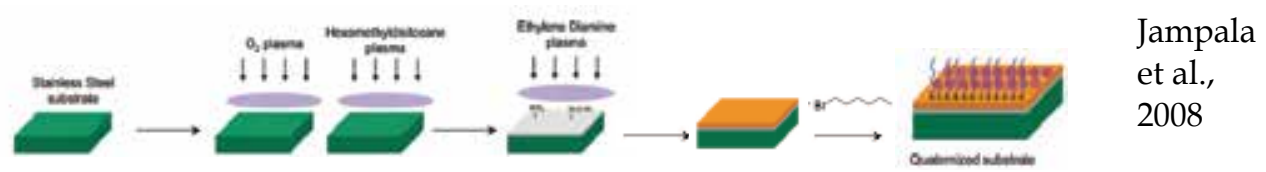

Fig. 1. Methods for casting antimicrobial films on surfaces. Adapted from Tiller et al., 2001; Pereira et al., 2008. Adapted with permission from Jampala et al., 2008. Copyright 2008 American Chemical Society. Reprinted from Biomaterials, Shukla, A.; Fleming, K.E.; Chuang, H.F.; Chau, T.M.; Loose, C.R.; Stephanopoulos, G.N. \& Hammond, P.T., Controlling the release of peptide antimicrobial agents from surfaces, 31, 2348-2357, Copyright (2010), with permission from Elsevier.

Surfaces modified by liposomes have already been recognized as an interesting and promising delivery vehicle for active and passive drug targeting purposes (Catuogno \& Jones, 2003; Pinto-Alphandary et al., 2000; Jones, 2005). Liposomes can either disrupt on adsorption or adsorb intact, or a combination of both processes can occur (Carmona-Ribeiro, 1992; Carmona-Ribeiro \& Lessa, 1999; Carmona-Ribeiro, 2003; Carmona-Ribeiro, 2010a,b). Hence, systems based on vesicles deposited on the surface of a biomedical device could limit 
drug delivery to the area immediately surrounding the device, avoiding side effects in the rest of the organism (Vermette et al., 2002). Various strategies of liposome deposition on surfaces are available such as immobilization by hydrophobic interactions, covalent linkage and specific binding (Brochu et al., 2004). Pasquardini et al. (2008) reported the prevention of bacterial adhesion and colonization of polymeric surfaces through the immobilization of liposomes on polystyrene material. The surface was functionalized based on the deposition of covalently coupled lipid structures from antibiotic loaded vesicles, using either deposition of cationic vesicles on negatively charged surfaces or formation of covalent linkages between functionalized lipids and amines enriched surfaces. The lipid film, which was deposited on the polymeric surface, was used for loading and delivering a specific active agent, which was rifampicin, in a cationic liposome, to Staphylococcus epidermidis (Pasquardini et al., 2008). An artificial bone scaffold combined with liposomes was recently developed for therapy and prevention of refractory bacterial infections (Zhu et al., 2010). The porous $\beta$-tricalcium phosphate $(\beta-\mathrm{TCP})$ was combined with liposomal gentamicin to form a complex drug carrier, which could release an initial high dose of antibiotic from the matrix, and a further sustained release of free gentamicin from the liposome allowing treatment and prevention of post-operative osteomyelitis (Zhu et al., 2010).

Pathogenic bacteria secrete virulence factors such as toxins and lipases that actively damage cell membranes and tissues around infected wounds, while nonpathogenic bacteria do not (or not at high concentration) (DiRita et al., 1991; Zhang \& Austin, 2005). On this basis a 'smart' wound dressing system modified with antimicrobials encapsulated on lipid vesicles was developed, which only released an encapsulated antimicrobial agent in the presence of pathogenic bacteria, without responding to commensal/harmless bacteria (Zhou et al., 2010). The specificity towards pathogenic bacteria is particularly desirable given the importance of the normal microflora in providing a natural defense against infection (Tagg \& Dierksen, 2003). Importantly, this would minimize the evolutionary pressure for the selection of antibiotic resistant microorganisms and prolong the efficacy and shelf life of the encapsulated antimicrobial (Hecker et al., 2003). Figure 2 illustrates this responsive antimicrobial system.

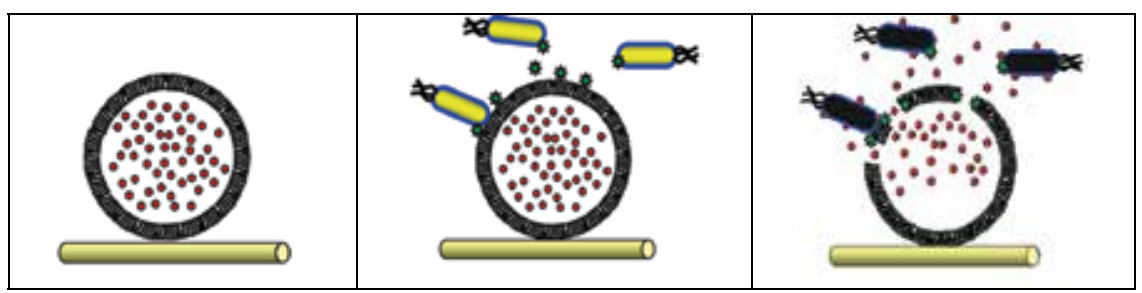

Fig. 2. Responsive antimicrobial system based on liposomal encapsulation of antimicrobials and drug release triggered by toxin secretion from pathogenic bacteria. Adapted with permission from Zhou et al., 2010. Copyright 2010 America Chemical Society.

Hydrogel matrices such as carbopol 940 or poly(ethylene glycol) gelatin incorporated liposomes improving the viscosity of the topical formulation and its retention time at the site of administration (Hosny, 2010). The bioadhesive properties of the gel ensured a sustained release of antibiotics from the liposomes. Alternatively, plain broad-spectrum antimicrobials, without any carriers, have been incorporated into devices (Tebbs \& Elliott, 1993; Bach et al., 1999; Donelli et al., 2002; Zalewska \& Ginalska, 2009; Francolini \& Donelli, 
2010). The coating of device surfaces with one or two antimicrobial substances or entrapping of these agents within the device material are approaches often used to obtain devices with different antimicrobial spectra and durations of the antimicrobial effect (Donelli \& Francolini, 2001; Darouiche, 2008; Zilberman \& Elsner, 2008). These are eluted with the aim of preventing biofilm formation by killing early colonizing bacteria. However, sufficient antibiotic must be incorporated for the "user-lifetime" of the device, and such incorporation must not damage the properties of the material (Danese, 2002). The technique of delivery must guarantee a rapid release of the antibiotic from the carrier and local drug levels well above the minimal inhibitory concentration (MIC). The drug release must be restricted to a limited period of time to prevent development of resistant bacterial strains and bactericidal should be favoured over bacteriostatic antibiotics (Schmidmaier et al., 2006). One of the main drawbacks of most available antimicrobial-coated devices is the burst release of the adsorbed antibiotics in the first few hours, followed by a long-lasting phase of slow release at low concentrations (Munson et al., 2004). This behaviour can develop antimicrobial resistance (Danese, 2002).

Antimicrobial polyurethane systems were developed containing two antibiotics, cefamandole nafate and rifampicin (RIF), selected by their action spectrum and their functional groups to interact with the suitably functionalized polymer (Ruggeri et al., 2007). In other similar instances, polyethylene glycol (PEG) was used as a pore forming agent (Kim et al., 2000; Meier et al., 2004). Although PEG is biologically inactive, the channels formed inside the polymeric matrix facilitated drug flow. Hence antibiotics released from these antimicrobial polyurethane systems inhibited the bacterial growth and exhibited a synergistic action when both cefamandole nafate and rifampicin antibiotics were present. In particular, PEG10000containing polymer was active against the RIF-resistant Staphylococcus aureus strain up to 23 days. These results suggest that the combined entrapping of antibiotics and pore formers in these novel polymer systems could be promising to prevent bacterial colonization (Ruggeri et al., 2007). Figure 3 illustrates the prevention of microbial colonization on such surfaces.
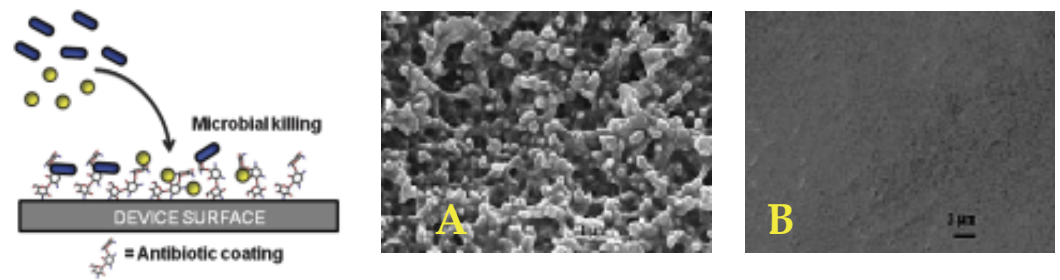

Fig. 3. Colonization of cocci (in yellow) and bacillus (in blue) on bare (A) or antibioticscoated polyurethane surface (B). Adapted with permission from Ruggeri et al., 2007, Journal of Biomedical Materials Research, and from Francolini \& Donelli, 2010, FEMS Immunology and Medical Microbiology. Copyright 2007 and 2010 John Wiley \& Sons.

Hybrid antimicrobial biomaterials with potential to be applied as orthopaedic implants were recently prepared through immobilization of aminoglycoside antibiotics (amikacin or gentamicin) on hydroxyapatite ceramics (HAp), showing activity against S. aureus, $S$. epidermidis, Pseudomonas aeruginosa and resistance to biofilm formation (Zalewska \& Ginalska, 2009). In fact, polymeric materials from both natural and synthetic origins are widely recognized as carriers for effective delivery of antimicrobial agents to treat the infections associated with orthopaedic implants. Resorbable polymeric materials such as 
polylactides (Mader et al., 1997; Schmidmaier et al., 2006), copolymers of lactide and glycolide (Mader et al., 1997; Ambrose et al., 2003), polycaprolactone (Burd et al., 2001; Le Rey et al., 2003), hydroxyapatite and glass ceramics (Saito et al., 2002; Makinen et al., 2005; Zalewska \& Ginalska, 2009), calcium sulfate (Nelson et al., 2002), and fibrin sealant implants (Mader et al., 2002) have already been investigated for use as antibiotic delivery systems. Prevention and treatment of osteomyelitis, particularly associated with orthopaedic implant surgery, have been the focus of many studies, since in most surgical procedures that include the incorporation of implants, the tissue-implant surface is especially prone to microbial contamination. Degradable polymer implant coating with antibiotics have been developed (Schmidmaier et al., 2006). For local antibiotic therapy, titanium K-wires to be implanted into the medullary canals of rat tibiae were coated with poly(D,L-Lactide) (PDLLA) loaded with gentamicin. Thereby, the onset of infections was prevented in $80-90 \%$ of animals thus treated (Schmidmaier et al., 2006). Since the PDLLA coating degrades by hydrolysis within 3-6 months of implantation with the products of degradation metabolized in the citric acid cycle (Hutmacher et al., 1996; Schmidmaier et al., 2001; Park et al., 2009), this local application of gentamicin from PDLLA-coated implants might support systemic antibiotic prophylaxis preventing implant-associated osteomyelitis.

Usnic acid as an alternative antimicrobial agent for device coating or impregnation has been loaded on polymer surfaces since it has the desirable properties of poor solubility in biological fluids and is not recomended for use in clinics for therapy. Polyurethanes adsorbed usnic acid and thereby there was inhibition of $S$. aureus biofilm formation (Francolini et al., 2004). Antiseptics have also been used to develop catheter materials (BrunBuisson et al., 2004; Ostendorf et al., 2005; Rupp et al., 2005). A hydrophilic catheter incorporated with iodine, leading to a polyvinylpyrrolidone-iodine complex on the inner and outer surfaces of the catheter inhibited adhesion of Staphylococcus spp., Escherichia coli, Pseudomonas aeruginosa and Candida albicans, during the time of iodine release (Jansen et al., 1992). Catheters incorporated with benzalkonium chloride also demonstrated a long-lasting antimicrobial activity against Staphylococcus spp., Gram-negative bacteria and C. albicans (Tebbs \& Elliott, 1994). A polyurethane-based catheter impregnated with minute amounts of the antiseptic chlorhexidine and silver sulfadiazine was developed (Heard et al., 1998). This catheter was firstly coated only on the external surface and exhibited antimicrobial properties for nearly 15 days. A second generation of chlorhexidine-silver sulfadiazine was coated both internally and externally, and exhibited enhanced chlorhexidine activity, with a marked decrease in the colonization on these catheters (Brun-Buisson et al., 2004; Ostenford et al., 2005; Rupp et al., 2005).

Advantages of polymeric antimicrobial agents, when compared to conventional antimicrobial agents of low molecular weight, are their nonvolatile character, chemical stability, and low permeation through the skin of a man or animal. Thus, they may enhance the efficacy of some existing antimicrobial agents and minimize the environmental problems accompanying the residual toxicity of the agents, in addition to prolonging their lifetime (Akashi et al., 2001; Chen \& Cooper, 2002; Gottenbos et al., 2002). Synthetic polymers with functional groups, especially when the functional group is a antimicrobial, active group, such as the quaternary nitrogen, are receiving considerable attention (Kenawy \& Mahmoud, 2003; Li et al., 2006; Pereira et al., 2008; Melo et al., 2010).

Quaternary ammonium salts are commonly employed as disinfectants, and effective against a wide variety of Gram-positive and Gram-negative bacteria (Tapias et al., 1994; Campanhã et al., 1999; Gilbert \& Moore, 2005; Carmona-Ribeiro et al., 2006). Various cationic and 
antimicrobial architectures have been tested such as polyelectrolyte layers (Tiller et al., 2001; Thome et al., 2003; Codling et al., 2003; Cen et al., 2003; Li et al., 2006; Vieira \& CarmonaRibeiro, 2008), hyperbranched dendrimers (Chen \& Cooper, 2000; Chen \& Cooper, 2002; Abid et al., 2010) and long-chained amphiphiles (Abel et al., 2002; Haldar et al., 2005). The deposition of organic monolayers onto solid surfaces containing quaternary ammonium groups has been shown to prevent deposition and growth of bacterial biofilms (Kugler et al., 2005). Molecules with a net positive charge are able to kill microorganisms both in solution (Fidai et al., 1997; Friedrich et al., 2000) or upon attachment or adsorption to surfaces (Isquith et al., 1972; Endo et al., 1987; Tiller et al., 2001; Thome et al., 2003; Kugler et al., 2005; Pereira et al., 2008) or particles (Vieira et al., 2003; Vieira \& Carmona-Ribeiro, 2008). Particularly interesting were the cationic liposomes (Tapias et al., 1994; Sicchierolli et al., 1995; Campanhã et al., 1999) or cationic bilayer fragments composed solely of dioctadecyldimethylammonium bromide or DODAB due to their intrinsic microbicidal property (Vieira \& Carmona-Ribeiro, 2001; Lincopan et al., 2003; Lincopan et al., 2005).

Polymeric bactericides are more potent than their monomeric counterparts (Kenawy et al., 2007). Surfaces with cations deposited on them were shown to kill microbes upon contact in the 1980s (Speier \& Malek, 1982) especially when treated with hydrophobic polycations (Klibanov, 2007). These cationic materials electrostatically attract a microorganism cell towards the treated surface, resulting in the puncturing of microbial cell envelope and subsequent cell death (Klibanov, 2007). Impregnation of polymers with quaternary ammonium compounds (QAC) was also achieved from deposition of alternate anionic and cationic polyelectrolyte layers where cetyltrimethylammonium bromide (CTAB) was the antimicrobial agent included in the cationic layer (Dvoracek et al., 2009). Films exposure to humidity allowed $\mathrm{CTAB}$ diffusion out of the film and bacterial growth inhibition in neighbouring regions. On silicon wafers, hybrid films, produced from spin-coating of a chloroformic solution of poly(methylmethacrylate) (PMMA) polymer and DODAB cationic lipid, exhibited remarkable antimicrobial activity against E. coli (Pereira et al., 2008). Antimicrobial PMMA/DODAB films are illustrated in Figure 4.
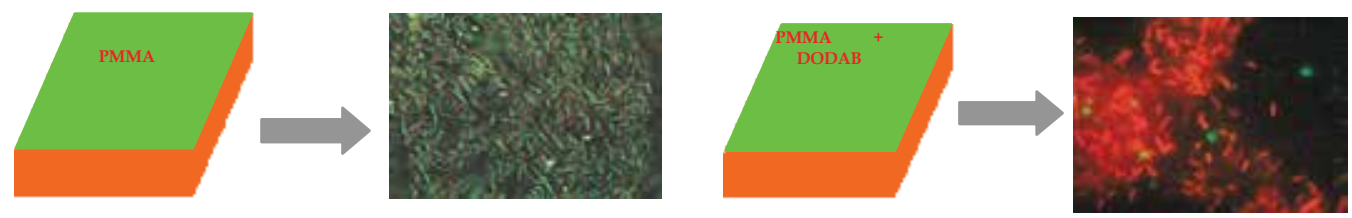

Fig. 4. PMMA films impregnated with a quaternary ammonium (QAC) lipid DODAB kill E. coli upon contact. In green, live, and in red, dead E. coli cells. Adapted with permission from Pereira et al., 2008. Copyright 2008 American Chemical Society.

Polymers of pyridinium derivatives were highly effective against Gram-positive bacteria (Kawabata \& Nishiguchi, 1988). Pyridinium derivatives with alkyl bromides were covalently attached to glass slides and designed to create surfaces that kill airborne bacteria on contact (Tiller et al., 2001). These surfaces were able to kill more than $90 \%$ of deposited $S$. aureus cells and more than $99 \%$ of deposited S. epidermidis, P. aeruginosa and E. coli cells in a dry state. The bacteria cells were sprayed onto the surfaces to simulate the deposition of airborne bacteria. These tethered amphipatic polycations, as well as polimyxin B and soluble cationic antimicrobials, probably share a similar mechanism of attacking bacteria, by displacing the divalent cations that hold together the negatively charged surface of the 
lipopolysaccharide network, thereby disrupting the outer membrane of Gram-negative bacteria. It is also possible that after destroying the outer membrane permeability barrier, the cationic groups of the tethered polymers further penetrate into the inner membrane, producing leakage (Vaara, 1992). Regarding Gram-positive bacteria, the action of immobilized polycations probably requires penetration of the cationic groups across the thick cell wall to reach the cytoplasmic membrane (Friedrich et al., 2000). Bromide salts of quaternized polyvinylpyridine (QPVP) with linear aliphatic chains of 2 and 5 carbon atoms were adsorbed onto silicon wafers, and lyzozyme molecules were adsorbed onto these polycations (Silva et al., 2009). The antimicrobial effect of lyzozyme bounded to the pyridinium derivative layers or to silicon wafers was evaluated with enzymatic assays using Micrococcus luteus. After 15 min of interaction with bacteria, pure QPVP with 5 carbons presented the best antimicrobial action, followed by pure QPVP with 2 carbons, mixtures of lyzozyme and QPVP with 5 carbons, pure lyzozyme and mixtures of QPVP with 2 carbons and the enzyme. When quaternary ammonium salts are linked to the polymer backbone by longer spacers, as in the case of QPVP with 5 carbons, their larger mobility favoured the biocidal effect. After one hour of interaction, all systems yielded $100 \%$ of death.

Antimicrobial silver particles alone or in combination with other metals or elements, such as carbon or platinum (Ranucci et al., 2003) or copper (Mclean et al., 1993) were also used to impregnate biomaterials (Davenas et al., 2002). Thin polymeric films prepared by the LbL method assembled oppositely charged polyelectrolytes, loaded with silver nanoparticles and presented differential cytotoxicity representing a good approach to manage microbial burden in wounds without impairment of wound healing (Agarwal et al., 2010). Impregnation of biodegradable polymer matrix with silver nanoparticles showed strong bactericidal effect against E. coli, S. aureus and P. aeruginosa (Bryaskova et al., 2010).

Silver has also been extensively used for the development of infection-resistant catheters. Polyurethane catheters in which carbon, silver and platinum particles are incorporated led to an electrochemically driven release of silver ions in the outer and inner vicinities of the catheter surface, demonstrating low catheter-related bloodstream infections (Ranucci et al., 2003). Silver-containing zeolite compounds received approval of Food and Drug Administration (FDA) for being used as food contact surfaces (Joerger, 2007). Silver-zeolites have already been incorporated into polymeric films yielding antimicrobial properties (Kamisoglu et al., 2008; Zampino et al., 2008; Fernández et al., 2010). Polymer composites of plasticized poly(vinylchloride) pellets with silver zeolites demonstrated activity against $S$. epidermidis and E. coli (Zampino et al., 2008), while polyurethane composites with silver zeolites showed antimicrobial action against $E$. coli (Kamisoglu et al., 2008) and polylactid acidpolylactide (PLA)/silver zeolite composites also presented activity against S. aureus and E. coli, with silver being effectively released from the films (Fernández et al., 2010). The silvercontaining materials usually rely on the diffusion of $\mathrm{Ag}^{+}$ions from the material and their subsequent action on adherent microbes as broad spectrum antimicrobials (Lansdown, 2006).

\subsection{Coatings with covalent modifications}

The surfaces of medical devices can be simply modified with the application of external coating substances onto them. Thereby, alterations of material surfaces may lead to changes in specific and non-specific interactions with microorganisms and, thus, reduce microbial adherence. Medical devices made out of a material that would be antiadhesive or at least colonization resistant would be the most suitable candidates to avoid colonization and subsequent infection (Duran, 2000; Chandra et al., 2005; Hou et al., 2007). 
One possible approach to inhibit microbial contamination of surfaces is to prepare a surface to which microbes find it hard to become attached. This is a preventive strategy, where the aim is to prevent microbial adhesion to the surface in the first place (Page et al., 2009). One well established method for preventing the adhesion of microbes, proteins and mammalian cells to surfaces is to coat them with a layer of poly(ethylene glycol) (PEG) (Page et al., 2009). PEG modification of polyurethane surfaces inhibited microbial adhesion (Park et al., 1998; Ostuni et al., 2001; Hou et al., 2007). The current method involves the deposition of a selfassembled monolayer, over a substrate, followed by functionalization of the monolayer with PEG. PEG polymeric surfaces are antimicrobial firstly because of the steric repulsion between PEG and the microbial cell envelope. The dynamic movement of PEG chains tethered to the surface, coupled with their lack of binding sites further hamper microbe adhesion (Page et al., 2009). For protecting stainless steel surfaces against protein and/or bacterial adhesion, thin films including the glycosidase hen egg white lysozyme (HEWL) and/or PEG were covalently bound to flat substrates pretreated with poly(ethylene imine) (PEI) (Caro et al., 2009). The ability of these modified surfaces to prevent protein adsorption and bacterial adhesion together with their biocide properties were tested employing bovine serum albumin (BSA), and the bacteria Listeria ivanovii and Micrococcus luteus. The cografting of PEG and HEWL resulted in a surface with both antiadhesive and antibacterial properties (Caro et al., 2009). Figure 5 illustrates these antiadhesive and antibacterial surfaces grafted with HEWL and PEG.
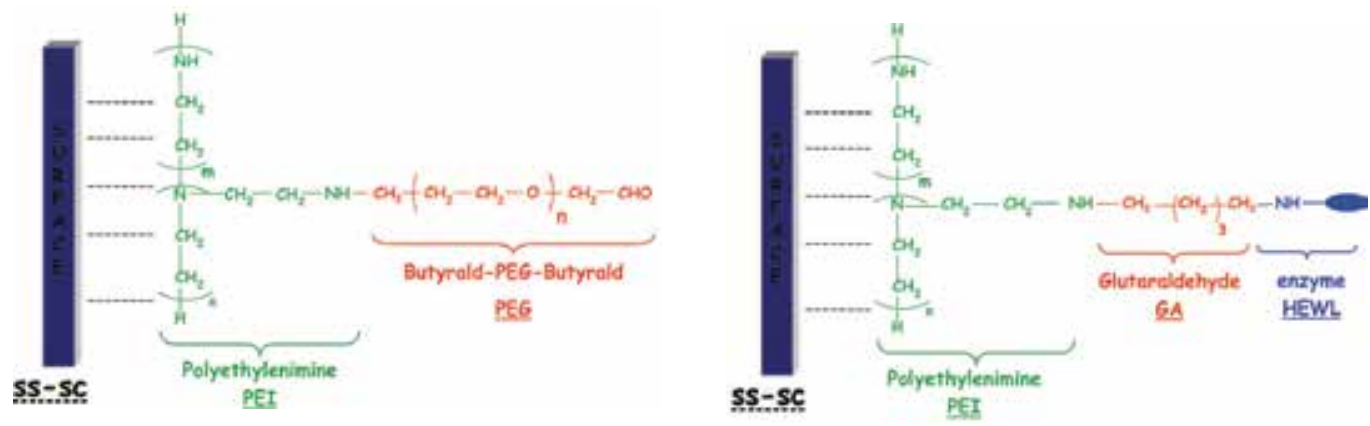

Fig. 5. Antiadhesive and antibacterial surfaces on stainless steel (SS) with graftings of poly(ethylene glycol) (PEG) or hen egg white lyzozyme (HEWL). Adapted with permission from Caro et al., 2009. Copyright 2009 American Chemical Society.

Inhibition of $C$. albicans biofilm formation was achieved by adding $6 \%$ polyethylene oxide (PEO) to polyurethane surfaces (Chandra et al., 2005). Similarly, biofilm formation by $C$. albicans and Candida tropicalis was inhibited on a silicone rubber voice prosthesis treated with a colloidal palladium/tin solution (resulting in a thin metal coat) (Dijk et al., 2000). On similar surfaces of hydrophobic polyurethanes modified with hydrophilic polyethylene oxide, adhesion of S. epidermidis was abolished (Patel et al., 2003; Patel et al., 2007). These modified surfaces significantly inhibited S. exidermidis biofilm formation over $48 \mathrm{~h}$ in vitro (Patel et al., 2007).

Polymers with zwitterionic head groups were also applied as surface coatings, preventing microbial contamination on surfaces. The zwitterionic nature of the polymer head group mimics that found in the lipid bilayer of biological membranes imparting biocompatibility and non-thrombogenic character to these materials. Several examples of this concept are 
available from the literature such as polymers based on phosphorylcholine (Lewis, 2000; Rose et al., 2005; Hirota et al., 2005), sulfobetain and carboxybetaine (Cheng et al., 2007). The zwitterionic head groups increased hydrophilicity of the material, leading to reversible interactions between incident microbes and the surface and discouraging adhesion of cells, both mammalian and microbial (Cheng et al., 2007). Similarly, surfaces containing immobilized long-chain N-alkylated polyvinylpyridines and structurally unrelated Nalkylated polyethylenimines were reported to be lethal to $S$. aureus, S. epidermidis, $P$. aeruginosa and E. coli (Lin et al., 2002). The structure-activity analysis revealed that for surfaces to be bactericidal, the immobilized long polymeric chains have to be hydrophobic, but not excessively so, and positively charged.

Grafted or smeared on the surface, surfactants or polymers carrying electric charges could also modify the physico-chemical properties of the interface and decrease the binding ability of the colonizing organisms (Mireles et al., 2001). Synthetic vascular grafts such as polytetrafluoroethylene (PFTE) prostheses are easily accessible to pathogens after inserted into the patient. The lipophilicity of these PTFE grafts has been modulated with benzalkonium chloride (Harvey \& Greco, 1981; Greco et al., 1982) or tridodecylmethylammonium chloride (Harvey et al., 1982), or incorporating drugs into biodegradable polymer carriers (Gollwitzer et al., 2003). Recently, new lipid-based formulations to incorporate antibiotics for anti-infective action in grafts were developed. In this case, PFTE grafts were coated with lipophilic agents such as poly-lactid acid or tocopherol acetate as carriers for gentamicin and teicoplanin, in order to release high drug concentrations locally and completely inhibit bacterial colonization on the implant (Matl et al., 2008). A recent study also reported the covalent attachment of quaternary ammonium groups to stainless steel and porous filter paper (cellulose) surfaces, through low-pressure plasma-enhanced functionalization (Jampala et al., 2008). The grafting of quaternary ammonium groups on these surfaces yielded stable and very efficient bactericidal properties, with activity against $S$. aureus and Klebsiella pneumoniae.

Another different and recent approach is the concept of modifying a surface with bacteriophages, in order to produce an antimicrobial surface (Curtin \& Donlan, 2006). Bacteriophages are viruses that infect prokaryotic cells, which contain a core nucleic acid, usually double-stranded DNA (dsDNA), within a protein or lipoprotein capsid (Guttman et al., 2004; Hanlon, 2007). As obligate parasites of bacteria, the bacteriophages bind to microbial surfaces, injecting their genetic material and replicating within the bacterial host. If phage replication is a lytic process, it will result in the lysis of the host cell (Sulakvelidze et al., 2001). The characteristics of lytic phages, such as target specificity, rapid bacterial killing, and amplification at the site of infection, make them possible candidates as antimicrobial therapeutic agents (Deresinski, 2009). A phage-modified surface is certainly an interesting antimicrobial approach, especially because microorganisms currently resistant to antibiotics do not show resistance to phages. However, a few problems have to be considered. Firstly, the inherent specificity of phages to bacterial species, and further, bacteria can become resistant to phages (Stone, 2002). Thus, phage-treated surfaces should constantly be monitored. One study demonstrated the successful use of a developed wound dressing, containing lytic bacteriophages, to treat some skin infections that were not responding to conventional antimicrobial therapy (Stone, 2002). A biodegradable polymer wound dressing impregnated with ciprofloxacin, benzocaine, chymotrypsin, bicarbonate, and 6 lytic phages (Pyophage) with activity against P. aeruginosa, S. aureus, E. coli, Streptococcus spp. and Proteus spp. was also reported (Markoishvili et al., 2002). Another research showed the action of 
catheters pre-treated with a coagulase-negative staphylococci phage reducing significantly S. epidermidis biofilm formation (Curtin \& Donlan, 2006). Finally, the use of bacteriophages has been recently reported as a promising approach in the control of $S$. epidermidis and $P$. aeruginosa biofilm formation when catheters are pretreated with a cocktail of bacteriophages, thus reducing the 48 -h mean biofilm cell density by $99.9 \%$, even if few biofilm isolates were reported to be resistant to these phages (Fu et al., 2010). Approximate $90 \%$ reduction in both Proteus mirabilis and E. coli biofilm formation on bacteriophage-treated catheters when compared with untreated controls followed impregnation of hydrogel-coated catheter sections with a lytic bacteriophage (Carson et al., 2010).

\subsection{Biodegradable antimicrobial materials}

There has been a growing interest over the past few years in applications of biopolymers due to their renewable, sustainable and biodegradable properties (Zivanovic et al., 2007). One of the most popular biopolymers is chitosan. Chitosan is a cationic biopolymer obtained by $\mathrm{N}$-deacetylation of chitin, which is known to be the second most abundant biopolymer in nature and is the major component of exoskeleton of crustaceans (Roberts \& Wood, 2000). This biopolymer has been found to be nontoxic, biodegradable, biocompatible in addition to having antimicrobial characteristics (Park et al., 2002; Jayakumar et al., 2007). In view of these qualities, chitosan films have been used as a packaging material for the quality preservation of a variety of food products (Park et al., 2004). Blending of chitosan and polyethylene oxide (PEO) produced films with good antimicrobial effect against E. coli (Zivanovic et al., 2007). Chitosan-based films have the potential to be used in the food industry as active packaging materials to inhibit food-borne pathogens and in the pharmaceutical industry for controlled release of active compounds (Zivanovic et al., 2007; Noel et al., 2010).

Different theories have been put forward to explain the antimicrobial mode of action of chitosan such as chitosan interaction with intracellular targets, eg DNA (Rabea et al., 2003), chitosan chelating activity (Rabea et al., 2003) or chitosan perturbation of the cell membrane (Helander et al., 2001; Zakrzewska et al., 2005; Je \& Kim, 2006). Others considered that a sequence of rather "untargeted" molecular events would take place simultaneously or successively. The initial contact between the polycationic chitosan macromolecule and the negatively charged cell wall polymers driven by electrostatic interaction between chitosan and teichoic acids in the cell wall would disrupt the equilibrium of cell wall dynamics, and cause ultimate cell death (Raafat et al., 2008).

Bioactive chitosan films can incorporate other antimicrobial agents, enabling to improve its antimicrobial efficacy (Quintavalla \& Vicini 2002). A degradable chitosan sponge was loaded with the antibiotics amikacin or vancomycin for therapy after a traumatic injury or surgery with sustained release of the antibiotics for 72 hours, representing very high release levels needed for preventing early-stage infection (Noel et al., 2010). Chitosan-coated plastic films, alone or loaded with antimicrobial agents, were evaluated for their effect against Listeria monocytogenes, a food-borne pathogen with ability to survive and grow at refrigeration temperatures, tolerant to relatively high concentrations of salt and able to cause high fatality rate associated with listeriosis (Ye et al., 2008). These chitosan-coated films inhibited this pathogen growth in a concentration-dependent manner whereas chitosancoated films impregnated with antibiotics were considerably more effective against $L$. monocytogenes. The antimicrobial activity of chitosan film proved against food pathogenic 
bacteria (E. coli, S. aureus, Salmonella typhimurium, L. monocytogenes and Bacillus cereus) has also been enhanced by incorporation of garlic oil, potassium sorbate and nisin (Pranoto et al., 2005). Edible films or coatings are prepared from proteins, polysaccharides and lipids (Cagri et al., 2004) and reduce the risk of pathogen growth on food surfaces (Quattara et al., 2000; Pranoto et al., 2005; Seydim \& Sarikus, 2006; Maizura et al., 2007). As chitosan is a potentially edible material, it has been used as a coating material for different types of foods (Coma et al., 2002; Coma et al., 2003; Zivanovic et al., 2005; Fernandez-Saiz et al., 2006). Incorporation of natural spices such as oregano, rosemary, garlic and lemongrass essential oils into edible films has been used to inhibit the growth of microorganisms (Quattara et al., 2000; Pranoto et al., 2005; Seydim \& Sarikus, 2006; Maizura et al., 2007). Addition of essential oil of Mexican oregano (Lippia berlandieri Schauer) as antimicrobial agent to edible films of chitosan or starch inhibited Aspergillus niger and Penicillium spp. growth at low concentrations in the films hampering mould growth (Avila-Sosa et al., 2010).

Polymeric bioactive films laced with an assortment of antimicrobial agents, such as nisin (Kim et al., 2002; Lee et al., 2003; Mauriello et al., 2005; Nguyen et al., 2008), essential oils (Pranoto et al., 2005; López et al., 2007; Avila-Sosa et al., 2010) and bacteriocins (An et al., 2000; Mauriello et al., 2004; Ercolini et al., 2006; Ghalfi et al., 2006) have been described. Addition of the antimicrobial peptide nisin efficiently inhibited growth of L. monocytogenes in films of gelatin and corn zein (Ku \& Song, 2007). Several reviews are available on preparation, characterization and determination of antimicrobial activity of these films and novel materials (Cutter, 2002; Quintavanalla \& Vicini, 2002; Cagri et al., 2004; Cha \& Chinnan, 2004; Cutter, 2006; Joerger, 2007; Dutta et al., 2009). Nisin has been the antimicrobial most frequently found in films for food packaging (Kim et al., 2002; Lee et al., 2003; Mauriello et al., 2005; Nguyen et al., 2008). Its small molecular size allows the production of films that release this peptide upon contact with food or liquid (Gill \& Holley, 2000; Cutter et al., 2001). A self-assembled bacterial cellulose film containing nisin prevented L. monocytogenes and total aerobic bacteria growth on the surface of vacuum-packaged processed meat products (Nguyen et al., 2008). These cellulose pellicles were produced by Gluconacetobacter xylinus $\mathrm{K} 3$ and then impregnated with nisin, yielding active cellulose films with potential applicability as antimicrobial packaging films. Biodegradable polylactid acid (PLA) polymeric films impregnated with nisin also killed foodborne L. monocytogenes, E. coli O157:H7 and Salmonella enteritidis and was proposed as a good material to make bottles or films, or coatings for use in liquid or solid food packaging (Jin \& Zhang, 2008). Similarly to nisin, some antimicrobial films have also been prepared from bacteriocins to prevent food contamination with L. monocytogenes (Mauriello et al., 2004; Ercolini et al., 2006; Ghalfi et al., 2006).

The desire for natural ingredients and the realization that plants harbour antimicrobial compounds have led to the production of a number of films with extracts from plants (Pranoto et al., 2005; Kim et al., 2006; Seydim \& Sarikus, 2006). In fact, plants have exceptional ability to produce cytotoxic agents and there is an ecological rationale that antimicrobial natural products should be present or synthesized in plants following microbial attack to protect the producer from pathogenic microbes in its environment (Gibbons, 2005). Moreover, natural products are both fundamental sources of new chemical diversity and integral components of today's pharmaceutical compendium and more than 300 natural metabolites with antimicrobial activity have been reported in the period 20002008 (Saleem et al., 2010). As such, there has been an increase in the use of essential oils as an alternative to conventional synthetic antimicrobial agents. Essential oils that contain higher concentrations of phenolic compounds, such as carvacrol, eugenol, and thymol also 
possess strong antibacterial properties against foodborne pathogens and display a wide range of other biological effects, including antioxidant and antimicrobial properties. The mode of action is considered to be the disturbance of the cytoplasmic membrane, disrupting the proton motive force, electron flow, and active transport, and/or coagulation of bacteria cell contents (Burt, 2004). López et al. (2007) prepared flexible films of polypropylene and polyethylene/ethylene vinyl alcohol copolymer added of the essential oil of cinnamon (Cinnamomum zeylanicum), oregano (Origanum vulgare) and clove (Syzigium aromaticum) and determined their activity against a wide range of microoganisms such as Gram-negative or positive bacteria, moulds and yeasts showing specially more pronounced antifungal activities that persisted for more than two months after films preparation (López et al., 2007). Oregano and cinnamon essential oils were recently incorporated on the same polymeric material completely inhibiting L. monocytogenes, Salmonella choleraesuis, C. albicans and Aspergillus flavus growth (Gutiérrez et al., 2010).

The enzyme lysozyme has been another natural choice for the preparation of antimicrobial films (Park et al., 2004; Souza et al., 2010). Lysozyme is a food grade antimicrobial enzyme with bacteriostatic, bacteriolytic and bactericidal activity, particularly against Gram-positive bacteria, and efficient in controlling the growth of a great number of food pathogens (Souza et al., 2010). In humans, lysozyme is found in a wide variety of fluids, such as tears, breast milk, and respiratory and saliva secretions, as well as in cells of the innate immune system, including neutrophils, monocytes, macrophages, and epithelial cells participating of the innate defense response against invading microorganisms (Jolles \& Jolles, 1984). This enzyme acts on bacteria by hydrolyzing the $\beta-1,4$ glycosidic bonds between $\mathrm{N}$ acetylmuramic acid (MurNAc) and N-acetylglucosamine (GlucNAc), resulting in degradation of peptidoglycan (PG), and subsequent microbial cell lysis (Schindler et al., 1977). The effective incorporation and release of lysozyme in chitosan films was used to reinforce the antimicrobial activity of chitosan (Park et al., 2004). Lysozyme has already been embodied in several biodegradable matrices yielding antimicrobial films and surfaces (Park et al., 2004; Fernández et al., 2008). In films of sodium caseinate lysozyme was released in a controlled manner so that sustained antimicrobial activity against S. aureus and Micrococcus lysodeikticus could be achieved (Souza et al., 2010).

\section{Antimicrobial particles}

\subsection{Inorganic, metal and composite particles}

The use of geological nanomaterials to heal skin infections has been known since the earliest recorded history, and specific clay minerals may prove valuable in the treatment of bacterial diseases, including infections for which there are no effective antibiotics, such as Buruli ulcer and multidrug-resistant infections (Williams \& Haydel, 2010). A French green clay (rich in Fe-smectite) has been used in clinics for healing Buruli ulcer, a necrotizing fasciitis ('flesh-eating' infection) caused by Mycobacterium ulcerans (Falkinham et al., 2009). However, little is known about the physicochemical properties involved in the antibacterial activity of many minerals.

The mineral $\mathrm{CsAg}_{2}$ demonstrated broad bactericidal activity against pathogenic Escherichia coli, extended-spectrum beta-lactamase (ESBL) E. coli, Salmonella enterica serovar Typhimurium, Pseudomonas aeruginosa and Mycobacterium marinum, and a combined bacteriostatic/bactericidal effect against Staphylococcus aureus, penicillin-resistant S. aureus, methicillin-resistant $S$. aureus (MRSA) and Mycobacterium smegmatis, whereas another 
mineral with similar structure and bulk crystal chemistry, $\mathrm{CsAr}_{2}$, had no effect on or even enhanced bacterial growth (Haydel et al., 2008). This mineral particulate heated to 200 or 500 ${ }^{\circ} \mathrm{C}$ still retained bactericidal activity, whereas heated or nonheated cation-exchanged $\mathrm{CsAg} \mathrm{O}_{2}$ no longer killed E. coli. Natural mineral mixtures were recently identified with antibacterial activity against a broad-spectrum of bacterial pathogens (Cunningham et al., 2010). Mineralderived aqueous leachates also exhibited antibacterial activity, revealing that chemical, not physical, mineral characteristics were responsible for the observed activity. Chelation of these minerals with EDTA or desferrioxamine eliminated or reduced antibacterial action suggesting a role of an acid-soluble metal species, particularly $\mathrm{Fe}(3+)$ or other sequestered metal cations, in mineral toxicity. Testing the bactericidal effect of the heated product, many toxins were eliminated from consideration (e.g., microbes, organic compounds, volatile elements) and several redox-sensitive refractory metals that are common among antibacterial clays were identified (Williams \& Haydel, 2010).

Inorganic active agents with antimicrobial activity can be based on a variety of inorganic nanostructured materials, such as titanium dioxide (Fu et al., 2005), silver (Jeong et al., 2005a; Rai et al., 2009) and silver-based nanostructured materials (Nishino \& Kanno, 2008; Kittler et al., 2009), zinc oxide (Li et al., 2007), copper (Cubillo et al., 2006), gallium (Valappil et al., 2008) or gold (Park et al., 2006; Zhang et al., 2008) plus their composites (Sambhy et al., 2006).

Metallic and inorganic particles can be loaded into different organic carriers, like liposomes (Park et al., 2005), nano- and micro-capsules (Shim et al., 2002) or dendrimers (Raveendran et al., 2006) finding many applications in the industry of fabrics (Gorensek et al., 2010; Dastjerdi \& Montazer, 2010), plastic (Roe et al., 2008; Xu et al., 2010) or biomaterials for drug delivery (Sharma et al., 2004; Pandey \& Khuller, 2004; Hardi-Ianderer et al., 2008).

Titanium dioxide nanoparticles have antibacterial (Fu et al., 2005; Daoud et al., 2005) and self-cleaning properties (Bozzi et al., 2005). Copper nanoparticles embedded into submicron particles of sepiolite $\left(\mathrm{Mg}_{8} \mathrm{Si}_{12} \mathrm{O}_{30}(\mathrm{OH})_{4}\left(\mathrm{H}_{2} \mathrm{O}\right)_{4} \cdot 8 \mathrm{H}_{2} \mathrm{O}\right)$ also demonstrated strong bactericidal properties (Cubillo et al., 2006) despite the lower antibacterial activity of copper when compared to silver nanoparticles (Pape et al., 2002). Grace \& Pandian (2007) have used gold nanoparticles as carriers core coated by antibiotics like streptomycin, gentamycin and neomycin showing that gold nanocomposites have an intense antibacterial efficiency against various Gram-negative and Gram-positive bacteria, like E. coli, P. aeruginosa, S. aureus and Micrococcus luteus. They concluded that metal nanoparticles may change the metabolite pathway and the release mechanism of bacterial cells. Therefore, Au/drug nanocomposites were more efficient than drug alone. Park et al. (2006) loaded gold nanoparticles inside lipid liposomes, reporting an increased fluidity and permeability of barrier of the lipid and provided a kind of thermally sensitive liposome. Consequently, these systems showed potential as controlled release delivery system at particular temperatures (Park et al., 2006). Silver has been employed since ancient times to fight infections and control spoilage (Tokumaru et al., 1984). Silver nanoparticles are antibacterial and multi-functional displaying low toxicity to human cells (Jeong et al., 2005a; Rai et al., 2009; Dastjerdi et al., 2009). Its antimicrobial effect at low concentrations is therapeutic against over 650 diseasecausing organisms in the body (Jeong et al., 2005a,b; Lok, 2006). The ability of silver to prevent biofilm formation has also been demonstrated (Stobie et al., 2008). The most common synthesis of silver nanoparticles is the chemical reduction of a silver salt solution by a reducing agent such as $\mathrm{NaBH}_{4}$, citrate, or ascorbate (Nickel et al., 2000; Leopold \& Lendl, 2003; Khanna \& Subbarao, 2003; Sondi et al., 2003). 
Nanocomposites from polymers and silver particles have also been described; silver introduction into poly(styrene-co-acrylic acid) copolymer enhanced antibacterial activity by increasing ionic mobility (da Silva Paula et al., 2009). Silver nanoparticles inside phosphatebased, biodegradable ceramic particles were released in the presence of a growing microorganism (Loher et al., 2008). This effect was based on the microorganism requirements for mineral uptake during growth, creating a flux of calcium, phosphate and other ions to the microorganism. The growing microorganism dissolved the carrier releasing the silver nanoparticles. These biodegradable silver carriers in materials and polymer coatings enabled the creation of self-sterilizing surfaces (Loher et al., 2008). Metal nanoparticles with bactericidal effects can be affixed on various surfaces for prevention or protection purposes in specific applications, such as infirmaries, clothing, different surfaces, food protection and packing and water treatment (Ruparelia et al., 2008). Contrary to effects of ionic silver, the antimicrobial activity of colloidal silver particles is influenced by particle dimensions: the smaller the particles, the greater the antimicrobial effect due to its larger surface area to get in contact with the bacterial cells (Morones et al., 2005; Panacek et al., 2006; Pal et al., 2007).

In spite of several reports on the antimicrobial activity of silver nanoparticles (Sondi \& Salopek-Sondi, 2004; Pal et al., 2007; Kim et al., 2007; Travan et al., 2009; Li et al., 2010), the mechanism of inhibitory effects of Ag ions on microorganisms is not yet fully elucidated. Some studies reported that the positive charge on the Ag ion would be crucial for antimicrobial activity (Dragieva et al., 1999; Hamouda et al., 1999; Dibrov et al., 2002). However, negatively charged silver nanoparticles also killed Gram-negative bacteria in a nanoparticle concentration -dependent manner (Sondi \& Salopek-Sondi, 2004). The activity was also closely associated with the formation of "pits" in the cell wall of bacteria plus nanoparticles incorporation, accumulation and permeability increase of the bacterial cell membrane (Sondi \& SalopekSondi, 2004). Damage of bacterial cell membrane with observations of pits and gaps on the cells was related to reduction of activity of some enzymes, leakage of sugars and proteins and cell death ( $\mathrm{Li}$ et al., 2010). Silver nanoparticle shape also affected antibacterial effect against $E$. coli: truncated triangular silver nanoplates displayed stronger biocidal action than spherical or rod-shaped nanoparticles (Pal et al., 2007).

The use of microbial cells for the biosynthesis of nanosized materials has emerged as a novel approach for the synthesis of metal nanoparticles, particularly based on the main reaction of reduction/oxidation, where microbial enzymes with reducing or anti-oxidant properties are usually responsible for reduction of metal ions compounds into their respective metalic particles (Gericke \& Piches, 2006a; Prathna et al., 2010). Metal particles can be obtained from biosynthesis by microorganisms and plants (Durán et al., 2005; Mohanpuria et al., 2008). Bacteria are known to produce inorganic materials either intra- or extracellularly. Microorganisms are considered as a potential biofactory for the synthesis of gold (Ahmad et al., 2003a; Ahmad et al., 2003b; Sastry et al., 2003; Gericke \& Piches, 2006b), silver (Fu et al., 2000; Fu et al., 2006; Gericke \& Piches, 2006a) and cadmium sulphide nanoparticles (Fu et al.,1999).

Silver nanoparticles were biosynthesized by Klebsiella pneumoniae (Shahverdi et al., 2007), Staphylococcus aureus (Nanda \& Saravanan, 2009), Escherichia coli (Gurunathan et al., 2009) or Brevibacterium casei (Kalishwaralal et al., 2010). In combination with antibiotics, such as vancomycin and clindamycin, silver nanoparticles biosynthesized by $K$. pneumoniae exhibited enhanced activities against S. aureus (Shahverdi et al., 2007). Curiously, silver particles synthesized by $S$. aureus exhibited activity against resistant strains of Staphylococcus 
sp. such as the methicillin-resistant $S$. aureus (MRSA) and methicillin-resistant S. epidermidis (MRSE) (Nanda \& Saravanan, 2009).

Fungi have also been widely studied for the biosynthesis of nanoparticles, and when compared to bacteria, they could be used as a source for the production of large amounts of nanoparticles. This is associated with the fact that fungi secrete large amounts of proteins, which directly translate to higher productivity of nanoparticle formation (Mohanpuria et al., 2008). Several fungus species have been employed to synthesize silver nanoparticles: Phaenerochaete chrysosporium (Vigneshwaran et al., 2006), Phoma glomerata (Birla et al., 2009), Trichoderma viride (Fayaz et al., 2010) and Aspergillus clavatus (Saravanan \& Nanda, 2010; Verma et al., 2010). The biosynthesized nanoparticles showed antimicrobial activities alone (Verma et al., 2010; Saravanan \& Nanda, 2010) or combined with antibiotics (Birla et al., 2009; Fayaz et al., 2010). Several plants have also been investigated for their role in the synthesis of nanoparticles (Torresday et al., 2002; Bali et al., 2006). The advantage of using plants to synthesize nanoparticles is that they are easily available, safe to handle and possess a broad variability of metabolites that may act in reduction reactions (Prathna et al., 2010). Gold (Torresday et al., 2002; Song et al., 2009), silver, nickel, cobalt, zinc and copper nanoparticles were obtained from biosynthesis by plants (Bali et al., 2006). Antibacterial properties of silver nanoparticles synthesized by plants like Azadirachta indica (Tripathi et al., 2009) or Acalypha indica (Krishnaraj et al., 2010) were recently reported. These particles were incorporated onto cotton disks, showing activity against $E$. coli. Leaf extracts were also used to synthesize silver nanoparticles with antimicrobial activity against water born pathogens such as E. coli and Vibrio cholerae.

\subsection{Polymeric, lipid-based and hybrid particles}

Biocompatible and biodegradable polymers have been extensively used in clinics for controlled drug release. Polymeric nanoparticles can be formed through self-assembly of copolymers, consisting of hydrophilic and hydrophobic segments or through linear polymers, such as poly (alkylacrylates) and poly (methylmethacrylate). A variety of biodegradable polymers have been used to form nanoparticles, including poly(lactic acid) (PLA), poly(glycolid acid) (PGA), poly(lactide-co-glycolide) (PLGA) and polyethylene glycol (PEG) (Sharma et al., 2004; Pandey \& Khuller, 2004; Hardi-Ianderer et al., 2008; Zhang et al., 2010). Antimicrobial drugs can be adsorbed to the nanoparticles during polymerization or covalently conjugated to the nanoparticles surface after they are formed (Zhang et al., 2010). Polystyrene (PS) and poly(styrene-co-styrene sulfonate) particles coated with silver nanoparticles by gama-irradiation induced reduction of $\mathrm{Ag}$ ions yielding microbicidal composite particles against $S$. aureus (Oh et al., 2006). Another study described PS particles coated with poly(ethylene-co-butylene) copolymer containing a polymethacrylate block activated with amino or octyl bromide bactericidal moieties (Lenoir et al., 2005). The antimicrobial activity was directly related to the concentration of coated PS particles. Amphotericin B (AmB)-loaded poly( $\varepsilon$-caprolactone) nanospheres have therapeutic efficacy against Leishmania donovani (Espuelas et al., 2002) and C. albicans (Espuelas et al., 2003), when compared to free drug. Rifampicin-loaded polybutylcyanoacrylate nanoparticles have also shown enhanced antibacterial activity both in vitro and in vivo against S. aureus and Mycobacterium avium due to an effective delivery of drugs to macrophages (Skidan et al., 2003). Chitosan, a natural biopolymer, has antimicrobial and antifungal activity (Sudarshan et al., 1992; Jeon et al., 2001). Chitosan nanoparticles prepared 
and loaded with antimicrobials or antibiotics (Portero et al., 2002) or metals (Qi et al., 2004) further enhance their antimicrobial action. Chitosan nanoparticles themselves or with adsorbed copper ions inhibited bacterial growth, with copper-loaded ones exhibiting higher activity due to higher surface charge density enhancing the affinity with the negatively charged bacteria membrane (Qi et al., 2004).. Polymeric particles of poly(4-vinyl pyridine), synthesized and chemically modified to become positively charged, were used for in situ silver and copper metal nanoparticle synthesis and presented antimicrobial action against $S$. aureus, P. aeruginosa, E. coli and Bacillus subtilis (Ozay et al., 2010).

Charged polymers or polyelectrolytes have often been used to produce nanostructured particles (Vieira \& Carmona-Ribeiro, 2008; Melo et al., 2010). Cationic polymers can be potent antimicrobial agents (Codling et al., 2003; Kuegler et al., 2005). Cationic poly(arylene ethylene) conjugated polyelectrolytes have recently been reported as potent dark biocidals against $P$. aeruginosa, due to its high lipophilicity and the presence of accessible quaternary ammonium groups (Corbitt et al., 2009). The layer-by-layer (LbL) procedure (Decher \& Hong, 1991) was used to produce hybrid antimicrobial and cationic particles from dioctadecyldimethylammonium bromide (DODAB) bilayer fragments (BF) supporting consecutive layers of the anionic polymer carboxymethylcellulose (CMC) and the cationic polyelectrolyte poly(diallyldimethylammonium) chloride (PDDA) (Melo et al., 2010). Both cationic microbicides, DODAB and PDDA, were combined in a single supramolecular assembly. These assemblies in form of small or large particles were obtained from small or large DODAB BF concentrations, respectively. The assemblies DODAB BF/CMC/PDDA exhibited potent antimicrobial activity against $P$. aeruginosa and $S$. aureus. The antimicrobial effect was similar for particles with 100 or $500 \mathrm{~nm}$ of mean diameter and dependent only on the amount of positive charges on particles (Melo et al., 2010). These hybrid particles also delivered AmB to C. albicans (Vieira \& Carmona-Ribeiro, 2008). Cationic lipid, antibiotic and cationic polyelectrolyte nanostructured in each particle effectively attacked the fungus. Figure 6 shows these assemblies which were microbicidal with or without drug.
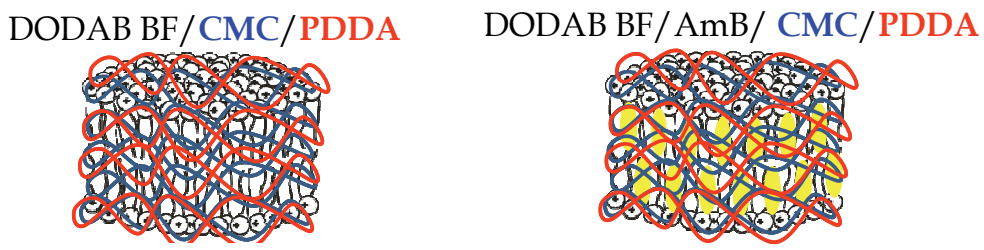

Fig. 6. Antimicrobial particles of cationic lipid (DODAB) and polyelectrolytes (CMC and PDDA), with or without amphotericin B (AmB). Adapted from Vieira \& Carmona-Ribeiro, 2008 and adapted with permission from Melo et al., 2010. Copyright 2010 American Chemical Society.

Other interesting approaches were immobilization of bacteriophages active against a variety of food-borne bacteria by physisorption to modified, cationic silica particles (Cademartiri et al., 2010) or use of low-density lipoproteins (LDLs) from human plasma for delivering drugs inside the cells and treat intracellular infections (Hu et al., 2000). Biopolymer particles from lipoproteins can readily be obtained from human plasma by density gradient ultracentrifugation (Kader et al., 1998). A lipid core is surrounded by a monolayer of phospholipids, in which cholesterol and apolipoprotein-B are present. Other human plasma 
lipoproteins, the high-density lipoproteins (HDLs) particles, have also been related with antimicrobial properties against Staphylococcus epidermidis, due to apolipoprotein A1 (Tada et al., 1993) or related with protection against trypanosome infection, due to native human HDLs containing haptoglobin-related protein (Hpr), apolipoprotein L-I (apoL-I) and apolipoprotein A-I (apoA-I) (Shiflet et al., 2005).

Dendrimers also possess several unique properties that make them a good nanoparticle platform for antimicrobial drug delivery. They are highly ordered and regularly branched globular macromolecules, with a core, layers of branched repeat units emerging from the core and functional end groups on the outer layer of repeat units (Grayson \& Frechet, 2001). The branched nature of dendrimers provides huge surface area to size ratio, allowing great reactivity with microorganisms and drug loading capacity (Florence, 2005). Moreover, using antimicrobial drugs to synthesize dendrimers, they can become a potent antimicrobial for themselves. Dendrimer biocides may contain quaternary ammonium salts as functional end groups displaying greater antimicrobial activity against bacteria than small drug molecules, due to a high density of active antimicrobials on the dendrimer surfaces. The polycationic structure of dendrimer biocides facilitates the initial electrostatic adsorption to negatively charged bacteria, increasing membrane permeability and allowing more dendrimers to enter the bacterial cell (Chen et al., 2000; Chen \& Cooper, 2002). Dendrimers have also been used as a vehicle to develop antimicrobial properties in textile fabrics (Ghosh et al., 2010; Klaykruayat et al., 2010). The poly(amidoamine) (PAMAM) dendrimer was modified to obtain quaternary ammonium groups as antimicrobial moieties or loaded with silver compounds. Both modified PAMAM structures were applied to cotton and nylon fabrics, exhibiting significant biocidal activity against $S$. aureus for each type of modified dendrimer (Ghosh et al., 2010). Cotton fabrics were impregnated with chitosan modified with antimicrobial PAMAM dendrimers, which imparted good activity against $S$. aureus to the fabrics when compared to fabrics with unmodified chitosan (Klaykruayat et al., 2010).

Dendrimeric structures were effective against mature biofilms, completely inhibiting E. coli (Hou et al., 2009) or P. aeruginosa (Johansson et al., 2008) biofilm formation and inducing complete dispersion of both bacterial established mature biofilms, in a clear advantage with the majority of antimicrobial agents that are ineffective against already formed biofilms. Furthermore, dendrimers design can mimick the active conformation of linear antimicrobial peptides (Janiszewska \& Urbanczyk-Lipkowska, 2007; Bruschi et al., 2010). The synthesis of a family of these peptidic dendrimers also showed antimicrobial properties against $S$. aureus, E. coli and C. albicans (Janiszewska \& Urbanczyk-Lipkowska, 2007). Pini et al. (2005) reported the synthesis of an antimicrobial peptide in monomeric and dendrimeric form, obtaining activity against $E$. coli of the dendrimeric peptide much higher than that of the monomeric form. In fact, multimeric peptides offer several advantages with respect to their monomeric counterparts, due to improved stability in the presence of degrading enzymes as peptidases and proteases (Bruschi et al., 2010). A recent review reported the current state of therapeutic potential of the dendrimer systems in wound healing, bone mineralization, tissue repair, anticoagulant, anti-inflammatory and anticancer therapy (Gajbhiye et al., 2009).

Among the classical cationic surfactants, quaternary ammonium compounds (QACs) are the most useful antiseptics and disinfectants (Merianos, 1991; Frier, 1971). Since 1935 the antibacterial activity of the long-chained quaternary ammonium salts has been disclosed (Domagk, 1935). The fourth generation of quaternary antimicrobials included several mono- 
and dialkyl dimethylammonium and polymeric quaternary ammonium salts (Petrocci et al., 1979). QACs are membrane active agents (Hugo \& Frier, 1969; Furhop \& Wang, 2004) that can cause lysis of spheroplasts and protoplasts suspended in sucrose (Salton, 1968; Davies \& Field, 1969; Denyer, 1995; Russel et al., 1999). The cationic agents hypothetically react with phospholipid components in the cytoplasmic membrane, thereby producing membrane distortion and protoplast lysis under osmotic stress (Cabral, 1992; Russel \& Chopra, 1996). Another possible mechanism for QACs action might be their inhibition and blockade of potassium channels in Gram-negative bacteria (Raja \& Vales, 2009). The positive charge on microbial cells has been often correlated with the biocidal action (Isquith et al., 1972; Endo et al., 1987; Tapias et al., 1994; Sicchierolli et al., 1995; Fidai et al., 1997; Friedrich et al., 2000; Campanhã et al., 2001; Kugler et al., 2005). Dioctadecyldimethylammonium bromide (DODAB) (Tapias et al., 1994; Campanhã et al., 1999; Pereira et al., 2008; Melo et al., 2010), cetyltrimethylammonium bromide (CTAB) (Vieira \& Carmona-Ribeiro, 2006; Dvoracek et al., 2009) and benzyldimethyldodecylammonium chloride (BDMDAC) (Ferreira et al., 2010) are some examples of quaternary ammonium compounds used to prepare antimicrobial particles.

Supramolecular assemblies of cationic lipid such as the bilayer fragments (BF) or the large bilayer vesicles have already been established as antimicrobial agents (Tapias et al., 1994; Sicchierolli et al., 1995; Martins et al., 1997; Campanhã et al., 1999; Carmona-Ribeiro, 2000; Campanhã et al., 2001; Lincopan et al., 2003; Carmona-Ribeiro, 2003; Carmona-Ribeiro et al., 2006; Vieira \& Carmona-Ribeiro, 2008). In particular, DODAB is a cationic bilayer-forming synthetic lipid with a high chemical stability and well-described anti-infective properties (Carmona-Ribeiro et al., 2006). Adsorption of DODAB cationic bilayers onto bacteria cells changes the sign of the cell surface potential from negative to positive, with a clear relationship between positive charge on bacterial cells and cell death (Campanhã et al., 1999). DODAB BF also affected viability of Candida albicans (Campanhã et al., 2001; Vieira \& Carmona-Ribeiro, 2006). Simultaneous determination of C. albicans viability and eletrophoretic mobility as a function of DODAB concentration also yielded good correlation between yeast surface charge and cell viability. Micromolar DODAB concentrations effectively killed bacteria, but DODAB concentrations required to kill yeast cells were much higher than those required to kill bacteria. Mammalian cells in culture were still more resistant to DODAB than fungi (Carmona-Ribeiro et al., 1997). DODAB indeed exhibits differential cytotoxicity, an important property for therapeutic uses. DODAB bilayer fragments $(\mathrm{BF})$, by themselves or combined with particles, can produce lipid-based biomimetic assemblies or particles with antimicrobial activity. Synthetic amphiphile bilayers prepared from DODAB or other synthetic lipid, sodium dihexadecyl phosphate (DHP), were deposited onto oppositely charged polystyrene microspheres, forming bilayer covered lattices (Carmona-Ribeiro \& Midmore, 1992). These homodisperse, DODAB bilayer-covered polystyrene sulfate (PSS) particles were combined with DNA, yielding supramolecular assemblies of PSS/DODAB/DNA (Rosa et al., 2008). Over a low concentration range of DNA, PSS/DODAB/DNA assemblies were cationic, colloidally stable and highly cytotoxic against $E$. coli cells, while from DNA concentration corresponding to charge neutralization, neutral or anionic assemblies, PSS/DODAB/DNA exhibited low colloid stability, high polydispersity and low antimicrobial activity.

Other important application of lipid based biomimetics refers to formulation of hydrophobic drugs. Aqueous miconazole (MCZ) aggregates were solubilized and/or colloidally stabilized by bilayer-forming synthetic lipids such as DODAB or DHP dispersions (Pacheco 
\& Carmona-Ribeiro, 2003). Drug particles became colloidally stable in the presence of charged bilayer fragments. At high drug to lipid molar proportion $(\mathrm{P})$, when bilayer fragments covered drug particles, formulations were stable and highly effective. At low $\mathrm{P}$, the drug became soluble in its monomeric form at the borders of the bilayer fragments (Vieira \& Carmona-Ribeiro, 2001; Pacheco \& Carmona-Ribeiro, 2003; Lincopan et al., 2003). The formulations were also effective and stable despite the toxicity due to the large concentration of cationic lipid (Lincopan et al., 2005; Lincopan et al., 2006). The results showed that synthetic bilayer fragments offered extra solubilization sites useful as receptive surfaces at their hydrophobic borders. The MCZ particles covered with DODAB BF showed a synergistic action between lipid and drug against $C$. albicans (Lincopan \& CarmonaRibeiro, 2006). At high $\mathrm{P}$, addition of chaotropic $\mathrm{K}_{2} \mathrm{HPO}_{4}$ converted $\mathrm{MCZ}$ aggregates into negatively charged particles with affinity for cationic lipid, which then surrounded each drug particle with a cationic layer. In these formulations DODAB and MCZ acted synergistically against yeast. Biomimetic PSS/DODAB/DNA and MCZ drug particles are illustrated in Figure 7.

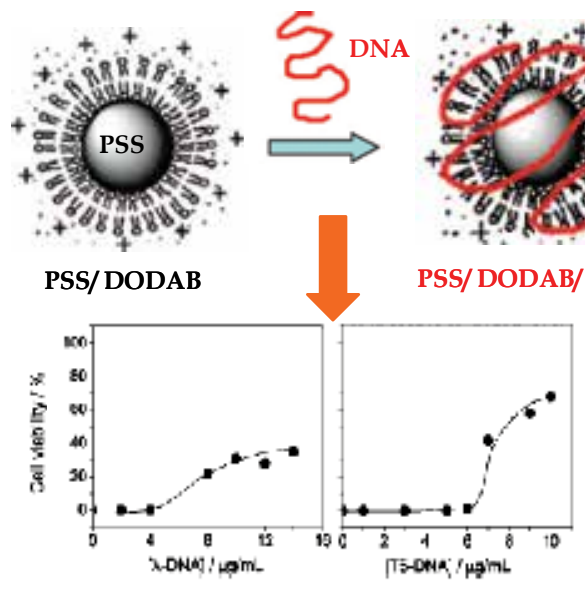

A

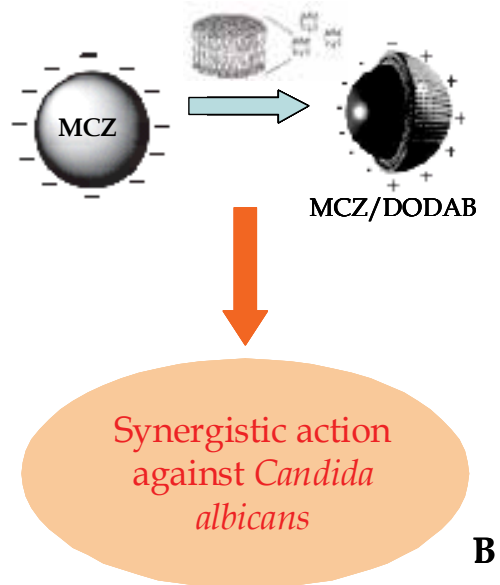

Fig. 7. Biomimetic particles: PSS/DODAB/DNA against E. coli (A); MCZ/DODAB against C. albicans (B). Adapted with permission from Rosa et al., 2008. Copyright 2008 American Chemical Society. Adapted with permission from Lincopan \& Carmona-Ribeiro, 2006. Copyright 2006 Oxford University Press.

Lipids such as fatty acids, triglycerides, steroids, partial glycerides and waxes can also be used to produce solid nanoparticles (Zhang et al., 2010). As in oil-in-water nanoemulsions where a dispersed oil phase is stabilized in a water phase by an emulsifying layer of surfactant, nanoparticles composed of solid lipids such as stearic acid or solid lipid nanoparticles (SLN) (Müller et al., 2000) have been stabilized with polymers or surfactants such as poloxamer 188, polysorbate 80 , lecithin, polyglycerol methylgluco distearate, sodium cocoamphoacetate or saccharose fatty acid esters and used to carry drugs, peptides or proteins (Martins et al., 2007). Advantages of SLN are their composition (biocompatible compounds), the fast and effective production process, including the possibility of large scale production, the avoidance of organic solvents in the production procedures, and the possibility to produce concentrated lipid suspensions. However, the drug loading capacity of conventional SLN is limited because of the formation of a perfect lipid crystal matrix and 
other colloidal structures such as micelles, liposomes, mixed micelles and drug nanocrystals might be also present in the aqueous dispersion (Wissing et al., 2004). The preparation of SLN involves a first step of emulsification in hot water by stirring $10 \%$ of melted solid lipid such as stearic acid, $15 \%$ of surfactant and up to $10 \%$ of co-surfactant via microemulsions. Next, the warm microemulsion is dispersed under stirring in excess cold water. Finally, ultrafiltration or liophylization remove water excess and increases the SLN concentration (Fundaro et al., 2000; Igartua et al., 2000; Mehnert \& Mäder, 2001).

SLNs are considered good drug carriers to obtain sustained release of antibiotics (FaustinoVega et al., 2009; Han et al., 2009). SLNs can act as promising carriers for sustained ciprofloxacin release in infections (Jain \& Banerjee, 2008) or to enhance the bioavailability of tobramycin from antibiotic-loaded SLN in the aqueous humor for topical ocular delivery (Cavalli et al., 2002). SLNs also represent a good carrier for intracerebral delivery of drugs, since these nanocarriers can not only mask the blood brain barrier limiting characteristics, but may also protect the drug from chemical and enzymatic degradation (Tiwari \& Amiji, 2006). Besides, reduction of toxicity of drugs to peripheral organs can also be achieved with the SLN delivering the drugs directly to the central nervous system. Another possible application of SLNs is to deliver azole antifungal drugs to superficial fungal infection patients (Gupta et al., 2008). SLNs can also facilitate the delivery of anti-tuberculosis drugs such as rifampicin, isoniazid and pyrazinamide to the lungs as well as to the lymphatic system (Pandey \& Khuller, 2005). Nimje et al. (2009) reported the selective delivery of rifabutin, another antituberculosis drug, to alveolar tissues, using drug-loaded solid lipid nanoparticles, increasing the therapeutic margin of safety and reducing side effects.

\section{Liposomes in antimicrobial chemotherapy}

Many infections are localized within phagocytic cells in the reticuloendothelial system (liver and spleen), in the blood stream, or in granuloma in various tissues and are possibly targets for liposomal drug delivery and therapy (Richarson, 1983). Examples of such infectious diseases are brucellosis, leprosy, tuberculosis, and listeria, all of them caused by intracellular bacteria.

Liposomes have been extensively used as carriers of antimicrobial and antineoplastic drugs (Lopez-Berestein, 1987). They are usually produced from naturally occurring, biodegradable and non-toxic phospholipids (Furneri et al., 2000). Liposomes have been designed to release drugs into an extracellular or intracellular compartment to reach their site of action (Fielding, 1991). The ability of liposomes to alter drug distribution depends largely on their size and surface properties (Fielding, 1991). Thus, liposomal encapsulation of antibiotics helps to increase their therapeutic index with mode of action related to increasing the drug concentration at the site of infection and/or reducing its toxicity (Schiffelers et al., 2001a). Organs rich in cells from the reticuloendothelial system (RES) preferentially take up liposomes, e.g. liver, spleen, lung and bone marrow (Gregoriadis, 1976a; Gregoriadis, 1976b). Targeting of liposomal antibiotic to bone marrow might achieve a high concentration of the drug in bone tissues. For extracellular bacteria, the enhanced antibacterial effect may be due to a fusion mechanism of the liposomal formulation with bacteria. The phagocytosis of antibiotic-loaded liposomes yields therapeutic intracellular drug concentrations and consequently enhanced killing of intracellular microorganisms, such as S. aureus, E. coli, Brucella abortus and Mycobacterium avium (Schiffelers et al., 2001a). 
Most studies regarding liposomal antibiotics deal with aminoglycosides, quinolones, polypeptides, and $\beta$-lactams (Drulis-Kawa \& Dorotkiewicz-Jach, 2010). The many advantages of liposomes as antibiotic carriers are improved pharmacokinetics and biodistribution, decreased toxicity, enhanced activity against intracellular pathogens, target selectivity, enhanced activity against extracellular pathogens, and efectiveness in overcoming bacterial drug resistance. The variety of liposomal formulations allows the design of effective antibiotic formulations and subsequent therapeutic success (Abeylath \& Turos, 2008; Jia et al., 2008).

Traditional antibiotic therapy of staphylococcal osteomyelitis by a single drug or a drug combination is ineffective in producing complete sterilization of infected bones. Ciprofloxacin and vancomycin were encapsulated in a cationic, anionic or neutral liposomal formulation (Kadry et al., 2004). Cationic liposomes entrapped the highest percentage of antibiotics, and enhanced antibacterial activity above that of the free antibiotics; they were used for therapeutic trials to treat chronic staphylococcal osteomyelitis induced in rabbits. These liposomal formulations showed much lower nephrotoxicity than that induced by free drugs. Several other papers describe liposomal formulations against pathogenic microorganisms such as P. aeruginosa (Okusanya et al., 2009), K. pneumoniae (Gubernator et al., 2007), E. coli and S. aureus (Beaulac et al., 1998). The antibiotics chosen for encapsulation were mostly fluoroquinolones and aminoglycosides.

Encapsulation of gentamicin in liposomes can be used to achieve intracellular delivery and broaden the clinical utility of this drug. pH-dependent liposomal fusion with cells could be achieved due to the presence of phosphatidylethanolamine (PE) and the $\mathrm{pH}$-sensitive lipid N-succinyldioleoyl-PE (Cordeiro et al., 2000). The pharmacokinetics and biodistribution of the free and liposomal gentamicin were examined in mice bearing a systemic Salmonella enteric serovar Typhimurium infection. Encapsulation of gentamicin in $\mathrm{pH}$-sensitive liposomes significantly increased the concentrations of the drug in plasma compared to those of free gentamicin.

Liposomes of DMPC/CHOL (molar ratio 2:1) containing gentamicin showed better activity against $P$. aeruginosa than the free drug (Rukholm et al., 2006). For a highly resistant $P$. aeruginosa strain there was a 16-fold reduction in MIC for the liposomal gentamicin. Similar results in MIC reduction were obtained for liposomes of DPPC/CHOL (molar ratio 2:1) containing amikacin, gentamicin, and tobramicin (Mugabe et al., 2006). Long-circulating liposome encapsulated gentamicin demonstrated superior antibacterial activity over the free drug in a single-dose study of immunocompetent rats with K. pneumoniae pneumonia (Schiffelers et al., 2001b). Multilamellar liposomes carried gentamicin for treatment of mice lethally infected with Brucella abortus (Vitas et al., 1997). The use of free or liposomal gentamicin in liposomes with a negative net charge did not produce a protective effect. Only the cationic liposomes had a therapeutic effect against infection. Pulmonary delivery of rifampicin encapsulated in liposomes was reported (Deol \& Kuller., 1997; Vyas et al., 2004; Zaru et al., 2007; Changsan et al., 2009). Lung-specific Stealth liposomes made of phosphatidylcholine, cholesterol, dicetylphosphate, O-steroyl amylopectin and monosialogangliosides/ distearylphosphatidylethanolamine-poly (ethylene glycol) 2000 for the targeted delivery of anti-tuberculosis drugs to the lung have been described (Deol \& Kuller, 1997). Modification of surface of stealth liposomes by tagging O-stearylamylopectin resulted in the increased affinity of these liposomes towards lung tissue of mice. Regarding tissue distribution, these liposomes showed more accumulation in lungs than in reticuloendothelial system of the normal and tuberculous mice. Isoniazid and rifampicin 
encapsulated in liposomes were less toxic to peritoneal macrophages than the free drugs. The same formulations administered at one-third of the recommended doses showed a sustained release of the drugs in the plasma (5 days), lungs, liver and spleen ( 7 days) (Labana et al., 2002). Vyas et al. (2004) formulated aerosolized liposomes incorporating rifampicin via a cast-film method employing egg phosphatidylcholine- and cholesterolbased liposomes. Liposomes coated with alveolar macrophage-specific ligands demonstrated preferential accumulation in alveolar macrophages, maintaining high concentrations of rifampicin in the lungs even after $24 \mathrm{~h}$ after inhalation. Other tuberculostatic drugs such as pyrazinamide (El-Ridy et al., 2007) and rifabutin (Gaspar et al., 2008) were also formulated in liposomes stressing the great versatility and potential of the nanocarriers. Rifampicin-encapsulating liposomes were nontoxic to respiratory associated cells, including bronchial epithelial cells, small airway epithelial and alveolar macrophages (Changsan et al., 2009). Furthermore, the liposomes did not activate alveolar macrophages to produced interleukin-1, tumor necrosis factor- $\alpha$, or nitric oxide at a level that would cascade to other inflammatory effects. The MIC against Mycobacterium bovis was smaller for liposomes containing rifampicin than for free rifampicin.

Liposomal formulations for important antifungal drugs such as amphotericin B (AmB) were first described by Lopez-Berestein and coworkers (Lopez-Berestein, 1987). Systemic fungal infections are often the cause of mortality in patients with hematological malignancies and certain other conditions associated with profound immunosuppression. The majority of such infections are caused by Aspergillus and Candida species (Potter, 2005). Voriconazole and lipid-associated AmB have been shown to be effective in the first-line therapy (Potter, 2005). Nebulized liposomal AmB formulations are effective, safe, and convenient for the prevention of Aspergillus infection in lung transplant patients (Monforte et al., 2010). A novel method was developed to incorporate polyene antibiotics, nystatin and AmB, into liposomes prepared from the mixture of phosphatidylcholine and cholesterol (7: 3) or phosphatidylcholine, cholesterol, and cardiolipin (7: 3: 1) plus the amphiphilic polymer Nvinylpyrrolidone showing higher antifungal activity than non-immobilized antifungal antibiotics (Yamskov et al., 2008). Other water-soluble complexes of AmB and polyvinylpyrrolidone were compared with $\mathrm{AmB}$ for antifungal activity, and were less haemolytic and cytotoxic than AmB showing cytotoxicity similar to AmBisome (Charvalos et al., 2006). AmB-loaded cationic liposome gels were formulated with 1, 2-dioleoyl-snglycero-3-phosphoethanolamine (DOPE), 1, 2-dioleoyl-3-trimethylammonium-propane (DOTAP), and cholesterol $(\mathrm{CH})$ at a molar ratio of DOPE: DOTAP: $\mathrm{CH}$ of 4:5:1 in thermosensitive gel composed of poloxamer 407 and poloxamer 188. AmB-loaded cationic liposome gels were more stable and less toxic than free AmB. These gels containing cationic liposome may become useful for vaginal delivery of AmB (Kang et al., 2010).

Disadvantages of liposomal antibiotics are associated with chemical and physical instability mainly due to the hydrolysis of ester bonds or the oxidation of unsaturated acyl chains of the lipids used to construct the liposomal vesicles (Sharma \& Sharma, 1997; Storm \& Crommelin, 1998; Carmona-Ribeiro, 2003). Besides hydrolysis, peroxidation of unsaturated acyl chain bonds is also possible (Storm \& Crommelin, 1998). Oxidation and/or hydrolysis can be prevented by adding antioxidant components or by freeze-drying or by storage at low temperature (Storm \& Crommelin, 1998). The physical instability of liposomal drugs leads to drug leakage from the lipid vesicles. Under physiological conditions, stability is usually low and depends on the interaction of the liposomal membranes with components of body fluids (Gregoriadis, 1995). This is a very unfavourable situation, especially as the 
best results of antibacterial activity of liposomal drugs in vitro are observed for positively charged or fluid liposomes (Drulis-Kawa et al., 2006). The presence of anionic lipids in liposomal vesicles also favours the binding of serum proteins to the vesicle surface (Briones et al., 2008).

Encapsulation efficiency depends on the type of lipids and on the hydrophobic-hydrophilic character of the drug. There are several instances of low encapsulation efficiency of antibiotics depending on type of the lipid. Gubernator et al. (2007) obtained meropenem and gentamicin (hydrophilic drugs) encapsulation efficiency in the range of $2.7-5.7 \%$ for a cationic fluid formulation. Lutwyche et al. (1998) showed that $25-33 \%$ of total gentamicin was associated with the outer surface of anionic liposomes composed of DOPE lipid, so a gentamicin encapsulation capacity of $2.8 \%$ was obtained in the anionic formulation DOPE/DOPS/PEG. Low encapsulation efficiency was also obtained by others (Lutwyche et al., 1998; Omri \& Ravaoarinoro, 1996) making liposomal formulations much more expensive than conventional antibiotic treatment (Kshirsagar et al., 2005).

Since the major requirement to form a supramolecular assembly of the bilayer type is a cylindrical molecular geometry (Israelachvili et al., 1977), bilayer vesicles and liposomes can be obtained not only from expensive phospholipids but also from several other synthetic amphiphiles such as dialkyldimethylammonium bromide or chloride (Kunitake et al., 1977), sodium dihexadecylphosphate (Mortara et al., 1978, Carmona-Ribeiro et al., 1991) and many other molecules (Furhhop \& Fristch, 1986; Segota \& Tezak, 2006). For synthetic lipids such as dioctadecyldimethylammonium bromide (DODAB), chemical stability is superior to the one exhibited by the phospholipids since the hydrocarbon chains are saturated and ester functionalities are absent from DODAB chemical structure. The properties and applications of vesicles and bilayer fragments composed solely of synthetic lipids have been reviewed in the literature (Carmona-Ribeiro, 1992; Carmona-Ribeiro, 2001; Carmona-Ribeiro, 2003; Carmona-Ribeiro, 2006; Carmona-Ribeiro, 2007; Carmona-Ribeiro, 2010a; Carmona-Ribeiro, 2010b).

DODAB bilayers adsorb or become adsorbed onto negatively charged biomolecules such as proteins (Carvalho \& Carmona-Ribeiro, 1998; Lincopan \& Carmona-Ribeiro, 2009), DNA (Kikuchi \& Carmona-Ribeiro, 2000; Rosa et al., 2008), biological structures such as microorganisms (Martins et al., 1997; Campanhã et al., 1999; Campanhã et al., 2001; Pacheco et al., 2004; Carmona Ribeiro, 2006) or mammalian cells (Carmona-Ribeiro et al., 1997) or drugs (Vieira \& Carmona-Ribeiro, 2001; Lincopan et al., 2003; Pacheco \& Carmona-Ribeiro, 2003; Lincopan et al., 2005; Carmona-Ribeiro, 2006; Lincopan \& Carmona-Ribeiro, 2006; Vieira et al., 2006; Vieira \& Carmona-Ribeiro, 2008). In antimicrobial chemotherapy, DODAB revealed excellent microbicidal properties (Vieira \& Carmona-Ribeiro, 2001; Pacheco \& Carmona-Ribeiro, 2003; Lincopan et al., 2003; Lincopan et al., 2005; Vieira et al., 2006; Carmona-Ribeiro, 2006; Lincopan \& Carmona-Ribeiro, 2006; Vieira \& Carmona-Ribeiro, 2008) besides outstanding versatility to formulate several antimicrobial drugs (Vieira \& Carmona-Ribeiro, 2001; Lincopan et al., 2003; Pacheco \& Carmona-Ribeiro, 2003; Lincopan et al., 2005; Vieira et al., 2006; Lincopan \& Carmona-Ribeiro, 2006; Carmona-Ribeiro, 2006; Vieira \& Carmona-Ribeiro, 2008). AmB and MCZ self-assemble and solubilize at hydrophobic sites of DODAB bilayer fragments in water solution exhibiting in vivo therapeutic activity (Vieira \& Carmona-Ribeiro, 2001; Pacheco \& Carmona-Ribeiro, 2003; Lincopan et al., 2003; Lincopan et al., 2005; Vieira et al., 2006; Carmona-Ribeiro, 2006). In order to formulate hydrophobic drugs with the DODAB lipid at high drug-to-lipid molar ratios, the "sticky" property of chaotropic dihydrogen phosphate anion converted MCZ or 
AmB drug particles into negatively charged particles (Figure 7). Thereafter, anionic drug particles could be coated by the DODAB cationic lipid (Lincopan \& Carmona-Ribeiro, 2006; Vieira et al., 2006). These formulations were tested against Crytpococcus neoformans and Candida albicans and were very effective. Coalescence of bilayer fragments around drug granules encapsulated drug particles at high drug-to-lipid molar ratios (Pacheco \& Carmona-Ribeiro, 2003; Lincopan \& Carmona-Ribeiro 2006; Vieira et al., 2006; Vieira \& Carmona-Ribeiro, 2008). In vivo activity of the DODAB/AmB formulation against systemic candidiasis was evaluated from survival and tissue burden experiments in comparison to the classical drug formulation Fungizone (Lincopan et al., 2003). Effective AmB dose in the novel DODAB/AmB formulation was lower than AmB dose in Fungizone but gave the same therapeutic result: 100\% survival (Lincopan et al., 2003). From tissue burden experiments, DODAB/AmB efficacy was also equivalent to the one exhibited by Fungizone regarding elimination of Candida albicans colonization in spleen and kidneys. In contrast to Fungizone, which is the traditional $\mathrm{AmB}$ formulation using deoxycholate, the novel formulation exhibited low nephrotoxicity (Lincopan et al., 2005). Synthetic and charged bilayer fragments are opening new perspectives for delivery of water insoluble drugs. In the specific case of the synthetic cationic lipid DODAB, bilayer fragments present antimicrobial activity, solubilize fungicides such as $\mathrm{AmB}$ and $\mathrm{MCZ}$, stabilize hydrophobic drug particles, are therapeutically effective in vivo, and sometimes exhibit synergism with the drug carried.

\section{Antimicrobial peptides}

Antimicrobial peptides (AMPs) are widely distributed in nature, being produced by bacteria, plants, and a wide variety of animals - both vertebrates and invertebrates (Zasloff, 2002; Brogden, 2005; Pereira, 2006; Rossi, 2008). These compounds are also considered to be key players in innate immunity against microorganisms (Devine et al., 2002; Song et al., 2005). Although AMPs produced by animal and plants and those produced by bacteria certainly function in entirely different settings, the production of bacterial AMPs may also be thought of as a type of defense, since the peptides kill invading bacteria that compete with the AMP-producer for nutrients. The AMPs produced by bacteria seem overall to be more potent than the ones produced by eukaryotes, the former peptides being active at picoto nanomolar concentrations and the latter at micromolar concentrations (Fimland et al., 2005). AMPs are generally small peptides consisting of 5-50 amino acid residues and are highly positively charged (Hancock, 1998) amphipathic molecules with well defined hydrophobic and hydrophilic regions (Zasloff, 2002; Toke, 2005). AMP's found in nature exhibit a wide variety of structures and amino acid sequences with amphiphilic nature and positive charge as the only common factors between them (Melo et al., 2009). These properties permit the peptide to fold into an amphiphilic structure in three dimensions, often upon contact with membranes, so they form separate patches rich in positively charged and hydrophobic amino acids. Folded peptides fall into four broad structural groups: $\beta$-sheet peptides stabilized by two to four disulfide bridges (for example, human aand $\beta$-defensins, plectasin or protegrins); $\alpha$-helical peptides (for example, LL-37, cecropins or magainins); extended structures rich in glycine, proline, tryptophan, arginine and/or histidine (for example, indolicidin); and loop peptides with one or disulfide bridge (for example, bacteriocins) (Hancock \& Sahl, 2006). Among the bacteriocins of Gram-positive bacteria, there is a particular group, the lantibiotics (lanthionine-containing peptide antibiotics), which are characterized by thioether-based intramolecular rings resulting from 
post-translational modifications of serine (or threonine) and cysteine residues (for example, nisin and mersacidin) (McAuliffe et al., 2001). Lanthionine rings, some of which represent conserved binding motifs for recognition of specific targets, create segments of defined spatial structures in the peptides (Hsu et al., 2004). These ring structures also provide stability against proteases and against the antigen-processing machinery, since antibodies against highly cross-bridged antibiotics are very difficult to obtain.

Hundreds of peptide antibiotics have been described in the past half-century (Perlman \& Bodansky, 1971; Kleinkauf \& Dohren, 1988; Hancock et al., 1995). AMPs belong to two classes. They can be nonribosomally (gramicidins, polymyxins, bacitracins, glycopeptides, etc.) or ribosomally synthesized peptides. The former are often drastically modified and are largely produced by bacteria, whereas the latter are produced by all living species (including bacteria) as a major component of the natural host defense molecules of these species (Perlman \& Bodansky, 1971; Kleinkauf \& Dohren, 1988).

Non-ribosomally synthesized peptides can be described as peptides elaborated in bacteria, fungi, and streptomycetes that contain two or more moieties derived from amino acids (Perlman \& Bodansky, 1971; Kleinkauf \& Dohren, 1988). By definition even the longer peptidic molecules in this class are made on multienzyme complexes rather than being synthesized on ribosomes. Many of the antibiotics used in our society are peptide derived. For example, the natural penicillins can be dissected into residues of mono substituted acetic acid, L-cysteine and D-valine, while cephalosporin C, the basic building block of many semi synthetic cephalosporins comprises D-a-aminoadipic acid, L-cysteine, a,b-dehydrovaline, and acetic acid. The glycopeptides class of antibiotics including vancomycin and teicoplanin have sugarsubstituted peptide backbones (Hancock \& Chapple, 1999). Another example is daptomycin lipopeptide, an important reserve antibiotic against multiple resistant Gram positive bacteria.

Cationic peptides such as polymyxin B (net charge of +5 ) and gramicidin $S$ (net charge of +2) exhibit different selectivities. The first is selective to Gram-negative bacteria whereas the second exhibited activity against Gram-positive and Gram-negative bacteria plus Candida albicans (Kondejewski et al., 1996). The cationic antimicrobial peptides act on cells by selfpromoting their uptake across the cytoplasmic membrane interfering with the cytoplasmic membrane functionality as a barrier. In contrast, the gram-positive-specific antibiotic bacitracin works by inhibiting the transfer of cytoplasmically synthesized peptidoglycan precursors to bactoprenol pyrophosphate. Other antibiotic peptides of nonribosomal origin, the streptogramins, are protein synthesis inhibitors (Hancock \& Chapple, 1999).

Ribosomally synthesized peptides are produced by eukaryotes and represent crucial components of their defense systems against microorganisms, being widely distributed in nature and produced by mammals, birds, amphibians, insects, plants, and microorganisms. Although they form a diverse group of peptides as judged by their primary structures, they are often cationic, amphiphilic and most of them kill bacteria by permeabilizing their cell membranes. Their positive charge presumably facilitates interactions with the negatively charged bacterial phospholipid-containing membranes and or acidic bacterial cell walls, whereas their amphiphilic character enables membrane permeabilization. Classification from chemical functionalities may be used for these AMPs from a high content of a certain amino acid, most often proline, intramolecular disulfide bridges, and content of a-helical structure (Hancock \& Chapple, 1999; Papagianni, 2003).

Antibiotics primarily generated by bacteria and fungi have led to dramatic improvement in the ability to treat infectious diseases and significant increase in food animal production. They represent one of the major scientific and medical advances of the 20th century (Gordon 
et al., 2005; McPhee \& Hancock, 2005). Although antibiotic therapy is still the first choice to combat microbial infections in humans and animals, the prevalence of bacterial resistance to conventional antibiotics is a growing public health concern. This has driven the search for new antimicrobials that are broadly effective and less likely to induce antimicrobial resistance (Sang \& Blecha, 2008). While conventional antibiotics are active only against bacteria and/or fungi, AMPs have a broader range of applications against bacteria, fungi, parasites, enveloped viruses and cancer. A large variety of AMPs synthesized by bacteria belong to the group of bacteriocins which are not ribosomally synthesized (Papagianni, 2003). Bacteriocins are small, heat stable peptides that bacteria use to compete against other bacteria of the same species (narrow spectrum) or against bacteria of other genera (broad spectrum) (Cotter et al., 2005). The majority of Class I and Class II bacteriocins are active in the nanomolar range against Gram-positive bacteria in closely related species or in a broadspectrum manner for many species. The most promising bacteriocins as antibiotics are produced by lactic acid bacteria (LAB) with the core genera including Lactobacillus, Lactococcus, Leuconostoc, Pediococcus and Streptococcus. Examples of such peptides are nisin (Cotter et al., 2005; Dufour et al., 2007), mersacidin (pre-clinical test to treat Gram-positive infections) (Hancock \& Sahl, 2006) and lacticin (against mastitis infections) (Gardiner et al., 2007). Table 1 illustrates AMPs diversity.

\begin{tabular}{|c|c|c|c|c|}
\hline Origin & Class & Examples & Antimicrobial activity & Reference \\
\hline Lactic Acid & Class I and II & Lantibiotics & Nanomolar range, activity against & Willey et al.,2007 \\
\hline Bacteria LAB & Bacteriocins & $\begin{array}{l}\text { Class I: nisin, mersacidin; } \\
\text { non-lantabiotics } \\
\text { Class II: pediocin, PA1, } \\
\text { enterocin AS48 }\end{array}$ & $\begin{array}{l}\text { closely related or broad-spectrum } \\
\text { Gram-positive bacteria }\end{array}$ & Field et al.,2008 \\
\hline $\begin{array}{l}\text { Bacteria } \\
\text { (E.coli) }\end{array}$ & Bacteriocins & Colicins, microcins & $\begin{array}{l}\text { Nanomolar range, activity against } \\
\text { Enterobacteriaceae }\end{array}$ & $\begin{array}{l}\text { Duquesne et al.,2007 } \\
\text { Nes et al.,2007 }\end{array}$ \\
\hline Fungi & Fungal Defensins & Plectasin & $\begin{array}{l}\text { Activity multiple resistant Gram- } \\
\text { positive }\end{array}$ & Mygind et al.,2005 \\
\hline Plants & Plant Defensins & Ib-AMP1-4 and cyclotides & $\begin{array}{l}\text { Micromolar range: antifungal, } \\
\text { anti-HIV, anti parasites }\end{array}$ & $\begin{array}{l}\text { Colgrave et al.,2008 } \\
\text { Ireland et al.,2008 } \\
\text { Marcos et al.,2008 }\end{array}$ \\
\hline $\begin{array}{l}\text { Insects/ } \\
\text { amphibians }\end{array}$ & $\begin{array}{l}\text { Insect/ } \\
\text { amphibian } \\
\text { cationic } \\
\text { peptides }\end{array}$ & $\begin{array}{l}\text { Cecropin A, mellitin, } \\
\text { magainins, temporins }\end{array}$ & $\begin{array}{l}\text { Micromolar range } \\
\text { active against } \\
\text { multidrug-resistant } \\
\text { bacteria }\end{array}$ & $\begin{array}{l}\text { Bechinger, 1997; } \\
\text { Giacometti et al.,2003 }\end{array}$ \\
\hline $\begin{array}{l}\text { Arachnida/ } \\
\text { vertebrates }\end{array}$ & $\begin{array}{l}\text { Venom toxins/ } \\
\text { b-defensins }\end{array}$ & $\begin{array}{l}\text { Defensin-like toxins } \\
\text { (DLTs) } \\
\text { in venom, and b-defensins }\end{array}$ & $\begin{array}{l}\text { Micromolar range, } \\
\text { active against } \\
\text { multidrug-resistant } \\
\text { bacteria mostly in a } \\
\text { salt-dependent } \\
\text { manner }\end{array}$ & $\begin{array}{l}\text { Yeaman \& Yount, 2007; } \\
\text { Warren et al.,2008 }\end{array}$ \\
\hline Mammals & $\begin{array}{l}\text { a-Defensins } \\
\text { q-Defensins } \\
\text { b-Defensins }\end{array}$ & $\begin{array}{l}\text { Human neutrophil } \\
\text { defensins, } \\
\text { enteric and epithelial } \\
\text { defensins }\end{array}$ & $\begin{array}{l}\text { Micromolar range } \\
\text { active against } \\
\text { multidrug-resistant } \\
\text { bacteria, and fungi and viruses }\end{array}$ & $\begin{array}{l}\text { Selsted \& Ouellette, 2005; } \\
\text { Lehrer, } 2007\end{array}$ \\
\hline $\begin{array}{l}\text { Higher } \\
\text { vertebrates }\end{array}$ & Cathelicidins & $\begin{array}{l}\text { Human LL-37, porcine } \\
\text { PR-39, bovine indolicidin }\end{array}$ & $\begin{array}{l}\text { Micromolar range } \\
\text { active against } \\
\text { multidrug-resistant } \\
\text { bacteria, and fungi and viruses }\end{array}$ & Zanetti, 2005 \\
\hline Humans & Others & $\begin{array}{l}\text { Lactoferricin, and } \\
\text { antimicrobial domain of } \\
\text { lysozyme }\end{array}$ & $\begin{array}{l}\text { Micromolar range } \\
\text { active against } \\
\text { multidrug-resistant } \\
\text { bacteria }\end{array}$ & Brogden, 2005 \\
\hline
\end{tabular}

Table 1. Antimicrobial peptides diversity of origin, class and antimicrobial activity. Adapted from Sang \& Blecha, 2008. 
Bacillus species are efficient AMPs factories. Their AMPs are active against Gram-positive microorganisms, with some presenting broader activity against Gram-negative bacteria and fungi (Katz \& Demain, 1977). The soil isolate B. cereus 8A produces an antibacterial substance, cerein 8A, which inhibits several Gram-positive bacteria including Bacillus spp., Streptococcus spp. and Listeria monocytogenes (Bizani \& Brandelli, 2002). Iturins are lipopeptides produced by Bacillus subtilis that show antifungal activities against various pathogenic yeasts and molds. The antifungal activity of iturin is related to their interaction with the cytoplasm membrane of target cells leading to an increase in $\mathrm{K}^{+}$permeability (Maget-Dana \& Peypoux, 1994). Another antifungal lipopeptide complex produced by the Bacillus subtilis, the fengycin, was found inhibitory to filamentous fungi but not yeast (Vanittanakom et al., 1986). The mechanism of fengycin action was revealed as a two-state transition controlled by the lipopeptide concentration - one state being monomeric, not deeply anchored and nonperturbing lipopeptide and other burried, aggregated form responsible for membrane leakage and bioactivity (Deleu et al., 2008).

A soil microorganism identified as Bacillus megaterium was found to produce several antibiotics (Pueyo et al., 2009). Analysis both by electron spray ionization (ESI) and matrixassisted laser desorption ionization-time of flight (MALDI-TOF) mass spectrometry (MS) identified these substances as lipopeptides. Predominant peaks at $\mathrm{m} / \mathrm{z} 1,041$ and $\mathrm{m} / \mathrm{z} 1,065$ revealed ions which were compatible with surfactins and lichenysins, respectively. Two other ions $\mathrm{m} / \mathrm{z}$ 1,057 and $\mathrm{m} / \mathrm{z}$ 1,464 were further studied by collision-induced dissociation (CID) unveiling an iturin $A$ at the first and fengycins $A$ and $B$ at the second $\mathrm{m} / \mathrm{z}$ peaks. The CID spectrum of the $\mathrm{m} / \mathrm{z} 1,464$ ion also suggested the existence of fengycins $A$ and $B$ variants in which Ile was changed to Val in the position 10 of the peptide moiety. This mixture of lipopeptides isolated from B. megaterium was very effective against $B$. cereus. The culture did not grow after the exposure to $12 \mu \mathrm{g} / \mathrm{mL}$ of the lipopeptides mixture for 30 minutes.

Many AMPs are produced by fungi. The most widely used and historic antibiotic to date, penicillin, was from the fungus Penicillium chrysogenum, previously named Penicillium notatum. Plectasin is the first identified fungal defensin derivated from Pseudoplectania nigrella, a black saprophytic asomycetes. This defensin was active against antibiotic-resistant strains of Streptococcus pneumoniae with rates similar to penicillin and vancomycin presented, efficacy in treating peritonitis and pneumonia in mice, and showed therapeutic potential as antifungal compound (Mygind et al., 2005). Anafp, another antifungal peptide obtained from the culture supernatant of Aspergillus niger inhibited various yeast strains as well as filamentous fungi at low concentrations (Lee et al., 1999). A novel antifungal peptide named 'AcAFP' from Aspergillus clavatus exhibited thermostability and is promising due to its thermostability at $100^{\circ} \mathrm{C}$ for $1 \mathrm{~h}$ and strong inhibitory activity against mycelial growth of several molds including Fusarium oxysporum, Fusarium solani, Aspergillus niger, Botrytis cinera and Alternaria solani (Skouri-Gargouri \& Gargouri, 2008). A 6.0-kDa antimicrobial peptide from Aspergillus clavatus ES1, designated as AcAMP, was isolated by a one-step heat treatment, was sensitive to proteolytic enzymes, stable between $\mathrm{pH} 5.0$ and 10.0, and heat resistant $\left(15 \mathrm{~min}\right.$ at $100^{\circ} \mathrm{C}$ ) (Hajji et al., 2010). AcAMP exhibited antibacterial activity against several Gram-positive and -negative bacteria. Based on all these features, AcAMP can be considered as a promising new member of the restraint family of ascomycete antimicrobial peptides that might be used in biological control of plant diseases and also for potential applications in food preservation. 
The mode of action of the cationic and amphiphilic AMPs has been associated with their electrostatic attraction to the microbial cell surfaces, which contain negatively charged and acidic polymers, such as lipopolysaccharides (Gram negative bacteria), and wall-associated teicoic acids (Gram-positive bacteria). They transit the outer membrane of the Gram negative bacteria via self-promoted uptake (Hancock \& Lehrer, 1998). Subsequently these peptides contact the anionic surface of the cytoplasmic membrane and insert in a manner such that they initially straddle the interface of the hydrophilic head groups and the fatty acyl chains of membrane phospholipids. After insertion into the membrane, antimicrobial peptides act by either disrupting the physical integrity of the bilayer, via membrane thinning, transient poration and/or disruption of the barrier function, or translocate across the membrane and act on internal targets (Hancock \& Sahl, 2006).

Several complex and controversial models describe these subsequent events, including the reorientation of peptide molecules perpendicular to the membrane to form either barrel-stave or toroidal channels, the breakdown of membrane integrity as a result of the swamping of membrane charge by a 'carpet' of peptides at the interface, the detergent-like dissolution of patches of membrane and the formation of peptide-lipid aggregates within the bilayer (Jenssen et al., 2006). Each of these successfully predicts the ability of cationic antimicrobial peptides to break down the cytoplasmic membrane, but only the toroidal channel and aggregate models explain the action of certain peptides on cytoplasmic targets. Indeed, the action of many peptides cannot be explained by disruption of membrane permeability barriers, as discussed in several reviews (Yeaman \& Yount, 2003; Jenssen et al., 2006; Peschel \& Sahl, 2006). Figure 8 shows mechanisms of action for conventional antibiotics and AMPs.

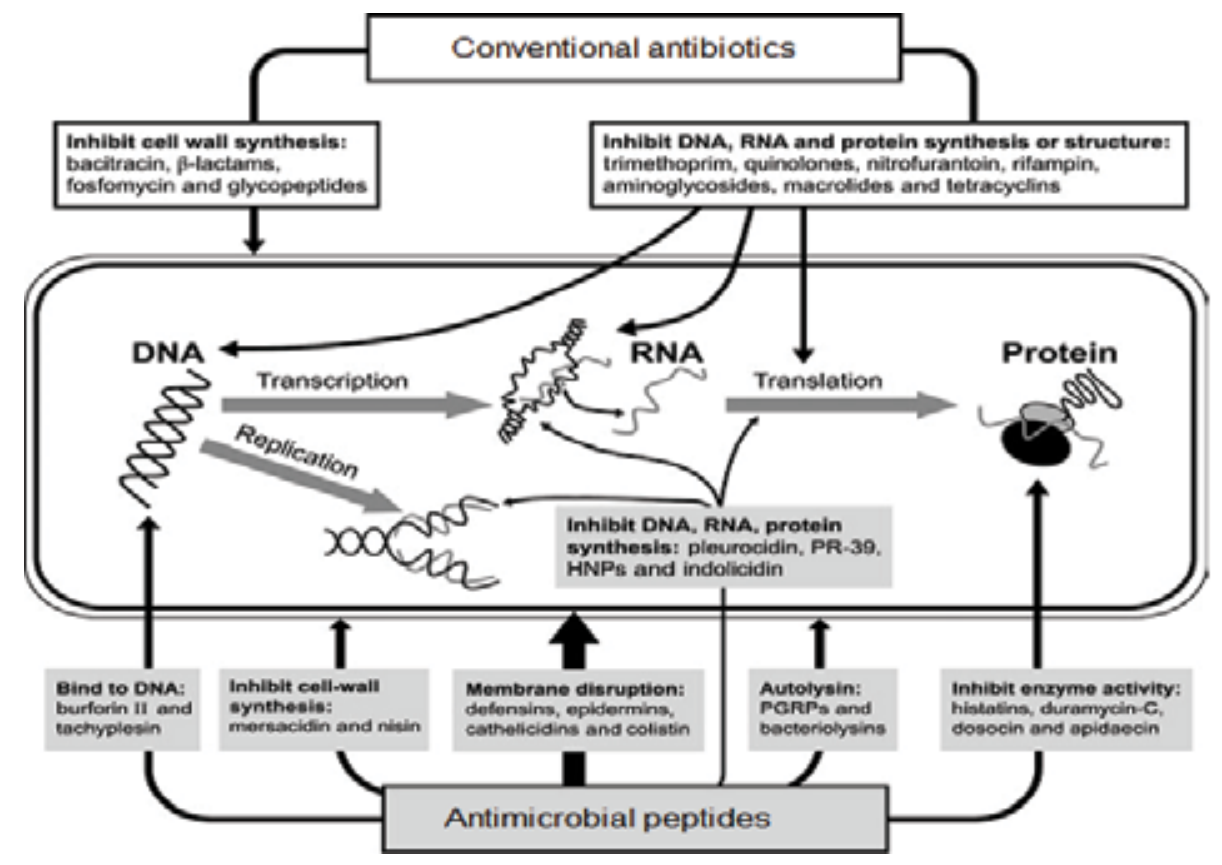

Fig. 8. Mechanism of action for conventional antibiotics and AMPs. Reproduced with permission from Sang, Y. \& Blecha, F., Antimicrobial peptides and bacteriocins: alternatives to traditional antibiotics, Animal Health Research Reviews, 9, 2, 227-235, 2008. Copyright 2008 Cambridge Journals. 
Bacitracin, $\beta$-lactams and glycopeptides action is related to the inhibition of cell wall synthesis whereas trimethoprim, quinolones, nitrofurantoin, rifampin, aminoglycosides, macrolides and tetracyclines inhibit DNA, RNA or protein synthesis. However, recent observations suggest that AMPs besides insert and damage of the cytoplasmatic membranes of target cells, peptides may also interact with intracellular targets such as DNA and RNA, presumably interfering with their metabolic functions and thus leading to cell death (Brogden, 2005; Hale \& Hancock, 2007). They can alter cytoplasmic membrane septum formation; inhibit cell wall synthesis; inhibit nucleic acid and protein synthesis; or inhibit enzymatic activity (Brogden, 2005).

Antibiotics and AMPs have a mixed history so does the development of their applications. Polymyxin B and gramicidin S have been used in the clinic and as topical over-the-counter medicines for a long time, and the cationic lantibiotic nisin has been used as an antimicrobial food additive. In contrast, despite several series of clinical trials, only one of the new generation (designer) cationic antimicrobial peptides has demonstrated efficacy in phase 3 clinical trials. Nevertheless, given their exceptionally broad activity spectra, which for a single peptide can include activity against Gram-negative and Gram-positive bacteria, fungi as well as viruses and parasites, still substantial interest remains in exploiting the potential of these molecules (Hancock \& Sahl, 2006).

Some AMP-based antibiotic formulations are at preclinical stages with some proceeding to clinical trials (Andrès \& Dimarcq, 2005; Gordon et al., 2005; McPhee \& Hancock, 2005; Hancock \& Sahl, 2006). Nisin, a LAB lantibiotic, is one of few examples of AMP-based antibiotic therapies that have been commercialized. AMP-based drugs derived from insect cecropin B and bovine indolicidin have progressed to clinical trials to treat wounds or skinrelated infections in humans, applications that may also be used in veterinary medicine (Hancock \& Sahl, 2006; Scott et al., 2007). Some drugs under testing are derivatives of AMPs that have been modified to improve their antimicrobial activity. These modifications include introducing non-natural residues like D-amino acids, addition of C-terminal amidation and catalysis of cyclic formation, which are believed to improve stability and activity against targeted micro-organisms as shown in natural bacteriocins, plant cyclotides and primate qdefensins (Lehrer, 2007; Bansal et al., 2008; Ireland et al., 2008). Optimized design of synthetic peptides based on knowledge from natural AMP studies (the concept of 'designer AMPs') may provide a feasible way to increase novel drug development (Scott et al., 2007; Jenssen et al., 2008). Short antimicrobial peptides with nine and eleven residues were developed against several clinically important bacterial and fungal pathogens such as E. coli, P. aeruginosa, S. aureus, C. albicans and Fusarium solani (Qi et al., 2010). Twelve analogues of previously reported peptides BP76 (KKLFKKILKFL) and Pac-525 (KWRRWVRWI) were designed, synthesized, and tested for their antimicrobial activities. Two of eleven amino acid peptides, P11-5 (GKLFKKILKIL) and P11-6 (KKLIKKILKIL), have very low MICs of 3.1-12.5 $\mu \mathrm{g} / \mathrm{mL}$ against all five pathogens. The MICs of these two peptides against $S$. aureus, $C$. albicans and F. solani are four to ten times lower than the corresponding MICs of the reference peptide BP76. P9-4 (KWRRWIRWL), newly designed nine-amino acid analogue, also has particularly low MICs of 3.1-6.2 microgram/mL against four of the tested pathogens; these MICs are two to eight times lower than those reported for Pac-525 (6.2-50 micrograms/mL). These new peptides (P11-5, P11-6 and P9- 4) also exhibit improved stability in the presence of salts, and have low cytotoxicity as shown by the haemolysis and MTT assays. From the results of field-emission scanning electron microscopy, membrane depolarization and dye-leakage assays, were propose that these peptides exert their action 
by disrupting lipid membranes. Molecular dynamics simulation studies confirm that P11-6 peptide maintains relatively stable helical structure and exerts more perturbation action on the order of acyl tail of lipid bilayers (Qi et al., 2010). A series of AMP's incorporating the un-natural amino-acids Tic-Oic have been developed. Herein the in vitro activity of these peptides, including ten new compounds, against eight potential bio-terrorism bacteria agents and three other bacterial strains were tested. These peptides exhibit a wide range of organism potency and selectivity (Venugopal et al., 2010).

Endogenous antibiotics are antimicrobial peptides called host defense peptides and participate in multiple aspects of immunity (inflammation, wound repair, and regulation of the adaptive immune system) as well as in maintaining homeostasis (Auvynet \& Rosestein, 2009; Pathan et al., 2010). The possibility of utilizing these multifunctional molecules to effectively combat the ever-growing group of antibiotic-resistant pathogens has intensified research aimed at improving their antibiotic activity and therapeutic potential, without the burden of an exacerbated inflammatory response, but conserving their immunomodulatory potential. Because of their wide involvement in inflammatory response and the emerging role of inflammation in atherosclerosis, antimicrobial peptides have been proposed to represent an important link between inflammation and the pathogenesis of atherosclerotic cardiovascular diseases (Li, 2009). The synthesis of AMPs and the development of analogues is an option for their use in humans. Another interesting approach was to induce the endogenous production of these peptides, which would avoid the possible toxicity and adverse systemic reactions, as well as the difficulty to deliver them in integral form to the desired sites of action (Guani-Guerra et al., 2010). The increasing incidence of antibioticresistant bacterial infections is one of the greatest challenges faced by modern medicine with an obvious need for new effective and safe treatments. Thanks to AMPs multifunctional properties, the development of resistance by microorganisms towards AMPs is more difficult. Eventually, AMPs may become useful therapeutic tools.

\section{Conclusion}

Antimicrobial films and surfaces have been produced from impregnation of materials and coatings with antimicrobials, deposition of coatings with antimicrobial covalent modifications and biodegradable materials. These films prevent adhesion and colonization of pathogenic microorganisms and are important for designing biomedical devices and food packaging. Antimicrobial particles have been obtained from inorganic, metal and composite materials, polymers, lipids and a variety of hybrid combinations. They are important in disinfection, sterilization and in impregnation of materials to become antimicrobials. In therapy against infectious diseases, antimicrobial particles, liposomes and antimicrobial peptides provided several instances of improvement of the therapeutic index for a variety of formulations. The future will probably witness important novel developments in applied research regarding antimicrobial hybrid and composite systems by themselves or in efficient combinations with drugs.

\section{Acknowledgments}

Financial support from Conselho Nacional de Desenvolvimento Científico e Tecnológico $(\mathrm{CNPq})$ is gratefully acknowledged. $\mathrm{LB}$ and LDM are recipients of MSc CNPq fellowships. 


\section{References}

Abel, T.; Cohen, J.I.; Engel, R.; Filshtinskaya, M.; Melkonian, A. \& Melkonian, K. (2002). Preparation and investigation of antibacterial carbohydrate-based surfaces. Carbohydr.Res., 337, 24, 2495-2499.

Abeylath, S. C. \& Turos, E. (2008). Drug delivery approaches to overcome bacterial resistance to $\beta$-lactam antibiotics. Expert Opin. Drug Deliv., 5, 9, 931-949.

Abid, C.K.V.Z.; Chattopadhyay, S.; Mazumdar, N. \& Singh, H. (2010). Synthesis and characterization of quaternary ammonium PEGDA dendritic copolymer networks for water disinfection. J. Appl. Polym. Sci., 116, 3, 1640-1649.

Agarwal, A.; Weis, T.L.; Schurr, M.J.; Faith, N.G.; Czuprynski, C.J.; McAnulty, J.F.; Murphy, C.J. \& Abbott, N.L. (2010). Surfaces modified with nanometer-thick silverimpregnated polymeric films that kill bacteria but support growth of mammalian cells. Biomaterials, 31, 680-690.

Ahmad, A.; Senapati, S.; Khan, M.I.; Kumar, R. \& Sastry, M. (2003a). Extracellular biosynthesis of monodisperse gold nanoparticles by a novel extremophilic actinomycete, Thermomonospora sp. Langmuir, 19, 3550-3553.

Ahmad, A.; Senapati, S.; Khan, M.I.; Kumar, R.; Ramani, R.; Srinivas, V. \& Sastry, M. (2003b). Intracellular synthesis of gold nanoparticles by a novel alkalotolerant actinomycete, Rhodococcus species. Nanotechnology, 14, 824- 828.

Akashi, A.; Matsuya, Y.; Unemori, M. \& Akamine, A. (2001). Release profile of antimicrobial agents from a-tricalcium phosphate cement. Biomaterials, 22, 20, 2713-2717.

Ambrose, C. G.; Gogola, G. R.; Clyburn, T. A.; Raymond, A. K.; Peng, A. S. \& Mikos, A. G. (2003). Antibiotic microspheres: preliminary testing for potential treatment of osteomyelitis. Clin.Orthop. Relat. Res., 415, 279-285.

Andrès, E. \& Dimarcq, J. L. (2005). Clinical development of antimicrobial peptides. Int. J. Antimicrob. Agents, 25, 5, 448-449.

Arai, T.; Benny, O.; Joki, T.; Menon, L. G.; Machluf, M.; Abe, T.; Carroll, R. S. \& Black, P. M. (2010). Novel local drug delivery system using thermoreversible gel in combination with polymeric microspheres or liposomes. Anticancer Res., 30, 1057-1064.

Auvynet, C. \& Rosenstein, Y. (2009). Multifunctional host defense peptides: antimicrobial peptides, the small yet big players in innate and adaptive immunity. FEBS J. 276, 22, 6497-6508.

Avila-Sosa, R.; Hernández-Zamoran, E.; López-mendoza, I.; Palou, E.; Munguía, M.T.J.; Nevárez-Moorillón, G.V. \& López-Malo, A. (2010). Fungal inactivation by Mexican oregano (Lippia berlandieri Schauer) essential oil added to amaranth, chitosan, or starch edible films. J. Food Sci., 75, 3, M127-M133.

Bach, A.; Eberhardt, H.; Frick, A.; Schmidt, H.; Bottinger, B.W. \& Martin, E. (1999). Efficacy of silver-coating central venous catheters in reducing bacterial colonization. Crit. Care Med., 27.; 515-520.

Bali, R.; Razak, N.; Lumb, A. \& Harris, A.T. (2006). The synthesis of metal nanoparticles inside live plants. IEEE Xplore, 4143372, 224-227.

Bansal, P.S.; Torres, A.M. Crossett, B.; Wong, K.K.; Kok, J.M.; Geraghty, D,P.; Vandenberg, J.I. \& Kuchel, P.W. (2008). Substrate specific of platypus venom L-toD-peptide isomerase. J. Biol. Chem., 283, 8969-8975. 
Beaulac, C., Sachetelli, S. \& Lagace, J. (1998). In vitro bactericidal efficacy of sub-MIC concentrations of liposome-encapsulated antibiotic against Gram-negative and Gram-positive bacteria. J. Antimicrob. Chemther., 41, 35-41.

Bechinger, B. (1997). Structure and functions of channel-forming peptides: magainins, cecropins, melittin and alamethicin. J. Memb. Biol., 156, 3, 197-211.

Birla, S.S.; Tiwari, V.V.; Gade, A.K.; Ingle, A.P.; Yadav, A.P. \& Raí, M.K. (2009). Fabrication of silver nanoparticles by Phoma glomerata and its combined effect against Escherichia coli, Pseudomonas aeruginosa and Staphylococcus aureus. Lett. Appl. Microbiol., 48, 173-179.

Bizani, D. \& Brandelli, A. (2002). Characterization of a bacteriocins produced by a newly isolated Bacillus sp. strain 8A. J. Appl. Microbiol., 93, 3, 512-519.

Bozzi, A.; Yuranova, T. \& Kiwi, J. (2005). Self-cleaning of wool-polyamide and polyester textile by $\mathrm{TiO}_{2}$-rutile modification under daylight irradiation at ambient temperature. J. Photochem. A 172.; 27-43.

Briones, E.; Colino, C. I. \& Lanao, J.M. (2008). Delivery systems to increase the selectivity of antibiotics in phagocytic cells. J. Controlled Release, 125, 3, 210-227.

Brochu, H.; Polidori, A.; Pucci, B. \& Vermette, P. (2004). Drug delivery systems using immobilized intact liposomes: a comparative and critical review. Curr. Drug Deliv., $1,3,299-312$.

Brogden, K.A, (2005). Antimicrobial peptides: pore formers or metabolic inhibitors in becteria? Nat. Rev. Microbiol., 3, 238-250.

Brun-Buisson, C.; Doyon, F.; Sollet, J.P.; Cochard, J.F.; Cohen, Y. \& Nitenberg, G. (2004). Prevention of intravascular catheter-related infection with newer chlorhexidinesilver-sulfadiazine-coated catheters. Intensive Care Med., 30, 837-843.

Bruschi, M.; Pirri, G.; Giuliani, A.; Nicoletto, S.F.; Baster, I.; Scorciapino, M.A.; Casu, M. \& Rinaldi, A.C. (2010). Synthesis, characterization, antimicrobial activity and LPSinteraction properties of SB041, a novel dendrimeric peptide with antimicrobial properties. Peptides, 31, 8, 1459-1467.

Bryaskova, R.; Pencheva, D.; Kale, G.M.; Lad, U. \& Kantardjiev, T. (2010). Synthesis, characterization and antibacterial activity of PVA/TEOS/Ag-Np hybrid thin films. J. Colloid Interface Sci., 349, 77-85.

Burd, T.A.; Anglen, J.O.; Lowry, K.J.; Hendricks, K.J. \& Day, D. (2001). In vitro elution characteristics of tobramycin from bioabsorbable polycaprolactone bead. J. Orthop. Trauma, 15, 424-428.

Burt, S.A. (2004). Essential oils: their antibacterial properties and potential applications in foods: a review. Int. J.Food Microbiol., 94, 3, 223-253.

Cabral, J.P.S. (1992). Mode of antibacterial action of dodine (dodecylguanidine monoacetate) in Pseudomonas seryngae. Can.J.Microbiol., 38, 2, 115-123.

Cademartiri, R.; Anany, H.; Gross, I.; Bhayani, R.; Griffiths, M. \& Brook, M.A. (2010). Immobilization of bacteriophages on modified silica particles. Biomaterials, 31, 19041910.

Cagri, A.; Ustunol, Z. \& Ryser, E.T. (2004). Antimicrobial edible films and coatings. J. Food Prot., 67, 4, 833-848.

Campanhã, M.T.N.; Mamizuka, E.M. \& Carmona-Ribeiro, A.M. (1999). Interactions between cationic liposomes and bacteria: the physical-chemistry of the bactericidal action. J. Lipid Res., 40, 8, 1495-1500. 
Campanhã, M.T.N.; Mamizuka, E.M. \& Carmona-Ribeiro, A.M. (2001). Interactions between cationic vesicles and Candida albicans. J. Phys. Chem. B, 105, 8230-8236.

Campoccia, D.; Montanaro, L.; Speziale, P. \& Arciola, C.R. (2010). Antibiotic-loaded biomaterials and the risks for the spread of antibiotic resistance following their prophylactic and therapeutic clinical use. Biomaterials, 31, 6363-6377.

Carmona-Ribeiro, A.M. (2010). Biomimetic nanoparticles: Preparation, Characterization and Biomedical Applications. Int. J. Nanomed., 5, 1, 249-259

Carmona-Ribeiro, A.M. (2000). Interactions between cationic liposomes and drugs or biomolecules. Anais da Academia Brasileira de Ciências, 72, 1, 39-43.

Carmona-Ribeiro, A.M. (2010). Lipid-based biomimetics in drug and vaccine delivery. In: Biomimetics, Learning from Nature. Amitava Mukherjee (1 Ed.), v. 1, 507-534, Olajnica, Vukovar, Croatia: IN-TEH.

Carmona-Ribeiro, A.M. (2007). Biomimetic particles in drug and vaccine delivery. J. Liposome Res., 17, 3-4, 165-172.

Carmona-Ribeiro, A.M. (2006). Lipid bilayer fragments and disks in drug delivery. Curr. Med. Chem., 13, 12, 1359-1370.

Carmona-Ribeiro, A.M.; Vieira, D.B. \& Lincopan, N. (2006). Cationic surfactants and lipids as anti-infective agents. Anti-Infective Agents Med. Chem., 5, 1, 33-54.

Carmona-Ribeiro, A.M. (2003). Bilayer-forming synthetic lipids: drugs or carriers?. Curr. Med. Chem., 10, 22, 2425-2446.

Carmona-Ribeiro, A.M. (2001). Bilayer vesicles and liposomes as interface agents. Chem. Soc. Rev., 30, 4, 241-247.

Carmona-Ribeiro, A.M. \& Lessa, M.M. (1999). Interactions between bilayer vesicles and latex. Colloids Surf. A, 153, 355-361.

Carmona-Ribeiro, A.M.; Ortis, F.; Schumacher, R.I. \& Armelin, M.C.S. (1997). Interactions between cationic vesicles and cultured mammalian cells. Langmuir, 13, 8, 2215-2218.

Carmona-Ribeiro, A.M. (1992). Synthetic amphiphile vesicles. Chem. Soc. Rev., 21, 3, 209-214.

Carmona-Ribeiro, A.M. \& Midmore, B.R. (1992). Synthetic bilayer adsorption onto polystyrene microspheres. Langmuir, 8, 801-806.

Carmona-Ribeiro, A.M.; Castuma, C.E.; Sesso, A. \& Schreier, S. (1991). Bilayer structure and stability in dihexadecyl phosphate dispersions. J. Phys Chem., 95, 13, 5361-5366.

Caro, A.; Humblot, V.; Méthivier, C.; Minier, M.; Salmain, M. \& Pradier, C. (2009). Grafting of lysozyme and/or poly (ethylene glycol) to prevent biofilm growth on stainless steel surfaces. J. Phys. Chem. B, 113, 2101-2109.

Carson, L.; Gorman, S.P. \& Gilmore, B.F. (2010). The use of lytic bacteriophages in the prevention and eradication of biofilms of Proteus mirabilis and Escherichia coli. FEMS Immunol. Med. Microbiol., 59, 447-455.

Carvalho, L.A. \& Carmona-Ribeiro, A.M. (1998). Interactions between cationic vesicles and serum proteins. Langmuir, 14, 21, 6077-6081.

Catuogno, C. \& Jones, M.N. (2003). The antibacterial properties of solid supported liposomes on Streptococcus oralis biofilms. Int.J.Pharm., 257, 1-2, 125-140.

Cavalli, R.; Gasco, M.R.; Chetoni, P.; Burgalassi, S. \& Saettone, M.F. (2002). Solid lipid nanoparticles (SLN) as ocular delivery system for tobramycin. Int. J. Pharm., 238, 12, 241-245. 
Cen, L.; Neoh, K.G. \& Kang, E.T. (2003). Surface functionalization technique for conferring antibacterial properties to polymeric and cellulosic surfaces. Langmuir, 19, 24, 10295-10303.

Cha, D.S. \& Chinnan, M.S. (2004). Biopolymer-based antimicrobial packaging: A review. Crit. Rev. Food Sci. Nut., 44, 4, 223-237.

Chandra, J.; Patel, J.D.; Li, J.; Zhou, G.; Mukherjee, P.K.; McCormick, T.S.; Anderson, J.M. \& Ghannoum, M.A. (2005). Modification of surface properties of biomaterials influences the ability of Candida albicans to form biofilms. Appl. Environ. Microbiol., $71,12,8795-8801$.

Changsan, N.; Nilkaeo, A.; Pungrassami, P. \& Srichana, T. (2009). Monitoring safety of liposomes containing rifampicin on respiratory cell lines and in vitro efficacy against Mycobacterium bovis in alveolar macrophages. J. Drug Targeting, 17, 10, 751762.

Charvalos, E.; Tzatzarakis, M.N.; Van Bambeke, F.; Tulkens, P. M.; Tsatsakis, A.M.; Tzanakakis, G.N. \& Mingeot- Leclercq, M.P. (2006). Water-soluble amphotericin B polyvinylpyrrolidone complexes with maintained antifungal activity against Candida spp. and Aspergillus spp. and reduced haemolytic and cytotoxic effects. J. Antimicrob. Chemother., 57, 2, 236-244.

Chen, C.Z. \& Cooper, S.L. (2002). Interactions between dendrimer biocides and bacterial membranes. Biomaterials, 23, 3359-3368.

Chen, C.Z.; Beck-Tan, N.C.; Dhurjati, P.; van Dyk, T.K.; LaRossa, R.A. \& Cooper, S.L. (2000). Quaternary ammonium functionalized poly(propylene imine) dendrimers as effective antimicrobials : structure-activity studies. Biomacromolecules, 1, 473-480.

Chen, C.Z.S. \& Cooper, S.L. (2000). Recent advances in antimicrobial dendrimers. Adv. Mater., 12, 11, 843-846.

Cheng, G.; Zhang, Z.; Chen, S.F.; Bryers, J.D. \& Jiang, S.Y. (2007). Inhibition of bacterial adhesion and biofilm formation on zwitterionic surfaces. Biomaterials, 28, 29, 41924199.

Codling, C.E.; Maillard, J.Y. \& Russell, A.D. (2003). Aspects of the antimicrobial mechanisms of action of a polyquaternium and an amidoamine. J. Antimicrob. Chemother., 51, 1153-1158.

Cohen, H.A.; Amir, J.; Matalon, A.; Mayan, R.; Beni, S. \& Barzilai, A. (1997). Stethoscopes and otoscopes - a potential vector of infection? Family Practice, 14, 6, 446-449.

Colgrave, M.L.; Kotze, A.C.; Huang, Y.H.; O'Grady, J.; Simonsen, S.M. \& Craik, D.J. (2008). Cyclotides: natural, circular plant peptides that possess significant activity against gastrointestinal nematode parasites of sheep. Biochemistry, 47, 20, 5581-5589.

Colton, M.B. \& Ehrlich, E. (1953). Bactericidal effect obtained by addition of antibiotics to dental cements and direct filling resins. J.Am. Dent. Assoc., 47, 5, 524-531.

Coma, V.; Deschamps, A. \& Martial-Gros, A. (2003). Bioactive packaging materials from edible chitosan polymer - antimicrobial activity assessment on dairy-related contaminants. J. Food Sci., 68, 9, 2788-2792.

Coma, V.; Martial-Gros, A.; Garreau, S.; Copinet, A.; Salin, F. \& Deschamps, A. (2002). Edible antimicrobial films based on chitosan matrix. J.Food Sci., 67, 3, 1163-1169.

Corbitt, T.S.; Ding, L.; Ji, E.; Ista, L.K.; Ogawa, K.; Lopez, G.P.; Schanze, K.S. \& Whitten, D.G. (2009). Light and dark biocidal activity of cationic poly(aryleneethylene) conjugated polyelectrolytes. Photochem. Photobiolog.Sci., 8, 998-1005. 
Cordeiro, C.; Wiseman, D.J.; Lutwyche, P.; Uh, M.; Evans, J.C.; Finlay, B.B. \& Webb, M.S. (2000). Antibacterial Efficacy of Gentamicin Encapsulated in pH-Sensitive Liposomes against an In Vivo Salmonella enterica serovar Typhimurium Intracellular Infection Model. Antimicrob. Agents Chemother., 44, 3, 533-539.

Cotter, P.D.; Hill, C. \& Ross, R.P. (2005). Bacteriocins: developing innate immunity for food. Nat. Rev. Microbiol., 3, 10, 777-788.

Cubillo, E.; Pecharromán, C.; Aguilar, E.; Santarén, J. \& Moya, J.S. (2006). Antibacterial activity of copper monodispersed nanoparticles into sepiolite. J.Mater. Sci., 41, 5208-5212.

Cui, X.; Li, C.M.; Bao, H.; Zheng, X. \& Lu, Z. (2008). In situ fabrication of silver nanoarrays in hyaluronan/PDDA layer-by-layer assembled structure. J. Colloid Interface Sci., 327, 459-465.

Cunningham, T.M.; Koehl, J.L..; Summers, J.S,. \& Haydel, S.E. (2010). pH-Dependent metal ion toxicity influences the antibacterial activity of two natural mineral mixtures. PLoS One., 5, 3, 9456.

Curtin, J.J. \& Donlan, R.M. (2006). Using bacteriophages to reduce formation of catheterassociated biofilms by Staphylococcus epidermidis. Antimicrob. Agents Chemother., 50, $4,1268-1275$.

Cutter, C.N. (2006). Opportunities for bio-based packaging technologies to improve the quality and safety of fresh and further processed muscle foods. Meat Sci., 74, 1, 131142.

Cutter, C.N. (2002). Microbial control by packaging: A review. Crit. Rev. Food Sci. Nutr., 42, 2, 151-161.

Cutter, C.N.; Willett, J.L. \& Siragusa, G.R. (2001). Improved antimicrobial activity of nisinincorporated polymer films by formulation change and addition of food grade chelator. Lett. Applied Microbiol., 33, 325-328.

da Silva Paula, M.M.; Franco, C.V.; Baldin, M.C.; Rodrigues, L.; Barichello, T.; Savi, G.D.; Bellato, L.F.; Fiori, M.A. \& da Silva, L. (2009). Synthesis, characterization and antibacterial activity studies of poly-\{styrene-acrylic acid $\}$ with silver nanoparticles. Mat. Sci. Eng. C., 29, 2, 647-650.

Danese, P.N. (2002). Antibiofilm approaches: prevention of catheter colonization. Chem. Biol., 9, 8, 873-880.

Daoud, W.A.; Xin, J.H. \& Zhang, Y.H. (2005). Surface functionalization of cellulose fibers with titanium dioxide nanoparticles and their combined bactericidal activities. Surf. Sci., 599, 69-75.

Darouiche, R.O. (2008). Prevention of infections associated with vascular catheters. Int. J. Artif. Organs, 31, 810-819.

Dastjerdi, R \& Montazer, M. (2010). A review on the application of inorganic nanostructured materials in the modification of textiles: Focus on anti-microbial properties. Colloids Surf. B, 79, 5-18.

Dastjerdi, R.; Mojtahedi, M. R. M. \& Shoshtari, A. M. (2009). Comparing the effect of three processing methods for modification of filament yarns with inorganic nanocomposite filler and their bioactivity against Staphylococcus aureus. Macromol. Res., 17, 6, 378-387.

Dastjerdi, R.; Montazer, M. \& Shahsavan, S. (2009). A new method to stabilize nanoparticles on textile surfaces. Colloids Surf. A, 345, 202-210. 
Davenas, J.; Thevenard, P.; Philippe, F. \& Arnaud, M. N. (2002). Surface implantation treatments to prevent infection complications in short term devices. Biomol. Eng., 19, 2-6, 263-268.

Davies, A.; Field, B. S. (1969). Action of biguanides, phenols and detergents on Escherichia coli and its sphereoplasts. J. Appl. Bacteriol., 32, 2, 233-243.

Decher, G. \& Hong, J. D. (1991). Buildup of ultrathin multilayer films by a self-assembly process: II. Consecutive adsorption of anionic and cationic bipolar amphiphiles and polyelectrolytes on charged surfaces. Ber. Bunsen Ges., 95, 1430-1434.

Decher, G. (1997). Fuzzy nanoassemblies: toward layered polymeric multicomposites. Science, 277, 1232-1237.

Deleu, M.; Paquot, M. \& Nylander, T. (2008). Effect of fengycin, a lipopeptide produced by Bacillus subtilis, on model biomembranes. Biophys. J., 94, 7, 2667-2679.

Denyer, S.P. (1995). Mechanisms of action of antibacterial biocides. Int. Biodeterior. Biodegrad., 36, 227-245.

Deol, P. \& Khuller, G. K. (1997). Lung specific stealth liposomes: stability, biodistribution and toxicity of liposomal antitubercular drugs in mice.Biochim. Biophys. Acta, 1334, 2-3, 161-172.

Deresinski, S. (2009). Bacteriophage therapy: Exploiting smaller fleas. Clin. Infect. Dis., 48, 8, 1096-1101.

Devine, D. A. \& Hancock, R.E.W. (2002). Cationic peptides: Distribution and mechanisms of resistance. Curr.Pharm. Des., 8, 9, 703-714

Dibrov, P.; Dzioba, J.; Gosink, K. K. \& Hase, C. C. (2002). Chemiosmotic mechanism of antimicrobial activity of $\mathrm{Ag}(+)$ in Vibrio cholerae. Antimicrob.Agents Chemother., 46, 2668-2670.

Diefenbeck, M.; Muckley, T. \& Hofmann, G. O. (2006). Prophylaxis and treatment of implant-related infections by local application of antibiotics. Injury, 37(Suppl 2), S95-S104.

Dijk, F.; Westerhof, M.; Busscher, H. J.; van Luyn, M. J. \& Der Mei, H. C. (2000). In vitro formation of oropharyngeal biofilms on silicone rubber treated with a palladium/tin salt mixture. J. Biomed. Mater. Res., 51, 408-412.

DiRita, V.J.; Parsot, C.; Jander, G. \& Mekalanos, J.J. (1991). Regulatory cascade controls virulence in Vibrio cholerae. Proc. Nat. Acad. Sci. USA., 88, 5403-5407.

Domagk, G. (1935). Eine neue klasse von disinfektionsmitteln. Dtsch. Med. Wonchenschr., 61, 829-832.

Donelli, G. \& Francolini, I. (2001). Efficacy of antiadhesive, antibiotic and antiseptic coatings in preventing catheter-related infections: review. J. Chemother., 13, 595-606.

Donelli, G.; Francolini, I.; Piozzi, A.; Di Rosa, R. \& Marconi, W. (2002). New polymerantibiotic systems to inhibit bacterial biofilm formation: a suitable approach to prevent central venous catheter-associated infections. J. Chemother., 14, 5, 501-507.

Dragieva, I.; Stoeva, S.; Stoimenov, P.; Pavlikianov, E. \& Klabunde, K. (1999). Complex formation in solutions for chemical synthesis of nanoscaled particles prepared by borohydride reduction process. Nanostruct. Mater., 12, 267-270.

Drulis-Kawa, Z. \& Dorotkiewicz-Jach, A. (2010). Liposomes as delivery systems for antibiotics. Int. J. Pharm., 387, 1-2, 187-198. 
Drulis-Kawa, Z.; Gubernator, J.; Dorotkiewicz-Jach, A.; Doroszkiewicz, W. \& Kozubek, A. (2006). In vitro antimicrobial activity of liposomal meropenem against $P$. aeruginosa strains. Int. J. Pharm., 315, 1-2, 59- 66.

Dufour,A.; Hindré, T. ; Haras, D. \& Le Pennec, J.P. (2007). The biology of lantibiotics from the lacticin 481 group is coming of age. FEMS Microbiol. Rev., 31, 2, 134-167.

Duquesne, S.; Destoumieux-Garzón, D.; Peduzzi, J. \& Rebuffat, S. (2007). Microcins, geneencoded antibacterial peptides from enterobacteria. Nat. Prod. Report, 24, 4, 708-734.

Duran, L.W. (2000). Preventing medical device related infections. Med.Dev. Technol., 11, 6, 14-17.

Durán, N.; Marcato, P. D.; Alves, O. L.; Souza, G. I. H. \& Esposito, E. (2005). Mechanistic aspects of biosynthesis of silver nanoparticles by several Fusarium oxysporum strains. J. Nanobiotechnol., 3, 8.

Dutta, P.K.; Tripathi, S.; Mehotra, G. K. \& Dutta, J. (2009). Perspectives for chitosan based antimicrobial films in food applications. Food Chem., 114, 1173-1182.

Dvoracek, C. M.; Sukhonosova, G.; Benedik, M. J. \& Grunlan, J. C. (2009). Antimicrobial behavior of polyelectrolyte -surfactant thin film assemblies. Langmuir, 25, 17, 1032210328.

El-Ridy, M. S.; Mostafa, D. M.; Shehab, A.; Nasr, E. A. \& Abd El-Alim, S. (2007). Biological evaluation of pyrazinamide liposomes for treatment of Mycobacterium tuberculosis. Int. J. Pharm., 330, 1-2, 82-88.

El-Sayed, I. H.; Huang, X. \& El-Sayed, M. A. (2005). Surface plasmon resonance scattering and absorption of anti-EGFR antibody conjugated gold nanoparticles in cancer diagnostics: applications in oral cancer. Nano Lett., 5, 5, 829-834.

Endo, Y.; Tani, T. \& Kodama, M. (1987). Antimicrobial activity of tertiare amine covalently bonded to a polystyrene fiber. Appl. Environ. Microbiol., 53, 2050-2055.

Ercolini, D.; La Storia, A.; Villani, F. \& Mauriello, G. (2006). Effect of a bacteriocin-activated polythene film on Listeria monocytogenes as evaluated by viable staining and epifluorescence microscopy. J. Appl. Microbiol., 100, 4, 765-772.

Espuelas, M.S.; Legrand, P.; Campanero, M.A.; Appel, M.; Cheron, M.; Gamazo, C.; Barratt, G. \& Irache, J.M. (2003). Polymeric carriers for amphotericin B: in vitro activity, toxicity and therapeutic efficacy against systemic candidiasis in neutropenic mice. J. Antimicrob. Chemother. 52.; 419-427.

Espuelas, M. S.; Legrand, P.; Loiseau, P. M.; Bories, C.; Barratt, G. \& Irache, J. M. (2002). In vitro antileishmanial activity of amphotericin B loaded in poly (epsiloncaprolactone) nanospheres. J. Drug Target., 10, 593-599.

Falkinham, J.O.; Wall, T.E ; Tanner, J.R. ; Tawaha, K. ; Alali, F.Q ; Li, C. \& Oberlies, N.H. (2009). Proliferation of antibiotic-producing bacteria and concomitant antibiotic production as the basis for the antibiotic activity of Jordan's red soils. Appl. Environ. Microbiol., 75, 9, 2735-41.

Faustino-Vega, A.; Alvarez-Polo, M.A.; Gasca, B. \& Bernad-Bernad, M.J. (2009). Influence of three different colloidal systems on the oxytetracycline-lecithin behavior. Pharmazie, 64, 8, 505-509.

Fayaz, A.M.; Balaji, K.; Girilal, M.; Yadav, R.; Kalaichelvan, P.T. \& Venketesan, R. (2010). Biogenic synthesis of silver nanoparticles and their synergistic effect with antibiotics: a study against gram-positive and gram-negative bacteria. Nanomedicine, 6, 103-109. 
FDA approval of Listeria-specific bacteriophage preparation on ready-to-eat (RTE) meat and poultry products, http://www.cfsan.fda.gov/wdms/opabacqa.html; 2006.

Feng, K.; Sun, H.; Bradley, M. A.; Dupler, E. J.; Giannobile, W. V. \& Ma, P. X. (2010). Novel antibacterial nanofibrous PLLA scaffolds. J. Controlled Release, in press.

Fernández, A.; Cava, D.; Ocio, M.J. \& Lagarón, J.M. (2008). Perspectives for biocatalysts in food packaging. Trends Food Sci. Technol., 19, 198-206.

Fernández, A.; Soriano, E.; Hernández-Muñoz, P. \& Gavara, R. (2010). Migration of antimicrobial silver from composites of polylactide with silver zeolites. J. Food Sci., 75, 3, E186-E193.

Fernandez-Saiz, P.; Ocio, M.J. \& Lagaron, J.M. (2006). Film forming process and biocide assessment of high-molecular-weight chitosan as determined by combined ATRFTIR spectroscopy and antimicrobial assays. Biopolymers, 83, 6, 577-583.

Ferreira, C.; Rosmaninho, R.; Simões, M.; Pereira, M. C.; Bastos, M. M. S. M.; Nunes, O. C.; Coelho, M. \& Melo, L. F. (2010). Biofouling control using microparticles carrying a biocide. Biofouling, 26, 2, 205-212.

Fidai, S.; Farer, S. W. \& Hancock, R. E. W. (1997). Interaction of cationic peptides with bacterial membranes. Met. Mol. Biol., 78, 187-204.

Field, D.; Connor, P. M. O.; Cotter, P. D.; Hill, C. \& Ross, R. P. (2008). The generation of nisin variants with enhanced activity against specific Gram positive pathogens. Mol. Microbiol., 69, 1, 218-230.

Fielding, R.M. (1991). Liposomal drug delivery: advantages and limitations from a clinical pharmacokinetics and therapeutic perspective. Clin. Pharmacol., 21, 3, 155-164.

Fimland, G.; Johnsen, L.; Dalhus. \& Jonnissen-Meyer, J. (2005). Pediocin-like antimicrobial peptides (class IIa bacteriocins) and their immunity proteins: biosynthesis, structure, and mode of action. J. Pept. Sci., 11, 11, 688- 696.

Florence, A.T. (2005). Dendrimers: a versatile targeting platform. Adv. Drug Deliv. Rev., 57, 2104-2105.

Francolini, I. \& Donelli, G. (2010). Prevention and control of biofilm-based medical-devicerelated infections. FEMS Immmunol. Med. Microbiol., 59, 227-238.

Francolini, I.; Norris, P.; Piozzi, A.; Donelli, G. \& Stoodley, P. (2004). Usnic acid, a natural antimicrobial agent able to inhibit bacterial biofilm formation on polymer surfaces. Antimicrob. Agents Chemother., 48, 4360-4365.

Frank, A.; Rath, S. K. \& Venkatraman, S. S. (2005). Controlled release from bioerodible polymers: effect of drug type and polymer composition. J. Controlled Release, 102, 333-344.

Friedrich, C. L.; Moyles, D.; Beverige, T. J. \& Hancock, R. E. (2000). Antibacterial action of structurally diverse cationic peptides on gram-positive bacteria. Antimicrob. Agents Chemother., 44, 2086-2092.

Frier, M. (1971). Derivatives of 4-amino-quinaldinium and 8-hydroxyquinoline. In: Hugo WB, ed., Inhibition and destruction of the microbial cell (Academic Press, London) pp. 107-120.

Fu, G.; Vary, P. \& Lin, C.T. (2005). Anatase TiO2 nanocomposites for antimicrobial coatings. J. Phys. Chem. B, 109, 18, 8889-8898.

Fu, J.K.; Liu, Y.; Gu, P.; Tang, D.L.; Lin, Z.Y.; Yao, B.X. \& Weng, S.Z. (2000). Spectroscopic characterization on the biosorption and bioreduction of $\mathrm{Ag}(\mathrm{I})$ by Lactobacillus $s p$. A09. Acta Phys.Chim. Sin., 16, 9, 770-782. 
Fu, J.K.; Zhang, W.D.; Liu, Y.Y.; Lin, Z.Y.; Yao, B.X. \& Weng, S.Z. (1999). Characterization of adsorption and reduction of noble metal ions by bacteria. Chem. J.Chin.Univ., 20, 9, 1454.

Fu, M.; Li, Q.; Sun, D.; Lu, Y.; He, N.; Deng, X.; Wang, H. \& Huang, J. (2006). Rapid preparation process of silver nanoparticles by bioreduction and their characterizations. Chin. J. Chem. Eng., 14, 1, 114-117.

Fu, W.; Forster, T.; Mayer, O.; Curtin, J.J.; Lehman, S.M. \& Donlan, R.M. (2010). Bacteriophage cocktail for the prevention of biofilm formation by Pseudomonas aeruginosa on catheters in an in vitro model system. Antimicrob. Agents Chemother., $54,397-404$.

Fuhrhop, A. H. \& Wang, T.Y. (2004). Bola amphiphiles. Chem. Rev., 104, 6, 2901-2937.

Fuhrhop, J -H. \& Fritsch, D. (1986). Bolaamphiphiles form ultrathin, porous and unsymmetric monolayer lipid membranes. Acc. Chem. Res., 19, 130-137.

Fundaro, A. ; Cavalli, R. ; Bargoni, A. ; Vighetto, D. ; Zara, G. P. \& Gasco, M. R. (2000). Nonstealth and stealth solid lipid nanoparticles (SLN) carrying doxorubicin: pharmacokinetics and tissue distribution after iv administration to rats. Pharmacol. Res., 42, 4, 337-343.

Furneri, P.M. (2000). Ofloxacin loaded liposomes: in vitro acivity and drug accumulation in bacteria. Antimicrob. Agents Chemother., 44, 2458-2464.

Furno, F.; Morley, K.S.; Wong, B.; Sharp, B.L.; Arnold, P.L.; Howdle, H.J.; Bayston, R.; Brown, P.D.; Winship, P.D. \& Reid, H.J. (2004). Silver nanoparticles and polymeric medical devices: a new approach to prevention of infection? J. Antimicrob. Chemother., 54, 1019-1024.

Gajbhiye, V.; Palanirajan, V.K.; Tekade, R.K. \& Jain, N.K. (2009). Dendrimers as therapeutic agents: a systematic review. J. Pharm. Pharmacol., 61, 8, 989-1003.

Gardiner, G.E.; Rea, M.C.; O’Riordan, B.; O'Connor, P.; Morgan, S.M.; Lawlor, P.G.; Lynch, P.B.; Cronin, M.; Ross, R.P. \& Hill, C. (2007). Fate of the two-component lantibiotic lacticin 3147 in the gastrointestinal tract. Appl. Environ. Microbiol., 73, 21, 7103-7109.

Gaspar, M.M.; Cruz, A.; Penha, A.F.; Reymão, J.; Sousa, A.C.; Eleutério, C.V.; Domingues, S.A.; Fraga, A.G.; Longatto Filho, A. Cruz, M.E.M. \& Pedrosa, J. (2008). Rifabutin encapsulated in liposomes exhibits increased therapeutic activity in a model of disseminated tuberculosis. Int. J. Antimicrob. Chemoter., 31, 1, 37-45.

Gericke, M. \& Pinches, A. (2006). Biological synthesis of metal nanoparticles. Hydrometallurgy, 83, 132-140. a

Gericke, M. \& Pinches, A. (2006). Microbial production of gold nanoparticles. Gold Bull., 39, $1,22-28 . \mathrm{b}$

Ghalfi, H.; Allaoui, A.; Destain, J.; Benkerroum, N. \& Thonart, P. (2006). Bacteriocin activity by Lactobacillus curvatus CWBI-B28 to inactivate Listeria monocytogenes in coldsmoked salmon during $4^{\circ} \mathrm{C}$ storage. J. Food Prot., 69, 5, 1066-1071.

Ghosh, S.; Yadav, S.; Vasanthan, N. \& Sekosan, G. (2010). A study of antimicrobial property of textile fabric treated with modified dendrimers. J. Appl. Polym. Sci., 115, 2, 716722.

Giacometti, A.; Cirioni, O.; Kamysz, W.; D’Amato. G.; Silvestri, C.; Del Prete, M.S.; Łukasiak, J. \& Scalise, G. (2003). Comparative activities of cecropin A, melittin, and cecropin A-melittin peptide $\mathrm{CA}(1-7) \mathrm{M}(2-9) \mathrm{NH} 2$ against multidrug-resistant nosocomial isolates of Acinetobacter baumannii. Peptides, 24, 9, 1315-1318. 
Gibbons, S. (2005). Plants as a source of bacterial resistance modulators and anti-infective agents. Phytochem. Rev., 4, 1, 63-78.

Gilbert, P. \& Moore, L.E. (2005). Cationic antiseptics: diversity of action under a commom epithet. J. Appl. Microbiol., 99, 4, 703-715.

Gill, A.O. \& Holley, R.A. (2000). Surface application of lysozyme, nisin, and EDTA to inhibit spoilage and pathogenic bacteria on ham and bologna. J. Food Prot., 63, 10, 13381346.

Gollwitzer, H.; Ibrahim, K.; Meyer, H.; Mittelmeier, W.; Busch, R. \& Stemberger, A. (2003). Antibacterial poly(D,L-lactic acid) coating of medical implants using a biodegradable drug delivery technology. J. Antimicrob. Chemother., 51, 585-591.

Gordon, Y.J.; Romanoviski, E.G. \& MacDermott, A.M. (2005). A review of antimicrobial peptides and their therapeutic potential as anti-infective drugs. Curr. Eye Res., 30, 505-515.

Gorensek, M.; Gorjanc, M.; Bukosek, V.; Kovac, J.; Jovancic, P. \& Mihailovic, D. (2010). Functionalization of PET fabrics by corona and nano silver. Text. Res. J., 80, 3, 253262.

Gottenbos, B.; van. der Mei, H.C.; Klatter, F.; Nieuwenhuis, P. \& Busscher, H.J. (2002). In vitro and in vivo antimicrobial activity of covalently coupled quaternary ammonium silane coatings on silicone rubber. Biomaterials, 23, 6, 1417-1423.

Grace, A.N. \& Pandian, K. (2007). Antibacterial efficacy of aminoglycosidic antibiotics protected gold nanoparticles: a brief study. Colloids Surf. A, 297, 63-70.

Grayson, S.M. \& Frechet, J.M. (2001). Convergent dendrons and dendrimers: from synthesis to applications. Chem. Rev., 101, 3819-3868.

Greco, R.S.; Harvey, R.A.; Smilow, P.C. \& Tesoriero, J.V. (1982). Prevention of vascular prosthetic infection by a benzalkonium-oxacillin bonded polytetrafluoroethylene graft. Surg.Gynecol.Obstet., 155, 1, 28-32.

Gregoriadis, G. (1995). Engineering for drug delivery: progress and problems. Trends Biotechnol., 13, 12, 527-537.

Gregoriadis G (1976). The carrier potential of liposomes in biology and medicine (first of two parts). N. Engl. J. Med., 295, 13, 704-710.A

Gregoriadis G (1976). The carrier potential of liposomes in biology and medicine (second of two parts). N. Engl. J. Med., 295, 14, 765-770.B

Guani-Guerra, E.; Santos-Mendoza, T.; Lugo-Reyes, S. O. \& Teran, L. M. (2010). Antimicrobial peptides: General overview and clinical implications in human health and disease. Clin. Immunol., 135, 1, 1-11.

Gubernator, J.; Drulis-Kawa, Z.; Dorotkiewicz-Jach, A.; Dorotkiewicz, W.; \& Kozubek, A. (2007). In vitro antimicrobial activity of liposomes containing ciprofloxacin, meropenem and gentamicin against Gram-negative clinical bacterial strains. Lett. Drug Des. Discov., 4, 4, 297-304.

Gupta, A.K. \& Cooper, E.A. (2008). Update in antifungal therapy of dermatophytosis. Mycopathologia, 166, 353-367.

Gurunathan, S.; Kalishwaralal, K.; Vaidyanathan, R.; Deepak, V.; Pandian, S.R.K.; Muniyandi, J.; Hariharan, N. \& Eom, S.H. (2009). Biosynthesis, purification and characterization of silver nanoparticles using Escherichia coli. Colloids Surf. B, 74, 328-335. 
Gutiérrez, L.; Batlle, R.; Sánchez, C. \& Nerín, C. (2010). New approach to study the mechanism of antimicrobial protection of an active packaging. Food. Path. Dis., 7, 9, 1-8.

Guttman, B.; Raya, R. \& Kutter, E. (2004). Basic phage biology. In: Bacteriophages biology and applications, Kutter, E. \& Sulakvelidze, A.; 29-66, eds. CRC Press, New York.

Hajji, M.; Jellouli, K.; Hmidet, N.; Balti, R.; Sellami-Kamoun, A. \& Nasri, M. (2010). A highly thermostable antimicrobial peptide from Aspergillus clavatus ES1: biochemical and molecular characterization. J. Ind. Microbiol. Biothechnol., 37, 8, 805-813.

Haldar, J.; Kondaiah, P. \& Bhattacharya, S. (2005). Synthesis and antibacterial property of novel hydrolyzable cationic amphiphiles. Incorporation of multiple head groups leads to impressive antibacterial activity. J. Med.Chem., 48, 11, 3823-3831.

Hale, J.D.F. \& Hancock, R.E.W. (2007). Alternative mechanisms of action of cationic antimicrobial peptides on bacteria. Exp. Rev. Anti-Infectiv. Ther., 5, 6, 951-959.

Hamouda, T., Hayes, M.; Cao, Z.; Tonda, R.; Johnson, K.; Craig, W.; Brisker, J. \& Baker, J. (1999). A novel surfactant nanoemulsion with broad-spectrum sporicidal activity against Bacillus species. J. Infect. Dis., 180, 1939-1949.

Han, C.; Qi, C.M.; Zhao, B.K.; Cao, J.; Xie, S.Y.; Wang, S.L. \& Zhou, W.Z. (2009). Hydrogenated castor oil nanoparticles as carriers for the subcutaneous administration of tilmicosin: in vitro and in vivo studies. J. Vet. Pharmacol. Therap., $32,2,116-123$.

Hancock, R.E. \& Sahl, H.G. (2006). Antimicrobial and host-defense peptides as new antiinfective therapeutic strategies. Nat. Biotechnol., 24, 12, 1551-1557.

Hancock, R.E.W \& Chapple, D.S. (1999). Peptide Antibiotics. Antimicrob.Agents Chemother., 43, 6, 1317-1323.Hancock, R. E. W.; Falla, T.; \& Brown, M. H. (1995). Cationic bactericidal peptides. Adv. Microb. Physiol., 37, 135-175.

Hancock, R.E.W. \& Lehrer, R. (1998). Cationic peptides: a new source of antibiotics. Trends in Biotechnol., 16, 2, 82-88.

Hancock, R.E.W. (1998). The therapeutic potential of cationic peptides. Exp. Opin. Investig. Drugs, 7, 2, 167-174.

Hanlon, G.W. (2007). Bacteriophages: an appraisal of their role in the treatment of bacterial infections. Int.J. Antimicrob. Agents, 30, 118-128.

Harvey, R.A. \& Greco, R.S. (1981). The noncovalent bonding of antibiotics to a polytetrafluoroethylene-benzalkonium graft. Annals of Surgery, 194, 5, 642-647.

Harvey, R.A.; Alcid, D.V. \& Greco, R.S. (1982). Antibiotic bonding to polytetrafluoroethylene with tridodecylmethylammonium chloride. Surgery, 92, 504-512.

Haydel, S.E.; Remenih, C.M. \& Williams, L.B. (2008). Broad-spectrum in vitro antibacterial activities of clay minerals against antibiotic-susceptible and antibiotic-resistant bacterial pathogens. J. Antimicrob. Chemother., 61, 2, 353-61.

Heard, S.O.; Wagle, M.; Vijayakumar, E.; McClean, S.; Brueggemann, A.; Napolitano, L.M.; Edwards, L.P.; O'Connell, F.M.; Puyana, J.C. \& Doern, G.V. (1998). Influence of triple-lumen central venous catheters coated with chlorhexidine and silver sulfadiazine on the incidence of catheter-related bacteremia. Arch. Int.Med., 158, 1, 81-87. 
Hecker, M.T.; Aron, D.C.; Patel, N.P.; Lehmann, M.K. \& Donskey, C.J. (2003). Unnecessary use of antimicrobials in hospitalized patients: current patterns of misuse with an emphasis on the antianaerobic spectrum of activity. Arch. Int.Med., 163, 8, 972-978.

Heijink, A.; Yaszemski, M.J.; Patel, R.; Rouse, M.S.; Lewallen, D.G. \& Hanssen, A.D. (2006). Local antibiotic delivery with OsteoSet, DBX, and Collagraft. Clin. Orthop. Rel. Res., 451, 29-33.

Helander, I.M.; Nurmiaho-Lassila, E.-L.; Ahvenainen, R.; Rhoades, J. \& Roller. S. (2001). Chitosan disrupts the barrier properties of the outer membrane of Gram-negative bacteria. Int. J. Food Microbiol., 71, 235-244.

Hirota, K.; Murakami, K.; Nemoto, K. \& Miyake, Y. (2005). Coating of a surface with 2methacryloyloxyethyl phosphorylcholine (MPC) co-polymer significantly reduces retention of human pathogenic microorganisms. FEMS Microbiol. Lett., 248, 1, 37-45.

Hoiby, N.; Bjarnsholt, T.; Givskov, M.; Molin, S. \& Ciofu, O. (2010). Antibiotic resistance of bacterial biofilms. Int. J.Antimicrob. Agents, 35, 4, 322-332.

Hosny, K.M. (2010). Ciprofloxacin as ocular liposomal hydrogel. Am. Assoc. Pharm. Scient., $11,1,241-246$.

Hou, S.; Burton, E.A.; Simon, K.A.; Blodgett, D.; Luk, Y.Y. \& Ren, D. (2007). Inhibition of Escherichia coli biofilm formation by self-assembled monolayers of functional alkanethiols on gold. Appl. Environ. Microbiol., 73, 13, 4300-4307.

Hou, S.; Zhou, C.; Liu, Z.; Young, A.W.; Shi, Z.; Ren, D. \& Kallenbach, N.R. (2009). Antimicrobial dendrimer active against Escherichia coli biofilms. Bioorg. Med. Chem. Lett., 19, 18, 5478-5481.

Hsu, S.T.; Breukink, E.; Tischenko, E.; Lutters, M.A.; Kruijff, B.; Kaptein, R.; Bonvin, A.M. \& van Nuland, N.A. (2004). The nisin-lipid II complex reveals a pyrophosphate cage that providesa blueprint for novel antibiotics. Nat. Struct. Mol. Biol., 11, 963-967.

Hu, J.; Liu, H. \& Wang, L. (2000). Enhanced delivery of AZT to macrophages via acetylated LDL. J. Controlled Release, 69, 3, 327-335.

Hugo, W.B. \& Frier, M. (1969). Mode of action of the antibacterial compound dequalinium acetate. Appl. Microbiol., 17, 1, 118-127.

Hutmacher, D.; Hurzeler, M.B.; Schliephake, H. (1996). A review of material properties of biodegradable and bioresorbable polymers and devices for GTR and GBR applications. Int.J. Oral Maxillofacial Impl., 11, 5, 667-678.

Igartua, M. ; Saulnier, P. ; Heurtault, B. ; Pech, B. ; Proust, J.E. ; Pedraz, J.L. \& Benoit, J.P. (2002). Development and characterization of solid lipid nanoparticles loaded with magnetite. Int.J. Pharm., 233, 1-2, 149-157.

Ireland, D.C.; Wang, C.K.L.; Wilson, J.A.; Gustafson, K.R. \& Craik, D.J. (2008). Cyclotides as natural anti-HIV agents. Biopolymer. Pept. Sci. Sect., 90, 1, 51-60.

Isquith, A.J.; Abbott, E.A. \& Walters, P.A. (1972). Surface-bonded antimicrobial activity of an organosilicon quaternary ammonium chloride. Appl. Microbiol., 24, 859-863.

Israelachvili, J. N.; Mitchell, D. J. \& Ninham, B.W. (1977). Theory of self-assembly of lipids bilayers and vesicles. Biochim. Biophys. Acta, 470, 2, 185-201.

Jain, D. \& Banerjee, R. (2008). Comparison of ciprofloxacin hydrochloride-loaded protein, lipid, and chitosan nanoparticles for drug delivery. J. Biomed. Mater. Res., 86B, 1, $105-112$. 
Jampala, S.N.; Sarmadi, M.; Somers, E.B.; Wong, A.C.L. \& Denes, F.S. (2008). Plasmaenhanced synthesis of bactericidal quaternary ammonium thin layers on stainless steel and cellulose surfaces. Langmuir, 24, 16, 8583-8591.

Janiszewska, J. \& Urbanczyk-Lipkowska, Z. (2007). Amphiphilic dendrimeric peptides as model non-sequential pharmacophores with antimicrobial properties. J.Mol. Microbiol.Biotechnol., 13, 4, 220-225.

Jansen, B.; Jansen, S.; Peters, G. \& Pulverer, G. (1992). In vitro efficacy of a central venous catheter ('Hydrocath') loaded with teicoplanin to prevent bacterial colonization. J. Hosp.Infect., 22, 2, 93-107.

Jausovec, D.; Angelescu, D.; Voncina, B.; Nylander, T. \& Lindman, B. (2008). The antimicrobial reagent role on the degradation of model cellulose film. J. Colloid Interface Sci., 327, 1, 75-83.

Jayakumar, R., Nwe, N.T., Tokura, S. \& Tamura, H. (2007). Sulfated chitin and chitosan as novel biomaterials. Int. J. Biol. Macromol., 40, 175-181.

Je, J.-Y. \& Kim, S.-K. (2006). Chitosan derivatives killed bacteria by disrupting the outer and inner membrane. J. Agricult. Food Chem., 54, 6629-6633.

Jeon, Y.J.; Park, P.J. \& Kim, S.K. (2001). Antimicrobial effect of chitooligosaccharides produced by bioreactor. Carbohydr. Polym., 44, 71-76.

Jeong, S.H.; Hwang, Y.H. \& Yi, S.C. (2005). Antibacterial properties of padded PP/PE nonwovens incorporating nano-sized silver colloids. J. Mater. Sci., 40, 5413-5418. b

Jeong, S.H.; Yeo, S.Y. \& Yi, S.C. (2005). The effect of filler particle size on the antibacterial properties of compounded polymer/silver fibers. J. Mater. Sci., 40, 5407-5411. a

Jia., Y.; Joli, H. \& Omri, A. (2008). Liposomes as a carrier for gentamicin delivery: development and evaluation of the physicochemical properties. Int. J. Pharm., 359, $1-2,254-263$.

Jin, T. \& Zhang, N. (2008). Biodegradable polylactid acid polymer with nisin for use in antimicrobial food packaging. J. Food Sci., 73, 3, M127-M134.

Joerger, R.D. (2007). Antimicrobial films for food applications: a quantitative analysis of their effectiveness. Pack. Technol.Sci., 20, 231-273.

Johansson, E.M.V.; Crusz, S.A.; Kolomiets, E.; Buts, L.; Kadam, R.U.; Cacciarini, M.; Bartels, K.; DiBiggle, S.P.; Camara, M.; Williams, P.; Loris, R.; Nativi, C.; Rosenau, F.; Jaeger, K.; Darbre, T. \& Reymond, J. (2008). Inhibition and dispersion of Pseudomonas aeruginosa biofilms by glycopeptide dendrimers targeting the fucose-specific lectin LecB. Chem. Biol., 15, 12, 1249-1257.

Jolles P, Jolles J (1984) What's new in lysozyme research? Always a model system, today as yesterday. Mol. Cell. Biochem, 63, 165-189.

Jones, M.N. (2005). Use of liposomes to deliver bactericides to bacterial biofilms. Methods Enzymol., 391, 211-228.

Kader, A.; Davis, P.J.; Kara, M. \& Liu, H. (1998). Drug targeting using low density lipoprotein (LDL): physicochemical factors affecting drug loading into LDL particles. J.Controlled Release, 55, 2-3, 231-243.

Kadry, A.A.; Al-Suwayeh, S.A.; Abd-Allah, A.R.A. \& Bayomi, A.M. (2004). Treatment of experimental osteomyelitis by liposomal antibiotics. J.Antimicrob.Chemother., 54, 6, 1103-1108. 
Kalishwaralal, K.; Deepak, V.; Pandian, S.R.K.; Kottaisamy, M.; BarathManiKanth, S.; Kartikeyan, B. \& Gurunathan, S. (2010). Biosynthesis of silver and gold nanoparticles using Brevibacterium casei. Colloids Surf., B., 77, 257-262.

Kamisoglu, K.; Aksov, E.A.; Akata, B.; Hasirci, N. \& Bac, N. (2008). Preparation and characterizaton of antibacterial zeolite-polyurethane composites. J. Appl. Polymer Sci., 110, 2854-2861.

Kang, J.W.; Davaa, E.; Kim, Y-T. \& Park, J-S. (2010). A new vaginal delivery system of amphotericin B: a dispersion of cationic liposomes in a thermosensitive gel. J. Drug Targ., 18, 8, 637-644.

Katz, E. \& Demain, A.L. (1977). The peptide antibiotics of Bacillus: chemistry, biogenesis, and possible functions. Bacteriol. Rev., 41, 2, 449-474.

Kawabata, N. \& Nishiguchi, M. (1988). Antibacterial activity of soluble pyridinium-type polymers. Appl. Environ. Microbiol., 54, 10, 2532-2535.

Kenawy, E. \& Mahmoud, Y.G. (2003). Biologically active polymers, 6a: Synthesis and antimicrobial activity of some linear copolymers with quaternary ammonium and phosphonium groups. Macromol. Biosci., 3, 2, 107-116.

Kenawy, E.R.; Worley, S.D. \& Broughton, R. (2007). A new degradable hydroxamate linkage for $\mathrm{pH}$-controlled drug delivery. Biomacromolecules, 8, 1, 1359-1384.

Khanna, P.K. \& Subbarao, V.V.V.S. (2003). Nanosized silver powder via reduction of silver nitrate by sodium formaldehydesulfoxylate in acidic $\mathrm{pH}$ medium. Mater. Lett., 57, 2242-2245.

Kharlampieva, E.; Kozlovskaya, V. \& Sukhishvili, S.A. (2009). Layer-by-layer hydrogenbonded polymer films: from fundamentals to applications. Adv. Mater., 21, 30, 3053-3065.

Khor, E. \& Lim, L.Y. (2003). Implantable applications of chitin and chitosan. Biomaterials, 24, 2339-2349.

Kikuchi, I.S. \& Carmona-Ribeiro, A.M. (2000). Interactions between cationic vesicles and DNA. J. Phys. Chem. B., 104, 13, 2829-2835.

Kim, J.E.; Kim, S.R.; Lee, S.H.; Lee, C.H. \& Kim, D.D. (2000). The effect of pore formers on the controlled release of cefadroxil from a polyurethane matrix. Int. J. Pharm., 201, 1, 29-36.

Kim, J.S.; Kuk, E.; Yu, K.N.; Kim, J.; Park, S.J.; Lee, H.J.; Kim, S.H.; Park, Y.K.; Park, Y.H.; Hwang, C.; Kim, Y.; Lee, Y.; Jeong, D.H. \& Cho, M. (2007). Antimicrobial effects of silver nanoparticles. Nanomedicine, 3, 95-101.

Kim, K.M.; Lee, B.-Y.; Kim, Y.T.; Choi, S.-G.; Lee, J.; Cho, S.Y. \& Choi, W.-S. (2006). Development of antimicrobial edible film incorporated with green tea extract. Food Sci. Biotechnol., 15, 478-481.

Kim, Y.-M.; An, D.-S.; Park, H.-J.; Park, J.-M. \& Lee, D.S. (2002). Properties of nisinincorporated polymer coatings as antimicrobial packaging materials. Pack. Technol. Sci., 15, 247-254.

Kittler, S.; Greulich, C.; Köller, M. \& Epple, M. (2009). Synthesis of PVP-coated silver nanoparticles and their biological activity towards human mesenchymal stem cells. Material wissenschaft und Werkstofftechnik, 40, 4, 258-264.

Klaykruayat, B.; Siralertmukul, K. \& Srikulkit, K. (2010). Chemical modification of chitosan with cationic hyperbranched dendritic polyamidoamine and its antimicrobial activity on cotton fabric. Carbohydr. Polym., 80, 1, 197-207. 
Kleinkauf, H.; \& H. von Dohren, H. (1988). Peptide antibiotics, $\beta$-lactams and related compounds. Crit.Rev. Biotechnol., 8, 1, 1-32.

Klibanov, A.M. (2007). Permanently microbicidal materials coatings. J. Mater. Chem., 17, 24, 2479-2482.

Kondejewski, L.H.; Farmer, S.W.; Wishart, D.S.; Hancock, R.E.W. \& Hodges, R. S. (1996). Gramicidin $S$ is active against both gram-positive and gram-negative bacteria. Int. J. Pept. Prot. Res., 47, 6, 460-465.

Kopecek, J. (1977). Soluble biomedical polymers. Polimery w Medycynie, 7, 3, 191-221.

Krishnaraj, C.; Jagan, E.G.; Rajasekar, S.; Selvakumar, P.; Kalaichelvan, P.T. \& Mohan, N. (2010). Synthesis of silver nanoparticles using Acalypha indica leaf extracts and its antibacterial activity against water borne pathogens. Colloids Surf. B., 76, 50-56.

Kshirsagar, N. A.; Pandya, S. K.; Kirodian, B. G. \& Sanath, S. (2005). Liposomal drug delivery systems from laboratory to clinic. J. Postgrad. Med., 51, 5-15.

Ku, K. \& Song, K.B. (2007). Physical properties of nisin-incorporated gelatin and corn zein films and antimicrobial activity against Listeria monocytogenes. J. Microbiol.Biotechnol., 17, 3, 520-523.

Kuegler, R.; Bouloussa, O. \& Rondelez, F. (2005). Evidence of a charge-density threshold for optimum efficiency of biocidal cationic surfaces. Microbiology, 151, 1341-1348.

Kunitake, T.; Okahata, Y.; Tamaki, K.; Kumamaru, F. \& Takayanagi, M. (1977). Formation of the bilayer membrane from a series of quaternary ammonium salts. Chem.Lett., 6, 387-390.

Labana, S.; Pandey, R.; Sharma, S. \& Khuller, G.K. (2002). Chemoterapheutic activity against murine tuberculosis of once weekly administred drugs (isoniazida and rifampicin) encapsulated in liposomes. Int. J. Antimicrob. Agents, 20, 301-304.

Lansdown, A.B.G. (2006). Silver in healthcare: an enigma and pathological fascination. Bull. Royal Col. Pathol., 133, 36-38.

Le Rey, A.M.; Chiffoleau, S.; Iooss, P.; Grimandi, G.; Gouyette, A.; Daculsi, A. \& Merle, C. (2003). Vancomycin encapsulation in biodegradable poly(e-caprolactone) microparticles for bone implantation. Influence of the formulation process on size, drug loading, in vitro release and cytocompatibility. Biomaterials, 24, 443-449.

Lee, D.G.; Shin, S.Y.; Maeng, C-Y.; Jin, Z.Z.; Kim, K.L. \& Hahm K-S. (1999). Isolation and characterization of a novel antifungal peptide from Aspergillus niger. Biochem. Biophys. Res. Commun., 263, 3, 646-651.

Lee, C.H.; An, D.S.; Park, H.J. \& Lee, D.S. (2003). Wide-spectrum antimicrobial packaging materials incorporating nisin and chitosan in the coating. Pack. Technol Sci., 16, 3, 99-106.

Lehrer, R.I. (2007). Multispecific myeloid defensins. Curr. Opinion Hematol., 14, 1, 16-21.

Lejeune, P. (2003). Contamination of abiotic surfaces: what a colonizing bacterium sees and how to blur it. Trends Microbiol., 11, 4, 179-184.

Lenoir, S.; Pagnoulle, C.; Detrembleur, C.; Galleni, M. \& Jerome, R. (2005). Antimicrobial activity of polystyrene particles coated by photo-crosslinked block copolymers containing a biocidal polymethacrylate block. e-Polymers.

Leopold, N. \& Lendl, B. (2003). A new method for fast preparation of highly surfaceenhanced Raman scattering (SERS) active silver colloids at room temperature by reduction of silver nitrate with hydroxyalmine hydrochloride. J. Phys. Chem. B, 107, 5723-5727. 
Li, Q.; Chen, S.L. \& Jiang, W.C. (2007). Durability of nano ZnO antibacterial cotton fabric to sweat. J. Appl. Polym. Sci., 103, 412-416.

Li, W.; Xie, X.; Shi, Q.; Zeng, H.; OU-Yang, Y. \& Chen, Y. (2010). Antibacterial activity and mechanism of silver nanoparticles on Escherichia coli. Appl. Microbiol. Biotechnol., 85, 1115-1122.

Lincopan, N. \& Carmona-Ribeiro, A.M. (2009). Protein Assembly onto Cationic Supported Bilayers. J. Nanosci. Nanotechnol., 9, 6, 3578-3586.

Lincopan, N.; Espíndola, N.M.; Vaz, A.J.; Costa, M.H.B.; Faquim-Mauro, E.; CarmonaRibeiro, A.M. (2009). Novel immunoadjuvants based on cationic lipid: Preparation, characterization and activity in vivo. Vaccine, 5760-5771.

Lincopan, N. \& Carmona-Ribeiro, A.M. (2006). Lipid-covered drug particles: combined action of dioctadecyldimethylammonium bromide and amphotericin B or miconazole. J. Antimicrob. Chemother., 58, 1, 66-75.

Lincopan, N.; Borelli, P.; Fock, R.; Mamizuka, E.M.; Carmona-Ribeiro, A.M. (2006). Toxicity of an effective amphotericin B formulation at high cationic lipid to drug molar ratio. Exp. Toxicol. Pathol., 58, 2-3, 175-183.

Lincopan, N.; Mamizuka, E.M. \& Carmona-Ribeiro, A.M. (2005). Low nephrotoxicity of an effective amphotericin B formulation with cationic bilayer fragments. J. Antimicrob. Chemother., 55, 5, 727-734.

Lincopan, N.; Mamizuka, E.M. \& Carmona-Ribeiro, A.M. (2003). In vivo activity of a novel amphotericin B formulation with synthetic cationic bilayer fragments. J. Antimicrob. Chemother., 52, 3, 412-418.

Loher, S.; Schneider, O.D.; Maienfisch, T.; Bokorny, S. \& Stark, W.J. (2008). Micro-organismtriggered release of silver nanoparticles from biodegradable oxide carriers allows preparation of self-sterilizing polymer surfaces. Small, 4, 6, 824-832.

Lok, C. (2006). Proteomic analysis of the mode of antibacterial action of silver nanoparticles. J. Proteome Res., 5, 916-924.

López, P.; Sanchez, C.; Battle, R. \& Nerín, C. (2007). Development of flexible antimicrobial films using essential oils as active agents. J. Agricultur.Food Chem., 55, 8814-8824.

Lopez-Berestein, G.L. (1987). Liposomes as carriers of antimicrobial agents. Antimicrob. Agents Chemother., 31, 5, 675- 678.

Lutwyche, P.; Cordeiro, C.; Wiseman, D.J.; St-Louis, M.; Uh, M.; Hope, M.J.; Webb, M. S. \& Finlay, B. (1998). Intracellular delivery and antibacterial activity of gentamicin encapsulated in pH-sensitive liposomes. Antimicrob. Agents Chemother., 42, 10, 25112520.

Mader, J.T.; Calhoun, J. \& Cobos, J. (1997). In vitro evaluation of antibiotic diffusion from antibiotic-impregnated biodegradable beads and polymethylmetacrylate beads. Antimicrob. Agents Chemother., 41, 415-418.

Mader, J.T.; Stevens, C.M.; Stevens, J.H.; Roble, R.; Lathrop, J.T. \& Calhoun, J.H. (2002). Treatment of experimental osteomyelitis with a fibrin sealant antibiotic implant. Clin. Orthop. Rel. Res., 403, 58-72.

Maget-Dana, R. \& Peypoux, F. (1994). Iturins, a special class of pore forming lipopeptides: biological and physicochemical properties. Toxicology, 87, 1-3, 151-174.

Mah, T.F.C. \& O’Toole, G.A. (2001). Mechanisms of biofilm resistance to antimicrobial agents. Trends Microbiol., 9, 34-39. 
Maizura, M.; Fazilah, A.; Norziah, M.H. \& Karim, A.A. (2007). Antibacterial activity and mechanical properties of partially hydrolyzed sago starch-alginate edible film containing lemongrass oil. J. Food Sci., 72, 6, C324-C330.

Mäkinen, T.J.; Veiranto, M.; Lankinen, P.; Moritz, N.; Jalava, J.; Törmälä, P. \& Aro, H.T. (2005). In vitro and in vivo release of ciprofloxacin from osteoconductive bone defect filler. J. Antimicrob. Chemother., 56, 1063-1068.

Marcos, J.F.; Muñoz, A.; Pérez-Payá, E.; Misra, S. \& López-García, B. (2008). Identification and rational design of novel antimicrobial peptides for plant protection. An. Rev. Phytopathol., 46, 273-301.

Martins, L. M. S.; Mamizuka, E.M. \& Carmona-Ribeiro, A.M. (1997). Cationic Vesicles as Bactericides. Langmuir, 13, 21, 5583-5587.

Martins, S. ; Sarmento, B. ; Ferreira, D.C. \& Souto, E.B. (2007). Lipid-based colloidal carriers for peptide and protein delivery-liposomes versus lipid nanoparticles. Int. J. Nanomedicine, 2, 4, 595-607.

Matl, F.D.; Obermeier, A.; Repmann, S.; Friess, W.; Stemberger, A. \& Kuehn, K.D. (2008). New anti-infective coatings of medical implants. Antimicrob. Agents Chemother., 52, 6, 1957-1963.

Mauriello, G.; De Luca, E.; La Storia, A.; Villani, F. \& Ercolini, D. (2005). Antimicrobial activity of a nisin-activated plastic film for food packaging. Letters Appl. Microbiol., 41, 6, 464-469.

Mauriello, G.; Ercolini, D.; La Storia, A.; Casaburi, A. \& Villani, F. (2004). Development of polythene films for food packaging activated with an antilisterial bacteriocin from Lactobacillus curvatus 32Y. J. Appl. Microbiol., 97, 2, 314-322.

McAuliffe, O.; Ross, R.P. \& Hill, C. (2001). Lantibiotics: structure, biosynthesis and mode of action. FEMS Microbiol. Rev., 25, 3, 285-308.

McLaren, A.C. (2004). Alternative materials to acrylic bone cement for delivery of depot antibiotics in orthopaedic infections. Clin. Orthop. Rel. Res., 427, 101-106.

Mclean, R.J.; Hussain, A.A.; Sayer, M.; Vincent, P.J.; Hughes, D.J. \& Smith, T.J. (1993). Antibacterial activity of multilayer silver-copper surface film on catheter material. Can. J. Microbiol., 39, 9, 895-899.

McPhee, J.B. \& Hancock, R.E. (2005). Function and therapeutic potential of host defence peptides. J. Pept. Sci., 11, 11, 677-687.

Medintz, I.; Clapp, A.R.; Melinger, J.S.; Deschamps, J.R. \& Mattoussi, H. (2005). A reagentless biosensing assembly based on quantum dot-donor forster resonance energy transfer. Adv. Mater., 17, 20, 2450-2455.

Mehnert, W. \& Mäder, K. (2001). Solid lipid nanoparticles production, characterization and applications. Adv. Drug Deliv. Rev., 47, 2-3, 165-196.

Meier, M.M.; Kanis, L.A. \& Soldi, V. (2004). Characterization and drug-permeation profiles of microporous and dense cellulose acetate membranes: Influence of plasticizer and pore forming agent. Inter. J. Pharm., 278, 99-110.

Melo, L.D.; Mamizuka, E.M. \& Carmona-Ribeiro, A.M. (2010). Antimicrobial particles from cationic lipid and polyelectrolytes. Langmuir, 26, 14, 12300-12306.

Melo, M.N.; Ferre, R.; Castanho, M.A.R.B. (2009). Antimicrobial peptides: Linking partition, activity and high membrane-bound concentrations. Nat. Rev. Microbiol., 7, 3, 245250. 
Merianos, J.J. (1991). Quaternary ammonium antimicrobial compounds. In: Disinfection, sterilization, and preservation, S. S. Block, ed. (Lea \& Febiger, Philadelphia), pp. 225-255.

Mireles, J.R.; Toguchi, A. \& Harshey, R.M. (2001). Salmonella enterica serovar Typhimurium swarming mutants with altered biofilm-forming habilities: surfactin inhibits biofilm formation. J. Bacteriol., 183, 5848-5854.

Mohanpuria, P.; Rana, K.N. \& Yadav, S.K. (2008). Biosynthesis of nanoparticles: technological concepts and future applications. J. Nanopart. Res., 10, 507-517.

Monforte, V.; Ussetti, P.; Gavaldà, J.; Bravo, C.; Laporta, R.; Len, O.; Garcia-Gallo, C.L.; Tenorio, L.; Solé, J. \& Román, A. (2010). Feasibility, tolerability, and outcomes of nebulized liposomal amphotericin B for Aspergillus infection prevention in lung transplantation. J. Heart Lung Transp., 29, 5, 523-530

Morones, J.R; Elechiguerra, J.L.; Camacho, A.; Holt, K.; Kouri, J.B.; Ramirez, J.T. \& Yacaman, M.J. (2005). The bactericidal effect of silver nanoparticles. Nanotechnol., 16, 23462353.

Mortara, R.A.; Quina, F.H \& Chaimovich, H. (1978). Formation of closed vesicles from a simple phosphate diester. Preparation and some properties of vesicles of dihexadecyl phosphate. Biochem. Biophys. Res. Commun., 81, 4, 1080-1086.

Mugabe, C.; Halwani, M.; Azghani, A.O.; Lafrenie, R.M. \& Omri, A. (2006). Mechanism of enhanced activity of liposome-entrapped aminoglycosides against resistant strains of Pseudomonas aeruginosa. Antimicrob. Agents Chemother., 50, 6, 2016-2022.

Müller, R.H. ; Mäder, K. \& Gohla, S. (2000). Solid lipid nanoparticles (SLN) for controlled drug delivery - a review of the state of the art. Eur. J. Pharm. Biopharm., 50, 1, 161177.

Munson, E.L.; Heard, S.O. \& Doern, G.V. (2004). In vitro exposure of bacteria to antimicrobial impregnated-central venous catheters does not directly lead to the emergence of antimicrobial resistance. Chest, 126, 1628-1635.

Mygind, P.H.; Fischer, R.L.; Schnorr, K.M.; Hansen, M.T.; Sonksen, C.P.; Ludvigsen, S.; Raventos, D.; Buskov, S.; Christensen, B.; De Maria, L.; Taboureau, O.; Yaver, D.; Elvig-Jorgensen, G.; Sorensen, M.V.; Christensen, B.E.; Kjaerulff, S.; FridmodtMoller, N.; Lehrer, R.I.; Zasloff, M. \& Kristensen H. (2005). Plectasin is a peptide antibiotic with therapeutic potential from a saprophytic fungus. Nature, 437, 7061, 975-980.

Nanda, A. \& Saravanan, M. (2009). Biosynthesis of silver nanoparticles from Staphylococcus aureus and its antimicrobial activity against MRSA and MRSE. Nanomedicine, 5, 452456.

Neely, A.N. \& Maley, M.P. (2000). Survival of enterococci and staphylococci on hospital fabrics and plastic. J. Clin. Microbiol., 38, 2, 724-726.

Nelson, C.L. (2004). The current status of material used for depot delivery of drugs. Clin. Orthop. Rel. Res., 427, 72-78.

Nelson, C.L.; McLaren, S.G.; Skinner, R.A.; Smeltzer, M.S.; Thomas, J.R. \& Olsen, K.M. (2002). The treatment of experimental osteomyelitis by surgical debridement and the implantation of calcium sulfate tobramycin pellets. J. Orthop. Res., 20, 643-647.

Nes, I.F.; Diep, D.B. \& Holo, H. (2007). Bacteriocin diversity in Streptococcus and Enterococcus. J. Bacteriol., 189, 4, 1189-1198. 
Nguyen, V.T.; Gidley, M.J. \& Dykes, G.A. (2008). Potential of a nisin-containing bacterial cellulose film to inhibit Listeria monocytogenes on processed meats. Food Microbiol., $25,471-478$.

Nickel, U.; Castell, A.Z.; Poppl, K. \& Schneider, S. (2000). A silver colloid produced by reduction with hydrazine as support for highly sensitive surface-enhanced Raman spectroscopy. Langmuir, 16, 9087-9091.

Nimje, N.; Agarwal, A.; Saraogi, G.K.; Lariya, N.; Rai, G.; Agrawal, H.; Agrawal, G.P. (2009). Mannosylated nanoparticulate carriers of rifabutin for alveolar targeting. J. Drug Targeting, 17, 10, 777-787.

Nishino, J. \& Kanno, Y. (2008). An influence of concentration of polyvinylpyrrolidone on the morphology of silver metal formed from $\mathrm{AgNO}_{3}$ aqueous solution. J. Nanomater., 1, art. no. 592838.

Noel, S.P.; Courtney, H.S.; Bumgardner, J.D. \& Haggard, W.O. (2010). Chitosan sponges to locally deliver amikacin and vancomycin. Clin. Orthop. Relat. Res., 468, 2074-2080.

O'Hagan, D.T.; Ugozzoli, M.; Barackman, J.; Singh, M.; Kazzaz, J.; Higgins, K.; Vancott, T.C. \& Ott, G. (2000). Microparticles in MF59, a potent adjuvant combination for a recombinant protein vaccine against HIV-1. Vaccine, 18, 1793-1801.

Oh, S.; Byun, B.; Lee, S. \& Choi, S. (2006). Preparation of Ag-PS and Ag-PSS particles by Yirradiation and their antimicrobial efficiency against Staphylococcus aureus ATCC 6538 and Klebsiella pneumoniae ATCC 4352. Macromol. Res., 14, 2, 194-198.

Oie, S.; Hosokawa, I. \& Kamiya, A. (2002). Contamination of room door handles by methicillin-sensitive/methicillin-resistant Staphylococcus aureus. J. Hosp. Infect., 51, 2, 140-143.

Okusanya, Ó. O.; Bhavnani, S.M.; Hammel, J.; Minic, P.; Dupont, L.J.; Forrest, A.; Mulder, G. J.; Mackinson, C.; Ambrose, P. G. \& Gupta, R. (2009). Pharmacokinetic and pharmacodynamic evaluation of liposomal amikacin for inhalation in cystic fibrosis patients with chronic pseudomonal infection. Antimicrob. Agents Chemother., 53, 9, 3847-3854.

Omri, A. \& Ravaoarinoro, M. (1996). Preparation properties and the effects of amikacin netilmicin and tobramycin in free and liposomal formulations on Gram-negative and Gram-positive bacteria. Int. J. Antimicrob. Agents, 7, 1, 9-14.

Ostendorf, T.; Meinhold, A.; Harter, C.; Salwender, H.; Egerer, G.; Geiss, H.K.; Ho, A.D. \& Goldschmidt, H. (2005). Chlorhexidine and silver-sulfadiazine coated central venous catheters in haematological patients - a double-blind, randomised, prospective, controlled trial. Support Care Cancer, 13, 993-1000.

Ostuni, E.; Chapman, R.G.; Liang, M.N.; Meluleni, G.; Pier, G.; Ingber, D.E. \& Whitesides, G.M. (2001). Self-assembled monolayers that resist the adsorption of proteins and the adhesion of bacterial and mammalian cells. Langmuir, 17, 20, 6336-6343.

Ozay, O.; Akcali, A.; Otkun, M.T.; Silan, C.; Aktas, N. \& Sahiner, N. (2010). P(4-VP) based nanoparticles and composites with dual action as antimicrobial materials. Colloids Surf., B, 79, 460-466.

Pacheco, L. F.; Vieira, D. B.; Correia, F.M. \& Carmona-Ribeiro, A.M. (2004). Interactions between Cationic Bilayers and Candida albicans cells. Prog. Colloid Polym. Sci., 128, 175-177.

Pacheco, L.F. \& Carmona-Ribeiro, A.M. (2003). Effects of synthetic lipids on solubilization and colloid stability of hydrophobic drugs. J. Colloid Interface Sci., 258, 146-154. 
Page, K.; Wilson, M. \& Parkin, I.P. (2009). Antimicrobial surfaces and their potential in reducing the role of the inanimate environment in the incidence of hospitalacquired infection. J. Mater. Chem., 19, 3819-3831.

Pal, S.; Tak, Y.K. \& Song, J.M. (2007). Does the antibacterial activity of silver nanoparticles depend on the shape of the nanoparticle? A study of the Gram-negative bacterium Escherichia coli. Appl. Environ. Microbiol., 73, 6, 1712-1720.

Panácek, A.; Kvítek, L.; Prucek, R.; Kolár, M.; Vecerová, R.; Pizúrová, N.; Sharma, V.K.; Nevecná, T. \& Zboril, R. (2006). Silver colloid nanoparticles: synthesis, characterization, and their antibacterial activity. J. Phys. Chem. B, 110, 16248-16253.

Pandey, R. \& Khuller, G.K. (2004). Subcutaneous nanoparticle-based antitubercular chemotherapy in an experimental model. J. Antimicrob. Chemother., 54, 1, 266-268.

Pandey, R. \& Khuller, G.K. (2005). Solid lipid particle-based inhalable sustained drug delivery system against experimental tuberculosis. Tuberculosis (Edinb), 85, 227-234.

Papagianni, M. (2003). Ribosomally synthesized peptides with antimicrobial properties: biosynthesis, structure, function, and applications. Biotechnol. Adv., 21, 6, 465-499.

Pape, H.L.; Serena, F.S.; Contini, P.; Devillers, C.; Maftah, A. \& Leprat, P. (2002). Evaluation of the antimicrobial properties of an activated carbon fibre supporting silver using a dynamic method. Carbon, 40, 2947-2954.

Park, J.K.; Yeom, J.; Oh, E.J.; Reddy, M.; Kim, J.Y.; Cho, D.W.; Lim, H.P.; Kim, N.S.; Park, S.W.; Shin, H.I.; Yang, D.J.; Park, K.B. \& Hahn, S.K. (2009). Guided bone regeneration by poly(lactic-co-glycolic acid) grafted hyaluronic acid bi-layer films for periodontal barrier applications. Acta Biomater., 5, 3394-3403.

Park, K.D.; Kim, Y.S.; Han, D.K.; Kim, Y.H.; Lee, E.H.B.; Suh, H. \& Choi, K.S. (1998). Bacterial adhesion on PEG modified polyurethane surfaces. Biomaterials, 19, 7-9, 851-859.

Park, S.H.; Oh, S.G.; Munb, J.Y. \& Han, S.S. (2006). Loading of gold nanoparticles inside the DPPC bilayers of liposome and their effects on membrane fluidities. Colloids Surf., $B, 48,112-118$.

Park, S.H.; Oh, S.G.; Munb, J.Y. \& Hanb, S.S. (2005). Effects of silver nanoparticles on the fluidity of bilayer in phospholipid liposome. Colloids Surf., B, 44, 117-122.

Park, S.I.; Daeschel, M.A. \& Zhao, Y. (2004). Functional properties of antimicrobial lysozyme-chitosan composite films. J. Food Sci., 69, M215-M221.

Park, S.Y., Marsh, K.S. \& Rhim, J.W. (2002). Characteristics of different molecular weight chitosan films affected by the type of organic solvents. J. Food Sci., 67, 1, 194-197.

Pasquardini, L.; Lunelli, L.; Vanzetti, L.; Anderle, M. \& Pederzolli, C. (2008). Immobilization of cationic rifampicin-loaded liposomes on polystyrene for drug-delivery applications. Colloids Surf., B, 62, 265-272.

Patel, J.D.; Ebert, M.; Stokes, K.; Ward, R. \& Anderson, J.M. (2003). Inhibition of bacterial and leukocyte adhesion under shear stress conditions by material surface chemistry. J. Biomater. Sci., Polym. Ed., 14, 279-295.

Patel, J.D.; Ebert, M.; Ward, R. \& Anderson, J.M. (2007). S. epidermidis biofilm formation: Effects of biomaterial surface chemistry and serum proteins. J. Biomed. Mater. Res., $80 \mathrm{~A}, 742-751$.

Pathan, F.K.; Venkata, D.A. \& Panguluri, S.K. (2010). Recent patents on antimicrobial peptides. Recent Pat. DNA Gene Seq., 4, 1, 10-16. 
Pereira, A.H. (2006). Novel therapeutics based on cationic peptides. Curr. Pharm. Biotechnol., $7,4,229-234$

Pereira, E.M.A.; Kosaka, P.M.; Rosa, H.; Vieira, D.B.; Kawano, W.; Petri, D.F.S. \& CarmonaRibeiro, A.M. (2008). Hybrid materials from intermolecular associations between cationic lipid and polymers. J. Phys. Chem. B, 112, 31, 9301-9310.

Perlman, D. \& Bodanszky, M. (1971). Biosynthesis of peptide antibiotics. Annu. Rev. Biochem., 40, 449-464.

Peschel, A. \& Sahl, H.G.(2006). The co-evolution of host cationic antimicrobial peptides and microbial resistance. Nat. Rev. Microbiol., 4, 7, 529-536.

Petrocci, A.N.; Clarke, P.; Merianos, J. \& Green, H. (1979). Quaternary ammonium antimicrobial compounds: old and new. Dev. Ind. Microbiol., 20, 11-14.

Picart, C. (2008). Polyelectrolyte multilayer films: from physico-chemical properties to the control of cellular processes. Curr. Med. Chem., 15, 7, 685-697.

Pini, A.; Giuliani, A.; Falciani, C.; Runci, Y.; Ricci, C.; Lelli, B.; Malossi, M.; Neri, P.; Rossolini, G.M. \& Bracci, L. (2005). Antimicrobial activity of novel dendrimeric peptides obtained by phage display selection and rational modification. Antimicrob. Agents Chemother., 49, 7, 2665-2672.

Pinto-Alphandary, H.; Andremont, A. \& Couvreur, P. (2000). Targeted delivery of antibiotics using liposomes and nanoparticles: research and applications. Int. J. Antimicrob. Agents, 13, 3, 155-168.

Portero, A.; Remunan-Lopez, C.; Criado, M.T. \& Alonso, M.J.J. (2002). Reacetylated chitosan microspheres for controlled delivery of anti-microbial agents to the gastric mucosa. J. Microencapsulation, 19, 797-809.

Potter, M. (2005). Strategies for managing systemic fungal infection and the place of itraconazole. J. Antimicrob. Chemother., 56, 1, 49-54.

Pranoto, Y., Rakshit, S.K. \& Salokhe, V.M. (2005). Enhancing antimicrobial activity of chitosan films by incorporating garlic oil, potassium sorbate and nisin. LWT Food Sci. Technol., 38, 8, 859-865.

Prathna, T.C.; Mathew, L.; Chandrasekaran, N.; Raichur, A.M. \& Mukherjee, A. (2010). Biomimetic synthesis of nanoparticles: science, technology and applicability. In: Biomimetics, Learning from Nature, Amitava Mukherjee (1 Ed.), v. 1, 1-20, Olajnica, Vukovar, Croatia: IN-TEH.

Pueyo, M.T.; Bloch, C.Jr.; Carmona-Ribeiro, A.M. \& Mascio, P. (2009). Lipopeptides produced by a soil Bacillus megaterium strain. Microb. Ecol., 57, 2, 367-378.

Qi, L.; Xu, Z.; Jiang, X.; Hu, C. \& Zou, X. (2004). Preparation and antibacterial activity of chitosan nanoparticles. Carbohydr. Res., 339, 2693-2700.

Qi, X.; Zhou, C.; Li,P.; Xu, W.; Cao, Y.; Ling, H.; Chen, W.N.; Li, C.M.; Xu, R.; Lamrani, M.; Mub, Y.; Su, Leong, S.S.J.; Chang, M.W. \& Chan-Park, M.B. (2010). Novel short antibacterial and antifungal peptides with low cytotoxicity: Efficacy and action mechanisms. Biochem. Biophys. Res. Commun., 398, 594-600.

Quattara, B.; Simard, R.E.; Piette, G.; Begin, A. \& Holly, R.A. (2000). Diffusion of acetic and propionic acids from chitosan-based antimicrobial package films. J. Food Sci., 65, 5, 768-773.

Quintavalla, S. \& Vicini, L. (2002). Antimicrobial food packaging in the meat industry. Meat Sci., 62, 3, 373-380. 
Raafat, D.; von Barger, K.; Haas, A. \& Sahl, H. (2008). Insights into the mode of action of chitosan as an antibacterial compound. Appl. Environ. Microbiol., 74, 12, 3764-3773.

Rabea, E.I.; Badawy, M.E.-T.; Stevens, C.V.; Smagghe, G. \& Steurbaut, W. (2003). Chitosan as antimicrobial agent: applications and mode of action. Biomacromolecules, 4, 14571465.

Rai, M.; Yadav, A. \& Gade, A. (2009). Silver nanoparticles as a new generation of antimicrobials. Biotechnol. Adv., 27, 1, 76-83.

Raja, E.; Vales, E. (2009). Effects of sodium chloride on membrane fusion and on the formation of aggregates of potassium channel KcsA in Escherichia coli membrane. Biophys. Chem., 142, 1-3, 46-54.

Ranucci, M.; Isgro, G.; Giomarelli, P.P.; Pavesi, M.; Luzzani, A.; Cattabriga, I.; Carli, M.; Giomi, P.; Compostella, A.; Digito, A.; Mangani, V.; Silvestri, V. \& Mondelli, E. (2003). Catheter related infection trial (CRIT) group, impact of oligon central venous catheters on catheter colonization and catheter-related bloodstream infection. Crit. Care Med., 31, 1, 52-59.

Raveendran, P.; Goyal, A.; Blatchford, M.A. \& Wallen, S.L. (2006). Stabilization and growth of silver nanocrystals in dendritic polyol dispersions. Mater. Lett., 60, 897-900.

Richardson, V.J (1983). Liposomes in antimicrobial chemotherapy. J. Antimicrob. Chemother., $12,6,532-534$.

Roberts, G.A.F. \& Wood, F.A. (2000). Inter-source reproducibility of the chitin deacetylation process. Adv. Chitin Sci., 4, 34-39.

Roe, D.; Karandikar, B.; Bonn-Savage, N.; Gibbins, B. \& Roullet, J.B. (2008). Antimicrobial surface functionalization of plastic catheters by silver nanoparticles. J. Antimicrob. Chemother., 61, 4, 869-876.

Rosa, H.; Petri, D.F.S. \& Carmona-Ribeiro, A.M. (2008). Interactions between bacteriophage DNA and cationic biomimetic particles. J. Phys. Chem. B, 112, 16422-16430.

Rossi, L.M.; Rangasamy, P.; Zhang, J.; Qui, X-Q \& Wu, G.Y. (2008). Research advances in the development of peptides antibiotics. J. Pharm. Sci., 97, 3, 1060-1070.

Ruggeri, V.; Francolini, I.; Donelli, G. \& Piozzi, A. (2007). Synthesis, characterization, and in vitro activity of antibiotic releasing polyurethanes to prevent bacterial resistance. $J$. Biomed. Mater. Res. A, 81, 2, 287-298.

Rukholm, G.; Mugabe, C.; Azghani, A. O. \& Omri, A. (2006). Antibacterial activity of liposomal gentamicin against Pseudomonas aeruginosa: a time-kill study. Int. J. Antimicrob. Agents, 27, 3, 247-252.

Ruparelia, J.P.; Chatterjee, A.K.; Duttagupta, S.P.; Mukherji, S. (2008). Strain specificity in antimicrobial activity of silver and copper nanoparticles. Acta Biomater., 4, 707-716.

Rupp, M.E.; Lisco, S.J.; Lipsett, P.A.; Perl, T.M.; Keating, K.; Civetta, J.M.; Mermel, L.A.; Lee, D.; Dellinger, E.P.; Donahoe, M.; Giles, D.; Pfaller, M.A.; Maki, D.G. \& Sherertz, R. (2005). Effect of a second generation venous catheter impregnated with chlorhexidine and silver sulfadiazine on central catheter-related infections: a randomized, controlled trial. Ann. Intern. Med., 143, 570-580.

Russell, A.D. \& Chopra, I. (1996). Understanding antibacterial action and resistance (Ellis Horwood, Chichester).

Russell, A.D.; Hugo, W.B. \& Ayliffe, G.A.J. (1999). Principles and practice of disinfection, preservation and sterilization. (Blackwell Science, Oxford). 
Saito, T.; Takeuchi, R.; Hirakawa, K.; Nagata, N.; Yoshida, T.; Koshino, T.; Okuda, K.; Takema, M. \& Hori, T. (2002). Slow-releasing potential of vancomycin loaded porous hydroxyapatite blocks implanted into MRSA osteomyelitis. J. Biomed. Mater. Res., 63, 245-251.

Saleem, M.; Nazir, M.; Ali, M.S.; Hussain, H.; Lee, Y.S.; Riaz, N. \& Jabbar, A. (2010). Antimicrobial natural products: an update on future antibiotic drug candidates. Nat. Prod. Rep., 27, 2, 238-254.

Salton, M.R.J. (1968). Lytic agents, cell permeability, and monolayer penetrability. J. Gen. Physiol., 52, 1, 227-252.

Sang, Y. \& Blecha, F. (2008). Antimicrobial peptides and bacteriocins: alternatives to traditional antibiotics. Anim. Health Res. Rev., 9, 2, 227-235.

Saravanan, M. \& Nanda, A. (2010). Extracellular synthesis of silver bionanoparticles from Aspergillus clavatus and its antimicrobial activity against MRSA and MRSE. Colloids Surf., B, 77, 214-218.

Sastry, M.; Ahmad, A.; Khan, M.I. \& Kumar, R. (2003). Biosynthesis of metal nanoparticles using fungi and actinomycete. Curr. Sci., 85, 2, 162-170.

Schierholz, J.M.; Lucas, L.J.; Rump, A. \& Pulverer, G. (1998). Efficacy of silver coated medical devices. J. Hosp. Infect., 40, 4, 257-262.

Schiffelers, R.; Storn, G. \& Bakker-Woudenberg, I. (2001). Liposome-encapsulated aminoglycosides in pre-clinical and clinical studies. J. Antimicrob. Chemother., 48, 3, 333-344.

Schiffelers, R.M.; Storm, G. \& Bakker-Woudenberg, I.A.J.M. (2001). Host factors influencing the preferential localization of sterically stabilized liposomes in Klebsiella pneumoniae-infected rat lung tissue. Pharm. Res., 18, 6, 780-787.

Schindler M, Assaf Y, Sharon N, Chipman DM (1977) Mechanism of lysozyme catalysis: Role of ground-state strain in subsite D in hen egg-white and human lysozymes. Biochemistry, 16, 423-431.

Schmidmaier, G.; Lucke, M.; Wildemann, B.; Haas, N.P. \& Raschke, M. (2006). Prophylaxis and treatment of implant-related infections by antibiotic-coated implants: a review. Injury, 37, S105-S112.

Schmidmaier, G.; Wildemann, B.; Stemberger, A.; Haas, N.P. \& Raschke, M. (2001). Biodegradable poly(D,L-lactide) coating of implants for continuous release of growth factors. J. Biomed. Mater. Res., 58, 4, 449-455.

Scott, M.G.; Dullaghan, E.; Mookherjee, N.; Glavas, N.; Waldbrook, M.; Thomposon, A.; Wang, A.; Lee, K.; Doria, S.; Hamill, P., Yu, J.J.; Li, Y.; Donini, O.; Guarna, M.M.; Finlay, B.B.; North, J.R. \& Hancock, R.E. (2007). An anti-infective peptide that selectively modulates the innate immune response. Nat. Biotechnol., 25, 465-472.

Segota, S. \& Tezak, D. (2006). Spontaneous formation of vesicles. Adv. Colloid Interface Sci., $121,1-3,51-57$.

Selsted, M.E. \& Ouellette, A.J. (2005). Mammalian defensins in the antimicrobial immune response. Nat. Immunol., 6, 6, 551-557.

Seydim, A.C. \& Sarikus, G. (2006). Antimicrobial activity of whey protein-based edible films incorporated with oregano, rosemary and garlic essential oil. Food Res. Int., 39, 5, 639-644. 
Shahverdi, A.R.; Fakhimi, A.; Shahverdi, H.R. \& Minaian, S. (2007). Synthesis and effect of silver nanoparticles on the antibacterial activity of different antibiotics against Staphylococcus aureus and Escherichia coli. Nanomedicine, 3, 168-171.

Sharma, A. \& Sharma U.S. (1997). Liposomes in drug delivery: progress and limitations. Int. J. Pharm., 154, 2, 123-140.

Sharma, A.; Sharma, S. \& Khuller, G.K. (2004). Lectin-functionalized poly (lactide-coglycolide) nanoparticles as oral/aerosolized antitubercular drug carriers for treatment of tuberculosis. J. Antimicrob. Chemother., 54, 4, 761-766.

Shiflet, A.M.; Bishop, J.R.; Pahwa, A. \& Hajduk, S.L. (2005). Human high density lipoproteins are platforms for the assembly of multi-component innate immune complexes. J. Biol. Chem., 280, 38, 32578-32585.

Shim, J.W.; Kim, J.W.; Han, S.H.; Chang, I.S.; Kim, H.K.; Kang, H.H.; Lee, O.S. \& Suh, K.D. (2002). Zinc oxide/polymethylmethacrylate composite microspheres by in situ suspension polymerization and their morphological study. Colloids Surf., A, 207, 13, 105-111.

Shukla, A.; Fleming, K.E.; Chuang, H.F.; Chau, T.M.; Loose, C.R.; Stephanopoulos, G.N. \& Hammond, P.T. (2010). Controlling the release of peptide antimicrobial agents from surfaces. Biomaterials, 31, 8, 2348-2357.

Sicchierolli, S.M.; Mamizuka, E.M. \& Carmona-Ribeiro, A.M. (1995). Bacteria flocculation and death by cationic vesicles. Langmuir, 11, 2991-2995.

Silva, R.A.; Urzúab, M.D. \& Petri, D.F.S. (2009). Lysozyme binding to poly(4-vinyl-Nalkylpyridinium bromide). J. Colloid Interface Sci., 330, 310-316.

Skidan, I.N.; Gel'perina, S.E.; Severin, S.E. \& Guliaev, A.E. (2003). Enhanced activity of rifampicin loaded with polybutyl cyanoacrylate nanoparticles in relation to intracellularly localized bacteria. Antibiot. Khimioter., 48, 1, 23-26.

Skouri-Gargouri, H. \& Gargouri, A. (2008). First isolation of a novel thermostable antifungal peptide secreted by Aspergillus clavatus. Peptides, 29, 11, 1871-1877.

Sondi, I. \& Salopek-Sondi, B. (2004). Silver nanoparticles as antimicrobial agent: a case study on E. coli as a model from Gram-negative bacteria. J. Colloid Interface Sci., 275, 177182.

Sondi, I.; Goia, D.V. \& Matijevic, E.J. (2003). Preparation of highly concentrated stable dispersions of uniform silver nanoparticles. J. Colloid Interface Sci., 260, 75-81.

Song, J.Y.; Jang, H.K. \& Kim, B.S. (2009). Biological synthesis of gold nanoparticles using Magnolia kobus and Diopyros kaki leaf extracts. Process Biochem., 44, 1133-1138.

Song, Y.m.; Park,Y.; Lim, S.S.; Yang, S.T.; Woo, E.R.; Park,S., Lee, J.S.; Kim, J.I.;Hahm, K,S.; Kim, Y.; Shin, S.Y. (2005). Cell selectivity and mechanism of action of antimicrobial model peptides containing peptoid residues. Biochemistry, 44, 36, 12094-12106.

Souza, P.M.; Fernández, A.; López-carballo, G.; Gavara, R. \& Hernández-Muñoz, P. (2010). Modified sodium caseinate films as releasing carriers of lysozyme. Food Hydrocolloids, 24, 300-306.

Speier, J.L.\& Malek, J.R. (1982). Destruction of microorganisms by contact with solid surfaces. J. Colloid Interface Sci., 89, 1, 68-76.

Stobie, N.; Duffy, B.; McCormack, D.E.; Colreavy, J.; Hidalgo, M.; McHale, P. \& Hinder, S.J. (2008). Prevention of Staphylococcus epidermidis biofilm formation using a lowtemperature processed silver-doped phenyltriethoxysilane sol-gel coating. Biomaterials, 29, 963-969. 
Stone, R. (2002). Bacteriophage therapy: Stalin's forgotten cure. Science, 298, 5594, 728-731.

Storm, G. \& Crommelin, D.J.A. (1998). Liposomes: quo vadis? Pharm. Sci. Tecnol. Today, 1, 19-31.

Sudarshan, N.R.; Hoover, D.G. \& Knorr, D. (1992). Antibacterial action of chitosan. Food Biotechnol., 6, 3, 257-272.

Sulakvelidze, A.; Alavidze, Z. \& Morris, J.G. (2001). Bacteriophage therapy. Antimicrob. Agents Chemother., 45, 3, 649-659.

Tada, N.; Sakamoto, T.; Kagami, A.; Mochizuki, K. \& Kurosaka, K. (1993). Antimicrobial activity of lipoprotein particles containing apolipoprotein A1. Mol. Cell. Biochem., 119, 1-2, 171-178.

Tagg, J.R. \& Dierksen, K.P. (2003). Bacterial replacement therapy: adapting 'germ warfare' to infection prevention. Trends Biotechnol., 21, 5, 217-223.

Tamilvanan, S.; Venkateshan, N. \& Ludwig, A. (2008). The potential of lipid- and polymerbased drug delivery carriers for eradicating biofilm consortia on device-related nosocomial infections. J. Controlled Release, 128, 2-22.

Tapias, G.N.; Sicchierolli, S.M.; Mamizuka, E.M. \& Carmona-Ribeiro, A.M. (1994). Interactions between cationic vesicles and Escherichia coli. Langmuir, 10, 3461-3465.

Tebbs, S.E. \& Elliott, T.S. (1994). Modification of central venous catheter polymers to prevent in vitro microbial colonization. Eur. J. Clin. Microbiol., 13, 2, 111-117.

Tebbs, S.E. \& Elliott, T.S.J. (1993). A novel antimicrobial central venous catheter impregnated with benzalkonium chloride. J. Antimicrob. Chemother., 31, 261-271.

Thome, J.; Holländer, A.; Jaeger, W.; Trick, I. \& Oehr, C. (2003). Ultrathin antibacterial polyammonium coatings on polymer surfaces. Surf. Coat. Technol., 174-175, 584-587.

Tiller, J.C.; Liao, C.; Lewis, K. \& Klibanov, A.M. (2001). Designing surfaces that kill bacteria on contact. Proc. Nat. Acad. Sci. U.S.A., 98, 11, 5981-5985.

Tiwari, S.B. \& Amiji, M. (2006). A review of nanocarrier-based CNS delivery systems. Curr. Drug Deliv., 3, 2, 219-232.

Toke, O. (2005). Antimicrobial peptides: New candidates in the fight against bacterial infections. Biopolymers, 80, 6, 717-735.

Tokumaru, T., Shimizu, Y. \& Fox, C.L. (1984). Antiviral activities of silver sulfadiazine and ocular infection. Res. Commun. Chem. Pathol. Pharmacol., 8, 151-158.

Torresday, J.L.G.; Parsons, J.G.; Gomez, E.; Videa, J.P.; Troiani, H.E.; Santiago, P. \& Yacaman, M.J. (2002). Formation and growth of Au nanoparticles inside live alfa alfa plants. Nano Lett., 2, 4, 397-401.

Travan, A.; Pelillo, C.; Donati, I.; Marsich, E.; Benincasa, M.; Scarpa, T.;; Semeraro, S.; Turco, G.; Gennaro, R. \& Paoletti, S. (2009). Non-cytotoxic silver nanoparticlepolysaccharide nanocomposites with antimicrobial activity. Biomacromolecules, 10, 1429-1435.

Tripathi, A.; Chandrasekaran, N.; Raichur, A.M. \& Mukherjee, A. (2009). Antibacterial applications of silver nanoparticles synthesized by aqueous extract of Azadirachta indica (Neem) leaves. J. Biomed. Nanotechnol., 4, 3, 1-6.

Vaara, M. (1992). Agents that increase the permeability of the outer membrane. Microbiol. Rev., 56, 3, 395-411.

Valappil, S.P.; Ready, D.; Neel, E.A.A.; Pickup, D.M.; Chrzanowski, W.; O’Dell, A.; Newport, L.R.J.; Smith, M.E.; Wilson, M. \& Knowles, J.C. (2008). Antimicrobial gallium-doped phosphate-based glasses. Adv. Funct. Mater., 18, 732-741. 
Vanittanakom, N.; Loeffler, W.; Koch, U. \& Jung, G. (1986). Fengycin - a novel antifungal lipopeptide antibiotic produced by Bacillus subtilis F-29-3. J. Antibiot., 39, 7, 888-901.

Venugopal, D.; Klapper, D.; Srouji, A.H.; Bhonsle, J.B.; Borschel, R.; Mueller, A.; Russel, A.L.; Williams, B.C.; Hicks, R.P. (2010). Novel antimicrobial peptides that exhibit activity against select agents and other drug resistant bacteria. Bioorg. Med. Chem., 18, 14, 5137-5147.

Verma, V.C.; Kharwar, R.N. \& Gange, A.C. (2010). Biosynthesis of antimicrobial silver nanoparticles by the endophytic fungus Aspergillus clavatus. Nanomedicine, 1, 33-40.

Vermette, P.; Meagher, L.; Gagnon, E.; Griesser, H.J. \& Doillon, C.J. (2002). Immobilized liposome layers for drug deliver applications: inhibition of angiogenesis. J. Controlled Release, 80, 1-3, 179-195.

Vieira, D.B. \& Carmona-Ribeiro, A.M. (2008). Cationic nanoparticles for delivery of amphotericin B: preparation, characterization and activity in vitro. J. Nanobiotechnol., 6, 6.

Vieira, D.B.; Pacheco, L..F.; Carmona-Ribeiro, A.M. (2006). Assembly of a model hydrophobic drug into cationic bilayer fragments. J. Colloid Interface Sci., 293, 1, 240247.

Vieira, D.B. \& Carmona-Ribeiro, A.M. (2006). Cationic lipids and surfactants as antifungal agents: Mode of action. J. Antimicrob. Chemother., 58, 4, 760-767.

Vieira, D.B.; Lincopan, N.; Mamizuka, E.M. \& Carmona-Ribeiro, A.M. (2003). Competitive adsorption of cationic bilayers and chitosan on latex: optimal biocidal action. Langmuir, 19, 3, 924-932.

Vieira, D.B. \& Carmona-Ribeiro, A.M. (2001). Synthetic bilayer fragments for solubilization of amphotericin B. J. Colloid Interface Sci., 244, 2, 427-431.

Vigneshwaran, N.; Kathe, A.A.; Varadarajan, P.V.; Nachane, R.P. \& Balasubramanya, R.H. (2006). Biomimetics of silver nanoparticles by white rot fungus, Phaenerochaete chrysosporium. Colloids Surf. B, 53, 55-59.

Vitas, A. I.; Diáz, R. \& Gamazo, C. (1997). Protective effect of liposomal gentamicin against systemic acute murine brucellosis. Chemotherapy, 43, 3, 204-210.

Vyas, S.P.; Kannan, M. E.; Jain, S.; Mishra, V. \& Singh, P. (2004). Design of liposomal aerosols for improved delivery of rifampicin to alveolar macrophage. Int. J. Pharm., $269,1,37-49$.

Warren, W.C.; Hillier, L.W.; Marshall Graves, J.A.; Birney, E.; Ponting, C.P.; Grutzner, F.; Belov, K., Miller, W.; Clarke, L.; Chinwalla, A.T.; Yang, S-P.; Heger, A.; Locke, D.P.; Miethke, P.; Waters, P.D.; Veyrunes, F.; Fulton, L.; Fulton, B.; Graves, T.; Wallis, J.; Puente, X.; Lopez-Otin, C.; Ordonez, G.R.; Eichler, E.E.; Cheng, L.I.N.; Cheng, Z.E.; Deakin, J.E.; Alsop, A.; Thompson, K.; Kirby, P.; Papenfuss, A.T.; Wakefield, M.J.; Olender, T.; Lancet, D.; Huttley, G.A.; Smit Arian F.A.; Pask, A.; Temple-Smith, P.; Batzer, M.A.; Walker, J.A.; Konkel, M.K.; Harris, R.S.; Whittington, C.M.; Wong, E.S.W.; Gemmell, N.J.; Buschiazzo, E.; Vargas Jentzsch, I.M.; Merkel, A.; Schmitz, J.; Zemann, A.; Churakov, G.; Kriess, J.O.; Brosius, J.; Murchison, E.P.; Sachidanandam S.C.; Hannon,G.J.; Tsend- Ayush, E.; Mcmillan, D. \& Attenborough, R. (2008). Genome analysis of the platypus reveals unique signatures of evolution. Nature, 453, 7192, 175-183.

Willey, J.M. \& Van Der Donk, W.A. (2007). Lantibiotics: peptides of diverse structure and function. Annu. Rev. Microbiol., 61, 477-501. 
Williams, L.B. \& Haydel, S.E. (2010). Evaluation of the medicinal use of clay minerals as antibacterial agents. Int. Geol. Rev., 52, 7/8, 745-770.

Wissing, S.A. ; Kayser, O. \& Müller, R.H. (2004). Solid lipid nanoparticles for parenteral drug delivery. Adv. Drug Delivery Rev., 56, 9, 1257-1272.

Zanetti, M. (2005). The role of cathelicidins in the innate host defenses of mammals. Curr. Issues Mol. Biol., 7, 2, 179-196.

Zaru, M.; Mourtas, S.; Klepetsanis, P.; Fadda, A.M. \& Antimisiaris, S. G. (2007). Liposomes for drug delivery to the lungs by nebulization. Eur. J. Pharm. Biopharm., 67, 3, 655666.

Zasloff, M. (2002). Antimicrobial peptides of multicellular organisms. Nature, 415, 389-395.

Zhang, L.; Pornpattananangkul, D.; Hu, C.M.J. \& Huang, C.M. (2010). Development of nanoparticles for antimicrobial drug delivery. Curr. Med. Chem., 17, 6, 585-594.

Zhang, X.; Wyss, U.P.; Pichora, D. \& Goosen, M.F. (1994). Biodegradable controlled antibiotic release devices for osteomyelitis: optimization of release properties. $J$. Pharm. Pharmacol., 46, 718-724.

Zhang, X.H. \& Austin, B. (2005). Haemolysins in Vibrio species. J. Appl. Microbiol., 98, 10111019.

Zhang, Y.; Peng, H.; Huanga, W.; Zhou, Y. \& Yan, D. (2008). Facile preparation and characterization of highly antimicrobial colloid Ag or Au nanoparticles. J. Colloid Interface Sci., 325, 371-376.

Zhou, J.; Loftus, A.L.; Mulley, G. \& Jenkins, A.T. (2010). A thin film detection/response system for pathogenic bacteria. J. Am. Chem. Soc., 132, 6566-6570.

Zhu, C.T.; Xu, Y.Q.; Shi, J.; Li, J. \& Ding, J. (2010). Liposome combined porous $\beta$-TCP scaffold: Preparation, characterization, and anti-biofilm activity. Drug Delivery, 17, 6, 391-398.

Zilberman, M. \& Elsner, J.J. (2008). Antibiotic-eluting medical devices for various applications. J. Controlled Release, 130, 202-215.

Zivanovic, S.; Chi, S. \& Draughton, A.E. (2005). Antimicrobial activity of chitosan films enriched with essential oils. J. Food Sci., 70, 1, M45-M51.

Zivanovic, S.; Li, J.; Davidson, P.M. \& Kit, K. (2007). Physical, mechanical and antibacterial properties of chitosan/PEO blend films. Biomacromolecules, 8, 1505-1510. 


\title{
Biomimetic Adsorbents: Enrichment of Trace Amounts of Organic Contaminants (TAOCs) in Aqueous Solution
}

\author{
Chao-Hai Wei, Xiao-Xuan Zhang, Yuan Ren and Xu-Biao Yu \\ South China University of Technology \\ P.R. China
}

\section{Introduction}

The purification of the wastewater containing trace amounts of organic contaminants (TAOCs) is a very challenging subject in the field of environmental engineering. Although the concentration of TAOCs in wastewater is extremely low (in the range of $\mathrm{mg} / \mathrm{L}-\mathrm{ng} / \mathrm{L}$ ), the mutagenic and carcinogenic effects of TAOCs (e.g. PCBs, PAHs, PCDDS, EDCs, etc.) are non-negligible. Traditional technologies, such as biological degradation, chemical oxidation/reduction and coagulation, are less effective for this kind of wastewater both technically and economically owning to the low concentration of the contaminants. The adsorption technique has been found to be not only effective, but also practical in application for this kind of wastewater due to the high enrichment capacity of the adsorbents towards the contaminants. By the enrichment of adsorbents, the subsequent decomposition and detoxification of the contaminants become possible using other biological and chemical methods. Therefore, development of the adsorbents with high enrichment capacity to TAOCs is highly necessary.

It is well known that TAOCs in natural water body can be greatly enriched by the aquatic oragnism such as fish and shellfish, namely bioaccumulation. This is a very interesting phenomenon because it motivates the idea of developing biomimetic adsorbents originated from aquatic animals for removing TAOCs. The tissues of aquatic animals which enrich the maximum amount of organic contaminants are found in brain which mostly consists of lipid. This suggests that materials of biological lipid possess an excellent adsorption capacity towards TAOCs. TAOCs normally exhibit low water solubility but high compatibility with organic matters, as evidenced from a high $\log K_{\text {ow }}$ value (octanol - water partition coefficient). Hence, TAOCs can be enriched in the lipid through an interaction like the "solid phase extraction". The binding force between the solids and TAOCs is neither the covalent binding nor the electrostatic force, but a kind of hydrophobic bond. This type of adsorption does not have an obvious selectivity for the functional groups located at the molecules of TAOCs. Indeed, the molecular hydrophobility plays an important role in the adsorption process. In order to achieve an efficient enrichment, we need to find out the suitable biomimetic adsorbent which has a high organic compatibility towards TAOCs. In our previous studies (Zhang et al., 2010b), a biomimetic adsorbent prepared by poly-3hydroxybutyrate (PHB) exhibited high enrichment ability for trace amounts of 
chlorobenzene and o-chloronitrobenzene at a low level of specific surface area $\left(8.5 \mathrm{~m}^{2} / \mathrm{g}\right)$, confirming the assumption of the adsorption mechanism.

This chapter provides a review of this new type of adsorbents and their application in the removal of various organic pollutants. The fundamental processes involved in the technique are elaborated with a discussion of some recent novel concepts in biomimetic adsorbents. The preparation and characterization of biomimetic adsorbents and the evaluation of their adsorption capacity are subsequently described.

\section{Organic micro pollutant and control technology}

\subsection{Organic micro pollutant}

1. Source and generation mechanism

Organic micro pollutants have a wide range of wastewater sources produced from chemical, pharmaceutical and pesticide industries. Most of these pollutants are petroleum pollutants, phenols, ketones and hydrocarbon, etc. Although their solubility is very low, they can be stable in water for decades (Díaz-Cruz and Barceló, 2008).

As a representative of the industrial production, chemical industry is an important source of TAOCs. Many types of trace non-degradable organic compounds remaining in the tail water are discharged into the natural water. For example, polycyclic aromatic hydrocarbons (PAHs) are common pollutants, mainly from coal-based chemical production, garbage incineration and sudden oil leakage (Khim et al., 2001). In addition, naphthalenedisulfonates are widely-used chemical intermediates in the production and are detected both in industrial wastewater and domestic sewage (Díaz-Cruz and Barceló, 2008). TAOCs can not only be detected in industry wastewater but also in municipal wastewater, which main sources are chemical commodities such as cosmetic, cleaner and drug. Alkylphenol ethoxylates (APEOs), as a kind of common household and industrial cleanser as well as, and one of the main components of plastic products and prophylactic, can often be detected in the natural waters. APEOs and their degradation products have also become the common organic pollutants in water (Kolpin et al., 2002). Corcia et al. pointed out that APEOs could be partially decomposed in water treatment process, since approximately $40 \%$ of APEOs were discharged to water body as intermediate products (Corcia et al.2000).

As herbicides, insecticide and phytocide are widely used in modern agriculture, the pesticide residues are found to spread into the river, ground water and ocean with the rainfall. Postle et al. (2004) randomly tested chlorine acetanilide phytocide and its metabolites' from of 336 well in Wisconsin, America. They found that $38 \pm 5.0 \%$ of the wells were detected of these herbicides and the average concentrations of acetochlor ESA and alachlor OA are $0.15 \pm 0.082 \mu \mathrm{g} / \mathrm{L}$ and $1.8 \pm 0.60 \mu \mathrm{g} / \mathrm{L}$, respectively. The widely use of organochlorine pesticides in the last century causes an extensive ubiquitous distribution of them in the natural environment. For example, $7.06 \mathrm{mg} / \mathrm{kg}$ DDT was detected in the Antimora rostrata (found at $200 \mathrm{~m}$ depth of the Atlantic) fish liver and DDT concentration in the same order was detected in Atlantic cod (found in the shallow water of Canada Atlantic coast) (Barber et al., 1979).

In addition to the above direct sources of organic pollutants, TAOCs also can be produced during disinfection of drinking water as disinfection by-products (DBPs). The disinfection by chlorine which was initiated in the early of 20th century has such advantages as low cost and high efficiency. However, chlorine can react with the natural organics in water, leading to the formation of DBPs during the disinfection. Since the first discovery of trihalomethanes (THMs) by Rook at 1974, many studies on the generation mechanism and toxicity of DBPs 
have been conducted (Richardson et. al, 2002). The production of DBPs has an intimate connection with concentrations of bromide, iodide and natural organic matter (NOM) in water. Furthermore, the $\mathrm{pH}$ value of water affect the generated type of DBPs (Richardson et. al., 2002). In order to prevent the formation of THMs and haloacetic acids (HAAs), new disinfectants such as ozone, chlorine dioxide and chloramine have been used to instead chlorine. However, some new problems appeared. When using ozone as the disinfectant, bromate (a strong carcinogen) forms in spite of significant reduction of the formation of THMs and HAAs. (Richardson et al., 2002). Therefore, how to effectively reduce DBPs is a long-term consideration during the wastewater treatment.

2. Solution property

The wastewater containing organic micro pollutants is very different with common organic wastewater. The total organic matter content is much less than common organic wastewater which likes the lean phase solution. Such features of organic micro pollutants affect their conversion in the environment and the selection of the treatment technologies. Moreover, TAOCs are normally featured with high hydrophobility. For some organics such as dioxin, it is very difficult to test their solubility in water and is even difficult to be measured by experiments (Yang et al., 2006) ,but only calculated by theory methods. TAOCs also are normally lipophilic. When an organic solid is present in wastewater, the pollutants predominantly exist in the solid phase rather than in the water phase. Typically, the fat of aquatics (e.g., fish) usually has a high concentration of TAOCs. It is widely , although organics are hydrophobic and lipophilic, hydrophobility and lipophilia are conspicuous for TAOCs with complex molecular structures. The treatment technology for wastewater containing TAOCs should be adjusted according to their great change in solution. Therefore, it is worthy of consideration and investigation on how to treat such kind of wastewater efficiently.

3. Typical pollutants

There are a variety of typical organic micro pollutants, such as persistent organic pollutants (POPs), environmental endocrine disputing chemicals (EDCs), and DBPs.

POPs are a group of organic substances, which are toxic, persistent, bioaccumulative and prone to long-scale migration (Lohmann et al., 2007). POPs mainly include halogenated aromatic compounds (polychlorinated biphenyls (PCBs), polychlorinated dibenzo- $p$-dioxins and-furans (PCDD/Fs)), polybromodiphenyl ethers (PBDEs) and organochlorine insecticide (such as dichlorodiphenyltrichlorothane (DDT) and its metabolites). Most of POPs are hydrophobic, lipophilic, and easily to bioaccumulate at he top of the food chain. There is a large number of literature concerning POPs hazard on organism (Armitage and Gobas, 2007; Lohmann and Muir, 2010; Gioia et al., 2007; Kellyn et al., 2005). POPs are generally carcinogenic, environmental hormonal and immune system nocuous. Currently, the harm of POPs to human body is still under investigation. The hazard mechanism of POPs will be revealed by the further study.

EDCs present in environment can disrupt the procreation for human and animals, the metabolism and growth of embryo or children and the function of nerves (Kandarakis et al., 2009). PCBs, organo-chlorine pesticide, unpersistent pesticide, phthalate, bisphenol A, nonyl phenols, brominated flame retardant and some other new pollutants are all included in the category of EDCs. Natural or artificial-synthesized estrogens, male hormone, cortisol and organotin are widely detectable in the drainage basins. Previous studies have shown that, not only the procreation problem and abnormal rate of embryo, but also the deterioration of male sperm and testosterone are related to EDCs. Besides, it may lead to spermary cancer 
and thyroid cancer. Thus the harmful effect of EDCs on male health becomes a concerned environmental problem.

DBPs are mainly generated from reaction of disinfectant and natural organic pollutants or man-made pollutants (e.g., bromine and iodine) (Hebert et al., 2010). Most of the pathology studies concern with the two main kinds of DBPs: THMs and HAAs. Because chlorine disinfection increases the risk of urinary bladder and colon cancer, World Health Organization (WHO) and some developed countries have made strict criteria to limit the discharge of DBPs to minimize the harm to human beings.

4. Effect on drinking water safety

For human beings, TAOCs have the greastest threat on the drinking water safety. These TAOCs dissolve in drinking water during water migration. Many countries have established a lot of strict criterions to control the content of TAOCs. U.S. EPA made a list of 120 kinds of priority pollutants in water quality standard, 115 belonging to among them are TAOCs. However, drinking water in many countries are still facing tremendous risks due to the high transitivity and stability of TAOCs. By analyzing the micro pollutants in the sediment of Seine in France, Carpentier et al. (2002) found that, the concentrations of PCB and PAHs reached moderate level of pollution. Götz et al. (1996) analyzed 145 kinds of pollutants in water of Elbe in Hamburg and found that the concentrations of 1,2-dichloroethane, 1,4dichlorobenzene, hexachlorobenzene $(\mathrm{HCB})$ and nitrobenzene were greater than those depicted in the water criteria of Germany. The improper disposal of hospital sewage containing large amounts of disinfectant, drugs and organic solvents also cause the potential threat to drinking water safety (Laber et al., 1999). In developing countries, septic tanks are generally used for storing hospital sewage, which increases the risk of the pollution to groundwater. Emmanuel et al. (2009) evaluated the pollution risk of the hospital sewage collection device (as shown in Fig.1) by monitoring COD, chloroform, dichloromethane, dibromochloromethane, dichlorobromomethane and tribromomethane. Results showed that the concentrations of organic pollutants in groundwater of the hospitals using these devices were greater than those in others. This implies a high security risk of directly drinking water.

\subsection{Biological enrichment}

In the polluted natural water, aquatic animals' adipose tissues act as the "natural sorbent" due to the high hydrophobic characteristic of TAOCs. The relationship of TAOCs migration behavior and aquatic animals enrichment (See Fig.2) indicates that, aquatic animals are the important site were micro pollutants are enriched during the food chain transmission.

PCBs are important species of POPs, and have been widely studied on enrichment behaviors with respect to many kinds of aquatic animals. Magnusson et. al. (2006) studied accumulation of 29 nonplanar and 11 coplanar congeners of PCBs in 9 seabed mollusks and found that the accumulation of PCBs in different aquatic animals didn't have linear correlation with concentrations of pollutants, but strongly dependent on the specific kind, figure and longevity of animals. Ribeiro et al. (2008) studied the residual concentration of PCBs in eel living in Nature Camargue Reserve and found that 10 out of the 70 detected PCBs were dioxin-like PCBs. PCBs had a high concentration that was up to $29.6 \mathrm{pg} / \mathrm{g}$ (dry weight) with $22 \%$ and $29 \%$ of PCBs consisted in liver and fat of eels, respectively, which suggested that it has potential risk for eating these eels. Pierce et al. (2008) studied PCBs concentrations in the fat of living dolphins in coast of Western Europe and their effect on 


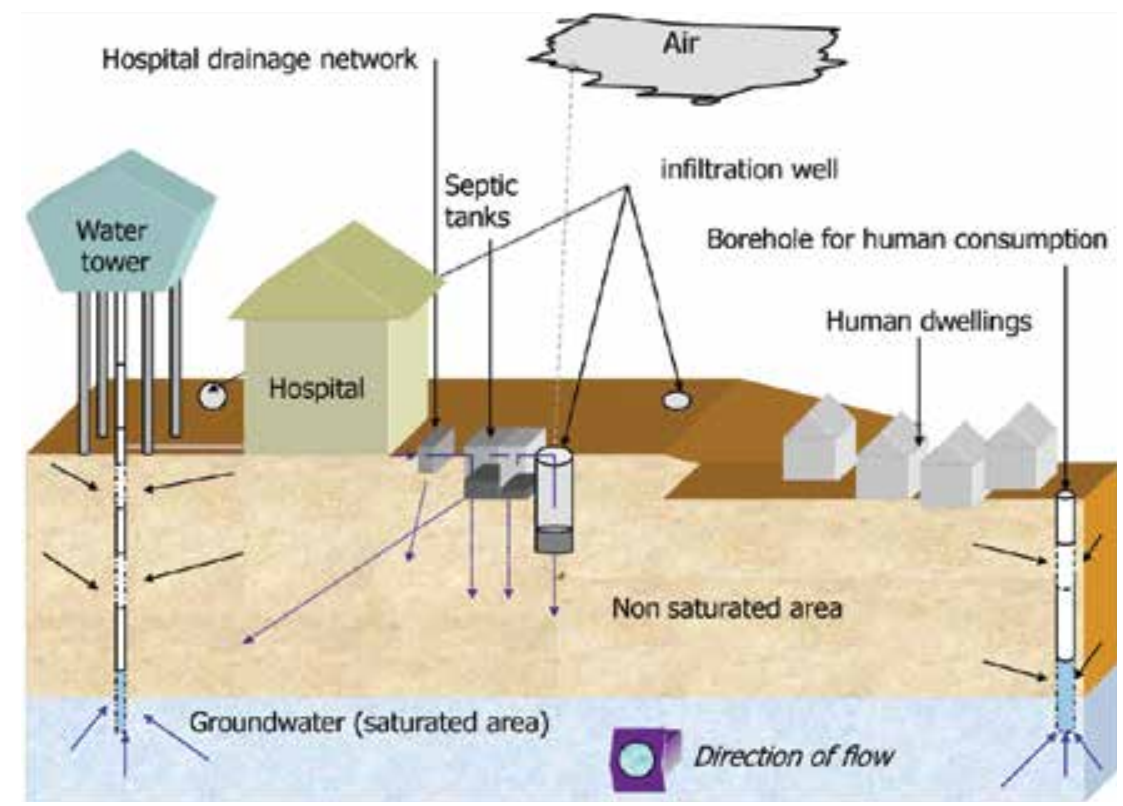

Fig. 1. Graphic representation of the scenario studied. (Emmanuel et al., 2009)
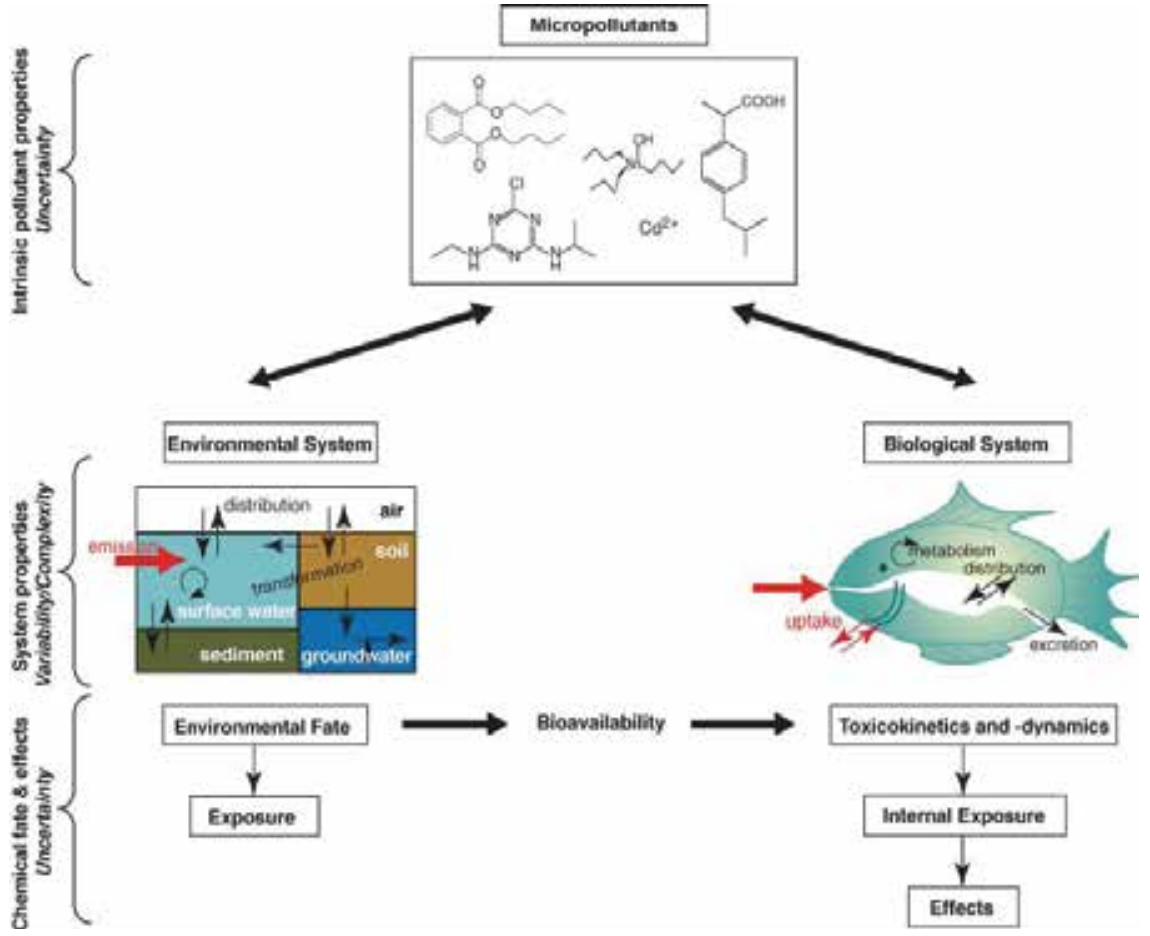

Fig. 2. Consistent exposure and effect assessment is possible if processes in the environmental system and in the organisms (biological system) are treated with the same modeling structure and tools. (Schwarzenbach et al., 2006) 
reproduction and death of dolphins. PCBs concentrations were found to exceed the safe level in fat of every Atlantic dolphins and porpoises analyzed. Concentrations of PCBs in porpoises died of disease or parasitic were be higher than that of normal porpoises.

Many previous studies (Ikemoto et al., 2008; Voutsas et al., 2002; Liu et al., 2007) indicated that contents of many micro pollutants in aquatic animals were much greater than normal, and that these micro pollutants were considered to affect the metabolism. It can be concluded from micro pollutants enrichment behavors of aquatic animals. Concentrations of pollutants in animals are not only much greater than that of normal environment, but also greater than that adsorbed by other substrates. In nature, many organisms have extraordinary abilities, such as high hydrophobility self-cleaning lotus leaves, and adhesive forces of lizards. These all can be considered as bionic behaviour and are being under investigation. The super enrichment ability for aquatic animals to absorb organic pollutants is not a natural phenomenon but appears after exposure to the increasing pollutants in water. Nevertheless, that is also a hint for organic pollutants control: novel absorbents imitated from the super absorbing behaviors of aquatic animals will provide a new approach for pollutant treatment.

\subsection{Control technology}

1. Adsorption technology

Adsorption technology plays an important role in the wastewater treatment process. In recent years, adsorption technology has been developed rapidly and concentrated on the protection and recycling of water resource. For TAOCs, carbonaceous adsorbents are the most used materials due to their abundant micropore structure and hydrophobic surface. There are various kinds of carbonaceous adsorbents such as activated carbon, activated carbon fiber, carbon nanotube. Zhang et al. (2010) studied the adsorption of three TAOCs by four types of carbonaceous adsorbents [a granular activated carbon (HD4000), an activated carbon fiber (ACF10), two single-wall carbon nanotubes (SWNT, SWNT-HT), and a multiwalled carbon nanotube (MWNT)] with different structural characteristics but similar surface polarities. Isotherm results demonstrated that the molecular sieving and micropore effects played an important role in the adsorption of TAOCs onto carbonaceous porous adsorbents. ACF10 and HD4000 with greater microporous volumes exhibited higher adsorption affinities to low molecular-weight TAOCs than SWNT and MWNT with greater mesopore and macropore volumes. The adsorption behavior of organic pollutants onto activated carbons has been widely studied. The amount of adsorption not only depends on the properties of pollutants (hydrophobility, polarity, aromaticity, etc, but also has a strong relation with the surface properties of activated carbon (Ridder et al., 2010). Furthermore, the particle size of activated carbon has an important effect on the removal ability, especially in the practical use. Crowin et al. (2010) investigated the effect of particle size on the reduction of granular activated carbon (GAC) adsorption capacity for trace organic contaminants by dissolving organic matter and demonstrating the lower adsorption capacity per mass of adsorbent in relation to the larger GAC particles. On the preparation of activated carbons, many novel methods have been developed in order to optimize the surface properties for better performance regarding pollutant removal and their regeneration. Ji et al. (2009) synthesized a microporous carbon with very high specific surface area and narrow pore size distribution using $\mathrm{Y}$ zeolite as a template. The synthesized - microporous carbon showed extraordinarily high adsorption affinity (comparable or higher than activated carbons and carbon nanotubes) for phenol, 1,3- 
dichlorobenzene, and 1,3-dinitrobenzene, and very fast adsorption/desorption kinetics. These adsorption properties were attributed to the large hydrophobic surface area and the regular-shaped, open and interconnected three-dimensional pore structure of the synthesized microporous carbon. In the case of practical treatment, the effect of NOM on adsorption is important. Yu et al. (2009) investigated preloading effects of NOM on adsorption capacity and kinetics under conditions and concentrations relevant to drinking water treatment. The isotherms demonstrated that all compounds were significantly negatively impacted by NOM fouling.

Although activated carbon has a good adsorption capacity for organic pollutant, the expensive price and relatively weak affinities for trace amount pollutants limit its wide application. Many studies have been conducted to search the substitutes of activated carbon including agricultural wastes, natural and modified clays, organic polymer, etc. Nowadays, these new adsorbents can not readily replace activated carbon but the development of adsorbents with greater cost performance for TAOCs adsorption has become a new trend.

2. Membrane technology

Membrane technology has gained interest in the last decades in water purification applications. In addition to their use for desalination of seawater and brackish water, the technologies such as nanofiltration and reverse osmosis membrane offer good removal efficiencies (Verliefde et al., 2009). The treatment efficiency of membrane is related to the molecular size directly but not the molecular weight. The non-polar molecule has better removal efficiency than polar molecules. Agenson et al. (2003) compared the removal of DBPs, EDCs, and plastics additives by nanofiltration and reverse osmosis at low pressure $(100-300 \mathrm{kPa})$. Their results indicated that reverse osmosis exhibited better removal capability to VOCs than nanofiltration membrane which removal rate was $20 \%$ only. By the multiple linear regression, they predicted that larger molecular size and higher hydrophobility favored the removal. In the treatment of membrane, the control factors include diffusion, adsorption, partition and desorption (Ducom et al., 1999; Chellam et al., 2001; Nghiem et al., 2004; Lee et al., 2001). Due to the close relationship between the removal rate and the physicochemical properties of pollutants, some pollutants can not be treated well (Bellona et al., 2004). Especially, for the large-scale production such as drinking water purification, the poor performance for TAOCs will bring about a series of problems (Verliefde et al., 2009). Moreover, the high cost of membrane and its regeneration restrict its large scale application.

3. Membrane bioreactor (MBR)

MBR is considered as the next-generation technology of wastewater treatment, which is expected to replace the traditional activated sludge process. MBR possesses such advantages as small footprints and a superior effluent quality (Wever et al., 2007). Wever et al. (2007) compared MBR and conventional activated sludge systems (CAS) for micro pollutant degradation in laboratory-scale experiments with synthetic and real domestic wastewater. MBR treatment can significantly enhance the removal of the micro pollutants, and reduce the lag phases for degradation, implying that they may respond quicker to variable influent concentrations. Nghiem et al. (2009) conducted laboratory-scale experiments to investigate the removal mechanisms of MBR system for trace organic contaminants. Results indicated that removal efficiency of specific trace organic contaminants strongly depended on their physicochemical properties. Approximately $90 \%$ of bisphenol A was removed, while, the removal efficiency of sulfamethoxazole was only about $50 \%$ under the same condition.. Both biodegradation and adsorption to the sludge were thought to be responsible for the 
removal of bisphenol A, which is a relatively hydrophobic organic compound. In contrast, the latter mechanism was absent for sulfamethoxazole as this compound is rather hydrophilic. Chu et al. (2010) investigated the feasibility of treating micro polluted surface water for drinking water production with a bio-diatomite dynamic membrane reactor (BDDMR) at a laboratory-scale-discontinuous-flow mode. Results indicated that the BDDMR was effective in removing trihalomethanes' formation potential (THMFP) at a hydraulic retention time (HRT) of $3.5 \mathrm{~h}$ due to its high concentrations of mixed liquor suspended solids (MLSS) and mixed liquor volatile suspended solids (MLVSS).

4. Other technologies

Because of the great difficulty in treating TAOCs, single technology can not meet the purification purpose. Thus the combination of different technologies is employed. Reungoat et al. (2010) assessed the removal of organic micro pollutants and the concurrent reduction of their biological activity in a full-scale reclamation plant treating secondary effluent. The treatment consists of 6 stages: denitrification, pre-ozonation, coagulation/flocculation/ dissolved air flotation and filtration (DAFF), main ozonation, activated carbon filtration and final ozonation for disinfection. The process is shown in Fig. 3. Results showed that, among the 54 micro pollutants quantified in the influent water, 50 were removed to below their limit for quantification $(0.01 \mu \mathrm{g} / \mathrm{L})$ which represents that more than $90 \%$ of pollutants were removed. The key processes responsible for the plant's performances were the coagulation/flocculation/DAFF, main ozonation and activated carbon filtration. Hollender et al. (2009) studied the removal efficiency for 220 micro pollutants from a municipal wastewater treatment plant (WWTP) upgraded with post-ozonation followed by sand filtration. During post-ozonation, compounds with activated aromatic moieties, amine functions, or double bonds such as sulfamethoxazole, diclofenac, or carbamazepine with second-order rate constants for the reaction with ozone $>10^{4} \mathrm{M}^{-1} \mathrm{~s}^{-1}$ at $\mathrm{pH} 7$ (fast reacting) were eliminated to concentrations below the detection limit for an ozone dose of $0.47 \mathrm{~g} \mathrm{O}_{3} / \mathrm{g}$ dissolved organic carbon (DOC). Compounds more resistant to oxidation by ozone such as atenolol and benzotriazole were increasingly eliminated with increasing ozone doses, resulting in $>85 \%$ removal for a medium ozone dose $\left(\sim 0.6 \mathrm{~g} \mathrm{O}_{3} / \mathrm{g}\right.$ DOC). Only a few micro pollutants such as some X-ray contrast media and triazine herbicides with second-order rate constants $<10^{2} \mathrm{M}^{-1} \mathrm{~s}^{-1}$ (slowly reacting) persisted to a large extent. With a medium ozonedose, only 11 of micro pollutants 55 detected in the secondary effluent were found at $>100 \mathrm{ng} / \mathrm{L}$. The energy requirement for the additional post-ozonation step was about 0.035 $\mathrm{kWh} \mathrm{m}^{-3}$, which corresponded to $12 \%$ of a typical medium-sized nutrient removal plant $(5 \mathrm{~g}$ $\left.\mathrm{DOC} / \mathrm{m}^{3}\right)$.

In comparison with the ordinary municipal sewage and industrial wastewater, organic micro polluted wastewater has the characteristics of low load and poor nutrient. Thus, some energy-effective eco-treatment technologies have been adopted. Among them, the constructed wetland is one of a representative technology. An engineered constructed wetland, fed with wastewater effluent, was investigated with respect to the control of organic micro pollutants (Park et al., 2009). The levels of 30 different micro pollutants, including pharmaceuticals, endocrine disruptors and personal care products, were measured using solid phase extraction, followed by liquid chromatography/tandem mass spectrometer. Only 9 out of the 30 chemicals exhibited relatively high concentrations in the effluent samples. Furthermore, analyses of the removal efficiencies and two characteristic parameters (logKow and $\mathrm{pKa}$ ) showed no evidence supporting hydrophobic and electrostatic interactions for the control of micro pollutants. 


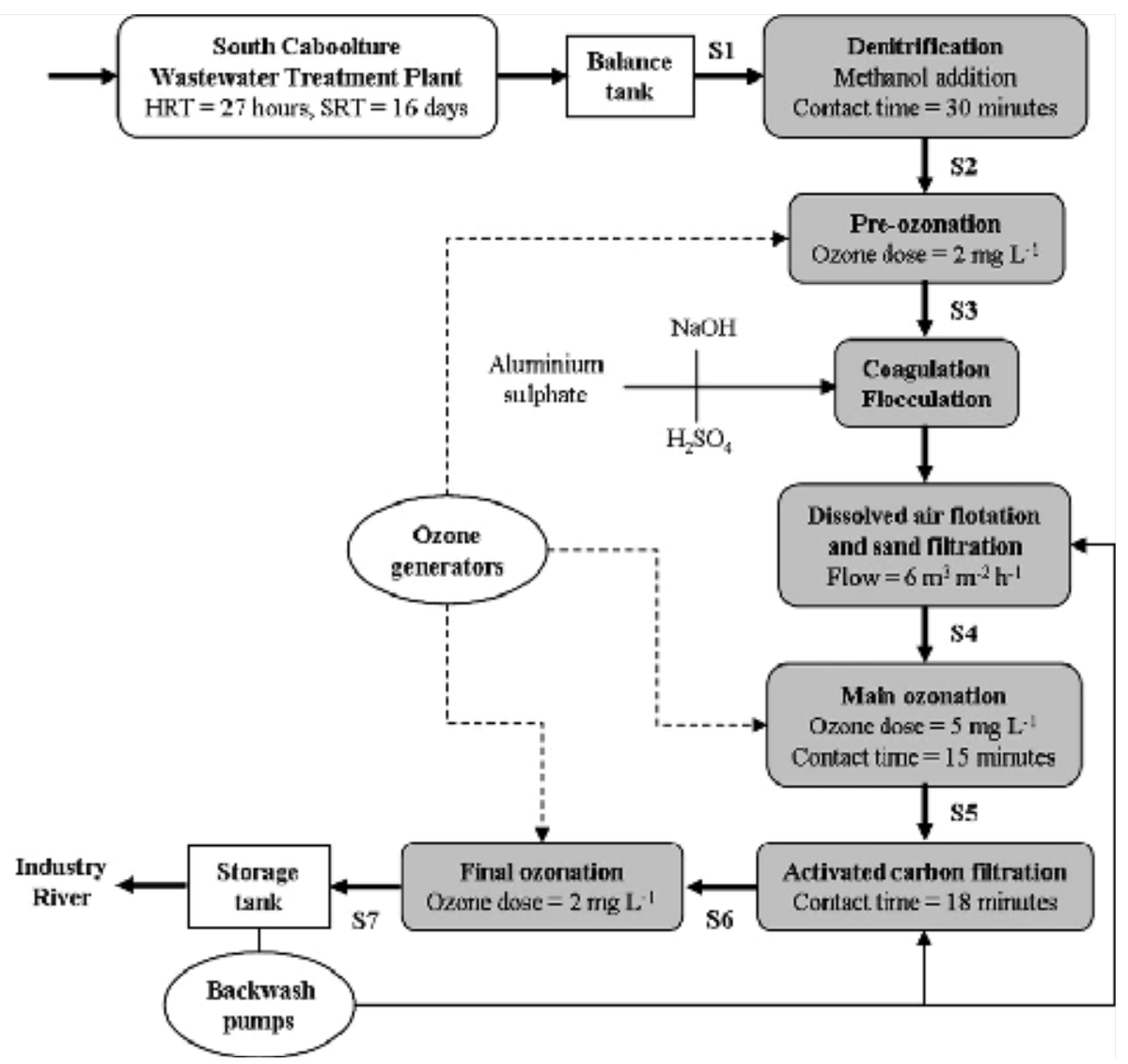

Fig. 3. South Caboolture Water Reclamation Plant. S1 to S7: sampling locations. HRT: hydraulic retention time. SRT: sludge retention time. (Reungoat et al., 2010)

\section{Biomimetic adsorbent}

\subsection{Definition of biomimetic adsorbent}

The study of biomimetic phenomena has a long history. Plenty of knowledge through imitating nature is available. For example, the pot experiments showed that the peanut (Arachis hypogaea), soybean (Glycine max) and sesame (Sesamum indicum) had great bioaccumulativity for DDT. The order of DDT concentration in each grain was: peanut $>$ sesame > soybean, which indicated that the concentration of DDT had a positive correlation with the oil contents of grains (Wei et al., 2006). More generally, the water plant with large rich fat skin can adsorb lipophilic OCPs (Ockenden et al., 1998). The concentration of PCBs in blubber of neonate Delphinapterus leucas, Orcinus orca, adult Delphinapterus leucas and Balaena mystice was 17563, 78000, 59000 and $354 \mathrm{ng} / \mathrm{g}$, respectively, which was 1-3 orders of magnitude higher than that in other tissues (Liu et al., 2008). Also, the concentration of DDT in blubber was 1-2 orders of magnitude higher than that in other tissues, and was 2230, 98600, 56000 and $377 \mathrm{ng} / \mathrm{g}$, respectively (Liu et al., 2008). The measured concentrations of PCBs and DDT in different organs were in the ascending order: blubber, liver, kidney, brain and muscle (Liu et al., 2008). BCF lipid (bioconcentration factor based on lipid content) of 13 
OCPs in 18 fish species from Qiantang River in China ranged from 1000 to 251189, and the bioaccumulation capacity of fish organ was high when the lipid content of organ was high (Zhou et al., 2007). The methods of POPs entering organism body contained breathing in from air, inhaling by skin and feeding food. The POPs eliminating processes included 4 methods. The first one was breathing out, but the discharged amount of method was very small and can be ignored. The second one was discharge by dejecta. Breathing in from air, inhaling by skin and feeding food are the main ways of POPs entering organism body. The third one was metabolism. Youth biology can metabolize POPs in some extent, but the metabolic function may become slow with the increasing age. The fourth one was transference. For example, POPs in fat of mammals can be transferred to milk. The absorption rate is much higher than the elimination rate, so that some special pollutants transfer to and accumulate in lipid and protein tissues of plants and animals. The process that people simulate these natural phenomena to prepare adsorbent to remove pollutants is biomimetic adsorption, and therefore the adsorbent is denoted as biomimetic adsorbent.

Triolein has a high accumulation ability for trace POPs in water (Chiou, 1985). People design and prepare biomimetic adsorbents through imitating the cell structure and using the accumulation ability of lipid for POPs. The biomimetic fat cell (BFC) was synthesized with a hydrophobic nucleolus-triolein and hydrophilic membrane-polyamide (Song et al., 2007). CA-triolein was prepared by embedding triolein in the cellulose acetate (CA) spheres (Liu et al., 2007). Zhang et al. (2010b) found that the lipophilic poly-3-hydroxybutyrate (PHB) produced by many microorganisms contains the same functional groups of the lipid. They prepared PHBBMA with PHB to enrich the trace chlorobenzene and o-nitrochlorobenzene from water and found that all these biomimetic adsorbents had a good enrichment effect for low-concentration POPs.

The adsorption materials prepared by the molecular imprinting technology represent another kind of biomimetic adsorbents. According to the principle of the interaction force or template structure between two kinds of molecule or group, a selective adsorbent was prepared with a template of adsorbate or analogue. For example, imprinting polymer prepared with dibenzothiophene sulphone (DBTS) as the template can separate organosulfur compound such as dibenzothiophene (DBT) and benzothiophene (BT) from the mixture (Castro et al., 2001). Researchers also used mimetic antibody and enzyme as the template to prepare adsorption materials which adsorbed special pollutants according to the interaction between antibody and antigen, and the interaction between enzyme and substrate (Cormack and Mosbach, 1999).

As can be seen from the above materials, the whole or part of some plants and animals in nature can enrich specific substances. Their characters, such as the structure, group interaction and principle of enrichment, are simulated to design and prepare adsorbents. This biomimetic adsorbent can quickly and effectively realize the adsorption/enrichment function of organisms in nature.

\subsection{Adsorption principles of biomimetic adsorbent}

The adsorption principle of biomimetic adsorbent is the same or similar as the enrichment mechanism of organisms. The enrichment process is a balance process of adsorption, absorption, metabolism and storage. For example, the processes of algae enriching trace element include physical adsorption, biosorption, surface deposition, active transport and passive diffusion. Biosorption and active transport are the main ways of bioconcentration, accounting for $80 \%$ and $20 \%$ of the total enrichment amount, respectively. Biosorption is a 
process that organisms adsorb metal ions, non-metallic compounds and solid particles from the solution by complexation and ion exchange. The complexation is the combination of metal ions and the negative function groups of biological ligands. For example, the strong complexation is favorable between carboxyl, sulfonic, thiol, amino, hydroxyl, etc. and different metal ions, such as $\mathrm{Cu}^{2+}, \mathrm{Al}^{3+}$ ( $\mathrm{Li}$ et al., 1998). Some metal ions are adsorbed by algae, while other ions are released. The charge number of released ions such as $\mathrm{Ca}^{2+}, \mathrm{Mg}^{2+}, \mathrm{H}^{+}$is approximately equal to that of adsorbed ions, making the biosorption as an ion exchange process. For most algaes, carboxyl and sulfonic are the main groups involved in the ion exchange process, as alginate and sulphate polysaccharide have a significant ion exchange capacity (Li et al., 1998). Active transport is an intracellular active absorption process related to metabolism, which need energy and some specific enzyme. This is a unique way of enriching trace elements by live organisms. The process, however, is very complicated and associated with the algae fat, sugar, protein, inorganic salt, etc. Irgolic et al. (1971) showed that arsenic are actively involved in the lipid metabolism in some algaes. It can replace the phosphorus atom or nitrogen atom in phosphatidylethanolamine (PE) and phosphatidylcholine (PC) to enter phospholipid biosynthesis process and form some arsenic fats, such as arsenicphosphatidylethanolamine (As-PE) and arsenic-phosphatidylcholine (As-PC). Arsenic in algaes is also involved in glucose metabolism, resulting in the formation of arsenic sugar. Edmonds and Francesconi (1983), Pillips and Depledg (1985) considered that arsenic sugar was transformed from S-adenosylmethionine, and the latter thought the translation occurred in the process of As-PC synthesis by the As-PE. According to the mechanism of the formation of selenoprotein, selenium can be combined with lipid, polysaccharide and protein in the algae. Selenate and sulfate have the same assimilation pathway in the algal. Selenite enteres the algae and replaces the sulphur atom in cysteine (Cys) and methionine (Met) to form selenocysteine ([Se]Cys) and selenomethionine ([Se]Met). The process allowed toxic selenate transfer to a large number of non-toxic selenium compounds.

Biomimetic adsorption is inspired from nature system. The mechanisms of BFC, CA-triolein absorbent and PHBBMA are their dissolution for adsorbates. Triolein-embedded sorbent consists of the supporting materials and the surrounding triolein-embedded cellulose eacetate membrane by embedding triolein into CA spheres. Triolein has a high dissolution capacity for trace lipophilic pollutants in water. A cellulose acetate polymer can be easily molded into different forms such as membranes, fibers and spheres, and its hydrophilicity improves the accessibility of aqueous solutions to the surface of the film. PHB is analogous to the lipid with strong affinity for organic pollutants, so PHBBMA has excellent compatibility with lipophilic chemicals. At the same time, the porous structure of PHBBMA can be regarded as an uptake process of fish. For biomimetic adsorbents, the role forces include strong affinity between adsorbent and adsorbate, hydrophobic force, van der Waals force and principle of "like dissolves like". In the nature system, lipophilic chemicals are always accumulated in the lipid of organism because the pollutants are absorbed by organisms through skin, gill, and feeding, while they are difficult to depurate by metabolism. Therefore, TAOCs are found in almost all plants, animals and human, especially in lipid organs. This phenomenon can inspire people to prepare biomimetic adsorbents to eliminate the pollutants. An important adsorption mechanism of imprinting adsorbent is the strict chimeric structures between adsorbent and adsorbate.

\subsection{Evaluation of biomimetic adsorption}

The principle of biomimetic adsorption is different from that of traditional adsorption. The traditional adsorption only happens on the interface of adsorbent and water. The surface 
area, namely active site, plays most important role in such adsorption. The biomimetic adsorption, however, occurs not only on the interface but also in the inner of adsorbent, because pollutants can be dissolved in the lipid phase. The adsorption isotherms like Langmuir, Freundliuch are useful for predicting the interface adsorption processes, but may not be suitable to interpret the biomimetic adsorption processes.

Adsorption quantity and adsorption rate are two important parameters for evaluating the efficiency of adsorption. The adsorption amount highly depends on the surface area, pore size distribution and properties of surface groups. In nature, the bioconcentration of pollutants that includes surface infiltration and food intake, is also influenced by environment, metabolism and growth of aquatic organisms. The discharge of pollutants from animal bodies.

The models of bioconcentration of pollutants in aquatic animals include steady-state model, two-compartment model and biodynamic model. The steady-state model is based on the balance between biology and water. The biology grows for long time in water, so that many processes approximately reach balance. However, concentrations of some heavy metals are difficult to reach a steady state. Therefore, the steady-state model cannot be applicable for this case. The bioconcentration of heavy metals can be depicted by the mass transfer model. According to conservation of mass, bioaccumulation and metabolism of heavy metals are expressed as:

Enrichment rate of substances in individual $=$ individual input rate - output rate + net generation (conversion) rate

Heavy metals cannot be formed and transformed by life activities, giving rise to the simplified model of the bioaccumulation of heavy metals:

$$
\text { Enrichment }=\text { input }- \text { output }
$$

As a consequence, the interaction process of biology and water can be described by twocompartment model (Croisetiere, 2005). Assuming the accumulation of pollutants in vivo is approximated as two-phase distribution process of pollutants between biology and water, the enrichment and discharge processes can be described by the first-order dynamics (Kahle et al., 2002). Compared with the steady-state model, the two-compartment model afford the calculation of the dynamics parameters of heavy metals at the theory equilibrium before reaching the balance. The biodynamic model includes the absorption process of heavy metals from water and food, the discharge process of heavy metals from organisms, and the dilution due to organisms growing (Luoma et al., 2005). This model includes comprehensive consideration, but the parameter measurement is complex and the practical application is inconvenient.

Biomimetic adsorbent is designed based on the enrichment process of pollutants. The process is similar to that related to the two-compartment model. In order to evaluate the accumulation ability of biomimetic adsorbent, we propose a concept of enrichment factor (EF) which is a dimensionless ratio of the concentrations of adsorbate on biomimetic adsorbent and those in the solution at equilibrium:

$$
\mathrm{EF}=\frac{q_{\mathrm{e}} \rho}{c_{\mathrm{e}}}
$$


where $q_{\mathrm{e}}(\mathrm{mg} / \mathrm{g})$ and $c_{\mathrm{e}}(\mathrm{mg} / \mathrm{L})$ are the concentrations of adsorbate on biomimetic adsorbent and in the solution at equilibrium, respectively. $\rho$, solution density, is about $1000 \mathrm{~g} / \mathrm{L}$.

Although biomimetic adsorbents have been well developed, there is a lack of effective evaluation method as conventional adsorption isotherms and adsorption kinetics are widely applied to describe this adsorption process. The principle of POPs adsorbed on biomimetic adsorbent is "like dissolves like", and POPs concentrations are usually very low. This urges to develop the new evaluation method for biomimetic adsorption.

\section{Application of biomimetic adsorbent}

\subsection{Preparation and application of biomimetic adsorbent}

Polyhydroxyalkanoates (PHAs) are biodegradable polyesters as carbon and energy sources, which are sorted in microorganisms in the form of granules. Zhang et al. (2010a; 2010b) successfully prepared a new biomimetic adsorbent with high porosity named as PHBBMA using $\mathrm{PHB}$, a representative compound of PHAs, produced from microorganism degrading pollutants. As shown in Fig. 4, a modified double emulsion solvent evaporation technique was used for preparing this adsorbent.

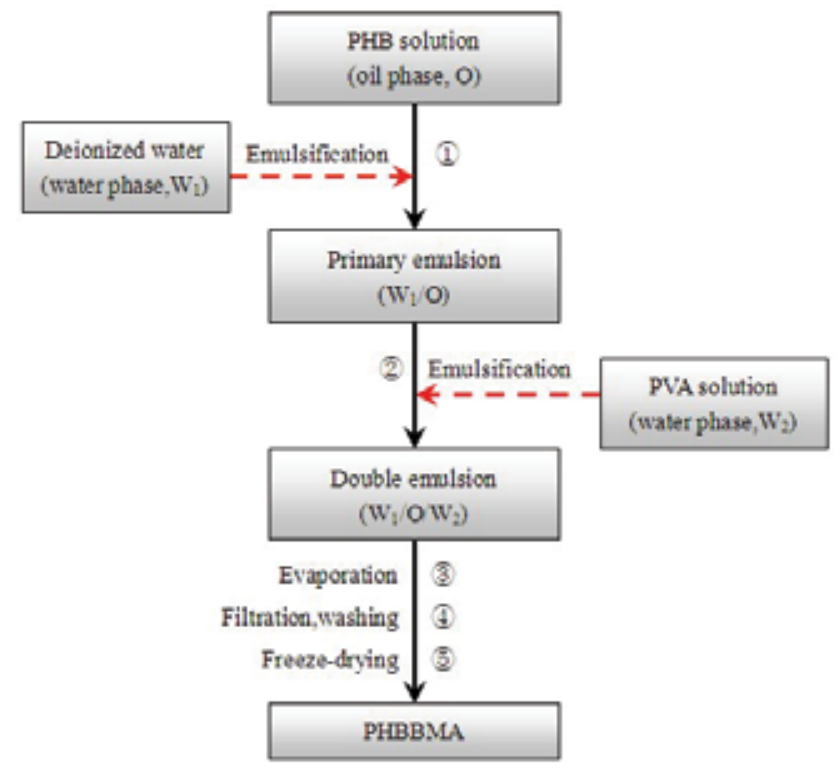

Fig. 4. Diagram of PHBBMA preparation. 1, 2, 3, 4, and 5 express the operation order.

(Zhang et al., 2010a)

The textural and surface characteristics of PHBBMA were shown in Fig.5. The results indicated that PHBBMA was composed of spherical particles with rough surface and micropores. The diameter of the particles was 100-200 $\mu \mathrm{m}$. The BET surface area, total pore volume, and average pore diameter were $8.45 \mathrm{~m}^{2} / \mathrm{g}, 0.0105 \mathrm{~cm}^{3} / \mathrm{g}$, and $4.9 \mathrm{~nm}$, respectively. PHBBMA has a wide pore distribution with diameter ranging from 2 to $90 \mathrm{~nm}$. Few particles were collapsed because the thin PHB walls broke up at the pressure when the big cavities were produced by big water particles (W1). PHBBMA particle size and pore diameter were affected by many factors including the concentrations of oil phase and extra-water 

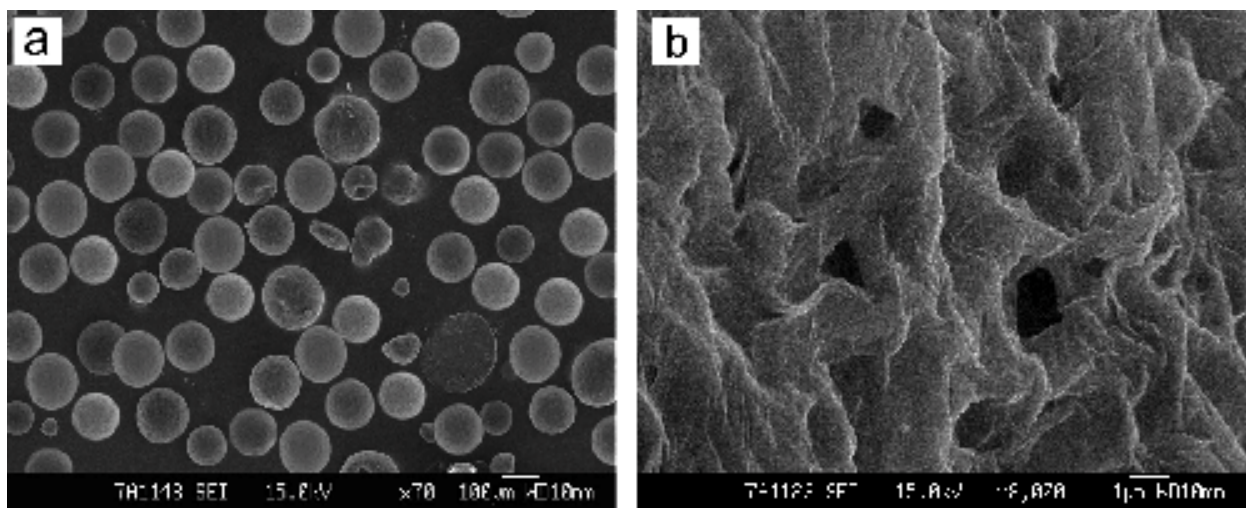

Fig. 5. Scanning electron micrographs of PHBBMA .(a) whole particles $(\times 70)$ and (b) surface of particle $(\times 8000)$. ( Zhang et al., 2010b)

phase $\left(\mathrm{W}_{2}\right)$, the volume ratio of $\mathrm{W}_{1}$ and oil phase, the volume ratio of oil phase and $\mathrm{W}_{2}$, and the stirring speed.

Among the organochlorine compounds $(\mathrm{OClCs})$, chlorobenzene $(\mathrm{CB})$ is structurally similar to $o$-nitrochlorobenzene $(o-\mathrm{NCB})$. It is always released to the environment during manufacturing and application processes as a carrier, a solvent and/or an intermediate (Adebusoye et al., 2007). In addition, $\mathrm{CB}$ and $o-\mathrm{NCB}$ with low concentrations are easily enriched in lipid in organisms owing to their strong hydrophobic and lipophilic properties. Zhang et al. (zhang (b) et al., 2010) evaluates the adsorption capacity of PHBBMA using CB and $o$-NCB as target compounds in their extreme low concentration.

Adsorption isotherms of $\mathrm{CB}$ and $o-\mathrm{NCB}$ on PHBBMA under different temperature are shown in Fig.6. It was clear that the adsorption amount of $\mathrm{CB}$ was always greater than that of $o-\mathrm{NCB}$ under the same conditions. This is because that the adsorption amount has a positive correlation with the affinity between PHBBMA and the compounds. CB had stronger affinity than $o-\mathrm{NCB}$ due to the absence of a hydrophilic group $\left(-\mathrm{NO}_{2}\right)$. Furthermore, the adsorption capacity decreased when the temperature increased for both $\mathrm{CB}$ and $o-\mathrm{NCB}$. The acting force between the PHBBMA and $\mathrm{CB}$ or $o-\mathrm{NCB}$ is van der Waals Force and hydrophobic force, suggesting that the process of PHBBMA adsorption is a physical adsorption.
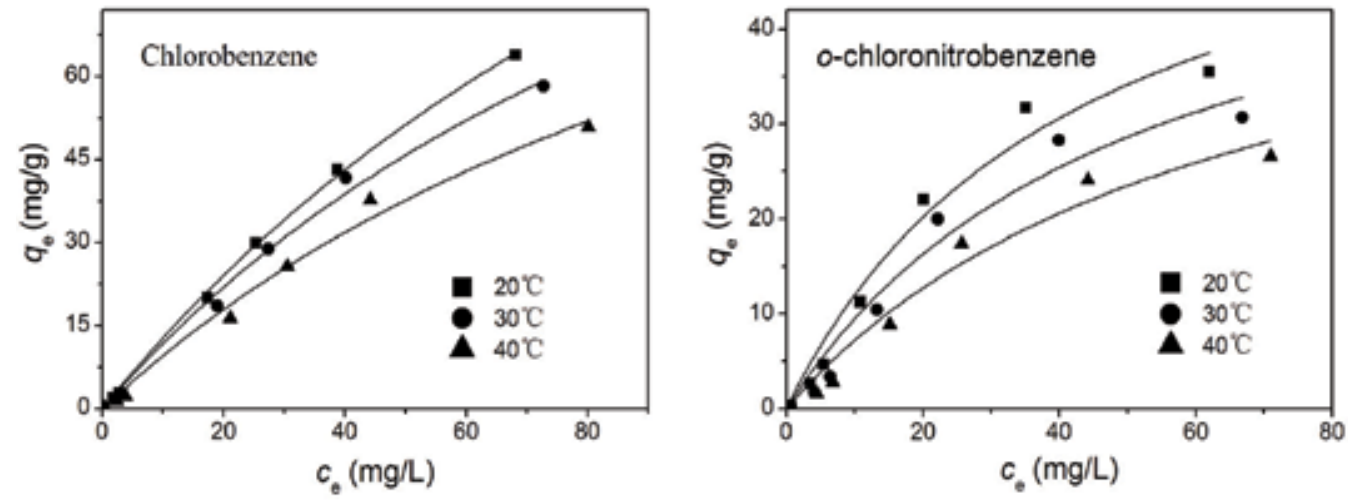

Fig. 6. Langmuir adsorption isotherms for CB and o-NCB onto PHBBMA. (Zhang et al., 2010b) 
EF was employed as a criterion for the evaluation of the adsorption ability for different pollutants. The EF of PHBBMA for CB and $o-\mathrm{NCB}$ was calculated by equilibrium data from experiments and Sips model fitting curves. Fig. 7 presents the variety of EF as a function of different equilibrium concentrations. For both $\mathrm{CB}$ and $o-\mathrm{NCB}, \mathrm{EF}$ showed a pattern that increased at first and then decreased with the increasing equilibrium concentrations. It can be interpreted by the fact that there are enough available active adsorption sites at the surface of PHBBMA in the case of low equilibrium concentrations. The value of $q_{\mathrm{e}}$ increased faster than $c_{\mathrm{e}}$, indicating that EF increased with the increase of $c_{\mathrm{e}}$. In the case of high concentrations, however, adsorbent was gradually saturated, resulting in a slow increase in $q_{\mathrm{e}}$ and a decrease in EF with the increase of $c_{\mathrm{e}}$. The change of EF versus temperature for $\mathrm{CB}$ and $o$-NCB had the same trends, i.e. $\mathrm{EF}_{20^{\circ} \mathrm{C}}\left(\mathrm{EF}\right.$ at $\left.20^{\circ} \mathrm{C}\right)>\mathrm{EF}_{30^{\circ} \mathrm{C}}>\mathrm{EF}_{40^{\circ} \mathrm{C}}$ because the increasing temperature significantly increases aqueous solubility, although it causes a decrease in $K_{\text {ow }}$ (Finizio et al., 2001). High $K_{o w}$ and low aqueous solubility imply strong lipophilic and hydrophobic properties, causing the easy adsorption of compounds for PHBBMA.
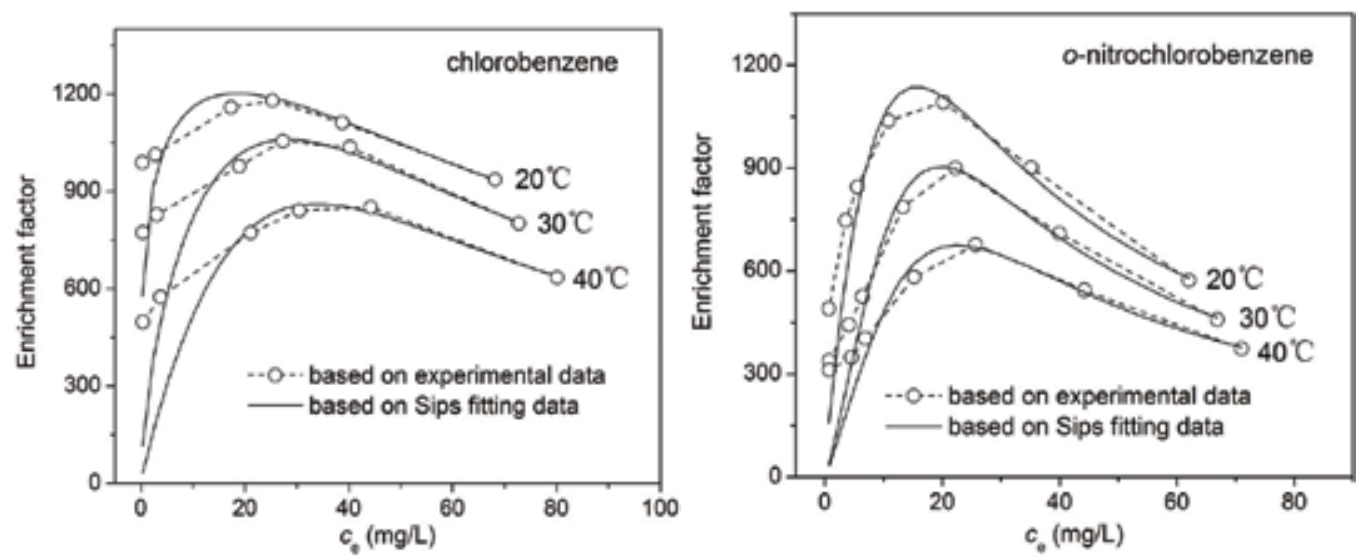

Fig. 7. EF variety of PHBBMA for $\mathrm{CB}$ and $o-\mathrm{NCB}$ with equilibrium concentrations at different temperatures. (Zhang et al., 2010b)

\subsection{Preparation and application of lipoid adsorbent}

Triolein is one of the most important lipid components and the main materials for soap preparation. According to the principle of similarity and intermiscibility, triolein has a strong absorbability for trace level of hydrophobic organic pollutants. Previous studies (Liu et al., 2007; Liu et al., 2009; Huo et al., 2005; Ru et al., 2007; Qu, 2008) showed the preparation of a biomimetic adsorbent by embedding triolein into cellulose acetate (CA) spheres. The section structure is shown in Fig. 8. Triolein was wrapped entirely into CA spheres through optimizing the preparation method, and thus it can not be expelled during the adsorption process. The hydrophilicity of (CA polymer improved the accessibility of trace organic pollutants to the surface of the adsorbent. In addition, the formation of mesh structures of CA (Fig. 9) provided enough channels for organic pollutant molecular transfer through CA. This design weakens the diffusion and the mass transfer obstacle between the lipid phase and the water phase by providing a hydrophilic and porous film at the top surface of the obtained biomimetic adsorbent. 


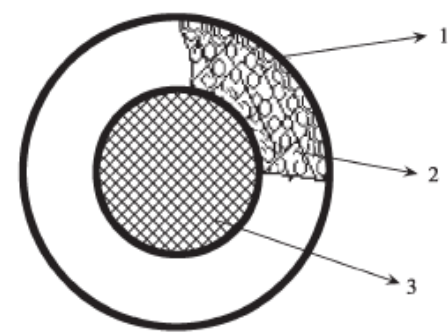

Fig. 8. Designed structure of triolein-embedded sorbent. (1) cellulose acetate membrane, (2) triolein and (3) supporting materials. (Qu, 2008)

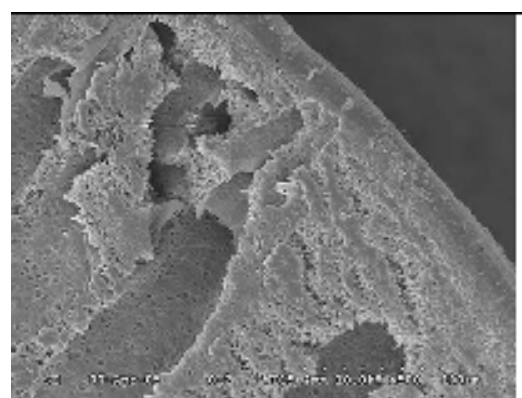

(a)

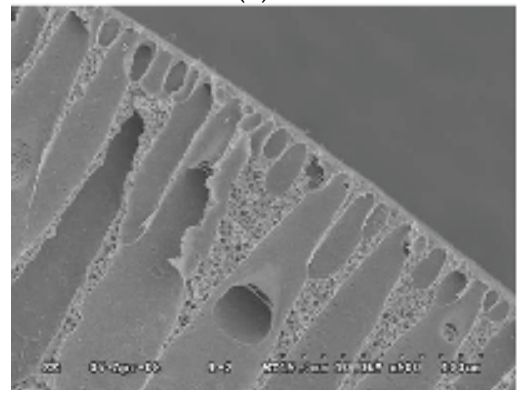

(c)

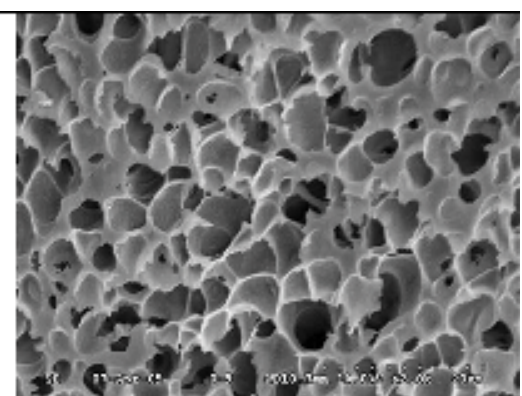

(b)

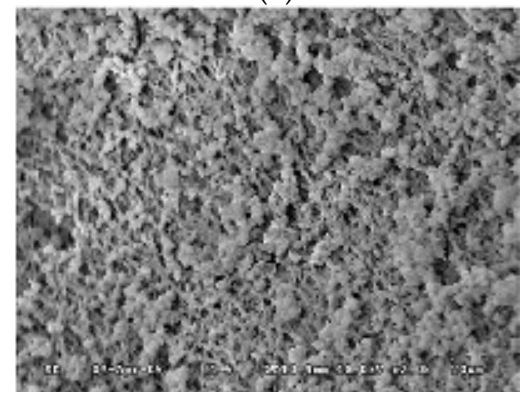

(d)

Fig. 9. SEM micrographs of cross-sections of CA and CA-triolein adsorbents. (a) CA adsorbent $(\times 500)$, (b) CA adsorbent $(\times 2000)$, (c) CA-triolein adsorbent containing $2.0 \%$ $(\mathrm{w} / \mathrm{w})$ triolein $(\times 500)$, and $(\mathrm{d})$ CA-triolein adsorbent containing $2.0 \%(\mathrm{w} / \mathrm{w})$ triolein $(\times 2000)$. (Liu et al., 2007)

Liu et al. (2007) investigated the adsorption efficiency for selected POPs (e.g. aldrin, dieldrin, endrin and heptachlor epoxide) by this biomimetic adsorbent. The comparison of CA-triolein and granular activated carbon (GAC) for dieldrin removal was also performed. Results showed that the adsorption capacity of CA-triolein was larger than that of GAC, although GAC had high removal rate in the first $4 \mathrm{~h}$ of adsorption. Adsorption isotherms of the selected POPs exhibited linear isotherms, which indicated that the POPs were mainly adsorbed onto CA-triolein by a partition mechanism. Partitioning of organic compounds between water and triolein may be similar to that between organic solvent and water. The partition coefficient of POPs was closely related to their hydrophobicity. The higher the hydrophobicity of POPs was, the larger the adsorption amount was. Furthermore, the 
reusability of the biomimetic absorbent was tested. Although the CA-triolein was regenerated five times, the removal efficiencies were almost unchanged.

Due to the particular adsorbed amount and bioaffinity, layer by later (LBL)-assembled hydrogel films have been applied in the research of sustained release of medicines and genetic science. Ding et al. (2009) loaded alginate sodium (ALG) and chitosan to weakly cross-link melamine formaldehyde (MF) colloidal particles, preparing a kind of saccate biomimetic adsorbent. 2,4-dichlorophenol and salicylic acid were adsorbed by this adsorbent. Fig. 10 shows the SEM pictures the adsobents before and after adsorption. The gap between two shells was filled after adsorption. This phenomenon indicated that the organic componds entered into the internal of bursa. The cliff of bursa was gradually uplifted with the increase of adsorption, making the adsorbent like a balloon filled with gas. Fig. 11 shows that the adsorption isotherms of DCP and SA cpmpiled with the Langmuir equation.
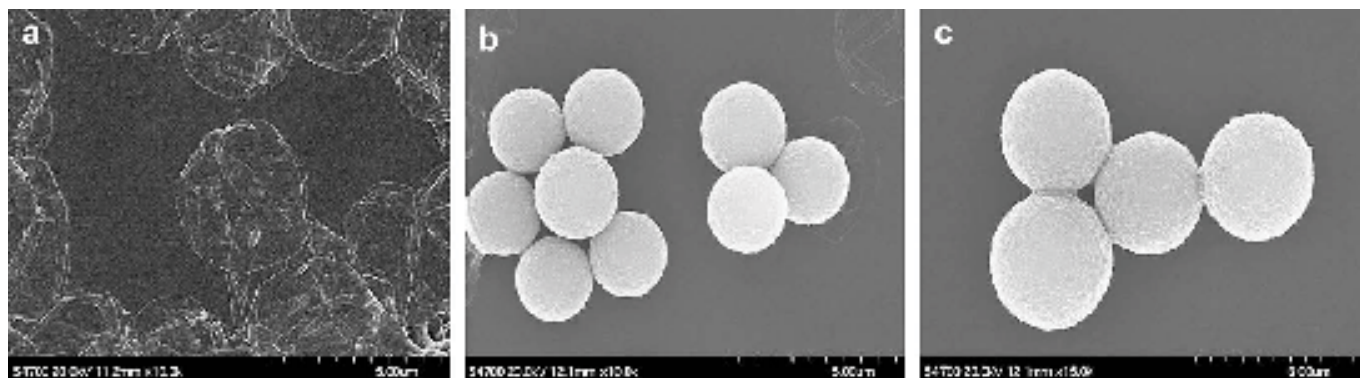

Fig. 10. SEM images of the (ALG/CHI)5 hollow shells (a) and shells upon incubating the preformed shells in various organic solutions in pure water medium: (b) DCP; (c) SA. Scale bars are $5 \mathrm{~mm}$ for a, $5 \mathrm{~mm}$ for $\mathrm{b}$ and $3 \mathrm{~mm}$ for c. (Ding et al., 2009)

There are a lot of materials that can serve as the biomimetic adsorbents are in nature. If these materials are successfully used to manufacture the commercial adsorption materials, they will have such a distinct advantage as high performance-to-price ratio. Baruch-Teblum (2010) used the a-cyclodextrin as the basic material and adopted the method of miniemulsion polymerization to produce a series of controlled-size nanoparticles under the link of isophorone diisocyanate (Fig.12). In the molecular structure of cyclodextrin, there was hydrophobic channels (as shown in Fig.13), which could adsorb organic pollutants especially for the pollutants with benzene ring in the interface of water-nanoparticle. Toluene and phenol were tested as the model pollutants to evaluate adsorption capacity. Results indicated that the nanoparticles exibited good adsorption. Compared with inorganic adsorbents, biomass materials can be made into different shapes such as granule, powder and membrane depending on the used conditions needed.

In addition to the nature polymer, super hydrophobic and lipophilic adsorption materials were synthesized in the laboratory. Although some materials did not have large specific surface area, their adsorption capacities were much higher than the porous materials, showing unique advantages of biomass adsorbents in adsorption principles and mechanism. Zhang et al. (2009) produced a super hydrophobic polydivinylbenzene with high specific surface area and pore volume, controllable pore size distribution, super hydrophobicity and super lipotropy. The adsorption capacity for organic pollutants was compared with activated carbon and Amberlite XAD-4 resin, as illustrated in Fig 14. It could found that the 
adsorption capacity of polydivinylbenzene was much higher than that of activated carbon and Amberlite XAD-4 resin, suggesting good potential for applications.

Activated carbon is dominant in the field of adsorption materials. However, activated carbon can not meet the requirement of purification of wastewater containing TAOCs. Biomimetic adsorption materials are now reported frequently owing to their higher adsorption capacity than activated carbon. With the development of bionics and material science, the biomimetic adsorbents can see more applications to remove TAOCs on a large scale.

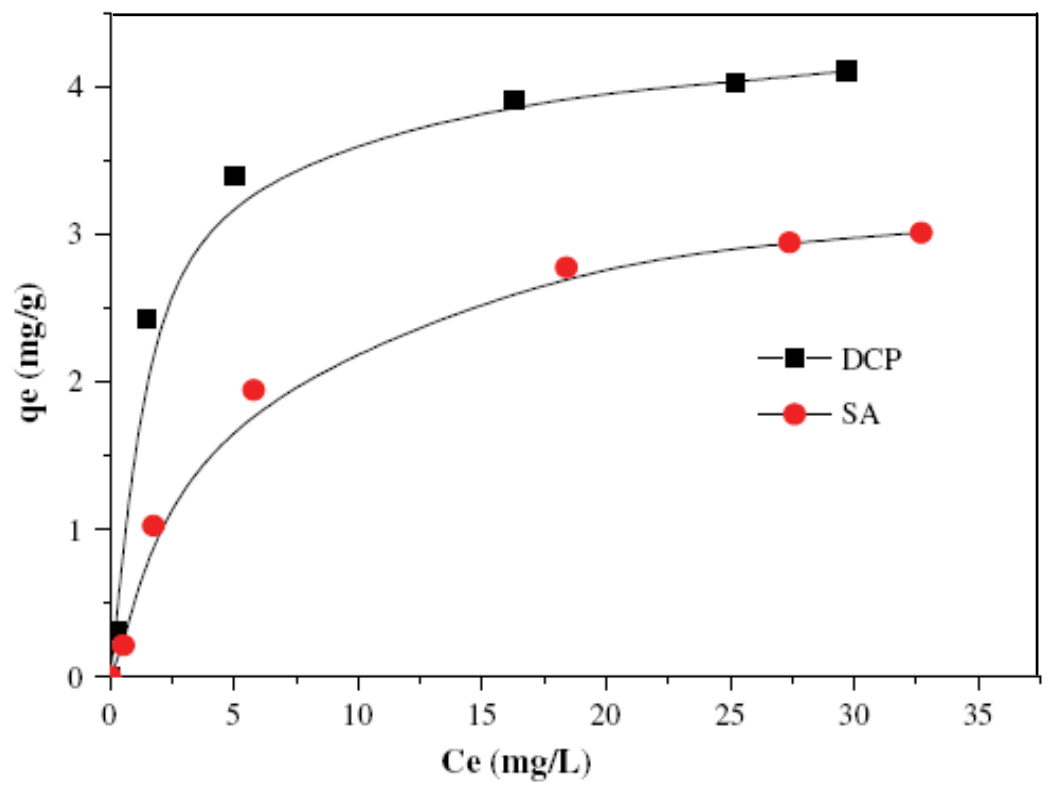

Fig. 11. Loading isotherms of DCP and SA into ALG/CHI shells: experimental equilibrium uptakes and the Langmuir model fitting ( $\mathrm{pH} 7 ; 25^{\circ} \mathrm{C}$ ). (Ding et al., 2009)
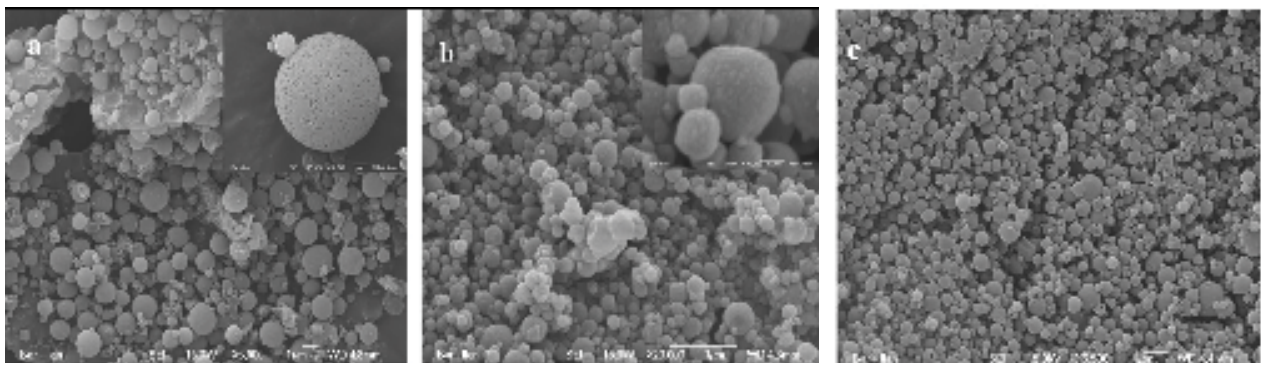

Fig. 12. HR-SEM images of (a) sample A, (b) sample B, and (c) sample D. (Baruch-Teblum et al., 2010) 

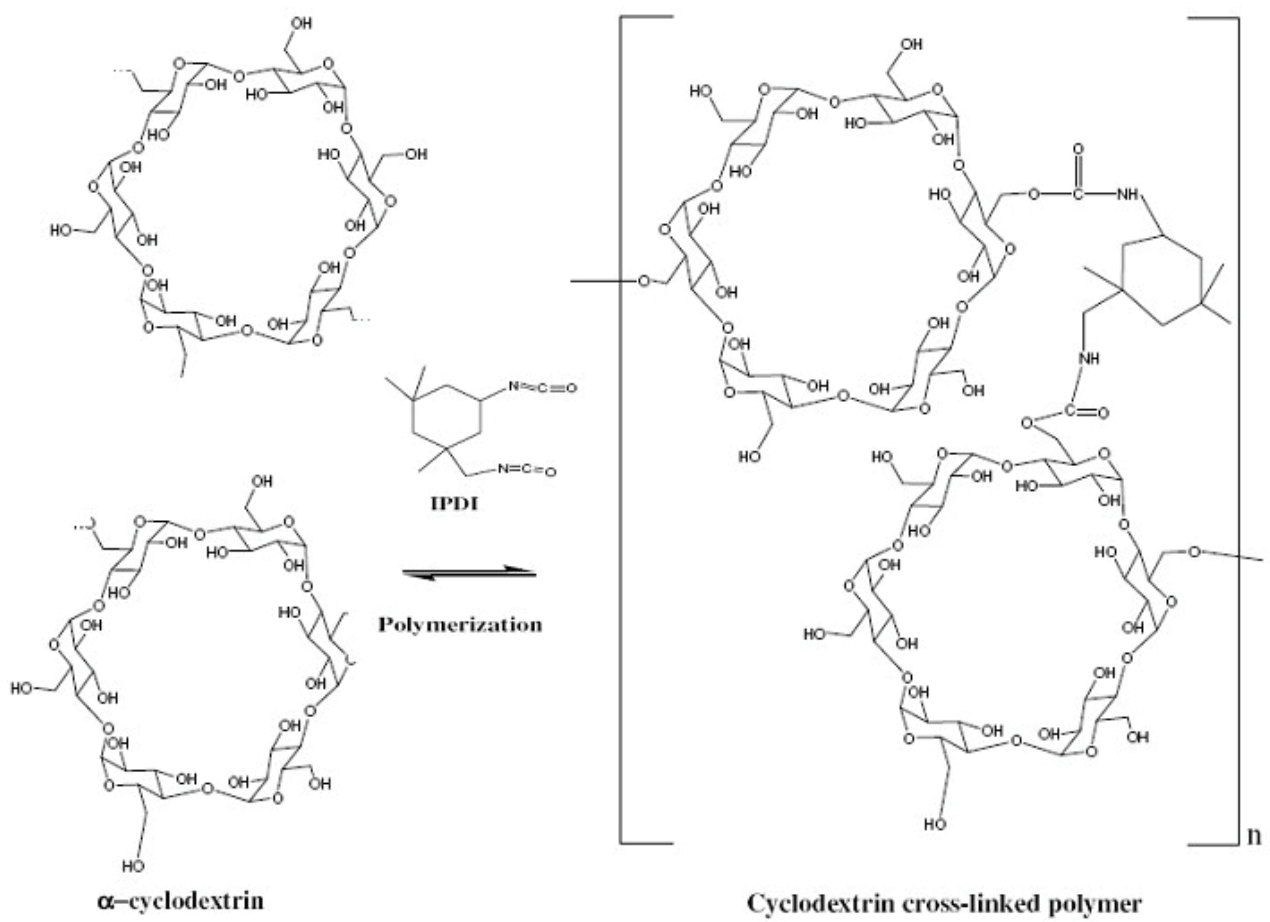

(Cyclodextrin-isophoronecarbamate copolymer)

Fig. 13. Copolymerization of cyclodextrine-isophoronecarbamate copolymer. (BaruchTeblum et al., 2010)
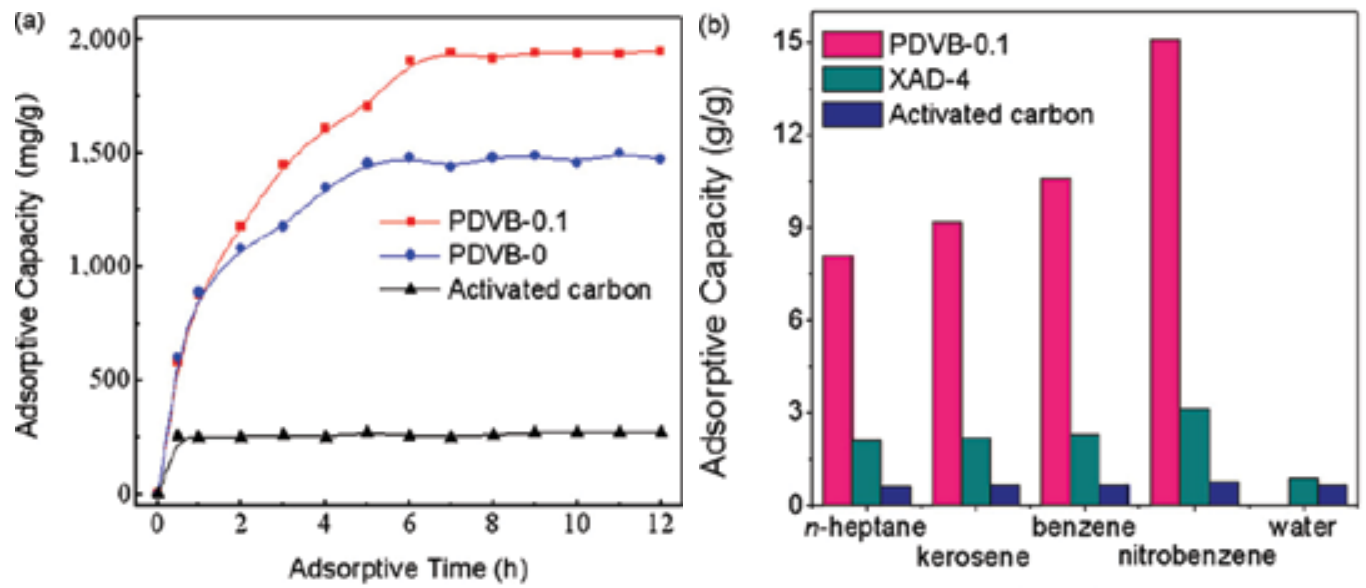

Fig. 14. Dependence of adsorptive capacity for benzene on adsorption time over various samples. (b) Saturated adsorptive capacity of probing molecules in liquid phase over various samples. (Zhang et al., 2009) 


\section{Biomimetic adsorption and advanced treatment}

Although pollutants can be effectively separated from the aqueous phase by adsorbents, an improper treatment of saturated adsorbent will result in secondary pollution. Biological treatment is one of the most common technique for wastewater treatment, as evidenced from the fact that $65 \%$ of wastewater all over the world is treated by biological process (the percentage is up to $95 \%$ particularly for the municipal sewage). However, biodegradation is affected by two factors: the solubility and concentration of pollutants. Organic compounds with higher solubility are more easy to be degraded by biomass than those with lower solubility. This is why PCB compounds with high chlorine content and with lower solubility are more difficultly to be degraded than those with low chlorine content. Furthermore, if the concentration of pollutant is too low, there will be a lack of nutrients needed for the biomass and the enzyme will not work efficiently. In general, the low concentration of contaminants can not make full use of the degradation ability and satisfy the growth requires of microorganisms. In contrast, the microorganism will be toxic damaged if the concentration of pollutant is too high. In the low concentration range, the degradation rate is linearly enhanced with the increase of contaminants concentration until the maximal concentration value is reached. Therefore, the coupling of adsorption and microbiological degradation for the treatment of POPs will break the balance constantly, thus the contaminants will be removed constantly by the new dynamic process.

Vogt et al. (2004) reported that chlorobenzene can be adsorbed by granular active carbon from ground water and then degraded by biomass. The adsorptive capacity of chlorobenzene on granular active carbon is up to $450 \mathrm{mg} / \mathrm{g}$ and permineralization of chlorobenzene was achieved through biomass. The amount of the lipophilic POPs on active carbon and bentonite is limited, but can be significantly improved by addition of PHBBMA and other lipoid adsorbents. The treatment efficiency of refractory wastewater is not satisfactory by the way of biological technique owing to two issues. On the one hand, many pollutants (e.g. POPs) are foreign substances for environment, thus the microorganism is lack of corresponding enzyme system to produce degrading enzyme. On the other hand, the amount of functional microorganism in sludge is not enough to degrade the pollutants. The first issue can be resolved by long-time acclimation because this allows the formation of proper enzyme secreted from microorganism to degrade POPs. To overcome the second problem, polymerase chain reaction-denaturing gradient gel electrophoresis (PCR-DGGE) technique can be explored to isolate functional microorganisms bacteria in sludge, and therefore higher efficiency will be obtained by the introduction of functional microbial into the biological treatment system. The conception of PHBBMA, a kind of biomimetic adsorbent, combines the adsorption technology for TAOCs and immobilized cell technology of microorgnism, which use PHAs as the main material and cyclodextrin, tween, polyving alcohol as the excipient. This adsorbent is the immobilized cell support with adsorption capacity, which can embed single or hybrid functional bacteria. TAOCs can be enriched on the surface of PHBBMA, which not only impulses the process of microbial degradation, but also improves the adaptability and resistance of functional bacteria. Furthermore, it is also easy to separate biomass from the aqueous solution. With the pre-enrichment of biomimetic adsorbents for POPs, followed by the enhanced biodegradation of functional bacteria, TAOCs can be detoxificated rapidly and thoroughly. This coupling technology, namely biomimetic adsorption-functional microbial degradation technology, is proved to be a possible practical technology. 
Moreover, the combination of adsorption and chemical degradation such as adsorptioncatalytic wet oxidation/reduction process and, adsorption-catalytic supercritical water oxidation/reduction may be effective in removing various contaminants with different concentrations. During the control of industrial wastewater, the advanced oxidation/ reduction often served for the pretreatment process, advanced treatment and emergency of highly toxic and non-biodegradable pollutants. It includes photocatalytic oxidation, wet (supercritical) oxidation, Fenton oxidation, hydrogenation reduction and metal reduction, etc. Photocatalytic oxidation that utilizes the power of ultraviolet or visible light and semiconductor catalyst can produce $\mathrm{OH}$ radical, which possesses high oxidation capacity for degrading of many organic pollutants. Wet oxidation, especially the condition of supercritical water, can mineralize organic compounds to $\mathrm{CO}_{2}$ and $\mathrm{H}_{2} \mathrm{O}$ with in a very short time ( $\sim 5 \mathrm{~min})$, which is an efficient advanced technology in treating the wastewater containing concentrated, toxic and non-biodegradable contaminants. Fenton technique is based on the reaction of $\mathrm{Fe}^{2+}$ and $\mathrm{H}_{2} \mathrm{O}_{2}$, which can produce $\mathrm{OH}$ radical to oxidize the organic pollutants. In contrary, advanced reduction technology is able to remove the functional groups such as halogene and nitryl by the reductive substance, which can significantly decrease the toxicity of the organic pollutants. Based on the above principles, to prepare the composite materials combined with biomimetic adsorbents and catalysts, and coupled with the mentioned advanced oxidation or reduction processes is capable of achieving the transform and degradation of the TAOCs. Furthermore, the adsorbents can be regenerated automatically without special treatment, thus a development route of biomimetic adsorbents which integrates multi-coupling technologies needs to be constructed.

\section{Acknowledgements}

The authors thank the State Key Program of National Natural Science of China (No. 21037001) and the Hi-Tech Research and Development Program (863) of China (No. 2006AA06Z378) for supporting this work.

\section{References}

Adebusoye, A.; Picardal, W. \& Ilori O. (2007). Aerobic degradation of di- and trichlorobenzenes by two bacteria isolated from polluted tropical soils, Chemosphere, 66, 10, 1939-1946, 0045-6535.

Agenson, K.; Oh, J. \& Urase, T. (2003). Retention of a wide variety of organic pollutants by different nanofiltration/reverse osmosis membranes: controlling parameters of process. Journal of Membrane Science, 225, 1-2, 91-103, 0376-7388.

Ahmed, E.N. \& Aly, M.A.A. (2004). Organochlorine contamination in some marketable fish in Egypt. Chemosphere, 54, 1401-1406, 0045-6535.

Andersen, G.; Kovacs, K.M.; Lydersen, C.; Skaare, J.U.; Gjertz, I. \& Jenssen, B.M. (2001). Concentrations and patterns of organochlorine contaminants in white whales (Delphinapterus leucas) from Svalbard, Norway. Science of the Total Environment, 264, 3, 267-281, 0048-9697.

Armitage J. \& Gobas, F. (2007). A terrestrial food-chain bioaccumulation model for POPs. Environmental Science and Technology, 41, 11, 4019-4025, 0013-936X. 
Azza, K.; Ahmed, E.N.; Tarek, O.S.; Amany, E.S. \& Aly M.A.A. (2004). Polychlorinated biphenyls and chlorinated pesticides in mussels from the Egyptian Red Sea coast. Chemosphere, 54, 1407-1412, 0045-6535.

Barber, R. \& Warlen, S. (1979). Organochlorine insecticide residues in deep sea fish from $2500 \mathrm{~m}$ in the Atlantic Ocean. Environmental Science and Technology, 13, 9, 1146-1148. 0013-936X.

Baruch-Teblum, E.; Mastai, Y.; \& Landfester K., (2010). Miniemulsion polymerization of cyclodextrin nanospheres for water purification from organic pollutants. European Polymer Journal, 46, 8, 1671-1678, 0014-3057.

Bellona, C.; Drewes, J.; Xu, P. \& Amy, G. (2004). Factors affecting the rejection of organic solutes during NF/RO treatment-a literature review. Water Research, 38, 12, 2795, 0043-1345.

Betts, K.; Pelley, J. \& Renner, R. (2005). A new record for PBDEs in people | POPs treaty targets further chemicals | Canada's research funding system works | Do male frogs naturally have female traits? I Are low levels of lead in water risky. Environmental Science and Technology, 39, 14, 296A-298A, 0013-936X.

Braunegg, G.; Lefebvre, G. \& Genser, K.F. (1998). Polyhydroxyalkanoates, biopolyesters from renewable resources: physiological and engineering aspects. Journal of Biotechnology, 65, 127-161, 0168-1656.

Carpentier, S.; Moilleron, R.; Beltran, C.; Hervé, D. \& Thévenot, D. (2002). Quality of dredged material in the river Seine basin (France). II. Micropollutants. The Science of the Total Environment, 299, 1-3, 57-72, 0048-9697.

Castro, B.; Whicombe, M.J.; Vulfson, E.N.; Vazquez-Duhalt, R. \& Bárzana, E. (2001). Molecular imprinting for the selective adsorption of organosulphur compounds present in fuels. Analytica Chimica Acta, 435, 83-90, 0003-2670.

Chellam, S. \& Taylor J. (2001). Simplified analysis of contaminant rejection during groundand surface water nanofiltration under the information collection rule. Water Research, 35, 10, 2460-2474, 0043-1345.

Chen, G.Q. \& Wu, Q. (2005). The application of polyhydroxyalkanoates as tissue engineering materials. Biomaterials, 26, 33, 6565-6578, 0412-9612.

Chiou, T. (1985). Partition coefficients of organic compounds in lipid-water systems and correlations with fish bioconcentration factors. Environmental Science and Technology, 19, 57-62, 0013-936X.

Chu, H.; Cao, D.; Dong, B. \& Qiang, Z. (2010). Bio-diatomite dynamic membrane reactor for micro-polluted surface water treatment. Water Research, 44, 5, 1573-1579, 0043-1345.

Corcia, A.; Cavallo, R.; Crescenzi, C. \& Nazzari, M. (2000). Occurrence and Abundance of Dicarboxylated Metabolites of Nonylphenol Polyethoxylate Surfactants in Treated Sewages. Environmental Science and Technology, 34, 18, 3914-3919, 0013-936X.

Diamanti-Kandarakis, E.; Bourguignon P.; Giudice C.; Hauser, R.; Prins, S.; Soto, M.; Zoeller, T. \& Gore, C. (2009). Endocrinedisruptingchemicals: an Endocrine Society scientific statement. Endocrine Reviews, 30, 4, 293-342, 0163-769X.

Díaz-Cruz, M. \& Barceló, D. (2008). Trace organic chemicals contamination in ground water recharge. Chemosphere, 72, 3, 333-342, 0045-6535.

Ding, Y.; Zhao, Y.; Tao, X.; Zheng, Y. \& Chen, J. (2009). Assembled alginate/chitosan microshells for removal of organic pollutants. Polymer, 50, 13, 2841-2846, 0032-3861. 
Ducom, G. \& Cabassud, C. (1999). Interests and limitations of nanofiltration for the removal of volatile organic compounds in drinking water production. Desalination, 124, 1-3, 115-123, 0011-9164.

Edmonds, J.S. \& Francesconi, K.A. (1983). Arsenic-containing ribofuranosides: isolation from brown kelp Ecklonia radiata and nuclear magnetic resonance spectra. Journal of the Chemical Society Perkin Transactions 1, 1, 2375-2382, 0368-1769.

Ferm, V. H.; Saxon A. \& Smith W. (1971). The teratogenicprofile of sodium arsenate in the golden hamster.Archives of Environmental Health, 22, 577- 560, 0003-9896.

Finizio, A. \& Guardo, D. (2001). Estimating temperature dependence of solubility and octanol-water partition coefficient for organic compounds using RP-HPLC. Chemosphere, 45, 6-7, 1063-1070, 0045-6535.

Gao, B.; Gao, Y. \& Li, Y. (2010). Preparation and chelation adsorption property of composite chelating material poly(amidoxime) $/ \mathrm{SiO}_{2}$ towards heavy metal ions. Chemical Engineering Journal, 158, 3, 542-549, 1385-8947.

Gioia, R.; Sweetman, A. \& Jones, K. (2007). Coupling Passive Air Sampling with Emission Estimates and Chemical Fate Modeling for Persistent Organic Pollutants (POPs): A Feasibility Study for Northern Europe. Environmental Science and Technology, 41, 7, 2165-2171, 0013-936X.

Götz, R.; Bauer, O.; Friesel, P. \& Roch, K. (1998). Organic trace compounds in the water of the River Elbe near Hamburg Part I. Chemosphere, 36, 9, 2085-2101, 0045-6535.

Hebert, A.; Forestier, D.; Lenes, D.; Benanou, D.; Jacob, S.; Arfi, C.; Lambolez L. \& Levi, Y. (2010). Innovative method for prioritizing emerging disinfection by-products (DBPs) in drinking water on the basis of their potential impact on public health. Water Research, 44, 10, 3147-3165, 0043-1354.

Hollender, J.; Zimmermann, S; Koepke, S.; Krauss, M.; McArdell, C.; Ort, C.; Singer, H.; Gunten U. \& Siegrist, H. (2009). Elimination of Organic Micropollutants in a Municipal Wastewater Treatment Plant Upgraded with a Full-Scale Post-Ozonation Followed by Sand Filtration. Environmental Science and Technology, 43, 20, 78627869, 0013-936X.

Huo, J.; Liu, H.; Qu, J.; Wang, Z.; Ge, J. \& Liu, H. (2005). Preparation and characteristic of triolein-embedded composite sorbents for water purification. Separation and Purification Technology, 44, 1, 37-43, 1383-5866.

Ikemoto, T.; Tu, N.; Watanabe, M.; Okuda, N.; Omori, K.; Tanabe, S.; Tuyen B. \& Takeuchi, I. (2008). Analysis of biomagnification of persistent organic pollutants in the aquatic food web of the Mekong Delta, South Vietnam using stable carbon and nitrogen isotopes. Chemosphere, 72, 1, 104-114, 0045-6535.

Ji, L.; Liu, F.; Xu, Z.; Zheng, S. \& Zhu, D. (2009). Zeolite-Templated Microporous Carbon As a Superior Adsorbent for Removal of Monoaromatic Compounds from Aqueous Solution. Environmental Science and Technology, 43, 20, 7870-7876, 0013-936X.

Khardenavis, A.A.; Kumar, M.S.; Mudliar, S.N. \& Chakrabarti, T. (2007). Biotechnological conversion of agro-industrial wastewaters into biodegradable plastic, ploy $\beta$ hydroxybutyrate. Bioresource Technology, 98, 18, 3579-3584, 0960-8524.

Khim, J.; Lee K.; Kannan, K.; Villeneuve, D.; Giesy, J. \& Koh, C. (2001). Trace Organic Contaminants in Sediment and Water from Ulsan Bay and Its Vicinity, Korea. Archives of Environmental Contamination and Toxicology, 40, 2, 141-150, 1432-0703. 
Kolpin, D.; Furlong, E.; Meyer, M.; Thurman, E.; Zaugg, S.; Barber, L. \& Buxton, H. (2002). Pharmaceuticals, Hormones, and Other Organic Wastewater Contaminants in U.S. Streams, 1999-2000: A National Reconnaissance. Environmental Science and Technology, 36, 6, 1202-1211, 0013-936X.

Laber, J.; Raimund, H. \& Shrestha, R. (1999). Two-stage constructed wetland for treating hospital wastewater in Nepal. Water Science and Technology, 40, 3, 317-324, 02731223.

Lee, S.; Lueptow \& R.M. (2001). Membrane rejection of nitrogen compounds. Environmental Science and Technology, 35, 14, 3008-3018, 0013-936X.

Li, Z.; Guo, S.; Li, L. \& Cai, M. (1998). Mechanisms of trace elements' bioaccumulation by algae. Journal of South China University of Technology, 26, 2, 33-37, 1000-565X. (in Chinese).

Liu, H.; Qu, J.; Dai, R.; Ru, J. \& Wang, Z. (2007). A biomimetic absorbent for removal of trace level persistent organic pollutants from water. Environmental Pollution, 147, 2, 337342, 0269-7491, 0469-7291.

Liu, H.; Gan, J. \& Jia, X. (2008). Progress and status of research on persistent organochlorine compounds in cetaceans. South China Fisheries Science, 4, 5, 74-80, 1673-2227.

Liu, H.; Ru, J.; Qu, J.; Dai, R.; Wang, Z. \& Hu, C. (2009). Removal of persistent organic pollutants from micro-polluted drinking water by triolein embedded absorbent. Bioresource Technology, 100, 12, 2995-3002, 0960-8524.

Liu, W.; Chen, J.; Lin, X.; Fan Y. \& Tao, S. (2007). Residual concentrations of micropollutants in benthic mussels in the coastal areas of Bohai Sea, North China. Environmental Pollution, 146, 2, 0269-7491, 0469-7291.

Lohmann, R.; Breivik, K.; Dachs, J. \&. Muir, D. (2007). Global fate of POPs: Current and future research directions. Environmental Pollution, 150, 1, 150-165, 0269-7491.

Lohmann, R. \& Muir, D. (2010). Global Aquatic Passive Sampling (AQUA-GAPS): Using Passive Samplers to Monitor POPs in the Waters of the World. Environmental Science and Technology, 44, 3, 860-8643, 0013-936X.

Magnusson, K.; Ekelund, R. Grabic, R.; \& Bergqvist, P. (2006). Bioaccumulation of PCB congeners in marine benthic infauna Marine. Environmental Research, 61, 4, 379-395.

Mary, G. \& Birgit, M.B. (1999). Contaminant residue levels in arctic wolves (Canis lupus) from the Yukon Territory, Canada. Science of the Total Environment, 243-244, 00489697.

Muir, D.; Savinova, T.; Savinov, V.; Alexeeva, L.; Potelov, V. \& Svetochev, V. (2003). Bioaccumulation of PCBs and chlorinated pesticides in seals, fishes and invertebrates from the White Sea, Russia. Science of the Total Environmennt, 306, 111131, 0048-9697.

Nghiem, L.D.; Schäfer, A.I., Elimelech \& M., (2004). Removal of natural hormones by nanofiltration membranes: measurement, modeling, and mechanisms. Environmental Science and Technology, 38, 6, 1888-1896, 0013-936X.

Nghiem, L.; Tadkaew, N.\& Sivakumar, M. (2009). Removal of trace organic contaminants by submerged membrane bioreactors. Desalination, 236, 1-3, 127-134, 0011-9164.

Ockenden, W.A.; Steinnes, E.; Parker, C. \& Jones, K.C. (1998). Observations on persistent organic pollutants in plants: implications for their use as passive air samplers and for POP cycling. Environmental Science and Technology, 32, 18, 2721-2726, 0013-936X. 
Perihan, B.K. \& Hulya, B.O. (2004). A survey to determine levels of chlorinated pesticides and PCBs in mussels and seawater from the Mid-Black Sea Coast of Turkey. Marine Pollution Bulletin, 48, 1076-1083, 0025-326X.

Phillips, D.J.H. \& Depledge, M.H. (1985). Metabolic pathways involving arsenic in marine organisms: A unifying hypothesis. Marine Environmental Research, 17, 1, 1-12, 0141 1136.

Postle, J.; Rheineck, B.; Allen, P.; Baldock, J.; Cook, C.; Zogbaum, R. \& VandenBrook, J. (2004). Chloroacetanilide Herbicide Metabolites in Wisconsin Groundwater: 2001 Survey Results. Environmental Science and Technology, 38, 20, 5339-5343, 0013-936X.

$\mathrm{Qu}$, J. (2008). Research progress of novel adsorption processes in water purification: A review. Journal of Environmental Sciences, 20, 1, 1-3, 1001-0742, 1878-7320.

Ren, Y.; Zhang, M. \& Zhao, D. (2008). Synthesis and properties of magnetic Cu(II) ion imprinted composite adsorbent for selective removal of copper. Desalination, 228, 13, 135-149, 0011-9164.

Reungoat, J.; Macova, M.; Escher, B.; Carswell, S.; Mueller J. \& Keller, J. (2010). Removal of micropollutants and reduction of biological activity in a full scale reclamation plant using ozonation and activated carbon filtration. Water Research, 44, 2, 625-637, 00431354.

Ribeiro, C.; Vollaire, Y.; Coulet, E. \& Roche, H. (2008). Bioaccumulation of polychlorinated biphenyls in the eel (Anguilla anguilla) at the Camargue Nature Reserve - France. Environmental Pollution, 153, 2, 424-431, 0469-7291.

Richardson, S.; Simmons, J.\& Rice, G. (2002). Disinfection Byproducts: The Next Generation. Environmental Science and Technology, 36, 9, 198A-205A, 0013-936X.

Ridder, D.; Villacorte, L.; Verliefde, A.; Verberk, J.; Heijman, S.; Amy, G. \& Dijk, J. (2010). Modeling equilibrium adsorption of organic micropollutants onto activated carbon. Water Research, 44, 10, 3077-3086, 0043-1354.

Ru, J.; Liu, H.; Qu, J.; Wang, A. \& Dai, R. (2007). Removal of dieldrin from aqueous solution by a novel triolein-embedded composite adsorbent. Journal of Hazardous Materials, 141, 1, 61-69, 0304-3894.

Sakellarides, T.M.; Konstantinou, I.K.; Hela, D.G.; Lambropoulou, D.; Dimou, A. \& Albanis, T.A. (2006). Accumulation profiles of persistent organochlorines in liver and fat tissues of various waterbird species from Greece. Chemosphere, 63, 1392-1409, 0045 6535.

Schwarzenbach, R.; Escher, B.; Fenner, K.; Hofstetter, T.; Johnson, C.; Gunten U. \& Wehrli, B. (2006). The Challenge of Micropollutants in Aquatic Systems. Science, 313, 5790, 1072-1077, 0036-8075.

Sivaraj, R.; Siva, K.S.; Senthil, K.P. \& Subburam, V. (2001). Carbon from cassava peel, an agricultural waste, as an adsorbent in the removal of dyes and metal ions from aqueous solution. Bioresource Technology, 80, 233-235, 0960-8524.

Song, L.; Zhao, Y.; Wang, G.; Li, B.; Niu, D. \& Chai, X. (2007). Biomimetic fat cell (BFC) preparation and for lindane removal from aqueous solution. Journal of Hazardous Materials, 146, 289-294, 0304-3894.

Verliefde, A.; Cornelissen, E.; Heijman, S.; Verberk, J.; Amy, G.; Bruggen, B. \& Dijk, J. (2009). Construction and validation of a full-scale model for rejection of organic micropollutants by NF membranes. Journal of Membrane Science, 339, 1-2, 10-20, 0376-7388. 
Vincent, J.F.V.; Bogatyreva, O.A.; Bogatyrev, N.R.; Bowyer, A. \& Pahl, A.K. (2006). Biomimetics: its practice and theory. Journal of the Royal Society Interface, 3, 9, 471482, 1742-5689

Vogt, C.; Alfreider A.; Lorbeer, H.; Hoffmann, D.; Wuensche L. \& Babel, W. (2004) Bioremediation of chlorobenzene-contaminated ground water in an in situ reactor mediated by hydrogen peroxide. Journal of Contaminant Hydrology, 68, 1-2, 542$549,0169-7722$.

Voutsas, E.; Magoulas, K. \& Tassios, D. (2002). Prediction of the bioaccumulation of persistent organic pollutants in aquatic food webs. Chemosphere, 48, 7, 645-651, 0045-6535.

Wei, F.; Dong, Y.; An, Q.; Zhang, T.; Liu, D. (2006). Uptake and accumulation of weathered DDT by oil plants. Ecology and Environment, 15, 6, 1188-1191.

Wever, H.; Weiss, S.; Reemtsma, T.; Vereecken, J.; Müller, J.; Knepper, T.; Rörden, O.; Gonzalez, S.; Barcelo D. \& Hernando, M. (2007). Comparison of sulfonated and other micropollutants removal in membrane bioreactor and conventional wastewater treatment. Water Research, 41,4, 935-945, 0043-1354.

Yang, G.; Zhang, X.; Wang, Z.; Liu H. \& Ju, X. (2006). Estimation of the aqueous solubility $(-\operatorname{lgSw})$ of all polychlorinated dibenzo-furans (PCDF) and polychlorinated dibenzo-p-dioxins (PCDD) congeners by density functional theory. Journal of Molecular Structure: THEOCHEM, 30, 1, 25-33, 0166-1280.

Yu, Z.; Peldszus, S. \& Huck, P. (2009). Adsorption of Selected Pharmaceuticals and an Endocrine Disrupting Compound by Granular Activated Carbon. 1. Adsorption Capacity and Kinetics. Environmental Science and Technology, 43, 5, 1467-1473, 0013936X.

Zhang, S.; Shao, T.; Kose, H. \& Karanfil, T. (2010). Adsorption of Aromatic Compounds by Carbonaceous Adsorbents: A Comparative Study on Granular Activated Carbon, Activated Carbon Fiber, and Carbon Nanotubes. Environmental Science and Technology, 44, 16, 6377-6383, 0013-936X.

Zhang, X.; Wei, C.; He, Q. \& Ren, Y. (2010a). Preparation and characterization of biomimetic adsorbent from poly-3-hydroxybutyrate. Journal of Environmental Sciences, 22, 8, 1267-1272, 1878-7320.

Zhang, X.; Wei, C.; He, Q.; Ren, Y. (2010b). Enrichment of chlorobenzene and onitrochlorobenzene on biomimetic adsorbent prepared by poly-3-hydroxybutyrate (PHB). Journal of Hazardous Materials, 177, 1-3, 508-515, 0304-3894.

Zhang, Y.; Wei, S.; Liu, F.; Du, Y.; Liu, S.; Ji, Y.; Yokoi, T.; Tatsumi, T. \& Xiao, F. (2009). Superhydrophobic nanoporous polymers as efficient adsorbents for organic compounds. Nano Today, 4, 2, 135-142, 1748-0132.

Zhang, Z.; Hong, H.S.; Zhou, J.L.; Huang, J. \& Yu, G. (2003). Fate and assessment of persistent organic pollutants in water and sediment from Minjiang River Estuary, Southeast China. Chemosphere, 52, 9, 1423-1430, 0045-6535

Zhou, R.B.; Zhu, L.Z. \& Kong, Q.X. (2007). Persistent chlorinated pesticides in fish species from Qiantang River in East China. Chemosphere, 68, 838-847, 0045-6535. 


\title{
Spectro-Electrochemical Investigation of the bc $_{1}$ Complex from the yeast Saccharomyces cerevisiae using Surface Enhanced B-Band Resonance Raman Spectroscopy
}

\author{
Denise Schach ${ }^{1,2}$, Marc Großerüschkamp ${ }^{1,2}$, Christoph Nowak ${ }^{1,2}$, \\ Wolfgang Knoll2 and Renate L. C. Naumann ${ }^{1,2}$ \\ ${ }^{1}$ Max Planck Institute for Polymer Research, Ackermannweg 10, 55128 Mainz, \\ ${ }^{2}$ Austrian Institute of Technology GmbH - AIT, Donau-City Str. 1, 1220 Vienna, \\ ${ }^{1}$ Germany \\ ${ }^{2}$ Austria
}

\section{Introduction}

The ubiquinol-cytochrome c oxidoreductase ( $\mathrm{bc}_{1}$ complex), also denoted complex III of the respiratory chain, is present in the inner mitochondrial membrane of eukaryotic organisms and many aerobic bacteria. It catalyzes electron transfer from ubiquinol to cytochrome $\mathrm{c}$ coupled to the electrogenic translocation of protons across the membrane. The proton electrochemical gradient thus generated is used for the synthesis of ATP. The crystal structure of the bacterial and the mitochondrial $b_{1}$ complex was determined by X-ray diffraction.(Xia, Yu et al. 1997; Hunte, Koepke et al. 2000; Lange and Hunte 2002; Palsdottir, Lojero et al. 2003; Berry, Huang et al. 2004; Solmaz and Hunte 2008) Both are present as a dimer, the mitochondrial complex differs from the bacterial one by a larger number of subunits extending into the aqueous phase. The elements essential for the function of the enzyme such as the location and orientation of the redox centers, however, are very similar. Three of the four prosthetic groups of the $b_{1} c_{1}$ complex, heme $b_{1}$ and $b_{h}$ and cytochrome $c_{1}$, are metalloporphyrins. Vibrational modes of metalloporphyrins have been extensively studied by Resonance Raman (RR) spectroscopy, since specific modes are very sensitive to the redox state of the heme structure.(Kitagawa, Kyogoku et al. 1975; Spiro 1975; Kitagawa, Ozaki et al. 1978; Spiro 1978) RR spectra were obtained mostly from B-band (Soret) excitations,(Spiro 1988) whereas Q-band excited spectra are less intensive and informative at low protein concentrations.(Le Moigne, Schoepp et al. 1999) Soret excitation and Q-band resonance was applied for investigations of the bacterial (Hobbs, Kriauciunas et al. 1990; Le Moigne, Schoepp et al. 1999) and mitochondrial bc1 complex (Gao, Qin et al. 1998), respectively. In these cases the redox state of the hemes was altered by adding soluble redox compounds such as ascorbate and sodium hydrosulphite to the protein, which was present in the detergent-solubilized form. By contrast in the present investigation we use electrochemistry to direct electron transfer (ET) into the enzyme reconstituted into a lipid bilayer, a method that we had introduced successfully in the case of cytochrome $c$ 

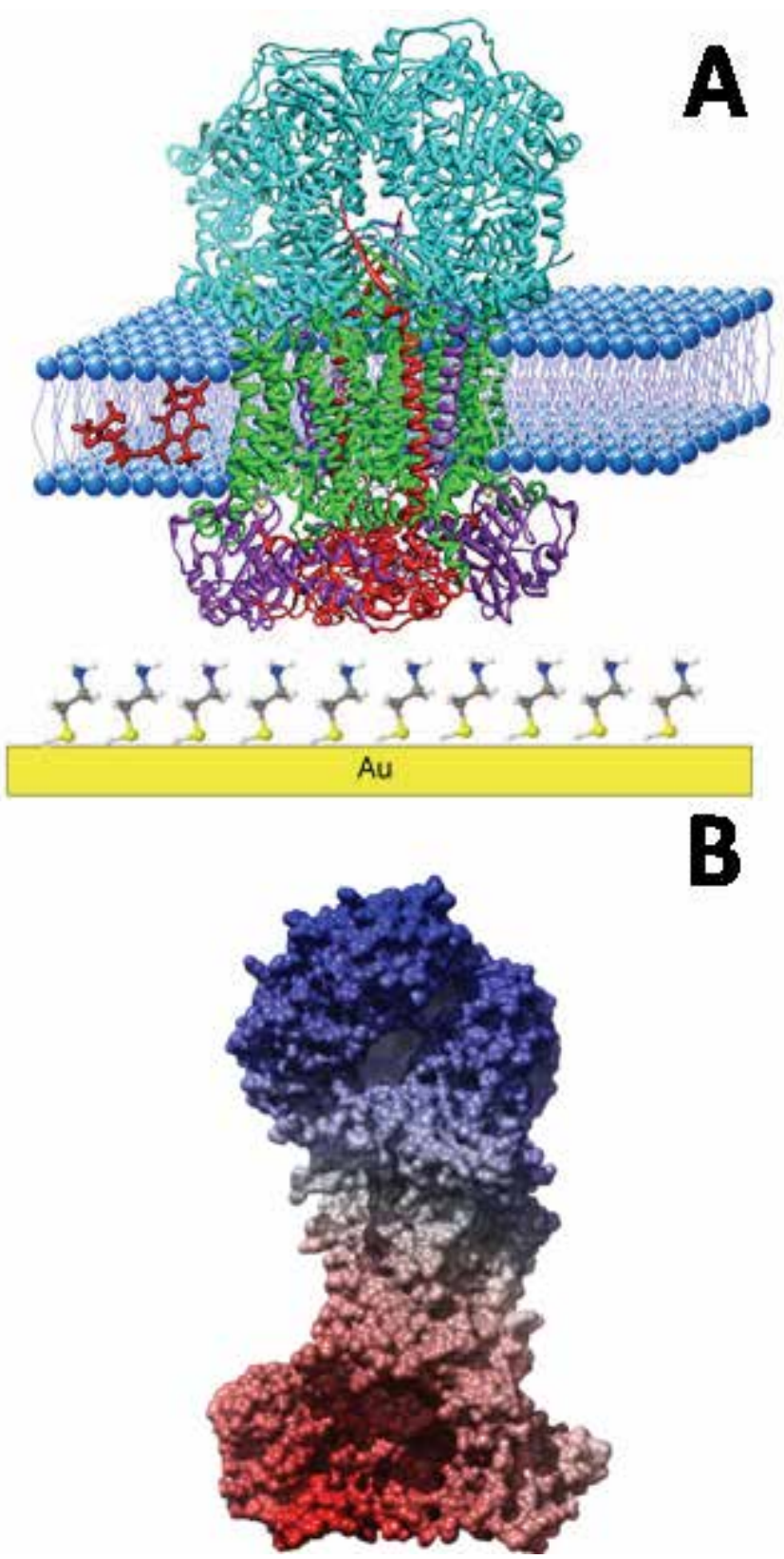

B

Fig. 1. Ubiquinol-cytochrome c oxidoreductase . (A) Cytochrome $b_{1}$ complex from the yeast Saccharomyces cerevisiae (Lange and Hunte 2002) adsorbed onto a cysteamine functionalized electrode and reconstituted into a bilayer lipid membrane.The protein is assembled with the intermembrane side directed toward the electrode. Ubiquinone $Q_{10}$ (dark red) is incorporated into the bilayer lipid membrane. (B) Electrostatic surface of the monomer of the yeast $b c_{1}$ complex(Lange, Nett et al. 2001) in the equivalent orientation. The intermembrane side comprises a negatively charged surface (red) whereas the matrix site comprises a positively charged surface (blue). 
oxidase.(Friedrich, Robertson et al. 2008; Nowak, Schach et al. 2009; Schach, Nowak et al. 2010) For this purpose the $b_{1}$ complex from the yeast Saccharomyces cerevisiae was adsorbed on a monolayer of cysteamine (CA) self assembled on template stripped gold (TSG) and alternatively the top plane of a silver rod modified with silver nanoparticles (Fig. 1A). This way the $b_{1}$ complex is immobilized in a preferred orientation due to electrostatic interaction between the negatively charged surface of the protein and the positively charged CA layer (Fig. 1B shows the electrostatic surface charge distribution). The silver rod works at the same time as an electrode and due to the modification with the nanoparticles as the active surface for purposes of Surface Enhanced Resonance Raman Spectroscopy (SERRS).(Grosserueschkamp, Nowak et al. 2009) After immobilization the protein is reconstituted into a protein-tethered bilayer lipid membrane (ptBLM) using in-situ dialysis of micelles prepared from a lipid with the help of a detergent. The formation of the ptBLM is controlled by surface plasmon resonance (SPR) and electrochemical impedance spectroscopy (EIS) (Fig. 2A, B, C, respectively). Cyclic voltammetry (CV) measurements (Fig. 3) as well as potentiometric titrations are performed, i.e. SERR spectra of the biomimetic membrane system are measured at different potentials applied to the electrode (Fig. 4-6). Spectra are analyzed on the basis of previous investigations of the $b_{1} c_{1}$ complex in different oxidation states obtained by adding reducing compounds in solution.(Hobbs, Kriauciunas et al. 1990; Le Moigne, Schoepp et al. 1999) These data are used to find out whether or not electrochemical reduction/oxidation of the $\mathrm{bc}_{1}$ complex can be accomplished under the experimental conditions described above.

\section{Materials and methods}

Electrochemical measurements (EIS and CV) were performed on template stripped gold (TSG) electrodes, whereas potentiometric titrations followed by SERRS were conducted on the top plane surfaces of silver rods modified with AgNPs.

Modification of the silver electrodes was done as described.(Grosserueschkamp, Nowak et al. 2009) The top planes of $12 \mathrm{~mm}$ thick silver rods were polished down to an rms roughness of 3-5 nm.(Grosserueschkamp, Friedrich et al. 2009) Self-assembled monolayers (SAMs) of cysteamine (CA) were formed on the silver rods by immersion into an aqueous solution of CA $(10 \mathrm{mM} / \mathrm{l})$ for $1 \mathrm{~h}$. After thorough rinsing with water the modified silver surfaces were immersed for two hours into a suspension of silver nanoparticles of $40 \mathrm{~nm}$ diameter. Thereafter the silver rods were again rinsed with water.

Immobilization of the protein and reconstitution into a lipid bilayer: Either TSG electrodes or the above mentioned silver electrodes modified with nanoparticles were immersed for $1 \mathrm{~h}$ into a solution of CA $10 \mathrm{mM} / \mathrm{L}$. The excess CA was removed by rinsing the surfaces with water. The $\mathrm{bc}_{1}$ complex from the yeast Saccharomyces cerevisiae was expressed and purified according to the method of H. Pálsdóttir and C. Hunte.(Pálsdóttir and Hunte 2003) The protein was adsorbed by exposing either the gold electrode or the silver surfaces for $1 \mathrm{~h}$ to a solution of $\mathrm{bc}_{1}$ complex $(0.1 \mu \mathrm{M} / \mathrm{l})$ in phosphate / dodecyl-D-maltoside (DDM) buffer $\left(\mathrm{K}_{2} \mathrm{HPO}_{4} 0.05 \mathrm{M} / 1, \mathrm{KCl} 0.1 \mathrm{M} / 1, \mathrm{pH}=7,0.1 \% \mathrm{DDM}\right)$. The protein solution was removed by cautious rinsing with PBS / DDM buffer. The detergent solution was then replaced by a lipid-containing PBS / DDM buffer $\left(\mathrm{K}_{2} \mathrm{HPO}_{4}\right.$ (Sigma Aldrich) $0.05 \mathrm{M} / \mathrm{l}, \mathrm{KCl}$ (Sigma Aldrich) $0.1 \mathrm{M} / \mathrm{l}, \mathrm{pH}=7,0.1 \%$ DDM (Sigma Aldrich), DiPhyPC (Avanti Polar Lipids) $0.05 \mathrm{mg} / \mathrm{ml}$ ). Ubiquinone $\mathrm{Q}_{10}$ (Fluka) was added if mentioned in the text to the lipidcontaining PBS at a concentration of $0.025 \mathrm{mg} / \mathrm{ml}$. Biobeads (Bio-Rad Laboratories $\mathrm{GmbH}$, 
Munich, Germany) were added to initiate dialysis. Membrane formation was finished after 24 hours. Thereafter the excess lipid buffer and the biobeads were removed by rinsing with fresh PBS buffer.

Surface Plasmon Resonance (SPR): SPR was performed in a custom-made setup using the Kretschmann-configuration. The glass slide (LaSFN9 glass from Hellma Optik, Jena, refractive index $n=1.85$ at $633 \mathrm{~nm}$ ) was optically matched to the base of a $90^{\circ}$ glass prism (LaSFN9). Monochromatic light from a He/Ne Laser, (Uniphase, San Jose, CA, $\lambda=632.8$ $\mathrm{nm}$ ) was directed through the prism and collected by a custom made photodiode detector. Reflectivity at a fixed angle of incidence transferred into a thickness yields the time course of protein binding and reconstitution.

Electrochemistry: Electrochemical measurements were performed using an Autolab instrument (PGSTAT302) equipped with an FRA2-Module for electrochemical impedance measurements, an ECD-module amplifier for low-currents, an ADC750 module for rapid scan measurements and a SCAN-GEN module for analog potential scanning. Electrochemical impedance spectroscopy (EIS) data were recorded in a frequency range of $50 \mathrm{kHz}-3 \mathrm{mHz}$ with an excitation amplitude of $10 \mathrm{mV}$ and a bias potential of $0 \mathrm{~V}$ against an $\mathrm{Ag} \mid \mathrm{AgCl}, \mathrm{KCl}_{\text {sat }}$ reference electrode. Data were analyzed by the complex nonlinear fitting algorithm supplied in the data processing software ZVIEW (Version 2.6, Scribner Associates, Inc.). Cyclic voltammetry experiments were conducted with IR drop compensation. Measurements under anaerobic conditions were performed in a buffer solution containing $\mathrm{K}_{2} \mathrm{HPO}_{4} 0.05 \mathrm{Mol} / \mathrm{l}, \mathrm{KCl} 0.1 \mathrm{Mol} / \mathrm{l}, \mathrm{pH}=7$ and the oxygen trap consisting of glucose $(0.3 \% \mathrm{w} / \mathrm{w})$ (Sigma Aldrich), glucose oxidase $(75 \mu \mathrm{g} / \mathrm{ml})$ (Sigma Aldrich) and catalase $(12.5 \mu \mathrm{g} / \mathrm{ml}$ ) (Sigma Aldrich). This solution was flushed with Ar purged from oxygen by bubbling through the oxygen trap containing buffer solution. All electrochemical measurements were taken in a three electrode configuration with gold as the working electrode, an $\mathrm{Ag} \mid \mathrm{AgCl}, \mathrm{KCl}_{\text {sat }}$ reference and a platinum wire as the counter electrode. All electrode potentials are quoted versus SHE.

Spectro-electrochemical Raman measurements: The measurements were performed under anaerobic conditions as described above for electrochemical measurements. The spectra were recorded at room temperature. The setup, used for the Raman measurements was described in detail in a previous publication.(Grosserueschkamp, Friedrich et al. 2009) The $413 \mathrm{~nm}$ emission line of a $\mathrm{Kr}^{+}$laser (Innova 90C, Coherent) was used for the Soret excitation. After passing a premonochromator (Laserspec III, Spectrolab Research Laboratory, Newbury, England) the laser beam was coupled into a confocal Raman microscope (LabRam, HR800, HORIBA Jobin Yvon) equipped with a water immersion objective (Olympus LUMPLFL, $100 \mathrm{XW}, \mathrm{WD}=1.5, \mathrm{NA}=1, \mathrm{BFOBJ}$ ). By this means the laser beam with a power of $3 \mathrm{~mW}$ was focused on top of the prepared plane of the silver rod. The scattered light was filtered by a holographic notch filter and guided to an 1800 grooves $/ \mathrm{mm}$ grating providing spectral resolution. The spectra were imaged onto a liquid nitrogen cooled back-illuminated CCD detector (Symphony, Jobin Yvon). Spectro-electrochemical measurements were taken in a three electrode configuration with silver as the working electrode, an $\mathrm{Ag} \mid \mathrm{AgCl}, \mathrm{KCl}_{\text {sat }}$ reference and a platinum wire as the counter electrode. Potentials were applied by using a software controlled (GPES, Autolab) potentiostat (Autolab, PGSTAT302, Eco Chemie, B.V., Utrecht, Netherlands). All electrode potentials are quoted versus SHE. 


\section{Results}

\subsection{SPR/EIS measurements}

Adsorption of the $\mathrm{bc}_{1}$ complex solubilized in DDM on the monolayer of CA was recorded by SPR (Fig. 2A). The kinetic trace shows a thickness increase due to $b_{1}$ complex adsorption, however, saturation was not attained indicating unspecific adsorption. In order to avoid too much unspecific binding, the adsorption process was cut short at about the beginning of the saturation and the excess protein was washed off with DDM containing PBS buffer. In-situ dialysis initiated by adding biobeads was indicated by a further increase of the reflectivity definitely reaching saturation after a period of $20 \mathrm{hrs}$. This is characteristic for the exchange of detergent by lipid molecules in the protein monolayer as we had observed in the case of proteins specifically adsorbed using the his-tag technology. The thickness of the protein layer obtained from fitting the angle scans of $10 \mathrm{~nm}$ (inset of Fig. 2A) indicates a submonolayer of $\mathrm{bc}_{1}$ complex considering a thickness of the protein of $18 \mathrm{~nm}$ according to X-ray data (Chase and Parkinson 1991; Feng and Tachikawa 2008) (Table 1). A further thickness increase by $2 \mathrm{~nm}$ after dialysis characterizes the formation of lipid bilayer patches between immobilized proteins. These findings are confirmed by electrochemical impedance spectroscopy showing a decrease of the capacitance and an increase of the resistance after dialysis (Fig. 2B, C). The sealing resistance in the order of magnitude of several $M \Omega \mathrm{cm}^{2}$ is well in the range of resistances, that we had found in the case of ptBLMs based on his-tagged proteins (Table 1). The decrease of the capacitance and the increase of the resistance can be explained in terms of the difference in the dielectric constants of the protein $(\varepsilon=20)$ and lipid $(\varepsilon=2)$. The capacitance decreases since detergent and water are replaced by lipid molecules. We conclude that a densely packed mixed protein/lipid bilayer had been formed on the surface.

\subsection{Cyclic voltammetry}

Cyclic voltammograms were performed at $\mathrm{pH}=7$ under anaerobic conditions. They showed a slow process at around $-400 \mathrm{mV}$ vs. SHE (Fig. 3), which was attributed to the uptake of two electrons and two protons by ubiquinone $\mathrm{Q}_{10}$ to form ubihydroquinone. Midpoint potentials from $+265 \mathrm{mV}$ to $+300 \mathrm{mV}$ and from $+190 \mathrm{mV}$ to $+290 \mathrm{mV}$ were reported for heme $\mathrm{c}_{1}$ and the Rieske center, respectively, whereas $-30 \mathrm{mV}$ to $+100 \mathrm{mV}$, and $-90 \mathrm{mV}$ to $-30 \mathrm{mV}$ were found for heme $b_{H}$ and $b_{L}$, respectively.(Hobbs, Kriauciunas et al. 1990; Le Moigne, Schoepp et al. 1999) So obviously, the b-type hemes do not exchange electrons directly with the electrode. In order to find out about ubiquinone $\mathrm{Q}_{10}$, we added this coenzyme to the lipid/DDM buffer solution used for dialysis. Ubiquinone is thus incorporated into the mixed protein-lipid layer, as shown by two well defined cathodic peaks at about $-0.35 \mathrm{~V}$ and $-0.55 \mathrm{~V}$ in the $\mathrm{CV}$. These peaks were attributed to the stepwise electrochemical reduction of ubiquinone $Q_{10}$ to ubisemiquinone and ubihydroquinone, respectively. The cathodic peaks decrease with successive scans, which is considered as an indication of the reduced species of ubiquinone $\mathrm{Q}_{10}$ being consumed by the enzyme (see Fig. 3, inset). The cathodic peaks are accompanied by a couple of small peaks at $+250 \mathrm{mV}$ and $+350 \mathrm{mV}$, which are tentatively attributed to the heme $\mathrm{c}_{1}$. It should be noted that ubiquinone $\mathrm{Q}_{10}$ neither in the oxidized nor reduced form added to the aqueous buffer solution gives rise to any response in the CVs. Hence the presence of these peaks in the CV alone are a clear indication of a lipid layer around the proteins. Ubiquinone $\mathrm{Q}_{10}$ dissolved in the lipid phase can thus exchange electrons with the electrode to form ubisemiquinone and ubihydroquinone. The formation of the reduced forms of ubiquinone $\mathrm{Q}_{10}$ opens the possibility of the hemes being reduced electrochemically mediated by ubihydroquinone. 

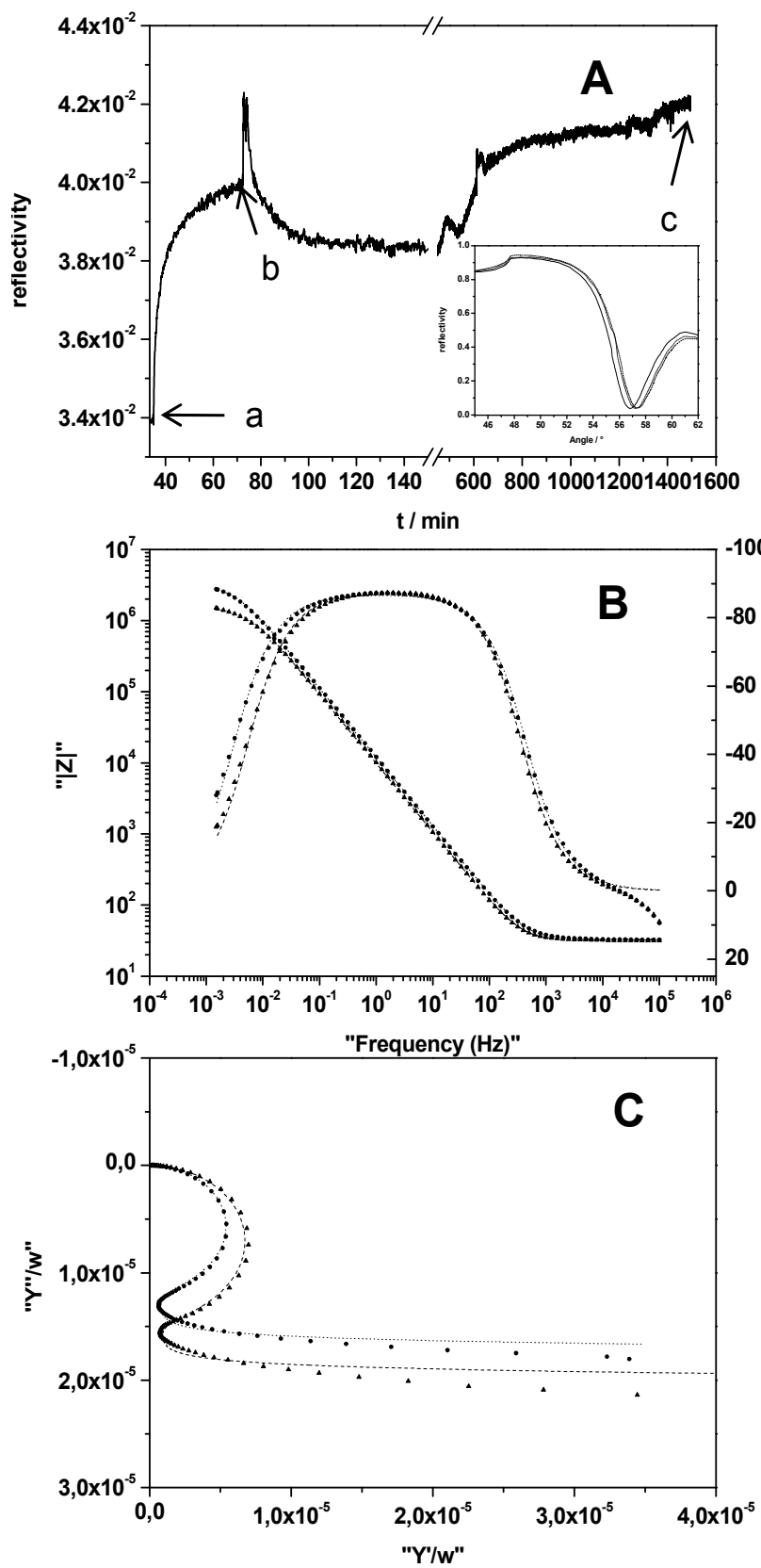

Fig. 2. Immobilization of $\mathbf{b c}_{1}$ complex and reconstitution into the ptBLM. (A) Kinetic trace of the SPR spectrum at a fixed angle of incidence $\Theta=54^{\circ}$ showing the adsorption of the $b_{1}$ complex in solubilized form before (a) and after (b) addition of the protein and the reconstitution of a lipid bilayer (c) after the addition of biobeads to the lipid-detergent containing buffer solution. (B) Bode plot and (C) frequency normalized admittance plot of electrochemical impedance spectra after adsorption of $b_{1} c_{1}$ complex (solid triangles) and after reconstitution (solid circles). 


\begin{tabular}{|c|c|c|c|c|}
\hline & $\mathrm{C} / \mu \mathrm{F} \mathrm{cm}^{-2}$ & $\mathrm{R} / \mathrm{M} \Omega \mathrm{cm}^{2}$ & $\begin{array}{c}\Delta \mathrm{d} / \mathrm{nm} \\
\text { (theory) }\end{array}$ & $\begin{array}{c}\Delta \mathrm{d} / \mathrm{nm} \\
(\text { exp.) }\end{array}$ \\
\hline spacer molecule & & & 0.6 & 0.5 \\
\hline $\mathrm{bc}_{1}$ complex & $17.1+/-0.1$ & $1.5+/-0.2$ & $\begin{array}{c}18.0 \\
\text { (Hunte, Koepke } \\
\text { et al. 2000) }\end{array}$ & 10.0 \\
\hline lipid bilayer & $14.2+/-0.1$ & $3.1+/-0.4$ & 2.0 & 2.0 \\
\hline
\end{tabular}

Table 1. Data from EIS and SPR measurements of the different layer structures

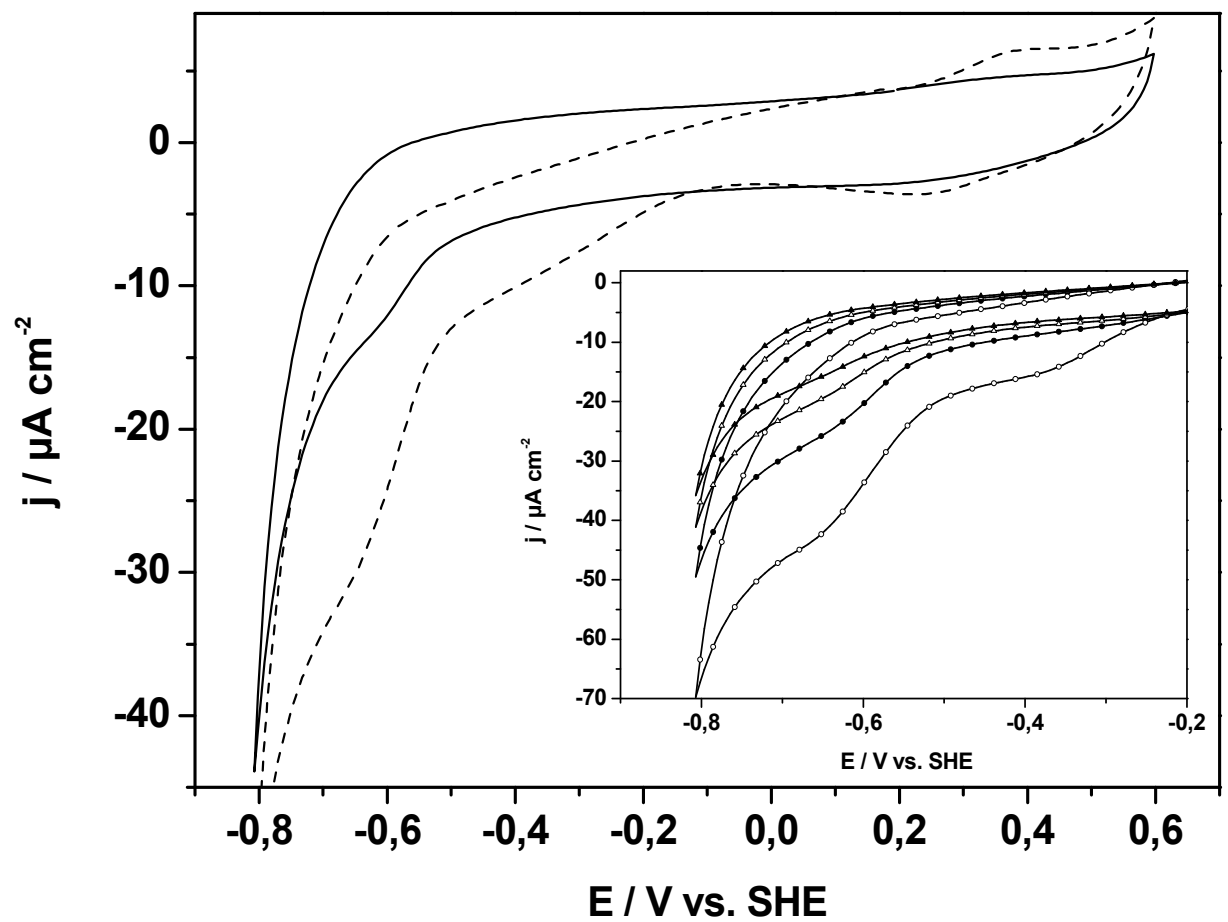

Fig. 3. Cyclic voltammograms of $\mathbf{b c}_{\mathbf{1}}$ complex adsorbed onto a functionalized electrode and reconstituted into a bilayer lipid membrane (see Fig.1) with additional ubiquinone $\mathrm{Q}_{10}$ (dashed line and inset) and without additional ubiquinone $\mathrm{Q}_{10}$ (solid line) taken under anaerobic conditions ( $2^{\text {nd }}$ scans at scan rate $0.05 \mathrm{Vs}^{-1}$ for either $\mathrm{CV}$ ). The inset shows evolution of the CVs after insertion of ubiquinone $Q_{10}$ to the protein-lipid layer. $1^{\text {st }}$ scan (open circles), $2^{\text {nd }}$ scan (solid circles), $3^{\text {rd }}$ scan (open triangles) and $4^{\text {th }}$ scan (solid triangles).

\subsection{Resonance Raman study}

SERR spectra were recorded at $250 \mathrm{mV}$ for the oxidized heme species and at $-400 \mathrm{mV}$ for the reduced heme species (Fig. 4A-D). At these potentials the enzyme was found to be in the fully oxidized and reduced form, respectively, see below for the details. The spectra were 
sub-divided into different ranges for better interpretation. Tentative band assignments were performed according to SERR spectra obtained from two different sources both using the bacterial bc 1 complex (Hobbs, Kriauciunas et al. 1990; Le Moigne, Schoepp et al. 1999) from Rhodospirillum rubrum under conditions mentioned above (Table 2).

In general, frequency modes observed upon B-band excitations over the entire frequency range showed band profiles with higher intensities at the negative potential than at the positive potential (Fig. 4). This phenomenon is associated with the potential dependent surface enhancement effect, which is well known to result from two main effects: an electromagnetic effect and a chemical effect.(Chase and Parkinson 1991; Feng and Tachikawa 2008) On the basis of intensity versus potential profiles measured in electrochemical environments, it has been shown, that the charge transfer mechanism dominates the intensity of SERR spectra of molecules adsorbed on a silver electrode. (Lombardi, Birke et al. 1986; Osawa, Matsuda et al. 1994)

Low Frequency Region $\left(250\right.$ - $\left.450 \mathrm{~cm}^{-1}\right)$

SERR spectra of the $b_{1}$ complex measured in this region are shown in (Fig. 4A). Distortion of the heme groups effect the majority of bands occurring at these frequencies.(Hu, Morris et al. 1993) In general the region below $1000 \mathrm{~cm}^{-1}$ comprises modes with less enhancement since these are stronger coupled to ring deformations and stretching of bonds to the central metal iron than to $\pi-\pi^{*}$ excitations, as it is the case in the high frequency region. Furthermore a natural falloff of Raman intensity with decreasing frequency is noted in literature.(Choi and Spiro 1983)

The $v_{9}$ mode, a $\delta\left(C_{\alpha}-C_{m}\right)$ sym vibration, (Spiro, Czernuszewicz et al. 1989) is displayed in the measured spectra at $263 \mathrm{~cm}^{-1}$ for the oxidized state and at $265 \mathrm{~cm}^{-1}$ for the reduced state. The $v_{9}$ mode is a peripheral heme mode that occurs distinctly for b-type hemes.

For the reduced heme species five prominent overlapping bands arise between $343 \mathrm{~cm}^{-1}$ and $416 \mathrm{~cm}^{-1}$, whereas two bands of this profile, at $343 \mathrm{~cm}^{-1}$ and $407 \mathrm{~cm}^{-1}$ only persist in the spectra recorded for the oxidized state. The band at $343 \mathrm{~cm}^{-1}$ appears for either of the redox states. In both cases it can be assigned to the $v_{8}$ mode of b- and c-type cyt. The $v_{8}$ mode, a skeletal in-plane heme mode, is known to be the strongest low-frequency band in metalloporphyrin spectra. (Hu, Smith et al. 1996) This was confirmed by the present investigation.

Either the $v_{8}$ or the $v_{9}$ mode consist of a combination of iron-N (pyrrole) stretching and $\mathrm{C}_{\mathrm{b}^{-}}$ pyrrole-substituent deformation.(Hu, Morris et al. 1993) Previous measurements taken at different excitation wavelengths show three neighboring bands between $344 \mathrm{~cm}^{-1}$ and 356 $\mathrm{cm}^{-1}$, that are assigned respectively to the $v_{8}$ modes of the heme $b_{\mathrm{H}}$, heme $c_{1}$ and heme $b_{1 \cdot(\mathrm{Le}}$ Moigne, Schoepp et al. 1999) The spectrum presented here comprises only two bands at $344 \mathrm{~cm}^{-1}$ and $356 \mathrm{~cm}^{-1}$. So a distinction between the hemes was not possible.

Additionally we propose to assign the shoulder of the band referring to the $v_{8}$ mode at 356 $\mathrm{cm}^{-1}$ to the $v_{50}$ mode, a heme skeletal mode of the reduced cyt $\mathrm{c}_{1}$. It seems to be not existent in the spectra for the oxidized state. The region between $377 \mathrm{~cm}^{-1}$ and $416 \mathrm{~cm}^{-1}$ is dominated by deformation modes of peripheral heme groups. For the reduced species we find three bands at $377 \mathrm{~cm}^{-1}, 403 \mathrm{~cm}^{-1}$ and $416 \mathrm{~cm}^{-1}$, that have been assigned to $\delta_{\mathrm{CPr}}$ of heme b, $\delta_{\mathrm{CCaCb} 2,4}$ of heme $c_{1}$ and $\delta_{C C V n}$ of heme b, respectively. In the oxidized state there is one band at 407 $\mathrm{cm}^{-1}$ left, which we assigned to $\delta_{\mathrm{CCVn}}$ of heme b.(Le Moigne, Schoepp et al. 1999) 
In general peripheral heme modes show sensitivity to vibrations of peripheral groups, therefore they can be considered as fingerprint vibrations identifying the heme types. The assignment of bands particularly in the low frequency region demonstrates that we can distinguish between signals originating from b- or c-type hemes. We can also conclude that reduction and oxidation of both types of hemes are reduced and oxidized in this particular potential window.

Mid Frequency Region (450 - $1250 \mathrm{~cm}^{-1}$ )

The mid frequency region (Fig. $4 \mathrm{~B}$ and $\mathrm{C}$ ) can be sub-divided into two parts. The part between $450 \mathrm{~cm}^{-1}$ and $920 \mathrm{~cm}^{-1}$ is dominated by heme skeletal modes, whereas the spectrum between $920 \mathrm{~cm}^{-1}$ and $1250 \mathrm{~cm}^{-1}$ comprises mostly peripheral heme modes. The region between $600 \mathrm{~cm}^{-1}$ and $850 \mathrm{~cm}^{-1}$ is remarkably rich due to narrow overlapping bands with strong intensities and good signal-to-noise ratio.

A notably large band particularly for the reduced state was displayed at $681 \mathrm{~cm}^{-1}$. This band was assigned to the $v_{7}$ mode exclusively of b-type hemes.(Le Moigne, Schoepp et al. 1999) It is clearly distinguished from the $v_{7}$ mode of heme $c_{1}$, which appears with less intensity at $697 \mathrm{~cm}^{-1}$ and is only existent for the reduced species.(Le Moigne, Schoepp et al. 1999) This was confirmed by a comparative measurement of the bc1 complex with cytochrome $c$ both in the reduced state (Fig. 5, spectrum a, b, respectively). The $v_{7}$ mode of the c- type heme in cyt c produces a band at a higher frequency, $693 \mathrm{~cm}^{-1}$, however, with less intensity. The $v_{7}$ mode, an in-plane skeletal mode, originates from a symmetrical pyrrole deformation.

The further deconvolution of the $v_{7}$ envelope in the reduced state reveals strongly overlapping bands originating from heme skeletal modes. Two signals at $650 \mathrm{~cm}^{-1}$ and 663 $\mathrm{cm}^{-1}$ can be allocated to $\gamma_{20}$. A second shoulder of the $v_{7}$ envelope appears at $635 \mathrm{~cm}^{-1}$, which can be assigned to $v_{48}$. Other shoulders are found at higher frequencies, such as $716 \mathrm{~cm}^{-1}$, $747 \mathrm{~cm}^{-1}$ and $783 \mathrm{~cm}^{-1}$ are induced respectively by $\gamma_{5}, v_{15}$ and $v_{6}$. The modes $v_{15}$ and $v_{6}$ are both pyrrole breathing modes.(Spiro, Czernuszewicz et al. 1989) A slight signal in the lower frequency region at $460 \mathrm{~cm}^{-1}$ was assigned to $\gamma_{22}$.

The region between $850 \mathrm{~cm}^{-1}$ and $1250 \mathrm{~cm}^{-1}$ contains few bands of higher intensity. We find low signals at $920 \mathrm{~cm}^{-1}, 1147 \mathrm{~cm}^{-1}$ and $1170 \mathrm{~cm}^{-1}$. The first two frequencies can be assigned to the heme skeletal modes of reduced cytochrome $c_{1}, v_{46}$ and $v_{43}$, respectively. The latter frequency was assigned in the reduced and the oxidized state to the heme skeletal mode $v_{30}$ of cytochromes $b$ and $c_{1}$. The $v_{30}$ mode originates from an asymmetrical deformation of the pyrrole half ring, whereas the $v_{46}$ mode corresponds to $\delta$ (pyrrole) asym and the $v_{43}$ mode is effected by a $\mathrm{C}-\mathrm{Y}$ stretching mode, $v\left(\mathrm{C}_{\beta}-\mathrm{Y}\right)_{\text {sym. }}$ (Spiro, Czernuszewicz et al. 1989) Peripheral modes of b- and c-type hemes, $\delta_{\mathrm{CPr}}, v_{5}$ and $v_{14}$ give rise to bands at $968 \mathrm{~cm}^{-1}$ (red.state)/ 972 $\mathrm{cm}^{-1}$ (ox. state), $1113 \mathrm{~cm}^{-1}$ (red. state) and $1130 \mathrm{~cm}^{-1}$ (red. and ox. state), respectively. Two peripheral modes of b-type hemes produce bands at $1006 \mathrm{~cm}^{-1}$ (ox. state) according to $\delta_{\mathrm{CVn}}$ and at $1222 \mathrm{~cm}^{-1}$ (reduced and oxidized state) according to $v_{13}$. We assign a slight signal occuring at $1087 \mathrm{~cm}^{-1}$ in the reduced state to another peripheral heme mode, $\delta_{\mathrm{CCH} 3}$ of the ctype heme.(Le Moigne, Schoepp et al. 1999)

More bands were observed in the present investigation, that were not observed in previous studies of the $b_{1}$ complex, e.g. in the paper of Moigne et al.(Le Moigne, Schoepp et al. 1999) These are the bands at $503 \mathrm{~cm}^{-1}, 560 \mathrm{~cm}^{-1}, 715 \mathrm{~cm}^{-1}, 741 \mathrm{~cm}^{-1}, 785 \mathrm{~cm}^{-1}$ and $885 \mathrm{~cm}^{-1}$, which appear in the spectrum of the oxidized species and the bands at $518 \mathrm{~cm}^{-1}, 546 \mathrm{~cm}^{-1}, 562 \mathrm{~cm}^{-1}$, $602 \mathrm{~cm}^{-1}, 734 \mathrm{~cm}^{-1}, 1020 \mathrm{~cm}^{-1}$ and $1052 \mathrm{~cm}^{-1}$ in the spectrum of the reduced state. 
We conclude from the results presented in this section that we have isolated a band at 681 $\mathrm{cm}^{-1}$, which selectively demonstrates the reduction of the b-type hemes.

High Frequency Region (1250 - $\left.1700 \mathrm{~cm}^{-1}\right)$

SERR spectra of the $b_{1}$ complex measured in this region are shown in (Fig. 4D). In agreement with spectra of other metalloporphyrins and heme proteins they comprise particularly modes, which are strongly enhanced and resonant with dominant Q- and B electronic transitions. Mainly in-plane ring modes coupled to $\pi-\pi^{*}$ excitations generate these bands.(Choi and Spiro 1983) They are produced by heme skeletal vibrations and they are barely assignable distinctly to the b- or $\mathrm{c}_{1}$-type hemes applying Soret excitation.

Contrary to the lower frequency regions the high frequency region comprises modes, which are sensitive to oxidation or spin state, such as the heme skeletal modes $v_{2}, v_{3}, v_{4}$ and $v_{10}$. A small general lowering of some frequencies is caused by reduction from $\mathrm{Fe}(\mathrm{III})$ to $\mathrm{Fe}(\mathrm{II})$. This process can be explained in terms of changes in $\pi$ back donation, which can be understood as an electronic transition. Larger shifts of some frequencies are induced by conversion from low- to high-spin state.(Spiro and Strekas 1974) As in previous studies the $v_{4}$ mode serves as an oxidation state marker, whereas the $v_{2}$ mode is rather sensitive to the spin state. Either, the $v_{3}$ and the $v_{10}$ mode are sensitive to both, the oxidation and the spin state.(Spiro and Strekas 1974) The frequency according to the $v_{4}$ mode is located at $1374 \mathrm{~cm}^{-1}$ in the oxidized state. Reduction gives rise to a discrete shift in frequency from $1374 \mathrm{~cm}^{-1}$ to $1358 \mathrm{~cm}^{-1}$. From the intensity of either band one can read out the concentration of reduced or oxidized species. A residual contribution of the reduced species is still apparent in the spectrum of the fully oxidized protein due to photoreduction of a fraction of the heme sites. Laser intensity dependent photoreduction has been observed before.(Hobbs, Kriauciunas et al. 1990) The band originating from the $v_{4}$ mode is the strongest in the high frequency region, especially in the reduced state. Calculations predict, that the $v_{4}$ mode consists of $36 \%$ of $\left(\mathrm{C}_{\alpha}-\mathrm{C}_{\beta}\right)$ stretching and deformation of the pyrrole half ring, the latter plays a crucial role for the sensitivity to the oxidation state.(Kozlowski, Bingham et al. 2008) Likewise the modes $v_{2}, v_{3}$ and $v_{10}$ originate from $C-C$ stretching, namely $v\left(C_{\beta}-C_{\beta}\right), v\left(C_{\alpha}-C_{m}\right)_{\text {sym }}$ and $v\left(C_{\alpha}-C_{m}\right)$ asym, respectively.(Spiro, Czernuszewicz et al. 1989; Hu 1993)

4In comparison to the $v_{4}$ mode the contribution of the $v_{3}$ mode is rather small. For the correspondent frequency a discrete shift from $1502 \mathrm{~cm}^{-1}$ in the oxidized state to $1492 \mathrm{~cm}^{-1}$ in the reduced state can be observed.

Contrary to the modes $v_{3}, v_{4}$ and $v_{10}$, by reducing the potential the frequency according to the $v_{2}$ mode underlies a shift to a higher value in terms of spin state transitions, which has already been observed previously.(Grosserueschkamp, Friedrich et al. 2009) In our spectra the corresponding band observed at $1581 \mathrm{~cm}^{-1}$ and $1589 \mathrm{~cm}^{-1}$ account for oxidized and reduced species, respectively.

For the reduced species we assigned three shoulders of the band referring to $v_{2}$ at $1559 \mathrm{~cm}^{-}$ $1,1606 \mathrm{~cm}^{-1}$ and at $1620 \mathrm{~cm}^{-1}$ to the $v_{38}$ mode, a $C_{\beta}-C_{\beta}$ stretching, to the $v_{37}$ mode, an asym. $\mathrm{C}_{\alpha}-\mathrm{C}_{\mathrm{m}}$ stretching, and to the $v_{10}$ mode, respectively.(Spiro, Czernuszewicz et al. 1989) In the oxidized state there is no contribution from the $v_{38}$ and the $v_{37}$ mode. The $v_{10}$ mode however generates a slight signal, which is discretely shifted to $1631 \mathrm{~cm}^{-1}$ for the oxidized species. A shoulder of the band assigned to $v_{4}$ at $1390 \mathrm{~cm}^{-1}$ in the reduced state can be assigned to the $v_{29}$ mode, originating from a pyrrole quarter ring deformation. A small band occurring for the reduced state at $1466 \mathrm{~cm}^{-1}$ was assigned to the $v_{28}$ mode, a symmetrical $C_{\alpha}-C_{m}$ stretching mode.(Spiro, Czernuszewicz et al. 1989) 

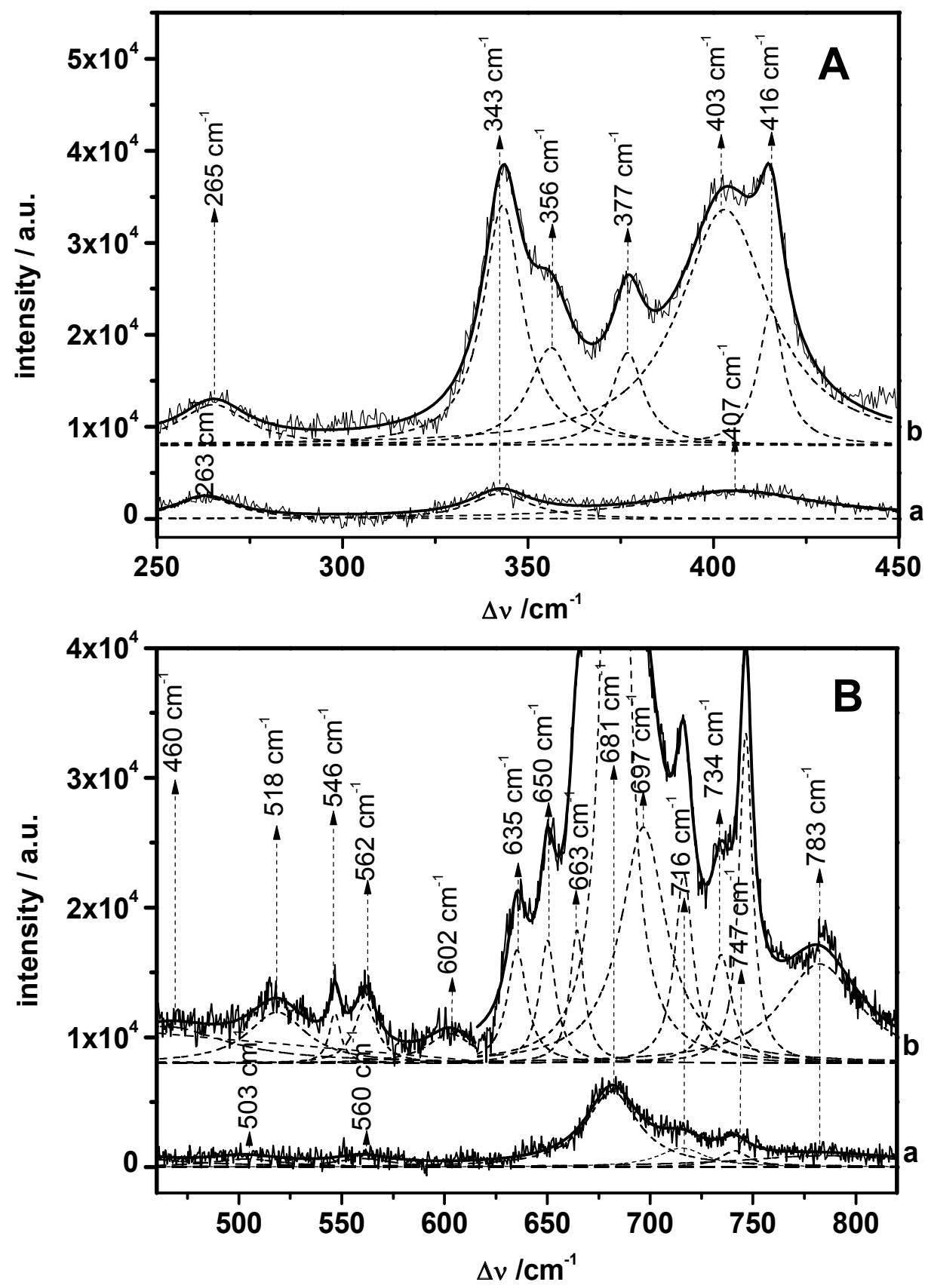

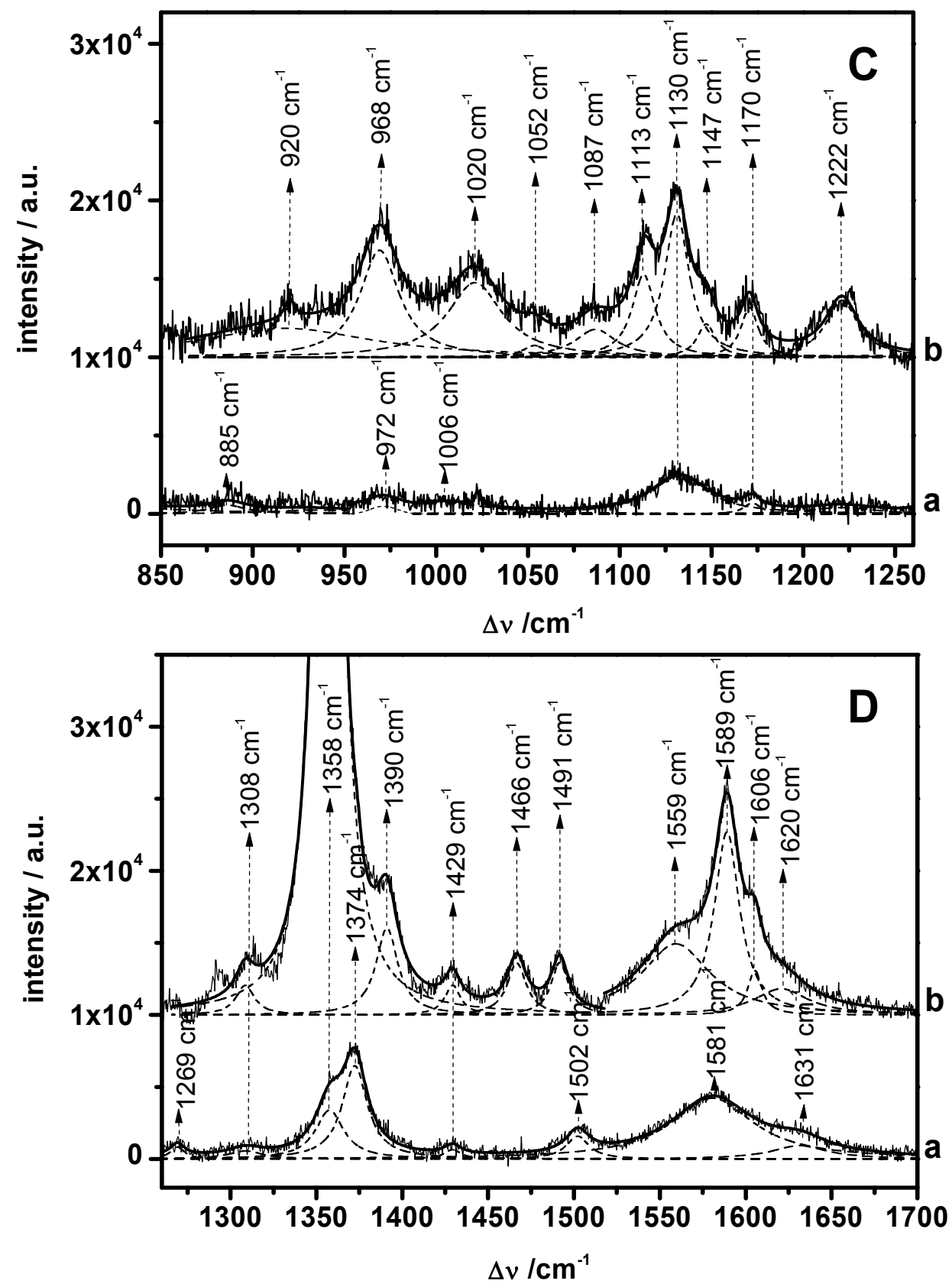

Fig. 4. SERR spectra of the $\mathbf{b c}_{1}$ complex adsorbed onto a functionalized electrode and reconstituted into a bilayer lipid membrane (see Fig.1) with ubiquinone $\mathrm{Q}_{10}$ added, recorded under anaerobic conditions at oxidizing potential $250 \mathrm{mV}$ (a) and reducing potential $-400 \mathrm{mV}(\mathrm{b})$ in the low- $(\mathrm{A})$, the mid- $(\mathrm{B}, \mathrm{C})$ and high frequency region $(\mathrm{D})$. Deconvoluted spectra (dashed lines) and envelope (solid line). 


\begin{tabular}{|c|c|c|c|c|c|}
\hline frequency & mode assignment & redox state & frequency & mode assignment & redox state \\
\hline 343 & $v^{\prime} 8\left(\mathrm{bHH}_{H}+\mathrm{c}_{1}\right), v_{8}\left(\mathrm{~b}_{\mathrm{H}}+\mathrm{c}_{1}\right)$ & red, ox & 263 & $v^{\prime} 9(\mathrm{~b})$ & ox \\
\hline 356 & $V^{\prime}{ }_{50}\left(\mathrm{c}_{1}\right)$ & red & 265 & $v^{\prime} 9(\mathrm{~b})$ & red \\
\hline 635 & $v^{\prime} 48\left(c_{1}\right)$ & red & 377 & $\delta^{\prime} \mathrm{CPr}(\mathrm{b}), \delta^{\prime} \mathrm{CPr}(\mathrm{b})$ & red \\
\hline 650 & $\gamma^{\prime} 20\left(\mathrm{c}_{1}\right)$ & red & 403 & $\delta^{\prime} \mathrm{CCaCb} 2,4\left(\mathrm{c}_{1}\right)$ & red \\
\hline 663 & $\gamma^{\prime} 20\left(\mathrm{c}_{1}\right)$ & red & 407 & $\delta^{\prime} \mathrm{CCVn}\left(\mathrm{bL}_{\mathrm{L}}\right), \delta_{\mathrm{CCVn}}\left(\mathrm{bl}_{\mathrm{L}}\right)$ & ox \\
\hline 681 & $v^{\prime} 7(b), v_{7}(b)$ & red, ox & 416 & $\delta^{\prime} \mathrm{CCVn}\left(\mathrm{b}_{\mathrm{H}}\right), \delta \mathrm{CCVn}\left(\mathrm{b}_{\mathrm{H}}\right)$ & red \\
\hline 697 & $v^{\prime} 7\left(\mathrm{c}_{1}\right)$ & red & $681 / 696$ & $v^{\prime} \operatorname{Cs}\left(\mathrm{c}_{1}\right), v \operatorname{cs}\left(\mathrm{c}_{1}\right)$ & red, ox \\
\hline 716 & $\gamma^{\prime} 5\left(\mathrm{~b}+\mathrm{c}_{1}\right)$ & red, ox & 968 & $\delta^{\prime} \mathrm{CPr}\left(\mathrm{b}+\mathrm{c}_{1}\right), \delta_{\mathrm{CPr}}\left(\mathrm{b}+\mathrm{c}_{1}\right)$ & red \\
\hline 747 & $v^{\prime} 15\left(b+c_{1}\right)$ & red, ox & 972 & $\delta^{\prime} \mathrm{CPr}\left(\mathrm{b}+\mathrm{c}_{1}\right), \delta \mathrm{CPr}\left(\mathrm{b}+\mathrm{c}_{1}\right)$ & ox \\
\hline 783 & $v^{\prime} 6\left(\mathrm{c}_{1}\right)$ & red, ox & 1006 & $\delta^{\prime} \mathrm{CVn}_{\mathrm{n}}(\mathrm{b}), \delta_{\mathrm{CVn}}(\mathrm{b})$ & ox \\
\hline 920 & $v^{\prime} 46\left(c_{1}\right)$ & red & 1087 & $\delta{ }^{\prime}{ }^{\prime} \mathrm{CH}_{3}\left(\mathrm{c}_{1}\right)$ & red \\
\hline 1147 & $v^{\prime} 43\left(c_{1}\right)$ & red & 1113 & $v^{\prime} 5\left(b+c_{1}\right)$ & red \\
\hline 1170 & $v_{30}^{\prime}\left(b_{H}+c_{1}\right), v_{30}\left(b_{H}+c_{1}\right)$ & red, ox & 1130 & $v^{\prime} 14\left(b+c_{1}\right), v_{14}\left(b+c_{1}\right)$ & red, ox \\
\hline 1358 & $v^{\prime} 4\left(b+c_{1}\right)$ & red, ox & 1222 & $v^{\prime}{ }_{13}(b), v_{13}(b)$ & red, ox \\
\hline 1374 & $v_{4}\left(b+c_{1}\right)$ & ox & 1308 & $\delta^{\prime}{ }^{\mathrm{CHVn}}(\mathrm{b})$ & red, ox \\
\hline 1390 & $v^{\prime} 29\left(b+c_{1}\right)$ & red & 1429 & $\delta^{\prime} \mathrm{CH}_{2} \mathrm{Vn}(\mathrm{b})$ & red, ox \\
\hline 1466 & $v^{\prime} 28\left(b+c_{1}\right)$ & red & & & \\
\hline 1491 & $v_{3}^{\prime}\left(b+c_{1}\right)$ & red & & & \\
\hline 1502 & $v_{3}\left(b+c_{1}\right)$ & ox & & & \\
\hline 1559 & $v^{\prime} 38\left(b+c_{1}\right)$ & red & & & \\
\hline 1581 & $v_{2}^{\prime}\left(b+c_{1}\right), v_{2}\left(b+c_{1}\right)$ & ox & & & \\
\hline 1589 & $v_{2}^{\prime}\left(c_{1}\right), v_{2}\left(c_{1}\right)$ & red & & & \\
\hline 1606 & $v^{\prime} 37\left(b+c_{1}\right)$ & red & & & \\
\hline 1620 & $v^{\prime} 10\left(c_{1}\right)$ & red & & & \\
\hline 1631 & $v_{10}\left(b+c_{1}\right)$ & ox & & & \\
\hline
\end{tabular}

Table 2. Frequency $\left(\mathrm{cm}^{-1}\right)$ of Heme Skeletal Modes (left) and Peripheral Heme Modes (right) observed in SERRS of electrochemically reduced/ oxidized bc complex. $v^{\prime}{ }_{x}, \delta^{\prime}{ }_{x}, \gamma^{\prime}{ }_{x}, v_{x}, \delta_{x}, \gamma_{x}$ correspond to the reduced and oxidized state, respectively.

Only two bands at $1308 \mathrm{~cm}^{-1}$ and at $1429 \mathrm{~cm}^{-1}$ occur in the high frequency region, which originate from peripheral heme modes of the b hemes, $\delta_{\mathrm{CHVn}}$ and $\delta_{\mathrm{CH} 2 \mathrm{Vn}}$, respectively. We found only one band at $1269 \mathrm{~cm}^{-1}$ in the high frequency region, which was not considered in previous studies.(Le Moigne, Schoepp et al. 1999)

In summary, the high frequency region contains bands, which originate from all types of hemes. Of particular significance are the marker bands at $1374 \mathrm{~cm}^{-1}$ and at $1358 \mathrm{~cm}^{-1}$ indicating the redox state of all of the hemes. We conclude therefrom that the $b_{1} c_{1}$ complex is fully reduced within this particular potential window. However, applying Soret excitation in this particular region it is hard to distinguish between signals from different cytochromes. Nevertheless some modes of this region, such as the $v_{2}, v_{3}, v_{4}$ and $v_{10}$ mode give useful information on oxidation and spin state. 


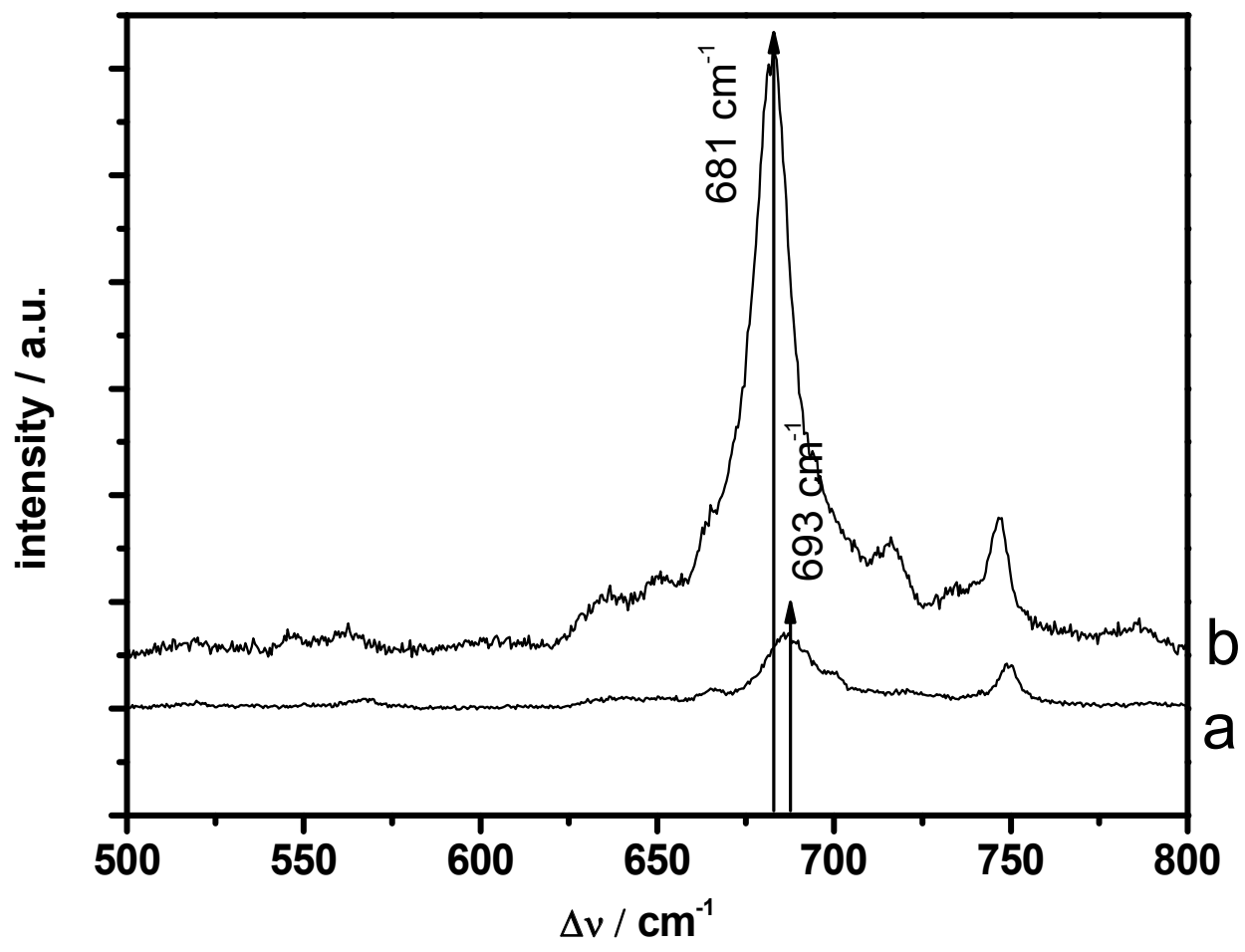

Fig. 5. Comparison between SERRS in the low frequency region of reduced cytochrome $c$ (a) and reduced bc $\mathrm{c}_{1}$ complex (b) under anaerobic conditions recorded at $-400 \mathrm{mV}$ vs. SHE.

\section{Potentiometric titration}

Based on these results, potentiometric titrations were conducted followed by SERRS, i.e. the potential was altered stepwise between $+200 \mathrm{mV}$ and $-400 \mathrm{mV}$. Fig. 6 shows the spectra as a function of potential, in the spectral range $1200-1770 \mathrm{~cm}^{-1}$ (A) and $250-970 \mathrm{~cm}^{-1}$ (B). The most prominent band refers to the $v_{4}$ mode, a skeletal heme mode of all the hemes, $b_{\mathrm{L}}, b_{H}$ and $c_{1}$, present in the $b_{1}$ complex. The discrete shift from $1374 \mathrm{~cm}^{-1}$ to $1357 \mathrm{~cm}^{-1}$ indicates that the oxidized form is progressively transformed into the reduced form, respectively. At $+200 \mathrm{mV}$ and $-300 \mathrm{mV}$ the hemes seem to be completely oxidized and reduced, respectively. Closer inspection of the spectra even at slightly higher and lower potentials, show small contributions of the reduced form at the positive edge of the potential window, whereas the oxidized form seems to be completely removed at $-400 \mathrm{mV}$ (compare Fig. $4 \mathrm{D}$ spectra $\mathrm{a}$ and $\mathrm{b}$ showing the deconvoluted SERR spectra in the fully oxidized and reduced state of the enzyme, respectively). We can conclude that the reduction of all the hemes does take place under our experimental conditions. 

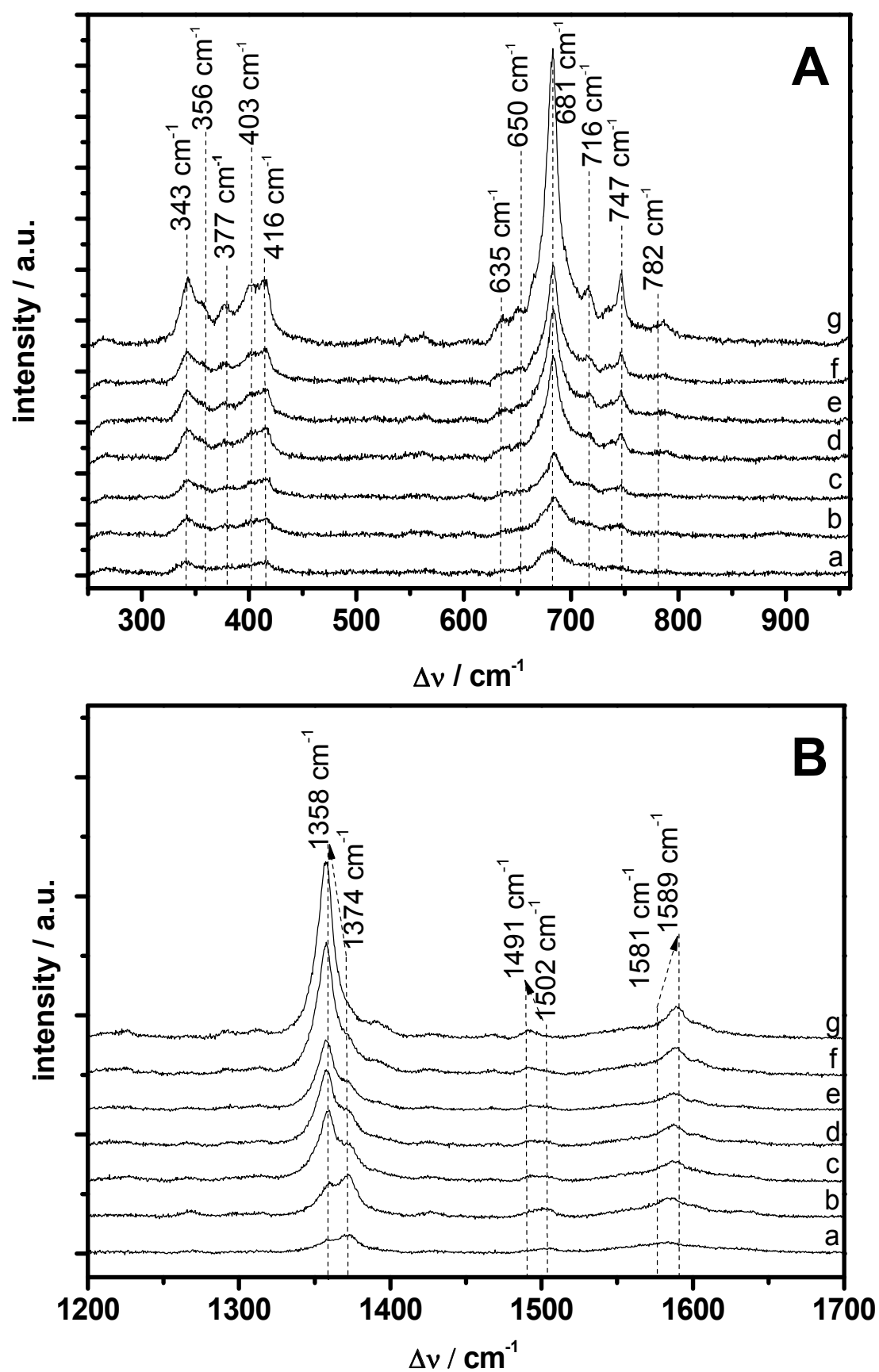

Fig. 6. SERR spectra of the $\mathbf{b c}_{1}$ complex adsorbed onto a functionalized electrode and reconstituted into a bilayer lipid membrane (see Fig.1) with additional ubiquinone $\mathrm{Q}_{10}$ recorded under anaerobic conditions as a function of potential taken at $200 \mathrm{mV}$ (a), $100 \mathrm{mV}$ (b), $0 \mathrm{mV}(\mathrm{c}),-130 \mathrm{mV}(\mathrm{d}),-150 \mathrm{mV}(\mathrm{e}),-170 \mathrm{mV}$ (f) and $-300 \mathrm{mV}$ (g) for the lower- (A) and the higher frequency region (B). 

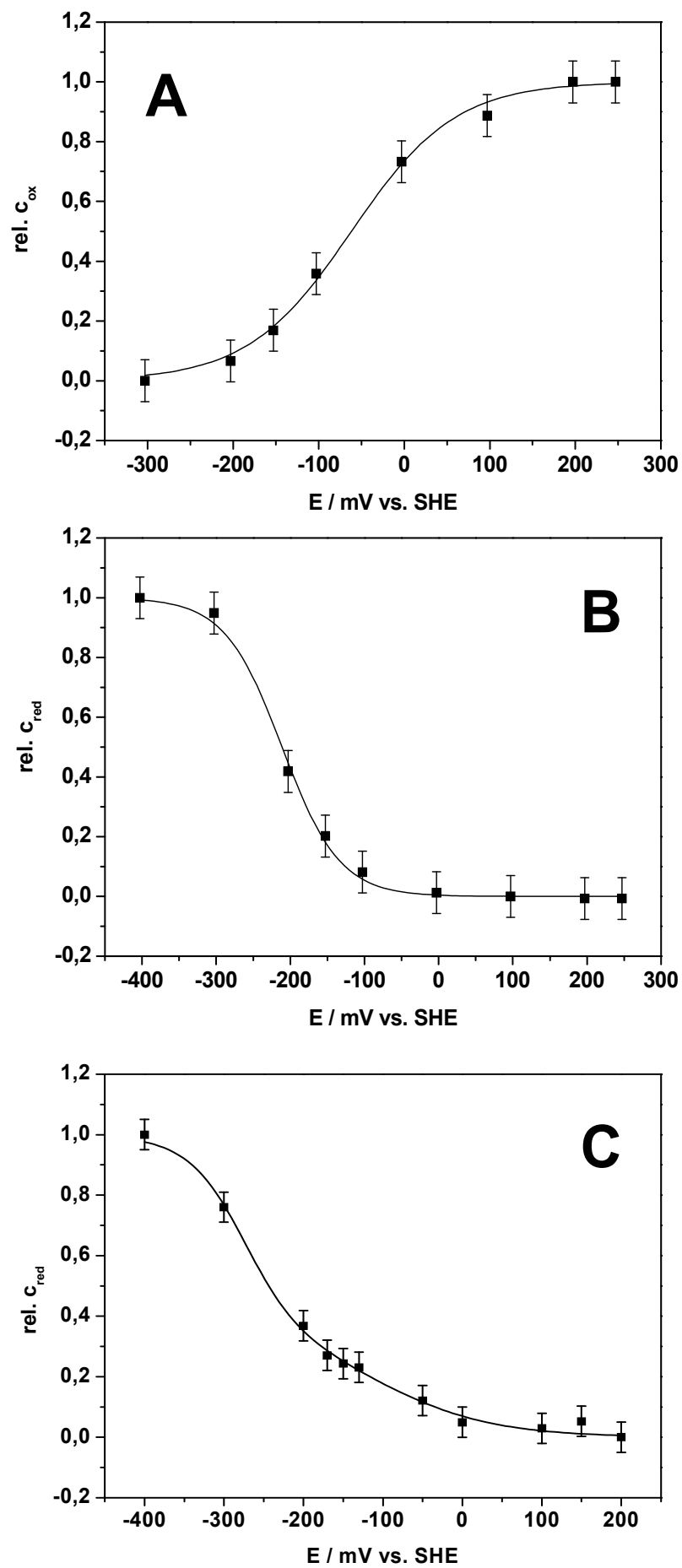

Fig. 7. Normalized concentration versus potential plots derived from the $v_{4}(A)$ and $v_{4}^{\prime}$ mode (B) and the $v_{7}^{\prime}$ mode $(C)$. 
The intensities of the bands referring to $v^{\prime}{ }_{4}$ and $v_{4}$ correspond to the relative concentration of reduced and oxidized species. Based on this relation we plotted the normalized concentrations of each species as a function of potential (Figure 7). The sigmoid function

$$
\text { rel } c_{\text {red }}=\frac{1}{\exp \left(\left(E-E_{i p}\right) \frac{n F}{R T}\right)+1}
$$

was fitted to the data, where rel $c_{\text {red }}$ is the relative concentration derived from the intensity $I_{\text {SERRS }}$ of the bands at 1359 and $1374 \mathrm{~cm}^{-1}$, respectively. $E_{\mathrm{ip}}$ is the potential of the inflection point, which reflects the midpoint potential $E_{\mathrm{m}}$ of the redox center, and $\varphi$ is a scaling factor. Ideally, $\varphi=R T / F \approx 26 \mathrm{mV}$, and $E_{\mathrm{ip}}=E_{\mathrm{m}}$ in accordance with the Nernst equation. Evaluating the band of $v_{4}^{\prime}$ revealed a midpoint potential of $E_{m}=-211 \mathrm{mV}$ and a scaling factor of $\varphi=38$ $\mathrm{mV}$ (see Fig. 7B). The evaluation of the band according to $v_{4}$ resulted in $E_{m}=-62 \mathrm{mV}$ and a scaling factor of $\varphi=61 \mathrm{mV}$ (see Fig. 7A). The sigmoid functions obtained as a function of potentials clearly indicate that electrochemical ET into the enzyme does take place. However, we see different $\mathrm{E}_{\mathrm{m}}$ values depending on the the band used for the evaluation. This can be explained in terms of different contributions of the three different heme species to the two marker bands.

This assumption is consistent with the potential dependence of a band that is truely selective, such as the band at $681 \mathrm{~cm}^{-1}$, which had been shown above to represent only the $\mathrm{b}$ hemes. In this case equation 2

$$
\text { rel } c_{\text {red }}=\frac{1-c_{1-2}}{\exp \left(\left(E-E_{i p, 1}\right) \frac{n F}{R T}\right)+1}+\frac{c_{1-2}}{\exp \left(\left(E-E_{i p, 2}\right) \frac{n F}{R T}\right)+1}
$$

had to be used to be fitted to the data, showing a two-step process (Fig. 7C) with two inflection points, $E_{\mathrm{m} 1}=-275 \mathrm{mV}$ and $\mathrm{E}_{\mathrm{m} 2}=-111 \mathrm{mV}$. $\mathrm{c}_{1-2}$ denotes the relative concentration of reduced species at the first reduction step. $E_{\mathrm{m} 1}$ and $E_{\mathrm{m} 2}$ correspond roughly to the two different $\mathrm{E}_{\mathrm{m}}$ values found for the overall marker bands $v_{4}$ described above. Concerning the different heights of the steps, they have to be considered in terms of the sensitivity of the band amplitude from the electric field, rather than in terms of different concentrations. Other bands gave a similar result (not shown), e.g. the bands at 343 and $403 \mathrm{~cm}^{-1}$. One could speculate about a stepwise reduction, for example of the $b$ hemes. The two $E_{m}$ values, however, are still within the potential window comprising ubisemiquinone rather than ubihydroquinone. This had been shown above (see the CVs Fig. 3) to be reduced at more negative potentials.

\section{Conclusion}

In general, SERR spectra obtained by electrochemical reduction/oxidation of the $b_{1}$ complex adsorbed to the SERR-active surface correspond to the spectra of the solubilized enzyme.(Le Moigne, Schoepp et al. 1999) Therefore we feel entitled to conclude from our results that electrochemical reduction/oxidation of all of the hemes of the $b_{1}$ complex does take place. However, direct electron transfer to one of the hemes, for example cytochrome $c_{1}$, which is nearest to the surface, seems to be excluded. Instead the hemes appear to take up 
the electrons via ubiquinone. $Q_{10}$, on the other hand, seems to exchange electrons with the electrode, even though the lipid phase is separated from the surface by about $3 \mathrm{~nm}$ as deduced from the dimensions of the hydrophilic and hydrophobic domain of the $b_{1}$ complex. However, $\mathrm{Q}_{10}$ will distribute between lipid and protein matrix where it may find a tunnel pathway to the electrode, possibly via $c_{1}$ which is closest to the electrode (Fig. 1). This would explain the electrochemical reduction of $Q_{10}$ taking place in two steps at around -350 $\mathrm{mV}$ and $-550 \mathrm{mV}$. The two steps are considered in terms of the ubisemiquinone and ubihydroquinone species, respectively. As deduced from these potentials, ubisemiquinone alone appears to mediate electron transfer into the hemes. Potentials as highly negative as $600 \mathrm{mV}$ required for the reduction to ubihydroquinone are obviously not needed to reduce the hemes. However, within the potential window investigated the hemes are possibly also reduced in two steps at around $-90 \mathrm{mV}$ and around $-200 \mathrm{mV}$ each. The first value corresponds quite nicely to midpoint potentials reported for the $b$ hemes, whereas the second one was not reported so far. In this context, we have to bear in mind that we are dealing with the electrochemical situation where reduction/oxidation may take place far from the thermodynamic potential due the kinetic limitations. This applies in particular to the reduction ubiquinone $(\mathrm{Q})$ to ubisemiquinone $(\mathrm{Q} \bullet-)$ and later to ubihydroquinone $\left(\mathrm{Q}^{2-}\right)$ the peak potentials of which are highly likely shifted to negative potentials. A large variety of midpoint potentials of the $\mathrm{Q} / \mathrm{Q} \bullet^{-}$and the $\mathrm{Q} \bullet^{-} / \mathrm{Q}^{2-}$ couple were reported in biochemical literature varying from $-600 \mathrm{mV}$ to $-160 \mathrm{mV}$ for $\mathrm{Q} / \mathrm{Q}^{-}{ }^{-}$and $+800 \mathrm{mV}$ to $+280 \mathrm{mV}$ for $\mathrm{Q} \bullet^{-} / \mathrm{Q}^{2-}$ (Osyczka, Moser et al. 2005; Grammel and Ghosh 2008). In these cases the $\mathrm{Q}^{-}{ }^{-} / \mathrm{Q}^{2-}$ couple is considered to be at a higher (more positive) potential than the $\mathrm{Q} / \mathrm{Q} \bullet^{-}$ couple. This is highly unlikely not only in the light of the present results, but also with respect to standard potentials known from electrochemical literature. For example the standard potential of $\mathrm{BQ} / \mathrm{BQ} \bullet^{-}$and the $\mathrm{BQ} \bullet{ }^{-} / \mathrm{BQ}^{2-}$ couples of benzoquinone are given in a textbook as $-540 \mathrm{mV}$ and $-140 \mathrm{mV}$, respectively, however, in a non-aqueous solution (Bard and Faulkner 2001). Irrespective of the absolute values, the $Q / Q \bullet^{-}$is always at a higher potential than the $\mathrm{Q} \bullet-/ \mathrm{Q}^{2-}$ couple, consistent with thermodynamics. The uptake of the first electron requires less energy than that of the second electron. We deduce from this consideration that the peak at around $-350 \mathrm{mV}$ corresponds to the $\mathrm{Q} / \mathrm{Q} \bullet^{-}$couple whereas the peak at $-550 \mathrm{mV}$ corresponds to the $\mathrm{Q} \bullet{ }^{-} / \mathrm{Q}^{2-}$ couple of ubiquinone $\mathrm{Q}_{10}$ at $\mathrm{pH}=7$. Unfortunately, midpoint potentials cannot be deduced from these data since a scan rate study is not feasible due the disappearance of the peaks with time. We can conclude, however, that under our experimental conditions, ubisemiquinone alone is sufficient to reduce the hemes and consequently also cyt $\mathrm{c}_{1}$, which has a more positive potential anyway. The occurrence of the ubisemiquinone is consistent with the corrected version of the $\mathrm{Q}$ cycle brought forward by the group of Dutton.(Osyczka, Moser et al. 2005) In this version the bifurcation of the electron pathway to $\mathrm{FeS}$ and $\mathrm{cyt}_{\mathrm{c}} \mathrm{c}_{1}$ on the one hand and the $\mathrm{b}$ hemes on the other occurs simultaneously at the $\mathrm{Q}_{0}$ site, with ubisemiquinone having a negligible lifetime. Interestingly, we observe the ubisemiquinone only with the $\mathrm{bc}_{1}$ complex immobilized by electrostatic attraction as described above. In this case we consider the cyt $c_{1}$ side directed towards the electrode, bearing in mind that speculations regarding orientation are merely tentative. Anyhow, bifurcation is prevented because cyt $c_{1}$ is in the reduced state together with ubiquinone $\mathrm{Q}_{10}$. Hence the $\mathrm{Q}^{-}{ }^{-}$species can be observed in the $\mathrm{CV}$. This could be considered as an indication of the $\mathrm{Q}_{0}$ site, particularly since ubisemiquinone is quickly consumed by the enzyme, see the evolution of the CVs with time (Fig.3, inset). In the case of 
the $b_{1}$ complex immobilized via a his-tag attached to the side opposite to $c_{1}$ we observe only the two-electron uptake directly to ubihydroquinone at around $-550 \mathrm{mV}$ (data not shown) without the intermediate step to the ubisemiquinone. This is consistent with data from the Jeuken group, who investigated ubiquinone $\mathrm{Q}_{10}$ incorporated in a tethered bilayer lipid membrane. They also observe the two-electron uptake at potentials of $-400 \mathrm{mV}$ to -500 $\mathrm{mV}$ depending on protonation states.(Jeuken, Bushby et al. 2006)

Summarizing hemes $b_{\mathrm{L}}, \mathrm{b}_{\mathrm{H}}$ and $\mathrm{c}_{1}$ are reduced via $\mathrm{QH} \bullet$ under electrochemical control. However, catalytic currents were not observed, which is not surprising considering that cyt $c$ is also reduced at the same potential together with the $b$ hemes.

As regards the SERR spectra, that we observe under these conditions, they show a much improved resolution and sensitivity as compared to the spectra in solution. This can be explained in terms of the surface enhancement effect described earlier for a silver electrode modified with Ag nanoparticles.(Grosserueschkamp, Nowak et al. 2009) Different mechanisms contribute to the enhanced Raman scattering of molecules adsorbed on rough metal substrates. The electro-magnetic enhancement effect (Jeanmaire and Van Duyne 1977) (EMEE) and the chemical enhancement effect (CEE)(Albrecht and Creighton 1977), the latter also known as charge transfer effect, are of great interest in this context. The EMEE is based on collective electron oscillations in resonance with the exciting laser wavelength yielding high electromagnetic field enhancement in the proximity of the metal surface.(Moskovits 1985) In the literature such electron oscillations are often referred to as localized surface plasmons (LSP), particularly if they are excited within nanoscopic metal structures such as colloids or rough surface bumps. The EMEE is capable of enhancing the intensity of Raman spectra of all molecules in close proximity to the metal substrate. Contrary to the EMEE the CEE only occurs if the probed molecules are adsorbed on the surface. The underlying mechanism is a charge-transfer resonance between the metal substrate and the adsorbed molecule. Assuming the Fermi level of the metal is located between the ground state and an excited state of the molecule, charge transfer transitions from the Fermi level to the excited state, as well as transitions from the ground state to the Fermi level, can contribute to the CEE.(Lombardi, Birke et al. 1986; Lombardi and Birke 2008)

Even though these effects are well understood, it is difficult to differentiate between contributions from EMEE and CEE. EM enhancement has to be considered particularly if the molecules are adsorbed to a metal substrate modified with nanoparticles. The Ag surface used in the present study had been optimized with respect to the enhancement factor using cytochrome $\mathrm{c}$ as a benchmark system.(Grosserueschkamp, Nowak et al. 2009) The good enhancement could be confirmed in the case of the $b_{1}$ complex. In this case, the enhancement is further reinforced by externally applied electric fields. This is clearly demonstrated by the comparatively high amplitude of the bands, particularly in the negative range of potentials. This is due to electrochemical properties of the Ag surface, which is sensitive to anodic dissolution at the positive edge of the potential window. Therefore, the potential window extends much more into the range below zero than above zero. In addition chemical enhancement may also play a role, particularly since the molecules are arranged on the surface in a strict orientation due to electrostatic interaction. This orientation is further supported by the lipid molecules inserted in between the protein entities. These effects taken together seem to overcome the traditionally poor signal-to-noise ratios in Raman spectra which are an inherent problem of the weak scattering process, particularly since in spectro-electrochemistry we are probing merely a monolayer of molecules. 
As a further advantage of spectro-electrochemistry, the redox state of the protein can be easily manipulated, so that a titration can be conducted. The regular changes recorded in this case further highlight the quality of the spectra which can be presented without any additional treatment.

Since the marker band for the $v_{4}$ mode shows sensitivity to the redox state, we conclude, that the potential dependent changes in intensity of the displayed bands are not exclusively generated by the charge transfer mechanism but also by a genuine reduction and oxidation of the hemes.

\section{Acknowledgement}

We are grateful to Carola Hunte, University of Freiburg, Germany, for critical discussions of the results and also for providing us with the sample of the $b_{1}$ complex the yeast Saccharomyces cerevisiae.

\section{References}

Albrecht, M. and J. A. Creighton (1977). "Anomalously Intense Raman-Spectra of Pyridine at a Silver Electrode." JACS 99(15): 5215-5217.

Bard, A. J. and L. R. Faulkner (2001). Electrochemical Methods: Fundamentals and Applications, John Wiley \& Sons.

Berry, E. A., L. S. Huang, et al. (2004). "X-Ray Structure of Rhodobacter Capsulatus Cytochrome bc (1): Comparison with its Mitochondrial and Chloroplast Counterparts." Photosynth Res 81(3): 251-75.

Chase, B. and B. Parkinson (1991). "A study of the wavelength and potential dependence of surface enhanced Raman scattering on copper, silver, and gold electrodes." The Journal of Physical Chemistry 95(20): 7810-7813.

Choi, S. and T. G. Spiro (1983). "Out-of-plane deformation modes in the resonance Raman spectra of metalloporphyrins and heme proteins." JACS 105(11): 3683-3692.

Feng, M. and H. Tachikawa (2008). "Surface-Enhanced Resonance Raman Spectroscopic Characterization of the Protein Native Structure." Journal of the American Chemical Society 130(23): 7443-7448.

Friedrich, M. G., J. W. Robertson, et al. (2008). "Electronic wiring of a multi-redox site membrane protein in a biomimetic surface architecture." Biophys J 94(9): 3698-3705.

Gao, F., H. Qin, et al. (1998). "Q-band resonance Raman spectra of oxidized and reduced mitochondrial bc1 complexes." Biochemistry 37(27): 9751-8.

Grammel, H. and R. Ghosh (2008). "Redox-State Dynamics of Ubiquinone-10 Imply Cooperative Regulation of Photosynthetic Membrane Expression in Rhodospirillum rubrum." Journal of Bacteriology 190, No.14: 4912-4921.

Grosserueschkamp, M., M. G. Friedrich, et al. (2009). "Electron transfer kinetics of cytochrome c probed by time-resolved surface-enhanced resonance Raman spectroscopy." J Phys Chem B 113(8): 2492-7.

Grosserueschkamp, M., C. Nowak, et al. (2009). "Silver Surfaces with Optimized Surface Enhancement by Self-Assembly of Silver Nanoparticles for Spectroelectrochemical Applications." Journal of Physical Chemistry C 113(41): 17698-17704. 
Hobbs, D. D., A. Kriauciunas, et al. (1990). "Resonance Raman spectroscopy of cytochrome bc1 complexes from Rhodospirillum rubrum: initial characterization and reductive titrations." Biochim Biophys Acta 1018(1): 47-54.

$\mathrm{Hu}$, S. (1993). "Comment on Cooper and Kennedy Flow-Insensitive Interprocedural Summary Information Computation Algorithm." Sigplan Notices 28(5): 3-8.

Hu, S., I. K. Morris, et al. (1993). "Complete assignment of cytochrome c resonance Raman spectra via enzymic reconstitution with isotopically labeled hemes." Journal of the American Chemical Society 115(26): 12446-12458.

Hu, S., K. M. Smith, et al. (1996). "Assignment of Protoheme Resonance Raman Spectrum by Heme Labeling in Myoglobin." Journal of the American Chemical Society 118(50): 12638-12646.

Hunte, C., J. Koepke, et al. (2000). "Structure at 2.3 A resolution of the cytochrome bc(1) complex from the yeast Saccharomyces cerevisiae co-crystallized with an antibody Fv fragment." Structure 8(6): 669-84.

Jeanmaire, D. L. and R. P. Van Duyne (1977). "Surface raman spectroelectrochemistry: Part I. Heterocyclic, aromatic, and aliphatic amines adsorbed on the anodized silver electrode." Journal of Electroanalytical Chemistry 84(1): 1-20.

Jeuken, L. J. C., R. J. Bushby, et al. (2006). "Proton transport into a tethered bilayer lipid membrane." Electrochemistry Communications 9(4): 610-614.

Kitagawa, T., Y. Kyogoku, et al. (1975). "Resonance Raman scattering from hemoproteins. Effects of ligands upon the Raman spectra of various C-type cytochromes." J Biochem 78(4): 719-28.

Kitagawa, T., Y. Ozaki, et al. (1978). "Resonance Raman studies on the ligand-iron interactions in hemoproteins and metallo-porphyrins." Adv Biophys 11: 153-96.

Kozlowski, P. M., J. R. Bingham, et al. (2008). "Theoretical Analysis of Core Size Effect in Metalloporphyrins." Journal of physical Chemistry A 112(50): 12781-12788.

Lange, C. and C. Hunte (2002). "Crystal structure of the yeast cytochrome bc1 complex with its bound substrate cytochrome c." Proc Natl Acad Sci U S A 99(5): 2800-5.

Lange, C., J. H. Nett, et al. (2001). "Specific roles of protein-phospholipid interactions in the yeast cytochrome bc1 complex structure." EMBO J 20(23): 6591-600.

Le Moigne, C., B. Schoepp, et al. (1999). "Distinct structures and environments for the three hemes of the cytochrome bc1 complex from Rhodospirillum rubrum. A resonance Raman study using B-band excitations." Biochemistry 38(3): 1066-76.

Lombardi, J. R. and R. L. Birke (2008). "A unified approach to surface-enhanced Raman spectroscopy." Journal of Physical Chemistry C 112(14): 5605-5617.

Lombardi, J. R., R. L. Birke, et al. (1986). "Charge-transfer theory of surface enhanced Raman spectroscopy: Herzberg--Teller contributions." The Journal of Chemical Physics 84(8): 4174-4180.

Moskovits, M. (1985). "Surface-Enhanced Spectroscopy." Review of Modern Physics 57(3): 783-826.

Nowak, C., D. Schach, et al. (2009). "Oriented Immobilization and Electron Transfer to the Cytochrome c Oxidase." J.Solid State Electrochemistry DOI 10.1007/s10008-0101032-x.

Osawa, M., N. Matsuda, et al. (1994). "Charge transfer resonance Raman process in surfaceenhanced Raman scattering from p-aminothiophenol adsorbed on silver: HerzbergTeller contribution." The Journal of Physical Chemistry 98(48): 12702-12707. 
Osyczka, A., C. C. Moser, et al. (2005). "Fixing the Q cycle." TRENDS in Biochemical Sciences 30 No.4: 176-182.

Pálsdóttir, H. and C. Hunte (2003). Purification of the Cytochrome bc1 Complex from Yeast. Membrane Protein Purification and Crystallization (Second Edition). H. Carola, J. Gebhard Von and S. Hermann. San Diego, Academic Press: 191-203.

Palsdottir, H., C. G. Lojero, et al. (2003). "Structure of the yeast cytochrome bc1 complex with a hydroxyquinone anion Qo site inhibitor bound." J Biol Chem 278(33): 3130311.

Schach, D., C. Nowak, et al. (2010). "Modeling direct electron transfer to a multi-redox center protein: cytochrome c oxidase." J. of Electroanalytical Chemistry DOI10.1016/j.jelechem.2010.07.009.

Solmaz, S. R. and C. Hunte (2008). "Structure of complex III with bound cytochrome c in reduced state and definition of a minimal core interface for electron transfer." J Biol Chem 283(25): 17542-9.

Spiro, T. G. (1975). "Resonance Raman spectroscopic studies of heme proteins." Biochim Biophys Acta 416(2): 169-89.

Spiro, T. G. (1978). "Resonance Raman spectra of hemoproteins." Methods Enzymol 54: 23349.

Spiro, T. G. (1988). Biological applications of Raman spectroscopy, Vol. 3: Resonance Raman spectra of heme proteins and other metalloproteins.

Spiro, T. G., R. S. Czernuszewicz, et al. (1989). "Metalloporphyrin structure and dynamics from resonance raman spectroscopy." Coordination Chemistry Reviews 100: 541571.

Spiro, T. G. and T. C. Strekas (1974). "Resonance Raman spectra of heme proteins. Effects of oxidation and spin state." J Am Chem Soc 96(2): 338-45.

Xia, D., C. A. Yu, et al. (1997). "Crystal structure of the cytochrome bc1 complex from bovine heart mitochondria." Science 277(5322): 60-6. 


\title{
Self-Oscillating Gel as Novel Biomimetic Materials
}

\author{
Ryo Yoshida \\ Department of Materials Engineering, Graduate School of Engineering \\ The University of Tokyo, \\ Japan
}

\section{Introduction}

Polymer gel is a research field of polymer science which has made rapid progress during the past 20-30 years. Since the discovery of "volume phase transition" phenomena (Tanaka, 1978) as a turning point, many researchers have developed several kinds of stimuliresponsive polymer gels that exhibit reversible swelling-deswelling change in response to environmental changes such as solvent composition, temperature, $\mathrm{pH}$ change, etc. Especially, from the late 1980's or early 1990's, new functional gels which sense an external signal (sensor function), judge it (processor function) and take action (actuator function), have been developed as "intelligent gels" or "smart gels", and their applications have been demonstrated. As typical examples, many biomedical applications to actuator (artificial muscle), drug delivery systems (DDS), tissue engineering, purification or separation systems, biosensor, shape memory materials, molecular recognition systems, etc., have been extensively studied (Yoshida, 2005; Ottenbrite, et al., 2010; Miyata, 2002; Osada \& Khokhlov, 2002). And also, the gels has used in the field of micromachines and nanotechnology $[9,10]$. In addition to new synthetic methods to give unique functions by molecular design in nanoorder scale including supramolecular design, the design and construction of micro or nano material systems with the biomimetic functions have been attempted.

One of the characteristic behavior in living systems is autonomous oscillation, that is, spontaneous changes with temporal periodicity (called "temporal structure") such as heartbeat, brain waves, pulsatile secretion of hormone, cell cycle, and biorhythm. Although several stimuli-responsive polymer systems have been studied from the standpoint of biomimetics, polymer systems undergoing self-oscillation under constant condition without any on-off switching of external stimuli are still undeveloped. If autonomous polymer systems resembling living organisms can be realized by using completely synthetic polymers, unprecedented biomimetic materials may be created.

The author developed a novel polymer gel that cause autonomous mechanical oscillation without an external control in a completely closed solution. In order to realize the autonomous polymer system, the Belousov-Zhabotinsky (BZ) reaction, which is well-known for exhibiting temporal and spatiotemporal oscillating phenomena (Field, et al., 1985; Epstein \& Pojman, 1998), was focused. The BZ reaction is often analogically compared with the TCA cycle which is a key metabolic process taking place in the living body, and it is recognized as a chemical model for understanding several autonomous phenomena in 
biological systems. The overall process of the BZ reaction is the oxidation of an organic substrate, such as malonic acid (MA) or citric acid, by an oxidizing agent (bromate ion) in the presence of a strong acid and a metal catalyst. In the course of the reaction, the catalyst undergoes spontaneous redox oscillation. When the solution is homogeneously stirred, the color of the solution periodically changes, like a neon sign, based on the redox changes of the metal catalyst. When the solution is placed as a thin film in stationary conditions, concentric or spiral wave patterns develop in the solution. The wave of oxidized state propagating in the medium at a constant speed is called a "chemical wave". The significance of the BZ reaction has been recognized as a chemical model for understanding some aspects of biological phenomena, such as glycolytic oscillations or biorhythms, cardiac fibrillation, self-organization of amoeba cells, pattern formation on animal skin, visual pattern processing on retina, etc.

The author attempted to convert the chemical oscillation of the BZ reaction into a mechanical change in gels and generate an autonomous swelling-deswelling oscillation under non-oscillatory outer conditions. A copolymer gel consisting of Nisopropylacrylamide (NIPAAm) and ruthenium tris(2,2'-bipyridine) ( $\left.\mathrm{Ru}(\mathrm{bpy})_{3}\right)$ was prepared. $\mathrm{Ru}(\mathrm{bpy})_{3}$, acting as a catalyst for the $\mathrm{BZ}$ reaction, is pendent to the polymer chains of NIPAAm. Poly(NIPAAm) is a well known thermosensitive polymer which exhibits a lower critical solution temperature (LCST) of approximately $32^{\circ} \mathrm{C}$, and the homopolymer gel undergoes a volume phase transition at that temperature. For the poly(NIPAAm-co-Ru(bpy) 3 ) gel, the oxidation of $\mathrm{Ru}(\mathrm{bpy})_{3}{ }^{2+}$ moiety caused not only an increase in the swelling degree of the gel, but also a rise in the volume phase transition temperature. As a result, it is expected that the gel undergoes a cyclic swelling-deswelling change when the $\mathrm{Ru}(\mathrm{bpy})_{3}$ moiety is periodically oxidized and reduced under constant temperature. When the gel is immersed in an aqueous solution containing the substrates of the $\mathrm{BZ}$ reaction (malonic acid, nitric acid, and sodium bromate) except for the catalyst, the substrates penetrates into the polymer network and the BZ reaction occurs in the gel. Consequently, periodic redox changes induced by the BZ reaction produce periodic swelling-deswelling changes in the gel (Fugure 1).

Since being first reported in 1996 as a "self-oscillating gel" (Yoshida et al., 1996; 1997), the author have been systematically studying the self-oscillating polymer and gel as well as their applications to biomimetic or smart materials (Figure 2). In fact, as applications to autonomic biomimetic actuators, ciliary motion actuators (Tabata et al., 2002, 2003) and selfwalking gels (Maeda et al. 2007), etc. were realized. As an autonomic microconveyor, mass transport surface by utilizing peristaltic motion of the self-oscillating gel was also designed (Murase et al., 2009, 2010). In the case of the uncrosslinked linear polymer, the polymer undergoes spontaneous cyclic soluble-insoluble changes and the transmittance of the polymer solution oscillates autonomously (Yoshida et al., 2002a). In addition, submicronsized self-oscillating microgel beads were prepared by a precipitation polymerization method (Suzuki et al., 2008, 2009; Suzuki \& Yoshida, 2008a, 2008b, 2010; Taniguchi et al., 2010). In order to realize nano-actuators that exhibit autonomous oscillation on a nanometer scale (nano-oscillator) by utilizing the linear polymer chain or the microgels, their oscillating behavior were investigated through the optical transmittance or viscosity changes (Suzuki et al., 2009; Taniguchi et al., 2010; Hara \& Yoshida, 2008) of the polymer solution or microgel dispersions. 


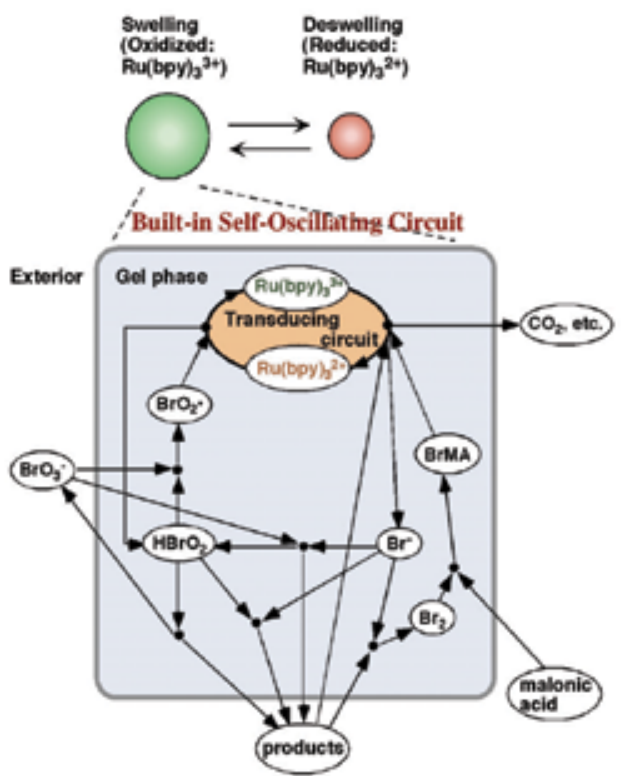

Reaction network of the $\mathrm{BZ}$ reaction (FKN mechanism)
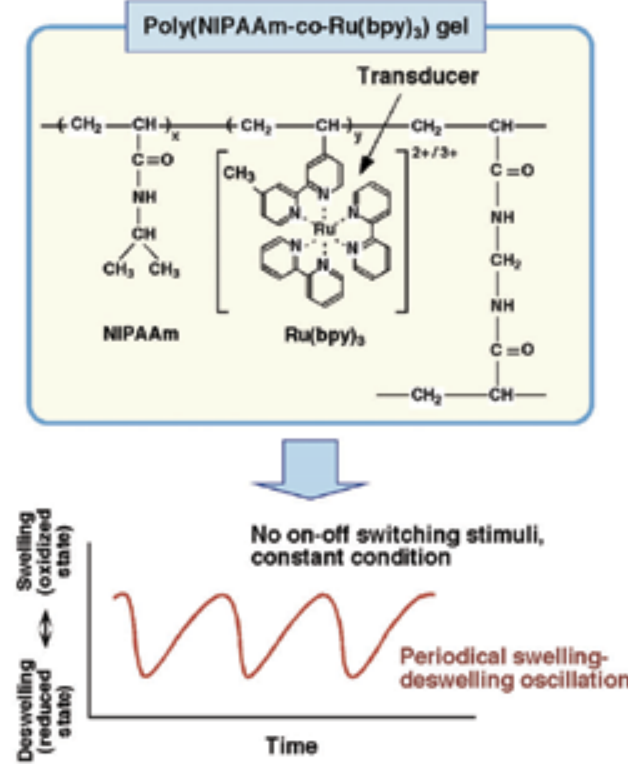

Fig. 1. Mechanism of self-oscillation for poly(NIPAAm-co-Ru(bpy) $3^{2+}$ ) gel coupled with the Belousov-Zhabotinsky reaction.

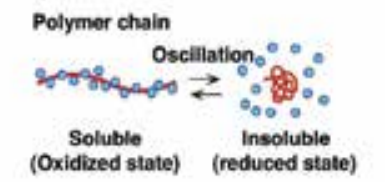

Self-oscillation of polymer chains

\section{Self-oscillating gel}
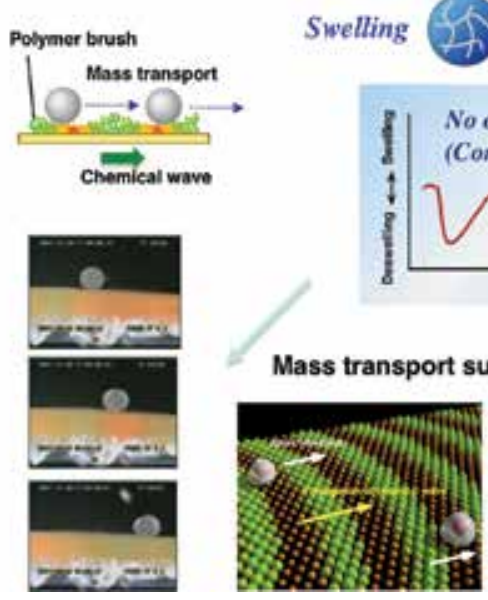

- Transmittance oscillation

- Viscosity oscillation

Self-flocculating/ dispersing oscillation of microgels

\section{Swelling}

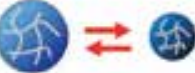

Deswelling

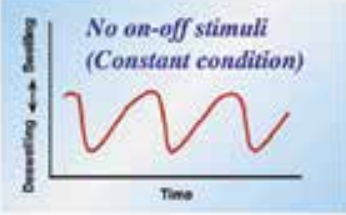

\section{Peristaltic \\ motion}

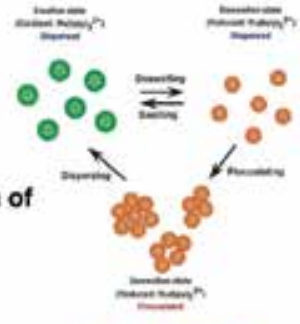

Fig. 2. Development of self-oscillating polymers and gels.

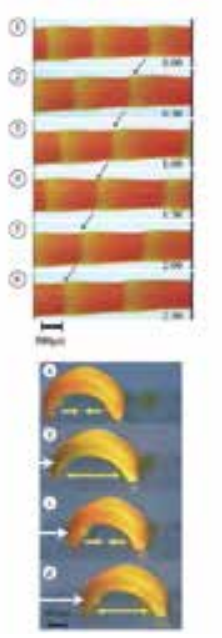




\section{Self-oscillating behavior of the gel}

\subsection{Self-oscillation of gel with smaller size than chemical wavelength and control of oscillating behavior}

Figure 3 shows the observed oscillating behavior under a microscope for the small cubic poly(NIPAAm-co-Ru(bpy) $3^{2+}$ ) gel (each length of about $0.5 \mathrm{~mm}$ ). In miniature gels sufficiently smaller than the wavelength of the chemical wave (typically several $\mathrm{mm}$ ), the redox change of ruthenium catalyst can be regarded to occur homogeneously without pattern formation (Yoshida et al., 2000). Due to the redox oscillation of the immobilized $\mathrm{Ru}(\mathrm{bpy}) 3^{2+}$, mechanical swelling-deswelling oscillation of the gel autonomously occurs with the same period as for the redox oscillation. The volume change is isotropic and the gel beats as a whole, like a heart muscle cell. The chemical and mechanical oscillations are synchronized without a phase difference (i.e., the gel exhibits swelling during the oxidized state and deswelling during the reduced state).

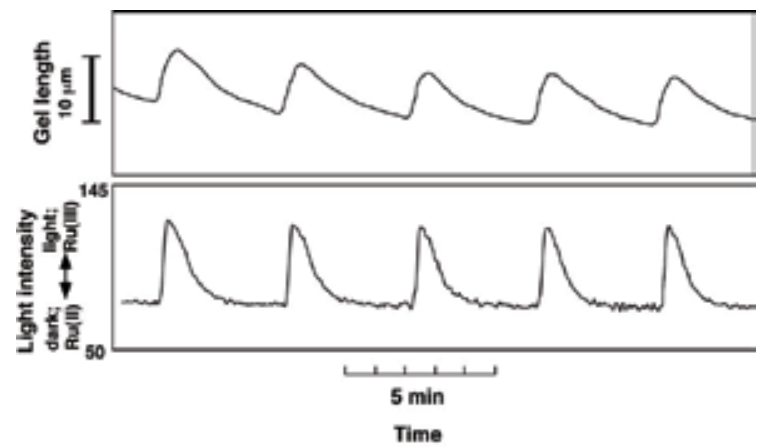

Fig. 3. Periodic redox changes of the miniature cubic poly(NIPAAm-co-Ru(bpy) $3^{2+}$ ) gel (lower) and the swelling-deswelling oscillation (upper) at $20^{\circ} \mathrm{C}$. Color changes of the gel accompanied by redox oscillations (orange: reduced state, light green: the oxidized state) were converted to 8-bit grayscale changes (dark: reduced, light: oxidized) by image processing. Transmitted light intensity is expressed as an 8-bit grayscale value. Outer solution: $[\mathrm{MA}]=62.5 \mathrm{mM} ;\left[\mathrm{NaBrO}_{3}\right]=84 \mathrm{mM}$; $\left[\mathrm{HNO}_{3}\right]=0.6 \mathrm{M}$. (Yoshida et al., 2000)

Typically, the oscillation period increases with a decrease in the initial concentration of substrates. Further, in general, the oscillation frequency (the reciprocal of the period) of the $\mathrm{BZ}$ reaction tends to increase as the temperature increases, in accordance with the Arrhenius equation. The swelling-deswelling amplitude of the gel increases with an increase in the oscillation period and amplitude of the redox changes. Therefore the swelling-deswelling amplitude of the gel is controllable by changing the initial concentration of substrates as well as temperature.

As inherent behavior of the BZ reaction, the abrupt transition from steady state (nonoscillating state) to oscillating state occurs with a change in controlling parameter such as chemical composition, light, etc. By utilizing these characteristics, reversible on-off regulation of self-beating triggered by addition and removal of MA was successfully achieved (Yoshida et al., 2003). We showed the oscillating behavior of the gel when the stepwise concentration change in MA was repeated between lower concentration $(10 \mathrm{mM})$ in steady state and higher concentration $(25 \mathrm{mM})$ in oscillating state. At $10 \mathrm{mM}$, the redox oscillation does not occur and consequently the gel exhibited no swelling-deswellng 
changes. Then the concentration was quickly increased to $25 \mathrm{mM}$. Immediately after increasing concentration, the gel started self-beating. The beating stopped again as soon as the concentration was decreased back to the initial value. In these ways, reversible on-off regulation of self-beating triggered by MA was successfully achieved. Since there are some organic acid which can be the substrate for the $\mathrm{BZ}$ reaction (e.g., citric acid), the same regulation of beating is possible by using those organic acid instead of MA. As the gel has thermosensitivity due to the NIPAAm component, the beating rhythm can be also controlled by temperature. By utilizing the thermosensitivity, on-off control of the selfoscillation is also possible. At lower temperature, the oscillation does not occur because there is no remarkable difference in hydrophilicity of the polymer between the redox states, but the difference becomes remarkable at higher temperature and cause self-oscillation (Ito et al., 2003).

It is well known that the period of oscillation is affected by light illumination for the $\mathrm{Ru}(\mathrm{bpy}) 3^{2+}$-catalysed BZ reaction. Therefore we can intentionally make a pacemaker with a desired period (or wavelength) by local illumination of laser beam to the gel, or we can change the period (or wavelength) by local illumination to a pacemaker which already exists in the gel (Yoshida et al., 2002b). Optical on-off control of the self-oscillating motion of the gel were demonstrated (Takeoka et al., 2003; Shinohara et al., 2008a, 2008b).

\subsection{Peristaltic motion of gels with propagation of chemical wave}

When the gel size is larger than chemical wavelength, the chemical wave propagates in the gel by coupling with diffusion of intermediates. Then peristaltic motion of the gel is created. Figure 4 shows the cylindrical gel which is immersed in an aqueous solution containing the three reactants of the BZ reaction. The chemical waves propagate in the gel at a constant speed in the direction of the gel length (Maeda et al., 2008a). Considering the orange ( $\mathrm{Ru}(\mathrm{II}))$ and green $(\mathrm{Ru}(\mathrm{III}))$ zones represent simply the shrunken and swollen parts respectively, the locally swollen and shrunken parts move with the chemical wave, like the peristaltic motion of living worms.

The tensile force of the cylindrical poly(NIPAAm-co-Ru(bpy) $3^{2+}$ ) gel with oscillation was also measured (Sasaki et al., 2003). Further, swelling behavior of the gel during selfoscillation was observed using CCD camera and the force due to the BZ reaction was evaluated using semiconductor gauge (Aoki et al., 2007). Self-oscillating swelling-deswelling behavior in samples with different swelling ratio was evaluated by this apparatus, and mechanical parameters such as Young's modulus, stress amplitude and oscillating period were quantitatively estimated. Dependency of initial length in samples on Young's modulus seems to be related to the propagation of chemical wave in the samples.

\section{Design of biomimetic actuator using self-oscillating polymer and gel}

\subsection{Ciliary motion actuator using self-oscillating gel}

Recently, microfabrication technologies such as photolithography are also attempted for preparation of microgels. Since any shape of gel can be created by these methods, application as a new manufacturing method for soft microactuator, microgel valve, gel display, etc. is expected. Microfabrication of gel has also been attempted by photolithography for application to such micro-devices (Yoshida et al., 2006a, 2006b). 


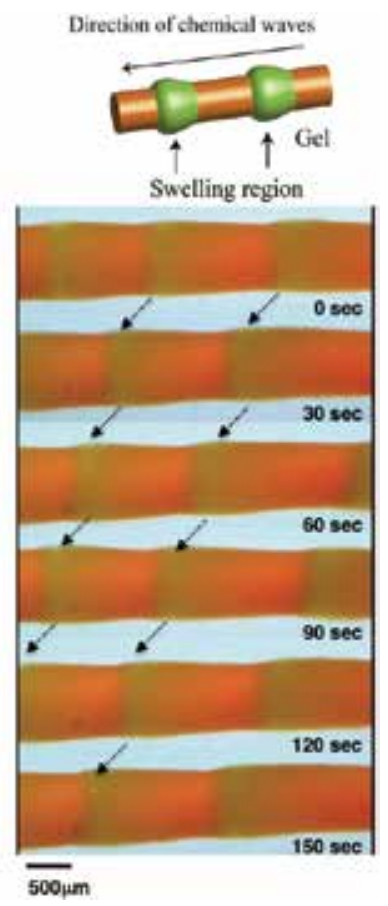

Fig. 4. Time course of peristaltic motion of poly(NIPAAm-co-Ru(bpy) $3^{2+}$-co-AMPS) gel in a solution of the $\mathrm{BZ}$ substrates at $18^{\circ} \mathrm{C}$. The green and orange colors correspond to the oxidized and reduced states of the Ru moiety in the gel, respectively (Maeda et al., 2008).

One of the promising fields of the MEMS is micro actuator array or distributed actuator systems. The actuators, which have a very simple actuation motion such as up and down motion, are arranged in an array form. If their motions are random, no work is extracted from this array. However, by controlling them to operate in a certain order, they can generate work as a system. A typical example of this kind of actuation array is a ciliary motion micro actuator array. There have been many reports on this system. Although various actuation principles have been proposed, all the previous work is based on the concept that the motion of actuators is controlled by external signals. If a self-oscillating gel plate with a micro projection structure array on top were realized, it would be expected that the chemical wave propagation would create dynamic rhythmic motion of the structure array. This proposed structure could exhibit spontaneous dynamic propagating oscillation producing a ciliary motion array (Figure 5)(Tabata et al., 2002, 2003).

A gel plate with micro projection array was fabricated by molding. First, moving mask deep-X-ray lithography was utilized to fabricate a PMMA plate with a truncated conical shape microstructure array. This step was followed by evaporation of a Au seed layer and subsequent electroplating of nickel to form the metal mold structure. Then, a PDMS mold structure was duplicated from the Ni structure and utilized for gel molding. The formation of gel was carried out by vacuum injection molding. A structure with a height of $300 \mu \mathrm{m}$ and bottom diameter of $100 \mu \mathrm{m}$ was successfully fabricated by the described process. The propagation of chemical reaction wave and dynamic rhythmic motion of the micro projection array were confirmed by chemical wave observation and displacement measurements. Motion of the top with $5 \mu \mathrm{m}$ range in both lateral and vertical directions, 
and elliptical motion of the projection top were observed. The feasibility of the new concept of the ciliary motion actuator made of self-oscillating polymer gel was successfully confirmed. The actuator may serve as a micro-conveyer to transport objects on the surface.

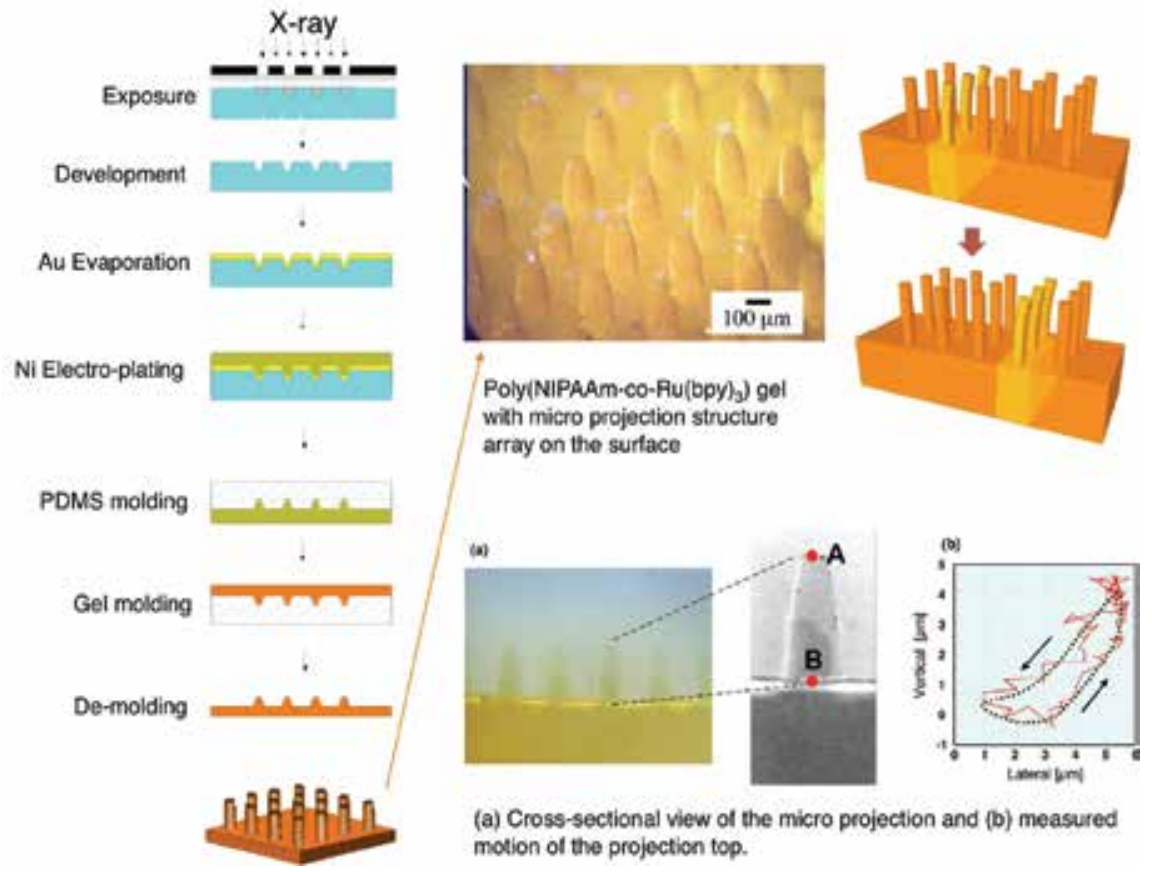

Fig. 5. Fabrication of ciliary motion actuator (artificial cilia) using self-oscillating gel (Tabata et al., 2002, 2003).

\subsection{Self-walking gel}

Further, we successfully developed a novel biomimetic walking-gel actuator made of selfoscillating gel (Maeda et al., 2007). To produce directional movement of gel, asymmetrical swelling-deswelling is desired. For these purposes, as a third component, hydrophilic 2acrylamido-2'-methylpropanesulfonic acid (AMPS) was copolymerized into the polymer to lubricate the gel and to cause anisotropic contraction. During polymerization, the monomer solution faces two different surfaces of plates; a hydrophilic glass surface and a hydrophobic Teflon surface. As the thickness of the spacer is thin $(0.5 \mathrm{~mm})$, the surface property of the plate may affect the distribution of the monomer in the solution. Since $\mathrm{Ru}(\mathrm{bpy}) 3^{2+}$ monomer is hydrophobic, it easily migrates to the Teflon surface side. As a result, a non-uniform distribution along the height is formed by the components, and the resulting gel has gradient distribution for the content of each component in the polymer network.

In order to convert the bending and stretching changes to one-directional motion, we employed a ratchet mechanism. A ratchet base with an asymmetrical surface structure was fabricated. On the ratchet base, the gel repeatedly bends and stretches autonomously resulting in the forward motion of the gel, while sliding backwards is prevented by the teeth of the ratchet. Figure 6 shows successive profiles of the "self-walking" motion of the gel like a looper in the BZ substrate solution under constant temperature. The walking velocity of the gel actuator was approximately $170 \mu \mathrm{m} / \mathrm{min}$. Since the oscillating period and the 
propagating velocity of chemical wave change with concentration of substrates in the outer solution, the walking velocity of the gel can be controlled. By using the gel with gradient structure, other type of actuator which generates a pendulum motion is also realized (Maeda et al., 2008b).

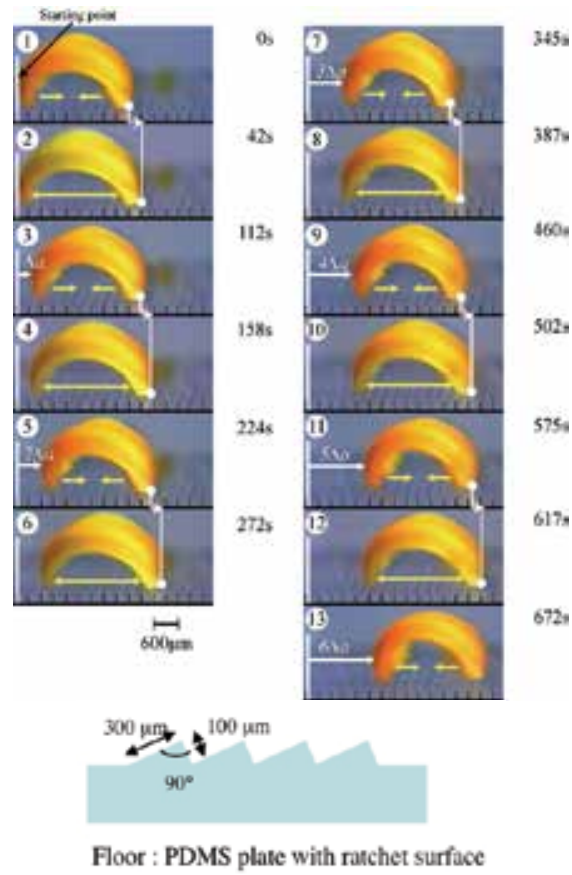

Fig. 6. Time course of self-walking motion of the gel actuator. During stretching, the front edge can slide forward on the base, but the rear edge is prevented from sliding backwards. Oppositely, during bending, the front edge is prevented from sliding backwards while the rear edge can slide forward. This action is repeated, and as a result, the gel walks forward. Outer solution: $[\mathrm{MA}]=62.5 \mathrm{mM},\left[\mathrm{NaBrO}_{3}\right]=84 \mathrm{mM},\left[\mathrm{HNO}_{3}\right]=0.894 \mathrm{M}, 18^{\circ} \mathrm{C}$ (Maeda et al., 2007).

\subsection{Mass transport surface utilizing peristaltic motion of gel}

Further, we attempted to transport an object by utilizing the peristaltic motion of the selfoscillating gel (Murase et al., 2009a, 2009b, 2010). In order to control the transportability, it is necessary to enhance the driving force as a conveyer. It is effective to copolymerize AMPS to poly(NIPAAm-co-Ru(bpy) $3^{2+}$ ) gel network to generate large amplitude of volume change of the self-oscillating gel. For the design of mass transport surface, the influence of the AMPS's feed ratio on the swelling-deswelling properties of poly(NIPAAm-co$\mathrm{Ru}(\mathrm{bpy}) 3^{2+}$-co-AMPS) gels and mass transportability of the gels were investigated.

The gel had a microphase-separated structure when the AMPS's feed ratio was less than 5 mol $\%$ due to the effect of the poor solvent in the polymerization process. On the other hand, when the AMPS's feed ratio is more than $10 \mathrm{~mol} \%$, the gel had a homogeneous structure. The microphase-separated structure highly improved the swelling-deswelling kinetics and generated swelling-deswelling amplitude more than $10 \%$ of the gel thickness, that was approximately 10 times larger than that of the gel with a homogeneous network structure. 
As a model object, a cylindrical poly(acrylamide) (PAAm) gel was put on the gel surface. It was observed that the PAAm gel was transported on the gel surface with the propagation of the chemical wave as it rolled (Figure 7) when the AMPS's feed ratio was low (less than 2.5 mol \%). It was found that the cylindrical PAAm gel was not transported if the inclination angle of the wave front was less than approximately $0.05 \mathrm{rad}$. The mass transportability does not depend on the velocity of the chemical wave but on the diameter of the cylindrical PAAm gel and the inclination angle of the wave front. We have proposed a model to describe the mass transport phenomena based on the Hertz contact theory, and the relation between the transportability and the peristaltic motion was investigated for the cases that the loaded gel cargos were gel bead and cylindrical gel (Murase et al., 2010). It was found that the surface design to form grooves was efficient to increase contact areas when the wave front of the peristaltic motion reaches the loaded cargo and useful to transport not only cylindrical cargos but also spherical cargos in desired directions.

The functional gel surface generating autonomous and periodic peristaltic motion has a potential for several new applications such as a conveyer to transport soft materials, a formation process for ordered structures of micro- and/or nanomaterials, a self-cleaning surface and so on.
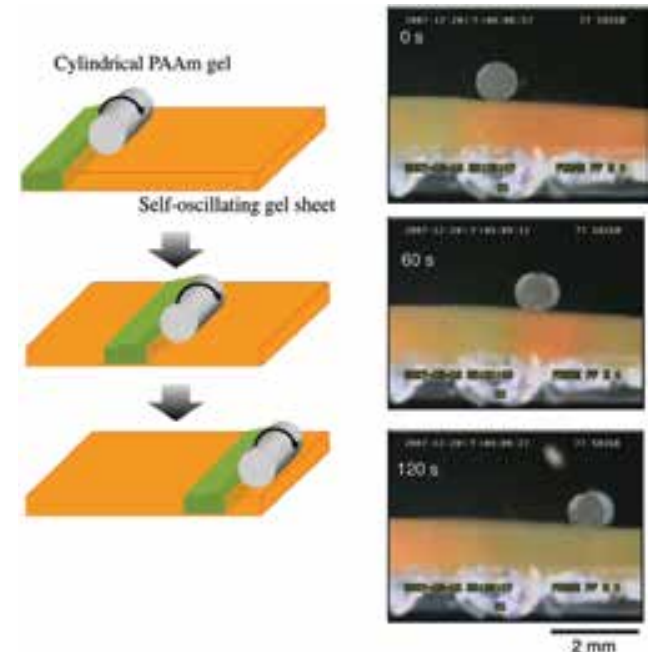

Fig. 7. Schematic illustration of mass transport on the peristaltic surface (left) and observed transport of cylindrical PAAm gel on the poly(NIPAAm-co-Ru(bpy) $3^{2+}$-co-AMPS) gel sheet in a solution of the $\mathrm{BZ}$ substrates $\left(\mathrm{MA}, \mathrm{NaBrO}_{3}\right.$ and $\left.\mathrm{HNO}_{3}\right)$ (right) (Murase et al., 2009).

\subsection{Control of chemical wave propagation through gap junction in self-oscillating gel array}

A chemomechanical actuator utilizing a reaction-diffusion wave across gap junction was constructed toward a novel mircoconveyer by micropatterned self-oscillating gel array (Tateyama et al., 2008). Unidirectional propagation of the chemical wave the BZ reaction was induced on gel arrays. In the case of using a triangle-shaped gel as an element of the array, the chemical wave propagated from the corner side of the triangle gel to the plane side of the other gel (C-to-P) across the gap junction, whereas it propagated from the plane side to the corner side (P-to-C) in the case of the pentagonal gel array. Numerical analysis 
based on theoretical model was done for understanding the mechanism of unidirectional propagation in triangle and pentagonal gel arrays. By fabricating different shapes of gel arrays, control of the direction is possible. The swelling and deswelling changes of the gels followed the unidirectional propagation of chemical wave.

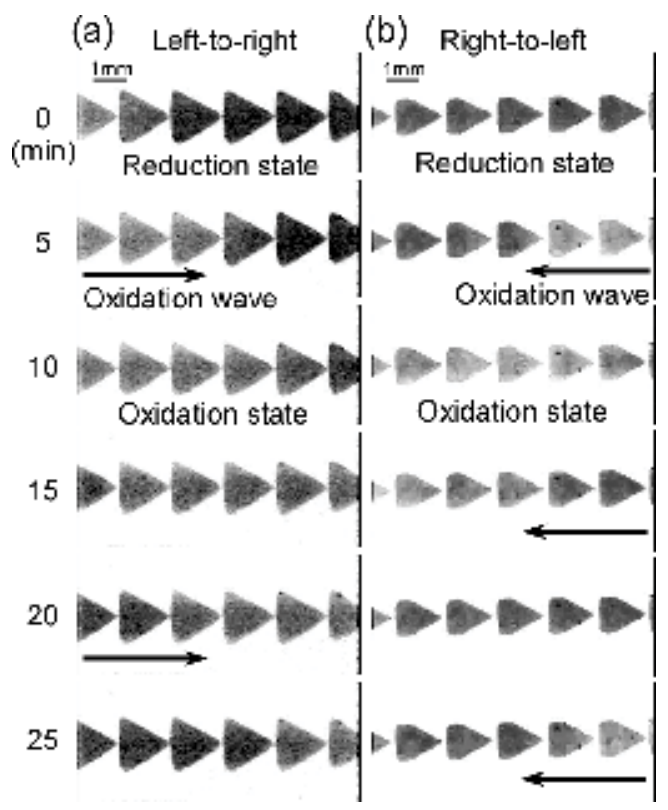

Fig. 8. Propagating behavior of the chemical wave on the (a) triangle gel array and (b) pentagonal gel array (Tateyama et al., 2008).

\section{Self-oscillating polymer chains and microgels as "nano-oscillator"}

\subsection{Self-oscillation of polymer chains with rhythmical soluble-insoluble changes}

In self-oscillating gel, redox changes of $\mathrm{Ru}(\mathrm{bpy}) 3^{2+}$ catalyst are converted to conformational changes of polymer chain by polymerization. The conformational changes are amplified to macroscopic swelling-deswelling changes of the polymer network by crosslinking. Further, when the gel size is larger than chemical wavelength, the chemical wave propagates in the gel by coupling with diffusion. Then peristaltic motion of the gel is created. In this manner, a synchronization process exists from microscopic to macroscopic level in the self-oscillating gel.

The periodic changes of linear and uncrosslinked polymer chains can be easily observed as cyclic transparent and opaque changes for the polymer solution with color changes due to the redox oscillation of the catalyst (Yoshida et al., 2002a). Figure 9(a) shows the oscillation profiles of transmittance for a polymer solution which consists of linear poly(NIPAAm-co$\left.\mathrm{Ru}(\text { bpy })_{3}{ }^{2+}\right), \mathrm{MA}, \mathrm{NaBrO}_{3}$ and $\mathrm{HNO}_{3}$ at constant temperatures. The wavelength $(570 \mathrm{~nm})$ at the isosbestic point of reduced and oxidized states was used to detect the optical transmittance changes based on soluble-insoluble changes of the polymer, not on the redox changes of the $\mathrm{Ru}(\mathrm{bpy})_{3}$ moiety. Synchronized with the periodical changes between $\mathrm{Ru}(\mathrm{II})$ and $\mathrm{Ru}(\mathrm{III})$ states of the $\mathrm{Ru}(\mathrm{bpy}) 3^{2+}$ site, the polymer becomes hydrophobic and hydrophilic, and exhibits cyclic soluble-insoluble changes. 
By grafting the polymers or arraying the gel beads on the surface of substrates, we have attempted to design self-oscillating surfaces as nano-conveyers. The self-oscillating polymer was covalently immobilized on a glass surface and self-oscillation was directly observed at a molecular level by AFM (Ito et al., 2006). The self-oscillating polymer with $N$-succinimidyl group was immobilized on an aminosilane-coupled glass plate. While no oscillation was observed in pure water, nano-scale oscillation was observed in an aqueous solution containing the BZ substrates. The amplitude was about $10-15 \mathrm{~nm}$ and the period was about $70 \mathrm{sec}$, although some irregular behavior was observed due to no stirring. The amplitude was less than that in solution, as observed by DLS (23.9 and $59.6 \mathrm{~nm}$ ). This smaller amplitude may be because the structure of the immobilized polymer was a loop-train-tail: the moving regions were shorter than that of the soluble polymer. The amplitude and frequency were controlled by the concentration of reactant, as observed in the solution. Here nano-scale molecular self-oscillation was observed for the first time. The oscillation polymer chain may be used as a component of a nano-clock or nano-machine.

(a)

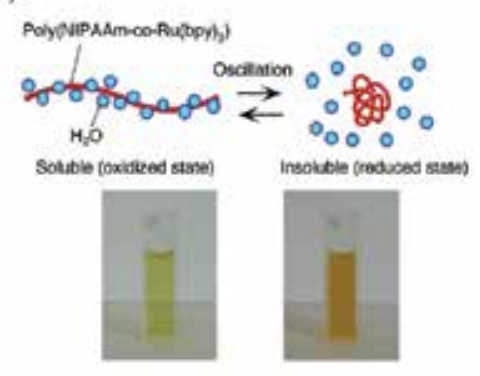

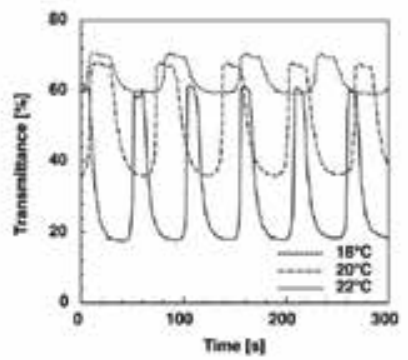

(b)

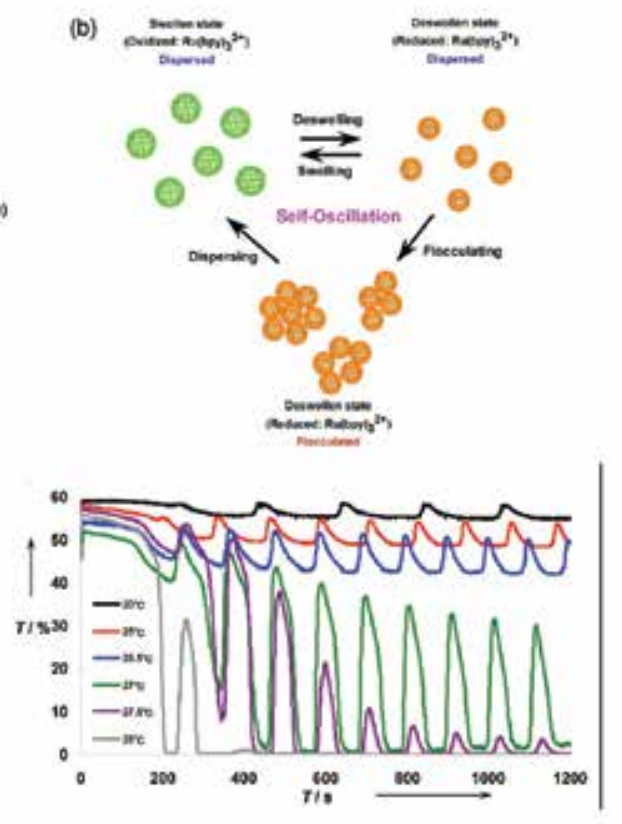

Fig. 9. (a) Oscillating profiles of optical transmittance for poly(NIPAAm-co-Ru(bpy) $3^{2+}$ ) solution at several temperatures (Yoshida et al., 2002). (b) Self-oscillating profiles of optical transmittance for microgel dispersions at several temperatures. The microgels were dispersed in aqueous solutions containing $\mathrm{MA}(62.5 \mathrm{mM}), \mathrm{NaBrO}_{3}(84 \mathrm{mM})$, and $\mathrm{HNO}_{3}$ (0.3M). Microgel concentration was $0.25 \mathrm{wt} \%$ (Suzuki et al, 2008).

\subsection{Self-flocculating/dispersing oscillation of microgels}

We then prepared submicron-sized poly(NIPAAm-co-Ru(bpy) $3^{2+}$ ) gel beads by surfactantfree aqueous precipitation polymerization, and analyzed the oscillating behavior (Suzuki et al., 2008, 2009; Suzuki \& Yoshida, 2008a, 2008b, 2010; Taniguchi et al., 2010; Sakai \& Yoshida, 2004). Figure $9(\mathrm{~b})$ shows the oscillation profiles of transmittance for the microgel 
dispersions. At low temperatures $\left(20-26.5^{\circ} \mathrm{C}\right)$, on raising the temperature, the amplitude of the oscillation became larger. The increase in amplitude is due to increased deviation of the hydrodynamic diameter between the $\mathrm{Ru}(\mathrm{II})$ and $\mathrm{Ru}(\mathrm{III})$ states. Furthermore, a remarkable change in waveform was observed between 26.5 and $27^{\circ} \mathrm{C}$. Then the amplitude of the oscillations dramatically decreased at $27.5^{\circ} \mathrm{C}$, and finally the periodic transmittance changes could no longer be observed at $28^{\circ} \mathrm{C}$. The sudden change in oscillation waveform should be related to the difference in colloidal stability between the $\mathrm{Ru}(\mathrm{II})$ and $\mathrm{Ru}(\mathrm{III})$ states. Here, the microgels should be flocculated due to lack of electrostatic repulsion when the microgels were deswollen. The remarkable change in waveform was only observed at higher dispersion concentrations (greater than $0.225 \mathrm{wt.} \%$ ). The self-oscillating property makes microgels more attractive for future developments such as microgel assembly, optical and rheological applications, etc.

\subsection{Viscosity oscillation of polymer solution and microgel dispersion}

In the case of the self-oscillating polymer solution or the mircogel dispersion, the solubility changes or the swelling-deswelling changes can be measured as viscosity changes as well as optical transmittance changes of the solution or the dispersion. Actually, we succeeded in observing the viscosity self-oscillation for the polymer solution induced by the BZ reaction at the constant temperature (Hara \& Yoshida, 2008). The viscosity self-oscillation was originated by the difference between solubilities of the polymer chain in the reduced and oxidized states. The effects of the polymer concentration and the temperature of the viscosity self-oscillation were investigated. As a result, the viscosity self-oscillating behavior significantly depended on the polymer concentration and the temperature of the polymer solution. The period of the viscosity self-oscillation decreased with increasing temperature in accordance with the Arrenius equation.

Recently, we have achieved autonomously oscillating viscosity in a microgel dispersion using autonomously oscillating microgels (Suzuki et al., 2009; Taniguchi et al., 2010). We found out that viscosity oscillation occurs in two different manners, exhibiting a simple pulsatile waveform or a complex waveform with two peaks per period (Figure 10(a)). It was suggested that the difference in waveform is due to the difference in oscillating manner of the microgels: swelling/deswelling or dispersing/flocculating oscillation as mentioned before. We can control rhythm and amplitude of the oscillation using these two phenomena of the microgels. In order to characterize the viscosity oscillation, two types of the microgels were synthesized by changing the feed ratio of $\mathrm{Ru}(\mathrm{bpy})_{3}$ and cross-linker. Viscosity of the microgel dispersions at high salt concentrations could be controlled by changing concentrations of the microgels. Autonomously oscillating viscosity was only measured when concentration of the microgels was high. The amplitude of the oscillation became bigger with increasing concentrations of the microgels. By adjusting the concentration of the substrates for the BZ reaction, we could achieve the constant oscillation for a long time. Moreover, with increasing $\mathrm{Ru}(\mathrm{bpy})_{3}$ and decreasing the cross-linker, microgels showed a high degree of swelling/deswelling oscillation, resulting in bigger amplitudes of autonomously oscillating viscosity (Figure 10(b)).

This technology could be applied in many applications as electro- or magnetic- rheological (ER or MR) fluids have been done. In particular, the dispersion with autonomously oscillating viscosity may be used as a micropump, which could realize novel microfluidics devices. 
(a)
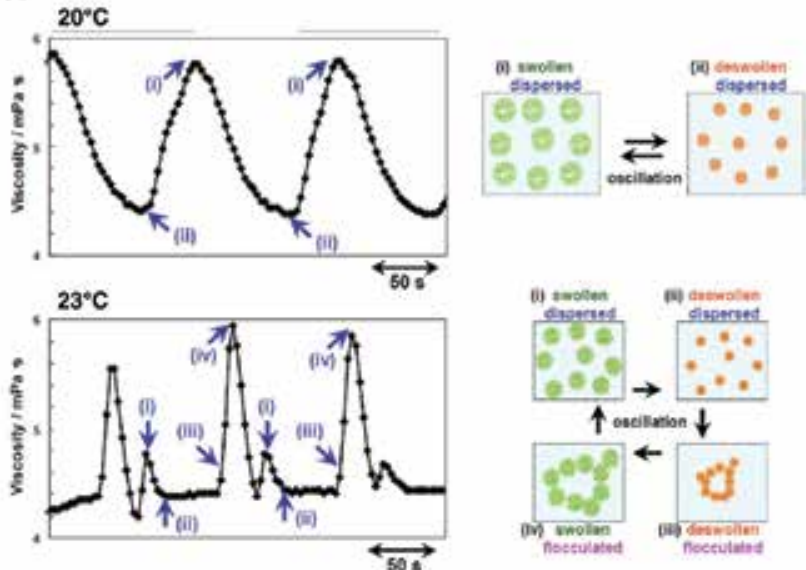

(b)

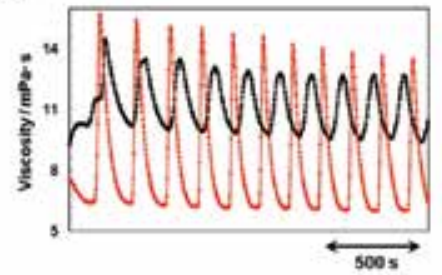

Fig. 10. (a) Two different types of oscillating waveforms observed at $20^{\circ} \mathrm{C}$ and $23^{\circ} \mathrm{C}$. The numbers in each oscillating profiles refer to the corresponding cartoons (Suzuki et al., 2009). (b) Autonomously oscillating profiles of viscosity in the microgel dispersions measured at $15^{\circ} \mathrm{C}$. for NIPAAm:Ru(bpy) $3: \mathrm{BIS}=95: 1: 4$ (black line) and 96:2:2 microgels (red line). The microgels $(5.0 \mathrm{wt} \%)$ were dispersed in aqueous solutions containing $\mathrm{MA}(100 \mathrm{mM}), \mathrm{NaBrO}_{3}$ $(150 \mathrm{mM})$, and $\mathrm{HNO}_{3}(500 \mathrm{mM})$. Both data were taken at constant shear rate of $132 \mathrm{~s}^{-1}$ (Taniguchi et al., 2010).

\subsection{Fabrication of microgel beads monolayer}

As discussed in the previous section, we have been interested in the construction of micro/nano-conveyers by grafting or arraying self-oscillating polymer or gel beads. For this purpose, a fabrication method for organized monolayers of microgel beads was investigated (Sakai et al., 2007). A 2D close-packed array of thermosensitive microgel beads was prepared by double template polymerization. First, a 2D colloidal crystal of silica beads with $10 \mathrm{~mm}$ diameter was obtained by solvent evaporation. This monolayer of colloidal crystal can serve as the first template for preparation of macroporous polystyrene. The macroporous polystyrene trapping the crystalline order can be used as a negative template for fabricating a gel bead array. By this double template polymerization method, functional surfaces using thermosensitive PNIPAAm gel beads were fabricated. It was observed that topography of the surface changed with temperature. The fabrication method demonstrated here was so versatile that any kind of gel beads could be obtained. This method may be a key technology to create new functional surface. Actually, monolayer of self-oscillating microgel beads was fabricated by this method and the chemical wave propagation on the monolayer was observed. 


\section{Attempts of self-oscillation under physiological conditions}

\subsection{Self-oscillation of polymer chains under acid-free conditions}

So far, the author had succeeded in developing a novel self-oscillating polymer (or gel) by utilizing the BZ reaction. However, the operating conditions for the self-oscillation are limited to conditions under which the BZ reaction occurs. For practical applications as functional bio- or biomimetic materials, it is necessary to design a self-oscillating polymer which acts under biological environments. To cause self-oscillation of polymer systems under physiological conditions, BZ substrates other than organic ones, such as malonic acid and citric acid, must be built into the polymer system itself. Therefore, we took the two steps; the first step is to design a novel self-oscillating polymer chains with incorporated $\mathrm{pH}$-control sites, that is, the polymer chains which exhibit rhythmic oscillations in an aqueous solutions containing only the two BZ substrates, without using acid as an added agent. For this purpose, 2-acrylamido-2-methylpropanesulfonic acid (AMPS) was incorporated into the poly(NIPAAm-co- $\mathrm{Ru}(\mathrm{bpy}) 3_{3^{2+}}$ ) chain as the $\mathrm{pH}$ control site (Hara \& Yoshida, 2005a, 2005b).

The self-oscillating transmittance change for the solutions of poly(NIPAAm-co-Ru(bpy) $3^{2+}$ co-AMPS) $\left(20: 10: 70 \mathrm{wt} \%\right.$ in feed) at three constant temperatures $\left(18,21\right.$ and $\left.24^{\circ} \mathrm{C}\right)$ were demonstrated. Under acid-free conditions and in the presence of only two BZ substrates (malonic acid and sodium bromate), we succeeded in causing soluble-insoluble selfoscillation of a polymer solution. Oscillating behavior were remarkably influenced by the temperature, polymer concentration, and composition.

\subsection{Self-oscillation under oxidant-free conditions}

As the next step, we attempted to introduce the oxidizing agent into the polymer. Methacrylamidopropyltrimethylammonium chloride (MAPTAC), with a positively charged group, was incorporated into the poly(NIPAAm-co-Ru(bpy) $3^{2+}$ ) as a capture site for an anionic oxidizing agent (bromate ion) (Hara et al., 2005). The bromate ion was introduced into the MAPTAC-containing polymer through ion-exchange. Under the conditions in which only two BZ substrates (malonic acid and sulfuric acid) were present, solubleinsoluble self-oscillation of the polymer was observed.

In the self-oscillating polymer solution system induced by the BZ reaction, self-oscillation was achieved without addition of oxidizing agent by utilizing the MAPTAC-containing polymer which included sodium bromate as a counter ion. The self-oscillating behavior was controllable by temperature. The polymer has two advantages because of the higher LCST; one is self-oscillation around body temperature, and the other is oscillation for a longer time without intermolecular aggregation among the polymer chains in the reduced state.

\subsection{Self-oscillation under acid- and oxidant-free condition}

Further, we have synthesized a quarternary copolymer which includes both $\mathrm{pH}$-control and oxidant-supplying sites in the poly(NIPAAm-co-Ru(bpy) 3 ) chain at the same time (Hara \& Yoshida, 2008b). In the polymer, AMPS was incorporated as a $\mathrm{pH}$-control site, and MAPTAC with a positively charged group was incorporated as a capture site for an anionic oxidizing agent (bromate ion). By using the polymer, self-oscillation under biological conditions where only the organic acid (malonic acid) exists was actually achieved (Figure 11). Other than malonic acid, citric acid or malic acid, which is biorelated organic acid, can be a substrate of the BZ reaction. For practical medical use, however, it would be necessary 
to avoid exchange of toxic ion such as bromate to outside of the gel. In addition, working temperature must be improved. The design of gel for biomedical application is under investigation.
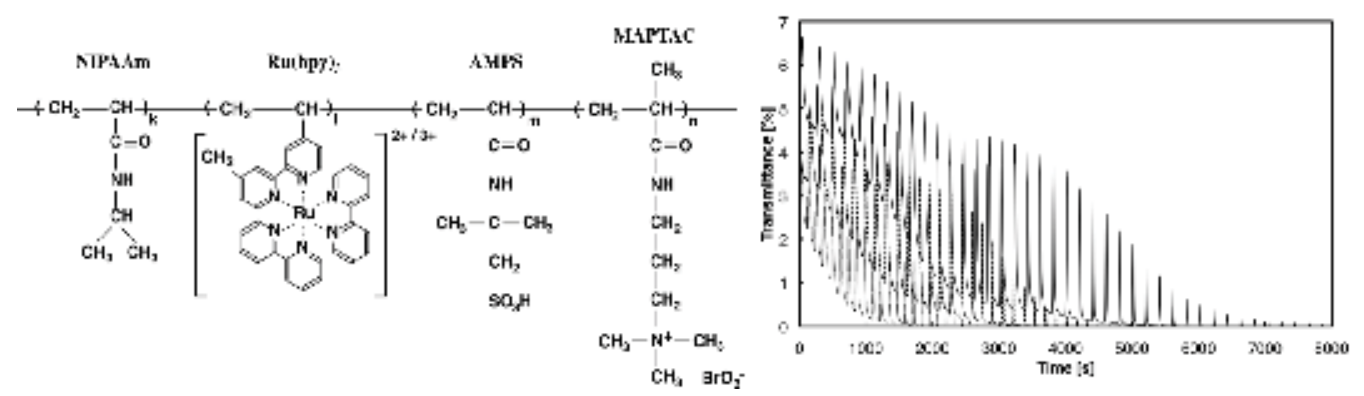

Fig. 11. Chemical structure of poly(NIPAAm-co-Ru(bpy) $3^{2+}$-co-AMPS-co-MAPTAC) (upper) and the oscillating profiles of the optical transmittance for the polymer solution at $12^{\circ} \mathrm{C}$ when only MA (0.7M: fine dotted line, $0.5 \mathrm{M}$ : rough dotted line, $0.3 \mathrm{M}$ : solid line) is added to the solution (lower) (Hara \& Yoshida, 2008).

\section{References}

Aoki, R., Enoki, M. and Yoshida, R. (2007). Key Eng. Mater. 353, 2235-2238.

Epstein, I.R., Pojman, J.A. (1998). An Introduction to Nonlinear Chemical Dynamics: Oscillations, Waves, Patterns, and Chaos, Oxford University Press, New York.

Field R.J.; Burger, M. Eds. (1985). Oscillations and Traveling Waves in Chemical Systems, John Wiley \& Sons, New York.

Hara, Y. and Yoshida, R. (2008). (a) J. Chem. Phys. 128, 224904. (b) J. Phys. Chem. B 112, 84278429.

Hara, Y. and Yoshida, R. (2005). (a) Langmuir 21, 9773-9776. (b) J. Phys. Chem. B 109, 94519454.

Hara, Y., Sakai, T., Maeda, S., Hashimoto, S. and Yoshida, R. (2005). J. Phys. Chem. B 109, 23316-23319.

Ito, Y., Nogawa, N. and Yoshida, R. (2003). Langmuir 19, 9577-9579.

Ito, Y., Hara, Y., Uetsuka, H., Hasuda, H., Onishi, H., Arakawa, H., Ikai, A. and Yoshida, R. (2006). J. Phys. Chem. B 110, 5170-5173.

Maeda, S., Hara, Y., Sakai, T., Yoshida, R. and Hashimoto, S. (2007). Adv. Mater. 19, 34803484.

Maeda, S., Hara, Y., Yoshida, R. and Hashimoto, S. (2008). (a) Angew. Chem. Int. Ed. 47, 66906693. (b) Macromol. Rapid Commun. 29, 401-405.

Miyata, T. (2002). Stimuli-responsive polymer and gels: in Supramolecular Design for Biological Applications, Yui, N. Ed.; CRC Press, Boca Raton, pp.191-225.

Murase, Y., Maeda, S., Hashimoto, S. and Yoshida, R. (2009a). Langmuir 25, 483-489.

Murase, Y., Takeshima, R. and Yoshida, R. (2009b). Trans. Mater. Res. Soc. Japan 34, 171-174 .

Murase, Y., Hidaka, M. and Yoshida, R. (2010). Sensors and Actuators B 149, 272-283.

Osada, Y., Khokhlov, A.R., Eds. (2002). Polymer Gels and Networks, Marcel Dekker, New York. 
Ottenbrite, R.M., Park, K., Okano, T. and Peppas, N.A. (Eds) (2010). Biomedical Applications of Hydrogels Handbook, Springer, New York.

Sakai, T. and Yoshida, R. (2004). Langmuir 20, 1036-1038.

Sakai, T., Takeoka, Y., Seki, T. and Yoshida, R. (2007). Langmuir 23, 8651-8654.

Sasaki, S., Koga, S., Yoshida, R. and Yamaguchi, T. (2003). Langmuir 19, 5595-5600.

Shinohara, S., Seki, T., Sakai, T., Yoshida, R. and Takeoka, Y. (2008). (a) Angew. Chem. Int. Ed. 47, 9039-9043. (b) Chem. Commun. 4735-4737.

Suzuki, D., Sakai, T. and Yoshida, R. (2008). Angew. Chem. Int. Ed. 47, 917-920.

Suzuki, D. and Yoshida, R. (2008). (a) J. Phys. Chem. B 112, 12618-12624. (b) Macromolecules 41, 5830-5838.

Suzuki, D., Taniguchi, H. and Yoshida, R. (2009). J. Am. Chem. Soc. 131, 12058-12059.

Suzuki, D. and Yoshida, R. (2010). Polym. J. 42, 501-508.

Tabata, O., Hirasawa, H., Aoki, S., Yoshida, R. and Kokufuta, E. (2002). Sensors and Actuators A 95, 234-238.

Tabata, O., Kojima, H., Kasatani, T., Isono, Y. and Yoshida, R. Chemo-mechanical actuator using self-oscillating gel for artificial cilia, Proceedings of the International Conference on MEMS 2003, pp.12-15 (2003).

Takeoka, Y., Watanabe, M. and Yoshida, R. J. Am. Chem. Soc. (2003). 125, 13320-13321.

Tanaka, T. Phys. Rev. Lett. 1978, 40, 820-823.

Taniguchi, H., Suzuki, D. and Yoshida, R. (2010). J. Phys. Chem. B 114, 2405-2410.

Tateyama, S., Shibuta, Y. and Yoshida, R. (2008). J. Phys. Chem. B 112, 1777-1782.

Yoshida, R., Takahashi, T., Yamaguchi, T. and Ichijo, H. (1996). J. Am. Chem. Soc. 118, 51345135.

Yoshida, R., Takahashi, T., Yamaguchi, T. and Ichijo, H. (1997). Adv. Mater. 9, 175-178.

Yoshida, R., Tanaka, M., Onodera, S., Yamaguchi, T. and Kokufuta, E. (2000). J. Phys. Chem. A 104, 7549-7555.

Yoshida, R., Sakai, T., Ito, S. and Yamaguchi, T. (2002a). J. Am. Chem. Soc. 124, 8095-8098.

Yoshida, R., Sakai, T., Tabata, O. and Yamaguchi, T. (2002b). Sci. Tech. Adv. Mater. 3, 95-102.

Yoshida, R., Takei, K. and Yamaguchi, T. (2003). Macromolecules 36, 1759-1761.

Yoshida, R. (2005). Curr. Org. Chem. 9, 1617-1641.

Yoshida, R., Omata, K., Yamaura, K., Ebata, M., Tanaka, M., Takai, M. (2006a). Lab on a Chip 6, 1384-1386.

Yoshida, R., Omata, K., Yamaura, K., Sakai, T., Hara, Y., Maeda, S. and Hashimoto, S. (2006b). J. Photopolymer Sci. Tech. 19, 441-444.

Yoshida, R. (2008). Bull. Chem. Soc. Jpn 81, 676-688.

Yoshida, R., Sakai, T., Hara, Y., Maeda, S., Hashimoto, S., Suzuki, D. and Murase, Y. J. Controlled Release (2009). 140, 186-193.

Yoshida, R. (2010). Adv. Mater. 22, 3463-3483. 


\title{
Non-Calcium Inorganic Materials Fabrication by Surface-Immobilized Organic Molecular Template
}

\author{
Peng Yang1, Wantai Yang1, Xu Zhang2 and Jinchun Chen ${ }^{2}$ \\ ${ }^{1}$ College of Materials Science and Engineering, \\ Beijing University of Chemical Technology, Beijing, 100029, \\ ${ }^{2}$ College of Life Science and Technology, \\ Beijing University of Chemical Technology, Beijing, 100029, \\ P. R. China
}

\section{Introduction}

It has been extensively reported that organic molecules, i.e. biological molecules (CrookesGoodson et al, 2008) and polymers (Xu et al, 2007), could function as structural templating and/or selective adsorption at certain crystal faces, which may control and mediate the final crystal morphology. However, most of literatures are concentrated on the mediation ability of soluble free organic molecules in solutions. For practical applications, for example, tissue engineering, biomaterials fabrication and organic/inorganic hybrid film, a more straightforward way is to investigate such work on surfaces, that means, explore the mediation ability of surface-immobilized organic molecular template.

Until now, most of attentions are paid on the calcium-based inorganic material fabrication by such surface-immobilized organic molecular template (Tugulu et al, 2006; Kumar et al, 2010). However, besides calcium mineral, natural system also provides good biomineralization models for other kinds of useful inorganic materials such as silicate, barite, copper, iron and gold. The researches on the fabrication of these non-calcium materials by surface-immobilized organic molecular template have been not extensively investigated (Coffman et al, 2004; Kim et al, 2004 \& 2005; Morin et al, 2007). Therefore, our aim is to extend the content of the biomineralization mediated by surface-immobilized organic molecular template from classical calcium to a wide range of inorganic and metal materials through rationally engineering surface molecular template from small functional groups to macromolecules. We for the first time initiated this program in 2005 (Zhang et al, 2005) for the synthesis of silver nanoparticles mediated by surface-immobilized peptides, and after that, a series of researches in this field were reported to exploit the mediation ability of surface-immobilized organic molecule at different molecular scale, including small functional groups for the synthesis of titanium, zinc and silicon oxide films (Yang et al, 2007 and 2008; Gan et al, 2010), oligomer-grade peptides for the synthesis of gold, silver, platinum nanoparticles and superstructures (Zhang et al, 2006 and 2008; Wang et al, 2007; Li et al, 2007) and macromolecular brush for the fabrication of zinc oxide quantum dot and organic/inorganic hybrid nanocomposite film (Zou et al, 2009). 
In this chapter, we will systematically summarize the data obtained from our group. Engineered surface organic molecular template from our group could be categorized into three levels, depends on the size of surface immobilized molecule on substrates (Figure 1): level (1). small functional groups-mediated inorganic oxide deposition, growth and assembly; level (2). biomolecules such proteins, enzymes and peptides could be immobilized on small functional group-functionalized surface and these surfaceimmobilized biomolecules (i.e. inorganic-binding peptide) are further used as organic template to mediate noble metal deposition and growth; level (3). macromolecular (polymer) brush-mediated formation of semiconductor quantum dots in polymer matrix to fabricate organic/inorganic hybrid nanocomposites.

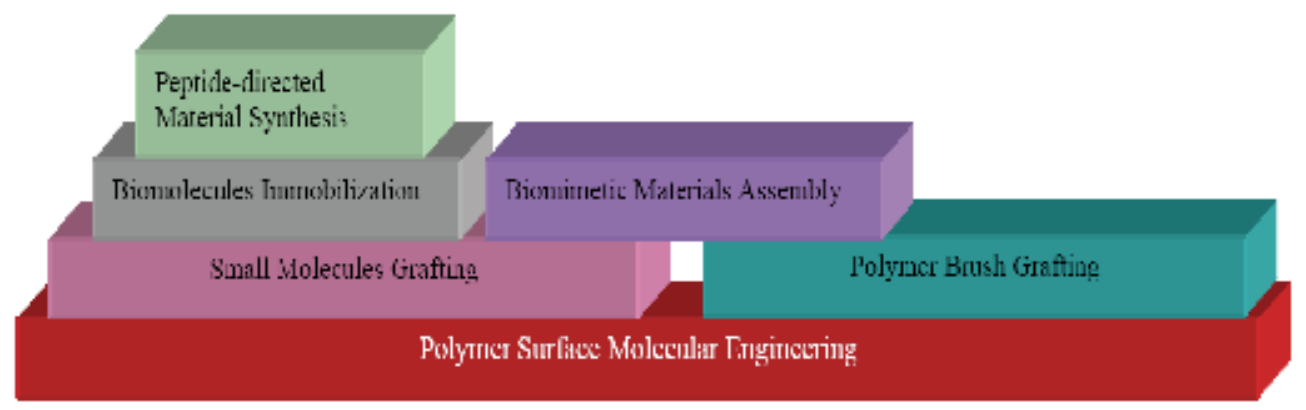

Fig. 1. Inorganic material fabrication mediated by surface-immobilized organic molecular template from our group. These templates could be categorized into three types: small molecules, peptide and polymer brush.

\section{Results and discussion}

\subsection{Level 1: Small molecule-mediated inorganic oxide film formation}

Firstly, surface-grafted small functional group is introduced. This is the smallest functional organic template for directing inorganic material deposition and growth on surfaces. Nevertheless, their mediation ability is not compromised by their tiny size and often very useful in advanced material and devices fabrication process. In 2003-2010, we continuously reported three novel confined photochemical reaction systems on polymer surface (Yang et al, 2003-2008; Gan et al, 2009 and 2010), which are eligible to convert directly C-H on polymer surface to well-defined reactive sulfate, hydroxyl and carboxyl monolayers. These organic functional groups are further used as molecular template to direct the nucleation and micro/nano-scale assembly of inorganic and metal oxides including silicon oxide $\left(\mathrm{SiO}_{\mathrm{x}}\right)$, titatnium dioxide $\left(\mathrm{TiO}_{2}\right)$ and zinc oxide $(\mathrm{ZnO})$. The resulting $\mathrm{TiO}_{2}$ and $\mathrm{ZnO}$ deposition could form isolated array (positive pattern) or continuous micronetwork (negative pattern, supported macroporous film). The latter is especially promising for preparation of highefficient solar cells because nanoparticles, photosensizers or complex salts could be conveniently incorporated into the holes of macroporous film from negative pattern. Moreover, this macroporous negative film has enough mechanical strength that supports its free transfer from original deposited support to any other substrates by simple adhesion technique. The resulting $\mathrm{SiO}_{x}$ deposition not only could form order array but also could form a transparent, ultraflat and continuous film on polymer substrate with widely tunable thickness and strong bonding strength. 


\subsubsection{Methods and chemistry to introduce functional small molecular monolayer on polymer surface}

In 2003, we for the first time found that when a very thin persulfate salt aqueous solution layer $(\mu \mathrm{m})$ was sandwiched between two polymer films and strong UV light irradiated the assembly from the side transparent to UV light, a fast surface hydrophilic modification method for most of commercial polymeric materials was developed (Figure 2) (Yang et al, 2003). For example, irradiating for $90 \mathrm{~s}$ and using $30 \mathrm{wt} \%$ ammonium persulfate, the static surface water contact angles of polymeric substrates decreased from $100^{\circ}$ to $44^{\circ}$ for low-density polyethylene (LDPE), from $107^{\circ}$ to $34^{\circ}$ for high-density polyethylene (HDPE), and from $73^{\circ}$ to $15^{\circ}$ for poly(ethylene terephthalate) (PET). The increases in surface hydrophilicity came from the formation of a sulfate salt group $\left(\mathrm{SO}_{4}-\mathrm{NH}_{4}{ }^{+}\right)$-ionized surface, which was characterized by $\mathrm{X}$-ray Photoelectron Spectroscopy (XPS) and Attenuated Total Reflection Fourier Transform Infrared spectroscopy (ATR-FTIR). The surface topography of the modified polymer substrates were observed by Scanning Electron Microscope (SEM) and Atomic Force Microscope (AFM), and no visible etching effects to original surface were found.

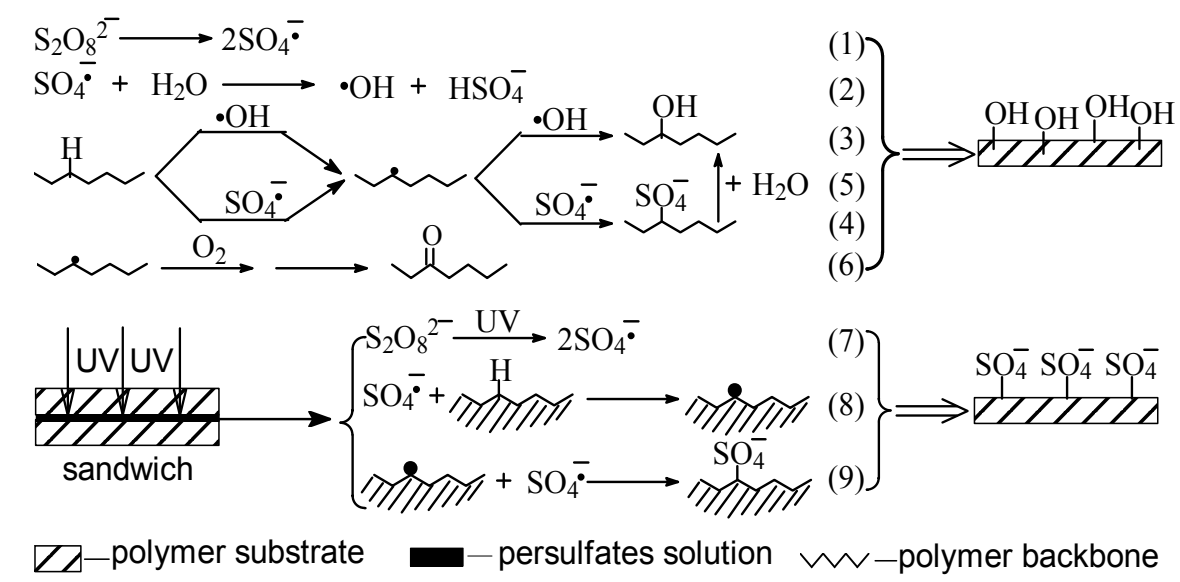

Fig. 2. The possible reaction model for surface oxidation by persulfates: the conventional and the "sandwich" methods. Reproduced from (Yang et al, 2003). Copyright from Elsevier. Reproduced with permission.

A possible reaction model named by 'confined photo-catalytic oxidation' (CPO) was put forward to interpret the above results. Conventional photooxidation route of persulfate is that water $\left(\mathrm{H}_{2} \mathrm{O}\right)$ molecules are firstly oxidized by persulfates to form hydroxyl radicals, which undertake further oxidization to organic substances. However, in such sandwiching setup, due to the role of $\mathrm{CPO}$, a new reaction route is achieved where persulfates dissociate directly to form sulfate anion radicals under UV irradiation, the resulting sulfate radicals quickly oxidize polymer surface by abstracting hydrogen atoms from polymer surface, and then the formed surface radicals couple with other sulfate anion radicals to fabricate an array of sulfate anion groups covalently attached on the outmost surface. This strategy provides an effective surface hydrophilic and functionalization method suitable for most of commercial polymeric materials with the advantages of fast, mild (to environment and substrate), facile and low cost technological process. Based on $\mathrm{CPO}$, we further develop a very simple method to create hydrophilic/hydrophobic hybrid polymer surface, through 
"CPO" under the regional control by a photomask (Figure 3). The resulting wettability or surface molecular template patterns have been used to fabricate polyaniline (PANI) (Yang et al, 2006), $\mathrm{TiO}_{2}$ and $\mathrm{ZnO}$ micropatterns.
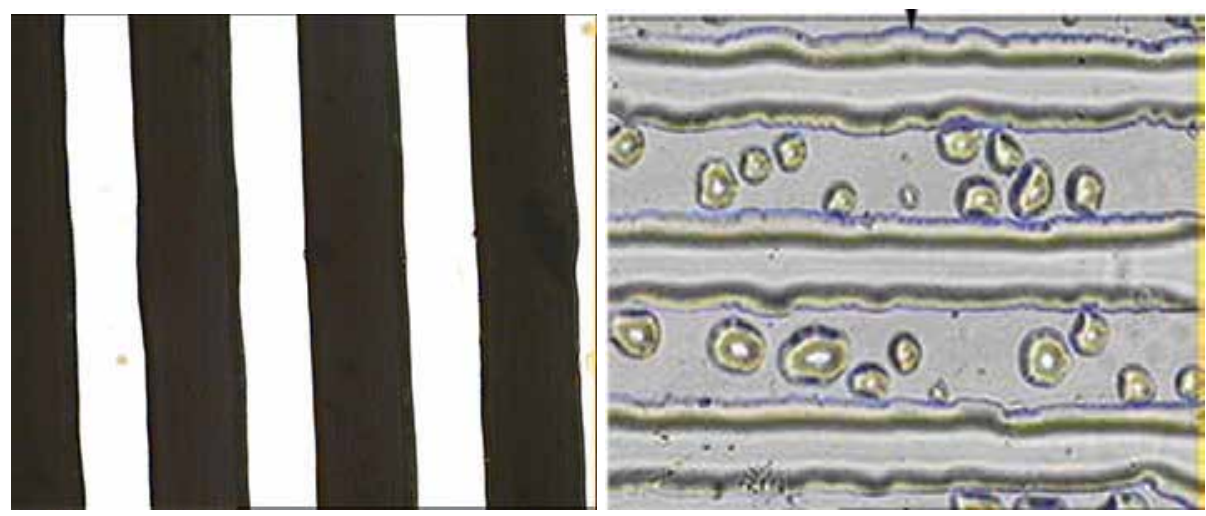

Fig. 3. The wettability pattern obtained by CPO method. The left image is a photomask used in $\mathrm{CPO}$ reaction to control the irradiation/non-irradiation areas: the white bars are UV transparent regions which will result in the corresponding UV-exposed regions (i.e. CPO reaction occurred) on polymer surface, producing a hydrophilic region, as shown in parallel water condensation lines in right image; in contrast, the black regions in the photomask are metallic parts which will shield UV light and thereby result in non-irradiated parts on underlying polymer substrate, producing original hydrophobic surface, as shown in right image (the spaces between hydrophilic lines). Reproduced from (Yang et al, 2006). Copyright from Wiley-VCH Verlag $\mathrm{GmbH} \& \mathrm{Co}$. KGaA. Reproduced with permission.

Moreover, the formed sulfate groups on surfaces could be readily hydrolyzed to form hydroxyl monolayer, which has been demonstrated by XPS, contact angle (CA) measurement (Figure 4) and ATR-FTIR (Figure 5). As shown in Figure 4, when sulfated surface was soaked in water, the surface contact angle increased gradually and reached a plateau after about 10 hours. It has been known that polymer surfaces hydrophilized by oxidizing treatments should not decay when store in water or high-energy media. The conflict between present results and this theory revealed that surface sulfate groups actually converted to hydroxyl groups during this soaking process because of the difference in surface free energy between the sulfate anion and hydroxyl groups. The conversion of sulfate to hydroxyl group-implanted surfaces resulted in the continuous increase of the CA on the treated surface and became constant when the hydrolysis reached equilibrium. This judgment was further strongly supported by ATR-FTIR (Figure 5). We used a classical reaction to tell the existence of hydroxyl groups on the surface, that is, allowed the sulfated surface after soaking in water to be incubated in trifluoroacetic anhydride (TFAA) solution under certain conditions. If there are a plenty of hydroxyl groups on the surface, such incubation would result in an effective reaction between surface hydroxyl group and TFAA in the solution, producing characteristic ester and C-F signals in ATR-FTIR spectra. The results were as we expected. The spectrum of the sample after soaking (c) displayed an ester carbonyl bond at $1789 \mathrm{~cm}^{-1}$ and a C-F bond at $1222 \mathrm{~cm}^{-1}$, both of which could not be found in the spectra of the samples before soaking $(b, d)$ and the sample for the untreated LDPE (a). XPS (data not shown here) was also consistent with ATR-FTIR results, and both of them 


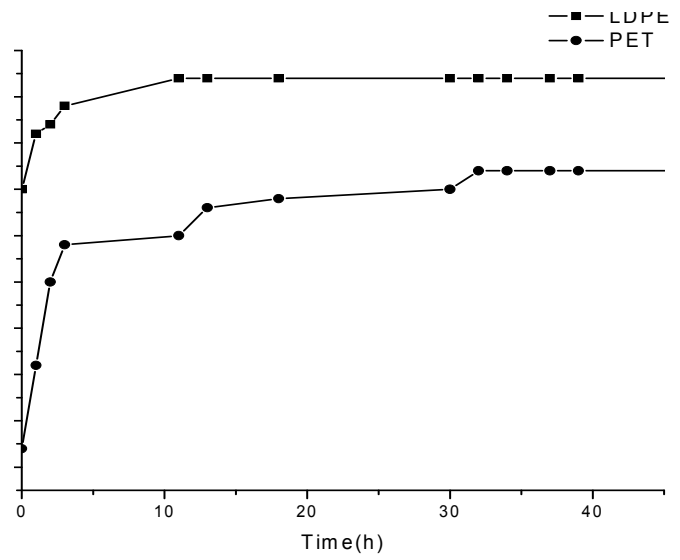

Fig. 4. Surface hydrophilicity changes upon soaking modified surface in water. Reproduced from (Yang et al, 2007). Copyright from American Chemical Society. Reproduced with permission.

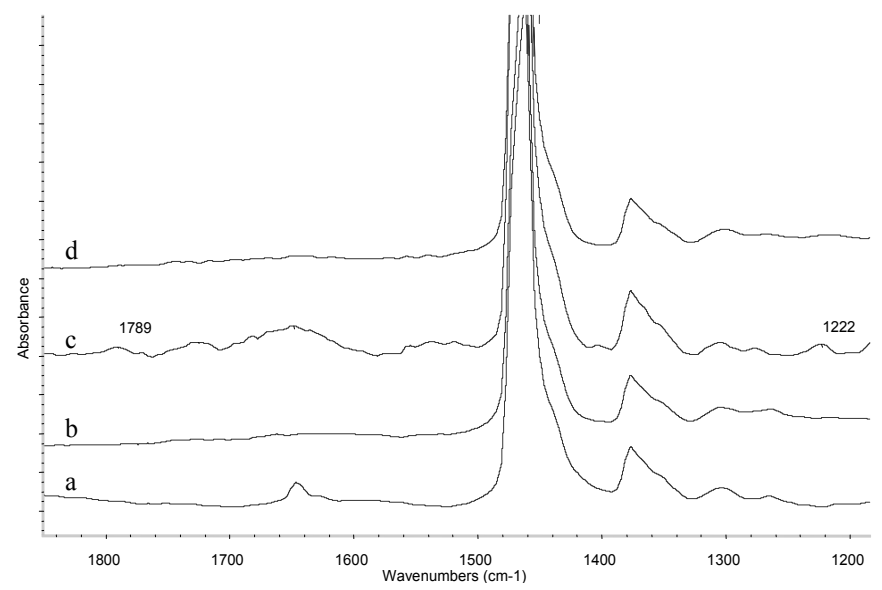

Fig. 5. ATR-FTIR spectra: (a) LDPE: unirradiated; (b) LDPE: irradiated; (c) the derivation of modified LDPE: irradiated, soaked into water for $14 \mathrm{~h}$, and then treated with TFAA at $30^{\circ} \mathrm{C}$, dried under reduced pressure at $20^{\circ} \mathrm{C}$ for $24 \mathrm{~h}$; (d) the derivation of modified LDPE: irradiated, and then treated with TFAA at $30^{\circ} \mathrm{C}$, dried under reduced pressure at $20^{\circ} \mathrm{C}$ for 24h. (Irradiation conditions: LDPE films; irradiation time is 90s; the conc. of $\left(\mathrm{NH}_{4}\right)_{2} \mathrm{~S}_{2} \mathrm{O}_{8}$ is $30 \%$, UV-intensity is $6500 \mu \mathrm{w} / \mathrm{cm}^{2}$ ). Reproduced from (Yang et al, 2007). Copyright from American Chemical Society. Reproduced with permission.

$$
\mathrm{SO}_{4}^{-}+\mathrm{H}_{2} \mathrm{O} \longrightarrow \mathrm{OH}_{\mathrm{H}^{+}}^{\mathrm{OH}}+\mathrm{SO}_{4}^{-}
$$

Fig. 6. Hydrolysis reaction scheme of grafted sulfate groups on polymer surface. 
plus CA measurements indicated strongly that the polar sulfate groups on the CPO-treated surface converted to hydroxyl groups after slow hydrolysis in water (Figure 6).

After successfully introducing anion sulfate and neutral hydroxyl groups on surfaces, we also developed two novel approaches to introduce amine groups on surfaces, because these amine groups could potentially provide positive charge and reactivity towards proteins and silanes. The chemistry and methods used to fabricate such aminated surface are based on newly-developed UV-induced Surface Aminolysis Reaction (USAR) and silanization on

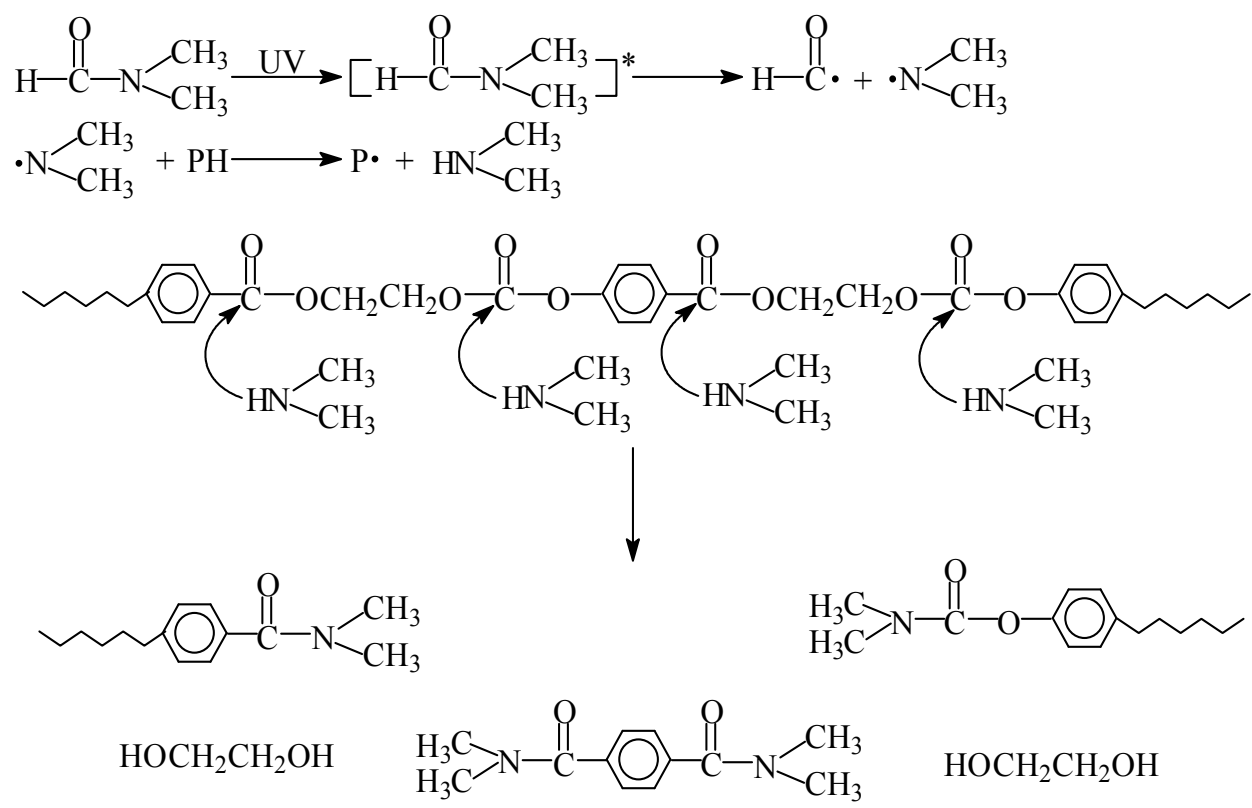

Fig. 7. A plausible reaction mechanism of USAR to introduce positive amine on PET surface. Reproduced from (Yang et al, 2005). Copyright from Wiley-VCH Verlag GmbH \& Co. KGaA. Reproduced with permission.

hydroxyl-modified surface by CPO. In the former reaction, a thin film of $\mathrm{N}, \mathrm{N}$ dimethylformamide (DMF) liquid is sandwiched between two polyester films, and then transferred to UV field for irradiation. It has been known that dimethylamine and formaldehyde would be released very slowly when DMF is irradiated by visible light. However, in such confined reaction setup, this reaction is obviously accelerated largely. Systemic investigations reveal that after UV irradiation for short time (2-16min), DMF undergoes photodissociation to give out amine molecules (dimethylamine), which could insitu attack ester bond along PET chains to incorporate tertiary-amine functional groups at PET chain end (Figure 7). With additional photomask, the region to be aminated could be designed and controlled with micron-meter precision, as shown in Figure $8 \mathrm{~b}$ by selective staining from Sumikaron red dye. After positive amines incorporation, we further demonstrated that peptides, enzymes and proteins could be immobilized on such surface with keeping their biological activity for catalysis and biomineralization. For example, as shown in Figure 8c, when the enzyme, horseradish peroxide (HRP), was immobilized on aminated surface (circular regions), the enzyme could effectively catalyze the reaction with the standard substrate, 3-amino-9-ethylcarbazole (AEC) to produce precipitations which 
was deposited on circular aminated regions (line-type particles). Besides this direct aminolysis on polyester surface, a more general approach is also developed by us to introduce primary amine groups on most of polymer surface. This is a derivation reaction on hydroxyl-modified surface by CPO, that is, when hydroxyl monolayer is formed on surfaces by $\mathrm{CPO}$, the further silanization reaction with any other silane reagent could give us a well-defined functionalized surface (Gan et al, 2009). We have used this kind of surface to immobilize antibody effectively.

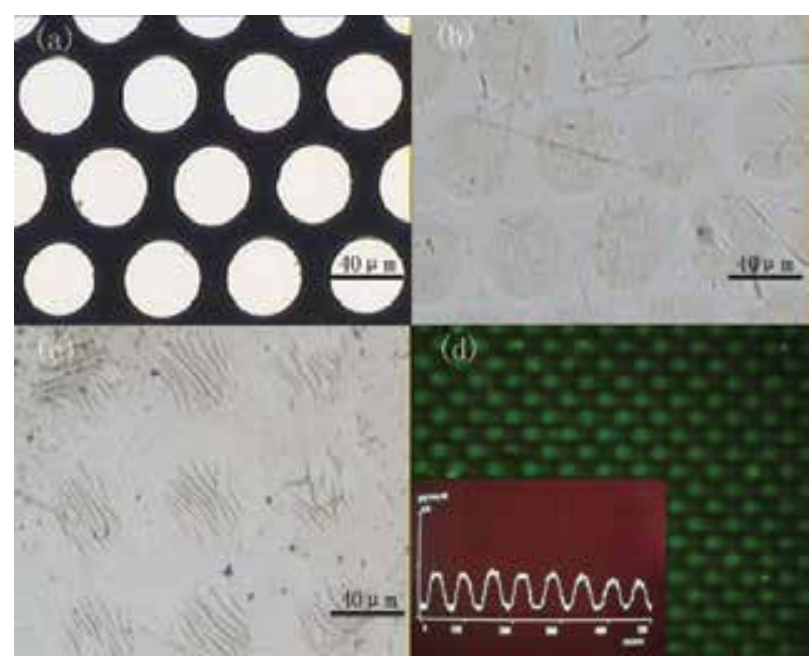

Fig. 8. (a). Micrograph of metallic(Cr) photomask. the diameter of circle hole is $40 \mu \mathrm{m}$. (b). Micrograph of Sumikaron red stained microprocessing surface. Irradiation time for USAR is $16 \mathrm{~min}$, and aminated film was stained by a water solution of Sumikaron red, followed by ultrasonic washing. (c). Micrograph of patterned aminated surface after electrostatic selfassembling HRP. (d). Fluorescent micrograph of patterned aminated surface after electrostatic self-assembling IgG. Irradiation time for USAR is $16 \mathrm{~min}$. The inset in Figure 8(d) was the line profile of fluorescence intensity of the FITC-IgG pattern in Figure 8(d). Reproduced from (Yang et al, 2005). Copyright from Wiley-VCH Verlag GmbH \& Co. KGaA. Reproduced with permission.

\subsection{2 $\mathrm{TiO}_{2}$ film fabrication on sulfated and hydroxylated polymer surface by biomimetic nucleation and growth in aqueous solution. (Yang et al, 2007)}

For plastic electronics and optics, the fabrication of smooth, transparent and stable crackfree inorganic oxide films (and patterning) on flexible polymeric substrates with strong bonding strength and controllable thickness from nanometers to micrometers is a key but still remains a challenge. From this section, we will continuously introduce a series of novel approaches developed by us to fabricate high quality $\mathrm{TiO}_{2}, \mathrm{ZnO}$ and $\mathrm{SiO}_{\mathrm{x}}$ films on polymer substrate by a mild biomimetic interface-directed nucleation and growth process.

The fabrication of $\mathrm{TiO}_{2}$ films on substrates especially flexible polymers is extremely important due to their great potential in photocatalysis, energy conversion, and electrooptical techniques. Such films are often fabricated by sol-gel method where gelation at high temperature is needed to ensure the quality of crystals. In contrast, low-temperature biomimetic nucleation and growth in aqueous solution by surface-immobilized functional 
groups could provide an alternative way to fabricate high quality $\mathrm{TiO}_{2}$ film on weak polymer substrate where high temperature annealing must be avoided.

For this aim, we firstly fabricated a wettability-patterned polypropylene surface by CPO. As shown in Figure 9, after CPO modification, the biaxially oriented polypropylene (BOPP) film surface was incubated in a mixture solution of $\left(\mathrm{NH}_{4}\right)_{2} \mathrm{TiF}_{6}$ and $\mathrm{H}_{3} \mathrm{BO}_{3}$ at certain low temperature, molar ratio and incubation time. Under deposition condition (c), an anatase $\mathrm{TiO}_{2}$ film could be selectively deposited on a hydrophilic region with keeping original hydrophobic regions being undisturbed (no deposition). Figure 10 presented an optical microscope image of this positive pattern. AFM analysis indicated that the thickness of such film under deposition condition (c) was $350 \mathrm{~nm}$. A 3D AFM profile image further showed that the obtained $\mathrm{TiO}_{2}$ film consisted of crystal fusions from dense column arrays. Such column-type crystal growth implied that the deposition process was dominated by a preferential $\mathrm{c}$ axis (normal line direction on the surface) oriented crystallization growth. (Masuda et al, 2003; Dutschke et al, 2003; Strohm et al, 2005). The resulting patterned $\mathrm{TiO}_{2}$ film possessed significant bonding strength with underling substrates and good line edge acuity. Actually, when standard 3M adhesive tape peeling experiment was applied on this positive pattern film, the film could sustain very well. Such selective deposition could be attributed to the preferential nucleation and growth on sulfate group implanted (i.e. irradiated hydrophilic) regions. This functional group-mediated inorganic oxide deposition has been observed and developed in selfassembled monolayer (SAM) on inorganic and metal substrates, and our approach provided an alternative way to do this on polymer substrate where SAM is difficultly constructed. Obviously, this biomimetic approach provided an effective solution toward the microfabrication on various inert polymer substrates.

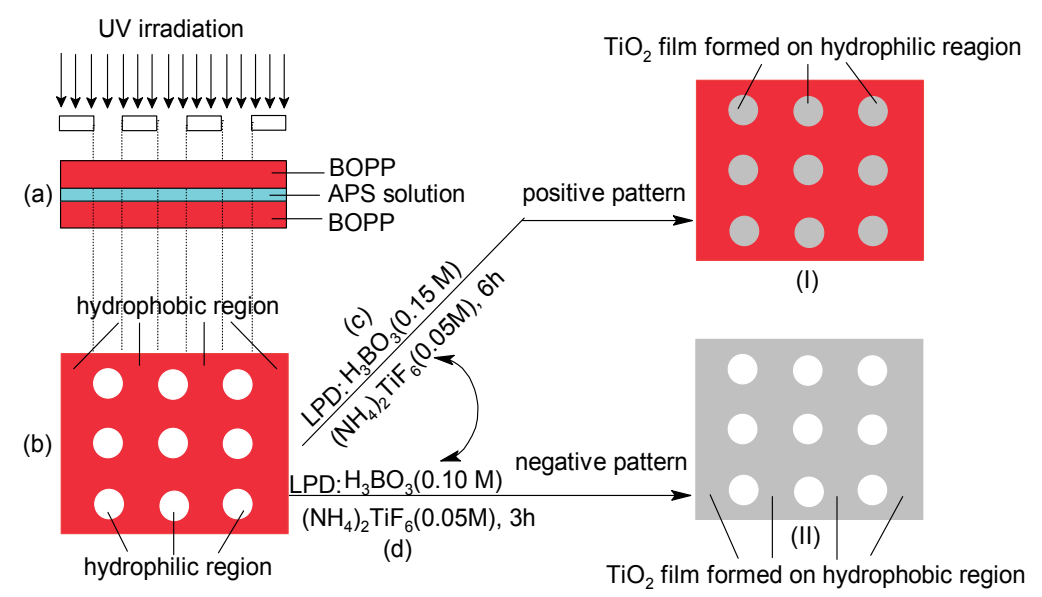

Fig. 9. The schematic process of $\mathrm{TiO}_{2}$ micropattern on BOPP film. (a). A thin layer of ammonium persulfate (APS) aqueous solution was sandwiched between two BOPP films, and selective UV irradiation was conducted by using a photomask; (b). CPO took place in the irradiated region, and the resulting wettability-patterned surface was utilized to fabricate positive (I) pattern under positive condition (c) and negative (II) pattern under negative condition (d) respectively. Reproduced from (Yang et al, 2007). Copyright from American Chemical Society. Reproduced with permission. 

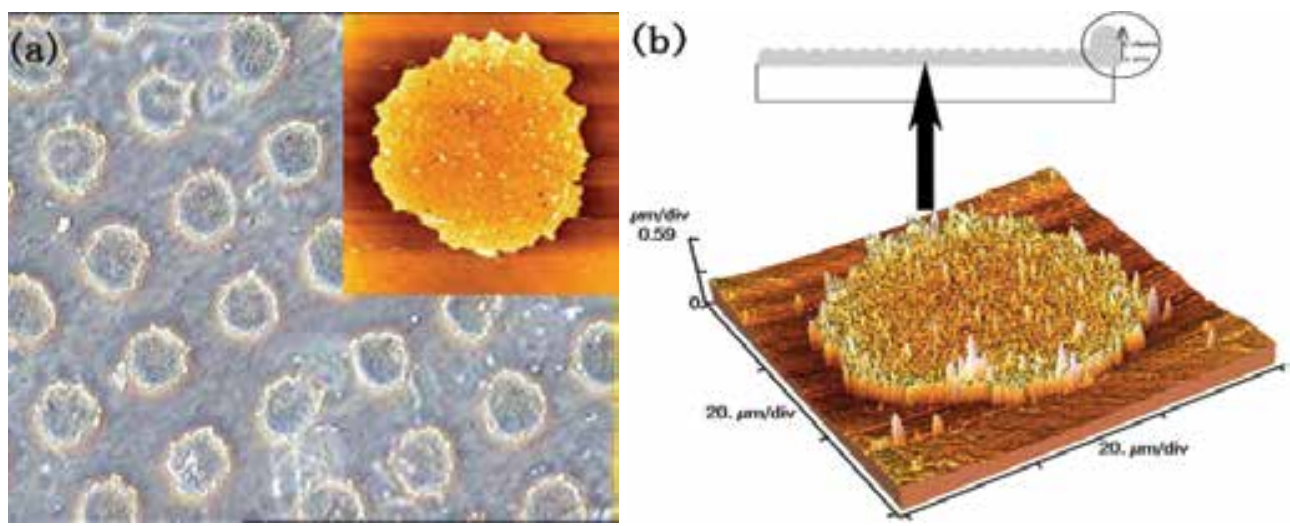

Fig. 10. Optical and AFM images of positive pattern on BOPP surface obtained by condition (c). (a). Optical image of positive pattern, the inert picture in (a) showed a detailed circle $\mathrm{TiO}_{2}$ deposition layer by AFM; (b). 3D profile AFM image of positive pattern (a), the upper picture in (b) showed the schematic column growth of $\mathrm{TiO}_{2}$ crystalline particle along c-axis, this column-pattern could be found clearly in the lower 3D profile AFM picture.

Reproduced from (Yang et al, 2007). Copyright from American Chemical Society. Reproduced with permission.
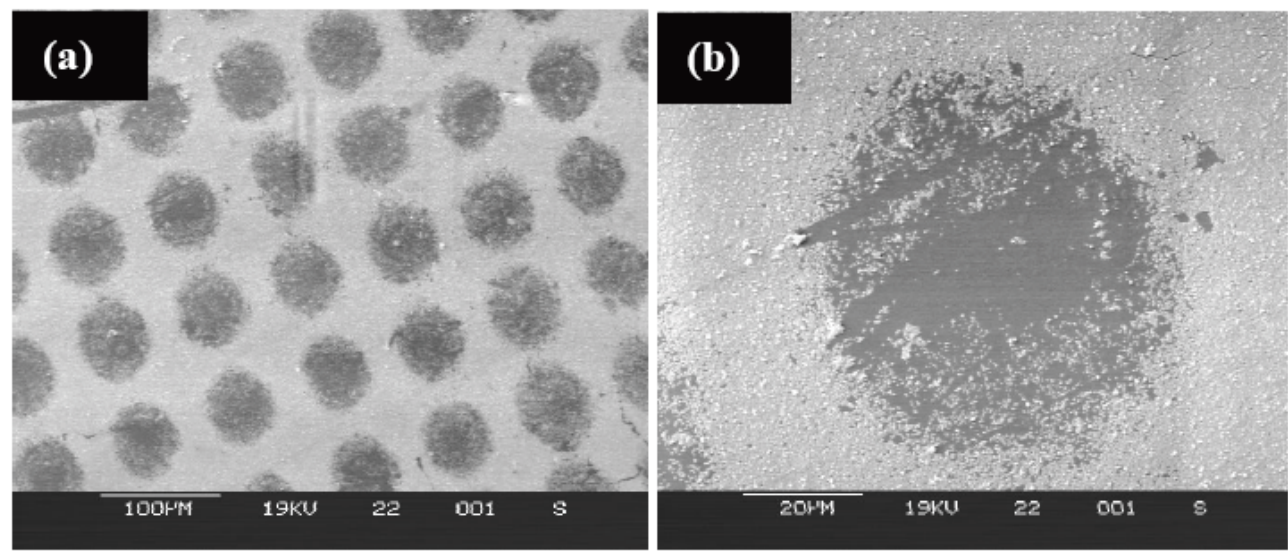

Fig. 11. SEM images of the negative $\mathrm{TiO}_{2}$ pattern on $\mathrm{BOPP}$ surface obtained by condition (d): (a). SEM image of negative pattern; (b). an enlarged image in circle region in (a). Reproduced from (Yang et al, 2007). Copyright from American Chemical Society. Reproduced with permission.

More surprisingly, we also found, for the first time, that under deposition condition (d), instead of deposition on hydrophilic regions, the deposition of $\mathrm{TiO}_{2}$ film would take a phase inversion process, that is, the deposition was only selectively retained on the hydrophobic region with keeping hydrophilic regions being undisturbed (no deposition) to form a negative pattern. Figure 11 presented a typical negative pattern obtained by such method.

This is a very interesting and novel phenomenon found firstly by us, and further investigation on the mechanism showed that the hydrolysis reaction of active sulfate groups and the resulting affinity change towards $\mathrm{TiO}_{2}$ crystals during this process played a key role 
(Figure 12). Such negative patterns refute the conventional opinion that only hydrophilic regions favor the formation of $\mathrm{TiO}_{2}$ films and could be used to fabricate large areas $\left(\mathrm{mm}^{2}\right)$ of interconnected $\mathrm{TiO}_{2}$ micronetworks, which are obviously difficult to obtain by conventional metallic masks. Such negative pattern also could be freely transferred to any other substrate by adhesive tape peeling (Figure 13) and could be used as a flexible photomask for photochemical reactions (Figure 14).

To summary, the present method that surface functionality mediated $\mathrm{TiO}_{2}$ deposition is expected to provide new strategies in the fabrication of flexible positive $\mathrm{TiO}_{2}$ array and negative macro/mesoporous $\mathrm{TiO}_{2}$ interconnected films on flexible organic substrates, without the use of complex photolithography procedures. We further found that this method is also suitable for fabrication of other inorganic oxide films such as $\mathrm{ZnO}$ and $\mathrm{SiO}_{2}$, as described below. Further exploration on other kinds of inorganic oxide film fabrication by such method and incorporation of functional molecules into the positive film and the pores formed in negative film for functional device fabrication may be suggested as the next direction of this research.

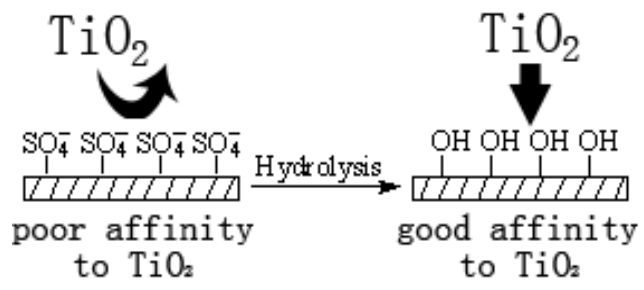

Fig. 12. The chemical change of functional groups on oxidized surface by hydrolysis reaction. It was found that sulfate groups had poor affinity to titanium layer $\left(\mathrm{TiO}_{2}\right)$, while hydroxyl groups had good affinity to $\mathrm{TiO}_{2}$. Reproduced from (Yang et al, 2007). Copyright from American Chemical Society. Reproduced with permission.

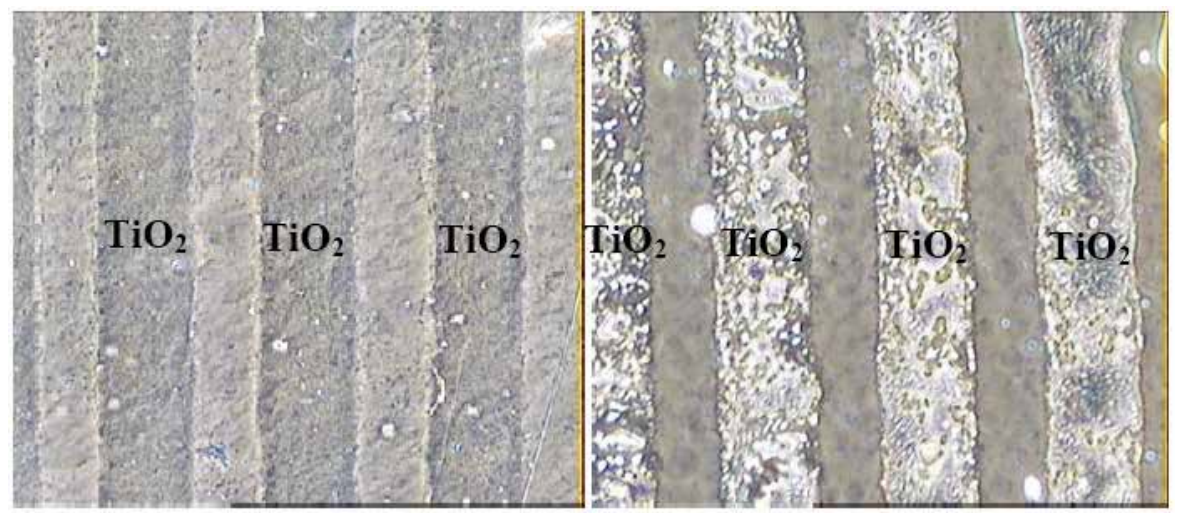

Fig. 13. Negative $\mathrm{TiO}_{2}$ pattern formed on BOPP substrate and subsequent peeling for transferring such pattern onto an adhesive tape. (a). optical images of the negative $\mathrm{TiO}_{2}$ micropattern formed on BOPP surface by condition (d) (using a photomask with parallel line pattern). (b). optical images of the negative $\mathrm{TiO}_{2}$ micropattern on the 3M Scotch ${ }^{\circledR}$ adhesive tape after peeling the $\mathrm{TiO}_{2}$ pattern in (a) from BOPP substrate. Reproduced from (Yang et al, 2007). Copyright from American Chemical Society. Reproduced with permission. 


\subsection{2 $\mathrm{ZnO}$ film fabrication on sulfated, hydroxylated and caroxylated polymer surfaces by biomimetic nucleation and growth in aqueous solution. (Yang et al, 2008)}

Similar to patterned $\mathrm{TiO}_{2}$ deposition, patterned $\mathrm{ZnO}$ deposition on polymer substrates has also received great attentions because of its promising potential in photocatalysis, energy conversion, and electro-optical techniques. Our research indicated the functionality pattern formed by $\mathrm{CPO}$ method could be also used to mediate the growth of $\mathrm{ZnO}$ crystals on polymer substrates under mild conditions, giving positive and negative patterns on BOPP and PET substrates respectively (Figure 15). The fabrication was achieved by incubating functionalized polymer film in a mixture solution of $\mathrm{Zn}\left(\mathrm{NO}_{3}\right)_{2}$ and hexamethylenetetramine (HMT) at suitable temperature and concentration (Yang et al, 2008).
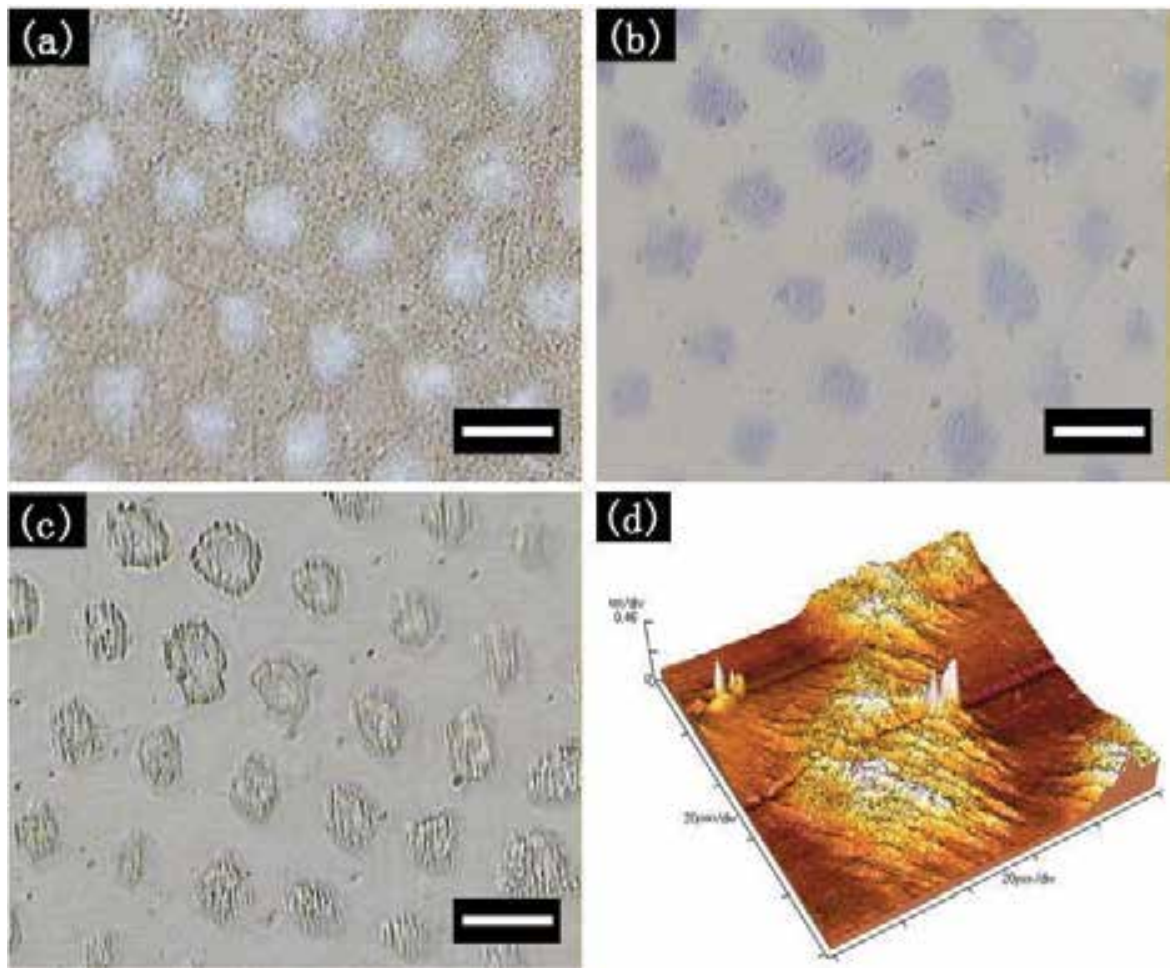

Fig. 14. Micropatterning of a BOPP surface with patterned photograft polymerization products of acrylic acid (AA) by using the negative $\mathrm{TiO}_{2}$ pattern on the BOPP film as a photomask. (a). Phase contrast microscope image of the negative $\mathrm{TiO}_{2}$ pattern on the BOPP film. (b). Optical microscope image of the patterned poly(AA) (PAA) grafts on the BOPP surface after staining with toluidine blue. (c). Phase contrast microscope image of the patterned PAA grafts on the BOPP surface. (d). 3D AFM image of a circle from the PAA grafted region on the BOPP surface. The scale bars in (a)-(c) are $80 \mu \mathrm{m}$. Reproduced from (Yang et al, 2007). Copyright from American Chemical Society. Reproduced with permission.

Similar to the column-type growth behavior observed in $\mathrm{TiO}_{2}$ deposition system, $\mathrm{ZnO}$ film also grew along c-axis from the surface. However, fusion among these columns is not observed in $\mathrm{ZnO}$ material, and separated $\mathrm{ZnO}$ rods are obtained in the resulting $\mathrm{ZnO}$ film. 
We found that the rods having the typical size around 500-750nm in diameter and $2.5 \mu \mathrm{m}$ in length, was selectively obtained on sulfated and hydroxylated regions of BOPP substrate, resulting in a positive $\mathrm{ZnO}$ pattern which was corresponding to the irradiated regions by UV (Figure 16 and 17). In contrast, for reactive polyesters such as PET, the ZnO rods selectively remained on the unmodified original regions, creating negative patterns which were corresponding to the unirradiated regions (Figure 18 and 19). X-ray Diffraction (XRD) pattern was further used to characterize the crystal morphology of $\mathrm{ZnO}$ rods obtained on sulfated and hydroxylated BOPP as well as PET surfaces (Figure 20). Interestingly, different dominating crystal direction was found on four kinds of surfaces. On BOPP-OH surface, preferential c-axis direction was found since (002) plane had the maximum intensity. When this substrate was changed to $\mathrm{BOPP}_{-} \mathrm{SO}_{4}{ }^{-}$, this c-axis orientation was lowered and as a result, both of c-axis direction as (002) and nearly parallel directions to the substrate as (100), (101) co-dominated in the crystal morphology. When the substrate was further changed to PET from BOPP, dominated crystal direction was found around the direction parallel to the substrate since the planes as (100) and (101) had the higher signal intensity. The detailed reason for this substrate-dependent crystal morphology change is needed to be investigated in the future, and different crystallization orientation on original BOPP and PET films may become a possible reason.

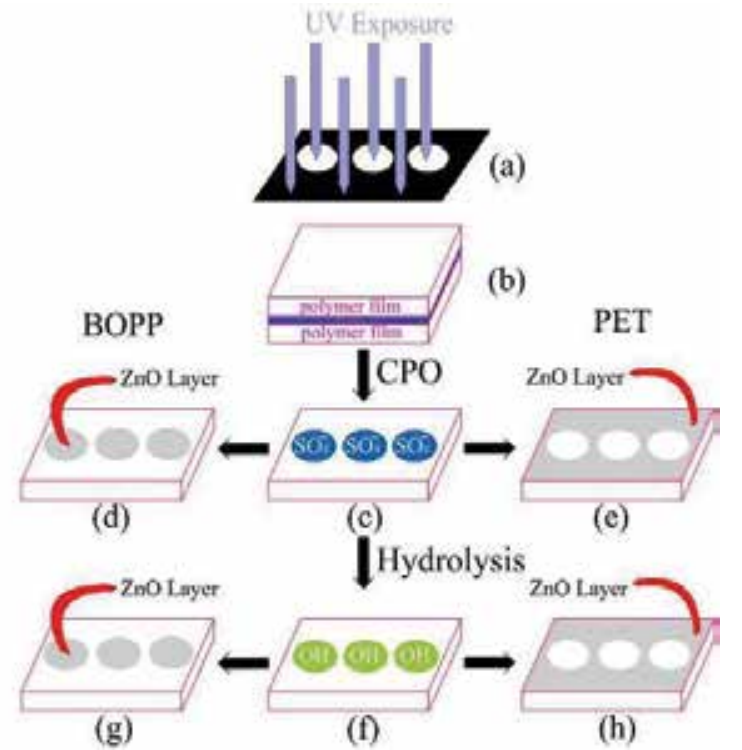

Fig. 15. The growth scheme of the ZnO layer on patterned functionalized polymer surfaces: (a) UV rays transported selectively through a photomask with circular holes; (b) a sandwich setup consisting of two polymer films with a persulfate ammonium solution in the middle, irradiated by UV light whose route was controlled by the photomask; (c) following the patterned irradiation, the sulfate groups could be selectively grafted onto the irradiated regions, which could be further transformed into hydroxyl groups by a hydrolysis reaction; (f) the $\mathrm{ZnO}$ layer selectively remained on the sulfated and hydroxylated regions of the BOPP film $(\mathrm{d}, \mathrm{g})$, whereas for PET, the growth of the $\mathrm{ZnO}$ layer selectively remained on the unirradiated regions (e, h). Reproduced from (Yang et al, 2008). Copyright from Wiley-VCH Verlag GmbH \& Co. KGaA. Reproduced with permission. 
Moreover, the photoluminescence property of a ZnO crystal was also investigated. Near UV and visible luminescence from $\mathrm{ZnO}$ crystals were detected by fluorescence spectroscopy (Figure 21). Strong UV and visible-light luminescence originated from band edge emission $(395 \mathrm{~nm})$ and the oxygen vacancy $(450-600 \mathrm{~nm})$ respectively (Masuda et al, 2006; Wu et al, 2001; Kang et al, 2003), showing a potential for polymer-based display devices. The mechanism for the above-mentioned pattern formation also involves in the mediation ability of surface functional monolayers. On BOPP substrates, functionalized sulfated and hydroxylated regions were found to favor the formation of a $\mathrm{ZnO}$ layer through possible electrostatic and hydrogen coordination between functional groups and $\mathrm{ZnO}$ crystals (Chaudhary et al, 2004; Zubkov et al, 2005; Saito et al, 2001). As mentioned above, the sulfate groups on the surface were not stable species which would undertake a slow hydrolysis to give hydroxyl groups. This process also released protons as byproduct $\left(\mathrm{H}^{+}\right)$(Yang et al, 2003; House et al, 1962; Wilmarth et al, 1962; Bamford et al, 1994). This newly formed $\mathrm{H}^{+}$
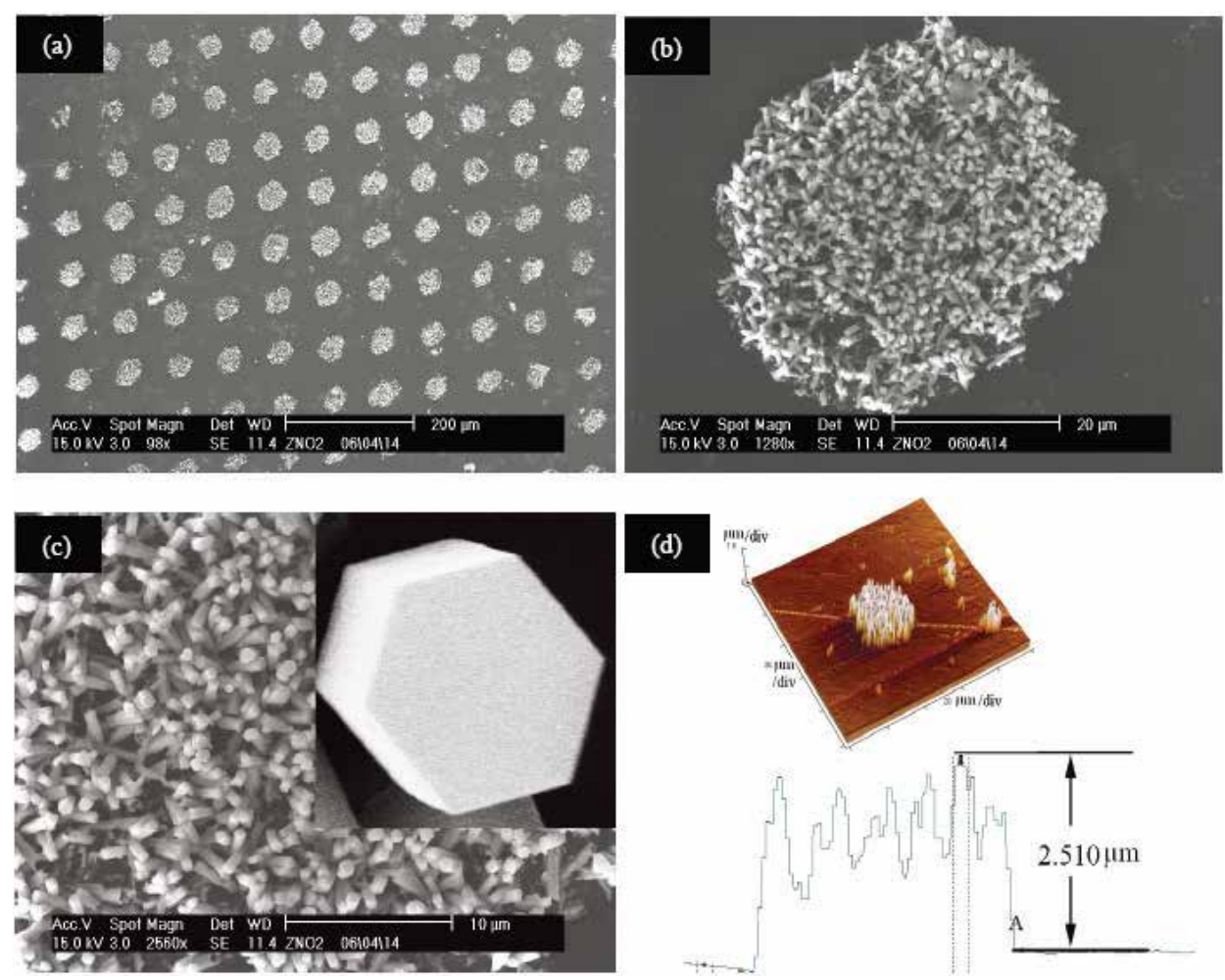

Fig. 16. (a-c) SEM and (d) AFM images of a patterned ZnO deposition on a sulfated BOPP surface via ultrasonic washing. (a) The resulting film showed a regular circle pattern where the $\mathrm{ZnO}$ layer could be found solely on the irradiated region. The magnifications $(\mathrm{b}, \mathrm{c})$ showed that the diameter of the deposited region and a single $\mathrm{ZnO}$ pillar was about $40 \mu \mathrm{m}$ and $680 \mathrm{~nm}$, respectively. The cross-sectional shape of the ZnO pillar was typically hexagonal (inset picture in (c)). (d) An AFM image of a single circular pattern indicated a thickness of the $\mathrm{ZnO}$ layer on the sulfated area of about $2.5 \mu \mathrm{m}$. Reproduced from (Yang et al, 2008). Copyright from Wiley-VCH Verlag GmbH \& Co. KGaA. Reproduced with permission. 
would decrease the extent of supersaturation of the solution as a result of neutralization with $\mathrm{OH}^{-}$formed by the hydrolysis of HMT. Accordingly, during the same time scale, the extent of deposition reaction for $\mathrm{ZnO}$ growth on the sulfated BOPP surface would be lower than that on the hydroxylated BOPP surface. This analysis was well demonstrated by the following experiment: under equivalent conditions $\left(0.1 \mathrm{M}, 90^{\circ} \mathrm{C}, 48 \mathrm{~h}\right)$, the density of the $\mathrm{ZnO}$ rods on a sulfated BOPP surface was $0.8 / \mu^{2}$, whereas this value increased to $1.3 / \mu^{2}$ on the hydroxylated surface.

For the negative patterns on PET substrates, Koumoto et al (Masuda et al, 2006) suggested the reason for this was because the $\mathrm{ZnO}$ crystal surface could become hydrophobic by the adsorption of certain hydrophobic organic molecules used in the solution, and then the hydrophobic $\mathrm{ZnO}$ crystallites naturally preferred to be deposited on the hydrophobic unirradiated PET surface. We analyzed this phenomenon from another point of view, that is, the effect of surface functional group plays an important role on this pattern formation.
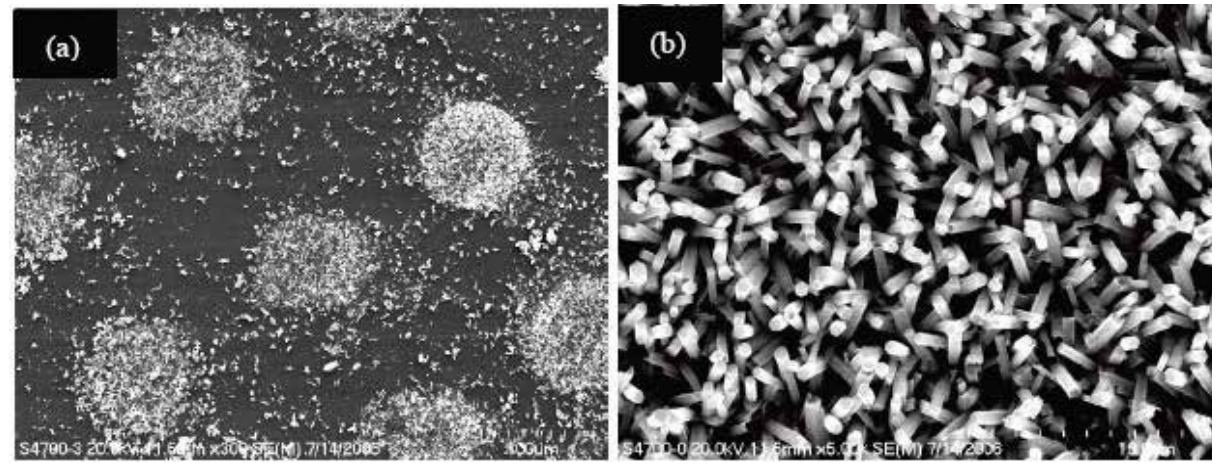

Fig. 17. (a) A SEM micrograph of a patterned $\mathrm{ZnO}$ deposition on a hydroxylated BOPP surface and (b) a magnification of one circular region. By ultrasonic washing, the ZnO pillar became selective to the hydroxylated BOPP region whereas a few particles dispersed into the unirradiated region. The average diameter of the $\mathrm{ZnO}$ rods was $500 \mathrm{~nm}$. Reproduced from (Yang et al, 2008). Copyright from Wiley-VCH Verlag GmbH \& Co. KGaA. Reproduced with permission.

Not like inert BOPP, PET is a relatively reactive substrate containing abundant ester bonds, which are capable to undergo hydrolysis reactions in aqueous environments (Karayannidis et al, 2007). Similar to our consideration, Pizem et al (Pizem et al, 2005) also pointed out that during the solution deposition process of a titanium film on polyimide substrates, reactive imide groups could be partially hydrolyzed and thereby influence the acidic oxide deposition solution. In our work, we found that the existence of polar sulfate and hydroxyl groups on irradiated PET regions would facilitate the alkaline hydrolysis to release more $\mathrm{COOH}$ groups in a weak base environment than unirradiated regions, because grafting of sulfate and hydroxyl groups onto the $a$ site of the glycol ester (COOCH2CH2OOC) along PET chains would effectively increase the rate constant of the alkaline hydrolysis (Schmeer et al, 1990; Bruice et al, 1961 and 1962), whereas this effect was not obvious on unirradiated regions without such polar groups. The existence of $\mathrm{COOH}$ groups on substrates would weaken the deposition of the $\mathrm{ZnO}$ layer (Hsu et al, 2005) because these $\mathrm{COOH}$ endgroups on polymer surface could firstly be deprotonated and negatively charged, and then preferentially bonded with HMT molecules to create electrostatic and/or steric screening for 

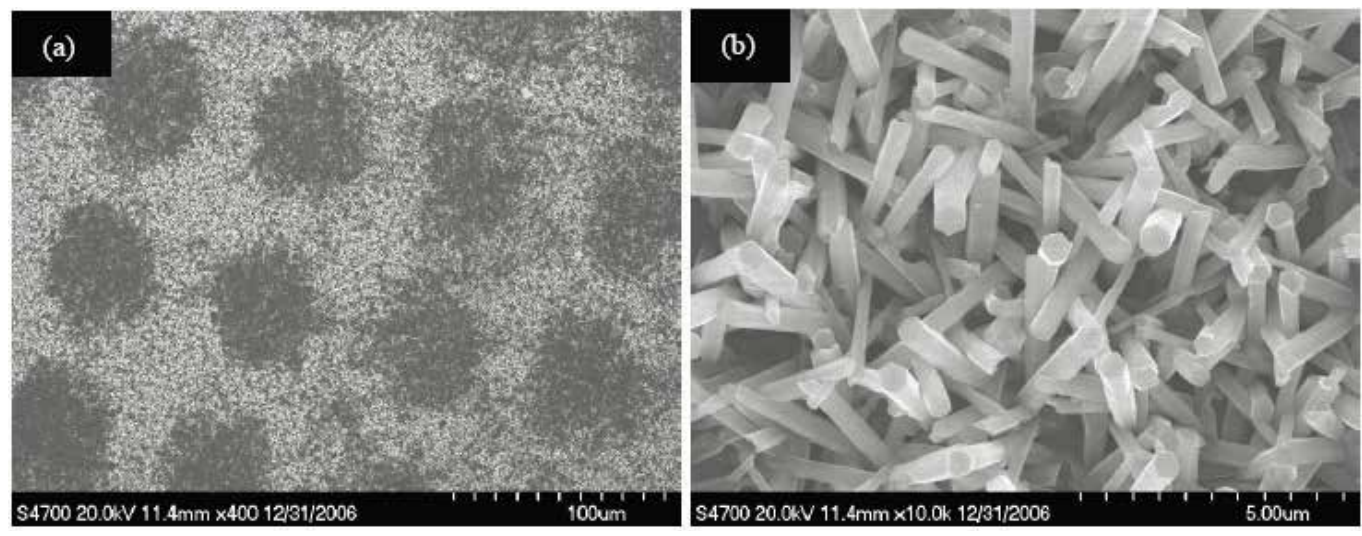

Fig. 18. SEM micrographs of $\mathrm{ZnO}$ deposition on patterned sulfated PET surface and the magnification displaying the detailed deposition on sulfated (the dark regions in (a)) and unirradiated regions ((b) and the bright regions in (a)). Very little deposition was found on the sulfated regions represented by a circular pattern. Rather, the deposition was mainly found to form a continuous network on the unirradiated regions. The average diameter of the $\mathrm{ZnO}$ rods was about $750 \mathrm{~nm}$. Reproduced from (Yang et al, 2008). Copyright from Wiley-VCH Verlag GmbH \& Co. KGaA. Reproduced with permission.
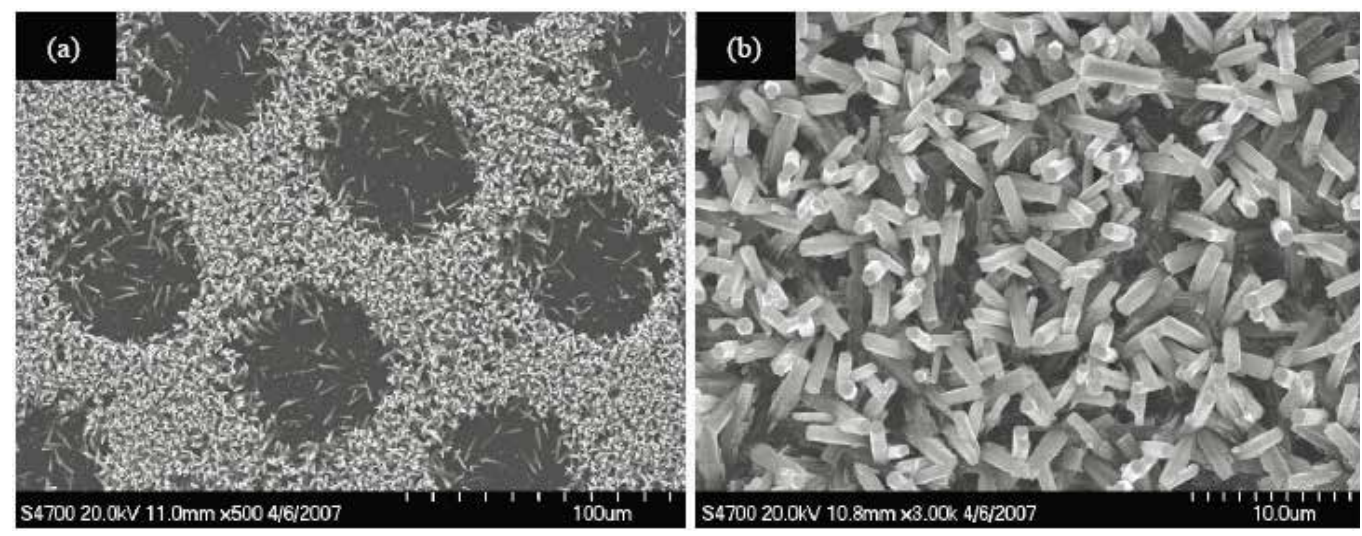

Fig. 19. SEM micrographs of the $\mathrm{ZnO}$ deposition on a patterned hydroxylated PET surface and the magnification displaying the detailed deposition on hydroxylated (the dark regions in (a)) and unirradiated regions ((b) and the bright regions in (a)). The average diameter of $\mathrm{ZnO}$ rods was about $750 \mathrm{~nm}$. Reproduced from (Yang et al, 2008). Copyright from Wiley$\mathrm{VCH}$ Verlag $\mathrm{GmbH} \& \mathrm{Co}$. KGaA. Reproduced with permission. 

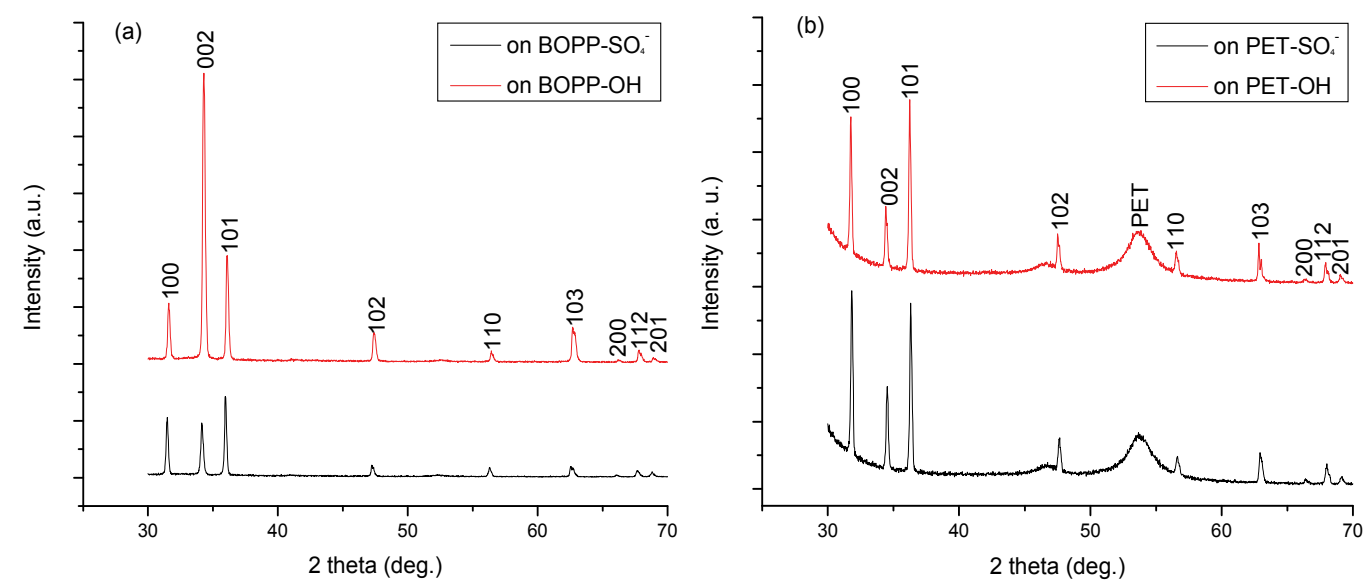

Fig. 20. XRD patterns of $\mathrm{ZnO}$ rods deposited on substrates of (a) sulfated BOPP (lower curve in (a), BOPP-SO $4^{-}$), hydroxylated BOPP (upper curve in (a), BOPP-OH), and (b) sulfated PET (lower curve in (b), PET-SO $4^{-}$) and hydroxylated PET (upper curve in (b), PET-OH). Reproduced from (Yang et al, 2008). Copyright from Wiley-VCH Verlag GmbH \& Co. KGaA. Reproduced with permission.
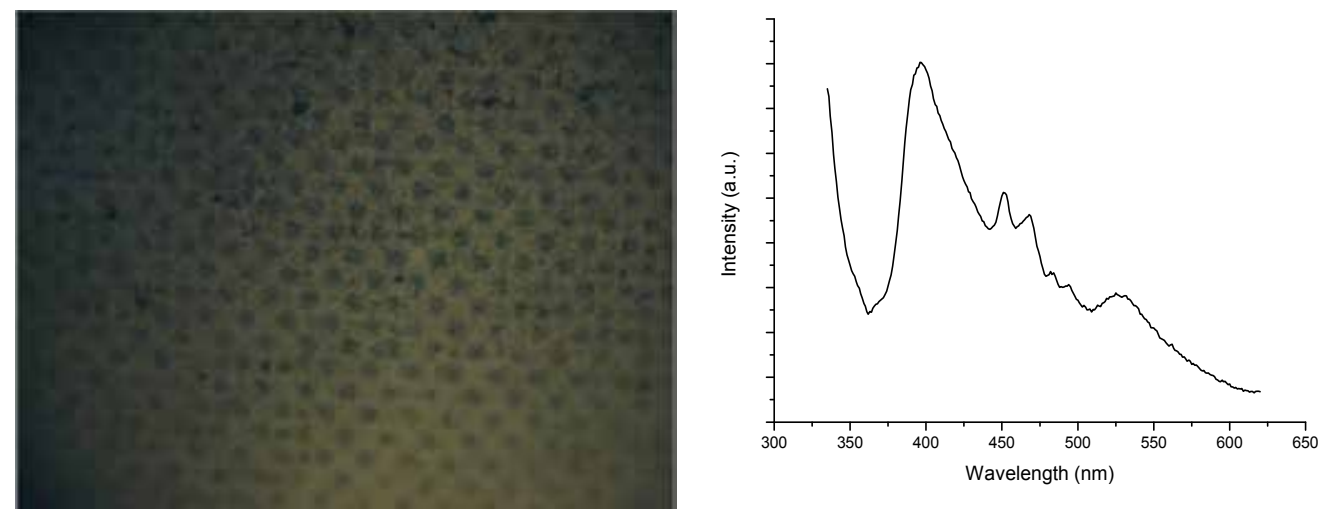

Fig. 21. A photoluminescence image of a patterned $\mathrm{ZnO}$ deposition on a sulfated BOPP substrate under UV light illumination $(365 \mathrm{~nm})$. The corresponding emission spectra of $\mathrm{ZnO}$ deposition layer is shown in right picture (the excitation wavelength is $325 \mathrm{~nm}$ ). Reproduced from (Yang et al, 2008). Copyright from Wiley-VCH Verlag GmbH \& Co. KGaA. Reproduced with permission.

inhibiting the $\mathrm{ZnO}$ nucleation. However, this statement is still not fully confirmed because recently Morin et al reported a reverse phenomenon that $\mathrm{COOH}$-enriched PET surface actually induced the formation of $\mathrm{ZnO}$ nanorods patterning (Morin et al, 2007).

To summarize this section, surface functional small molecule-mediated ability is demonstrated again to be used for the fabrication of positive and negative $\mathrm{ZnO}$ film on polymer substrates. Such patterned $\mathrm{ZnO}$ deposition is very sensitive to the surface functional group changes. Patterned $\mathrm{ZnO}$ films made up of vertical arrayed rods typically $500-680 \mathrm{~nm}$ in diameter, were found to form on sulfated and hydroxylated BOPP surfaces. When the substrate was changed from BOPP to polyester, the $\mathrm{ZnO}$ deposition with vertical 
$\mathrm{ZnO}$ rods typically $750 \mathrm{~nm}$ in diameter would selectively grow on unirradiated regions because of the transformation from sulfated or hydroxylated to carboxylated regions on PET substrate during the deposition process, the latter group was considered as a suppressing moiety for hindering the $\mathrm{ZnO}$ deposition. The present results gave us the $\mathrm{ZnO}$ film with microrods array. Since $\mathrm{ZnO}$ nanorods and their array have found great applications in laser and photo-energy devices, the next possible direction for this research is to optimize the reaction condition to fabricate $\mathrm{ZnO}$ nanorods array on polymer substrates by such surface functional group-mediated growth. Morin et al has presented such an approach on flexible polymer substrates. (Morin et al, 2007)

\subsection{3 $\mathrm{SiO}_{\mathrm{x}}$ film fabrication on hydroxylated polymer surfaces by biomimetic interface- directed sol-gel. (Gan et al, 2010)}

Among versatile inorganic oxides, silica oxide film as $\mathrm{SiO}_{x}$ is especially important because this semiconductor material could provide crucial properties in devices or serve as a base layer for further multilayer construction. In this section, we describe a new interfacedirected sol-gel method to fabricate flexible high quality silicon oxide film onto commodity plastics (Figure 22). Such fabrication strategy relies on CPO process described above. Firstly, $\mathrm{CPO}$ reaction was used on $\mathrm{BOPP}$ surface to introduce a hydroxyl monolayer. We found that this kind of hydroxyl monolayer could serve as nucleation and growth site for subsequent

\section{Whole-area deposition:}

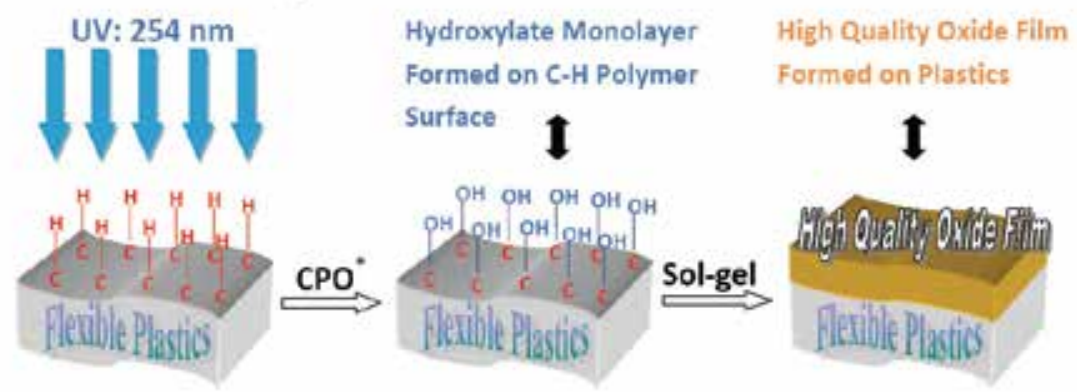

Area-selective deposition:

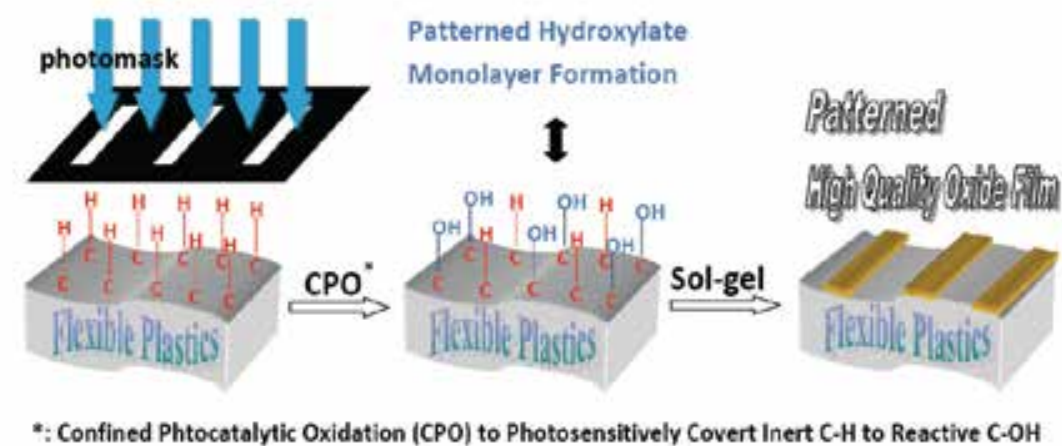

Fig. 22. The schematic process to fabricate $\mathrm{SiO}_{x}$ and its patterning on BOPP-OH surface. Reproduced from (Yang et al, 2010). Copyright from Science in China Chemistry. Reproduced with permission. 
sol-gel reaction on the surface. Different from commonly used sol-gel technique where bulk sol-gel reaction occurs simultaneously, surface hydroxyl-directed sol-gel reaction in this work presents the unique character as surface-initiating cascade growing. Surface hydroxyl groups were capable of capturing silanol groups to initiate surface gelation. Such interface condensation reaction was possibly catalyzed by trace water and acidic molecules adsorbed onto hydrophilic hydroxyl-modified surface. The latter physisorption could become energetically favorable through the possible formation of hydrogen bond and/or protonation between trace water/acid and surface hydroxyl groups. This kind of surface condensation reaction seemed to express higher reactivity than the condensation in the Sisol, because the preferential surface growth was observed at the early deposition stage to form 4-6 nm silica layer. As a result, the deposition on the surface proceeded along a more controlled two-step way. In the first step, the deposition was initiated by the surface hydroxyl groups which could be reflected by the thin thickness data $(4-6 \mathrm{~nm})$ detected in the early stage (10-40 min). This process would last at least $40 \mathrm{~min}$ to form enough reactive sites.
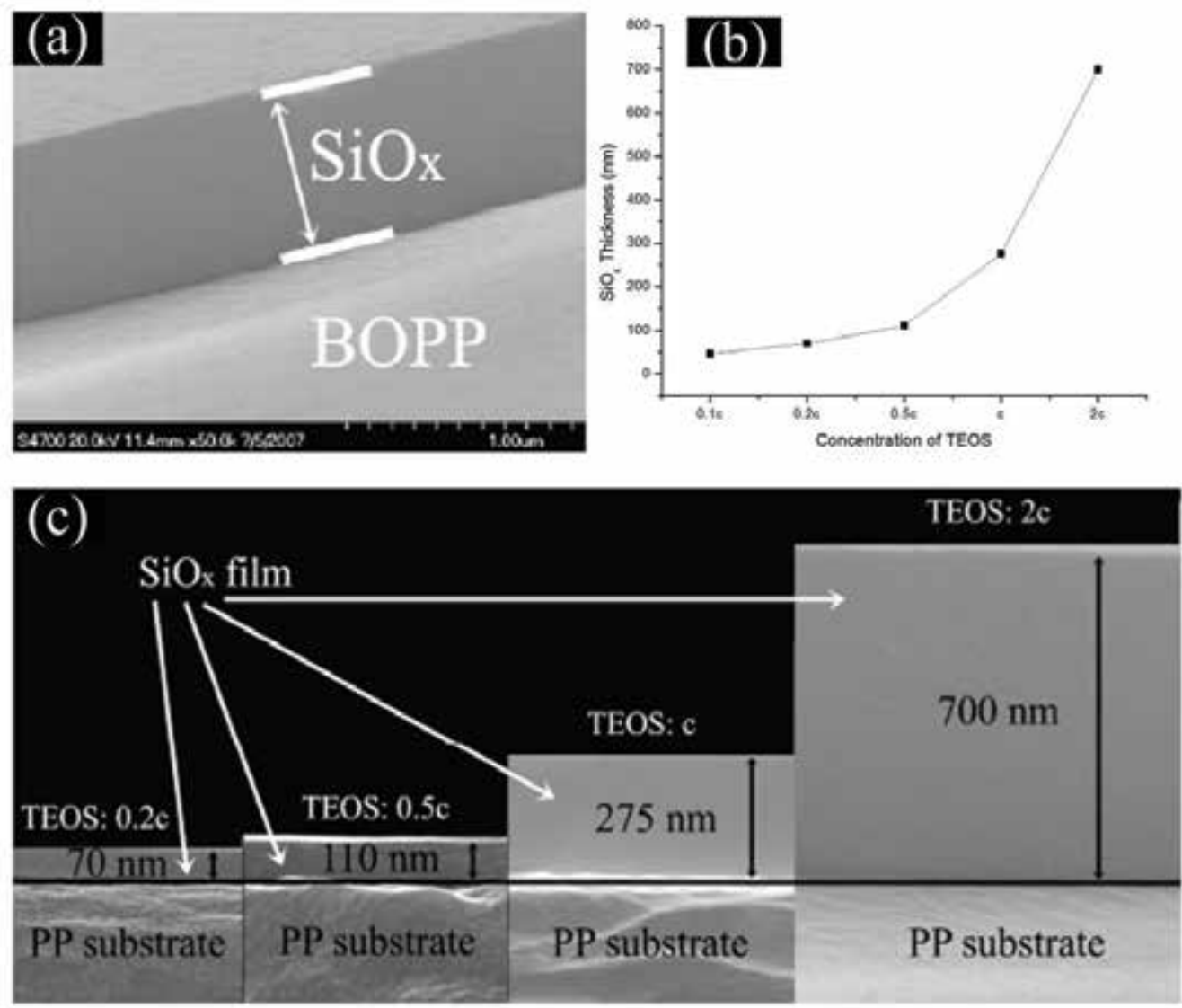

Fig. 23. A typical cross-sectional FE-SEM image (a) of $\mathrm{SiO}_{x}$ deposition on $\mathrm{BOPP}-\mathrm{OH}$ surface $\left(\mathrm{BOPP}-\mathrm{OH} / \mathrm{SiO}_{\mathrm{x}}\right)$, and the controlling on the deposition thickness was facilely achieved by changing TEOS concentration, as plotted in (b) and visualized in (c). Reproduced from (Yang et al, 2010). Copyright from Science in China Chemistry. Reproduced with permission. 
After $40 \mathrm{~min}$, the mature thin silica layer served as nucleation and growth templates to gradually thicken the silica layer. Similarly, Hozumi and coworkers (Hozumi et al, 2003) also found an ultrathin SiOx layer $(\sim 1 \mathrm{~nm})$, termed as "nanoskin", could form on photosensitive polymer surface during vacuum UV enhanced vapor deposition, which facilitated further deposition of solid and ultraflat inorganic oxide.

The resulting silica was crack-free with strong covalent bonding with underlying polymer substrates, and also had homogeneous morphology with ultralow roughness, highly optical transparency, tunable thickness from $\mathrm{nm}$ to $\mu \mathrm{m}$, and easy patterning ability. As shown in Figure 23, a typical cross-sectional image of $\mathrm{BOPP}-\mathrm{OH} / \mathrm{SiO}_{\mathrm{x}}$ film indicated that a clear and even interface between $\mathrm{SiO}_{x}$ and BOPP phases. Homogeneous $\mathrm{SiO}_{x}$ surface also could be partially revealed from this side-view observation. Accordingly, the thickness of $\mathrm{SiO}_{\mathrm{x}}$ layer could be directly obtained from these cross-sectional pictures. We further found that the thickness could be flexibly tuned by simply changing the sol-gel condition [e.g. tetraethylorthosilicate (TEOS) concentration and gelation time] from nanometer to micrometer, and this ability is very crucial for practical applications. The effect of TEOS concentration on the thickness increase was not a linear relationship, but a typical one observed in inorganic oxide sol-gel process.

The smooth surface morphology of resulting silica film was further revealed by AFM (Figure 24). Comparing with pristine BOPP and BOPP-OH films, the $\mathrm{BOPP}-\mathrm{OH} / \mathrm{SiO}_{x}$ surface showed very even and homogeneous morphology in large scale. The defects such as cracks, pinholes commonly observed in other methods did not appear under the observation field. Further measurement illustrated that ultra-smooth surface with extremely low root mean square (RMS) $(\sim 8 \AA)$ over large area $\left(25 \mu \mathrm{m}^{2}\right)$ was obtained after $\mathrm{SiO}_{\mathrm{x}}$ layer formed onto BOPP-OH surface. The RMS value on such ultraflat surface was lower than BOPP and $\mathrm{BOPP}-\mathrm{OH}$ surfaces before the deposition and even better than that on commercial quartz surface (around $10 \AA$ ). Because the fabrication of such ultrasmooth surface only utilized the well-developed sol-gel technique and universal CPO reaction on polymer surface, this method provided us with a very simple and scalable approach to quickly fabricate ultraflat oxide film on polymeric materials. The resulting film also possessed superior optical transparence (Figure 25). Comparing with blank $\mathrm{BOPP}$ film, $\mathrm{BOPP}-\mathrm{OH} / \mathrm{SiO}_{\mathrm{x}}$ film with different thickness only had very little decrease $(<3 \%)$ in the transmittance at $600 \mathrm{~nm}$, even in the case that the thickness of $\mathrm{SiO}_{\mathrm{x}}$ increased to $300 \mathrm{~nm}$. As a result, no discernible difference could be told between pristine $\mathrm{BOPP}$ and $\mathrm{BOPP}-\mathrm{OH} / \mathrm{SiO}_{\mathrm{x}}$ films, after direct observation by eyes on the characters ("BUCT") behind two kinds of films.
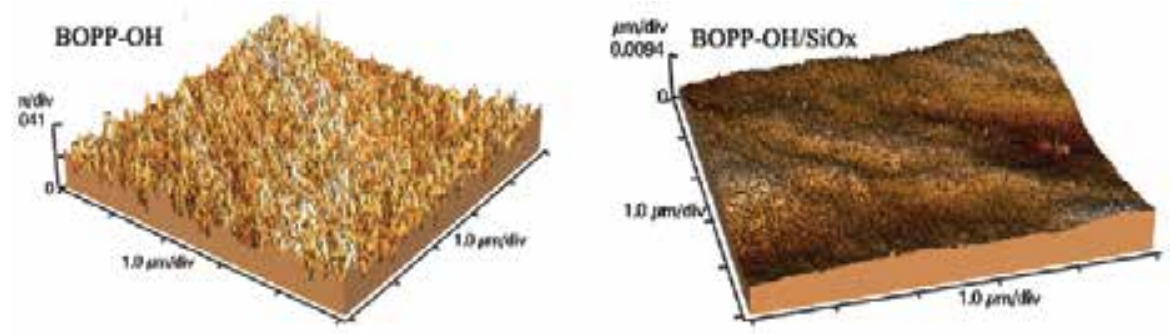

Fig. 24. Surface roughness evaluation by AFM on BOPP-OH before/after $\mathrm{SiO}_{x}$ deposition. Reproduced from (Yang et al, 2010). Copyright from Science in China Chemistry.

Reproduced with permission. 


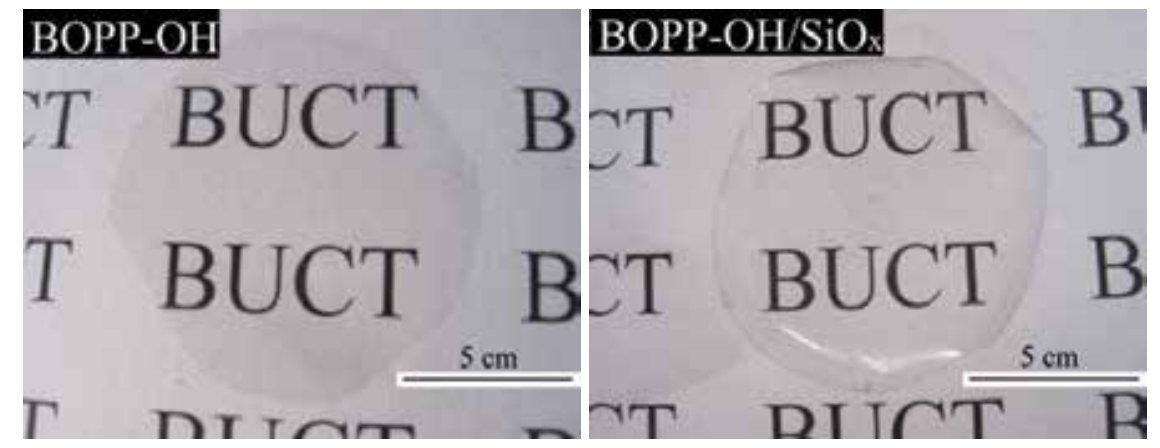

Fig. 25. The optical image after putting a BOPP-OH or $\mathrm{BOPP}-\mathrm{OH} / \mathrm{SiO}_{x}$ film on a paper with "BUCT" characters on it. The two pictures presented the contrast between optical micrograph of BOPP-OH (left) and $\mathrm{BOPP}-\mathrm{OH} / \mathrm{SiO}_{x}$ film (right). The clear observation on underlying characters ("BUCT") proved excellent optical transparency in visible light could be obtained on BOPP-OH/SiO film. Reproduced from (Yang et al, 2010). Copyright from Science in China Chemistry. Reproduced with permission.

The film could be easily patterned by applying ultrasonic or adhesive tape peeling on the asdeposited film, because the big coating adhesion difference existed between hydroxylated (covalent bonding) and unmodified regions (physisorbed). For this aim, patterned hydroxylated surface was firstly fabricated by $\mathrm{CPO}$ under a control of a photomask. After the same $\mathrm{SiO}_{x}$ sol-gel deposition on such patterned hydroxylation surface, the pattern was finally formed through mechanical destabilization from either ultrasonic washing or $3 \mathrm{M}$ adhesive tape peeling to remove weak deposition on unmodified regions. As shown in Figure 26, either adhesive tape peeling (left) or ultrasonic agitation (right) could give us clear circular or stripe patterns with extremely low line edge variation, presenting good copies from the photomasks used. The feature size of such patterns was also homogeneous with few defects. Moreover, a large-area homogeneous pattern $\left(\mathrm{cm}^{2}\right)$ could be also obtained with good fidelity by such a process. The cracking of inorganic layer under the deformation of organic substrates is a common problem when preparing hybrid composite film, however, no discernible cracks were observed on $\mathrm{SiO}_{x}$ layer when mechanically bending $\mathrm{BOPP}-\mathrm{OH}$ substrate because of strong interfacial bonding and low internal stress buried in $\mathrm{SiO}_{x}$ layer.
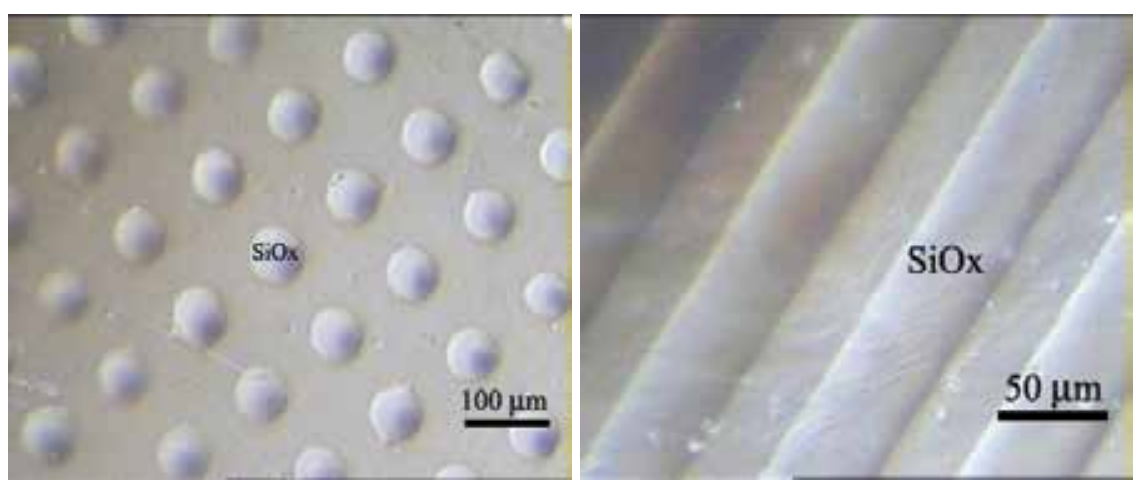

Fig. 26. Optical images of patterned $\mathrm{SiO}_{\mathrm{x}}$ layer on $\mathrm{BOPP}-\mathrm{OH}$ surface fabricated by adhesive tape peeling (left) and ultrasonic agitation (right). Reproduced from (Yang et al, 2010). Copyright from Science in China Chemistry. Reproduced with permission. 
To summarize this section, our strategy provides a simple and effective way to fabricate ultraflat silica film on polymer substrate with strong bonding strength and easy patterning ability, excluding the requirements of clean room and vacuum devices so as to fulfill lowcost and fast fabrication demands. Future research along this direction may be suggested as follows: 1). Explore the possibility to extend this method for other oxide material fabrication. For example, we have successfully achieved $\mathrm{TiO}_{2}$ film fabrication by such method (Figure 27). 2). Explore the possibility to prepare hybrid multilayer structure by using silica film as a base layer. 3). Explore the possibility to develop the applications of this material. Herein, two application examples from such high quality $\mathrm{SiO}_{x}$ layer onto plastics are given but should not be limited within these. One is that oxygen permeation rate of $\mathrm{SiO}_{\mathrm{x}}$ deposited polymer film decreases 25 times than pristine polymer substrate, which is good for the
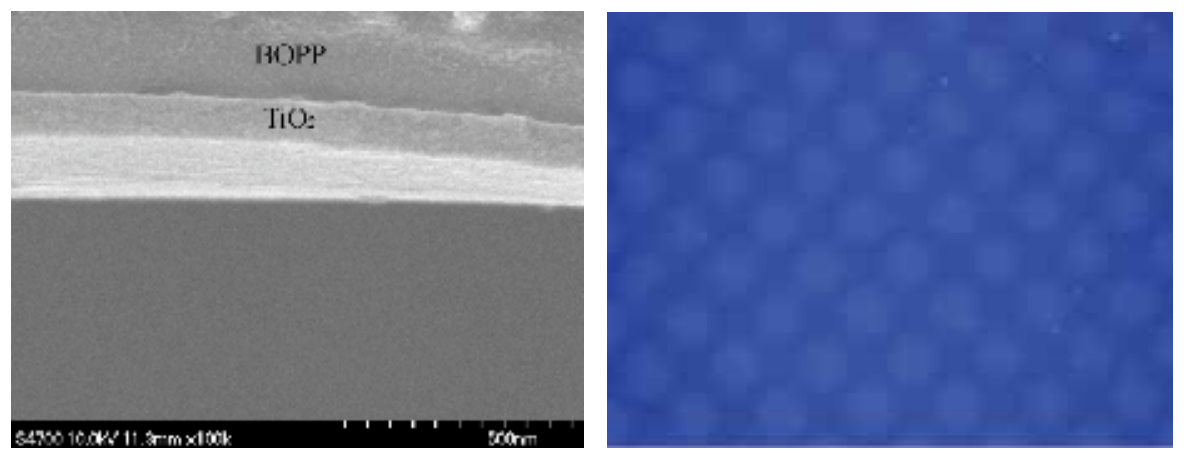

Fig. 27. Cross-sectional Field Emission (FE)-SEM image of $\mathrm{BOPP}-\mathrm{OH} / \mathrm{TiO}_{2}$ film (left) and fluorescent image (under UV excitation) of $\mathrm{TiO}_{2}$ micropatterning on $\mathrm{BOPP}-\mathrm{OH}$ surface (right) fabricated by our interface-directed sol-gel. Reproduced from (Yang et al, 2010). Copyright from Science in China Chemistry. Reproduced with permission.
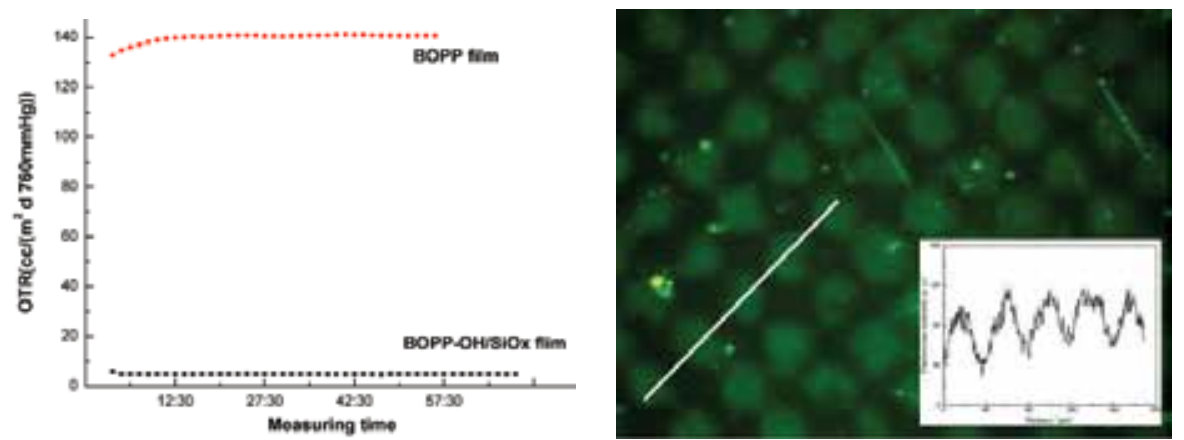

Fig. 28. Two application examples on $\mathrm{BOPP}-\mathrm{OH} / \mathrm{SiO}_{\mathrm{x}}$ samples. On the left, oxygen permeability of BOPP-OH film with only $150 \mathrm{~nm}$ thick $\mathrm{SiO}_{x}$ could get 25 times decrease than that of pristine BOPP. On the right, fluorescence microscope image demonstrated that patterned FITC-IgG immobilization could be easily achieved on patterned $\mathrm{BOPP}-\mathrm{OH} / \mathrm{SiO}_{\mathrm{x}}$ through the decoration of amine-terminated silane, 3-aminopropyltriethoxysilane (APTES) and further activation by glutaraldehyde on the surface. The profile intensity on selected region (inset picture) showed around 3:1 signal-to-background ratio could be obtained by this type of protein immobilization. Reproduced from (Yang et al, 2010). Copyright from Science in China Chemistry. Reproduced with permission. 
potential packaging materials (Figure 28 left). The other one is that silanization monolayer, for example, 3-aminopropyltriethoxysilane (APTES), could be successfully constructed onto the resulting silica layer through classical silanization reaction, which is applicable for many potential purposes, for instance, proteins could be accordingly immobilized onto plastic support with effective signal-to-background ratio (Figure 28 right).

\subsection{Level 2: Peptide-mediated nanocrystal and superstructures formation}

Following the researches performed by small functional groups, a larger molecule, that is, inorganic-binding peptide (IBP) with oligomer grade was further used to mediate metal and inorganic material growth. IBP belongs to one kind of functional biomolecules which is capable to function as biomimetic template for material synthesis. Recently, Naik et al extenstively reviewed this field on biomolecules-mediated biomimetic material fabrication (Dickerson et al, 2008). The IBP is a short polypeptide capable to selectively reduce certain inorganic ions from solutions to form nanoparticles. In our work, four types of peptides, NPSSLFRYLPSD (AG4), AYSSGAPPMPPF (AG3), MHGKTQATSGTIQS (MS14) and DRTSTWR (PT2) have been successfully immobilized on aminated polymer surface formed by USAR method and utilized to direct the formation of shape-controlled noble metals (Pt, $\mathrm{Ag}$, and $\mathrm{Au}$ ) micro/nano assemblies on flexible polymer substrates. Triangle, square, sphere, and hexagon as well as superamolecular assembly structure of particles have been observed in these peptide-directed inorganic material synthesis.

Firstly, silver nanoparticles were synthesized by AG3 and AG4 peptides (Zhang et al, 2005, 2006 and 2008). Among various IBPs, AG3 (AYSSGAPPMPPF) is a special peptide sequence that specifically and selectively binds to silver (Naik et al, 2002) in a solution. Inspired by this pioneering research, we immobilized AG3 on aminated PET film surface modified by USAR. As mentioned above, the biomolecule immobilized by USAR could keep its biological activity after immobilization on the aminated surface through electrostatic coordination. After that, the substrate with AG3 immobilized was incubated directly in silver nitrate solution without any other reducing regents added. Silver crystallites with the size being 1-4 $\mu \mathrm{m}$ were found on the film surface after finishing the incubation (Figure 29). The shape of silver microcrystals was diverse including hexagonal, triangle and cubic. Hexagonal and triangular crystallites were commonly observed in such IBP-mediated biomimetic synthesis in a solution, while the cubical shape is seldom reported in the literatures so far. The possible in-plane confinement from the substrate on the nucleation and growth of the crystal may be attributed to the formation of this novel morphology. Although AG3-mediated silver synthesis is effective, the size and morphology of silver crystals are quite broad. In order to narrow the size and morphology distribution for better controlling on this synthesis, we switched from the use of AG3 to AG4, because the latter also expressed strong biomineralization effect on silver (Naik et al, 2002). After similar peptide immobilization and incubation procedures, silver microcrystals with the size being 1-2 $\mu \mathrm{m}$ were found on PET film (Figure 30). Besides these big particles, AFM scanning on the surface (Figure 31) also revealed the existence of some nanoparticles with the size being around $40 \mathrm{~nm}$ on the surface. The shapes of these crystals mainly included triangular and square. Accordingly, based on our results, it seems difficult to obtain uniform silver particles if only peptides were used in this biomimetic synthesis system.

For addressing this challenge, we developed a new approach that combines an organic matrix with IBP to perform such synthesis. This idea is actually inspired by organic matrices in some life systems which can operate as templates for biosynthesis of various materials 


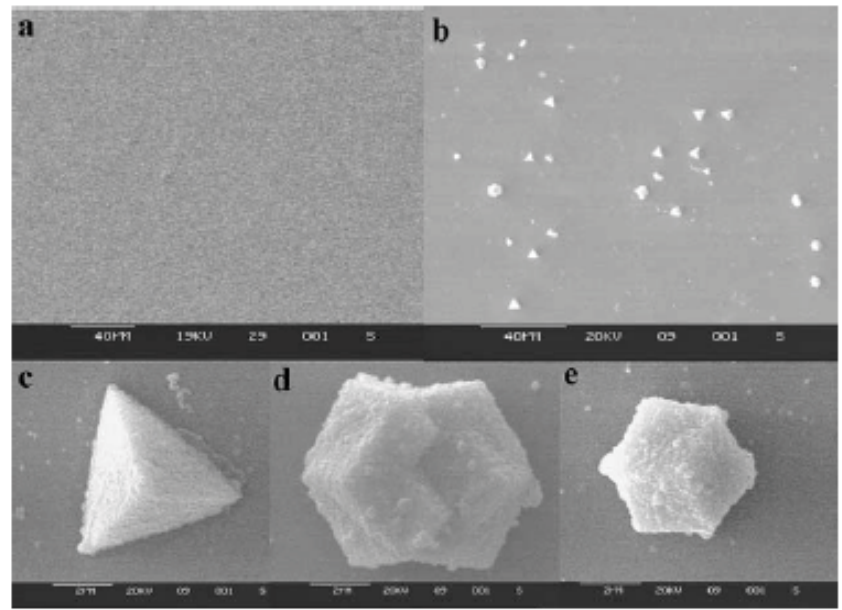

Fig. 29. Silver crystallite morphologies observed under SEM. (a) There was not any crystallite on the blank PET film (control, bar length $40 \mu \mathrm{m}$ ). (b) Various silver crystallites (white dots, bar length $40 \mu \mathrm{m}$ ). (c) Triangular silver crystallite (bar length $2 \mu \mathrm{m})$. (d) Hexagonal silver crystallite (bar length $2 \mu \mathrm{m}$ ). (e) Cubic lattice crystallite (bar length $2 \mu \mathrm{m}$ ). Reproduced from (Zhang et al, 2005). Copyright from Elsevier. Reproduced with permission.
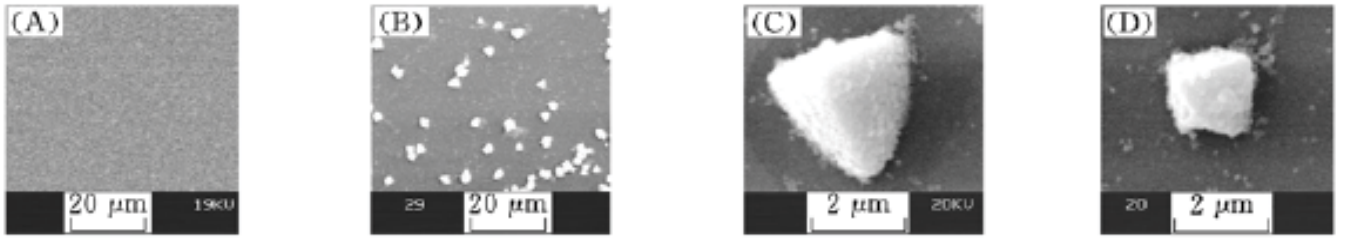

Fig. 30. SEM images of silver particles formed on (A) control (original PET without the peptide AG4 immobilized); (B). PET with AG4 immobilized. (C) and (D) are enlarged images from (B) to show two typical types of silver crystals formed in (B). Reproduced from (Zhang et al, 2006). Copyright from Chemical Journal of Chinese Universities. Reproduced with permission.
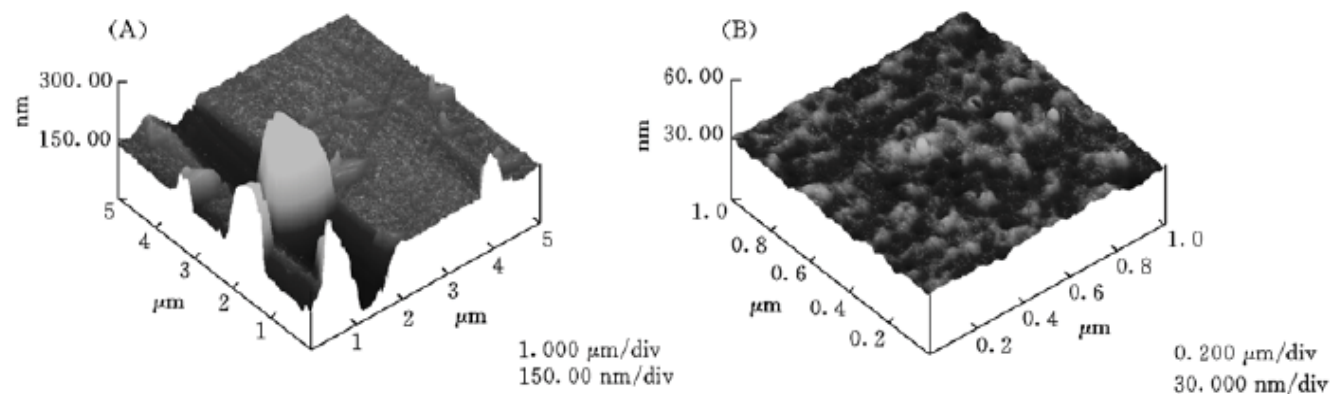

Fig. 31. AFM image on silver particle formed on PET substrate with AG4 immobilized. Reproduced from (Zhang et al, 2006). Copyright from Chemical Journal of Chinese Universities. Reproduced with permission. 
with uniform size and morphology. In our experiment, chitosan was chosen to serve as this kind of matrix. Chitosan is found to function as the main cell wall constitution of some fungi in the nature and made of 3D gridding structures from the self-organization of linear polymers. Such 3D grid texture is expected to provide good host spaces for metal ions and their in situ reduction reaction, and thereby induces the formation of uniform crystals with well controlled size and morphology (Huang et al, 2004). Based on this design, the chitosan and peptide AG4 solutions were firstly mixed well before adding silver nitrate solution. The composition in chitosan is a linear polysaccharide chain consisting of randomly distributed copolymer from D-glucosamine and N-acetyl-D-glucosamine. Therefore, abundant amines along chitosan chain are considered to form coordination bonding with silver ions, which can serve as nucleation sites in the reducing environment provided by AG4. As shown in Figure 29, the resulting silver crystals expressed low polydispersity on morphology and size. This control from chitosan was concentration-dependent. When the concentration of chitosan was in the range of $(0.01 \%-0.1 \%)$, the size change of silver nanoparticles was not obvious, with nanoparticles being about $20 \mathrm{~nm}$ in average size (Figure 32a, b). With further increase of chitosan concentration above $0.1 \%$, the size of silver nanoparticles became smaller with the diameter being around $5 \mathrm{~nm}$ (Figure 32c, d). When the chitosan concentration is changed, the host space volume and nucleation sites for silver growth is correspondingly changed, which could be responsible for the size change of the silver nanoparticles.

Besides silver, we also extended this surface-immobilized IBP-based reaction to other peptide system for other material synthesis. For example, the polypeptide sequence MS14 (MHGKTQATSGTIQS) (Brown et al, 1997 and 2001; Braun et al, 2002; Kulp et al, 2004; Tamerler et al, 2006) was used for biomimetic preparation of gold nanoparticles and their aggregates (Wang et al, 2007). As shown in Figure 33, MS14-mediated reduction of $\mathrm{HAuCl}_{4}$ on PET surface resulted in single-crystallite formed on the surface, presenting hexagonal, triangular and quadrangular morphology with 1-3 $\mu \mathrm{m}$ in size. There were also small spherical particles, approximately $200 \mathrm{~nm}$ in size found on the same substrate. Further effort to combine organic matrix with MS14 for controllable preparation of uniform gold nanoparticles could be suggested as the next future work. After successful preparation of gold and silver crystals, another kind of important noble metal, platinum crystals were also prepared by the peptide PT2(DRTSTWR) (Sarikaya et al, 2003) immobilized on PET surface. Platinum microparticles with the size being 1-2 $\mu \mathrm{m}$ were observed on PT2-functionalized PET surface (Li et al, 2007).

To summarize this section, surface-immobilized peptides have expressed certain potential to in situ reduce and fabricate metal nanocrystals on the film surface. However, some questions and problems must be addressed: 1). Exact biomimetic reaction mechanism must be elucidated; 2). The size and morphology of the resulting crystals must be well controlled. Some organic compounds such as chitosan, PAA have showed certain potential for this controlling ability. In this aspect, we actually found that the size and morphology of particles obtained by surface-immobilized peptide was often much wider than that of particles obtained by same peptide but freely dissolved in a solution. For example, in the case that the synthesis of Pt crystals, instead of the use of surface immobilized PT2, simple use of a mixture solution of free PT2 and $\mathrm{PtCl}_{4}$ could give us much smaller $\mathrm{Pt}$ nanoparticles ranging from $1.5-2 \mathrm{~nm}$. The question that whether the surface immobilization process would affect the biological mediation ability of peptides and nucleation as well as growth process of crystals needs to be clearly anwered. 

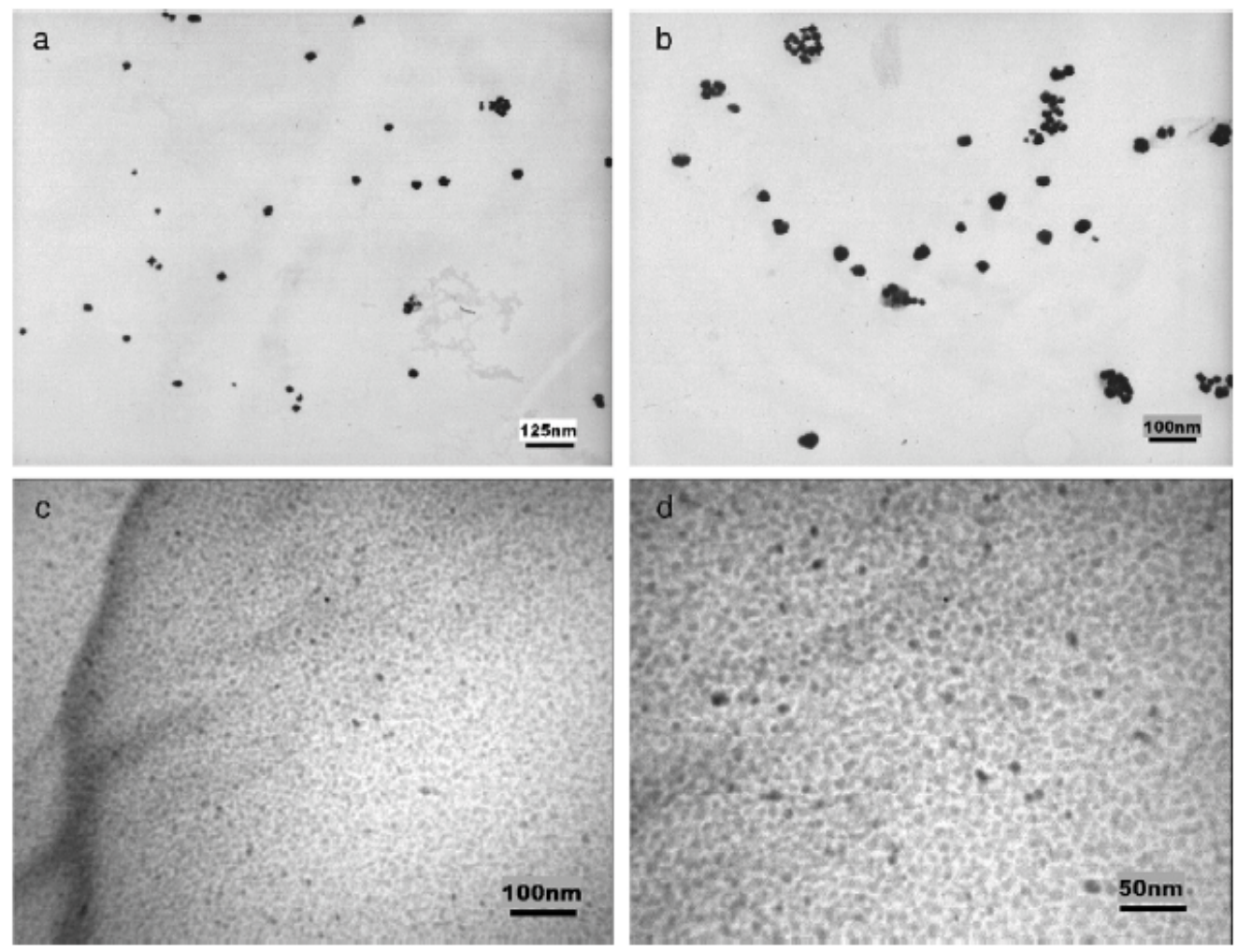

Fig. 32. Transmission Electron Microscope (TEM) images of silver nanoparticles prepared with various chitosan concentrations (wt. \%) without adjusting silver ion concentration. (a) $0.05 \%$; (b) $0.1 \%$; (c) $0.5 \%$; (d) $1 \%$. Reproduced from (Zhang et al, 2008). Copyright from Elsevier. Reproduced with permission.

\subsection{Level 3: Polymer brush-mediated quantum dot formation (Zou et al, 2009)}

Finally, we reached the macromolecular grade for this kind of research. In this aspect, we utilized photo-initiated surface grafting systems to construct large-area functional acidic polymer brush, PAA on arbitrary polymer substrate, and further test for inorganic material growth showed that surface-immobilized polymer brush was able to mediate the formation of $\mathrm{ZnO}$ quantum dots (QD) (Figure 34) and patterning within polymer brush regions. To our best of knowledge, before our report, the formation of $\mathrm{ZnO}$ quantum dot mediated by surface-immobilized polymer brush is never reported. In our experiment, a typical two-step biomimetic procedure was employed: firstly, the film with PAA brush (BOPP-g-PAA) was soaked in zinc chloride solution and achieved a saturated complexation between zinc ions and carboxyl groups in PAA chains. After that, the film with zinc complexed was incubated in HMT solution under suitable temperature and time, and finally, ZnO QDs were in situ formed within PAA brush on BOPP surface, resulting in an organic/inorganic hybrid nanocomposite film (BOPP-g-PAA/ ZnO).

As shown in Figure 35, FE-TEM directly demonstrated the formation of ZnO QD after incubating polymer brush-grafted film in HMT solution. The size of these nanoparticles ranging from 4-5 nm was in the regime of quantum size of $\mathrm{ZnO}$. For single $\mathrm{ZnO} \mathrm{QD}$, a 


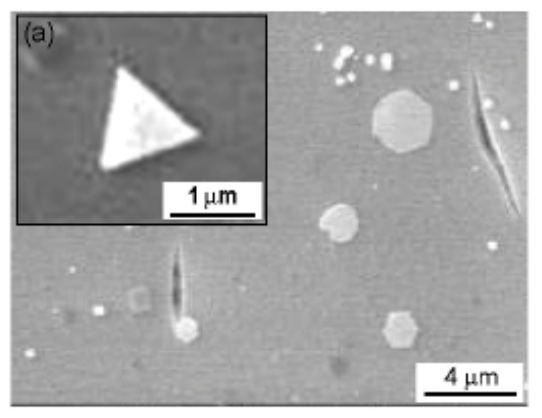

(b)
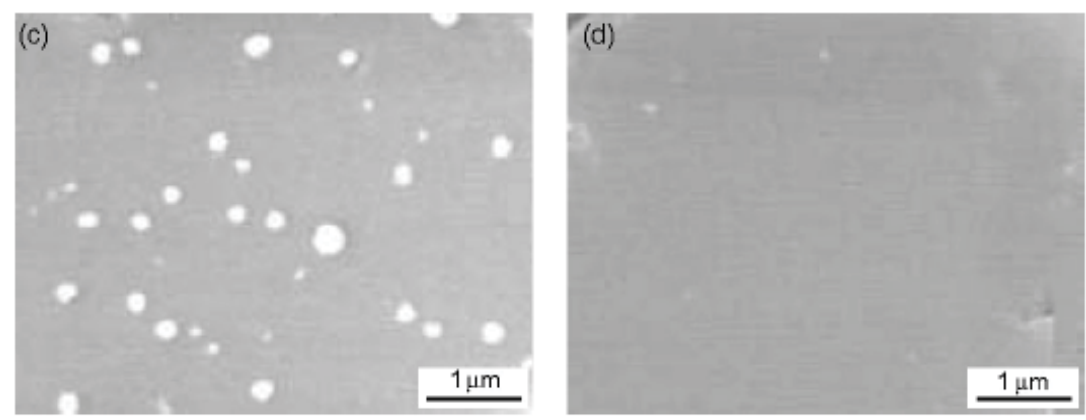

Fig. 33. SEM image and schematic illustration. (a) Various (including triangular, hexagonal and cubic lattice) single crystallites formed on PET and not moved away with $\operatorname{ddH}_{2} \mathrm{O}$. (b) Schematic illustration of (a). (c) Spherical particles deposited on blank PET film. (d) Control: there were no particles on the blank PET film after washing with $\mathrm{ddH}_{2} \mathrm{O}$. Reproduced from (Wang et al, 2007). Copyright from Wiley-VCH Verlag GmbH \& Co. KGaA. Reproduced with permission.

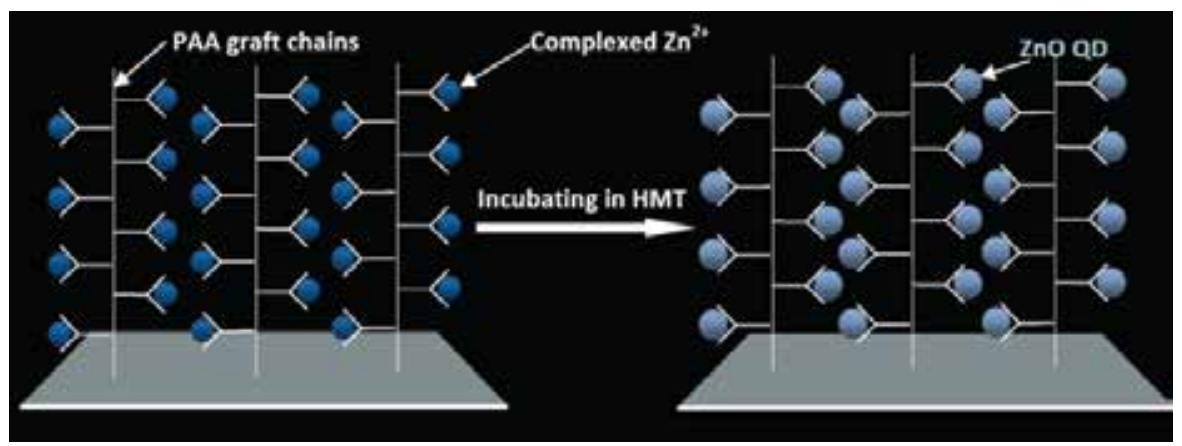

Fig. 34. The schematic process of ZnO QD formation mediated by surface immobilized PAA brushes. PAA chains grafted surface (white tree-like structures) was soaked in $\mathrm{Zn}\left(\mathrm{NO}_{3}\right)_{2}$ aqueous solution $(0.1 \mathrm{M})$ for $1 \mathrm{hr}$ to achieve the complex between carboxylic groups (from grafted PAA chains) and $\mathrm{Zn}^{2+}$ (symbolized by dark blue spheres). After washing, the surface with complexed $\mathrm{Zn}^{2+}$ was incubated in HMT aqueous solution $(0.1 \mathrm{M})$ for 2 days at $90^{\circ} \mathrm{C}$. HMT provided $\mathrm{OH}^{-}$source for the hydrolysis and precipitation of $\mathrm{ZnO}$ to transform complexed $\mathrm{Zn}^{2+}$ to $\mathrm{ZnO}$ QD (light blue spheres). Reproduced from (Zou et al, 2009). Copyright from Wiley-VCH Verlag $\mathrm{GmbH} \& \mathrm{Co}$. KGaA. Reproduced with permission. 

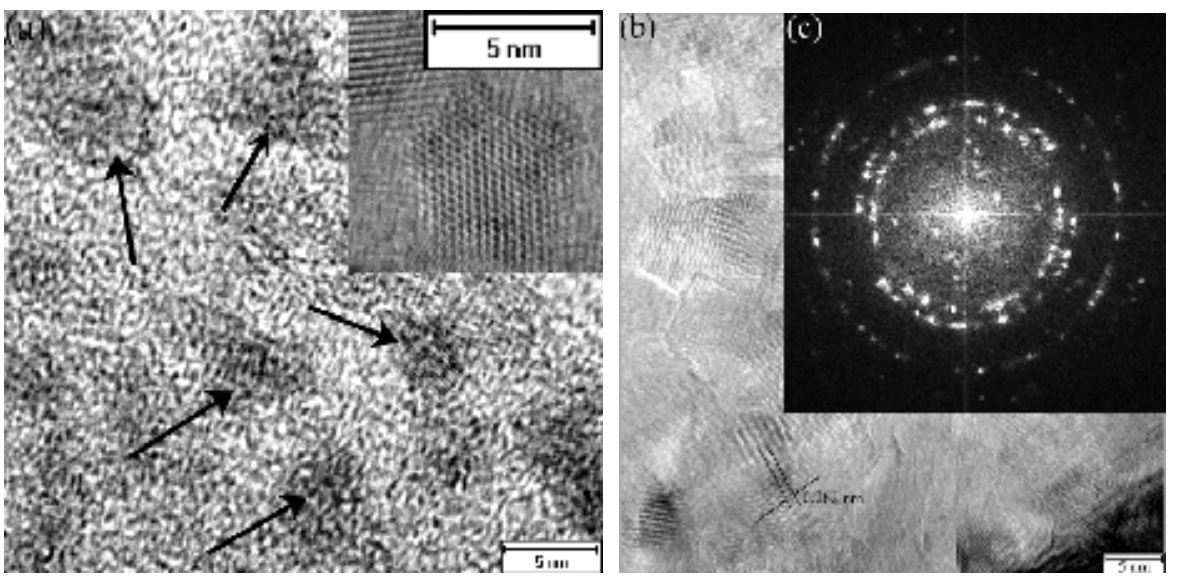

Fig. 35. FE-TEM, High-resolution (HR)-TEM images ( $a, b)$, and SAED pattern (c) of ZnO QD obtained by our method. Inset picture in (a) showed clearly an enlarged ZnO QD with hexagonal wurtize-type lattice. Reproduced from (Zou et al, 2009). Copyright from Wiley$\mathrm{VCH}$ Verlag $\mathrm{GmbH} \& \mathrm{Co}$. KGaA. Reproduced with permission.
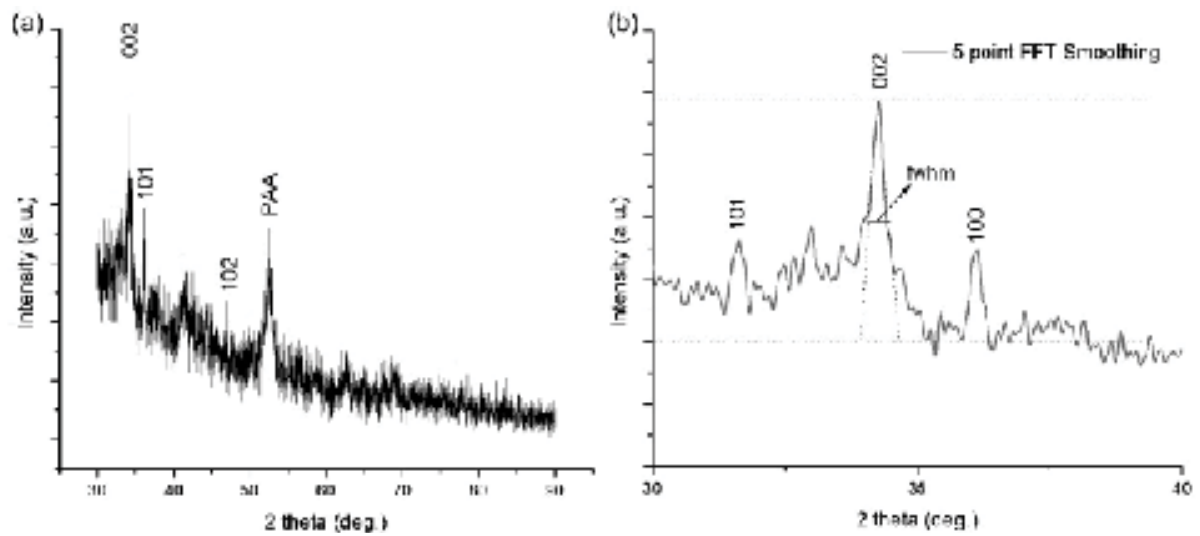

Fig. 36. (a) XRD pattern of BOPP-g-PAA/ZnO QD nanocomposite film and (b) the corresponding fitting curve by 5-point FFT smoothing. The (002), (101) and (102) diffraction patterns could be matched with the standard pattern of a wurtzite $\mathrm{ZnO}$ crystal from the JCPDS database. A preferred orientation was observed to be the (002) orientation. The peak at 528 is ascribed to organic component (PAA). Reproduced from (Zou et al, 2009).

Copyright from Wiley-VCH Verlag GmbH \& Co. KGaA. Reproduced with permission.

uniform hexagonal lattice structure was observed, which signified a single crystal nature without defects. This single crystal nature was also supported by the dotted Selected Area Electron Diffraction (SAED) pattern. (002) direction was found to be the brightest spots in SAED pattern, which revealed that the preferential growth direction for this $\mathrm{ZnO}$ QD was (002) direction (c-axis). The spacing distance between two diffraction planes in single QD was estimated as $0.262 \mathrm{~nm}$ from high-resolution(HR)-TEM image, this value was a typical $\mathrm{d}$ spacing of the (002) planes in a wurtize-type $\mathrm{ZnO}$. This wurtize type was further supported by XRD pattern of BOPP-g-PAA/ZnO film (Figure 36), where three characteristic diffraction 

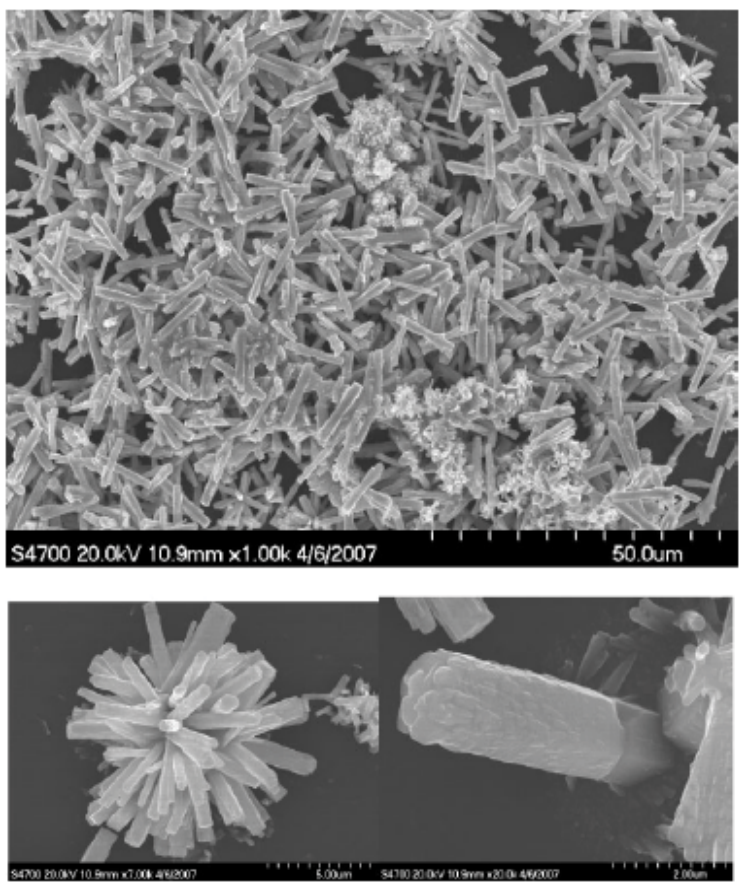

Fig. 37. ZnO particles grew on the surface without PAA biomimetic mediation. BOPP-gPAA film was directly placed into the mixed solution of $\mathrm{Zn}\left(\mathrm{NO}_{3}\right)_{2}$ and HMT $(0.1 \mathrm{M})$. The growth took place at $90^{\circ} \mathrm{C}$ for $48 \mathrm{~h}$. The lower two images are the amplification of the upper image to show two typical kinds of microcrystallites (flowers and rods), which are found on such a surface. Reproduced from (Zou et al, 2009). Copyright from Wiley-VCH Verlag $\mathrm{GmbH} \& \mathrm{Co}$. KGaA. Reproduced with permission.

peaks at (002), (101) and (102) were observed. The highest intensity at (002) plane reflected again that the resulting $\mathrm{ZnO} \mathrm{QD}$ had dominated c-axis direction.

It should be noted that the above-mentioned two-step biomimetic procedure was extremely necessary for the formation of $\mathrm{ZnO}$ QDs. As a control experiment, we deliberately used the same PAA grafts without pre-doping of $\mathrm{Zn}^{2+}$ for incubation in a mixture solution of zinc chloride and HMT. We found that in such a system, the mediation ability of PAA brush was diminished and only large rod-like microcrystallines on the underlying film were obtained (Figure 37). Therefore, we inferred that the effective separation of heterogeneous nucleation and growing process by two-step protocol in our method gave enough time and space to PAA, and thereby the coordination and mediation ability of PAA brushes could be fully reserved. In nature, biofabrication in organisms also often utilize this kind of process where carboxylate groups from biomolecules induce minerals formation within organic matrix through interacting with mineral ions (Estroff, 2008).

The resulting hybrid material from $\mathrm{ZnO} \mathrm{QD}$ and PAA expressed interesting photoluminescence property. Two excitation peaks at 323 and $339 \mathrm{~nm}$ in the spectrum of BOPP-g-PAA/ZnO film were observed (Figure 38a). These excitation peaks took an obvious blue shift compared with the band gap of bulk ZnO crystal at $365 \mathrm{~nm}$ (Abdullah et al, 2003; Mikrajuddin et al, 2002; Dijken et al, 2000; Brus, 1986), indicating the quantum effect of $\mathrm{ZnO}$ particles in the composite film. By using $325 \mathrm{~nm}$ as excitation light, we further investigated 
emission spectra of this composite film. An intensive emission in the range of 350-500 nm was found for the composite film, which was 5-fold higher than those from blank BOPP and BOPP-g-PAA films (Figure 38b). A slight split in the present emission was also observed at the near UV range as 380 and $400 \mathrm{~nm}$. Bound-excitation and vacancy-related transitions are responsible for the UV emission around $390 \mathrm{~nm}$ (Huang et al, 2001; Saito et al, 2002), and the splitting could be attributed to a consequence of the interfacial interaction of $\mathrm{ZnO}$ nanoparticles with the surrounding PAA material (Vollath et al, 2004). This interaction is facilitated by the formation of ester like linkage: $-(\mathrm{C}=\mathrm{O})-\mathrm{O}-(\mathrm{ZnO})$ (Ravindran et al, 2005; Wu et al, 1997; Hu et al, 1991). Our XPS data also gave the evidence for the formation of this kind of ester like linkage. Such ester like linkage could facilitate highly efficient electron
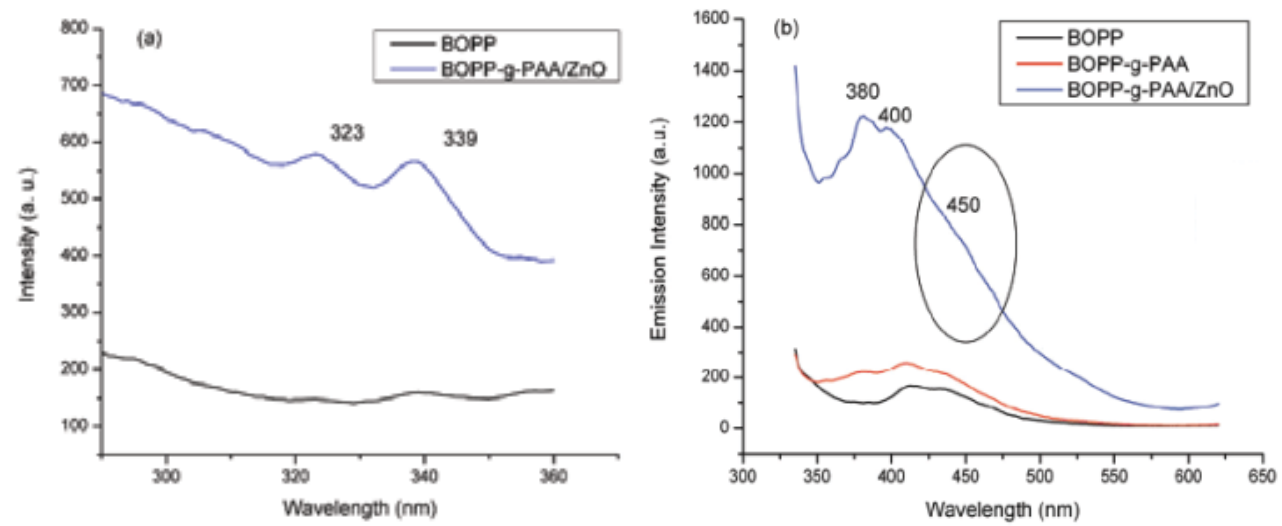

Fig. 38. Excitation (a) and emission spectra (b) of several films. In excitation spectra, the emission wavelength was settled as $385 \mathrm{~nm}$. For BOPP-g-PAA/ZnO, two maximum peaks are at about 323 and $339 \mathrm{~nm}$. In emission spectra, excitation wavelength was $325 \mathrm{~nm}$. Three much higher maximum peaks at about $380 \mathrm{~nm}, 400 \mathrm{~nm}$ and unconspicuous shoulder at 450 $\mathrm{nm}$ were found in the curve for BOPP-g-PAA/ $\mathrm{ZnO}$ composite film. Reproduced from (Zou et al, 2009). Copyright from Wiley-VCH Verlag GmbH \& Co. KGaA. Reproduced with permission.

transportation, resulting in the split on the emission peak. We also noticed that defectrelated transition usually observed at long wavelength like $600 \mathrm{~nm}$ was not observed in our composite film, indicating ZnO QD in our nanocomposite film had high quality with few defects. This judgment was also consistent with HR-TEM observations (Figure 35). The slight shoulder peak at $450 \mathrm{~nm}$ implied that such composite film had the ability for blue light emission devices (Figure 39).

Our hybrid nanocomposite film also possessed excellent optical transparency (Figure 40). Comparing with pristine and BOPP-g-PAA films, the introduction of ZnO QDs in PAA just got a little decrease in the transmittance percent. Actually, high transmittance percent (about $90 \%$ ) in the wavelength between 400 to $900 \mathrm{~nm}$ could be obtained on BOPP-g-PAA/ZnO films. This reason why high transmittance could be kept after ZnO QD introduced was because of a homogeneous dispersion of $\mathrm{ZnO}$ QDs in the polymer matrix with a mean diameter of no more than $5 \mathrm{~nm}$ (as revealed by TEM), therefore possible light scattering and diffraction from large particles and aggregates was effectively eliminated. This highly optical transparence is very important for advanced opto-electrical devices. Another 
important requirement for opto-electrical device is the ability to large scale patterning/array of functional nanocomposite film. This is easily achieved by our method because of the use of photo-initiated polymerization. A photomask which can effectively control irradiation path on the surface was simply applied during the photografting polymerization of AA on the surface. As a result, patterned PAA polymer brush could be obtained (Wang et al, 2005) and then region-selective fabrication of the above $\mathrm{ZnO} Q \mathrm{QD} / \mathrm{PAA}$ nanocomposite hybrid could be easily achieved. As shown in Figure 41, the pattern of PAA/ZnO QD composite film could form on the underlying substrate in a large area $\left(5 \mathrm{~mm}^{2}\right)$, which is especially appealing in macroelectronics (Morin et al, 2007; Forrest, 2004; Henzie et al, 2006; Reuss et al, 2006). The circular nanocomposite film array distributed evenly across the surface with few defects and good size homogenous. The thickness of the composite film was revealed as $2.8 \mu \mathrm{m}$ by AFM profile analysis, only a little higher than pure PAA film before $\mathrm{ZnO}$ incorporation $(2.5 \mu \mathrm{m})$. This fact reflected that instead of simply adding upon PAA layer, the newly formed ZnO QDs mainly filled into the free spaces inside PAA film, because simple adding upon PAA layer would result in an obvious thickness increase.

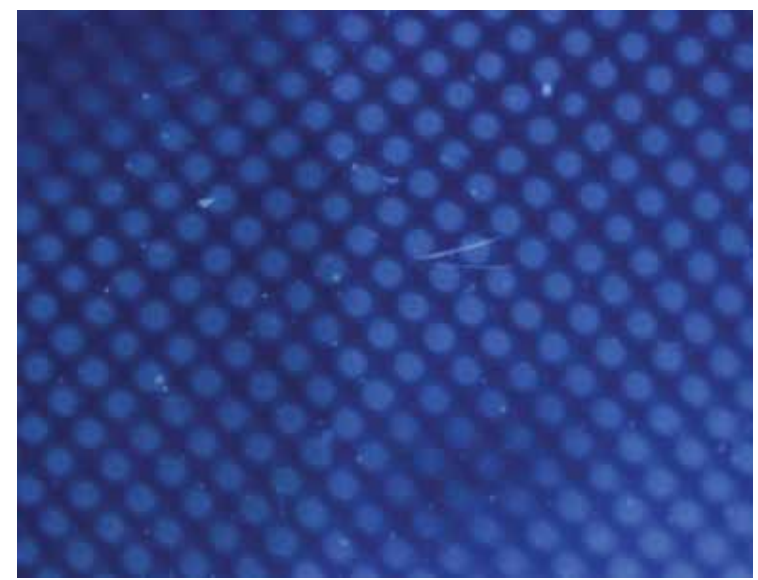

Fig. 39. The fluorescence microscopy image of patterned PAA/ZnO QD arrays on BOPP surface. Using UV exciting light, every circle area as PAA/ZnO QD hybrid represents a composite region emitting blue light. Reproduced from (Zou et al, 2009). Copyright from Wiley-VCH Verlag GmbH \& Co. KGaA. Reproduced with permission.

We also found that PAA was not an exclusive template for this biomimetic fabrication. When PAA was replaced by other similar acidic polymers with the same carboxyl groups, similar enhanced fluorescent emission was observed. In contrast, control experiments showed that switching the use of polymer brush from these carboxyl-contained acidic polymers to ester polymers (i.e. deactivation of carboxyl to ester) would result in very weak fluorescent enhancement or even lowered fluorescent emission compared with pristine polymer films before $\mathrm{ZnO}$ introducing. Such contrast strongly implies that carboxyl group plays a key role for the formation of $\mathrm{ZnO}$ QD in our biomimetic synthesis design, and any acidic polymers having carboxyl groups could become candidates for this synthesis. This finding agrees with the common accepted assumption that carboxyl group (and other functional groups)-based interaction on inorganic surface would be basic and central molecular tailoring mode in biomineralization process. 


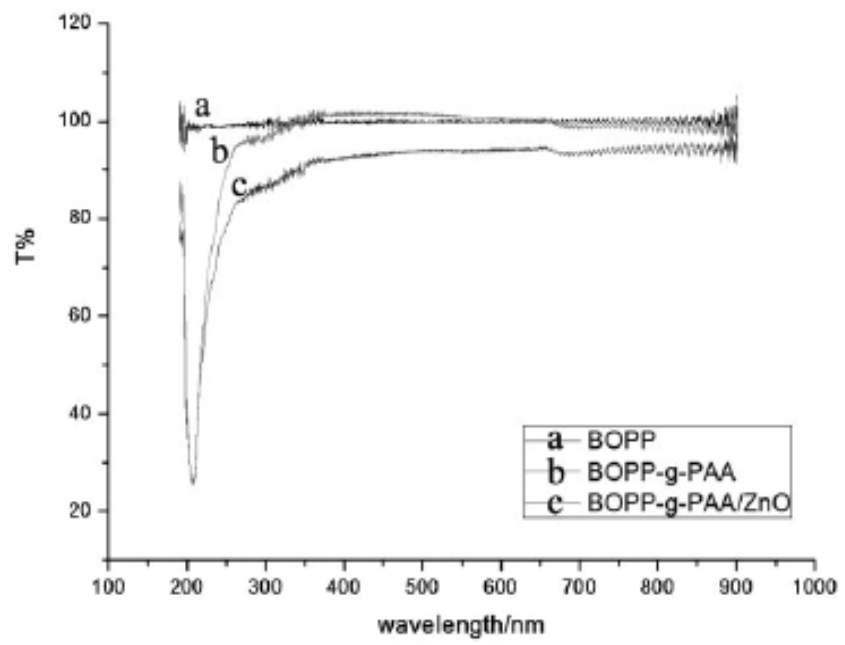

Fig. 40. The UV-vis transmittance spectra of three samples: BOPP (a), BOPP-g-PAA (b) and BOPP-g-PAA/ZnO QD (c). The thickness of BOPP, BOPP-g-PAA and BOPP-g-PAA/ZnO is around 25.7, 28.2, 28.5mm respectively. Reproduced from (Zou et al, 2009). Copyright from Wiley-VCH Verlag GmbH \& Co. KGaA. Reproduced with permission.
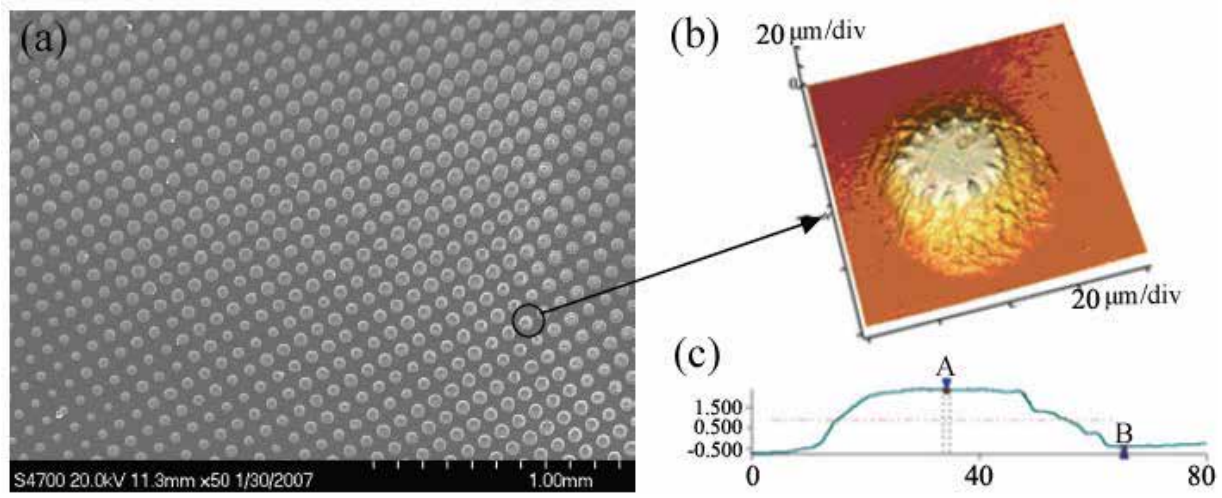

Fig. 41. FE-SEM micrograph (a) and AFM profile (b, c) of patterned PAA/ZnO arrays on BOPP surface. Reproduced from (Zou et al, 2009). Copyright from Wiley-VCH Verlag $\mathrm{GmbH} \& \mathrm{Co}$. KGaA. Reproduced with permission.

In conclusion, our results finally demonstrated that polymer brush grown on polymer substrate for example PAA used in this work had great potential to fabricate $\mathrm{ZnO}$ QD by a biomimetic chemical approach. The resulting ZnO QD was in situ embedded into acidic polymer templates, giving rise to the formation of a hybrid nanocomposite film. Such mediation ability came from surface photografted acidic polymer where the complex interaction between carboxyl groups in PAA and $\mathrm{Zn}^{2+}$ played a key role. Because the $\mathrm{ZnO}$ QD was homogeneously dispersed in polymer matrix, the final polymer/ZnO QD nanocomposite was a highly transparent inorganic/organic hybrid film which could be constructed on arbitrary inorganic and organic substrates. Patterning of such nanocomposite film could be also very easily achieved by simply applying a photomask in this 
photopolymerization process. The resulting patternable array of nanocomposite film had good quality and large area. Possible next work along this direction may be suggested to explore other kinds of polymer brushes for biomimetic material fabrication, since the field about polymer brush-mediated biomineralization, especially non-calcium material biomineralization is still in the early developing stage.

\section{Summary and outlook}

All of the above research results could produce inorganic and metal oxide micropatterning as well as organic/inorganic hybrid film array on soft matter surface, which would contribute to the current fast development of flexible polymer-based biomedical, energetical and optical sensors. We believe that our systematical explorations on non-calcium inorganic material fabrication mediated by surface-immobilized organic molecular template could find its potential in the fundamental and engineering researches on polymer-supported advanced technologies, biomineralization, inorganic nanocrystals and macroelectronics realms. Obviously, the future development direction in this field should be located at extensive investigation and engineering of the mediation ability of various functional groups, polypeptide, polymer brush immobilized on surfaces for the inorganic and metal materials fabrication and patterning achieving.

\section{References}

Abdullah, M.; Lenggoro, I. W.; Okuyama, K.; Shi, F. G. (2003). In Situ Synthesis of Polymer Nanocomposite Electrolytes Emitting a High Luminescence with a Tunable Wavelength. J. Phys. Chem. B 107, 1957-1961, ISSN 1089-5647.

Brown, S. (1997). Metal-recognition by repeating polypeptides. Nat. Biotechnol. 15, 269-272.

Brown, S. (2001). Protein-mediated particle assembly. Nano Lett. 1, 391-394, ISSN 1530-6992.

Brus, L. (1986). Electronic wave functions in semiconductor clusters: experiment and theory. J. Phys. Chem. 90, 2555-2560, ISSN 0022-3654.

Bamford, C. H.; AILamee, G. K. (1994). Studies in polymer surface functionalization and grafting for biomedical and other applications. Polymer 35, 2844-2852, ISSN 0032 3861.

Bruice, T. C.; Fife, T. H. (1961). The nature of neighboring hydroxyl group assistance in the alkaline hydrolysis of the ester bond. Tetrahedron Lett. 2, 8, 263-266, ISSN 0040-4039.

Bruice, T. C.; Fife, T. H. (1962). Hydroxyl Group Catalysis. III. ${ }^{1}$ The Nature of Neighboring Hydroxyl Group Assistance in the Alkaline Hydrolysis of the Ester Bond. J. Am. Chem. Soc. 84, 1973-1979, ISSN 0002-7863.

Braun, R.; Sarikaya, M.; Schulten, K. (2002). Genetically engineered gold-binding polypeptides: Structure prediction and molecular dynamics. J. Biomater. Sci. Polym. Ed. 13, 747-757, ISSN 0920-5063.

Chaudhary, S.; Kim, H. J.; Singh, K. V.; Ozkan, M. (2004). Fluorescence Microscopy Visualization of Single-Walled Carbon Nanotubes Using Semiconductor Nanocrystals. Nano Lett. 4, 2415-2419, ISSN 1530-6992.

Coffman, E. A.; Melechko, A. V.; Allison, D. P.; Simpson, M. L.; Doktycz, M. J. (2004). Surface Patterning of Silica Nanostructures Using Bio-Inspired Templates and 
Directed Synthesis. Langmuir 20, 20, 8431-8436, ISSN 0743-7463 (print), 1520-5827 (online)

Crookes-Goodson, W.; Slocik, J. J. M.; Naik, R. R. (2008). Bio-directed Synthesis and Assembly of Nanomaterials. Chem. Soc. Rev. 37, 11, 2403-2412, ISSN 0306-0012 (print), 1460-4744 (online)

Dutschke, A.; Diegelmann, C.; Löbmann, P. (2003). Nucleation and Growth of $\mathrm{TiO}_{2}$ Thin Films on Modified Polystyrene Surfaces. Chem. Mater. 15, 3501-3506, ISSN 08974756.

Dickerson, M. B.; Sandhage, K. H.; Naik, R. R. (2008). Protein- and Peptide-Directed Syntheses of Inorganic Materials. Chem. Rev. 108, 4935-4978, ISSN 0009-2665.

van Dijken, A.; Meulenkamp, E. A.; Vanmaekelbergh, D.; Meijerink, A. (2000). Identification of the transition responsible for the visible emission in $\mathrm{ZnO}$ using quantum size effects. J. Lumin. 90, 123, ISSN 0022-2313.

Estroff, L. A. (2008). Introduction: Biomineralization. Chem. Rev. 11, 4329-4331. This is a speical issue of Chemical Reviews, including 23 papers ranging from pp. 4329 to pp. 4978. ISSN 0009-2665.

Forrest, S. R. (2004). The path to ubiquitous and low-cost organic electronic appliances on plastic. Nature 428, 911-918, ISSN 0028-0836.

Gan, S. H.; Yang, P.; Yang, W. T. (2010). Interface-directed Sol-gel: Direct fabrication of the Covalently Attached Ultraflat Inorganic Oxide Pattern on Functionalized Plastics. Sci. China-Chem. 53, 1, 173-182, ISSN 1674-7291 (print), 1862-2771 (online)

Gan, S. H.; Yang, P.; Yang, W. T. (2009). Photoactivation of Alkyl C-H and Silanization: A Simple and General Route to Prepare High-Density Primary Amines on Inert Polymer Surfaces for Protein Immobilization. Biomacromolecules 10, 5, 1238-1243, ISSN 1525-7797.

House, D. A. (1962). Kinetics and Mechanism of Oxidations by Peroxydisulfate. Chem. Rev. 62, 185-203, ISSN 0009-2665.

Henzie, J.; Barton, J. E.; Stender, C. L.; Odom, T. W. (2006). Large-area nanoscale patterning: Chemistry meets fabrication. Acc. Chem. Res. 39, 249-257, ISSN 0001-4842.

Huang, M. H.; Mao, S.; Feick, H.; Yan, H.; Wu, Y.; Kind, H.; Weber, E.; Russo, R.; Yang, P. (2001). Room-Temperature Ultraviolet Nanowire Nanolasers. Science 292, 18971899, ISSN 1095-9203.

Hozumi, A.; Masuda, T.; Sugimura, H.; Kameyama, T. (2003). Oxide Nanoskin Formed on Poly(methyl methacrylate). Langmuir 19, 18, 7573-7579, ISSN 0743-7463.

Hu, H.; Saniger, J.; Garcia-Alejandre, J.; Castaňo, V. M. (1991). Fourier transform infrared spectroscopy studies of the reaction between polyacrylic acid and metal oxides. Mater. Lett. 12, 281-285, ISSN 0167-577X.

Hsu, J. W. P.; Tian, Z. R.; Simmons, N. C.; Matzke, C. M.; Voigt, J. A.; Liu, J. (2005). Directed spatial organization of zinc oxide nanorods. Nano Lett. 5, 83-86, ISSN 1530-6992.

Huang, H. Z.; Yuan, Q.; Yang, X. (2004). Preparation and characterization of metal-chitosan nanocomposites. Colloids Surf., B Biointerfaces 39, 31-37, ISSN 0927-7765.

Karayannidis, G. P.; Achilias, D. S. (2007). Chemical recycling of poly(ethylene terephthalate). Macromol. Mater. Eng. 292, 128-146, ISSN $1438-7492$ (print), 14392054 (online). 
Kumar, S.; Ito, T.; Yanagihara, Y.; Oaki, Y.; Nishimura, T.; Kato, T. (2010). Crystallization of Unidirectionally Oriented Fibrous Calcium Carbonate on Thermo-Responsive Polymer Brush Matrices. CrystEngComm 12, 2021-2024, ISSN 1466-8033.

Kang, J. S.; Kang, H. S.; Pang, S. S.; Shim, E. S.; Lee, S. Y. (2003). Investigation on the origin of green luminescence from laser-ablated $\mathrm{ZnO}$ thin film. Thin Solid Films 443, 1, 5-8, ISSN 0040-6090.

Kim, D. J.; Lee, K. B.; Chi, Y. S.; Kim, W. J.; Paik, H. J.; Choi, I. S. (2004). Biomimetic Formation of Silica Thin Films by Surface-Initiated Polymerization of 2(Dimethylamino)ethyl Methacrylate and Silicic Acid. Langmuir, 20, 19, 7904-7906, ISSN 0743-7463 (print), 1520-5827 (online)

Kim, D. J.; Lee, K. B.; Lee, T. G.; Shon, H. K.; Kim, W. J.; Paik, H. J.; Choi, I. S. (2005). Biomimetic Micropatterning of Silica by Surface-Initiated Polymerization and Microcontact Printing. Small 1, 10, 992 - 996, ISSN 1613-6810 (print), 1613-6829 (online)

Kulp, III J. L.; Sarikaya, M.; Evans, J. S. (2004). Molecular characterization of a prokaryotic polypeptide sequence that catalyzes Au crystal formation. J. Mater. Chem. 14, 23252332, ISSN 0959-9428 (print), 1364-5501 (online).

Li. F.; Wang, Z.; Chen, J. C.; Yang, W. T. (2007). Exploiting Preparation of Platinum Nanocrystals with Peptide PT2 (DRTSTWR). Acta Chimica Sinica 65, 22, 2644-2648, ISSN 0567-7351

Morin, S. A.; Amos, F. F.; Jin, S. (2007). Biomimetic Assembly of Zinc Oxide Nanorods onto Flexible Polymers. J. Am. Chem. Soc. 129, 45, 13776-13777, ISSN 0002-7863 (print), 1520-5126 (online)

Masuda, Y.; Kinoshita, N.; Sato, F.; Koumoto, K. (2006). Site-Selective Deposition and Morphology Control of UV- and Visible-Light-Emitting ZnO Crystals. Cryst. Growth Design 6, 75-78, ISSN 1528-7483.

Mikrajuddin, A.; Lenggoro, I. W.; Okuyama, K.; Shi, F. G. (2002). Luminescent Polymer Electrolytes. Prepared by Growing $\mathrm{ZnO}$ Nanoparticles in the Matrix of Polyethylene Glycol. J. Electrochem. Soc. 149, H107-H112, ISSN 0013-4651 (print), 1945-7111 (online).

Masuda, Y.; Sugiyama, T.; Seo, W. S.; Koumoto, K. (2003). Deposition Mechanism of Anatase $\mathrm{TiO}_{2}$ on Self-Assembled Monolayers from an Aqueous Solution. Chem. Mater. 15, 2469-2476, ISSN 0897-4756.

Naik, R. R.; Stringer, S. J.; Agarwal, G.; Jones, S. E.; Stone, M. O. (2002). Biomimetic synthesis and patterning of silver nanoparticles. Nat. Mater. 1, 169-172, ISSN 1476-1122 (print), 1476-4660 (online)

Pizem, H.; Gershevitz, O.; Goffer, Y.; Frimer, A. A.; Sukenik, C. N.; Sampathkumaran, U.; Milhet, X.; Mcllwain, A.; De Guire, M. R.; Meador, M. A. B.; Sutter, J. K. (2005). Titania Deposition on PMR-15. Chem. Mater. 17, 3205-3213, ISSN 0897-4756.

Reuss, R. H.; Hopper, D. G.; Park, J. G. (2006). Macroelectronics. MRS Bull. 31, 447-450, ISSN 0883-7694.

Ravindran, S.; Ozkan, C. S. (2005). Self-assembly of ZnO nanoparticles to electrostatic coordination sites of functionalized carbon nanotubes. Nanotechnology 16, 11301136, ISSN 0957-4484 (print), 1361-6528 (online) 
Sarikaya. M.; Candan, T.; Alexk, Y. (2003). Molecular biomimetics: nanotechnology through biology. Nat. Mater. 9, 577-585, ISSN 1476-1122 (print), 1476-4660 (online)

Saito, N.; Haneda, H.; Seo, W. S.; Koumoto, K. (2001). Selective deposition of ZnF(OH) on self-assembled monolayers in $\mathrm{Zn}-\mathrm{NH}_{4} \mathrm{~F}$ aqueous solutions for micropatterning of zinc oxide. Langmuir 17, 1461-1469, ISSN 0743-7463.

Saito, N.; Haneda, H.; Sekiguchi, T.; Ohashi, N.; Sakaguchi, I.; Koumoto, K. (2002). LowTemperature Fabrication of Light-Emitting Zinc Oxide Micropatterns Using SelfAssembled Monolayers. Adv. Mater. 14, 418-421, ISSN 1521-4095.

Strohm, H.; Löbmann, P. (2005). Liquid-Phase Deposition of TiO2 on Polystyrene Latex Particles Functionalized by the Adsorption of Polyelectrolytes. Chem. Mater. 17, 6772-6780, ISSN 0897-4756.

Schmeer, G.; Riembauer, S.; Barthel, J. (1990). The influence of hydrophobic solvation on the alkaline hydrolysis of ethyl esters of polar substituted 2-methylpropionic acids in water. J. Solution Chem. 19, 1175-1189, ISSN 0095-9782 (print), 1572-8927 (online)

Tugulu, S.; Harms, M.; Fricke, M.; Volkmer, D.; Klok, H. A. (2006). Polymer Brushes as Ionotropic Matrices for the Directed Fabrication of Microstructured Calcite Thin Films. Angew. Chem., Int. Ed. 45, 44, 7458-7461, ISSN 1433-7851 (print), 1521-3773 (online)

Tamerler, C.; Oren, E. E.; Duman, M.; Venkatasubramanian, E.; Sarikaya, M. (2006). Adsorption Kinetics of an Engineered Gold Binding Peptide by Surface Plasmon Resonance Spectroscopy and a Quartz Crystal Microbalance. Langmuir 22, 77127718, ISSN 0743-7463.

Vollath, D.; Szabó, D. V.; Schlabach, S. (2004). Oxide/Polymer Nanocomposites as New Luminescent Materials. J. Nanoparticle Res. 6, 181-191, ISSN 1572-896X.

Wang, Z.; Chen, J. C.; Yang, P.; Yang, W. T. (2007) Biomimetic Synthesis of Gold Nanoparticles and Their aggregates using a Polypeptide Sequence. Appl. Organometal. Chem. 21, 8, 645-651, ISSN 0268-2605.

Wilmarth, W. K.; Haim, A. (1962). in Peroxide Reaction Mechanism, (Ed: Edwards, J. O.), Wiley Interscience, New York, p. 175.

Wu, H. S.; Jone, H. C. (1997). Kinetic Study and Characterization of Poly(acrylic acid) and Metal Oxide. Ind. Eng. Chem. Res. 36, 2006-2011, ISSN 0888-5885 (print), 1520-5045 (online)

Wang, Y.; Lai, H. H.; Bachman, M.; Sims, C. E.; Li, G. P.; Allbritton, N. L. (2005). Covalent micropatterning of poly(dimethylsiloxane) by photografting through a mask. Anal. Chem. 77, 7539-7546, ISSN 0003-2700.

$\mathrm{Wu}, \quad$ X. L.; Siu, G. G.; Fu, C. L.; Ong, H. C. (2001). Photoluminescence and cathodoluminescence studies of stoichiometric and oxygen-deficient $\mathrm{ZnO}$ films. Appl. Phys. Lett. 78, 2285-2287, ISSN 0003-6951.

Xu, A. W.; Ma, Y.; Cölfen, H. (2007). Biomimetic Mineralization. J. Mater. Chem. 17, 5, 415449, ISSN 0959-9428 (print), 1364-5501 (online)

Yang, P.; Deng, J. Y.; Yang, W. T. (2003). Confined photo-catalytic oxidation: a fast surface hydrophilic modification method for polymeric materials. Polymer 44, 23, 71577164, ISSN 0032-3861. 
Yang, P.; Xie, J.; Yang, W. T. (2006). A Simple Method to Fabricate a Conductive Polymer Micropattern on an Organic Polymer Substrate. Macro. Rapid. Commn. 27, 418-423, ISSN 1521-3927.

Yang, P.; Yang, M.; Zou, S. L.; Xie, J. Y.; Yang, W. T. (2007). Positive and Negative TiO2 Micropatterns on Organic Polymer Substrates. J. Am. Chem. Soc. 129, 6, 1541-1552, ISSN 0002-7863 (print), 1520-5126 (online)

Yang, P.; Zhang, X.; Yang, B.; Zhao, H.; Chen, J.; Yang, W. T. (2005). Facile Preparation of a Patterned, Aminated Polymer Surface by UV-Light-Induced Surface Aminolysis. Adv. Funct. Mater. 15, 1415-1425, ISSN 1616-301X.

Yang, P.; Zhang, X.; Xie, J.; Chen, J.; Yang, W. T. (2006). Micro/Nanoscale Well and Channel Fabrication on Organic Polymer Substrates via a Combination of Photochemical and Alkaline Hydrolysis Etchings. Biomacromolecules 2006, 7, 10, 2770-2775, ISSN 1525-7797.

Yang, P.; Zou, S. L.; Yang, W. T. (2008). Positive and Negative ZnO Micropatterning on Functionatized Polymer Surfaces. Small 4, 9, 1527-1536, ISSN 1613-6810 (print), 1613-6829 (online)

Zou, S; Bai, HD; Yang, P; Yang, WT. (2009) A Biomimetic Chemical Approach to Facile Preparation of Large-Area, Patterned, ZnO Quantum Dot/Polymer Nanocomposites on Flexible Plastics. Macro. Chem. Phys. 210, 18, 1519-1527, ISSN 1022-1352 (print), 1521-3935 (online)

Zhang, X.; Chen, J. C.; Yang, P.; Yang, W. T. (2005). Biomimetic Synthesis Silver Crystallite by Peptide AYSSGAPPMPPF Immobilized on PET film in vitro. J. Inorg. Biochem. 99, 8, 1692-1697, ISSN 0162-0134

Zhang, X.; Chen, J. C.; Yang, P.; Yang, W. T. (2006). Silver Biomimetic Mineralization in vitro with Inorganic-binding peptide AG4 (NPSSLFRYLPSD) Immobilized on PET Film. Chem. J. Chinese Universities 27, 1, 5-8, ISSN 0251-0790

Zubkov, T.; Lucassen, A. B.; Freeman, D.; Feldman, Y.; Cohen, S. R.; Evmenenko, G.; Dutta, P.; van der Boom, M. E. (2005). Photoinduced deprotection and $\mathrm{ZnO}$ patterning of hydroxyl-terminated siloxane-based monolayers. J. Phys. Chem. B 109, 14144-14153, ISSN 1089-5647.

Zhang, X.; Yang, P.; Yang, W. T.; Chen, J. C. (2008).The Bio-inspired Approach to Controllable Biomimetic Synthesis of Silver Nanoparticles in Organic Matrix of Chitosan and Silver-binding Peptide (NPSSLFRYLPSD). Mater. Sci. Eng. CBiomimetic Supramol. Syst. 28, 2, 237-242, ISSN 0928-4931 


\title{
Biomimetic Applications of Metal Systems Supported by Scorpionates
}

\author{
Maura Pellei and Carlo Santini \\ University of Camerino, School of Science and Technology - Chemistry Division \\ Via S. Agostino 1, 62032 Camerino, Macerata,
}

Italy

\section{Introduction}

It is well established that a number of metal ions are essential to life (Fraústo da Silva \& Williams, 2001; Bertini et al., 2006). A major determinant of their functional relevance in living systems is that a substantial fraction of enzymes requires metal for its catalytic activity. A wide variety of metal-dependent enzymes is found in nature which acts in fundamental biological processes, including photosynthesis, respiration and nitrose fixation (Bertini et al., 2001). Over the years, a wealth of knowledge on enzymes has been accumulated, including data on three-dimensional structures, kinetic and biochemical properties, and reaction mechanisms (Andreini et al., 2008). As metalloprotein chemistry is governed by the environment close to the metal center(s), a fertile field of investigation is concerned with the preparation of low molecular weight complexes that mimic the structural or functional features of protein active sites. The synthetic analogue or model approach provides insights into bioinorganic systems through the synthesis and study of closely related 'model' compounds. It garners structural, electronic, spectroscopic, and chemical information crucial to a complete understanding of enzyme behavior. Scorpionates have been extensively used in biomimetic chemistry as "spectator ligands" but are not directly involved in the metal-based reactivity. A common approach for obtaining synthetic analogs of the type $[\{X Y Z\} M-L]\left(M=\right.$ metal; $\mathrm{L}=\mathrm{OH}, \mathrm{H}_{2} \mathrm{O}, \mathrm{Cys}$, etc. $)$ involves the application of tridentate ligands which incorporate the requisite $X, Y$, and $Z$ donor groups to mimic the protein residues that bind metals at the active site. In particular, tripodal ligands in which the $X, Y$, and $Z$ groups are attached to a common tetrahedral (or trigonal pyramidal) center have proven to be of particular benefit for several reasons: a) tripodal ligands enforce the "facial" binding that is required to create a tetrahedral metal center, b) tripodal ligands typically possess only a single relevant binding conformation, c) as a consequence of the directional nature of tripodal ligands, it is possible to incorporate substituents that directly influence the steric environment about the metal center, and d) the substituents on these ligands can be readily modified to provide a mean to influence both the size of the coordination pocket and the electronic properties of the metal center. One of the most versatile tripodal ligand typology that can be utilized for biomimetic purposes is represented by the scorpionates. The azole rings of these ligands can in fact be considered as good models of the histidine residues of proteins, and their spatial disposition provides the steric arrangements found in many active sites. In addition, from a synthetic point of view, 
the steric and electronic properties of these ligands can be easily modulated by placing appropriate substituents in close proximity of the $\mathrm{N}$ donor atoms. In recent years, complexes of scorpionate ligands were successfully used to mimic the activity of enzymes containing metals. With this ever-growing wealth of scorpionate-supported coordination and bioinorganic chemistry, this issue would provide a valuable resource for chemists and biologist, clarifying the properties of metal complexes with scorpionate ligands with biological activity or used as models for active sites of enzymes and proteins.

This Chapter analyzes the chemical diversity exhibited by some metal complexes (such as copper and zinc complexes) supported by scorpionate ligands and the overall progress on synthetic analogs of enzyme centers, focusing primarily on systems in which coordination spheres contain poly(pyrazolyl)borate ligands.

\section{Poly(pyrazolyl)borate ligands}

Since poly(pyrazolyl)borate ligands were discovered in 1966 by Trofimenko (Trofimenko, 1966), their coordination chemistry has been extensively developed with particular interest arising out of the ability of this class of ligands to modify or control the steric and electronic environment about the metal center by variation of the pyrazolyl groups (Trofimenko, 1999). Apart Trofimenko's papers (Trofimenko, 1971; Trofimenko, 1972; Niedenzu \& Trofimenko, 1986; Trofimenko, 1986; Trofimenko, 1993), a number of reviews and chapters were devoted to chemistry of poly(pyrazolyl)borate ligands (Edelmann, 2001; Ward et al., 2001; Webster \& Hall, 2003; Pettinari \& Santini, 2004; Dias \& Fianchini, 2007; Spicer \& Reglinski, 2009; Pellei et al., 2010; Santini et al., 2010) and related metal complexes. In 2003 the scorpionate ligands and their father Trofimenko were guests of honor at a symposium to celebrate 35 years of chemistry with poly(pyrazolyl)borates and related ligands (Ritter, 2003). The special issues 2-3 (Vol. 23, 2004) of Polyhedron were dedicated to this topic, the first paper being presented by Trofimenko on development of scorpionate ligands system from its genesis (Trofimenko, 2004). In 2008 a book dedicated to Swiatoslaw Trofimenko has been published by the Imperial College Press (Pettinari, 2008). The special issue 12 (Vol. 362, 2009) of Inorganica Chimica Acta was dedicated to Dr. Swiatoslaw Trofimenko to honour and remember his strong contribution to chemistry and 40 years of work accomplished with the poly(pyrazolyl)borates and related tripodal ligands.

\subsection{Poly(pyrazolyl)borate properties}

The fundamental feature in all poly(pyrazolyl)borate complexes is the six-membered ring within a more general structure $\mathrm{RR}^{\prime} \mathrm{B}(\mu-\mathrm{pz})_{2} \mathrm{M}(\mathrm{L})_{\mathrm{n}}$ (Fig. 1). For these ligands and their constituents we adopt an extensive nomenclature based on the abbreviation proposed in ref. (Pellei et al., 2010).

Because of the bond angles and distances involved, the $\mathrm{B}(\mu-\mathrm{pz})_{2} \mathrm{M}$ ring has almost nearly a boat conformation. In Fig. $1 a, R$ and $R^{\prime}$ are different: the pseudoequatorial $R^{\prime}$ is pointing away from the metal roughly along the B-M axis, but the pseudoaxial $\mathrm{R}$ is directed towards the metal, and may bond to it, interact with it, or simply screen it towards other ligands. $\mathrm{R}$ may be $\mathrm{H}$, alkyl, aryl, OR, SR, $\mathrm{NMe}_{2}$ or another pyrazolyl group with unspecified substituents $\left(\mathrm{pz}^{\mathrm{x}}\right)$. It was this feature that prompted Trofimenko to coin the term "scorpionates" for polypyrazolylborates, as the coordination behavior of the RR'B $(\mu-p z)_{2}$ ligands closely resembles the hunting habits of a scorpion: this creature grabs its prey with 
two identical claws (coordination of $\mathrm{M}$ through the two 2-N atoms of the $\mathrm{B}(\mu-\mathrm{pz})_{2}$ groups), and then may, or may not, proceed to sting it with its overarching tail (the $R^{\prime}$ group).

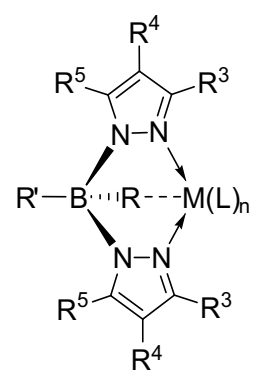

(a)

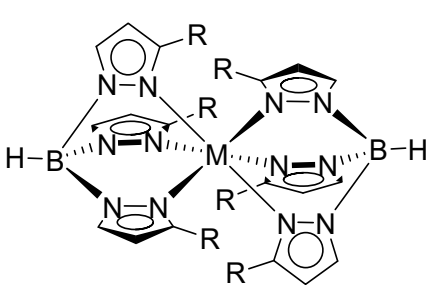

(b)

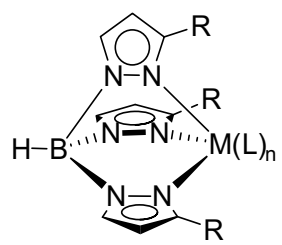

(c)

Fig. 1. General structure of: a) poly(pyrazolyl)borate complexes $R^{\prime} B\left(p z^{R 3, R 4, R 5}{ }_{2} M(L) n\right.$; b) $\left[\mathrm{HB}\left(\mathrm{pz}^{\mathrm{R}}\right)_{3}\right]_{2} \mathrm{M}$ complexes; c) $\left[\mathrm{HB}\left(\mathrm{pz}^{\mathrm{R}}\right)_{3}\right] \mathrm{M}(\mathrm{L})_{\mathrm{n}}$ complexes

Tris(pyrazolyl)borates generally coordinate as tridentate ligands $\kappa^{3}-\mathrm{N}_{,} \mathrm{N}^{\prime}, \mathrm{N}^{\prime \prime}$ (Fig. 1b and 1c), through three nitrogen atoms of the pyrazole rings thereby providing effective steric shielding of the metal center. In a $\mathrm{M}\left[\mathrm{HB}\left(\mathrm{pz}^{\mathrm{R}}\right)_{3}\right]$ fragment the $\mathrm{R}$ substituents protrude in space past the metal, enveloping it, and forming a protective pocket of varying size and shape. The evaluation of the size of the variously substituted $\left[\mathrm{HB}\left(\mathrm{pz}^{\mathrm{R}}\right)_{3}\right]^{-}$ligands is more important for developing an understanding the influence of pyrazolyl ring substituents on the chemistry of their metal complexes. Ligand size can be evaluated by the concept of cone angle $(\theta)$, which was originally introduced for phosphine ligands (Tolman, 1977). The smaller the cone angle, and the larger the wedge angle, the easier it is for other ligands coordination to the metal. Because of this feature, the proper choice of 3-R substituents does adjust the steric accessibility of the coordinated metal, in this fashion controlling the coordination chemistry of the $\left[\mathrm{HB}\left(\mathrm{pz}^{\mathrm{R}}\right)_{3}\right] \mathrm{M}$ species (Trofimenko, 1999). All of the $\left[\mathrm{HB}\left(\mathrm{pz}^{\mathrm{R}}\right)_{3}\right]^{-}$ ligands have a cone angle $(\theta)$ larger then $180^{\circ}$, and the trends in the values of these angles were consistent with the trends in the coordination chemistry of the $\left[\mathrm{HB}\left(\mathrm{pz}^{\mathrm{R}}\right)_{3}\right]^{-}$ligands. Indeed, the ligands of small cone angle (i.e. $\left[\mathrm{HB}(\mathrm{pz})_{3}\right],\left[\mathrm{HB}\left(\mathrm{pz}^{\mathrm{Me}}\right)_{3}\right]$, etc.) are characterized by a strong tendency to form $\left[\mathrm{HB}\left(\mathrm{pz}^{\mathrm{R}}\right)_{3}\right]_{2} \mathrm{M}$ (Fig. 1b) complexes with divalent first row transition metals, and the inability to form stable $\left[\mathrm{HB}\left(\mathrm{pz}^{\mathrm{R}}\right)_{3}\right] \mathrm{M}(\mathrm{L})_{\mathrm{n}}$ species (Fig. 1c) (Trofimenko, 1999; Pettinari \& Santini, 2004; Pellei et al., 2010; Santini et al., 2010).

Ligands having an intermediate cone angle (i.e. $\left[\mathrm{HB}\left(\mathrm{pz}^{\mathrm{iPr}}\right)_{3}\right],\left[\mathrm{HB}\left(\mathrm{pz}^{\mathrm{Ph}}\right)_{3}\right]$, etc.) are capable of forming both $\left[\mathrm{HB}\left(\mathrm{pz}^{\mathrm{R}}\right)_{3}\right]_{2} \mathrm{M}$ and $\left[\mathrm{HB}\left(\mathrm{pz}^{\mathrm{R}}\right)_{3}\right] \mathrm{MX}$ species. The most sterically demanding ligands (i.e., $\left[\mathrm{HB}\left(\mathrm{pz}^{\mathrm{tBu}}\right)_{3}\right],\left[\mathrm{HB}\left(\mathrm{pz}^{\mathrm{Ms}}\right)_{3}\right]$, etc.) inhibit formation of $\left[\mathrm{HB}\left(\mathrm{pz}^{\mathrm{R}}\right)_{3}\right]_{2} \mathrm{M}$ and heavily favour four-coordinate compounds $\left[\mathrm{HB}\left(\mathrm{pz}^{\mathrm{R}}\right)_{3}\right] \mathrm{MX}$ with $\mathrm{C}_{3 \mathrm{v}}$-distorted tetrahedral geometries (Trofimenko et al., 1992). The cone angles depend not only on the ligand itself, but also on the length of the N-M bond. The combined results from experimental studies in which the structures, spectroscopic properties, and reactivity of a number of metal complexes were examined and in some cases directly compared, were summarized in a series according to effective steric bulk at a complexed metal center. Analougosly, comparison of the structure, physical and spectroscopic properties of similar compounds with homologous ligands provides insight into the relative electron-donating or electron-withdrawing capabilities of $\left[\mathrm{HB}\left(\mathrm{pz}^{\mathrm{R}}\right)_{3}\right]$ - ligands (Kitajima \& Tolman, 1995). 


\section{Biomimetic chemistry of Scorpionates}

In the last decades the comprehension of the molecular mechanisms behind crucial enzymatic reaction has considerably advanced. The increasing number of available protein structures has allowed to elucidate many metal-based catalytic sites responsible of specific enzymes activity. In parallel with this, the employment of synthetic analogues (Holm \& Solomon, 2004), such as small molecular weight complexes that reproduce not only the structural aspects of the metals sites, but also their functioning, has permitted a better understanding of many biological processes. In addition, this has resulted also in the potential exploitation of these models in stoichiometrically simple but fundamental catalytic reactions such as nitrogen activation (Yandulov \& Schrock, 2003) or oxygen-evolving from water (Kanan \& Nocera, 2008). The design and synthesis of specific ligands that confer to the resulting complex desired structural and, possibly, functional properties is therefore of fundamental importance, and many new ligand systems were produced with this specific intent.

Scorpionate ligands have been extensively used in biomimetic chemistry as spectator ligands, which modulate the electronic and steric properties of the metal ion and of the co-ligands or actor ligands, but are not directly involved in the metal-based reactivity. Poly(pyrazolyl)borates can in many ways mimic histidine nitrogen ligation, so, it is possible to synthesize simple models for active sites of bio-organic macromolecules such as enzymes (Parkin, 2004). In addition, from a synthetic point of view, the steric and electronic properties of these ligands can be easily modulated by placing opportune substituents in close proximity of the $\mathrm{N}$ donor atoms. Different steric hindrance produces in some cases complexes that exhibit different nuclearity.

\subsection{Copper biomimetic systems}

The biological role of copper is made evident by its involvement in many crucial biological functions. These can be classified as follows: dioxygen activation and transport (Solomon et al., 1994; Solomon et al., 1996; Solomon et al., 2001), electron transfer (Colman et al., 1978; Durley et al., 1993; Hart et al., 1996; Shibata et al., 1999), nitrite reduction (Wasser et al., 2002) and copper delivery, storage and detoxification (Henkel \& Krebs, 2004; Calderone et al., 2005). In most cases copper exerts its function by means of its redox properties, i.e., the ability to cycle between +1 and +2 oxidation states. The geometry and the type of donor atoms surrounding the metal are fundamental in determining the functional properties of the Cu-proteins (Holm et al., 1996). The copper centers can be classified according to their geometry, donor set and nuclearity. At present, at least seven structural motives have been recognized (Koval et al., 2006), among which are the classical T1 (plastocyanin), T2 (copper nitrite reductase), and T3 (oxy-hemocyanin, oxy-Hc) arrangements (Fig. 2).

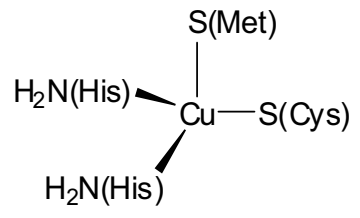

T1

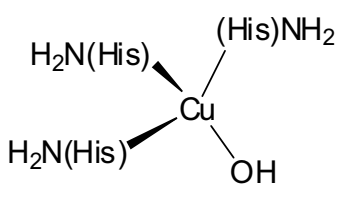

T2

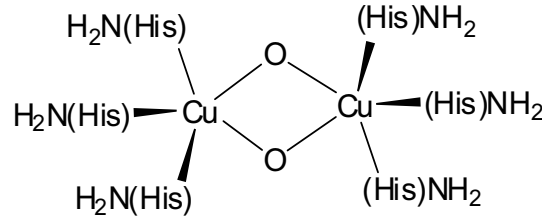

T3

Fig. 2. Some examples of T1, T2 and T3 copper sites. 
Model chemistry has been decisive in identifying the mode of peroxide coordination in oxyhemocyanin (oxy-Hc). Better spectroscopic models of oxy-Hc were obtained by using sterically encumbered tris(pyrazol-1-yl)borates as supporting ligands in $\mathrm{Cu}(\mathrm{I})$ complexes that can bind dioxygen. The peroxo complex $\left[\mathrm{HB}\left(\mathrm{pz}^{\mathrm{iPr}, \mathrm{PPr}}\right)_{3}\right]_{2} \mathrm{Cu}_{2}\left(\mathrm{O}_{2}\right)$ represents the first Xray characterized model of the oxy-Hc metal site that mimics well the spectral properties of the protein (Kitajima et al., 1989; Volbeda \& Hol, 1989; Hazes et al., 1993; Magnus et al., 1994; Cuff et al., 1998) (Fig. 3a).

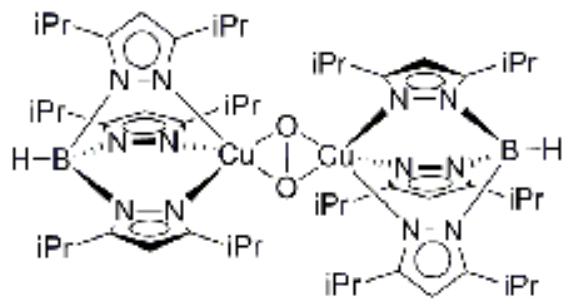

(a)

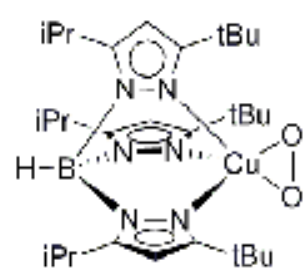

(b)

Fig. 3. Structure of: a) $\left.\left[\mathrm{HB}\left(\mathrm{pz}^{\mathrm{iPr}, \mathrm{PPr}}\right)_{3}\right]_{2} \mathrm{Cu}_{2}\left(\mathrm{O}_{2}\right) ; \mathrm{b}\right)\left[\mathrm{HB}\left(\mathrm{pz} z^{\mathrm{tBu}, \mathrm{Pr}}\right)_{3}\right] \mathrm{Cu}\left(\mathrm{O}_{2}\right)$.

The complex displayed an unprecedented coordination mode of the bridging peroxo anion, with a planar arrangement of the $\mathrm{Cu}-\mathrm{O}_{2}-\mathrm{Cu}$ moiety and with the peroxo group bridging side-on two metal centers $\left(\mu-\eta_{2}: \eta_{2}-\mathrm{O}_{2}{ }^{2-}\right.$ or $\mathbf{S P}$ coordination mode). It is notable that the reaction between $\left[\mathrm{HB}\left(\mathrm{pz}^{\mathrm{iPr}, \mathrm{PPr}}\right)_{3}\right]_{2} \mathrm{Cu}_{2}\left(\mathrm{O}_{2}\right)$ and $\mathrm{NaN}_{3} \cdot \mathrm{H}_{2} \mathrm{O}$ gave $\left[\mathrm{HB}\left(\mathrm{pz}^{\mathrm{iPr}, \mathrm{PPr}}\right)_{3}\right]_{2} \mathrm{Cu}_{2}(\mu-\mathrm{OH})(\mu-$ $\mathrm{N}_{3}$ ), a dinuclear copper(II) complex containing ( $\mu$-hydroxo)( $\mu$-1,3-azido) bridges (with the bridging hydroxide originating from water) (Kitajima et al., 1993), which mimics the physico-chemical properties of metazido-hemocyanin (Himmelwright et al., 1980; Pate et al., 1986). As for the functional properties of hemocyanin, the $\mathrm{Cu}$-site allows a reversible and fast $\mathrm{O}_{2}$-binding that renders $\mathrm{O}_{2}$-transport possible, while the $\mathrm{Cu}(\mathrm{I})$ model complexes with tris(pyrazolyl)borates bind $\mathrm{O}_{2}$ irreversibly to yield the SP species (Kitajima et al., 1988; Kitajima et al., 1989; $\mathrm{Hu}$ et al., 2000). On the other hand, $\mathrm{O}_{2}$-reactivity is modified when employing neutral tris(pyrazolyl)methanes $\left(\left[\mathrm{HC}\left(\mathrm{pz}^{\mathrm{R}, \mathrm{R}^{\prime}}\right)_{3}\right]\right)$ as supporting ligands in $\mathrm{Cu}(\mathrm{I})$ models. For example, the pseudo-tetrahedral complex $\left\{\left[\mathrm{HC}\left(\mathrm{pz}^{\mathrm{Me}, \mathrm{Me}}\right)_{3}\right] \mathrm{Cu}(\mathrm{MeCN})\right\}^{+}$binds $\mathrm{O}_{2}$ as a $\mathbf{S P}$ species in a reversible manner (Cvetkovic et al., 2001).

Oxytyrosinase (oxy-Tyr) has an active site very similar to oxy-Hc (Matoba et al., 2006). Models of oxy-Hc (SP peroxo complexes) have been tested as models for tyrosinase activity, in order to verify whether they are able to incorporate an oxygen atom into a substrate such as phenol. The model compound $\left[\mathrm{HB}\left(\mathrm{pz}^{\mathrm{Me}, \mathrm{Me}}\right)_{3}\right]_{2} \mathrm{Cu}_{2}\left(\mathrm{O}_{2}\right)$ reacts with hindered phenols, but yields diphenoquinones instead of benzoquinones (Kitajima et al., 1990). However, this behavior is not in contrast with tyrosinase biomimetics, because this enzyme also gives primarily diphenoquinones when hindered phenols are used as substrates (Pandey et al., 1990). Two mechanisms can explain this reactivity of $\left.\left[\mathrm{HB}\left(\mathrm{pz}^{\mathrm{Me}, \mathrm{Me}}\right)_{3}\right]_{2} \mathrm{Cu}_{2}\left(\mathrm{O}_{2}\right): 1\right)$ the spontaneous O-O bond cleavage of the peroxide to give a $\mathrm{Cu}(\mathrm{II})-\mathrm{O}-$ species that abstracts a hydrogen atom from phenol; 2) the acid/base substitution between the acidic phenol and the basic peroxide, affording a $\mathrm{Cu}(\mathrm{II})$-phenoxo species (equivalent to a $\mathrm{Cu}(\mathrm{I})$-phenoxy one) that undergoes reductive $\mathrm{Cu}-\mathrm{O}$ bond cleavage. In both the cases a phenoxyl radical is generated, which easily reacts to yield diphenoquinones. The alkylperoxo complex $\left[\mathrm{HB}\left(\mathrm{pz}^{\mathrm{iPr}, \mathrm{iPr}}\right)_{3}\right] \mathrm{Cu}(\mathrm{tBuOO})$ models the supposed hydroperoxo intermediate (Kitajima et al., 1993). 
A successful strategy to isolate $\mathrm{Cu}(\mathrm{II})-\mathrm{O}_{2}-$ complexes has been developed by employing sterically hindered tris(pyrazolyl)borates as supporting ligands. The steric hindrance and the anionic character of these ligands contribute to diminish the oxidizing ability of the mononuclear species towards the second equivalent of $\mathrm{Cu}(\mathrm{I})$, such that 1:1:1 $\left[\mathrm{HB}\left(\mathrm{pz}^{\mathrm{R}, \mathrm{R}^{\prime}}\right)_{3}\right]: \mathrm{Cu}: \mathrm{O}_{2}$ complexes could be isolated. In particular, by reacting the mononuclear complex $\left[\mathrm{HB}\left(\mathrm{pz}^{\mathrm{tBu}, \mathrm{iPr}}\right)_{3}\right] \mathrm{Cu}(\mathrm{DMF})$ with dioxygen, a diamagnetic mononuclear side-on superoxo complex (sS) (Fig. 4) $\left[\mathrm{HB}\left(\mathrm{pz}^{\mathrm{tBu}, \mathrm{iPr}}\right)_{3}\right] \mathrm{Cu}\left(\mathrm{O}_{2}\right)$, was crystallized (Fujisawa et al., 1994).

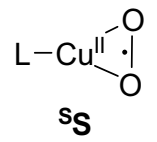

(a)

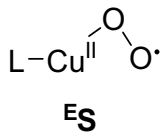

(b)

Fig. 4. a) Diamagnetic mononuclear side-on superoxo complex (SS); b) dioxygen in an endon coordinated superoxo form (ES).

A more hindered t-butyl group in place of a i-propyl group was decisive in defining the nuclearity of the product of oxygenation (Kitajima \& Tolman, 1995). As shown by spectroscopic measurements, solutions of $\left[\mathrm{HB}\left(\mathrm{pz}^{\mathrm{tBu}, i \mathrm{Pr}}\right)_{3}\right] \mathrm{Cu}\left(\mathrm{O}_{2}\right)$ (Fujisawa et al., 1994) (Fig. $3 b)$ contain small amounts of the corresponding binuclear SP species. When a bulkier $R$ substituent such as an adamantyl group was introduced on the pyrazolic arms of the scorpionate, in place of $t$-butyl, only the sS species $\left[\mathrm{HB}\left(\mathrm{pz}^{\mathrm{Ad}, \mathrm{iPr}}\right)_{3}\right]_{2} \mathrm{Cu}_{2}\left(\mathrm{O}_{2}\right)$ was detected, without any trace of the $\mathbf{S P}$ form (Chen et al., 2003).

Fungal galactose oxidase (GOase) is an extracellular copper enzyme that catalyzes the oxidation of various primary alcohols to aldehydes (Whittaker \& Whittaker, 1998; Whittaker, 2003; Whittaker, 2005). The crystal structure of the protein (Ito et al., 1994) reveals a mononuclear copper site, with two $\mathrm{N}$-donor histidines, two O-donor tyrosines (one axial and one equatorial) and an exogenous ligand (water or acetate) in a distorted squarepyramidal coordination. The catalytically active group is the modified tyrosine in the equatorial position (cofactor), which is covalently linked to a cysteine residue. Three oxidation levels of the metal site are involved in the mechanism of the enzyme: 1) the oxidized $\mathrm{Cu}(\mathrm{II})$-tyrosyl radical (GOase ${ }^{\mathrm{OX}}$ ), which is responsible for the two-electron oxidation of the substrate, 2) the $\mathrm{Cu}(\mathrm{I})$-tyrosine form (GOase ${ }^{\mathrm{RED}}$ ) that reduces $\mathrm{O}_{2}$ to $\mathrm{H}_{2} \mathrm{O}_{2}$, and 3) the $\mathrm{Cu}$ (II)-tyrosine form (GOase ${ }^{\mathrm{SEMI}}$ ), which is considered as an intermediate between the previous states. The related enzyme glyoxal oxidase, which catalyzes the oxidation of aldehydes to carboxylic acids, has a similar reactivity and active site composition (Chaudhuri \& Wieghardt, 2001). Modeling the $\mathrm{Cu}(\mathrm{II})$-phenoxo state (GOase ${ }^{\mathrm{OX}}$ ) in order to improve our understanding of the mechanistic details of the first rate-determining step of the catalytic cycle has been a challenge for biomimetic chemists (Wang et al., 1998; Thomas, 2007). Chelate effect, sulfur involvement and steric shielding have been employed to mimic the stability of the $\mathrm{Cu}(\mathrm{II})$-phenoxo moiety. In this context, tris(pyrazolyl)borates were used as supporting ligands in ternary complexes to mimic the diradical $\mathrm{Cu}(\mathrm{II})$-phenoxo state. The complex $\left[\mathrm{HB}\left(\mathrm{pz}^{\mathrm{Cum}, \mathrm{Me}}\right)_{3}\right] \mathrm{Cu}\left(\mathrm{O}(\mathrm{MeS}) \mathrm{C}_{6} \mathrm{H}_{4}\right) \quad\left(\mathrm{Cum}=\right.$ cumenyl, $\mathrm{C}_{6} \mathrm{H}_{4}(\mathrm{SMe}) \mathrm{OH}=2-$ (methylsulfanyl)phenol) of Fig. 5a, is a structural model of GOase ${ }^{\text {SEMI }}$ (Ruf \& Pierpont, 1998). The irreversible behavior of the phenoxo/phenoxy radical couple in $\left[\mathrm{HB}\left(\mathrm{pz}^{\mathrm{Cum}, \mathrm{Me}}\right)_{3}\right] \mathrm{Cu}\left(\mathrm{O}(\mathrm{MeS}) \mathrm{C}_{6} \mathrm{H}_{4}\right)$ is attributed to sulfur decoordination during the oxidative process. Also the complex $\left[\mathrm{HB}\left(\mathrm{pz}^{\mathrm{Ph}}\right)_{3}\right] \mathrm{Cu}(\mathrm{L}) \quad(\mathrm{L}=$ 2-hydroxy-3-methylsulfanyl-5methylbenzaldehyde, Fig. 5b) can be considered as a model of GOaseSEMI, wherein the co- 
ligand L mimics the thioether-substituted Tyr272 of the enzyme (Halcrow et al., 1998; Halcrow et al., 1999).

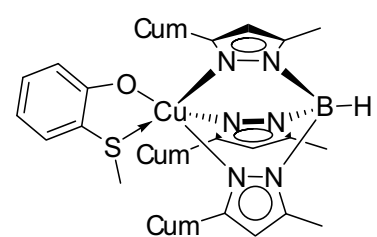

(a)

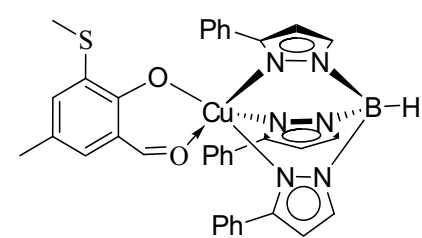

(b)

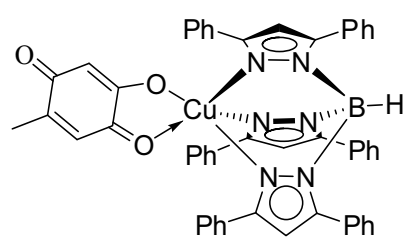

(c)

Fig. 5. Molecular structure of: a) $\left[\mathrm{HB}\left(\mathrm{pz}^{\mathrm{Cum}, \mathrm{Me}}\right)_{3}\right] \mathrm{Cu}\left(\mathrm{O}(\mathrm{MeS}) \mathrm{C}_{6} \mathrm{H}_{4}\right) ;$ b) $\left[\mathrm{HB}\left(\mathrm{pz}^{\mathrm{Ph}}\right)_{3}\right] \mathrm{Cu}(\mathrm{L})(\mathrm{L}=$ 2-hydroxy-3-methylsulfanyl-5-methylbenzaldehyde); c) $\left[\mathrm{HB}\left(\mathrm{pz}^{\mathrm{Ph}}, \mathrm{Ph}\right)_{3}\right] \mathrm{Cu}(\mathrm{L})(\mathrm{HL}=2$ hydroxy-5-methyl-1,4-benzoquinone).

Other $\left[\mathrm{HB}\left(\mathrm{pz}^{\mathrm{Ph}}\right)_{3}\right] \mathrm{Cu}^{\mathrm{II}}$ complexes containing chelate phenolates exhibit a similar arrangement around the copper center (Halcrow et al., 1999). [HB(pz $\left.\left.{ }^{\mathrm{Ph}}\right)_{3}\right] \mathrm{Cu}(\mathrm{L})$ was electrochemically oxidized to $\left\{\left[\mathrm{HB}\left(\mathrm{pz}^{\mathrm{Ph}}\right)_{3}\right] \mathrm{Cu}(\mathrm{L})\right\}^{+}$, which is a good spectroscopic model of the diradical $\mathrm{Cu}(\mathrm{II})$-phenoxo moiety of GOaseOx, being EPR silent and presenting comparable visible-NIR features (Whittaker et al., 1996). The electrochemical reversibility of the phenoxo/phenoxy radical redox process, not observed in the aforementioned $\left[\mathrm{HB}\left(\mathrm{pz}^{\mathrm{Cum}}, \mathrm{Me}\right)_{3}\right] \mathrm{Cu}\left(\mathrm{O}(\mathrm{MeS}) \mathrm{C}_{6} \mathrm{H}_{4}\right)$ model, probably implies conservation of the square pyramidal geometry also in the oxidised form $\left\{\left[\mathrm{HB}\left(\mathrm{pz}^{\mathrm{Ph}}\right)_{3}\right] \mathrm{Cu}(\mathrm{L})\right\}^{+}$, even though there is no structural evidence to support this hypothesis. Interestingly, by introducing bulky substituents such as $t$-butyl group in the para position of the phenolic co-ligand, a decrease in the kinetic stability of the electrochemically generated diradical $\mathrm{Cu}$ (II)-phenoxo species is observed (Sylvestre et al., 2005). The heteroscorpionate ligand (2-hydroxy-3-t-butylmethylphenyl)bis(3,5-dimethylpyrazolyl)methane (L1O) gave the ternary complex $[\mathrm{Cu}(\mathrm{L} 1 \mathrm{O})(\mathrm{OAc})]$ where the acetate ion is bound to the metal with both oxygen atoms. It has been proposed that the $\mathrm{N}_{2} \mathrm{O}$ donor set provided by L1O ligand serves as a mimic for two histine and the tyrosine residues (Warthen \& Carrano, 2003).

Copper amine oxidases (CuAOases) catalyze the aerobic oxidation of primary amines to aldehydes in some bacteria, yeasts, plants and mammals (Klinman, 1996; Mure et al., 2002). The catalytic site contains a T2 copper center, $\left[\mathrm{Cu}(\mathrm{His})_{3}\left(\mathrm{OH}_{2}\right)_{n}\right]^{+/ 2+}(\mathrm{n}=0$ or 2$)$, and the organic cofactor 2-(1-amino-1-carboxyethyl)-5-hydroxy-1,4-benzoquinone (TPQ). CuAOases were crystallized in two forms, TPQ-on (Parsons et al., 1995) and TPQ-off (Li et al., 1997) exhibiting the TPQ cofactor bound to the metal or 3-5 $\AA$ distant from it, respectively. The complex $\left[\mathrm{HB}\left(\mathrm{pz}^{\mathrm{Ph}, \mathrm{Ph}}\right)_{3}\right] \mathrm{Cu}(\mathrm{L})(\mathrm{HL}=2$-hydroxy-5-methyl-1,4-benzoquinone, Fig. 5c) (Foster et al., 2000) was synthesized and electrochemically characterized in order to model Cu/TPQ interactions in $\mathrm{CuAOases}$. In the structure of $\left[\mathrm{HB}\left(\mathrm{pz}^{\mathrm{Ph}, P h}\right)_{3}\right] \mathrm{Cu}(\mathrm{L})$, copper adopts a distorted square-pyramidal geometry, with a longer $\mathrm{Cu}-\mathrm{N}$ interaction in axial position, as was previously found for similar complexes ( $\mathrm{Li}$ et al., 2000) and in TPQ-on CuAOases (Nakamura et al., 1992; Speier et al., 1994).

Model complexes have been reported to reproduce the peculiar structure and spectroscopic features of blue copper proteins (Kitajima et al., 1990; Kitajima et al., 1992; Holland \& Tolman, 1999; Holland \& Tolman, 2000; Randall et al., 2000), or the function of these biological electron carriers (Rorabacher, 2004). The major synthetic challenges to yield structural/spectroscopic models have been: 1) obtain $\mathrm{Cu}(\mathrm{II})$-thiolate species, without the 
concomitant formation of disulfides and $\mathrm{Cu}(\mathrm{I})$ (Mandal et al., 1997), and 2) provide complexes exhibiting distorted tetrahedral coordination of $\mathrm{Cu}(\mathrm{II})$, which mostly prefers a tetragonal environment. Substituted tris(pyrazolyl)borate ligands were originally employed in order to fulfil these requirements (Kitajima \& Tolman, 1995), since they are tetrahedral enforcers and furnish enough electron density to copper to disfavor oxidation of thiolate coligands (Bruce \& Ostazewski, 1973; Churchill et al., 1975). Moreover, steric hindrance on $\left[\mathrm{HB}\left(\mathrm{pz}^{\mathrm{R}, \mathrm{R}^{\prime}}\right)_{3}\right]^{-}$prevents (a) the formation of $\left[\mathrm{HB}\left(\mathrm{pz}^{\mathrm{R}, \mathrm{R}^{\prime}}\right)_{3}\right]_{2} \mathrm{Cu}$ complexes and (b) the occurrence of direct $\mathrm{Cu}$ (II)-thiolate- $\mathrm{Cu}$ (II) bridges, which may be involved in the mechanism of disulfide formation (Lappin \& McAuley, 1978).

The complexes $\left[\mathrm{HB}\left(\mathrm{pz}^{\mathrm{Me}, \mathrm{Me}}\right)_{3}\right] \mathrm{CoI}(\mathrm{SR})$ (SR = p-nitrobenzenethiolate or O-ethylcysteinate), which are stable in solution only at low temperatures, represent the first spectroscopic models of the Co(II)-substituted blue Copper proteins active site (Thompson et al., 1979). $\mathrm{Cu}(\mathrm{II})$ complexes with $\mathrm{N}, \mathrm{N}^{\prime}, \mathrm{S}\left(\mathrm{SR}^{\prime}\right)$ coordination motif, as commonly found in $\mathrm{T} 1$ sites, were isolated by using the $\mathrm{N}, \mathrm{N}^{\prime}, \mathrm{S}$-donor scorpionate ligand $\left[\mathrm{H}\left(\mathrm{SPh}{ }^{p-\mathrm{Me}}\right) \mathrm{B}\left(\mathrm{pz}^{\mathrm{Me}}, \mathrm{Me}\right)_{2}\right]^{-}$instead of $\left[\mathrm{HB}\left(\mathrm{pz}^{\mathrm{Me}, \mathrm{Me}}\right)_{3}\right]^{-}$(Thompson et al., 1980). The complexes exhibit similar spectroscopic features to the aforementioned $\left[\mathrm{HB}\left(\mathrm{pz}^{\mathrm{Me}, \mathrm{Me}}\right)_{3}\right] \mathrm{Cu}(\mathrm{SR})$ species. An increase in the steric bulk of the scorpionate substituents (i-propyl groups in place of methyl groups) allowed for the crystallization of a model of an oxidized $\mathrm{T} 1$ site in the form of the complex $\left[\mathrm{HB}\left(\mathrm{pz}^{\mathrm{iPr}, \mathrm{iPr}}\right)_{3}\right] \mathrm{Cu}(\mathrm{SR})\left(\mathrm{SR}=\mathrm{SC}_{6} \mathrm{~F}_{5}\right.$, Fig. 6a) (Kitajima et al., 1992).

The $\left[\mathrm{HB}\left(\mathrm{pz}^{\mathrm{iPr}, \mathrm{iPr}}\right)_{3}\right] \mathrm{Cu}(\mathrm{SR})$ complexes $\left(\mathrm{SR}=\mathrm{SC}_{6} \mathrm{~F}_{5}, \mathrm{SCMe}_{3}, \mathrm{SCPh}_{3}, \mathrm{~S}-\mathrm{tBu}\right)$ are good spectroscopic models for the oxidized T1 sites (Kitajima et al., 1990; Kitajima et al., 1992; Qiu et al., 1994). Spectroscopic measurements and DFT calculations on $\left[\mathrm{HB}\left(\mathrm{pz}^{\mathrm{iPr}, \mathrm{iPr}}\right)_{3}\right] \mathrm{Cu}\left(\mathrm{SCPh}_{3}\right)$ have led to the following observations (Randall et al., 2000): 1) there is a stronger and more covalent thiolate- $\mathrm{Cu}$ donor interaction in $\left[\mathrm{HB}\left(\mathrm{pz}^{\mathrm{iPr}, i \mathrm{Pr}}\right)_{3}\right] \mathrm{Cu}\left(\mathrm{SCPh}_{3}\right)$ than in the $\mathrm{T} 1$ site of plastocyanin; 2) there is a weaker ligand field in the model, consistent with a more regular tetrahedral geometry around the metal; 3) an increase in the effective nuclear charge of copper from plastocyanin to $\left[\mathrm{HB}\left(\mathrm{pz}^{\mathrm{iPr}, i \mathrm{Pr}}\right)_{3}\right] \mathrm{Cu}\left(\mathrm{SCPh}_{3}\right)$, which implies a weaker donor set in the model $\left(\mathrm{N}, \mathrm{N}, \mathrm{N}\right.$ from $\left.\left[\mathrm{HB}\left(\mathrm{pz}^{\mathrm{iPr}, \mathrm{Pr}}\right)_{3}\right]\right)$ relative to the $\mathrm{N}(\mathrm{His})_{2} \mathrm{~S}(\mathrm{Met})$ donor set in plastocyanin.

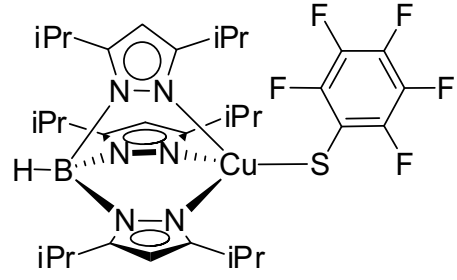

(a)

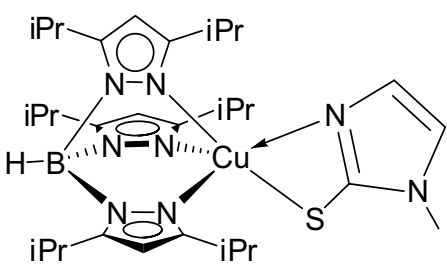

(b)

Fig. 6. Molecular structure of: a) $\left[\mathrm{HB}\left(\mathrm{pz}^{\mathrm{iPr}, \mathrm{iPr}}\right)_{3}\right] \mathrm{Cu}\left(\mathrm{SC}_{6} \mathrm{~F}_{5}\right)$; b) $\left[\mathrm{HB}\left(\mathrm{pz}^{\mathrm{iPr}, \mathrm{iPr}}\right)_{3}\right] \mathrm{Cu}\left(\mathrm{tim}^{\mathrm{Me}}\right)$.

A further $\mathrm{Cu}(\mathrm{II})$ complex with the $\left[\mathrm{HB}\left(\mathrm{pz}^{\mathrm{iPr}, \mathrm{iPr}}\right)_{3}\right]^{-}$supporting ligand was synthesized, using a thioxo-imidazole (tim ${ }^{\mathrm{Me}}$ ) as co-ligand (Basumallick et al., 2002). The resulting complex $\left[\mathrm{HB}\left(\mathrm{pz}^{\mathrm{iPr}, \mathrm{iPr}}\right)_{3}\right] \mathrm{Cu}\left(\mathrm{tim}^{\mathrm{Me}}\right)$ exhibits a square-pyramidal geometry around the copper ion, with a $\mathrm{N}_{4} \mathrm{~S}$ donor set (Fig. 6b). [HB(pziPr,iPr $\left.)_{3}\right] \mathrm{Cu}(\mathrm{SMeIm})$ can be considered a structural model of some mutant azurins (Canters \& Gilardi, 1993; Den Blaauwen \& Canters, 1993; den Blaauwen et al., 1993) and nitrosocyanin (a red copper protein) (Whittaker et al., 2000; Lieberman et al., 2001), both involving a pentacoordinated $\mathrm{Cu}$ (II) ion. The effect of the 
increase of the coordination number was investigated by comparing the structural/spectroscopic properties of $\left[\mathrm{HB}\left(\mathrm{pz}^{\mathrm{iPr}, \mathrm{Pr}}\right)_{3}\right] \mathrm{Cu}(\mathrm{SMeIm})$ with those of $\left[\mathrm{HB}\left(\mathrm{pz}^{\mathrm{iPr}, \mathrm{iPr}}\right)_{3}\right] \mathrm{Cu}\left(\mathrm{SCPh}_{3}\right)$.

The metalloenzyme copper nitrite reductase (CuNIR) plays a key role in the nitrogen cycle since it reduces nitrite ions $\left(\mathrm{NO}_{2}{ }^{-}\right)$to nitric oxide (NO). The resting state active site of CuNIR consists of a $\mathrm{Cu}(\mathrm{II})$ ion tetrahedrally coordinated by three $\mathrm{N}$-donor histidines and a water molecule (T2 site) (Godden et al., 1991). This site is connected to a T1 electron transfer site by a His-Cys bridge. A first hypothesis of the enzyme's catalytic mechanism, proposed by Averill (Hulse et al., 1989; Averill, 1996), contemplates the initial reduction of the metal center (with electrons shuttled from the T1 site) and subsequent binding of nitrite to $\mathrm{Cu}(\mathrm{I})$ by means of the $\mathrm{N}$ atom ( $\eta^{1-N}$ coordination). This $\eta^{1-N}$ coordination is supported by experimental evidence on model complexes (Halfen \& Tolman, 1994; Mahapatra et al., 1996). Subsequent protonation of the substrate would give water and a copper-nitrosyl complex, presumably an unstable $\{\mathrm{CuNO}\}^{10}$ species (Enemark \& Feltham, 1974), wherein NO is reasonably considered to be $\eta^{1}-\mathrm{N}$ end-on coordinated to the metal. The copper-nitrosyl intermediate is crucial for the catalytic mechanism of CuNIR, and for this reason some mononuclear $\mathrm{Cu}-\mathrm{NO}$ complexes were prepared to model this species. Copper complexes as models for nitrite reductase were examined in ref. (Wasser et al., 2002). The complex $\left[\mathrm{HB}\left(\mathrm{pz}^{\mathrm{tBu}}\right)_{3}\right] \mathrm{Cu}(\mathrm{NO})$ is the first structurally characterized mononuclear copper-nitrosyl complex (Fig. 7a) (Carrier et al., 1992; Ruggiero et al., 1993).

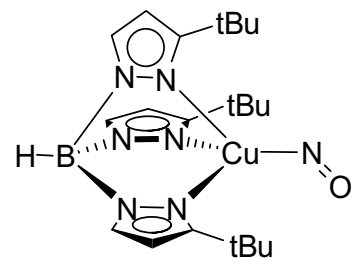

(a)

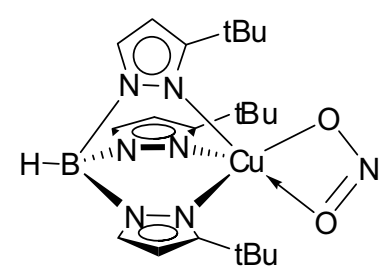

(b)

Fig. 7. Molecular structure of: a) $\left[\mathrm{HB}\left(\mathrm{pz}^{\mathrm{tBu}}\right)_{3}\right] \mathrm{Cu}(\mathrm{NO})$; b) $\left[\mathrm{HB}\left(\mathrm{pz}^{\mathrm{tBu}}\right)_{3}\right] \mathrm{Cu}\left(\mathrm{NO}_{2}\right)$.

The metal presents a pseudotetrahedral geometry and a $\eta^{1-} \mathrm{N}$ end-on $\mathrm{NO}$ coordination, supporting indirectly the $\eta^{1}-\mathrm{NO}$ end-on hypothesis for the $\{\mathrm{CuNO}\}^{10}$ species of CuNIR. Nitric oxide binding is reversible, since it is lost upon application of a vacuum or when purging with argon. Two other $\mathrm{Cu}$-nitrosyl complexes with scorpionates as supporting ligands, namely $\left[\mathrm{HB}\left(\mathrm{pz}^{\mathrm{tBu}, \mathrm{iPr}}\right)_{3}\right] \mathrm{Cu}(\mathrm{NO})$ and $\left[\mathrm{HC}\left(\mathrm{pz}^{\mathrm{tBu}, i \mathrm{Pr}}\right)_{3}\right] \mathrm{Cu}(\mathrm{NO})$, have been characterized in the solid state (Fujisawa et al., 2008). Both these species are structural analogues of $\left[\mathrm{HB}\left(\mathrm{pz}^{\mathrm{tBu}}\right)_{3}\right] \mathrm{Cu}(\mathrm{NO})$, exhibiting tetrahedral coordination of the metal, with a $\eta^{1}-\mathrm{N}$ end-on NO. Spectroscopic evidence and ab initio calculations have revealed that all the three complexes have a $\{\mathrm{CuNO}\}^{11}$ electronic structure, better described with a $\mathrm{Cu}(\mathrm{I})-\mathrm{NO}$. radical interaction characterized by a significant covalency. The sterically hindered scorpionate ligand $\left[\mathrm{HB}\left(\mathrm{pz}^{\mathrm{tBu}}\right)_{3}\right]^{-}$, as well as being a pseudotetrahedral enforcer, has the important role of stabilizing $\mathrm{NO}$ coordination, by protecting it from possible disproportionation into $\mathrm{N}_{2} \mathrm{O}$ and $\mathrm{NO}_{2}$ - A similar $\mathrm{NO}$ decomposition pathway occurs even in the enzyme, under conditions of high NO concentration (Jackson et al., 1991). NO dismutation in model complexes is strongly influenced by the nature of the substituents $R$ and $R^{\prime}$ on the $\left[H B\left(p z^{R, R^{\prime}}\right)_{3}\right]^{-}$ligand. Interesting, not only steric bulk (e.g., tBu group), but also the electron withdrawing 
character (e.g., $\mathrm{CF}_{3}$ group) of the $\left[\mathrm{HB}\left(\mathrm{pz}^{\mathrm{R}, \mathrm{R}^{\prime}}\right)_{3}\right]$-substituents tends to slow the rate of $\mathrm{NO}$ disproportionation. Mechanistic investigations suggest that the process occurs by an electrophilic attack of a second $\mathrm{NO}$ molecule on the initial $\mathrm{Cu}-\mathrm{NO}$ adduct, thus yielding a $\left[\mathrm{HB}\left(\mathrm{pz}^{\mathrm{R}, \mathrm{R}^{\prime}}\right)_{3}\right] \mathrm{Cu}(\mathrm{NO})_{2}$ adduct. Following $\mathrm{N}-\mathrm{N}$ coupling and O-atom transfer, $\mathrm{N}_{2} \mathrm{O}$ and $\mathrm{Cu}(\mathrm{II})-\left(\mathrm{NO}_{2}^{-}\right)$are generated.

The $\mathrm{Cu}(\mathrm{II})$-nitrito form of $\mathrm{CuNIR}$, which exhibits $\eta^{2}-\mathrm{O}, \mathrm{O}^{\prime}$ coordination of $\mathrm{NO}_{2}^{-}$to copper (Murphy et al., 1997; Veselov et al., 1998), is stabilized by hydrogen bond interactions with the protein-matrix side chains. A few $\mathrm{Cu}$ (II)-nitrito model complexes with hydrotris(pyrazolyl)borate co-ligands have been structurally characterized, showing either a symmetric or asymmetric $\eta^{2}-\mathrm{O}, \mathrm{O}$ coordination mode of $\mathrm{NO}_{2}^{-}$, depending on the pyrazolyl substituents. Bulky $\mathrm{R}$ substituents, such as $t$-butyl groups, favor an asymmetric array as found in the active site of CuNIR. This is observed in the complexes $\left[\mathrm{HB}\left(\mathrm{pz} \mathrm{Bu}_{3}\right] \mathrm{Cu}\left(\mathrm{NO}_{2}\right)\right.$ (Fig. 7b) (Tolman, 1991) and $\left[\mathrm{HB}\left(\mathrm{pz}^{\mathrm{tBu}, \mathrm{iPr}}\right)_{3}\right] \mathrm{Cu}\left(\mathrm{NO}_{2}\right)$ (Lehnert et al., 2007), whereas $\left[\mathrm{HB}\left(\mathrm{pz}^{\mathrm{iPr}, \mathrm{iPr}}\right)_{3}\right] \mathrm{Cu}\left(\mathrm{NO}_{2}\right)$ (Lehnert et al., 2007), $\left[\mathrm{HB}\left(\mathrm{pz}^{\mathrm{CF} 3}, \mathrm{Me}\right)_{3}\right] \mathrm{Cu}\left(\mathrm{NO}_{2}\right)$ and $\left[\mathrm{HB}\left(\mathrm{pz}^{\mathrm{Me}}, \mathrm{Me}\right)_{3}\right] \mathrm{Cu}\left(\mathrm{NO}_{2}\right)$ (Schneider et al., 1998) show a symmetric bidentate nitrite coordination. When using a neutral tris(pyrazolyl)methane as a co-ligand, two $\mathrm{NO}_{2}$ - ions are coordinated to $\mathrm{Cu}(\mathrm{II})$ : one nitrite is $\eta^{1-} \mathrm{O}$ and the second one is $\eta^{1}-\mathrm{N}$ bound (Lehnert et al., 2007).

Recently it has been discovered that the nitrite ion is bound in a tridentate fashion in the reduced form of $\mathrm{CuNIR}$, with its oxygen atoms coordinated to $\mathrm{Cu}(\mathrm{I})$ and an additional weak $\mathrm{Cu}-\mathrm{N}$ interaction (Antonyuk et al., 2005). This binding mode would allow minimal structural rearrangements between the reacting $\eta^{2}-\mathrm{O}, \mathrm{O}^{\prime} \mathrm{NO}_{2^{-}}$and the incipient side-on coordinated NO (\{CuNO $\}^{10}$ species). The spectroscopic properties of the model complexes $\left[\mathrm{HB}\left(\mathrm{pz}^{\mathrm{tBu}, \mathrm{iPr}}\right)_{3}\right] \mathrm{Cu}(\mathrm{NO})$ and $\left[\mathrm{HC}\left(\mathrm{pz}^{\mathrm{tBu}, \mathrm{iPr}}\right)_{3}\right] \mathrm{Cu}(\mathrm{NO})$, in which $\mathrm{NO}$ is end-on bound to the metal, were compared to those of CuNIR (Usov et al., 2006). This has allowed for the determination of the binding mode of the $\{\mathrm{CuNO}\}^{11}$ species in protein solutions as strongly bent end-on but not side-on (Fujisawa et al., 2008).

Comprehensive reviews describing structural and functional aspects of copper dioxygen models have been reported (Kitajima \& Moro-oka, 1994; Lewis \& Tolman, 2004; Mirica et al., 2004). The employment of scorpionate ligands in copper biometics (Kitajima, 1992; Kitajima \& Tolman, 1995; Gennari \& Marchiò, 2009) and to understand copper protein active-sites chemistry (Tolman, 2006) was reviewed in the past.

\subsection{Zinc biomimetic systems}

Zinc is essential to all forms of life and it is probably the most bio-relevant metal. Human beings contain an average of approximately 2 to $3 \mathrm{~g}$ of zinc and it has been found that $3 \%$ of the human genome contain the code for zinc finger proteins (Klug, 1999). There are about 400 three-dimensional structures for zinc proteins, representing all six fundamental enzyme classes: oxidoreductases (alcohol dehydrogenase family), transferases, hydrolases (carboxypeptidase family), lyases (carbonic anhydrase family), isomerases, and ligases (Auld, 2006). Thus, zinc is involved in a wide variety of metabolic processes including carbohydrate, lipid, protein, and nucleic acid synthesis and degradation. Mononuclear zinc enzymes were currently studied by several bioinorganic research groups with biomimetic complexes. Recent and substantial reviews by G. Parkin (Parkin, 2000; Parkin, 2004; Parkin, 2007), H. Vahrenkamp (Vahrenkamp, 2007) and N. Burzlaff (Fischer et al., 2009) cover almost all aspects of synthetic zinc enzyme analogues. Thus, here we will summarise some aspects of synthetic analogues of zinc enzymes that feature scorpionate ligands. 
The molecular characterization of zinc sites in biological systems came first through replacing the spectroscopically silent zinc with the chromophoric metal cobalt. In particular, $\mathrm{Co}(\mathrm{II})$ substitution has been widely employed as a tool for conversion of spectroscopically silent $\mathrm{Zn}$ (II)-containing proteins into still-functioning enzymes, but for which optical (absorption, magnetic circular dichroism (MCD)) and magnetic resonance (nuclear magnetic resonance (NMR), electron paramagnetic resonance (EPR), electron nuclear double resonance (ENDOR)) spectroscopic techniques all can be productively applied to understand enzyme structure and function (Maret \& Vallee, 1993; Bertini et al., 2001). Recent examples involved high-frequency and -field electron paramagnetic resonance (HFEPR) investigation of cobalt-substituted zinc enzymes (Krzystek et al., 2010), EPR/ENDOR studies of a Co-substituted Zn finger protein, TF IIIA (Walsby et al., 2003), MCD studies of a variety of Co-substituted Zn enzymes and model compounds (Larrabee et al., 1997). In the past the substitution of zinc in enzymes by cadmium was also attempted, in an effort to take advantage of ${ }^{113} \mathrm{Cd}$ NMR spectroscopy in gaining structural and mechanistic information (Coleman, 1992). These efforts were abandoned because it became too obvious that cadmium and zinc have essentially nothing in common in terms of ligand preferences, coordination numbers or stabilities.

Zinc enzymes in the resting state almost without exception contain zinc-bound water (Vallee \& Auld, 1993). The tetrahedral $\left[\{X Y Z\} Z n-O_{n}\right]$ zinc centre is the most common structural feature found for the active site of zinc enzymes. In these enzymes zinc is bound to the protein by three amino acid residues. The zinc binding residues $\mathrm{X}, \mathrm{Y}$ and $\mathrm{Z}$ are either histidine, glutamate, aspartate or cysteine.

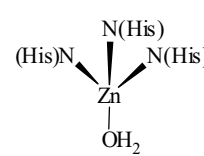

(a)

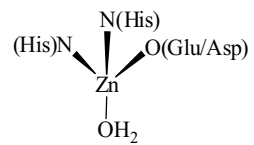

(b)

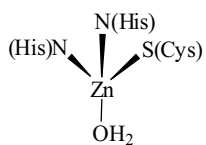

(c)

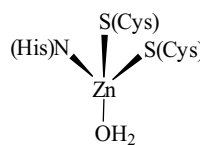

(d)

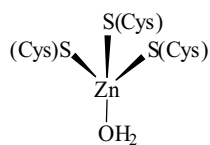

(e)

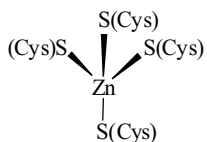

(f)

Fig. 8. Coordination motifs in mononuclear zinc enzymes: a) carbonic anhydrase, matrix metalloproteases; b) thermolysin, carboxypeptidase A; c) plant peptide deformylases (PDFs); d) alcoholdehydrogenase, cobalamine independent methionine synthase; e) 5aminolevulinate dehydratase; f) Ada DNA repair protein.

Almost all triple combinations of these residues are found in zinc enzymes. During the resting state, the fourth position of the $\left[\{\mathrm{XYZ}\} \mathrm{Zn}-\mathrm{OH}_{n}\right]$ active site is usually occupied by water or a hydroxido ligand (Fig. 8) (Parkin, 2004; Kraatz \& Metzler-Nolte, 2006). In many cases, characteristically in the alcoholdehydrogenase $(\mathrm{ADH})$ and cobalamine independent methionine synthase (CAIMS) group, the water molecule only represents the vacant coordination site which during catalysis is occupied by the substrate. More importantly, though, and exclusively in the carbonic anhydrase (CA) and matrix metalloproteases (MMP) group, the water ligand is the functional reagent which is deprotonated to become the powerful $\mathrm{Zn}-\mathrm{OH}$ nucleophile which is responsible for the efficiency of the hydrolytic zinc enzymes. Infact, during their catalytic cycle the tetrahedral or pseudotetrahedral geometries of these zinc enzymes are quite flexible and change between a tetra-coordinated and a penta-coordinated zinc centre. Especially the coordination of a carboxylate donor e.g. a glutamate can easily vary in the so called carboxylate shift between a monodentate and a bidentate coordination. This flexibility is thought to be essential for the hydrolytic activity of many of the zinc enzymes. 
Bioinorganic coordination chemists who are trying to model such active sites by simple ligands and zinc complexes are therefore facing several problems: a) the flexibility mentioned above is difficult to mimic without a surrounding protein pocket; b) amino acids on their own coordinate zinc ion not only via the amino acid residue but also via the $\mathrm{N}$ - or C-terminus of the sequence; c) protected amino acids or small oligopeptides are often not bulky enough to prevent the formation of bisligand complexes or hydroxido bridged dinuclear zinc complexes, this is also the case for imidazoles or thiols, which tend to form oligo- or poly-nuclear complexes. During studies by coordination chemists aimed at the modelling of zinc enzymes, many polydentate ligands have been designed which leave room for just one water ligand in the coordination sphere of zinc (Parkin, 2004). In those cases where the acidities of these complexes have been determined they provide evidence for the coordination number/ $\mathrm{p} K_{\mathrm{a}}$ relationship. In particular, $\mathrm{LZn}\left(\mathrm{OH}_{2}\right)$ complexes with $\mathrm{L}$ being a tetradentate ligand have $\mathrm{p} K_{\mathrm{a}}$ values at or above 8 (Mareque-Rivas et al., 2004). But, most strikingly, all attempts to prepare $\mathrm{LZn}\left(\mathrm{OH}_{2}\right)$ complexes with tripodal scorpionates ligands have resulted in the isolation of their deprotonated derivatives, the $\mathrm{LZn}-\mathrm{OH}$ complexes.

Tripodal scorpionate ligands have been applied successfully in zinc model complexes. Their key advantages are a "facial" binding causing tetrahedral to octahedral zinc centre and a rather rigid geometry compared to the often very flexible macrocyclic ligands. As Figure 9 shows, they can form 4-, 5-, or 6-coordinate zinc complexes depending on the counteranions present (Rombach et al., 1999).

Basing on borderline properties of zinc, mixed hard and soft ligand environments make for perfectly stable complexes, purely hard or soft ligand environments making zinc unwilling to accept the ligand thiolate. This is one of the most important properties governing the biological chemistry of zinc (Vahrenkamp, 2007). Modifications regarding the sterical hindrance can easily be employed to many scorpionate ligands to prevent dimer formation e.g. by attachment of bulky residues (R groups) to the ligands. In recent studies functional groups are attached to the ligands to mimic the enzyme pockets more closely. This allows the stabilisation of coordinated substrates, water or hydroxido ligands by hydrogen bridges. Similar interactions are found in enzymes.

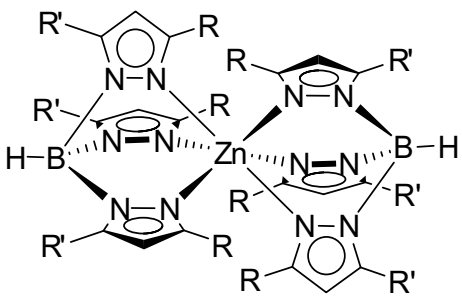

(a)

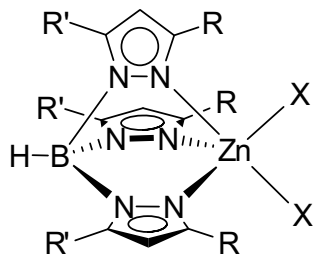

(b)

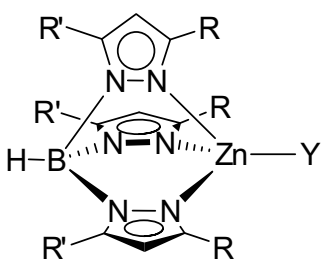

(c)

Fig. 9. Pyrazolylborate zinc complexes with 4-, 5-, and 6-coordinated zinc: a) with non coordinating anions; $b$ ) with hard coligands $\mathrm{X} ; \mathrm{c}$ ) with soft coligands $\mathrm{Y}$.

Most models for mononuclear zinc enzymes described so far consist of tripod ligands with three or four $\mathrm{N}$ donors to mimic mononuclear zinc enzymes with three zinc binding histidine residues (e.g. the matrix metalloproteases or carbonic anhydrase) (Parkin, 2000; Parkin, 2004). Often sterically hindered hydrotris(pyrazol-1-yl)borate ligands are used to mimic this tris(histidine) motif by three pyrazol-1-yl donors. Especially the works of $\mathrm{H}$. 
Vahrenkamp and G. Parkin have helped to elucidate several biocatalytic mechanisms with poly(azolyl)borate-zinc models. Scorpionate zinc complexes have been recognised by various research groups as versatile tools in the quest for new zinc binding groups suitable for enzyme inhibitors. The synthesis of such structural enzyme (inhibitor) models usually starts from the hydroxido complexes.

The most difficult task, which is not fully achieved as yet, is the isolation of a $\left\{\left[\mathrm{HB}\left(\mathrm{pz}^{\mathrm{R}}\right)_{3}\right] \mathrm{Zn}\left(\mathrm{OH}_{2}\right)\right\}^{+}$cation (Ruf et al., 1996; Bergquist \& Parkin, 1999) $\left(\left[\mathrm{HB}\left(\mathrm{pz}^{\mathrm{R}}\right)_{3}\right]\right.$ denotes any one substituted tris(pyrazolyl)borate ligand), the main reason for which is the hydrolytic destruction of the $\left[\mathrm{HB}\left(\mathrm{pz}^{\mathrm{R}}\right)_{3}\right]^{-}$ligands in acidic media. In all cases studied, the $\left[\mathrm{HB}\left(\mathrm{pz}^{\mathrm{R}}\right)_{3}\right] \mathrm{Zn}-\mathrm{OH}$ complexes are the easiest to prepare (Alsfasser et al., 1993; Ruf et al., 1996; Ruf \& Vahrenkamp, 1996). In just a few cases they could be induced to release water and form the dinuclear complexes $\left\{\left[\mathrm{HB}\left(\mathrm{pz}^{\mathrm{R}}\right)_{3}\right] \mathrm{Zn}-\mathrm{OH}-\mathrm{Zn}\left[\mathrm{HB}\left(\mathrm{pz}^{\mathrm{R}}\right)_{3}\right]\right\}^{+}$(Ibrahim et al., 2006); full deprotonation with formation of the neutral molecular species $\left\{\left[\mathrm{HB}\left(\mathrm{pz}^{\mathrm{R}}\right)_{3}\right] \mathrm{Zn}-\mathrm{O}-\right.$ $\left.\mathrm{Zn}\left[\mathrm{HB}\left(\mathrm{pz}^{\mathrm{R}}\right)_{3}\right]\right\}$ have been achieved in one case (Ruf \& Vahrenkamp, 1996).

The $\left[\mathrm{HB}\left(\mathrm{pz}^{\mathrm{R}}\right)_{3}\right] \mathrm{Zn}-\mathrm{OH}$ complexes owe their stability both to the fact that the sterically laden $\left[\mathrm{HB}\left(\mathrm{pz}^{\mathrm{R}}\right)_{3}\right]^{-}$ligands enforce a low coordination number and that they create a hydrophobic pocket and an encapsulation of the $\mathrm{Zn}-\mathrm{OH}$ unit. Increasing the polarity (hardness) near zinc (e.g. by a pyridyl substituted ligand) allows the coordination number to be increased; as a result, observed in the enzyme-substrate model complex $\left[\mathrm{HB}\left(\mathrm{pz}^{\mathrm{Py}}, \mathrm{Me}\right)_{3}\right] \mathrm{Zn}\left(\mathrm{OH}_{2}\right) \mathrm{OPO}(\mathrm{OPh})_{2}$ (Weis et al., 1998), the hydrolytic agent water and the organophosphate (employed to model the substrate) are attached to zinc at the same time.

The $\left[\mathrm{HB}\left(\mathrm{pz}^{\mathrm{R}}\right)_{3}\right] \mathrm{Zn}-\mathrm{OH}$ complexes were shown to be able to perform, in a stoichiometric way, all hydrolytic reactions catalyzed by zinc enzymes (Vahrenkamp, 1999; Parkin, 2004). Bearing voluminous organic substituents, they suffer from two limitations with respect to catalytic action. They are hydrophobic and do not dissolve in water, thereby rendering the reagent $\mathrm{H}_{2} \mathrm{O}$ unavailable, and they form very stable complexes with the carboxylates and phosphates resulting from hydrolysis. On one hand this is unfavourable and still requires efforts in order to overcome it, but on the other hand it has provided important compounds for the structure correlation based mechanistic findings.

The $\left[\mathrm{HB}\left(\mathrm{pz}^{\mathrm{R}, \mathrm{R}^{\prime}}\right)_{3}\right] \mathrm{Zn}(\mathrm{OH})$ complexes $\left(\mathrm{R}=\mathrm{tBu}, \mathrm{Ph}, \mathrm{Cum}, \mathrm{R}^{\prime}=\mathrm{H}, \mathrm{Me}, \mathrm{tBu}\right)$ (Fig. 10a) (Alsfasser et al., 1991; Kitajima et al., 1993; Looney et al., 1993; Ruf \& Vahrenkamp, 1996), including $\left[\mathrm{HB}\left(\mathrm{pz}^{\mathrm{R}}\right)_{3}\right]^{-}$incorporating hydrogen bonding accepting ester substituents, react as functional models according to the carbonic anhydrase mechanism (Looney et al., 1993; Rombach \& Vahrenkamp, 2001; Hammes et al., 2002; Bergquist et al., 2003; Lipton et al., 2003; Lipton \& Ellis, 2007) and form bicarbonate from $\mathrm{CO}_{2}$. Hydrogensulfido, thiolato, phenolato and alcoholato $\left[\mathrm{HB}\left(\mathrm{pz}^{\mathrm{R}}\right)_{3}\right]^{-}$complexes are accessed by substitution reactions with $\mathrm{H}_{2} \mathrm{~S}$, thiols, phenols or alcohols (Ruf \& Vahrenkamp, 1996; Bergquist et al., 2000; Brand et al., 2001).

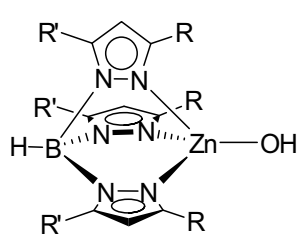

(a)

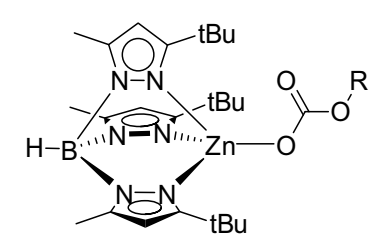

(b)

Fig. 10. Structure of: a) hydroxido complexes $\left.\left[\mathrm{HB}\left(\mathrm{pz}^{\mathrm{R}, \mathrm{R}^{\prime}}\right)_{3}\right] \mathrm{Zn}(\mathrm{OH}) ; \mathrm{b}\right)$

$\left[\mathrm{HB}\left(\mathrm{pz}^{\mathrm{tBu}, \mathrm{Me}}\right)_{3}\right] \mathrm{Zn}(\mathrm{OCOOR})$ as a carbonic anhydrase model. 
The complex $\left\{\left[\mathrm{HB}\left(\mathrm{pz}^{\mathrm{Ph}, \mathrm{Me}}\right)_{3}\right]_{2} \mathrm{Zn}_{2}\left(\mathrm{H}_{3} \mathrm{O}_{2}\right)\right\} \mathrm{ClO}_{4}$ (Puerta \& Cohen, 2002) is important for the relevance of its structure in terms of hydrolytic zinc enzymes, that use hydrogen-bond stabilized water nucleophiles to perform peptide bond cleavage.

With dialkyl carbonate the hydroxido complexes form monoalkyl carbonato complexes (Fig. 10b) (Ruf et al., 1996; Vahrenkamp, 1999). These are also accessible by a reaction of alcoholato complexes with $\mathrm{CO}_{2}$, a reaction that is analogous to the carbonic anhydrase catalysis. This even allows the catalytic formation of dialkyl carbonate from alcohols and $\mathrm{CO}_{2}$ under pressure (Vahrenkamp, 1999). Binding studies with sulfonamide inhibitors have also been described (Hartmann \& Vahrenkamp, 1994; Brombacher \& Vahrenkamp, 2004).

The protective pocket provided by the pyrazolylborate ligands $\left[\mathrm{HB}\left(\mathrm{pz}^{\mathrm{Cum}, \mathrm{Me}}\right)_{3}\right]^{-}$and $\left[\mathrm{HB}\left(\mathrm{pz}^{\mathrm{Ph}}, \mathrm{Me}\right)_{3}\right]^{-}$has made it possible to obtain stable and inert zinc complexes $\left[\mathrm{HB}\left(\mathrm{pz}^{\mathrm{R}}\right)_{3}\right] \mathrm{Zn}$ base of the anionic nucleobases (i.e. thymine, uracil, dihydrouracil, cytosine, adenine, guanine, diaminopurine, xanthine, hypoxanthine) in their deprotonated forms (Badura \& Vahrenkamp, 2002) and to investigate the reactivity of aminoacids toward zinc (Rombach et al., 2002). The complex $\left[\mathrm{HB}\left(\mathrm{pz}^{\mathrm{Cum}, \mathrm{Me}}\right)_{3}\right] \mathrm{Zn}(\mathrm{OH})$ has been reported to cleave activated amides and esters (Ruf \& Vahrenkamp, 1996). Other hydroxido complexes, e.g. $\left[\mathrm{HB}\left(\mathrm{pz}^{\mathrm{Cum}, \mathrm{Me}}\right)_{3}\right] \mathrm{Zn}(\mathrm{OH})$, are able to hydrolyse amides (Vahrenkamp, 1999; Parkin, 2004). This indicates that such model complexes are also structural and functional models for matrix metalloproteases (MMPs). Therefore, matrix metalloproteases model properties are also utilised for binding studies with protease inhibitors (Ruf et al., 1996; Vahrenkamp, 1999). In these binding studies the coordination properties of the zinc binding groups of MMP inhibitors are investigated. In particular the pioneering work of Cohen (Puerta \& Cohen, 2002; Puerta \& Cohen, 2003; Jacobsen \& Cohen, 2004; Puerta et al., 2004; Puerta et al., 2005; Puerta et al., 2006; Jacobsen et al., 2007) and Vahrenkamp (Ruf et al., 1996; Tekeste \& Vahrenkamp, 2006; Tekeste \& Vahrenkamp, 2006) in this field has to be highlighted.

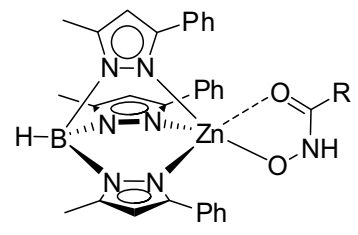

(a)

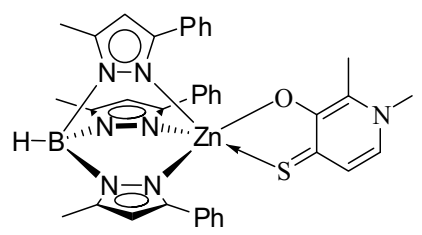

(b)

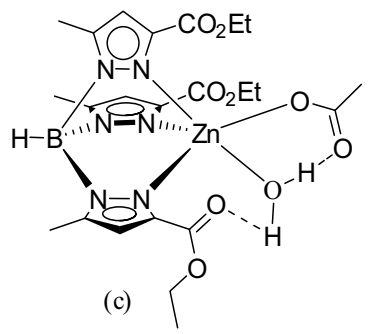

(c)

Fig. 11. a) Model complex for MMPs with bound hydroxamate inhibitor; b) Bidentate $\mathrm{k}^{2}$ coordination of pyridinethione in biomimetic zinc complex $\left.\left[\mathrm{HB}\left(\mathrm{pz}^{\mathrm{Ph}, \mathrm{Me}}\right)_{3}\right] \mathrm{ZnL} ; \mathrm{c}\right)$ structure of $\left[\mathrm{HB}\left(\mathrm{pz} \mathrm{CO}^{\mathrm{CE}}, \mathrm{Me}\right){ }_{3}\right] \mathrm{Zn}\left(\mathrm{H}_{2} \mathrm{O}\right)(\mathrm{OAc})$.

Common zinc binding groups applied successfully in peptidase inhibitors have been either a carboxylic acid, a hydroxamic acid or a thiol functionality. Reactions of $\left[\mathrm{HB}\left(\mathrm{pz}^{\mathrm{R}, \mathrm{R}^{\prime}}\right)_{3}\right] \mathrm{Zn}(\mathrm{OH})$ complexes with carboxylic acids, hydroxamic acids or thiols yield carboxylato, hydroxamato (Fig. 11a) and thiolato complexes (Ruf et al., 1996; Parkin, 2004). These complexes may be understood as biomimetic complexes that model the coordination environment of these zinc binding groups bound to the catalytic zinc ion in the matrix metalloproteases. Cohen and coworkers first utilized coordination complexes to model drug-protein interactions of inhibitors to MMPs of which the binding mode was not known so far. The focus of this work was to predict the drug-protein interaction of non- 
hydroxamate inhibitors without the need for elaborate drug synthesis or protein structure determination. They reported the binding of $\beta$-mercaptoketone and $\beta$-mercaptoamide drugs in a bidentate fashion, while $\beta$-mercaptothiols bound exclusively in a monodentate manner, contrary to prior expectations (Puerta \& Cohen, 2002). A whole series of publications by the same authors extended this concept to four different groups of chelators: hydroxypyridinones, pyrones, hydroxypyridinethiones and thiopyrones (Puerta \& Cohen, 2003; Puerta et al., 2004; Puerta et al., 2005; Puerta et al., 2006; Jacobsen et al., 2007). Each of the tested small molecules was able to displace the hydroxide ligand in the $\left[\mathrm{HB}\left(\mathrm{pz}^{\mathrm{Ph}}, \mathrm{Me}\right)_{3}\right] \mathrm{ZnOH}$ model complex of the active site and to coordinate the zinc(II) in a bidentate fashion (Puerta \& Cohen, 2003; Puerta et al., 2004; Puerta et al., 2006; Jacobsen et al., 2007) (Fig. 11b). These compounds represented the zinc chelating portion of new MMP inhibitors (MPIs), lacking the critical peptidomimetic backbone. The zinc binding groups (ZBGs) listed above were found to have a greater affinity than acetohydroxamic acid (AHA) for the zinc(II) ion the model complex. Furthermore, the inhibitory activity of these ZBGs was compared with that of AHA (Puerta et al., 2006). All the new compounds were found to be more potent inhibitors of MMP-3 than AHA (Puerta et al., 2004). This finally resulted in the development of potent and selective pyrone-based inhibitors (Puerta et al., 2005).

Other binding studies by the Cohen group reported on $\kappa^{1}$-coordination for zinc complexes $\left[\mathrm{HB}\left(\mathrm{pz}^{\mathrm{Ph}, \mathrm{Me}}\right)_{3}\right] \mathrm{ZnL}$ bearing 2-thenylmercaptan, salicylamide and thiosalicylic acid, but on $\kappa^{2}$ coordination in case of methyl salicylate, methyl thiosalicylate or 2-hydroyacetophenone ligands. Thus, it was possible to reveal the mode of binding of many ZBGs (Jacobsen \& Cohen, 2004). Interesting, Cohen and coworkers applied the $\left[\mathrm{HB}\left(\mathrm{pz}^{\mathrm{Ph}, \mathrm{Me}}\right)_{3}\right] \mathrm{ZnOH}$-fragment to identify ZBGs for the lethal factor of anthrax (Jacobsen et al., 2006). Vahrenkamp and coworker observed both, a bidentate and a monodentate coordination to a $\left[\mathrm{HB}\left(\mathrm{pz}^{\mathrm{Ph}, \mathrm{Me}}\right)_{3}\right] \mathrm{ZnOH}$ fragment, in case of 2-mercaptophenol (Tekeste \& Vahrenkamp, 2006). Vahrenkamp also reported on further studies regarding the coordination manner of $\alpha$-keto carboxylic acids, $\alpha$ - and $\beta$-diketones, $\beta$-mercapto amines and alcohols as well as mercaptopropionic acid (Tekeste \& Vahrenkamp, 2006).

Recent studies try to attach functional groups to the scorpionate ligands of the zinc models, in order to mimic the catalytically important glutamic acid of the $\underline{\mathrm{HEx}} \underline{\mathrm{H}}$ zincin sequence. In some examples, the carboxylate complex $\left[\mathrm{HB}\left(\mathrm{pz}^{\mathrm{CO} 2 \mathrm{Et}, \mathrm{Me}}\right)_{3}\right] \mathrm{Zn}\left(\mathrm{H}_{2} \mathrm{O}\right)(\mathrm{OAc})$ and the amidate complex $\left[\mathrm{HB}\left(\mathrm{pz}^{\mathrm{Fu}}, \mathrm{Me}\right)_{3}\right] \mathrm{Zn}\left(\mathrm{H}_{2} \mathrm{O}\right)\left(\mathrm{NHCOCF}_{3}\right)$, ester or furyl functionalities form hydrogen bridges to water or an amidate, respectively (Fig. 11c) (Hammes et al., 2002; Maldonado Calvo \& Vahrenkamp, 2006).

Chelating ligands belonging to the poly(pyrazolyl)borates can be regarded as relatively hard donor ligands having two or three azole nitrogen atoms that can be involved in metal coordination. The analogous poly(methimazolyl)borate anions (methimazole $=1$-methyl$1 H$-imidazole-2(3H)-thione) have recently been reviewed by Reglinski and Spicer (Spicer \& Reglinski, 2009) in order to explore the properties of a softer coordination environment in an anionic ligand, provided in this case by thione sulfur atoms of the methimazole rings. The two ligand systems also differ fundamentally in the number of atoms linking the central boron atom to the donor atoms. The structural consequences of this difference are displayed in the dimensions of the chelate rings formed upon metal complex formation and the molecular symmetry generated as a consequence of the conformations adopted by these rings. Thus, complexes of the tris(pyrazolyl)borates contain three six-membered chelate rings and the resulting complexes exhibit a local coordination environment of $C_{3 \mathrm{v}}$ symmetry, while poly(methimazolyl)borates complexes contain three eight-membered chelate rings, 
thus generating a more flexible twisted or propeller-like ligand conformation and local $C_{3}$ symmetry.

The $\left[\mathrm{HB}\left(\operatorname{tim}^{\mathrm{R}}\right)_{3}\right]$ ligand systems (Fig 12a) provide a useful platform for obtaining synthetic analogues of zinc enzymes and proteins that have sulfur rich active sites such 5aminolevulinate dehydratase (ALAD) and the Ada DNA repair protein (Penner-Hahn, 2007) (Fig 8f).

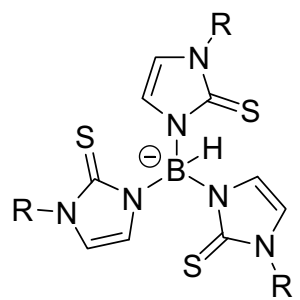

(a)

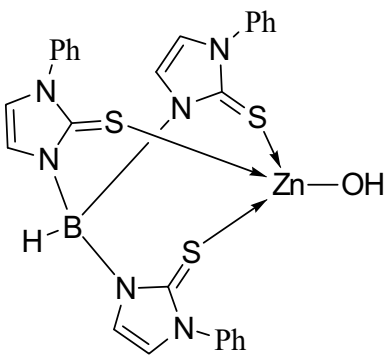

(b)

Fig. 12. Structure of: a) $\left[\mathrm{HB}\left(\operatorname{tim}^{\mathrm{R}}\right)_{3}\right]$ ligands; b) $\left[\mathrm{HB}\left(\mathrm{tim}^{\mathrm{Ph}}\right)_{3}\right] \mathrm{ZnOH}$ complex, synthetic analogue of ALAD.

The three sulfur donors of the $\left[\mathrm{HB}\left(\operatorname{tim}^{\mathrm{R}}\right)_{3}\right]$ ligand system may be used to emulate the three cysteine residues in ALAD. In particular, the zinc hydroxide complex $\left[\mathrm{HB}\left(\mathrm{tim}^{\mathrm{Ph}}\right)_{3}\right] \mathrm{ZnOH}$ complex (Bridgewater \& Parkin, 2001) (Fig 12b) is the first tetrahedral zinc hydroxide complex supported by a [S3] donor ligand to be structurally characterized by X-ray diffraction.

The mechanism of action of ALAD involves displacement of the aqua ligand by the substrate 5-aminolevulinic acid (ALA). A simple indication that displacement of the aqua ligand in such a coordination environment is facile is provided by protonation of $\left[\mathrm{HB}\left(\mathrm{tim}^{\mathrm{Ph}}\right)_{3}\right] \mathrm{ZnOH}$ with $\mathrm{HClO}_{4}$ in acetonitrile, in which the incipient aqua ligand is displaced by MeCN to give $\left\{\left[\mathrm{HB}\left(\operatorname{tim}^{\mathrm{Ph}}\right)_{3}\right] \mathrm{Zn}(\mathrm{NCMe})\right\}^{+}$(Bridgewater \& Parkin, 2000; Bridgewater \& Parkin, 2001).

Simple synthetic analogues for the $\left[(\mathrm{Cys})_{4} \mathrm{Zn}\right]$ motif of the Ada protein (Penner-Hahn, 2007) are provided by the thiolate complexes $\left[\mathrm{HB}\left(\mathrm{tim}^{\mathrm{R}}\right)_{3}\right] \mathrm{ZnSPh}(\mathrm{R}=\mathrm{Ph}$ or $\mathrm{tBu})$, in which the $\left[\mathrm{HB}\left(\operatorname{tim}^{\mathrm{R}}\right)_{3}\right]$ ligand serves the role of the three cysteine residues that remain bound to zinc during the course of the alkylation reaction (Bridgewater et al., 2000; Ibrahim et al., 2005; Melnick et al., 2006). The thiolate complexes $\left[\mathrm{HB}\left(\mathrm{tim}^{\mathrm{R}}\right)_{3}\right] \mathrm{ZnSCH}_{2} \mathrm{C}(\mathrm{O}) \mathrm{N}(\mathrm{H}) \mathrm{Ph}(\mathrm{R}=\mathrm{Ph}, \mathrm{tBu})$, which incorporate an $\mathrm{N}-\mathrm{H}$ hydrogen bonding functionality, provide a more refined structural analogue for the Ada protein (Morlok et al., 2005).

Alkylation of a zinc-cysteine thiolate residue is the key step in the mechanism of action of the Ada DNA repair protein and may be modeled by the reactivity of $\left[\mathrm{HB}\left(\operatorname{tim}^{\mathrm{R}}\right)_{3}\right] \mathrm{ZnSR}$ towards alkylating agents such as MeI. Indeed, $\left[\mathrm{HB}\left(\operatorname{tim}^{\mathrm{R}}\right)_{3}\right] \mathrm{ZnSR}$ complexes react rapidly with MeI to yield [HB(timR $\left.)_{3}\right] \mathrm{ZnI}$ and RSMe (Bridgewater et al., 2000; Ibrahim et al., 2005; Melnick et al., 2006). A comprehensive evaluation of the reactivity of a series of zinc thiolate complexes, namely $\left[\mathrm{HB}\left(\mathrm{pz}^{\mathrm{R}, \mathrm{R}^{\prime}}\right)_{3}\right] \mathrm{ZnSR},\left[\mathrm{HB}(\mathrm{pz})_{2}\left(\operatorname{tim}^{\mathrm{R}}\right)\right] \mathrm{ZnSR},\left[\mathrm{HB}\left(\mathrm{pz}^{\mathrm{R}}\right)\left(\mathrm{tim}^{\mathrm{R}}\right)_{2}\right] \mathrm{ZnSR}$, and $\left[\mathrm{HB}\left(\operatorname{tim}^{\mathrm{R}}\right)_{3}\right] \mathrm{ZnSR}$, in which the supporting ligand presents $\left[\mathrm{N}_{3}\right],\left[\mathrm{N}_{2} \mathrm{~S}\right],\left[\mathrm{NS}_{2}\right]$ and $\left[\mathrm{S}_{3}\right]$ donor arrays, demonstrates that the reactivity towards thiolate alkylation increases by four orders of magnitude across the series (Rombach et al., 2006).

In addition to the widespread use of tris(pyrazolyl)hydroborato ligands, their bis(pyrazolyl)hydroborato counterparts, $\left[\mathrm{H}_{2} \mathrm{~B}\left(\mathrm{pz}^{\mathrm{R}, \mathrm{R}^{\prime}}\right)_{2}\right]$, , were employed to prepare three- 
coordinate derivatives, e.g. $\left[\mathrm{H}_{2} \mathrm{~B}\left(\mathrm{pz}^{\mathrm{R}, \mathrm{R}^{\prime}}\right)_{2}\right] \mathrm{ZnR}$, (Gorrell et al., 1990; Looney et al., 1995) that may be functionalized by insertion of unsaturated molecules (e.g. $\mathrm{R}_{2} \mathrm{CO}, \mathrm{CO}_{2}$ and $\mathrm{R}_{2} \mathrm{CS}$ ) into the $\mathrm{B}-\mathrm{H}$ group to obtain compounds that feature new facially tridentate $\left[\mathrm{N}_{2} \mathrm{O}\right]$ and $\left[\mathrm{N}_{2} \mathrm{~S}\right]$ donor ligands that have potential for modeling certain zinc enzymes (Gorrell et al., 1990; Dowling \& Parkin, 1996; Ghosh \& Parkin, 1998). Vahrenkamp synthesized the bis(pyrazolyl)(thioimidazolyl)borate ligands, as $\left[\mathrm{N}_{2} \mathrm{~S}\right]$ donor ligands namely $\left[\mathrm{HB}\left(\mathrm{pz}^{\mathrm{x}}\right)_{2}\left(\text { tim }^{\mathrm{x}}\right)\right]^{-}$(Fig. 13a) (Benkmil et al., 2004).

The $\left[\mathrm{NS}_{2}\right]$ donor bis(thioimidazolyl)(pyrazolyl)borate ligands (Santini et al., 2010), $\left[\mathrm{HB}\left(\mathrm{pz}^{\mathrm{x}}\right)\left(\operatorname{tim}^{\mathrm{x}}\right)_{2}\right]^{-}$, can be prepared essentially by the same procedure used for the species $\left[\mathrm{HB}\left(\mathrm{pz}^{\mathrm{x}}\right)_{3}\right]^{-}$and $\left[\mathrm{HB}\left(\operatorname{tim}^{\mathrm{x}}\right)_{3}\right]^{-}$, which consists of the high-temperature reaction between $\mathrm{KBH}_{4}$ and a stoichiometric amount of the pyrazole and/or thioimidazole. The ligand $\mathrm{Li}\left[\mathrm{HB}(\mathrm{pz})\left(\mathrm{tim}^{\mathrm{Me}}\right)_{2}\right]$ (Fig. 13b) has been prepared by Parkin and applied successfully for modeling aspects of the bioinorganic chemistry of zinc enzymes. The molecular structure of $\left[\mathrm{HB}(\mathrm{pz})\left(\mathrm{tim}^{\mathrm{Me}}\right)_{2}\right] \mathrm{ZnI}$ was determined by X-ray diffraction, thereby demonstrating that the complex is indeed mononuclear with a distorted tetrahedral coordination geometry that resembles the active site of LADH (Kimblin et al., 1997; Kimblin et al., 1999; Kimblin et al., 2000).

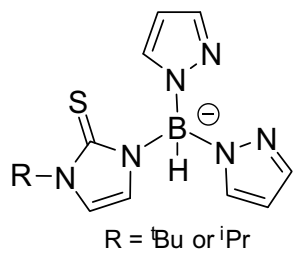

(a)

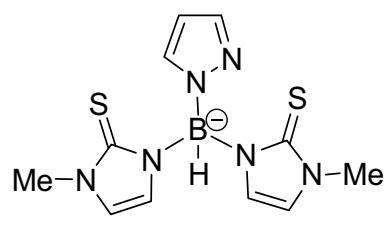

(b)<smiles></smiles>

(c)

Fig. 13. Structure of a) $\left[\mathrm{HB}(\mathrm{pz})_{2}\left(\operatorname{tim}^{\mathrm{R}}\right)\right]^{-}$, b) $\left[\mathrm{HB}(\mathrm{pz})\left(\operatorname{tim}^{\mathrm{Me}}\right)_{2}\right]^{-}$and c) $\left[\mathrm{HB}\left(\mathrm{Seim}^{\mathrm{R}}\right)_{3}\right]^{-}$ligands.

An important development with respect to the use of this class of $\left[\mathrm{NS}_{2}\right]$ donor ligands was provided by isolation of the ethanol complex, $\left\{\left[\mathrm{HB}\left(\mathrm{pz}^{\mathrm{Ph}, \mathrm{Me}}\right)(\mathrm{tim}-\mathrm{An})_{2}\right] \mathrm{Zn}(\mathrm{HOEt})\right\} \mathrm{ClO}_{4} \cdot \mathrm{EtOH}$ (Seebacher et al., 2001; Shu et al., 2003; Ibrahim et al., 2005) (timo-An = 1-(o-anisyl)-2thioimidazole) which provides the best structural model to date for LADH. Interestingly, the sulfur rich coordination environment provided by poly(thioimidazolyl)borate ligand stabilizes coordination of the alcohol to zinc, thereby suggesting that one of the reasons why LADH utilizes a sulfur rich coordination environment is to promote coordination of the alcohol relative to that of water. A recent and substantial review by J. Reglinski and M. D. Spicer (Reglinski \& Spicer, 2009) covers almost all aspects of chemistry of methimazole based soft scorpionate ligands with special refererence to their use in biomimetic chemistry (alc. dehydrogenase, Ada repair proteins, 5-aminolevulinate dehydratase, sulfite oxidase, hydrogenase, MerB) and their emerging use as radiopharmaceuticals (rhenium, technetium).

Since the poly(thioimidazolyl)borate ligands (Garner et al., 1996) have proven to be versatile, with a large variety of $\left[\mathrm{HB}\left(\operatorname{tim}^{\mathrm{R}}\right)_{3}\right]^{-}$- derivatives having been prepared, Parkin et al. envisioned that a similar series of ligands that features an $\mathrm{Se}_{3}$-donor or $\mathrm{Se}_{2}$-donor array should be accessible and thereby provide a set of ligands with modified electronic properties (Minoura et al., 2006). Indeed, the tris(2-seleno-1-R-imidazolyl)borate ligands, $\left[\mathrm{HB}\left(\text { Seim }{ }^{\mathrm{R}}\right)_{3}\right]($ Guziec \& Guziec, 1994) $(\mathrm{R}=\mathrm{Me}, \mathrm{Mes})$, have been obtained (Fig. 13c) and used to synthesize a variety of metal (zinc, cadmium and mercury) complexes (Landry et al., 
2007). The related [Se $]$-donor bis(2-seleno-1-methylimidazolyl)hydroborato ligand, $\left[\mathrm{H}_{2} \mathrm{~B}\left(\mathrm{Seim} \mathrm{Me}_{2}\right)_{2}\right]$, has also been synthesized and investigated (Landry et al., 2007; Landry \& Parkin, 2007). Interesting, whereas $\left\{\left[\mathrm{H}_{2} \mathrm{~B}\left(\mathrm{Seim}^{\mathrm{Me}}\right)_{2}\right] \mathrm{ZnI}\right\}_{2}$ exists as a dimer, the sulfur counterpart $\left[\mathrm{H}_{2} \mathrm{~B}\left(\mathrm{tim}^{\mathrm{Me}}\right)_{2}\right] \mathrm{ZnI}$ is a monomer (Kimblin et al., 1997; Kimblin et al., 2000). Another interesting aspect of $\left\{\left[\mathrm{H}_{2} \mathrm{~B}\left(\mathrm{Seim} \mathrm{Me}_{2}\right] \mathrm{ZnI}\right\}_{2}\right.$ is that the bridging entity is one of the 2seleno-1-methylimidazolyl groups rather than the halide ligands.

Several attempts to model the mononuclear active site with small biomimetic zinc complexes ended either in bisligand complexes $\left[\mathrm{ZnL}_{2}\right]$ or the formation of oligo- or poly-nuclear complexes (Parkin, 2004). C. J. Carrano and B. S. Hammes applied bulky tert-butyl groups in ortho position to a phenolate donor to overcome these problems. With their anionic tripodal $\mathrm{N}, \mathrm{N}, \mathrm{O}$ ligand (3-tert-butyl-2-hydroxy-5-methylphenyl)bis(3,5-dimethylpyrazol-1-yl)methane, $\left[\mathrm{HC}\left(\mathrm{pz}^{\mathrm{Me} 2}\right)_{2}\left(\mathrm{C}_{6} \mathrm{H}_{2} \mathrm{OH}(\mathrm{tBu}) \mathrm{Me}\right)\right]$, they obtained several tetrahedral zinc complexes with halogeno, acetato or thiolato ligands (Fig. 14a) (Hammes \& Carrano, 1999). With two nitrogen donors and an oxygen donor this ligand is well suited to mimic the active sites of thermolysin or carboxypeptidase A, although the zinc(II) in these models is bound by a phenolato donor instead of a carboxylato donor (Smith et al., 2003; Smith et al., 2005).

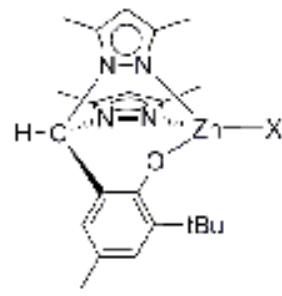

(a)
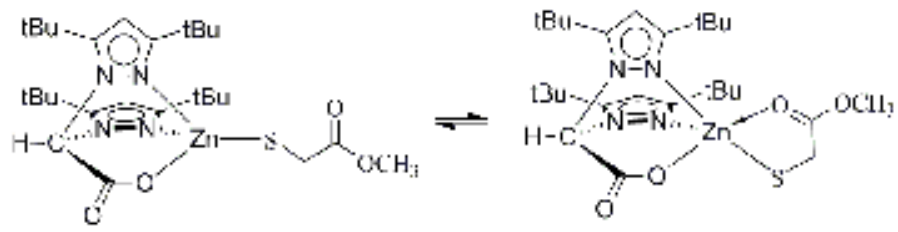

(b)

Fig. 14. a) Structure of $\left[\mathrm{HC}\left(\mathrm{pz}^{\mathrm{Me} 2}\right)_{2}\left(\mathrm{C}_{6} \mathrm{H}_{2} \mathrm{O}(\mathrm{tBu}) \mathrm{Me}\right)\right] \mathrm{ZnX}$ complexes; b) $\kappa^{1 /} / \kappa^{2}$ equilibrium in hydroxamato complexes.

Alkyl zinc complexes bearing various bis(pyrazol-1-yl)acetato ligands $\left(\mathrm{HC}\left(\mathrm{pz}^{\mathrm{R}, \mathrm{R}^{\prime}}\right)_{2}(\mathrm{COO})^{-}\right)$ offer an easy access to better structural model complexes for zinc peptidases with a 2-His-1carboxylate motif. Starting from methyl complexes $\left[\left(\mathrm{HC}\left(\mathrm{pz}^{\mathrm{R}, \mathrm{R}^{\prime}}\right)_{2}(\mathrm{COO}) \mathrm{ZnCH}_{3}\right]\right.$ so far several complexes with carboxylato-, thiolato- and also hydroxamato-ligands have been obtained by methane releasing reactions with carboxylic acids, thiols and hydroxamic acids (Beck et al., 2001; Hegelmann et al., 2003; Smith et al., 2003; Smith et al., 2005). According to the NMR spectra these complexes often exhibit a $\kappa^{1} / \kappa^{2}$ equilibrium (Fig. 14b).

Thiolates, carboxylates and hydroxamates are the most common zinc binding groups used in zinc peptidase inhibitors such as ACE inhibitors. Most of these models for carboxypeptidases have been characterised by X-ray structure determinations (Beck et al., 2001; Hegelmann et al., 2003; Smith et al., 2003; Smith et al., 2005), the model complexes helping to develop new zinc binding groups for potential peptidase inhibitors.

\subsection{Other relevant biomimetic metal systems}

In recent years complexes of scorpionate ligands were successfully used to mimic the activity of enzymes containing various metals such as vanadium, manganese, iron, cobalt, nickel, molybdenum and tungsten.

Vanadium. An important aim of the vanadium chemistry is to model vanadium histidine interactions thought to be present in the enzyme haloperoxidase (Butler, 1999). The 
occurrence of vanadium in living systems and the relevant chemistry have been reviewed (Etienne, 1996). Recently, the vanadium complexes of general type $\left\{\mathrm{VO}\left(\mathrm{O}_{2}\right)\left[\mathrm{HB}(\mathrm{pz})_{3}\right](\mathrm{Hpz})\right\}$ and $\left\{\mathrm{VO}\left(\mathrm{O}_{2}\right)\left[\mathrm{pzB}(\mathrm{pz})_{3}\right](\mathrm{Hpz})\right\}$ were synthesized, characterized and indicated as model for haloperoxidase (VHPO) enzymes (Holmes \& Carrano, 1991; Xing et al., 2007), that are able to catalyze the oxidation of halides to corresponding hypohalous acids, which readily undergo halogenation of organic substrates or conversion of hydrogen peroxide to singlet oxygen and generation of halides. The pseudooctahedral V(IV) complexes with tris(pyrazolyl)borate ligands, $\left\{\left[\mathrm{HB}(\mathrm{pz})_{3}\right] \mathrm{V}(\mathrm{O}) \mathrm{Cl}\right\} \cdot \mathrm{DMF}$ and $\left\{\left[\mathrm{HB}\left(\mathrm{pz}^{\mathrm{Me}, \mathrm{Me}}\right)_{3}\right] \mathrm{V}(\mathrm{O}) \mathrm{Cl}\right\} \cdot \mathrm{DMF}$ were found to exhibit potent biological activity (Ghosh et al., 1999).

Manganese. Mimicking the activity of manganese superoxide dismutase and of various binuclear manganese enzymes active in redox functions- was approached with $\left[\mathrm{HB}\left(\mathrm{pz}^{\mathrm{iPr}, \mathrm{iPr}}\right)_{3}\right] \mathrm{Mn}(\mathrm{OBz})$ and related binuclear complexes (Sheats et al., 1987; Kitajima et al., 1991; Kitajima et al., 1993) such as $\left[\mathrm{HB}\left(\mathrm{pz}^{\mathrm{iPr}, \mathrm{PPr}}\right)_{3}\right] \mathrm{Mn}_{2}(\mu-\mathrm{FOBz})_{3}\left(\mathrm{Hpz}^{\mathrm{iPr}, \mathrm{iPr}}\right)_{2}$ (Singh et al., 2006). Dehydrative condensation of the dinuclear $\mathrm{Mn}(\mathrm{III})$-bis $(\mu-\mathrm{oxo})$ complex $\left\{\left[\mathrm{HB}\left(\mathrm{pz} \mathrm{Pr}^{\mathrm{iP}, \mathrm{Pr}}\right)_{3}\right] \mathrm{Mn}(\mu-\right.$ O) $\}_{2}$ with $\mathrm{H}_{2} \mathrm{O}_{2}$ in the presence of 2-methylimidazole yielded the imidazole-containing peroxomanganese(III) complex $\left[\mathrm{HB}\left(\mathrm{pz}^{\mathrm{iPr}, i \mathrm{Pr}}\right)_{3}\right] \mathrm{Mn}\left(\mu_{2}-\mathrm{O}_{2}\right)\left(\mathrm{Him}^{\mathrm{Me}}\right)$. This complex may mimic the essential role of the "distal" histidine residue in the hemoglobin/myoglobin (Singh et al., 2006).

Iron. In the area of iron-containing enzymes, the behavior of the oxo-bridged di-iron enzyme hemerythrin was approximated with complexes such as $\left\{\left[\mathrm{HB}(\mathrm{pz})_{3}\right] \mathrm{Fe}\right\}_{2}(\mu-\mathrm{O})(\mu-$ OOCR) and $\left\{\left[\mathrm{HB}(\mathrm{pz})_{3}\right] \mathrm{Fe}\right\}_{2}(\mu-\mathrm{OH})(\mu-\mathrm{OOCR})$ (Armstrong \& Lippard, 1984; Armstrong et al., 1984; Czernuszewicz et al., 1987), while the complex $\left[\mathrm{HB}\left(\mathrm{pz}^{\mathrm{iPr}, \mathrm{Pr} r}\right)_{3}\right] \mathrm{Fe}(\mathrm{OBz})\left(\mathrm{CH}_{3} \mathrm{CN}\right)$ was regarded as a synthetic model for the dioxygen binding site of nonheme iron proteins (Kitajima et al., 1990). The aliphatic a-keto carboxylate $\left[\mathrm{HB}\left(\mathrm{pz}^{\mathrm{Ph}, \mathrm{Ph}}\right)_{3}\right] \mathrm{Fe}^{\mathrm{II}}\left(\mathrm{O}_{2} \mathrm{CC}(\mathrm{O}) \mathrm{CH}_{3}\right)$ and the carboxylate complexes $\left[\mathrm{HB}\left(\mathrm{pz}^{\mathrm{Ph}, \mathrm{Ph}}\right)_{3}\right] \mathrm{Fe}{ }^{\mathrm{II}}(\mathrm{OBz})$ and $\left.\left.\mathrm{HB}\left(\mathrm{pz}^{\mathrm{Ph}}, \mathrm{Ph}\right)_{3}\right] \mathrm{Fe}^{\mathrm{II}}(\mathrm{OAc})\left(\mathrm{Hpz}^{\mathrm{Ph} 2}\right)\right]$ were synthesized and studied to clarified the key role that the a-keto functionality plays in oxygen activation by a-keto acid-dependent iron enzymes (Mehn et al., 2003). The complex $\left.\left[\mathrm{HB}\left(\mathrm{pz}^{\mathrm{iPr}, \mathrm{iPr}}\right)_{3}\right] \mathrm{Fe}(\mathrm{OOPtn})\right](\mathrm{Ptn}=$ Pterinperoxo$)$ has been described as a relevant model for potential intermediates in pterin-dependent hydroxylases (Lehnert et al., 2003). Other related complexes were regarded as structural and functional models of catechol dehydrogenases (Ogihara et al., 1998) and as peroxo intermediate in the methane monooxygenase hydroxylase reaction cycle (Kim \& Lippard, 1996). The complex $\left[\mathrm{HB}\left(\mathrm{pz}^{\mathrm{Me}}, \mathrm{Me}\right)_{3}\right] \mathrm{Fe}($ catecholate $)\left(\mathrm{Hpz}^{\mathrm{Me}, \mathrm{Me}}\right)$ has been prepared and its reactivity toward oxygen investigated as model for the catechol dioxygenases (Yat et al., 2003).

Cobalt. The cobalt complexes $\left[\mathrm{HB}\left(\mathrm{pz}^{\mathrm{R}}\right)_{3}\right] \mathrm{Co}(\mathrm{X})\left(\mathrm{X}=\mathrm{N}_{3}, \mathrm{NCS} ;\left[\mathrm{HB}\left(\mathrm{pz}^{\mathrm{R}}\right)_{3}\right]=\left[\mathrm{HB}\left(\mathrm{pz}^{\mathrm{Me}, \mathrm{Me}}\right)_{3}\right]\right.$ or $\left.\left[\mathrm{HB}\left(\mathrm{pz}^{\mathrm{Ph}}\right)_{3}\right]\right)$ have been used as catalysts in the bicarbonate dehydration reaction in the presence of inhibitors (Sun et al., 2004; Sun et al., 2004).

Nikel. Monomeric five-coordinate Ni-cysteine complexes of tris(3,5-disubstituted pyrazolyl)borates $\left(\left[\mathrm{HB}\left(\mathrm{pz}^{\mathrm{Me}, \mathrm{Me}}\right)_{3}\right]^{-}\right.$and $\left.\left[\mathrm{HB}\left(\mathrm{pz}^{\mathrm{Ph}, \mathrm{Me}}\right)_{3}\right]^{-}\right)$and L-cysteine (diethyl ester and amino acid forms) were studied as being of relevance to the nickel component of the active site in several hydrogenase enzymes, which participate in the bio-generation of hydrogen and methane, as well as in nitrogen fixation (Desrochers et al., 1999). Hydrotris(3-phenyl-5methylpyrazoyl)boratonickel(II) complexes with organoxanthate or dithiocarbamate coligands equilibrate between $k^{2}$ - and $k^{3}$-chelation modes of the scorpionate ligand in solution, connecting $\mathrm{N}_{2} \mathrm{~S}_{2}$ square-planar and $\mathrm{N}_{3} \mathrm{~S}_{2}$ pyramidal ligand fields and a spin crossover. The complexes also exhibit quasi-reversible oxidations at low anodic potentials, thus modeling the structure, dynamics, and redox reactivity of the reduced nickel 
superoxide dismutase (NiSOD) active site (Ma et al., 2008). The use of analogous sterically less demanding hydrotris(3,5-dimethylpyrazolyl)borate ligands allow to obtain paramagnetic pentacoordinate $\mathrm{N}_{3} \mathrm{~S}_{2}$ structures for both xanthate and dithiocarbamate coligands in the solid state (Ma et al., 2009). However, these structures exhibit variable distortion towards a trigonal bipyramidal geometry due to enhanced rotation of the dithioacid chelates relative to the scorpionate face. Evidence was found nonetheless for retention of the spin equilibrium and one-electron redox couples in solution. These observations allow to consider steric effects arising from a pattern of 3-pyrazole ring substitution on the structure and dynamics of the biomimetic complexes.

Molybdenum and tungsten. The group 6 elements molybdenum and tungsten are the only second and third row transition metals essential to all forms of life on Earth. Molybdenum is found at the active sites of nitrogenase and all of the more than 50 known Mo-MPT enzymes $(\mathrm{MPT}=$ 'molybdopterin' or Metal-binding Pterin ene-1,2-diThiolate) that play vital roles in plant, animal, and human health, the carbon, sulfur, and nitrogen cycles, biofeedback systems, and the control of global climate (Hille, 1996; Tunney et al., 2004). The Mo-MPT enzymes feature active sites composed of a single (mononuclear) Mo atom coordinated by one or two MPT-based ligands; tungsten is also associated with MPT-based ligands in all its known biological manifestations. Chemical approaches to molybdenum enzyme sites have been directed toward mimicking a portion of the structural center in order to ascertain the role of that particular feature of the center on the chemical reactivity and the spectroscopic properties of the center. Existing sources (Holm, 1987; Holm, 1990; Enemark \& Young, 1994; Stiefel, 1997; Young \& Wedd, 1997; Young \& Young, 1997; Hille et al., 1998; Fischer \& Burgmayer, 2002; Enemark et al., 2004) provide background to earlier work in this area. The enzymology and other aspects of molybdenum biochemistry have been extensively considered elsewhere (Hille, 1996; Hille, 2002; Moura et al., 2004; Brondino et al., 2006; Schwarz \& Mendel, 2006). The action of oxygen-atom transfer enzymes which contain tris(pyrazolyl)borate-based molybdenum and tungsten pterin enzyme models centres was discussed in a review of Young and Wedd (Young \& Wedd, 1997). In a recent review we analyzed the overall progress on synthetic analogues of these enzyme centers and dissected the contributions of systems in which coordination spheres contain poly(pyrazolyl)borate ligands (Pellei et al., 2009).

\subsubsection{Nitrogenase and related synthetic models having pyrazolylborate anions}

The most extensively studied nitrogenase enzyme contains iron and molybdenum metals, and is called molybdenum nitrogenase (Burgess \& Lowe, 1996; Smith, 1999; Christiansen et al., 2001; Igarashi \& Seefeldt, 2003). In growth conditions where molybdenum concentration is low, a nitrogenase depending on iron and vanadium is expressed (Eady, 1996; Eady, 2003; Rehder, 2003; Crans et al., 2004). When both molybdenum and vanadium are unavailable, a third type of nitrogenase is expressed that contains iron as the only transition metal (Eady, 1996; Krahn et al., 2002; Siemann et al., 2002). Mo-nitrogenase is the only one for which both detailed structural and mechanistic data are available (Howard \& Rees, 1996; Mayer et al., 1999; Einsle et al., 2002). The enzymatic complex comprises two proteins: the iron-protein (Fe-protein) and the molybdenum iron-protein (MoFe-protein). There are three catalytically necessary metal-sulfur clusters in iron-molybdenum nitrogenase: the $\mathrm{Fe}_{4} \mathrm{~S}_{4}$ cluster in the Feprotein, the FeMo-cofactor and the P-cluster. The iron-molybdenum cofactor (FeMo-co) of nitrogenase (Kim \& Rees, 1992; Chan et al., 1993; Peters et al., 1997; Mayer et al., 1999; Einsle et al., 2002) is one of the most fascinating exhibits in bioinorganic chemistry, because it is 
here that the enzyme somehow catalytically cleaves the strong triple bond of $\mathrm{N}_{2}$ to give ammonia (Lowe et al., 1993). The FeMo-co has been observed in three redox states (Peters et al., 1997; Mayer et al., 1999; Schmid et al., 2002). Two different structures are known for the P-cluster and are assigned to different cluster core oxidation states. In the reduced or PN (fully reduced) state, the $\mathrm{Fe}_{8} \mathrm{~S}_{7}$ cluster can be described as two $\mathrm{Fe}_{4} \mathrm{~S}_{4}$ cubes sharing one common hexacoordinate sulfur atom, the iron atoms being linked to the protein by cysteinate ligands, two of them bridging the subcubes (Fig. 15a). In the oxidized state POx state $\left(\mathrm{P}^{2+}\right)$, oxidized by two electrons relative to $\mathrm{PN}^{\mathrm{N}}$, the central sulfur atom loses two bonds with two iron atoms in one of the subcubes, thus becoming more open. The tetrahedral coordination of these two iron atoms is then completed by extra ligations from neighboring cysteine or serine residues (Peters et al., 1997; Mayer et al., 1999; Einsle et al., 2002).
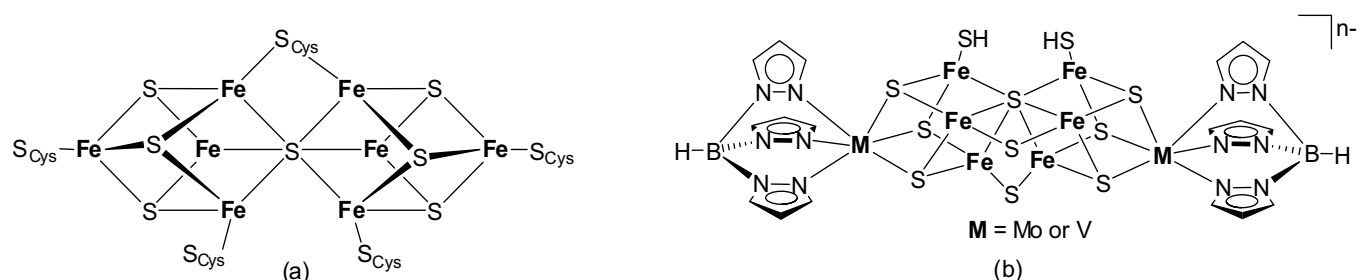

(b)

Fig. 15. a) Structure of the P-clusters of nitrogenase in the reduced state $\left[\mathrm{Fe}_{8}\left(\mu_{2}-\mathrm{S}_{\mathrm{Cys}}\right)_{2}\left(\mu_{3}-\right.\right.$ $\left.\mathrm{S})_{6}\left(\mu_{6}-\mathrm{S}\right)\right]$ core, (P-Cluster, $\mathrm{P}^{\mathrm{N}}$ state); b) molybdenum- or vanadium-containing $\mathrm{P}^{\mathrm{N}}$-cluster topological analogue of the $\mathrm{PN}^{\mathrm{N}}$ cluster of nitrogenase.

Synthetic chemists have created iron-sulfur clusters that mimic the unusual distribution of iron atoms in PN (Huang et al., 1997; Osterloh et al., 1999; Osterloh et al., 2001; Zhang et al., 2002; Zhang \& Holm, 2003; Zuo et al., 2003; Lee \& Holm, 2004; Zhang \& Holm, 2004). In particular monomeric clusters $\left\{\left[\mathrm{HB}(\mathrm{pz})_{3}\right]_{2} \mathrm{M}_{2} \mathrm{Fe}_{6} \mathrm{~S}_{9}\right\}^{\mathrm{n}} ;(\mathrm{M}=\mathrm{Mo}, \mathrm{n}=3 ; \mathrm{M}=\mathrm{V}, \mathrm{n}=4)$ are stabilized by the tris(pyrazolyl)borate ligand, showing that the cluster topology found in $\mathrm{PN}^{\mathrm{N}}$ can exist as a free entity in solution (Zhang et al., 2002).

The species in Fig. 15b, is an example of a topological analog of the $\mathrm{Fe}_{8} \mathrm{~S}_{7} \mathrm{P}$-cluster with the $\left[\mathrm{Mo}_{2} \mathrm{Fe}_{6} \mathrm{~S}_{7}\right]^{5-}$ core, isolated as the crystalline $\mathrm{Et}_{4} \mathrm{~N}^{+}$salt (Zhang et al., 2002; Zhang \& Holm, 2003).

The P-cluster structure could be stabilized in other molecules, and in particular the vanadium-containing $\mathrm{PN}^{\mathrm{N}}$-cluster topological analogue is reported (Hauser et al., 2002; Zhang et al., 2002; Zuo et al., 2003) (Fig. 15b). The clusters have a crystallographically imposed twofold axis which contains atom $\mu_{6}-\mathrm{S}$ and is perpendicular to the $\mathrm{Fe}_{4}$ plane in the center of the molecule. The $\mu_{6}-S$ atom and its associated interactions constitute the most extraordinary part of the structures of the clusters reported in Fig. 15b. Sextuply-bridging sulfur atoms are not unprecedented in synthetic clusters, but they are rarely encountered. A best-fit superposition of the $\mathrm{V}_{2} \mathrm{Fe}_{6} \mathrm{~S}_{9}$ core of $\mathrm{V}$-species in Fig. $15 \mathrm{~b}$ with the core atoms of the $\mathrm{PN}^{\mathrm{N}}$ cluster of nitrogenase (K. pneumoniae MoFe protein) (Mayer et al., 1999) leads to an rms deviation of $0.33 \AA$ in atom positions (Lee \& Holm, 2004). The corresponding deviation for Mo-species is $0.38 \AA$. One source of the deviation between synthetic clusters and the $\mathrm{PN}$ cluster is the significantly larger $\mathrm{Fe}-\left(\mu_{6}-\mathrm{S}\right)-\mathrm{Fe}$ angle of $158^{\circ}$ and its attendant effect on atom positions in the native cluster. The differences of a $\mathrm{Mo}_{2} \mathrm{Fe}_{6}$ or a $\mathrm{V}_{2} \mathrm{Fe}_{6}$ instead of an $\mathrm{Fe}_{8}$ metal content and two $\mu_{2}-S$ atoms instead of two $\mu_{2}-S_{\text {Cys }}$ bridges notwithstanding. Therefore, clusters reported in Fig. 15b are the excellent examples of molecular topological analogues of the $\mathrm{PN}^{\mathrm{N}}$ cluster of nitrogenase. 
High-nuclearity metal-sulfur clusters may function as precursors to other clusters related in structure to the P-cluster $\left(\mathrm{Fe}_{8} \mathrm{~S}_{9}\right)$ and FeMo-cofactor cluster $\left(\mathrm{MoFe}_{7} \mathrm{~S}_{9}\right)$ of nitrogenase (Zhou et al., 2002; Berlinguette et al., 2006). In particular, the double cubane $\left[\mathrm{HB}(\mathrm{pz})_{3}\right]_{2} \mathrm{Mo}_{2} \mathrm{Fe}_{6} \mathrm{~S}_{8}\left(\mathrm{PEt}_{3}\right)_{4}$ system has been investigated as model for the reactivity of the nitrogenase PN cluster (Zhang et al., 2002; Zhang \& Holm, 2003; Zuo et al., 2003); the complex sustains terminal ligand substitution with retention of the $\mathrm{Mo}_{2} \mathrm{Fe}_{6}\left(\mu_{3}-\mathrm{S}\right)_{6}\left(\mu_{4}-\mathrm{S}\right)_{2}$ core structure and rearrangement to the $\mathrm{Mo}_{2} \mathrm{Fe}_{6}\left(\mu_{2}-\mathrm{S}\right)_{2}\left(\mu_{3}-\mathrm{S}\right)_{6}\left(\mu_{6}-\mathrm{S}\right)$ topology of the nitrogenase $\mathrm{PN}^{\mathrm{N}}$ cluster (Peters et al., 1997; Mayer et al., 1999) upon reaction with certain nucleophiles. Distinct processes for the conversion of double cubanes to PN-type clusters are documented, affording the products $\left\{\left[\mathrm{HB}(\mathrm{pz})_{3}\right]_{2} \mathrm{Mo}_{2} \mathrm{Fe}_{6} \mathrm{~S}_{9}(\mathrm{SR})_{2}\right\}^{3-}$ (Zhang \& Holm, 2004), $\left\{\left[\mathrm{HB}(\mathrm{pz})_{3}\right]_{2} \mathrm{Mo}_{2} \mathrm{Fe}_{6} \mathrm{~S}_{9}(\mathrm{SH})_{2}\right\}^{3-}$ (Berlinguette \& Holm, 2006), $\left\{\left[\mathrm{HB}(\mathrm{pz})_{3}\right]_{2} \mathrm{Mo}_{2} \mathrm{Fe}_{6} \mathrm{~S}_{8}(\mathrm{OMe})_{3}\right\}^{3-}$ (Zhang \& Holm, 2004), $\left\{\left[\mathrm{HB}(\mathrm{pz})_{3}\right]_{2} \mathrm{Mo}_{2} \mathrm{Fe}_{6} \mathrm{~S}_{7}(\mathrm{OMe})_{4}\right\}^{2-}$ (Zhang \& Holm, 2004), $\left\{\left[\mathrm{HB}(\mathrm{pz})_{3}\right]_{2} \mathrm{Mo}_{2} \mathrm{Fe}_{6} \mathrm{~S}_{9}(\mathrm{OH})_{2}\right\}^{3-}$ (Hlavinka et al., 2007), $\left\{\left[\mathrm{HB}(\mathrm{pz})_{3}\right]_{2} \mathrm{Mo}_{2} \mathrm{Fe}_{6} \mathrm{~S}_{9}(\mathrm{OMe})_{2}\left(\mathrm{H}_{2} \mathrm{O}\right)\right\}^{3-}$ (Hlavinka et al., 2007), $\left\{\left\{\left[\mathrm{HB}(\mathrm{pz})_{3}\right]_{2} \mathrm{Mo}_{2} \mathrm{Fe}_{6} \mathrm{~S}_{9}\left(\mu_{2}-\mathrm{O}\right)\right\}_{2}\right\}^{5-}$ and $\left\{\left[\mathrm{HB}(\mathrm{pz})_{3}\right]_{2} \mathrm{Mo}_{2} \mathrm{Fe}_{6} \mathrm{~S}_{8} \mathrm{Q}(\mathrm{QH})_{2}\right\}^{3-}$ $(\mathrm{Q}=\mathrm{S}, \mathrm{Se})$ in which HQ- is a terminal ligand and $\mathrm{Q}^{2-}$ is a $\mu_{2}$-bridging atom in the core (Hlavinka et al., 2007). The reverse transformation of a $\mathrm{P}^{\mathrm{N}}$-type cluster to an edge-bridged double cubane has been demonstrated by the reaction of $\left\{\left[\mathrm{HB}(\mathrm{pz})_{3}\right]_{2} \mathrm{Mo}_{2} \mathrm{Fe}_{6} \mathrm{~S}_{8}(\mathrm{OMe})_{3}\right\}^{3-}$ with $\mathrm{Me}_{3} \mathrm{SiX}$ to afford $\left\{\left[\mathrm{HB}(\mathrm{pz})_{3}\right]_{2} \mathrm{Mo}_{2} \mathrm{Fe}_{6} \mathrm{~S}_{8} \mathrm{X}_{4}\right\}^{2-}\left(\mathrm{X}=\mathrm{Cl}^{-}, \mathrm{Br}-\right)$ (Zhang \& Holm, 2004).

In biomimetic research, many fewer heterometal $\mathrm{MFe}_{3} \mathrm{~S}_{4}$ cubane-type clusters have been synthesized with vanadium or tungsten than with molybdenum because of the wellestablished structural relationship of the latter to the molybdenum coordination unit in the nitrogenase MoFe-protein (Lee \& Holm, 2003; Lee \& Holm, 2004). A structural relationship appears to exist between $\mathrm{VFe}_{3} \mathrm{~S}_{4}$ clusters and the vanadium site in VFe-proteins (Eady, 1996; Eady, 2003). Far fewer vanadium than molybdenum single cubanes (SC) clusters, containing the $\left[\mathrm{VFe}_{3}\left(\mu_{3}-\mathrm{S}\right)_{4}\right]$ core of idealized trigonal symmetry, have been prepared since their inception in 1986-1987 (Kovacs \& Holm, 1986; Kovacs \& Holm, 1987; Kovacs \& Holm, 1987), and the first and only examples of vanadium edge-bridged double cubanes (EBDC), with the core $\left[\mathrm{V}_{2} \mathrm{Fe}_{6} \mathrm{~S}\left(\mu_{3}-\mathrm{S}\right)_{6}\left(\mu_{4}-\mathrm{S}\right)_{2}\right]$ of idealized centrosymmetry, were obtained in 2002 (Zhang et al., 2002). The two cluster types may be generalized as $\left\{\left[\mathrm{HB}\left(\mathrm{pz}^{\mathrm{R}}\right)_{3}\right] \mathrm{VFe}_{3} \mathrm{~S}_{4} \mathrm{X}_{3}\right\}^{z}$ and $\left\{\left[\mathrm{HB}\left(\mathrm{pz}^{\mathrm{R}}\right)_{3}\right]_{2} \mathrm{~V}_{2} \mathrm{Fe}_{6} \mathrm{~S}_{8} \mathrm{X}_{4}\right\}^{z}$ of variable oxidation level $z$ with diverse scorpionate ligands (hydrotris(pyrazolyl)borate or tris(pyrazolyl)methanesulfonate) bound to the octahedral heterometal site and ligands $X(X=$ phosphine, thiolate, or halide $)$ at the tetrahedral iron sites. These matters are best pursued with clusters in which $\left[\mathrm{HB}\left(\mathrm{pz}^{\mathrm{R}}\right)_{3}\right]^{-}$is constant, facilitating the isolation of clusters of either heterometal with the same charge $z$. Infact, the tris(pyrazolyl)borate ligand conforms to the trigonal symmetry of the SC core and in synthesis generally affords SCs with $z=-2$ and EBDCs with $z=-3$ or -4 in species carrying monoanionic ligands X (Fomitchev et al., 2002; Hauser et al., 2002; Zhang \& Holm, 2003; Zuo et al., 2003; Berlinguette et al., 2006; Pesavento et al., 2007).

Recently, a series of single cubane and edge-bridged double cubane clusters containing the cores $\left[\mathrm{VFe}_{3}\left(\mu_{3}-\mathrm{S}\right)_{4}\right]^{2+}$ and $\left[\mathrm{V}_{2} \mathrm{Fe}_{6}\left(\mu_{3}-\mathrm{S}\right)_{6}\left(\mu_{4}-\mathrm{S}\right)_{2}\right]^{2+}$ have been prepared (Scott \& Holm, 2008) by ligand substitution of the phosphine clusters $\left\{\left[\mathrm{HB}(\mathrm{pz})_{3}\right] \mathrm{VFe}_{3} \mathrm{~S}_{4}\left(\mathrm{PEt}_{3}\right)_{3}\right\}^{+}$and $\left[\mathrm{HB}(\mathrm{pz})_{3}\right]_{2} \mathrm{~V}_{2} \mathrm{Fe}_{6} \mathrm{~S}_{8}\left(\mathrm{PEt}_{3}\right)_{4}$. The single cubanes $\left\{\left[\mathrm{HB}(\mathrm{pz})_{3}\right] \mathrm{VFe}_{3} \mathrm{~S}_{4} \mathrm{~L}_{3}\right\}^{2-}$ and double cubanes $\left\{\left[\mathrm{HB}(\mathrm{pz})_{3}\right] \mathrm{V}_{2} \mathrm{Fe}_{6} \mathrm{~S}_{8} \mathrm{X}_{4}\right\}^{4-}\left(\mathrm{X}=\mathrm{F}, \mathrm{N}_{3}, \mathrm{CN}, \mathrm{PhS}\right)$ are shown by $\mathrm{X}$-ray structures to have trigonal symmetry and centrosymmetry, respectively. Single cubanes form the three-member electron transfer series $\left\{\left[\mathrm{HB}(\mathrm{pz})_{3}\right] \mathrm{VFe}_{3} \mathrm{~S}_{4} \mathrm{X}_{3}\right\}^{3-2-, 1-}$. The ligand dependence of redox potentials and electron distribution in cluster cores as sensed by ${ }^{57} \mathrm{Fe}$ isomer shifts $(\delta)$ have been determined (Scott \& Holm, 2008). 
Examples of $\mathrm{WFe}_{3} \mathrm{~S}_{4}$ clusters, nearly all in the form of tungsten-bridged double cubanes, were prepared nearly simultaneously with molybdenum containing clusters in the early development of M-Fe-S cluster chemistry (Wolff et al., 1980; Armstrong et al., 1982; Palermo et al., 1982). Few others have been prepared since that time (Coucouvanis et al., 1992; Raebiger et al., 1997). The structures of tungsten-iron-sulfur clusters have been explored using reactions based on $\left\{\left[\mathrm{HB}\left(\mathrm{pz}^{\mathrm{Me}, \mathrm{Me}}\right)_{3}\right] \mathrm{WS}_{3}\right\}$ - as precursor, which reacts with $\mathrm{FeCl}_{2}, \mathrm{NaSEt}$ and $\mathrm{S}$ affording the cubane cluster $\left\{\left[\mathrm{HB}\left(\mathrm{pz}^{\mathrm{Me}, \mathrm{Me}}\right)_{3}\right] \mathrm{WFe}_{3} \mathrm{~S}_{4} \mathrm{Cl}_{3}\right\}$-, which with $\mathrm{NaSEt}$ is converted to $\left\{\left[\mathrm{HB}\left(\mathrm{pz}^{\mathrm{Me}, \mathrm{Me}}\right)_{3}\right] \mathrm{WFe}_{3} \mathrm{~S}_{4}(\mathrm{SEt})_{3}\right\}$ - Treatment of $\left\{\left[\mathrm{HB}\left(\mathrm{pz}^{\mathrm{Me}, \mathrm{Me}}\right)_{3}\right] \mathrm{WFe}_{3} \mathrm{~S}_{4} \mathrm{Cl}_{3}\right\}-$ with $\mathrm{Et}_{3} \mathrm{P}$ yields the edge-bridged double $\left[\mathrm{HB}\left(\mathrm{pz}^{\mathrm{Me}, \mathrm{Me}}\right)_{3}\right]_{2} \mathrm{~W}_{2} \mathrm{Fe}_{6} \mathrm{~S}_{8}\left(\mathrm{PEt}_{3}\right)_{4}$ with the $\left[\mathrm{W}_{2} \mathrm{Fe}_{6}\left(\mu_{3}-\mathrm{S}\right)_{6}\left(\mu_{4^{-}}\right.\right.$ $\left.\mathrm{S})_{2}\right]$ core. The cubane cluster $\left.\left\{\left[\mathrm{HB}\left(\mathrm{pz}^{\mathrm{Me}, \mathrm{Me}}\right)_{3}\right] \mathrm{WFe}{ }_{3} \mathrm{~S}_{4} \mathrm{Cl}_{3}\right)\right\}$ - reacts also with an excess of $\mathrm{Et}_{3} \mathrm{P}$, $\mathrm{BH}_{4}^{-}$and $\mathrm{HS}^{-}$leading a mixture of products, from which $\left\{\left[\mathrm{HB}\left(\mathrm{pz}^{\mathrm{Me}, \mathrm{Me}}\right)_{3}\right]_{2} \mathrm{~W}_{2} \mathrm{Fe}_{5} \mathrm{~S}_{9} \mathrm{Na}(\mathrm{SH})(\mathrm{MeCN})\right\}^{3-}$ was identified. This cluster, as closely related $\left\{\left[\mathrm{HB}(\mathrm{pz})_{3}\right]_{2} \mathrm{Mo}_{2} \mathrm{Fe}_{6} \mathrm{~S}_{9}(\mathrm{SH})_{2}\right\}^{3-}$, exhibits a core topology $\left[\mathrm{W}_{2} \mathrm{Fe}_{5} \mathrm{Na}\left(\mu_{2}-\mathrm{S}\right)_{2}\left(\mu_{3}-\mathrm{S}\right)_{6}\left(\mu_{6}-\mathrm{S}\right)\right]$ very similar to the $\mathrm{PN}^{\mathrm{N}}$ cluster of nitrogenase (Hong et al., 2005).

\subsubsection{Hydrogenase and related synthetic models}

Hydrogenases comprise a fundamental group of bacterial enzymes that catalyze the reversible oxidation of dihydrogen to protons in aerobic and anaerobic microorganisms and, thus, play a key role in molecular bioenergetics (Fontecilla-Camps \& Ragsdale, 1999; Adams \& Stiefel, 2000; Matias et al., 2001; Carepo et al., 2002). Two classes of hydrogenases, [Fe]only $\mathrm{H}_{2}$ ases (Pandey et al., 2008) and [NiFe] $\mathrm{H}_{2}$ ases (Higuchi et al., 1999), have been studied widely, each containing their metals in a high sulfur density environment. The X-ray crystallographic studies of the active-site structure of [NiFe] $\mathrm{H}_{2}$ ases isolated from Desulfovibrio gigas, Desulfovibrio Vulgaris, Desulfovibrio fructosovorans, and Desulfovibrio desulfuricans ATCC27774 in combination with infrared spectroscopy have revealed an active site comprised of a heterobimetallic (Scys) ${ }_{2} \mathrm{Ni}(\mu \text {-Scys) })_{2}(\mu-\mathrm{X}) \mathrm{Fe}(\mathrm{CO})(\mathrm{CN})_{2}\left(\mathrm{X}=\mathrm{O}^{2-}, \mathrm{HO}_{2}^{-}, \mathrm{OH}-\right)$ cluster (Volbeda et al., 1995; Rousset et al., 1998; Garcin et al., 1999; Ogata et al., 2002; Chiou \& Liaw, 2008) (Fig. 16a). The bridging ligand $X$ was proposed to be an oxide, hydroxide, or hydroperoxide in the oxidized state and was found to be absent in the reduced state.

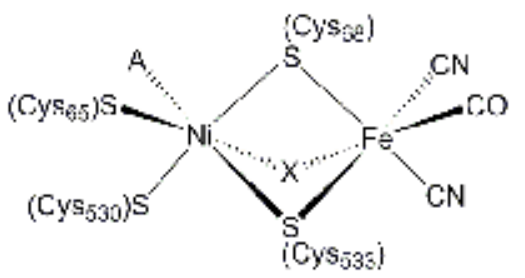

(a)

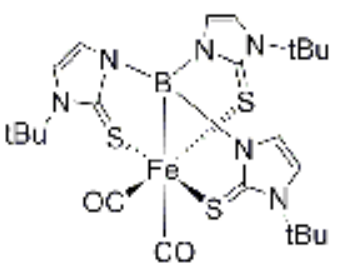

(b)

Fig. 16. a) Representation of the active site in the [NiFe] hydrogenase extracted from Desulfovibrio gigas showing the vacant coordination site $(\mathrm{A})$ and the additional bridging oxo or hydroxo ligand $(X)$ present only in the oxidized (inactive) form of the enzyme; $b$ ) structure of the boratrane $\left[\left\{\mathrm{B}\left(\mathrm{tim}^{\mathrm{tBu}}\right)_{3}\right\} \mathrm{Fe}(\mathrm{CO})_{2}\right]$.

These enzymes have become excellent targets within biomimetic chemistry. Modelling of hydrogenase enzymes requires the efficient synthesis of half-sandwich complexes of the form $\left[\mathrm{HB}\left(\operatorname{tim}^{\mathrm{R}}\right)_{3}\right] \mathrm{NiX}$ or $\left[\mathrm{H}_{2} \mathrm{~B}\left(\operatorname{tim}^{\mathrm{R}}\right)_{2}\right] \mathrm{NiX}$. The chemistry of nikel is driven by the formation of bis-ligand complexes such as $\left[\mathrm{HB}\left(\operatorname{tim}^{\mathrm{R}}\right)_{3}\right]_{2} \mathrm{Ni}$ (Garner et al., 2003; Senda et al., 2006) and 
$\left[\mathrm{H}_{2} \mathrm{~B}\left(\operatorname{tim}^{\mathrm{R}}\right)_{2}\right]_{2} \mathrm{Ni}$ (Alvarez et al., 2001; Alvarez et al., 2004). The $\left[\mathrm{H}_{2} \mathrm{~B}\left(\operatorname{tim}^{\mathrm{R}}\right)_{2}\right]_{2} \mathrm{Ni}$ complexes, having $\left[\mathrm{NiS}_{4} \mathrm{H}_{2}\right]$ cores and slightly distorted octahedral geometries resulting from the unexpected presence of two $\mathrm{Ni} \cdots \mathrm{H}-\mathrm{B}$ interactions, constitute unprecedented structural mimics that resemble the nickel coordination environment in the active form of [NiFe] hydrogenases. With $\left[\mathrm{HB}\left(\text { tim }^{\mathrm{Me}}\right)_{3}\right]_{2} \mathrm{Ni}$ it is possible to obtain the oxidised $\mathrm{Ni}(\mathrm{III})$ species $\left\{\left[\mathrm{HB}\left(\text { tim }^{\mathrm{Me}}\right)_{3}\right]_{2} \mathrm{Ni}\right\}^{+}$albeit in very low yield. By contrast, a $\mathrm{Ni}(\mathrm{III})$ species has not been isolated from studies of the analogous $\left[\mathrm{H}_{2} \mathrm{~B}\left(\operatorname{tim}^{\mathrm{R}}\right)_{2}\right]_{2} \mathrm{Ni}$ complex (Alvarez et al., 2001).

The use of a starting material containing a chelating diphosphine such as 1,2bis(diphenylphosphino)ethane (dppe) may prevent the addition of a second $\left[\mathrm{HB}\left(\operatorname{tim}^{\mathrm{R}}\right)_{3}\right]_{-}$ group to nickel and plausibly generate useful complexes such as $\left[\mathrm{HB}\left(\operatorname{tim}^{\mathrm{R}}\right)_{3}\right] \mathrm{NiX}$ or $\left[\mathrm{H}_{2} \mathrm{~B}\left(\mathrm{tim}^{\mathrm{R}}\right)_{2}\right] \mathrm{NiX}$, all while preserving the required soft donor ligand set around the metal center. Recently, using nickel phosphine complexes it was possible to obtain the species $\left[\kappa^{3}-\right.$ $\left.\mathrm{H}, \mathrm{S}, \mathrm{S}-\mathrm{HB}\left(\mathrm{tim}^{\mathrm{Tol}}\right)_{3}\right] \mathrm{Ni}(\mathrm{dppe}) \mathrm{Cl},\left[\mathrm{HB}\left(\mathrm{tim}^{\mathrm{Tol}}\right)_{3}\right] \mathrm{Ni}(\mathrm{NO})$ and $\left[\kappa^{3}-\mathrm{H}, \mathrm{S}, \mathrm{S}-\mathrm{H}_{2} \mathrm{~B}\left(\mathrm{tim}^{\mathrm{Me}}\right)_{2}\right] \mathrm{Ni}\left(\mathrm{PPh}_{3}\right)(\mathrm{NO})$ (Alvarez et al., 2004; Maffett et al., 2007).

The simple chemistry of iron is also driven by the formation of bis-ligand species $\left[\mathrm{HB}\left(\mathrm{tim}^{\mathrm{Me}}\right)_{3}\right]_{2} \mathrm{Fe}$ (Senda et al., 2006). Although $\left[\mathrm{HB}\left(\mathrm{tim}^{\mathrm{Me}}\right)_{3}\right]^{-}$produces an octahedral $\mathrm{S}_{6} \mathrm{Fe}$ species $\left[\kappa^{3}-\mathrm{S}, \mathrm{S}, \mathrm{S}-\mathrm{HB}\left(\mathrm{tim}^{\mathrm{Me}}\right)_{3}\right]_{2} \mathrm{Fe}$ (Senda et al., 2006), the $\left[\mathrm{HB}\left(\mathrm{tim}^{\mathrm{Ph}}\right)_{3}\right]^{-}$ligand generated a different isomer namely $\left[\kappa^{3}-\mathrm{H}, \mathrm{S}, \mathrm{S}-\mathrm{HB}\left(\mathrm{tim}^{\mathrm{Ph}}\right)_{3}\right]_{2} \mathrm{Fe}$ (Kimblin et al., 2001). By altering the stoichiometry and reaction conditions it was possible to produce half sandwich $\left[\mathrm{HB}\left(\text { tim }^{\mathrm{Me}}\right)_{3}\right] \mathrm{FeCl}$ complexes and chloro bridged $\left\{\left[\mathrm{HB}\left(\text { tim }^{\mathrm{Me}}\right)_{3}\right] \mathrm{Fe}(\mu-\mathrm{Cl})\right\}_{2}$ dimers of iron (Senda et al., 2006). The iron centres in hydrogenase are organometallic in nature, containing $\mathrm{Fe}-\mathrm{C} \equiv \mathrm{O}$ moieties. Although carbonyl complexes of manganese can be produced (Bailey et al., 2003) as yet we have been unable to directly and simply produce the analogous iron complexes. Parkin et al. used a circuitous route to these species, which pass through a boratrane $\left[\left\{\kappa^{4} \mathrm{~B}, \mathrm{~S}, \mathrm{~S}^{\prime}, \mathrm{S}^{\prime \prime}\right.\right.$ $\left.\left.\mathrm{B}\left(\mathrm{tim}^{\mathrm{tBu}}\right)_{3}\right\}\right] \mathrm{Fe}(\mathrm{CO})_{2}$ species (Fig. 16b). The molecular structure of $\left[\left\{\kappa^{\mathrm{B}} \mathrm{B}, \mathrm{S}, \mathrm{S}^{\prime}, \mathrm{S}^{\prime \prime}-\right.\right.$ $\left.\mathrm{B}\left(\operatorname{tim}^{\mathrm{tBu}}\right)_{3}\right\} \mathrm{Fe}(\mathrm{CO})_{2}$ has been determined by X-ray diffraction, which clearly reveals the presence of an $\mathrm{Fe} \rightarrow \mathrm{BX}_{3} \sigma$-interaction. Significantly, $\left[\left\{\kappa^{4} \mathrm{~B}, \mathrm{~S}, \mathrm{~S}^{\prime}, \mathrm{S}^{\prime \prime}-\mathrm{B}\left(\mathrm{tim}^{\mathrm{tBu}}\right)_{3}\right\}\right] \mathrm{Fe}(\mathrm{CO})_{2}$ exhibits novel reactivity towards a variety of reagents that results in eradication of the Fe-B bond via a formal 1,2-addition process and the formation of B-functionalized tris(mercaptoimidazolyl)borate derivatives, $\left[\mathrm{XB}\left(\mathrm{tim}^{\mathrm{tBu}}\right)_{3}\right] \mathrm{FeY}$ (Figueroa et al., 2006).

It is noted that the reports generated which related to hydrogenase chemistry are thus far fragmented. However, if they can be linked a viable model of hydrogenase could potentially be generated. For example, species such as $\left[\kappa^{3}-\mathrm{H}, \mathrm{S}, \mathrm{S}-\mathrm{HB}\left(\mathrm{tim}^{\mathrm{Tol}}\right)_{3}\right] \mathrm{Ni}(\mathrm{dppe}) \mathrm{Cl}$ contain a metal (boro)hydride interaction (Alvarez et al., 2001; Alvarez et al., 2004). Furthermore the hydride can be induced to leave the boron and a metal mediated hydride transfer can be initiated. As with much bio-mimetic chemistry, modelling reactions parallel the respective enzymes to a point (e.g. replacement of $\mathrm{NAD}^{+}$by benzylnicotinamide chloride in zinc alcohol dehydrogenase studies) and the manner in which the electrons are managed in the hydrogenase model reaction is unlikely to mimic that of the enzyme as there is no pathway for them to be sequestered. In this case it is likely that they will be stored in a bond such as the B-H bond (Reglinski \& Spicer, 2009).

\section{Acknowledgments}

This work was financially supported by Ministero dell'Istruzione dell'Università e della Ricerca (PRIN 20078EWK9B). We are grateful to CIRCMSB (Consorzio Interuniversitario di Ricerca in Chimica dei Metalli nei Sistemi Biologici). 


\section{References}

Adams, M.W.W. \& Stiefel, E.I. (2000). Organometallic iron: the key to biological hydrogen metabolism. Curr. Opin. Chem. Biol., 4, 214-220.

Alsfasser, R., Ruf, M., Trofimenko, S. \& Vahrenkamp, H. (1993). An L3ZnOH complex as a functional model of the enzyme carbonic anhydrase. Chem. Ber., 126, 703-710.

Alsfasser, R., Trofimenko, S., Looney, A., Parkin, G. \& Vahrenkamp, H. (1991). A mononuclear zinc hydroxide complex stabilized by a highly substituted tris(pyrazolyl)hydroborato ligand: analogies with the enzyme carbonic anhydrase. Inorg. Chem., 30, 4098-4100.

Alvarez, H.M., Krawiec, M., Donovan-Merkert, B.T., Fouzi, M. \& Rabinovich, D. (2001). Modeling Nickel Hydrogenases: Synthesis and Structure of a Distorted Octahedral Complex with an Unprecedented $\left[\mathrm{NiS}_{4} \mathrm{H}_{2}\right]$ Core. Inorg. Chem., 40, 5736-5737.

Alvarez, H.M., Tanski, J.M. \& Rabinovich, D. (2004). Poly(mercaptoimidazolyl)borate chemistry and the predominance of $\mathrm{k}^{3}-\mathrm{S}, \mathrm{S}, \mathrm{H}$ over $\mathrm{k}^{2}-\mathrm{S}, \mathrm{S}$ or $\mathrm{k}^{3}-\mathrm{S}, \mathrm{S}, \mathrm{S}$ coordination modes: unexpected formation of square pyramidal Ni(II) complexes. Polyhedron, 23, 395-403.

Andreini, C., Bertini, I., Cavallaro, G., Holliday, G.L. \& Thornton, J.M. (2008). Metal ions in biological catalysis: from enzyme databases to general principles. J. Biol. Inorg. Chem., 13, 1205-1218.

Antonyuk, S.V., Strange, R.W., Sawers, G., Eady, R.R. \& Hasnain, S.S. (2005). Atomic resolution structures of resting-state, substrate- and product-complexed $\mathrm{Cu}$-nitrite reductase provide insight into catalytic mechanism. Proc. Natl. Acad. Sci. U. S. A., 102, 12041-12046.

Armstrong, W.H. \& Lippard, S.J. (1984). Reversible protonation of the oxo bridge in a hemerythrin model compound. Synthesis, structure, and properties of $(\mu$ hydroxo)bis( $\mu$-acetato)bis[hydrotris(1-pyrazolyl)borato]diiron(III) $\left[\left(\mathrm{HB}(\mathrm{pz})_{3}\right) \mathrm{Fe}(\mathrm{OH})\left(\mathrm{O}_{2} \mathrm{CCH}_{3}\right)_{2} \mathrm{Fe}\left(\mathrm{HB}(\mathrm{pz})_{3}\right)_{2}\right]^{+}$. J. Am. Chem. Soc., 106, 4632-4633.

Armstrong, W.H., Mascharak, P.K. \& Holm, R.H. (1982). Doubly bridged double cubanes containing $\mathrm{MFe}_{3} \mathrm{~S}_{4}$ clusters. Synthesis, structure, and conversion to spin-quartet single clusters in solution. J. Am. Chem. Soc., 104, 4373-4383.

Armstrong, W.H., Spool, A., Papaefthymiou, G.C., Frankel, R.B. \& Lippard, S.J. (1984). Assembly and characterization of an accurate model for the diiron center in hemerythrin. J. Am. Chem. Soc., 106, 3653-3667.

Auld, D.S. (2006). Zinc Enzymes, John Wiley \& Sons, Ltd.

Averill, B.A. (1996). Dissimilatory nitrite and nitric oxide reductases. Chem. Rev., 96, 29512964.

Badura, D. \& Vahrenkamp, H. (2002). Pyrazolylborate-Zinc-Nucleobase-Complexes, 2: Preparations and Structures of TpCum,MeZn and TpPh,MeZn Complexes. Inorg. Chem., 41, 6013-6019.

Badura, D. \& Vahrenkamp, H. (2002). Pyrazolylborate-Zinc-Nucleobase-Complexes, 3: Base Pairing Studies. Inorg. Chem., 41, 6020-6027.

Bailey, P.J., Lorono-Gonzales, D.J., McCormack, C., Parsons, S. \& Price, M. (2003). Trismethimazolylhydroborate $(\mathrm{Tm})$ complexes of ruthenium and manganese. Inorg. Chim. Acta, 354, 61-67.

Basumallick, L., DeBeer George, S., Randall, D.W., Hedman, B., Hodgson, K.O., Fujisawa, K. \& Solomon, E.I. (2002). Spectroscopic comparison of the five-coordinate 
$\left[\mathrm{Cu}(\mathrm{SMeIm})\left(\mathrm{HB}\left(3,5-\mathrm{iPr} \mathrm{Pr}_{2}\right)_{3}\right)\right]$ with the four-coordinate $\left[\mathrm{Cu}\left(\mathrm{SCPh}_{3}\right)(\mathrm{HB}(3,5-\right.$ $\left.\left.\mathrm{iPr}_{2} \mathrm{pz}\right)_{3}\right)$ ]: effect of c.n. increase on a blue copper type site. Inorg. Chim. Acta, 337, 357-365.

Beck, A., Weibert, B. \& Burzlaff, N. (2001). Monoanionic N,N,O-scorpionate ligands and their iron(II) and zinc(II) complexes: models for mononuclear active sites of nonheme iron oxidases and zinc enzymes. Eur. J. Inorg. Chem., 521-527.

Benkmil, B., Ji, M. \& Vahrenkamp, H. (2004). Bis(pyrazolyl)(thioimidazolyl)borate Ligands: The Missing Member in the N3...S3 Scorpionate Series. Inorg. Chem., 43, 8212-8214.

Bergquist, C., Fillebeen, T., Morlok, M.M. \& Parkin, G. (2003). Protonation and Reactivity towards Carbon Dioxide of the Mononuclear Tetrahedral Zinc and Cobalt Hydroxide Complexes, $\left[\mathrm{Tp}^{\mathrm{But}, \mathrm{Me}}\right] \mathrm{ZnOH}$ and $\left[\mathrm{Tp}^{\mathrm{But}, \mathrm{Me}}\right] \mathrm{CoOH}$ : Comparison of the Reactivity of the Metal Hydroxide Function in Synthetic Analogues of Carbonic Anhydrase. J. Am. Chem. Soc., 125, 6189-6199.

Bergquist, C. \& Parkin, G. (1999). Protonation of the Hydroxide Ligand in a Synthetic Analogue of Carbonic Anhydrase, [T $\left.\mathrm{Tp}^{\mathrm{But}, \mathrm{Me}}\right] \mathrm{ZnOH}$ : Inhibition of Reactivity Towards $\mathrm{CO}_{2}$. J. Am. Chem. Soc., 121, 6322-6323.

Bergquist, C., Storrie, H., Koutcher, L., Bridgewater, B.M., Friesner, R.A. \& Parkin, G. (2000). Factors influencing the thermodynamics of zinc alkoxide formation by alcoholysis

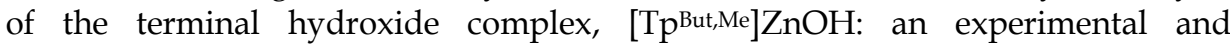
theoretical study relevant to the mechanism of action of liver alcohol dehydrogenase. J. Am. Chem. Soc., 122, 12651-12658.

Berlinguette, C.P. \& Holm, R.H. (2006). Edge-Bridged $\mathrm{Mo}_{2} \mathrm{Fe}_{6} \mathrm{~S}_{8}$ to $\mathrm{P}^{\mathrm{N}}-\mathrm{Type} \mathrm{Mo}_{2} \mathrm{Fe}_{6} \mathrm{~S}_{9}$ Cluster Conversion: Structural Fate of the Attacking Sulfide/Selenide Nucleophile. J. Am. Chem. Soc., 128, 11993-12000.

Berlinguette, C.P., Miyaji, T., Zhang, Y. \& Holm, R.H. (2006). Precursors to Clusters with the Topology of the PN Cluster of Nitrogenase: Edge-Bridged Double Cubane Clusters $\left[(\mathrm{Tp})_{2} \mathrm{Mo}_{2} \mathrm{Fe}_{6} \mathrm{~S}_{8} \mathrm{~L}_{4}\right]^{\mathrm{z}}$. Inorg. Chem., 45, 1997-2007.

Bertini, I., Gray, H.B., Stiefel, E.I. \& Valentine, J.S. (2006). Biological Inorganic Chemistry: Structure and Reactivity 1ed; University Science Books.

Bertini, I., Luchinat, C. \& Piccioli, M. (2001). Paramagnetic probes in metalloproteins. Methods Enzymol., 339, 314-340.

Bertini, I., Sigel, A. \& Sigel, H. (2001). Handbook on Metalloproteins, CRC Press.

Brand, U., Rombach, M., Seebacher, J. \& Vahrenkamp, H. (2001). Functional Modeling of Cobalamine-Independent Methionine Synthase with Pyrazolylborate-Zinc-Thiolate Complexes. Inorg. Chem., 40, 6151-6157.

Bridgewater, B.M., Fillebeen, T., Friesner, R.A. \& Parkin, G. (2000). A zinc thiolate species which mimics aspects of the chemistry of the Ada repair protein and matrix metalloproteinases: the synthesis, structure and reactivity of the tris(2-mercapto-1phenylimidazolyl)hydroborato complex [TmPh]ZnSPh. Dalton, 4494-4496.

Bridgewater, B.M. \& Parkin, G. (2000). Lead Poisoning and the Inactivation of 5Aminolevulinate Dehydratase as Modeled by the Tris(2-mercapto-1phenylimidazolyl)hydroborato Lead Complex, $\left\{\left[\mathrm{Tm}{ }^{\mathrm{Ph}}\right] \mathrm{Pb}\right\}\left[\mathrm{ClO}_{4}\right]$. J. Am. Chem. Soc., 122, 7140-7141.

Bridgewater, B.M. \& Parkin, G. (2001). A zinc hydroxide complex of relevance to 5aminolevulinate dehydratase: The synthesis, structure and reactivity of the complex $\left[\mathrm{Tm}^{\mathrm{Ph}}\right] \mathrm{ZnOH}$. Inorg. Chem. Commun., 4, 126-129. 
Brombacher, H. \& Vahrenkamp, H. (2004). Pyrazolylborate-Zinc Alkoxide Complexes. 3. Acid-Base Reactions. Inorg. Chem., 43, 6054-6060.

Brondino, C.D., Romao, M.J., Moura, I. \& Moura, J.J.G. (2006). Molybdenum and tungsten enzymes: the xanthine oxidase family. Curr. Opin. Chem. Biol., 10, 109-114.

Bruce, M.I. \& Ostazewski, A.P.P. (1973). Group IB metal chemistry. I. Preparation and reactions of the carbonyl(hydrotripyrazol-1-ylborato)copper(I) complex. J. Chem. Soc., Dalton Trans., 2433-2436.

Burgess, B.K. \& Lowe, D.J. (1996). Mechanism of Molybdenum Nitrogenase. Chem. Rev., 96, 2983-3011.

Butler, A. (1999). Mechanistic considerations of the vanadium haloperoxidases. Coord. Chem. Rev., 187, 17-35.

Calderone, V., Dolderer, B., Hartmann, H.-J., Echner, H., Luchinat, C., Del Bianco, C., Mangani, S. \& Weser, U. (2005). The crystal structure of yeast copper thionein: The solution of a long-lasting enigma. Proc. Natl. Acad. Sci. U. S. A., 102, 51-56.

Canters, G.W. \& Gilardi, G. (1993). Engineering type 1 copper sites in proteins. FEBS Lett., $325,39-48$.

Carepo, M., Tierney, D.L., Brondino, C.D., Yang, T.C., Pamplona, A., Telser, J., Moura, I., Moura, J.J.G. \& Hoffman, B.M. (2002). ${ }^{17} \mathrm{O}$ ENDOR Detection of a Solvent-Derived $\mathrm{Ni}-(\mathrm{OHx})-\mathrm{Fe}$ Bridge That Is Lost upon Activation of the Hydrogenase from Desulfovibrio gigas. J. Am. Chem. Soc., 124, 281-286.

Carrier, S.M., Ruggiero, C.E., Tolman, W.B. \& Jameson, G.B. (1992). Synthesis and structural characterization of a mononuclear copper nitrosyl complex. J. Am. Chem. Soc., 114, 4407-4408.

Chan, M.K., Kim, J. \& Rees, D.C. (1993). The nitrogenase iron-molybdenum-cofactor and Pcluster pair: 2.2. A resolution structures. Science, 260, 792-794.

Chaudhuri, P. \& Wieghardt, K. (2001). Phenoxyl radical complexes. Prog. Inorg. Chem., 50, 151-216.

Chen, P., Root, D.E., Campochiaro, C., Fujisawa, K. \& Solomon, E.I. (2003). Spectroscopic and Electronic Structure Studies of the Diamagnetic Side-On CuII-Superoxo Complex $\mathrm{Cu}\left(\mathrm{O}_{2}\right)\left[\mathrm{HB}(3-\mathrm{R}-5-\mathrm{iPrpz})_{3}\right]$. Antiferromagnetic Coupling versus Covalent Delocalization. J. Am. Chem. Soc., 125, 466-474.

Chiou, T.-W. \& Liaw, W.-F. (2008). Mononuclear Nickel(III) Complexes $\left[\mathrm{Ni}{ }^{\mathrm{II}}(\mathrm{OR})\left(\mathrm{P}\left(\mathrm{C}_{6} \mathrm{H}_{3}-3-\right.\right.\right.$ $\left.\left.\left.\mathrm{SiMe}_{3}-2-\mathrm{S}\right)_{3}\right)\right]^{-}(\mathrm{R}=\mathrm{Me}, \mathrm{Ph})$ Containing the Terminal Alkoxide Ligand: Relevance to the Nickel Site of Oxidized-Form [NiFe] Hydrogenases. Inorg. Chem., 47, 7908-7913.

Christiansen, J., Dean, D.R. \& Seefeldt, L.C. (2001). Mechanistic features of the Mocontaining nitrogenase. Annu. Rev. Plant Physiol. Plant Mol. Biol., 52, 269-295.

Churchill, M.R., DeBoer, B.G., Rotella, F.J., Abu Salah, O.M. \& Bruce, M.I. (1975). Determination of the crystal structure and molecular geometry of [hydrotris(1pyrazolyl)borato]copper(I) carbonyl. Unique structural investigation of a coppercarbonyl linkage. Inorg. Chem., 14, 2051-2056.

Coleman, J.E. (1992). Zinc Proteins: Enzymes, Storage Proteins, Transcription Factors, and Replication Proteins. Annual Review of Biochemistry, 61, 897-946.

Colman, P.M., Freeman, H.C., Guss, J.M., Murata, M., Norris, V.A., Ramshaw, J.A.M. \& Venkatappa, M.P. (1978). X-ray crystal structure analysis of plastocyanin at $2.7 \AA$ resolution. Nature, 272, 319-324. 
Coucouvanis, D., Al-Ahmad, S.A., Salifoglou, A., Papaefthymiou, V., Kostikas, A. \& Simopoulos, A. (1992). Synthesis, characterization, and reactivity of new clusters that contain the $\left[\mathrm{MFe}_{3} \mathrm{~S}_{4}\right]$ core, $\mathrm{M}=$ molybdenum, tungsten. J. Am. Chem. Soc., 114, 2472-2482.

Crans, D.C., Smee, J.J., Gaidamauskas, E. \& Yang, L. (2004). The Chemistry and Biochemistry of Vanadium and the Biological Activities Exerted by Vanadium Compounds. Chem. Rev., 104, 849-902.

Cuff, M.E., Miller, K.I., Van Holde, K.E. \& Hendrickson, W.A. (1998). Crystal structure of a functional unit from Octopus hemocyanin. J. Mol. Biol., 278, 855-870.

Cvetkovic, M., Batten, S.R., Moubaraki, B., Murray, K.S. \& Spiccia, L. (2001). Copper(I) tris(pyrazolyl)methane complexes and their reactivity towards dioxygen. Inorg. Chim. Acta, 324, 131-140.

Czernuszewicz, R.S., Sheats, J.E. \& Spiro, T.G. (1987). Resonance Raman spectra and excitation profile for bis(acetato)bis(hydrotripyrazolylborato)oxodiiron, a hemerythrin analog. Inorg. Chem., 26, 2063-2067.

Den Blaauwen, T. \& Canters, G.W. (1993). Creation of type-1 and type-2 copper sites by addition of exogenous ligands to the Pseudomonas aeruginosa azurin His117Gly mutant. J. Am. Chem. Soc., 115, 1121-1129.

Den Blaauwen, T., Hoitink, C.W., Canters, G.W., Han, J., Loehr, T.M. \& Sanders-Loehr, J. (1993). Resonance Raman spectroscopy of the azurin His117Gly mutant. Interconversion of type 1 and type 2 copper sites through exogenous ligands. Biochemistry, 32, 12455-12464.

Desrochers, P.J., Cutts, R.W., Rice, P.K., Golden, M.L., Graham, J.B., Barclay, T.M. \& Cordes, A.W. (1999). Characteristics of Five-Coordinate Nickel-Cysteine Centers. Inorg. Chem., 38, 5690-5694.

Dias, H.V.R. \& Fianchini, M. (2007). Fluorinated Tris(Pyrazolyl)Borates and Silver(I) Complexes of Group 14 Ligands. Comments Inorg. Chem., 28, 73-92.

Dowling, C. \& Parkin, G. (1996). Elaboration of the bis(pyrazolyl)hydroborato ligand [Bp $\left.\mathrm{But}^{\mathrm{Bu}, \mathrm{Pri}}\right]$ into the $\mathrm{NNO}$ donor ligand, [(MeO)BpBut,Pri]: structure characterization of a complex in which the $\left[(\mathrm{MeO}) \mathrm{Bp}^{\text {But,Pri] }}\right.$ ligand models the binding of zinc to the peptide backbone in thermolysin. Polyhedron, 15, 2463-2465.

Durley, R., Chen, L., Lim, L.W., Mathews, F.S. \& Davidson, V.L. (1993). Crystal structure analysis of amicyanin and apoamicyanin from Paracoccus denitrificans at $2.0 \AA$ and 1.8 Å resolution. Protein Science, 2, 739-752.

Eady, R.R. (1996). Structure-function relationships of alternative nitrogenases. Chem. Rev., 96, 3013-3030.

Eady, R.R. (2003). Current status of structure function relationships of vanadium nitrogenase. Coord. Chem. Rev., 237, 23-30.

Edelmann, F.T. (2001). Versatile scorpionates-new developments in the coordination chemistry of pyrazolylborate ligands. Angew. Chem. Int. Ed. Engl., 40, 1656-1660.

Einsle, O., Tezcan, F.A., Andrade, S.L.A., Schmid, B., Yoshida, M., Howard, J.B. \& Rees, D.C. (2002). Nitrogenase MoFe-protein at $1.16 \AA$ resolution: A central ligand in the FeMo-cofactor. Science, 297, 1696-1700.

Enemark, J.H., Cooney, J.J.A., Wang, J.-J. \& Holm, R.H. (2004). Synthetic Analogues and Reaction Systems Relevant to the Molybdenum and Tungsten Oxotransferases. Chem. Rev., 104, 1175-1200. 
Enemark, J.H. \& Feltham, R.D. (1974). Principles of structure, bonding, and reactivity for metal nitrosyl complexes. Coord. Chem. Rev., 13, 339-406.

Enemark, J.H. \& Young, C.G. (1994). Bioinorganic chemistry of pterin-containing molybdenum and tungsten enzymes. Adv. Inorg. Chem., 40, 1-88.

Etienne, M. (1996). Hydridotris(pyrazolyl)borato complexes of the Group 5 metals: inorganic and organometallic chemistry. Coord. Chem. Rev., 156, 201-236.

Figueroa, J.S., Melnick, J.G. \& Parkin, G. (2006). Reactivity of the Metal $\rightarrow \mathrm{BX}_{3}$ Dative $\sigma$-Bond: 1,2-Addition Reactions of the $\mathrm{Fe} \rightarrow \mathrm{BX}_{3}$ Moiety of the Ferraboratrane Complex $\left[\mathrm{K}^{4-}\right.$ $\left.\mathrm{B}\left(\mathrm{mim}^{\mathrm{But}}\right)_{3}\right] \mathrm{Fe}(\mathrm{CO})_{2}$. Inorg. Chem., 45, 7056-7058.

Fischer, B. \& Burgmayer, S.J.N. (2002). Models for the pyranopterin-containing molybdenum and tungsten cofactors. Met. Ions Biol. Syst., 39, 265-316.

Fischer, N.V., Türkoglu, G. \& Burzlaff, N. (2009). Scorpionate complexes suitable for enzyme inhibitor studies. Current Bioactive Compounds, 5, 277-295.

Fomitchev, D.V., McLauchlan, C.C. \& Holm, R.H. (2002). Heterometal Cubane-Type $\mathrm{MFe}_{3} \mathrm{~S}_{4}$ Clusters $(\mathrm{M}=\mathrm{Mo}, \mathrm{V})$ Trigonally Symmetrized with Hydrotris(pyrazolyl)borate(1-) and Tris(pyrazolyl)methanesulfonate(1-) Capping Ligands. Inorg. Chem., 41, 958966.

Fontecilla-Camps, J.C. \& Ragsdale, S.W. (1999). Nickel-iron-sulfur active sites: hydrogenase and CO dehydrogenase. Adv. Inorg. Chem., 47, 283-333.

Foster, C.L., Liu, X., Kilner, C.A., Thornton-Pett, M. \& Halcrow, M.A. (2000). Complexes of 2-hydroxy-5-methyl-1,4-benzoquinone as models for the 'TPQ-on' form of copper amine oxidases. Dalton, 4563-4568.

Fraústo da Silva, J.J.R. \& Williams, R.J.P. (2001). The Biological Chemistry of the Elements - The Inorganic Chemistry of Life, Oxford University Press, New York.

Fujisawa, K., Tanaka, M., Moro-oka, Y. \& Kitajima, N. (1994). A Monomeric Side-On Superoxocopper(II) Complex: $\mathrm{Cu}\left(\mathrm{O}_{2}\right)\left(\mathrm{HB}(3-\mathrm{tBu}-5-\mathrm{iPrpz})_{3}\right)$. J. Am. Chem. Soc., 116, 12079-12080.

Fujisawa, K., Tateda, A., Miyashita, Y., Okamoto, K.-i., Paulat, F., Praneeth, V.K.K., Merkle, A. \& Lehnert, N. (2008). Structural and spectroscopic characterization of mononuclear copper(I) nitrosyl complexes: end-on versus side-on coordination of NO to copper(I). J. Am. Chem. Soc., 130, 1205-1213.

Garcin, E., Vernede, X., Hatchikian, E.C., Volbeda, A., Frey, M. \& Fontecilla-Camps, J.C. (1999). The crystal structure of a reduced [NiFeSe] hydrogenase provides an image of the activated catalytic center. Structure, 7, 557-566.

Garner, M., Lewinski, K., Pattek-Janczyk, A., Reglinski, J., Sieklucka, B., Spicer, M.D. \& Szaleniec, M. (2003). Structural and spectroscopic characterisation of bis-ligand complexes of iron(II), nickel(II) and nickel(III) with the hydrotris(methimazolyl)borate anion. Dalton Trans., 1181-1185.

Garner, M., Reglinski, J., Cassidy, I., Spicer, M.D. \& Kennedy, A.R. (1996). Hydrotris(methimazolyl)borate, a soft analog of hydrotris(pyrazolyl)borate. Preparation and crystal structure of a novel zinc complex. Chem. Commun., 19751976.

Gennari, M. \& Marchiò, L. (2009). Cu-Complexes with Scorpionate Ligands as Models for the Binding Sites of Copper Proteins. Current Bioactive Compounds, 5, 244-263.

Ghosh, P., D'Cruz, O.J., DuMez, D.D., Peitersen, J. \& Uckun, F.M. (1999). Structural and functional characterization of seven spermicidal vanadium(IV) complexes: 
potentiation of activity by methyl substitution on the cyclopentadienyl rings. $J$. Inorg. Biochem., 75, 135-143.

Ghosh, P. \& Parkin, G. (1998). Mimicking the binding of glutamate to zinc in thermolysin and carboxypeptidase: the synthesis of $\left[\eta^{3}-\left(\mathrm{HCO}_{2}\right) \mathrm{Bp} \mathrm{put}^{\mathrm{Br}} \mathrm{Pr}\right] \mathrm{ZnCl}$ by insertion of $\mathrm{CO}_{2}$ into a B-H bond of the bis(pyrazolyl)hydroborato zinc complex [Bp $\left.{ }^{\text {But,Pri }}\right] \mathrm{ZnCl}$. J. Chem. Soc., Dalton Trans., 2281-2284.

Godden, J.W., Turley, S., Teller, D.C., Adman, E.T., Liu, M.Y., Payne, W.J. \& LeGall, J. (1991). The 2.3 angstrom $x$-ray structure of nitrite reductase from Achromobacter cycloclastes. Science, 253, 438-442.

Gorrell, I.B., Looney, A., Parkin, G. \& Rheingold, A.L. (1990). [Bis(3-tertbutylpyrazolyl)hydroborato]zinc alkyl derivatives: competitive reactivity of zinccarbon and boron-hydrogen bonds. J. Am. Chem. Soc., 112, 4068-4069.

Guziec, L.J. \& Guziec, F.S., Jr. (1994). A Directed Metalation Route to the Selenium Analog of Methimazole. J. Org. Chem., 59, 4691-4692.

Halcrow, M.A., Lindy Chia, L.M., Davies, J.E., Liu, X., Yellowlees, L.J., McInnes, E.J.L. \& Mabbs, F.E. (1998). Spectroscopic characterization of a copper(II) complex of a thioether-substituted phenoxyl radical: a new model for galactose oxidase. Chem. Commun., 2465-2466.

Halcrow, M.A., LindyChia, L.M., Liu, X., McInnes, E.J.L., Yellowlees, L.J., Mabbs, F.E., Scowen, I.J., McPartlin, M. \& Davies, J.E. (1999). Syntheses, structures and electrochemistry of copper(II) salicylaldehyde/tris(3-phenylpyrazolyl)borate complexes as models for the radical copper oxidases. J. Chem. Soc., Dalton Trans., 1753-1762.

Halfen, J.A. \& Tolman, W.B. (1994). Synthetic Model of the Substrate Adduct to the Reduced Active Site of Copper Nitrite Reductase. J. Am. Chem. Soc., 116, 5475-5476.

Hammes, B.S. \& Carrano, C.J. (1999). Synthesis and Characterization of Pseudotetrahedral $\mathrm{N}_{2} \mathrm{O}$ and $\mathrm{N}_{2} \mathrm{~S}$ Zinc(II) Complexes of Two Heteroscorpionate Ligands: Models for the Binding Sites of Several Zinc Metalloproteins. Inorg. Chem., 38, 4593-4600.

Hammes, B.S., Luo, X., Carrano, M.W. \& Carrano, C.J. (2002). Zinc complexes of hydrogen bond accepting ester substituted trispyrazolylborates. Inorg. Chim. Acta, 341, 33-38.

Hart, P.J., Nersissian, A.M., Herrmann, R.G., Nalbandyan, R.M., Valentine, J.S. \& Eisenberg, D. (1996). A missing link in cupredoxins: crystal structure of cucumber stellacyanin at $1.6 \AA$ resolution. Prot. Sci., 5, 2175-2183.

Hartmann, U. \& Vahrenkamp, H. (1994). Pyrazolylborate-zinc complexes with drugs as ligands. Chem. Ber., 127, 2381-2385.

Hauser, C., Bill, E. \& Holm, R.H. (2002). Single- and Double-Cubane Clusters in the Multiple Oxidation States $\left[\mathrm{VFe}_{3} \mathrm{~S}_{4}\right]^{3+, 2+, 1+}$. Inorg. Chem., 41, 1615-1624.

Hazes, B., Magnus, K.A., Bonaventura, C., Bonaventura, J., Dauter, Z., Kalk, K.H. \& Hol, W.G. (1993). Crystal structure of deoxygenated Limulus polyphemus subunit II hemocyanin at $2.18 \AA$ resolution: clues for a mechanism for allosteric regulation. Prot. Sci., 2, 597-619.

Hegelmann, I., Beck, A., Eichhorn, C., Weibert, B. \& Burzlaff, N. (2003). Alkylzinc complexes with achiral and chiral monoanionic $\mathrm{N}, \mathrm{N}, \mathrm{O}$ heteroscorpionate ligands. Eur. J. Inorg. Chem., 339-347. 
Henkel, G. \& Krebs, B. (2004). Metallothioneins: Zinc, Cadmium, Mercury, and Copper Thiolates and Selenolates Mimicking Protein Active Site Features - Structural Aspects and Biological Implications. Chem. Rev., 104, 801-824.

Higuchi, Y., Ogata, H., Miki, K., Yasuoka, N. \& Yagi, T. (1999). Removal of the bridging ligand atom at the $\mathrm{Ni}-\mathrm{Fe}$ active site of [NiFe] hydrogenase upon reduction with $\mathrm{H}_{2}$, as revealed by X-ray structure analysis at $1.4 \AA$ resolution. Structure, 7, 549-556.

Hille, R. (1996). The Mononuclear Molybdenum Enzymes. Chem. Rev., 96, 2757-2816.

Hille, R. (2002). Molybdenum and tungsten in biology. Trends Biochem. Sci., 27, 360-367.

Hille, R., Retey, J., Bartlewski-Hof, U., Reichenbecher, W. \& Schink, B. (1998). Mechanistic aspects of molybdenum-containing enzymes. FEMS Microbiol. Rev., 22, 489-501.

Himmelwright, R.S., Eickman, N.C., LuBien, C.D. \& Solomon, E.I. (1980). Chemical and spectroscopic comparison of the binuclear copper active site of mollusc and arthropod hemocyanins. J. Am. Chem. Soc., 102, 5378-5388.

Hlavinka, M.L., Miyaji, T., Staples, R.J. \& Holm, R.H. (2007). Hydroxide-Promoted Core Conversions of Molybdenum-Iron-Sulfur Edge-Bridged Double Cubanes: OxygenLigated Topological PN Clusters. Inorg. Chem., 46, 9192-9200.

Holland, P.L. \& Tolman, W.B. (1999). Three-Coordinate Cu(II) Complexes: Structural Models of Trigonal-Planar Type 1 Copper Protein Active Sites. J. Am. Chem. Soc., $121,7270-7271$.

Holland, P.L. \& Tolman, W.B. (2000). A Structural Model of the Type 1 Copper Protein Active Site: $\mathrm{N}_{2} \mathrm{~S}$ (thiolate)S(thioether) Ligation in a $\mathrm{Cu}(\mathrm{II})$ Complex. J. Am. Chem. Soc., 122, 6331-6332.

Holm, R.H. (1987). Metal-centered oxygen atom transfer reactions. Chem. Rev., 87, 1401-1449.

Holm, R.H. (1990). The biologically relevant oxygen atom transfer chemistry of molybdenum: from synthetic analog systems to enzymes. Coord. Chem. Rev., 100, 183-221.

Holm, R.H., Kennepohl, P. \& Solomon, E.I. (1996). Structural and Functional Aspects of Metal Sites in Biology. Chem. Rev., 96, 2239-2314.

Holm, R.H. \& Solomon, E.I. (2004). Preface: Biomimetic Inorganic Chemistry. Chem. Rev., 104, 347-348.

Holmes, S. \& Carrano, C.J. (1991). Models for the binding site in bromoperoxidase: mononuclear vanadium $(\mathrm{V})$ phenolate complexes of the hydridotris $(3,5-$ dimethylpyrazolyl)borate ligand. Inorg. Chem., 30, 1231-1235.

Hong, D., Zhang, Y. \& Holm, R.H. (2005). Heterometal cubane-type $\mathrm{WFe}_{3} \mathrm{~S}_{4}$ and related clusters trigonally symmetrized with hydrotris(3,5-dimethylpyrazolyl)borate. Inorg. Chim. Acta, 358, 2303-2311.

Howard, J.B. \& Rees, D.C. (1996). Structural Basis of Biological Nitrogen Fixation. Chem. Rev., 96, 2965-2982.

Hu, Z., Williams, R.D., Tran, D., Spiro, T.G. \& Gorun, S.M. (2000). Re-engineering EnzymeModel Active Sites: Reversible Binding of $\mathrm{O}_{2}$ at Ambient Conditions by a Bioinspired Copper Complex. J. Am. Chem. Soc., 122, 3556-3557.

Huang, J., Goh, C. \& Holm, R.H. (1997). On the Existence in Solution of Doubly ThiolateBridged Double Cubanes Containing the Heterometal $\left[\mathrm{MFe}_{3} \mathrm{~S}_{4}\right]$ Core $(\mathrm{M}=\mathrm{Mo}, \mathrm{W})$. Inorg. Chem., 36, 356-361. 
Hulse, C.L., Tiedje, J.M. \& Averill, B.A. (1989). Evidence for a copper-nitrosyl intermediate in denitrification by the copper-containing nitrite reductase of Achromobacter cycloclastes. J. Am. Chem. Soc., 111, 2322-2323.

Ibrahim, M.M., He, G., Seebacher, J., Benkmil, B. \& Vahrenkamp, H. (2005). Biomimetic thiolate alkylation with zinc pyrazolylbis(thioimidazolyl)borate complexes. Eur. J. Inorg. Chem., 4070-4077.

Ibrahim, M.M., Perez Olmo, C., Tekeste, T., Seebacher, J., He, G., Calvo, J.A.M., Boehmerle,

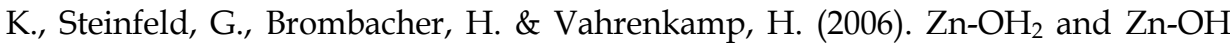
Complexes with Hydroborate-Derived Tripod Ligands: A Comprehensive Study. Inorg. Chem., 45, 7493-7502.

Ibrahim, M.M., Seebacher, J., Steinfeld, G. \& Vahrenkamp, H. (2005). Tris(thioimidazolyl)borate-Zinc-Thiolate Complexes for the Modeling of Biological Thiolate Alkylations. Inorg. Chem., 44, 8531-8538.

Igarashi, R.Y. \& Seefeldt, L.C. (2003). Nitrogen fixation: the mechanism of the Mo-dependent nitrogenase. Crit. Rev. Biochem. Mol. Biol., 38, 351-384.

Ito, N., Phillips, S.E.V., Yadav, K.D.S. \& Knowles, P.F. (1994). Crystal structure of a free radical enzyme, galactose oxidase. J. Mol. Biol., 238, 794-814.

Jackson, M.A., Tiedje, J.M. \& Averill, B.A. (1991). Evidence for a NO-rebound mechanism for production of $\mathrm{N}_{2} \mathrm{O}$ from nitrite by the copper-containing nitrite reductase from Achromobacter cycloclastes. FEBS Lett, 291, 41-44.

Jacobsen, F.E. \& Cohen, S.M. (2004). Using Model Complexes To Augment and Advance Metalloproteinase Inhibitor Design. Inorg. Chem., 43, 3038-3047.

Jacobsen, F.E., Lewis, J.A. \& Cohen, S.M. (2006). A New Role for Old Ligands: Discerning Chelators for Zinc Metalloproteinases. J. Am. Chem. Soc., 128, 3156-3157.

Jacobsen, F.E., Lewis, J.A., Heroux, K.J. \& Cohen, S.M. (2007). Characterization and evaluation of pyrone and tropolone chelators for use in metalloprotein inhibitors. Inorg. Chim. Acta, 360, 264-272.

Kanan, M.W. \& Nocera, D.G. (2008). In Situ Formation of an Oxygen-Evolving Catalyst in Neutral Water Containing Phosphate and $\mathrm{Co}^{2+}$. Science, 321, 1072-1075.

Kim, J. \& Rees, D.C. (1992). Crystallographic structure and functional implications of the nitrogenase molybdenum-iron protein from Azotobacter vinelandii. Nature, 360, 553-560.

Kim, K. \& Lippard, S.J. (1996). Structure and Mossbauer Spectrum of a ( $\mu$-1,2-Peroxo)bis( $\mu$ carboxylato)diiron(III) Model for the Peroxo Intermediate in the MMO Hydroxylase Reaction Cycle. J. Am. Chem. Soc., 118, 4914-4915.

Kimblin, C., Bridgewater, B.M., Churchill, D.G., Hascall, T. \& Parkin, G. (2000). Bis(mercaptoimidazolyl)(pyrazolyl)hydroborato Complexes of $\mathrm{Zn}, \mathrm{Cd}$ and Co: Struct. Evidence for the Enhanced Tendency of $\mathrm{Zn}$ in Biological Systems to Adopt Tetrahedral M[S 4 ] Coord. Inorg. Chem., 39, 4240-4243.

Kimblin, C., Bridgewater, B.M., Churchill, D.G. \& Parkin, G. (1999). Mononuclear tris(2mercapto-1-arylimidazolyl)hydroborato complexes of zinc, [TmAr]ZnX: structural evidence that a sulfur rich coordination environment promotes the formation of a tetrahedral alcohol complex in a synthetic analogue of LADH. Chem. Commun., 2301-2302. 
Kimblin, C., Bridgewater, B.M., Hascall, T. \& Parkin, G. (2000). The synthesis and structural characterization of bis(mercaptoimidazolyl)hydroborato complexes of $\mathrm{Li}, \mathrm{Tl}$ and Zn. J. Chem. Soc., Dalton Trans., 891-897.

Kimblin, C., Churchill, D.G., Bridgewater, B.M., Girard, J.N., Quarless, D.A. \& Parkin, G. (2001). Tris(mercaptoimidazolyl)hydroborato complexes of cobalt and iron, $\left[\mathrm{Tm}^{\mathrm{Ph}}\right]_{2} \mathrm{M} \quad(\mathrm{M}=\mathrm{Fe}, \mathrm{Co})$ : structural comparisons with their tris(pyrazolyl)hydroborato counterparts. Polyhedron, 20, 1891-1896.

Kimblin, C., Hascall, T. \& Parkin, G. (1997). Modeling the Catalytic Site of Liver Alcohol Dehydrogenase: Synthesis and Structural Characterization of $\left[\mathrm{HB}\left(\mathrm{tim}^{\mathrm{Me}}\right)_{2} \mathrm{pz}\right] \mathrm{ZnI}$. Inorg. Chem., 36, 5680-5681.

Kitajima, N. (1992). Synthetic approach to the structure and function of copper proteins. Adv. Inorg. Chem., 39, 1-77.

Kitajima, N., Fujisawa, K., Hikichi, S. \& Morooka, Y. (1993). Formation of ( $\mu$-hydroxo)( $\mu-$ azido) dinuclear copper complex from $\mu-\eta^{2}: \eta^{2}$-peroxo complex. J. Am. Chem. Soc., $115,7874-7875$.

Kitajima, N., Fujisawa, K. \& Morooka, Y. (1990). Tetrahedral copper(II) complexes supported by a hindered pyrazolylborate. Formation of the thiolato complex, which closely mimics the spectroscopic characteristics of blue copper proteins. J. Am. Chem. Soc., 112, 3210-3212.

Kitajima, N., Fujisawa, K., Morooka, Y. \& Toriumi, K. (1989). $\mu-\eta^{2}: \eta^{2}-$ Peroxo binuclear copper complex, $\left[\mathrm{Cu}\left(\mathrm{HB}\left(3,5-\left(\mathrm{Me}_{2} \mathrm{CH}\right)_{2} \mathrm{pz}\right)_{3}\right)\right]_{2}\left(\mathrm{O}_{2}\right)$. J. Am. Chem. Soc., 111, 8975-8976.

Kitajima, N., Fujisawa, K., Tanaka, M. \& Morooka, Y. (1992). X-ray structure of thiolatocopper(II) complexes bearing close spectroscopic similarities to blue copper proteins. J. Am. Chem. Soc., 114, 9232-9233.

Kitajima, N., Fukui, H., Morooka, Y., Mizutani, Y. \& Kitagawa, T. (1990). Synthetic model for dioxygen binding sites of non-heme iron proteins. X-ray structure of $\mathrm{Fe}(\mathrm{OBz})(\mathrm{MeCN})\left[\mathrm{HB}\left(3,5-\mathrm{iso}-\mathrm{Pr}_{2} \mathrm{pz}\right)_{3}\right]$ and resonance Raman evidence for reversible formation of a peroxo adduct. J. Am. Chem. Soc., 112, 6402-6403.

Kitajima, N., Hikichi, S., Tanaka, M. \& Morooka, Y. (1993). Fixation of atmospheric carbon dioxide by a series of hydroxo complexes of divalent metal ions and the implication for the catalytic role of metal ion in carbonic anhydrase. J. Am. Chem. Soc., 115, 54965508.

Kitajima, N., Katayama, T., Fujisawa, K., Iwata, Y. \& Morooka, Y. (1993). Synthesis, molecular structure, and reactivity of (alkylperoxo)copper(II) complex. J. Am. Chem. Soc., 115, 7872-7873.

Kitajima, N., Koda, T., Hashimoto, S., Kitagawa, T. \& Morooka, Y. (1988). An accurate synthetic model of oxyhemocyanin. J. Chem. Soc., Chem. Commun., 151-152.

Kitajima, N., Koda, T., Iwata, Y. \& Morooka, Y. (1990). Reaction aspects of a $\mu$-peroxo binuclear copper(II) complex. J. Am. Chem. Soc., 112, 8833-8839.

Kitajima, N. \& Moro-oka, Y. (1994). Copper-Dioxygen Complexes. Inorganic and Bioinorganic Perspectives. Chem. Rev., 94, 737-757.

Kitajima, N., Osawa, M., Tamura, N., Morooka, Y., Hirano, T., Hirobe, M. \& Nagano, T. (1993). Monomeric (benzoato)manganese(II) complexes as manganese superoxide dismutase mimics. Inorg. Chem., 32, 1879-1880. 
Kitajima, N., Osawa, M., Tanaka, M. \& Morooka, Y. (1991). A novel dioxygenase type oxygen insertion. Carbon-hydrogen bond oxidation of isopropyl groups in a dimanganese complex with molecular oxygen. J. Am. Chem. Soc., 113, 8952-8953.

Kitajima, N. \& Tolman, W.B. (1995). Coordination chemistry with sterically hindered hydrotris(pyrazolyl)borate ligands: organometallic and bioinorganic perspectives. Prog. Inorg. Chem., 43, 419-531.

Klinman, J.P. (1996). Mechanisms Whereby Mononuclear Copper Proteins Functionalize Organic Substrates. Chem. Rev., 96, 2541-2561.

Klug, A. (1999). Zinc Finger Peptides for the Regulation of Gene Expression. J. Mol. Biol., 293, 215-218.

Kovacs, J.A. \& Holm, R.H. (1986). Assembly of vanadium-iron-sulfur cubane clusters from mononuclear and linear trinuclear reactants. J. Am. Chem. Soc., 108, 340-341.

Kovacs, J.A. \& Holm, R.H. (1987). Heterometallic clusters: synthesis and reactions of vanadium-iron-sulfur single- and double-cubane clusters and the structure of $\left[\mathrm{V}_{2} \mathrm{Fe}_{6} \mathrm{~S}_{8} \mathrm{Cl}_{4}\left(\mathrm{C}_{2} \mathrm{H}_{4} \mathrm{~S}_{2}\right)_{2}\right]_{4}$. Inorg. Chem., 26, 702-711.

Kovacs, J.A. \& Holm, R.H. (1987). Structural chemistry of vanadium-iron-sulfur clusters containing the cubane-type $\left[\mathrm{VFe}_{3} \mathrm{~S}_{4}\right]^{2+}$ core. Inorg. Chem., 26, 711-718.

Koval, I.A., Gamez, P., Belle, C., Selmeczi, K. \& Reedijk, J. (2006). Synthetic models of the active site of catechol oxidase: Mechanistic studies. Chem. Soc. Rev., 35, 814-840.

Kraatz, H.-B. \& Metzler-Nolte, N. (2006). Concepts and Models in Bioinorganic Chemistry Wiley-VCH, Weinheim.

Krahn, E., Weiss, B.J.R., Krockel, M., Groppe, J., Henkel, G., Cramer, S.P., Trautwein, A.X., Schneider, K. \& Muller, A. (2002). The Fe-only nitrogenase from Rhodobacter capsulatus. J. Biol. Inorg. Chem., 7, 37-45.

Krzystek, J., Swenson, D.C., Zvyagin, S.A., Smirnov, D., Ozarowski, A. \& Telser, J. (2010). Cobalt(II) "Scorpionate" Complexes as Models for Cobalt-Substituted Zinc Enzymes. J. Am. Chem. Soc., 132, 5241-5253.

Landry, V.K., Buccella, D., Pang, K. \& Parkin, G. (2007). Bis- and tris(2-seleno-1methylimidazolyl)hydroborato complexes, $\left\{\left[\mathrm{Bse}^{\mathrm{Me}}\right] \mathrm{ZnX}\right\}_{2}(\mathrm{X}=\mathrm{Cl}, \mathrm{I}),\left[\mathrm{Bse}^{\mathrm{Me}}\right]_{2} \mathrm{Zn}$ and $\left[\mathrm{Tse}^{\mathrm{Me}}\right] \operatorname{Re}(\mathrm{CO})_{3}$ : Structural evidence that the [BseMe] ligand is not merely a "heavier" version of the sulfur counterpart, [BmMe]. Dalton Trans., 866-870.

Landry, V.K., Pang, K., Quan, S.M. \& Parkin, G. (2007). Tetrahedral nickel nitrosyl complexes with tripodal $\left[\mathrm{N}_{3}\right]$ and $\left[\mathrm{Se}_{3}\right]$ donor ancillary ligands: structural and computational evidence that a linear nitrosyl is a trivalent ligand. Dalton Trans., 820-824.

Landry, V.K. \& Parkin, G. (2007). Synthesis and structural characterization of [BseMe]Ni $\left(\mathrm{PPh}_{3}\right)(\mathrm{NO})$, a nickel complex with a bent nitrosyl ligand. Polyhedron, 26, 4751-4757.

Lappin, A.G. \& McAuley, A. (1978). Reactions between copper(II) and 2-mercaptosuccinic acid in aqueous perchlorate solution. J. Chem. Soc., Dalton Trans., 1606-1609.

Larrabee, J.A., Alessi, C.M., Asiedu, E.T., Cook, J.O., Hoerning, K.R., Klingler, L.J., Okin, G.S., Santee, S.G. \& Volkert, T.L. (1997). Magnetic Circular Dichroism Spectroscopy as a Probe of Geometric and Electronic Structure of Cobalt(II)-Substituted Proteins. J. Am. Chem. Soc., 119, 4182-4196.

Lee, S.C. \& Holm, R.H. (2003). Speculative synthetic chemistry and the nitrogenase problem. Proc. Natl. Acad. Sci. U. S. A., 100, 3595-3600. 
Lee, S.C. \& Holm, R.H. (2004). The Clusters of Nitrogenase: Synthetic Methodology in the Construction of Weak-Field Clusters. Chem. Rev., 104, 1135-1157.

Lehnert, N., Cornelissen, U., Neese, F., Ono, T., Noguchi, Y., Okamoto, K.-I. \& Fujisawa, K. (2007). Synthesis and Spectroscopic Characterization of Copper(II)-Nitrito Complexes with Hydrotris(pyrazolyl)borate and Related Coligands. Inorg. Chem., 46, 3916-3933.

Lehnert, N., Fujisawa, K. \& Solomon, E.I. (2003). Electronic Structure and Reactivity of HighSpin Iron-Alkyl- and -Pterinperoxo Complexes. Inorg. Chem., 42, 469-481.

Lewis, E.A. \& Tolman, W.B. (2004). Reactivity of Dioxygen-Copper Systems. Chem. Rev., 104, 1047-1076.

Li, P., Solanki, N.K., Ehrenberg, H., Feeder, N., Davies, J.E., Rawson, J.M. \& Halcrow, M.A. (2000). Copper(II) complexes of hydroquinone-containing Schiff bases. Towards a structural model for $\mathrm{Cu}$ amine oxidases. Dalton, 1559-1565.

Li, R., Chen, L., Cai, D., Klinman, J.P. \& Mathews, F.S. (1997). Crystallographic study of yeast copper amine oxidase. Acta Crystallogr. D Biol. Crystallogr., 53, 364-370.

Lieberman, R.L., Arciero, D.M., Hooper, A.B. \& Rosenzweig, A.C. (2001). Crystal Structure of a Novel Red Copper Protein from Nitrosomonas europaea. Biochemistry, 40, 5674-5681.

Lipton, A.S., Bergquist, C., Parkin, G. \& Ellis, P.D. (2003). Solid-State 67Zn NMR Spectroscopic Studies and ab Initio Molecular Orbital Calculations on a Synthetic Analogue of Carbonic Anhydrase. J. Am. Chem. Soc., 125, 3768-3772.

Lipton, A.S. \& Ellis, P.D. (2007). Modeling the Metal Center of Cys 4 Zinc Proteins. J. Am. Chem. Soc., 129, 9192-9200.

Looney, A., Han, R., Gorrell, I.B., Cornebise, M., Yoon, K., Parkin, G. \& Rheingold, A.L. (1995). Monomeric alkyl and hydride derivatives of zinc supported by poly(pyrazolyl)hydroborato ligation: synthetic, structural, and reactivity studies. Organometallics, 14, 274-288.

Looney, A., Han, R., McNeill, K. \& Parkin, G. (1993). Tris(pyrazolyl)hydroboratozinc hydroxide complexes as functional models for carbonic anhydrase: on the nature of the bicarbonate intermediate. J. Am. Chem. Soc., 115, 4690-4697.

Lowe, D.J., Fisher, K. \& Thorneley, R.N.F. (1993). Klebsiella pneumoniae nitrogenase: presteady-state absorbance changes show that redox changes occur in the molybdenum-iron protein that depend on substrate and component protein ratio; A role for P-centers in reducing dinitrogen? Biochem. J., 292, 93-98.

Ma, H., Chattopadhyay, S., Petersen, J.L. \& Jensen, M.P. (2008). Harnessing Scorpionate Ligand Equilibria for Modeling Reduced Nickel Superoxide Dismutase Intermediates. Inorg. Chem., 47, 7966-7968.

Ma, H., Wang, G., Yee, G.T., Petersen, J.L. \& Jensen, M.P. (2009). Scorpionate-supported models of nickel-dependent superoxide dismutase. Inorg. Chim. Acta, 362, 45634569.

Maffett, L.S., Gunter, K.L., Kreisel, K.A., Yap, G.P.A. \& Rabinovich, D. (2007). Nickel nitrosyl complexes in a sulfur-rich environment: the first poly(mercaptoimidazolyl)borate derivatives. Polyhedron, 26, 4758-4764.

Magnus, K.A., Hazes, B., Ton-That, H., Bonaventura, C., Bonaventura, J. \& Hol, W.G.J. (1994). Crystallographic analysis of oxygenated and deoxygenated states of 
arthropod hemocyanin shows unusual differences. Proteins: Structure, Function, and Genetics, 19, 302-309.

Mahapatra, S., Halfen, J.A., Wilkinson, E.C., Pan, G., Wang, X., Young, V.G., Jr., Cramer, C.J., Que, L., Jr. \& Tolman, W.B. (1996). Structural, Spectroscopic, and Theoretical Characterization of Bis( $\mu$-oxo)dicopper Complexes, Novel Intermediates in CopperMediated Dioxygen Activation. J. Am. Chem. Soc., 118, 11555-11574.

Maldonado Calvo, J.A. \& Vahrenkamp, H. (2006). A new tris(2-furyl) substituted pyrazolylborate ligand and its zinc complex chemistry. Inorg. Chim. Acta, 359, 40794086.

Mandal, S., Das, G., Singh, R., Shukla, R. \& Bharadwaj, P.K. (1997). Synthesis and studies of $\mathrm{Cu}(\mathrm{II})$-thiolato complexes: bioinorganic perspectives. Coord. Chem. Rev., 160, 191235.

Mareque-Rivas, J.C., Prabaharan, R. \& Parsons, S. (2004). Quantifying the relative contribution of hydrogen bonding and hydrophobic environments, and coordinating groups, in the zinc(II)-water acidity by synthetic modelling chemistry. Dalton Trans., 1648-1655.

Maret, W. \& Vallee, B.L. (1993). Cobalt as probe and label of proteins. Methods Enzymol., 226, 52-71.

Matias, P.M., Soares, C.M., Saraiva, L.M., Coelho, R., Morais, J., Le Gall, J. \& Carrondo, M.A. (2001). NiFe hydrogenase from Desulfovibrio desulfuricans ATCC 27774: gene sequencing, three-dimensional structure determination and refinement at $1.8 \mathrm{~A}$ and modelling studies of its interaction with the tetrahaem cytochrome c3. J. Biol. Inorg. Chem., 6, 63-81.

Matoba, Y., Kumagai, T., Yamamoto, A., Yoshitsu, H. \& Sugiyama, M. (2006). Crystallographic evidence that the dinuclear copper center of tyrosinase is flexible during catalysis. J. Biol. Chem., 281, 8981-8990.

Mayer, S.M., Lawson, D.M., Gormal, C.A., Roe, S.M. \& Smith, B.E. (1999). New Insights into Structure-function Relationships in Nitrogenase: A $1.6 \AA$ Resolution X-ray Crystallographic Study of Klebsiella pneumoniae MoFe-protein. J. Mol. Biol., 292, 871-891.

Mehn, M.P., Fujisawa, K., Hegg, E.L. \& Que, L., Jr. (2003). Oxygen activation by nonheme iron(II) complexes: alpha -Keto carboxylate versus carboxylate. J. Am. Chem. Soc., $125,7828-7842$.

Melnick, J.G., Zhu, G., Buccella, D. \& Parkin, G. (2006). Thiolate exchange in [Tm ${ }^{\mathrm{R}} \mathrm{ZnSR}^{\prime}$ complexes and relevance to the mechanisms of thiolate alkylation reactions involving zinc enzymes and proteins. J. Inorg. Biochem., 100, 1147-1154.

Minoura, M., Landry, V.K., Melnick, J.G., Pang, K., Marchio, L. \& Parkin, G. (2006). Synthesis and structural characterization of tris(2-seleno-1-mesitylimidazolyl) hydroborato complexes: A new type of strongly electron donating tripodal selenium ligand. Chem. Commun., 3990-3992.

Mirica, L.M., Ottenwaelder, X. \& Stack, T.D.P. (2004). Structure and Spectroscopy of CopperDioxygen Complexes. Chem. Rev., 104, 1013-1046.

Morlok, M.M., Janak, K.E., Zhu, G., Quarless, D.A. \& Parkin, G. (2005). Intramolecular NH...S Hydrogen Bonding in the Zinc Thiolate Complex [ Tm $\left.{ }^{\mathrm{Ph}}\right] \mathrm{ZnSCH}_{2} \mathrm{C}(\mathrm{O}) \mathrm{NHPh}$ : A Mechanistic Investigation of Thiolate Alkylation as Probed by Kinetics Studies and by Kinetic Isotope Effects. J. Am. Chem. Soc., 127, 14039-14050. 
Moura, J.J.G., Brondino, C.D., Trincao, J. \& Romao, M.J. (2004). Mo and W bis-MGD enzymes: nitrate reductases and formate dehydrogenases. J. Biol. Inorg. Chem., 9, 791-799.

Mure, M., Mills, S.A. \& Klinman, J.P. (2002). Catalytic Mechanism of the Topa Quinone Containing Copper Amine Oxidases. Biochemistry, 41, 9269-9278.

Murphy, M.E., Turley, S. \& Adman, E.T. (1997). Structure of nitrite bound to coppercontaining nitrite reductase from Alcaligenes faecalis. Mechanistic implications. J. Biol. Chem., 272, 28455-28460.

Nakamura, N., Kohzuma, T., Kuma, H. \& Suzuki, S. (1992). The first topa-containing copper(II) complex, $\left[\mathrm{Cu}\right.$ (DL-topa) $\left(\right.$ bpy) $\left.\left(\mathrm{H}_{2} \mathrm{O}\right)\right] \mathrm{BF}_{4} \cdot 3 \mathrm{H}_{2} \mathrm{O}$, as a model for the active site in copper-containing amine oxidase. J. Am. Chem. Soc., 114, 6550-6552.

Niedenzu, K. \& Trofimenko, S. (1986). Pyrazole derivatives of boron. Topics in Current Chemistry, 131, 1-37.

Ogata, H., Mizoguchi, Y., Mizuno, N., Miki, K., Adachi, S.-i., Yasuoka, N., Yagi, T., Yamauchi, O., Hirota, S. \& Higuchi, Y. (2002). Structural Studies of the Carbon Monoxide Complex of [NiFe]hydrogenase from Desulfovibrio vulgaris Miyazaki F: Suggestion for the Initial Activation Site for Dihydrogen. J. Am. Chem. Soc., 124, 11628-11635.

Ogihara, T., Hikichi, S., Akita, M. \& Moro-oka, Y. (1998). Synthesis, Structural Characterization, and Extradiol Oxygenation of Iron-Catecholato Complexes with Hydrotris(pyrazolyl)borate Ligands. Inorg. Chem., 37, 2614-2615.

Osterloh, F., Achim, C. \& Holm, R.H. (2001). Molybdenum-Iron-Sulfur Clusters of Nuclearities Eight and Sixteen, Including a Topological Analogue of the P-Cluster of Nitrogenase. Inorg. Chem., 40, 224-232.

Osterloh, F., Sanakis, Y., Staples, R.J., Munck, E. \& Holm, R.H. (1999). A molybdenum - iron - sulfur cluster containing structural elements relevant to the p-cluster of nitrogenase. Angew. Chem. Int. Edit., 38, 2066-2070.

Palermo, R.E., Power, P.P. \& Holm, R.H. (1982). Ligand substitution properties of the $\mathrm{MFe}_{3} \mathrm{~S}_{4}$ double-cubane cluster complexes $\left[\mathrm{Mo}_{2} \mathrm{Fe}_{6} \mathrm{~S}_{8}(\mathrm{SR})_{9}\right]^{3-}$ and $\left[\mathrm{M}_{2} \mathrm{Fe}_{7} \mathrm{~S}_{8}(\mathrm{SR})_{12}\right]^{3-}(\mathrm{M}$ = molybdenum, tungsten). Inorg. Chem., 21, 173-181.

Pandey, A.S., Harris, T.V., Giles, L.J., Peters, J.W. \& Szilagyi, R.K. (2008). Dithiomethylether as a Ligand in the Hydrogenase H-Cluster. J. Am. Chem. Soc., 130, 4533-4540.

Pandey, G., Muralikrishna, C. \& Bhalerao, U.T. (1990). Mushroom tyrosinase-catalysed coupling of hindered phenols: a novel approach for the synthesis of diphenoquinones and bisphenols. Tetrahedron Lett., 31, 3771-3774.

Parkin, G. (2000). The bioinorganic chemistry of zinc: synthetic analogues of zinc enzymes that feature tripodal ligands. Chem. Commun., 1971-1985.

Parkin, G. (2004). Synthetic Analogues Relevant to the Structure and Function of Zinc Enzymes. Chem. Rev., 104, 699-767.

Parkin, G. (2007). Applications of tripodal $\left[\mathrm{S}_{3}\right]$ and $\left[\mathrm{Se}_{3}\right] \mathrm{L}_{2} \mathrm{X}$ donor ligands to zinc, cadmium and mercury chemistry: Organometallic and bioinorganic perspectives. New J. Chem., 31, 1996-2014.

Parsons, M.R., Convery, M.A., Wilmot, C.M., Yadav, K.D.S., Blakeley, V., Corner, A.S., Phillips, S.E.V., McPherson, M.J. \& Knowles, P.F. (1995). Crystal structure of a quinoenzyme: copper amine oxidase of Escherichia coli at $2 \AA$ resolution. Structure, 3, 1171-1184. 
Pate, J.E., Thamann, T.J. \& Solomon, E.I. (1986). Resonance raman studies of the coupled binuclear copper active site in met azide hemocyanin. Spectroc. Acta Pt. A-Molec. Biomolec. Spectr., 42A, 313-318.

Pellei, M., Gioia Lobbia, G., Papini, G. \& Santini, C. (2010). Synthesis and Properties of Poly(pyrazolyl)borate and Related Boron-Centered Scorpionate Ligands. Part B: Imidazole-, Triazole- and Other Heterocycle-Based Systems. Mini-Rev. Org. Chem., 7, 173-203.

Pellei, M., Papini, G., Gioia Lobbia, G. \& Santini, C. (2009). Chemistry and Relevant Biomimetic Applications of Group 6 Metals Systems Supported by Scorpionates. Current Bioactive Compounds, 5, 321-352.

Penner-Hahn, J. (2007). Zinc-promoted alkyl transfer: a new role for zinc. Curr. Opin. Chem. Biol., 11, 166-171.

Pesavento, R.P., Berlinguette, C.P. \& Holm, R.H. (2007). Stabilization of reduced molybdenum-iron-sulfur single- and double-cubane clusters by cyanide ligation. Inorg. Chem., 46, 510-516.

Peters, J.W., Stowell, M.H.B., Soltis, S.M., Finnegan, M.G., Johnson, M.K. \& Rees, D.C. (1997). Redox-Dependent Structural Changes in the Nitrogenase P-Cluster. Biochemistry, 36, 1181-1187.

Pettinari, C. (2008). Scorpionates II: Chelating Borate Ligands Dedicated to Swiatoslaw Trofimenko, Imperial College Press.

Pettinari, C. \& Santini, C. (2004). Polypyrazolylborate and scorpionate ligands. In: McCleverty, J.A. and Meyer, T.J.(eds.) Comprehensive Coordination Chemistry II. Elsevier Pergamon, 159-210.

Puerta, D.T. \& Cohen, S.M. (2002). Elucidating Drug-Metalloprotein Interactions with Tris(pyrazolyl)borate Model Complexes. Inorg. Chem., 41, 5075-5082.

Puerta, D.T. \& Cohen, S.M. (2002). [(Tp" $\left.\left.\mathrm{Te}^{\mathrm{Me}}\right)_{2} \mathrm{Zn}_{2}\left(\mathrm{H}_{3} \mathrm{O}_{2}\right)\right] \mathrm{ClO}_{4}$ : a new $\mathrm{H}_{3} \mathrm{O}_{2}$ species relevant to zinc proteinases. Inorg. Chim. Acta, 337, 459-462.

Puerta, D.T. \& Cohen, S.M. (2003). Examination of Novel Zinc-Binding Groups for Use in Matrix Metalloproteinase Inhibitors. Inorg. Chem., 42, 3423-3430.

Puerta, D.T., Griffin, M.O., Lewis, J.A., Romero-Perez, D., Garcia, R., Villarreal, F.J. \& Cohen, S.M. (2006). Heterocyclic zinc-binding groups for use in next-generation matrix metalloproteinase inhibitors: potency, toxicity, and reactivity. J. Biol. Inorg. Chem., $11,131-138$.

Puerta, D.T., Lewis, J.A. \& Cohen, S.M. (2004). New Beginnings for Matrix Metalloproteinase Inhibitors: Identification of High-Affinity Zinc-Binding Groups. J. Am. Chem. Soc., 126, 8388-8389.

Puerta, D.T., Mongan, J., Tran, B.L., McCammon, J.A. \& Cohen, S.M. (2005). Potent, Selective Pyrone-Based Inhibitors of Stromelysin-1. J. Am. Chem. Soc., 127, 14148-14149.

Qiu, D., Kilpatrick, L., Kitajima, N. \& Spiro, T.G. (1994). Modeling blue copper protein resonance Raman spectra with thiolate-CuII complexes of a sterically hindered tris(pyrazolyl)borate. J. Am. Chem. Soc., 116, 2585-2590.

Raebiger, J.W., Crawford, C.A., Zhou, J. \& Holm, R.H. (1997). Reactivity of Cubane-Type $\left[(\mathrm{OC})_{3} \mathrm{MFe}_{3} \mathrm{~S}_{4}(\mathrm{SR})_{3}\right]^{3-}$ Clusters $(\mathrm{M}=\mathrm{Mo}, \mathrm{W})$ : Interconversion with Cuboidal $\left[\mathrm{Fe}_{3} \mathrm{~S}_{4}\right]$ Clusters and Electron Transfer. Inorg. Chem., 36, 994-1003.

Randall, D.W., DeBeer George, S., Hedman, B., Hodgson, K.O., Fujisawa, K. \& Solomon, E.I. (2000). Spectroscopic and electronic structural studies of blue copper model 
complexes. Part 1. Perturbation of the thiolate-Cu bond. J. Am. Chem. Soc., 122, 11620-11631.

Randall, D.W., DeBeer George, S., Holland, P.L., Hedman, B., Hodgson, K.O., Tolman, W.B. \& Solomon, E.I. (2000). Spectroscopic and Electronic Structural Studies of Blue Copper Model Complexes. 2. Comparison of Three- and Four-Coordinate $\mathrm{Cu}(\mathrm{II})$ Thiolate Complexes and Fungal Laccase. J. Am. Chem. Soc., 122, 11632-11648.

Reglinski, J. \& Spicer, M.D. (2009). The bioinorganic chemistry of methimazole based soft scorpionates. Current Bioactive Compounds, 5, 264-276.

Rehder, D. (2003). Biological and medicinal aspects of vanadium. Inorg. Chem. Commun., 6, 604-617.

Ritter, S.K. (2003). Pinch and sting: the scorpionates. Chem. Eng. News, 81, 40-43.

Rombach, M., Gelinsky, M. \& Vahrenkamp, H. (2002). Coordination modes of amino acids to zinc. Inorg. Chim. Acta, 334, 25-33.

Rombach, M., Maurer, C., Weis, K., Keller, E. \& Vahrenkamp, H. (1999). Evidence for a trajectory of hydrolytic reactions brought about by $\left[\mathrm{L}_{3} \mathrm{Zn}-\mathrm{OH}\right]$ species. Chem. Eur. J., 5, 1013-1027.

Rombach, M., Seebacher, J., Ji, M., Zhang, G., He, G., Ibrahim, M.M., Benkmil, B. \& Vahrenkamp, H. (2006). Thiolate Alkylation in Tripod Zinc Complexes: A Comparative Kinetic Study. Inorg. Chem., 45, 4571-4575.

Rombach, M. \& Vahrenkamp, H. (2001). Pyrazolylborate-Zinc-Hydrosulfide Complexes and Their Reactions. Inorg. Chem., 40, 6144-6150.

Rorabacher, D.B. (2004). Electron Transfer by Copper Centers. Chem. Rev., 104, 651-697.

Rousset, M., Montet, Y., Guigliarelli, B., Forget, N., Asso, M., Bertrand, P., Fontecilla-Camps, J.C. \& Hatchikian, E.C. (1998). [3Fe-4S] to [4Fe-4S] cluster conversion in Desulfovibrio fructosovorans [NiFe] hydrogenase by site-directed mutagenesis. Proc. Natl. Acad. Sci. U. S. A., 95, 11625-11630.

Ruf, M., Burth, R., Weis, K. \& Vahrenkamp, H. (1996). Zinc complexes of amino acids and peptides. Part 9. Coordination of cysteine and histidine derivatives to the pyrazolylborate-zinc unit. Chem. Ber., 129, 1251-1257.

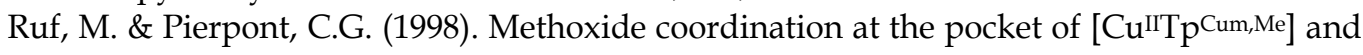
a simple model for the $\mathrm{Cu}$ center of galactose oxidase. Angew. Chem., Int. Ed., 37, 1736-1739.

Ruf, M. \& Vahrenkamp, H. (1996). Hydrolysis of esters and amides by the metallo nucleophile TpCum,MeZn-OH. Chem. Ber., 129, 1025-1028.

Ruf, M. \& Vahrenkamp, H. (1996). Small Molecule Chemistry of the Pyrazolylborate-Zinc Unit TpCum,MeZn. Inorg. Chem., 35, 6571-6578.

Ruf, M., Weis, K., Brasack, I. \& Vahrenkamp, H. (1996). Modeling transition state analogs and enzyme-inhibitor complexes of zinc-containing class II aldolases and metalloproteases. Inorg. Chim. Acta, 250, 271-281.

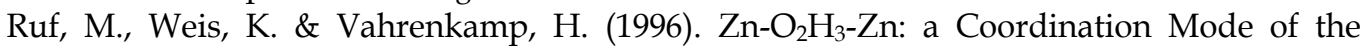
Hydrolytic Zinc-Aqua Function and a Possible Structural Motif for Oligozinc Enzymes. J. Am. Chem. Soc., 118, 9288-9294.

Ruggiero, C.E., Carrier, S.M., Antholine, W.E., Whittaker, J.W., Cramer, C.J. \& Tolman, W.B. (1993). Synthesis and structural and spectroscopic characterization of mononuclear copper nitrosyl complexes: models for nitric oxide adducts of copper proteins and copper-exchanged zeolites. J. Am. Chem. Soc., 115, 11285-11298. 
Santini, C., Pellei, M., Gioia Lobbia, G. \& Papini, G. (2010). Synthesis and Properties of Poly(pyrazolyl)borate and Related Boron-Centered Scorpionate Ligands. Part A: Pyrazole-Based Systems Mini-Rev. Org. Chem., 7, 84-124.

Schmid, B., Ribbe, M.W., Einsle, O., Yoshida, M., Thomas, L.M., Dean, D.R., Rees, D.C. \& Burgess, B.K. (2002). Structure of a cofactor-deficient nitrogenase MoFe protein. Science, 296, 352-356.

Schneider, J.L., Carrier, S.M., Ruggiero, C.E., Young, V.G., Jr. \& Tolman, W.B. (1998). Influences of Ligand Environment on the Spectroscopic Properties and Disproportionation Reactivity of Copper-Nitrosyl Complexes. J. Am. Chem. Soc., 120, 11408-11418.

Schwarz, G. \& Mendel, R.R. (2006). Molybdenum cofactor biosynthesis and molybdenum enzymes. Annu. Rev. Plant Biol., 57, 623-647.

Scott, T.A. \& Holm, R.H. (2008). $\mathrm{VFe}_{3} \mathrm{~S}_{4}$ Single and Double Cubane Clusters: Synthesis, Structures, and Dependence of Redox Potentials and Electron Distribution on Ligation and Heterometal. Inorg. Chem., 47, 3426-3432.

Seebacher, J., Shu, M. \& Vahrenkamp, H. (2001). The best structural model of ADH so far: a pyrazolylbis(thioimidazolyl)borate zinc ethanol complex. Chem. Commun., 10261027.

Senda, S., Ohki, Y., Hirayama, T., Toda, D., Chen, J.-L., Matsumoto, T., Kawaguchi, H. \& Tatsumi, K. (2006). Mono\{hydrotris(mercaptoimidazolyl)borato\} Complexes of Manganese(II), Iron(II), Cobalt(II), and Nickel(II) Halides. Inorg. Chem., 45, 99149925.

Sheats, J.E., Czernuszewicz, R.S., Dismukes, G.C., Rheingold, A.L., Petrouleas, V., Stubbe, J., Armstrong, W.H., Beer, R.H. \& Lippard, S.J. (1987). Binuclear manganese(III) complexes of potential biological significance. J. Am. Chem. Soc., 109, 1435-1444.

Shibata, N., Inoue, T., Nagano, C., Nishio, N., Kohzuma, T., Onodera, K., Yoshizaki, F., Sugimura, Y. \& Kai, Y. (1999). Novel insight into the copper-ligand geometry in the crystal structure of Ulva pertusa plastocyanin at $1.6-\AA$ resolution. Structural basis for regulation of the copper site by residue 88. J. Biol. Chem., 274, 4225-4230.

Shu, M., Walz, R., Wu, B., Seebacher, J. \& Vahrenkamp, H. (2003). New bis(mercaptoimidazolyl)(pyrazolyl)borate ligands and their zinc complex chemistry. Eur. J. Inorg. Chem., 2502-2511.

Siemann, S., Schneider, K., Drottboom, M. \& Muller, A. (2002). The Fe-only nitrogenase and the Mo nitrogenase from Rhodobacter capsulatus. A comparative study on the redox properties of the metal clusters present in the dinitrogenase components. Eur. J. Biochem., 269, 1650-1661.

Singh, U.P., Sharma, A.K., Hikichi, S., Komatsuzaki, H., Moro-oka, Y. \& Akita, M. (2006). Hydrogen bonding interaction between imidazolyl $\mathrm{N}-\mathrm{H}$ group and peroxide: Stabilization of $\mathrm{Mn}(\mathrm{III})$-peroxo complex $\mathrm{Tp} \mathrm{p}^{\mathrm{iPr} 2 \mathrm{Mn}}\left(\eta^{2}-\mathrm{O}_{2}\right)\left(\mathrm{im}^{\mathrm{MeH}}\right) \quad(\mathrm{imMeH}=2$ methylimidazole). Inorg. Chim. Acta, 359, 4407-4411.

Singh, U.P., Sharma, A.K., Tyagi, P., Upreti, S. \& Singh, R.K. (2006). Mononuclear manganese carboxylate complexes: Synthesis and structural studies. Polyhedron, 25, 3628-3638.

Smith, B.E. (1999). Structure, function, and biosynthesis of the metallosulfur clusters in nitrogenases. Adv. Inorg. Chem., 47, 159-218. 
Smith, J.N., Hoffman, J.T., Shirin, Z. \& Carrano, C.J. (2005). H-Bonding Interactions and Control of Thiolate Nucleophilicity and Specificity in Model Complexes of Zinc Metalloproteins. Inorg. Chem., 44, 2012-2017.

Smith, J.N., Shirin, Z. \& Carrano, C.J. (2003). Control of Thiolate Nucleophilicity and Specificity in Zinc Metalloproteins by Hydrogen Bonding: Lessons from Model Compound Studies. J. Am. Chem. Soc., 125, 868-869.

Solomon, E.I., Chen, P., Metz, M., Lee, S.-K. \& Palmer, A.E. (2001). Oxygen binding, activation, and reduction to water by copper proteins. Angew. Chem., Int. Ed., 40, 4570-4590.

Solomon, E.I., Sundaram, U.M. \& Machonkin, T.E. (1996). Multicopper Oxidases and Oxygenases. Chem. Rev., 96, 2563-2605.

Solomon, E.I., Tuczek, F., Root, D.E. \& Brown, C.A. (1994). Spectroscopy of Binuclear Dioxygen Complexes. Chem. Rev., 94, 827-856.

Speier, G., Tisza, S., Tyeklar, Z., Lange, C.W. \& Pierpont, C.G. (1994). Coligand-Dependent Shifts in Charge Distribution for Copper Complexes Containing 3,5-Di-tertbutylcatecholate and 3,5-Di-tert-butylsemiquinonate Ligands. Inorg. Chem., 33, 2041-2045.

Spicer, M.D. \& Reglinski, J. (2009). Soft Scorpionate Ligands Based on Imidazole-2-thione Donors. Eur. J. Inorg. Chem., 1553-1574.

Stiefel, E.I. (1997). Chemical keys to molybdenum enzymes. J. Chem. Soc., Dalton Trans., 39153924.

Sun, Y.-J., Zhang, L.Z., Cheng, P., Lin, H.-K., Yan, S.-P., Liao, D.-Z., Jiang, Z.-H. \& Shen, P.W. (2004). Experimental and theoretical studies of the dehydration kinetics of two inhibitor-containing half-sandwich cobalt(II) complexes. J. Mol. Catal. A: Chem., 208, 83-90.

Sun, Y.-J., Zhang, L.Z., Cheng, P., Lin, H.-K., Yan, S.-P., Liao, D.-Z., Jiang, Z.-H. \& Shen, P.W. (2004). Spectroscopic properties, catalytic activities and mechanism studies of $\left[\left(\mathrm{Tp} \mathrm{Ph}^{\mathrm{Ph}} \mathrm{Co}(\mathrm{X})\left(\mathrm{CH}_{3} \mathrm{OH}\right)_{\mathrm{m}}\right] \cdot \mathrm{nCH}_{3} \mathrm{OH}\right.$ : bicarbonate dehydration in the presence of inhibitors. Biophys. Chem., 109, 281-293.

Sylvestre, I., Wolowska, J., McInnes, E.J.L., Kilner, C.A. \& Halcrow, M.A. (2005). An unexpected destabilisation of copper(II) phenoxyl radical species by steric protection. Inorg. Chim. Acta, 358, 1337-1341.

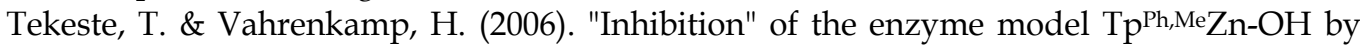
diketo compounds. Eur. J. Inorg. Chem., 5158-5164.

Tekeste, T. \& Vahrenkamp, H. (2006). Modeling Zinc Enzyme Inhibition with Functional Thiolate Ligands. Inorg. Chem., 45, 10799-10806.

Thomas, F. (2007). Ten years of a biomimetic approach to the copper(II) radical site of galactose oxidase. Eur. J. Inorg. Chem., 2379-2404.

Thompson, J.S., Sorrell, T., Marks, T.J. \& Ibers, J.A. (1979). Synthesis, structure, and spectroscopy of pseudotetrahedral $\mathrm{CoIIN}_{3}(\mathrm{SR})$ complexes. Active site approximations to the cobalt(II)-substituted type 1 copper proteins. J. Am. Chem. Soc., 101, 4193-4201.

Thompson, J.S., Zitzmann, J.L., Marks, T.J. \& Ibers, J.A. (1980). Synthesis and characterization of $\mathrm{M}(\mathrm{II})\left(\mathrm{N}_{2} \mathrm{~S}\right)(\mathrm{SR})$ complexes $(\mathrm{M}=\mathrm{Co}, \mathrm{Cu})$ : synthetic approximations to the active site in poplar plastocyanin. Inorg. Chim. Acta, 46, L101L105. 
Tolman, C.A. (1977). Steric effects of phosphorus ligands in organometallic chemistry and homogeneous catalysis. Chem. Rev., 77, 313-348.

Tolman, W.B. (1991). A model for the substrate adduct of copper nitrite reductase and its conversion to a novel tetrahedral copper(II) triflate complex. Inorg. Chem., 30, 48774880.

Tolman, W.B. (2006). Using synthetic chemistry to understand copper protein active sites: a personal perspective. J. Biol. Inorg. Chem., 11, 261-271.

Trofimenko, S. (1966). Boron-pyrazole chemistry. J. Am. Chem. Soc., 88, 1842-1844.

Trofimenko, S. (1971). Polypyrazolylborates, a new class of ligands. Acc. Chem. Res., 4, 17-22.

Trofimenko, S. (1972). Coordination chemistry of pyrazole-derived ligands. Chem. Rev., 72, 497-509.

Trofimenko, S. (1986). The coordination chemistry of pyrazole-derived ligands. Prog. Inorg. Chem., 34, 115-210.

Trofimenko, S. (1993). Recent advances in poly(pyrazolyl)borate (scorpionate) chemistry. Chem. Rev., 93, 943-980.

Trofimenko, S. (1999). Scorpionates: The Coordination Chemistry of Poly(pyrazolyl)borate Ligands. Imperial College Press: London.

Trofimenko, S. (2004). Scorpionates: genesis, milestones, prognosis. Polyhedron, 23, 197-203.

Trofimenko, S., Calabrese, J.C., Kochi, J.K., Wolowiec, S., Hulsbergen, F.B. \& Reedijk, J. (1992). Spectroscopic analysis, coordination geometry, and X-ray structures of nickel(II) compounds with sterically demanding tris(pyrazolyl)borate ligands and azide or (thio)cyanate anions. Inorg. Chem., 31, 3943-3950.

Tunney, J.M., McMaster, J. \& Garner, C.D. (2004). Molybdenum and tungsten enzymes. In: Wilkinson, G., Gillard, R.D. and McCleverty, J.A.(eds.) Compr. Coord. Chem. II. Elsevier Ltd., Oxford, UK, 459-477.

Usov, O.M., Sun, Y., Grigoryants, V.M., Shapleigh, J.P. \& Scholes, C.P. (2006). EPR-ENDOR of the $\mathrm{Cu}(\mathrm{I}) \mathrm{NO}$ Complex of Nitrite Reductase. J. Am. Chem. Soc., 128, 13102-13111.

Vahrenkamp, H. (1999). Transitions, Transition States, Transition State Analogs: Zinc Pyrazolylborate Chemistry Related to Zinc Enzymes. Acc. Chem. Res., 32, 589-596.

Vahrenkamp, H. (2007). Why does nature use zinc-a personal view. Dalton Trans., 4751-4759.

Vallee, B.L. \& Auld, D.S. (1993). Zinc: biological functions and coordination motifs. Acc. Chem. Res., 26, 543-551.

Veselov, A., Olesen, K., Sienkiewicz, A., Shapleigh, J.P. \& Scholes, C.P. (1998). Electronic Structural Information from Q-Band ENDOR on the Type 1 and Type 2 Copper Liganding Environment in Wild-Type and Mutant Forms of Copper-Containing Nitrite Reductase. Biochemistry, 37, 6095-6105.

Volbeda, A., Charon, M.H., Piras, C., Hatchikian, E.C., Frey, M. \& Fontecilla-Camps, J.C. (1995). Crystal structure of the nickel-iron hydrogenase from Desulfovibrio gigas. Nature, 373, 580-587.

Volbeda, A. \& Hol, W.G.J. (1989). Crystal structure of hexameric hemocyanin from Panulirus interruptus refined at 3.2 A resolution. J. Mol. Biol., 209, 249-279.

Walsby, C.J., Krepkiy, D., Petering, D.H. \& Hoffman, B.M. (2003). Cobalt-substituted zinc finger 3 of transcription factor IIIA: Interactions with cognate DNA detected by ${ }^{31} \mathrm{P}$ ENDOR spectroscopy. J. Am. Chem. Soc., 125, 7502-7503. 
Wang, Y., DuBois, J.L., Hedman, B., Hodgson, K.O. \& Stack, T.D.P. (1998). Catalytic galactose oxidase models: biomimetic $\mathrm{Cu}(\mathrm{II})$-phenoxyl-radical reactivity. Science, 279, 537-540.

Ward, M.D., McCleverty, J.A. \& Jeffery, J.C. (2001). Coordination and supramolecular chemistry of multinucleating ligands containing two or more pyrazolyl-pyridine 'arms'. Coord. Chem. Rev., 222, 251-272.

Warthen, C.R. \& Carrano, C.J. (2003). The Cu(II) acetate complex of the heteroscorpionate ligand (2-hydroxy-3-t-butyl-methylphenyl)bis(3,5-dimethylpyrazolyl)methane: a structural model for copper-substituted serralysin and astacin. J. Inorg. Biochem., 94, 197-199.

Wasser, I.M., de Vries, S., Moenne-Loccoz, P., Schroder, I. \& Karlin, K.D. (2002). Nitric Oxide in Biological Denitrification: $\mathrm{Fe} / \mathrm{Cu}$ Metalloenzyme and Metal Complex NOx Redox Chemistry. Chem. Rev., 102, 1201-1234.

Webster, C.E. \& Hall, M.B. (2003). De Novo design in organometallic chemistry: stabilizing iridium(V). Coord. Chem. Rev., 238-239, 315-331.

Weis, K., Rombach, M., Ruf, M. \& Vahrenkamp, H. (1998). (Pyrazolylborate)zinc organophosphate complexes resulting from hydrolytic cleavage of phosphate esters. Eur. J. Inorg. Chem., 263-270.

Whittaker, J.W. (2003). Free Radical Catalysis by Galactose Oxidase. Chem. Rev., 103, 23472363.

Whittaker, J.W. (2005). The radical chemistry of galactose oxidase. Arch. Biochem. Biophys., 433, 227-239.

Whittaker, J.W. \& Whittaker, M.M. (1998). Radical copper oxidases, one electron at a time. Pure and Applied Chemistry, 70, 903-910.

Whittaker, M., Bergmann, D., Arciero, D. \& Hooper, A.B. (2000). Electron transfer during the oxidation of ammonia by the chemolithotrophic bacterium Nitrosomonas europaea. Biochimica et Biophysica Acta, Bioenergetics, 1459, 346-355.

Whittaker, M.M., Kersten, P.J., Nakamura, N., Sanders-Loehr, J., Schweizer, E.S. \& Whittaker, J.W. (1996). Glyoxal oxidase from Phanerochaete chrysosporium is a new radical-copper oxidase. J. Biol. Chem., 271, 681-687.

Wolff, T.E., Berg, J.M., Power, P.P., Hodgson, K.O. \& Holm, R.H. (1980). Structural characterization of the iron-bridged "double-cubane" cluster complexes $\left[\mathrm{Mo}_{2} \mathrm{Fe}_{7} \mathrm{~S}_{8}\left(\mathrm{SC}_{2} \mathrm{H}_{5}\right)_{12}\right]^{3-}$ and $\left[\mathrm{M}_{2} \mathrm{Fe}_{7} \mathrm{~S}_{8}\left(\mathrm{SCH}_{2} \mathrm{C}_{6} \mathrm{H}_{5}\right)_{12}\right]^{4-}(\mathrm{M}=$ molybdenum, tungsten $)$ containing $\mathrm{MFe}_{3} \mathrm{~S}_{4}$ cores. Inorg. Chem., 19, 430-437.

Xing, Y., Zhang, Y., Sun, Z., Ye, L., Xu, Y., Ge, M., Zhang, B. \& Niu, S. (2007). Two new scorpionates vanadium haloperoxidases model complexes: Synthesis and structure of $\mathrm{VO}\left(\mathrm{O}_{2}\right)(\mathrm{pzH})\left(\mathrm{HB}(\mathrm{pz})_{3}\right)$ and $\mathrm{VO}\left(\mathrm{O}_{2}\right)(\mathrm{pzH})\left(\mathrm{B}(\mathrm{pz})_{4}\right)\left(\mathrm{pzH}=\right.$ pyrazole $\left.\left(\mathrm{C}_{3} \mathrm{H}_{4} \mathrm{~N}_{2}\right)\right)$. J. Inorg. Biochem., 101, 36-43.

Yandulov, D.V. \& Schrock, R.R. (2003). Catalytic Reduction of Dinitrogen to Ammonia at a Single Molybdenum Center. Science, 301, 76-78.

Yat, H.C., Lau, K.Y. \& Hung, K.L. (2003). Model complexes for catechol dioxygenases supported by a tridentate hydrotris(pyrazolyl)borate ligand. J. Inorg. Biochem., 96, 116.

Young, C.G. \& Wedd, A.G. (1997). Metal chemistry relevant to the mononuclear molybdenum and tungsten pterin enzymes. Chem. Commun., 1251-1257. 
Young, C.G. \& Young, C.G. (1997). Models for the molybdenum hydroxylases. J. Biol. Inorg. Chem., 2, 810-816.

Zhang, Y. \& Holm, R.H. (2003). Synthesis of a Molecular $\mathrm{Mo}_{2} \mathrm{Fe}_{6} \mathrm{~S}_{9}$ Cluster with the Topology of the PN Cluster of Nitrogenase by Rearrangement of an Edge-Bridged $\mathrm{Mo}_{2} \mathrm{Fe}_{6} \mathrm{~S}_{8}$ Double Cubane. J. Am. Chem. Soc., 125, 3910-3920.

Zhang, Y. \& Holm, R.H. (2004). Structural Conversions of Molybdenum-Iron-Sulfur EdgeBridged Double Cubanes and PN-Type Clusters Topologically Related to the Nitrogenase P-Cluster. Inorg. Chem., 43, 674-682.

Zhang, Y., Zuo, J.-L., Zhou, H.-C. \& Holm, R.H. (2002). Rearrangement of Symmetrical Dicubane Clusters into Topological Analogues of the P Cluster of Nitrogenase: Nature's Choice? J. Am. Chem. Soc., 124, 14292-14293.

Zhou, H.-C., Su, W., Achim, C., Rao, P.V. \& Holm, R.H. (2002). High-Nuclearity Sulfide-Rich Molybdenum-Iron-Sulfur Clusters: Reevaluation and Extension. Inorg. Chem., 41, 3191-3201.

Zuo, J.-L., Zhou, H.-C. \& Holm, R.H. (2003). Vanadium-Iron-Sulfur Clusters Containing the Cubane-type $\left[\mathrm{VFe}_{3} \mathrm{~S}_{4}\right]$ Core Unit: Synthesis of a Cluster with the Topology of the $\mathrm{PN}^{\mathrm{N}}$ Cluster of Nitrogenase. Inorg. Chem., 42, 4624-4631. 


\title{
Analogue CMOS Cochlea Systems: A Historic Retrospective
}

\author{
Andreas Katsiamis and Emmanuel Drakakis \\ Imperial College London \\ United Kingdom
}

\section{Introduction}

For more than twenty-five years, the bionics community (and particularly the VLSI engineering community) has been performing extensive research to understand, model and design in silicon the biological auditory system and specifically the inner ear or cochlea. The aim of this on-going effort is not only towards building the ultimate artificial speech processor or implant, but also to develop systems that can contribute towards a deeper understanding of the underlying engineering strategies that nature chose to espouse. For these reasons, certain parts of the VLSI engineering community believe that trying to mimic certain biological operations will, in principle, yield systems that are somewhat closer to nature's power-efficient computational ability.

However, engineers must identify what they should and what they should not directly replicate in an artificial system inspired from biology. As for example, it does not make sense to create commercial aircraft wings to flap like those of birds (even though unmanned flapping-wing aircrafts are currently being developed - check NASA's flapping solar aircraft) it is equally meaningful to argue that not all operations of the cochlea can or should be replicated in silicon in an exact manner. Abstractive operational or architectural simplifications dictated by logic and the available technology have been crucial for the successful implementation of useful hearing-type machines. Moreover, the biological cochlea is a threedimensional electro-hydro-mechanical system, whereas most electronic systems are onedimensional systems. Based on these thoughts, it would be wise to try and clarify three terms that are commonly used in the (bio-) engineering literature. Namely, these are:

- $\quad$ Neuromorphic: the system level architecture is designed in such a way in order to replicate 'exact' basic anatomical identified operations which embody several key features encountered in the biological system. The term 'neuromorphic' was introduced by Carver Mead in (Mead, 1989;Mead, 1990) .

- $\quad$ Bio-inspired: the design and/or operation are based on (inspired from) the engineering principles underlying its biological counterpart.

- Biomimetic: the behaviour/operation/response resembles the one directly observed from the biological system, applying completely, partially or not at all the engineering principles encountered in the biological system.

It should be therefore evident that a neuromorphic design is also bio-inspired and biomimetic, whereas a biomimetic (or bio-inspired) design is not necessarily neuromorphic. We shall provide tangible examples on this fact as the chapter unfolds. 
Since the late seventies, there we numerous attempts from several research laboratories to capture the main attributes of the biological cochlea in a way that are conveniently (i.e. computationally efficiently) replicated in silicon. This chapter attempts to present a rather detailed historical retrospective of nearly twenty-seven years of analogue VLSI cochlea development. The chapter is organized in ascending chronological in order to unravel the chain of events in order to appreciate how the research direction changed through time. If the reader wishes to skip all the details, s/he can refer to Table 1 and Fig. 32 at the end which summarizes the performances of the most important efforts.

\section{The early years: $1982-1992$}

1982: The first to sow the seeds for the coming generations of VLSI engineers was Richard F. Lyon who in 1982, while in Fairchild Artificial Intelligence Research Laboratory, proposed a VLSI-compatible computational model of filtering, detection and compression in the cochlea (Lyon, 1982), even though it seems that he had conceived the idea earlier - check one of his unpublished efforts circa 1978 (Lyon, 1978). His work made a clear link to the early Wegel and Lane (Wegel and Lane, 1924) and Fletcher (Fletcher, 1930) model of micromechanics (the one-dimensional passive transmission line model) and to the more recent model of nonlinear differential equations by Kim and his associates (Kim et al., 1973). In this pioneering work, the proposed model (which was later given the name: 'The Lyon's Cochlear Model' by Malcolm Slaney (Slaney, 1988)) cleanly separated the effects of mechanical filtering in the BM into time-invariant linear filtering based on a simple cascade/parallel filterbank network of second-order sections (or biquad filters). Lyon apart from BM filtering also discussed that detection and compression should be incorporated in the final model by means of half-wave rectification and a coupled nonlinear AGC network. Lyon's cochlea model can be described briefly as follows:

Wave propagation in distributed non-uniform media like the BM can - in principle - be modelled by a cascade of filters. Such a cascade will give rise to overall transfer functions with sharp roll-off slopes corresponding to higher-order structures. This kind of BM modelling relies upon the approximation that for a short BM section $\Delta x$, the parameters change only slightly and thus it behaves just as a short section would in a uniform medium. In other words, the non-uniform medium can be discretised with each portion assumed to be locally uniform. Each of these uniform portions can be modelled by a filtering stage which alters the magnitude and phase of excitation. The parameters of each successive filter (corresponding to a different $\Delta \mathrm{x}$ segment) will scale accordingly so that the collective response of the whole cascaded structure approximates the behaviour of the non-uniform medium.

In this first attempt, each BM segment was approximated as a lowpass notch (LPN) transfer function with the resonance frequencies, that determine the notch locations, changing approximately exponentially (modelling how the properties of the BM change lengthwise) from $20 \mathrm{kHz}$ at the input end (base) to $50 \mathrm{~Hz}$ at the output end (apex). The cascaded LPN filters conspired to create a collection of lowpass filters (LPF) with very steep highfrequency roll-off slopes. A resonator transfer function, a simple bandpass filter (BPF) was added at the output of each LPN output (tap) to convert BM displacement to BM velocity. The overall effect was a BM cochlea 'tuning curve' which qualitatively had an asymmetric BP shape much like the one Rhode observed in the early 70s.

This cascade/parallel filterbank architecture constitutes part of a single-input, multi-output system with a very useful property: the sum of the orders of the transfer functions from the 
input to each output (tap) greatly exceeds the sum of the orders of the component sections, thus achieving an economy of computation by using the same low-order section in many high-order transfer functions. The filter-cascade also enforces uni-directionality of energy (not allowing the introduction of reflections which could lead to instability) and achieves a high-gain pseudo-resonance by coupling the individual low-gain stages forming the cascade. A block diagram of the cascade/parallel architecture is shown in Fig. 1, whereas Fig. 2 shows the frequency responses of its constituent transfer functions.

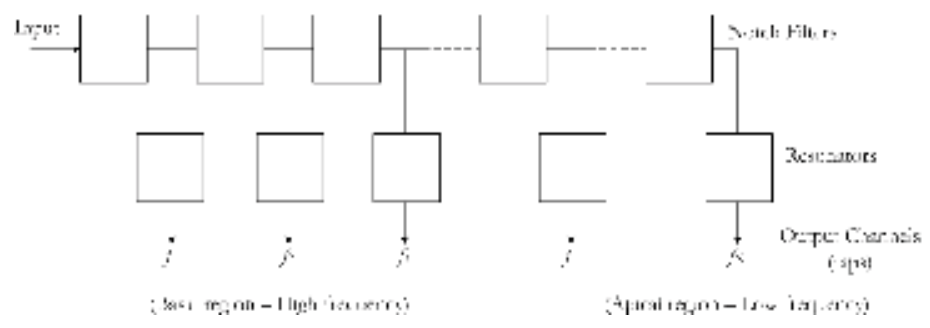

Fig. 1. Block diagram of the cascade/parallel filterbank for modelling travelling wave propagation in the BM; adapted from Lyon (Lyon, 1982).

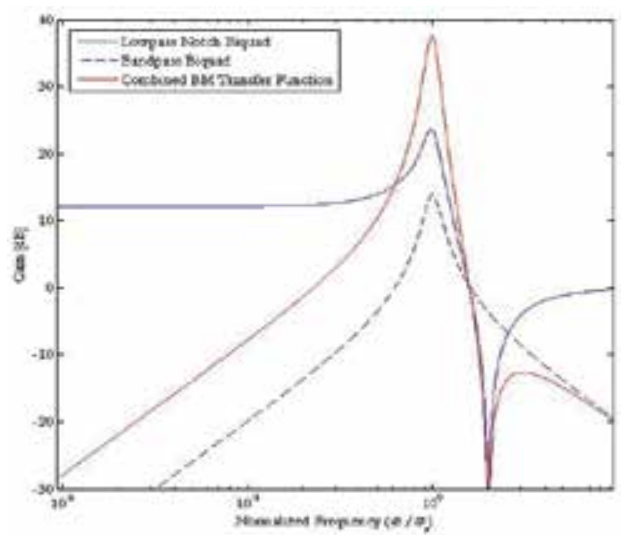

Fig. 2. Indicative transfer functions of the LPN, resonator and their combined response resembling the one obtained from a particular output tap.

1988: Even though Lyon's 1982 paper provided a very useful insight on how BM wave propagation could be modelled using basic second-order building blocks, his proposed AGC scheme, while carefully thought, seemed quite underdeveloped regarding a real-time neuromorphic circuit implementation. In his 1982 effort, all the filters forming the BM where linear time-invariant transfer functions with the AGC providing a straight gain variation at the output of each tap. Moreover the gain control responded to the system's measured output level and not the input level. This AGC scheme was quite different from what is really happening in reality since the peak gain of the cochlea filters changes dynamically with input level and not by providing separate amplification after the filtering stage. It took almost six years for the big contribution to arise. In 1988 the very first CMOS cochlea was presented. The paper 'An Analog Electronic Cochlea', co-authored with Carver Mead, won an IEEE best paper award and cemented the current views on how the basic functions of BM together with its vestibular apparatus can be modelled in silicon (Lyon and Mead, 1988a;Lyon and Mead, 
1988b). Even though the cornerstone was placed in 1982, it was this work that presented Lyon's modelling ideas in a more complete and unified way.

In this particular design effort, each BM segment was modelled as a LP biquad (although the initial 1982 model used LPN biquads) with no resonator stages connected to each of the outputs. The LP biquad (generally easier to design in silicon than a transfer function containing zeros) was a natural choice for modelling BM filtering, because it is passive and linear for low frequencies and provides gain near the peak or centre frequency (CF) while attenuating high frequencies.

Although, the frequency selective properties of the BM were modelled as a collection of linear, time-invariant transfer functions, sufficient evidence shows that the cochlea is highly nonlinear. The filtering architecture presented in this paper did not differ conceptually from the one presented in 1982. However, a scheme for a possible neuromorphic circuit implementation of the coupled AGC network was presented here for the first time. Lyon understood that for the purposes of speech analysis, the nonlinear behaviour of the cochlea could be adequately accounted for by lumping it in the compression mechanism of the AGC. Contrary to what he had shown in 1982, here he proposed that by adaptively varying the quality factors $(Q)$ of the individual filter stages in response to the input intensity level, overall large gain variations could be achieved for the combined response. Since the whole cascade structure is essentially a very high-order system, the $Q$ of the individual filters only need to vary by little in order to achieve large gain variations. In other words, the effect of the OHC-based AGC was modelled as a local fast-acting adaptation of the $Q$ for each BM stage: as a signal of particular frequency and amplitude travels along the cascade, each filter section will adjust its $Q$ value so that the collective pseudo-resonant transfer function tunes in accordance to the particular input excitation. In this way a large detected signal at one place could reduce the gain at nearby places and give rise to outstanding scale-invariant and matching properties along the cascade.

Lyon suggested at the time, that the actual local feedback for each stage would involve a half-wave rectifier connected directly after the stage's output tap (modelling the operation of the IHC, since IHC fire when they are bend in one direction and cease firing when they are bend in the other), a strength detector to extract the intensity of the input signal and some kind of a $Q$-decision circuit that will map that intensity level to an appropriate biasing value for setting the $Q$ of the particular filter stage. From all the above, it should be easy to see that Lyon's model is neuromorphic since the BM structure and its associate transducers are directly mapped onto circuit blocks that emulate their behaviour and operation.

The paper, apart from the thorough discussion on biological cochlea operation and mathematical modelling of wave propagation in non-uniform media, also discussed practical details on the circuit implementation of the biquad section. The LPF was designed in voltage-mode based on simple transconductance amplifiers (OTA) and capacitors (i.e. $\mathrm{g}_{\mathrm{m}}$ C) with all MOS devices operating in their weak inversion (WI) regime (see Fig. 3). Since in a WI MOS device a linear change in voltage corresponds to an exponential change in current, the exponential tapering of the cut-off frequencies was easily achieved by using a linear voltage gradient (through a resistive line) on the transistor gates. Finally, measured results from a 480-stage fabricated chip were provided showing LP constant- $Q$ (i.e. the individual $Q$ values were manually set off-chip) AC responses (see Fig. 4); no detailed discussion or results from an AGC or from an overall adaptively regulated system were provided. Even though Lyon proposed the architecture behind the aforementioned AGC scheme, he did not implement it, probably because of certain stability problems they were facing at the time. 
Detailed results on power consumption, linearity, noise, dynamic range (DR) etc., were also not given.

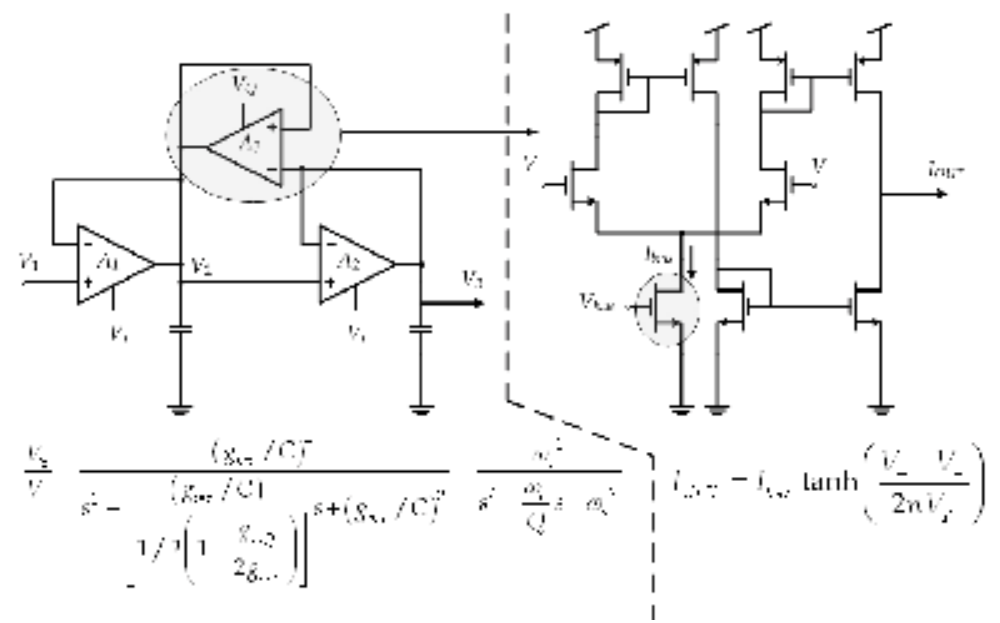

Fig. 3. The lowpass biquad circuit. The $\omega_{0}$ and $Q$ control inputs set the OTA bias currents for controlling the pole frequency and quality factor (and hence the peak gain) of the filter response. Amplifiers $A_{1}$ and $A_{2}$ have the same transconductance gm (left). The basic CMOS OTA circuit used for realizing amplifiers $A_{1}, A_{2}$ and $A_{3}$ (right). The implemented relations are shown beneath each circuit. Parameters $V_{T}$ and $n$ denote the thermal voltage and subthreshold slope parameter, respectively; adapted from Lyon (Lyon and Mead, 1988a).

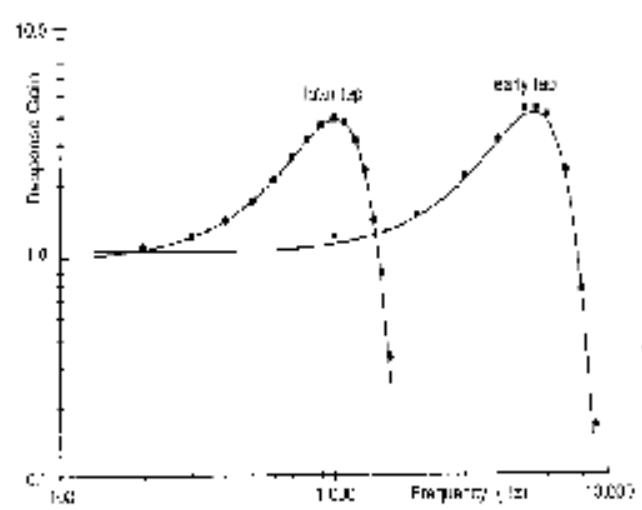

Fig. 4. Measured log-log frequency responses to two output taps (i.e. at particular BM locations) placed 120 stages apart. The stages $Q$ were manually adjusted to 0.79 ; from Lyon (Lyon and Mead, 1988a).

1989\&1990: One year later in Japan, Tatsuya Hirahara published a conference paper on a computational nonlinear model with adaptive $Q$ circuits (Hirahara and Komakine, 1989). Even though the word 'circuits' was used both in the title and numerous times in the text, no circuits or indications of how to build them were presented. The paper reported on system level ideas by showing the equations and basic transfer functions of the blocks comprising a 61-stage cascade. Hirahara's main idea was based on Lyon's cascade/parallel 1982 model, but included a model on the actual decision block that specified which input 
amplitude corresponded to which $Q$ for each filter stage of the cascade. In other words, it specified the law with which the filter stages adapted their gains at the presence of an input signal, essentially modelling the effect of OHC (see Fig. 5). To this author knowledge, this effort was one of the very first to elaborate on the $Q$-control law.
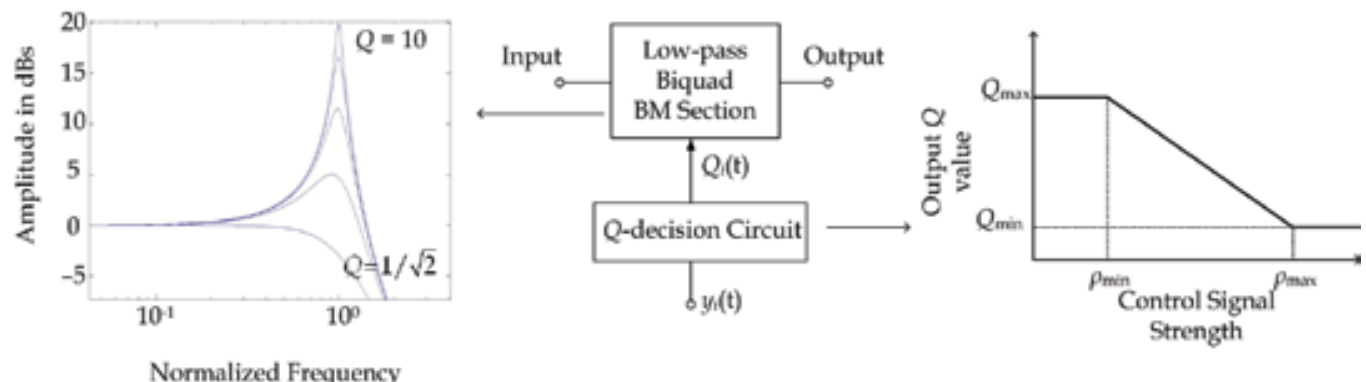

Fig. 5. Block diagram of the adaptive $Q$ function (middle), frequency response of a biquad LPF left) and input-output relationship of the $Q$ decision block which calculates the LP $Q$ values according to (input or output) signal strength. For a large control signal $\left(\rho_{\max }\right)$, the $Q$ value is minimum and the LP response becomes passive providing no gain at the peak frequency. On the other extreme $\left(\rho_{\text {min }}\right)$, the $Q$ value is maximum and the LP response becomes selective providing maximum gain for frequencies near the peak frequency. The relationship between the two extremes is linear; adapted from Hirahara (Hirahara and Komakine, 1989).

Around the same time John Lazzaro from Carver Mead's group at Caltech, continued Lyon's work and proposed silicon integrated circuits that modelled sensory transduction in the cochlea (Lazzaro and Mead, 1989a). His cochlea chip (based on filter-cascades and using the same $\mathrm{g}_{\mathrm{m}}-\mathrm{C}$ filters as the ones Lyon had used earlier) was neuromorphic because once again it captured the structure as well as the function of the cochlea with all the proposed subcircuits having anatomical correlates. In this effort, the very first CMOS IHC circuit model was presented consisting of a hysteretic differentiator that performed both logarithmic compression (proposed by Mead in (Mead, 1989)) and half-wave rectification. The function of the hysteretic differentiator was to convert BM displacement to BM velocity while enhancing the zero-crossings of the input waveform, thereby emphasizing its phase information. The input of the IHC was attached at each BM section tap, whereas the output was connected to a spiral-ganglion-neuron (SGN) circuit (a slightly modified version of the neuron circuit also proposed by Mead in (Mead, 1989)) that converted the (uni-directional) half-wave rectified current waveform into fixed-width, fixed-height voltage pulses (see Fig. 6). The average pulse rate and the temporal placement of each pulse reflected the average value and shape of the IHC half-wave rectified waveform, respectively. The pulsed output was then delayed by a time matched to the resonant frequency of its associated cochlea tap and consequently correlated (through a simple CMOS AND gate) with the initial pulsed waveform to create the final output. The resulting matched filter operation resembled in frequency a sharply tuned $\mathrm{BP}$ response with the maximum spike rate corresponding to the CF of the particular tap.

Lazzaro inserted pure sinusoidal tones to his cochlea chip and measured the rate (in spikes per second) from the output of each silicon auditory nerve. His results were useful additions to Lyon's initial BM frequency-response measurements because he managed to faithfully 
reproduce a variety of physiological auditory nerve responses. His integrated circuit model captured many essential features of data representation in the auditory nerve, in real-time and over a $60 \mathrm{~dB}$ of input DR. Indicative results are shown in Fig. 7. Nevertheless, as with Lyon's effort, Lazzaro still did not implement the $\mathrm{OHC}$ circuits to obtain responses from an adaptively regulated system.

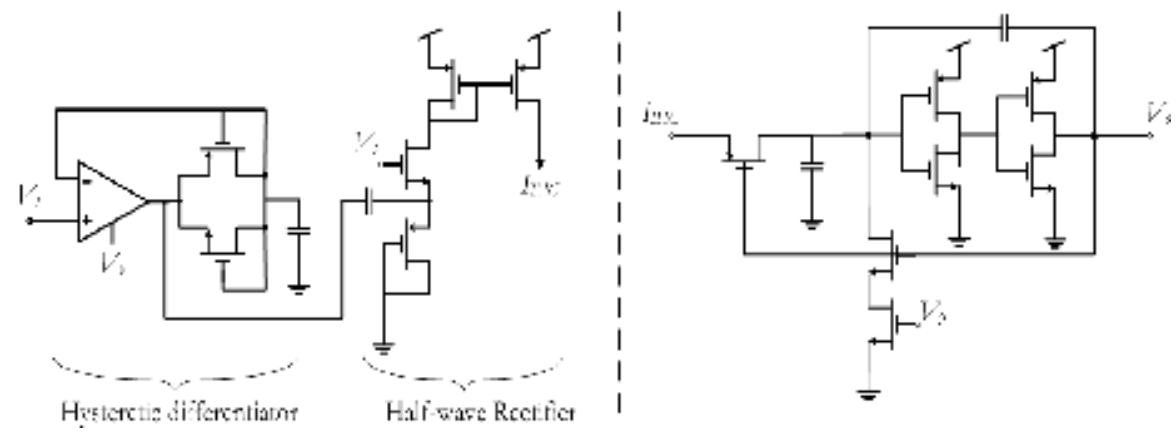

Fig. 6. The IHC (left) and SGN circuit models (right). The input $V_{i}$ from the BM filtering stage is a time-varying voltage waveform. The hysteretic differentiator, biased with a voltage $V_{y}$ performs time-differentiation and logarithmic compression. The output from the hysteretic differentiator goes to a half-wave current rectifier circuit. The uni-directional output current from the IHC circuit goes to the SGN circuit which converts it into fixedwidth, fixed-height voltage pulses at the output $V_{o}$. The bias voltage $V_{p}$ sets the pulse width with $V_{o}$ pulsating between the supply and ground; adapted from Lazzaro (Lazzaro and Mead, 1989a).

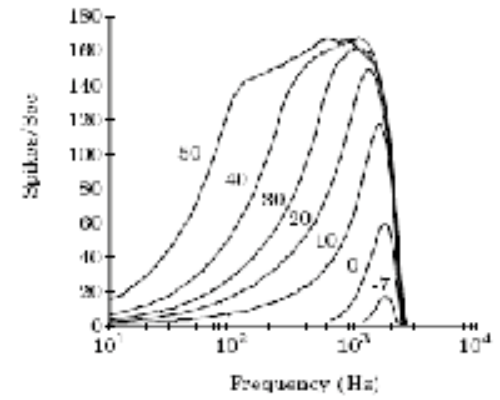

(a)

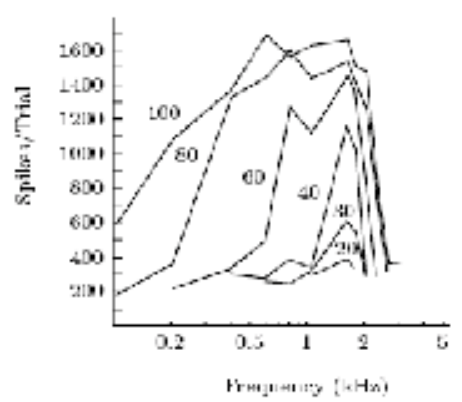

(1))

Fig. 7. (a) Measured chip plots showing the mean spike rate of a silicon auditory fibre as a function of pure tone frequency. (b) Physiological plots showing the number of neural discharges of an auditory fibre in the squirrel monkey in response to a 10sec pure tone. Tone amplitudes in dB are indicated on each plot; from Lazzaro (Lazzaro and Mead, 1989a).

Lazzaro's work did not stop there. He was also the first to apply his and Lyon's circuit modelling ideas for sound source localization (based on the passive localization system of the barn owl) (Lazzaro and Mead, 1990;Lazzaro, 1991) and for pitch perception (Lazzaro and Mead, 1989b). With the aid of Fig. 8, the operation of Lazzaro's localization system can be briefly described as follows:

Lazzaro integrated two cochlea cascades on the same chip which represented the left and right ears of the barn owl. The chip received its inputs from two separate signal generators 
and the two 62-stage $\mathrm{g}_{\mathrm{m}}-\mathrm{C}$ cascades performed frequency decomposition causing a maximum excitation at a tap corresponding to the frequency of the input waveforms. The previously presented IHC and SGN circuitry consequently rectified and converted the output signals at each tap into pulses. Each pulse from the SGN circuit propagated down a silicon axon circuit; the direction of propagation being from left-to-right from the SGN of the left cochlea and from right-to-left from the SGN of the right cochlea. Thus, when a sound appeared at both chip inputs, action potentials counter-propagated across the chip. Circuitwise, the axon was nothing more than a discrete delay line with the input being a fixedwidth, fixed-height pulse which travelled along its length, section by section, at a controllable velocity. At any point in time, only one section of the axon was firing. Correlator circuits laid in between each pair of antiparallel axons and at every discrete section that connected directly to both axons. The simultaneous appearance of pulses at both inputs of the correlator initiated a maximum output response. If only one input was present, the correlator generated no output. In this way, interaural time differences could map into a neural place code. The final section of the chip was a circuit that performed a winner-takeall-function (a modified version of the circuit was used by Misha Mahowald and Tobi Delbrück in (Mahowald and Delbriick, 1989)), producing a new map of interaural time differences in which only one neuron had significant energy. The chip then multiplexed this final map on a single wire for display on an oscilloscope.

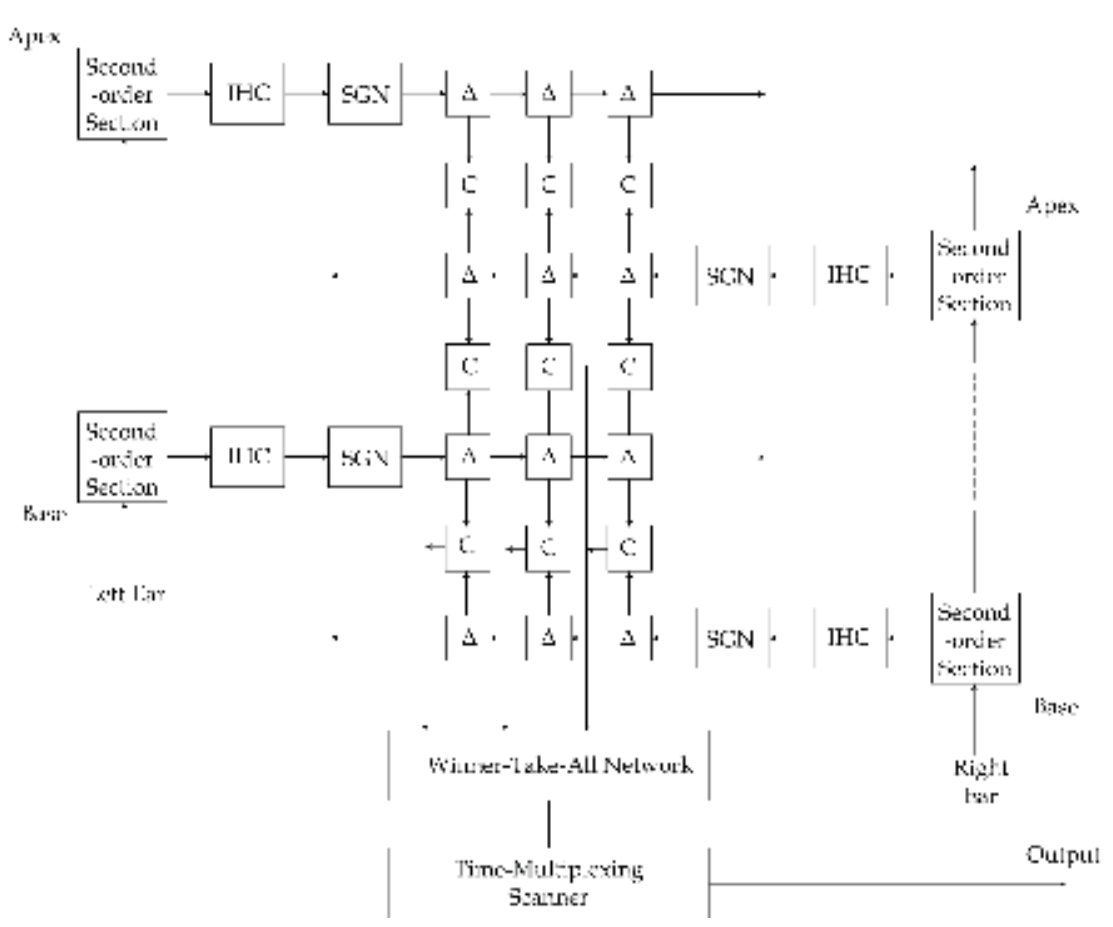

Fig. 8. Floorplan of the silicon model for sound localization of the owl. The square blocks marked with a ' $\Delta$ ' represent the discrete delay elements forming the silicon axons, whereas the square blocks marked ' $C$ ' in between every antiparallel axon are the correlators or coincidence detectors which take two inputs from the upper and lower axons; adapted from Lazzaro (Lazzaro and Mead, 1990). 
Lazzaro's contributions to the neuromorphic community were important because he managed to steer research towards a more application-specific direction. In the following years, quite a few researchers did significant work on the (re-)design and optimization of sound localization systems (using either neuromorphic or bio-inspired approaches) in an attempt to create artificial systems that could not only hear but also detect azimuthally or horizontally sound source locations. Applications were localization plays an important role include soundguided robots and automatic camera orientation in teleconference systems.

1991: Two years later, an interesting paper was published by Lyon which addressed for the first time the key problems they were facing at Caltech with the first generations of cochlea chips (Lyon, 1991). Lyon understood that the inherent exponential behaviour of MOS transistors in WI led - in general - to nonlinear filter circuits in which the small-signal and large-signal behaviours could be quite different. He proposed that in order to overcome problems like poor DR, excessive noise (accumulating naturally in a long cascade) and instability, they would need to come up with the design of inherently compressive filter stages. Quoting Lyon: 'Tests on early second-order filter stages, including a low-noise version based on the MOSIS lownoise analogue BiCMOS process, revealed a problematic nonlinear effect related to the saturation of the tanh nonlinearity of the transconductance amplifiers. The filter stages ended up with more gain for large signals than for small signals (i.e. they were "expansive"), and the result was that a given periodic input could lead to a pair of distinct periodic attractors. In a cascade of such filter stages, when the input became large enough to kick any stage into its large-signal mode, the final result was a chaotic output waveform resembling fractal mountains'. That was quite an interesting observation, firstly because identifying this kind of misbehaviour was non-trivial at a time where simulation tools (let alone accurate WI MOS transistors models) were not as developed as they are now, and secondly because the first paper on filters which perform signal compression to increase their DR and lower their power consumption (the so called companding signal processors) was published around that same year (Seevinck, 1990;Tsividis et al., 1990). A systematic formulation for the design of companding filters followed almost two years later (Frey, 1993).

In this paper, Lyon explored two different biquadratic architectures (based on cascades of two- and three-OTA topologies, called DIFF2 and DIFF3 respectively in (Lyon, 1991)) and incorporated source degeneration and capacitive division to widen the linear range of the OTA comprising the filters. However, contrary to the implementation of Fig. 3, these designs had their $Q$ and pole-frequency voltages interdependent leading to generally more difficult tuning schemes. Nevertheless, with these improvements, he managed to get a $40 \mathrm{~dB}$ pseudo-resonance gain in the cochlea response, rather than the $12 \mathrm{~dB}$ previously shown in Fig. 4 with a useable DR of about $40 \mathrm{~dB}$.

1992: After Lazzaro, the lead in the Caltech group was passed on to Lloyd Watts who was responsible for creating the first advancement in cochlea chip design since Lyon's original chip. Watts's overall design was based on a model of a passive (i.e. without an AGC network) 2-D cochlea (the first of its kind) (Watts, 1992). That model differs considerably from Lyon's 1-D filter-cascade, because it tries to replicate BM filtering together with the cochlea fluid. The fluid was modelled using a 2-D resistive network while the BM was modelled using $\mathrm{g}_{\mathrm{m}}-\mathrm{C}$ circuits which could mimic the BM impedance. Although the resulting system had ten times larger area than its 1-D equivalent, it had the advantages of exhibiting more realistic responses, being bi-directional (essentially modelling fluid reflections), faulttolerant and having a continuum limit. A conceptual diagram of his proposed 2-D cochlea 
model is shown in Fig. 9. Moreover, Watts was actually the first to propose a model for 'closing the loop' with $\mathrm{OHC}$ circuits, but his investigation ended prematurely. Quoting Watts: 'At the present time, the correct behaviour of the OHC circuit has not been verified at the system level, so we must leave the project as it is. Since there is still confusion in the auditory community about the form and sign of the mechanical feedback from the motile OHC...'.

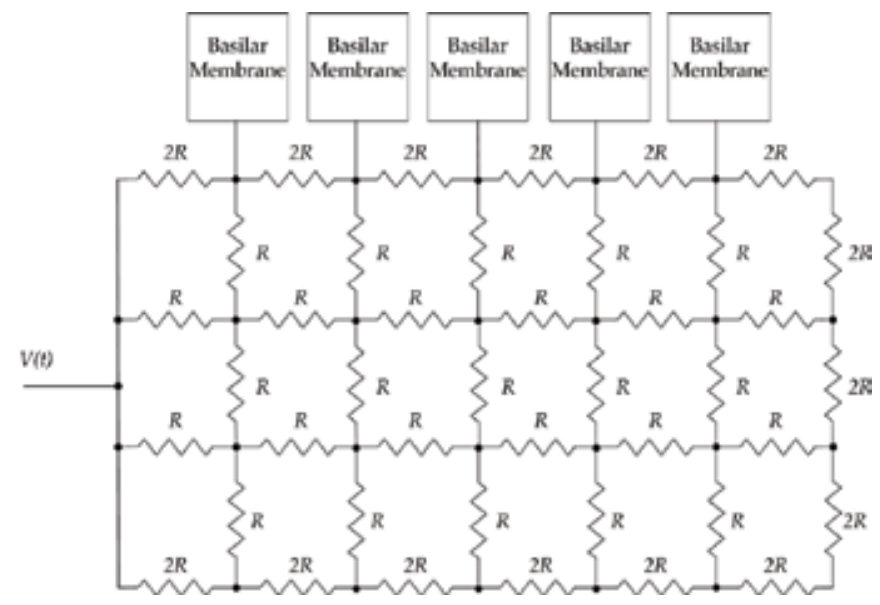

Fig. 9. The 2-D cochlea model. The cochlea fluid was modelled as a 2-D resistive network; adapted from Watts (Watts, 1992).

Apart from his 2-D contribution, Watts also thoroughly explored Lyon's 1-D cochlea. In (Watts et al. 1992), he not only discussed in detail the stability problems that Lyon had mentioned in his 1991 publication, but also elaborated on DR, matching and compactness; issues that were very superficially treated up to that point in time. Watts' improvements revolved around:

1. DR and stability: He found that by using one diode per side to degenerate the OTA, he could increase the input DR of the filter stage by 7.6dB. Moreover, by degenerating only the OTAs of the feed-forward path in Fig. 3, while keeping the feedback OTA narrow, he could eliminate the large-signal stability problem firstly addressed by Lyon one year earlier.

2. Improved Layout: Apart from applying obvious layout techniques like: a) making devices generally larger, b) placing match-sensitive devices close to each other and c) using common-centroid geometries, Watts realized that for a massively cascaded system such as the cochlea, one should work with the available chip area and mainly focus on the matching of critical devices. One example of his well-thought approach was the layout of the three transistors responsible for biasing the three OTAs in Fig. 3. Instead of duplicating each of the biasing transistors, laying them out in a hexagonal arrangement around a central point and connecting the pairs together in parallel, he realized that for equal capacitances in the filter, it was important only for the transconductance $g_{m Q}$ to match the sum of the two feed-forward $g_{m \tau}$ transconductances and not the individual transconductances (see transfer function in Fig. 3). Thus, he duplicated only the feedback biasing transistor and juxtaposed that pair with the feedforward biasing transistors in a 'pseudo-quad' formation as shown in Fig. 10. In addition, the initial Lyon cochlea relied on identical tilts on two resistive (polysilicon) 
lines which set the voltages $V_{\tau}$ and $V_{Q}$ to achieve uniform $Q$ at each stage. In Watts' design, he used only one resistive line for both the $\tau$ and $Q$ biasing transistors and controlled the actual $Q$ value by changing externally the source voltage of the $Q$ biasing transistor. In this way he was able to eliminate the mismatch for the case where two resistive lines were used and simplify considerably the actual testing procedure.
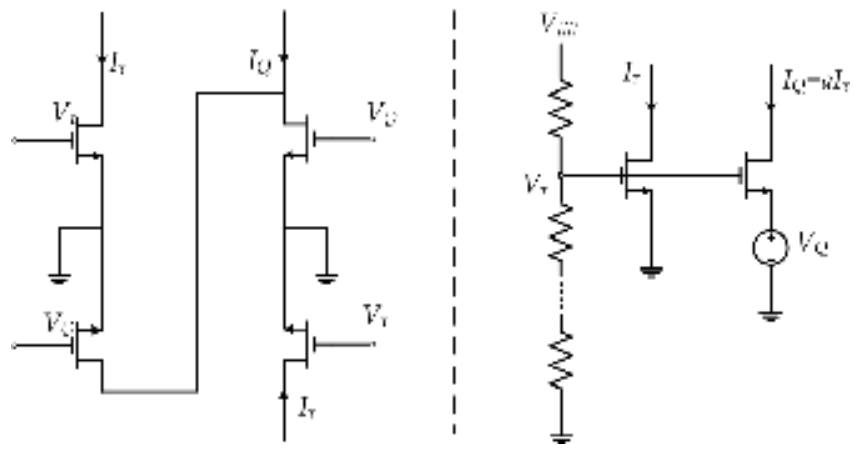

Fig. 10. The 'pseudo-quad' structure, formed by duplicating the $Q$ bias transistor and juxtaposing the resulting pair with the $\tau$ bias transistors (left). $Q$-source control by externally providing a voltage $V_{Q}$ to scale the feedback amplifier current $I_{Q}$ with respect to $I_{\tau}$ (right); adapted from Watts (Watts et al., 1992).

3. Compactness: Apart from the careful layout, Watts also considered how to save space. In particular, he noticed that the first feed-forward OTA shared a common output node together with the feedback OTA. Thus, he eliminated two redundant transistors by sharing a single current mirror between the two OTAs. Lastly, he observed that while the output $V_{3}$ was a LP version of the input $V_{1}$, the output current of the feed-forward OTA was related to $V_{3}$ by $I_{O U T}=s C V_{3}$. Thus, there was no need to devote extra circuitry for the differentiator circuit to convert BM displacement to BM velocity (i.e. from LPF to BPF response).

As a final point, Watts was actually one of the first to comment on the overall power consumption of his chip $(7.5 \mathrm{~mW} / 11 \mu \mathrm{W}$ for a 51-stage cascade with/without the circuits for making the results of the computation externally observable). Indicative measurements from his chip are shown in Fig. 12.

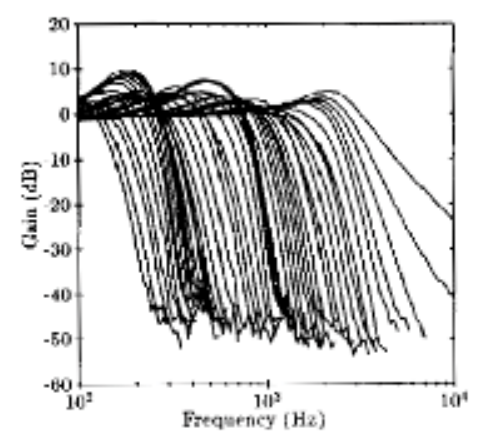

(a)

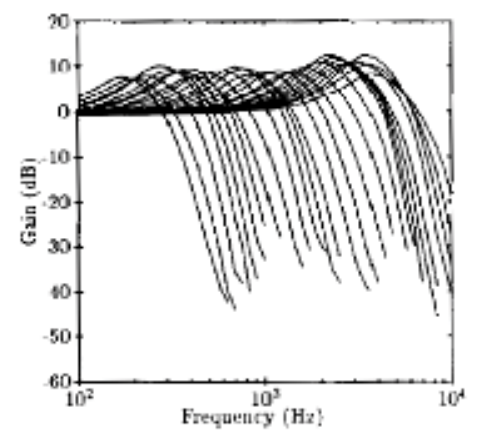

(b)

Fig. 11. Frequency response at each voltage tap. (a) Early layout. (b) Improved layout; from Watts (Watts et al., 1992). 
Up to that point, it seemed that most of the development of analogue VLSI cochlea design was undertaken by the people at Caltech. From 1992 onwards, research contributions from other university groups started to arise. For example, a journal paper was published in 1992 by Weimin Liu and Andreas Andreou from Johns Hopkins University (Liu et al. 1992), where he presented an analogue CMOS implementation of a model of the auditory periphery; his neuromorphic effort was heavily influenced by Lyon's and Lazzaro's previous efforts and in certain aspects extended and improved their modelling work. Liu designed a complete system that included, apart from the BM and its transducers, the filtering effect of the middle ear. His BM model was Lyon's cascade/parallel model but the actual arrangement of the transfer functions was slightly different: he used simple (passive) first-order LP filters to form a 30-stage cascade with a BP biquad connected at each tap; all filters were $g_{m}$-C operating in WI. As with Lyon's initial chip, tuning was achieved by using two polysilicon resistive lines. Liu also built a separate chip containing the circuits of IHC and synapses (based on the neurotransmitter substance reservoir model (Smith and Brachman, 1982)) and provided similar measured results to Lazzaro's (i.e. BM-tap frequency response and auditory-nerve firing rates). Although Liu's effort was important in its own right, it did not advance the field by presenting results from a system with AGC or from a detailed performance assessment (he only reported on the $15 \mu \mathrm{W}$ power consumption).

An interesting circuit contribution came from Jyphong Lin the same year, who used switchcapacitor (SC) techniques to design the biquad filters employed in his cochlea model. As far as we know, that was the first non $\mathrm{g}_{\mathrm{m}}$-C-based cochlea design effort. Lin published two separate conference papers in 1992 (the first dedicated solely on the design of the SC cochlea filters (Lin et al., 1992b) and the second on the design of a SC filterbank (Lin et al., 1992a)) and a complete Journal article with measured results in 1994 (Lin et al., 1994). The designs of his SC biquads were area-efficient, because they were synthesized using the chargedifferencing technique in which the time constants are controlled by both the product of capacitor ratios and the differences of the capacitor values, thus making the capacitor spread ratio small. His overall transfer function consisted of a cascade of two biquad filters; a LP together with a highpass (HP) response. The end result was an asymmetric BP shape similar to the one depicted in Fig. 2, with the added benefits (high precision and reliability) offered by the SC technique.

In his second conference paper, he designed a 32-channel filterbank to model BM filtering with each channel employing a $6^{\text {th }}$-order asymmetric BP response. In filterbank architectures the input is applied to all channels simultaneously, whereas in a filter-cascade the input is applied serially and gets successively filtered before reaching each output tap (see Fig. 12). For this reason, Lin's particular choice of modelling BM filtering together with his chosen asymmetric BP response may be classified as bio-inspired and bio-mimetic but not neuromorphic. What made his contribution interesting is that he used a biquad sharing technique (Chang and Tong, 1990) to achieve an efficiency of computation without the associate disadvantages of the filter-cascade architecture (like instability, sensitivity to mismatch, noise and offset accumulation etc.). In his dilating-biquad filterbank, each channel output was formed by the addition and scaling of three BP biquad responses from three separate channels, thus facilitating considerable area savings. In other words, instead of using 192 biquads, he used 34 biquads and 32 sum-gain amplifiers; 66 in total (see Fig. 13). The only problem with this scheme is that the three dilated biquads must have constant $Q$ values in order for the resulting higher-order response to maintain its shape and since all 
biquads are inter-connected, the whole filterbank must be of constant $Q$. This is a significant drawback for applications where a realistic, OHC-based, AGC needs to be incorporated.

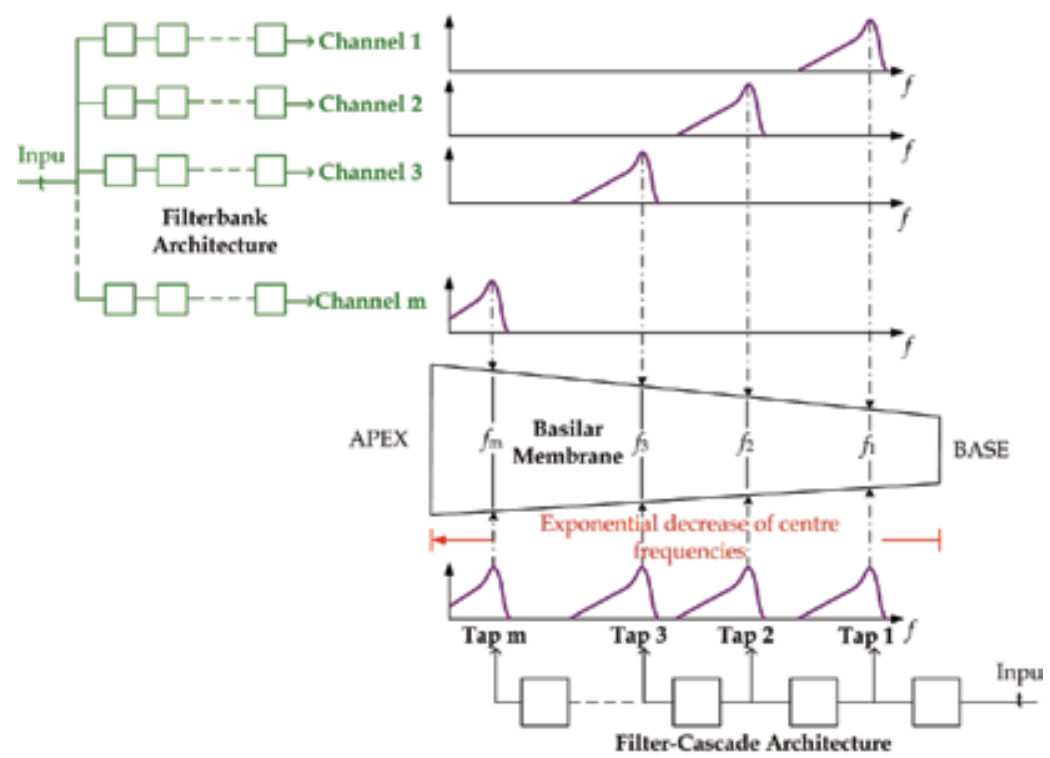

Fig. 12. Modelling BM filtering via neuromorphic (filter-cascade) and bio-inspired (filterbank) architectures.

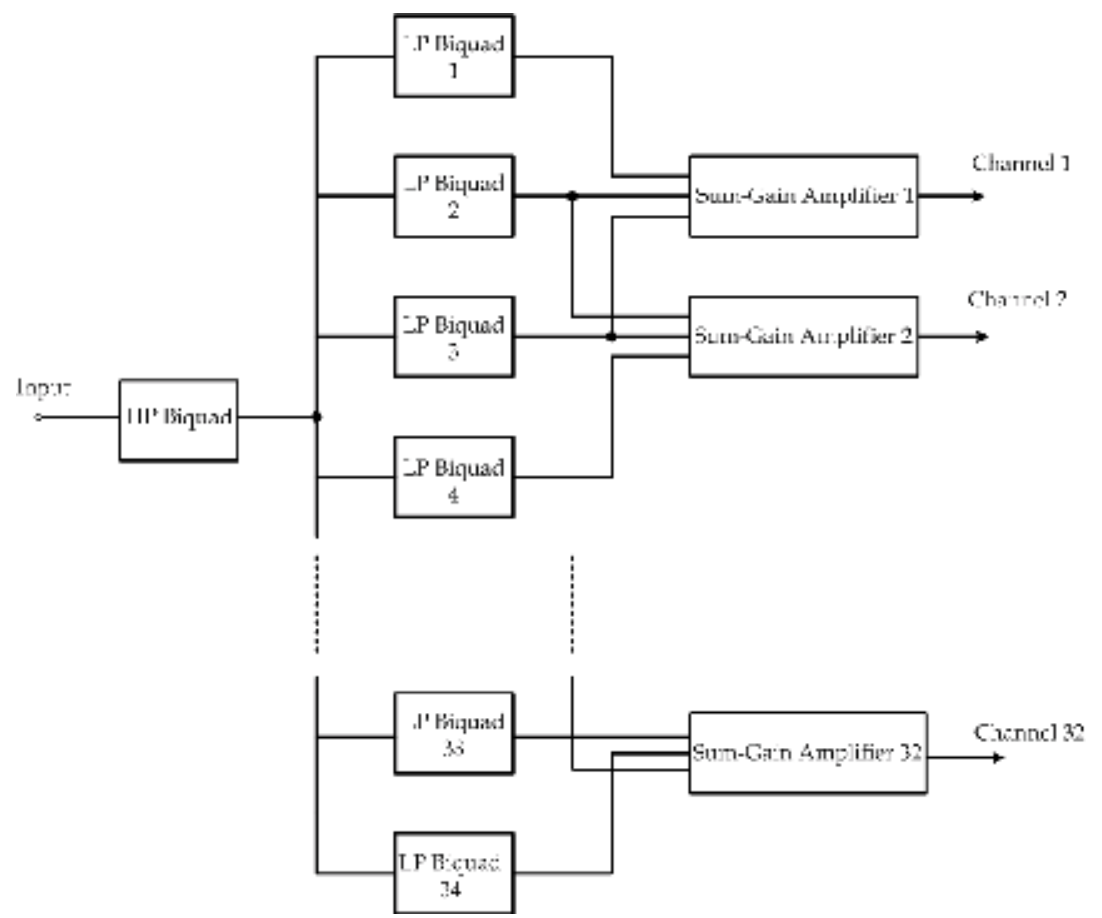

Fig. 13. Dilating-biquads filterbank; adapted from Lin (Lin et al., 1992a). 


\section{Increasing the performance: 1993 - 1998}

1993 E 1994: After Lazzaro's work, the second sound localization systems were presented in 1993 and 1994 by Neal Bhadkamkar from Stanford University (Bhadkamkar and Fowler, 1993;Bhadkamkar, 1994). The 1993 effort was based on a design that was an architectural duplicate of the biological system (i.e. neuromorphic), whereas the 1994 effort was not, because it tried to duplicate some of the functions of the real system without relying on strict architectural analogies.

The neuromorphic system consisted of two fabricated chips; one chip contained the circuitry to model the left and right side cochleae, IHC and auditory neurons, whereas the other chip contained circuitry to model the binaural cross-correlation activity of neurons in the superior olive of the brainstem. Even though the system-level ideas were identical to Lazzaro's and Lyon's, Bhadkamkar's 1993 circuit design choices were slightly different. For example, Bhadkamkar's 1993 design included separate voltages to control the propagation delays and cut-off frequencies of the stages, respectively. This fact, together with his choice of a more complex transfer function for each BM section, resulted in a multiresolution BM design where the frequency resolution could be increased without excessively increasing the propagation delay ${ }^{1}$ (Bhadkamkar, 1993). Similarly, the differentiator used for the IHC was different than Mead's hysteretic differentiator because it was designed to pump current into the SGN circuit during the discharge time of the capacitor. At low frequencies this occurred approximately half of every input cycle that it saw. At high frequencies, current was pumped in for a much smaller portion of the cycle. Thus, at these high frequencies, increases in the amplitude of an input sinusoid caused a sudden but temporary increase in the current that pumped into the SGN circuit. His SGN circuit was identical to Lazzaro's and Mead's but included a refractory period control i.e. freedom to control the time needed to elapse before permitting the SGN input current to have any effect. Moreover, his correlator chip was similar to that presented by Lazzaro, but his axons (delay-lines elements) received pulses in parallel and not serially to eliminate the accumulation of errors. Finally, no winner-take-all circuit was used; each output was summed together with the other corresponding outputs along all frequency channels and integrated using a leaky integrator. On the other hand, his 1994 bio-inspired system was designed using two separate parallel banks of simple $\mathrm{g}_{\mathrm{m}} \mathrm{C}$ BP filters. As mentioned previously this is not an accurate cochlea model but improves localization accuracy by removing the accumulation errors inherent in the cascade structure. The design of the IHC was based on a half-wave rectification, LP filtering and pulse-width modulation, with the pulse-width being a monotonic function of the amplitude of the LP-filtered, half-wave rectified signal. Thus, in this case he chose a pulse-width-modulation scheme for the neural encoding as opposed to the pulse-frequencymodulation used in his 1993 effort.

That same year the first current-mode cochleae designs arose; one from Christopher Abel (Abel et al., 1994) and one from Christofer Toumazou and Tor Sverre Lande (Toumazou et al. 1994). The former effort presented a novel implementation of a silicon cochlea based on

\footnotetext{
${ }^{1}$ One of the disadvantages of the cascade approach is that the propagation delay of the travelling wave from the input to a particular output tap is controlled by the same parameter that controls the cut-off frequency of the filters. This leads to the problem that the accumulated delay increases as a function of the number of filters per given frequency range, i.e. the larger the frequency resolution, the more the accumulated delay.
} 
discrete-time, switch-current biquads. Since the equivalent continuous-time cut-off frequency of a discrete-time filter is proportional to the sampling rate, reducing the sampling rate by a factor of two lowers the cut-off frequency by one octave. Abel exploited this fact and realized that a set of filters designed to cover one octave may be used again at a reduced sampling rate to cover a lower octave. This technique allowed the entire range of audio spectrum to be covered by one repeated set of filters, thereby avoiding the need of a wide-range of integrator time constants and allowing for great area savings.

Abel's architecture is shown in Fig. 14. In addition, unlike SC, the switch-current technique does not require linear capacitors and produces filters that are thus compatible with standard CMOS digital processes. Abel's contribution was really interesting because he not only used a totally different circuit technique (let alone signal representation) to design his system, but also he showed preliminary simulation results from a very simple $Q$-control circuit. To avoid any confusion, Abel's $Q$-control circuit was not intended to close-the-loop between each stage of his cascade. By $Q$-control circuit he meant a circuit where adjustment of his biquads $Q$ values could be possible, but this adjustment did not happen automatically according to signal strength.

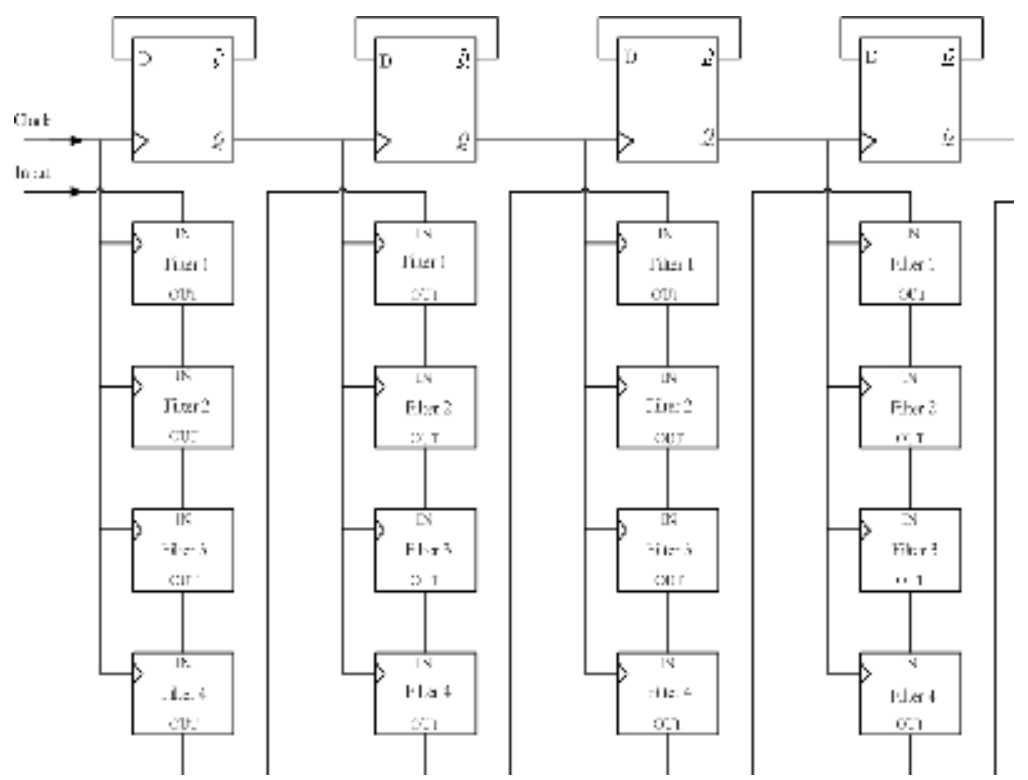

Fig. 14. The system level block-diagram of Abel's proposed design. A single D flip-flop acts to divide the clock frequency by two after each stage. This allows one set of four filters to cover multiple octaves; adapted from Abel (Abel et al., 1994).

Toumazou's contribution was quite substantial at the time. To this author's knowledge, his effort was the first true continuous-time current-mode implementation of a BM segment that employed the aforementioned companding technique (check Lyon's quote in 1991) to achieve high-DR and low-power dissipation. The companding strategy was based on the pioneering contributions of Douglas Frey (Frey, 1993;Frey, 1996)on the systematic synthesis of the so called log-domain filters published only a year before. The rationale behind this is that the signal is firstly compressed to an intermediate nonlinear voltage according to the logarithmic $I$-to- $V$ relationship of WI MOS devices. The compression generally leads to 
voltage swings that are small thereby allowing low-power operation. Also, through the application of the particular nonlinear compressive law, a wide range of signals could be accommodated at the input without needing to spend additional power (like in conventional $\mathrm{g}_{\mathrm{m}}-\mathrm{C}$ designs). After compression, the intermediate nonlinear voltage signal gets filtered and subsequently expanded at the output, using the exponential $V$-to- $I$ relationship of WI MOS devices and thus maintaining overall input-output linearity. Because of the fact that the compression law is based on the natural logarithm, the resulting filters were called log-domain filters. Toumazou's design demonstrated a simulated input DR of $80 \mathrm{~dB}$, while dissipating a mere $125 \mathrm{nW} /$ pole. Toumazou's contribution was quite valuable at the time, because a) it revealed the potential of current-mode design within the particular cochlea application and b) it showed results from the $1^{\text {st }}$ ever CMOS log-domain biquadratic filter.

1995 \& 1996: Around 1995, papers that focused more on performance started to arise. Paul Furth and Andreou from Johns Hopkins University published three papers spaced one year apart. His 1995 effort (Furth and Andreou, 1995a) detailed the design of a multiresolution analogue filterbank with the primary engineering constraint being power consumption. He presented a design strategy for hardware cochlea filterbank models addressing issues both at architectural- and circuit-design level. Architecture-wise, Furth used a slight variation of Liu's filterbank (see 1992). In particular, Furth added two cascaded identical BP biquads at the output of each of the 16 taps to increase the per-tap selectivity much like what Liu did in his $\mathrm{PhD}$ dissertation (Liu, 1992). Furth was actually one of the first to address in more detail the DR problem with $\mathrm{g}_{\mathrm{m}}-\mathrm{C}$ designs. Instead of designing a linearized OTA (with a tanh $I-V$ transconductance characteristic), he resorted to a CMOS Class-AB stage (with a sinh I-V transconductance characteristic) due to the facts that: a) it uses minimal number of components, b) it has a wide tunability range by tuning its supply voltages or substrate terminals and c) it contains no biasing elements, thereby rendering it the less noisy from all CMOS transconductor configurations at a given bias current (see Fig. 15). Furth consequently derived and numerically computed the maximum DR of a LP integrator employing two Class-AB transconductors. He showed that for a pure tone input, the maximum distortion-free DR (i.e. in which the distortion components equal the noise floor) is $44.3 \mathrm{~dB}$, whereas the maximum distortion-limited DR (for an output THD of $2 \%$ ) is $49.4 \mathrm{~dB}$. He then estimated the distortion-limited DR at the output of each tap to be $28.2 \mathrm{~dB}$. The power supply of his circuits was $1.5 \mathrm{~V}$ and the total power dissipation of a 16-tap filterbank was $355 \mathrm{nW}$; the lowest reported up to that point.

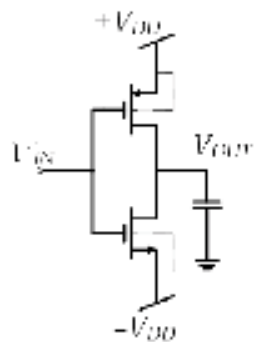

a)

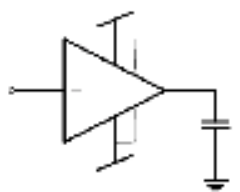

b)

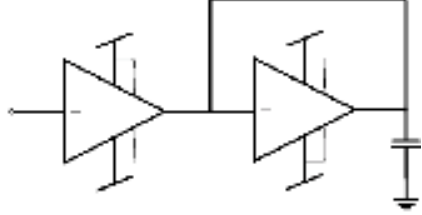

c)

Fig. 15. a) the CMOS Class-AB transconductor, b) its symbol and c) the topology of a LP integrator; adapted from Furth (Furth and Andreou, 1995a). 
In his 1996 effort (Furth and Andreou, 1996a), Furth took a different approach. He compared two differential-pair OTA employing respectively the single and double diffusive source degeneration technique (Boahen and Andreou, 1992). Circuit-wise the diffusor is a floating active MOS resistor placed in between the sources of a differential-pair. The diffusivity or conductivity of this resistor depends on the aspect ratio and it could be electronically controlled by varying the gate voltage. He found that the DR was $56.8 \mathrm{~dB}$ for the single diffusion OTA (a 13.2dB improvement over the basic differential-pair) but extra commonmode circuitry was required. On the other hand, the double diffusor OTA exhibited $3 \mathrm{~dB}$ less DR but without adding power or needing extra common-mode circuitry. Furth built the two OTA in a $2 \mu \mathrm{m}$ CMOS process using large device areas $(1377 \mu \mathrm{m} / 4.8 \mu \mathrm{m})$ and measured their relative DR performances, which came out being $10 \%$ lower than the aforementioned theoretical values. The diffusor source degenerated OTA with their corresponding $I-V$ transconductance relations are depicted in Fig. 16. All parameters have their usual meanings, while $m$ denotes here the aspect ratios of the diffusors.

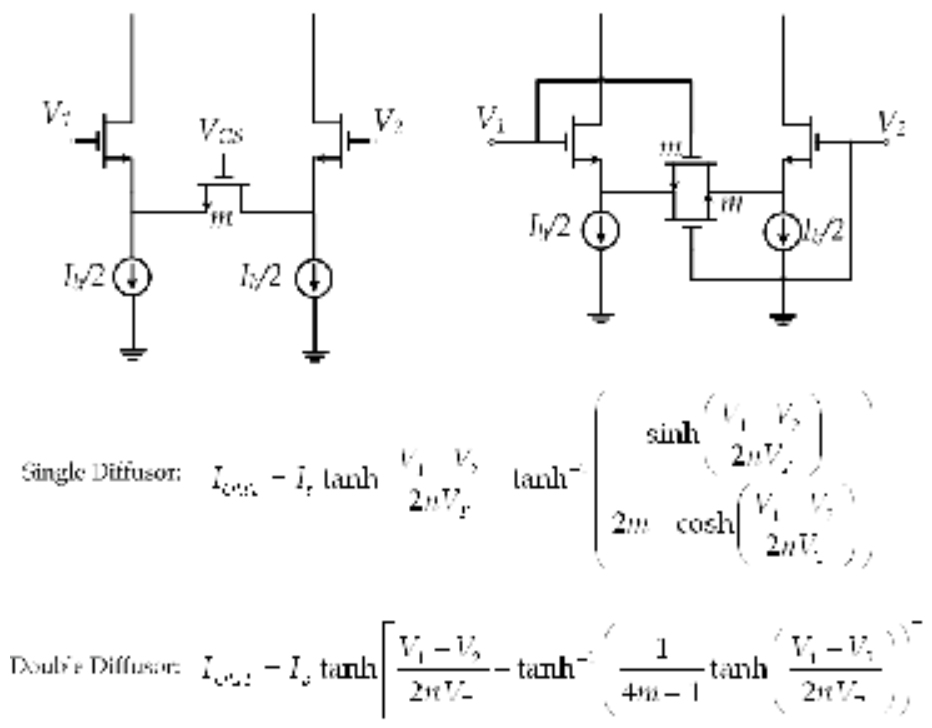

Fig. 16. Linearized OTA by means of single and double diffusive source degeneration; adapted from Furth (Furth and Andreou, 1996a).

In 1996, Jenn-Chyou Bor adopted the previous practice of other authors on SC filters and presented a SC cochlea cascade of 32 sections based on the transmission-line model of Zwislocki (Zwislocki, 1950). In essence, Bor resorted to the 2-D nonlinear partial differential equation describing the transmission-line cochlea model and by applying the principles of discrete-time signal processing and variable transformation, he reduced it into several 1-D equations that could be realized using circuits designed through the multiplexing SC technique. In his paper (Bor and Wu, 1996), he demonstrated through measurements a neuromorphic BM-cascade cochlea with very low sensitivity to process variations (see Fig. 17). The resulting filters operated from a $\pm 3 \mathrm{~V}$ supply and the single filter stage $\mathrm{DR}$ at $1 \mathrm{KHz}$ and $1 \%$ THD was measured $67 \mathrm{~dB}$ (the highest reported up to that date). Bor also provided a table with the errors from the measured peak gains and peak frequencies (i.e. CF) from theoretical values. Yet, no IHC, neuron or OHC-based AGC circuits were designed. 

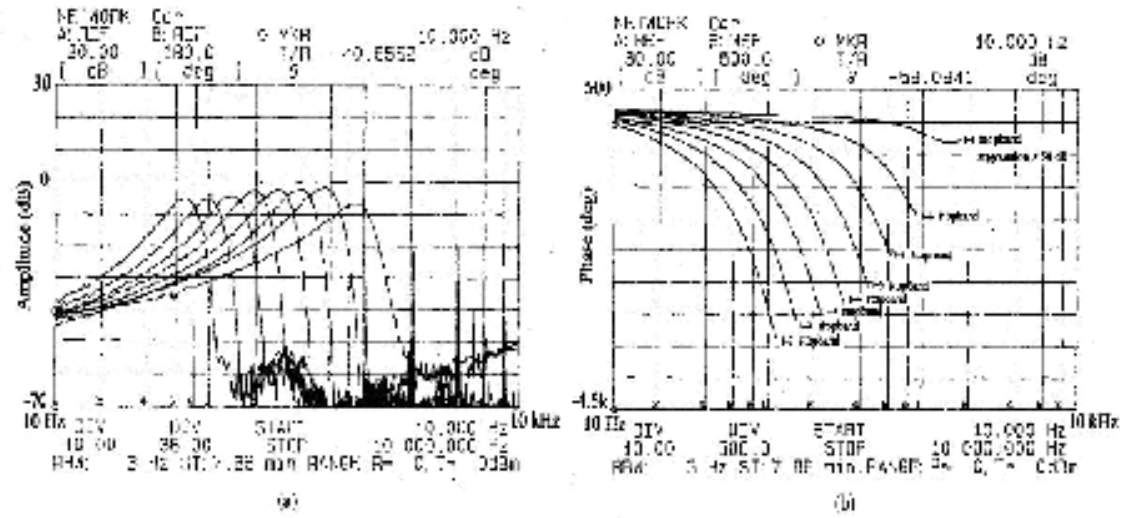

Fig. 17. Measured a) amplitude and b) phase responses from 8 taps of a 32-section SC filtercascade; from Bor (Bor and Wu, 1996).

Within Carver Mead's group, Rahul Sarpeshkar, the successor of Watts, took the rather challenging direction of designing a high performance neuromorphic cochlea cascade. As mentioned previously, the group's prior $\mathrm{g}_{\mathrm{m}} \mathrm{C}$ biquad designs had their large-signal stability limit smaller than their small-signal stability limit. This misbehaviour forced them to set the gains, for each stage of the cascade, at conservatively small values (i.e. having very low $Q$ ) in order to ensure that each stage remained stable at large signal levels. Consequently, this resulted in small pseudo-resonant peak gains (around 12dB in (Lyon and Mead, 1988a)) and input DRs between 20 - 40dB at best. Initially, Sarpeshkar focused on the careful design of the OTAs comprising the biquads and in (Sarpeshkar et al., 1996) he designed a wide linear range OTA (named the WLR) through the use of four linearization techniques extending the linear range from $75 \mathrm{mV}$ to $1-1.75 \mathrm{~V}$. With Fig. 18 as a reference, these linearization techniques were:

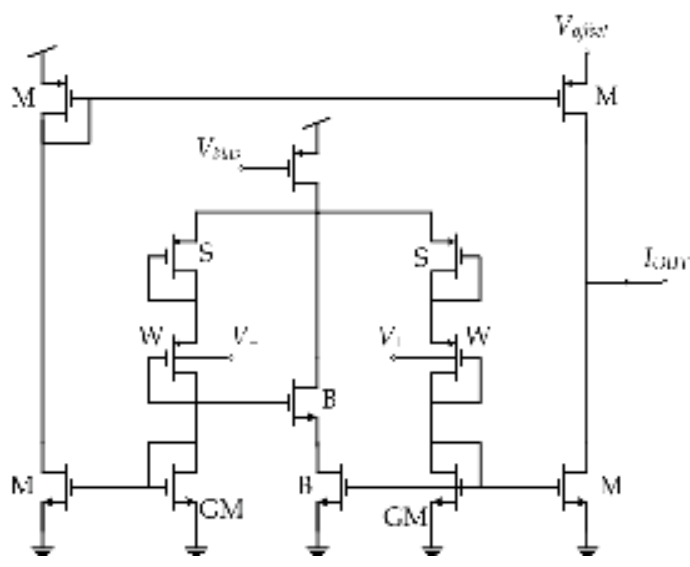

Fig. 18. The WLR OTA. The devices marked $S$ and GM denote the source and gate degeneration transistors, whereas the devices $\mathrm{W}$ and $\mathrm{M}$ denote the well-input and mirror transistors. The B transistors perform bump linearization. In addition, the voltage $V_{\text {bias }}$ sets the bias current of the amplifier, whereas $V_{\text {offset }}$ allows fine adjustment of the amplifier's output offset if necessary; adapted from Sarpeshkar (Sarpeshkar et al., 1996). 
1. Instead of using the gates of the $W$ devices as inputs he used their wells due to the well's lower transconductance. To avoid latch-up he used large common-mode voltages in the range $1-5 \mathrm{~V}$.

2. Devices $S$ lower the transconductance of the $W$ devices further through the common technique of source degeneration.

3. Devices GM lower the transconductance of the $W$ devices even further through gate degeneration. An increase in current in either of the two differential pair branches causes an increase in voltage of the GM devices that is fed-back to the gate of the W transistor and turns it off.

4. Bump linearization. The bump circuit was proposed by Delbrück in (Delbruck, 1991) and the way it linearizes the tanh function of an OTA is qualitatively similar to Katsuji Kimura's triple-tail technique (Kimura, 1995). The basic idea of the bump transistors is that they steal current from the two branches of the differential pair at low differential voltages, thereby reducing the transconductance. Moreover, by appropriately choosing their aspect ratios, odd-order harmonic distortion gets suppressed as compared to the tailor expansion of the standard tanh characteristic.

Sarpeshkar then incorporated the WLR to create biquad filters forming a 45-stage BM cascade. His filter design (a block diagram of which is shown in ) was based on that of Fig. 3 but with the following three modifications:

1. Since the well was used as the input, there were no explicit capacitors in the filter topology; he used the well capacitance. In addition, since the well-to-bulk reverse bias voltage was of the order of $3 \mathrm{~V}$, this capacitance was fairly constant without introducing excessive distortion at the output.

2. The feedback OTA (amplifier $A_{3}$ in Fig. 3) now implemented a non-monotonic function with a fuse-like characteristic. Recall that Watts was the one who linearized OTAs $A_{1}$ and $A_{2}$ while leaving $A_{3}$ narrow to eliminate the large signal instability. Sarpeshkar, on the other hand, linearized OTAs $A_{1}$ and $A_{2}$ and made the characteristic of $A_{3}$ to as such so that positive-feedback amplification shunts-off completely instead of simply saturating at large signal levels. Thus, at high-levels it effectively reduces the gain of the stage resembling the compressive mechanisms attributed to OHC operation. In essence, the fuse-like OTA performed static nonlinear compression. Note that OTAs with fuselike characteristics where presented earlier in (Kimura, 1994).

3. Finally, he followed Watts' ideas on compactness and realized that the feedback fuselike OTA shared the same differential inputs as the output feed-forward OTA and the same output as the first feed-forward OTA. Thus, he shared their differential pair and output mirror circuits, allowing the fuse-like OTA to be implemented by only four transistors.

Sarpeshkar's 1996 effort formed the basis of his forthcoming complete design presented two years later. His architectural and topological choices gave rise to biquad filters that exhibited input DR in excess of $60 \mathrm{~dB}$ i.e. an order of magnitude larger from the group's previous $\mathrm{g}_{\mathrm{m}}-\mathrm{C}$ efforts. However the topology shown in Fig. 19 had a very specific drawback; the noise per unit bandwidth increased with $Q$. This was because in that particular biquad topology, the $Q$ was obtained through the addition of positive-feedback currents (marked $I_{f b}$ in Fig. 19) which contributed additional shot noise. As it will be shown later on, his 1998 effort employed a different biquad topology (based on the DIFF2 structure which was discussed by Lyon in 1992 (Lyon, 1991)), although the WLR topology remained the same. 


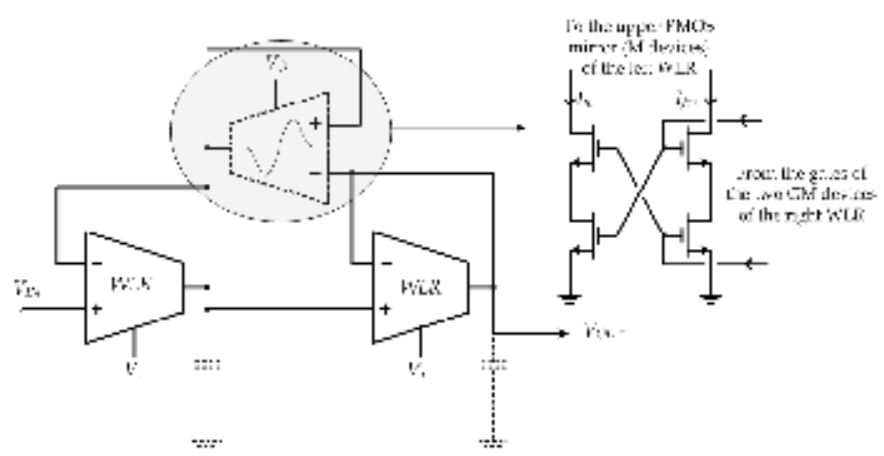

Fig. 19. A schematic block diagram of the fuse biquad. The dotted components indicate that they are not explicitly implemented in the actual circuit topology. The voltages $V_{\tau}$ and $V_{Q}$ set the pole frequency $\omega_{0}$ and quality factor $Q$ of the filter; adapted from Sarpeshkar (Sarpeshkar et al., 1996).

In the same year, Andre Van Schaik from EPFL's MANTRA Center for Neuromimetic Systems, together with Eric Vittoz addressed in detail the problem of matching in CMOS WI for the second time since Watts's 1992 effort (A.Van Schaik et al., 1996). Van Schaik used Compatible Lateral Bipolar Transistors (CLBTs) in a 104-stage cochlea cascade, to create the exponentially scaled currents when biased with a resistive line with a linear voltage gradient between its two ends. CLBTs can easily be made in a CMOS substrate and contrary to simple WI MOS devices, their collector current is independent of the CMOS technology's threshold voltage. The remaining mismatch is due to the geometry mismatch of the devices, but this parameter is much easier controlled than the variance of the threshold voltage. A CMOS CLBT can be obtained if the drain or source junctions of a MOS device gets forwardbiased in order to inject minority carriers to the local substrate. If the gate voltage is negative enough (for an n-channel MOS) then no current flows at the surface and the operation is purely bipolar (Vittoz, 1983). A diagram showing the carrier flow and bipolar operation of a MOS transistor is shown in Fig. 20.

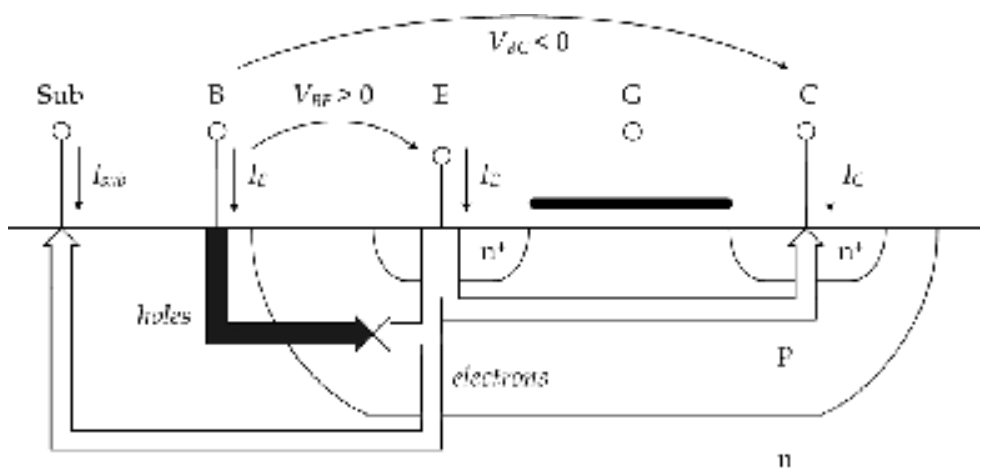

Fig. 20. Bipolar operation of a MOS device; adapted from Van Schaik (A.Van Schaik et al., 1996).

Much like Watts, Van Schaik identified that the biquad's biasing transistors (i.e. those that set the pole frequency and $Q$ ) had to be precisely matched. In prior designs, these transistors were simple MOS, thus a small variation in their threshold voltage would result in a large 
variation in their output current due to the (inherent in WI) exponential $V$-to- $I$ relationship. CLBTs offered a considerable improvement in the regularity of the frequency spacing of the cochlea filters which is of significant importance to long filter-cascade models, since one filter can distort the input signal for all the subsequent stages. Results showing the cascade's improved frequency response and spacing regularity are shown in Fig. 21. Finally, in 1996 Van Schaik published together with Ray Meddis an article describing preliminary designs of IHC and SGN circuits (van Schaik and Meddis, 1996) which were used later by the author in forthcoming publications.
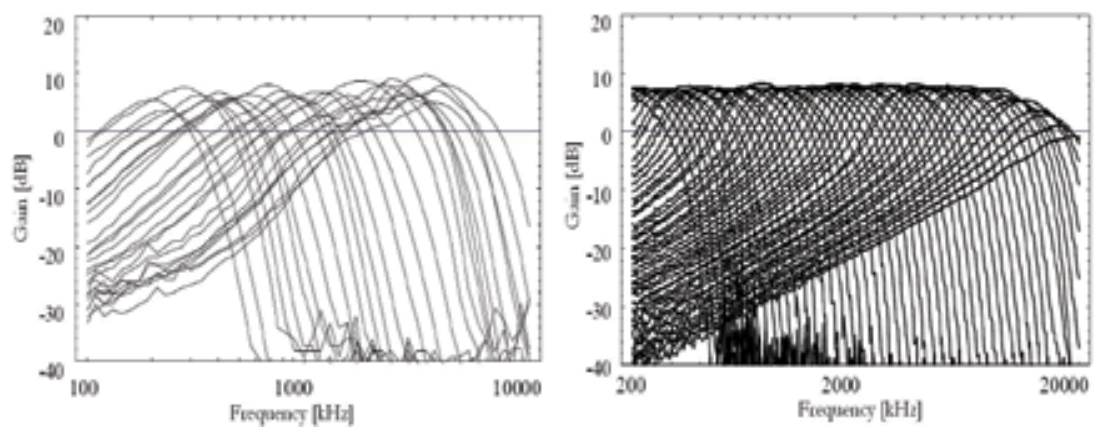

Fig. 21. Comparison between the 1992 design of Watts (left) with the improved design employing CLBTs (right); from Van Schaik (A.Van Schaik et al., 1996).

1997: As mentioned previously, Furth published his third paper in 1997 (Furth and Andreou, 1996b). Following Sarpeshkar's 1996 work on increasing the OTA linear range, that particular effort varied along the same lines and presented a new wide linear range OTA. Furth's OTA topology was based on the initial BiCMOS design of Liu and Andreou (Andreou and Liu, 1992) which incorporated an OTA operating in strong inversion, a bipolar Gilbert multiplier cell and a WI MOS current buffer. Liu claimed that the choice of the two different modes of operation together with the individual optimization of each block could lead to an increased overall performance regarding power consumption, DR, tuning range and area. In this effort, Furth made some modifications to Liu's and Andreou's design by linearizing the OTA by means of a double diffusor and by replacing the Gilbert multiplier cell by a WI MOS equivalent. In this way the problems associated with beta reductions, beta mismatch and/or large leakage currents of the bipolar devices could be alleviated. His simulations yielded a linear range of $\pm 260 \mathrm{mV}$ and an estimate (based on an empiric choice of noise floor value) for the DR of $66 \mathrm{~dB}$. His OTA design is shown in Fig. 22. One of the better contributions in analogue VLSI cochlea modelling came the same year from Eric Fragniére with Van Schaik and Vittoz (Fragniere et al., 1997). Together they proposed an analogue VLSI model of an active cochlea based on Lyon's silicon BM filtering implementation. Circuit modelling-wise they exploited the same $\mathrm{g}_{\mathrm{m}}-\mathrm{C}$ biquad design as the one proposed by Lyon and Watts in 1992 (i.e. the one with the two feed-forward OTAs degenerated with one-diode per side, while keeping the feedback OTA narrow), but they also provided a thorough analysis (with CMOS WI circuit implementations) on an adaptive AGC scheme for regulating the stages $Q$ values that was never reported before.

They implemented a feedback closed-loop AGC scheme, where the signal at an output tap is used to regulate the $Q$ of a specific preceding stage according to a square-root level compression law (and not a linear one like Hirahara presented in 1989). From their study they 


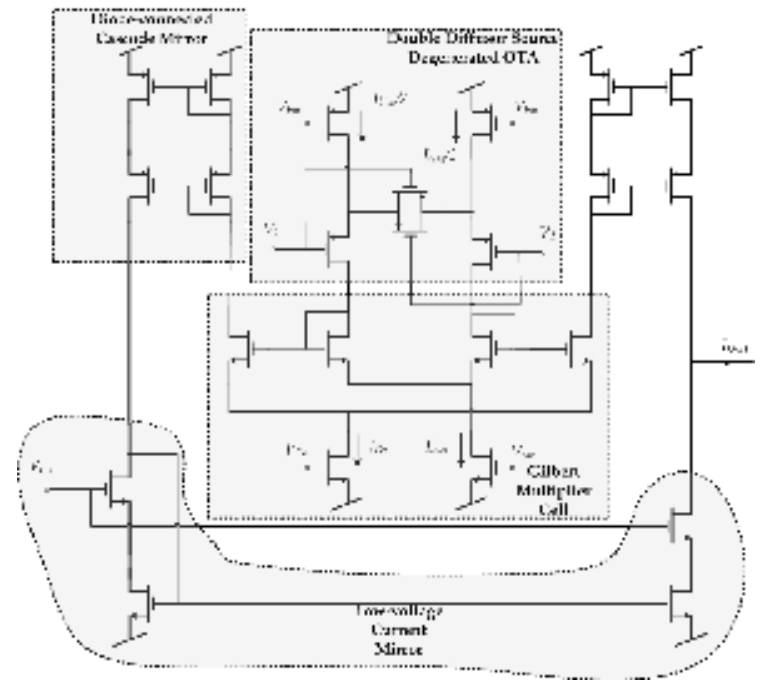

Fig. 22. Furth's wide linear range OTA implementation. The voltage $V_{G_{0}}$ sets the gain of the Gilbert Multiplier Cell, $V_{\text {sat }}$ is the biasing of the level shifter transistor, whereas $V_{\text {cas }}$ is the biasing of the low-voltage current mirror; adapted from Furth (Furth and Andreou, 1996b).

realized that in order to locally control the pseudo-resonant gain at the output of a particular stage, only the $Q$ of the stages participating to the build up of that pseudo-resonance had to be controlled. Their findings can be summarized in the following sentence: 'The output from any stage in the cascade must control the $Q$ of a stage located at a basal distance corresponding to $a$ $C F$ increase between one sixth and one third of an octave from that particular stage's output'. This conclusion was supported by physiological evidence which revealed that afferent fibres of IHC are paired with the afferent fibres of OHC located at one seventh to one third of an octave higher CF (Kim, 1986). In other words, they not only specified the law with which the output-tap's signal level was mapped to the stage $Q$ values, but they also provided a design framework for distributing the gains along the cascade. Fragniére's per stage local feed-back AGC scheme is shown in Fig. 23. The $V_{d i n}$ is the voltage output from the previous stage, whereas $V_{\text {dout }}$ is the output voltage representing $\mathrm{BM}$ displacement of the current stage. Following Watts observation on compactness (see 1992), $I_{\text {vout }}$ is a current signal representing BM velocity (i.e. it is the differentiation of $V_{\text {dout }}$ ) which consequently passes through a halfwave rectifier representing the action of the IHC. The output from the rectifier goes to a mean spiking rate (MSR) lossy integrator that computes the mean value of the half-wave rectified $I_{I H C}$; the output of the MSR $\left(i_{I H C_{-} D C}\right)$ is a quasi-DC signal that represents signal strength. The final stage is the $Q$ decision block (implemented by means of a simple WI translinear loop) that maps $I_{I H C_{-} D C}$ to the correct $I_{t \tau}$ and $I_{Q}$ values that set the $\omega_{0}$ and $Q$ of the filter. With Fig. 3 as a reference, the $Q$ decision circuit implements the following control law:

$$
Q \propto\left(\frac{g_{m Q}}{g_{m \tau}}\right) \Rightarrow \frac{I_{Q}}{I_{\tau}}=\left(\frac{I_{Q \max }}{i_{I H C_{-} D C}+I_{o}}\right),
$$

where $I_{Q \max }$ corresponds to the maximum $Q$ value for zero input, $I_{0}$ is a DC level ensuring loop stability for zero input and with $I_{Q}$ varying geometrically with signal strength implying a logarithmic compression-type mapping. 


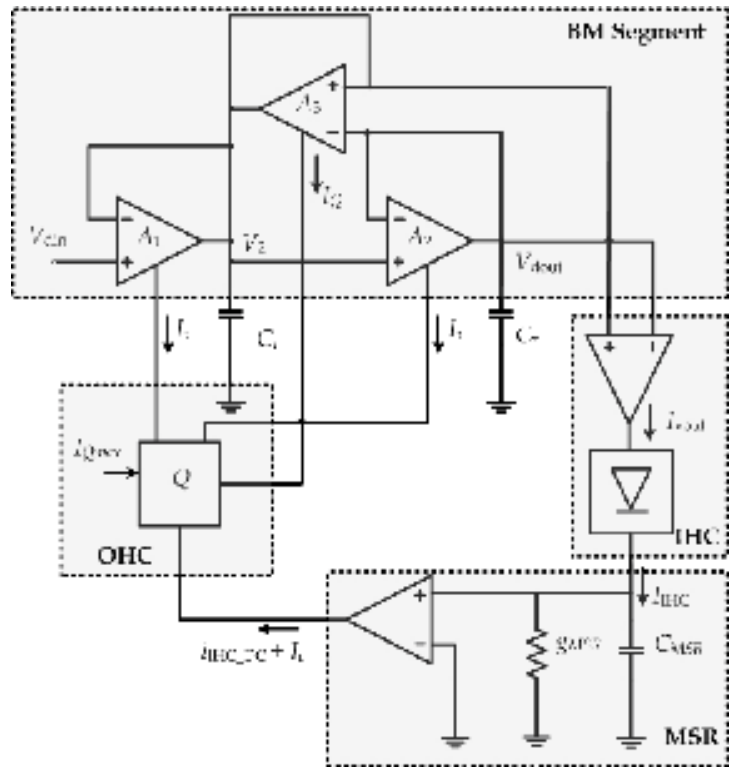

Fig. 23. Analogue model of one stage of the active cochlea cascade; adapted from Fragniére (Fragniere et al., 1997).

Finally, it should be mentioned that all the results shown in this work were simulated using mathematical software tools like MatLab and not from detailed circuit simulations or measurements. For this reason, Fragniére's contributions are considered significant but from the overall system-level modelling perspective and less from a circuits or performance evaluation perspective.

1998: Even though seventeen years had passed from Lyon's initial 1981 paper, still no publications detailing measured results from an 'active' silicon cochlea existed. Fragniére's efforts were indeed influential, but still his modelling ideas had to be demonstrated in an actual analogue hardware realization. The most complete single-piece of work on a neuromorphic silicon cochlea came in 1998 by Rahul Sarpeshkar from Carver Mead's lab. In (Sarpeshkar et al., 1998), he described an 117 -stage, $100 \mathrm{~Hz}-10 \mathrm{kHz}$, silicon cochlea that attained a DR of $61 \mathrm{~dB}$ (at $4 \%$ THD) at the CF of a typical stage while dissipating $0.5 \mathrm{~mW}$ of static power, by using the following techniques:

1. A modified version of the previously described WLR. Here he did not use source degeneration as in the design of Fig. 18, but used cascode circuitry to increase the DC gain and prevent low-frequency attenuation in the cochlea.

2. An improved biquad filter topology that is low-noise by overcoming the problem of his previous 1996 topology where the output noise scaled with $Q$.

3. DR gain control through a local, fast-acting feed-forward AGC. Architecture-wise Sarpeshkar's AGC loop is the same as Fragniere's but acts on the level of the stage's input and not output.

4. Automatic offset-compensation circuitry in each cochlea stage prevented offset accumulation along the cochlea cascade.

5. The architecture of three, overlapping 39-stage cochlea cascades (see Fig. 24). In this architecture the input is fed in parallel to tiny cochlea cascades whose corner frequencies overlapped by 1 octave. This particular architecture may be viewed as a 
hybrid of an architecture that has many parallel filters in a filterbank and one that has one filter-cascade with all filters in serial. Even though he partly deviated from a purely neuromorphic BM structure, he showed that by adopting this scheme he could reduce the group delay of his silicon cochlea and limit the accumulation of the $1 / f$ noise.

(a) Regular BM C.ariate

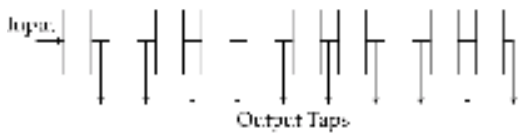

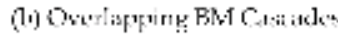

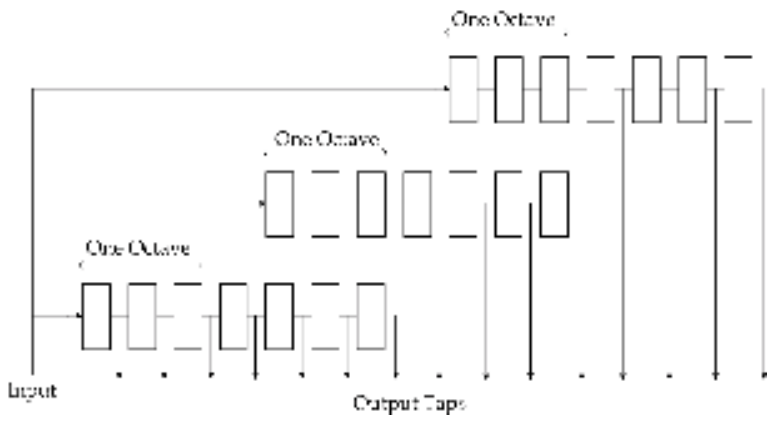

Fig. 24. Architecture of overlapping cascades. (a) In a standard filter-cascade the input is fed serially to all the stages, whereas in (b) the input is fed in parallel to tiny cochlea cascades that overlapped by one octave; adapted from Sarpeshkar (Sarpeshkar et al., 1998).

Moreover, Sarpeshkar presented a thorough evaluation of his system by presenting an arsenal of performance-related results like: amplitude and phase frequency responses, adaptation with/without AGC, harmonic-distortion levels at various taps, noise and distortion spectrums, spatial characteristics, etc. Finally, he provided insightful discussions on the comparison between his analogue CMOS WI implementation with a custom digital ASIC and on filter-cascade versus bandpass filterbank architectures. Sarpeshkar was able to demonstrate in silicon a variety of physiologically obtained results:

1. The frequency-to-place transformation as implemented by the amplification and propagation of travelling waves in the BM (much like the results of Fig. 4).

2. A compressive nonlinearity at and beyond the CF of a cochlea tap due to the dynamic and compressive action of the AGC with asymmetric attack and release responses to transient inputs.

3. Extension of the DR from $30 \mathrm{~dB}$ to $60+\mathrm{dB}$ due to adaptation and active amplification.

4. Sharp roll-off slopes (corresponding to $10^{\text {th- }}$ to $16^{\text {th }}$-order transfer functions), broadening of the frequency response and shift of the CF towards lower frequencies as input intensity is increased.

5. Masking of adjacent frequencies and harmonics due to the distributed nature of his AGC and compressive nonlinearities.

In conclusion, Sarpeshkar's 1998 effort remains to this day one of the most complete neuromorphic cochlea designs. His contributions demystified the silicon cochlea operation, made a clear link to all the modelling ideas Lyon had presented in the past and advanced the neuromorphic field with performance figures that serve to this day as benchmarks to future design efforts.

The other two 1998 contributions came from Timothy Edwards and Nagendra Kumar from the Johns Hopkins group. Edwards' work presented the second log-domain biquadratic filter implementation for audio frequency applications since Toumazou's 1994 effort (Edwards and Cauwenberghs, 1998). The BP transfer function was realized by using two 
integrators (one lossy and one lossless) with additional translinear loops to implement the feed-back gains. The overall design was BiCMOS and operated in Class-A. He also provided measured results regarding $\omega_{o}$ and $Q$ frequency response tunability from a $4^{\text {th }}$-order $\mathrm{BP}$ response. Yet, no noise or linearity results were reported. On the other hand, Kumar's work was one of the first attempts to apply a bio-inspired system to speech recognition experiments (Kumar et al., 1998). Kumar adopted Liu's proposed filterbank architecture and he consequently implemented it using Furth's linearized WI OTA presented in (Furth and Andreou, 1995b). In addition, due to its physiological plausibility, power signal processing capabilities and relative noise immunity, he used a zero-crossing-based signal representation for abstracting the auditory nerve fibre responses. Thus, his 15-channel filterbank chip essentially computed zero-crossing intervals and signal energies in frequency sub-bands. He consequently reported on speech recognition results using the TIDIGITS spoken digit database obtained from software simulation of the chip feature extraction algorithm.

\section{Recent years: 1999 - 2008}

1999: During 1999 there were two current-mode contributions; one from Todd Hinck and one from Timothy Edwards. Hinck's work was based on a current-mode implementation of the travelling-wave amplifier (TWamp) cochlea model. This model was proposed in 1993 by Hubbard (Hubbard, 1993) who hypothesized the existence of two separate wavepropagation modes in the cochlea. The TWamp model was realized using two activelycoupled transmission lines, each of which represented a different mode of energy propagation in the cochlea. The first transmission line was the same as in the classical travelling-wave model, whereas the second was composed of sections of a series inductor with a parallel shunt capacitor and resistor. Hinck's design strategy was quite similar to the one reported from Bor in 1996; He started from the transmission-line model of a BM segment $\Delta x$, he consequently converted it to a signal-flow-graph (SGF) and then replaced the SFG with current-mode equivalent blocks (i.e. gain and integrator blocks). In (Hinck et al. 1999), he showed simulation results from the frequency response of a 100-section cochlea with fixed $Q$ and estimated the DR to be $54 \mathrm{~dB}$ assuming a 100pA noise floor.

Finally, Edwards outlined the design of a current-mode filterbank front-end with signal feature extraction for acoustic pattern recognition using his 1998 work on log-domain filters (Edwards and Cauwenberghs, 1999). His feature extraction algorithm was based on rectification followed by smoothing and peak detection and tracking for which he showed current-mode circuits. He provided measured results from a 15-channel (each channel comprising two cascaded log-domain BP biquads) chip built using the $1.2 \mu \mathrm{m} \mathrm{BiCMOS}$ process with a measured DR of $35 \mathrm{~dB}$ and a power dissipation of $200 \mu \mathrm{W}$ from a $5 \mathrm{~V}$ supply. Moreover he was able to demonstrate that the action of the peak detector helped to increase the sharpening of the BP response (much like the difference in sharpening between the BMvelocity and neural tuning curves observed in the biological cochlea), compensating for the swallow low-frequency roll-off slopes typical of Class-A log-domain BP filters (see Fig. 25). 2000: In 2000, the first current-mode cochlea implant (CI) prosthesis system was presented by Walter Germanovix and Toumazou from Imperial College London (Germanovix and Toumazou, 2000). The function of a CI is to bypass the natural transducers (i.e. the IHC) which are damaged or missing in a diseased cochlea and consequently excite the SGN (via electrical stimulation) leading eventually to hearing perception. The proposed CI design was 


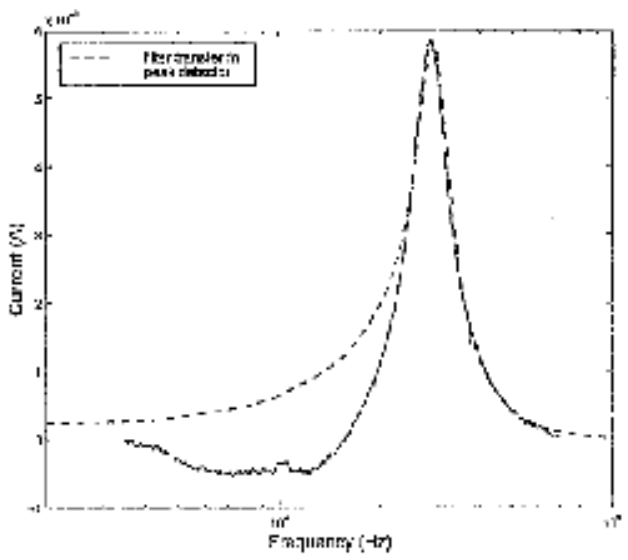

Fig. 25. Comparison between the measured transfer function of the BP filter of a single channel (dotted line), normalized to the current output of the peak detector and smoothing filter on the same channel (solid line); from Edwards (Edwards and Cauwenberghs, 1999).

switchable into two modes of operation: the single-channel and multi-channel modes. Singlechannel CIs code frequency based on the rate of firing of electrical pulses, whereas multichannel CIs use the place theory for coding frequency (i.e. the fact that different frequencies map at different places along the BM). Also, single-channel CIs usually employ the CA (Compressed Analogue) stimulation strategy, whereas multi-channel CIs employ the CIS (Continuous Interleaved Sampling). In the latter, each of the channels BP filtered envelopes of the input signal are encoded into non-overlapping biphasic electrical current pulses in order to eliminate any overlap between channels so that only one electrode is stimulating at a time.

Although multi-channel CIs are the prevailing devices around the world today, there are some arguments against the rational for their use. However, the cochleae of people with severe hearing impairment do not posses sufficient residual neuronal population to use tonotopic electrical stimulation through multiple electrodes. Therefore, there are arguments against the rational for using tonotopic stimulation strategies since multi-channel electrode systems aim to stimulate focal regions of dendrites along the BM, which might not exist in some/most cases (Namasivayan, 2004). For this reason, Germanovix attempted to create a versatile $\mathrm{CI}$ employing both aforementioned modes and stimulation strategies. The block diagram of his system is shown in Fig. 26.

One important feature of Germanovix' CI was the tone control circuit that follows the transconductance amplifier (OTA) in Fig. 26. The OTA (a simple tanh characteristic) was used to provide compression and sensitivity by converting the (wide) input DR (by varying its tail current) into an appropriate DR for the specific individual. Consequently, the tone control circuit allowed boost/cut of low/high frequencies to compensate deficiency in the diseased auditory system. The tone control circuit output was formed by subtracting the outputs of two parallel LP log-domain filters; one with low-frequency cut-off to emphasise the bass and one with high-frequency cut-off to emphasise the tremble. The operation of the tone control circuit is essentially similar to the bass/treble control of a Hi-Fi system. Even though Germanovix' contributions were only preliminary at the time, implementation-wise all his circuits were designed in CMOS WI, they were operating in the current mode and his BP sections were Class-A log-domain filters with a measured THD of $-40 \mathrm{~dB}$ (i.e. $1 \%$ ) at a modulation index of $35 \%$. No noise floor and power consumption figures were reported. 


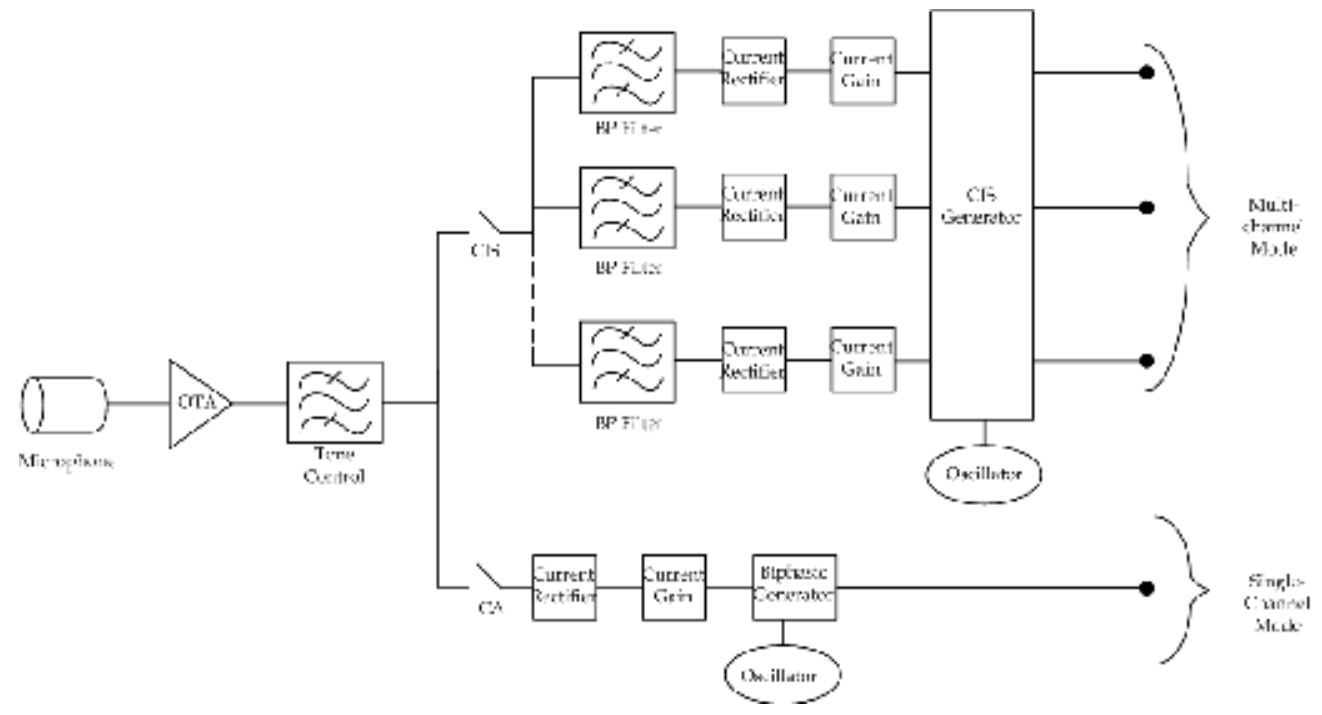

Fig. 26. Diagram of Germanovix' CI with switches between the CIS and CA modes of operation; adapted from Germanovix (Germanovix and Toumazou, 2000).

2001: In 2001 there were two contributions from André Van Schaik. In the first one (Schaik, 2001), he presented an electronic system that extracted the periodicity of a sound. The system was comprised from three analogue VLSI building blocks: a) an improved version of Watt's 1-D implementation utilizing 104 cascaded sections (the same $\mathrm{g}_{\mathrm{m}}-\mathrm{C}$ ones that Watt's used in (Watts et al., 1992)) with CLBTs for generating the biasing currents (built on a $4.77 \mathrm{~mm} \times 3.21 \mathrm{~mm}$ die chip), b) four IHC circuits (built on a $1 \mathrm{~mm}^{2}$ chip) and c) two spiking neuron chips (each chip built on a $2.5 \mathrm{~mm}^{2}$ and containing 32 neurons). Before explaining how his system performed periodicity extraction, let us briefly describe Van Schaik's IHC and neuron circuits, the initial versions of which were previously published by the author around 1996 (Van Schaik and Meddis, 1996).

His IHC circuit is the most bio-plausible contribution to date because it did not solely rely on half-wave rectification (like in all previous efforts) but attempted to replicate several other IHC characteristics/functions like input-output function asymmetry, temporal adaptation to constant stimulation etc. To appreciate Van Schaik's modelling choices, recall four interesting features of the biological auditory neuron fibre:

1. The spike rate of most auditory neuron fibres increase with increasing stimulus level but only over a narrow level of signal intensities (from 60 to 80 SPL). Above that level, the output saturates showing little or no further rate increase (see Fig. 27(left)). Moreover, the relation between the IHC cilia deflection and the percentage of open ion channels (or equivalently the IHC current to IHC membrane potential) has a sigmoidal form with a certain offset, so that only $20 \%$ of the channels are open at equilibrium.

2. The rate of firing is known to adapt to a new stimulus. The high rate at onset is quickly adapted to a lower rate within $3 \mathrm{msec}$ of the initial stimulus onset. In addition, when a persistent stimulus is present, the rate reduces even further but at a much slower pace (see Fig. 27(middle)).

3. IHC perform some kind of LP filtering with a cut-off frequency of $1 \mathrm{kHz}$ so that AC components of frequencies above about $4 \mathrm{kHz}$ are heavily attenuated and thus no information is available to the brain regarding the temporal properties of the input 
waveform. At frequencies well below $4 \mathrm{kHz}$, the IHC voltage has a noticeable AC component and its temporal structure can be extracted by the brain.

4. At low frequencies $(<3 \mathrm{kHz})$ the spike rate in an individual auditory neuron can be shown to be a half-wave rectified waveform (see Fig. 27(right)). In the absence of any input signal, the auditory neuron shows spontaneous action potential at a frequency almost as frequent as half of the maximum firing rate.

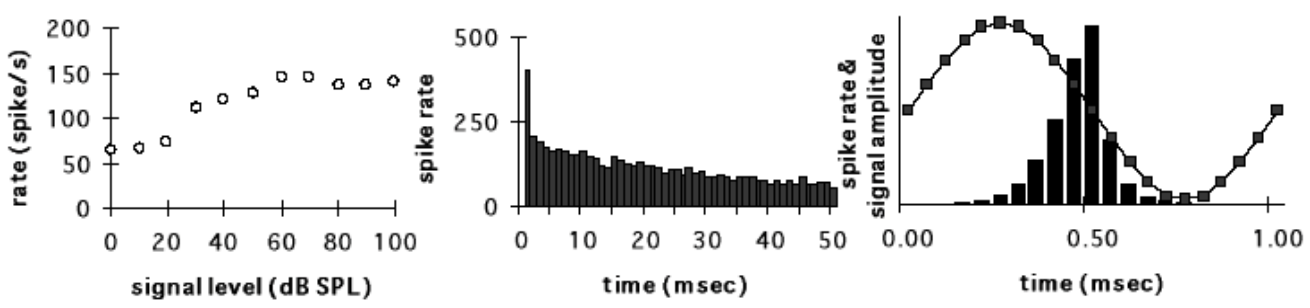

Fig. 27. (left) IHC rate-intensity function, (middle) IHC post stimulus time histogram showing adaptation to a persistent stimulus and (right) IHC period histogram shown with stimulating sinusoid; from Van Schaik (Van Schaik and Meddis, 1996).

Van Schaik's IHC model (shown in Fig. 28) consisted of three main processing stages that aim to model the four aforementioned features of an auditory neuron fibre: The first stage contained an asymmetric (sigmoid-like) compressive input-output function (realized by a simple OTA where one differential-pair transistor was $20 \%$ larger than the other - thus only $20 \%$ of the biasing current will flow through the smaller device when the input differential voltage is zero) followed by a bias circuit which adds bias to the membrane potential to guarantee spontaneous activity at the absence of any input (points 1 and 4). The second stage consisted of two first-order LP filters with a cut-off frequency of $1 \mathrm{kHz}$ or lower (point 3) and the final stage introduced adaptation to constant stimulation by subtracting a weighted LP filtered copy of the IHC output with the IHC output itself. By using a larger capacitor in the second filter (i.e. $C_{2}=3 C_{1}$ ), its output will react more slowly to an onset of $I_{\text {sig }}$ than $I_{1}$ would do (point 2). The final output current is given by $I_{\text {out }}=I_{1}-G I_{2}+I_{\text {spont }}$, where $G(<1)$ is the gain of the current mirror that controls the ratio of the system's peak response to the sustained response and $I_{\text {spont }}$ is a constant current level used to adapt the 'spontaneous' rate of firing (point 4).

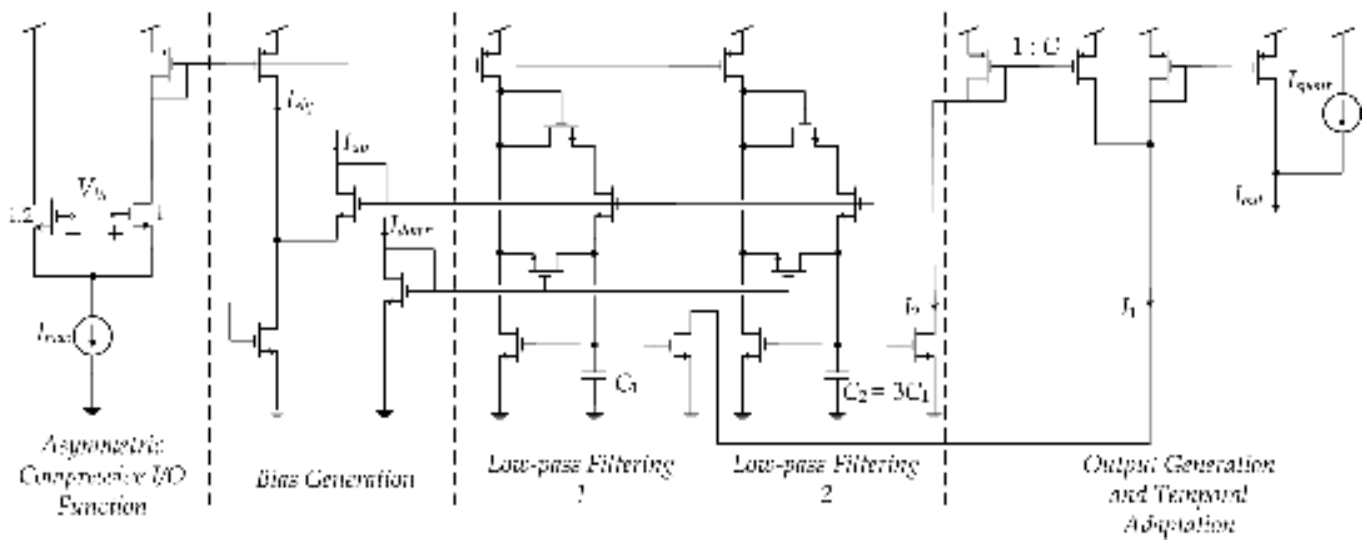

Fig. 28. Circuit diagram of the IHC stage; adapted from Van Schaik (Van Schaik, 2003). 
Implementation-wise, one of the main problems/challenges in modelling comparatively slow brain elements with analogue VLSl circuits is the realization of large time constants such as the ones for the IHC LP filtering. Van Schaik realized that since the linearity of the LP filter stage is not an important issue, large time constants could be realized using the current mirror arrangement of the two LP filter blocks in Fig. 28 (i.e. small capacitors appear larger via the Miller effect). This current mirror creates a non-linear LP filter for which $I_{u p}$ sets the maximum rise speed and $I_{\text {down }}$ sets the maximum fall speed of the voltage on the two capacitors. This construction allowed Van Schaik to obtain programmable time constants in the order of several milliseconds using capacitors in the pico-Farad range while using bias currents in the 100pA range. Moreover the time constants varied with input signal level for both the LP filter and adaptation stages. Fig. 29 shows a comparison of the output responses from his artificial IHC circuit relative to the biological ones. Clearly, Van Schaik's neuromorphic IHC circuit is able to faithfully reproduce the responses of its biological counterpart.

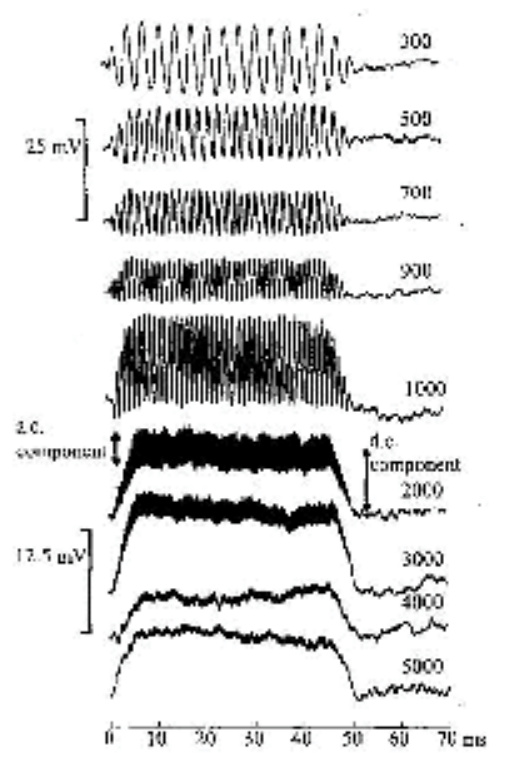

(a)

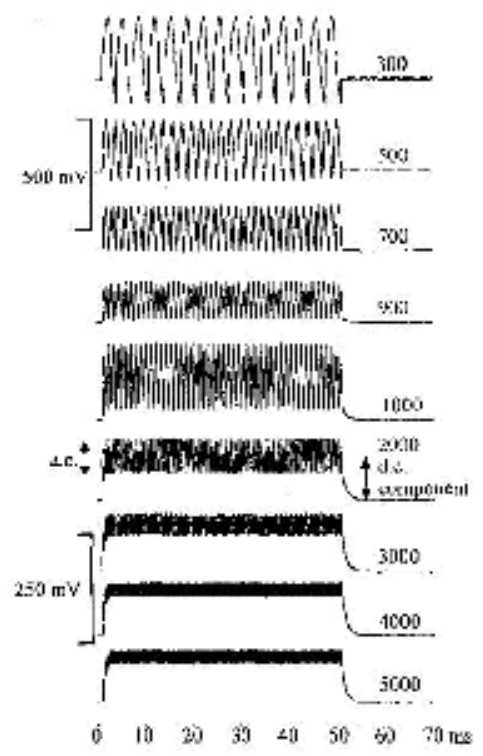

(b)

Fig. 29. Intracellular IHC voltage changes in (a) the biological IHC and (b) Van Schaik's circuit implementation for different frequencies of stimulation. For both cases, note the change of scale for the lower five traces; adapted from Van Schaik (van Schaik, 2003).

The final circuit was a silicon neuron (depicted in Fig. 30 above) which was designed after its biological counterpart. Recall that in the real neuron an increased sodium conductance creates the upswing of spike (i.e. activation through depolarization) and the delayed blocking of the sodium channels plus delayed increase of potassium conductances create the downswing (i.e. de-activation through re-polarization). Here, the membrane of the neuron was modelled by the membrane capacitance $C_{m e m}$ and the membrane leakage current is controlled by the current $I_{l e a k}$. In the absence of any input (i.e. $\left.I_{e x}=0\right)$ the membrane $\left(V_{m e m}\right)$ potential is drawn to its resting potential controlled by $V_{-}$. Excitatory inputs add charge to 


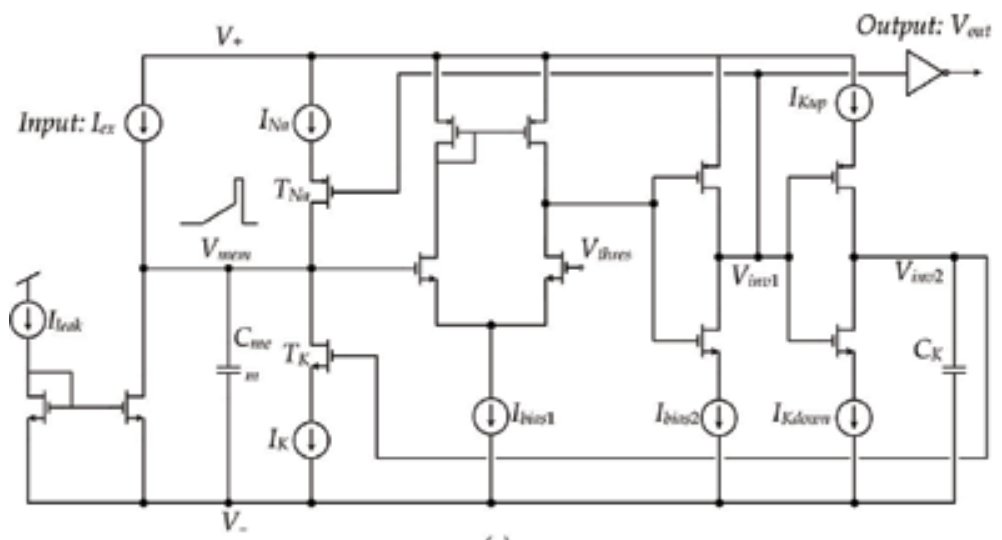

(a)

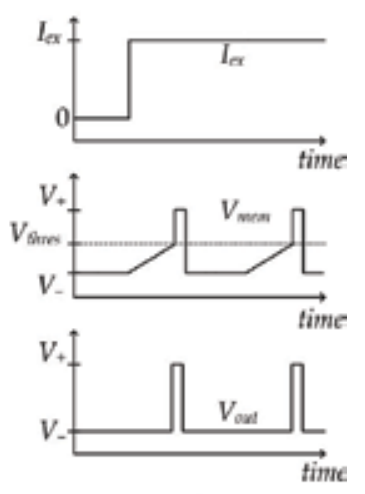

(b)

Fig. 30. (a) Circuit details of the VLSI neuron. (b) Example of the response of the membrane potential $\left(V_{\text {mem }}\right)$ and the output signal $\left(V_{\text {out }}\right)$ to a DC input current $\left(I_{\text {ex }}\right)$; from Van Schaik (Schaik, 2001).

$C_{m e m}$, whereas inhibitory inputs discharge $C_{m e m}$. If the excitatory input $\left(I_{e x}\right)$ becomes larger than $I_{\text {leak }}$ then $V_{\text {mem }}$ will rise from its resting potential. $V_{\text {mem }}$ is then compared with a controllable threshold voltage $\left(V_{\text {thres }}\right)$, using an OTA driving a high-impedance load. If $V_{\text {mem }}$ is larger than $V_{\text {thres, }}$, then the output of the OTA $\left(V_{\text {comp }}\right)$ will be high and thus the output of the first inverter $\left(V_{i n v 1}\right)$ will go low activating the PMOS $T_{N A}$ switch which injects 'sodium' current to $C_{m e m}$ effectively pulling $V_{m e m}$ up (i.e. the upswing of a spike ). At the same time, a second inverter has it output voltage $\left(V_{i n v 2}\right)$ high allowing the capacitor $C_{K}$ to be charged at a speed which is controlled by $I_{K u p}$. As soon as the voltage on $C_{K}$ goes high, the NMOS $T_{K}$ switch conducts allowing for the 'potassium' current $I_{K}$ to discharge $C_{m e m}$ and bring $V_{m e m}$ down to its resting value. The values of the currents $I_{K u p}$ and $I_{K d o w n}$ control the spike width and the neuron's refractory period respectively.

Van Schaik consequently used his improved BM cascade, IHC and neuron circuits to model periodicity extraction much like what Lazzaro had proposed and demonstrated in (Lazzaro and Mead, 1990). A major disadvantage of Lazzaro's implementation was the fact that in order to perform the matched filtering operation at the output of each SGN, each delay associated with a BM tap had to be matched precisely to the inverse of the CF of that tap. That meant that for a BM cascade whose CF changed exponentially from $4 \mathrm{kHz}$ to $100 \mathrm{~Hz}$, delays from $0.25 \mathrm{msec}$ to $10 \mathrm{~ms}$ had to be created. In the real cochlea these large variations in delay is unlikely to be provided by an axonal delay circuit because it would require a very large variation in axon length. Van Schaik observed that the phase of a pure tone of a given frequency on the BM increases from base to apex and the phase changes rapidly around the $\mathrm{CF}$. Moreover, the filter cascade acts as a delay line and each filter adds a delay which corresponds to $\pi / 2$ at the cut-off of that stage. Thus, if one monitors the output of filter $i$ and filter $i-4$, under the assumption that the cut-off frequency remains fairly constant between these four stages (i.e. for a high resolution BM cascade), then the delay would correspond to $2 \pi$ at the cut-off frequency. In essence, Van Schaik used the inherent delay of the BM cascade to perform the matched filtering operation at the output of the SGN and not through an additional axon circuit like Lazzaro. Van Schaik demonstrated through measurements that detecting these coincidences can yield very selective filters, i.e., filters responding only to a very narrow range of periodicities, but at the same time still responding after a few periods of the input 
signal. This turned out to be an advantage over traditional BP filters, where an increase in selectivity has to be traded off against a decrease in response time.

Van Schaik's second 2001 contribution (together with Fragniére) detailed the analogue current-mode VLSI implementation of a 2-D cochlea model using a grid of transistors to model cochlea fluid mass (Van Schaik and Fragniere, 2001). That particular model was based on the initial 1992 2-D model of Watts that was thoroughly investigated by Fragniére in (Fragniere, 1998) few years later. The two dimensions that were taken into account were the longitudinal direction along the BM from base to apex and the vertical direction which accounts for the liquid movement perpendicular to the BM. In order to achieve a compact VLSI implementation of the dense resistive network modelling the 2-D cochlea, Van Schaik used a single transistor implementation of the resistors. That transistor was operating in the pseudo-voltage domain and its resulting pseudo-conductance has been proven efficient in a variety of signal processing systems (Vittoz, 1997). To introduce temporal processing, the pseudo-conductance was used with a reactive component (a pseudo-transcapacitor where its differential input current is integrated into an output pseudo-voltage) yielding filters which are close relatives to log-domain filters.

2002 - 2004: From 2002 onwards Sarpeshkar's auditory research interest seemed to focus on fully-implantable CIs based on simple BP filterbanks. Together with his group at MIT, he published several conference and journal articles detailing the designs of the basic building blocks comprising a CI processor. He reported on the designs of a new BP filter (Salthouse and Sarpeshkar, 2003), envelope detector (Zhak et al., 2003), microphone preamplifier (Baker and Sarpeshkar, 2003) and A/D converter (Sit and Sarpeshkar, 2004) that were consequently used to realise a CI channel (Lu et al., 2003) and a CI processor (Baker et al., 2003). In 2005, all these designs were integrated to create one of the most complete analogue VLSI CIs to date. Sarpeshkar approach in this work was slightly different from what he had adopted in his earlier attempts. Here, he tried to create circuits that would eventually lead to a practical bio-inspired design having the potential towards possible commercialization. For this reason, his architectural and circuit design choices did not solely rely on increasing the performance but also on reliability and robustness. An example of this strategy can be seen in the design of his new BP filters. Sarpeshkar realized that while the linearization techniques used in his prior designs (from 1996 to 1998) successfully met the design requirements, the linear range could not be easily increased above the achieved value because the techniques do not scale. Recall that much of the achieved increase in linear range was due to the well as the input terminal. That makes the linear range a strong function of the subthreshold body-effect parameter $n$ which varies between processes and runs. Hence, Sarpeshkar tried to explore alternative strategies.

The BP filters used in this work were based on the design of Christopher Salthouse who used a capacitive-attenuation technique to extend the input linear range beyond $1 V_{p p}$ (Salthouse and Sarpeshkar, 2003). His design was based on two cascaded first-order sections; one LP and one HP built using the basic OTA. The linear range was increased by attenuating the input and feedback attenuating the output because the linear range of the OTAs is limited by their input swing rather than their output swing. Salthouse consequently extended his design to a differential topology and measured a cascade of two BP filters. He reported a $230 \mathrm{nW}$ power consumption with an input DR of $67.5 \mathrm{~dB}$ (5\% THD) at $100 \mathrm{~Hz}-200 \mathrm{~Hz}$ range and a $6.36 \mu \mathrm{W}$ with a DR of $65 \mathrm{~dB}(5 \% \mathrm{THD})$ at $5 \mathrm{kHz}-10 \mathrm{kHz}$ range. In 2002, David Graham with Paul Hasler from Georgia Tech presented a new very compact second-order section for cochlea modelling with high overall performance (Graham and 
Hasler, 2002). Their design was based on the DIFF2 structure discussed by Lyon in 1992 and on the autozeroing second-order section presented by the authors in (Hasler et al., ). The basic building block of their design is a capacitively-coupled current-conveyor $\left(\mathrm{C}^{4}\right)$ presented in (Hasler et al., 1999). In this paper, they presented only measured frequency response results from a 32-section $\mathrm{C}^{4} \mathrm{BP}$ filterbank, whereas a thorough analysis and characterization of the $\mathrm{C}^{4}$ structure was presented five years later (Graham et al., 2007). There, they demonstrated an impressive five-orders of magnitude frequency tunability with passband SNR generally ranging between $50-60 \mathrm{~dB}$. At $20 \mathrm{kHz}$ and with a $Q$ of 4 , the second-order $\mathrm{C}^{4} \mathrm{BP}$ section dissipated a mere $0.11 \mu \mathrm{W}$ of static power from a $3.3 \mathrm{~V}$ power supply and with a $Q$-programmability range of up to 9 . The simple second-order section occupied $2100 \mu \mathrm{m}^{2}$ in a $0.5 \mu \mathrm{m}$ CMOS process, whereas a tenth-order structure occupied an area of $13200 \mu \mathrm{m}^{2}$. Because of the fact the $C^{4}$ was so compact, a filterbank employing sixteen tenth-order BP filters occupied only $1.5 \mathrm{~mm} \times 1.5 \mathrm{~mm}$ including all biasing circuits. Finally, the authors were the first to use floating-gate transistors for the current-sources that set the time constants of the $\mathrm{C}^{4} \mathrm{BP}$ filters. Since the floating-gate transistor currents can be programmed very precisely $(< \pm 0.2 \%$ error), the responses of the BP filters were also set very precisely using a single calibration step.

In 2003, Ǿivid Noss, a student of Tor Sverre Lande from the University of Oslo, presented a low-voltage, low-power second-order filter consisting of six pseudo floating-gate transistors operating in the WI (Naess et al., 2003). Nœss recalled that in 1998 Sarpeshkar used offset adaptation circuitry in between each stage to limit the skew in offset/DC voltages through his BM cascade. By employing the pseudo floating-gate technique, such offset adaptation circuitry would not be necessary due to the capacitive coupling of the floating-gate nodes. The term 'pseudo' arises from the fact that the floating nodes are not truly floating but weakly connected. Nonetheless, the quiescent currents are still very low giving pseudo floating-gates the same advantage. Additional benefits of this technique include the possibility of operating them with very low-power, shifting the devices' threshold voltages as seen from their capacitive coupled input terminals and an easy initialization/programmability of their floating-gate voltages. Finally, Nœss provided preliminary frequency response simulation results from a cascade of six biquads and reported a $5 \mathrm{nW}$ power dissipation with a $-60 \mathrm{~dB}$ suppression of the second harmonic at $1 \mathrm{kHz}$ from a single second-order LP stage.

The two last contributions came from Ivan Grech from the University of Surrey who published two journals with detailed measurements on a new low-power log-domain CMOS filterbank for extracting localization spectral cues from two audio signals (Grech et al., 2003; Grech et al., 2004). As far as we are aware, Grech's implementation is the first logdomain realization of a front-end for 2-D sound source localization. Moreover, it is the first ever effort which employed high-performance log-domain biquads operating in Class-AB. All additional circuits were current-mode translinear loops used for performing mathematical operations like squaring, division and maximum value detection. The inputs to the system, which were left and right ear audio signals were converted to current signals and then splitted into complementary differential signals using the harmonic mean splitting law. These signals were then logarithmically compressed after having their level adjusted by a current-mode, open-loop AGC block which acted globally altering the $Q$ values of the subsequent BP filters. Each path was then processed by a parallel bank of 24 fourth-order BP log-domain filters whose CF changed from $50 \mathrm{~Hz}$ to $20 \mathrm{kHz}$ in an exponentially decreasing fashion. Envelop processing was realized at the outputs of each BP filter by squaring and LP 
filtering. Envelopes for CFs below $1 \mathrm{kHz}$ were further processed off-chip to extract interaural phase delay (IPD) ${ }^{2}$ cues, whereas higher frequency envelopes were used to extract interaural envelope delays (IED) ${ }^{3}$ cues. Finally, interaural intensity difference (IID) ${ }^{4}$ cues were extracted by dividing the envelopes of the left- and right-channels, whereas monaural ${ }^{5}$ cues were extracted by dividing the envelopes of adjacent filters of the same channel. Grech's design has one of the highest performances reported to date with a measured BP stage input DR of $62 \mathrm{~dB}$ at $1.8 \%$ THD (in excess of $80 \mathrm{~dB}$ for the $4-5 \%$ THD figures used by other authors), second-order intermodulation distortion products below $-40 \mathrm{~dB}$ and an overall power dissipation of $890 \mu \mathrm{W}$ for the whole system. Moreover, he used on-chip, temperature independent biasing and a $Q$-value digital calibration memory for manually resetting a uniform peak response to all the BP stages. It should be noted that the maximum $Q$ reported was 10.25 resulting in a measured passband gain of around $40 \mathrm{~dB}$.

2005 \& 2006: Five years after Germanovix' CI effort, Julio Georgiou, his successor in Toumazou's group at Imperial College London, was actually the first to validate a 16channel fully-implantable log-domain CI prosthesis system (Georgiou and Toumazou, 2005a). Georgiou's system occupied an area of $3.4 \mathrm{~mm} \times 6 \mathrm{~mm}$ and consisted of a) a mixedsignal chip that combined the speech processor (utilizing a 16-channel Class-A BP logdomain filterbank) and CIS stimulator, b) a rechargeable battery and c) a second chip for power management and charging circuits. The whole system was encapsulated in a hermetically sealed platinum case for biocompatibility reasons. Georgiou reported on a total of $126 \mu \mathrm{W}$ power consumption (including an off-chip microphone but excluding the CIS stimulation) and an input DR (at the BP pole frequencies) between $51-58 \mathrm{~dB}$ for a $4 \%$ THD. His filters were Class-A log-domain filters with a state-elimination circuitry (to eliminate additional existing DC operating points (Fox and Nagarajan, 1999)) and a nominal power dissipation of only $40 \mathrm{nW}$. It should be noted here that Georgiou performed rectification, compression and smoothing elegantly and with minimal power requirements by using a circuit that was identical to the nonlinear LP filtering stage used by Van Schaik in his IHC circuit model. Finally, Georgiou employed a digital AGC, in the form of saturation nonlinearity that acted globally on the input prior to being fed to the filterbank. In addition, it was also possible to externally program its attack and release time constants so that it responded more quickly to increasing amplitudes than to decreasing ones. On the other hand, Sarpeshkar's final CI was also reported in 2005 (Sarpeshkar et al. 2005). Their design achieved a total power consumption of $211 \mu \mathrm{W}$ (including microphone and stimulation circuits), a DR of $77 \mathrm{~dB}$ and occupied an area of $9.58 \mathrm{~mm} \times 9.23 \mathrm{~mm}$. The basic circuit building blocks comprising their design were:

1. A build-in microphone front-end (using the commercial JFET-buffered FG3329) electret microphone to transduce sound into an electrical signal. The microphone front-end was designed to have an $80 \mathrm{~dB}$ input DR and its total power consumption was at $100 \mu \mathrm{W}$ (almost half the power dissipation of the whole $\mathrm{CI}$ ).

\footnotetext{
2 IPD represents the time delay between the left and right channels for actual input signals of frequencies below $1 \mathrm{kHz}$.

${ }^{3}$ IED is the same as IPD only for higher frequencies.

${ }^{4}$ IID represent the difference in signal intensity between the left and right channels.

${ }^{5}$ Monaural cues are directly related to intensity differences between adjacent frequency bands of the same channel.
} 
2. An AGC circuit that was implemented by regulating the transconductance of a single transconductance-resistance variable gain amplifier (VGA). The signal from the microphone front-end was passed through an envelope detector (a cascade of a rectifier and LP filter) and consequently fed to a translinear circuit used to create the compressed output current for controlling the gain of the VGA. Moreover, the envelope detector had an asymmetry in its attack and release time constants. Their AGC had an effective $60 \mathrm{~dB}$ of DR and a power consumption of $30 \mu \mathrm{W}$.

3. A 16-channel filterbank employing Salthouse's fourth-order BP filters.

4. A logarithmic A/D with 7 -bit precision at $1 \mathrm{kHz}$ sampling rate with $3 \mu \mathrm{W} /$ channel.

5. High PSRR and temperature independent current and voltage biasing with a total power dissipation of $3 \mu \mathrm{W}$.

6. Digital I/O interface and CIS stimulator dissipating $32 \mu \mathrm{W}$.

The last 2005 contribution came from Christopher Galbraith who reported on a planar channelizer filter (i.e. a microwave multiplexer with a large number of output ports) based on a model of the mammalian cochlea operating at $20-90 \mathrm{MHz}$. Applications of such a bioinspired system could include wideband electronic warfare systems and radio astronomy and spectroscopy. The channelizer filter topology was derived from the transmission-line model of the cochlea and he reported measured results from a 20-channel prototype chip (Galbrdith et al., 2005;Galbraith et al., 2007). The second RF cochlea followed one year later from Sarpeshkar (Mandal et al., 2006).

Finally, one of the most recent neuromorphic efforts came from Bo Wen and Kwabena Boahen from Stanford University, where they presented a 2-D nonlinear cochlea model utilizing a novel active mechanism that realizes the frequency-selective OHC-based AGC (Wen and Boahen, 2006). Their AGC is based on a model of OHC electromotility through active bi-directional coupling that was initially proposed by Geisler and Sang in 1995 (Geisler and Sang, 1995) and later extended by the authors in (Wen and Boahen, 2003). Their hypothetical model includes both feed-forward and feed-backward interactions supported by the physiological evidence:

1. The force resulting from $\mathrm{OHC}$ contraction and elongation is exerted onto adjacent downstream BM segment due to the $\mathrm{OHC}$ basal tilt.

2. The $\mathrm{OHC}$ force is delivered onto adjacent upstream BM segment due to the apical tilt of the phalangeal process extending from the Deiter's cells' main trunk.

Thus, the OHC motile forces, through the microanatomy of the cochlea partition, feed forward and backward, in harmony with each other, resulting in bi-directional coupling between adjacent stages in the longitudinal direction. More specifically, due to the opposing action of the $\mathrm{OHC}$ forces on the $\mathrm{BM}$ and reticular lamina, the $i_{\text {th }}-1 \mathrm{BM}$ segment reinforces that of segment $i$, while the motion of segment $i^{\text {th }}+1$ opposes that of segment $i$.

Their cochlea processor was built along the same lines with Watts' and Van Schaik's 2-D implementations. Wen's fluid network was implemented by an array of diffusors (i.e. simple NMOS transistors in WI), while each BM segment was realized using pseudodifferential Class-AB log-domain filters. The active bi-directional coupling was achieved by exchanging currents between neighbouring second-order sections, thus realizing an AGC mechanism without needing to detect the signal amplitude and implement AGC loops. Wen reported results from a fabricated chip with $360 \mathrm{BM}$ segments and two 4680-element fluid grids $(360 \times 13)$ occupying $10.9 \mathrm{~mm}^{2}$ area in a $0.25 \mu \mathrm{m}$ CMOS technology. However, Wen's frequency responses seemed to be heavily affected by noise and mismatch resulting in quite 
low DR performances $(17-42 \mathrm{~dB})$, which generally are not indicative of pseudo-differential Class-AB log-domain implementations.

2007: In this year, most contriburions came from Van Schaik's group at the University of Sydney. The first one was a conference publication where his student Tara Julia Hamilton presented a BM resonator segment together with a feed-backward AGC control loop for 2-D cochlea implementations (Hamilton et al., 2007). The resonator stage was a Class-A logdomain filter whereas the feedback AGC loop was comprised of a peak detector, a decision circuit, a ramp generator and Sarpeshkar's WLR. The output signal level from the BM resonator was measured using the peak detector (again a variation of the nonlinear LP filter Van Schaik and Georgiou used in (Schaik, 2001) and (Georgiou and Toumazou, 2005b), respectively) and then fed into the decision circuit. The decision circuit was consisted of two current comparators with some additional digital logic for setting the ceiling and thresholds of the signal amplitudes (i.e. essentially specifying the $Q$-control law). In addition, the decision circuit employed hysteresis to avoid errors due to oscillations. The $Q$ value of the resonator stage was set by the WLR's output current whose value was controlled by the voltage from the ramp generator. A higher voltage generated a larger current and thus a larger $Q$ value. When the ramp generator's output voltage was equal to the supply voltage, the WLR's output current was set to give the maximum $Q$ value. Finally they reported preliminary simulated results on a) the manual $Q$ adaptation of the $\mathrm{BP}$ log-domain frequency response, $b$ ) transient responses from the outputs of each AGC processing stages and c) the actual nonlinear input-output compressive function of their AGC.

The final contributions came from Vincent Chan, Shih-Chii Liu and Van Schaik who presented a matched silicon cochlea pair with address event representation (AER) designed for sound localization applications (Vincent et al., 2007). In recent years, the AER interface has become the standard interface protocol for a plethora of neuromorphic (mostly vision) chips because it allows the use of discrete level (spikes), continuous-time events to convey information in a manner similar to the pulse code neural communication and processing schemes found in biological systems. The AER allows multiple devices to share a common data bus by using a hand-shaking protocol that arbitrates between transmitters to determine which transmitter can access the bus. The next transmission is not permitted unless an acknowledgment is returned from the receiver. This event-driven representation is ideal for communicating sparse events from many sources using a narrow channel, utilizing effectively the available power and bandwidth.

One of the prior cochlea designs that utilized such a scheme is (Abdalla and Horiuchi, 2005). Their system was comprised of four main building blocks: a) two matched, 32-section, constant- $Q$ and passive $(Q=0.75)$ silicon cochleae (identical to the one presented in []$), b)$ a new simplified version of Van Schaik's IHC circuit (due to the other's increased complexity and size), c) integrate-and-fire neurons for the AER and d) the AER interface circuitry to generate and communicate auditory nerve spikes. Thus, the output at each BM tap gets halfwave rectified and LP filtered (effect of IHC), and consequently integrated on the membrane capacitance of the integrate-and-fire neuron which fired a spike. The neuron's operation is similar to the one presented in (Schaik, 2001) with two additional voltage lines: the request (req) and acknowledgement (ack) which set and reset it, respectively: When a spike is generated, the req line goes low. A high pulse in the ack line resets the neuron, after which it enters a refractory period for a controllable duration. When a neuron makes a request (by pulling its req signal low), an on-chip arbiter arbitrates between all neurons making a request and sending off each neuron's address in sequence through two external hand- 
shaking signals. Once the neuron's address has been communicated off-chip, the arbiter sends a signal to the ack line which in turns resets the neurons.

The authors provided an analysis on the mismatch, offset and gain errors of their system with additional measured results on a sound localization experiment performed in a reverberant realistic environment. They placed a dummy head at the centre of a room facing a loudspeaker with two microphones inserted in its ears. The recorded sounds were played to the left and right cochleae through a soundcard. The dummy head was rotated from $-90^{\circ}$ to $+90^{\circ}$ in $5^{\circ}$ steps and interaural time delay (ITD) cues were extracted by performing crosscorrelation on the spike trains between all corresponding channels. Fig. 31 shows that the calculated delay had a sinusoidal shape with the maximum delays occurring close to the two $90^{\circ}$ angle extremes as expected.

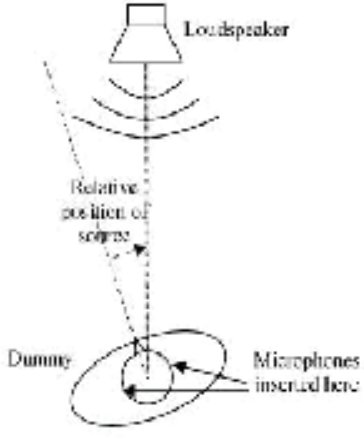

(a)

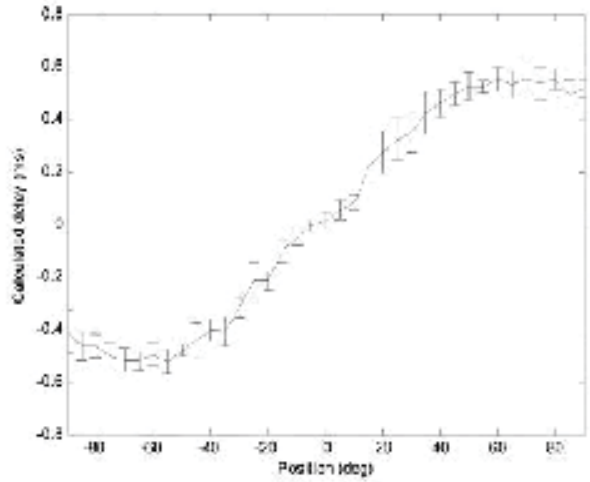

(1)

Fig. 31. (a) Experimental setup. The source was fixed but the dummy is free to rotate from $90^{\circ}$ to $+90^{\circ}$ in $5^{\circ}$ steps. Microphones were inserted into its ear to record the sound at each position. (b) ITD computed by cross-correlation of the spike trains from all channels of the two matched silicon cochleae; adapted from Chan (Vincent et al., 2007).

2009: Our final contribution is from Andreas Katsiamis, who designed a very-high dynamic range and low-power cochlea channel with focus on biorealism and performance (Katsiamis A.G. et al. 2009). The design implements a recently proposed transfer function, namely the One-Zero Gammatone Filter (or OZGF), which provides a robust foundation for modeling a variety of auditory data such as realistic passband asymmetry, linear low-frequency tail and level-dependent gain (Katsiamis A.G. et al., 2007). Moreover, the OZGF is attractive because it can be implemented efficiently in any technological medium - analogue or digital - using standard building blocks. The channel was synthesized using novel, low-power, Class-AB, log-domain, biquadratic filters employing MOS transistors operating in their weak inversion regime. In addition, his work details the design of a new low-power automatic gain control circuit that adapts the gain of the channel according to the input signal strength, thereby extending significantly its input dynamic range. The performance of a $4^{\text {th-order }}$ OZGF channel (equivalent to an $8^{\text {th }}$-order cascaded filter structure) was evaluated through both detailed simulations and measurements from a fabricated chip using the commercially available $0.35 \mu \mathrm{m}$ AMS CMOS process. The whole system was tuned at $3 \mathrm{kHz}$, dissipated a mere $4.46 \mu \mathrm{W}$ of static power, accommodated $124 \mathrm{~dB}$ (at $<5 \% \mathrm{THD}$ ) of input dynamic range at the center frequency and was set to provide up to $70 \mathrm{~dB}$ of amplification for small signals. 


\section{Summary and conclusion}

This chapter has hopefully served two main purposes: a) to form a detailed review of all the important developments on the modelling, design and experimental verification of CMOS VLSI cochlea processors, and b) to equip the reader with the general knowledge needed so that $\mathrm{s} /$ he can appreciate the design/modelling efforts presented in the following chapters.

This chapter discussed in considerable detail most of the advancements on the modelling and design of CMOS VLSI cochlea systems from 1982 until recently. From Lyon's initial 1982 and 1988 papers, there were numerous attempts from various research groups to extend and optimize the design of cochlea processors for applications including cue extraction for source localization, cochlea implants and general speech recognition frontends. In this chapter we reviewed the application of voltage mode $\left(\mathrm{g}_{\mathrm{m}}-\mathrm{C}\right)$, discrete-time (switch-capacitor and switch-current), current-mode (switch-current, log-domain and current-conveyor-based) and even microwave-RF circuit design techniques in pursue of cochlea processors of an increased overall performance. We also saw several variations in the architecture of the BM filtering (1- and 2-D BM cascade, simple BP filterbanks, parallel banks of cascaded filters, dilating-biquads filterbanks and variable clock-frequency filterbanks) and bio-plausible designs of IHC and neuron circuits together with several versions of the OHC-based AGC (static compression, feedback, feed-forward or both). In conclusion, the design of artificial cochlea systems able to exhibit a high overall performance with a high degree of biological fidelity was and very much still is a perpetual objective.

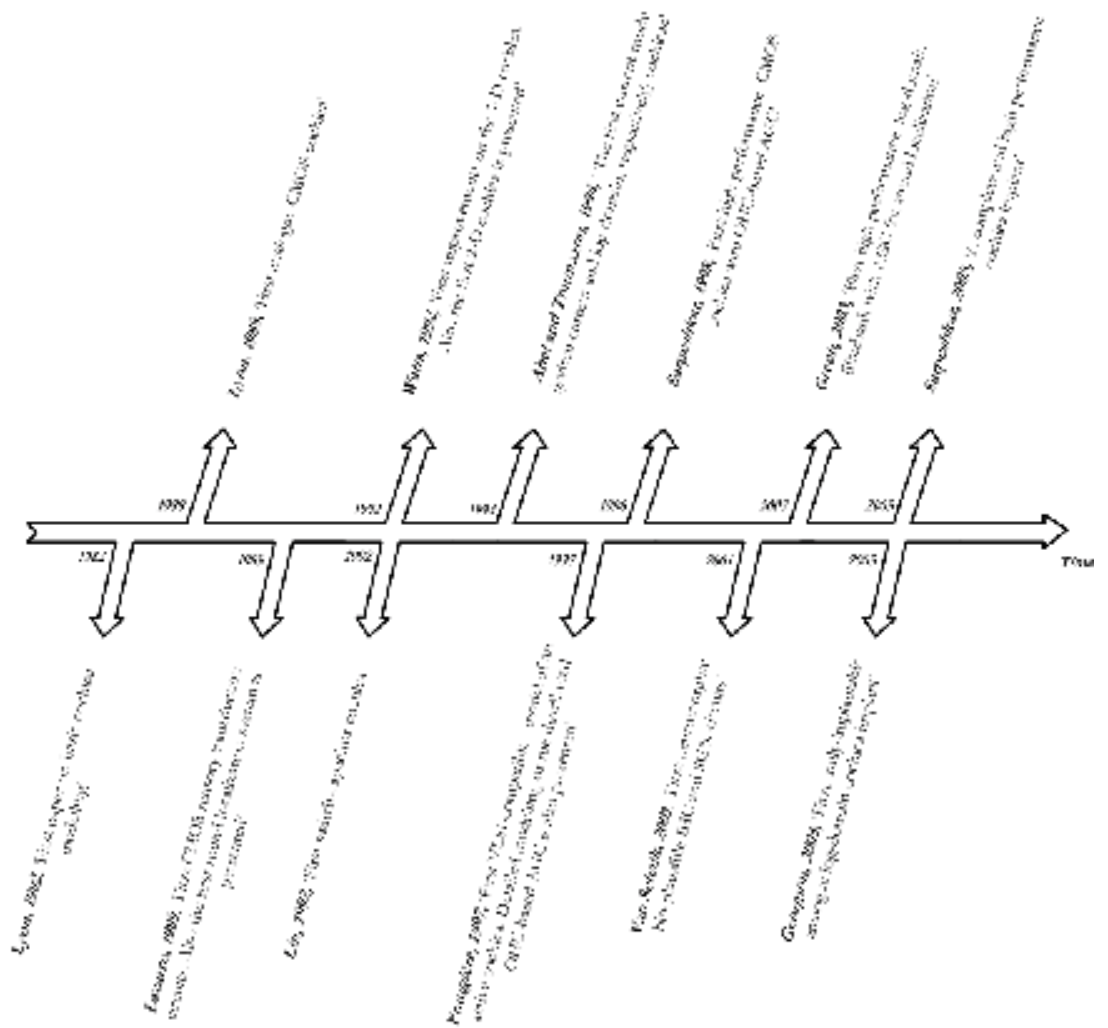

Fig. 32. Timeline of most important CMOS Cochlea Advancements. 


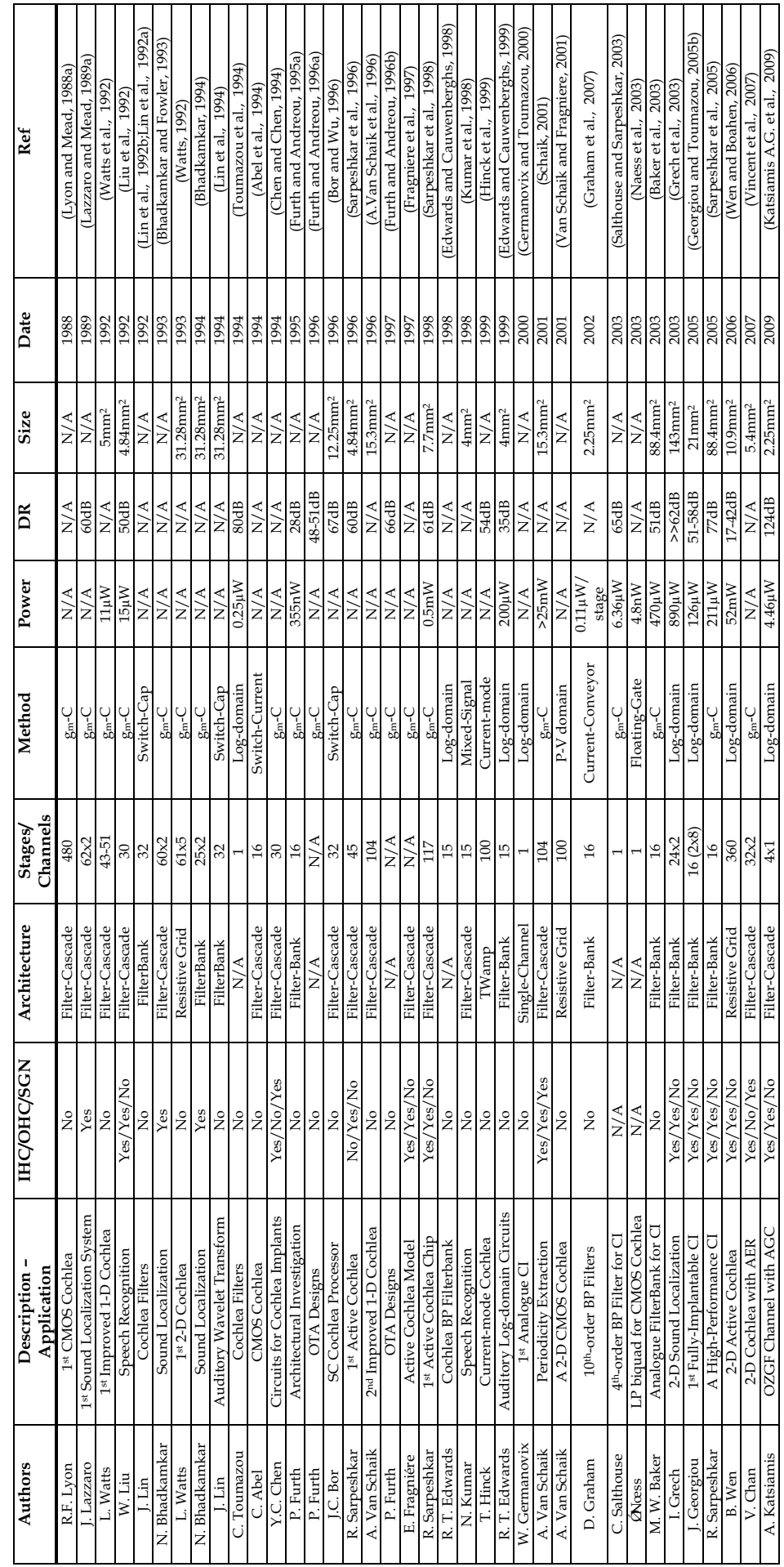

Table 1. Summary of Developments of Analogue VLSI Cochlea Systems 
One may be caught by surprise to realize the fact that the engineering principles the real cochlea is using are not conceptually far from what we had developed over the years to solve various engineering problems: Master/Slave architectures with automatic control for tuning and stabilization, compression for the accommodation of large inputs, gain adaptation to maintain signal integrity, rectification and filtering for strength detection, parallel processing (i.e. redundancy) to increase speed and robustness, to name just a few, are concepts that were developed by engineers who might had not 'made the link' with biological correlates. Yet, nature had all these ideas for millions of years and had already optimized them to realise systems which are able to perform with astonishing reliability in the haziest of environments. The cochlea is just one of those systems and to this author's opinion this is exactly the scientific contribution of neuromorphic engineering.

\section{Reference}

A.Van Schaik, E.Fragniere, E.Vittoz. 1996. Improved Silicon Cochlea using Compatible Lateral Bipolar Transistors, In: D.Touretzky et al., editor. Advances in Neural Information Processing Systems 8. Cambridge MA: MIT press. p 671-677.

Abdalla H, Horiuchi TK. 2005. An ultrasonic filterbank with spiking neurons. Circuits and Systems, 2005 ISCAS 2005 IEEE International Symposium on4201-4204.

Abel C, Park J, Ismail M, Lohiser B, Justice S, Bibyk S, Fiez T. 1994. A switched-current silicon cochlea. Neural Networks, 1994 IEEE World Congress on Computational Intelligence, 1994 IEEE International Conference on 3.

Andreou AG, Liu W. BiCMOS circuits for silicon cochleas. European Conference on Circuit Theory and Design503-508.

Baker MW, Lu TKT, Salthouse CD, Sit JJ, Zhak S, Sarpeshkar R. 2003. A 16-channel analog VLSI processor for bionic ears and speech-recognition front ends. Custom Integrated Circuits Conference, 2003 Proceedings of the IEEE 2003521-526.

Baker MW, Sarpeshkar R. 2003. A low-power high-PSRR current-mode microphone preamplifier. Solid-State Circuits, IEEE Journal of 38:1671-1678.

Bhadkamkar N. 1993. A Variable Resolution, Nonlinear Silicon Cochlea. Computer Systems Laboratory, Stanford University.

Bhadkamkar N, Fowler B. 1993. A sound localization system based on biological analogy. Neural Networks, 1993 , IEEE International Conference on1902-1907.

Bhadkamkar NA. 1994. Binaural source localizer chip using subthreshold analog CMOS. Neural Networks, 1994 IEEE World Congress on Computational Intelligence , 1994 IEEE International Conference on 3:1866-1870.

Boahen KA, Andreou AG. 1992. A contrast sensitive silicon retina with reciprocal synapses. Advances in Neural Information Processing Systems 4:764-772.

Bor JC, Wu CY. 1996. Analog electronic cochlea design using multiplexing switchedcapacitor circuits. Neural Networks, IEEE Transactions on 7:155-166.

Chang JS, Tong YC. 1990. A pole sharing technique for linear phase switched-capacitor filterbanks. Circuits and Systems, IEEE Transactions on 37:1465-1479.

Chen YC, Chen JH. 1994. An analog CMOS circuit for cochlea implant. Engineering in Medicine and Biology Society, 1994 Engineering Advances: New Opportunities for Biomedical Engineers Proceedings of the 16th Annual International Conference of the IEEE978-979. 
Delbruck T. 1991. Bump circuits for computing similarity and dissimilarity of analog voltages. Neural Networks, 1991 , IJCNN-91-Seattle International Joint Conference on 1.

Edwards RT, Cauwenberghs G. A second-order log-domain bandpass filter for audio frequency applications. Circuits and Systems, 1998.ISCAS '98.Proceedings of the 1998 IEEE International Symposium on 3, p 651-654. 1998.

Ref Type: Internet Communication

Edwards RT, Cauwenberghs G. 1999. Log-domain circuits for auditory signal processing. Circuits and Systems, 1999 42nd Midwest Symposium on 2.

Fletcher H. 1930. A SPACE-TIME PATTERN THEORY OF HEARING. The Journal of the Acoustical Society of America 1:311-343.

Fox RM, Nagarajan M. 1999. Multiple operating points in a CMOS log-domain filter. Circuits and Systems II: Analog and Digital Signal Processing, IEEE Transactions on 46:705-710.

Fragniere E. 1998. Analogue VLSI Emulation of the Cochlea. PhD thesis, Swiss Federal Institute of Technology (EPFL), Lausanne.

Fragniere E, van Schaik A, Vittoz EA. 1997. Design of an Analogue VLSI Model of an Active Cochlea. Analog Integrated Circuits and Signal Processing 13:19-35.

Frey DR. 1993. Log-domain filtering: an approach to current-mode filtering. Circuits, Devices and Systems, IEE Proceedings G 140:406-416.

Frey DR. 1996. Exponential state space filters: a generic current mode-design strategy. Circuits and Systems I: Fundamental Theory and Applications, IEEE Transactions on 43:34-42.

Furth PM, Andreou AG. 1995a. A design framework for low power analog filter banks. Circuits and Systems I: Fundamental Theory and Applications, IEEE Transactions on 42:966-971.

Furth PM, Andreou AG. 1995b. Linearised differential transconductors in subthreshold CMOS. Electronics Letters 31:545-547.

Furth PM, Andreou AG. 1996a. Cochlear models implemented with linearized transconductors. Circuits and Systems, 1996 ISCAS'96 'Connecting the World' , 1996 IEEE International Symposium on 3.

Furth PM, Andreou AG. 1996b. Translinear transconductor design for cochlear filter banks. Circuits and Systems, 1996 , IEEE 39th Midwest symposium on 2.

Galbraith C, Rebeiz GM, Drangmeister R. 2007. A Cochlea-based Preselector for UWB Applications. Radio Frequency Integrated Circuits (RFIC) Symposium, 2007 IEEE219-222.

GALBRDITH C, WHITE R, GROSH K, REBEIZ GM. 2005. A mammalian cochlea-based RF channelizing filter. IEEE MTT-S International Microwave Symposium digest 4:1935-1938.

Geisler CD, Sang C. 1995. A cochlear model using feed-forward outer-hair-cell forces. Hearing Research 86:132-146.

Georgiou J, Toumazou C. 2005a. A 126-/spl mu/W cochlear chip for a totally implantable system. Solid-State Circuits, IEEE Journal of 40:430-443.

Georgiou J, Toumazou C. 2005b. A 126-/spl mu/W cochlear chip for a totally implantable system. Solid-State Circuits, IEEE Journal of 40:430-443. 
Germanovix W, Toumazou C. 2000. Design of a micropower current-mode log-domain analog cochlearimplant. Circuits and Systems II: Analog and Digital Signal Processing, IEEE Transactions on 47:1023-1046.

Graham DW, Hasler P. 2002. Capacitively-coupled current conveyer second-order section for continuous-time bandpass filtering and cochlea modeling. Circuits and Systems, 2002 ISCAS 2002 IEEE International Symposium on 5.

Graham DW, Hasler PE, Chawla R, Smith PD. 2007. A Low-Power Programmable Bandpass Filter Section for Higher Order Filter Applications. Circuits and Systems I: Regular Papers, IEEE Transactions on 54:1165-1176.

Grech I, Micallef J, Vladimirova T. 2003. Low-Power Log-Domain CMOS Filter Bank for 2-D Sound Source Localization. Analog Integr Circuits Signal Process 36:99-117.

Grech I, Micallef J, Vladimirova T. 2004. Analog CMOS Chipset for a 2-D Sound Localization System. Analog Integrated Circuits and Signal Processing 41:167-184.

Hamilton TJ, Jin C, van Schaik A. 2007. A Basilar Membrane Resonator for an Active 2-D Cochlea. Circuits and Systems, 2007 ISCAS 2007 IEEE International Symposium on2387-2390.

Hasler P, Kucic M, Minch BA. 1999. A transistor-only circuit model of the autozeroing floating-gate amplifier. Circuits and Systems, 1999 42nd Midwest Symposium on 1.

Hasler P, Stanford T, Minch BA, Diorio C. An autozeroing floating-gate second-order section. Circuits and Systems, 1998 ISCAS'98 Proceedings of the 1998 IEEE International Symposium on 2.

Hinck T, Yang Z, Zhang Q, Hubbard AE. 1999. A current-mode implementation of a traveling wave amplifier modelsimilar to the cochlea. Circuits and Systems, 1999 ISCAS'99 Proceedings of the 1999 IEEE International Symposium on 2.

Hirahara T, Komakine T. 1989. A computational cochlear nonlinear preprocessing model with adaptive $Q$ circuits. Proceedings of IEEE International Conference on Acoustics, Speech and Signal Processing (ICASSP 89) 1:496-499.

Hubbard A. 1993. A traveling-wave amplifier model of the cochlea. Science 259:68-71.

Katsiamis A.G., Drakakis EM, Lyon R.F. A Biomimetic, $4.5 \mu \mathrm{W}, 120+\mathrm{dB}$, Log-domain Cochlea Channel with AGC. IEEE JOURNAL OF SOLID STATE CIRCUITS 44, p 1006-1022. 2009.

Ref Type: Journal (Full)

Katsiamis A.G., Drakakis EM, Lyon RF. 2007. Practical Gammatone-like Filters for Auditory Processing. EURASIP Journal on Audio, Speech, and Music Processing 2007, 15 Pages.

Kim DO. 1986. Active and nonlinear cochlear biomechanics and the role of outer-hair-cell subsystem in the mammalian auditory system. Hearing Research 22:105-114.

Kim DO, Molnar CE, Pfeiffer RR. 1973. A system of nonlinear differential equations modeling basilar-membrane motion. The Journal of the Acoustical Society of America 54:1517-1529.

Kimura K. 1994. Some circuit design techniques using two cross-coupled, emitter-coupled pairs. Circuits and Systems I: Fundamental Theory and Applications, IEEE Transactions on 41:411-423.

Kimura K. 1995. Circuit design techniques for very low-voltage analog functional blocks using triple-tail cells. Circuits and Systems I: Fundamental Theory and Applications, IEEE Transactions on 42:873-885. 
Kumar N, Himmelbauer W, Cauwenberghs G, Andreou AG. 1998. An analog VLSI chip with asynchronous interface for auditoryfeature extraction. Circuits and Systems II: Analog and Digital Signal Processing, IEEE Transactions on 45:600-606.

Lazzaro J. 1991. A silicon model of an auditory neural representation of spectralshape. SolidState Circuits, IEEE Journal of 26:772-777.

Lazzaro J, Mead C. 1989a. CIRCUIT MODELS OF SENSORY TRANSDUCTION IN THE COCHLEA. Analog VLSI Implementation of Neural Systems.

Lazzaro J, Mead C. 1989b. Silicon Modeling of Pitch Perception. Proceedings of the National Academy of Sciences of the United States of America 86:9597-9601.

Lazzaro J, Mead CA. 1990. A silicon model of auditory localization. Neural Computation 1:47-57.

Lin J, Ki WH, Edwards T, Shamma S, Inc CL, Fremont CA. 1994. Analog VLSI implementations of auditory wavelet transforms using switched-capacitor circuits. Circuits and Systems I: Fundamental Theory and Applications, IEEE Transactions on 41:572-583.

Lin J, Ki WH, Thompson K, Shamma S. 1992a. Cochlear filters design using a parallel dilating-biquadsswitched-capacitor filter bank. Circuits and Systems, 1992 ISCAS'92 Proceedings , 1992 IEEE International Symposium on 4.

Lin J, Ki WH, Thompson K, Shamma S. 1992b. Realization of cochlear filters by VLT switched capacitor biquads. Acoustics, Speech, and Signal Processing, 1992 ICASSP-92 , 1992 IEEE International Conference on 2.

Liu W. 1992. An Analog Cochlear Model Signal Representation and VLSI Realization.

Liu W, Andreou AG, Goldstein MH. 1992. Voiced-speech representation by an analog silicon model of the auditory periphery. Neural Networks, IEEE Transactions on 3:477-487.

Lu TKT, Baker M, Salthouse CD, Sit JJ, Zhak S, Sarpeshkar R. 2003. A micropower analog VLSI processing channel for bionic ears and speech-recognition front ends. Circuits and Systems, 2003 ISCAS'03 Proceedings of the 2003 International Symposium on 5.

Lyon R. 1982. A computational model of filtering, detection, and compression in the cochlea. Acoustics, Speech, and Signal Processing, IEEE International Conference on ICASSP '82 7:1282-1285.

Lyon RF. 1978. A Signal Processing Model of Hearing. found in 'http:/ / www dicklyon com'.

Lyon RF. 1991. Analog Implementations of Auditory Models. DARPA Workshop on Speech Recognition and Natural Language.

Lyon RF, Mead C. 1988a. An analog electronic cochlea. Acoustics, Speech, and Signal Processing [see also IEEE Transactions on Signal Processing], IEEE Transactions on 36:1119-1134.

Lyon RF, Mead CA. 1988b. A CMOS VLSI cochlea. Acoustics, Speech, and Signal Processing, 1988 ICASSP-88 , 1988 International Conference on2172-2175.

Mahowald MA, Delbriick T. 1989. Cooperative Stereo Matching Using Static and Dynamic Image Features. Analog VLSI Implementation of Neural Systems.

Mandal S, Zhak S, Sarpeshkar R. 2006. Circuits for an RF Cochlea. Circuits and Systems, 2006 ISCAS 2006 Proceedings 2006 IEEE International Symposium on4.

Mead C. 1989. Analog VLSI and neural systems. Addison-Wesley Longman Publishing Co., Inc. Boston, MA, USA.

Mead C. 1990. Neuromorphic electronic systems. Proceedings of the IEEE 78:1629-1636. 
Naess O, Olsen EA, Berg Y, Lande TS. 2003. A low voltage second order biquad using pseudo floating-gate transistors. Circuits and Systems, 2003 ISCAS'03 Proceedings of the 2003 International Symposium on 1.

Namasivayan AK. 2004. Cochlear Implant Technical Issues: Electrodes, Channels, Stimulation Modes and More. AudiologyOnline, Archives.

Salthouse CD, Sarpeshkar R. 2003. A practical micropower programmable bandpass filter for use in bionic ears. Solid-State Circuits, IEEE Journal of 38:63-70.

Sarpeshkar R, Lyon R.F., Mead C. 1998. A Low-Power Wide-Dynamic-Range Analog VLSI Cochlea. Analog Integrated Circuits and Signal Processing 16:245-274.

Sarpeshkar R, Lyon RF, Mead CA. 1996. An analog VLSI cochlea with new transconductance amplifiers and nonlinear gain control. Circuits and Systems, 1996 ISCAS '96, 'Connecting the World' , 1996 IEEE International Symposium on 3:292-296.

Sarpeshkar R, Salthouse C, Ji-Jon S, Baker MW, Zhak SM, Lu TKT, Turicchia L, Balster S. 2005. An ultra-low-power programmable analog bionic ear processor. Biomedical Engineering, IEEE Transactions on 52:711-727.

Schaik A. 2001. An Analog VLSI Model of Periodicity Extraction in the Human Auditory System. Analog Integrated Circuits and Signal Processing 26:157-177.

Seevinck E. 1990. Companding current-mode integrator: A new circuit principle for continuous-time monolithic filters. Electronics Letters 26:2046.

Sit JJ, Sarpeshkar R. 2004. A micropower logarithmic A/D with offset and temperature compensation. Solid-State Circuits, IEEE Journal of 39:308-319.

Slaney M. 1988. Lyon's Cochlear Model. Apple Computer, Advanced Technology Group.

Smith RL, Brachman ML. 1982. Adaptation in auditory-nerve fibers: A revised model. Biological Cybernetics 44:107-120.

Toumazou C, Ngarmnil J, Lande TS. 1994. Micropower log-domain filter for electronic cochlea. Electronics Letters 30:1839-1841.

Tsividis YP, Gopinathan V, Toth L. 1990. Companding in signal processing. Electronics Letters 26:1331-1332.

van Schaik A. 2003. A small analog VLSI inner hair cell model. Circuits and Systems, 2003 ISCAS'03 Proceedings of the 2003 International Symposium on 1.

Van Schaik A, Fragniere E. 2001. Pseudo-voltage domain implementation of a 2-dimensional silicon cochlea. Circuits and Systems, 2001 ISCAS 2001 The 2001 IEEE International Symposium on 3.

van Schaik A, Meddis R. 1996. The electronic ear; towards a blueprint. Neurobiology233250.

Vincent C, Shih-Chii L, Andre VS. 2007. AER EAR: A Matched Silicon Cochlea Pair With Address Event Representation Interface. Circuits and Systems I: Regular Papers, IEEE Transactions on 54:48-59.

Vittoz EA. 1983. MOS transistors operated in the lateral bipolar mode and their application in CMOS technology. Solid-State Circuits, IEEE Journal of 18:273-279.

Vittoz EA. 1997. Pseudo-Resistive Networks and their Applications to Analog Collective Computation. Proceedings of the 7th International Conference on Artificial Neural Networks1133-1150.

Watts L. 1992. Cochlear Mechanics: Analysis and Analog VLSI. PhD Thesis, California Institute of Technology. 
Watts L, Kerns DA, Lyon RF, Mead CA. 1992. Improved implementation of the silicon cochlea. Solid-State Circuits, IEEE Journal of 27:692-700.

Wegel RL, Lane CE. 1924. The Auditory Masking of One Pure Tone by Another and its Probable Relation to the Dynamics of the Inner Ear. Phys Rev 23:266.

Wen B, Boahen K. 2003. A linear cochlear model with active bi-directional coupling. Engineering in Medicine and Biology Society, 2003 Proceedings of the 25th Annual International Conference of the IEEE 3.

Wen B, Boahen K. 2006. A 360-Channel Speech Preprocessor that Emulates the Cochlear Amplifier. Solid-State Circuits, 2006 IEEE International Conference Digest of Technical Papers2268-2277.

Zhak SM, Baker MW, Sarpeshkar R. 2003. A low-power wide dynamic range envelope detector. Solid-State Circuits, IEEE Journal of 38:1750-1753.

Zwislocki J. 1950. Theory of the Acoustical Action of the Cochlea. The Journal of the Acoustical Society of America 22:778. 


\title{
Design Considerations for Magnetically Actuated Biomimetic Cilia
}

\author{
Benjamin Evans ${ }^{1}$ and Rich Superfine ${ }^{2}$ \\ ${ }^{1}$ Elon University \\ ${ }^{2}$ University of North Carolina at Chapel Hill
}

USA

\section{Introduction}

Biology has produced extraordinary solutions for functions necessary for life. Sensing and movement appear to be two such necessary functions. For both of these, biology has developed extensions from the cell body called cilia (Sleigh, 1962). For movement - either of the entire organism or for moving fluid and hence nutrients toward the stationary organism - nature has evolved motile cilia (Satir, Mitchell, \& Jekely, 2008). These remarkable structures, consisting of a highly organized collection of several hundred thousand proteins packed in a single 250 nanometer diameter cylinder 7 microns long, beat in regular bend shapes autonomously, fuelled by chemical energy (Nicastro, 2009; Satir \& Christensen, 2007). Cilia exist throughout biology, from single cell organisms to humans, and are essentially identical down to the protein level across this vast range of life forms. Within the human body, the literature on their significance to human health has exploded over the past decade (Cardenas-Rodriguez \& Badano, 2009). Cilia have long been understood to be the critical mechanism for fighting lung infections through the propulsion of mucus (Antunes \& Cohen, 2007; Boucher, 2007). More recently, cilia have been found to guide neurons within the brain and to be responsible for the left-right asymmetry of the human body (Okada \& Hirokawa, 2009; Sutherland \& Ware, 2009). Whether for organism propulsion or for its more complex physiological functions, the cilium is the primary manner in which the cell interacts with surrounding fluids (Cartwright, Piro, Piro, \& Tuval, 2008; Smith, Gaffney, \& Blake, 2009). Each of these issues: the structure, operation and function of cilia remains a major challenge for biological physics. Difficult unanswered questions remain in the protein-level organization of the cilium, the coordination of 4000 molecular motors to produce bend shapes and the structure-fluid interaction of beating, slender bodies with Newtonian and viscoelastic fluids.

From an engineering perspective, cilia present a challenge for replicating their function at the micrometer length scale. Applications of such structures may include microfluidics for pumping and mixing, sensing surfaces for measuring local fluid flows and active surfaces for energy applications and the inhibition of biofouling. We are far from having the ability to replicate the extraordinary, nanoscale architecture of the cilium. However, we have been successful recently at replicating the functional capability of cilia at the sub-micron length scale through a variety of advances in materials and fabrication strategies. We begin our discussion with magnetic materials as magnetic actuation offers flexibility in design and 
application to arrays of moving structures. With candidate materials in hand, we then describe fabrication strategies that permit well defined, highly elongated structures in large area arrays. We need to actuate these structures with bend shapes appropriate for moving fluids. We therefore describe the physics of the magnetic actuation of extended structures, including specific field generation schemes and the bend shapes that result. Throughout these sections we present general guidelines for the design space within which an engineer would operate.

While biology provides inspiration for engineering solutions, the resulting technology allows us to reflect an engineering understanding back onto biological design challenges. We therefore end this review with a discussion of the overall hydrodynamic, energy and force considerations that the biological cilia are designed to solve and compare our technical achievement with the remarkable molecular solution of biology. It will not surprise the reader that we remain in awe of the nanotechnology of life.

\section{Materials}

\subsection{Introduction}

The ideal material for a magnetic microactuator must be both flexible and magnetic. In any single material, these qualities are generally mutually exclusive. Magnetic materials are with rare exception metallic, and therefore of high modulus while soft polymeric materials may exhibit only a slight diamagnetism. We can, however, combine the flexibility of a polymer with the magnetization of a metal by developing a composite material in which magnetic particles are suspended within a polymer matrix. Depending on their modulus, such materials are referred to as either ferrogels (soft) or magnetic elastomers (firm). In this work, we will use the latter term to refer to both.

\subsection{Nanoparticle candidates}

Magnetic elastomers first began to appear in the literature about 20 years ago, and a wide range of such materials have now been described. The vast majority of these materials use iron oxide nanoparticles as their magnetic component (either magnetite: $\mathrm{Fe}_{3} \mathrm{O}_{4}$, or maghemite: $\mathrm{Fe}_{2} \mathrm{O}_{3}$ ), since procedures for their fabrication are simple and well-established (Bee, Massart, \& Neveu, 1995; Massart, 1981; Massart, Dubois, Cabuil, \& Hasmonay, 1995; van Ewijk, Vroege, \& Philipse, 1999). The room temperature saturation magnetizations of $480 \mathrm{kA} / \mathrm{m}$ for magnetite and $380 \mathrm{kA} / \mathrm{m}$ for maghemite differ by a relatively small proportion (Dunlop \& Ozdemir, 1997). While a few materials use cobalt nanoparticles or alloys of cobalt, iron, and nickel, saturation magnetizations of such materials are generally on par with those for iron oxides, and therefore the most significant factor in the magnetization of a composite material is not the choice of nanoparticle but the loading fraction of particles within the matrix.

Magnetic elastomers containing magnetite or maghemite nanoparticles are by far the most prevalent in the literature, and therefore calculations in this work will generally consider the loading material to be maghemite. Magnetization of maghemite nanoparticles as a function of applied field is shown in Fig. 1, below. Synthesis of stable aqueous solutions of iron oxide nanoparticles under acidic or alkaline conditions was first described by Massart (Massart, 1981), and such solvents are stable up to a concentration of about $5 \%$ nanoparticles by weight. These solutions can be made stable at higher concentrations at neutral $\mathrm{pH}$ with the addition of sodium citrate and tetramethylammonium hydroxide, or may be transferred to 
an organic phase (Dubois, Cabuil, Boue, \& Perzynski, 1999; van Ewijk et al., 1999). The former is particularly useful in the synthesis of hydrogel-based magnetic elastomers which are often crosslinked under conditions of controlled $\mathrm{pH}$, while the latter renders particles suitable for a hydrophobic matrix.

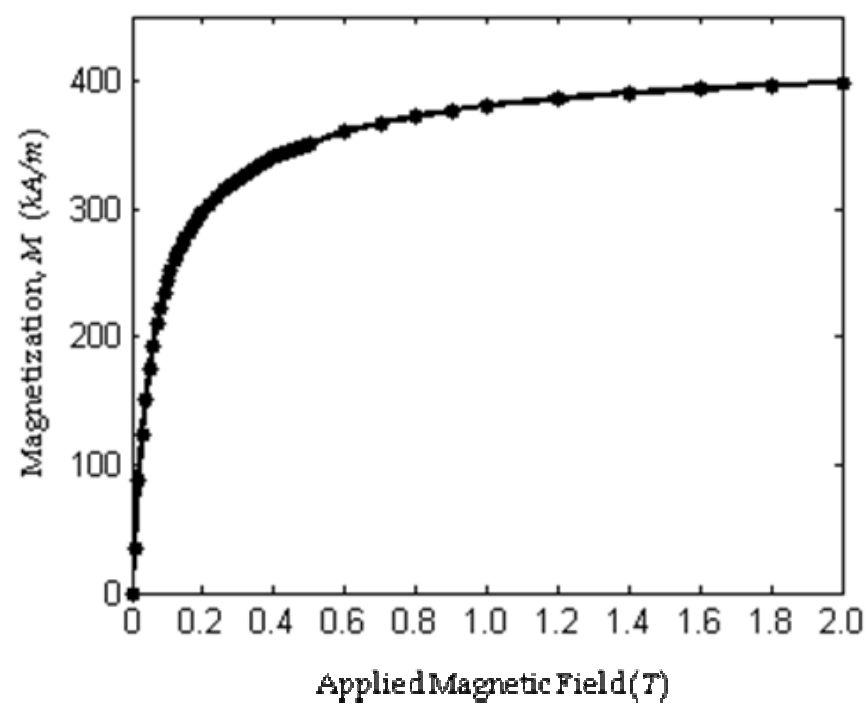

Fig. 1. Room-temperature magnetization of maghemite nanoparticles as a function of applied magnetic field, by our own SQUID measurements.

\subsection{Polymer candidates}

As for the polymer matrix, the most common choices by far are soft, hydrophilic magnetic elastomers such as those constructed of polyacrylamide (Caykara, Yoruk, \& Demirci, 2009; Galicia et al., 2003; Mayer, Cabuil, Lalot, \& Thouvenot, 2000), N-isopropylacrylamide (Xulu, Filipcsei, \& Zrinyi, 2000), polyvinyl alcohol (Barsi, Buki, Szabo, \& Zrinyi, 1996; Collin, Auernhammer, Gavat, Martinoty, \& Brand, 2003; H. Lin, Watanabe, Kimura, Hanabusa, \& Shirai, 2003; Mitsumata et al., 1999; Resendiz-Hernandez, Rodriguez-Fernandez, \& GarciaCerda, 2008; Szabo, Czako-Nagy, Zrinyi, \& Vertes, 2000; Zrinyi, Barsi, \& Buki, 1997), and their derivative copolymers (C. L. Lin, Chiu, \& Don, 2006). Also prevalent are gelatin (Saslawski, Weingarten, Benoit, \& Couvreur, 1988) and copolymers of polyethylene oxide (Qin et al., 2009; Wormuth, 2001). Significantly less attention has been given to hydrophobic magnetic elastomers, which include a few instances of polydimethylsiloxane (Evans et al., 2007; Fahrni, Prins, \& van Ijzendoorn, 2009; Jolly, Carlson, Munoz, \& Bullions, 1996; Varga, Feher, Filipcsei, \& Zrinyi, 2003) and polystyrene (Timonen et al., 2010).

A material which is to be useful in the fabrication of a microactuator must be chemically and osmotically compatible with its target environment, contain a large volume fraction of magnetic nanoparticles, and have a low modulus. Since the target environment for most biological and biomimetic applications is aqueous, a hydrophobic matrix is helpful in meeting the first requirement. A hydrophobic magnetic elastomer resists swelling in an aqueous environment, and nanoparticles effectively encapsulated in the hydrophobic matrix are unlikely to leech into an aqueous environment. The lack of swelling, however, generally 
gives hydrophobic elastomers a significantly higher modulus than their hydrophilic peers, which are usually fabricated in a swollen state and are able to remain so due to the presence of water as a good solvent.

We will therefore explore two different materials as candidates for magnetic microactuators. The first is a hydrogel consisting of maghemite $\left(\mathrm{Fe}_{2} \mathrm{O}_{3}\right)$ nanoparticles in a polyacrylamide matrix, and the second is a complex of maghemite nanoparticles in a hydrophobic siloxane matrix.

\subsection{Hydrogel-based composites}

Hydrogels are, by definition, any polymeric material which is swollen to a great degree, generally greater than $90 \%$, with water (Mathur, Moorjani, \& Scranton, 1996). They may be crosslinked, such as in an acrylamide gel or most polyvinyl alcohol hydrogels, or uncrosslinked, as in agarose. Although hydrogels are susceptible to osmotic effects while in an aqueous environment and are therefore likely to well or shrink unpredictably, they offer several advantages over silicone elastomers which make them attractive. Most intriguing is their affinity for water, which gives them an extremely low surface energy in an aqueous medium. This property has the potential to reduce the incidence of collapse due to selfadhesion which is prevalent in high-aspect-ratio hydrophobic structures (Evans et al., 2007; Roca-Cusachs et al., 2005). In addition, aqueous ferrofluids mix readily into many hydrogels, simplifying synthesis and allowing for relatively high concentrations of magnetic material. Finally, most hydrogels have very low elastic moduli $(1-100 \mathrm{kPa})$, resulting in very flexible structures, and in many cases the modulus may be tuned simply by adjusting the ratio of polymer to water.

Incorporating nanoparticles into a hydrogel results in a magnetic elastomer. In the case of a hydrogel, the magnetic nanoparticles can either be precipitated directly within a crosslinked matrix (H. Lin et al., 2003) or the polymer and particles may be synthesized separately and mixed together prior to crosslinking (Galicia et al., 2003; Mayer et al., 2000; Szabo et al., 2000).

A prime candidate for a hydrogel-based magnetic elastomer is a maghemite-impregnated polyacrylamide. In the fabrication of a polyacrylamide magnetic elastomer, a citratestabilized pH 7 maghemite nanoparticle ferrofluid can be mixed directly into an aqueous solution of acrylamide monomer. The low viscosity and absence of long-chain polymers at this stage allows the ferrofluid to mix well even at very high concentrations, after which polymerization and crosslinking are performed simultaneously, forming a gel in which the maghemite particles are trapped. In this manner, materials have been produced with maghemite concentrations as high as $18 \% \mathrm{v}$. (50\% wt.) and with elastic moduli as low as $10^{4}$ $\mathrm{Pa}$ (Galicia et al., 2003; Mayer et al., 2000). So stable is the ferrofluid that we have found that the upper limit on concentration results from a mechanical limitation - the fluid becomes to pasty to be workable much above $18 \%$ v., yet shows no sign of flocculation.

Following Galicia et al., a typical recipe for an acrylamide magnetic elastomer requires the acrylamide monomer (AM), a bisacrylamide crosslinker (N, N methylene bisacrylamide, or $\mathrm{BA})$, catalyst (ammonium persulfate, APS) and an initiator (tetramethylethylenediamine, TEMED). Polymerization and crosslinking necessarily occur simultaneously, as the symmetric bisacrylamide forms crosslinks by becoming incorporated in adjacent polymer chains during polymerization. Synthesis of a representative sample of a ferrofluid-acrylamide material (FFAAm) which contains $6 \% \mathrm{wt}$. acrylamide in water would proceed as follows:

The monomer (AM) and crosslinker (BA) are mixed in a ratio of 20 to 1 in a $20 \%$ wt. solution in deionized water (2.22 grams $\mathrm{AM}$ and 0.11 grams of $\mathrm{BA}$ into $10 \mathrm{~mL} \mathrm{H}_{2} \mathrm{O}$ ). This solution is 
diluted with a citrate-stabilized ferrofluid of a given maghemite concentration to produce a ferrofluid-acrylamide solution of $6 \%$ wt. polymer $(0.54 \mathrm{~mL}$ AM/BA mixture to $1.46 \mathrm{~mL}$ citrate-stabilized ferrofluid). Since oxygen is known to interfere with the free-radical decomposition products of the APS, the solution is degassed with argon for 10 minutes prior to crosslinking and the subsequent reaction is performed in a nitrogen environment. Crosslinking occurs upon the addition of $0.2 \%$ wt. APS ( $40 \mathrm{uL}$ of $10 \%$ APS in $\mathrm{H}_{2} \mathrm{O}$ ) and $0.2 \%$ TEMED, and is complete within a couple of minutes.

\subsection{Silicone-based composites}

Only a few instances of hydrophobic magnetic elastomers occur in the literature. These materials, however, have several advantages over their hydrophilic cousins when implemented in an aqueous environment: they are unlikely to interact chemically or osmotically with the environment and are less likely to leech nanoparticles. The scarcity of hydrophobic magnetic elastomers relative to their hydrophilic peers is likely the result of the additional complication of treating iron oxide nanoparticles to make them miscible in a non-polar matrix. (In-situ precipitation of nanoparticles within a crosslinked matrix has not been explored in this case, since current techniques precipitate nanoparticles in an aqueous solution.)

While larger particles (> 1 micron) can be mixed directly into a siloxane polymer by brute force, this results in a material that is heterogeneous at the microscale and therefore likely unsuitable for application in magnetic microactuators. To achieve homogeneity at the micron scale, great care must be taken to ensure the colloidal stability of the nanoparticles. Such stability may be achieved through the use of surfactants (Evans et al., 2007) or by strong adsorption of a hydrophobic chemical species on the surface of the particle (Mefford et al., 2008; Rutnakornpituk et al., 2002; Stevenson et al., 2001; Wilson, Goff, Riffle, Harris, \& St Pierre, 2005). A description of the preparation of the former follows.

Magnetite nanoparticles are first prepared via the coprecipitation of iron salts (Massart, 1981) and are further oxidized to form maghemite by heating in an acidic solution in the presence of iron nitrate. The particles are then grafted with oleic acid (van Ewijk et al., 1999). The presence of oleic acid serves to enhance the solubility of the nanoparticles in a non-polar solution; however, mixing directly with a silicone elastomer such as Dow Corning Sylgard 184 results in flocculation of the nanoparticles and further action is required to achieve a homogenous solution. Before mixing, hexadecane is added in a 1:1 v/v ratio with the oleic acid nanoparticle suspension and the entire product is diluted 5:1 in toluene. Sylgard 184 prepolymer is similarly diluted in toluene, and the two solutions are combined over the course of several minutes under the influence of immersion ultrasonication. The toluene is then removed by evaporation. After resting for several days, excess hexadecane and Sylgard 184 will separate from the composite. Decantation yields a stable suspension of maghemite nanoparticles in a poly(dimethyl siloxane) matrix. This material is produced without any curing agent in order to extend its shelf life.

\section{Fabrication}

\subsection{Template candidates}

The two primary strategies that have emerged for fabricating magnetic cila are self-assembly and template-based methodologies. In the first, an applied magnetic field is used to cause the material to assemble into an energetically-favorable arrangement. Minimization of magnetic 
energy at the expense of surface energy can lead to elongated structures which are suitable for magnetic actuation applications. These self-assembled structures may consist of either an uncrosslinked magnetic polymer composite (Timonen et al., 2010) or a series of magnetic beads (Furst, Suzuki, Fermigier, \& Gast, 1998; Singh, Laibinis, \& Hatton, 2005; Vilfan et al., 2010), which may or may not be crosslinked prior to the removal of the external field.

The most common template-based fabrication strategy is soft lithography, in which soft polymeric structures are templated in a photolithographic mold, which is generally constructed of photoresist on a silicon substrate. However, while soft lithography is capable of producing structures of very high aspect ratio in a plane parallel to the surface (length >> width), it is not well-suited to producing high-aspect ratio structures in a plane perpendicular to the surface (depth $>>$ width). This limitation may be attributed to photon scattering during the exposure of the resist. Furthermore, with soft materials such as silicones and hydrogels, photolithographic lift-off procedures may lead to structure collapse (Pokroy, Epstein, Persson-Gulda, \& Aizenberg, 2009; Roca-Cusachs et al., 2005; Zhang, Lo, Taylor, \& Yang, 2006).

Another common template for high-aspect-ratio microstructures is anodized aluminum oxide (AAO) (Chik \& Xu, 2004; McGary et al., 2006). AAO offers the advantage of a gentle removal of the template by dissolution and may be grown to a wide range of thicknesses, enabling the production of microstructures of various length. However, AAO imposes severe limits on both the diameter and the spacing of the microstructures, which may limit its application.

Track-etched membranes have also been used in the synthesis of nanostructures (Huczko, 2000; Hulteen \& Martin, 1997) - most notably metallic nanowires (Brumlik, Menon, \& Martin, 1994; Schonenberger et al., 1997) - and they present a third possibility for a template for the production of biomimetic cilia. These membranes generally consist of a very thin layer of polymer (most commonly polycarbonate) which is exposed to high-energy ions from a nuclear source or ion beam. As the ions travel through the polymer, they leave behind a track of broken bonds (about $10 \mathrm{~nm}$ diameter) surrounded by a halo of additional damage $(10-100 \mathrm{~nm})$ (Fischer \& Spohr, 1983). The particle tracks etch preferentially in a solution of sodium hydroxide to produce pores of a uniform and tunable diameter (Ferain \& Legras, 1997; Fischer \& Spohr, 1983). Polycarbonate track etched (PCTE) membranes are available in a variety of thicknesses and pore densities, allowing for the production of microstructures in a variety of lengths and inter-structure spacing. They are also soluble in organic solvents such as dichloromethane (DCM) and N-methylpyrrolidone (NMP), among others, allowing for the gentle removal of the membrane from the molded structures.

\subsection{Sample design considerations}

In its simplest manifestation, template-based fabrication of biomimetic cilia from one of the aforementioned materials would require filling the template with material, crosslinking the material, and then removing the membrane by dissolution. There are, however, several considerations which impose further requirements. For example, micro- and nanostructured materials are highly susceptible to collapse during drying due to surface tension forces. While such damage may be alleviated by the use of a critical point dryer, it is most convenient to process the samples in such a way that they remain submerged. We will present a fabrication method below which maintains a fluid environment at all times.

In addition, completed cilia arrays will be actuated by a magnetic source which should be as close as possible to the cilia array to allow for the largest possible applied fields. Cilia arrays 
should also be fabricated in such a way as to facilitate high-magnification optical microscopy. Fig. 2I presents a configuration which allows for both high-magnification optical microscopy and optimal placement of a magnetic source. In this configuration, the magnetic source is placed directly above the sample. This would block the light path in conventional transmission microscopy; however, a reflective gold-coated cover slip placed just above the sample allows for imaging in reflection. It should be noted that this is not traditional reflection microscopy as the incident light does not reflect directly off the sample itself, but is transmitted through the sample after reflecting from the gold coating. This configuration places an additional constraint on the sample configuration: in order to be close to both the magnetic source and the objective, the sample should be made as thin as possible.

We will present a fabrication method below which can be used to produce samples as thin as 500 microns. In this example, we will assume that the cilia are fabricated of a siliconebased magnetic elastomers templated in a polycarbonate track-etched membrane. Modifications are necessary, of course, for hydrogel-based magnetic elastomers such as acrylamide (see section 3.4), but the bulk of the processing remains the same.

\subsection{Fabrication of silicone-based microstructures}

Before beginning a discussion of the fabrication procedure, we should note that one critical element in the following is the adhesion of the silicone cilia to a glass substrate. Generally, silicone exhibits an affinity for glass - even when submerged in water, silicone cured in contact with a glass cover slip will readily adhere to the glass surface. However, an organic solvent such as that used to dissolve a template will quickly swell the silicone and cause it to release from the glass surface. In addition, the same solvent will often render epoxies and other adhesives useless. It is best then to secure the silicone directly to a firm glass substrate via a covalent bond. This may be done by oxidizing the surfaces of both the glass and the silicone with an oxygen plasma, ultraviolet light, or corona discharge (Berdichevsky, Khandurina, Guttman, \& Lo, 2004; Efimenko et al., 2005; Efimenko, Wallace, \& Genzer, 2002; Makamba, Kim, Lim, Park, \& Hahn, 2003), after which the surfaces will bond on contact via a condensation reaction.

A typical fabrication procedure for silicone-based biomimetic cilia would then proceed as follows. A polycarbonate track-etched (PCTE) membrane is selected with an appropriate thickness and pore density, and is etched in a sodium hydroxide solution to achieve the appropriate pore diameter. We find that a solution of $4 \mathrm{M} \mathrm{NaOH}$ heated to $80^{\circ} \mathrm{C}$ will preferentially etch the pores of a PCTE membrane to increase pore diameter at a rate of about $15 \mathrm{~nm} / \mathrm{min}$. After etching, the membrane is impregnated with liquid magnetic elastomer, and the surface of the membrane is cleaned thoroughly with a lab wipe moistened with ethanol (Fig. 2A).

A cover slip is prepared to receive the membrane by affixing a thin rectangular ring of silicone elastomer via the oxidation/condensation reaction described above. This ring will act as a spacer between the upper and lower cover slips in the final product, and should be sized accordingly. The impregnated membrane is then submerged in a small amount of uncured silicone elastomer containing curing agent and placed within the silicone ring (Fig. 2B). Care should be taken to ensure that the uncured silicone is in good contact with the silicone well - it is the well which is chemically bound to the glass; the encapsulating silicone is bound only to this well. The sample is then cured by heating to $80^{\circ} \mathrm{C}$ for one hour. While the silicone-nanoparticle composite described in Section 2.5 will contain no 

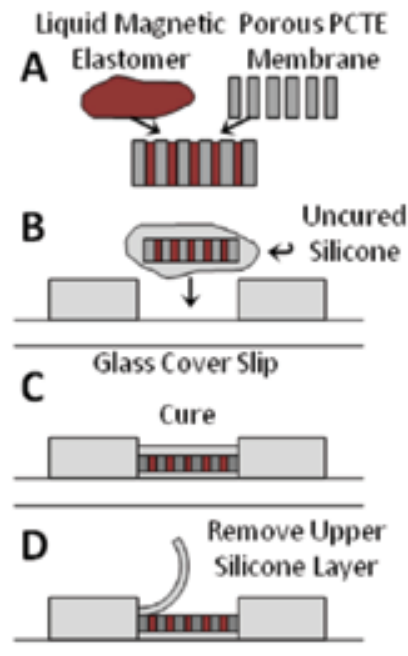

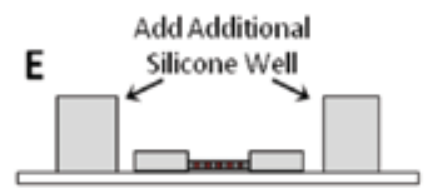

Dissolve Membrane; Replace Solvent

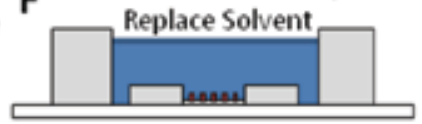

G

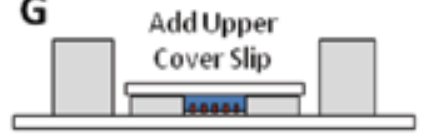

$\mathrm{H}$

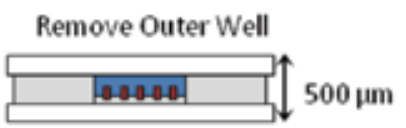

I

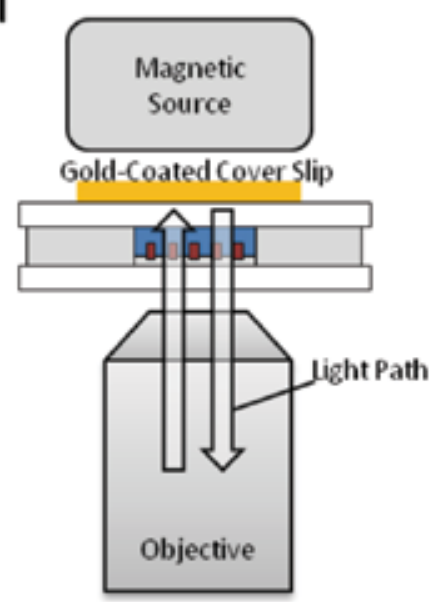

Fig. 2. A-F: Fabrication of silicone-based magnetic biomimetic cilia. I: Modified optical reflection microscopy for viewing biomimetic cilia under magnetic actuation.

curing agent, we have found that the curing agent contained in the surrounding silicone diffuses sufficiently into the composite material, resulting in reliable crosslinking. Finally, the sample is prepared for dissolution by exposing the upper face of the membrane via the removal of the upper layer of cured, encapsulating silicone (Fig. 2D). This layer may be cut carefully with a scalpel and peeled away.

The sample is quite robust only until the membrane is dissolved, and so after dissolution the exposed cilia should remain continually submerged in a liquid. The thin silicon spacer is not enough to reliably retain a liquid bath around the cilia, and so a larger temporary well is added just outside the small well (Fig. 2E). This well must be affixed via the oxidation/condensation reaction previously described to avoid release during dissolution, but will be removed with a razor blade later.

The membrane is then dissolved by submerging the entire sample in a bath of solvent, such as dichloromethane. We have found that heating the solvent results in quicker dissolution and a greater number of viable cilia in the final product, so it is advantageous to heat the solvent to just below its boiling point (about $40^{\circ}$ for dichloromethane) before dissolving the membrane. Since dichloromethane is immiscible with water, it is advisable to replace the solvent first with ethanol, after which it may be transferred to water.

Finally, a piece of cover slip is cut to fit within the outer well and is placed on top of the inner well, as shown in Fig. 2G. Excess fluid is removed, and the outer well can be carefully cut free with a razor. This upper cover slip is not permanently attached, but evaporative effects will produce a small vacuum and fix the cover slip in place quite well. For longer term storage, the entire sample may be submerged in a fluid bath. The completed sample can be designed to be less than 500 microns thick; this dimension is limited mostly by the dimensions of the upper and lower cover slips.

\subsection{Process modifications for hydrogels}

The fabrication procedure for hydrogel-based biomimetic cilia is very similar, but requires a few modifications. The most significant of these is the manner in which the polymer is 


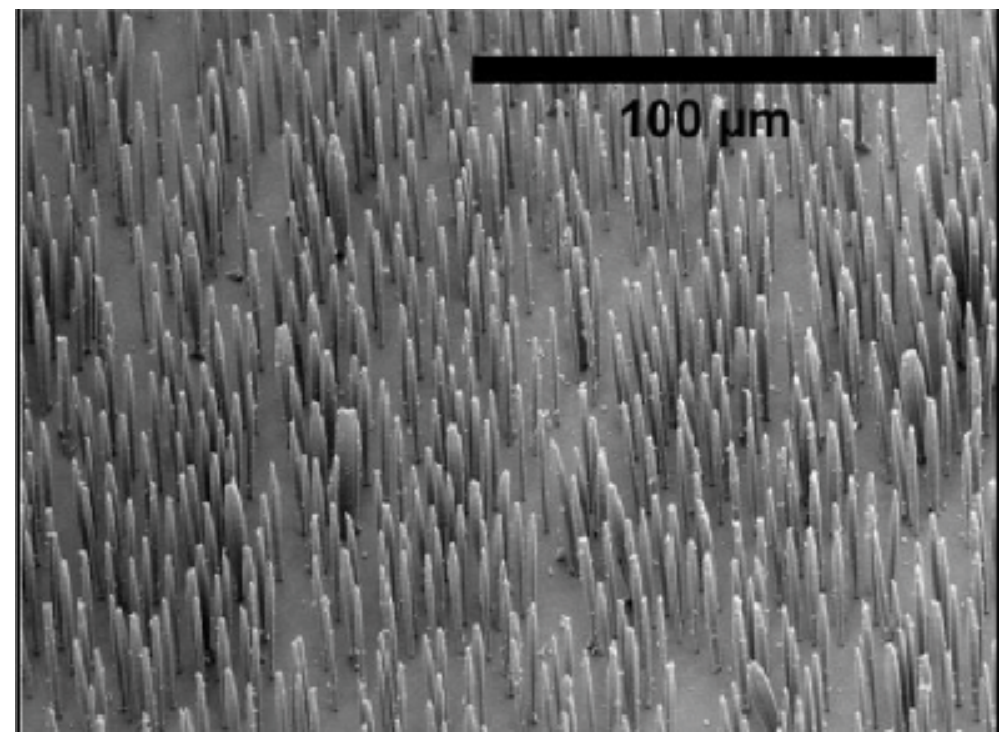

Fig. 3. Biomimetic cilia fabricated of a maghemite-nanoparticle / polydimethyl-siloxane composite by the techniques described in this section.

affixed to the glass substrate. The oxidation/condensation reaction that worked for the silicone materials is useless here, and it can be a challenge to find a suitable substitute. Sidorenko et al. have developed a method of bonding an acrylamide hydrogel to glass by first oxidizing the glass by plasma, corona, or UVO treatment and then using the available hydroxyl groups to bond poly(glycidyl methacrylate) (PGMA) to the surface. The epoxy groups of the PGMA are then functionalized with acrylic acid, which will become incorporated within in the polyacrylamide backbone upon polymerization, permanently bonding the polymer to the glass (Sidorenko, Krupenkin, Taylor, Fratzl, \& Aizenberg, 2007). In addition, prepolymers of hydrogel-based magnetic elastomers generally have a much lower viscosity than silicon-based elastomers, and so it is uncertain whether the uncured magnetic elastomer material would remain within the pores of the PCTE membrane upon submerging the sample in the encapsulating hydrogel layer (as in Fig. 2B). For this reason, the membrane is submerged in a bath of magnetic elastomer and the entire bath is cured; the membrane is then removed and cleaned before submersion in the encapsulating hydrogel layer.

\section{Actuator design}

\subsection{Introduction}

Since the advent of viable experimental models of cilia-like magnetic structures (Evans et al., 2007; Furst et al., 1998), a number of works have been presented which propose detailed computational models describing their actuation (Alexeev, Yeomans, \& Balazs, 2008; Downton \& Stark, 2009; Evans et al., 2007; Gauger, Downton, \& Stark, 2009; Shcherbakov \& Winklhofer, 2004). In addition, a few authors have published on the energy of rotating magnetic droplet (Cebers, 2002; Morozov, Engel, \& Lebedev, 2002). While many of these models take into account dynamics such as fluid-structure interactions and polymer viscosity, we will confine ourselves here to a quasi-static model. Such a model is 
independent of the application of biomimetic cilia structures and is more than suitable for providing a set of guidelines for the design of magnetic cilia.

\subsection{Actuation in a uniform field}

\subsubsection{A simple model}

We will assume the cilium is cantilevered perpendicular to the substrate, and the elastic energy therefore increases with displacement from the vertical (Fig. 4). Since magnetic elastomers are generally superparamagnetic and therefore have no remanence magnetization, any magnetic torque is the result of shape anisotropy. The shape anisotropy of a high-aspect-ratio cilium results in a demagnetizing field along its short axes, which affects both the direction and magnitude of the resulting magnetization. Specifically, the magnetic energy is minimized when the magnetization of the cilium lies along its long axis and this results in a torque which causes it to tend to align with an applied field.

Thus, we can write down the energy of a magnetic cilium as the sum of elastic and magnetic energies:

$$
U=\frac{1}{2} E I \int_{0}^{L} \frac{1}{R(s)^{2}} d s-\frac{\mu_{0} V}{2}\left[G^{2} M^{\prime}\left(\frac{\cos ^{2} \theta}{H+N_{x} M^{\prime}}+\frac{\sin ^{2} \theta}{H+N_{y} M^{\prime}}\right)-M^{\prime} H+2 \int_{0}^{H} M^{\prime}\left(H^{\prime}\right) d H^{\prime}\right]
$$

where $G$ and $H$ are related by

$$
\frac{1}{G^{2}}=\frac{\cos ^{2} \theta}{H+N_{x} M^{\prime}(H)}+\frac{\sin ^{2} \theta}{H+N_{y} M^{\prime}(H)}
$$

Here, the elastic energy is described by the Kirchhoff model (Landau \& Lifshitz, 1986) of a uniform elastic rod, where $E$ is the Young's modulus of the cilium, $I$ is the bending moment, and $R$ is the radius of curvature of the cilium which is in general a function of $s$, the distance along the contour of the cilium from its base. In the magnetic energy term (Morozov et al., 2002), $G$ is the applied magnetic field (in $A / m$ ), $M^{\prime}$ is the magnetization of the cilium, which in general is a function of $H$, the internal field (in $\mathrm{A} / \mathrm{m}$ ). The angle between the cilium and the applied field is given by $\theta$, and $N_{x}$ and $N_{y}$ are the demagnetization factors parallel and perpendicular to the axis of the cilium, respectively. $V$ is the volume of the cilium.

We have found that the magnetization of a magnetite-polymer composite is linear with respect to the volume fraction of magnetic particles even to very high loadings ( $90 \% \mathrm{wt}$.). Therefore to make explicit the effect of nanoparticle loading, we will henceforth write the magnetization of the composite material, $M^{\prime}$, in terms of the magnetization of the magnetic component, $M$, and the volume fraction of magnetic nanoparticles, $f$, or $M^{\prime}=M f$.

Much of the complexity in equations (1) and (2) stems from the fact that shape anisotropy affects both the direction and magnitude of the magnetization, $M$. However, the influence of anisotropy on the total energy due to its effect on the magnitude of $M$ is secondary to its effect on direction. If we therefore consider a simplification in which the magnitude of the magnetization is dependent only on the internal field, $H$ and not on the orientation of the cilium, then we can describe the magnetic energy with the Stoner-Wohlfarth model (Stoner, 1947), in which case the total energy becomes:

$$
U=\frac{1}{2} E I \int_{0}^{L} \frac{1}{R(s)^{2}} d s-\frac{1}{2} \mu_{0} M^{2} f^{2} V\left(N_{x}-N_{y}\right) \cos ^{2} \theta
$$


These two models converge for saturating fields, and in the case of small fields the StonerWohlfarth model will underestimate the calculated applied field magnitude by a factor of $\sqrt{1+\chi N_{x}}$, where $\chi=M / H$ is the dimensionless magnetic susceptibility. This factor is greatest for highly permeable materials and very small fields. In the extreme limit of a material which consists of $100 \%$ iron oxide and for fields approaching $0 \mathrm{~T}$, we find that the resulting error is approximately a factor of 2; in most cases the error is less than $10 \%$. This is more than sufficient accuracy for an investigation of design considerations of magnetic cilia, and this simplification will enable us to derive several useful expressions to guide design considerations.

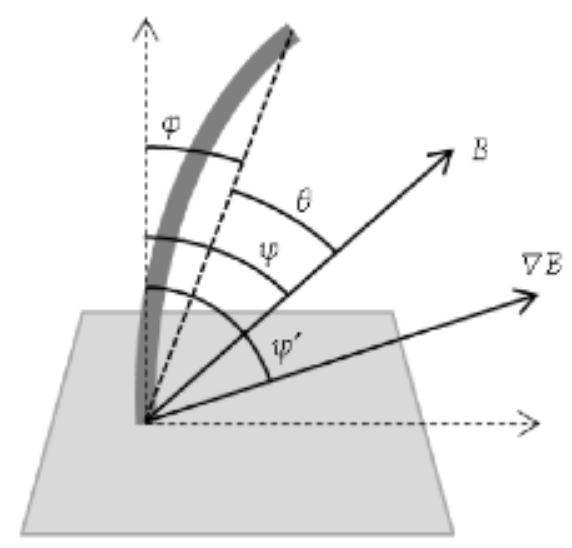

Fig. 4. Angle definitions used in the model. An upright elastic cilium which is cantilevered from a horizontal surface bends through an angle of $\varphi$ due to the presence of a magnetic field, $B$, and/or a magnetic field gradient, $\nabla \boldsymbol{B}$.

\subsubsection{Field requirements}

We would like to consider the necessary requirements for successful actuation of a magnetic cilia. Let us therefore define the lower limit of 'successful' action as a $10^{\circ}$ bend from the vertical, where the angle is defined to be between a line connecting the base of the cilium to the tip and the vertical (Fig. 4). In the case of a $10^{\circ}$ bend, it is reasonable to approximate the radius of curvature of the cilium as constant along the length, in which case we can allow $R$ $=L / 2 \varphi$ in Equation (3). In addition, in the limit of high aspect ratio $(L / 2 r>20)$, the difference between the demagnetization factors $N_{x}$ and $N_{y}$ approaches $1 / 2$. Since a typical epithelial cilium has an aspect ratio of about 35, we will henceforth assume that this is the case. Taking a derivative to find the torque on the cilia, we see

$$
\tau=\pi \frac{E r^{4}}{L} \varphi-\frac{1}{2} \mu_{0} M^{2} f^{2} V \cos (\psi-\varphi) \sin (\psi-\varphi)
$$

where we have used $\theta=\psi-\varphi$ to make the angular dependencies more explicit (Fig. 4). One interesting thing to note in this expression is that the magnetic torque on the cilium is always maximized when $\theta=45^{\circ}$. Let us assume that the field is applied in this optimal orientation; then the magnetization required to achieve a bending of $\varphi$ is given by the simple expression: 


$$
M=\frac{1}{f} \sqrt{\frac{E}{\mu_{0}}} \frac{2 r}{L} \varphi^{1 / 2}
$$

The applied field can be mapped to magnetization via the full magnetization curve of the chosen nanoparticle. In Fig. 5, we show the magnetic field required to bend a cilium of radius $100 \mathrm{~nm}$ and length 10 microns (typical of epithelial cilia) through an angle of $10^{\circ}$.

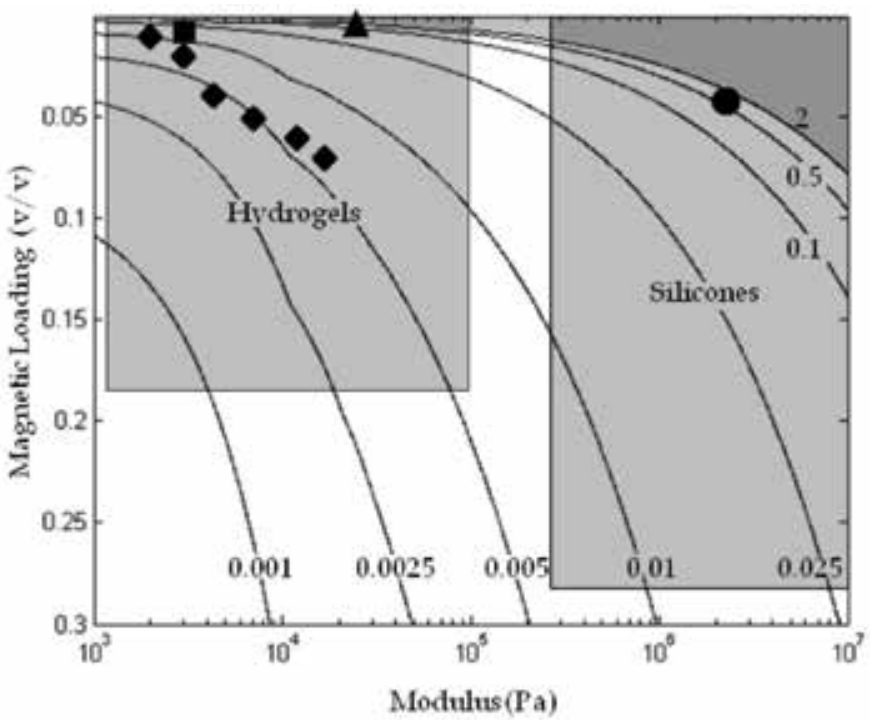

Fig. 5. The magnetic field in tesla (T) required to bend a 7-micron tall magnetic cilium of 100 $\mathrm{nm}$ radius through an angle of $10^{\circ}$. The cilium is loaded with maghemite nanoparticles. Superimposed on the plot are instances of reported magnetoelastomers. Diamonds: maghemite/polyacrylamide (Galicia et al., 2003); Square: magnetite/polyvinyl alcohol (Barsi et al., 1996); Triangle: M-300/polyvinyl alcohol (Collin et al., 2003); Circle: maghemite/ poly(dimethyl siloxane) (Evans et al., 2007).

In Fig. 5, we have highlighted the regions of the parameter space which are feasibly accessible with current materials. The glyphs indicate reported magnetic elastomers, which also appear in Table 1. The shaded areas indicate the range of moduli of common hydrogel and silicon elastomers independently of whether they have been employed in a magnetic nanoparticle composites, and the range of magnetic nanoparticle loading in hydrogel and silicone composites independently of whether the composite has been crosslinked. Most notable in the latter group is a poly(dimethyl siloxane) - magnetite composite fluid which contains nanoparticle loadings up to $28 \%$ v/v (Wilson et al., 2005). Similarly, polyacrylamide hydrogels with maghemite loading up to $18 \%$ have been described (Mayer et al., 2000), but moduli have not been reported for these materials.

It is interesting to note that in the region above $2 \mathrm{~T}$ in the extreme upper-right of the plot represents an area of the parameter space in which it is impossible to bend a cilium through the required angle with any field magnitude. This occurs because the applied field has fully saturated the cilium at this point, and further contributions have no effect. This saturation effect is not an artifact of our simplified model; though not quite so explicit, the limitation also exists in Equation (1). 


\subsubsection{Material design}

We have thus far described the magnetic field required to actuate a cilium of known composition and aspect ratio. However, from the point of view of materials design, it would be useful to have a figure of merit which indicates how well suited a material is for magnetic actuation applications, regardless of geometry. Such a figure could be used to optimize a material with respect to tunable parameters such as modulus and magnetic content. In the case of an optimally-applied field, we can solve Equation (5) for the bend angle $\varphi$, in which case

$$
\varphi=\frac{\mu_{0} M^{2} f^{2}}{E}\left(\frac{L}{2 r}\right)^{2}
$$

The second part of this expression is the square of the aspect ratio, and contains all of the geometrical parameters of the cilium. The first contains only material properties, and is therefore useful in designing materials for use in magnetic actuation devices. In this work, therefore, we refer to the first term as the magnetoelastic ratio. It is important to note that the dependencies on magnetization $M$ and Young's modulus $E$ are not necessarily independent, since adding magnetic content to the composite material generally results in an increase in modulus. Measurements of the modulus of a particular material as a function of particle loading can therefore be used to determine an optimal loading for maximal actuation.

\begin{tabular}{|c|c|c|c|c|}
\hline & & & \multicolumn{2}{|c|}{ Magnetoelastic Ratio } \\
\hline & $f$ & $E(\mathrm{kPa})$ & $\begin{array}{c}\text { Field } \\
\text { Bending }\end{array}$ & $\begin{array}{l}\text { Gradient } \\
\text { Bending }\end{array}$ \\
\hline Maghemite/Polyacrylamide* & $0.01-0.07$ & $2-16$ & 0.0650 & 1.680 \\
\hline Magnetite / Poly(vinyl alcohol) † & 0.009 & 3 & 0.0078 & 0.072 \\
\hline M-300 / Poly(vinyl alcohol) $\ddagger$ & 0.005 & $25^{* *}$ & 0.0003 & 0.048 \\
\hline Maghemite / Poly(dimethyl siloxane) § & 0.04 & 2500 & 0.0002 & 0.003 \\
\hline
\end{tabular}

Table 1. Magnetoelastic ratios for reported magnetic elastomers with nanoscale magnetic particles, for both field-dominated bending and gradient-dominated bending. *(Galicia et al., 2003); †(Barsi et al., 1996); $\ddagger($ Collin et al., 2003); §(Evans et al., 2007); **estimated from reported shear modulus.

The preceding table details the magnetoelastic ratios of extant composite materials for which both nanoparticle concentration and modulus were reported (Table 1). Magnetoelastic ratios as a function of particle loading for the first material on this table are shown in Fig. 7.

\subsection{Actuation by a field gradient \\ 4.3.1 Modeling}

We have thus far only considered the case of a constant magnetic field, in which the mechanism for actuation is the tendency for the long axis of the microactuator to align with the applied magnetic field due to shape anisotropy. However, another mode of actuation exists in the presence of a magnetic field gradient. Any paramagnetic material - regardless of geometry - will be attracted toward regions of higher field, i.e. along a magnetic field gradient. We can account for this type of bending in our energy model simply by adding a term for the energy of a magnetic moment in a magnetic field: $U=-m B \cos \alpha$, where $\alpha$ is the angle between the magnetic moment and the applied field. It can be shown that for the vast majority of magnetic nanoparticle composite materials, $\alpha \sim 0^{\circ}$; that is, the magnetic moment 
aligns with the applied field rather than with the easy axis of the rod (Evans et al., 2007). Thus, energy is minimized when an actuator bends toward a region of higher magnetic field, $B$.

To first order, we will assume that $m$ is constant with respect to a bend through an angle $\varphi$, and that the magnetic field $B$ has a constant gradient. Then upon integrating this energy over length of the rod, we find that the total energy is given by

$$
U=\frac{1}{2} E I \int_{0}^{L} \frac{1}{R(s)^{2}} d s-\frac{1}{2} \mu_{0} M^{2} f^{2} V\left(N_{x}-N_{y}\right) \cos ^{2}(\psi-\varphi)-\frac{1}{2} M f V \nabla B L \cos \left(\psi^{\prime}-\varphi\right)
$$

where the final term represents the contribution of the magnetic field gradient. In this term, $\psi^{\prime}$ is the angle of the magnetic field gradient relative to the vertical (Fig. 4), and for consistency we have replaced the magnetic moment $m$ with the magnetic moment per unit volume $M$ multiplied by the volume of the actuator, $V$.

\subsubsection{Gradient requirements}

The net torque on the microactuator is then

$$
\tau=\pi \frac{E r^{4}}{L} \varphi-\frac{1}{2} \mu_{0} M^{2} f^{2} V \cos (\psi-\varphi) \sin (\psi-\varphi)-\frac{1}{2} M f V \nabla B L \sin \left(\psi^{\prime}-\varphi\right)
$$

where we have again assumed constant curvature and high aspect ratio.

Bending within the field-dominated regime is described earlier in this document. In the case of gradient-dominated bending, we can turn off the magnetic field torque term in Equation (8) simply by requiring that the magnetic field direction align at all times with the axis of the actuator $(\psi=\varphi)$. Solving for the condition of zero net torque, we find that the magnetic field gradient necessary to bend an actuator through an angle $\varphi$ in a gradient-dominated regime is given by

$$
\nabla B=\frac{2 E}{M f} \frac{r^{2}}{L^{3}} \varphi
$$

In Fig. 6 we show the magnetic field gradient, in $\mathrm{T} / \mathrm{m}$, required to bend a cilium of radius $100 \mathrm{~nm}$ and length 7 microns (typical of epithelial cilia) through an angle of $10^{\circ}$.

\subsubsection{Materials design}

Furthermore, in the case of gradient-dominated bending we can develop a term analogous to the magnetoelastic ratio for field bending introduced in Section 4.2.3. To do so, we rewrite Equation (9) to solve for the bend angle $\varphi$ :

$$
\phi=\frac{M f}{2 E} \frac{L^{3}}{r^{2}} \nabla B
$$

The first term of this expression contains all of the material properties of the microactuator and is independent of geometrical considerations. As such, it serves as a figure of merit for the suitability of material for application in gradient-dominated magnetic microactuation applications. Magnetoelastic ratios of extant composite materials for gradient bending are reported in Table 1. 


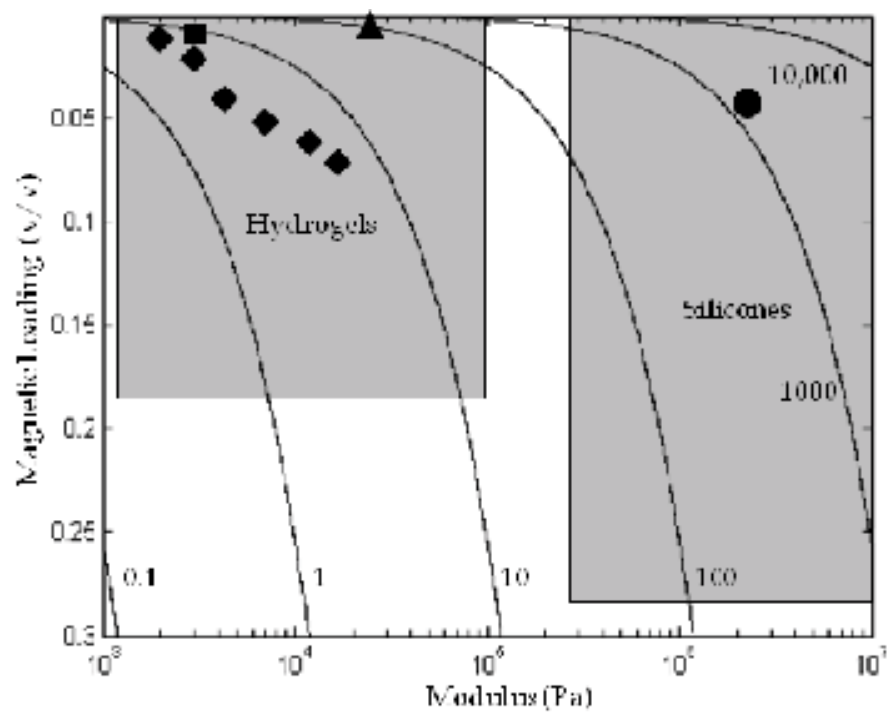

Fig. 6. The magnetic field gradient in tesla per meter $(\mathrm{T} / \mathrm{m})$ required to bend a 7-micron tall magnetic cilium of $100 \mathrm{~nm}$ radius through an angle of $10^{\circ}$. The cilium is loaded with maghemite nanoparticles, and is assumed to be magnetically saturated. Superimposed on the plot are instances of reported magnetoelastomers. Diamonds: maghemite / polyacrylamide (Galicia et al., 2003); Square: magnetite / polyvinyl alcohol (Barsi et al., 1996); Triangle: M-300 / polyvinyl alcohol (Collin et al., 2003); Circle: maghemite / poly(dimethyl siloxane) (Evans et al., 2007).

In Fig. 7, below, we plot the magnetoelastic ratios for both field bending and gradient bending for a maghemite / polyacrylamide magnetic elastomer reported by Galicia et al. Both curves show a clear optimization at a $4 \%$ nanoparticle loading; at higher nanoparticle concentrations, the increase in modulus due to the nanoparticle component outweighs the additional magnetization. Similarly, it is clear in Fig. 5 and Fig. 6 that the $f=0.04$ sample of this material is predicted to bend at the lowest values of magnetic field and field gradient, respectively.

\section{Actuation strategies}

\subsection{Introduction}

The biomimetic cilia in this discussion are designed to be actuated magnetically, and there are three possible means for producing a magnetic field: with a current-carrying wire, with a permanent magnet, and with an electromagnet. A current-carrying wire holds a certain amount of appeal, since such would provide a great degree of flexibility in producing a time-varying magnetic field. One can imagine an array of lithographed wires supplanted beneath the cilia array which might be individually addressed to produce a synchrony of temporally and spatially manipulated magnetic fields. While such an image is enticing, no one has yet achieved actuation of magnetic cilia with a current-carrying wire, although possibilities have been discussed in the literature (Fahrni, Prins, \& van IJzendoorn, 2009). We will discuss difficulties and possibilities for this sort of actuation below.

Permanent magnets, on the other hand, have been successfully used to actuate magnetic cilia arrays (Evans et al., 2007), and are attractive due to their relatively large fields and ease 


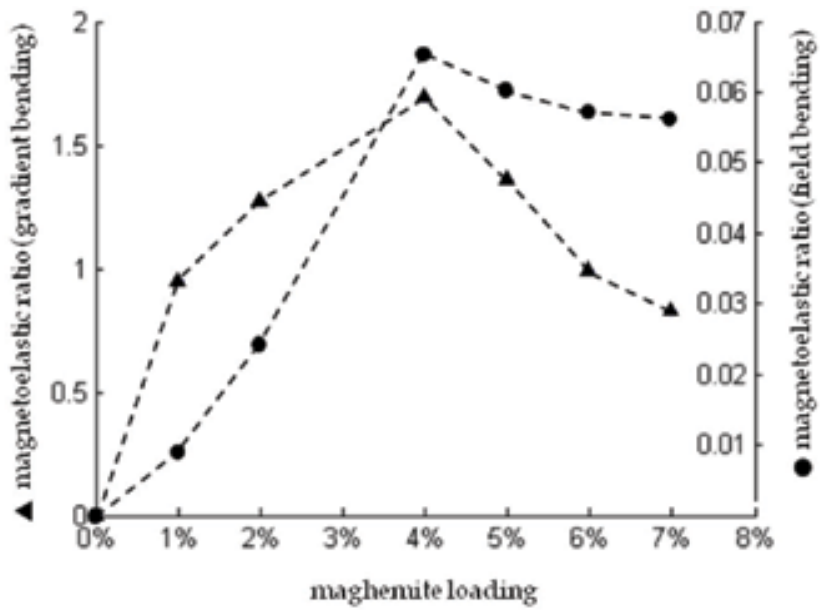

Fig. 7. Magnetoelastic ratios as a function of maghemite nanoparticle loading for a maghemite / polyacrylamide magnetic elastomer reported by (Galicia et al., 2003).

of implementation. A detailed discussion of actuation via permanent magnet will follow. Finally, electromagnets may be considered an extension of the prior two methods, combining the flexibility of the current-carrying wire with the strength of a permanent magnet. Excepting the case of superconductors, however, macroscopic wires or electromagnets can at best approach the field magnitudes generated by permanent magnets and will therefore not be considered in-depth in this work. To date no one has reported actuation with an electromagnet or electromagnet array, although such might be possible with an extant micromagnetic apparatus such as the 3DFM (Fisher et al., 2006).

\subsection{Field or gradient dominance}

Before we begin a discussion of these mechanisms, however, we first recognize that bending may occur in two distinct regimes - field-dominated or gradient-dominated - as previously described. To better understand the magnetic field geometries characteristic of each, we take the ratio of 'gradient' torque to 'field' torque, and find that

$$
R=\frac{2 \nabla B L}{M(B) \mu_{0} f}
$$

Here we have written the magnetization as function of magnetic field in order to make the role of the magnetic field magnitude explicit. Fig. 8 displays in the parameter space of applied field and field gradient contours on which the ratio $R$ is unity. Here we have selected a length $L$ of 7 microns and maghemite as the loading material. We have chosen three representative values of particle loading $f$, which cover the gamut of extant materials. The region to the upper-left of each $R=1$ contour is field-dominated; the region to the lowerright of each is gradient-dominated.

\subsection{Permanent magnets}

Perhaps the most straight-forward way to actuate a magnetic microstructure is by introducing a permanent magnet. Currently, rare earth magnets such as those constructed of neodymium-iron-boride (NIB) offer the largest fields of available magnets. With internal 


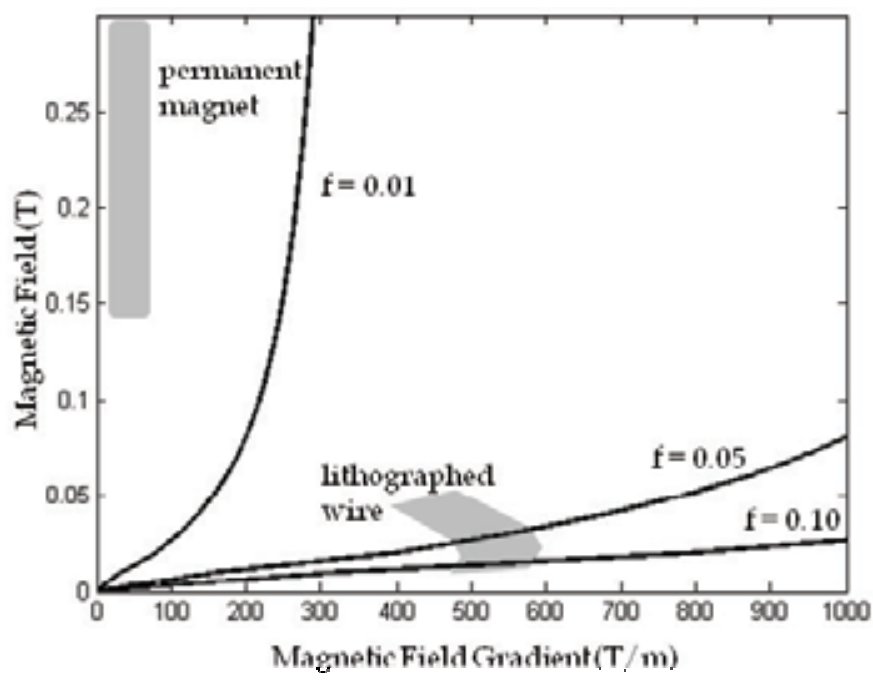

Fig. 8. Field-vs.-Gradient dominance for a cilium of length 7 microns. Lines represent $R=1$ contours, on which the torque due to the magentic field and torque due to the magnetic field gradient are equal. $\mathrm{R}=1$ lines are shown for different values of maghemite nanoparticle loading, $f$. Regions to the upper-left of each of these lines is field-dominated; regions to the lower-right are gradient-dominated. Shaded regions indicate regions of the parameter space accessible by permanent magnets and lithographed wires.

fields of approximately one tesla, the magnetic field available at the surface of such magnets is approximately 0.5 tesla. This is largely independent of the size of the magnet itself; however, the size of the magnet does determine how quickly the field decays with distance from the magnet - i.e. the gradient. From our own measurements, we find that the field strength at a reasonable working distance of 3-mm from various sizes of NIB magnets ranges from about $0.15-0.25$ tesla, and the magnetic field gradients at similar distances ranges from $30-60 \mathrm{~T} / \mathrm{m}$. Measurements were taken on 6-mm-thick cylindrical magnets with radii ranging from $25-50 \mathrm{~mm}$. This region is highlighted in Fig. 8; it is clear from this plot that magnetic actuation of biomimetic cilia with permanent magnets will generally be field-dominated. However, in certain circumstances the magnetic field gradient from a permanent magnet can be locally enhanced by affixing a sharp pole tip (Evans et al., 2007).

\subsection{Current-carrying wires}

The magnetic field and field gradient generated by a current-carrying wire is given by

$$
|B|=\frac{\mu_{0} I}{2 \pi r} ; \quad|\nabla B|=\frac{\mu_{0} I}{2 \pi r^{2}}
$$

where $I$ is the current through the wire and $r$ is the distance from its center. For the case of a single, macroscopic wire, both the field and field gradient generated are negligible in the context of magnetic actuation of biomimetic cilia. Constructions of multiple wires such as solenoids or Helmholtz coils certainly generate significantly larger magnetic fields; however these fields are similar in geometry and yet far weaker than those produced by permanent magnets, and will not be considered here. 
In the realm of the microscopic, however, one might imagine an array of lithographed wires on a substrate immediately beneath the cilia. In this case, we find that both the magnetic field and the magnetic field gradient may be large enough to achieve actuation. In considering the case of a lithographed wire, we will assume a gold wire of square cross section deposited on a silicon wafer. While the expressions above for magnetic field and magnetic field gradient produced by these wires are simple, the amount of current $I$ which such wires may carry is difficult to determine. In order to arrive at a reasonable estimate, we will assume that power is delivered to the wires via resistive heating and leaves the wire via conductive losses through the silicon substrate. In the steady-state these rates are equal, thus

$$
I^{2} R=\frac{A}{R_{T}} \Delta T
$$

where the expression on the right is Fourier's Conduction Law, in which $A$ is the contact area between the gold and the silicon, $R_{T}$ is the thermal resistance of the silicon, and $\Delta T$ is the difference in temperature between the hot wire and the cold bath below the silicon wafer. Due to the high thermal conductivity of the silicon, we will assume all conductive losses occur through the silicon and not the other three sides of the wire. In addition, we assume that the thermal resistance of the gold wire is negligible, which is valid as long as the wire is thin compared to the thickness of the silicon substrate. Rewriting the resistance in terms of the resistivity of the wire and its geometrical properties, we find that the steadystate current through the wire is given by

$$
I=\sqrt{\frac{d^{3}}{\rho R_{T}}\left(T_{H}-T_{C}\right)}
$$

where $d$ is the width of the square cross section of the gold wire and $\rho$ is the resistivity of the gold. Complicating matters considerably is the fact that both the resistivity of the gold and the thermal resistance of the silicon are temperature-dependent. In short, the resistivity of gold is linear with respect to temperature over a wide range ( $50 \leq T \leq 1000 \mathrm{~K}$ ) and is given by $\rho_{A u}(T)=8.7 \times 10^{-11} \mathrm{~T} \Omega m$ (Lide, 1994). The thermal resistance is given by integrating the reciprocal of the thermal conductivity of silicon through its depth; this thermal conductivity is also dependent on temperature, and is $k_{S i}(T)=6.83 \times 10^{5} \mathrm{~T}^{-1.45} \mathrm{Wm}^{-1} \mathrm{~K}^{-1}$ for temperatures greater than $30 \mathrm{~K}$ (Glassbrenner \& Slack, 1964). Assuming a constant thermal gradient through the silicon at steady-state and a minimal wafer thickness of $1 \mathrm{~mm}$, we can find the current through the wire as a function of $T_{H}$ and take the maximum. The resulting field and field gradient are plotted in Fig. 8 for wires ranging in size from 10 to 100 microns, with larger wires at the upper-left of the range and smaller wires at the lower-right. The field and gradient are sampled at a distance of 10 microns from the wire.

While no one has yet demonstrated actuation of biomimetic cilium with a current-carrying wire, the capability is enticing. However, certain considerations not yet presented must be taken into account. For example, the gradient bending calculations of Fig. 6 assume that the magnetic material is saturated; however, at the relatively low field magnitudes produced by lithographed current carrying wires, this is unlikely (see Fig. 1) and actuation will be significantly less than predicted. In addition, the direction of the gradient produced by a lithographed wire is unlikely to be optimal. Thoughtful design, however, and low-modulus, high-permeability materials may make such a system a reality. 


\section{Operation under a load}

\subsection{Introduction}

Whether biological or biomimetic, an actuator may often be called upon to carry a load - to pump a fluid or to transport a bacterial plaque - and it is to such load-bearing operation that we now turn our attention. In the case of biological cilia, we will show that each cilium operates with a reserve of excess power which may be called upon to support an additional load. After tapping this reserve, the cilium operates at the expense of reduced amplitude. Magnetic biomimetic cilia have no such reserve available, and yet we show in Section 6.3 that they may be designed to operate with a robustness similar to that of their biological peers. However, in order to compare the biological to the biomimetic, we must first develop an energy model for biological cilia. In this case, energy is expended in the form of elastic energy and work done against viscous drag forces, and energy is made available through the hydrolysis of ATP.

\subsection{Biological cilia}

\subsubsection{Elastic energy}

The elastic energy of a bent cilium is shown as the first term in Equation (3), where $R$ is the radius of curvature which in general is a function of distance along the length of the cilium. By assuming a constant curvature, we can rewrite the elastic energy in terms of the amplitude of the ciliary beat, $A$ :

$$
U_{E}=\frac{E I}{2 L^{2}} A^{2}
$$

The flexural rigidity, $E I$, of a biological cilium has been measured in three-point bending (Okuno \& Hiramoto, 1978) and stall-force experiments (Hill et al., 2010) to be approximately $7 \times 10^{-22} \mathrm{Nm}^{2}$. A typical airway epithelial cilia is about $7 \mu \mathrm{m}$ long and beats with a similar amplitude, yielding an elastic energy at full extension of about $1 \times 10^{-16} \mathrm{~J}$. This is in good agreement with values given for cilia of sabellaria and mytilus (Sleigh \& Holwill, 1969).

\subsubsection{Work done by viscous drag}

The energy lost to viscous drag during the effective stroke of a cilium has previously been shown to be (Sleigh \& Holwill, 1969)

$$
U_{D}=\frac{1}{3} C_{N} \omega^{2} L^{3} t ; \quad C_{N}=\frac{4 \pi \eta}{\ln \left(\frac{2 L}{d}\right)-\frac{1}{2}}
$$

where $C_{N}$ is the drag coefficient per unit length for flow normal to a cylinder.

For a 7-micron cilium of diameter $200 \mathrm{~nm}$ actuating in water $\left(\eta=8.9 \times 10^{-4} \mathrm{~Pa} \mathrm{~s}\right)$, the drag coefficient is $0.0015 \mathrm{~Pa}$ s. The time elapsed during the effective stroke has been shown to be about $33 \mathrm{~ms}$, and the drag energy is therefore approximately $2 \times 10^{-17} \mathrm{~J}$.

It is interesting to note that the energy lost to drag is an order of magnitude less than the elastic energy of a biological cilium. This tells us that the majority of the energy expenditure in a ciliary system goes toward bending the structure itself, rather than doing work on the fluid. 


\subsubsection{Available chemical energy}

Energy for a biological cilium is provided by the hydrolysis of ATP and converted into mechanical energy by dynein, a molecular motor. To estimate the total amount of chemical energy available to a biological cilium, we may begin with the assumption that the energy available from a single dynein during the effective stroke of the cilium is given by $U_{D}=e_{A T P} E_{A T P} k_{A T P} t_{B}$, where $E_{A T P}=1 \times 10^{-19} \mathrm{~J}$ is the chemical energy available from the hydrolysis of one ATP, $e_{A T P}$ is the efficiency with which dynein is able to convert this chemical energy into mechanical energy, $k_{\text {ATP }}$ is the rate of hydrolysis of ATP per dynein, and $t_{B}$ is the time elapsed during one stroke. Values of $k_{A T P}$ vary widely in the literature, but we will take $120 \mathrm{~s}^{-1}$ as an upper bound (Kon, Nishiura, Ohkura, Toyoshima, \& Sutoh, 2004), and the time elapsed during the effective stroke of a cilium is approximately $33 \mathrm{~ms}$. To arrive at a reasonable value of the conversion efficiency $e_{A T P}$, we note that given a stall force for a single dynein of $6 \mathrm{pN}$ (Shingyoji, Higuchi, Yoshimura, Katayama, \& Yanagida, 1998) and a step size of $8 \mathrm{~nm}$ (Holwill, 2001), the mechanical energy of a single dynein step cannot be more than the product of the two, or $4.8 \times 10^{-20} \mathrm{~J}$. Since this is just less than half of the chemical energy made available by the hydrolysis of ATP, the conversion efficiency $e_{\text {ATP }}$ can be at most 0.5 .

The number of dynein involved in the effective stroke of a cilium is a topic of active debate. However, most sources cite approximately 2000 outer arm dynein engaged in a 7-micron cilium (Holwill, 2001; Salathe, 2007), or a density of $2.9 \times 10^{8}$ dynein per meter. We can further refine this number by noting that since the dynein in a cilium are distributed among the nine microtubule doublets surrounding the center, not all are oriented to apply a maximum force toward the effective stroke. By geometrical considerations, we can estimate that $64 \%$ of the dynein are effective in any particular actuation ${ }^{1}$. The effective number of dynein involved in the ciliary beat is then $N_{D}=R_{D} n_{D} L$, where $R_{D}=0.64$ is the fraction of dynein which are effective, $n_{D}=2.9 \times 10^{8}$ is the number of dynein per meter, and $L=7$ microns is the length of the cilium. The total mechanical energy available to a cilium is then the number of dynein multiplied by the energy per dynein, or

$$
U_{M}=R_{D} n_{D} L e_{A T P} E_{A T P} k_{A T P} t_{B}
$$

Using the numbers previously quoted, this total amount of chemical energy which is available for conversion to mechanical energy is about $2.6 \times 10^{-16} \mathrm{~J}$, which is significantly greater than the sum of elastic and drag energies for a biological cilium.

\subsubsection{Beat amplitude as a function of applied load}

It is interesting to note that a biological cilium has available approximately $2.6 \times 10^{-16} \mathrm{~J}$ of useable chemical energy, yet expends only $1.2 \times 10^{-16} \mathrm{~J}$ in a typical effective stroke. We speculate that this energy surplus is an adaptation which will allow a cilium to apply

\footnotetext{
${ }^{1}$ Given that the microtubule doublets are spaced every $40^{\circ}$ (or $2 \Pi / 9$ radians) around the axis of the cilium and their component in the direction of the effective stroke is proportional to the cosine of their angular position, the effective number of dynein can be written as $n_{D}=\frac{n_{D}}{9} \sum_{n=0}^{8}\left|\cos \left(\frac{2 \pi n}{9}\right)\right|=0.64 n_{D}$
} 
additional force in the case of an increased load. Such a load may occur due to bacterial infection or changes in the viscoelasticity of the mucus due to illness. In the case of an increased loading at the tip of the cilium, it would be reasonable to expect that the cilium is able to apply all of its available chemical energy to elastic energy and work done on the increased load: $U_{M}=U_{E}+W_{L}$. Here we have considered drag forces to be negligible. The work done on the load is simply the force exerted by the load multiplied by the amplitude of the ciliary beat, or $W_{L}=F_{L} A$, and so by incorporating the elastic energy term from Equation (3), we can show that the amplitude of the effective stroke under an applied loading $F_{L}$ is given by

$$
A=\frac{L^{3}}{E I}\left[\sqrt{F_{L}^{2}+\frac{2}{L^{3}} E I U_{M}}-F_{L}\right]
$$

where $U_{M}$ is as described in Equation (17). The expected amplitude as a function of applied load is plotted in Fig. 9. The curved line (continued in dashes at the upper left) indicates the expected amplitude assuming all available chemical energy is used. Under normal conditions, however, a cilium works below capacity. This is indicated by the flat line at 7 microns. This reserve energy is fully expended at a load of about $30 \mathrm{pN}$, and so any additional efforts come at the expense of elastic energy and, hence, amplitude. The untapped chemical energy available an unloaded beat is given by the area of the grey box in Fig. 9 .

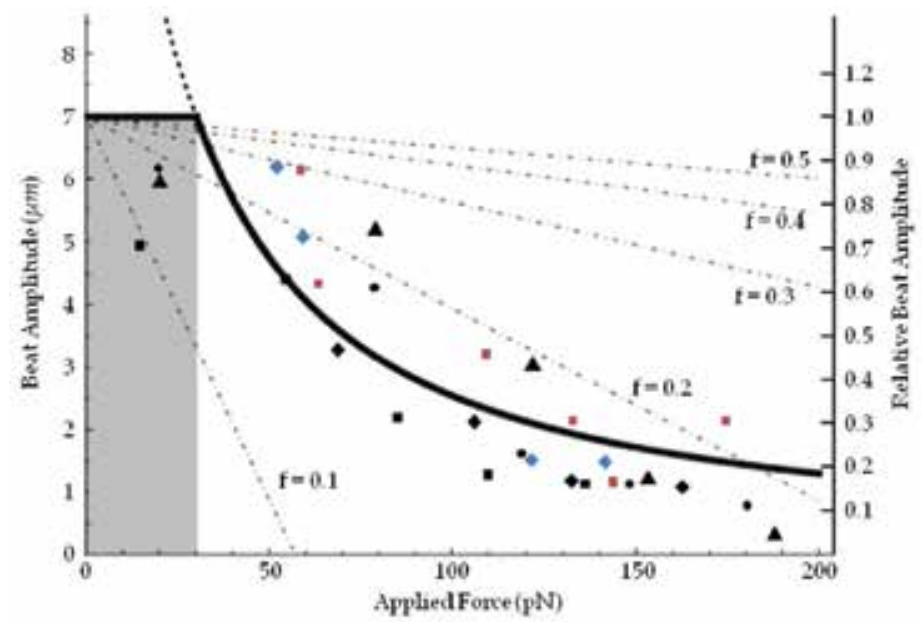

Fig. 9. Relative ciliary beat amplitude as a function of applied force at the tip of the cilium. Glyphs represent relative beat amplitude of human lung epithelial cilia taken from Hill (Hill et al., 2010). The dark black line indicates the beat amplitude of a biological cilium under an applied load predicted by Equation (18). Under $30 \mathrm{pN}$, the cilium has an additional energy reserve and beats as less than full capacity. This reserve (indicated by the area of the shaded box) is exhausted at about $30 \mathrm{pN}$. Dot-dashed lines represent relative beat amplitude of magnetic biomimetic cilia under applied load for various values of maghemite nanoparticle loading, $f$.

This prediction is in good agreement with measurements by Hill of the relative beat amplitude of human lung epithelial cilia under an applied load (Hill et al., 2010). This data is reproduced in Fig. 9 above, in which each set of glyphs represents a single cilium. Each data 
set is normalized by the unloaded amplitude and represented in the original work as a relative beat amplitude (right axis).

\subsection{Biomimetic cilia}

In the case of field-dominated bending, we can derive an expression similar to Equation (18) for biomimetic cilia by adding the torque due to an applied load at the tip of the cilium, $\tau=$ $F_{L} L$, to Equation (4). Solving for the amplitude in the case of zero net torque and normalizing with respect to unloaded beat amplitude, we find

$$
A=1-\frac{F_{L}}{\frac{\pi}{4} \mu_{0} M^{2} f^{2} r^{2}}
$$

Thus, the relative decrease in amplitude with applied load is independent of the modulus of the material and depends most significantly on nanoparticle loading. Relative beat amplitudes for biomimetic cilia of biological proportions $(r=100 \mathrm{~nm})$ and loaded with various volume fractions $f$ of maghemite are represented by the dashed lines in Fig. 9. It is clear that higher loadings result in more robust cilia, and loadings as low as $f=0.2$ match the load-handling capabilities of biological cilia.

\section{Conclusion}

We have described in this work the current state in the development of magnetically acutated biomimetic cilia, including promising candidate materials and proven fabrication techniques. In addition, we have described a predictive analytical model to aid in the design of field-generation mechanisms, materials for magnetic actuation and actuator geometry, and provide guidelines for design within the relevant parameter space. Finally, we have compared the abilities of these biomimetic cilia with that of their biological peers. While we are far from replicating the intricacies of biological cilia, the framework presented in this work will facilitate advances in the vibrant future of this field.

\section{References}

Alexeev, A., Yeomans, J. M., \& Balazs, A. C. (2008). Designing Synthetic, Pumping Cilia That Switch the Flow Direction in Microchannels. Langmuir, 24(21), 12102-12106.

Antunes, M. B., \& Cohen, N. A. (2007). Mucociliary clearance - a critical upper airway host defense mechanism and methods of assessment. Current Opinion in Allergy and Clinical Immunology, 7(1), 5-10.

Barsi, L., Buki, A., Szabo, D., \& Zrinyi, M. (1996). Gels with Magnetic Properties. In Progress in Colloid and Polymer Science (Vol. 102, pp. 57-63). Berlin: Springer.

Bee, A., Massart, R., \& Neveu, S. (1995). Synthesis of Very Fine Maghemite Particles. Journal of Magnetism and Magnetic Materials, 149(1-2), 6-9.

Berdichevsky, Y., Khandurina, J., Guttman, A., \& Lo, Y. H. (2004). UV/ozone modification of poly(dimethylsiloxane) microfluidic channels. Sensors and Actuators B-Chemical, 97(2-3), 402-408.

Boucher, R. C. (2007). Cystic fibrosis: a disease of vulnerability to airway surface dehydration. Trends in Molecular Medicine, 13(6), 231-240. 
Brumlik, C. J., Menon, V. P., \& Martin, C. R. (1994). Template Synthesis of Metal Microtubule Ensembles Utilizing Chemical, Electrochemical, and Vacuum Deposition Techniques. Journal of Materials Research, 9(5), 1174-1183.

Cardenas-Rodriguez, M., \& Badano, J. L. (2009). Ciliary Biology: Understanding the Cellular and Genetic Basis of Human Cillopathies. American Journal of Medical Genetics Part C-Seminars in Medical Genetics, 151C(4), 263-280.

Cartwright, J. H. E., Piro, N., Piro, O., \& Tuval, I. (2008). Fluid Dynamics of Nodal Flow and Left-Right Patterning in Development. Developmental Dynamics, 237(12), 3477-3490.

Caykara, T., Yoruk, D., \& Demirci, S. (2009). Preparation and Characterization of Poly(Ntert-butylacrylamide-co-acrylamide) Ferrogel. Journal of Applied Polymer Science, $112(2), 800-804$.

Cebers, A. (2002). Dynamics of an elongated magnetic droplet in a rotating field. Physical Review E, 66(6).

Chik, H., \& Xu, J. M. (2004). Nanometric superlattices: non-lithographic fabrication, materials, and prospects. Materials Science E Engineering R-Reports, 43(4), 103-138.

Collin, D., Auernhammer, G. K., Gavat, O., Martinoty, P., \& Brand, H. R. (2003). Frozen-in magnetic order in uniaxial magnetic gels: Preparation and physical properties. Macromolecular Rapid Communications, 24(12), 737-741.

Downton, M. T., \& Stark, H. (2009). Beating kinematics of magnetically actuated cilia. Epl, 85(4).

Dubois, E., Cabuil, V., Boue, F., \& Perzynski, R. (1999). Structural analogy between aqueous and oily magnetic fluids. Journal of Chemical Physics, 111(15), 7147-7160.

Dunlop, D. J., \& Ozdemir, O. (1997). Rock Magnetism: Fundamentals and Frontiers (1st ed.). Cambridge: Cambridge University Press.

Efimenko, K., Crowe, J. A., Manias, E., Schwark, D. W., Fischer, D. A., \& Genzer, J. (2005). Rapid formation of soft hydrophilic silicone elastomer surfaces. Polymer, 46(22), 9329-9341.

Efimenko, K., Wallace, W., \& Genzer, J. (2002). Surface Modification of Sylgard-184 Poly(dimetyl siloxane) Networks by Ultraviolet and Ultraviolet/Ozone Treatment. Journal of Colloid and Interface Science, 254(2), 306-315.

Evans, B. A., Shields, A. R., Carroll, R. L., Washburn, S., Falvo, M. R., \& Superfine, R. (2007). Magnetically actuated nanorod arrays as biomimetic cilia. Nano Letters, 7(5), 14281434.

Fahrni, F., Prins, M. W. J., \& van IJzendoorn, L. J. (2009). Magnetization and actuation of polymeric microstructures with magnetic nanoparticles for application in microfluidics. Journal of Magnetism and Magnetic Materials, 321(12), 1843-1850.

Fahrni, F., Prins, M. W. J., \& van Ijzendoorn, L. J. (2009). Micro-fluidic actuation using magnetic artificial cilia. Lab on a Chip, 9(23), 3413-3421.

Ferain, E., \& Legras, R. (1997). Characterisation of nanoporous particle track etched membrane. Nuclear Instruments $\mathcal{E}$ Methods in Physics Research Section B-Beam Interactions with Materials and Atoms, 131(1-4), 97-102.

Fischer, B. E., \& Spohr, R. (1983). Production and Use of Nuclear Tracks - Imprinting Structure on Solids. Reviews of Modern Physics, 55(4), 907-948.

Fisher, J. K., Cribb, J., Desai, K. V., Vicci, L., Wilde, B., Keller, K., et al. (2006). Thin-foil magnetic force system for high-numerical-aperture microscopy. Review of Scientific Instruments, 77(2), 9.

Furst, E. M., Suzuki, C., Fermigier, M., \& Gast, A. P. (1998). Permanently linked monodisperse paramagnetic chains. Langmuir, 14(26), 7334-7336. 
Galicia, J. A., Sandre, O., Cousin, F., Guemghar, D., Menager, C., \& Cabuil, V. (2003). Designing magnetic composite materials using aqueous magnetic fluids. Journal of Physics-Condensed Matter, 15(15), S1379-S1402.

Gauger, E. M., Downton, M. T., \& Stark, H. (2009). Fluid transport at low Reynolds number with magnetically actuated artificial cilia. European Physical Journal E, 28(2), 231-242.

Glassbrenner, C. J., \& Slack, G. A. (1964). THERMAL CONDUCTIVITY OF SILICON + GERMANIUM FROM 3 DEGREES K TO MELTING POINT. Physical Review aGeneral Physics, 134(4A), 1058-\&.

Hill, D. B., Swaminathan, V., Estes, A., Cribb, J., O'Brien, E. T., Davis, C. W., et al. (2010). Force Generation and Dynamics of Individual Cilia under External Loading. Biophysical Journal, 98(1), 57-66.

Holwill, M. E. J. (2001). Dynein motor activity during ciliary beating. In M. Salathe (Ed.), Cilia and Mucus: From Development to Respiratory Disease (pp. 19-25). New York: Marcel Dekker.

Huczko, A. (2000). Template-based synthesis of nanomaterials. Applied Physics a-Materials Science E Processing, 70(4), 365-376.

Hulteen, J. C., \& Martin, C. R. (1997). A general template-based method for the preparation of nanomaterials. Journal of Materials Chemistry, 7(7), 1075-1087.

Jolly, M. R., Carlson, J. D., Munoz, B. C., \& Bullions, T. A. (1996). The magnetoviscoelastic response of elastomer composites consisting of ferrous particles embedded in a polymer matrix. Journal of Intelligent Material Systems and Structures, 7(6), 613-622.

Kon, T., Nishiura, M., Ohkura, R., Toyoshima, Y. Y., \& Sutoh, K. (2004). Distinct functions of nucleotide-binding/hydrolysis sites in the four AAA modules of cytoplasmic dynein. Biochemistry, 43(35), 11266-11274.

Landau, L. D., \& Lifshitz, E. M. (1986). Theory of Elasticity, 3rd Edition. New York: Pergamon Press.

Lide, D. R. (1994). Handbook of Chemistry and Physics, 75th ed.: Lewis Publishing.

Lin, C. L., Chiu, W. Y., \& Don, T. M. (2006). Superparamagnetic thermoresponsive composite latex via W/O miniemulsion polymerization. Journal of Applied Polymer Science, 100(5), 3987-3996.

Lin, H., Watanabe, Y., Kimura, M., Hanabusa, K., \& Shirai, H. (2003). Preparation of magnetic poly(vinyl alcohol) (PVA) materials by in situ synthesis of magnetite in a PVA matrix. Journal of Applied Polymer Science, 87(8), 1239-1247.

Makamba, H., Kim, J. H., Lim, K., Park, N., \& Hahn, J. H. (2003). Surface modification of poly(dimethylsiloxane) microchannels. Electrophoresis, 24(21), 3607-3619.

Massart, R. (1981). Preparation of Aqueous Magnetic Liquids in Alkaline and Acidic Media. IEEE Transactions on Magnetics, 17(2), 1247-1248.

Massart, R., Dubois, E., Cabuil, V., \& Hasmonay, E. (1995). Preparation and Properties of Monodisperse Magnetic Fluids. Journal of Magnetism and Magnetic Materials, 149(12), 1-5.

Mathur, A. M., Moorjani, S. K., \& Scranton, A. B. (1996). Methods for synthesis of hydrogel networks: A review. Journal of Macromolecular Science-Reviews in Macromolecular Chemistry and Physics, C36(2), 405-430.

Mayer, C. R., Cabuil, V., Lalot, T., \& Thouvenot, R. (2000). Magnetic nanoparticles trapped in $\mathrm{pH} 7$ hydrogels as a tool to characterize the properties of the polymeric network. Advanced Materials, 12(6), 417-+.

McGary, P. D., Tan, L. W., Zou, J., Stadler, B. J. H., Downey, P. R., \& Flatau, A. B. (2006). Magnetic nanowires for acoustic sensors (invited). Journal of Applied Physics, 99(8). 
Mefford, O. T., Vadala, M. L., Goff, J. D., Carroll, M. R. J., Mejia-Ariza, R., Caba, B. L., et al. (2008). Stability of polydimethylsiloxane-magnetite nanoparticle dispersions against flocculation: Interparticle interactions of polydisperse materials. Langmuir, 24(9), 5060-5069.

Mitsumata, T., Ikeda, K., Gong, J. P., Osada, Y., Szabo, D., \& Zrinyi, M. (1999). Magnetism and compressive modulus of magnetic fluid containing gels. Journal of Applied Physics, 85(12), 8451-8455.

Morozov, K. I., Engel, A., \& Lebedev, A. V. (2002). Shape transformations in rotating ferrofluid drops. Europhysics Letters, 58(2), 229-235.

Nicastro, D. (2009). Cryo-Electron Microscope Tomography to Study Axonemal Organization. In Cilia: Structure and Motility (Vol. 91, pp. 1-39).

Okada, Y., \& Hirokawa, N. (2009). Observation of Nodal Cilia Movement and Measurement of Nodal Flow. In Cilia: Structure and Motility (Vol. 91, pp. 265-285).

Okuno, M., \& Hiramoto, Y. (1978). Direct Measurements of the Stiffness of Echinoderm Sperm Flagella. Journal of Experimental Biology, 79, 235-243.

Pokroy, B., Epstein, A. K., Persson-Gulda, M. C. M., \& Aizenberg, J. (2009). Fabrication of Bioinspired Actuated Nanostructures with Arbitrary Geometry and Stiffness. Advanced Materials, 21(4), 463-+.

Qin, J., Asempah, I., Laurent, S., Fornara, A., Muller, R. N., \& Muhammed, M. (2009). Injectable Superparamagnetic Ferrogels for Controlled Release of Hydrophobic Drugs. Advanced Materials, 21(13), 1354-1357.

Resendiz-Hernandez, P. J., Rodriguez-Fernandez, O. S., \& Garcia-Cerda, L. A. (2008). Synthesis of poly(vinyl alcohol)-magnetite ferrogel obtained by freezing-thawing technique.

Roca-Cusachs, P., Rico, F., Martinez, E., Toset, J., Farre, R., \& Navajas, D. (2005). Stability of microfabricated high aspect ratio structures in poly(dimethylsiloxane). Langmuir, 21(12), 5542-5548.

Rutnakornpituk, M., Thompson, M. S., Harris, L. A., Farmer, K. E., Esker, A. R., Riffle, J. S., et al. (2002). Formation of cobalt nanoparticle dispersions in the presence of polysiloxane block copolymers. Polymer, 43(8), 2337-2348.

Salathe, M. (2007). Regulation of mammalian ciliary beating. Annual Review of Physiology, 69, 401-422.

Saslawski, O., Weingarten, C., Benoit, J. P., \& Couvreur, P. (1988). MAGNETICALLY RESPONSIVE MICROSPHERES FOR THE PULSED DELIVERY OF INSULIN. Life Sciences, 42(16), 1521-1528.

Satir, P., \& Christensen, S. T. (2007). Overview of structure and function of mammalian cilia. Annual Review of Physiology, 69, 377-400.

Satir, P., Mitchell, D. R., \& Jekely, G. (2008). HOW DID THE CILIUM EVOLVE? In Ciliary Function in Mammalian Development (Vol. 85, pp. 63-82).

Schonenberger, C., vanderZande, B. M. I., Fokkink, L. G. J., Henny, M., Schmid, C., Kruger, M., et al. (1997). Template synthesis of nanowires in porous polycarbonate membranes: Electrochemistry and morphology. Journal of Physical Chemistry B, 101(28), 5497-5505.

Shcherbakov, V. P., \& Winklhofer, M. (2004). Bending of magnetic filaments under a magnetic field. Physical Review E, 70(6).

Shingyoji, C., Higuchi, H., Yoshimura, M., Katayama, E., \& Yanagida, T. (1998). Dynein arms are oscillating force generators. Nature, 393(6686), 711-714.

Sidorenko, A., Krupenkin, T., Taylor, A., Fratzl, P., \& Aizenberg, J. (2007). Reversible switching of hydrogel-actuated nanostructures into complex micropatterns. Science, 315(5811), 487-490. 
Singh, H., Laibinis, P. E., \& Hatton, T. A. (2005). Synthesis of flexible magnetic nanowires of permanently linked core-shell magnetic beads tethered to a glass surface patterned by microcontact printing. Nano Letters, 5(11), 2149-2154.

Sleigh, M. A. (1962). The Biology of Cilia and Flagella.

Sleigh, M. A., \& Holwill, M. E. J. (1969). Energetics of Ciliary Movement in Sabellaria and Mytilus. Journal of Experimental Biology, 50, 733-743.

Smith, D. J., Gaffney, E. A., \& Blake, J. R. (2009). Mathematical modelling of cilia-driven transport of biological fluids. Proceedings of the Royal Society a-Mathematical Physical and Engineering Sciences, 465(2108), 2417-2439.

Stevenson, J. P., Rutnakornpituk, M., Vadala, M., Esker, A. R., Charles, S. W., Wells, S., et al. (2001). Magnetic cobalt dispersions in poly(dimethylsiloxane) fluids. Journal of Magnetism and Magnetic Materials, 225(1-2), 47-58.

Stoner, E. C., Wohlfarth, E. P. (1947). A Mechanism of Magnetic Hysteresis in Heterogeneous Alloys. Phil. Trans. of the Royal Society of London, A240, 559-642.

Sutherland, M. J., \& Ware, S. M. (2009). Disorders of Left-Right Asymmetry: Heterotaxy and Situs Inversus. American Journal of Medical Genetics Part C-Seminars in Medical Genetics, 151C(4), 307-317.

Szabo, D., Czako-Nagy, I., Zrinyi, M., \& Vertes, A. (2000). Magnetic and Mossbauer studies of magnetite-loaded polyvinyl alcohol hydrogels. Journal of Colloid and Interface Science, 221(2), 166-172.

Timonen, J. V. I., Johans, C., Kontturi, K., Walther, A., Ikkala, O., \& Ras, R. H. A. (2010). A Facile Template-Free Approach to Magnetodriven, Multifunctional Artificial Cilia. Acs Applied Materials \& Interfaces, 2(8), 2226-2230.

van Ewijk, G. A., Vroege, G. J., \& Philipse, A. P. (1999). Convenient preparation methods for magnetic colloids. Journal of Magnetism and Magnetic Materials, 201, 31-33.

Varga, Z., Feher, J., Filipcsei, G., \& Zrinyi, M. (2003). Smart nanocomposite polymer gels.

Vilfan, M., Potocnik, A., Kavcic, B., Osterman, N., Poberaj, I., Vilfan, A., et al. (2010). Selfassembled artificial cilia. Proceedings of the National Academy of Sciences of the United States of America, 107(5), 1844-1847.

Wilson, K. S., Goff, J. D., Riffle, J. S., Harris, L. A., \& St Pierre, T. G. (2005). Polydimethylsiloxane-magnetite nanoparticle complexes and dispersions in polysiloxane carrier fluids. Polymers for Advanced Technologies, 16(2-3), 200-211.

Wormuth, K. (2001). Superparamagnetic latex via inverse emulsion polymerization. Journal of Colloid and Interface Science, 241(2), 366-377.

Xulu, P. M., Filipcsei, G., \& Zrinyi, M. (2000). Preparation and responsive properties of magnetically soft poly(N-isopropylacrylamide) gels. Macromolecules, 33(5), 17161719.

Zhang, Y., Lo, C. W., Taylor, J. A., \& Yang, S. (2006). Replica molding of high-aspect-ratio polymeric nanopillar arrays with high fidelity. Langmuir, 22(20), 8595-8601.

Zrinyi, M., Barsi, L., \& Buki, A. (1997). Ferrogel: a new magneto-controlled elastic medium. Polymer Gels and Networks, 5(5), 415-427. 


\title{
Monitoring the Intertidal Environment with Biomimetic Devices
}

\author{
Fernando P. Lima ${ }^{1,2}$, Nicholas P. Burnett ${ }^{1}$, Brian Helmuth ${ }^{1}$, Nicole Kish', \\ Kyle Aveni-Deforge ${ }^{1}$ and David S. Wethey ${ }^{1}$ \\ ${ }^{1}$ Department of Biological Sciences, 715 Sumter Street \\ University of South Carolina, Columbia, South Carolina 29208 \\ ${ }^{2}$ CIBIO, Centro de Investigação em Biodiversidade e Recursos Genéticos \\ Campus Agrário de Vairão, 4485-661 Vairão \\ 1 United States of America \\ ${ }^{2}$ Portugal
}

\section{Introduction}

Worldwide, the narrow bands of habitat between the tidemarks on coastal shores are inhabited by a variety of marine species which periodically have to cope with stressful terrestrial conditions during low tide (Raffaelli \& Hawkins, 1996; Denny \& Wethey, 2000; Harley, 2008). The interplay between biotic and abiotic factors acting over such small spatial scales results in outstandingly condensed and diverse communities, which are regarded as ideal natural models for studying the coupled role of physical and biological factors in determining the abundance and distribution of organisms (Raffaelli \& Hawkins, 1996).

Most intertidal invertebrates and algae are sessile or slow moving and cannot prevent being exposed to extreme stressful events, and thus are recognized as sensitive indicators of the effects of climate variability and climate change (Helmuth et al., 2006 and references within). Subsequently, temperature-driven mass mortalities of intertidal organisms such as limpets, mussels and barnacles have been observed in the field after exposure to periods of acute thermal stress (e.g., Lewis, 1954; Connell, 1961a; Crisp, 1964; Harley, 2008; Jones et al., 2010). Importantly, molecular and physiology studies have also recently shown the importance of sub-lethal stresses in shaping the distribution of intertidal species (e.g., Somero, 2002; Dahlhoff, 2004; Sorte \& Hofmann, 2004; Helmuth et al., 2005).

In addition to clines in thermal stress, the tidal cycle also generates steep gradients in light, oxygen and nutrient availability, wave force, salinity and desiccation. These abiotic factors have long been identified as drivers in the physiology and ecology of intertidal animals and plants (Broekhuysen, 1941; Smaldon \& Duffus, 1984; Denny \& Wethey, 2000; Santini et al., 2001; Firth \& Williams, 2009). Vertical clines in the strength of stressors, determined by the timing and duration of emersion/submersion (Foster, 1971) were firstly regarded as the main causative factors for the ubiquitous vertical substitution of species observed at scales of a few meters on rocky shores (Stephenson \& Stephenson, 1949; Lewis, 1964). Soon after, results from experimental ecology began to demonstrate the importance of biotic, selective pressures related to competition, predation, grazing, and recruitment in setting zonation 
patterns (Connell, 1961b; Hawkins et al., 1992). Connell (1972) suggested that the lower distributional limits of intertidal organisms were frequently set by biological interactions, while the upper limits were often set by desiccation and thermal factors. Later on, some authors showed that the strengths of some biotic interactions were themselves influenced by gradients in abiotic stress (Hodkinson, 1999; Wethey, 2002; Pincebourde et al., 2008; Yamane \& Gilman, 2009).

\section{Monitoring the environment at the scale of the organisms}

Recent advances in remote sensing and information technology, allied to the creation of huge data repositories, have provided the large volumes of environmental data needed to feed bio-climatic or process-based models, and thus have fueled numerous large-scale studies relating biogeographic patterns of intertidal organisms with changes in climate (e.g., Lima et al., 2007a; Lima et al., 2007b; Wethey \& Woodin, 2008; Berke et al., 2010; Jones, et al., 2010). It has become apparent, however, that these large-scale studies may be missing key environmental features only observed at the scale of the organisms (Helmuth et al., 2010). For example, when intertidal organisms are submerged, their body temperatures are in equilibrium with the surrounding water, but during aerial exposure other factors such as air temperature, wind speed, solar radiation and relative humidity come into play, interacting with the physical characteristics of the organisms such as their thermal inertia (Spotila et al., 1973), shape and color (Helmuth, 1999; Denny et al., 2006; Gilman et al., 2006) to determine the organism's body temperature. As a result, daily variations in body temperature can exceed $20^{\circ} \mathrm{C}$ (Helmuth \& Hofmann, 2001; Helmuth, 2002; Helmuth, et al., 2010), a range that is far greater than the yearly variation of coastal sea temperature at most locations. Daily variations in temperature may also be radically different between individuals just a few meters apart but located in surfaces facing different directions (Helmuth, 1998; 2002; Wethey, 2002; Helmuth, et al., 2005; Harley, 2008; Lima \& Wethey, 2009), and can depart significantly from measurements of air and surface temperature. Topography interacts with daily and seasonal variation in the incidence of sunlight, tide and weather, modifying heat exchanges between organisms and the environment, leading to large-scale geographic mosaics of thermal and desiccation stress (Gilman, et al., 2006; Helmuth, et al., 2006). Such topographic variation in stress has dramatic consequences for the distribution of species and for the intensity of their interactions (Wethey, 1984; 2002). Local, small-scale topography also strongly influences wave exposure. Wave forces are one of the major sources of natural disturbance in rocky shores, by directly creating patchiness by dislodging organisms (Sousa, 1984), by underpinning thermal regimes of organisms, by inducing behavioral responses that facilitate body temperature cooling (Fitzhenry et al., 2004), or by altering the timing of submersion (Harley \& Helmuth, 2003).

Thus, although it is clear that environmental data at the scale of the organisms should be explicitly used when describing, modeling and predicting biogeographic and ecological changes within these ecosystems, this requirement has been hindered by the lack of costeffective, self-contained instruments that can measure physiologically relevant aspects of the environment at the scale of the organisms. Here we describe four types of biomimetic sensors/loggers, specifically designed to capture different aspects of the coastal environment. These devices can be used to measure and log temperature, water loss (desiccation) and wave forces in the intertidal, as perceived by intertidal organisms. 


\section{Biomimetic temperature data loggers: robomussels and robolimpets}

In the intertidal environment, the most important abiotic determinant of organismal performance and distribution from micro to macroscales is indubitably temperature (Southward, 1958; Crisp, 1964; Lewis, 1964; Southward, 1995; Denny \& Wethey, 2000; Helmuth \& Hofmann, 2001; Gilman, et al., 2006; Wethey \& Woodin, 2008).

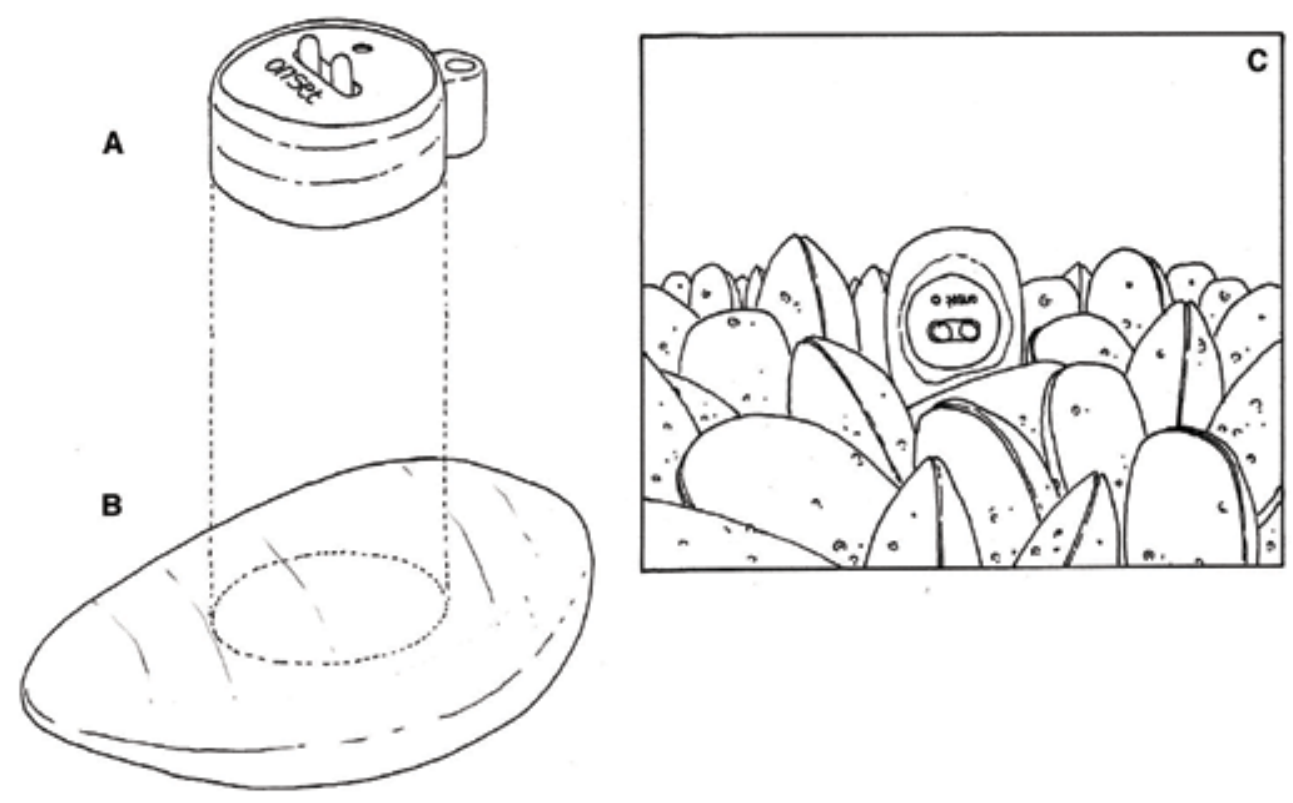

Fig. 1. Temperature logger mimicking a Mytilus californianus (robomussel). A: Onset TidbiT logger, B: Evercoat Everfix epoxy cast of a M. californianus, C: representation of a robomussel deployed in a mussel bed in the field.

Until recently, measuring body temperatures in the intertidal zone was inefficient or even impracticable at large spatial or temporal scales, as temperature loggers were too expensive, large, and required frequent servicing. The harshness of the intertidal zone, associated with rapidly varying temperatures, tremendous wave forces and hard substrata, was also problematic, often causing the loss of sensors and data loggers. Recent advances in sensor miniaturization resulted in a series of commercially-available data loggers that were rapidly adopted by intertidal ecologists and physiologists. Unfortunately, little consideration was given to what these instruments were actually measuring. As is true for organisms, the color, morphology, and mass of a temperature logger can significantly affect the temperature that it records. As a result, without carefully considering the characteristics of the instrument being deployed, a datalogger may easily record temperatures that have very little to do with the organism of interest. Instead, one must use biomimetic sensorsinstruments specifically matched to the physical characteristics of the organism, in this case case to the thermal characteristics. To overcome these problems, two types of biomimetic sensors/loggers have been developed: robomussels (Helmuth \& Hofmann, 2001; Fitzhenry, et al., 2004) and robolimpets (Lima \& Wethey, 2009). These are self-contained, rugged and 
miniaturized devices that can be easily and inexpensively built to mimic the thermal characteristics of intertidal organisms and record their thermal trajectories over a broad variety of temporal and spatial scales. Robomussels and robolimpets are biomimetic in the sense that they feature the thermal characteristics and visually resemble real organisms, being inconspicuous in the intertidal environment and thus less likely to be intentionally destroyed.

Robomussels (Fig. 1) are constructed following the methods described by Fitzhenry et al. (2004). Briefly, Evercoat Everfix epoxy resin (Fibre Glass-Evercoat Co. Inc., Cincinnati, OH) with a black coloring is poured into Smooth-Cast ${ }^{\circledR} 385$ molds created from Mytilus californianus shells. For each robomussel, a TidbiT logger (part \#UTBI-001, Onset Computer Corporation, Mass.) is embedded in the polyester resin, which after hardening is smoothed and shaped to that of a real mussel using a Dremel tool. TidbiT loggers have an accuracy of \pm $0.2^{\circ} \mathrm{C}$ over $0^{\circ}$ to $50^{\circ} \mathrm{C}$ and a resolution $0.02^{\circ} \mathrm{C}$ at $25^{\circ} \mathrm{C}$. The battery lasts for more than five years and the non-volatile memory stores approx. 42,000 12-bit temperature measurements, enough for more than 2.5 years with a sampling frequency of $30 \mathrm{~min}$ (which can be set from 1 second to 18 hours). Robomussels can be deployed in the field using A-788 Z-Spar Splash Zone Compound (Kop-Coat Inc, Pittsburgh, Pennsylvania, USA) to attach them to the rock or to any other substrata. Z-Spar sets in approximately $30 \mathrm{~min}$ and completely cures after a couple of hours (even underwater). TidbiT loggers are waterproof rated up to $300 \mathrm{~m}$, and thus no special care is needed to deal with immersion in seawater. Logger programming and data retrieval is done through the instruments' LEDs, which are exposed on the outside of the robomussel, using an USB optical communication cable and software from Onset Computer Corporation.
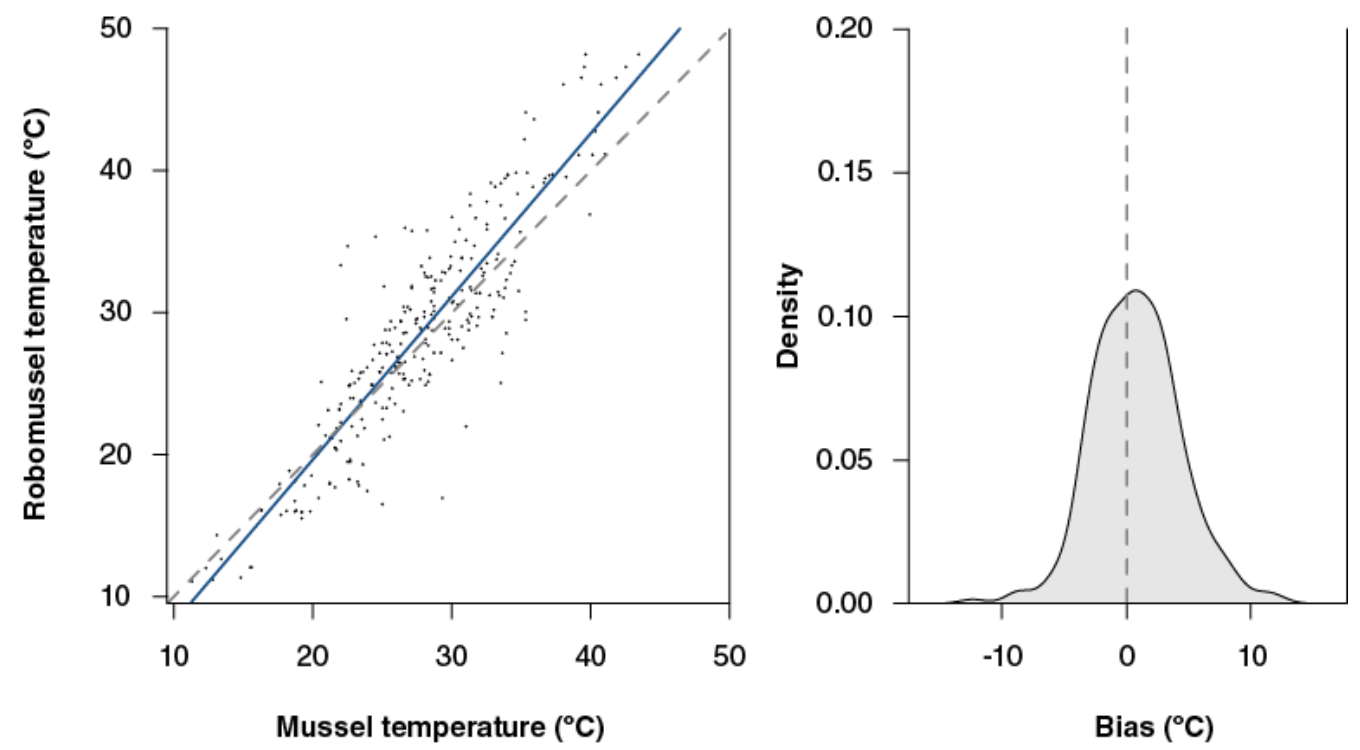

Fig. 2. Robomussel validation data (Root mean square error: $3.64^{\circ} \mathrm{C}$ ). Left: correlation between mussel body temperatures and robomussel measurements in a simulated mussel bed in the lab (276 pairs of measurements from 38 independent runs). The linear correlation $(R=0.89)$ is shown in blue and the reference dashed line $y=x$ is depicted in gray. Right: Density plot of the differences (bias) between each robomussel measurement and the correspondent body temperature (mean bias: $0.82^{\circ} \mathrm{C}$ ). 
Robomussel loggers have a thermal inertia comparable to that of a real mussel $74 \mathrm{~mm}$ in length. Under most field conditions, robomussels display temperatures within $1.5-2.0^{\circ} \mathrm{C}$ of adjacent, living mussels (Helmuth \& Hofmann, 2001). Notably, this compares very favorably to an unmodified Tibit logger, which displays an average error of $14^{\circ} \mathrm{C}$ to live mussels in the field (Fitzhenry et al. 2004). Fig. 2 shows some validation data acquired by comparing the body temperature of live mussels with robomussel measurements in a simulated mussel bed in the lab. In these conditions, loggers showed a linear correlation of 0.89 , with a root mean square error of $3.64{ }^{\circ} \mathrm{C}$ and a mean bias of $0.82{ }^{\circ} \mathrm{C}$.

One of the main shortcomings of the above described robomussels is a specific size size ( 74 $\mathrm{mm})$ where the specific heat $\times$ mass relationship crosses that of a real animal. Therefore, robomussels cannot be easily modified to mimic smaller animals, which in practice means that it is not possible to use this design to study other species of mussels such as M. edulis, M. galloprovincialis or M. trossulus. Also, the ability of robomussels to thermally match $M$. californianus seems to drop significantly when these loggers are used outside of a mussel bed (i.e., isolated robomussels do not perform as well as the others, B Helmuth, unpub. data). Finally, robomussels need to be entirely removed from the mussel bed each time they are serviced, which creates gaps and weakens the mussel bed. Nevertheless, data have shown that it is possible to obtain reasonably reliable results with the robomussel design, and that the errors in measurement are within the variability of what is recorded in the field within beds of a fixed substratum angle (Helmuth et al. 2006).

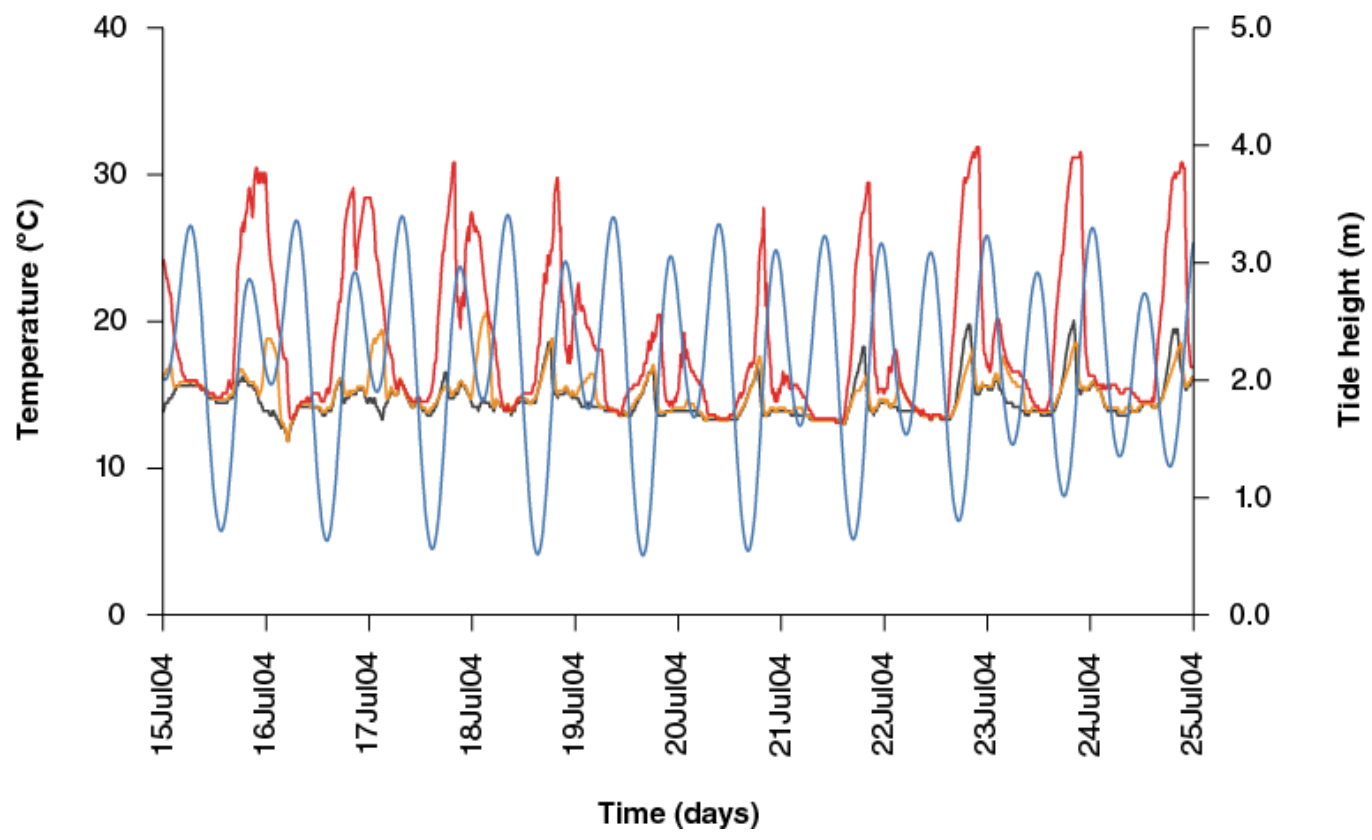

Fig. 3. Example of robomussel data from an exposed rocky shore in Seppings Island (SW Canada) taken between July 15th 2004 and July 25nd 2004. Times are in GMT. The black, orange and red lines show temperatures as measured by low-, mid- and high-intertidal loggers. Tidal heights are shown by the blue line.

Fig. 3 shows a 10-day long excerpt of the thermal profiles of three robomussels deployed in the low-, mid- and high- intertidal levels at a rocky shore in Seppings Island (Vancouver 
Island, British Columbia, Canada). The cyclic effect of the tide is quite clear, with maximum body temperatures occurring during low tide and minimum daily temperatures coinciding with high tides (e.g., in July 16th, the second high-tide peak caused a sudden drop in the temperature of the high-intertidal robomussel). It is even possible to observe how the daily tidal oscillations interact with solar radiation, influencing body temperatures. For instance, in the beginning of the data series, low tides were occurring early in the morning and so all three loggers showed a short delay before starting to heat, while towards the end of the plot low tides were happening later in the day, and thus robomussels' temperatures started to increase earlier. Also evident is the difference in temperature caused by the tidal height at which loggers were deployed. For example, the low-intertidal logger never exceeds $20^{\circ} \mathrm{C}$, while temperatures from the high-intertidal robomussel routinely reach $30^{\circ} \mathrm{C}$.

Robolimpets can be built following the methodology described in Lima \& Wethey (2009). Succinctly, these loggers consist of a lithium battery powering the circuit board from a DS1922L iButton (Dallas Semiconductor), embedded in a waterproof compound, inside an emptied real limpet shell (Fig. 4). Two exposed wires penetrating the shell serve as contacts for logger programming and subsequent data retrieval.
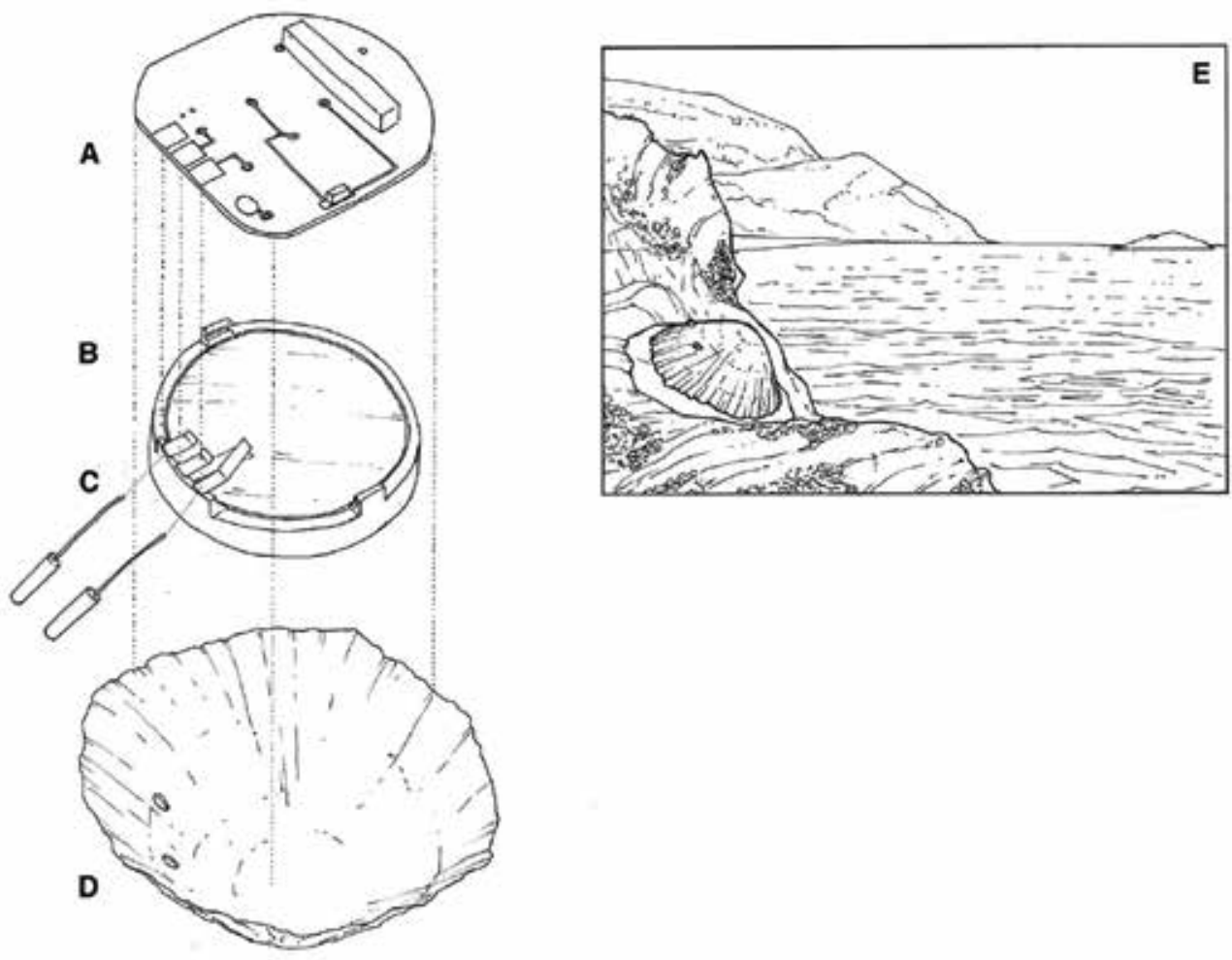

Fig. 4. Exploded view of the different parts used for building a temperature logger mimicking a Patella sp. (robolimpet). A: DS1922L iButton circuit board, B: DS1922L iButton battery, C: constantan wires (larger diameter) connected to wirewrap wires, D: Patella sp. shell with two holes to allow the constantan wires to protrude, E: representation of a robolimpet deployed in a rocky shore during low tide. 
The DS1922 iButton hardware can be programmed to record up to 8192 readings at $0.5^{\circ} \mathrm{C}$ (enough for more than 5 months with a sampling frequency of $30 \mathrm{~min}$ ) or 4096 readings at $0.0625^{\circ} \mathrm{C}$ resolution, measured at intervals from $1 \mathrm{~s}$ to $273 \mathrm{~h}$, with an accuracy of $\pm 0.5^{\circ} \mathrm{C}$. The first step in robolimpet assembly involves dissecting a DS1922L iButton. Using this procedure, even old, corroded iButtons can be recycled since the circuit board often remains perfectly functional despite deterioration of all other parts. iButtons have a stainless steel case that needs to be cut open with the help of a rotary tool fitted with an abrasive cutting wheel. For details on this procedure, see Lima \& Wethey (2009). The circuit board is then separated from the battery clip and thoroughly washed with water and isopropanol if necessary. The original lithium battery $(3 \mathrm{~V}, 40 \mathrm{mAh})$ may be kept or may be replaced by a higher-capacity one (e.g., Panasonic BR1255-1VC). Two pieces of AWG 30 wirewrap wire (Digikey part K329-ND) are then soldered to the appropriate terminals, connecting the circuit board to the outside contacts in the shell. The external contacts are made of 1.6-mm-diameter non-corrodible constantan thermocouple wire (part EXPPT- 14, Omega Engineering), and are passed through holes previously drilled into the shell using a Dremel Rotary Tool fitted with a high-speed cutter (Dremel part 569). All electronic parts must be waterproofed to prevent corrosion. Thus, 3M Scotchcast 2130 Flame Retardant Compound is poured into shells previously fitted with the electronic parts and allowed to fully harden for at least 24 hours before deployment. This material has previously been shown to offer appropriate levels of waterproofing without compromising the match between temperature trajectories of live animals and temperature trajectories recorded by the loggers (Lima \& Wethey, 2009).

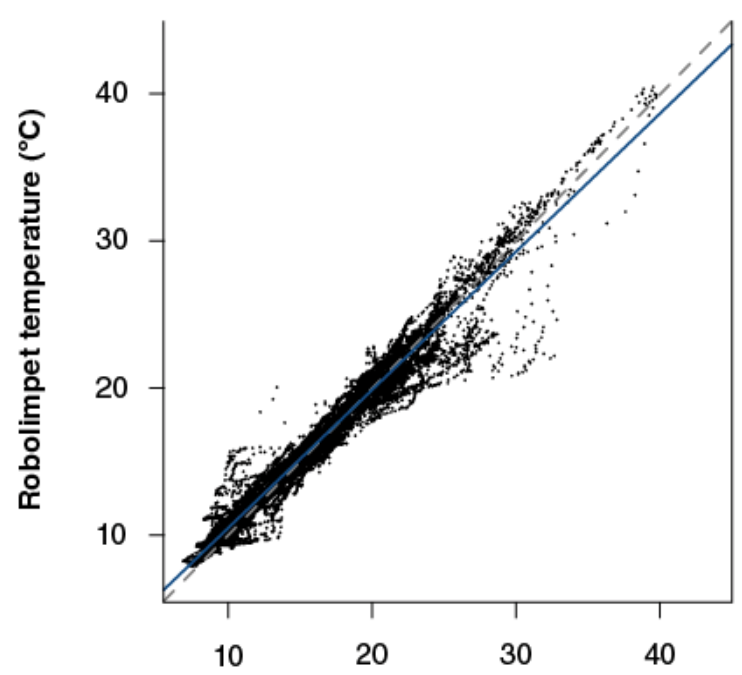

Limpet temperature $\left({ }^{\circ} \mathrm{C}\right)$

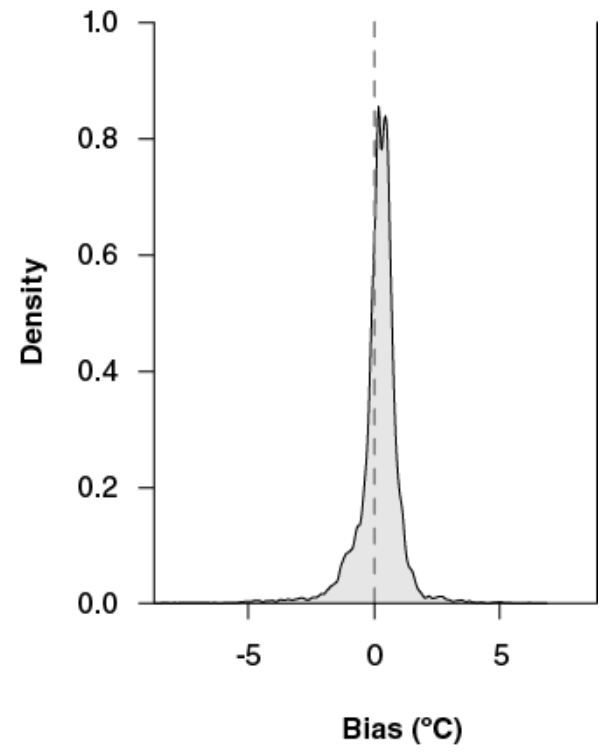

Bias $\left({ }^{\circ} \mathrm{C}\right)$

Fig. 5. Robolimpet validation data (Root mean square error: $0.94{ }^{\circ} \mathrm{C}$ ). Left: correlation between limpet body temperatures and robolimpet measurements in the field (25,355 paired measurements from 5 replicates taken between May 26 2008 and June $2^{\text {nd }} 2008$ at Friday Harbor, San Juan Island, Washington, USA). The linear correlation $(\mathrm{R}=0.98)$ is shown in blue and the reference dashed line $\mathrm{y}=\mathrm{x}$ is depicted in gray. Right: Density plot of the differences (bias) between each robolimpet measurement and the correspondent body temperature (mean bias: $0.16^{\circ} \mathrm{C}$ ). 
A connector cable can be made by stripping one end of a RJ-11 telephone cable and soldering two small alligator clips (Radio Shack, part 270-373) to the green and red wires (pins 3 and 4, respectively). These clips can then be connected to the robolimpet's negative and IO external contacts. The other end of the cable receives a RJ-11 telephone connector to be used with the 1-wire USB iButton adapter (Maxim IntegratedProducts part DS9490R - see Lima \& Wethey (2009) for more details). Robolimpets can be programmed in the field with a laptop computer using free OneWireViewer software (www.maxim-ic.com). The alternative is to use a computer-independent, portable reader such as the NS-71 (Newshift Lda., Leiria, Portugal) to download data to a Secure Digital (SD) card.

Robolimpets have been shown to mach very tightly the temperature profiles of live animals in the field. Fig. 5 shows some validation data on the relation between body temperature on five live Tectura persona and the corresponding robolimpet measurements over more than a week, in a rocky shore in San Juan Islands, Washington. The two datasets have a linear correlation of 0.98 , with a root mean square error of $0.94{ }^{\circ} \mathrm{C}$ and a mean bias of $0.16{ }^{\circ} \mathrm{C}$, which is smaller than the variability observed between live animals separated by a couple of meters (Lima \& Wethey, 2009). It is worth mentioning that these values were recorded by calibrated iButtons. Off-the-shelf iButtons are factory-calibrated, but since their calibration coefficients are stored in a volatile section of the memory and because it is very easy to momentarily disconnect de power from the circuit board while dehousing the electronics, calibration coefficients are often lost (this is obviously problematic when replacing the original battery). Calibration loss can be fixed by performing a 3-point calibration of the final assembled loggers under lab conditions. In this case, loggers are placed in a constant temperature chamber along with the probe of a precise thermocouple. The chamber is programmed to reach three specific temperatures (ideally, encompassing the full range of temperatures that robolimpets will likely experience in the field). Upon temperature stabilization, readings of the thermocouple $\left(\operatorname{Ref}_{1}, \operatorname{Ref}_{2}, \operatorname{Ref}_{3}\right)$ are compared with corresponding logs from the biomimetic sensors $\left(\log _{1}, \log _{2}, \log _{3}\right)$, and for each measured temperature $T$ in the field, a corrected $T^{\prime}$ can be derived as follows:

$$
\begin{gathered}
\operatorname{Dif}_{1}=\log _{1}-\operatorname{Ref}_{1} \\
\operatorname{Dif}_{2}=\log _{2}-\operatorname{Ref}_{2} \\
\operatorname{Dif}_{3}=\log _{3}-\operatorname{Ref}_{3} \\
a=\left(\operatorname{Dif}_{1}-\operatorname{Dif}_{3}\right)+\left(\left(\operatorname{Dif}_{2}-\operatorname{Dif}_{1}\right) \times\left(\operatorname{Ref}_{3}-\operatorname{Ref}_{1}\right) /\left(\operatorname{Ref}_{2}-\operatorname{Ref}_{1}\right)\right) \\
\beta=\left(\operatorname{Ref}_{3}\right)^{2}-\left(\operatorname{Ref}_{2} \times \operatorname{Ref}_{3}\right)+\left(\operatorname{Ref}_{2} \times \operatorname{Ref}_{1}\right)-\left(\operatorname{Ref}_{3} \times \operatorname{Ref}_{1}\right) \\
c=-\alpha / \beta \\
b=\left(\operatorname{Dif}_{2}-\mathrm{Dif}_{1}-c \times\left(\left(\operatorname{Ref}_{2}\right)^{2}-\left(\operatorname{Ref}_{1}\right)^{2}\right)\right) /\left(\operatorname{Ref}_{2}-\operatorname{Ref}_{1}\right) \\
a=\operatorname{Dif}_{1}-b \times \operatorname{Ref}_{1}-c \times\left(\operatorname{Ref}_{1}\right)^{2} \\
T^{\prime}=T-a-(b \times T)-\left(c \times T^{2}\right)
\end{gathered}
$$

Fig. 6 shows three examples of temperature profiles from robolimpets deployed for ten days in the low-, mid- and high-intertidal at a rocky shore in Biarritz (SW France). It is worth 
noticing that during spring low tides (in the first part of the plot), all loggers reached similar high temperatures peaks (exceeding $32{ }^{\circ} \mathrm{C}$ ), irrespectively of their vertical position on the shore. On the other hand, during the neap tides (in the last days), the low-shore logger was spending relatively longer periods immersed in the sea and consequently never reached temperatures as high as those measured by the high-tide robolimpet. This clearly illustrates that even low-shore inhabiting limpets can reach potentially stressful temperatures during spring low tides, but that that the frequency of stressful events experienced by each individual is highly dependent on its vertical position on the shore.

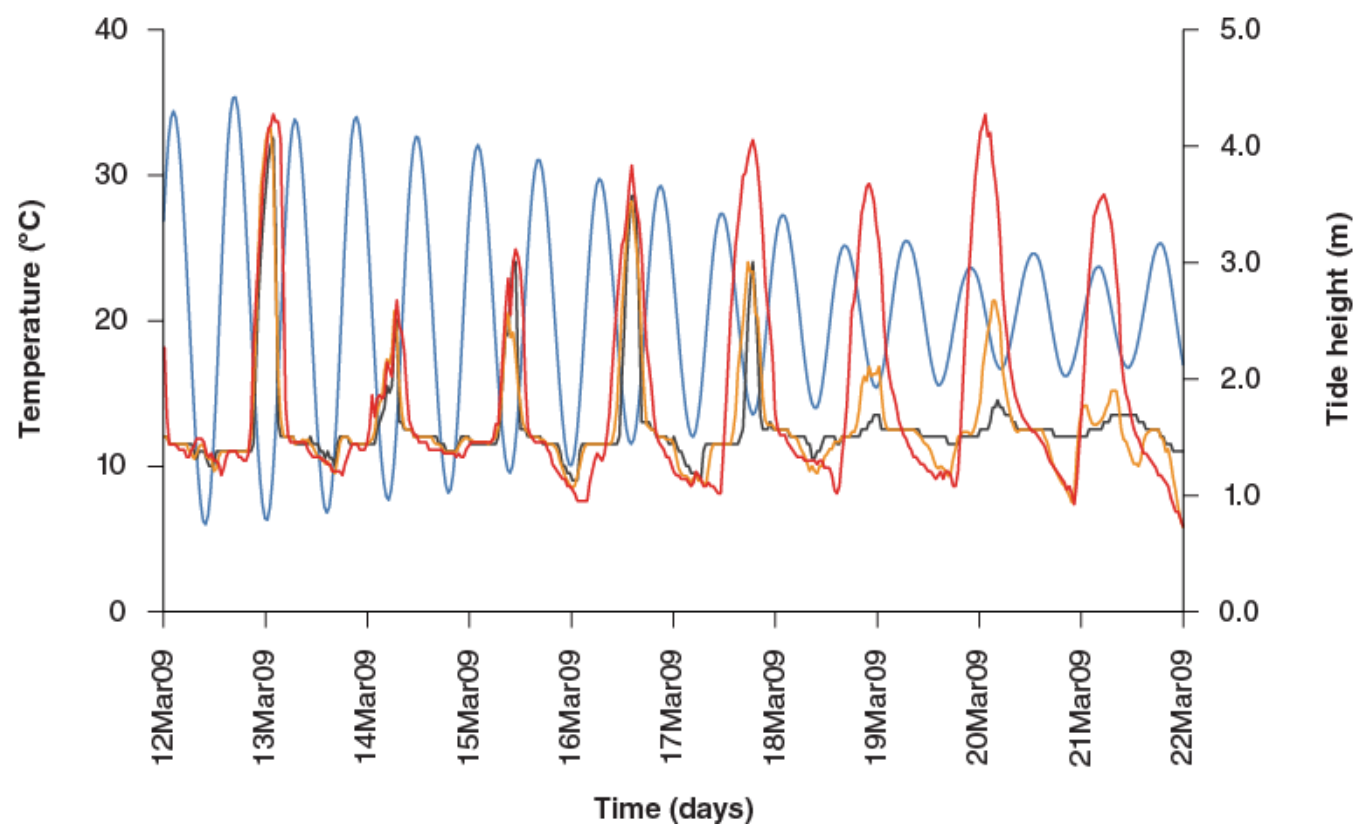

Fig. 6. Example of robolimpet data from an exposed rocky shore in Biarritz (SW France) taken between March 12 2009 and March 22 ${ }^{\text {th }}$ 2009. Times are in GMT. The black, orange and red lines show temperatures as measured by low-, mid- and high-intertidal loggers. Tidal heights are shown by the blue line.

In relation to their biomimetic characteristics, robolimpets outperformed robomussels in the sense that they not only show thermal profiles much closer to those displayed by live animals, but they also look more alike real animals (this is important because a higher mimetic capability makes them less likely to draw attention and thus less likely to be intentionally damaged). In addition, robolimpet electronics are much smaller and lightweighted (the dimensions of the coupled circuit board/battery do not exceed $14 \times 4 \mathrm{~mm}$, weighing approximately $1.5 \mathrm{~g}$ ). This allows for a much higher flexibility in the logger design, which can be adapted to mimic different limpet species or even other gastropods, bivalves, barnacles, and other groups (see, for instance, robobarnacles and small robomussels in Lima \& Wethey, 2009). Robolimpets are relatively inexpensive (each unit costs approximately 45 USD, substantially less than the cost of a robomussel, 130 USD) and thus for roughly the same cost it is possible to deploy $3 \times$ more loggers covering a much larger geographical area. Finally, data downloading/servicing in the field is easier to do with these loggers. 
Robolimpets have, however, some disadvantages over robomussels, namely a more complex and thus more time-consuming manufacturing process, and much smaller memory meaning that servicing must be done more frequently which may be problematic from the logistically and budgetary perspectives. Also, in robolimpets data are stored in a volatile section of the memory and thus are lost in the event of power failure. These loggers are also much more prone to leaking (at every six months, approximately one fourth of the loggers are lost), which can also be limiting when performing large-scale studies.

In sum, sensors/loggers mimicking the thermal characteristics of sessile intertidal species offer the opportunity to obtain data series with thousands of body temperatures along a wide range of microhabitats. Equally important is the ability to measure environments where these organisms do not occur, which may be extremely informative to understand the biogeographic patterns or the physiological requirements of a given species (Wethey, 2002; Lima et al., 2006).Taking measurements of body temperatures in areas where a given species does not occur is obviously problematic. The biomimetic loggers here described are a relatively easy way to overcome this problem and still measure temperatures that closely resemble those experienced by real organisms. They potentially allow building large-scale networks of sensors, obtaining fundamental data for ecological, physiological, biogeographical or even climate monitoring studies.

New advances are being made in these technologies, including a version of the robomussel that uses RFID technology to transmit data, thus eliminating the need to remove loggers after each deployment. By using materials with a higher mass and/or specific heat capacity, it will also be possible to manufacture smaller versions of the instrument to mimic smaller congeners ( 50mm).

\section{Biomimetic desiccation data loggers}

The dehydration, or desiccation, tolerances of an organism depend on the physiological response, whether it is the production of stress proteins (Benoit et al., 2010; Mizrahi et al., 2010) or change in energy metabolism (Churchill \& Storey, 1995; Santini, et al., 2001). These tolerances dictate how long an organism can survive without replenishing its water content, thus determining suitable habitat as a function of distance from a water source and also outlining appropriate organismal behavior. However, habitat cannot be generalized only by its proximity from water. Regardless of an organism's ability to combat or tolerate water loss, the severity of the desiccation it faces in any habitat is also dependent on meteorological factors. Air temperature, ambient humidity, substrate properties, and wind all affect evaporative water loss. In the intertidal zone, organisms face a choice of microclimates that can be restricted by vertical tidal height, maximizing feeding time (Borrero, 1987; Schneider et al., 2010), minimizing time vulnerable to predators and minimizing exposure to abiotic stresses (Petes et al., 2008; Smee et al., 2010).

The biomimetic desiccation data loggers - BDDL (also known as desiccation robolimpets) described in this section are designed to serve as a relative measure of potential desiccation stress that intertidal limpets are exposed to in a particular microclimate. The functional unit of each BDDL is a DS1923 Hygrochon iButton (Dallas Semiconductor) with simultaneous relative humidity $(\mathrm{RH})$ and temperature measuring capabilities. Thus, the sensors in these BDDL can record a relative measure of the change in water content of the soft tissue, as well as body temperature of the modeled organisms. Each DS1923 iButton was dissected, modified, and encased in a limpet shell following the general methodology used for the production of robolimpets (Lima \& Wethey, 2009, see also previous section). 


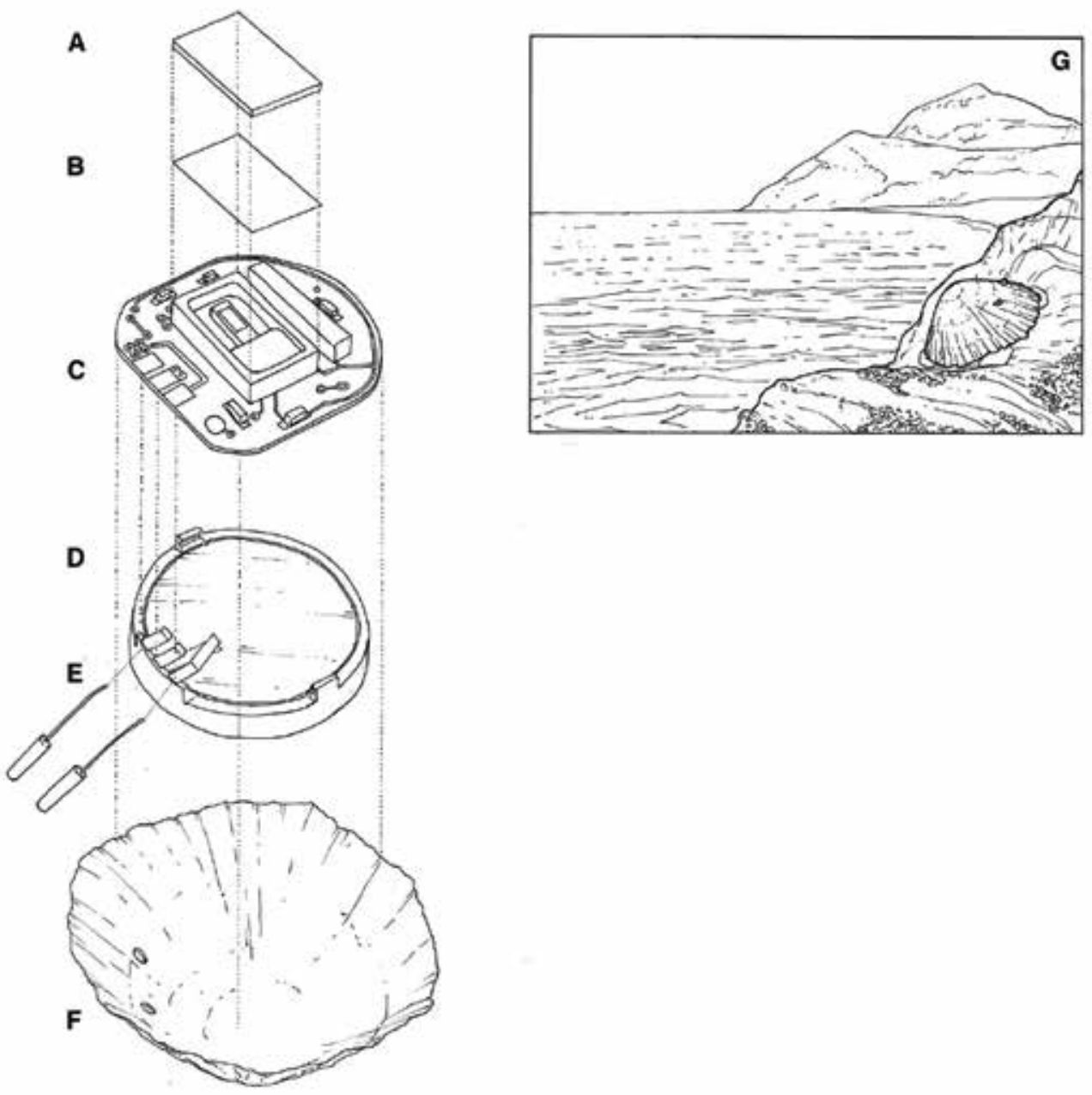

Fig. 7. Humidity logger mimicking a Patella sp. A: viscose pad, B: Tyvek polyethylene fiber paper, C: DS1923 iButton circuit board, D: DS1923 iButton battery, E: constantan wires (larger diameter) connected to wirewrap wires. F: Patella sp. shell with two holes to allow the constantan wires to protrude, G: Representation of a humidity sensor deployed in a rocky shore during low tide.

The RH sensor of the DS1923 iButtons is a structurally delicate thermoset polymer capacitive sensing element (Pan et al., 2010) and therefore must be protected from physical abrasions, which was done by covering it with a single layer of Tyvek polyethylene fiber paper (DuPont, Wilmington, DE) adhered with super glue control gel (Loctite, Westlake, $\mathrm{OH})$ around the edge of the humidity sensor casing. Tyvek polyethylene fiber paper is a tough, durable and tear-resistant material unaffected by contact with liquid water but permeable to water vapor. The coupled electronic circuit/battery from the DS1923 was encased in the limpet shell with the RH sensor on the ventral face (where the limpet would normally contact the substrate, see Fig. 7) and all the electronic parts waterproofed with 3M Scotchcast 2130 Flame Retardant Compound. Caution was taken to prevent the Tyvek and 
$\mathrm{RH}$ sensor from being covered by the potting compound, facilitating the diffusion of ambient water vapor into the RH sensor. To mimic water-saturated soft tissues of the live organisms, a $1 \times 1 \mathrm{~cm}$ piece of viscose (Boat Towl, Paddling.net, Caledonia, MI), a nonbiodegradable, highly absorptive fabric, was placed over the face of the humidity sensor and the cloth glued around its edges to the set Scotchcast Compound. Prior to sampling this fabric was saturated with freshwater and the evaporation of the water was monitored with the RH sensor. This design allows meteorological events (e.g., wind, solar radiation) to act on the soft tissue mimic just as those factors would act on the body of real limpets, and be measured by the RH sensor.

In contrast to temperature robolimpets, desiccation loggers cannot be completely attached to the substrate surface because that would prevent water vapor transfer between the ventral face of the biomimetic logger and the surrounding environment. Desiccation loggers were therefore temporarily attached to the rocky surfaces using small amounts of modeling clay placed on the edge of the shell, leaving enough small gaps for water vapor exchange (Fig. $7 \mathrm{G}$ ).

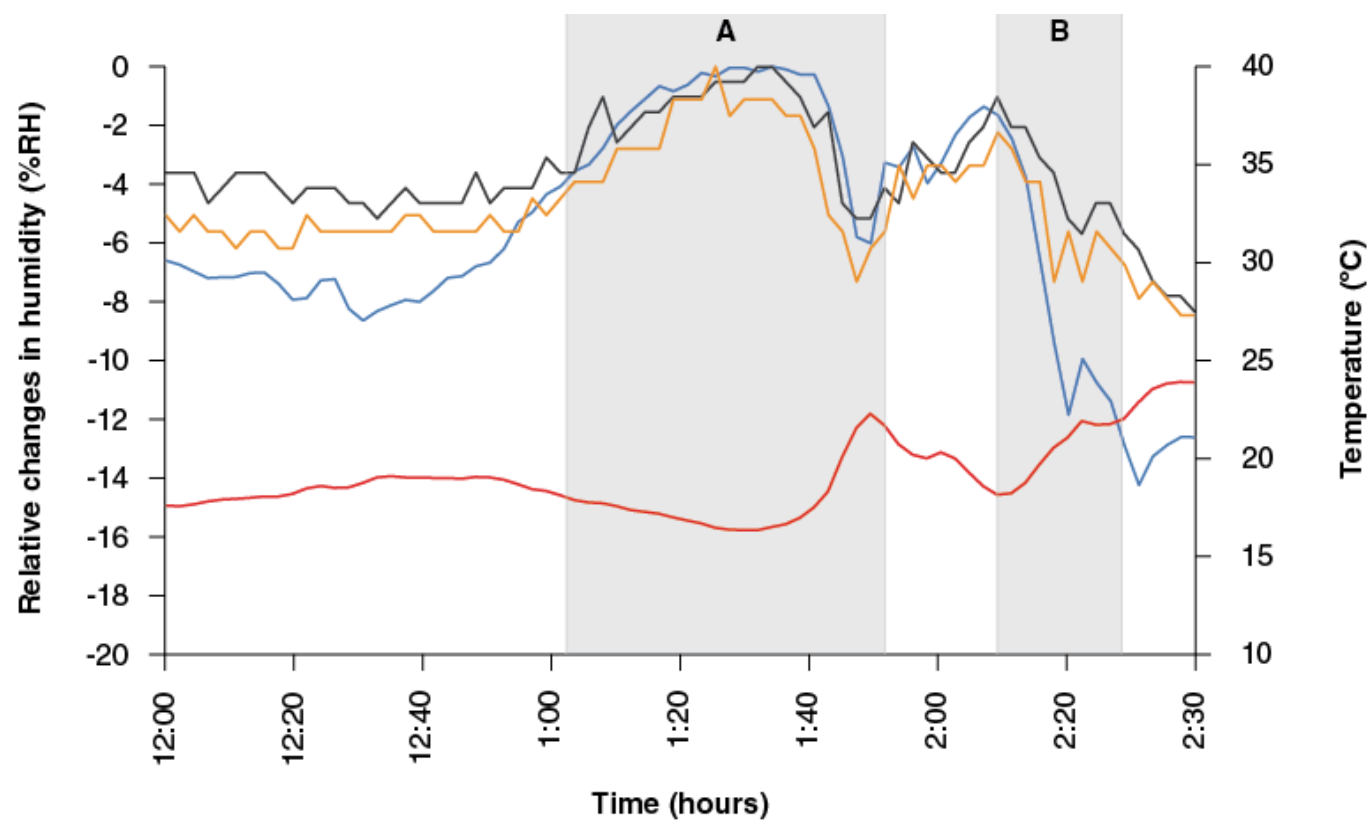

Fig. 8. Comparison between water loss measured in a live limpet (Patella vulgata - black line) and water loss as recorded by two biomimetic desiccation loggers (blue and orange lines) on a mid-intertidal crevice on a rocky shore at Le Croisic (NW France) on June $14^{\text {th }} 2010$. The temperature trajectory of the robolimpet is shown in red. A 45-minute rain storm occurred during period $\mathrm{A}$, followed by sun at period $\mathrm{B}$.

The described BDDL were compared against live limpet desiccation rates, measured in vivo via relative humidity monitoring of the mantle cavity. Neither the BDDL nor the in vivo measurements provide a direct estimate of the quantity of water lost due to evaporation. Rather, the sensor and the live animals behave like "a wet sponge that can lose some water but remain wet." As expected, both live limpets and the BDDL have fully saturated (100\% $\mathrm{RH}$ ) internal humidity when shaded or when there is no wind. However, abrupt peaks in humidity were recorded during deployments and were associated with the local weather 
conditions, such as gusts of wind, sun radiation or precipitation (Figs. 8 and 9). It is important to note that these changes were synchronous between each BDDL and its respective live limpet measurement. Gradual, longer lasting changes in the recorded humidity of live limpets and BDDL were indicative of precipitation (Figs. 8) and the presence of solar radiation (Fig. 9), both of which could be dually verified by changes in the body temperature. In fact, during the field deployment at Le Croisic (SW France), changes in $\mathrm{RH}$ were inversely correlated with changes in body temperature (Fig. 8).

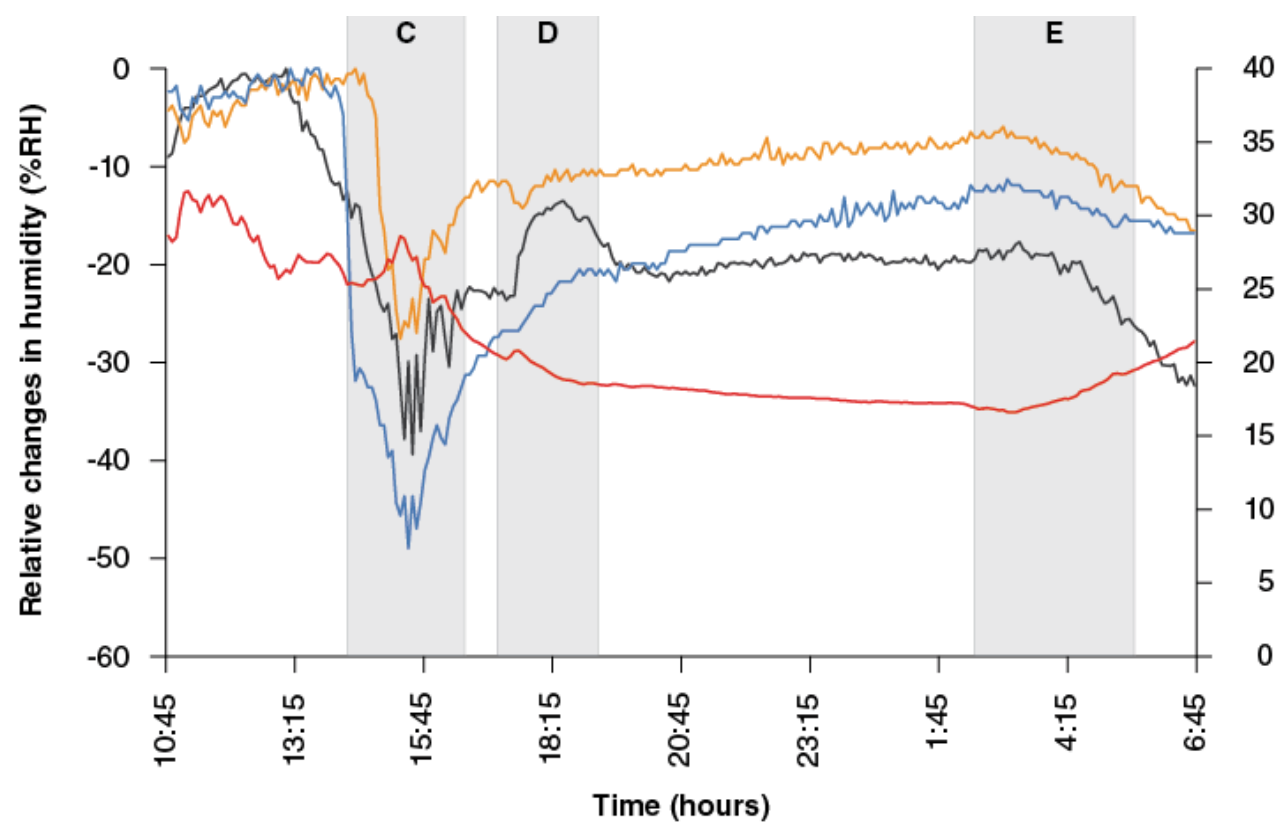

Fig. 9. Comparison between water loss measured in a live limpet (Patella vulgata - black line) and water loss as recorded by two biomimetic desiccation loggers (blue and orange lines) on a supralitoral rocky surface at Lavra (NW Portugal) on June 23rd-24th 2010 . The temperature trajectory of the limpet is shown in red. Period C corresponds to strong afternoon sun, which was followed by sunset at D. Temperatures decreased gradually during the night, but rose at sunrise (E), causing evaporative water loss in the live animal and in the biomimetic loggers.

On the most basic level, BDDL can measure the frequency and relative magnitude of desiccation stress associated with wind and larger meteorological events as experienced by the study organism, in any possible microclimate. Wind, in combination with low humidity, has been suggested as a dominant physical stressor on some rocky shores (Bertness et al., 2006; Bazterrica et al., 2007). The scope of quantifiable parameters is limited in this data logger, allowing several options for interpretation. A catalogue of the data loggers' behaviors referenced against real meteorological events, specific to any study site, can easily be compiled, allowing for a more accurate interpretation of data. The electronic basis of BDDL also presents an obstacle for long term and widespread field monitoring. The humidity sensor acts a gateway for salt to enter the electronics of the DS1923 iButton and thus salt water corrosion of the device is inevitable for long term field monitoring. The $\mathrm{RH}$ sensor has an accuracy of $\pm 3.5 \% \mathrm{RH}$, but according to the manufacturer (Honeywell - 
http://sensing.honeywell.com), a repeated exposure of the $\mathrm{RH}$ sensor to high humidity environments can cause a reversible 3\% shift in the measurements. The DS1923 iButton also costs approximately 85 USD, making each data logger relatively expensive, especially when considering its susceptibility to corrosion and anticipated short field life.

Employing BDDL in the study of intertidal ecology and climate change can provide insights into the importance of protection from desiccating physical stress for a specific organism. This tool, in combination with other biomimetic data loggers mentioned in this chapter, can provide a more refined representation of the effects of microclimate in the physical stress of organisms, a key to understand and forecast how long-term global climate change can influence the biogeography and ecology of intertidal species worldwide.

\section{Biomimetic wave force data loggers}

Disturbance by wave forces is a major factor influencing the distribution, abundance, activities and temporal dynamics of intertidal organisms. Such forces cause erosion of individuals, create patches of bare space, set limits to body size, and limit the foraging abilities of consumers (Lewis, 1964; Dayton, 1971; Koehl, 1976; Menge, 1976; Sousa, 1979; Paine \& Levin, 1981; Denny et al., 1985; Denny, 1995; Gaylord, 2000; Wethey, 2002; Denny et al., 2003; Carrington et al., 2009).

From the point of view of an organism in the shore, it is the total force which determines its ability to resist erosion, so direct measurements of both lift and drag components of force are essential for predicting survival or death. Still, there is relatively poor quantitative knowledge of these forces on the spatial and temporal scales over which they operate. Maximum forces have been estimated with spring-scale dynamometers since the 1970's (Jones \& Demetropoulos, 1968; Denny, et al., 1985; Denny, et al., 2003; Helmuth \& Denny, 2003), but these measure maxima over time scales of days to weeks. Instantaneous forces on one or more axes have been measured on models of organisms (Denny, 1982; Denny, 1995), or live organisms (Boller \& Carrington, 2006) but these have been limited to short deployments. A common approach has been to estimate or measure water velocities at the substratum and use equations for drag and lift to predict the forces that should have occurred during those conditions (Denny, et al., 1985; Denny, 1995; Denny \& Wethey, 2000; Gaylord, 2000), but the extent to which the theoretical estimates of wave forces and wave heights actually represent conditions experienced by organisms in the surf zone is not known (e.g., Helmuth \& Denny, 2003). Also, because big waves and correspondingly big forces are rare and difficult to measure, most authors calculate from probability distributions the maximum forces that organisms would likely experience in a year, rather than actually measuring them (e.g., Denny, et al., 1985; Denny, 1995).

Therefore there is a clear need for instruments that would make continuous direct measurements of drag and lift forces, wave heights, and their rates of change, over a wide range of habitats, organisms, and hydrodynamic conditions from calm to extreme storms. Such instruments should be relatively cheap, and be capable of long-term deployments in the field. Here, we describe a three-axis force sensor designed to measure wave forces and wave heights on a time and size scale appropriate for organisms inhabiting the surf zone of rocky intertidal shores. The instrument is capable of continuous deployments of several months duration. The sensor/logger package consists of a three axis force transducer connected to a resin model of a mussel (Mytilus edulis), a custom-made data logger in a waterproof housing and respective communication cables (Fig. 10). 

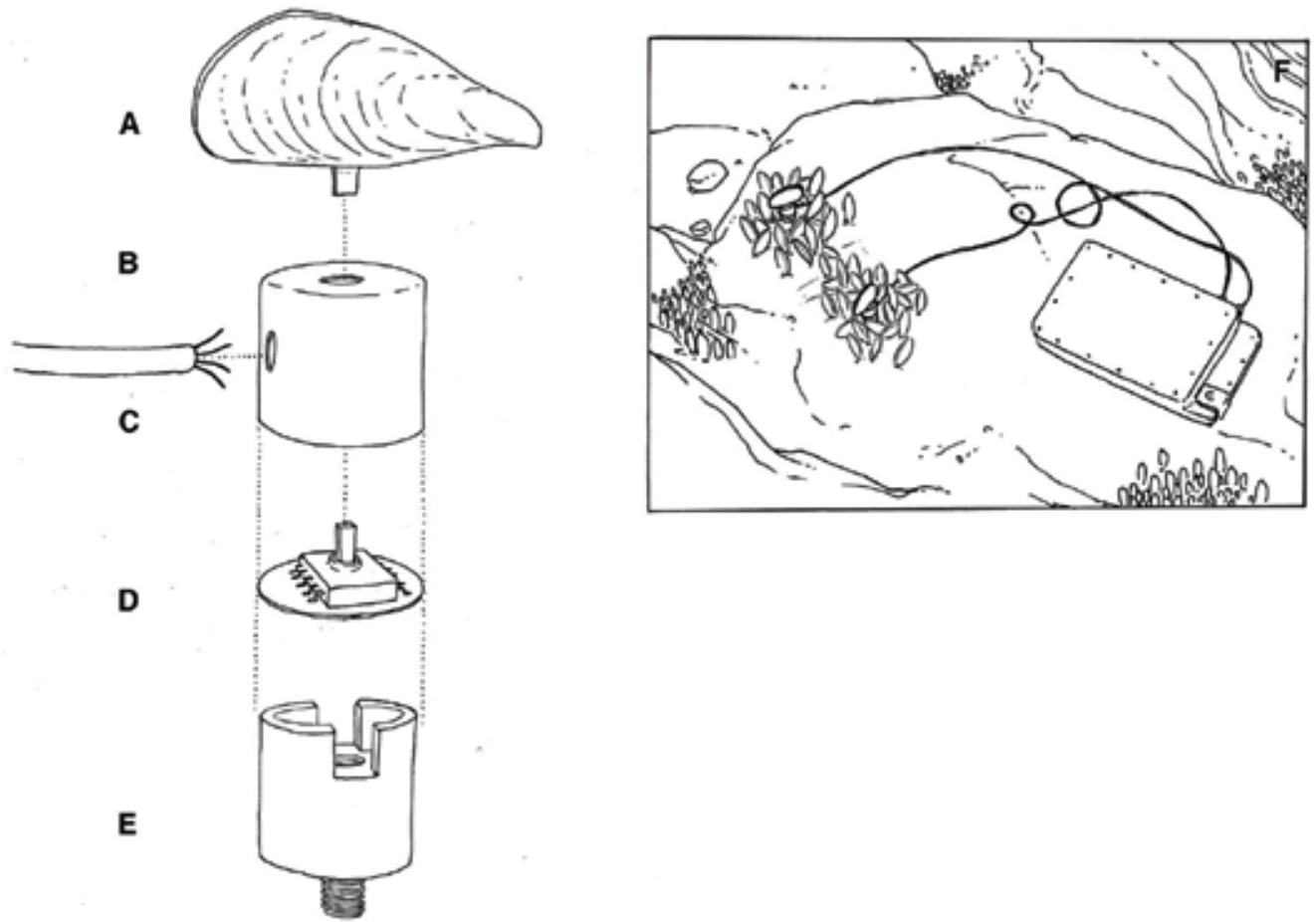

Fig. 10. Wave force logger mimicking a Mytilus edulis. A: polyester mussel model, B: copper pipe cap, C: sensor cable, D: printed circuit board with "SurfStick" sensor, E: polycarbonate plug with threaded rod for attachment. F: two wave force loggers deployed in musselbeds in the rocky intertidal together with an aluminum housing protecting the data logger.

The three axis force transducer was based on the CTS Corp Series 109 SurfStik (CTS Corp, Berne, Indiana, USA), a surface mount device with four strain gauges arranged around a $1.78 \mathrm{~mm}$ square ceramic post designed for use as the pointing device in notebook computer keyboards (Fig 10 D). These devices are rated to withstand forces of $200 \mathrm{~N}$ and $40 \mathrm{~N}$ in the vertical and horizontal directions, respectively. The SurfStick was soldered to a small circular printed circuit board, and embedded with epoxy within a standard copper cap for $1 / 2 " 12.7 \mathrm{~mm})$ ID copper water pipe, with the ceramic post protruding through a hole in the center of the flat face of the cap. A shielded signal cable was passed through a hole in the side of the cap. The base of the copper cap was sealed by a polycarbonate plastic plug including a slot for the cable, and a threaded hole on the exposed side.

A polyester resin model of a blue mussel Mytilus edulis was molded with a square brass tube protruding from the ventral surface where byssal threads emerge from the shell of a live animal (Fig. 10 A). The brass tube was fitted over the ceramic post of the force sensor and secured with Devcon 2-ton epoxy (Devcon Corp, Danvers, Massachusetts, USA). The sensor was calibrated by hanging weights from the top edge of the shell in orientations parallel and perpendicular to the ceramic post.

The strain gauges were arranged in 3 Wheatstone bridge circuits (Fig. 11), so that 3-axis forces could be measured. For x-axis forces, the east and west resistors were arranged as one 
half of a bridge, sensed from a connection between $\mathrm{E}$ and W. For $\mathrm{y}$-axis forces, the north and south resistors were placed in a half-bridge, sensed from a connection between $\mathrm{N}$ and $\mathrm{S}$. The other half of these bridges were pairs of $10 \mathrm{~K} \Omega 1 \%$ metal film resistors on opposite sides of an 11-turn cermet $100 \Omega$ potentiometer (Fig 11, "trm_x, trm_y"). The zero point for each sensor was adjusted with the potentiometer. The z-axis measurement used all four strain gauges together configured as a single arm of another Wheatstone bridge. The other arms of the $\mathrm{z}$ bridge were $10 \mathrm{~K} \Omega 1 \%$ metal film resistors, and an 11-turn cermet $100 \Omega$ potentiometer was used to set the zero (Fig 11, "trm_z"). The bridges were excited with a precision voltage reference (Burr Brown INA125), and the signals were amplified by Burr Brown INA125 and INA2126 instrumentation amplifiers. The circuit (Fig. 11) was produced on a two-layer printed circuit card (ExpressPCB Corp, Santa Barbara, California, USA).

Water depth was measured with a stainless steel absolute pressure sensor (Sensym ICT Series19, 30 psi), excited and amplified by a Burr Brown INA125 instrumentation amplifier. The pressure sensor was mounted in the instrument housing.

Analog signals were digitized at $40 \mathrm{~Hz}$ and recorded using a Persistor CF2 (Persistor Instruments, Bourne, Massachusetts, USA) microprocessor-based data logger, with a BurrBrown 16-bit analog to digital converter (ADS8344, on a Persistor R216 "recipe card"). Data were stored on 2GB compact flash cards (as used in digital cameras). The data logger is $5 \mathrm{~cm}$ $x 7.5 \mathrm{~cm}$, and the analog amplifier card is a similar size. In order to reduce power drain, the CF2 microprocessor (Motorola 68332) is run at $4 \mathrm{MHz}$, and awakened from sleep-mode by a programmable interrupt timer running at $40 \mathrm{~Hz}$ for data acquisition. The system draws approximately $5 \mathrm{~mA}$ and is powered with three $3 \mathrm{~V}$ lithium C-cells ( 5 ampere-hour), enough for deployments up to 90 days. The ability to make continuous measurement for several months is a great advantage over similar force loggers which are limited to less than 1-hour deployments by data storage capacity (Boller \& Carrington, 2006).

Analog and digital electronics boards and power supplies were mounted in a low profile aluminum housing $(5 \mathrm{~cm} \times 23 \mathrm{~cm} \times 15 \mathrm{~cm}$, including $1.3 \mathrm{~cm}$ thick lid and $1.6 \mathrm{~cm}$ thick base). The sensor cable was attached to the housing with waterproof 4-pin connectors (Type 9104.14 and 9104.54, Ikelite Corp, Indianapolis, Indiana, USA). The instrument package was designed to withstand impact from wave-borne rocks, and to resist drag and lift forces. It was attached to the rock by six $6.35 \mathrm{~mm}$ diameter stainless steel wedge anchors placed in holes drilled in the rock. The electronics package was attached approximately $0.5 \mathrm{~m}$ away from the sensor so that it would not disrupt fluid flow around the sensor to any greater extent than the variable topography in the intertidal zone. A sacrificial zinc anode was attached to the housing to prevent corrosion. In SW England, five housings were experimentally deployed in the surf zone of exposed to moderately exposed rocky shores for a total of 120 housing-days with no damage or failures. Wave heights on top of the housings ranged from $0.5 \mathrm{~m}$ to $3 \mathrm{~m}$ during the deployments.

The sensor was attached to the rock by drilling a $3 / 4^{\prime \prime}$ diameter hole in the rock within a patch of mussels, filling the hole with Z-Spar Splash Zone Compound (Kop-Coat Inc, Pittsburgh, Pennsylvania, USA), screwing a $1 \mathrm{~cm}$ length of threaded rod into the base of the sensor, and pressing the threaded rod and base of the sensor cap into the Z-Spar until the mussel model was level with the height of the surrounding mussel bed. The cable was anchored to the rock with cable ties attached to stainless steel wedge anchors. During the deployment period, only 1 mussel sensor broke after being hit by a wave-borne object. 


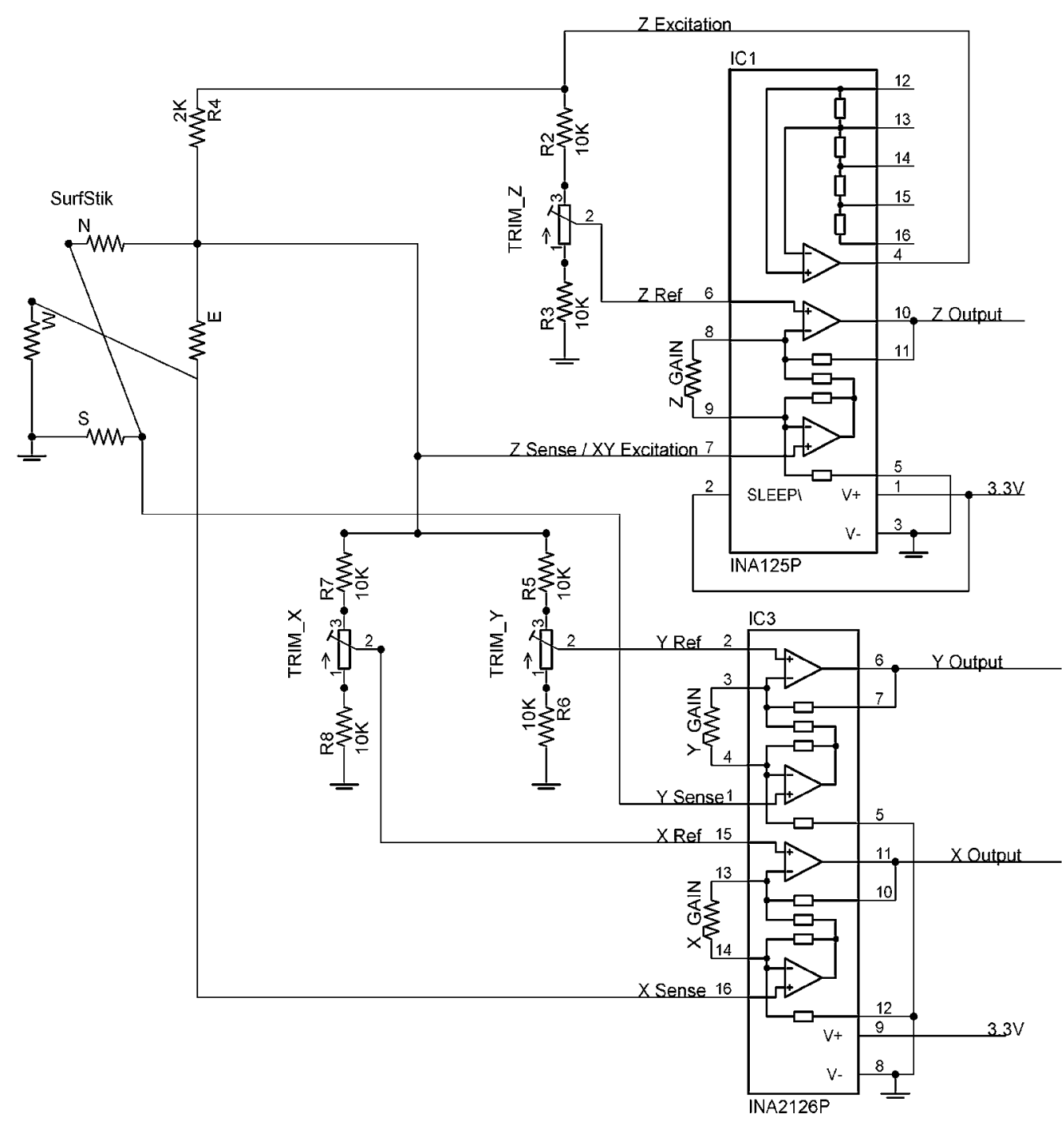

Fig. 11. Schematic Diagram of force sensor circuit. On the left hand side of the circuit is the CTS "SurfStik", with strain gauge resistors on the four sides $(\mathrm{N}, \mathrm{S}, \mathrm{E}, \mathrm{W})$ of the ceramic post. 

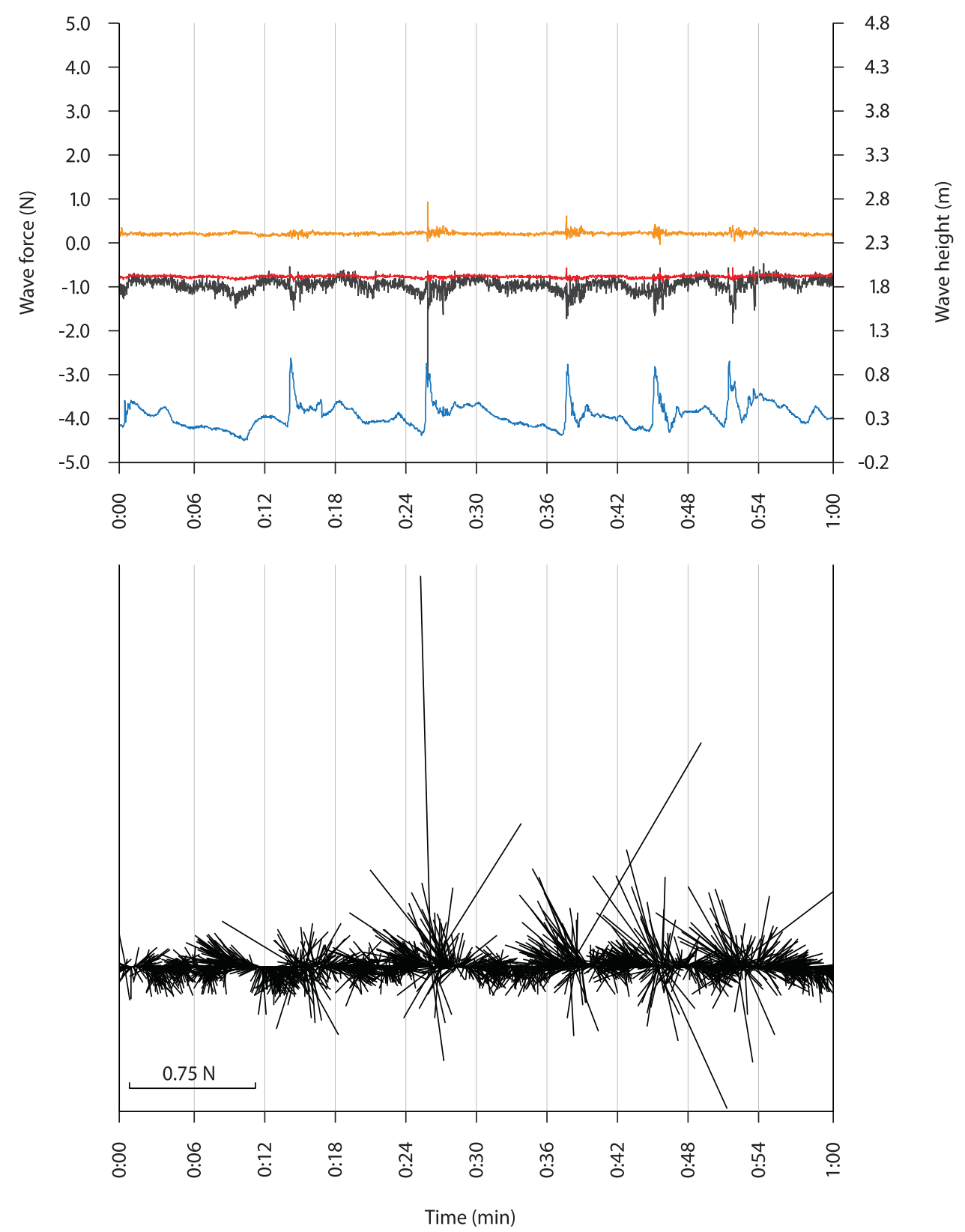

Fig. 12. Data from a mussel-shaped wave force sensor, acquired on an exposed rocky shore in Lansallos (SW England) in February 19th 2003. Top Panel: three axis force record and wave height during a 1 minute period. Red $=x$, orange $=y$, black $=\mathrm{z}$ (scale on left in newtons), blue $=$ wave height (scale on right, in meters). Lift forces are displayed as negative on the force axis. Note that drag forces (red and orange) are smaller than lift forces, and that not all waves of equivalent height generate equivalent forces. Bottom Panel: vector plot of horizontal forces during the same 1 minute period. Note the rotational motion as waves cross the sensor. 
The system has an effective resolution of $0.1 \mathrm{~N}$ in the vertical direction and $0.05 \mathrm{~N}$ in the horizontal, taking into account electrical noise. The maximum forces recorded were $55 \mathrm{~N}$ impact (downward), 8N lift (upward), and $4 \mathrm{~N}$ drag (horizontal). In order to detect the forces on a wave-by wave basis, a zero crossing detector was used to find the beginning and end of each wave in 1 min sections of the data stream. The maximum and minimum water heights in each wave were used to determine wave height. The maximum force along all three axes results from highly complex interactions between animals and waves in the surf zone. Of particular note, lift forces tended to be 3 to 4 times larger than drag forces because the sensor was deployed within mussel beds, where individuals are sheltered from drag by their neighbors.

Lift forces are significant in this circumstance because skimming flow of water moves rapidly over the surface of the bed, and water is stagnant at the bottom of the bed. The velocity difference between the top and the bottom of the bed is presumably responsible for generating lift. This result is consistent with the Pitot-tube observations of Denny (1987) on the pressure differences between the top and bottom of a mussel bed during flow. From the point of view of mussels compacted in extensive beds, therefore, lift is far more important than drag. Drag forces are probably important only for exposed individuals located outside from the mussel bed.

Water velocities and accelerations were large when waves crossed over the sensor package (Fig. 12). In the figure, a $0.75 \mathrm{~m}$ wave had a vertical water velocity of $13 \mathrm{~m} \mathrm{~s}^{-1}$, and a vertical acceleration of $300-500 \mathrm{~ms}^{-2}$, all associated with flow reversals and acceleration reversals within less than a second. These directly measured values are similar to those calculated from surf-zone force records by Denny et al., (1985). In larger waves, accelerations on the order of $1000 \mathrm{~ms}^{-2}$ were observed.

Not all waves of the same size and shape generate the same magnitude of forces on individuals. Thus two waves can be of the same size, and generate forces that differ by a factor of 5 or 10 (fig. 12). The reason for this is that the mussel is in a fixed location, so it is sensing the environment in an Eulerian manner. Hence, the exact size and trajectory of each wave determines how it will react with an individual point on the bottom. Waves with slightly different trajectories will sweep specific locations on the bottom with different velocities, creating different forces on the animal or sensor. The average of a large number of waves will be similar to the expected value of force or velocity, but the individual wave may generate much larger or much smaller forces. These results and those of Gaylord (1999; 2000) and Helmuth \& Denny (2003) indicate that there is a strongly probabilistic relationship between wave height and water velocity or wave force. For instance, among waves of height $0.5 \mathrm{~m}$ to $0.6 \mathrm{~m}$, the 50 th median value of water velocity was $2.59 \mathrm{~ms}^{-1}$, whereas the $90 \mathrm{th}$, 95th, and 99th percentiles of velocity were 3.98, 4.49, and $5.66 \mathrm{~ms}^{-1}$, respectively. Thus, calculations of average conditions or the use of regressions of wave height to velocity will estimate the conditions experienced only in the median wave in the overall distribution, and will underestimate the larger velocities and forces in half of the waves experienced by organisms in the surf zone. These velocities in the upper half of the distribution can be 2 to 5 times greater than the median, and therefore forces that scale with the square of velocity can be 4 to 25 times greater than the median. These data reinforce the idea that it is necessary to have reliable measurements of the distributions of forces generated by waves in order to be able to fully understand their influence on intertidal communities.

The described biomimetic wave force data logger provides an inexpensive method for directly measuring the distributions of lift and drag forces, wave heights, vertical water 
velocities and accelerations on time and spatial scales appropriate for studies of the effects of disturbance on intertidal communities. This sensor system can be adapted to study the effect of waves on other sedentary intertidal organisms, by changing the geometry of the sensor head to mimic barnacles, gastropods, algae, and other groups. Because it measures wave height simultaneously with forces, it avoids the tenuous link between offshore measurements of waves, and extrapolation via theory to the environment of the surf zone.

\section{Acknowledgements}

All drawings by André L. Araújo. The authors would also like to thank Jerry Hilbish, Nuno Queiroz and Rui Seabra for their help during fieldwork and for their insightful suggestions that often resulted in design improvements. Funding was provided by NOAA (NA04NOS4780264), NASA (NNG04GE43G and NNX07AF20G), National Science Foundation (IBN 0131308) and Fundação para a Ciência e a Tecnologia - FCT (PTDC/MAR/099391/2008 and SFRH/BPD/34932/2007) grants, and a University of South Carolina Residential Mini-Grant. Brian Bittner of CTS Corp provided evaluation samples of the SurfStik. Arthur Illingworth and Allen Frye designed and built the waterproof housings for the wave sensors.

\section{References}

Bazterrica, M. C.; Silliman, B. R.; Hidalgo, F. J.; Crain, C. M. \& Bertness, M. D. (2007). Limpet grazing on a physically stressful Patagonian rocky shore, Journal of Experimental Marine Biology and Ecology, 353, 1, 22-34, 0022-0981

Benoit, J. B.; Lopez-Martinez, G.; Phillips, Z. P.; Patrick, K. R. \& Denlinger, D. L. (2010). Heat shock proteins contribute to mosquito dehydration tolerance, Journal of Insect Physiology, 56, 2, 151-156, 0022-1910

Berke, S. K.; Mahon, A. R.; Lima, F. P.; Halanych, K. M.; Wethey, D. S. \& Woodin, S. A. (2010). Range shifts and species diversity in marine ecosystem engineers: patterns and predictions for European sedimentary habitats, Global Ecology and Biogeography, $19,2,223-232,1466-8238$

Bertness, M. D.; Crain, C. M.; Silliman, B. R.; Bazterrica, M. C.; Reyna, M. V.; Hildago, F. \& Farina, J. K. (2006). The community structure of Western Atlantic Patagonian rocky shores, Ecological Monographs, 76, 3, 439-460, 0012-9615

Boller, M. L. \& Carrington, E. (2006). In situ measurements of hydrodynamic forces imposed on Chondrus crispus Stackhouse, Journal of Experimental Marine Biology and Ecology, 337, 2, 159-170, 0022-0981

Borrero, F. J. (1987). Tidal height and gametogenisis: reproductive variation among populations of Geukensia demissa, Biology Bulletin, 173, 1, 160-168, 0006-3185

Broekhuysen, G. J. (1941). A preliminary investigation of the importance of desiccation, temperature and salinity as factors controlling the vertical distribution of certain intertidal marine gastropods in False Bay, South Africa, Transactions of the Royal Society of South Africa, 28, 255-292, 0035-919X

Carrington, E.; Moeser, G. M.; Dimond, J.; Mello, J. J. \& Boller, M. L. (2009). Seasonal disturbance to mussel beds: Field test of a mechanistic model predicting wave dislodgment, Limnology and Oceanography, 54, 3, 978-986, 0024-3590 
Churchill, T. \& Storey, K. (1995). Metabolic effects of dehydration on an aquatic frog, Rana pipiens, Journal of Experimental Biology, 198, 1, 147-154, 0022-0949

Connell, J. H. (1961a). Effects of competition, predation by Thais lapillus, and other factors on natural populations of the barnacle Balanus balanoides, Ecological Monographs, 31, 61104, 0012-9615

Connell, J. H. (1961b). The influence of intra-specific competition and other factors on the distribution of the barnacle Chthamalus stellatus, Ecology, 42, 4, 710-723, 0012-9658

Connell, J. H. (1972). Community interactions on marine rocky intertidal shores, Annual Review of Ecology and Systematics, 3, 169-192, 0066-4162

Crisp, D. J., Ed. (1964). The effects of the severe winter of 1962-63 on marine life in Britain, Journal of Animal Ecology, 33, 179-210, 0021-8790

Dahlhoff, E. P. (2004). Biochemical Indicators of Stress and Metabolism: Applications for Marine Ecological Studies, Annual Review of Physiology, 66, 1, 183-207, 0066-4278

Dayton, P. K. (1971). Competition, disturbance and community organization: the provision and subsequent utilization of space in a rocky intertidal community, Ecological Monographs, 41, 4, 357-389, 0012-9615

Denny, M. (1995). Predicting Physical Disturbance: Mechanistic Approaches to the Study of Survivorship on Wave-Swept Shores, Ecological Monographs, 65, 4, 371-418, 00129615

Denny, M.; Daniel, T. \& Koehl, M. (1985). Mechanical limits to size in wave-swept organisms, Ecological Monographs, 55, 69-102, 0012-9615

Denny, M. W. (1982). Forces on intertidal organisms due to breaking ocean waves: design and application of a telemetry system, Limnology and Oceanography, 27, 178-183, 0024-3590

Denny, M. W. (1987). Lift as a mechanism of patch initiation in mussel beds, Journal of Experimental Marine Biology and Ecology, 113, 3, 231-245, 0022-0981

Denny, M. W.; Miller, L. P. \& Harley, C. D. G. (2006). Thermal stress on intertidal limpets: long-term hindcasts and lethal limits, The Journal of Experimental Biology, 209, 24202431, 0022-0949

Denny, M. W.; Miller, L. P.; Stokes, M. D.; Hunt, L. J. H. \& Helmuth., B. S. T. (2003). Extreme water velocities: Topographical amplification of wave-induced flow in the surf zone of rocky shores, Limnology and Oceanography, 48, 1-8, 0024-3590

Denny, M. W. \& Wethey, D. S. (2000). Physical processes that generate patterns in marine communities, In: Marine Community Ecology, M. Bertness; S. Gaines \& M. Hay, (Ed), 1-37, Sinauer Associates, Sunderland, Massachusetts

Firth, L. B. \& Williams, G. A. (2009). The influence of multiple environmental stressors on the limpet Cellana toreuma during the summer monsoon season in Hong Kong, Journal of Experimental Marine Biology and Ecology, 375, 1-2, 70-75, 0022-0981

Fitzhenry, T.; Halpin, P. M. \& Helmuth, B. (2004). Testing the effects of wave exposure, site, and behavior on intertidal mussel body temperatures: Applications and limits of temperature logger design, Marine Biology, 145, 2, 339-349, 0025-3162

Foster, B. A. (1971). Desiccation as a factor in the intertidal zonation of barnacles, Marine Biology, 8, 12-29, 0025-3162

Gaylord, B. (1999). Detailing agents of physical disturbance: wave-induced velocities and accelerations on a rocky shore, Journal of Experimental Marine Biology and Ecology, $239,1,85-124,0022-0981$ 
Gaylord, B. (2000). Biological Implications of Surf-Zone Flow Complexity, Limnology and Oceanography, 45, 1, 174-188, 0024-3590

Gilman, S. E.; Wethey, D. S. \& Helmuth, B. (2006). Variation in the sensitivity of organismal body temperature to climate change over local and geographic scales, Proceedings of the National Academy of Sciences, 103, 25, 9560-9565, 1091-6490

Harley, C. (2008). Tidal dynamics, topographic orientation, and temperature-mediated mass mortalities on rocky shores, Marine Ecology Progress Series, 371, 37-46, 0171-8630

Harley, C. D. G. \& Helmuth, B. S. T. (2003). Local- and regional-scale effects of wave exposure, thermal stress, and absolute versus effective shore level on patterns of intertidal zonation, Limnology and Oceanography, 48, 4, 1498-1508, 0024-3590

Hawkins, S. J.; Hartnoll, R. G.; Kain, J. M. \& Norton, T. A. (1992). Plant-animal interactions on hard substrata in the North-east Atlantic, In: Plant-Animal Interactions in the Marine Benthos, D. John; S. J. Hawkins \& J. Price, (Ed), 1-32, Clarendon Press, 0-19857754-O, Oxford

Helmuth, B. (1998). Intertidal Mussel Microclimates: Predicting the Body Temperature of a Sessile Invertebrate, Ecological Monographs, 68, 29-52, 0012-9615

Helmuth, B. (1999). Thermal biology of rocky intertidal mussels: Quantifying body temperatures using climatological data, Ecology, 80, 1, 15-34, 0012-9658

Helmuth, B. (2002). How do we measure the environment? Linking intertidal thermal physiology and ecology through biophysics, Integrative and Comparative Biology, 42, 4, 837-845, 1540-7063

Helmuth, B.; Broitman, B. R.; Yamane, L.; Gilman, S. E.; Mach, K.; Mislan, K. A. S. \& Denny, M. W. (2010). Organismal climatology: analyzing environmental variability at scales relevant to physiological stress, Journal of Experimental Biology, 213, 6, 9951003, 0022-0949

Helmuth, B. \& Denny, M. W. (2003). Predicting wave exposure in the rocky intertidal zone: do bigger waves always lead to larger forces?, Limnology and Oceanography, 48, 1338-1345, 0024-3590

Helmuth, B.; Kingsolver, J. G. \& Carrington, E. (2005). Biophysics, physiological ecology, and climate change: does mechanism matter?, Annual Review of Physiology, 67, 177201, 0066-4278

Helmuth, B.; Mieszkowska, N.; Moore, P. \& Hawkins, S. J. (2006). Living on the Edge of Two Changing Worlds: Forecasting the Responses of Rocky Intertidal Ecosystems to Climate Change, Annual Review of Ecology, Evolution, and Systematics, 37, 1, 373-404, $1543592 X$

Helmuth, B. S. T. \& Hofmann, G. E. (2001). Microhabitats, thermal heterogeneity and patterns of physiological stress in the rocky intertidal zone, Biological Bulletin, 201, 374-384, 0006-3185

Hodkinson, I. D. (1999). Species response to global environmental change or why ecophysiological models are important: a reply to Davis et al, Journal of Animal Ecology, 68, 6, 1259-1262, 0021-8790

Jones, S. J.; Lima, F. P. \& Wethey, D. S. (2010). Rising environmental temperatures and biogeography: poleward range contraction of the blue mussel, Mytilus edulis L., in the western Atlantic, Journal of Biogeography, no-no, 1365-2699 
Jones, W. E. \& Demetropoulos, A. (1968). Exposure to wave action: Measurements of an important ecological parameter on rocky shores on Anglesey, Journal of Experimental Marine Biology and Ecology, 2, 1, 46-63, 0022-0981

Koehl, M. (1976). Effects of sea anemones on the flow force they encounter, Journal of Experimental Biology, 69, 87-105, 0022-0949

Lewis, J. R. (1964). The ecology of rocky shores, English University Press Ltd., 0340213604, London

Lewis, R. (1954). Observations on a high level population of limpets, Journal of Animal Ecology, 23, 85-100, 0021-8790

Lima, F. P.; Queiroz, N.; Ribeiro, P. A.; Hawkins, S. J. \& Santos, A. M. (2006). Recent changes in the distribution of a marine gastropod, Patella rustica Linnaeus, 1758, and their relationship to unusual climatic events, Journal of Biogeography, 33, 812-822, 03050270

Lima, F. P.; Ribeiro, P. A.; Queiroz, N.; Hawkins, S. J. \& Santos, A. M. (2007a). Do distributional shifts of northern and southern species of algae match the warming pattern?, Global Change Biology, 13, 2592-2604, 1354-1013

Lima, F. P.; Ribeiro, P. A.; Queiroz, N.; Xavier, R.; Tarroso, P.; Hawkins, S. J. \& Santos, A. M. (2007b). Modelling past and present geographical distribution of the marine gastropod Patella rustica as a tool for exploring responses to environmental change, Global Change Biology, 13, 2065-2077, 1354-1013

Lima, F. P. \& Wethey, D. S. (2009). Robolimpets: measuring intertidal body temperatures using biomimetic loggers, Limnology and Oceanography-Methods, 7, 347-353, 15415856

Menge, B. A. (1976). Organization of the New England Rocky Intertidal Community: Role of Predation, Competition, and Environmental Heterogeneity, Ecological Monographs, 46, 4, 355-393, 0012-9615

Mizrahi, T.; Heller, J.; Goldenberg, S. \& Arad, Z. (2010). Heat shock proteins and resistance to desiccation in congeneric land snails, Cell Stress and Chaperones, 15, 4, 351-363, 1355-8145

Paine, R. T. \& Levin, S. A. (1981). Intertidal Landscapes: Disturbance and the Dynamics of Pattern, Ecological Monographs, 51, 2, 145-178, 0012-9615

Pan, H.; Qing, Y. \& 2010, L. P.-Y. (2010). Direct and indirect measurement of soil suction in the laboratory, Electronic Journal of Geotechnical Engineering, 15, 1-14, 1089-3032

Petes, L.; Mouchka, M.; Milston-Clements, R.; Momoda, T. \& Menge, B. (2008). Effects of environmental stress on intertidal mussels and their sea star predators, Oecologia, 156, 3, 671-680, 0029-8549

Pincebourde, S.; Sanford, E. \& Helmuth, B. (2008). Body temperature during low tide alters the feeding performance of a top intertidal predator, Limnology and Oceanography, $53,4,1562-1573,0024-3590$

Raffaelli, D. \& Hawkins, S. J. (1996). Intertidal Ecology, Chapman and Hall, 0412299607, London

Santini, G.; Bruschini, C.; Pazzagli, L.; Pieraccini, G.; Moneti, G. \& Chelazzi, G. (2001). Metabolic responses of the limpet Patella caerulea (L.) to anoxia and dehydration, Comparative Biochemistry and Physiology - Part A: Molecular E Integrative Physiology, 130, 1, 1-8, 1095-6433 
Schneider, K. R.; Van Thiel, L. E. \& Helmuth, B. (2010). Interactive effects of food availability and aerial body temperature on the survival of two intertidal Mytilus species, Journal of Thermal Biology, 35, 4, 161-166, 0306-4565

Smaldon, P. R. \& Duffus, J. H. (1984). The effects of temperaure, $\mathrm{pH}$ and salinity on the maturation of gametes and fertilisation in Patella vulgata L, Journal of Molluscan Studies, 50, 3, 232-235, 0260-1230

Smee, D. L.; Ferner, M. C. \& Weissburg, M. J. (2010). Hydrodynamic sensory stressors produce nonlinear predation patterns, Ecology, 91, 5, 1391-1400, 0012-9658

Somero, G. N. (2002). Thermal physiology and vertical zonation of intertidal animals: Optima, limits, and costs of living, Integrative and Comparative Biology, 42, 4, 780-789, 1540-7063

Sorte, C. J. B. \& Hofmann, G. E. (2004). Changes in latitudes, changes in aptitudes: Nucella canaliculata (Mollusca : Gastropoda) is more stressed at its range edge, Marine Ecology Progress Series, 274, 263-268, 0171-8630

Sousa, W. P. (1979). Disturbance in Marine Intertidal Boulder Fields: The Nonequilibrium Maintenance of Species Diversity, Ecology, 60, 6, 1225-1239, 0012-9658

Sousa, W. P. (1984). Intertidal mosaics: patch size, propagule availability, and spatially variable patterns of succession, Ecology, 65, 6, 1918-1935, 0012-9658

Southward, A. J. (1958). Note on the temperature tolerance of some intertidal marine animals in relation to environmental temperatures and geographic distribution., Journal of the Marine Biological Association of the United Kingdom, 37, 49-66, 0025-3154

Southward, A. J. (1995). The importance of long time-series in understanding the variability of natural systems, Helgoländer Meeresuntersuchungen, 49, 329-333, 0017-9957

Spotila, J. R.; Lommen, P. W.; Bakken, G. S. \& Gates, D. M. (1973). A Mathematical Model for Body Temperatures of Large Reptiles: Implications for Dinosaur Ecology, The American Naturalist, 107, 955, 391-404, 00030147

Stephenson, T. A. \& Stephenson, A. (1949). The universal features of zonation between tidemarks on rocky coasts, Journal of Ecology, 38, 289-305, 0022-0477

Wethey, D. S. (1984). Sun and shade mediate competition in the barnacles Chthamalus and Semibalanus - a field experiment, Biological Bulletin, 167, 1, 176-185, 0006-3185

Wethey, D. S. (2002). Biogeography, competition, and microclimate: the barnacle Chthamalus fragilis in New England, Integrative and Comparative Biology, 42, 872-880, 1540-7063

Wethey, D. S. \& Woodin, S. (2008). Ecological hindcasting of biogeographic responses to climate change in the European intertidal zone, Hydrobiologia, 606, 1, 139-151, 00188158

Yamane, L. \& Gilman, S. E. (2009). Opposite responses by an intertidal predator to increasing aquatic and aerial temperatures, Marine Ecology-Progress Series, 393, $27-$ $36,0171-8630$ 


\title{
Nanoparticle Synthesis in Vesicle Microreactors
}

\author{
Peng Yang and Rumiana Dimova \\ Department of Theory and Bio-Systems, Max Planck Institute of Colloids and Interfaces, \\ Science Park Golm, 14424, Potsdam, \\ Germany
}

\section{Introduction}

Numerous microorganisms such as E-coli and yeast are capable of synthesizing inorganic micro- or nano-structures including gold, silver, CdS, ZnS and calcium/silicon-based materials in their intra- or extra-cellular matrix (Sanchez et al, 2005; Mandal et al, 2006; Bhattacharya et al, 2005). Even though the widespread speculations propose that a few enzymes or peptides present in the organic matrix (mainly in cell walls and periplasmic space) act as reducing and nucleation sites (Mukherjee et al, 2001; Ahmad et al, 2002; Dameron et al, 1989; Umetsu et al, 2005; Kröger et al, 2006; Naik et al, 2002), the molecular basis for the biosynthesis of these materials is not well established. Currently, there are two main directions of research in this field. The first one, as widely developed by Naik's group, for review see e.g. (Dickerson et al, 2008), is to use the technique of phage display library to screen potential peptide sequences which could selectively recognize inorganic and metal ions for material synthesis. Alternatively, the second direction is to directly perform chemical and physical structural analysis on biomolecules from biological organisms (Evans, 2008; Killian et al, 2008; George et al, 2008) and eventually mimic them by synthetic molecules (Kato et al, 2010; Meldrum and Cölfen et al, 2008; Sommerdijk et al, 2008). A progressive step along this second direction was recently reported by Nagasawa, Kato et al about calcium carbonate synthesis mediated by the Pif protein found in the pearl oyster Pinctada Fucata (Suzuki et al, 2009).

Although the above-described in vitro studies have been extensively reported, in vivo tests of the material mediation abilities of the biological and synthetic molecules may offer a potential for biologically inspired material synthesis (Naik et al, 2008). For this purpose, another key focus besides functional templating molecules is how to encapsulate (envelop) these molecules in biological organisms for performing targeted mediation functions. Nature already gives us a good answer to this question, that is, the cell membrane provides a good encapsulation function of enveloping and microcompartmentalization of reactive and functional molecules (Sweeney et al, 2004; Kloepfer et al, 2005; Mukherjee et al, 2001). Thus, a desirable future research direction aims at encapsulating functional or reactive molecules in cell membranes and/or analogous model systems for evaluating their biomimetic mediation ability for material fabrication in vitro and in vivo. For instance, Iverson et al reported an interesting finding that semiconductor CdS nanocrystals, around $2-5 \mathrm{~nm}$ in diameter could be formed intracelluarly by E-coli (Sweeney et al, 2004). However, direct use of the cell imposes obstacles, because of the following factors: First, cells, even the simplest 
form, have complex supramolecular structure, which usually shields the desired reaction from direct observation. Second, their fragile body also makes it difficult to apply harsh characterization techniques on them, which otherwise are widely employed in artificial materials science. Therefore, a good approach to overcome these difficulties is to use simplified cell analogs as an alternative to perform such studies.

Vesicles could be considered as an unique kind of "a simplified cell", i.e. an artificial container enclosed by a self-assembled envelop (Dimova et al, 2006 and 2007; Smith et al, 2007; Tang et al, 2006; Christensen et al, 2007; Hales et al, 2006; Venturolia et al, 2006; Jelinek et al, 2007; Luisi, 2007; Morigaki et al, 2007; Walde et al, 2010). This kind of self-assembled envelope mimics the basic skeleton of the cell membrane, while excluding many other complex functional blocks embedded in it. Accordingly, this kind of structure is very interesting and promising for biologically inspired research and future applications. It has been demonstrated that vesicles are able to provide a very flexible model for cell-based functional researches, such as photosynthesis, enzyme function profiling, gene synthesis and expression etc. (Tung et al, 2003; Walde et al, 2001; Yukito et al, 1996; Luisi 2007). Whereas direct research on material synthesis in cells is difficult and complex, performing similar reactions in vesicles could be expected to be much simpler and straightforward. Moreover, the construction of functional artificial cells becomes possible by performing bottom-up synthetic reactions in vesicles. In this chapter, we introduce one of the first attempts to construct such a model system for step-by-step investigation toward cell-based nanoparticle synthesis.

The molecules to form such bilayer self-assembled vesicular structures are various including diblock copolymers, lipids, polypeptides and surfactants (Discher et al, 1999, Pochan et al, 2004; Lorenceau et al, 2005; Angelova et al, 1986; Tangirala et al, 2007; González-Pérez et al, 2007; Holowka et al, 2007; Bellomo et al, 2004). Here, we focus on lipid vesicles. Lipid bilayer vesicles can be multilamellar or unilamellar, with various sizes ranging from nanometers to micrometers. The difference between multilamellar and unilamellar vesicles, as the names suggest, is that the former consists of a cavity enclosed by a multi-layer structure, while the latter represents a container formed by a single bilayer. Multilayer vesicles could provide a good accommodation space for ions and other reactive solutes binding to their multilayer walls, while high quality unilamellar vesicles with a single bilayer structure provide a closer analogue to cells than multilamellar vesicles. Unilamellar vesicles could be further categorized according to their sizes into three types: small unilamellar vesicles (SUV), large unilamellar vesicles (LUV) and giant unilamellar vesicles (GUV). The former two categories have sizes up to hundreds of nanometers while the latter one has dimensions in the range up to hundred micrometers. Various methods such as hydration, extrusion and electroformation have been developed for vesicle preparation. The hydration method usually produces a mixture of multilamellar and unilamellar vesicles. Extrusion can be used to obtain SUV and LUV suspensions with a narrow size distribution and diameters in the hundred-nanometer range. Electroformation provides a convenient way to produce high quality and large amount of GUVs with diameters up to hundred micrometers (Angelova et al, 1986; Dimova et al, 2006; Walde et al, 2010).

Material synthesis in lipid vesicles is inherently related to the properties of the lipid membrane. From the viewpoint of a cellular analogue, the lipid membrane in unilamellar vesicles has maximum structural proximity to the plasma membrane, thereby providing the most suitable type of simplified artificial cellular system for bio-inspired material synthesis research. However, some significant differences exist between pure lipid vesicles and the 
cell membrane. For example, the cell membrane is a complex assembly including many glycoproteins, peptides, and enzymes. These biomolecules can be involved in particular biological functions such as stimuli-responsive ion transport and gating through ion channels with on/off control across the cell membrane (Swartz, 2008). In contrast, the pure lipid membrane in unilamellar vesicles is a simple permeation barrier. Because of the absence of regulating proteins, in its natural state and without extra physical or (bio) chemical stimuli, this kind of lipid membrane is impermeable to ions and macromolecules, while water can freely permeate through the membrane to ensure osmotic balance. This lack of permeability to ions and other solutes allows lipid vesicles to function as a new type of confined micro- or nano-biological reactors for micro- or nano-material synthesis.

The synthesis in confined micro/nano-containers (Shchukin et al, 2004) can be divided into two main types: one involves exact templating where the product has the same shape and size as the container, and the other - not. Exact templating is usually achieved in SUVs and LUVs, and until now, nearly most of the reported syntheses of particles in the nanometer range have been realized in this way (Korgel et al, 1996 and 2000; Rafaeloff et al, 1985; Khramov et al, 1993). However, in order to obtain a clear picture about bio-fabrication processes in cells with much bigger sizes e.g. in the micrometer scale, such synthesis in nano-reactors does not provide a suitable model. Certainly, an important factor affecting the reaction and the final product is the dimensions of the physical confinement in which this reaction is carried out. Thus, some significant features of the underlying mechanism of biofabrication in cells may be lost when using LUV or SUV nanoreactors as a reaction model. On the contrary, performing inorganic synthesis reactions in GUVs should be expected to shed light on cell-based nanoparticle synthesis and the corresponding mechanism, since GUVs have dimensions in the cell-size scale (micrometer).

Until now, the investigations on various biological activities using GUVs as prototypes of cells have been widely performed covering many aspects: preparation (Angelova et al, 1986; Larsen et al, 2003; Takakura et al, 2003; Mohanty et al, 2003; Pautot et al, 2003), membrane related processes like fusion, fission, budding (Walde et al, 2010; Wang et al, 2010; Hanczyc et al, 2004), cellular processes and mechanisms like adhesion, communication, endocytosis, exocytosis (Marrink et al, 2003; Haque et al, 2001; Chen et al, 2005; Rustom et al, 2004; Menger et al, 1992, 1997 and 2002; Hanczyc et al, 2003; Espinoza et al, 1999; Ichikawa et al, 2004; Davidson et al, 2003), structure and shape transformation (Suezaki, 2002; Sasaki et al, 2004; Boon et al, 2002; Lee et al, 2005; Hamada et al, 2005; Tomšiè et al, 2005; Brückner et al, 2001), drug release (Menger et al, 1998; Barragan et al, 2001; Park et al, 2000; Sun et al, 2003; Vandenburg et al, 2002), micromanipulation (Karlsson et al, 2001; Marmottant et al, 2003), compartmentation (Jesorka et al, 2005; Bucher et al, 1998; Nomura et al, 2001) and microreactors (Vriezema et al, 2005; Tung et al, 2003; Walde et al, 2001; Morgan et al, 1997; Esch et al, 1986; Kang et al, 2003; Krafft et al, 2001; Moffitt et al, 1995; Kommareddi et al, 1996; Rassy et al, 2005; Regev et al, 2004; Faure et al, 2003; Nishikawa et al, 2004; Kim et al, 2000; Wu et al, 2005; Monnard et al, 2003; Fischer et al, 2002; Tsumoto et al, 2001; Nomura et al, 2003; Noireaux et al, 2004; Yu et al, 2001). The last application, namely GUVs as microreactors, has been developed in some realms such as enzymecatalyzed reactions (Walde et al, 2001; Yukito et al, 1996), photosynthesis reaction (Tung et al, 2003), biochemical reaction (Luisi, 2007), polymerization (Morgan et al, 1997; Esch et al, 1986; Kang et al, 2003; Krafft et al, 2001), inorganic particle synthesis (Moffitt et al, 1995; Kommareddi et al, 1996; Rassy et al, 2005; Regev et al, 2004; Faure et al, 2003; Nishikawa et al, 2004; Kim et al, 2000; Wu et al, 2005), gene (Monnard, 2003; Fischer et al, 2002; Tsumoto 
et al, 2001) and protein synthesis (Nomura et al, 2003; Noireaux et al, 2004; Yu et al, 2001). The new research aspect of nanoparticle synthesis in GUVs described in this chapter pertains also to this category. The reaction volume for such bio/chemical reactions in GUVs is typically on the order of femto- or picoliters, depending on the sizes of the GUVs used. Obviously, the features of the tiny nanometer products of such reactions would depend on the overall shape of the container, and the reaction initiation and pathway. Syntheses in GUVs also have the unique exclusive advantage that the vesicle containers and the corresponding product inside them may be monitored in real time under a light microscope. This on-line monitoring enables us to capture in situ some important information about material growth and reaction kinetics relevant among others to cell-based synthesis and representing frontier research topics in current chemical and material sciences.

The research based on the use of GUVs as microreactors for synthesis of inorganic nanoparticles inside GUVs was firstly initiated in 2006, and reported officially in 2009 (Yang et al, 2009). To the best of our knowledge, the studies introducing similar approaches are scarce. Namely, a proposal concerning the possibility of using electroporation of GUVs in order to synthesize inorganic nanoparticles in the vicinity of the lipid membrane was made by Schelly (Schelly 2007). Thus, the breakthrough reported in 2009 is the first report on the use of GUVs as confined containers for performing the synthesis of inorganic semiconductor nanocrystals. Briefly, we succeeded in inducing, controlling and directly observing the formation of CdS quantum dots and nanoparticles in GUV, an artificial cell system whereby the membrane container remains intact. Our study, for the first time, extended confined vesicular reactions to micrometer-scale cell-size reactors for the synthesis of nanomaterials. Differently from the drastic experimental conditions used previously for the synthesis of nanoparticles in vesicles, the processes we employed in this report are quite simple and mild, and effectively mimic intracellular mixing and membrane fusion, which naturally occur in cells.

One of the implementation pathways is to induce the reaction between two reactants loaded in two different GUVs. One GUV contains a solution of $\mathrm{CdCl}_{2}$ and the other contains $\mathrm{Na}_{2} \mathrm{~S}$. The synthesis of CdS nanoparticles is then simply initiated by conducting electrofusion between these two kinds of vesicles. This electrofusion-based synthesis is a good model for cellular fusion-based reaction systems. Another pathway involves an attempt to mimic the cell culturing-based systems, often used in bacteria-based inorganic nanoparticle synthesis. GUVs are in-situ grown in electroformation chambers, where they remain attached to the substrate via lipid nanotubes. The initial solution contained in the GUVs is $\mathrm{Na}_{2} \mathrm{~S}$, and the synthesis reaction of CdS nanoparticles is initiated by gradually replacing the external solution with $\mathrm{CdCl}_{2}$ solution, which enters the GUVs via the nanotubes. This is a good mimic to bacteria culturing, however, with accelerated speed. Below, we describe these two protocols in details. We emphasize that they can be applied to arbitrary reactants (not necessarily $\mathrm{CdCl}_{2}$ and $\mathrm{Na}_{2} \mathrm{~S}$ ) provided suitable concentration conditions are selected.

\section{Experimental section}

Growing GUVs via Electroformation. Giant vesicles made of L- $\alpha$-phosphatidylcholine from egg yolk (Egg-PC, Sigma, St. Louis, MO) were grown using the electroformation method (Angelova et al, 1986, Dimova 2006). In principle, the choice for the lipid is not limited to this lipid and any phosphatidylcholine (or a mixture of phosphatidylcholines), which is in the fluid phase at room temperature may be employed. Egg-PC was our choice because it is 
a natural and abundant lipid. The procedure for electroformation used here is described in detail elsewhere (Riske et al, 2005). Briefly, Egg-PC was dissolved in chloroform to form 2 $\mathrm{mg} / \mathrm{ml}$ lipid solution. For observation of the vesicles with fluorescence microscopy the following dyes were used: 1,1'-dioctadecyl-3,3,3',3'-tetramethylindocarbocyanine perchlorate $\left(\mathrm{DiIC}_{18}\right)$ from Molecular Probes (Leiden, The Netherlands; excitation wavelength at $551 \mathrm{~nm}$ and emission wavelength at $569 \mathrm{~nm}$ ) was added to the lipid solution at concentration $0.1 \mathrm{~mol} \%$, and perylene from Sigma-Aldrich (Steinheim, Germany; with excitation wavelength at $440 \mathrm{~nm}$ and emission wavelength at $450 \mathrm{~nm}$ ) was added at concentration 0.4 mol \%. Typically, for the $\mathrm{CdCl}_{2}$-loaded vesicles we used $\mathrm{DiIC}_{18}$ as fluorescence marker and for the $\mathrm{Na}_{2} \mathrm{~S}$-loaded vesicles we used perylene. A small drop $(\sim 50 \mu \mathrm{l})$ of lipid solution was placed onto a glass slide coated with indium tin oxide (ITO) and spread evenly on the surface. Two such coated ITO glasses were placed in a vacuum desiccator at room temperature for at least $2 \mathrm{~h}$ to evaporate the organic solvent. For the vesicles grown in the presence of $\mathrm{CdCl}_{2}$, we found out that drying at higher temperature $\left(60^{\circ} \mathrm{C}\right)$ yields more and bigger vesicles. A closed chamber was assembled from the two ITO glasses (the sides with conductive coating were facing each other) and a $1 \mathrm{~mm}$ thick rectangular Teflon spacer with two holes as solution inlet and outlet. The $\mathrm{CdCl}_{2-}$ and $\mathrm{Na}_{2} \mathrm{~S}$-loaded vesicles for the electrofusion protocol were typically grown in parallel. The growing solutions of $\mathrm{CdCl}_{2}$ or $\mathrm{Na}_{2} \mathrm{~S}$ in 100 mOsm sucrose were introduced through the inlet to fill the respective chamber. The chambers were connected in parallel to an alternating current (AC) field function generator (Agilent 33220A $20 \mathrm{MHz}$ function/arbitrary waveform generator) and an alternating voltage with amplitude of $0.7 \mathrm{~V}$ (peak-to-peak) and frequency of $10 \mathrm{~Hz}$ was applied for $4 \mathrm{~h}$. The magnitude of the $\mathrm{AC}$ voltage was a key parameter for the formation of $\mathrm{CdCl}_{2}$-loaded vesicles. In $0.03 \mathrm{mM} \mathrm{CdCl}_{2}$ solution, $\mathrm{GUV}$ s were formed at low voltage $(<1.7$ $\mathrm{V})$. At higher voltages, the vesicles would start rupturing. However, for electroformation of $\mathrm{Na}_{2} \mathrm{~S}$-loaded vesicles, even in $3 \mathrm{mM} \mathrm{Na} 2 \mathrm{~S}$ solution, this effect was not observed. Presumably, $\mathrm{CdCl}_{2}$ affects the membrane properties making the bilayers either more fragile or inducing additional tension, as the binding of $\mathrm{Cd}^{2+}$ effectively impeded the separation of lipid film, which is an essential condition for electroformation of GUVs.

Electrofusion Protocol for Synthesis of CdS Quantum Dots. After the completion of the electroformation process, the vesicles were detached from the ITO glass substrate by lowering the field frequency to $5 \mathrm{~Hz}$ and setting the voltage to $0.5 \mathrm{~V}$ for $20 \mathrm{~min}$. Then, the vesicles were removed from the electroformation chamber, diluted up to 10-40 times with isotonic glucose solution, and transferred into an electrofusion chamber (Eppendorf, Germany). The effective dilution is important for lowering the concentration of $\mathrm{CdCl}_{2}$ or $\mathrm{Na}_{2} \mathrm{~S}$ outside the vesicles and to ensure no interference of neighboring vesicles in the image when confocal imaging is performing. The glucose-sucrose asymmetry creates a refraction index difference between the interior and the exterior of the vesicles. The glucose-sucrose asymmetry also creates a density difference stabilizing the vesicles at the bottom of the chamber. The latter enhances the contrast of the phase-contrast microscopy images (vesicle images appear dark on a bright background). The electrofusion chamber consists of a Teflon frame with two cylindrical electrodes (500 $\mu \mathrm{m}$ in diameter) with a gap distance of $200 \mu \mathrm{m}$. The chamber was connected to a Multiporator (Eppendorf, Germany) to align the vesicles (AC field of $3 \mathrm{~V}, 2 \mathrm{MHz}$ for $90 \mathrm{~s}$ applied a few times) and electrofuse them (direct current pulses of field strength $0.5-2 \mathrm{kV} / \mathrm{cm}$ and $150-300 \mu$ duration). The observation of the vesicles was performed with an Axiovert135 microscope (Zeiss, Jena, Germany) equipped with 20x and 40x phase-contrast objectives. The fusion process was also monitored by a 
laser scanning confocal microscope (Leica, TCS SP5, Germany) with excitation at $458 \mathrm{~nm}$ (Ar laser) for perylene and $561 \mathrm{~nm}$ (DPSS laser) for DiIC 18 .

Batch Electrofusion for Synthesis of CdS Quantum Dots in Large Scale. Before doing batch electrofusion, the vesicles swelled in $\mathrm{CdCl}_{2}$ solution were incubated with an ion-exchange resin (Amberlite IR-120, $\mathrm{H}^{+}$form, Sigma-Aldrich, Germany) to remove the $\mathrm{Cd}^{2+}$ ions in the vesicle exterior media. The resin was carefully washed and activated according to the instructions of the manufacturer. Before use, the exchanging ability of the resin was checked by UV-Vis spectra to guarantee the highest performance of the resin. After removing the cadmium ions outside the GUVs, the vesicles solution with $\mathrm{CdCl}_{2}$ entrapped in the vesicles was mixed with the solution of vesicles loaded with $\mathrm{Na}_{2} \mathrm{~S}$. The mixture was then exposed several times to electric pulses in a helix fusion chamber (Eppendorf, Germany). Immediately after that, the solution was analyzed with a series of techniques including fluorescence spectra analysis (luminescence spectrometer LS50B with excitation at $400 \mathrm{~nm}$, Perkin Elmer, Beaconsfield, England) and transmission electron microscopy (TEM) (Omega 912 TEM, Carl Zeiss, Oberkochen, Germany, with $100 \mathrm{kV}$ accelerating voltage).

Slow Content Exchange Protocol for Synthesis of CdS Nanoparticles. A programmable syringe pump was used to slowly exchange the solution in the exterior of the vesicles in the electroformation chamber at a controlled rate. To ensure complete solution replacement, at least four times the volume of the chamber was flowed through it. We typically electroformed the vesicles in $\mathrm{Na}_{2} \mathrm{~S}$ aqueous solutions and then replaced these solutions with $\mathrm{CdCl}_{2}$ solutions. The osmotic balance across the vesicle membranes, and thus the preservation of the vesicle volumes was ensured by the presence of e.g. $100 \mathrm{mM}$ glucose in the salt solutions. Because the salt concentrations used were very low (on the order of 1 $\mathrm{mM}$ ), the osmolarity of the initial $\mathrm{Na}_{2} \mathrm{~S}$ solution and the exchanging solution are nearly the same as confirmed by osmolarity measurements (Osmomat030, Gonotec, Berlin). During and after the exchange, the solution flown out of the chamber and the one inside the chamber were carefully collected and examined with fluorescent spectroscopy and TEM.

\section{Results and discussion}

\subsection{Electrofusion protocol}

For the general interest, before reporting results from the electrofusion protocol, we will introduce a short discussion on the background about this protocol.

For the simplest " $\mathrm{A}+\mathrm{B}$ " type reaction (that is, the reaction is initiated when reactant $\mathrm{A}$ encounters reactant B), three models could be summarized to describe possible protocols when utilizing vesicles as microreactors (Figure 1). In Model 1, all reactants are incorporated into the vesicle interior or the membrane bilayer and the reaction occurs at the membrane or inside the vesicle. In Model 2, reactant A (such as an enzyme) is incorporated into the vesicle membrane or interior and reactant $B$ (such as a substrate) is dispersed in the media surrounding the vesicles. The reaction is then initiated when reactant $B$ diffuses across the membrane. Actually, nearly all of the work reported in the literature is based on these two approaches (Korgel et al, 1996 and 2000; Ralaeloff et al, 1985; Khramov et al, 1993). Up to now, there are only few reports on reactions realized via the fusion of two vesicles encapsulating reactant $\mathrm{A}$ and $\mathrm{B}$, respectively (Model 3). The corresponding feature of this model is that the triggering mechanism for the reaction between $A$ and $B$ to occur is vesicle fusion. 


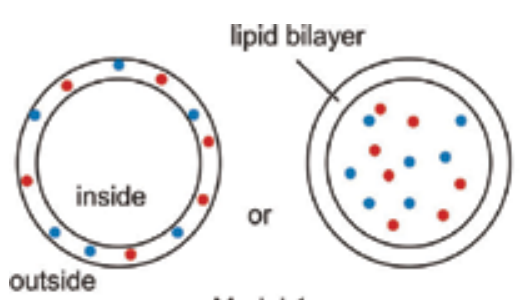

Model 1

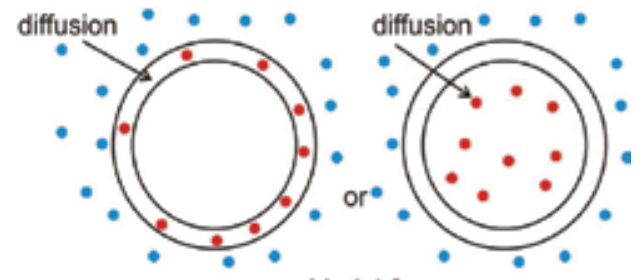

Model 2

- reactant A reactant B
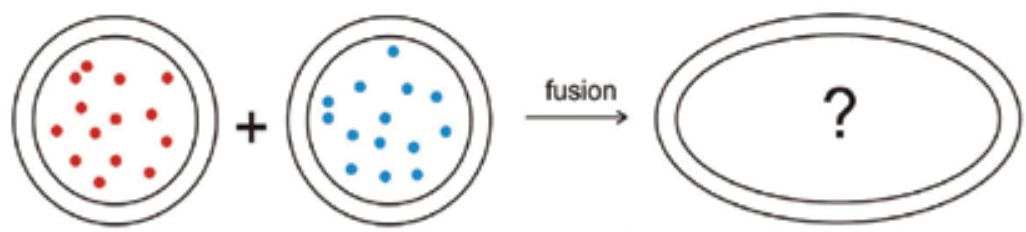

Model 3

Fig. 1. Three models for reactions taking place in vesicles.

In nature, fusion is a spontaneous and important event, by which a myriad of physicochemical processes occur (Leabu et al, 2006; Chernomordik et al, 2006; Jahn et al, 2006, Wang et al, 2006; Tsaadon et al, 2006; Ogura et al, 1995; Wilmut et al, 1997) such as fertilization from egg-sperm fusion, formation of multinucleated muscle cells from fusion of myoblasts during embryonic development, signaling cascades. Fusion has been also widely observed in artificial and natural colloidal systems from crystal growth via droplet condensation to lipid vesicle (Strömberg et al, 2000; Riske et al, 2006) or polymersome (Zhou et al, 2004 and 2005; Yan et al, 2004; Vriezema et al, 2003) recombination.

For fusion-initiated nanoparticle synthesis, the starting complementary reactants are separately loaded into different colloids, and then the reaction is triggered by the fusion of these reactive colloids to make the reactants meet each other. An instant benefit for this strategy is that the precise temporal and spatial control on the synthesis process could be easily achieved: these colloidal cartridges could be stored or transported to any place and then the reaction can be initiated at a desired time point or location. Successful examples of this approach have been reported for fabrication of semiconductor nanoparticles in microfluidic channels through the fusion of droplets containing the starting reactants (Shestopalov et al, 2004; Hung et al, 2006). Moreover, in a recent report, Beaune et al described potential bioimaging ability for tumor treatment when vesicles are entrapped with fluorescent magnetic nanoparticles and quantum dots (Beaune et al, 2007).

An important condition for successful fusion-initiated reactions is to stimulate the vesicles in order to obtain an effective fusion event since vesicle fusion is inefficient in the absence of external stimuli. Fusion can be induced artificially by means of membrane stress (Cohen et al, 1982; Shillcock et al, 2005), ions or organic reagents (Estes et al, 2006; Neil et al, 1993; Lentz, 2007; Haluska et al, 2006; Kunishima et al, 2006), DNA (Stengel et al, 2007), proteins (Jahn et al, 2006; Peters et al, 1999 and summary by Walde et al, 2001), laser beams (Steubing et al, 1991; Weber et al, 1992; Kulin et al, 2003) or electric fields (Strömberg et al, 2000). The last one is termed electrofusion. Among these fusion methods, electrofusion becomes increasingly important due to its reliable, fast and easy handling procedure (Riske et al, 2006). This method utilizes discrete intense electric pulses $(\mathrm{kV} / \mathrm{cm})$ of short duration $(\mu \mathrm{s})$. 
The electrical breakdown of the membranes of cells or vesicles which are in contact can lead to fusion. The process is completed within milliseconds. Due to its general applicability and mild conditions, electrofusion has been extensively used and the procedures further developed for many years (Strömberg et al, 2000) in a wide variety of biological experiments with cells and vesicles, from the creation of hybridomas and new cell lines to in vitro fertilization and the production of cloned offspring, like the sheep Dolly and her equals (Zimmermann et al, 1986). Genetic and biochemical reactions, such as DNA transcription, translation and gene expression, are often achieved by fusion. According to Zimmermann (Zimmermann et al, 1986), the electrofusion process could even have been an important step in evolution after the first simple cells had come into being. Therefore, it is expected that the research on electrofusion-based reactions and chemistry in vivo or in vitro, could have a great development potential in many fields such as origin of life and birth, biomineralization, bioengineering, cell science, tissue engineering, bio-inspired and new materials synthesis.

Electrofusion-based reactions in GUVs strongly rely on the physical behavior (deformation, poration and fusion) of these vesicles subjected to either alternating current field or strong direct current (DC) pulses. In recent years, our group has systematically investigated this topic by experimental and theoretical modeling (Riske et al, 2005; Riske et al, 2006; Haluska et al, 2006; Dimova et al, 2007; Aranda et al, 2008; Riske et al, 2009; Vlahovska et al, 2009; Dimova et al, 2009; Yamamoto et al, 2010). Based on the findings from our and other research groups, successful electrofusion is based on four important features of the membrane and the vesicles: 1) The lipid membrane is impermeable to reactants (ions and macromolecules), while water can freely permeate through the membrane to assure osmotic balance; 2) Fast and effective fusion could be initiated with a short electric pulse with characteristics above the poration threshold of the membrane; 3) Leakage of reactants during the fusion process is negligible (Riske et al, 2005); 4) Vesicles with complementary reactants can be brought together to form a reactive couple for electrofusion. Feature 1) - 3) stem from the intrinsic properties of lipid membranes, while the last one, feature 4), is inherent to giant vesicles and can be achieved either via micromanipulation or exposure of the GUV suspension to AC fields causing vesicle alignment due to dielectric screening.
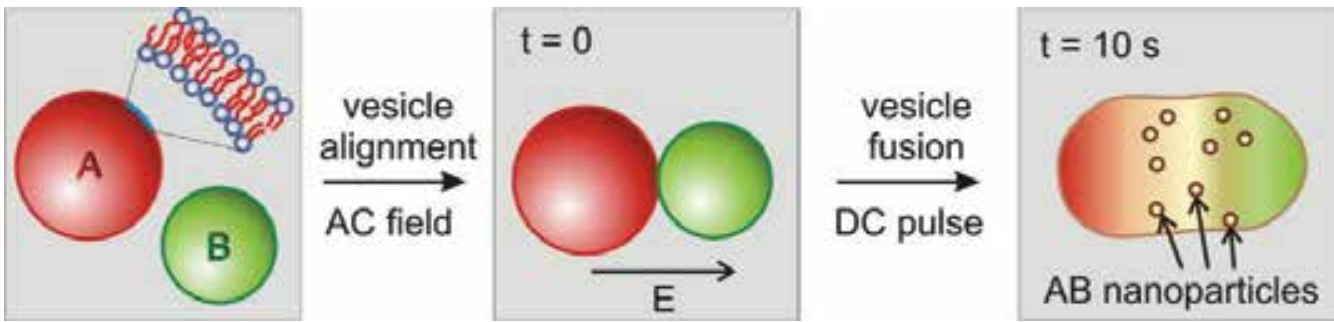

Fig. 2. Electrofusion of vesicles as a method for nanoparticle synthesis: vesicles containing reactant A or B are mixed (in A- and B-free environment) and subjected to an AC field to align them in the direction of the field and bring them close together. A DC pulse initiates the electrofusion of the two vesicles and the reaction between $A$ and $B$ proceeds to the formation of nanoparticles encapsulated in the fused vesicle. Reproduced from (Yang et al, 2009). Copyright Wiley-VCH Verlag GmbH \& Co. KGaA. Reproduced with permission.

The concept of utilizing electrofusion of two GUVs to initiate a reaction was first proposed by Chiu, Zare et al (Chiu et al, 1999), who used a microelectrode to trigger electrofusion. The study showed that ultrafast reaction kinetics could be conveniently investigated because 
electrofusion takes place only within milliseconds and the mixing of reactants upon fusion could be very fast ( $\mu \mathrm{s})$. Kulin et al (Kulin et al, 2003) further pointed out that this method may be a useful tool for quantitative studies in combinatorial chemistry involving only femtoliters of reagents. In the latter report ultraviolet laser light was used to fuse two liposomes $(10 \mu \mathrm{m}$ in size) containing chemical reagents and the encapsulated materials could be accurately conserved without leakage to the external media.

Different from the above-mentioned approaches, we used the electrofusion process to conduct nanoparticle synthesis. As shown in Figure 2, two couples of hybrid GUVs, a $\mathrm{CdCl}_{2}$-loaded GUV in red color (A, Cd-GUV) and $\mathrm{Na}_{2} \mathrm{~S}$-loaded GUV in green color (B, Na-GUV) could be effectively aligned by AC field and fused with a DS pulse. Similar to pearl-chain formation in cell suspensions subjected to an AC field (Zimmermann, 1986), the two types of vesicles are brought together and align along the direction of an exogenous AC field. This field-induced self-arrangement makes reactive vesicles match well for the CdS synthesis reaction: half of the aligned vesicle couples are A-B couples. After that, a strong DC pulse (typically pulses of 0.5 $2 \mathrm{kV} / \mathrm{cm}$ field strength and $150-300 \mu$ s duration suffice) is applied to initiate electrofusion between $\mathrm{A}$ and B. Electrofusion proceeds smoothly to form one hybrid GUV where mixing of $\mathrm{CdCl}_{2}$ and $\mathrm{Na}_{2} \mathrm{~S}$ occurs for the $\mathrm{CdS}$ nanoparticle synthesis. The product, in this case, quantumdot-like CdS nanoparticles, is visualized under laser excitation as a fluorescent bright spot in the fusion zone (Figure 3). Subsequent inspection of vesicle sections above and below the
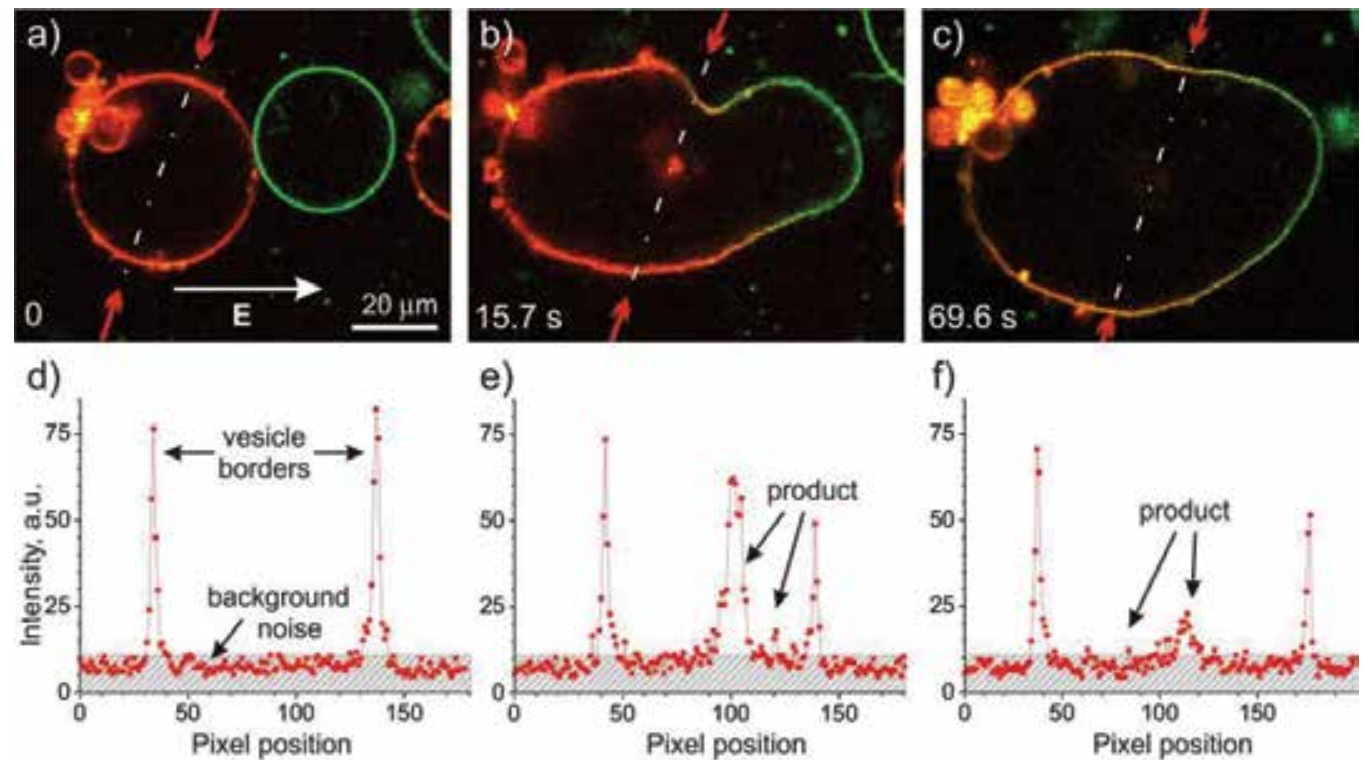

Fig. 3. a) - c) Electrofusion of vesicles as a method for nanoparticle synthesis: confocal scans of vesicles loaded with $0.3 \mathrm{mM} \mathrm{Na} \mathrm{S}_{2}$ (green) and $0.3 \mathrm{mM} \mathrm{CdCl}_{2}$ (red) undergoing fusion. d) - f) Intensity line profiles along the dash-dotted lines indicated by red arrows in a) - c), respectively. The direction of the field is indicated in a) with an arrow. Before fusion, a), d), the vesicle interior shows only background noise similar to the external solution as indicated by the shaded zone in d). After fusion, b), c), e), f), fluorescence from the product is detected in the interior of the fused vesicle. The time after applying the pulse is indicated on the micrographs. The fluorescence signal was acquired in the ranges $565-765 \mathrm{~nm}$ (red channel) and 462 - 558 nm (green channel). Reproduced from (Yang et al, 2009). Copyright Wiley$\mathrm{VCH}$ Verlag $\mathrm{GmbH} \& \mathrm{Co}$. KGaA. Reproduced with permission. 
scanning plane showed no presence of membrane inclusions (free vesicles or membrane fractions) in the fusion zone. Therefore, the source of fluorescence is provided by the formed nanoparticles. The intensity from this spot increases gradually from $0 \mathrm{~s}$ (the starting time point of electrofusion), and reaches maximum around $10 \mathrm{~s}$. We thus infer that the reaction begins at the electrofusion point and quickly reaches a balance around $10 \mathrm{~s}$. This signal then decays over time (Figure 3 and 4). The mechanism behind the decay of the signal from the CdS product is still not clear at present and under investigation.

During the investigation on this electrofusion approach, we also studied the effect of salt concentration on the process of vesicle electroformation and on the effectiveness of electrofusion. In the concentration range from $0.00003 \mathrm{mM}$ to $3 \mathrm{mM}, \mathrm{Na}_{2} \mathrm{~S}$ solutions showed weak effect on the electroformation of GUVs. In contrast, $\mathrm{CdCl}_{2}$ solutions showed obvious salt-related influence on the electroformation, particularly at concentrations above $0.03 \mathrm{mM}$. The total effect could be summarized as shrinking of the diameter of Cd-GUVs and decrease in the vesicle yield. The reason for this could be related to the binding of $\mathrm{Cd}^{2+}$ ions to the polar headgroups of the lipids in the membrane effectively impeding the separation (swelling) of the lipid film from the substrate, which is an essential condition for electroformation of GUVs. We further found that fusion of GUVs loaded with $\mathrm{CdCl}_{2}$ solution at concentration above around $0.1 \mathrm{mM}$ is not effective and was rather inhibited (Figure 5). On the other hand, GUVs loaded with $\mathrm{Na}_{2} \mathrm{~S}$ at similar concentrations showed
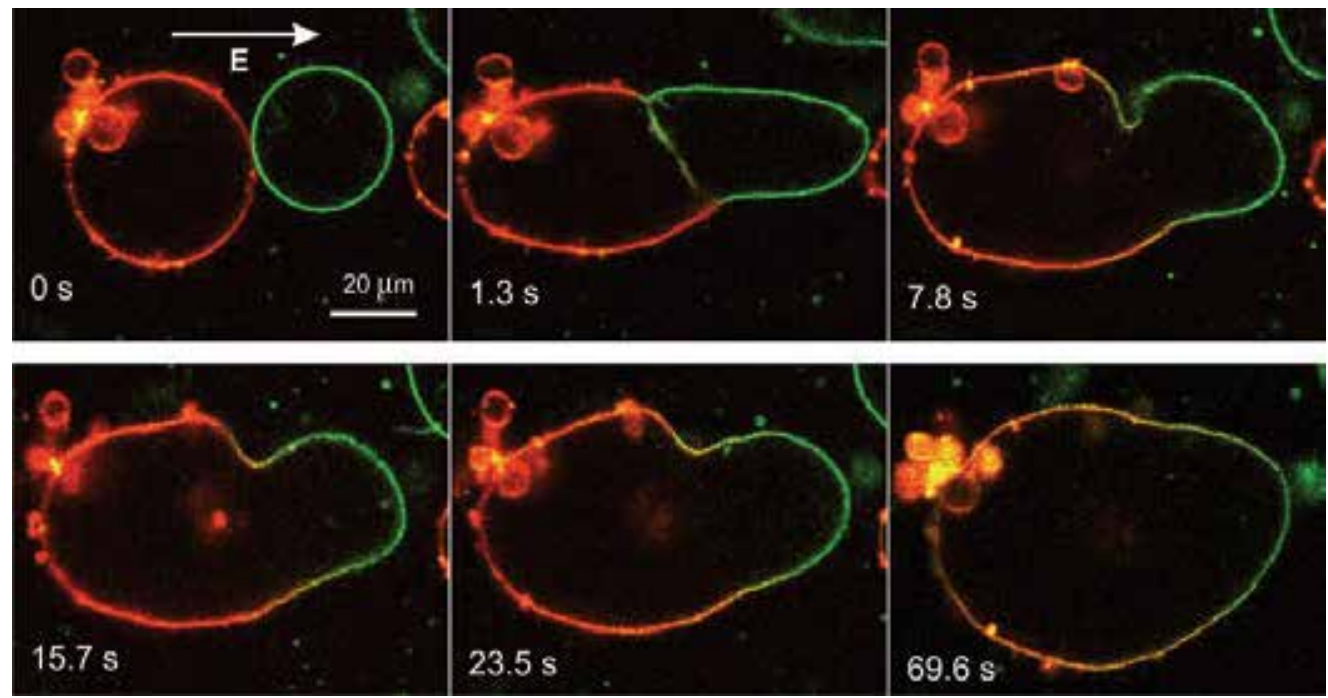

Fig. 4. Confocal scans of vesicles loaded with $0.3 \mathrm{mM} \mathrm{Na}_{2} \mathrm{~S}$ (green) and $0.3 \mathrm{mM} \mathrm{CdCl}_{2}$ (red) undergoing fusion after being exposed to an electric pulse (excitation wavelengths: $458 \mathrm{~nm}$ and $561 \mathrm{~nm}$ ). The fluorescence signal has been acquired in the ranges $565-765 \mathrm{~nm}$ (red channel) and 462 - $558 \mathrm{~nm}$ (green channel). The field direction is indicated by the arrow in the first snapshot. The acquisition time is indicated on every image, where $t=0 \mathrm{~s}$ corresponds to the last snapshot before applying the pulse. Several seconds after fusion, a fluorescence signal is detected from the product in the vesicle interior (note that only fluorescence from objects located in the focal plane is visible in the images; particles out of focus are not detected). The signal, both from the membrane and from the vesicle interior, decays with time, and the mechanism for the latter decay is under investigation. 


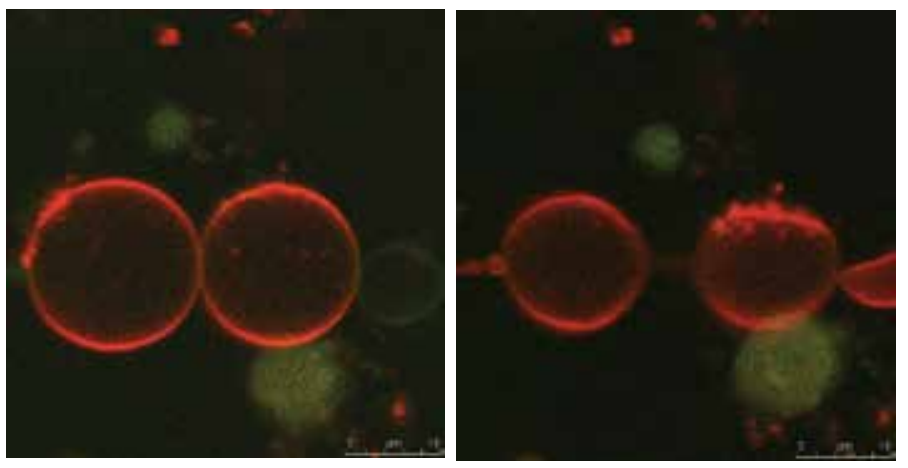

Fig. 5. Confocal scans of the electrofusion between two GUVs loaded with $3 \mathrm{mM} \mathrm{CdCl}_{2}$ solution. As shown in this figure, the electrofusion between two GUVs- $\mathrm{CdCl}_{2}$ could not occur under high salt concentrations, e.g. 0.3 and $3 \mathrm{mM}$. However, the electrofusion of $\mathrm{CdCl}_{2}$ loaded vesicles could proceed under lower salt concentration such as $0.03 \mathrm{mM}$.
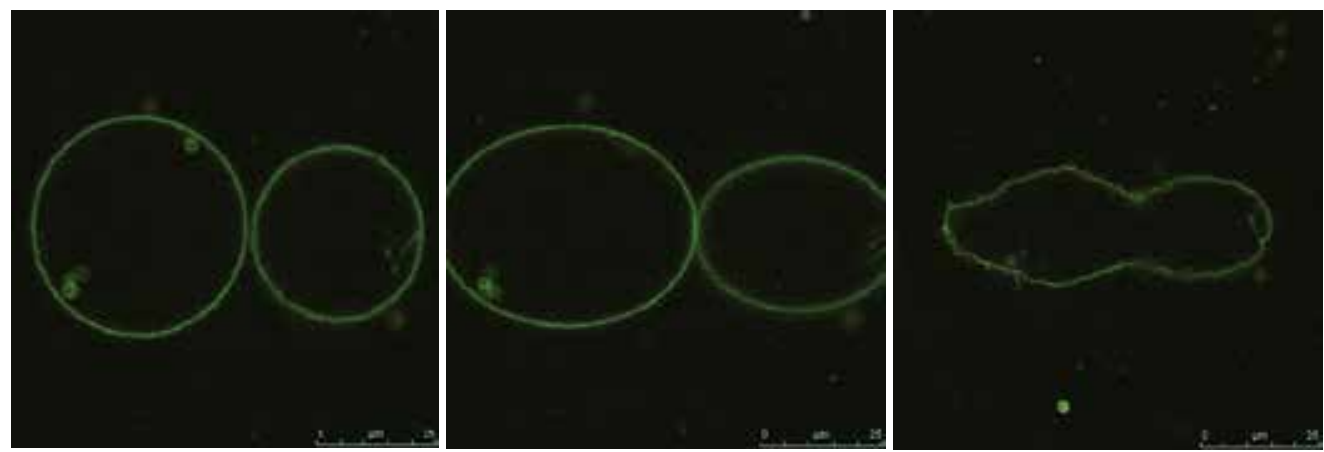

Fig. 6. Confocal scans of the electrofusion between two GUVs loaded with $3 \mathrm{mM} \mathrm{Na} 2 \mathrm{~S}$ solution. As shown in this figure, the electrofusion between two GUVs encapsulating $\mathrm{Na}_{2} \mathrm{~S}$ could proceed smoothly under high salt concentrations.

normal electrofusion behavior (Figure 6). A possible interpretation for the inability of CdGUVs to fuse is that $\mathrm{Cd}^{2+}$ ions could influence the mechanical properties of the membrane and in particular hindering the formation of a fusion pore (through its divalent binding to the pore surface), which is a necessary intermediate stage during electrofusion. However, attempts for electrofusion between two hybrid GUVs (a Cd-GUV and a Na-GUV) loaded with $0.3 \mathrm{mM}$ salt solutions, demonstrated that electrofusion could proceed smoothly. This showed that the commonly accepted fusion pore-mediated mechanism may not be necessarily valid for electrofusion.

After successfully exploring single electrofusion events, we applied this method to batch electrofusion in order to achieve large-scale synthesis of nanomaterials. The latter is beneficial for practical applications. In addition, analytical methods like TEM, selected area electron diffraction (SAED) and fluorescence measurements are easier to apply to samples containing a large fraction of fused vesicles and thus a larger amount of the fusion product. In order to increase the yield from the electrofusion protocol we applied a special electrofusion chamber where a larger fraction of vesicles could be fused. This was achieved by increasing the volume of the concentrated vesicle solution located between the two 
electrodes. For this purpose, we used a helix fusion chamber (Eppendorf, Germany) (Figure 7 ), which consists of two coiled helical electrodes with a gap distance of $250 \mu \mathrm{m}$ and a total inter-electrode volume of $250 \mu \mathrm{l}$. This volume is more than 20 times larger than the one between the two straight cylindrical electrodes used for the microscopy experiments, thus allowing higher yields.

Another important prerequisite for the successful batch electrofusion is to remove the free $\mathrm{Cd}^{2+}$ ions from the exterior of the $\mathrm{CdCl}_{2}$-loaded vesicles before mixing them with the $\mathrm{Na}_{2} \mathrm{~S}-$ loaded ones. The reason is that the presence of reactive $\mathrm{Cd}^{2+}$ ions in the GUV exterior would result in an undesired reaction between $\mathrm{Cd}^{2+}$ and $\mathrm{S}^{2-}$ outside the vesicles when mixing the two vesicle populations. Apart from that, this undesired reaction would further influence the osmotic balance across the vesicle membrane potentially leading to vesicle rupture. Therefore, the $\mathrm{CdCl}_{2}$-loaded vesicles were placed in contact with an ion-exchange resin (Amberlite). The resin was thoroughly washed beforehand to remove small molecular impurities ( 3 times in deionized water), then activated according to the instructions of the manufacturer (acid wash with $2 \mathrm{~N} \mathrm{HCl}$, at least 3 times), and rinsed at least 10 times with deionized water to $\mathrm{pH}>5$. The vesicles electroformed in $\mathrm{CdCl}_{2}$ solution were then added to the resin at optimized condition $(200 \mathrm{mg}$ resin for $2 \mathrm{ml} \mathrm{CdCl}$-loaded GUV solution under very slow stirring for $1 \mathrm{hr}$ ). After the removal of the $\mathrm{Cd}^{2+}$ ions outside the vesicles, $1 \mathrm{ml}$ solution of Cd-GUV was mixed with $1 \mathrm{ml}$ solution of $\mathrm{Na}-\mathrm{GUV}$. Half of the mixture was used as a reference sample, while the rest was subjected to electrofusion in the helix fusion chamber in 4 portions of $250 \mu \mathrm{l}$ each. The AC field $(9 \mathrm{~V}, 2 \mathrm{MHz})$ was applied to each portion for about $15 \mathrm{~min}$, followed by one pulse of $300 \mathrm{~V}$ and $150 \mu$ s duration. The 4 aliquots were then collected and subjected to further analysis using TEM and fluorescence spectroscopy.
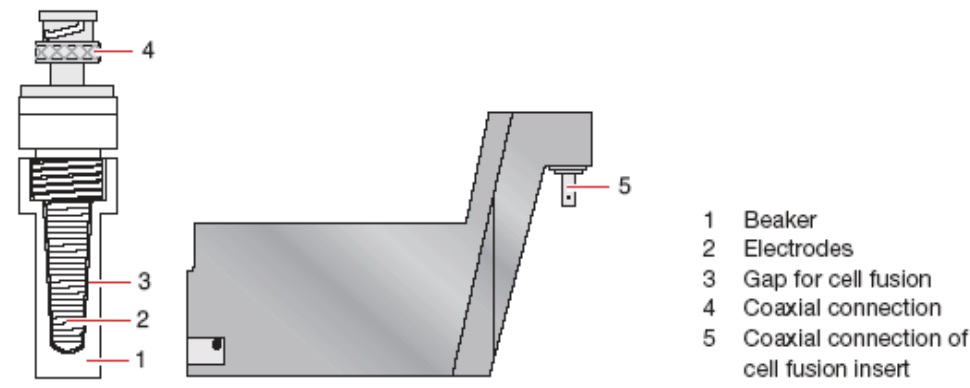

Fig. 7. The schematic illustration of the helix electrofusion chamber. Two populations of GUVs loaded with $\mathrm{CdCl}_{2}$ or $\mathrm{Na}_{2} \mathrm{~S}$ are mixed and filled into the space of beaker (1). After sealing, the chamber is connected to an electronic function generator providing AC or DC fields. After setting the desired AC and DC programs, the helix electrode (2) in the beaker is used to apply AC and DC fields for GUV alignment and electrofusion. After the filed application, the solution in the beaker is gently taken out and subjected to further spectroscopy analysis.

The TEM images from the mixed samples before applying a DC pulse showed that they contained only some irregular formless sediment (Figure 8), which is commonly found in samples produced by the direct mixing of $\mathrm{CdCl}_{2}$ and $\mathrm{Na}_{2} \mathrm{~S}$ solutions without any confinements. The SAED pattern showed two polydisperse diffraction rings, which are due to multiple overlapping from the following CdS crystalline lines: (100), (002), (101) for the inner diffraction ring and (110), (103), (112) for the outer ring (Li et al, 2003). This implies 
that the product was polycrystalline with poor crystalline orientation. Polycrystalline CdS products before electrofusion originate from the TEM sample preparation process: during the preparation process, Cd-GUVs and Na-GUVs break upon the evaporation of the water, and their $\mathrm{CdCl}_{2}$ and $\mathrm{Na}_{2} \mathrm{~S}$ content is released so they can react with each other along common mixing ways. In contrast, in the samples to which a DC pulse was applied, we found only dispersed tiny particles (Figure 9). Their diameters are lower than $10 \mathrm{~nm}$ and their SAED pattern showed the unique feature of monocrystallines (dotted pattern). The diameter of these CdS nanocrystals is lower than the Bohr radius of a CdS particle, and therefore in the quantum dot size regime. As also observed in the above-described confocal microscopy experiments, the emerging fluorescent emission in the interior of the fused vesicle formed upon the electrofusion of a Cd-GUV and a Na-GUV is attributed to the formation of these monocrystallines with quantum confinement effect for fluorescence emission. The fluorescent spectra discussed below further supported the photoluminecent property from these CdS quantum dots.
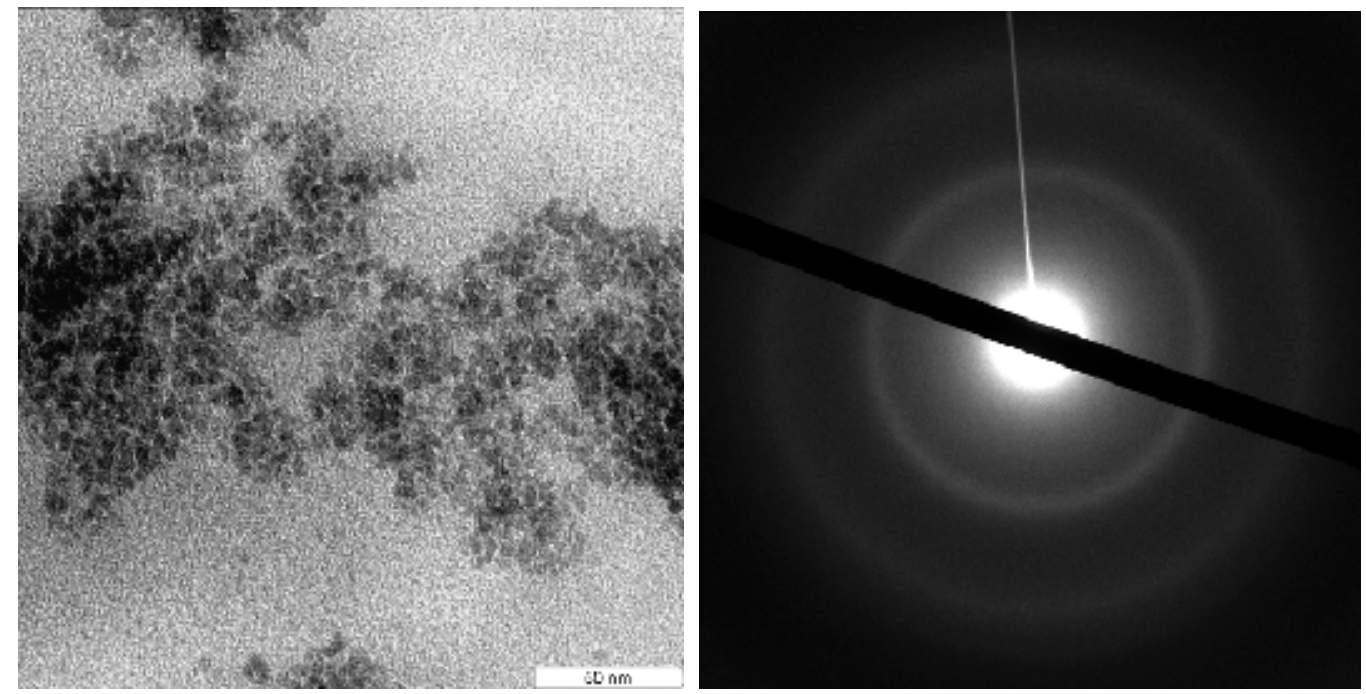

Fig. 8. A TEM image and a SAED pattern from a solution of mixed $\mathrm{Na}_{2} \mathrm{~S}$ - and $\mathrm{CdCl}_{2}$-loaded vesicles not subjected to electrofusion. The reagent concentration in the vesicles was $0.3 \mathrm{mM}$. Only irregular sediments with pour crystalline structure are formed due to vesicle rupture upon deposition on the TEM grid and subsequent content mixing.

Fluorescence spectra further demonstrated the formation of CdS nanocrystallines (Figure 10). Before applying a DC pulse, the fluorescence signal showed only a very small peak around $460 \mathrm{~nm}$, which is from the Na-GUV solution itself. After applying the DC pulse to the mixed solution, enhanced fluorescence signal was observed at $460 \mathrm{~nm}$, which can be attributed to the band gap emission and suggests high quality of the nanocrystals (Korgel et al, 2000; Khramov et al, 1993; Gratt et al, 2003). The enhanced fluorescence signal confirmed that a reaction has occurred after applying the pulse. In addition, the peak site around 460 $\mathrm{nm}$ demonstrated blue shift from the fluorescence peak of bulk CdS materials $(600 \mathrm{~nm})$, which further reflects quantum confinement effects by the $10 \mathrm{~nm}$ particles observed in TEM. It should be mentioned that this enhanced fluorescence had poor reproducibility when the vesicle solutions were not handled carefully. This could be attributed to the fragility of 

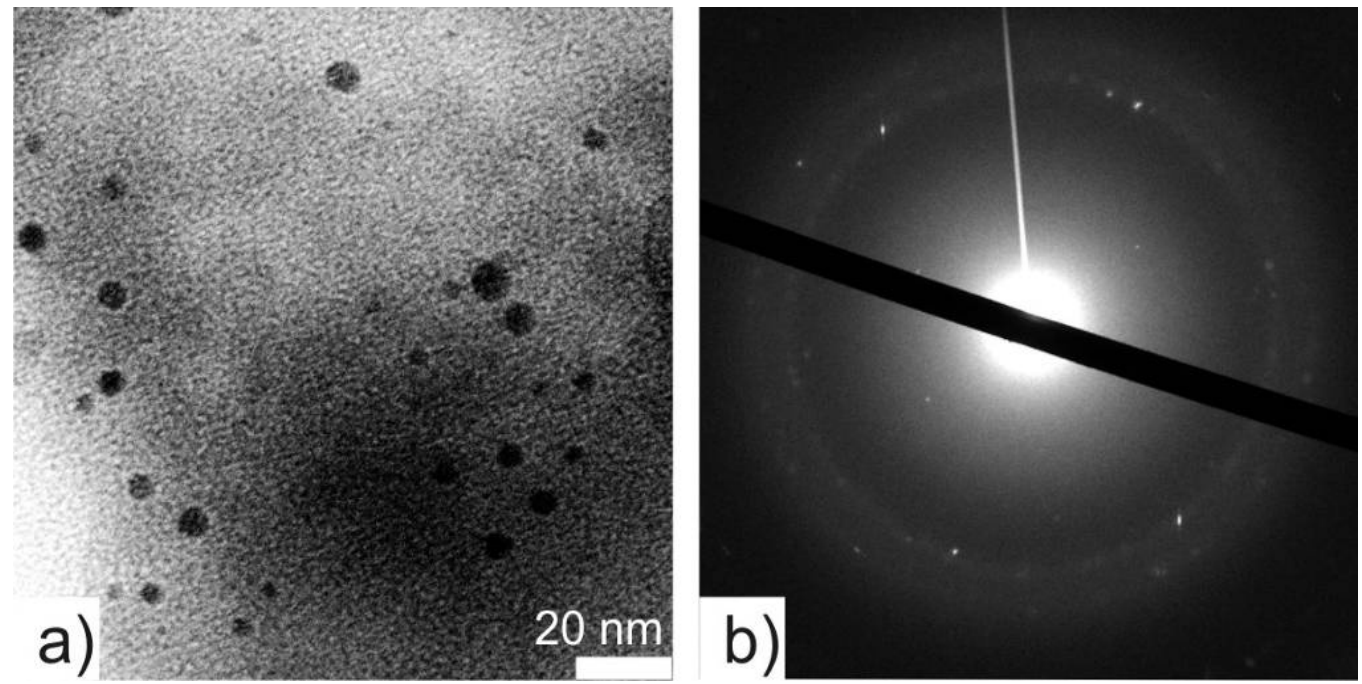

Fig. 9. a) TEM image and b) SAED pattern from the product in the solutions after vesicle fusion. The salt concentration in the vesicles was $0.3 \mathrm{mM}$. Dispersed single-crystalline nanoparticles with diameter between 4 and $8 \mathrm{~nm}$ are detected. Reproduced from (Yang et al, 2009).Copyright Wiley-VCH Verlag GmbH \& Co. KGaA. Reproduced with permission.

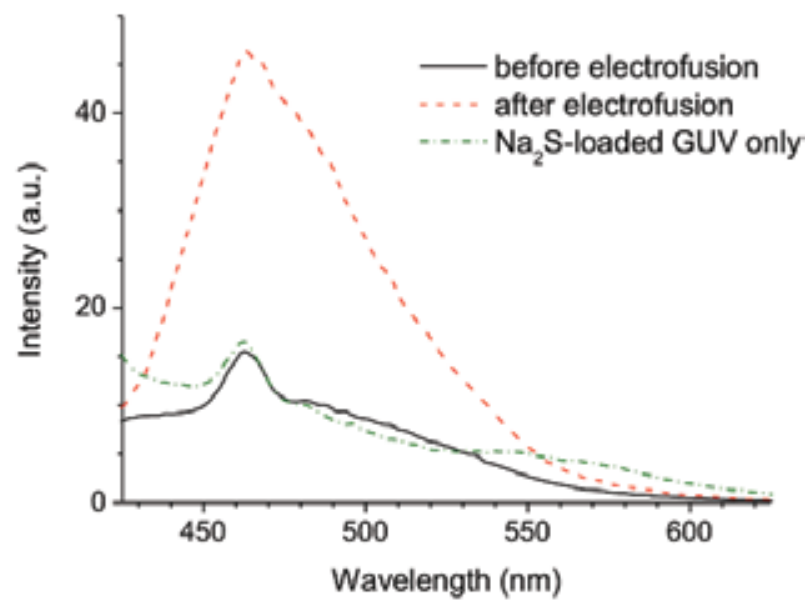

Fig. 10. Fluorescent spectra of the samples before (solid black curve) and after electrofusion (dashed red curve). As a control test, the fluorescence signal from $\mathrm{Na}_{2} \mathrm{~S}$-loaded GUV solution is also plotted (dash-dotted green curve). The excitation wavelength is $400 \mathrm{~nm}$. The resulting fluorescent emission at around $460 \mathrm{~nm}$ is strongly enhanced after electrofusion.

GUVs, which often results in undesirable breaking of the membranes and release of reactants. The absence of emission at higher wavelengths (up to $700 \mathrm{~nm}$ ) associated with deep trapped states due to surface or core defects, indicates the high quality of the nanocrystals. It should be also noted that for the fluorescent spectra measurements, the excitation wavelength was $400 \mathrm{~nm}$ while the confocal scans in Fig. 3-4 were obtained by excitation with a laser at $458 \mathrm{~nm}$. Therefore, the emission wavelength under confocal scanning exhibits a red-shift compared to that in the fluorescent spectra measurements. 
Before we proceed with the second protocol for nanoparticle synthesis in GUVs, we will address some common features, advantages and application perspectives of the electrofusion approach.

Obviously, this protocol provides us with a visualizing analytical tool to follow the reaction kinetics with high temporal resolution (Figure 3-4). Previously, we have demonstrated that fast fusion processes can be visualized at microsecond timescales using high-speed digital imaging (Haluska et al, 2006). This novel and facile electrofusion method is suitable not only for inorganic nanoparticle synthesis, but also for the online monitoring of ultrafast physicochemical processes such as photosynthesis, enzyme catalysis and photopolymerization, which at present requires complex and abstracted spectroscopy techniques. Our results also show that even without the mediation of biomacromolecules, nanoparticles can still be synthesized in biological compartments. This outcome provides a new insight in the developing research on biomineralization mechanisms.

Theoretically, the electrofusion-initiated reaction could be extended to chemical reactions with any stoichiometric ratio, because a large amount of vesicles can be aligned in the electric field, so that chain or step-type reactions could be achieved by entrapping $A_{1}-A_{n}$ reactants into 1-n vesicles respectively. For this purpose, a key condition is the controlled vesicle arrangement to encode them at pre-designed pattern. As shown above, AC fieldinduced alignment of GUVs shows the potential to control this prearrangement. However, it is difficult to control the number and the type of the aligned vesicles in the case where more than two vesicles need to be fused. Other methods have been already developed in this direction. For example, preliminary experimental and theoretical models have been reported based on vesicle recognition by biotin-avidin and electrostatic interactions (Farbman-Yogev et al, 1998; Walker et al, 1997 and Chiruvolu et al, 1994). We propose an alternative protocol, which may be more easily accessible, namely, first patterning the vesicles onto a planar surface and then fusing these pre-organized vesicles with a second vesicle population to complete the reactions (Lee et al, 2008; Christensen et al, 2007; Kim et al, 2008). The first step has been achieved by some smart designs, e.g. direct microcontact printing of vesicles (Liu et al, 2007) and ink-jet printing (Hauschild et al, 2005).

A possibly problematic feature of the electrofusion-induced reaction which may hinder the wide application of this approach is that vesicles are difficult to prepare by the electroformation method if high reactant (salt) concentrations are required. The reason is that the ions in the solutions could bind to the lipid headgroups suppressing the interbilayer separation and vesicle swelling. This effect was also observed in our experiments, showing that high concentrations of $\mathrm{Cd}^{2+}$ hinder electroformation and electrofusion of GUV$\mathrm{Cd}$. To overcome the problem facing electroformation at high salt concentration, Estes and Mayer et al developed a two-step approach: first, electroforming vesicles in solutions of low ionic strength and second, exchanging the solution inside the vesicles with a high ionic strength solution (Estes et al, 2006). More recently, a modification of the electroformation method was reported where high frequency AC field $(500 \mathrm{~Hz})$ was used to achieve effective electroformation under physiological conditions with sodium salts. (Montes et al, 2007; Pott et al, 2008) Concerning the difficulty of achieving electrofusion when hindered due to ion binding to the membrane, other fusion protocols may be utilized such as those based on the use of fusogenic molecules and laser beams. Further possible improvement of the protocol and allowing a better control over the reaction involves the use of monodisperse vesicles rather than polydisperse GUV samples used at present. The procedures of micro-contact printing (Taylor et al, 2003; Liu et al, 2007) and extrusion (Tangirala et al, 2007) have shown 
certain potential to prepare vesicles or droplet populations with low polydispersity. Other interesting and effective methods, which have emerged recently include ink-jet printing for small vesicles (50-200 nm) (Hauschild et al, 2005), double emulsion template made by glasscapillary microfluidics (Shum et al, 2008; Kuroiwa et al, 2009) and direct pulsed microfluidic jetting (Stachowiak et al, 2008).

Fusion-based synthesis is a highly multidisciplinary and open project, with the close interplay between chemistry (biological, polymer and inorganic) and physics (biomembrane and polymer physics). This field can be broadened by exploring systematically a series of reactions initiated by the fusion of vesicles containing separated reactants. Some unexplored reactions include, but are not limited to, the biosynthesis of genes (RNA and DNA), proteins and carbohydrates in vesicles; enzyme-catalyzed reactions; biomineralization processes; host-guest interactions. The above investigations may open new ways of biosynthesis and give fundamental and valuable information about cell bioactivity during life origin, growth, organ and tissue formation; mimic bioactivities of a living cell; provide fundamental information for the role of functional interfaces in biomineralzation in cells and some important aspects such as the detailed role of proteins in the synthesis of inorganic products, and finally, evaluate the biomineralization process in vivo.

\subsection{Solution exchanging protocol}

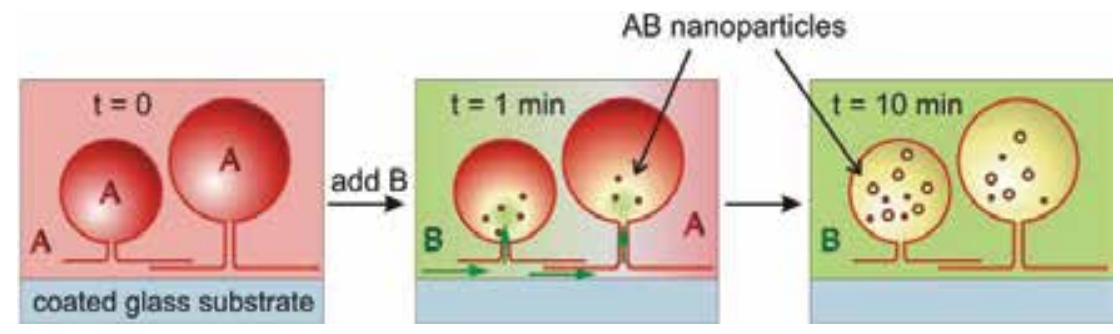

Fig. 11. Slow content exchange method for performing inorganic nanomaterial synthesis in GUVs: Vesicles formed in the presence of $\mathrm{A}\left(\mathrm{Na}_{2} \mathrm{~S}\right)$ are still connected via nanotubes to the glass substrate of the electroformation chamber (see also the Fig. 12). The thickness of the nanotubes (tens of $\mathrm{nm}$ ) and the size of the vesicles (tens of $\mu \mathrm{m}$ ) are not in scale. Reactant $\mathrm{B}$ $\left(\mathrm{CdCl}_{2}\right)$ is slowly injected in the chamber. After diffusing through the nanotubes into the vesicle interior, $\mathrm{B}$ reacts with $\mathrm{A}$ to produce nanoparticles. The approximate timescales of the events are indicated in the pictures. Reproduced from (Yang et al, 2009). Copyright Wiley$\mathrm{VCH}$ Verlag GmbH \& Co. KGaA. Reproduced with permission.

Another protocol we used is based on the solution exchange around $\mathrm{Na}_{2} \mathrm{~S}$-loaded GUV with a solution of $\mathrm{CdCl}_{2}$ (Figure 11). In this approach, a microreactor array was formed on an ITO surface where the GUVs in $\mathrm{Na}_{2} \mathrm{~S}$ solution were attached to the substrate via nanotubes. The freshly prepared $\mathrm{CdCl}_{2}$ solution could be gradually introduced into these vesicles via the nanotubes connecting them to the ITO surface. These nanotubes or tethers provide a good bridge connecting the GUV interior and the external media. They are clearly visible from microscopy images (Figure 12). The visualization of the tethers is achieved by preparing the vesicles on cylindrical platinum electrodes where side view observation of the growing vesicles is possible with xy-scans (contrary to the case when the vesicles are grown on ITO plates where z-scans are necessary to observe the tethers; the latter was not possible due to the large thickness of the ITO substrates used). Typically, these tethers can be broken (releasing the vesicles) if during the vesicle electroformation the frequency of the applied 
AC field is reduced to about $5 \mathrm{~Hz}$ for ten minutes. For the protocol used here, we did not apply this latter step. Instead, the electroformation chamber that contained the vesicles tether-bound to the substrate was connected to a polyvalent syringe pump (Lambda Vit-fit, the Czech Republic), which slowly injected $\mathrm{CdCl}_{2}$ solution into the chamber containing the $\mathrm{Na}_{2} \mathrm{~S}$-loaded GUVs without detaching the vesicles from the substrate. The injection speed influences the stability, shape and transformation of the attached GUVs (Estes et al, 2005 and 2006). The optimal injection speed was about $1 \mathrm{ml} / \mathrm{min}$. At this speed, the vesicles not connected to the ITO substrate via nanotubes flow out of the chamber. At the same time, the injection speed cannot be very quick in order to prevent the rupture of the surface-attached vesicles. As a result, the total amount of vesicles decreases.

After the introduction of $\mathrm{CdCl}_{2}$ into the chamber, the cadmium ions slowly diffuse into the vesicle interior via the nanotubes. In this way, the CdS product is expected to form in the confined space inside the GUVs. Of course, the reaction proceeds also outside the GUVs because of the present $\mathrm{Na}_{2} \mathrm{~S}$ in the vesicle exterior. Thus, the solution initially flown out of the chamber contains the CdS produced in the vesicle exterior. To ensure complete replacement of the external solution, the total injection volume was at least four times the volume of the chamber. Finally, an AC field with low frequency $(5 \mathrm{~Hz})$ was applied for 20 min to detach the GUVs from the ITO substrate and this sample was collected separately for inspecting the product formed within the vesicles.

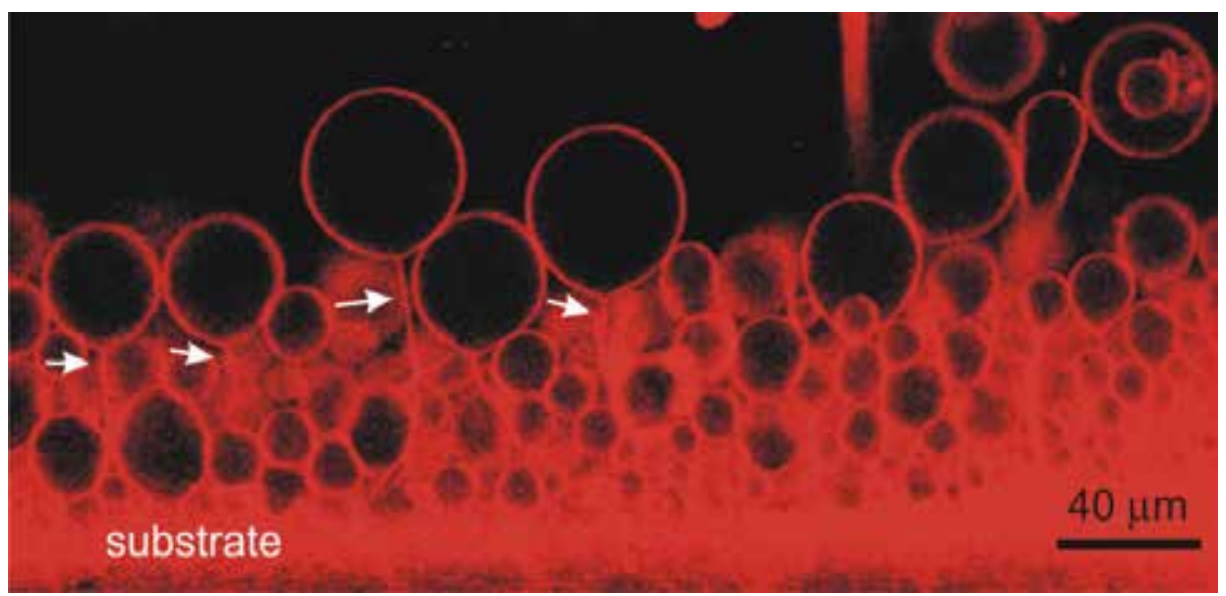

Fig. 12. Confocal scan through a chamber with vesicles electroformed on cylindrical electrodes. At the end of the preparation procedure, the vesicles remain connected to the substrate (electrode) via nanotubes (indicated by arrows). Note that tethers, which are slightly behind or in front of this scanning plane, are not imaged.

The fluorescence spectra of the solutions collected from the chamber (Figure 13) clearly indicated that after exchange, the solution in the chamber with GUV grafted to the surface showed an obvious increase of the fluorescence signal (red dashed line) compared to that from the solution flown out of the chamber before detaching the GUVs (black solid line). The fluorescent peaks emerged at two different sites: 460 and $570 \mathrm{~nm}$. Compared with the fluorescent peak from bulk CdS material $(600 \mathrm{~nm})$, obvious fluorescent blue shift showed that certain quantum confinement effect existed in the samples from the chamber. This indicated that CdS nanocrystallines are present in the sample after the solution exchange. 
We performed a parallel (reference) experiment to reflect the key role of the grafted GUVs. The same injection protocol was performed in a chamber containing no GUVs (i.e. using blank ITO glass without lipid coating). The obtained result showed that the solution in the chamber without GUVs present yields fluorescence signal (green dotted line) similar to that flown first outside the chamber. Both of these signals are also similar to the signal intensity obtained from the direct mixing of the two salt solutions.

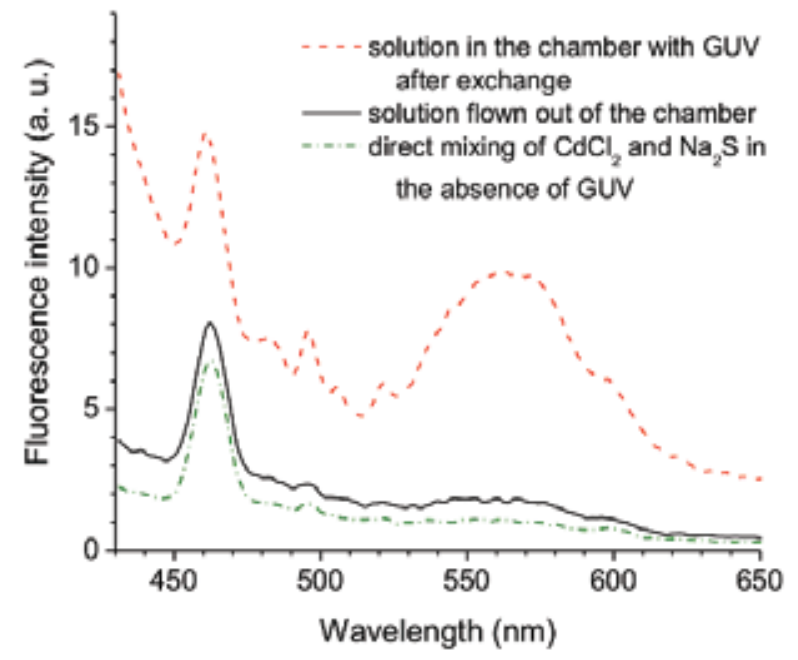

Fig. 13. Fluorescent spectra of the sample containing the vesicles and the one flown out of the chamber. The solution in the chamber with GUVs after the content exchange protocol (dashed red curve) has significant absorption in the range 460-600 $\mathrm{nm}$ due to the formed nanoparticles. This signal is not observed from the solution flown out of the chamber (solid black curve). For comparison, the fluorescence spectra from direct mixing of $\mathrm{CdCl}_{2}$ and $\mathrm{Na}_{2} \mathrm{~S}$ solutions with the same concentration $(0.3 \mathrm{mM})$ is also plotted (dash-dotted green curve).

TEM images (Figure 15) provided further evidence for the formation of CdS nanoparticles by this solution exchange method. The nanoparticles had diameters around $50 \mathrm{~nm}$ and were polycrystalline as demonstrated by the two diffraction rings in the corresponding SAED pattern (Gorer et al, 1998). In contrast, the solution flown out of the chamber, which consisted of a mixture of the introduced $\mathrm{CdCl}_{2}$ and the reagent $\mathrm{Na}_{2} \mathrm{~S}$ from the vesicle exterior, contained only irregular sediment with poor polycrystalline structure (Figure 15). For the slow content exchange protocol, our setup did not allow us to perform confocal microscopy scans and image analysis as in Fig. 3-4 because of the large thickness of the substrate. Thus we were not able to identify whether the nanoparticles were formed in the vesicle volume or in the nanotubes connecting them with the substrate. However, the drastic difference between the morphology of CdS material formed inside and outside the vesicles strongly suggested that templating role was present in this exchange system.

Besides the chemical reaction discussed above, the solution exchange method also provides an alternative way to prepare GUVs with a desired salt entrapped at a high concentration. For example, one could first form GUV populations at a low salt concentration, and then slowly exchange the content of the tethered vesicles with a solution of high salt concentration. We further noticed that when high concentration of $\mathrm{CdCl}_{2}$ solution, e.g. 3 $\mathrm{mM}$, is used as an exchange solution, the vesicles tend to adhere to each other. This finding 
supports our explanation for the observation that Cd-GUVs could not be effectively grown and self-electrofused at high $\mathrm{Cd}^{2+}$ concentration (e.g. $3 \mathrm{mM}$ ). Presumably, electrostatic interactions of cadmium ions with the zwitterionic headgroups of the lipids increased the inter-membrane interaction and stiffness. Similar behavior from other divalent ions such $\mathrm{Ca}^{2+}, \mathrm{Mg}^{2+}$ have been also previously found to induce tension on the membranes and accordingly, a marked increase in the membrane-membrane interactions between adjacent GUVs (Akashi et al, 1996; Bockmann et al, 2004; Sinn et al, 2006; Pabst et al, 2007).
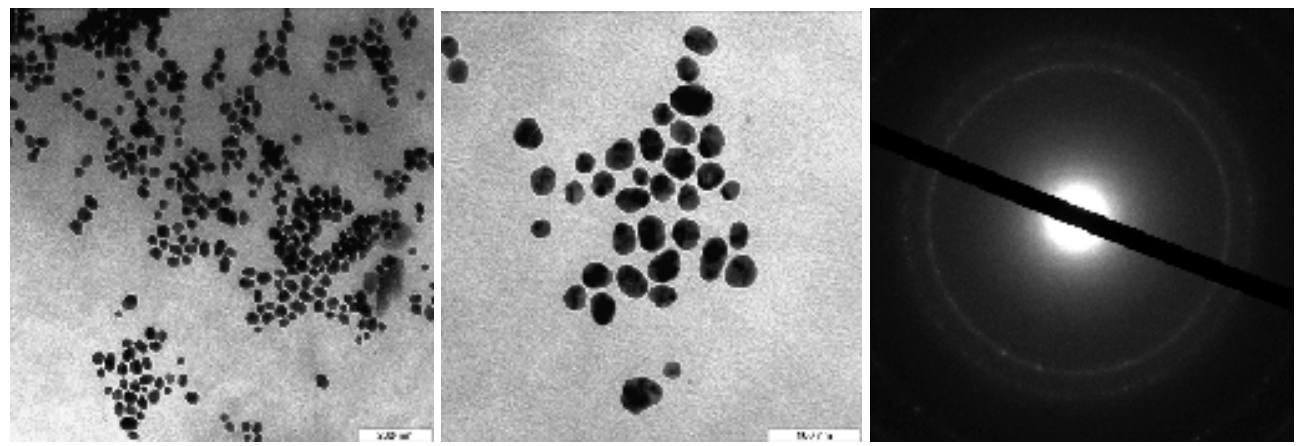

Fig. 14. TEM images (two left images) and a SAED pattern (right) from polycrystalline CdS nanoparticles in the chamber after the slow solution exchange. The salt concentration in the exchange solution and in the vesicles was $0.3 \mathrm{mM}$. The diameter of the nanoparticles is around $50 \mathrm{~nm}$. Reproduced from (Yang et al, 2009). Copyright Wiley-VCH Verlag GmbH \& Co. KGaA. Reproduced with permission.
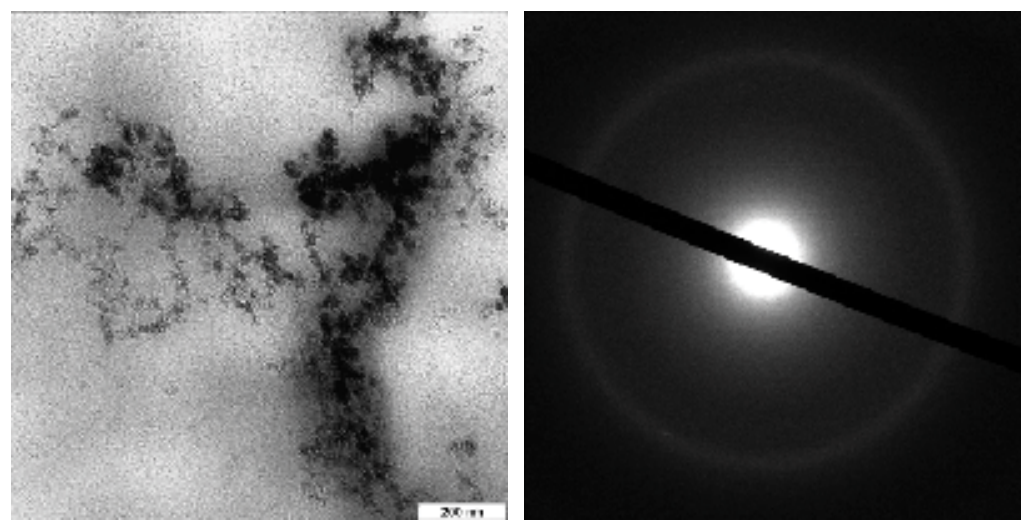

Fig. 15. TEM image (left) and SAED pattern (right) from sediments with poor polycrystalline structure in the solution flown out of the chamber. The reagent concentration in the exchange solution and in the vesicles was $0.3 \mathrm{mM}$.

\section{Conclusion and remarks}

To summarize, our results suggest that the possible mechanism of cell-based nanoparticle synthesis, whether intra- or extra-cellular, may not necessarily be only peptide- or proteindriven or regulated. Simple chemical mixing of subpicoliter volumes due to fusion of carrier vesicles with cell membranes or slow influx in the intracellular space may be the possible 
pathway of these syntheses. With the two protocols discussed above, the sizes of synthesized CdS nanoproducts could be tuned from 4 to $50 \mathrm{~nm}$ in diameter using two different protocols. The application and further improvement of the protocols may be sought in the following directions: 1) enhancing the yield and product quality for the electrofusion protocol can be achieved by applying batch electrofusion in optimized chambers; 2) optimization of the chamber substrate used in the solute exchanging protocol may allow us to elucidate the location (nanotubes of GUVs) for nanoparticle formation; 3) bio-organic/inorganic hybrid nanocomposite materials may also be prepared by our methods; 4) constructing GUV microreactor arrays on substrates can be employed for the construction of functional devices containing inorganic nano-functional blocks; 5) unexplored reactions, besides nanoparticle synthesis in GUV, include but are not limited to: biosynthesis of genes (RNA and DNA), proteins and carbohydrates in vesicles; enzymecatalyzed reactions; biomineralization processes; host-guest interactions.

\section{References}

Angelova, M. I.; Dimitrov, D. S. (1986). Liposome Electro formation. Faraday Discuss. Chem. Soc. 81, 303-311, ISSN 0956-5000.

Akashi, K.; Miyata, H.; Itoh, H.; Kinosita, Jr.,K. (1996). Preparation of giant liposomes in physiological conditions and their characterization under an optical microscope. Biophys. J. 71, 3242-3250, ISSN 0006-3495.

Ahmad, A.; Mukherjee, P.; Mandal, D.; Senapati, S.; Khan, M. I.; Kumar, R.; Sastry, M. (2002). Enzyme Mediated Extracellular Synthesis of CdS Nanoparticles by the Fungus, Fusarium oxysporum. J. Am. Chem. Soc. 124, 12108-12109, ISSN 0002-7863.

Aranda, S.; Riske, K. A.; Lipowsky, R.; Dimova, R. (2008). Morphological transitions of vesicles induced by AC electric fields. Biophys. J., 95, L19-L21, ISSN 0006-3495

Beaune, G.; Dubertret, B.; Clément, O.; Vayssettes, C.; Cabuil, V.; Ménager, C. (2007). Giant vesicles containing magnetic nanoparticles and quantum dots: feasibility and tracking by fiber confocal fluorescence microscopy. Angew. Chem. Int. Ed. 46, 54215424, ISSN 1433-7851.

Bockmann, R. A.; Grubmuller, H. (2004). Multistep Binding of Divalent Cations to Phospholipid Bilayers: A Molecular Dynamics Study. Angew. Chem. Int. Ed. 43, 1021-1024, ISSN 1433-7851 (print), 1521-3773 (online).

Bhattacharya, D.; Gupta, R. K. (2005). Nanotechnology and Potential of Microorganisms. Critical Reviews in Biotechnology 25, 4, 199-204, ISSN 0738-8551.

Bucher, P.; Fischer, A.; Luisi, P. L.; Oberholzer, T.; Walde, P. (1998). Giant vesicles as biochemical compartments: the use of microinjection techniques. Langmuir 14, 27122721, ISSN 0743-7463.

Barragan, V.; Menger, F. M.; Caran, K. L.; Vidil, C.; Morèreb, A.; Monterob, J. L. (2001). A mannose-6-phosphonate-cholesterylamine conjugate as a specific molecular adhesive linking cancer cells with vesicles. Chem. Commun. 1, 85-86, ISSN 13597345.

Brückner, E.; Sonntag, P.; Rehage, H. (2001). Light-induced shape transitions of unilamellar vesicles. Langmuir 17, 2308-2311, ISSN 0743-7463. 
Boon, J. M.; Shukla, R.; Smith, B. D.; Licini, G.; Scrimin, P. (2002). Selective Phosphatidylethanolamine Translocation across Vesicle Membranes Using Synthetic Translocases. Chem. Commun. 260-261, ISSN 1359-7345.

Bellomo, E. G.; Wyrsta, M. D.; Pakstis, L.; Pochan, D. J.; Deming, T. J. (2004). Stimuliresponsive polypeptide vesicles by conformation-specific assembly. Nat. Mater. 3, 244-248, ISSN 1476-1112.

Cohen, F. S.; Akabas, M. H.; Finkelstein, A. (1982). Osmotic swelling of phospholipid vesicles causes them to fuse with a planar phospholipid bilayer membranes. Science 217, 458-460, ISSN 0036-8675.

Chen, E. H.; Olson, E. N. (2005). Unveiling the Mechanisms of Cell-Cell Fusion. Science 308, 369-373, ISSN 0036-8675.

Christensen, S. M.; Stamou, D. (2007). Surface-based lipid vesicle reactor systems: fabricationand applications. Soft Matter 275, 3, 828-836, ISSN 1744-683X (print), 1744-6848 (online).

Chiruvolu, S.; Walker, S.; Israelachvili, J.; Schmitt, F. J.; Leckband, D.; Zasadzinski, J. A. (1994). Higher-order self-assembly of vesicles by site-specific binding. Science 264, 1753-1756, ISSN 0036-8075.

Chiu, D. T.; Wilson, C. F.; Ryttsén, F.; Strömberg, A.; Farre, C.; Karlsson, A.; Nordholm, S.; Gaggar, A.; Modi, B. P.; Moscho, A.; Garza-López, R. A.; Orwar, O.; Zare, R. N. (1999). Chemical transformations in individual ultrasmall biomimetic containers. Science 283, 1892-1895, ISSN 0036-8075.

Chernomordik, L. V.; Zimmerberg, J.; Kozlov, M. M. (2006). Membranes of the world unite. J. Cell Biology 175, 201-207, ISSN 1540-8140.

Dimova, R.; Aranda, S.; Bezlyepkina, N.; Nikolov, V.; Riske, K. A.; Lipowsky, R. (2006). A practical guide to giant vesicles. Probing the membrane nanoregime via optical microscopy. J. Phys.: Condens. Matter. 18, S1151-S1176, ISSN 0953-8984 (print), 1361648X (online).

Dimova, R.; Bezlyepkina, N.; Jordö, MD; Knorr, RL.; Riske, KA; Staykova, M.; Vlahovaska, PM.; Yamamoto, T.; Yang, P.; Lipowsky, R. (2009). Soft Matter 5, 3201-3212, ISSN 1744-683X (print), 1744-6848 (online).

Davidson, M.; Karlsson, M.; Sinclair, J.; Sott, K.; Orwar, O. (2003). Nanotube-vesicle networks with functionalized membranes and interiors. J. Am. Chem. Soc. 125, 374378, ISSN 0002-7863.

Dimova, R.; Riske, K. A.; Aranda, S.; Bezlyepkina, N.; Knorr, R. L.; Lipowsky, R. (2007). Giant vesicles in electric fields. Soft Matter 3, 817-827, ISSN 1744-683X (print), 17446848 (online).

Dameron, C. T.; Reese, R. N.; Mehra, R. K.; Kortan, A. R.; Carroll, P. J.; Steigerwald, M. L.; Brus, L. E.; Winge, D. R. (1989). Biosynthesis of cadmium sulphide quantum semiconductor crystallites. Nature 338, 596-597, ISSN 0028-0836.

Dickerson, M. B.; Sandhage, K. H.; Naik, R. R. (2008). Protein- and Peptide-Directed Syntheses of Inorganic Materials. Chem. Rev. 108, 4935-4978, ISSN 0009-2665.

Discher, B.; Won, Y.-Y.; Ege, D.; Lee, J. C-M.; Bates, F. S.; Discher, D. E.; Hammer, D. A. (1999). Polymersomes: Tough Vesicles Made from Diblock Copolymers. Science 284, 1143-1146, ISSN 0036-8675. 
Espinoza, H. A.; Chen, Y.; Dan, N.; Lubensky, T. C.; Nelson, P.; Ramos, L.; Weitz, D. A. (1999) Electrostatic repulsion of positively charged vesicles and negatively charged objects. Science 285, 394-397, ISSN 0036-8675.

Evans, J. S. (2008). Tuning in to mollusk shell nacre- and prismatic-associated protein terminal sequences. Implications for biomineralization and the construction of high performance inorganic-organic composites. Chem. Rev. 108, 4455-4462, ISSN 00092665.

Estes, D. J.; Lopez, S. R.; Fuller, A. O.; Mayer, M. (2006). Triggering and visualizing the aggregation and fusion of lipid membranes in microfluidic chambers. Biophys. J. 91, 233-243, ISSN 0006-3495.

Estes, D. J.; Mayer, M. (2005). Giant liposomes in physiological buffer using electroformation in a flow chamber. Biochim. Biophys. Acta. 1712, 152-160, ISSN 0006-3002.

Esch, van J.; Roks, M. F. M.; Nolte, R. J. M. (1986). Membrane-bound cytochrome P-450 mimic. Polymerized vesicles as microreactors. J. Am. Chem. Soc. 108, 6093-6094, ISSN 0002-7863.

Farbman-Yogev, I.; Bohbot-Raviv, Y.; Ben-Shaul, A. (1998). A Statistical-Thermodynamic Model For Cross-Bridge Mediated Condensation of Vesicles. J. Phys. Chem. A 102, 9586-9592, ISSN 0022-3654.

Faure, C.; Derré, A.; Neri, W. (2003). Spontaneous formation of silver nanoparticles in multilamellar vesicles. J. Phys. Chem. B 107, 4738-4746, ISSN 1089-5647 (print), 15205207 (online).

Fischer, A.; Franco, A.; Oberholzer, T. (2002). Giant Vesicles as Microreactors for Enzymatic mRNA Synthesis. ChemBioChem 3, 409-417, ISSN 1439-4227.

Gratt, J. A.; Cohen, R. E. (2003). Optical properties of block copolymers containing pendant carbazole groups and in situ synthesized CdS nanoclusters. J. Appl. Polym. Sci. 88, 177-182, ISSN 1097-4628.

Gorer, S.; Ganske, J. A.; Hemminger, J. C.; Penner R. M. (1998). Size-selective and epitaxial electrochemical/chemical synthesis of sulfur-passivated cadmium sulfide nanocrystals on graphite. J. Am. Chem. Soc. 120, 9584-9593, ISSN 0002-7863.

González-Pérez, A.; Schmutz, M.; Waton, G.; Romero, M. J.; Krafft, M. P. (2007). Isolated Fluid Polyhedral Vesicles. J. Amer. Chem. Soc. 129, 4, 756-757, ISSN 0002-7863.

George, A.; Veis, A. (2008). Phosphorylated proteins and control over apatite nucleation, crystal growth, and inhibition. Chem. Rev. 108, 4670-4693, ISSN 0009-2665.

Hung, L. H.; Choi, K. M.; Tseng, W. Y.; Tan, Y. C.; Shea, K. J.; Lee, A. P. (2006). Controlled droplet fusion in microfluidic devices. Lab Chip, 6, 174-178, ISSN 1473-0189.

Hanczyc, M. M.; Fujikawa, S. M.; Szostak, J. W. (2003). Experimental models of primitive cellular compartments: encapsulation, growth, and division. Science 302, 618-622, ISSN 0036-8675.

Hanczyc, M. M.; Szostak, J. W. (2004). Replicating vesicles as models of primitive cell growth and division. Curr. Opin. Chem. Biol. 8, 6, 660-664, ISSN 1367-5931.

Hauschild, S.; Lipprandt, U.; Rumplecker, A.; Borchert, U.; Rank, A.; Schubert, R.; Förster, S. (2005). Direct preparation and loading of lipid and polymer vesicles using inkjet printers. Small 1, 1177-1180, ISSN 1613-6829. 
Haque, M. E.; McCoy, A. J.; Glenn, J.; Lee, J.; Lentz, B. R. (2001). Effects of Hemagglutinin "Fusion Peptide" on Poly(ethylene glycol)-Mediated Fusion of Phosphatidylcholine Vesicles. Biochemistry 40, 14243-14251, ISSN 0006-2960 (print), 1520-4995 (online).

Hales, K.; Pochan, D. J. (2006). Using polyelectrolyte block copolymers to tune nanostructure assembly, Current Opinion in Colloid \& Interface Science 11, 330-336, ISSN 1359-0294.

Haluska, C. K.; Riske, K. A.; Artzner, V. M.; Lehn, J. M.; Lipowsky, R.; Dimova, R. (2006). Time scales of membrane fusion revealed by direct imaging of vesicle fusion with high temporal resolution. Proc. Natl. Acad. Sci. U.S.A. 103, 15841-15846, ISSN 00278423.

Holowka, E. P.; Sun, V.Z.; Kamei, D. T.; Deming, T. J. (2007). Polyarginine segments in block copolypeptides drive both vesicular assembly and intracellular delivery. Nat. Mater. 6, 52-57, ISSN 1476-1112.

Hamada, T.; Sato, Y. T.; Yoshikawa, K.; Nagasaki, T. (2005). Reversible photo-switching in a cell-sized vesicle Langmuir 21, 7626-7628, ISSN 0743-7463.

Ichikawa, S.; Walde, P. (2004). Phospholipase D-mediated aggregation, fusion, and precipitation of phospholipid vesicles. Langmuir 20, 941-949, ISSN 0743-7463.

Jelinek, R.; Kolusheva, S. (2007). Biomolecular Sensing with Colorimetric Vesicles. Top. Curr.Chem. 277, 155-180, ISSN 0340-1022 (print), 1436-5049 (online).

Jesorka, A.; Markström, M.; Karlsson, M.; Orwar, O. (2005). Controlled Hydrogel Formation in the Internal Compartment of Giant Unilamellar Vesicles. J. Phys. Chem. B 109, 14759-14763, ISSN 1089-5647 (print), 1520-5207 (online).

Jahn, R.; Scheller, R. H. (2006). SNAREs - engines for membrane fusion. Nat. Rev. Mol. Cell Biol. 7, 631-643, ISSN 1471-0072.

Kröger, N.; Dickerson, M. B.; Ahmad, G.; Cai, Y.; Haluska, M. S.; Sandhage, K. H.; Poulsen, N.; Sheppard, V. C. (2006). Bioenabled Synthesis of Rutile $\left(\mathrm{TiO}_{2}\right)$ at Ambient Temperature and Neutral pH. Angew. Chem. Int. Ed. 45, 7239-7243, ISSN 1433-7851 (print), 1521-3773 (online).

Kulin, S.; Kishore, R.; Helmerson, K.; Locascio, L. (2003). Optical manipulation and fusion of liposomes as microreactors. Langmuir 19, 8206-8210, ISSN 0743-7463.

Kuroiwa, T.; Kiuchi, H.; Noda, K.; Kobayashi, I.; Nakajima, M.; Uemura, K.; Sato, S.; Mukataka, S.; Ichikawa, S. (2009). Controlled preparation of giant vesicles from uniform water droplets obtained by microchannel emulsification with bilayerforming lipids as emulsifiers. Microfluid Nanofluid 6, 811-821, ISSN 1613-4982.

Kim, P.; Lee, B. K.; Lee, H. Y.; Kawai, T.; Suh, K. Y. (2008). Molded Nanowell Electrodes for Site-Selective Single Liposome Arrays. Adv. Mater. 20, 31-36, ISSN 0935-9648.

Kloepfer, J. A.; Mielke, R. E.; Nadeau, J. L. (2005). Uptake of CdSe and CdSe/ZnS Quantum Dots into Bacteria via Purine-Dependent Mechanisms. Appl. Environ. Microb. 71, 5, 2548-2557, ISSN 0099-2240.

Korgel, B. A.; Monbouquette, H. G. (2000). Controlled Synthesis of Mixed Core and Layered $(\mathrm{Zn}, \mathrm{Cd}) \mathrm{S}$ and $(\mathrm{Hg}, \mathrm{Cd}) \mathrm{S}$ Nanocrystals within Phosphatidylcholine Vesicles. Langmuir 16, 3588-3594, ISSN 0743-7463.

Korgel, B. A.; Monbouquette, H. G. (1996). Synthesis of size-monodisperse CdS nanocrystals using phosphatidylcholine vesicles as true reaction compartments. J. Phys. Chem. 100, 346-351, ISSN 0022-3654. 
Kim, D.; Oh, S.; Yi, S.; Bae, S.; Moon, S. (2000). Preparation of indium-tin oxide particles in shear-induced multilamellar vesicles (spherulites) as chemical reactors. Chem. Mater. 12, 996-1002, ISSN 0897-4756.

Khramov, M. I.; Parmon, V. N. (1993). Synthesis of ultrafine particles of transition-metal sulfides in the cavities of lipid vesicles and the light-stimulated transmembrane electron-transfer catalyzed by these particles. J. Photochem. Photobiol. A: Chem. 71, 279-284, ISSN 1010-6030.

Kang, S. Y.; Seong, B. S.; Han, Y. S.; Jung, H. T. (2003). Self-Organization of Amphiphilic Polymer in Vesicle Bilayers Composed of Surfactant Mixtures. Biomacromolecules 4, 360-365, ISSN 1526-7797.

Krafft, M. P.; Schieldknecht, L.; Marie, P.; Giulieri, F.; Schmutz, M.; Poulain, N.; Nakache, E. (2001). Fluorinated vesicles allow intrabilayer polymerization of a hydrophobic monomer, yielding polymerized microcapsules. Langmuir 17, 2872-2877, ISSN 07437463.

Karlsson, M.; Sott, K.; Cans, A. S.; Karlsson, A.; Karlsson, R.; Orwar, O. (2001). Micropipetassisted formation of microscopic networks of unilamellar lipid bilayer nanotubes and containers. Langmuir 17, 6754-6758, ISSN 0743-7463.

Kato, T.; Sakamoto, T.; Nishimura, T. (2010). Macromolecular Templating for the Formation of Inorganic-Organic Hybrid Structures. MRS Bulletin 35, 127, ISSN 0883-7694.

Kunishima, M.; Tokaji, M.; Matsuoka, K.; Nishida, J.; Kanamori, M.; Hioki, K.; Tani, S. (2006). Spontaneous membrane fusion induced by chemical formation of ceramides in a lipid bilayer. J. Am. Chem. Soc. 128, 14452-14453, ISSN 0002-7863.

Kommareddi, N. S.; Tata, M.; John, V. T.; McPherson, G. L.; Herman, M. F.; Lee, Y. S.; O'Connor, C. J.; Akkara, J. A.; Kaplan, D. L. (1996). Synthesis of superparamagnetic polymer-ferrite composites using surfactant microstructures. Chem. Mater. 8, 801809, ISSN 0897-4756.

Killian, C. E.; Wilt, F. H. (2008). Molecular aspects of biomineralization of the echinodermendoskeleton. Chem. Rev. 108, 4463-4474, ISSN 0009-2665.

Lentz, B. R. (2007). PEG as a tool to gain insight into membrane fusion. Eur. Biophys. J. 36, 315-326, ISSN 0175-7571.

Leabu, M. (2006). Membrane fusion in cells: molecular machinery and mechanisms. J. Cell. Mol. Med. 10, 423-427, ISSN 1582-1838.

Luisi, P. L. (2007). Chemical Aspects of Synthetic Biology. Chemistry E Biodiversity 4, 603-621, ISSN 1612-1872.

Lee, C. H.; Chang, Y. F.; Tsai, C. H.; Wang, P. H. (2005). Optical measurement of the deformation of giant lipid vesicles driven by a micropipet electrode. Langmuir 21, 7186-7190, ISSN 0743-7463.

Lee, J.; Kim, H. J.; Kim, J. (2008). Polydiacetylene liposome arrays for selective potassium detection. J. Am. Chem. Soc. 130, 5010-5011, ISSN 0002-7863.

Larsen, G.; Ortiz, V. R.; Minchow, K.; Barrero, A.; Loscertales, I. G. (2003). A Method for Making Inorganic and Hybrid (Organic/Inorganic) Fibers and Vesicles with Diameters in the Submicrometer and Micrometer Range via Sol-Gel Chemistry and Electrically Forced Liquid Jets. J. Am. Chem. Soc. 125, 1154-1155, ISSN 0002-7863. 
Lorenceau, E.; Utada, A. S.; Link, D. R.; Cristobal, G.; Joanicot, M.; Weitz, D. A. (2005). Generation of Polymerosomes from Double-Emulsions. Langmuir 21, 9183-9186, ISSN 0743-7463.

Li, H. L.; Zhu, Y. C.; Chen, S. G.; Palchik, O.; Xiong, J. P.; Koltypin, Y.; Gofer, Y.; Gedanken, (2003). A Novel Ultrasound-Assisted Approach to the Synthesis of CdSe and CdS Nanoparticles. J. Solid State Chem. 172, 102-110, ISSN 0022-4596.

Liu, X.; Zhao, R.; Zhang, Y.; Jiang, X.; Yue, J.; Jiang, P.; Zhang, Z. (2007). Using giant unilamellar lipid vesicle micro-patterns as ultrasmall reaction containers to observe reversible ATP synthesis/hydrolysis of F0F1-ATPase directly. Biochimica et Biophysica Acta 1770, 1620-1626, ISSN 0304-4165.

Mandal, D.; Bolander, M. E.; Mukhopadhyay, D.; Sarkar, G.; Mukherjee, P. (2006). The use of microorganisms for the formation of metal nanoparticles and their application. Appl. Microbiol. Biotechnol. 69, 485-492, ISSN 0175-7598 (print), 1432-0614 (online).

Marmottant, P.; Hilgenfeldt, S. (2003). Controlled vesicle deformation and lysis by single oscillating bubbles. Nature 423, 153-156, ISSN 0028-0836.

Marrink, S. J.; Mark, A. E. (2003). The Mechanism of Vesicle Fusion as Revealed by Molecular Dynamics Simulations. J. Am. Chem. Soc. 125, 11144-11145, ISSN 0027863.

Meldrum, FC.; Cölfen, H. (2008). Controlling Mineral Morphologies and Structures inBiological and Synthetic Systems. Chem. Rev. 108, 4332-4432, ISSN 0009-2665.

Menger, F. M.; Balachander, N. (1992). Chemically-induced aggregation, budding, and fusion in giant vesicles: direct observation by light microscopy. J. Am. Chem. Soc. 114, 5862-5864, ISSN 0002-7863.

Menger, F. M.; Keiper, J. S. (1997). Electrostatic layering of giant vesicles. Angew. Chem. Inter. Ed. 36. 22, 2489-2491, ISSN 1433-7851 (print), 1521-3773 (online).

Menger, F. M.; Keiper, J. S. (1998). Digitonin as a Chemical Trigger for the Selective Transformation of Giant Vesicles. Angew. Chem. Int. Ed. 37, 24, 3433-3435, ISSN 1433-7851 (print), 1521-3773 (online).

Menger, F. M.; Keiper, J. S. (1998). Giant Vesicles: Micromanipulation of Membrane Bilayers. Adv. Mater. 10, 888-890, ISSN 0935-9648.

Menger, F. M.; Seredyuk, V. A.; Yaroslavov, A. A. (2002). Angew. Chem. Int. Ed. 41, 8, 13501352, ISSN 1433-7851 (print), 1521-3773 (online).

Moffitt, M.; Eisenberg, A. (1995). Size Control of. Nanoparticles In Semiconductor-Polymer Composites. 1. Control via Multiplet Aggregation Numbers in. Styrene-based Random Ionomers Chem. Mater. 7, 1178-1184, ISSN 0897-4756.

Mohanty, A.; Dey, J. (2003). A giant vesicle forming single tailed chiral surfactant for enantioseparation by micellar electrokinetic chromatography. Chem. Commun. 1384-1385, ISSN 1359-7345.

Monnard, P. A. (2003). Liposome-entrapped polymerases as models for microscale/nanoscale bioreactors. J. Membrane. Biol. 191, 87-97, ISSN 1432-1424.

Montes, L. R.; Alonso, A.; Goñi, F. M.; Bagatolli, L. A. (2007). Giant unilamellar vesicles electroformed from native membranes and organic lipid mixtures under physiological conditions. Biophys. J. 93, 3548-3554, ISSN 0006-3495.

Morgan, J. D.; Johnson, C. A.; Kaler, E. W.(1997). Polymerization of Equilibrium Vesicles. Langmuir 13, 6447-6451, ISSN 0743-7463. 
Morigaki, K.; Walde, P. (2007). Fatty acid vesicles. Current Opinion in Colloid $\mathcal{E}$ Interface Science 12, 75-80, ISSN 1359-0294.

Mukherjee, P.; Ahmad, A.; Mandal, D.; Senapati, S.; Sainkar, S. R.; Khan, M. I.; Ramani, R.; Parischa, R.; Ajayakumar, P. V.; Alam, M.; Sastry, M.; Kumar, R. (2001). Bioreduction of AuCl4y Ions by the Fungus, Verticillium sp. and Surface Trapping of the Gold Nanoparticles Formed. Angew. Chem. Int. Ed. 40, 19, 3585-3588, ISSN 1433-7851 (print), 1521-3773 (online).

Noireaux, V.; Libchaber, A. (2004). A vesicle bioreactor as a step toward an artificial cell assembly. Proc. Nat. Aca. Si. USA 101, 17669-17674, ISSN 0027-8423.

Nishikawa, H.; Morita, T.; Sugiyama J.; Kimura, S. (2004). Formation of gold nanoparticles in microreactor composed of helical peptide assembly in water. J. Colloid. Inter. Sci. 280, 506-510, ISSN 0021-9797.

Naik, R. R.; Stringer, S. J.; Agarwal, G.; Jones, S. E.; Stone, M. O. (2002). Biomimetic synthesis and patterning of silver nanoparticles. Nat. Mater. 1, 169-172, ISSN 1476-1122 (print), 1476-4660 (online)

Nomura, S. M.; Tsumoto, K.; Hamada, T.; Akiyoshi, K.; Nakatani, Y.; Yoshikawa, K. (2003). Gene expression within cell-sized lipid vesicles. ChemBioChem 4, 1172-1175, ISSN $1439-4227$.

Nomura, S. M.; Yoshikawa, Y.; Yoshikawa, K.; Dannenmuller, O.; Chasserot-Golaz, S.; Ourisson, G.; Nakatani, Y. (2001). Towards Proto-Cells : Primitive Lipid Vesicles EncapsulatingGiant DMA and ItsHistone Complex. ChemBioChem 6, 457-459, ISSN 1439-4227.

Neil, G. A.; Zimmermann, U. (1993). Electrofusion. Methods Enzymol. 220, 174-196, ISSN 0076-6879.

Ogura, A.; Yanagimachi, R. (1995). Spermatids as male gametes. Reprod. Fertil. Dev. 7, 155159, ISSN 1031-3613.

Peters, C.; Andrews, P. D.; Stark, M. J. R.; Cesaro-Tadic, S.; Glatz, A.; Podtelejnikov, A.; Mann, M.; Mayer, A. (1999). Control of the Terminal Step of Intracellular Membrane Fusion by Protein Phosphatase 1. Science 285,1084-1087, ISSN 0036-8075.

Park, J. M.; Aoyama, S.; Zhang, W.; Nakatsuji, Y.; Ikeda, I. (2000). Photodimerisation of a styrylpyrazine amphiphile suppresses the release of glucose entrapped in its mixed vesicle with DPPC. Chem. Commun. 3, 231-232, ISSN 1359-7345.

Pochan, D. J.; Chen, Z.; Cui, H.; Hales, K.; Qi, K.; Wooley, K. L. (2004). Toroidal Triblock Copolymer Assemblies. Science 306, 94-97, ISSN 0036-8675.

Pautot, S.; Frisken, B. J.; Weitz, D. A. (2003). Production of unilamellar vesicles using an inverted emulsion. Langmuir 19, 2870-2879, ISSN 0743-7463.

Pabst, G.; Hodzic, A.; Strancar, J.; Danner, S.; Rappolt, M.; Laggner, P. (2007). Rigidification of neutral lipid bilayers in the presence of salts. Biophys. J. 93, 2688-2696, ISSN 00063495.

Pott, T.; Bouvrais, H. ; Meleard, P. (2008). Giant unilamellar vesicle formation under physiologically relevant conditions. Chem. Phys. Lipids 154, 115-119, ISSN 00093084 .

Regev, O.; Backov, R.; Faure, C. (2004). Gold nanoparticles spontaneously generated in onion-type multilamellar vesicles. Bilayers-Particle coupling imaged by Cryo-TEM. Chem. Mater. 16, 5280-5285, ISSN 0897-4756. 
Rassy, H. E; Belamie, E.; Livage, J.; Coradin, T. (2005). Onion phases as biomimetic confined media for silica nanoparticles growth. Langmuir 21, 8584-8587, ISSN 0743-7463.

Riske, K. A.; Bezlyepkina, N.; Lipowsky, R.; Dimova, R. (2006). Electrofusion of model lipid membranes viewed with high temporal resolution. Biophys. Rev. Lett. 4, 387-400, ISSN 1793-0480.

Riske, K. A.; Dimova, R. (2005). Electro- deformation and -poration of giant vesi- cles viewed with high temporal resolution. Biophys. J. 88, 1143-1155, ISSN 0006-3495.

Riske, K. A.; Knorr, R. L.; Dimova, (2009). Bursting of charged multicomponent vesiclessubjected to electric pulses. Soft Matter, 5, 1983-1986, ISSN 1744-683X (print), 1744-6848 (online)

Rustom, A.; Saffrich, R.; Markovic, I.; Walther, P.; Gerdes, H. H. (2004). Nanotubular highways for intercellular organelle transport. Science 303, 1007-1010, ISSN 00368675.

Rafaeloff, R.; Tricot, Y. M.; Nome, F.; Fendler, J. H. (1985). Colloid-catalyst-coated semiconductors in surfactant vesicles. In situ generation of rhodium-coated cadmium sulfide particles in dimethyldioctadecylammonium halide surfactant vesicles and their utilization in photosensitized charge separation and hydrogen generation. J. Phys. Chem. 89, 533-537, ISSN 0022-3654.

Schelly, ZA. (2007). Subnanometer size uncapped quantum dots via electroporation of synthetic vesicles. Colloids Surf B Biointerfaces 56, 1-2, 281-284, ISSN 0927-7765.

Suezaki, Y. (2002). Theoretical Possibility of Cuplike Vesicles for Aggregates of Lipid and Bile Salt Mixture. J. Phys. Chem. B 106, 13033-13039, ISSN 1089-5647 (print), 15205207 (online).

Swartz, K. J. (2008). Sensing voltage across lipid membranes. Nature 456, 18, 891-897, ISSN 0028-0836.

Sinn, C.; Antonietti, M.; Dimova, R. (2006). Binding of calcium to phosphatidylcholinephosphatidylserine membranes. Colloid Surf. A-Physicochem. Eng. Asp. 283, 410-419, ISSN 0927-7757.

Sanchez, C.; Arribart, H.; Madeleine, M. \& Guille, G. (2005). Biomimetism and bioinspirationas tools for the design of innovative materials and systems. Nat. Mater. 4, 277-288, ISSN 1476-1122 (print), 1476-4660 (online).

Sun, B.; Chiu, D. T. (2003). Spatially and temporally resolved delivery of stimuli to single Cells. J. Am. Chem. Soc. 125, 3702-3703, ISSN 0002-7863.

Steubing, R. W.; Cheng, S.; Wright, W. H.; Numajiri, Y.; Berns, M. W. (1991). Laser induced cell fusion in combination with optical tweezers: the laser cell fusion trap. Cytometry 12, 505-510, ISSN 1552-4930.

Shillcock, J. C.; Lipowsky, R. (2005). Tension-induced fusion of bilayer membranes and vesicles. Nat. Mater. 4, 225-228, ISSN 1476-1122.

Shum, H. C.; Lee, D.; Yoon, I.; Kodger, T.; Weitz, D. A. (2008). Double Emulsion Templated Monodisperse Phospholipid Vesicles. Langmuir 24, 7651-7653, ISSN 0743-7463.

Sweeney, R. Y.; Mao, C.; Gao, X.; Burt, J. L.; Belcher, A. M.; Georgiou, G.; Iverson, B. L. (2004). Bacterial Biosynthesis of Cadmium Sulfide Nanocrystals. Chemistry $\mathcal{E}$ Biology 11, 1553-1559, ISSN 1074-5521.

Strömberg, A.; Ryttsén, F.; Chiu, D. T.; Davidson, M.; Eriksson, P. S.; Wilson, C. F.; Orwar, O.; Zare, R. N. (2000). Manipulating the genetic identity and biochemical surface 
properties of individual cells with electric-field-induced fusion. Proc. Natl. Acad.Sci. U.S.A. 97, 7-11, ISSN 0027-8423.

Stachowiak, J. C.; Richmond, D. L.; Li, T. H.; Liu, A. P.; Parekh, S. H.; Fletcher, D. A. (2008). Unilamellar vesicle formation and encapsulation by microfluidic jetting. Proc. Natl. Acad. Sci. U.S.A. 105, 12, 4697-4702, ISSN 0027-8423.

Smith, A.-S.; Seifert, U. (2007). Vesicles as a model for controlled (de-)adhesion of cells: athermodynamic approach. Soft Matter 275, 3, 275-289, ISSN 1744-683X (print), 1744-6848 (online).

Sasaki, Y.; Shukla, R.; Smith, B. D. (2004). Facilitated phosphatidylserine flip-flop across vesicle and cell membranes using urea-derived synthetic translocases. Org. Biomol. Chem. 2, 214 -219, ISSN 1477-0520.

Suzuki, M.; Saruwatari, K.; Kogure, T.; Yamamoto, Y.; Nishimura, T.; Kato, T.; Nagasawa, H.(2009). An Acidic Matrix Protein, Pif, Is a Key Macromolecule for Nacre Formation. Science 325, 1388-1390, ISSN 0036-8675.

Shchukin, D. G.; Sukhorukov, G. B. (2004). Nanoparticle Synthesis in Engineered Organic Nanoscale Reactors. Adv. Mater. 16, 671-682, ISSN 0935-9648.

Shestopalov, I.; Tice, J. D.; Ismagilov, R. F. (2004). Multi-Step Chemical Reactions Performed On Millisecond Time Scale In a Microfluidic Droplet-Based System. Lab Chip 4, 316321, ISSN 1473-0189.

Sommerdijk, NAJM; de With, G. (2008). Biomimetic CaCO3 mineralization using designer molecules and interfaces. Chem. Rev. 108, 4499-4550, ISSN 0009-2665.

Stengel, G.; Zahn, R.; Höök, F. (2007). DNA-induced programmable fusion of phospholipid vesicles. J. Am. Chem. Soc. 129, 9584-9585, ISSN 0002-7863.

Tomšiè, N.; Babnik, B.; Lombardo, D.; Mavcic, B.; Kandušer, M.; Iglic, A.; Iglic, V. K. J. (2005). Chem. Inf. Model. 2005, 45, 1676-1679, ISSN 1549-9596.

Tsaadon, A.; Eliyahu, E.; Shtraizent, N.; Shalgi, R. (2006). When a sperm meets an egg: block to polyspermy. Mol. Cell. Endocrinol. 252, 107-114, ISSN 0303-7207.

Tsumoto, K.; Nomura, S. M.; Nakatani, Y.; Yoshikawa, K. (2001). Giant Liposome as a Biochemical Reactor: Transcription of DNA and Transportation by Laser Tweezers. Langmuir 17, 7225-7228, ISSN 0743-7463.

Taylor, P.; Xu, C.; Fletcher, P. D. I.; Paunov, V. N. (2003). A novel technique for preparation of monodisperse giant liposomes. Chem. Commun. 14, 1732-1733, ISSN 1359-7345.

Tangirala, R.; Revanur, R.; Russell, T. P.; Emrick, T. (2007). Sizing nanoparticle-covered droplets by extrusion through track-etch membranes. Langmuir 23, 965-969, ISSN 0743-7463.

Takakura, K.; Toyota, T.; Sugawara, T. (2003). A Novel System of Self-Reproducing Giant Vesicles. J. Am. Chem. Soc. 125, 8134-8140, ISSN 0002-7863.

Tang, Z.; Wang, Y.; Podsiadlo, P.; Kotov, N. A. (2006). Biomedical Applications of Layer-byLayer Assembly: From Biomimetics to Tissue Engineering. Adv. Mater. 18, 32033224, ISSN 0935-9648.

Tung, C.; Wu, L.; Zhang, L.; Chen, B. (2003). Supramolecular Systems as Microreactors: Control of Product Selectivity in Organic Phototransformation. Acc. Chem. Res. 36, 39-47, ISSN 0001-4842.

Umetsu, M.; Mizuta, M.; Tsumoto, K.; Ohara, S.; Takami, S.; Watanabe, H.; Kumagai, I.; Adschiri, T. (2005). Bioassisted Room-Temperature Immobilization and 
Mineralization of Zinc Oxide-The Structural Ordering of ZnO Nanoparticles into a Flower-Type Morphology. Adv. Mater. 17, 2571-2575, ISSN 0935-9648.

Vlahovska, P. M.; Gracia, R. S.; Aranda-Espinoza S.; Dimova, R. (2009). Electrohydrodynamic model of vesicle deformation in alternating electric fields. Biophys. J., 96,4789-4803, ISSN 0006-3495.

Vriezema, D. M.; Aragonés, M. C.; Elemans, J. A. A. W.; Cornelissen, J. J. L. M.; Rowan, A. E.; Nolte, R. J. M. (2005). Self-Assembled Nanoreactors. Chem. Rev. 105, 1445-1489, ISSN 0009-2665.

Vriezema, D. M.; Hoogboom, J.; Velonia, K.; Takazawa, K.; Christianen, P. C. M.; Maan, J. C.; Rowan, A. E.; Nolte, R. J. M. (2003). Vesicles and polymerized vesicles from thiophene-containing rod-coil block copolymers. Angew. Chem. Int. Ed. 42, 772-776, ISSN 1433-7851.

Vandenburg, Y. R.; Smith, B. D.; Bironb, E.; Voyer, N. (2002). Membrane disruption ability of facially amphiphilic helical peptides. Chem. Commun. 16, 1694-1695, ISSN 13597345.

Venturolia, M.; Sperotto, M. M.; Kranenburg, M.; Smit, B. (2006). Mesoscopic models of biological membranes. Physics Reports 437, 1-54, ISSN 0370-1573.

Weber, G.; Greulich, K. O. (1992). Manipulation of cells, organelles and genomes by laser microbeam and optical trap. Int. Rev. Cytol. 133, 1-41, ISSN 0074-7696.

Walde, P.; Ichikawa, S. (2001). Enzyme inside Lipid Vesicles: Preparation, Reaction and Application. Biomol. Eng. 18, 143-177, ISSN 1389-0344.

Walde, P.; Cosentino, K.; Engel, H.; Stano, P. (2010). Giant Vesicles: Preparations and Applications. ChemBioChem. 11, 848-865, ISSN 1439-4227.

Wang, Y.Y.; Kuang, A.; Russell, S. D.; Tian, H. Q. (2006). In vitro fertilization as a tool for investigating sexual reproduction of angiosperms. Sex Plant Reprod. 19, 103-115, ISSN 0934-0882.

Wang, Z.; Yasuhara, K.; Ito, H.; Mukai, M.; Kikuchi, J. (2010). Budding and Fission of Cationic Binary Lipid Vesicles Induced by the Incorporation of Pyranine. Chem. Lett. 39, 1, 54-55, ISSN 0366-7022.

Wilmut, I.; Schnieke, A. E.; McWhir, J.; Kind, A. J.; Campbell, K. H. S. (1997). Viable Offspring Derived from Fetal and Adult Mammalian Cells. Nature 385, 810-813, ISSN 0028-0836.

Wu, S.; Zeng, H.; Schelly, Z. A. (2005). Growth of Uncapped, Subnanometer Size Gold Clusters Prepared via Electroporation of Vesicles. J. Phys. Chem. B 109, 40, 1871518718, ISSN 1089-5647 (print), 1520-5207 (online).

Walker, S. A.; Zasadzinski, J. A. (1997). Electrostatic Control of Ligand-Receptor Induced Vesicle Aggregation. Langmuir 13, 5076-5081, ISSN 0743-7463.

Yamamoto, T.; Aranda-Espinoza, S.; Dimova R.; Lipowsky, R. (2010). Stability of spherical vesicles in electric fileds. Langmuir, 26, 12390-12407, ISSN 0743-7463.

Yukito, M.; Kikuchi, J.; Hisaeda, Y.; Hayashida, O. (1996). Artificial Enzymes. Chem. Rev. 96, 721-758, ISSN 0009-2665.

Yu, W.; Sato, K.; Wakabayashi, M.; Nakaishi, T.; Ko-Mitamura, E. P.; Shima, Y.; Urabe, I.; Yomo, T. (2001). Synthesis of functional protein in liposome. J. Biosci. Bioeng. 92, 590-593, ISSN 1389-1723. 
Yang, P.; Lipowsky, R.; Dimova, R. (2009). Nanoparticle formation in giant vesicles: synthesis in biomimetic compartments. Small 5, 18, 2033-2037, ISSN 1613-6829.

Yan,D.; Zhou,Y.; Hou,J.(2004). Supramolecular Self-assembly of Macroscopic Tubes. Science 303, 65, ISSN 0036-8675.

Zhou,Y.; Yan,D.(2004).Supramolecular Self-Assembly of Giant Polymer Vesicles With Controlled Size. Angew. Chem. Int. Ed. 43, 4896, ISSN 1433-7851.

Zhou, Y.; Yan, D.(2005).Real-Time Membrane Fission of Giant Polymer Vesicles. Angew. Chem. Int. Ed. 44, 3223, ISSN 1433-7851.

Zhou, Y.; Yan, D. (2005).Real-Time Membrane Fusion of Giant Polymer Vesicles. J. Am. Chem. Soc. 127, 10468, ISSN 0002-7863.

Zimmermann, U. (1986). Electrical breakdown, electropermeabilization and electrofusion. Rev. Physiol. Biochem. Pharmacol. 105, 176-256, ISSN 0303-4240. 


\title{
Biologically Inspired Locomotion Control of a Climbing Robot
}

\author{
Reinaldo de Bernardi ${ }^{1}$, Arturo Forner-Cordero ${ }^{2}$ and José Jaime Da Cruz ${ }^{1}$ \\ ${ }^{1}$ Department of Telecommunications and Control, São Paulo University \\ ${ }^{2}$ Department of Mechatronics, São Paulo University \\ Brazil
}

\section{Introduction}

Gait generation is the formulation and selection of a sequence of coordinated leg and body motions that propel a legged robot along a desired path.

Free gaits are the ones in which any leg is permitted to move at any time and fixed, or regular, gaits are those ones in which a specific pattern of leg movement is imposed. All animals move with fixed gaits (Pal \& Jayarajan, 1991; Wettergreen \& Thorpe, 1992; Pack \& Kang, 1999).

Online gait generation for robots with multiple degrees of freedom (DOF) is still a difficult problem, in particular for non-steady state locomotion, that is, when the robot has to move in non-structured environments with continuous variations of the speed, direction, and type of locomotor behavior (Crespi et al., 2008; Diedam et al., 2008). However, in nature, the motion of the legs follows stereotyped patterns with small variations from cycle to cycle related to the adaptation or entrainment with the environment (Taga, 1994; Forner-Cordero et al, 2006).

This work addresses the problem of controlling a four legged climbing robot using a control architecture based on several coupled Central Pattern Generators (CPG).

CPGs are neural circuits found in both invertebrate and vertebrate animals that can produce rhythmic patterns of neural activity even in the absence of excitatory inputs (Delcomyn, 1980; Ijspeert, 2008). The term central indicates that sensory feedback from the peripheral nervous system is not necessary to generate the rhythmical patterns, nevertheless, sensory feedback is important for the modulation of these patterns and entrain with the environment (Brown, 1914; Grillner, 1985; Collins \& Stewart, 1993; Duysens \& Van de Crommert, 1998).

CPGs are fundamental building blocks for the motion neural circuits which present several interesting properties including distributed control, the ability to deal with redundancies, fast control loops and disturbance rejection. They also allow modulation of locomotion by simple control signals (Fukuoka, Kimura, \& Cohen, 2003; Ijspeert, 2008).

The purpose of CPG models is to exhibit limit cycle behavior, i.e. to produce stable rhythmic patterns. When this is the case, the system rapidly returns to its normal rhythmic behavior after transient perturbations of the state variables, thus providing robustness against perturbations.

The choice of CPG is justified by the cited robustness in the generation of patterns. In addition, the artificial CPG, like its biological counterpart, can produce coordinated patterns of rhythmic activity while being modulated by control parameters (Matsuoka, 1987). 
These properties, when transferred to mathematical models, make CPGs interesting building blocks for locomotion controllers in robots and have been well studied by a number of researchers with possible applications to the control of walking machines (Inagaki, Yuasa \& Arai, 2003; Ishii, Masakado, \& Ishii, 2004; Inagaki, Yuasa \& Suzuki, 2006; Liu et al, 2007; Ijspeert, Crespi \& Ryczko, 2007; Aoi \& Tsuchiya, 2007; Morimoto, Endo \& Nakanishi, 2008).

\section{The four legged robot platform: Kamanbaré}

\subsection{Overview of the platform}

Purporting the main goal of climbing trees for environmental research applications, a bioinspired robotic platform named Kamanbaré was designed and built (Bernardi \& Da Cruz, 2007). The project's main application is climbing trees for non-invasive research purposes, reaching points that may represent a risk to humans.

The mechanical structure of the Kamanbare platform consists of a central rigid body with four identical legs distributed symmetrically, Fig 1. Each leg comprises three links connected by three rotating joints and fixed to the central body. Each joint has $1 \mathrm{DOF}$. Identical motor and reduction groups are responsible by the rotary movements. Fig. 2 shows the kinematic configuration of a leg.
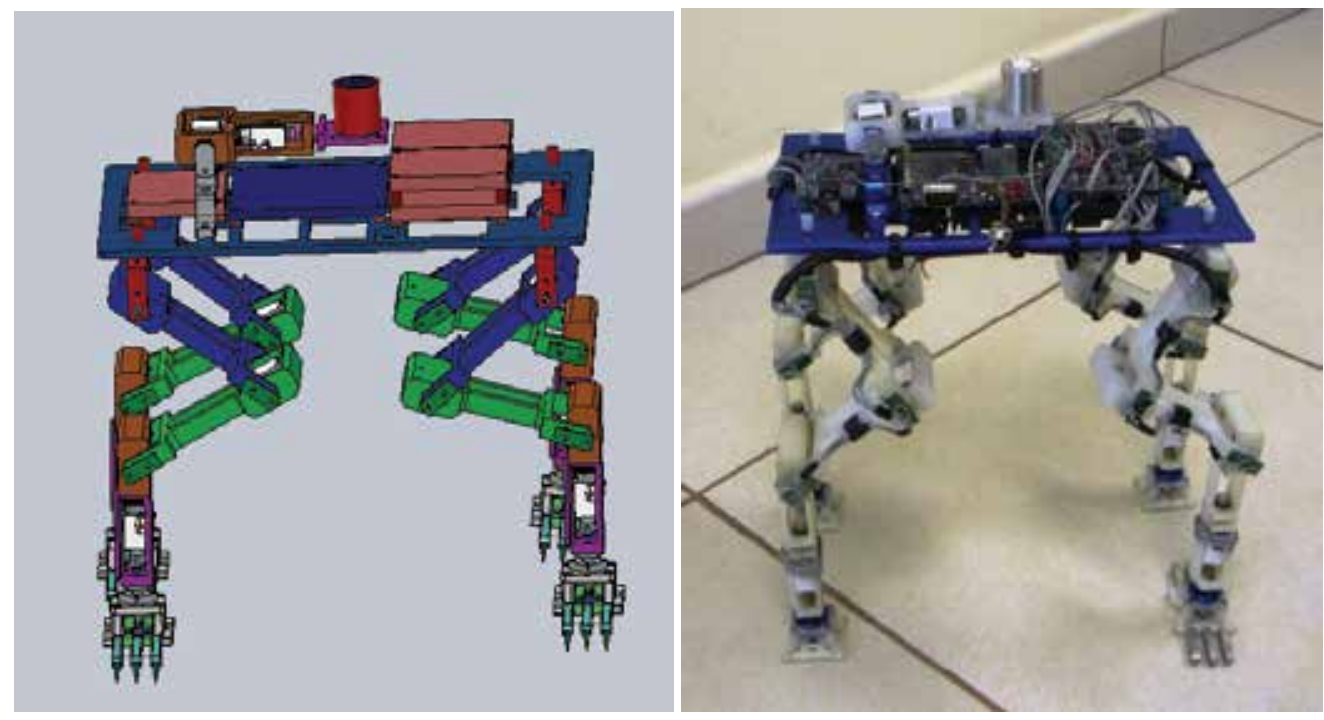

Fig. 1. Kamanbaré robot. Left: CAD model. Right: prototype picture.

\subsection{Hardware architecture}

The computations of locomotion strategies, motion control algorithms, sensor information processing and communication with the base station must be done in real-time. This significant computer load requires an advanced architecture processor running a very efficient operating system, therefore the ARM9 core running a Real Time Linux were chosen. 


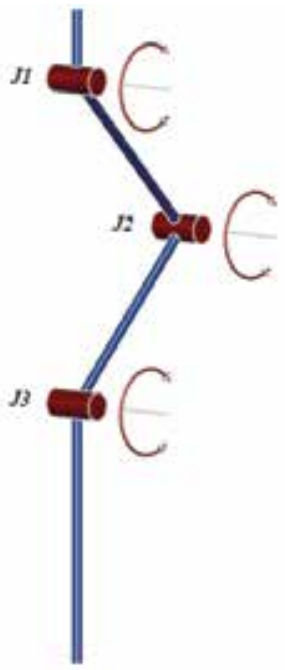

Fig. 2. Kinematic configuration of a leg.

Due to the control complexity present in this particular robotic platform, a main board for the execution of the highest hierarchical level control activities was considered.

As a solution for the main board, the TS-7250 (Technologic Systems, USA) was selected. The main reasons for this choice were: it is compact, it contains different standard interfaces, and it is based on the EP9302 Cirrus Logic processor, with an ARM9 core, Fig. 3. The EP9302 implements an advanced processor core: $200 \mathrm{MHz}$ ARM920T with support for a memory management unit (MMU). This ensemble allows the use of a high-level operating system, in this case Linux. The ARM920T core has a 32-bit architecture with a 5-stage pipeline, offering high performance and low energy consumption levels. With a $200 \mathrm{MHz}$ clock, the TS-7250 module offers a performance approximately twice as fast as similar boards based on 586core processors.

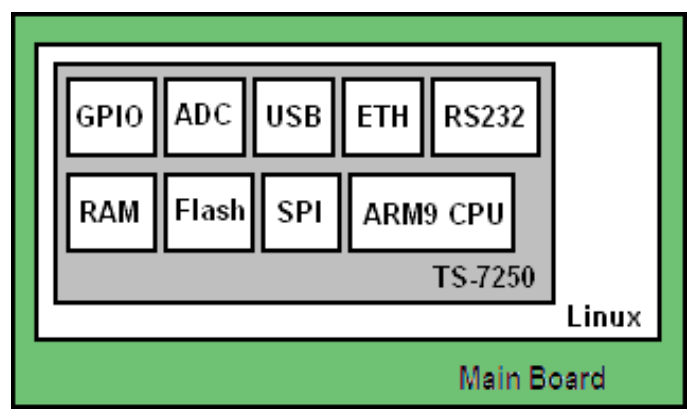

Fig. 3. Main board diagram.

Other motor control boards were also developed using a Texas Instruments microcontroller of the MSP430 family and specific integrated circuits to implement the power actuation module, based on the so-called H-bridge technique.

To implement the control systems for the Kamanbare platform, an electronic architecture was defined. Initially only one joint was considered as represented in Fig. 4, where the main components are shown: a DC motor, a potentiometer and a micro switch. 


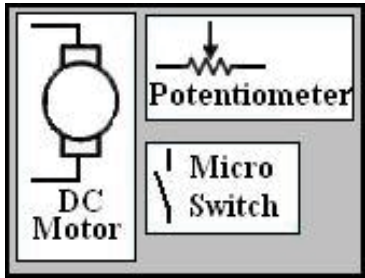

Fig. 4. Schematic representation of a joint.

Thus, for control purposes, the need for a PWM output (motor control), an analog input (potentiometer reading, indicating the joint angle), and a digital input (reading the limits of the joint course) was ascertained. One copy of the joint control system described above was built for each joint.

As the robot has four legs, it was decided to distribute the control to each leg. Thus, each leg control module needs four groups as mentioned, namely, three for the joints, and one for controlling the opening and closing of the claw.

A motor control board was developed for this specific purpose, Fig. 5, based on the MSP430F149 Texas Instruments microcontroller and on the L298 integrated circuit $(\mathrm{H}-$ bridge).

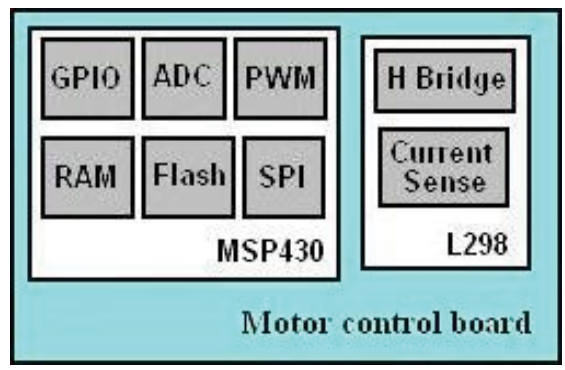

Fig. 5. Motor control board diagram.

Thus, the general hardware architecture for the Kamanbaré platform was deployed according to the diagram shown in Fig. 6.

\subsection{Control software architecture}

A control software architecture was implemented for local control of the Kamanbare platform. This architecture corresponds to the robot's functional organization.

Based on the hardware architecture presented above, the development of the following systems was accomplished according to Fig. 7. This model is based on the architecture implemented for the MARIUS robot (Pascoal et al., 1997) and has the following main components described below:

- General Control System: this system receives trajectory reference information from the Mission Control System. It controls all the robot's movements, sending the appropriate commands to the Actuators Control System. Problems occurring in the path, such as obstacles or absence of support points for the paws, are handled by this system.

- Mission Control System: this system is the main module, with the highest hierarchical level of the platform. It is responsible for receiving commands via the Communication System, and for distributing them to the systems involved. It also stores information on 
the general status of the platform (battery voltage, position of the legs, angles of joints, etc.) keeping them available. This system gathers information from the Environmental Inspection System to be subsequently forwarded to a base station.
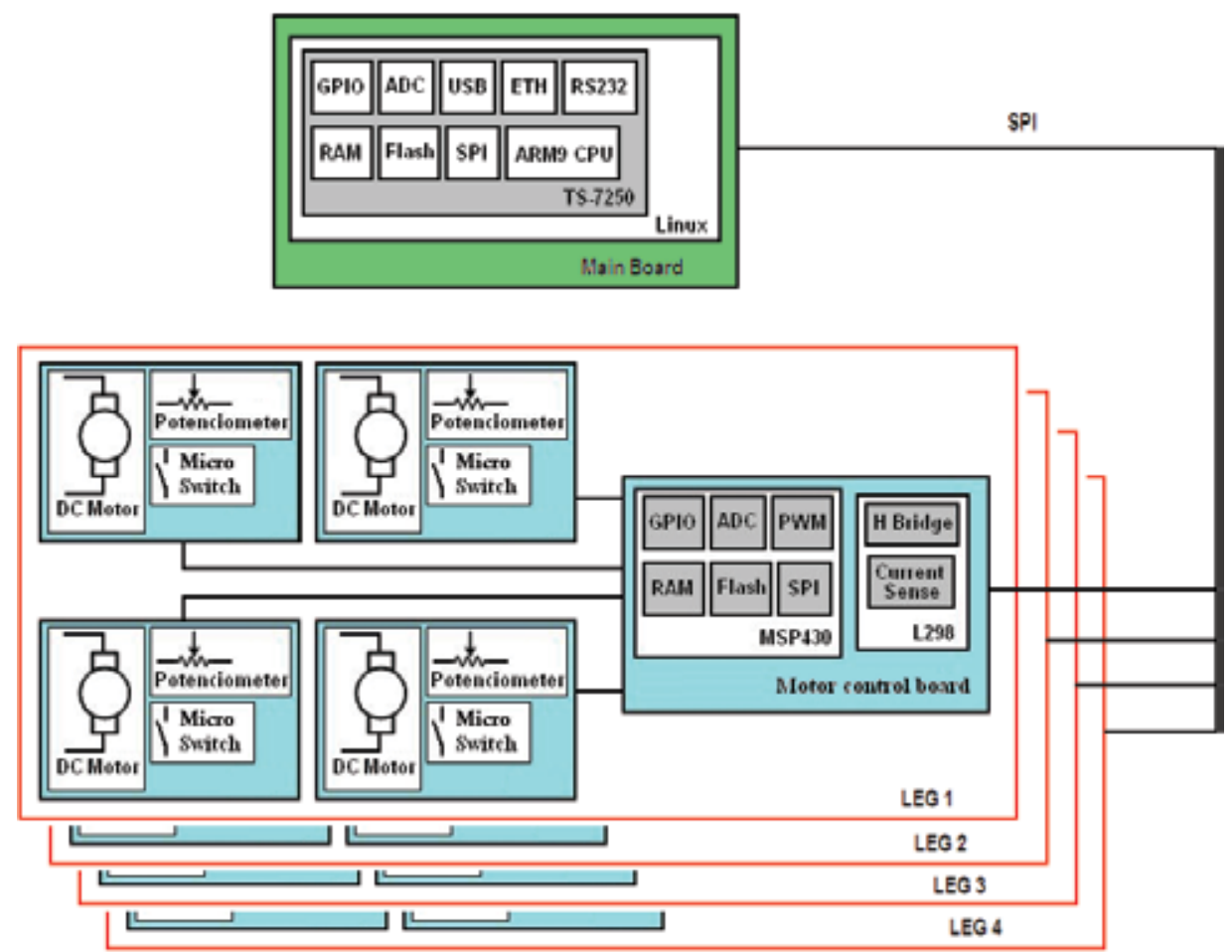

Fig. 6. Hardware architecture of the Kamanbaré platform.

- General Control System: this system receives trajectory reference information from the Mission Control System. It controls all the robot's movements, sending the appropriate commands to the Actuators Control System. Problems occurring in the path, such as obstacles and absence of support points for the paws, are handled by this system.

- Mission Control System: this system is the main module, with the highest hierarchical level of the platform. It is responsible for receiving commands via the Communication System, and for distributing them to the systems involved. It also stores information on the general status of the platform (battery voltage, position of the legs, angles of joints, etc.) keeping them available. This system gathers information from the Environmental Inspection System to be subsequently forwarded to a base station.

- Communication System: this system is the module in charge of the communication interfaces existing in the platform, managing communications and exchanging data with the Mission Control System.

- Environmental Inspection System: this system is responsible for gathering data from the installed sensors and for controlling any additional hardware required for that purpose as well. All acquired data are sent to the Mission Control System. 


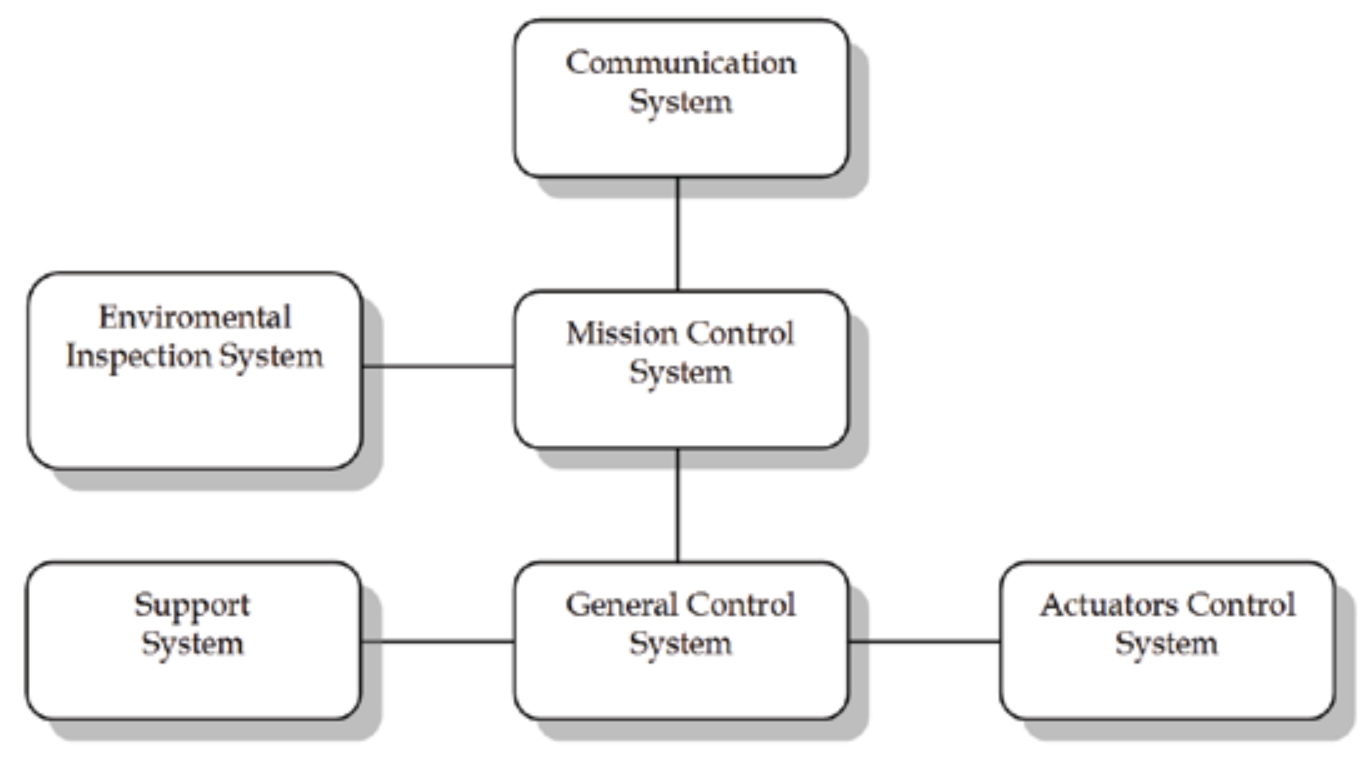

Fig. 7. Kamanbaré's control software architecture.

\subsection{Kamanbaré Simulink models}

The CAD model of the Kamanbaré robotic platform, described in section 2, was designed using Solidworks ${ }^{\circledR}$ (Dassault Systèmes SolidWorks Corp). Thus a Simulink Kamanbaré model was generated using the SolidWorks ${ }^{\circledR}$-to-SimMechanics $\left(\right.$ MATLAB $^{\circledR}$, Simulink ${ }^{\circledR}$, The MathWorks Inc.) translator. This translation process is based on two major steps: exportation of the CAD assembly into a physical modeling XML format file and importation of the generated XML file into a SimMechanics model in Simulink ${ }^{\circledR}$.

For the translation procedure, some configurations like the name of the joints and legs were adopted as well the desired sense of movement, as shown in Fig. 8.

The complete model obtained for the Kamanbaré platform is depicted in Fig. 9. For a better understanding and visualization the legs were represented as model blocks.

Since all four legs are identical, one detailed leg model block, namely, the Front Left Leg, is described in Fig. 10.

A Gait Generator was also implemented with the main function of providing the correct angle references for joints as functions of time. This block is presented and detailed in section 3 .

\section{Gait}

A gait is a repetitive (quasi-cyclical: consider small variations from cycle to cycle to adapt to ground irregularities) pattern of foot placements (Forner-Cordero et al., 2006). It is usual to assume that each leg is sufficiently specified as a two-state device: on the ground and off it. The legs on the ground are supporting and propelling the robot, and those in the air are being retracted. 


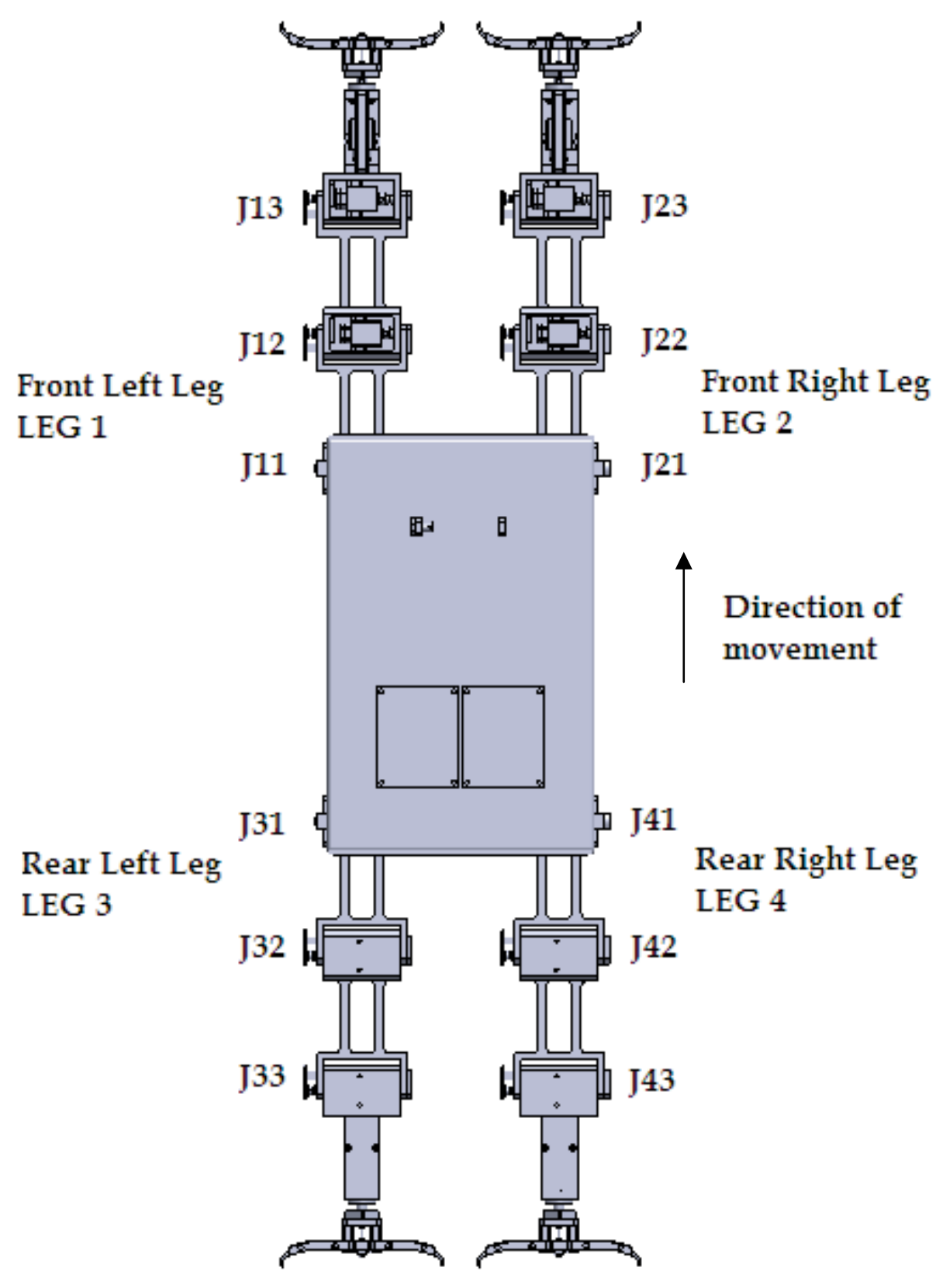

Fig. 8. Kamanbaré's configuration of joints: top view. 


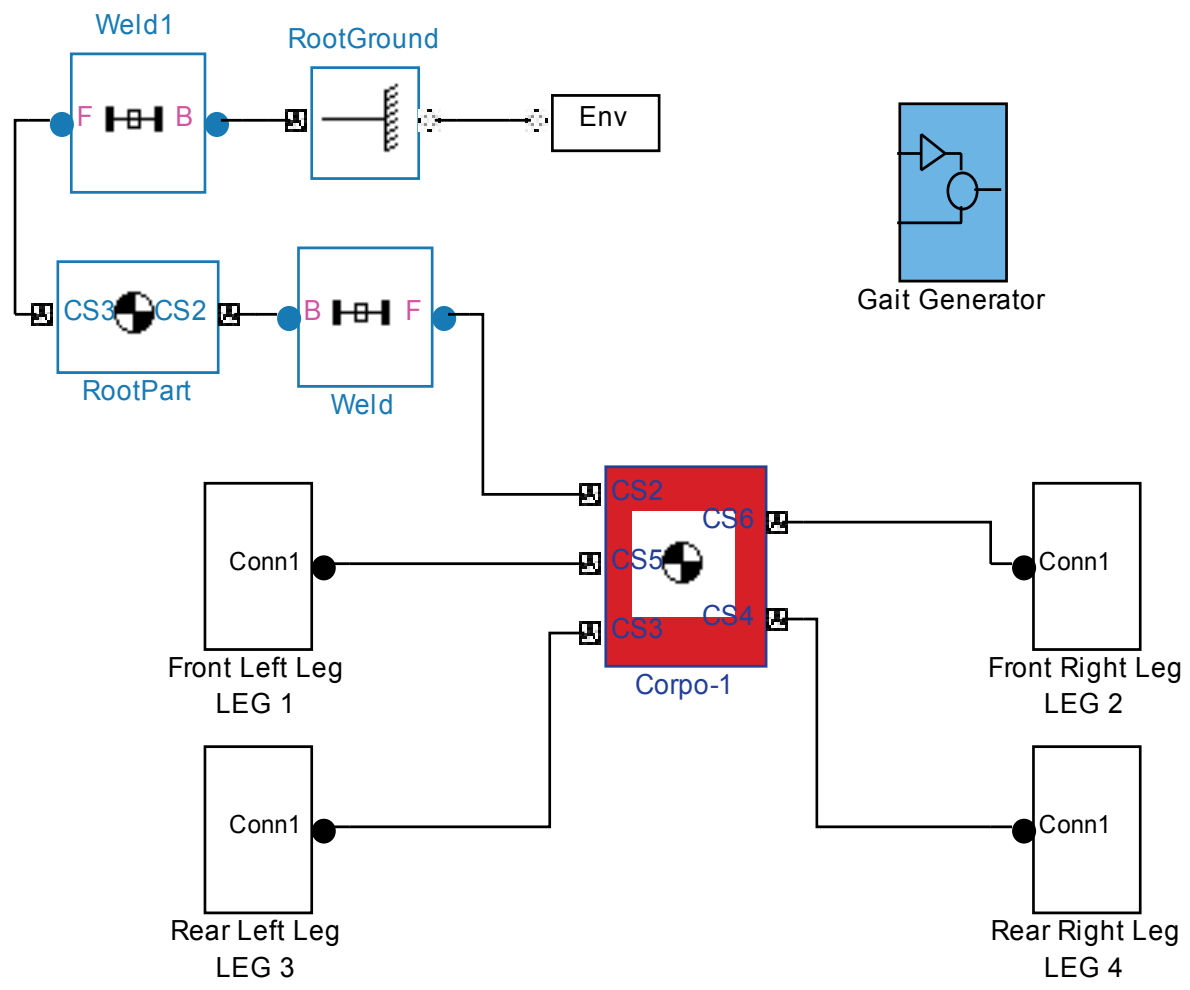

Fig. 9. Kamanbaré’s Simulink model.

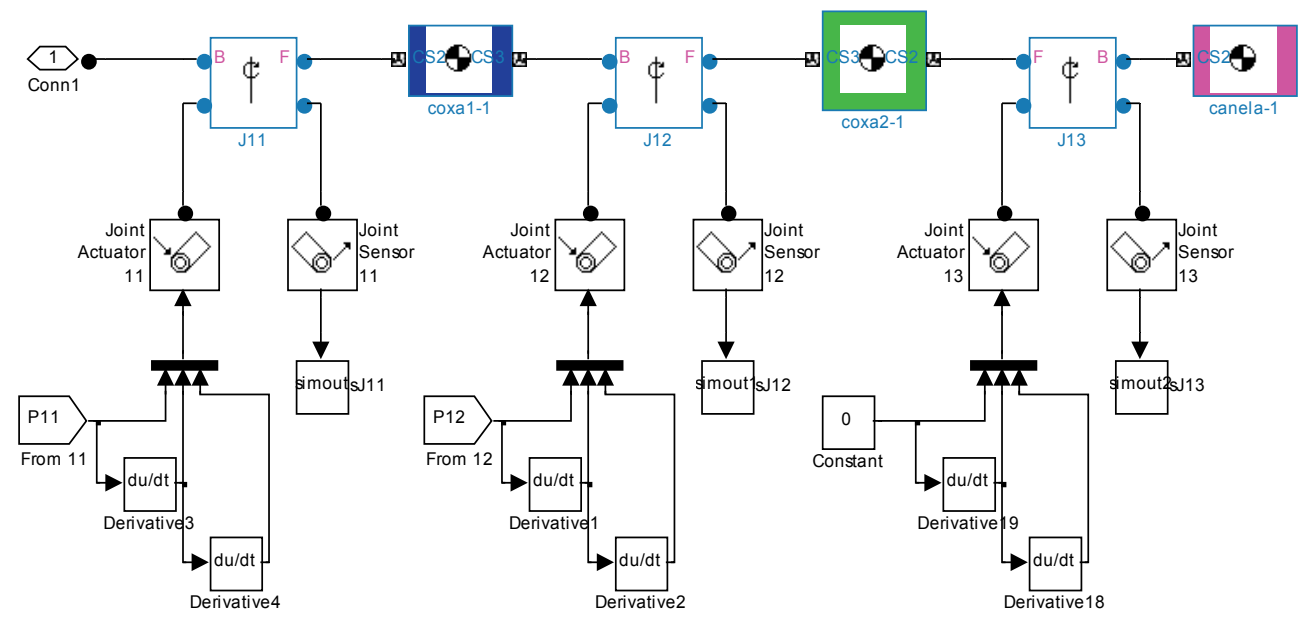

Fig. 10. Simulink Leg model: Front Left. 
The concept of gait assumes a regular progression forwards or backwards and can be expressed as a function of time or distance. Fig. 10 presents a plan view of a walker's footfalls at four successive times, $t_{1}, t_{2}, t_{3}$ and $t_{4}$, as it walks with a diagonal gait in which the front left (FL) and rear right (RR) legs move as a pair, and the front right (FR) and rear left $(R L)$, as a second pair. At times $t_{2}$ and $t_{4}$ the walker alternates from one support pair to the other (Todd, 1985).

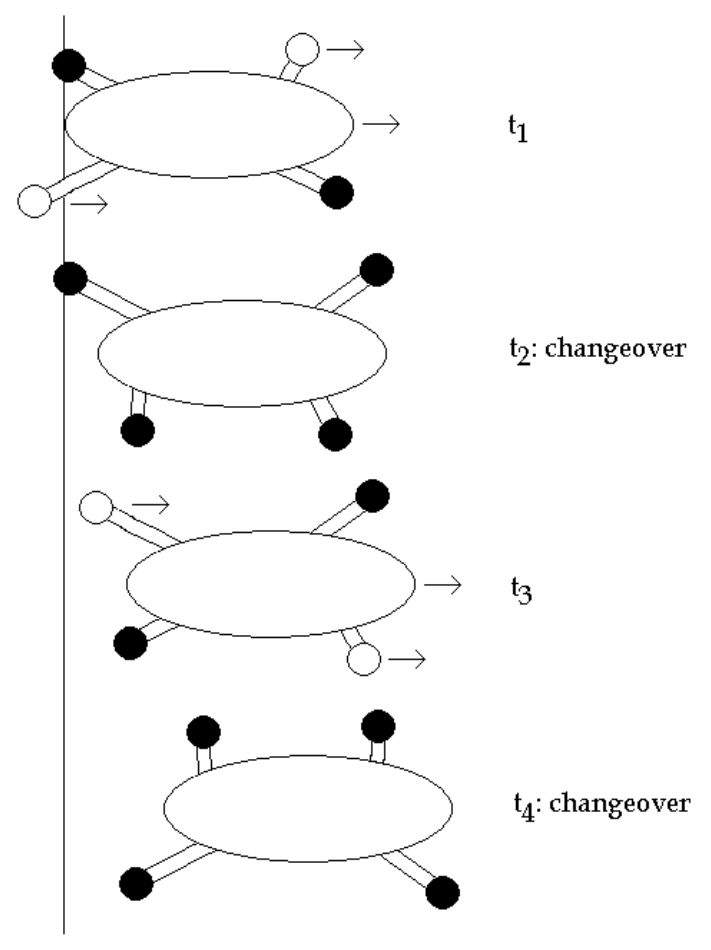

Fig. 11. A walker's footfalls at four successive times. A black circle represent a foot on the ground (adapted from Todd, 1985).

Fig. 12 shows the gait as a function of time. Each bar represents the time during which a foot is on the ground. This is called a gait diagram.

Another way normally used to represent a gait is shown in Fig. 13 where each wave represents the joint angles as a function of time for a set of corresponding joints, for example, all the four hips joints of a robot.

Quadrupeds can adopt a number of different gaits, depending upon their speed of locomotion and the terrain. Three of the more common gaits are: Walk, Trot, and Bound (Collins \& Richmond, 1994). These gaits are shown schematically in Fig. 14. In the Walk, which is a slow-speed gait, the limbs move a quarter period out of turn, in a figure-eight wave. In the Trot, which is a medium-speed gait, diagonal limbs, e.g., front right and rear left, move together and in phase, and pairs of diagonal limbs move half a period out of phase with one another. The Bound, which is a fast-speed gait, is similar to the trot, except that front and rear limbs, respectively, move together and in phase. 


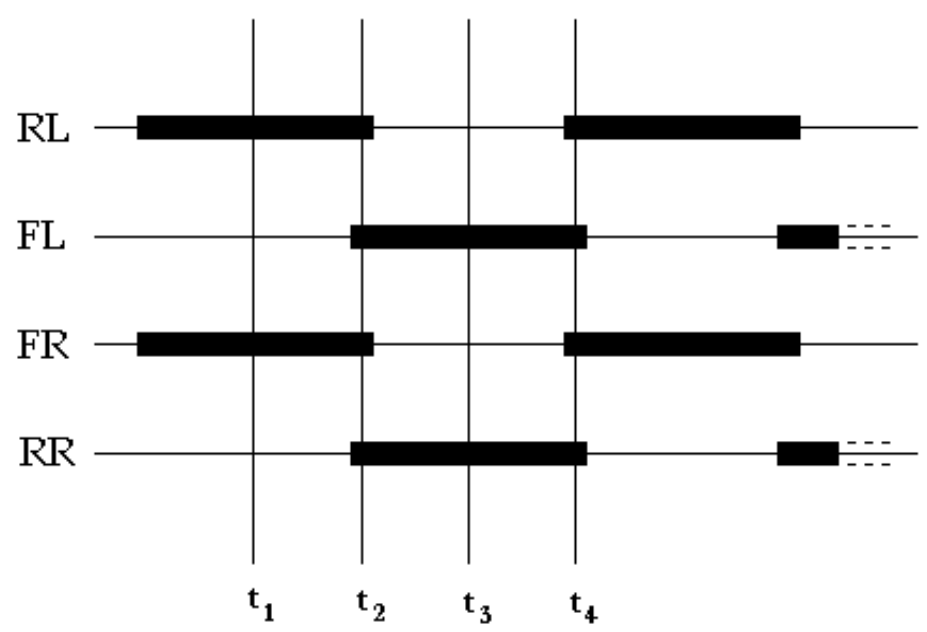

Fig. 12. Gait diagram (adapted from Todd, 1985).

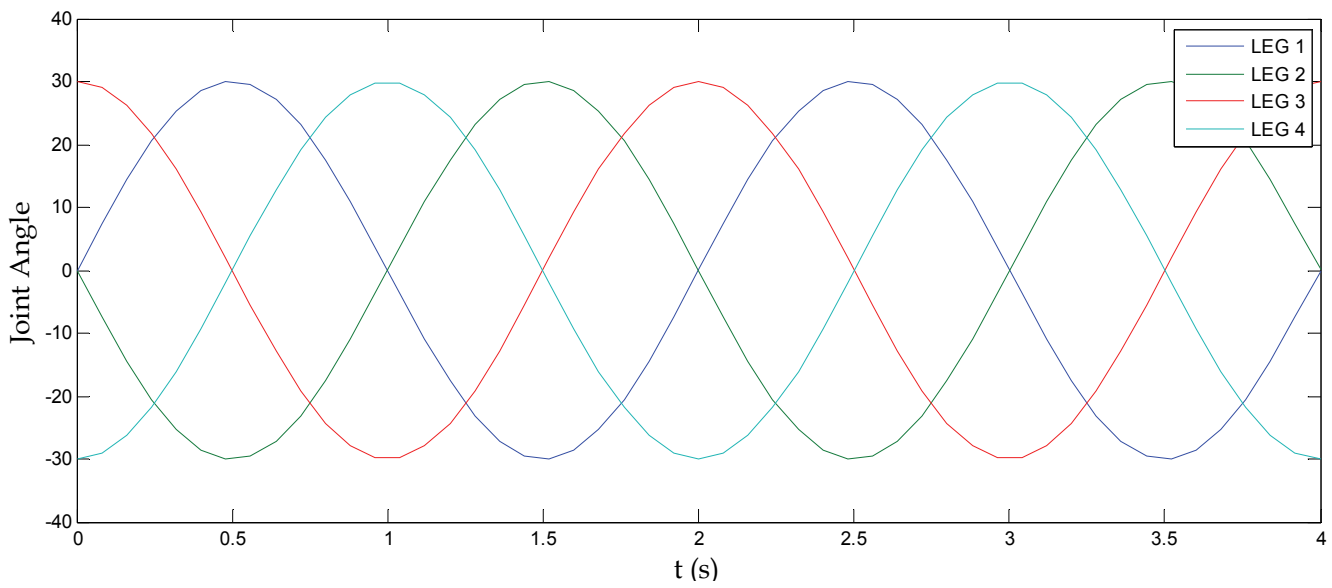

Fig. 13. Gait represented in the form of joint angles as functions of time.
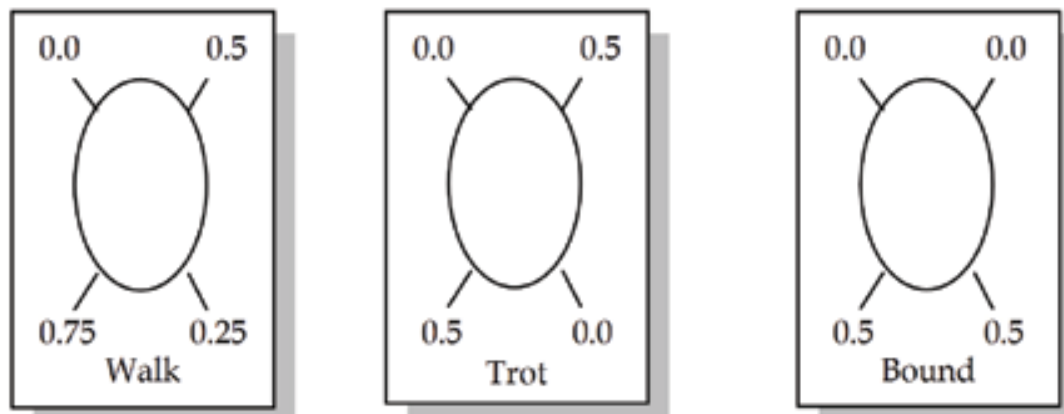

Fig. 14. Phase relations for three common quadruped gaits: Walk, Trot and Bound (adapted from Collins \& Richmond, 1994). 
In the present work, a CPG model was considered to be a locomotion control for only the particular gait mode Walk.

\section{CPG controller architecture}

\subsection{Matsuoka nonlinear neural oscillators}

The model of Matsuoka's nonlinear neural oscillator (Matsuoka, 1985, 1987; Liu et al, 2007) consists of two first-order coupled differential equations, one representing the membrane potential of the neuron and the other one, the degree of neuron fatigue, where the output of the neuron is nonlinear logic.

$$
\begin{gathered}
T_{r} \frac{d u_{i}}{d t}+u_{i}=\sum_{j=1}^{n} w_{i j} y_{j}+u_{0}-b v_{i}+\text { feed }_{i} \\
T_{a} \frac{d v_{i}}{d t}+v_{i}=y_{i} \\
y_{i}=\min \left\{u_{i}, \text { sat_value }\right\}
\end{gathered}
$$

Which equation represents:

1. Membrane potential;

2. Rate of neuron fatigue;

3. Non-linear output (saturation function).

The mathematical neuron model has two state variables and a few constant parameters. The first state variable is the inner variable $u_{i}$, corresponding to the membrane potential of the neuron. The second state variable is $v_{i}$, representing the degree of adaptation or self-inhibition in the $i$-th neuron, $b$ is the adaptation constant, and $y_{i}$ is the output of the $i$-th neuron. Subscripts $i, j$ denote the neuron number. $T r$ is the time constant specifying the rise time for a step input and the frequency of the output is proportional to $1 / T r$. Ta is the time constant specifying the adaptation time lag, $w_{i j}$ denotes the inhibitory synaptic connection weight from the $j$-th neuron to the $i$-th neuron, $w_{i j} \leq 0$ for $i \neq j$, and $w_{i j}=0$ for $i=j . \Sigma\left(w_{i j} y_{j}\right)$ represents the total input from neurons inside a neural network, $u_{0}$ is the constant drive input and feed is an input feedback sensor signal to the $i$-th neuron representing internal sensory information and interaction between the robot and its environment; it is mainly used in a closed-loop CPG model or else it is set to zero. Input feed $d_{i}$ may be any number of inputs applied to the $i$-th neuron model, which may be either proprioceptive signals or signals from other neurons. Time constants $\mathrm{Tr}$ and $\mathrm{Ta}$ change frequency and the input $u_{0}$ changes amplitude. Fig. 15 shows the general Matsuoka neuron model described by the equations presented above.

Assuming that the Matsuoka oscillator consists of two neurons with four state variables (Liu et al., 2007), two variables, $u_{i}$ and $u_{j}$, represent the inner state of each neuron and the other two state variables, $v_{i}$ and $v_{j}$, represent the degree of adaptation for each neuron. These neurons, linked reciprocally, alternately inhibit and excite each other to produce an oscillation as output. Such activity accounts for the alternating and mutually inhibition of the flexor and extensor muscles at joints during walking. The extensor and flexor are physiologically driven based on the output of each neuron. Self-inhibition is governed by $b \bullet v_{i}$ and $b \bullet v_{j}$ connections and mutual inhibition by $w_{i j} y_{j}$ and $w_{j i} y_{i}$ connections, as shown in Fig. 16. 


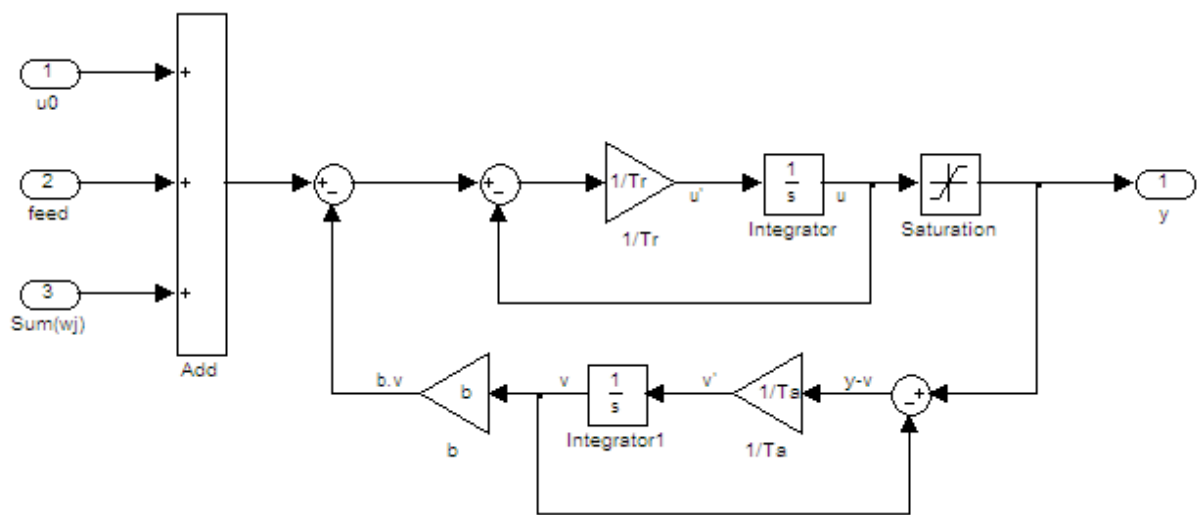

Fig. 15. Matsuoka neuron model (adapted from Liu et al, 2007).

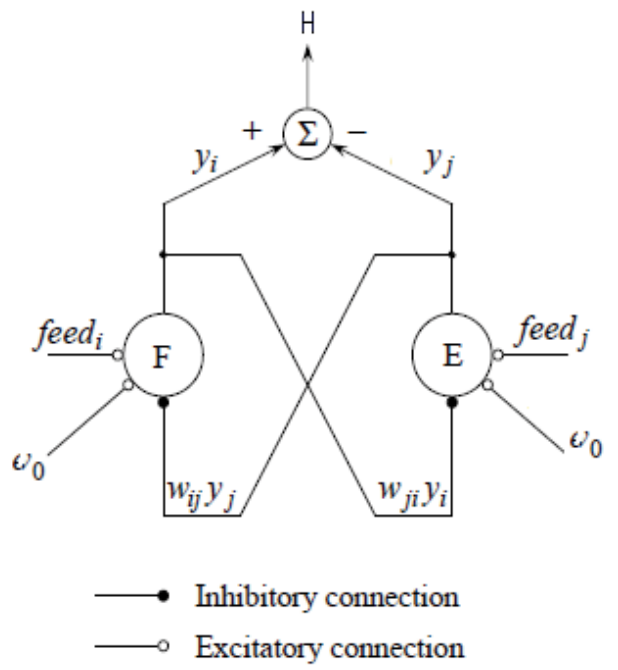

Fig. 16. Oscillator with an extensor (E) and a flexor (F) (adapted from Liu et al, 2007).

The oscillator output $H=y_{i}-y_{j}$, represents the algebraic sum of the output signal from each neuron. $H$ may be used as a motor command to drive a 1 DOF joint, where $H<0$ corresponds to extensor neuron activity, and $H>0$ corresponds to flexor neuron activity. In this work the output of oscillator $H$ is used to represent the desired angle of a joint.

\subsection{CPG network configuration}

The aim of the CPG network design was that one neural oscillator corresponded to the hip joint of each leg (Ishii, Masakado \& Ishii, 2004). The basic structures of CPG network and the output signals are presented in Fig. 17. Each neural oscillator consists of an extensor neuron and a flexor neuron. There is only one motor that can rotate in both directions, therefore, both neuron outputs are combined to drive the motor. The output of each neural oscillator determines the motion of hip joint of each leg whether the leg is in the support phase or in the swing phase. 
The numbers 1,2 correspond to the neurons of front left leg (LEG1), 3, 4 for the front right leg (LEG2), 5, 6 for the rear left leg (LEG3), and 7, 8 for the rear right leg (LEG4), respectively. The $w_{12}$ is the weight between the flexor neuron and the extensor neuron of LEG1 and $w_{13}$ is the weight between the flexor neuron of LEG1 and the flexor neuron of LEG3. If $w_{i j}$ is positive, the connection $i j$ excites the other neuron, if negative, it suppresses the other one, and if zero means no connection.

\subsection{CPG Simulink model}

The CPG network presented in Fig. 17 was implemented in Simulink ${ }^{\circledR}$ as shown in Fig. 18 below. The connectors P11, P12, P21, P22, P31, P32, P41 and P42 are signals to the Kamanbaré leg joints, where P11 contains the angle reference to joint 1 of LEG1, J11 as shown in Fig. 8, with the corresponding connection with P11 in Fig. 10. In the same way P12 represents the joint 2 of the LEG1 (J12), P21 represents the joint 1 of the LEG2 (J21) and so on.

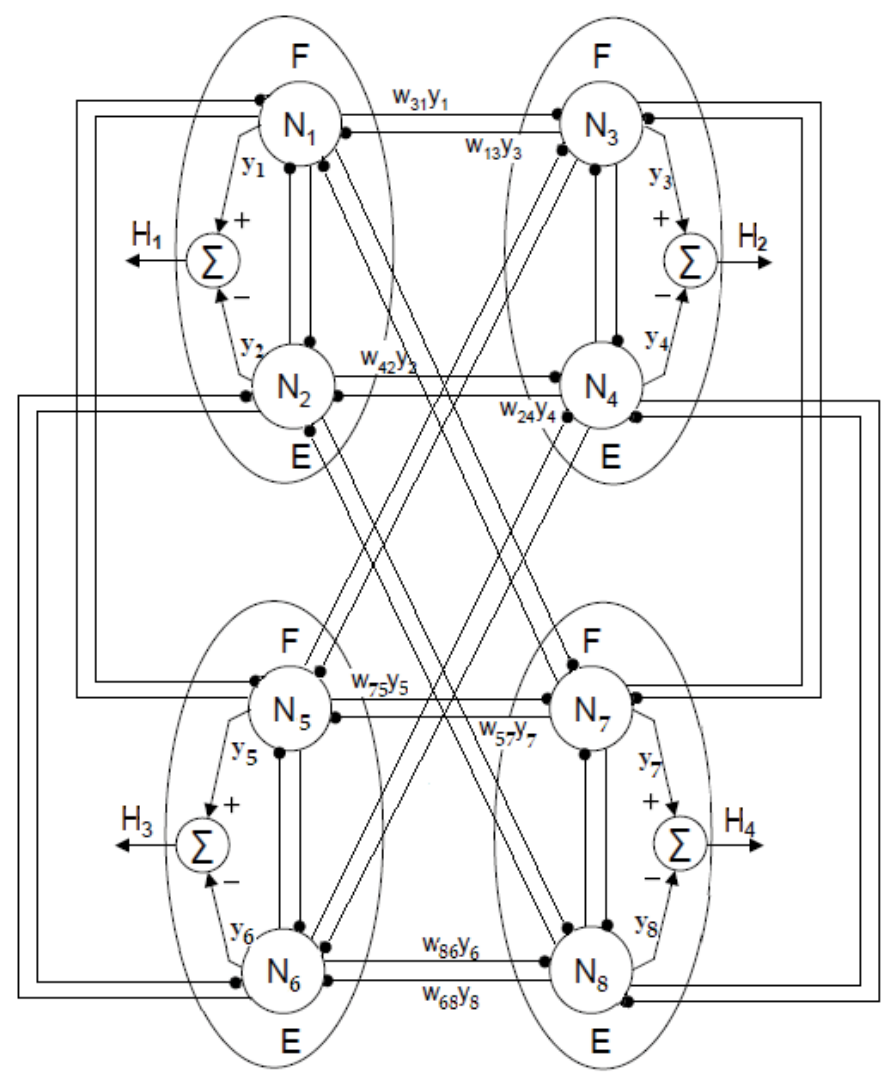

Fig. 17. The CPG network (adapted from Liu et al, 2007 and Ishii, Masakado \& Ishii, 2004). 


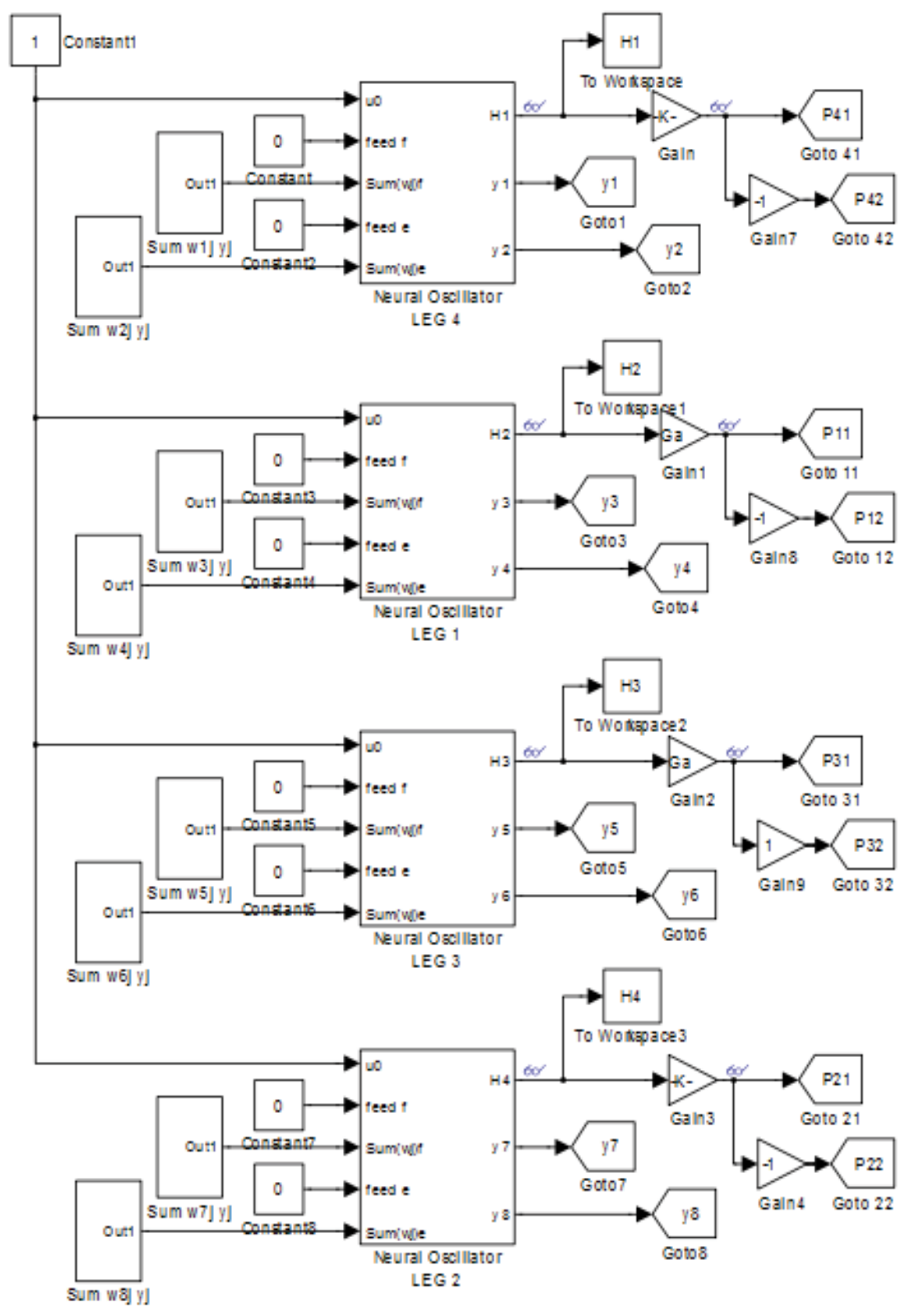

Fig. 18. The Simulink CPG network model.

The content of Neural Oscillators blocks of Fig. 18 is the corresponding implementation of the oscillator presented in Fig. 16 with two neurons: Flexor and Extensor, as shown in Fig. 19. 


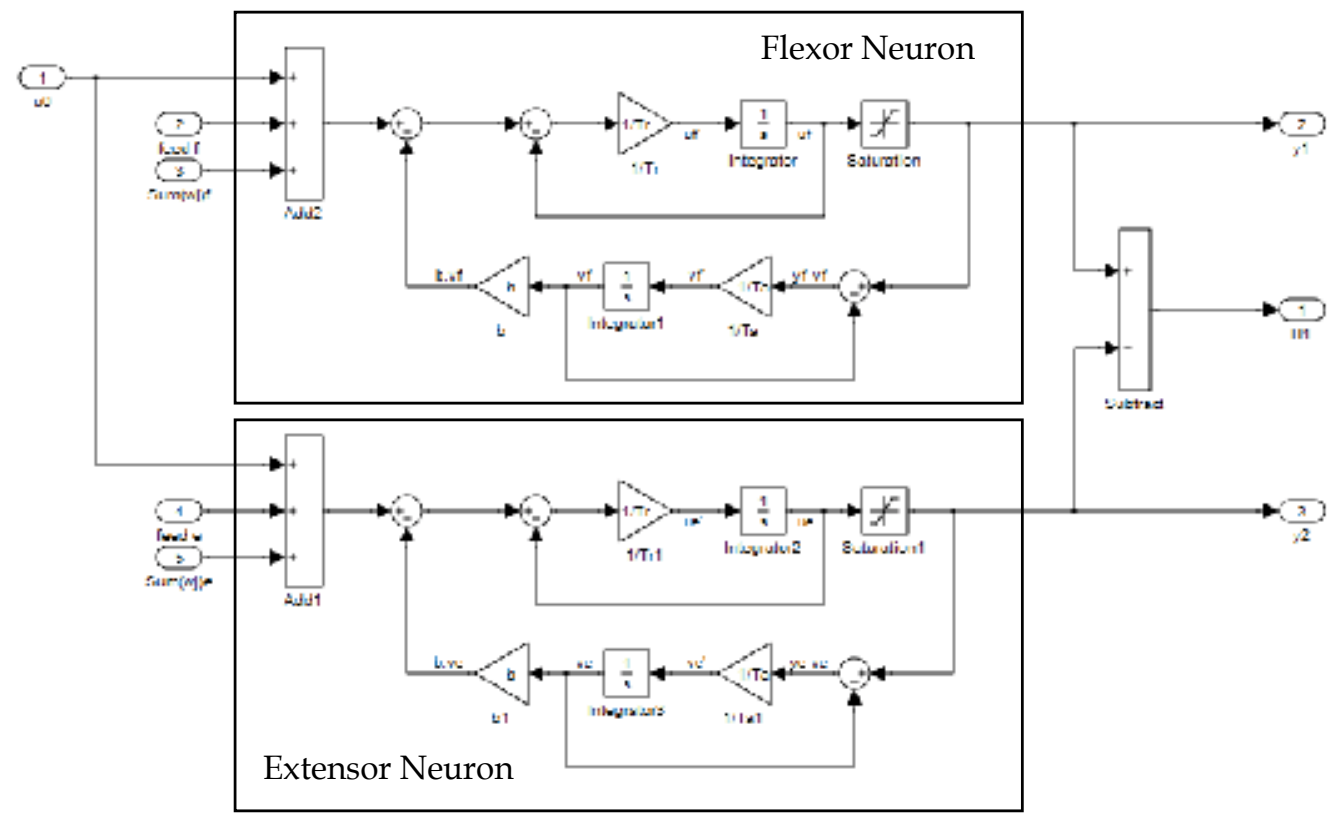

Fig. 19. Oscillator model.

\section{Simulation results}

\subsection{Overview}

Regarding the gait generation only a simple locomotion where the robot follows a straight path using the Walk quadruped mode gait was implemented. No waiting time will be introduced, besides those produced by the legs when searching for the support points, therefore this gait will be at the highest speed allowed by the surface.

The simulations combined the mechanical models of the machine presented in Figs 8-10 with the controller presented in Figs 18-19. The mechanical model receives the inputs from the controller, performs the simulated motion and feeds back the sensory information to the controller. In this way, the tuning of the CPG parameters can be performed before the implementation on the real Kamambaré.

\subsection{CPG initial parameter setup}

Once the parameter tuning of a CPG network is a difficult process as there is no established procedure (Son et al., 2006; Hattori et al., 2010; Costa et al., 2010), the initial parameters as chosen spending a low effort of time.

In this way, the described CPG was simulated considering the parameters: $\operatorname{Tr}=0.05, \mathrm{Ta}=$ $0.5, b=2.0, u_{0}=1.0$, feed $_{i}=0.0$. The weighting matrix $w_{i j}(i=1,2, \ldots, 8$ and $j=1,2, \ldots, 8)$ was chosen as 


$$
w_{i j}=\left[\begin{array}{rrrrrrrr}
0.0 & -2.0 & -1.0 & 0.0 & -1.0 & 0.0 & -1.0 & 0.0 \\
-2.0 & 0.0 & 0.0 & -1.0 & 0.0 & -1.0 & 0.0 & -1.0 \\
-1.0 & 0.0 & 0.0 & -2.0 & -1.0 & 0.0 & -1.0 & 0.0 \\
0.0 & -1.0 & -2.0 & 0.0 & 0.0 & -1.0 & 0.0 & -1.0 \\
-1.0 & 0.0 & -1.0 & 0.0 & 0.0 & -2.0 & -1.0 & 0.0 \\
0.0 & -1.0 & 0.0 & -1.0 & -2.0 & 0.0 & 0.0 & -1.0 \\
-1.0 & 0.0 & -1.0 & 0.0 & -1.0 & 0.0 & 0.0 & -2.0 \\
0.0 & -1.0 & 0.0 & -1.0 & 0.0 & -1.0 & -2.0 & 0.0
\end{array}\right]
$$

The first simulation result obtained using the parameters above is shown in Fig. 20.

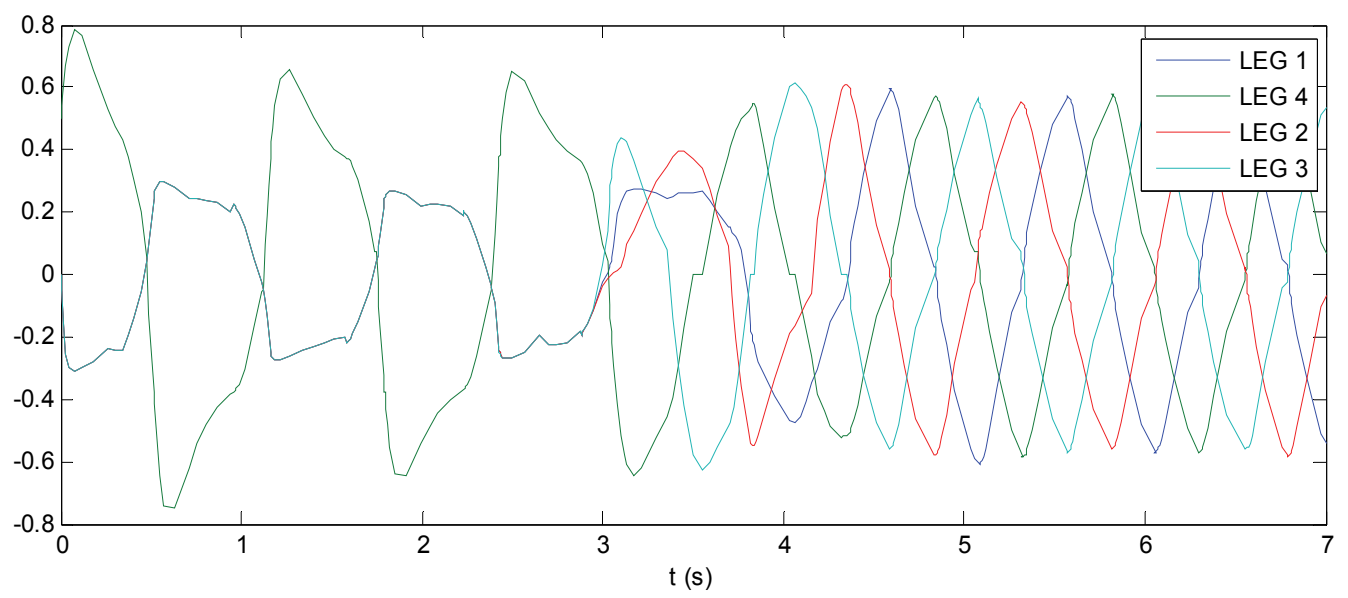

Fig. 20. Output response in identical and mutual inhibitory coupling between four Matsuoka oscillators.

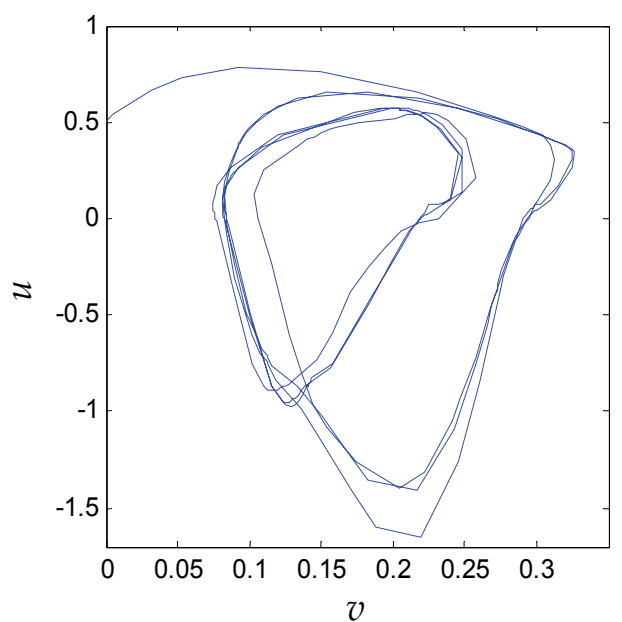

Fig. 21. Phase plot of the time evolution of the linear oscillator. 
Analysing Fig. 20, certain initial transient of the oscillators can be clearly noted in the first 4 seconds of simulation. In order to better verify this behaviour, a phase plot of the state variables $u$ and $v$ is presented in Fig. 21.

The initial transient is obviously undesirable, since it will produce a non-acceptable robot behavior, e.g movements of the legs joints. In this way, tuning of the CPG parameters turns out to be mandatory.

\subsection{Parameter tuning}

The parameter tuning of a CPG network is a difficult process since it implies in a task of changing a substantial number of interrelated parameters.

In this work a trial-and-error procedure was adopted in order to find a set of the parameters that provided the required control signals.

Thus, after several iterations and tests for analyzing the gait wave form, it was concluded that the initial transient obtained was directly correlated to the values used as initial conditions in the integrator blocks of the neural oscillator's models.

Once these initial conditions were adjusted, the complete neural system showed a stable behaviour. The following set of parameters has then been adopted: $\operatorname{Tr}=0.4, T a=0.5, b=4.0, u_{0}$ $=1.0$, feed $_{i}=0.0$ and the weighting matrix $w_{i j}(i=1,2, \ldots, 8$ and $j=1,2, \ldots, 8)$ chosen as

$$
w_{i j}=\left[\begin{array}{rrrrrrrr}
0.0 & -1.8 & -1.2 & 0.0 & -1.2 & 0.0 & -1.2 & 0.0 \\
-1.8 & 0.0 & 0.0 & -1.2 & 0.0 & -1.2 & 0.0 & -1.2 \\
-1.2 & 0.0 & 0.0 & -1.8 & -1.2 & 0.0 & -1.2 & 0.0 \\
0.0 & -1.2 & -1.8 & 0.0 & 0.0 & -1.2 & 0.0 & -1.2 \\
-1.2 & 0.0 & -1.2 & 0.0 & 0.0 & -1.8 & -1.2 & 0.0 \\
0.0 & -1.2 & 0.0 & -1.2 & -1.8 & 0.0 & 0.0 & -1.2 \\
-1.2 & 0.0 & -1.2 & 0.0 & -1.2 & 0.0 & 0.0 & -1.8 \\
0.0 & -1.2 & 0.0 & -1.2 & 0.0 & -1.2 & -1.8 & 0.0
\end{array}\right]
$$

The final simulation result obtained is shown in Fig. 22, where a significant reduction of the initial transient time to about $0.8 \mathrm{sec}$ can be observed. This improved behaviour can also be checked in Fig. 23 by the analysis of the phase plot of the states variables $u$ and $v$.

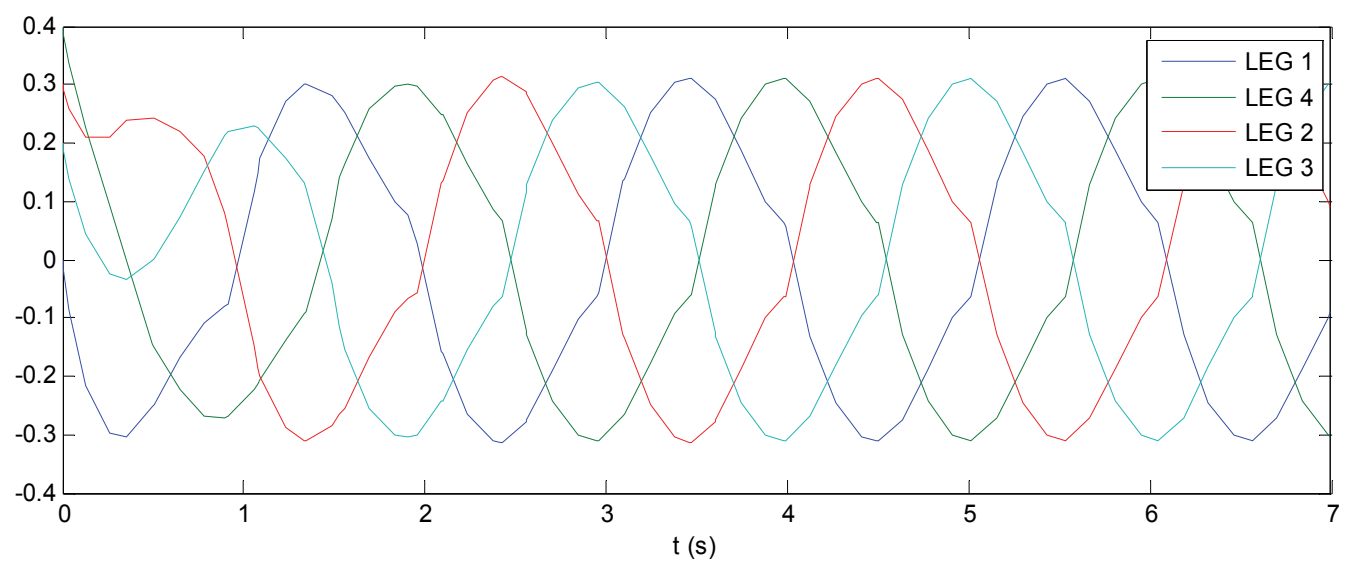

Fig. 22. Output response using the tuned set of parameters. 


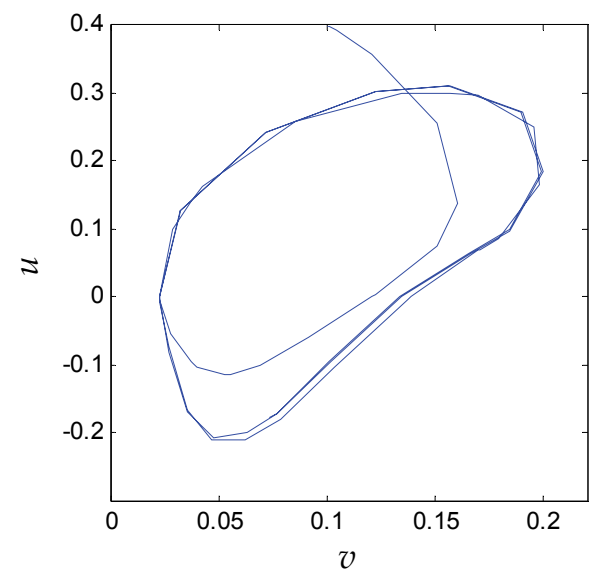

Fig. 23. Phase plot of the time evolution of the linear oscillator.

\section{Conclusions}

The main purpose of this work was to design and implement a CPG based control consisting of a network of mutually inhibited and coupled Matsuoka oscillators and to demonstrate that the CPG model can be useful for the online generation of gaits in the Kamanbare robot. The implementation of the CPG controller was proven to be feasible.

Nevertheless the parameters tuning is a quite complex task due to the large number of interrelated variables. In a first approach an optimization method was used to tune one half of the entire model but results obtained were not useful or appropriate to be applied to the full system implementation. Thus a trial-and-error procedure was adopted in order to find a good set of parameters to generate a gait that produced a smooth movement of the robot leg joints.

Future work will address first on the improvement of the CPG and the Kamanbare models including the joint sensor feedback connection to provide a closed loop control. An optimization method will also be developed in order to obtain a better setting for the CPG parameters.

\section{References}

Aoi, S. \& Tsuchiya, K. (2007). Gait transition from quadrupedal to bipedal locomotion of an oscillator-driven biped robot. In: de Pina Filho, ed. Humanoid Robots, New Developments. ISBN 978-3-902613-02-8, I-Tech, Vienna, Austria.

Bernardi, R. de \& Da Cruz, J. J. (2007). Kamanbaré: A Tree-climbing Biomimetic Robotic Platform for Environmental Research. International Conference on Informatics in Control, Automation \& Robotics. Angers, France.

Brown, T. G. (1914). On the nature of the fundamental activity of the nervous centres; together with an analysis of the conditioning of rhythmic activity in progression, and a theory of the evolution of function in the nervous system. The Journal of Physiology. Volume 48, pp. 18-46.

Collins, J. J. \& Stewart, I. N. (1993). Coupled nonlinear oscillators and the symmetries of animal gaits. Journal of Nonlinear Science. Volume 3, nº 1, pp. 349-392. 
Collins, J. J. \& Richmond, S. A. (1994). Hard-wired central pattern generators for quadrupedal locomotion. Journal of Biological Cybernetics. Volume 71, pp. 375-385.

Costa, L.; Rocha, A. M. A. C.; Santos, C. P. \& Oliveira, M. (2010). A Global Optimization Stochastic Algorithm for Head Motion Stabilization during Quadruped Robot Locomotion. Proceedings of the 2nd International Conference on Engineering Optimization.

Crespi, A.; Lachat, D.; Pasquier, A. \& Ijspeert, A. J. (2008). Controlling swimming and crawling in a fish robot using a central pattern generator. Autonomous Robot. Volume 25, pp. 3-13.

Delcomyn, F. (1980). Neural basis for rhythmic behavior in animals. Science.Volume 210, $\mathrm{n}^{\mathrm{o}}$ 4469, pp. 492-498.

Diedam, H.; Dimitrov, D.; Wieber, P. B.; Mombaur, K. \& Diehl, M. (2008). Online Walking Gait Generation with Adaptive Foot Positioning through Linear Model Predictive Control. IEEE/RSJ International Conference on Intelligent Robots and Systems. Nice, France, pp. 1121-1126.

Duysens, J. \& Van de Crommert, H. W. A. A. (1998). Neural control of locomotion. Part 1: The central pattern from cats to humans. Gait and Posture. Volume 7, pp. 131-141.

Forner-Cordero, A.; Koopman, H. J. F. M. \& Helm, F. C. T. (2006) Describing gait as a sequence of states. Journal of Biomechanics. Volume 39, n 5, pp. 948-957.

Fukuoka, Y.; Kimura, H. \& Cohen, A. H. (2003). Adaptive Dynamic Walking of a Quadruped Robot on Irregular Terrain Based on Biological Concepts. The International Journal of Robotics Research. Volume 22, n 3-4, pp. 187-202.

Grillner, S. (1985). Neurobiological bases of rhythmic motor acts in vertebrates. Science. Volume 228, nº 4696, pp. 143-149.

Hattori, Y.; Suzuki, M.; Soh, Z.; Kobayashi, Y. \& Tsuji, T. (2010). A Novel Tuning Method for Neural Oscillators with a Ladder-Like Structure Based on Oscillation Analysis. Artificial Neural Networks - ICANN 2010. Lecture Notes in Computer Science. Volume 6352, pp. 401-410.

Ijspeert, A. J. (2008). Central pattern generators for locomotion control in animals and robots: A review. Neural Networks. Volume 21, pp. 642-653.

Ijspeert, A. J.; Crespi, A. \& Ryczko, D. (2007). From swimming to walking with a salamander robot driven by a spinal cord model. Science. Volume 315(5817), pp. 1416-1420.

Inagaki S.; Yuasa H. \& Arai T. (2003). CPG model for autonomous decentralized multilegged robot system-generation and transition of oscillation patterns and dynamics of oscillators. Robotics and Autonomous Systems Volume 44(3-4), pp. 171-179.

Inagaki S.; Yuasa H. \& Suzuki T. (2006). Wave CPG model for autonomous decentralized multi-legged robot: Gait generation and walking speed control. Robotics and Autonomous Systems Volume 54(2), pp. 118-126.

Ishii, T.; Masakado, S. \& Ishii, K. (2004). Locomotion of a quadruped robot using CPG. Proceedings of the IEEE International Joint Conference on Neural Networks. Volume 4, pp. 3179-3184, Budapest.

Liu, G. L.; Habib, M. K.; Watanabe, K. \& Izumi, K. (2007). The Design of Central Pattern Generators Based on the Matsuoka Oscillator to Generate Rhythmic Human-Like Movement for Biped Robots. Journal of Advanced Computational Intelligence and Intelligent Informatics. Volume 11, $\mathrm{n}^{\circ}$ 8, pp. 946-955. 
Matsuoka, K. (1985). Sustained oscillations generated by mutually inhibiting neurons with adaptation. Journal of Biological Cybernetics. Volume 52, pp. 367-376.

Matsuoka, K. (1987). Mechanisms of frequency and pattern control in the neural rhythm generators. Journal of Biological Cybernetics, Volume 56, pp. 345-353.

Morimoto, J.; Endo, G. \& Nakanishi, J. (2008). A biologically inspired biped locomotion strategy for humanoid robots: Modulation of sinusoidal patterns by a coupled oscillator model. IEEE Transactions on Robotics Volume 24(1), pp. 185-191.

Pack, D. J. \& Kang, H. (1999), Free gait control for a quadruped walking robot. Laboratory Robotics and Automation. Volume 11, pp. 71-81.

Pal, P. K. \& Jayarajan, K. (1991). Generation of Free Gait - A Graph Search Approach. IEEE Transactions on Robotics and Automation. Volume 7, n 3, pp. 299-305.

Pascoal, A.; Oliveira, P.; Silvestre, C.; Bjerrum, A.; Ishloy, A.; Pignon, J. P.; Ayela, G. \& Petzelt, C. (1997). MARIUS: An Autonomous Underwater Vehicle for Coastal Oceanography. IEEE Robotics \& Automation Magazine. pp. 46-57.

Son, Y.; Kamano, T.; Yasuno, T.; Suzuki, T. \& Harada, H. (2006). Generation of adaptive gait patterns for quadruped robot with CPG network including motor dynamic model. Electrical Engineering in Japan. Volume 155, Issue 1, pp. 35-43.

Taga, G. (1994). Emergence of bipedal locomotion through entrainment among the neuromusculo-skeletal system and the environment. Physica D: Nonlinear Phenomena. Volume 75, Issue 1-3, pp. 190-208.

Todd, D. J. (1985). Walking machines: an introduction to legged robots. Published by Kogan Page Ltd.

Wettergreen, D. \& Thorpe, C. (1992). Gait Generation for Legged Robots, Proceedings of the IEEE International Conference on Intelligent Robots and Systems. Volume 2, pp. 14131420. 



\section{Edited by Anne George}

The interaction between cells, tissues and biomaterial surfaces are the highlights of the book "Biomimetic Based Applications". In this regard the effect of nanostructures and nanotopographies and their effect on the development of a new generation of biomaterials including advanced multifunctional scaffolds for tissue engineering are discussed. The 2 volumes contain articles that cover a wide spectrum of subject matter such as different aspects of the development of scaffolds and coatings with enhanced performance and bioactivity, including investigations of material surface-

cell interactions. 